Key Words:

Environmental Dosimetry

Reference Person

Retention Permanent

\title{
SITE SPECIFIC REFERENCE PERSON PARAMETERS AND DERIVED CONCENTRATION STANDARDS FOR THE SAVANNAH RIVER SITE
}

\author{
Daniel K. Stone
}

G. Timothy Jannik

REPORT DATE: MARCH 2013

Savannah River National Laboratory

Savannah River Nuclear Solutions

Aiken, SC 29808

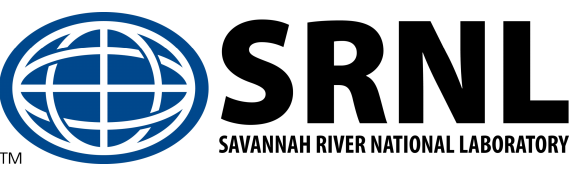




\section{DISCLAIMER}

This work was prepared under an agreement with and funded by the U.S. Government. Neither the U.S. Government or its employees, nor any of its contractors, subcontractors or their employees, makes any express or implied:

1. warranty or assumes any legal liability for the accuracy, completeness, or for the use or results of such use of any information, product, or process disclosed; or

2. representation that such use or results of such use would not infringe privately owned rights; or

3. endorsement or recommendation of any specifically identified commercial product, process, or service.

Any views and opinions of authors expressed in this work do not necessarily state or reflect those of the United States Government, or its contractors, or subcontractors.

Printed in the United States of America

Prepared for

U.S. Department of Energy 
Key Words:

Environmental Dosimetry

Reference Person

Retention Permanent

\title{
SITE SPECIFIC REFERENCE PERSON PARAMETERS AND DERIVED CONCENTRATION STANDARDS FOR THE SAVANNAH RIVER SITE
}

\author{
Daniel K. Stone
}

G. Timothy Jannik

REPORT DATE: MARCH 2013

Savannah River National Laboratory

Savannah River Nuclear Solutions

Aiken, SC 29808 


\section{REVIEWS AND APPROVALS}

Daniel K. Stone, Oregon State University

Date

G. Timothy Jannik, Environmental Sciences

Date

Eduardo B. Farfan, Peer Review, Environmental Sciences

Date

John J. Mayer, II, Manager, Environmental Sciences

Date

Alice M. Murray, Manager, Environmental Sciences \& Biotechnology

Date

- iii - 


\section{TABLE OF CONTENTS}

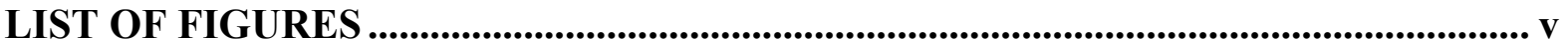

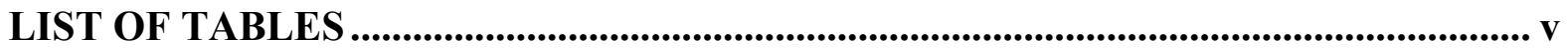

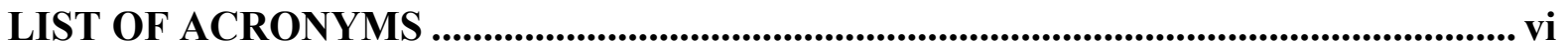

1.0 EXECUTIVE SUMMARY ......................................................................................................... 1

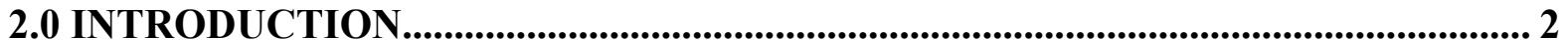

3.0 Dose and Dose Coefficients ............................................................................................ 3

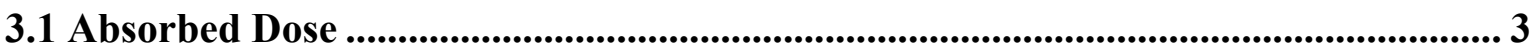

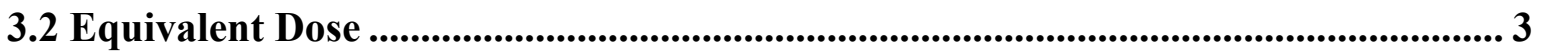

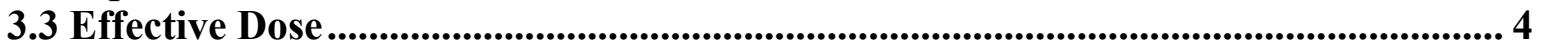

3.3.1 Committed Effective Dose ........................................................................................ 4

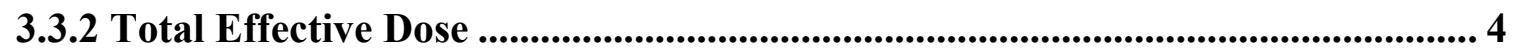

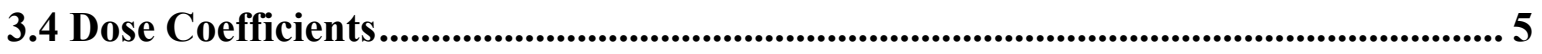

3.4.1 Internal Dose Coefficients .............................................................................. 5

3.4.2 External Dose Coefficients.............................................................................. 5

4.0 Maximally Exposed Individual and Reference Person................................................... 6

4.1 Maximally Exposed Individual..................................................................................... 7

4.2 Reference person...................................................................................................................... 7

4.3 Typical Person................................................................................................9

5.0 Development of Reference Person Parameters ............................................................... 10

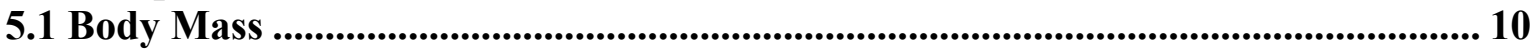

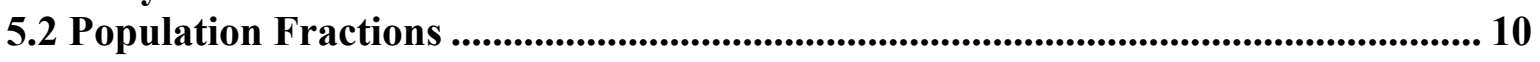

5.3 Exposure Pathways.......................................................................................... 11

5.3.1 Ingestion ......................................................................................................... 11

5.3.2 Inhalation ............................................................................................................................ 15

5.3.3 Immersion in Air and Submersion in Water ................................................... 16

5.3.4 Ground Shine............................................................................................ 16

6.0 Derived Concentration Standards (DCS) ........................................................................... 17

6.1 Calculation of Intake Rates and Reference Dose Coefficients ............................... 17

6.1.1 SRS-Specific DCS Results .............................................................................................. 18

7.0 Comparison \& Results......................................................................................... 19

7.1 Reference Intake Comparisons ........................................................................ 19

7.2 Comparison of Internal Dose Calculations ................................................................. 20

7.3 Comparison of External Dose Calculations .................................................................... 21

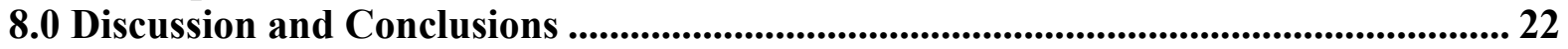

8.1 Use of Reference Person Parameters and Dose Coefficients ................................... 22

8.2 Use of SRS-Specific Derived Concentration Standards ........................................... 22

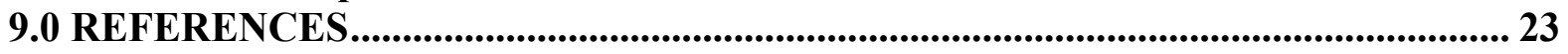

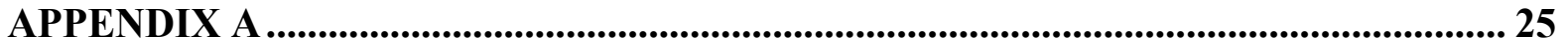

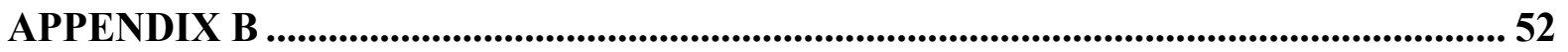

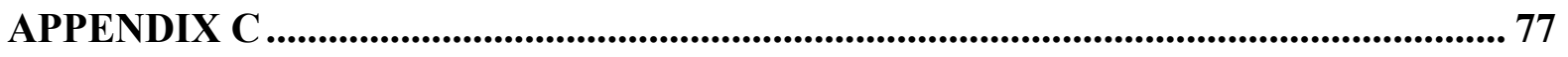

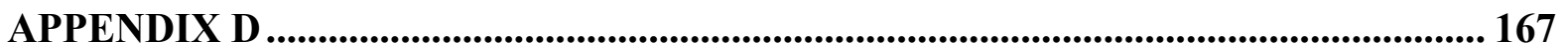




\section{LIST OF FIGURES}

Figure 1. Exposure Pathways to Humans from Atmospheric and Aqueous Releases...... 6

\section{LIST OF TABLES}

Table 1. Radiation Weighting Factors (ICRP 60) ................................................................. 3

Table 2. Tissue Weighting Factors (ICRP 60).......................................................................... 4

Table 3. ICRP 89 Reference Values for Body Mass ...................................................... 10

Table 4. U.S. and SC/GA 2010 population fractions ................................................... 11

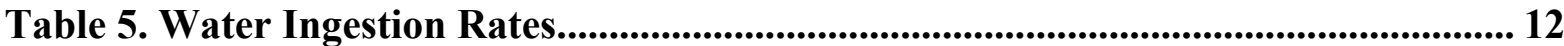

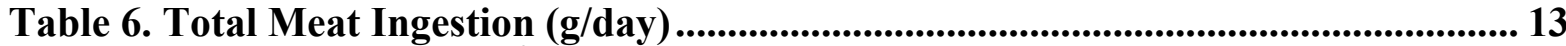

Table 7. Dairy Ingestion ( $\mathrm{cm}^{3} /$ day) ....................................................................................... 13

Table 8. Consumption Rates for Freshwater Fish and Saltwater Invertebrates ............ 14

Table 9. Total Produce Ingestion Rates (g/day) ........................................................... 15

Table 10. Inhalation Rates ........................................................................................ 16

Table 11. Comparison of Reference and Typical Person Intakes with Current Intakes 19 Table 12. Comparison of Representative and Typical Doses with Current Dose

Calculations for Unit Concentrations of Tritium Oxide ............................................ 20

Table 13. External Dose Calculation Comparisons ........................................................ 21 


\section{LIST OF ACRONYMS}

$\begin{array}{ll}\text { ASER } & \begin{array}{l}\text { Annual Site Environmental Report } \\ \text { Committed Effective Dose }\end{array} \\ \text { CED } & \text { Derived Concentration Standard } \\ \text { DCS } & \text { Department of Energy } \\ \text { DOE } & \text { Effective Dose } \\ \text { ED } & \text { Environmental Protection Agency } \\ \text { EPA } & \text { Federal Guidance Report } \\ \text { FGR } & \text { International Commission on Radiation Protection } \\ \text { ICRP } & \text { Maximully Exposed Individual } \\ \text { MEI } & \text { Nuclear Regulatory Commission } \\ \text { NRC } & \text { Savannah River Site } \\ \text { SRS } & \text { Savannah River National Laboratory } \\ \text { SRNL } & \text { Savannah River Nuclear Solutions } \\ \text { SRNS } & \text { Total Effective Dose } \\ \text { TED } & \end{array}$




\subsection{EXECUTIVE SUMMARY}

The purpose of this report is twofold. The first is to develop a set of behavioral parameters for a reference person specific for the Savannah River Site (SRS) such that the parameters can be used to determine dose to members of the public in compliance with Department of Energy (DOE) Order 458.1 "Radiation Protection of the Public and the Environment." A reference person is a hypothetical, gender and age aggregation of human physical and physiological characteristics arrived at by international consensus for the purpose of standardizing radiation dose calculations. DOE O 458.1 states that compliance with the annual dose limit of $100 \mathrm{mrem}(1 \mathrm{mSv})$ to a member of the public may be demonstrated by calculating the dose to the maximally exposed individual (MEI) or to a representative person. Historically, for dose compliance, SRS has used the MEI concept, which uses adult dose coefficients and adult male usage parameters. Beginning with the 2012 annual site environmental report, SRS will be using the representative person concept for dose compliance. The dose to a representative person will be based on 1) the SRS-specific reference person usage parameters at the $95^{\text {th }}$ percentile of appropriate national or regional data, which are documented in this report, 2) the reference person (gender and age averaged) ingestion and inhalation dose coefficients provided in DOE Derived Concentration Technical Standard (DOE-STD-1196-2011), and 3) the external dose coefficients provided in the DC_PAK3 toolbox.

The second purpose of this report is to develop SRS-specific derived concentration standards (DCSs) for all applicable food ingestion pathways, ground shine, and water submersion. The DCS is the concentration of a particular radionuclide in water, in air, or on the ground that results in a member of the public receiving $100 \mathrm{mrem}(1 \mathrm{mSv})$ effective dose following continuous exposure for one year. In DOE-STD-1196-2011, DCSs were developed for the ingestion of water, inhalation of air and submersion in air pathways, only. These DCSs are required by DOE O 458.1 to be used at all DOE sites in the design and conduct of radiological environmental protection programs. In this report, DCSs for the following additional pathways were considered and documented: ingestion of meat, dairy, grains, produce (fruits and vegetables), seafood, submersion in water and ground shine. These additional DCSs were developed using the same methods as in DOE-STD-1196-2011 and will be used at SRS, where appropriate, as screening and reference values. 


\subsection{INTRODUCTION}

The U.S. Department of Energy (DOE) Order 458.1 (DOE 2011) states that compliance with the annual dose limit of 100 mrem $(1 \mathrm{mSv})$ to a member of the public may be demonstrated by calculating the dose to the maximally exposed individual (MEI) or to a representative person. Historically, for dose compliance, the Savannah River Site (SRS) has used the MEI concept, which uses adult dose coefficients and adult male usage parameters. Beginning with the 2012 annual site environmental report, SRS will be using the representative person concept for dose compliance.

The dose to a representative person will be based on 1) the SRS-specific reference person usage parameters at the $95^{\text {th }}$ percentile of appropriate national or regional data, which are documented in this report, 2) the reference person (gender and age averaged) ingestion and inhalation dose coefficients provided in DOE Derived Concentration Technical Standard (DOE-STD-1196-2011), and 3) the external dose coefficients provided in the DC_PAK3 toolbox, which can be accessed at http://www.epa.gov/rpdweb00/federal/techdocs.html. The reference person is weighted based on sex and age and this weighting is based on the six age groups documented in International Commission on Radiological Protection (ICRP) Report 89 (ICRP 2002): Infant (0 years), 1 year, 5 years, 10 years, 15 years, and Adult. The various age- and gender-specific intake rates were proportioned to correspond with these respective age groupings.

Also developed in this report are SRS-specific derived concentration standards (DCSs) for all applicable food ingestion pathways, ground shine, and water submersion. The DCS is the concentration of a particular radionuclide in water, in air, or on the ground that results in a member of the public receiving 100 mrem $(1 \mathrm{mSv})$ effective dose following continuous exposure for one year. In DOE-STD-1196-2011, DCSs were developed for the ingestion of water, inhalation of air, and submersion in air pathways, only. These DCSs are required by DOE O 458.1 to be used at all DOE sites in the design and conduct of radiological environmental protection programs. In this report, DCSs for the following additional pathways were considered and documented: ingestion of meat, dairy, grains, produce (fruits and vegetables), freshwater fish, and saltwater invertebrates, submersion in water, and ground shine. These additional DCSs were developed using the same methods as in DOESTD-1196-2011 and will be used at SRS, where appropriate, as screening and reference values. 


\subsection{DOSE AND DOSE COEFFICIENTS}

Dose is an important quantity in studying the impacts of radiation on humans and the environment. There are three dose quantities, defined in ICRP Publication 60 (ICRP 1991), used in radiation protection.

\subsection{ABSORBED DOSE}

Absorbed dose, D, is the total energy absorbed in to a mass of any material, and is a measurable quantity. It is defined in ICRP (1991) as the quotient of the mean energy (d $\varepsilon$ ) deposited into a volume of mass $(\mathrm{dm})$, seen in eqn 1 . This quantity does not account for the type of radiation being absorbed or material type absorbing the radiation. The standard unit for absorbed dose is the gray (Gy), which is equivalent to 1 joule per kilogram $\left(\mathrm{J} \mathrm{kg}^{-1}\right)$. The conventional unit is the rad, which is equivalent to $0.01 \mathrm{~Gy}$.

$$
D=\frac{\mathrm{d} \varepsilon}{d m}
$$

\subsection{EQUIVALENT DOSE}

Equivalent dose, $\mathrm{H}$, is a radiation weighted dose. Different types of radiation have very different biological impacts. Alpha radiation can be more damaging than gamma rays if the alpha emitting radionuclide is distributed within the biological material. To adjust the dose to account for these differences, the absorbed dose $\mathrm{D}$ is multiplied by a radiation weighting factor, $\mathrm{w}_{\mathrm{R}}$. The total equivalent dose is the sum of the dose multiplied by the radiation factor for each radionuclide, seen in eqn 2.

$$
H=\sum_{i=0}^{n} D * w_{r}
$$

Due to the weighting factor, equivalent dose is not a measurable quantity. The units for equivalent dose are the Sievert (Sv) and the Roentgen Equivalent Man (rem). One Sv is equivalent to $100 \mathrm{rem}$. Table 1 presents the radiation weighting factors, $\mathrm{w}_{\mathrm{r}}$, documented in ICRP (1991).

Table 1. Radiation Weighting Factors (ICRP 60)

\begin{tabular}{|l|c|}
\hline Type of Radiation & $\mathrm{W}_{\mathrm{r}}$ \\
\hline Photons & 1 \\
\hline Electrons & 1 \\
\hline Neutrons & \\
\hline$-\quad$ Less than $10 \mathrm{keV}$ & 5 \\
\hline-10 to $100 \mathrm{keV}$ & 10 \\
\hline$-\quad 100 \mathrm{keV}$ to $2 \mathrm{MeV}$ & 20 \\
\hline$-\quad 2 \mathrm{MeV}$ to $20 \mathrm{MeV}$ & 10 \\
\hline$-\quad$ Greater than $20 \mathrm{MeV}$ & 5 \\
\hline Alpha Particles and Fission Fragments & 20 \\
\hline
\end{tabular}




\subsection{EFFECTIVE DOSE}

Effective dose, ED, is a tissue weighted dose. While equivalent dose is determined by the type of radiation, the effective dose is determined by what organ or tissue is receiving dose. Different types of tissue respond differently to radiation. Organs and tissues with fast cell cycles receive more damage that those of with slow cell cycles receiving the same effective dose. The tissue weighting factors are based on risk of total detriment, which includes probability of fatal and non-fatal cancer, probability of hereditary effects, and probability of length of life loss. The effective dose is calculated by multiplying the equivalent dose by a tissue weighting factor. This calculation is performed for each tissue and summed to calculate the total effective dose seen in eqn 3 .

$$
E=\sum H * w_{t}
$$

This dose quantity is also unable to be measured. The units for the effective dose are the $\mathrm{Sv}$ and the rem, the same as the equivalent dose. The tissue weighting factors, $\mathrm{w}_{\mathrm{t}}$, documented in ICRP (1991) are presented in Table 2.

Table 2. Tissue Weighting Factors (ICRP 60)

\begin{tabular}{|l|c|}
\hline Organ or Tissue & $\mathrm{W}_{\mathrm{t}}$ \\
\hline Gonads & 0.2 \\
\hline Colon, red marrow, stomach & 0.12 \\
\hline $\begin{array}{l}\text { Bladder, Breast, Esophagus, Liver, Thyroid, } \\
\text { Remainder }\end{array}$ & 0.05 \\
\hline Bone surface and Skin & 0.01 \\
\hline
\end{tabular}

\subsubsection{Committed Effective Dose}

The committed effective dose (CED) is the dose received from an internal source of exposure to an individual over a given time. The CED is the effective dose for members of the public integrated over the course of 50 years for adults and 70 years for children and infants (ICRP 103). The definition of the CED has changed from previous ICRP publications. The committed equivalent dose is calculated using eqn 4.

$$
H_{T}(\tau)=\int_{0}^{\tau} H_{T}(t) d t
$$

where $\tau$ is the time in which the dose is integrated over. The committed effective dose can simply be calculated by multiplying $H_{T}(\tau)$ by a tissue weighting factor in the table above.

\subsubsection{Total Effective Dose}

The total effective dose (TED) is the sum of the effective dose from external exposures and the CED. The TED is used to demonstrate compliance with required dose limits. For members of the public, the doses can be determined by use of human health input parameters, population fractions, and dose coefficients. The combination of these factors comprises the representative person dose, which will be discussed in the next section. 


\subsection{DOSE COEFFICIENTS}

In DOE STD-1196-2011, reference-person dose coefficients are provided for the ingestion of water, inhalation of air, and air immersion pathways. These coefficients were derived using the Dose and Risk Calculation (DCAL) System (Eckerman et al. 2006) and incorporate the nuclear decay data of ICRP Publication 107 (ICRP 2008). DCAL has been one of four software packages used in the production of ICRP publications of dose coefficients for inhaled and ingested radionuclides during the past twenty years. DCAL also was used in the production of Federal Guidance Reports 12 and 13 (EPA 1993, 1999).

\subsubsection{Internal Dose Coefficients}

The ingestion and inhalation dose coefficients for a reference person were developed using the age specific dose coefficients documented in the DC_PAK3 toolbox, which can be accessed at http://www.epa.gov/rpdweb00/federal/techdocs.html. The ingestion dose coefficients documented in DOE-STD-1196-2011 were developed for the water ingestion pathway, only. However, formal clarification that these water ingestion dose coefficients may be used for all ingestion pathways was provided by DOE-HQ in a memorandum from Andrew Lawrence (Director of the Office of Environmental Protection, Sustainability Support and Corporate Safety Analysis and the Office of Health, Safety, and Security) to David Moody (Manager of DOE-SR) dated January 9, 2013. This memorandum is attached in Appendix A. Also provided in Appendix A are the ingestion dose coefficients that will be used at SRS for demonstrating compliance with DOE O 458.1.

In Appendix B, the reference-person dose coefficients to be used at SRS for the air inhalation pathway are provided. These coefficients were taken directly from DOE-STD-1196-2011. However, only the ICRP 72 (ICRP 1996) recommended lung absorption type dose coefficients are listed. For the elements that do not have an ICRP 72 recommended absorption type, the maximum dose coefficient was selected and included in the Appendix B list. For tritium, the dose coefficient shown is for the oxide (vapor) form. For carbon, it is the dioxide form.

\subsubsection{External Dose Coefficients}

For external exposure pathways (ground shine, shoreline exposure, air immersion, and water submersion), age specific dose coefficients have not been developed. Therefore, there are no "reference person" external dose coefficients. The external dose coefficients to be used at SRS for demonstrating compliance with DOE O 458.1 are provided in Appendix C. These coefficients are taken from the DC_PAK3 toolbox and are based on FGR 12 methods, but like the internal dose coefficients they have been updated to incorporate the ICRP 107 decay data. The air-immersion dose coefficients are also documented in Table A-3 of DOE-STD1196-2011. 


\subsection{MAXIMALLY EXPOSED INDIVIDUAL AND REFERENCE PERSON}

During routine operations at SRS, limited amounts of radioactive materials are released to the environment through atmospheric and liquid pathways. These releases potentially result in a radiation dose commitment to offsite people. The principal pathways by which people are exposed to releases of radioactivity are:

- Inhalation

- Ingestion

- Skin absorption

- External exposure

Figure 1 is a simplified representation of the principal exposure pathways.

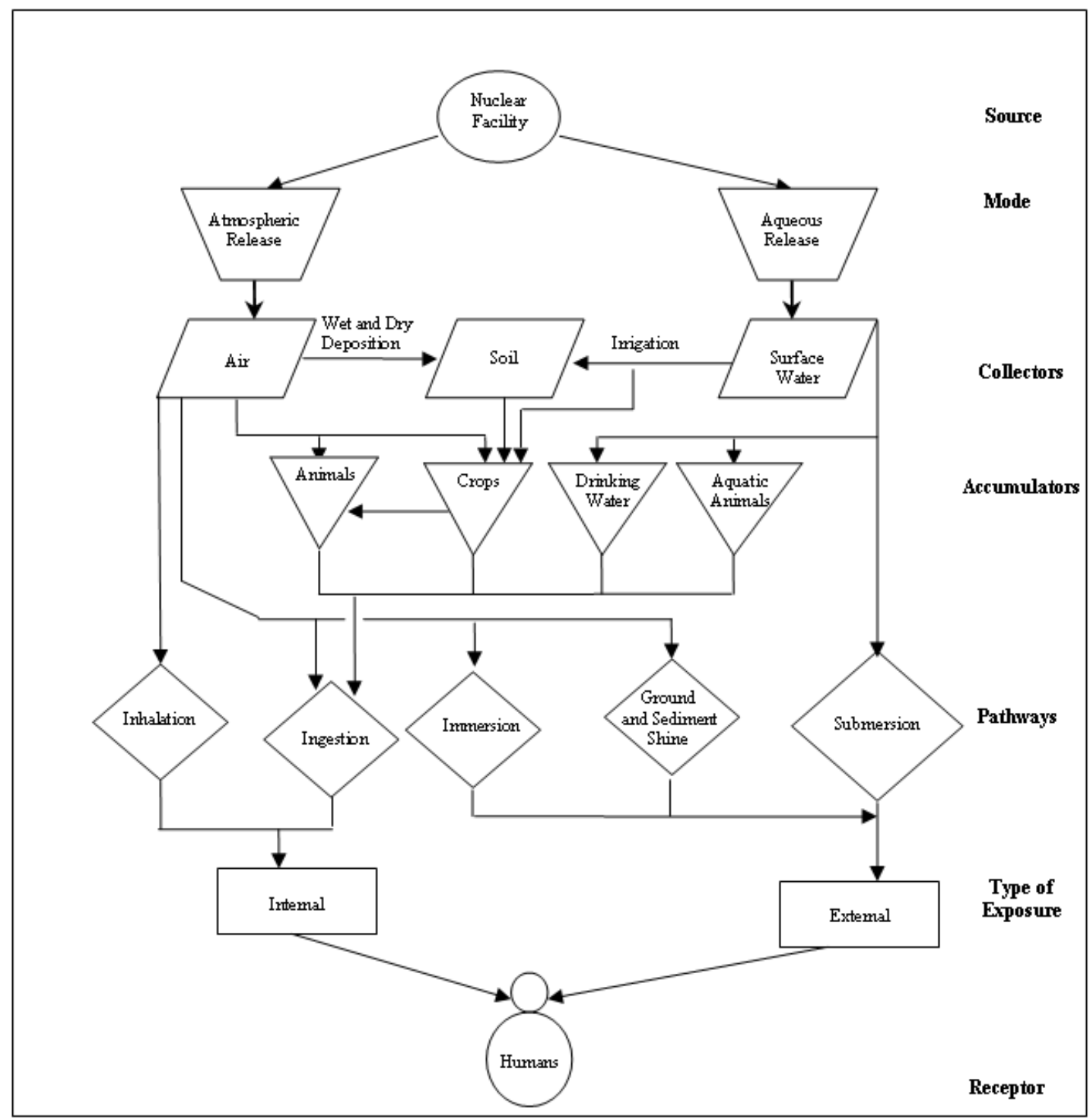

Figure 1. Exposure Pathways to Humans from Atmospheric and Aqueous Releases 
At SRS, the potential effects of routine radioactive releases have been assessed annually since operations began. Since 1972, annual offsite dose estimates have been published in site environmental reports, which are made available to the public. For all routine environmental dose calculations performed since 1978, SRS has used environmental transport models based on codes developed by the U.S. Nuclear Regulatory Commission (NRC) (NRC 1977). The NRC based transport models use DOE accepted methods, consider all significant exposure pathways, and permit detailed analysis of the effects of routine operations.

As previously discussed, DOE O 458.1 states that compliance with the annual dose limit of $100 \mathrm{mrem}(1 \mathrm{mSv})$ to a member of the public may be demonstrated by calculating the dose to the maximally exposed individual (MEI) or to a representative person.

\subsection{MAXIMALLY EXPOSED INDIVIDUAL}

When calculating radiological doses to the public from routine releases, SRS currently uses the concept of the MEI. For the MEI doses, SRS has been using adult male consumption rates for air, food, and drinking water and adult male usage parameters to estimate external exposures to radionuclides. These MEI consumption and usage rates were based on NRC NUREG values (NRC 1977) or on historical SRS-specific values (Hamby 1991). All current applicable land- and water-use parameters used in the dose calculations are documented in Jannik et al. (2010). These parameters include 1) local characteristics of food production, 2) river recreational activities, 3) meat, milk, and vegetable consumption rates, and 4) other human usage parameters required in the SRS dosimetry models. In addition, the preferred elemental bioaccumulation and transfer factors to be used in human health exposure calculations at SRS are documented in this report.

SRS conservatively combines the airborne pathway and liquid pathway MEI dose estimates, even though the two doses are calculated for hypothetical individuals residing at different geographic locations. This is done to demonstrate compliance (which is documented annually in the SRS annual site environmental report (ASER)), with the DOE O 458.1 (DOE 2011) all-pathway TED standard of 100 mrem per year (1 mSv per year).

\subsection{REFERENCE PERSON}

Beginning with the 2012 ASER, SRS will be using the representative person concept for dose compliance. The dose to a representative person will be based on the reference person (gender and age averaged) dose coefficients provided in DOE Derived Concentration Technical Standard (DOE-STD-1196-2011) and on the SRS-specific reference person usage parameters at the $95^{\text {th }}$ percentile of appropriate national or regional data, which are documented in this report. 
The following is a brief history of the representative person concept.

- Standard Man

- Standard Man was first introduced in ICRP Publication 2 (1960)

- Based on accepted "average" biological and dosimetric parameters

- Used to calculate "consistent and reproducible" doses to workers

- Reference Man

- Update from Standard Man to Reference Man in ICRP 23 (1974)

- Improved biokinetic models and better dosimetric models

- Still primarily focused on an adult worker

- Information widely used

- Reference Man (Individual)

- Reference Male and Female updated in ICRP 89 (2002)

- Includes 6 age ranges for specific Reference Values

- Provides the data for determining Reference Individual (Person)

- Representative Person

- ICRP 101 (2005) was published with the introduction of the Representative Person

- This term is equivalent of, and replaces, "average member of the critical group"

- ICRP 103 Recommendations (2007) formally recommends using the Representative Person

- DOE Order 458.1 (2011) allows use of Representative Person and defines Reference Person

In DOE O 458.1, the reference person is defined as A hypothetical aggregation of human (male and female) physical and physiological characteristics arrived at by international consensus for the purpose of standardizing radiation dose calculations. The reference person, therefore, is an age and gender aggregation of the twelve age-specific reference individuals documented in ICRP 89.

Also in DOE O 458.1, the representative person is defined as An individual receiving a dose that is representative of the more highly exposed individuals in the population. This term is equivalent of, and replaces, "average member of the critical group." However, in ICRP 101 (ICRP 2006), the definition is extended to include the Average value for the more highly exposed group $\mathbf{O R}$ the $95^{\text {th }}$ percentile of appropriate national or regional data. The OR is highlighted for emphasis. At SRS, the reference person who is at the $95^{\text {th }}$ percentile of national usage data will be used as a replacement for the MEI. The appropriate national usage data were taken from the U.S. Environmental Protection Agency's (EPA) Exposure Factors Handbook: 2011 Edition (EPA 2011). 


\subsection{TYPICAL PERSON}

As an extension of the reference person, and to show compliance with collective dose requirements in DOE O 458.1, SRS has defined the concept of the "Typical Person." The typical person is a hypothetical reference person that is typical of the entire population group and it is established at the $50^{\text {th }}$ percentile (median) of the national data. These national median data also were taken from EPA (2011). The median (as opposed to the mean) is better suited for skewed distributions, which are typical for human intake rates, to derive at central tendency since it is much more robust and sensible. 


\subsection{DEVELOPMENT OF REFERENCE PERSON PARAMETERS}

The following section describes and documents how the SRS reference person intake and usage parameters were developed.

\subsection{BODY MASS}

For ingestion rates other than water, reference-individual body masses were required to determine the reference-person ingestion rate of these pathways. These body masses are from ICRP (2002) and are shown in Table 3.

Table 3. ICRP 89 Reference Values for Body Mass

\begin{tabular}{|l|c|c|}
\hline & \multicolumn{2}{|c|}{ Mass $(\mathrm{kg})$} \\
\hline Age & Male & Female \\
\hline Newborn & 3.5 & 3.5 \\
\hline 1 year & 10 & 10 \\
\hline 5 years & 19 & 19 \\
\hline 10 years & 32 & 32 \\
\hline 15 years & 56 & 53 \\
\hline Adult & 73 & 60 \\
\hline
\end{tabular}

\subsection{POPULATION FRACTIONS}

The use of population fractions allows the reference person to be a weighted-average based on age and sex, such that it is an aggregation of male and female from six different age groups. The population fractions were determined using the current US census data (Census 2010).

Population data for the US as a whole and for the South Carolina (SC) and Georgia (GA) counties surrounding the Savannah River Site (within an $80 \mathrm{~km}$ radius) are shown in Table 4. The SC counties included are Aiken, Allendale, Bamberg, Barnwell, Calhoun, Colleton, Edgefield, Hampton, Lexington, McCormick, Orangeburg, and Saluda. The GA counties included are Bulloch, Burke, Columbia, Emanuel, Jefferson, Jenkins, McDuffie, Richmond, and Screven. These population fractions also are used in the calculation of the reference intake and reference dose coefficients, used in the calculation of the DCSs in section 6.0. 
Table 4. U.S. and SC/GA 2010 population fractions

\begin{tabular}{|c|c|c|c|c|c|}
\hline \multirow{2}{*}{ Age Group } & \multirow{2}{*}{ Age $\mathrm{x},(\mathrm{y})$} & \multicolumn{2}{|c|}{ US 2010} & \multicolumn{2}{c|}{$\begin{array}{c}\text { SC/GA Counties } \\
2010\end{array}$} \\
\cline { 3 - 6 } & & Male & Female & Male & Female \\
\hline new born & $\mathrm{x}<1$ & 0.006524 & 0.006251 & 0.006770 & 0.006511 \\
\hline 1 year & $1 \leq \mathrm{x}<3$ & 0.013354 & 0.012800 & 0.013421 & 0.013240 \\
\hline 5 year & $3 \leq \mathrm{x}<7$ & 0.026979 & 0.025833 & 0.027484 & 0.026269 \\
\hline 10 year & $7 \leq \mathrm{x}<12$ & 0.033949 & 0.032489 & 0.034591 & 0.033192 \\
\hline 15 year & $12 \leq \mathrm{x}<17$ & 0.034765 & 0.033087 & 0.035017 & 0.033156 \\
\hline Adult & 17 and older & 0.376036 & 0.397933 & 0.369306 & 0.401043 \\
\hline
\end{tabular}

As the 2010 demographic data show, people living in the surrounding SC/GA counties are slightly younger and more female than the general US population.

\subsection{EXPOSURE PATHWAYS}

There are many ways an individual member of the public may be exposed to radioactive materials. In the DOE Technical Standard 1196, the pathways analyzed were ingestion of water, inhalation of air, and external exposure from immersion in air. The pathways analyzed in this report were food and water ingestion, inhalation, and external exposure by immersion in air, submersion in water, and ground shine. As previously discussed, for each pathway, $95^{\text {th }}$ and $50^{\text {th }}$ percentile intake rates were taken from EPA (2011) and used for the reference person and typical person scenarios, respectively.

\subsubsection{Ingestion}

Ingestion is any intake of a radionuclide that enters the body through the gastrointestinal tract. The ingestion pathways included in this report are water, meat, produce (includes vegetables, fruits, and grains), freshwater fish, saltwater invertebrates, and dairy. The reference/typical person ingestion rates are weighted average, changing based on the population fractions and intake rates of each age group and gender.

For most ingestion rates, the $95^{\text {th }}$ and $50^{\text {th }}$ percentile rates were taken directly from EPA (2011). An exception to this was freshwater fish and saltwater invertebrates, where the mean intake is used instead of the $50^{\text {th }}$ percentile because there were no data provided at this level for young people. In the case of water ingestion, the intake rates are the same with the exception of the 15-year old and Adult age groups where the female intake rate is $75 \%$ that of the male intake rate as recommended by ICRP (2002). The water ingestion rate reported in EPA (2011) is assumed to be the male ingestion rate. Dose from ingestion is calculated by multiplying the annual ingestion rate by the reference-person ingestion dose coefficients, which are tabulated in Appendix A. 


\subsubsection{Intake Rates}

The ingestion intake rates for water, meat, dairy, freshwater fish, saltwater invertebrates, and produce are shown in Tables 5, 6, 7, 8, and 9, respectively. Weighted averages based on the multiple age group intake rates provided in EPA (2011) were used to select the appropriate rates for each of the six age groups recommended in ICRP (2002).

\subsection{Water Ingestion}

The daily water ingestion rates shown in Table 5 were taken from Table 3-10 of EPA (2011).

Table 5. Water Ingestion Rates

\begin{tabular}{|c|c|c|c|c|c|}
\hline \multirow{2}{*}{ Age Group } & \multirow{2}{*}{ Age $\mathrm{x},(\mathrm{y})$} & \multicolumn{4}{|c|}{ Water Intake $(\mathrm{L} / \mathrm{d})$} \\
\cline { 3 - 6 } & & \multicolumn{2}{|c|}{$50 \%$} & \multicolumn{2}{c|}{$95 \%$} \\
\cline { 3 - 6 } & $\mathrm{x}<1$ & 0.364 & 0.364 & 1.1195 & 1.1195 \\
\hline New Born & $1 \leq \mathrm{x}<3$ & 0.3295 & 0.3295 & 0.98 & 0.98 \\
\hline 1 year & $3 \leq \mathrm{x}<7$ & 0.438 & 0.438 & 1.2 & 1.2 \\
\hline 5 year & $7 \leq \mathrm{x}<12$ & 0.503 & 0.503 & 1.409 & 1.409 \\
\hline 10 year & $12 \leq \mathrm{x}<17$ & 0.663 & 0.49725 & 1.96 & 1.47 \\
\hline 15 year & 17 and older & 1.04175 & 0.781313 & 2.808 & 2.106 \\
\hline Adult & \multicolumn{2}{|c|}{0.81} & & 2.2 \\
\hline Reference Person & Mge and Gender Combined) & & & \\
\hline
\end{tabular}

The daily $95 \%$ reference person and 50\% typical person intake rates convert to about $800 \mathrm{~L} / \mathrm{y}$ and $300 \mathrm{~L} / \mathrm{y}$, respectively. The current MEI and average-population intake rates from Jannik et al. (2010) are $730 \mathrm{~L} / \mathrm{y}$ and $337 \mathrm{~L} / \mathrm{y}$, respectively.

\subsection{Meat Ingestion}

Previously at SRS, the meat consumption pathway was assumed to be entirely beef (Jannik et al. 2010). This assumption was based on the fact that most pigs and chicken in the SRS area were raised in enclosures and not directly exposed to deposited radionuclides on the ground and fodder. However, a developing local (and national) trend is for farmers and suburban residents to raise free-range chickens and pigs. Therefore, the SRS meat consumption pathway will now include all meats. Table 6 shows the meat ingestion rates by age, sex, and intake percentile and were taken from Tables 11-3 of EPA (2011). 
Table 6. Total Meat Ingestion (g/day)

\begin{tabular}{|c|c|c|c|c|c|}
\hline \multirow{4}{*}{ Age Group } & \multirow{4}{*}{ Age $x,(y)$} & \multicolumn{4}{|c|}{ Total Meat Ingestion } \\
\hline & & \multicolumn{4}{|c|}{ g/day } \\
\hline & & \multicolumn{2}{|c|}{$50 \%$} & \multicolumn{2}{|c|}{$95 \%$} \\
\hline & & Male & Female & Male & Female \\
\hline new born & $\mathrm{x}<1$ & 0 & 0 & 18.9 & 18.9 \\
\hline 1 year & $1 \leq x<3$ & 34 & 34 & 100 & 100 \\
\hline 5 year & $3 \leq x<7$ & 62.7 & 62.7 & 161.5 & 161.5 \\
\hline 10 year & $7 \leq x<12$ & 80 & 80 & 204.8 & 204.8 \\
\hline 15 year & $12 \leq x<17$ & 95.2 & 90.1 & 263.2 & 249.1 \\
\hline Adult & 17 and older & 105.85 & 78 & 262.8 & 201 \\
\hline \multicolumn{2}{|c|}{$\begin{array}{c}\text { Reference Person } \\
\text { (Age and Gender Combined) }\end{array}$} & \multicolumn{2}{|c|}{86} & \multicolumn{2}{|c|}{221} \\
\hline
\end{tabular}

The daily $95 \%$ reference person and 50\% typical person intake rates for meat ingestion convert to about $81 \mathrm{~kg} / \mathrm{y}$ and $32 \mathrm{~kg} / \mathrm{y}$, respectively. The current MEI and average-population intake rates are $81 \mathrm{~kg} / \mathrm{y}$ and $43 \mathrm{~kg} / \mathrm{y}$, respectively.

\subsection{Dairy Ingestion}

Table 7 shows the daily consumption rates of dairy and were taken from Table 11-3 of EPA (2011). The density of milk was required to convert the mass reported in $\mathrm{kg}$ in EPA (2011) to cubic centimeters. The density used for this conversion was $1.03 \mathrm{~kg} / \mathrm{L}$, which came from the National Institute of Standards and Technology at:

http://www.nist.gov/pml/wmd/pubs/upload/3-35-13-hb44-final.pdf.

Table 7. Dairy Ingestion $\left(\mathrm{cm}^{3} / \mathrm{day}\right)$

\begin{tabular}{|c|c|c|c|c|c|}
\hline \multirow{2}{*}{ Age Group } & \multirow{2}{*}{ Age $\mathrm{x},(\mathrm{y})$} & \multicolumn{5}{|c|}{ Dairy Ingestion $\left(\mathrm{cm}^{3} /\right.$ day $)$} \\
\cline { 3 - 6 } & & Male & Female & Male & Female \\
\cline { 3 - 6 } & $\mathrm{x}<1$ & 22 & 22 & 147 & 147 \\
\hline new born & $1 \leq \mathrm{x}<3$ & 380 & 380 & 919 & 919 \\
\hline 1 year & $3 \leq \mathrm{x}<7$ & 336 & 336 & 854 & 854 \\
\hline 5 year & $7 \leq \mathrm{x}<12$ & 335.5 & 335.5 & 988 & 988 \\
\hline 10 year & 291 & 276 & 1059 & 1002 \\
\hline 15 year & $12 \leq \mathrm{x}<17$ & 167 & 140 & 705 & 609 \\
\hline Adult & 17 and older & \multicolumn{3}{|c|}{715} \\
\hline $\begin{array}{c}\text { Reference Person } \\
\text { (Age and Gender Combined) }\end{array}$ & \multicolumn{2}{|c|}{188} & \multicolumn{2}{c|}{} \\
\hline
\end{tabular}


The daily $95 \%$ reference person and 50\% typical person intake rates for dairy consumption convert to about $260 \mathrm{~L} / \mathrm{y}$ and $69 \mathrm{~L} / \mathrm{y}$, respectively. The current MEI and population intake rates are $230 \mathrm{~L} / \mathrm{y}$ and $120 \mathrm{~L} / \mathrm{y}$, respectively.

\subsection{Freshwater Fish and Saltwater Invertebrate Ingestion}

For freshwater fish consumption, it is assumed that the fish are harvested in the Savannah River below SRS at the Highway 301 Bridge. For saltwater invertebrates, it is assumed that the shellfish are harvested near the mouth of the Savannah River. The fish and invertebrate ingestion rates are included in Table 8 and were taken from Tables 10-7 and 10-9 of EPA (2011).

Table 8. Consumption Rates for Freshwater Fish and Saltwater Invertebrates

\begin{tabular}{|c|c|c|c|c|c|}
\hline \multicolumn{6}{|c|}{ Seafood Consumption Rates (g/day) } \\
\hline \multicolumn{6}{|c|}{ Mean } \\
\hline \multirow{3}{*}{ Age Group } & \multirow{3}{*}{ Age $x,(y)$} & \multirow{2}{*}{\multicolumn{2}{|c|}{$\frac{\text { Freshwater (Finfish) }}{\text { g/day }}$}} & \multicolumn{2}{|c|}{ Saltwater (Shellfish) } \\
\hline & & & & \multicolumn{2}{|c|}{ g/day } \\
\hline & & Male & Female & Male & Female \\
\hline new born & $\mathrm{x}<1$ & 0.105 & 0.105 & 0 & 0 \\
\hline 1 year & $1 \leq \mathrm{x}<3$ & 2.2 & 2.2 & 0.4 & 0.4 \\
\hline 5 year & $3 \leq x<7$ & 3.4675 & 3.4675 & 0.95 & 0.95 \\
\hline 10 year & $7 \leq x<12$ & 5.12 & 5.12 & 1.6 & 1.143 \\
\hline 15 year & $12 \leq \mathrm{x}<17$ & 5.936 & 5.936 & 1.802 & 1.802 \\
\hline Adult & 17 and older & 10.5 & 10.2 & 3.9 & 3.3 \\
\hline \multicolumn{2}{|c|}{$\begin{array}{c}\text { Reference Person } \\
\text { (Age and Gender Combined) }\end{array}$} & \multicolumn{2}{|c|}{9.0} & \multicolumn{2}{|c|}{3.0} \\
\hline \multicolumn{6}{|c|}{$95 \%$} \\
\hline new born & $\mathrm{x}<1$ & 0 & 0 & 0 & 0 \\
\hline 1 year & $1<\mathrm{x}<3$ & 12 & 12 & 0 & 0 \\
\hline 5 year & $3<x<7$ & 5.486 & 5.486 & 0.95 & 0.95 \\
\hline 10 year & $7<x<12$ & 35.2 & 35.2 & 6.4 & 6.4 \\
\hline 15 year & $12<x<17$ & 43.68 & 43.68 & 2.24 & 2.24 \\
\hline Adult & 17 and older & 80.3 & 76.65 & 32.85 & 25.55 \\
\hline \multicolumn{2}{|c|}{$\begin{array}{c}\text { Reference Person } \\
\text { (Age and Gender Combined) }\end{array}$} & \multicolumn{2}{|c|}{66} & \multicolumn{2}{|c|}{23} \\
\hline
\end{tabular}

In EPA (2011), Table 10-13 shows that the South Atlantic region (of which South Carolina is a member) is higher than the overall national intake values. To account for these regionally higher fish intakes, freshwater fish and saltwater invertebrate consumption at the SRS is assumed to be $10 \%$ higher than the overall national values presented in Table 8 . Accounting for this 10 percent increase, the daily $95 \%$ reference person and $50 \%$ typical person intake rates for freshwater fish ingestion convert to about $24 \mathrm{~kg} / \mathrm{y}$ and $3.7 \mathrm{~kg} / \mathrm{y}$, respectively. The current MEI intake rates are $19 \mathrm{~kg} / \mathrm{y}$ and $9 \mathrm{~kg} / \mathrm{y}$, respectively. The daily 95\% reference person and 50\% typical person intake rates for saltwater invertebrates, 
converts to about $9.0 \mathrm{~kg} / \mathrm{y}$ and $1.5 \mathrm{~kg} / \mathrm{y}$, respectively. The current MEI intake rates for saltwater shellfish are $8 \mathrm{~kg} / \mathrm{y}$ and $2 \mathrm{~kg} / \mathrm{y}$, respectively.

\subsection{Produce Ingestion}

Total Produce includes fruits, vegetables, and grains. Body weight was required to determine a daily individual consumption rate presented in Table 9 and the intake rates were taken from Tables 9-3 (for fruits and vegetables) and 12-3 (for grains) of EPA (2011).

Table 9. Total Produce Ingestion Rates (g/day)

\begin{tabular}{|c|c|c|c|c|c|}
\hline \multirow{3}{*}{ Age Group } & \multirow{3}{*}{ Age $x,(y)$} & \multicolumn{4}{|c|}{ 5.3.1.1.5.1 Total Produce Ingestion (g/day) } \\
\hline & & \multicolumn{2}{|c|}{$50 \%$} & \multicolumn{2}{|c|}{$95 \%$} \\
\hline & & male & female & male & female \\
\hline new born & $\mathrm{x}<1$ & 27.3 & 27.3 & 170.45 & 170.45 \\
\hline 1 year & $1 \leq x<3$ & 171 & 171 & 493 & 493 \\
\hline 5 year & $3 \leq x<7$ & 228.475 & 228.475 & 691.125 & 691.125 \\
\hline 10 year & $7 \leq x<12$ & 262.4 & 262.4 & 873.6 & 873.6 \\
\hline 15 year & $12 \leq x<17$ & 342.72 & 324.36 & 1040.48 & 984.74 \\
\hline Adult & 17 and older & 328.5 & 261 & 1029.3 & 825 \\
\hline \multicolumn{2}{|c|}{$\begin{array}{c}\text { Reference Person } \\
\text { (Age and Gender Combined) }\end{array}$} & \multicolumn{2}{|c|}{284} & \multicolumn{2}{|c|}{892} \\
\hline
\end{tabular}

The daily $95 \%$ reference person and 50\% typical person intake rates for produce consumption convert to about $320 \mathrm{~kg} / \mathrm{y}$ and $100 \mathrm{~kg} / \mathrm{y}$, respectively. The current MEI and average-population intake rates are $319 \mathrm{~kg} / \mathrm{y}$ and $184 \mathrm{~kg} / \mathrm{y}$, respectively. In Table 9-5 (Per Capita Intake of Individual Fruits and Vegetables) of EPA (2011), only mean intake rates are provided for leafy vegetable consumption by the various age groups. Based on the mean consumption rates, the ratio of leafy to total vegetable consumption is about 23 percent. Therefore, as part of the daily $95 \%$ reference person and 50\% typical person total produce consumption rates, it is assumed that leafy vegetables account for $31 \mathrm{~kg} / \mathrm{y}$ and $11 \mathrm{~kg} / \mathrm{y}$, respectively.

\subsubsection{Inhalation}

The inhalation exposure pathway includes any intake that enters the body through the respiratory system via the bloodstream. There are three absorption types as published in ICRP 66; F, M, S. The F type absorption has a quick biological lifetime, M type absorption has an intermediate biological lifetime, and $\mathrm{S}$ type absorption has a very slow biological lifetime. The class of the radionuclide depends on its chemical compound. Generally, class F materials are absorbed in greater fractions in the body than class $\mathrm{S}$.

As with the ingestion pathways, the weighted averages based on the multiple age group inhalation rates provided in Table 6-4 of EPA (2011) were used to select the appropriate rates for each of the six age groups recommended in ICRP 89. 
Table 10. Inhalation Rates

\begin{tabular}{|c|c|c|c|c|c|}
\hline \multirow{2}{*}{ Age Group } & \multirow{2}{*}{ Age $\mathrm{x},(\mathrm{y})$} & \multicolumn{4}{|c|}{ Inhalation } \\
& & \multicolumn{3}{|c|}{$95 \%$} & \multicolumn{2}{|c|}{$50 \%$} \\
\cline { 3 - 6 } & & Male & Female & Male & Female \\
\cline { 3 - 6 } & $\mathrm{x}<1$ & 5.04 & 4.75 & 3.8 & 3.61 \\
\hline new born & $1 . \mathrm{x}<3$ & 6.56 & 6.36 & 5.12 & 4.78 \\
\hline 1 year & $3 . \mathrm{x}<7$ & 10.185 & 9.675 & 8.12 & 7.64 \\
\hline 5 year & $7 . \mathrm{x}<12$ & 13.87 & 12.61 & 10.59 & 9.84 \\
\hline 10 year & $12 .<17$ & 23.26 & 17.56 & 17.23 & 13.28 \\
\hline 15 year & 17 and older & 20.808 & 16.128 & 16.158 & 12.548 \\
\hline Adult & \multicolumn{2}{|c|}{17.4} & \multicolumn{2}{|c|}{13.5} \\
\hline \multicolumn{2}{|c|}{ Reference Person } & & &
\end{tabular}

The daily $95 \%$ reference person and 50\% typical person intake rates for inhalation of air convert to about $6400 \mathrm{~m}^{3} / \mathrm{y}$ and $5000 \mathrm{~m}^{3} / \mathrm{y}$, respectively. The current MEI and averagepopulation inhalation rates are $8000 \mathrm{~m}^{3} / \mathrm{y}$ and $5548 \mathrm{~m}^{3} / \mathrm{y}$, respectively. To calculate a dose from inhalation, the annual inhalation rate is multiplied by the reference-person dose coefficients, which are tabulated in Appendix B.

\subsubsection{Immersion in Air and Submersion in Water}

External doses can be received from immersion in air and submersion in water. The exposure time for immersion in air is conservatively set at $365.25 \mathrm{~d} / \mathrm{y}$. Exposure time from submersion in water is determined by the activity. For exposure from boating, a reduction factor of 0.5 is used to account for what is effectively a semi-infinite plain and for shielding provided by the boat. EPA (2011) does not provide exposure factor data for swimming or boating on rivers and lakes. Therefore, the exposure times documented in Jannik, et al. (2010) will continue to be used. For boating, the maximum time per year is 44 hours per year and the average time is 22 hours per year. The maximum annual exposure time for swimming is 14 hours and the average time is 7 hours. The external immersion and submersion dose coefficients were taken from the DC_PAK3 toolbox (http://www.epa.gov/rpdweb00/federal/techdocs.html) and are compiled in Appendix C.

\subsubsection{Ground Shine}

Ground shine is the external dose received from radioactive material deposited on the ground. The exposure time is conservatively set at $365.25 \mathrm{~d} / \mathrm{y}$. An additional ground shine pathway includes exposure to Savannah River sediment. To calculate dose from shoreline pathways, the ground-shine dose coefficient is multiplied by a dose reduction factor. Due to SRS being located along the Savannah River, the river-shoreline dose reduction factor of 0.2 is used as recommended in EPA (1993). The external ground-shine dose coefficients were taken from the DC_PAK3 toolbox (http://www.epa.gov/rpdweb00/federal/techdocs.html) and are compiled in Appendix C. 


\subsection{DERIVED CONCENTRATION STANDARDS (DCS)}

The DCS is the concentration of a particular radionuclide in water, in air, or on the ground that results in a member of the public receiving $100 \mathrm{mrem}(1 \mathrm{mSv})$ effective dose following continuous exposure for one year. In DOE-STD-1196-2011, DCSs were developed for the ingestion of water, inhalation of air and submersion in air pathways, only. These DCSs are required by DOE O 458.1 to be used at all DOE sites in the design and conduct of radiological environmental protection programs. In this report, DCSs for the following additional pathways were considered and documented: ingestion of meat, dairy, grains, produce (fruits and vegetables), seafood, submersion in water and ground shine. These additional DCSs were developed using the same methods as in DOE-STD-1196-2011 and will be used at SRS, where appropriate, as screening and reference values.

\subsection{CALCULATION OF INTAKE RATES AND REFERENCE DOSE COEFFICIENTS}

Reference intake rates and reference dose coefficients are calculated using the population fractions and intake rates for each associated age group for both male and female. The reference intake rate is calculated by summing the male intake rate multiplied by the male population fraction and the female intake rate multiplied by the female population fraction of each age group shown here in eqn 5 ,

$$
I R_{\text {ref }}=\sum_{n=1}^{6}\left(U_{\text {male }}(\text { age }) * f_{\text {male }}(\text { age })+U_{\text {female }}(\text { age }) * f_{\text {female }}(\text { age })\right.
$$

Where "U" is the age specific intake rate and "f" is the population fraction of that particular age group. Reference dose coefficients are calculated using the reference intake rate as seen in eqn 6.

$$
\begin{aligned}
& D C_{\text {ref }} \\
& =\frac{\sum_{n=1}^{6}\left[\left(U_{\text {male }}(\text { age }) * f_{\text {male }}(\text { age })+U_{\text {female }}(\text { age }) * f_{\text {female }}(\text { age })\right) * D C_{\text {age }}\right]}{I R_{\text {ref }}}
\end{aligned}
$$

where $D C_{a g e}$ is the dose coefficient of a particular age group. The dose coefficient converts the activity present in a medium an individual is exposed to into an effective dose. The units of the dose coefficient are either the $\mathrm{Sv} / \mathrm{Bq}$ or $\mathrm{rem} / \mu \mathrm{Ci}$. The dose coefficients used in this report were tabulated in DOE-STD-1196-2011 and calculated in this manner.

The DCS's for each pathway of ingestion are calculated in a similar manner. Four components are needed to calculate the DCS. The dose constraint is the dose an individual will receive when exposed to the DCS. This dose is the annual dose limit $(E C)$ recommended and used by DOE of $1 \mathrm{mSv}$ or $100 \mathrm{mrem}$. The reference intake $\left(I R_{r e f}\right)$ discussed previously is calculated for the ingestion pathway of interest in the units of volume or mass per day. The reference dose coefficient $\left(D C_{r e f}\right)$ is calculated as shown previously and used to convert the 
dose of the constraint into an activity. The DCS is calculated over the course of a year or 365 days $(t)$. The derived concentrations are calculated using eqn 7 .

$$
D C S_{\text {ing }}=\frac{E C}{t * I R_{r e f} * D C_{r e f}}
$$

Depending on the type of food being ingested, the DCS will result in units of activity per unit volume or unit mass.

The inhalation DCS is calculated similarly to that of the ingestion DCS's shown in eqn 8 .

$$
D C S_{i n h}=\frac{E C}{t * I R_{r e f} * D C_{r e f}}
$$

where: EC is the annual dose limit of $1 \mathrm{mSv}$ for the dose constraint. The reference-inhalation intake rate is calculated using eqn 5 and the reference-inhalation dose coefficient is calculated using eqn 6 .

The DCS for both air immersion and water submersion are calculated by eqn 9,

$$
D C S=\frac{E C}{t * D C}
$$

Where the dose constraint (EC) is the annual dose limit of $1 \mathrm{mSv}$. The dose coefficient is in units of Sv- $\mathrm{m}^{3}$ per Bq-sec, and time over the course of a year $(\mathrm{t})$ is $3.1536 \times 10^{7}$ seconds. The dose coefficients are from the DC_PAK3 toolbox (http://www.epa.gov/rpdweb00/federal/techdocs.html).

The calculation of the DCS for ground shine is similar to that of air and water submersion and eqn 9 can be used. The units of the DCS are in activity per unit area $\left(\mathrm{Bq} / \mathrm{cm}^{2}\right)$; the dose coefficient is in units of $\mathrm{Sv} \mathrm{m}^{2}$ per Bq-sec.

\subsubsection{SRS-Specific DCS Results}

The SRS-Specific DCSs for all pathways are tabulated in the Appendix D. Comparisons for water ingestion, inhalation, and air submersion are the only comparisons made with DOESTD-1196-2011. The major changes between current water ingestion and air inhalation DCS are the updated nuclear data in the dose coefficients and reference intake values based on EPA 2011. The major difference in the current and new air immersion DCS is the updated nuclear data in the dose rate coefficients. 


\subsection{COMPARISON \& RESULTS}

The following is a summary of the SRS-specific Reference Person ( $95^{\text {th }}$ percentile) and Typical Person $\left(50^{\text {th }}\right.$ percentile) intake rates. Comparisons to the current MEI and average population values are provided, as is an example of how the overall dose estimates will be affected.

\subsection{REFERENCE INTAKE COMPARISONS}

The reference intake values calculated in this report changed from the values that were previously used, which are listed in Tables 10 and 11 of Jannik et al. (2010). The $95^{\text {th }}$ percentile values showed no change or slight increases except for inhalation and leafy vegetables, which decreased by $20 \%$ and $28 \%$, respectively, from the current adult male (MEI) values. However, for the $50^{\text {th }}$ percentile values there was a noticeable decrease in all of the intake rates because of the effect of age and gender weighting and use of the median as opposed to the mean.

Table 11. Comparison of Reference and Typical Person Intakes with Current Intakes

\begin{tabular}{|c|c|c|c|c|}
\hline \multicolumn{5}{|c|}{$95 \%$} \\
\hline & & Reference Person & Current MEI & Difference \\
\hline Air & $\mathrm{m} 3 / \mathrm{y}$ & 6400 & 8000 & $-20.0 \%$ \\
\hline Water & $\mathrm{L} / \mathrm{y}$ & 800 & 730 & $9.6 \%$ \\
\hline Meat & $\mathrm{kg} / \mathrm{y}$ & 81 & 81 & $0.0 \%$ \\
\hline Leafy Vegetables & $\mathrm{kg} / \mathrm{y}$ & 31 & 43 & $-27.9 \%$ \\
\hline Other Produce & $\mathrm{kg} / \mathrm{y}$ & 289 & 276 & $0.5 \%$ \\
\hline Milk/Dairy & $\mathrm{L} / \mathrm{y}$ & 260 & 230 & $13 \%$ \\
\hline Freshwater Fish & $\mathrm{kg} / \mathrm{y}$ & 24 & 19 & $26.3 \%$ \\
\hline Saltwater Invertebrate & $\mathrm{kg} / \mathrm{y}$ & 9.0 & 8 & $12.5 \%$ \\
\hline \multicolumn{5}{|c|}{$50 \%$} \\
\hline & & Typical Person & Current Avg. & Difference \\
\hline Air & $\mathrm{m} 3 / \mathrm{y}$ & 5000 & 5548 & $-9.9 \%$ \\
\hline Water & $\mathrm{L} / \mathrm{y}$ & 300 & 337 & $-11.0 \%$ \\
\hline Meat & $\mathrm{kg} / \mathrm{y}$ & 32 & 43 & $-26.3 \%$ \\
\hline Leafy Vegetables & $\mathrm{kg} / \mathrm{y}$ & 11 & 21 & $-47.6 \%$ \\
\hline Other Produce & $\mathrm{kg} / \mathrm{y}$ & 89 & 163 & $-45.4 \%$ \\
\hline Milk/Dairy & $\mathrm{L} / \mathrm{y}$ & 69 & 120 & $-42.3 \%$ \\
\hline Freshwater Fish & $\mathrm{kg} / \mathrm{y}$ & 3.7 & 9 & $-58.9 \%$ \\
\hline Saltwater Invertebrate & $\mathrm{kg} / \mathrm{y}$ & 1.5 & 2 & $-25.0 \%$ \\
\hline
\end{tabular}




\subsection{COMPARISON OF INTERNAL DOSE CALCULATIONS}

Using the reference intakes shown in Table 11 and the internal dose coefficients for tritium oxide from DOE-STD-1196-2011, dose calculations were performed for a unit concentration $(1 \mathrm{~Bq} / \mathrm{L}$ or $1 \mathrm{~Bq} / \mathrm{kg})$ and are compared to the current dose values in Table 12 . Tritium oxide typically accounts for over $99 \%$ of the total releases from SRS.

Table 12. Comparison of Representative and Typical Doses with Current Dose Calculations for Unit Concentrations of Tritium Oxide

\begin{tabular}{|c|c|c|c|}
\hline \multicolumn{4}{|c|}{$95 \%$} \\
\hline & $\begin{array}{l}\text { Representative } \\
\text { Dose (Sv) }\end{array}$ & $\begin{array}{l}\text { Current Dose } \\
\text { (Sv) }\end{array}$ & Difference \\
\hline Air & $1.85 \mathrm{E}-07$ & $2.16 \mathrm{E}-07$ & $-14.22 \%$ \\
\hline Water & $1.68 \mathrm{E}-08$ & $1.31 \mathrm{E}-08$ & $27.85 \%$ \\
\hline Meat & $1.70 \mathrm{E}-09$ & $1.46 \mathrm{E}-09$ & $16.67 \%$ \\
\hline Total Produce & $6.72 \mathrm{E}-09$ & $5.74 \mathrm{E}-09$ & $17.03 \%$ \\
\hline Milk/Dairy & $5.46 \mathrm{E}-09$ & $4.14 \mathrm{E}-09$ & $31.88 \%$ \\
\hline Freshwater Fish & $5.04 \mathrm{E}-10$ & $3.42 \mathrm{E}-10$ & $47.37 \%$ \\
\hline Saltwater Invertebrate & $1.89 \mathrm{E}-10$ & $1.44 \mathrm{E}-10$ & $31.25 \%$ \\
\hline \multicolumn{4}{|c|}{$50 \%$} \\
\hline & Typical Dose (Sv) & $\begin{array}{l}\text { Current Dose } \\
\text { (Sv) }\end{array}$ & Difference \\
\hline Air & $1.45 \mathrm{E}-07$ & $1.50 \mathrm{E}-07$ & $-3.37 \%$ \\
\hline Water & $6.30 \mathrm{E}-09$ & $6.07 \mathrm{E}-09$ & $3.86 \%$ \\
\hline Meat & $6.72 \mathrm{E}-10$ & $7.74 \mathrm{E}-10$ & $-13.18 \%$ \\
\hline Total Produce & $2.10 \mathrm{E}-09$ & $3.31 \mathrm{E}-09$ & $-36.59 \%$ \\
\hline Milk/Dairy & $1.45 \mathrm{E}-09$ & $2.16 \mathrm{E}-09$ & $-32.92 \%$ \\
\hline Freshwater Fish & $7.77 \mathrm{E}-11$ & $1.62 \mathrm{E}-10$ & $-52.04 \%$ \\
\hline Saltwater Invertebrate & $3.15 \mathrm{E}-11$ & $3.60 \mathrm{E}-11$ & $-12.50 \%$ \\
\hline
\end{tabular}

In general, the reference-person dose coefficients are larger than the currently used ICRP 72 adult dose coefficients. For tritium oxide, the difference is about $17 \%$ more for ingestion and $7 \%$ more for inhalation. Therefore, for the $95 \%$ intake parameters that increased (Table 11) the dose difference is even larger (Table 12). For the 50\% intake parameters (all which decreased), the percentage dose difference is smaller. 


\subsection{COMPARISON OF EXTERNAL DOSE CALCULATIONS}

Using the current exposure times from Jannik et al. (2010) and the external dose coefficients for cesium-137 (including barium-137m) from the DC PAK3 toolbox, dose calculations were performed for a unit concentration $\left(1 \mathrm{~Bq} / \mathrm{m}^{2}\right.$ or $\left.1 \mathrm{~Bq} / \mathrm{m}^{3}\right)$ and compared to the current dose values in Table 1. The external dose coefficients are tabulated in Appendix C. There are only minor changes due to minor differences in the updated ICRP 107 decay data. Cesium137 is an important beta-gamma emitter at SRS.

Table 13. External Dose Calculation Comparisons

\begin{tabular}{|l|c|c|c|}
\hline & Updated Dose & Current Dose & Difference \\
\hline Air Immersion & $8.51 \mathrm{E}-07$ & $9.08 \mathrm{E}-07$ & $-6.30 \%$ \\
\hline Water Submersion & $1.84 \mathrm{E}-09$ & $1.97 \mathrm{E}-09$ & $-6.72 \%$ \\
\hline Ground Shine & $1.83 \mathrm{E}-08$ & $1.85 \mathrm{E}-08$ & $-1.05 \%$ \\
\hline
\end{tabular}




\subsection{DISCUSSION AND CONCLUSIONS}

In this report, the SRS-specific reference person intake parameters have been determined and the internal dose coefficients have been established (based on DOE direction) and documented. The external pathway exposure parameters remained unchanged from Jannik et al. (2010). However, the external exposure dose coefficients were updated to those that incorporate the ICRP 107 decay data. In addition, the SRS-specific derived concentration standards for all applicable exposure pathways have been calculated and provided for reference.

\subsection{USE OF REFERENCE PERSON PARAMETERS AND DOSE COEFFICIENTS} The SRS-specific reference person intake parameters (provided in Table 11) will be used in conjunction with the reference person ingestion (Appendix A) and inhalation (Appendix B) dose coefficients to calculate the representative person dose at SRS. This representative person dose will be used for demonstrating compliance with the DOE O 458.1 public dose limit of $100 \mathrm{mrem} / \mathrm{y}(1 \mathrm{mSv} / \mathrm{y})$.

\subsection{USE OF SRS-SPECIFIC DERIVED CONCENTRATION STANDARDS}

In DOE-STD-1196-2011, DCSs were developed for the ingestion of water, inhalation of air and submersion in air pathways. These DCSs are required by DOE O 458.1 to be used at all DOE sites in the design and conduct of radiological environmental protection programs.

In this report, DCSs for the following additional pathways were considered and documented: ingestion of meat, dairy, grains, produce (fruits and vegetables), seafood, submersion in water and ground shine. These additional DCSs were developed using the same methods as in DOE-STD-1196-2011 and will be used at SRS, where appropriate, only as screening and reference values. 


\subsection{REFERENCES}

Census 2010. Single Years of Age and Sex: 2010. Table QT-P2, U.S. Census Bureau, Washington, DC.

U.S. Department Of Energy. Radiation Protection of the Public and the Environment. DOE O 458.1; U.S. DOE, Washington, DC; 2011.

U.S. Department Of Energy. Derived Concentration Technical Standards. DOE-STD-11962011; U.S. DOE, Washington, DC; 2011.

Eckerman KF and Leggett RW. DC_PAK3. Oak Ridge National Laboratory.

$<$ http://www.epa.gov/rpdweb00/federal/techdocs.html >

Eckerman KF, Leggett RW, Cristy M, Nelson CB, Ryman JC, Sjoreen AL, Ward RC. User's guide to the DCAL System. Oak Ridge, TN: Oak Ridge National Laboratory; ORNL/ TM2001/190; 2006. <http://www.epa.gov/rpdweb00/assessment/dcal.html>

U.S. Environmental Protection Agency. External Exposure to Radionuclides in Air, Water, and Soil. Federal Guidance Report No. 12, EPA 402-R-93-81, Washington, DC, 1993.

U.S. Environmental Protection Agency. Cancer Risk Coefficients for Environmental Exposure to Radionuclides. Federal Guidance Report No. 13, EPA 402-R-99-001, Washington, DC, 1999.

U.S. Environmental Protection Agency. Exposure Factors Handbook 2011 Edition (Final). EPA/600/R-09/052F; U.S. Environmental Protection Agency, Washington, DC, 2011.

International Commission on Radiological Protection. Permissible Dose for Internal Radiation. ICRP Publication 2, Ann. ICRP 2; 1959.

International Commission on Radiological Protection. Report on the Task Group on Reference Man. ICRP Publication 23, Ann. ICRP 23; 1975.

International Commission on Radiological Protection. 1990 recommendations of the International Commission on Radiological Protection. ICRP Publication 60, Ann. ICRP 21(1Y3); 1991.

International Commission on Radiological Protection. Human Respiratory Tract Model for Radiological Protection. ICRP Publication 66. Ann. ICRP 24 (1-3); 1994.

International Commission on Radiological Protection. Age-dependent Doses to the Members of the Public from Intake of Radionuclides - Part 5 Compilation of Ingestion and Inhalation Coefficients. ICRP Publication 72. Ann. ICRP 26 (1); 1995. 
International Commission on Radiological Protection. Basic Anatomical and Physiological Data for Use in Radiological Protection Reference Values. ICRP Publication 89. Ann. ICRP $32(3-4) ; 2002$.

International Commission on Radiological Protection. Assessing Dose of the Representative Person for the Purpose of the Radiation Protection of the Public. ICRP Publication 101a. Ann. ICRP 36 (3); 2006.

International Commission on Radiological Protection. The 2007 Recommendations of the International Commission on Radiological Protection. ICRP Publication 103. Ann. ICRP 37 $(2-4) ; 2007$.

International Commission on Radiological Protection. Nuclear Decay Data for Dosimetric Calculations. ICRP Publication 107. Ann. ICRP 38 (3); 2008.

Jannik GT et al. Land and Water use Characteristics and Human Health Input Parameters for use in Environmental Dosimetry and Risk Assessments as the Savannah River Site. , SRNLSTI-2010-00447, Aiken, SC: Savannah River National Laboratory; 2010.

Hamby, D.M. Land and water use characteristics in the vicinity of the Savannah River Site. National Technical Information Service. Washington, DC: Report No. DE91013543XSP; 1991.

National Institute of Standards and Technology. NIST Handbook 44 - 2012 Edition, Section 3.35; 2012. http://www.nist.gov/pml/wmd/h44-12.cfm

U.S. Nuclear Regulatory Commission. Regulatory Guide 1.109 Calculation of Annual Doses to Man From Routine Releases of Reactor Effluents for the Purpose of Evaluating Compliance with 10 CFR 50, Appendix I. Washington, DC: U.S. NRC; 1977. 


\section{APPENDIX A}

\section{REFERENCE PERSON \\ INGESTION DOSE COEFFICIENTS}

Appendix A tabulates the ingestion dose coefficients to be used at SRS. The dose coefficients are from DOE Standard (DOE-STD-1196-2011): Derived Concentration Technical Standard.

Also included is a copy of the formal clarification from DOE-HQ that allows the use of these dose coefficients for all ingestion pathways. 


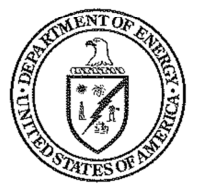

Department of Energy

Washington, DC 20585

January 9, 2013

MEMORANDUM FOR DAVID C. MOODY

MANAGER

SAVANNAH RIVER OPERATIONS OFFICE

FROM

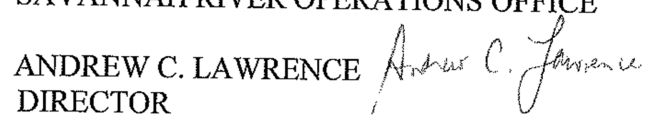

OFFICE OF ENVIRONMENTAL PROTECTION, SUSTAINABILITY

SUPPORT AND CORPORATE SAFETY ANALYSIS OFFICE OF HEALTH, SAFETY AND SECURITY

SUBJECT:

Proper Use of Reference Person Water-Ingestion Derived Dose Coefficients

The Office of Health, Safety and Security (HSS) has reviewed the Savannah River Operations Office (SR) memorandum, dated November 20, 2012. In this memo you requested approval to use the Reference Person water-ingestion weighted dose coefficients contained in the Department of Energy (DOE) standard (STD) DOE-STD-1196-2011, Derived Concentration Technical Standard (DCS), for all ingestion pathway dose calculations.

The dose coefficients for the Reference Person in Appendix A, Tables A-1 and A-2, and the dose rate coefficients in Table A-3 are "DOE-approved dose coefficients" per DOE Order (O) 458.1, Radiation Protection of the Public and the Environment, Section 4.e.(7). They may be used by $\mathrm{SR}$ and all other DOE offices for calculating doses from effluent emissions to demonstrate compliance with the Order. Alternatives to these coefficients require approval as specified in DOE O 458.1. The dose coefficients presented in the last column for "Reference Person" of Table A-1, are the "DOE approved dose coefficients" for all ingestion pathways. See the attachment for additional details.

We appreciate your efforts in calculating and comparing site-specific dose coefficients with the dose coefficients for water-ingestion from the DCS. Results of this effort illustrate that the differences between the two sets of values are relatively minor and thus provide further justification that the data tables provided in Appendix A of the DCS are sufficient and appropriate for use throughout the DOE complex. There is no need to develop a new set of dose coefficients that are site specific for demonstration of compliance with DOE O 458.1.

For additional information on the DCS please contact Mr. Edward Regnier of the Office of Environmental Policy and Assistance at (202)586-5027 or edward.regnier@hq.doe.gov.

Attachment

cc: David Huizenga, EM-1

Tracy Mustin, EM-1

Printed with soy ink on recycled paper 


\section{Attachment \\ Proper use of Reference Person Water-Ingestion Derived Dose Coefficients}

BACKGROUND: The DOE Standard, Derived Concentration Technical Standard, DOE-STD1196-2011, was developed largely to provide:

(1) The Derived Concentration Standards (Table 5 of the Standard) to support determinations for the need to use of Best Available Technology (BAT) for controlling liquid effluent discharges per DOE Order (O) 458.1, section 4.g.(5); and

(2) Approved dose coefficients (Appendix A of the standard) for calculating doses to the public in order to demonstrate compliance with DOE O 458.1 dose limits and constraints.

The Derived Concentration Standards are radiological quantities to be used in the design and conduct of radiological environmental protection programs at DOE facilities and sites. If liquid discharges from a DOE facility would contain concentrations of radionuclides that exceed the applicable Derived Concentration Standards in Table 5 then treatment of the effluent by BAT needs to be applied. Compliance with DOE O 458.1 dose limits and constraints is required whether or not BAT treatment is needed.

The tables of DOE-approved dose coefficients for a Reference Person, provided in Appendix A of the Standard, for ingestion of water, inhalati on, and submersion, are to be used for estimating doses to the public for demonstrating compliance with the Order.

DISCUSSION: The Derived Concentration Standards and the dose coefficients in DOE-STD1196 reflect the current state of knowledge and practice in radiation protection. The effective dose coefficients for Reference Person, which are both age- and gender-weighted, were developed utilizing dose methodologies from the International Commission on Radiological Protection (ICRP) 60, Recommendations of the International Commission on Radiological Protection, published in 1991. The effective dose coefficients' development used new sophisticated biokinetic and dosimetric models, data from ICRP 72 (ICRP 1996), Age Dependent Doses to Members of the Public and from Intake of Radionuclides; ICRP 89 (ICRP 2002), Basic Anatomical and Physiological Data for Use in Radiological Protection; ICRP 107(ICRP 2008), Nuclear Decay Data for Dosimetric Calculations; and the Environmental Protection Agency's Federal Guidance Report 13, Cancer Risk Coefficients for Environmental Exposure to Radionuclides.

Dose coefficients for ingestion of water can be used for all forms of ingestion for a Reference Person. As with previous dose coefficients derived under ICRP 26/30 dose methodologies, they are sufficient and appropriate for use throughout the DOE complex.

DOE dose limits and constraints contained in DOE O 458.1 are intended to protect the general public from long-term exposure. The Order achieves this through use of a Reference Person that takes into account the ages and the gender of the population being protected. It is not necessary or appropriate under typical situations to estimate separate doses to various age groups or genders in order to achieve long term protection. Derivation of doses using the Standard's dose 
SRNL-STI-2013-00115

coefficients for Reference Person is sufficient and appropriate to assure adequate protection from radiation exposure to the public for chronic exposures (a lifetime). The dose limits in DOE O 458.1 and implementation of the ALARA process provide an ample margin of safety to ensure the public is protected from DOE operations. While there is a requirement to regulate on the basis of yearly dose for compliance purposes, the main purpose is to minimize the lifetime radiological risk to the public. Calculating the dose to every age group individually is not warranted by the data quality objectives (DQOs) needed to determine protection of the population over their lifetime nor by the precision (or lack thereof) of the data and assumptions which are used in such calculations.

Columns other than the Reference Person column within the appendices for all pathways (ingestion, inhalation, and submersion) are primarily for informational purposes, and DOE does not recommend their use under normal circumstances. The dose limits and constraints in the Order are applicable to a Reference Person, not specific individuals. DOE neither requires nor advocates the calculation of separate doses to each of the six age groups listed in Tables A-1, A-2, and A-3 of the Standard. The ICRP 103, The 2007 Recommendations of the International Commission on Radiological Protection, recognizes this and states:

"The Commission's risk estimates are called 'nominal' because they relate to the exposure of a nominal population of females and males with a typical age distribution and are computed by averaging over age groups and both sexes. The dosimetric quantity recommended for radiological protection, effective dose, is also computed by age- and sex-averaging."

As new information with a sound technical basis is developed by national and international advisory groups on radiological protection of the public and the environment, we will continue to update our standards to reflect the best available science.

Further, the dose coefficients used within the Standard are for a Reference Person that is a member of the general public. Requirements for assessment of occupational exposures of DOE workers are specified in 10 Code of Federal Regulations 835, Occupational Radiation Protection. Guidance on meeting these requirements is found in DOE Guide 441.1-1C, Radiation Protection Programs Guide for Use with Title 10, Code of Federal Regulations, Part 835, Occupational Radiation Protection. Likewise dose coefficients in the Standard may not be appropriate for dose reconstruction analysis or individual dose assessments. 
Table A-1 Ingestion Dose Coefficients

\begin{tabular}{|c|c|c|c|}
\hline Nuclide & $\begin{array}{l}\text { Ingestion } \\
\text { Dose } \\
\text { Coefficient } \\
(\mathrm{Sv} / \mathrm{Bq})\end{array}$ & $\begin{array}{l}\text { Ingestion } \\
\text { Dose } \\
\text { Coefficient } \\
\text { (mrem/Ci) }\end{array}$ & $\begin{array}{l}\text { Ingestion } \\
\text { Dose } \\
\text { Coefficient } \\
\text { (mrem/pCi) }\end{array}$ \\
\hline H-3 & $2.10 \mathrm{E}-11$ & $7.77 \mathrm{E}+04$ & $7.77 \mathrm{E}-08$ \\
\hline $\mathrm{Be}-7$ & $3.48 \mathrm{E}-11$ & $1.29 \mathrm{E}+05$ & $1.29 \mathrm{E}-07$ \\
\hline Be-10 & $1.56 \mathrm{E}-09$ & $5.77 \mathrm{E}+06$ & $5.77 \mathrm{E}-06$ \\
\hline $\mathrm{C}-11$ & $3.10 \mathrm{E}-11$ & $1.15 \mathrm{E}+05$ & $1.15 \mathrm{E}-07$ \\
\hline C-14 & $6.33 \mathrm{E}-10$ & $2.34 \mathrm{E}+06$ & $2.34 \mathrm{E}-06$ \\
\hline F-18 & $6.24 \mathrm{E}-11$ & $2.31 \mathrm{E}+05$ & $2.31 \mathrm{E}-07$ \\
\hline $\mathrm{Na}-22$ & $3.88 \mathrm{E}-09$ & $1.44 \mathrm{E}+07$ & 1.44E-05 \\
\hline $\mathrm{Na}-24$ & $5.46 \mathrm{E}-10$ & $2.02 \mathrm{E}+06$ & $2.02 \mathrm{E}-06$ \\
\hline Mg-28 & $2.82 \mathrm{E}-09$ & $1.04 \mathrm{E}+07$ & $1.04 \mathrm{E}-05$ \\
\hline Al-26 & $4.59 \mathrm{E}-09$ & $1.70 \mathrm{E}+07$ & $1.70 \mathrm{E}-05$ \\
\hline Si-31 & $2.13 \mathrm{E}-10$ & $7.88 \mathrm{E}+05$ & $7.88 \mathrm{E}-07$ \\
\hline Si-32 & $7.99 \mathrm{E}-10$ & $2.96 \mathrm{E}+06$ & $2.96 \mathrm{E}-06$ \\
\hline P-32 & $3.39 \mathrm{E}-09$ & $1.25 \mathrm{E}+07$ & $1.25 \mathrm{E}-05$ \\
\hline P-33 & $3.37 \mathrm{E}-10$ & $1.25 \mathrm{E}+06$ & $1.25 \mathrm{E}-06$ \\
\hline S-35 & $1.74 \mathrm{E}-10$ & $6.44 \mathrm{E}+05$ & $6.44 \mathrm{E}-07$ \\
\hline S-38 & $4.34 \mathrm{E}-10$ & $1.61 \mathrm{E}+06$ & $1.61 \mathrm{E}-06$ \\
\hline Cl-34m & $1.37 \mathrm{E}-10$ & $5.07 \mathrm{E}+05$ & $5.07 \mathrm{E}-07$ \\
\hline $\mathrm{Cl}-36$ & $1.24 \mathrm{E}-09$ & $4.59 \mathrm{E}+06$ & $4.59 \mathrm{E}-06$ \\
\hline Cl-38 & $1.56 \mathrm{E}-10$ & $5.77 \mathrm{E}+05$ & $5.77 \mathrm{E}-07$ \\
\hline Cl-39 & $1.13 \mathrm{E}-10$ & $4.18 \mathrm{E}+05$ & $4.18 \mathrm{E}-07$ \\
\hline $\mathrm{K}-40$ & $8.22 \mathrm{E}-09$ & $3.04 \mathrm{E}+07$ & $3.04 \mathrm{E}-05$ \\
\hline K-42 & $5.89 \mathrm{E}-10$ & $2.18 \mathrm{E}+06$ & $2.18 \mathrm{E}-06$ \\
\hline $\mathrm{K}-43$ & $3.20 \mathrm{E}-10$ & $1.18 \mathrm{E}+06$ & $1.18 \mathrm{E}-06$ \\
\hline $\mathrm{K}-44$ & $1.11 \mathrm{E}-10$ & $4.11 \mathrm{E}+05$ & $4.11 \mathrm{E}-07$ \\
\hline K-45 & $6.48 \mathrm{E}-11$ & $2.40 \mathrm{E}+05$ & $2.40 \mathrm{E}-07$ \\
\hline $\mathrm{Ca}-41$ & $2.96 \mathrm{E}-10$ & $1.10 \mathrm{E}+06$ & $1.10 \mathrm{E}-06$ \\
\hline Ca-45 & $1.04 \mathrm{E}-09$ & $3.85 \mathrm{E}+06$ & $3.85 \mathrm{E}-06$ \\
\hline $\mathrm{Ca}-47$ & $2.05 \mathrm{E}-09$ & $7.59 \mathrm{E}+06$ & $7.59 \mathrm{E}-06$ \\
\hline Sc-43 & $2.91 \mathrm{E}-10$ & $1.08 \mathrm{E}+06$ & $1.08 \mathrm{E}-06$ \\
\hline Sc-44 & $4.72 \mathrm{E}-10$ & $1.75 \mathrm{E}+06$ & $1.75 \mathrm{E}-06$ \\
\hline Sc-44m & $3.26 \mathrm{E}-09$ & $1.21 \mathrm{E}+07$ & $1.21 \mathrm{E}-05$ \\
\hline Sc-46 & $1.88 \mathrm{E}-09$ & $6.96 \mathrm{E}+06$ & $6.96 \mathrm{E}-06$ \\
\hline Sc-47 & $7.46 \mathrm{E}-10$ & $2.76 \mathrm{E}+06$ & $2.76 \mathrm{E}-06$ \\
\hline Sc-48 & $2.14 \mathrm{E}-09$ & $7.92 \mathrm{E}+06$ & 7.92E-06 \\
\hline Sc-49 & $1.10 \mathrm{E}-10$ & $4.07 \mathrm{E}+05$ & 4.07E-07 \\
\hline Ti-44 & 7.41E-09 & $2.74 \mathrm{E}+07$ & $2.74 \mathrm{E}-05$ \\
\hline Ti-45 & $2.01 \mathrm{E}-10$ & $7.44 \mathrm{E}+05$ & $7.44 \mathrm{E}-07$ \\
\hline V-47 & $8.37 \mathrm{E}-11$ & $3.10 \mathrm{E}+05$ & $3.10 \mathrm{E}-07$ \\
\hline
\end{tabular}




\begin{tabular}{|c|c|c|c|}
\hline Nuclide & $\begin{array}{l}\text { Ingestion } \\
\text { Dose } \\
\text { Coefficient } \\
\text { (Sv/Bq) }\end{array}$ & $\begin{array}{c}\text { Ingestion } \\
\text { Dose } \\
\text { Coefficient } \\
\text { (mrem/Ci) }\end{array}$ & $\begin{array}{c}\text { Ingestion } \\
\text { Dose } \\
\text { Coefficient } \\
\text { (mrem/pCi) }\end{array}$ \\
\hline V-48 & $2.54 \mathrm{E}-09$ & $9.40 \mathrm{E}+06$ & $9.40 \mathrm{E}-06$ \\
\hline V-49 & $2.53 \mathrm{E}-11$ & $9.36 \mathrm{E}+04$ & $9.36 \mathrm{E}-08$ \\
\hline $\mathrm{V}-50$ & $3.75 \mathrm{E}-09$ & $1.39 \mathrm{E}+07$ & $1.39 \mathrm{E}-05$ \\
\hline $\mathrm{Cr}-48$ & $2.47 \mathrm{E}-10$ & $9.14 \mathrm{E}+05$ & $9.14 \mathrm{E}-07$ \\
\hline Cr-49 & $8.13 \mathrm{E}-11$ & $3.01 \mathrm{E}+05$ & $3.01 \mathrm{E}-07$ \\
\hline Cr-51 & $5.03 \mathrm{E}-11$ & $1.86 \mathrm{E}+05$ & $1.86 \mathrm{E}-07$ \\
\hline Mn-51 & $1.23 \mathrm{E}-10$ & $4.55 \mathrm{E}+05$ & $4.55 \mathrm{E}-07$ \\
\hline Mn-52 & $2.26 \mathrm{E}-09$ & $8.36 \mathrm{E}+06$ & $8.36 \mathrm{E}-06$ \\
\hline Mn-52m & $9.17 \mathrm{E}-11$ & $3.39 \mathrm{E}+05$ & $3.39 \mathrm{E}-07$ \\
\hline Mn-53 & $4.14 \mathrm{E}-11$ & $1.53 \mathrm{E}+05$ & $1.53 \mathrm{E}-07$ \\
\hline Mn-54 & $8.90 \mathrm{E}-10$ & $3.29 \mathrm{E}+06$ & $3.29 \mathrm{E}-06$ \\
\hline Mn-56 & $3.41 \mathrm{E}-10$ & $1.26 \mathrm{E}+06$ & $1.26 \mathrm{E}-06$ \\
\hline $\mathrm{Fe}-52$ & $1.83 \mathrm{E}-09$ & $6.77 \mathrm{E}+06$ & $6.77 \mathrm{E}-06$ \\
\hline Fe-55 & $5.51 \mathrm{E}-10$ & $2.04 \mathrm{E}+06$ & $2.04 \mathrm{E}-06$ \\
\hline Fe-59 & $2.74 \mathrm{E}-09$ & $1.01 \mathrm{E}+07$ & $1.01 \mathrm{E}-05$ \\
\hline Fe-60 & $1.48 \mathrm{E}-07$ & $5.48 \mathrm{E}+08$ & $5.48 \mathrm{E}-04$ \\
\hline Co-55 & $1.27 \mathrm{E}-09$ & $4.70 \mathrm{E}+06$ & $4.70 \mathrm{E}-06$ \\
\hline Co-56 & 3.44E-09 & $1.27 \mathrm{E}+07$ & $1.27 \mathrm{E}-05$ \\
\hline Co-57 & $3.13 \mathrm{E}-10$ & $1.16 \mathrm{E}+06$ & $1.16 \mathrm{E}-06$ \\
\hline Co-58 & 1.01E-09 & $3.74 \mathrm{E}+06$ & $3.74 \mathrm{E}-06$ \\
\hline Co-58m & $3.10 \mathrm{E}-11$ & $1.15 \mathrm{E}+05$ & $1.15 \mathrm{E}-07$ \\
\hline Co-60 & $5.49 \mathrm{E}-09$ & $2.03 \mathrm{E}+07$ & $2.03 \mathrm{E}-05$ \\
\hline Co-60m & $2.27 \mathrm{E}-12$ & $8.40 \mathrm{E}+03$ & $8.40 \mathrm{E}-09$ \\
\hline Co-61 & $1.00 \mathrm{E}-10$ & $3.70 \mathrm{E}+05$ & $3.70 \mathrm{E}-07$ \\
\hline Co-62m & $6.48 \mathrm{E}-11$ & $2.40 \mathrm{E}+05$ & $2.40 \mathrm{E}-07$ \\
\hline $\mathrm{Ni}-56$ & $1.08 \mathrm{E}-09$ & $4.00 \mathrm{E}+06$ & $4.00 \mathrm{E}-06$ \\
\hline $\mathrm{Ni}-57$ & $1.17 \mathrm{E}-09$ & $4.33 \mathrm{E}+06$ & $4.33 \mathrm{E}-06$ \\
\hline Ni-59 & $7.96 \mathrm{E}-11$ & $2.95 \mathrm{E}+05$ & $2.95 \mathrm{E}-07$ \\
\hline Ni-63 & $1.98 \mathrm{E}-10$ & $7.33 \mathrm{E}+05$ & $7.33 \mathrm{E}-07$ \\
\hline Ni-65 & $2.46 \mathrm{E}-10$ & $9.10 \mathrm{E}+05$ & $9.10 \mathrm{E}-07$ \\
\hline $\mathrm{Ni}-66$ & $4.15 \mathrm{E}-09$ & $1.54 \mathrm{E}+07$ & $1.54 \mathrm{E}-05$ \\
\hline $\mathrm{Cu}-60$ & $9.33 \mathrm{E}-11$ & $3.45 \mathrm{E}+05$ & $3.45 \mathrm{E}-07$ \\
\hline $\mathrm{Cu}-61$ & $1.49 \mathrm{E}-10$ & $5.51 \mathrm{E}+05$ & $5.51 \mathrm{E}-07$ \\
\hline $\mathrm{Cu}-64$ & $1.59 \mathrm{E}-10$ & $5.88 \mathrm{E}+05$ & $5.88 \mathrm{E}-07$ \\
\hline $\mathrm{Cu}-67$ & $4.35 \mathrm{E}-10$ & $1.61 \mathrm{E}+06$ & $1.61 \mathrm{E}-06$ \\
\hline $\mathrm{Zn}-62$ & $1.22 \mathrm{E}-09$ & $4.51 \mathrm{E}+06$ & $4.51 \mathrm{E}-06$ \\
\hline Zn-63 & $1.06 \mathrm{E}-10$ & $3.92 \mathrm{E}+05$ & $3.92 \mathrm{E}-07$ \\
\hline Zn-65 & $4.77 \mathrm{E}-09$ & $1.76 \mathrm{E}+07$ & $1.76 \mathrm{E}-05$ \\
\hline Zn-69 & $4.14 \mathrm{E}-11$ & $1.53 \mathrm{E}+05$ & $1.53 \mathrm{E}-07$ \\
\hline Zn-69m & $4.33 \mathrm{E}-10$ & $1.60 \mathrm{E}+06$ & $1.60 \mathrm{E}-06$ \\
\hline
\end{tabular}




\begin{tabular}{|c|c|c|c|}
\hline Nuclide & $\begin{array}{c}\text { Ingestion } \\
\text { Dose } \\
\text { Coefficient } \\
\text { (Sv/Bq) }\end{array}$ & $\begin{array}{c}\text { Ingestion } \\
\text { Dose } \\
\text { Coefficient } \\
\text { (mrem/Ci) }\end{array}$ & $\begin{array}{c}\text { Ingestion } \\
\text { Dose } \\
\text { Coefficient } \\
\text { (mrem/pCi) }\end{array}$ \\
\hline Zn-71m & $3.10 \mathrm{E}-10$ & $1.15 \mathrm{E}+06$ & $1.15 \mathrm{E}-06$ \\
\hline Zn-72 & $1.82 \mathrm{E}-09$ & $6.73 \mathrm{E}+06$ & $6.73 \mathrm{E}-06$ \\
\hline Ga-65 & $4.86 \mathrm{E}-11$ & $1.80 \mathrm{E}+05$ & $1.80 \mathrm{E}-07$ \\
\hline Ga-66 & $1.59 \mathrm{E}-09$ & $5.88 \mathrm{E}+06$ & $5.88 \mathrm{E}-06$ \\
\hline Ga-67 & $2.60 \mathrm{E}-10$ & $9.62 \mathrm{E}+05$ & $9.62 \mathrm{E}-07$ \\
\hline Ga-68 & $1.36 \mathrm{E}-10$ & $5.03 \mathrm{E}+05$ & $5.03 \mathrm{E}-07$ \\
\hline Ga-70 & $4.22 \mathrm{E}-11$ & $1.56 \mathrm{E}+05$ & $1.56 \mathrm{E}-07$ \\
\hline Ga-72 & $1.47 \mathrm{E}-09$ & $5.44 \mathrm{E}+06$ & $5.44 \mathrm{E}-06$ \\
\hline Ga-73 & $3.59 \mathrm{E}-10$ & $1.33 \mathrm{E}+06$ & $1.33 \mathrm{E}-06$ \\
\hline Ge-66 & $1.28 \mathrm{E}-10$ & $4.74 \mathrm{E}+05$ & $4.74 \mathrm{E}-07$ \\
\hline Ge-67 & $7.91 \mathrm{E}-11$ & $2.93 \mathrm{E}+05$ & $2.93 \mathrm{E}-07$ \\
\hline Ge-68 & $1.69 \mathrm{E}-09$ & $6.25 \mathrm{E}+06$ & $6.25 \mathrm{E}-06$ \\
\hline Ge-69 & $2.61 \mathrm{E}-10$ & $9.66 \mathrm{E}+05$ & $9.66 \mathrm{E}-07$ \\
\hline Ge-71 & $1.58 \mathrm{E}-11$ & $5.85 \mathrm{E}+04$ & $5.85 \mathrm{E}-08$ \\
\hline Ge-75 & $6.19 \mathrm{E}-11$ & $2.29 \mathrm{E}+05$ & $2.29 \mathrm{E}-07$ \\
\hline Ge-77 & $4.21 \mathrm{E}-10$ & $1.56 \mathrm{E}+06$ & $1.56 \mathrm{E}-06$ \\
\hline Ge-78 & $1.47 \mathrm{E}-10$ & $5.44 \mathrm{E}+05$ & $5.44 \mathrm{E}-07$ \\
\hline As-69 & $7.26 \mathrm{E}-11$ & $2.69 \mathrm{E}+05$ & $2.69 \mathrm{E}-07$ \\
\hline As-70 & $1.76 \mathrm{E}-10$ & $6.51 \mathrm{E}+05$ & $6.51 \mathrm{E}-07$ \\
\hline As-71 & $5.86 \mathrm{E}-10$ & $2.17 \mathrm{E}+06$ & $2.17 \mathrm{E}-06$ \\
\hline As-72 & $2.42 \mathrm{E}-09$ & $8.95 \mathrm{E}+06$ & $8.95 \mathrm{E}-06$ \\
\hline As-73 & $3.51 \mathrm{E}-10$ & $1.30 \mathrm{E}+06$ & $1.30 \mathrm{E}-06$ \\
\hline As-74 & $1.68 \mathrm{E}-09$ & $6.22 \mathrm{E}+06$ & $6.22 \mathrm{E}-06$ \\
\hline As-76 & $2.11 \mathrm{E}-09$ & $7.81 \mathrm{E}+06$ & $7.81 \mathrm{E}-06$ \\
\hline As-77 & $5.27 \mathrm{E}-10$ & $1.95 \mathrm{E}+06$ & $1.95 \mathrm{E}-06$ \\
\hline As-78 & $2.61 \mathrm{E}-10$ & $9.66 \mathrm{E}+05$ & $9.66 \mathrm{E}-07$ \\
\hline Se-70 & $1.25 \mathrm{E}-10$ & $4.63 \mathrm{E}+05$ & $4.63 \mathrm{E}-07$ \\
\hline Se-72 & $8.23 \mathrm{E}-09$ & $3.05 \mathrm{E}+07$ & $3.05 \mathrm{E}-05$ \\
\hline Se-73 & $2.78 \mathrm{E}-10$ & $1.03 \mathrm{E}+06$ & $1.03 \mathrm{E}-06$ \\
\hline Se-73m & $3.67 \mathrm{E}-11$ & $1.36 \mathrm{E}+05$ & $1.36 \mathrm{E}-07$ \\
\hline Se-75 & $3.35 \mathrm{E}-09$ & $1.24 \mathrm{E}+07$ & $1.24 \mathrm{E}-05$ \\
\hline Se-79 & $4.68 \mathrm{E}-09$ & $1.73 \mathrm{E}+07$ & $1.73 \mathrm{E}-05$ \\
\hline Se-81 & $3.61 \mathrm{E}-11$ & $1.34 \mathrm{E}+05$ & $1.34 \mathrm{E}-07$ \\
\hline Se-81m & $7.16 \mathrm{E}-11$ & $2.65 \mathrm{E}+05$ & $2.65 \mathrm{E}-07$ \\
\hline Se-83 & $5.87 \mathrm{E}-11$ & $2.17 \mathrm{E}+05$ & $2.17 \mathrm{E}-07$ \\
\hline Br-74 & $1.07 \mathrm{E}-10$ & $3.96 \mathrm{E}+05$ & $3.96 \mathrm{E}-07$ \\
\hline Br-74m & $1.76 \mathrm{E}-10$ & $6.51 \mathrm{E}+05$ & $6.51 \mathrm{E}-07$ \\
\hline Br-75 & $1.03 \mathrm{E}-10$ & $3.81 \mathrm{E}+05$ & $3.81 \mathrm{E}-07$ \\
\hline Br-76 & $5.92 \mathrm{E}-10$ & $2.19 \mathrm{E}+06$ & $2.19 \mathrm{E}-06$ \\
\hline Br-77 & $1.20 \mathrm{E}-10$ & $4.44 \mathrm{E}+05$ & $4.44 \mathrm{E}-07$ \\
\hline
\end{tabular}




\begin{tabular}{|c|c|c|c|}
\hline Nuclide & $\begin{array}{l}\text { Ingestion } \\
\text { Dose } \\
\text { Coefficient } \\
\text { (Sv/Bq) }\end{array}$ & $\begin{array}{l}\text { Ingestion } \\
\text { Dose } \\
\text { Coefficient } \\
\text { (mrem/Ci) }\end{array}$ & $\begin{array}{c}\text { Ingestion } \\
\text { Dose } \\
\text { Coefficient } \\
\text { (mrem/pCi) }\end{array}$ \\
\hline $\mathrm{Br}-80$ & $4.18 \mathrm{E}-11$ & $1.55 \mathrm{E}+05$ & $1.55 \mathrm{E}-07$ \\
\hline $\mathrm{Br}-80 \mathrm{~m}$ & $1.55 \mathrm{E}-10$ & $5.74 \mathrm{E}+05$ & $5.74 \mathrm{E}-07$ \\
\hline $\mathrm{Br}-82$ & $6.75 \mathrm{E}-10$ & $2.50 \mathrm{E}+06$ & $2.50 \mathrm{E}-06$ \\
\hline $\mathrm{Br}-83$ & $5.98 \mathrm{E}-11$ & $2.21 \mathrm{E}+05$ & $2.21 \mathrm{E}-07$ \\
\hline $\mathrm{Br}-84$ & $1.18 \mathrm{E}-10$ & $4.37 \mathrm{E}+05$ & $4.37 \mathrm{E}-07$ \\
\hline Rb-78 & $9.29 \mathrm{E}-11$ & $3.44 \mathrm{E}+05$ & $3.44 \mathrm{E}-07$ \\
\hline Rb-79 & $6.58 \mathrm{E}-11$ & $2.43 \mathrm{E}+05$ & $2.43 \mathrm{E}-07$ \\
\hline $\mathrm{Rb}-81$ & $6.31 \mathrm{E}-11$ & $2.33 \mathrm{E}+05$ & $2.33 \mathrm{E}-07$ \\
\hline $\mathrm{Rb}-81 \mathrm{~m}$ & $1.25 \mathrm{E}-11$ & $4.63 \mathrm{E}+04$ & $4.63 \mathrm{E}-08$ \\
\hline $\mathrm{Rb}-82 \mathrm{~m}$ & $1.63 \mathrm{E}-10$ & $6.03 \mathrm{E}+05$ & $6.03 \mathrm{E}-07$ \\
\hline Rb-83 & $2.14 \mathrm{E}-09$ & $7.92 \mathrm{E}+06$ & $7.92 \mathrm{E}-06$ \\
\hline Rb-84 & $3.52 \mathrm{E}-09$ & $1.30 \mathrm{E}+07$ & $1.30 \mathrm{E}-05$ \\
\hline $\mathrm{Rb}-84 \mathrm{~m}$ & $9.16 \mathrm{E}-12$ & $3.39 \mathrm{E}+04$ & $3.39 \mathrm{E}-08$ \\
\hline Rb-86 & $3.81 \mathrm{E}-09$ & $1.41 \mathrm{E}+07$ & $1.41 \mathrm{E}-05$ \\
\hline $\mathrm{Rb}-87$ & $2.05 \mathrm{E}-09$ & $7.59 \mathrm{E}+06$ & $7.59 \mathrm{E}-06$ \\
\hline Rb-88 & $1.22 \mathrm{E}-10$ & $4.51 \mathrm{E}+05$ & $4.51 \mathrm{E}-07$ \\
\hline $\mathrm{Rb}-89$ & $5.95 \mathrm{E}-11$ & $2.20 \mathrm{E}+05$ & $2.20 \mathrm{E}-07$ \\
\hline Sr-80 & $5.01 \mathrm{E}-10$ & $1.85 \mathrm{E}+06$ & $1.85 \mathrm{E}-06$ \\
\hline Sr-81 & $8.84 \mathrm{E}-11$ & $3.27 \mathrm{E}+05$ & $3.27 \mathrm{E}-07$ \\
\hline Sr-82 & $8.43 \mathrm{E}-09$ & $3.12 \mathrm{E}+07$ & $3.12 \mathrm{E}-05$ \\
\hline Sr-83 & $6.46 \mathrm{E}-10$ & $2.39 \mathrm{E}+06$ & $2.39 \mathrm{E}-06$ \\
\hline Sr-85 & $7.94 \mathrm{E}-10$ & $2.94 \mathrm{E}+06$ & $2.94 \mathrm{E}-06$ \\
\hline Sr- $85 \mathrm{~m}$ & $7.53 \mathrm{E}-12$ & $2.79 \mathrm{E}+04$ & $2.79 \mathrm{E}-08$ \\
\hline Sr-87m & $3.86 \mathrm{E}-11$ & $1.43 \mathrm{E}+05$ & $1.43 \mathrm{E}-07$ \\
\hline Sr-89 & $3.61 \mathrm{E}-09$ & $1.34 \mathrm{E}+07$ & $1.34 \mathrm{E}-05$ \\
\hline Sr-90 & $3.60 \mathrm{E}-08$ & $1.33 \mathrm{E}+08$ & $1.33 \mathrm{E}-04$ \\
\hline Sr-91 & $8.47 \mathrm{E}-10$ & $3.13 \mathrm{E}+06$ & $3.13 \mathrm{E}-06$ \\
\hline Sr-92 & $5.44 \mathrm{E}-10$ & $2.01 \mathrm{E}+06$ & $2.01 \mathrm{E}-06$ \\
\hline Y-84m & $1.79 \mathrm{E}-10$ & $6.62 \mathrm{E}+05$ & $6.62 \mathrm{E}-07$ \\
\hline Y-85 & $2.43 \mathrm{E}-10$ & $8.99 \mathrm{E}+05$ & $8.99 \mathrm{E}-07$ \\
\hline $\mathrm{Y}-85 \mathrm{~m}$ & $4.94 \mathrm{E}-10$ & $1.83 \mathrm{E}+06$ & $1.83 \mathrm{E}-06$ \\
\hline Y-86 & $1.22 \mathrm{E}-09$ & $4.51 \mathrm{E}+06$ & $4.51 \mathrm{E}-06$ \\
\hline Y-86m & $7.17 \mathrm{E}-11$ & $2.65 \mathrm{E}+05$ & $2.65 \mathrm{E}-07$ \\
\hline Y-87 & $7.12 \mathrm{E}-10$ & $2.63 \mathrm{E}+06$ & $2.63 \mathrm{E}-06$ \\
\hline Y-87m & $2.89 \mathrm{E}-10$ & $1.07 \mathrm{E}+06$ & $1.07 \mathrm{E}-06$ \\
\hline Y-88 & 1.61E-09 & $5.96 \mathrm{E}+06$ & $5.96 \mathrm{E}-06$ \\
\hline Y-90 & $3.70 \mathrm{E}-09$ & $1.37 \mathrm{E}+07$ & $1.37 \mathrm{E}-05$ \\
\hline Y-90m & $2.35 \mathrm{E}-10$ & $8.70 \mathrm{E}+05$ & $8.70 \mathrm{E}-07$ \\
\hline Y-91 & $3.27 \mathrm{E}-09$ & $1.21 \mathrm{E}+07$ & $1.21 \mathrm{E}-05$ \\
\hline Y-91m & $1.46 \mathrm{E}-11$ & $5.40 \mathrm{E}+04$ & $5.40 \mathrm{E}-08$ \\
\hline
\end{tabular}




\begin{tabular}{|c|c|c|c|}
\hline Nuclide & $\begin{array}{c}\text { Ingestion } \\
\text { Dose } \\
\text { Coefficient } \\
(\mathbf{S v} / \mathbf{B q})\end{array}$ & $\begin{array}{l}\text { Ingestion } \\
\text { Dose } \\
\text { Coefficient } \\
\text { (mrem } / \mathbf{C i})\end{array}$ & $\begin{array}{c}\text { Ingestion } \\
\text { Dose } \\
\text { Coefficient } \\
\text { (mrem/pCi) }\end{array}$ \\
\hline Y-92 & $6.79 \mathrm{E}-10$ & $2.51 \mathrm{E}+06$ & $2.51 \mathrm{E}-06$ \\
\hline Y-93 & $1.60 \mathrm{E}-09$ & $5.92 \mathrm{E}+06$ & $5.92 \mathrm{E}-06$ \\
\hline Y-94 & $1.13 \mathrm{E}-10$ & $4.18 \mathrm{E}+05$ & $4.18 \mathrm{E}-07$ \\
\hline Y-95 & $5.71 \mathrm{E}-11$ & $2.11 \mathrm{E}+05$ & $2.11 \mathrm{E}-07$ \\
\hline Zr-86 & $1.10 \mathrm{E}-09$ & $4.07 \mathrm{E}+06$ & $4.07 \mathrm{E}-06$ \\
\hline Zr-87 & $2.51 \mathrm{E}-10$ & $9.29 \mathrm{E}+05$ & $9.29 \mathrm{E}-07$ \\
\hline Zr-88 & $5.37 \mathrm{E}-10$ & $1.99 \mathrm{E}+06$ & $1.99 \mathrm{E}-06$ \\
\hline Zr-89 & $1.02 \mathrm{E}-09$ & $3.77 \mathrm{E}+06$ & $3.77 \mathrm{E}-06$ \\
\hline Zr-93 & $1.00 \mathrm{E}-09$ & $3.70 \mathrm{E}+06$ & $3.70 \mathrm{E}-06$ \\
\hline Zr-95 & $1.26 \mathrm{E}-09$ & $4.66 \mathrm{E}+06$ & $4.66 \mathrm{E}-06$ \\
\hline Zr-97 & $2.80 \mathrm{E}-09$ & $1.04 \mathrm{E}+07$ & $1.04 \mathrm{E}-05$ \\
\hline $\mathrm{Nb}-88$ & $9.43 \mathrm{E}-11$ & $3.49 \mathrm{E}+05$ & $3.49 \mathrm{E}-07$ \\
\hline $\mathrm{Nb}-89$ & $3.69 \mathrm{E}-10$ & $1.37 \mathrm{E}+06$ & $1.37 \mathrm{E}-06$ \\
\hline $\mathrm{Nb}-89 \mathrm{~m}$ & $1.80 \mathrm{E}-10$ & $6.66 \mathrm{E}+05$ & $6.66 \mathrm{E}-07$ \\
\hline Nb-90 & $1.62 \mathrm{E}-09$ & $5.99 \mathrm{E}+06$ & $5.99 \mathrm{E}-06$ \\
\hline Nb-91 & $6.22 \mathrm{E}-11$ & $2.30 \mathrm{E}+05$ & $2.30 \mathrm{E}-07$ \\
\hline Nb-91m & $5.65 \mathrm{E}-10$ & $2.09 \mathrm{E}+06$ & $2.09 \mathrm{E}-06$ \\
\hline $\mathrm{Nb}-92$ & $1.27 \mathrm{E}-09$ & $4.70 \mathrm{E}+06$ & $4.70 \mathrm{E}-06$ \\
\hline $\mathrm{Nb}-92 \mathrm{~m}$ & $6.26 \mathrm{E}-10$ & $2.32 \mathrm{E}+06$ & $2.32 \mathrm{E}-06$ \\
\hline $\mathrm{Nb}-93 \mathrm{~m}$ & $1.78 \mathrm{E}-10$ & $6.59 \mathrm{E}+05$ & $6.59 \mathrm{E}-07$ \\
\hline $\mathrm{Nb}-94$ & 2.23E-09 & $8.25 \mathrm{E}+06$ & $8.25 \mathrm{E}-06$ \\
\hline $\mathrm{Nb}-95$ & $7.50 \mathrm{E}-10$ & $2.78 \mathrm{E}+06$ & $2.78 \mathrm{E}-06$ \\
\hline $\mathrm{Nb}-95 \mathrm{~m}$ & $8.34 \mathrm{E}-10$ & $3.09 \mathrm{E}+06$ & 3.09E-06 \\
\hline Nb-96 & $1.42 \mathrm{E}-09$ & $5.25 \mathrm{E}+06$ & $5.25 \mathrm{E}-06$ \\
\hline $\mathrm{Nb}-97$ & $9.18 \mathrm{E}-11$ & $3.40 \mathrm{E}+05$ & $3.40 \mathrm{E}-07$ \\
\hline $\mathrm{Nb}-98 \mathrm{~m}$ & $1.42 \mathrm{E}-10$ & $5.25 \mathrm{E}+05$ & $5.25 \mathrm{E}-07$ \\
\hline Mo-90 & $2.77 \mathrm{E}-10$ & $1.02 \mathrm{E}+06$ & $1.02 \mathrm{E}-06$ \\
\hline Mo-91 & $8.03 \mathrm{E}-11$ & $2.97 \mathrm{E}+05$ & $2.97 \mathrm{E}-07$ \\
\hline Mo-93 & $3.12 \mathrm{E}-09$ & $1.15 \mathrm{E}+07$ & $1.15 \mathrm{E}-05$ \\
\hline Mo-93m & $1.47 \mathrm{E}-10$ & $5.44 \mathrm{E}+05$ & $5.44 \mathrm{E}-07$ \\
\hline Mo-99 & $7.73 \mathrm{E}-10$ & $2.86 \mathrm{E}+06$ & $2.86 \mathrm{E}-06$ \\
\hline Mo-101 & $5.33 \mathrm{E}-11$ & $1.97 \mathrm{E}+05$ & $1.97 \mathrm{E}-07$ \\
\hline Mo-102 & $9.32 \mathrm{E}-11$ & $3.45 \mathrm{E}+05$ & $3.45 \mathrm{E}-07$ \\
\hline Tc-93 & $8.84 \mathrm{E}-11$ & $3.27 \mathrm{E}+05$ & $3.27 \mathrm{E}-07$ \\
\hline Tc-93m & $4.11 \mathrm{E}-11$ & $1.52 \mathrm{E}+05$ & $1.52 \mathrm{E}-07$ \\
\hline Tc-94 & $2.53 \mathrm{E}-10$ & $9.36 \mathrm{E}+05$ & $9.36 \mathrm{E}-07$ \\
\hline Tc-94m & $1.36 \mathrm{E}-10$ & $5.03 \mathrm{E}+05$ & $5.03 \mathrm{E}-07$ \\
\hline Tc-95 & $2.26 \mathrm{E}-10$ & $8.36 \mathrm{E}+05$ & $8.36 \mathrm{E}-07$ \\
\hline Tc-95m & 7.19E-10 & $2.66 \mathrm{E}+06$ & $2.66 \mathrm{E}-06$ \\
\hline Tc-96 & $1.38 \mathrm{E}-09$ & $5.11 \mathrm{E}+06$ & $5.11 \mathrm{E}-06$ \\
\hline
\end{tabular}




\begin{tabular}{|c|c|c|c|}
\hline Nuclide & $\begin{array}{c}\text { Ingestion } \\
\text { Dose } \\
\text { Coefficient } \\
(\mathbf{S v} / \mathbf{B q})\end{array}$ & $\begin{array}{l}\text { Ingestion } \\
\text { Dose } \\
\text { Coefficient } \\
\text { (mrem/Ci) }\end{array}$ & $\begin{array}{c}\text { Ingestion } \\
\text { Dose } \\
\text { Coefficient } \\
\text { (mrem/pCi) }\end{array}$ \\
\hline Tc-96m & $1.58 \mathrm{E}-11$ & $5.85 \mathrm{E}+04$ & $5.85 \mathrm{E}-08$ \\
\hline Tc-97 & $9.47 \mathrm{E}-11$ & $3.50 \mathrm{E}+05$ & $3.50 \mathrm{E}-07$ \\
\hline Tc-97m & $7.70 \mathrm{E}-10$ & $2.85 \mathrm{E}+06$ & $2.85 \mathrm{E}-06$ \\
\hline Tc-98 & $2.43 \mathrm{E}-09$ & $8.99 \mathrm{E}+06$ & $8.99 \mathrm{E}-06$ \\
\hline Tc-99 & $9.00 \mathrm{E}-10$ & $3.33 \mathrm{E}+06$ & $3.33 \mathrm{E}-06$ \\
\hline Tc-99m & $2.91 \mathrm{E}-11$ & $1.08 \mathrm{E}+05$ & $1.08 \mathrm{E}-07$ \\
\hline Tc-101 & $2.50 \mathrm{E}-11$ & $9.25 \mathrm{E}+04$ & $9.25 \mathrm{E}-08$ \\
\hline Tc-104 & $1.09 \mathrm{E}-10$ & $4.03 \mathrm{E}+05$ & $4.03 \mathrm{E}-07$ \\
\hline Ru-94 & $1.25 \mathrm{E}-10$ & $4.63 \mathrm{E}+05$ & $4.63 \mathrm{E}-07$ \\
\hline Ru-95 & $7.75 \mathrm{E}-11$ & $2.87 \mathrm{E}+05$ & $2.87 \mathrm{E}-07$ \\
\hline Ru-97 & $1.94 \mathrm{E}-10$ & $7.18 \mathrm{E}+05$ & $7.18 \mathrm{E}-07$ \\
\hline Ru-103 & $9.41 \mathrm{E}-10$ & $3.48 \mathrm{E}+06$ & $3.48 \mathrm{E}-06$ \\
\hline $\mathrm{Ru}-105$ & $3.76 \mathrm{E}-10$ & $1.39 \mathrm{E}+06$ & $1.39 \mathrm{E}-06$ \\
\hline Ru-106 & $9.59 \mathrm{E}-09$ & $3.55 \mathrm{E}+07$ & $3.55 \mathrm{E}-05$ \\
\hline Rh-97 & $6.19 \mathrm{E}-11$ & $2.29 \mathrm{E}+05$ & $2.29 \mathrm{E}-07$ \\
\hline Rh-97m & $5.99 \mathrm{E}-11$ & $2.22 \mathrm{E}+05$ & $2.22 \mathrm{E}-07$ \\
\hline Rh-99 & $7.39 \mathrm{E}-10$ & $2.73 \mathrm{E}+06$ & $2.73 \mathrm{E}-06$ \\
\hline Rh-99m & $8.46 \mathrm{E}-11$ & $3.13 \mathrm{E}+05$ & $3.13 \mathrm{E}-07$ \\
\hline Rh-100 & $8.48 \mathrm{E}-10$ & $3.14 \mathrm{E}+06$ & $3.14 \mathrm{E}-06$ \\
\hline Rh-101 & $6.90 \mathrm{E}-10$ & $2.55 \mathrm{E}+06$ & $2.55 \mathrm{E}-06$ \\
\hline Rh-101m & $2.72 \mathrm{E}-10$ & $1.01 \mathrm{E}+06$ & $1.01 \mathrm{E}-06$ \\
\hline Rh-102 & $1.59 \mathrm{E}-09$ & $5.88 \mathrm{E}+06$ & $5.88 \mathrm{E}-06$ \\
\hline Rh-102m & 3.32E-09 & $1.23 \mathrm{E}+07$ & $1.23 \mathrm{E}-05$ \\
\hline Rh-103m & $5.11 \mathrm{E}-12$ & $1.89 \mathrm{E}+04$ & $1.89 \mathrm{E}-08$ \\
\hline Rh-105 & $5.02 \mathrm{E}-10$ & $1.86 \mathrm{E}+06$ & $1.86 \mathrm{E}-06$ \\
\hline Rh-106m & $2.18 \mathrm{E}-10$ & $8.07 \mathrm{E}+05$ & $8.07 \mathrm{E}-07$ \\
\hline Rh-107 & $3.20 \mathrm{E}-11$ & $1.18 \mathrm{E}+05$ & $1.18 \mathrm{E}-07$ \\
\hline $\begin{array}{l}\text { Pd-98 } \\
\end{array}$ & $8.25 \mathrm{E}-11$ & $3.05 \mathrm{E}+05$ & $3.05 \mathrm{E}-07$ \\
\hline Pd-99 & $4.59 \mathrm{E}-11$ & $1.70 \mathrm{E}+05$ & $1.70 \mathrm{E}-07$ \\
\hline Pd-100 & $1.18 \mathrm{E}-09$ & $4.37 \mathrm{E}+06$ & $4.37 \mathrm{E}-06$ \\
\hline Pd-101 & $1.22 \mathrm{E}-10$ & $4.51 \mathrm{E}+05$ & $4.51 \mathrm{E}-07$ \\
\hline $\mathrm{Pd}-103$ & $2.63 \mathrm{E}-10$ & $9.73 \mathrm{E}+05$ & $9.73 \mathrm{E}-07$ \\
\hline Pd-107 & $5.29 \mathrm{E}-11$ & $1.96 \mathrm{E}+05$ & $1.96 \mathrm{E}-07$ \\
\hline Pd-109 & $7.69 \mathrm{E}-10$ & $2.85 \mathrm{E}+06$ & $2.85 \mathrm{E}-06$ \\
\hline Pd-111 & $6.75 \mathrm{E}-11$ & $2.50 \mathrm{E}+05$ & $2.50 \mathrm{E}-07$ \\
\hline Pd-112 & $3.54 \mathrm{E}-09$ & $1.31 \mathrm{E}+07$ & $1.31 \mathrm{E}-05$ \\
\hline Ag-101 & $4.27 \mathrm{E}-11$ & $1.58 \mathrm{E}+05$ & $1.58 \mathrm{E}-07$ \\
\hline Ag-102 & $5.36 \mathrm{E}-11$ & $1.98 \mathrm{E}+05$ & $1.98 \mathrm{E}-07$ \\
\hline Ag-103 & $4.96 \mathrm{E}-11$ & $1.84 \mathrm{E}+05$ & $1.84 \mathrm{E}-07$ \\
\hline Ag-104 & 7.63E-11 & $2.82 \mathrm{E}+05$ & $2.82 \mathrm{E}-07$ \\
\hline
\end{tabular}




\begin{tabular}{|c|c|c|c|}
\hline Nuclide & $\begin{array}{l}\text { Ingestion } \\
\text { Dose } \\
\text { Coefficient } \\
\text { (Sv/Bq) }\end{array}$ & $\begin{array}{l}\text { Ingestion } \\
\text { Dose } \\
\text { Coefficient } \\
\text { (mrem/Ci) }\end{array}$ & $\begin{array}{c}\text { Ingestion } \\
\text { Dose } \\
\text { Coefficient } \\
\text { (mrem/pCi) }\end{array}$ \\
\hline Ag-104m & $8.74 \mathrm{E}-11$ & $3.23 \mathrm{E}+05$ & $3.23 \mathrm{E}-07$ \\
\hline Ag-105 & $5.91 \mathrm{E}-10$ & $2.19 \mathrm{E}+06$ & $2.19 \mathrm{E}-06$ \\
\hline Ag-106 & $4.21 \mathrm{E}-11$ & $1.56 \mathrm{E}+05$ & $1.56 \mathrm{E}-07$ \\
\hline Ag-106m & $1.83 \mathrm{E}-09$ & $6.77 \mathrm{E}+06$ & $6.77 \mathrm{E}-06$ \\
\hline $\mathrm{Ag}-108 \mathrm{~m}$ & $2.95 \mathrm{E}-09$ & $1.09 \mathrm{E}+07$ & $1.09 \mathrm{E}-05$ \\
\hline $\mathrm{Ag}-110 \mathrm{~m}$ & $3.55 \mathrm{E}-09$ & $1.31 \mathrm{E}+07$ & $1.31 \mathrm{E}-05$ \\
\hline Ag-111 & $1.73 \mathrm{E}-09$ & $6.40 \mathrm{E}+06$ & $6.40 \mathrm{E}-06$ \\
\hline $\mathrm{Ag}-112$ & $5.73 \mathrm{E}-10$ & $2.12 \mathrm{E}+06$ & $2.12 \mathrm{E}-06$ \\
\hline $\mathrm{Ag}-113$ & $5.40 \mathrm{E}-10$ & $2.00 \mathrm{E}+06$ & $2.00 \mathrm{E}-06$ \\
\hline Ag-115 & $8.30 \mathrm{E}-11$ & $3.07 \mathrm{E}+05$ & $3.07 \mathrm{E}-07$ \\
\hline Cd-104 & $1.34 \mathrm{E}-10$ & $4.96 \mathrm{E}+05$ & $4.96 \mathrm{E}-07$ \\
\hline Cd-105 & $5.47 \mathrm{E}-11$ & $2.02 \mathrm{E}+05$ & $2.02 \mathrm{E}-07$ \\
\hline Cd-107 & $8.59 \mathrm{E}-11$ & $3.18 \mathrm{E}+05$ & $3.18 \mathrm{E}-07$ \\
\hline Cd-109 & $2.52 \mathrm{E}-09$ & $9.32 \mathrm{E}+06$ & $9.32 \mathrm{E}-06$ \\
\hline Cd-111m & $1.81 \mathrm{E}-11$ & $6.70 \mathrm{E}+04$ & $6.70 \mathrm{E}-08$ \\
\hline Cd-113 & $2.63 \mathrm{E}-08$ & $9.73 \mathrm{E}+07$ & $9.73 \mathrm{E}-05$ \\
\hline Cd-113m & $2.57 \mathrm{E}-08$ & $9.51 \mathrm{E}+07$ & $9.51 \mathrm{E}-05$ \\
\hline Cd-115 & $1.90 \mathrm{E}-09$ & $7.03 \mathrm{E}+06$ & 7.03E-06 \\
\hline Cd-115m & $4.35 \mathrm{E}-09$ & $1.61 \mathrm{E}+07$ & $1.61 \mathrm{E}-05$ \\
\hline Cd-117 & $3.73 \mathrm{E}-10$ & $1.38 \mathrm{E}+06$ & $1.38 \mathrm{E}-06$ \\
\hline Cd-117m & $3.71 \mathrm{E}-10$ & $1.37 \mathrm{E}+06$ & $1.37 \mathrm{E}-06$ \\
\hline Cd-118 & $2.50 \mathrm{E}-10$ & $9.25 \mathrm{E}+05$ & $9.25 \mathrm{E}-07$ \\
\hline In-107 & $5.29 \mathrm{E}-11$ & $1.96 \mathrm{E}+05$ & $1.96 \mathrm{E}-07$ \\
\hline In-108 & $9.72 \mathrm{E}-11$ & $3.60 \mathrm{E}+05$ & $3.60 \mathrm{E}-07$ \\
\hline In- $108 \mathrm{~m}$ & $1.06 \mathrm{E}-10$ & $3.92 \mathrm{E}+05$ & 3.92E-07 \\
\hline In-109 & $7.46 \mathrm{E}-11$ & $2.76 \mathrm{E}+05$ & $2.76 \mathrm{E}-07$ \\
\hline In-110 & $3.03 \mathrm{E}-10$ & $1.12 \mathrm{E}+06$ & $1.12 \mathrm{E}-06$ \\
\hline In-110m & $1.34 \mathrm{E}-10$ & $4.96 \mathrm{E}+05$ & $4.96 \mathrm{E}-07$ \\
\hline In-111 & $3.79 \mathrm{E}-10$ & $1.40 \mathrm{E}+06$ & $1.40 \mathrm{E}-06$ \\
\hline In-112 & $1.40 \mathrm{E}-11$ & $5.18 \mathrm{E}+04$ & $5.18 \mathrm{E}-08$ \\
\hline In-112m & $2.28 \mathrm{E}-11$ & $8.44 \mathrm{E}+04$ & 8.44E-08 \\
\hline In-113m & $3.92 \mathrm{E}-11$ & $1.45 \mathrm{E}+05$ & $1.45 \mathrm{E}-07$ \\
\hline In-114m & $5.81 \mathrm{E}-09$ & $2.15 \mathrm{E}+07$ & $2.15 \mathrm{E}-05$ \\
\hline In-115 & $3.55 \mathrm{E}-08$ & $1.31 \mathrm{E}+08$ & $1.31 \mathrm{E}-04$ \\
\hline In- $115 \mathrm{~m}$ & $1.19 \mathrm{E}-10$ & $4.40 \mathrm{E}+05$ & $4.40 \mathrm{E}-07$ \\
\hline In-116m & $8.24 \mathrm{E}-11$ & $3.05 \mathrm{E}+05$ & $3.05 \mathrm{E}-07$ \\
\hline In-117 & $4.01 \mathrm{E}-11$ & $1.48 \mathrm{E}+05$ & $1.48 \mathrm{E}-07$ \\
\hline In- $117 \mathrm{~m}$ & $1.68 \mathrm{E}-10$ & $6.22 \mathrm{E}+05$ & $6.22 \mathrm{E}-07$ \\
\hline In-119m & $6.20 \mathrm{E}-11$ & $2.29 \mathrm{E}+05$ & $2.29 \mathrm{E}-07$ \\
\hline Sn-108 & $2.85 \mathrm{E}-11$ & $1.05 \mathrm{E}+05$ & $1.05 \mathrm{E}-07$ \\
\hline
\end{tabular}




\begin{tabular}{|c|c|c|c|}
\hline Nuclide & $\begin{array}{l}\text { Ingestion } \\
\text { Dose } \\
\text { Coefficient } \\
\text { (Sv/Bq) }\end{array}$ & $\begin{array}{c}\text { Ingestion } \\
\text { Dose } \\
\text { Coefficient } \\
\text { (mrem/Ci) }\end{array}$ & $\begin{array}{c}\text { Ingestion } \\
\text { Dose } \\
\text { Coefficient } \\
\text { (mrem/pCi) }\end{array}$ \\
\hline Sn-109 & $2.38 \mathrm{E}-11$ & $8.81 \mathrm{E}+04$ & $8.81 \mathrm{E}-08$ \\
\hline Sn-110 & $4.91 \mathrm{E}-10$ & $1.82 \mathrm{E}+06$ & $1.82 \mathrm{E}-06$ \\
\hline Sn-111 & $2.78 \mathrm{E}-11$ & $1.03 \mathrm{E}+05$ & $1.03 \mathrm{E}-07$ \\
\hline Sn-113 & $1.01 \mathrm{E}-09$ & $3.74 \mathrm{E}+06$ & $3.74 \mathrm{E}-06$ \\
\hline Sn-113m & $4.39 \mathrm{E}-12$ & $1.62 \mathrm{E}+04$ & $1.62 \mathrm{E}-08$ \\
\hline Sn-117m & $9.70 \mathrm{E}-10$ & $3.59 \mathrm{E}+06$ & $3.59 \mathrm{E}-06$ \\
\hline Sn-119m & $4.87 \mathrm{E}-10$ & $1.80 \mathrm{E}+06$ & $1.80 \mathrm{E}-06$ \\
\hline Sn-121 & $3.17 \mathrm{E}-10$ & $1.17 \mathrm{E}+06$ & $1.17 \mathrm{E}-06$ \\
\hline Sn-121m & $5.30 \mathrm{E}-10$ & $1.96 \mathrm{E}+06$ & $1.96 \mathrm{E}-06$ \\
\hline Sn-123 & 2.92E-09 & $1.08 \mathrm{E}+07$ & $1.08 \mathrm{E}-05$ \\
\hline Sn-123m & $5.18 \mathrm{E}-11$ & $1.92 \mathrm{E}+05$ & $1.92 \mathrm{E}-07$ \\
\hline Sn-125 & 4.19E-09 & $1.55 \mathrm{E}+07$ & $1.55 \mathrm{E}-05$ \\
\hline Sn-126 & $6.39 \mathrm{E}-09$ & $2.36 \mathrm{E}+07$ & $2.36 \mathrm{E}-05$ \\
\hline Sn-127 & $2.64 \mathrm{E}-10$ & $9.77 \mathrm{E}+05$ & $9.77 \mathrm{E}-07$ \\
\hline Sn-128 & $2.04 \mathrm{E}-10$ & $7.55 \mathrm{E}+05$ & $7.55 \mathrm{E}-07$ \\
\hline Sb-115 & $3.15 \mathrm{E}-11$ & $1.17 \mathrm{E}+05$ & $1.17 \mathrm{E}-07$ \\
\hline Sb-116 & $3.96 \mathrm{E}-11$ & $1.47 \mathrm{E}+05$ & $1.47 \mathrm{E}-07$ \\
\hline Sb-116m & $8.13 \mathrm{E}-11$ & $3.01 \mathrm{E}+05$ & $3.01 \mathrm{E}-07$ \\
\hline Sb-117 & $2.37 \mathrm{E}-11$ & $8.77 \mathrm{E}+04$ & $8.77 \mathrm{E}-08$ \\
\hline Sb-118m & $2.65 \mathrm{E}-10$ & $9.81 \mathrm{E}+05$ & $9.81 \mathrm{E}-07$ \\
\hline Sb-119 & $1.12 \mathrm{E}-10$ & $4.14 \mathrm{E}+05$ & $4.14 \mathrm{E}-07$ \\
\hline Sb-120 & $1.92 \mathrm{E}-11$ & $7.10 \mathrm{E}+04$ & $7.10 \mathrm{E}-08$ \\
\hline $\mathrm{Sb}-120 \mathrm{~m}$ & $1.56 \mathrm{E}-09$ & $5.77 \mathrm{E}+06$ & $5.77 \mathrm{E}-06$ \\
\hline Sb-122 & $2.31 \mathrm{E}-09$ & $8.55 \mathrm{E}+06$ & $8.55 \mathrm{E}-06$ \\
\hline Sb-124 & 3.37E-09 & $1.25 \mathrm{E}+07$ & $1.25 \mathrm{E}-05$ \\
\hline Sb-124n & $1.27 \mathrm{E}-11$ & $4.70 \mathrm{E}+04$ & $4.70 \mathrm{E}-08$ \\
\hline Sb-125 & $1.47 \mathrm{E}-09$ & $5.44 \mathrm{E}+06$ & 5.44E-06 \\
\hline Sb-126 & $3.48 \mathrm{E}-09$ & $1.29 \mathrm{E}+07$ & $1.29 \mathrm{E}-05$ \\
\hline Sb-126m & $5.00 \mathrm{E}-11$ & $1.85 \mathrm{E}+05$ & $1.85 \mathrm{E}-07$ \\
\hline Sb-127 & $2.26 \mathrm{E}-09$ & $8.36 \mathrm{E}+06$ & $8.36 \mathrm{E}-06$ \\
\hline Sb-128 & $1.06 \mathrm{E}-09$ & $3.92 \mathrm{E}+06$ & $3.92 \mathrm{E}-06$ \\
\hline Sb-128m & $4.44 \mathrm{E}-11$ & $1.64 \mathrm{E}+05$ & $1.64 \mathrm{E}-07$ \\
\hline Sb-129 & $5.72 \mathrm{E}-10$ & $2.12 \mathrm{E}+06$ & $2.12 \mathrm{E}-06$ \\
\hline $\mathrm{Sb}-130$ & $1.22 \mathrm{E}-10$ & $4.51 \mathrm{E}+05$ & $4.51 \mathrm{E}-07$ \\
\hline $\mathrm{Sb}-131$ & $1.47 \mathrm{E}-10$ & $5.44 \mathrm{E}+05$ & $5.44 \mathrm{E}-07$ \\
\hline Te-114 & $8.71 \mathrm{E}-11$ & $3.22 \mathrm{E}+05$ & $3.22 \mathrm{E}-07$ \\
\hline Te-116 & $2.58 \mathrm{E}-10$ & $9.55 \mathrm{E}+05$ & $9.55 \mathrm{E}-07$ \\
\hline Te-117 & $6.77 \mathrm{E}-11$ & $2.50 \mathrm{E}+05$ & $2.50 \mathrm{E}-07$ \\
\hline Te-118 & 4.13E-09 & $1.53 \mathrm{E}+07$ & $1.53 \mathrm{E}-05$ \\
\hline Te-119 & $2.24 \mathrm{E}-10$ & $8.29 \mathrm{E}+05$ & $8.29 \mathrm{E}-07$ \\
\hline
\end{tabular}




\begin{tabular}{|c|c|c|c|}
\hline Nuclide & $\begin{array}{c}\text { Ingestion } \\
\text { Dose } \\
\text { Coefficient } \\
\text { (Sv/Bq) }\end{array}$ & $\begin{array}{c}\text { Ingestion } \\
\text { Dose } \\
\text { Coefficient } \\
\text { (mrem/Ci) }\end{array}$ & $\begin{array}{c}\text { Ingestion } \\
\text { Dose } \\
\text { Coefficient } \\
\text { (mrem/pCi) }\end{array}$ \\
\hline Te-119m & $8.96 \mathrm{E}-10$ & $3.32 \mathrm{E}+06$ & $3.32 \mathrm{E}-06$ \\
\hline Te-121 & $5.65 \mathrm{E}-10$ & $2.09 \mathrm{E}+06$ & $2.09 \mathrm{E}-06$ \\
\hline Te-121m & $3.03 \mathrm{E}-09$ & $1.12 \mathrm{E}+07$ & $1.12 \mathrm{E}-05$ \\
\hline Te-123 & $1.49 \mathrm{E}-09$ & $5.51 \mathrm{E}+06$ & $5.51 \mathrm{E}-06$ \\
\hline Te-123m & $1.87 \mathrm{E}-09$ & $6.92 \mathrm{E}+06$ & $6.92 \mathrm{E}-06$ \\
\hline Te-125m & $1.22 \mathrm{E}-09$ & $4.51 \mathrm{E}+06$ & $4.51 \mathrm{E}-06$ \\
\hline Te-127 & $2.30 \mathrm{E}-10$ & $8.51 \mathrm{E}+05$ & $8.51 \mathrm{E}-07$ \\
\hline Te-127m & $3.40 \mathrm{E}-09$ & $1.26 \mathrm{E}+07$ & $1.26 \mathrm{E}-05$ \\
\hline Te-129 & $8.51 \mathrm{E}-11$ & $3.15 \mathrm{E}+05$ & $3.15 \mathrm{E}-07$ \\
\hline Te-129m & $4.25 \mathrm{E}-09$ & $1.57 \mathrm{E}+07$ & $1.57 \mathrm{E}-05$ \\
\hline Te-131 & $1.21 \mathrm{E}-10$ & $4.48 \mathrm{E}+05$ & $4.48 \mathrm{E}-07$ \\
\hline Te-131m & $2.69 \mathrm{E}-09$ & $9.95 \mathrm{E}+06$ & $9.95 \mathrm{E}-06$ \\
\hline Te-132 & $5.36 \mathrm{E}-09$ & $1.98 \mathrm{E}+07$ & $1.98 \mathrm{E}-05$ \\
\hline Te-133 & $1.01 \mathrm{E}-10$ & $3.74 \mathrm{E}+05$ & $3.74 \mathrm{E}-07$ \\
\hline Te-133m & $3.64 \mathrm{E}-10$ & $1.35 \mathrm{E}+06$ & $1.35 \mathrm{E}-06$ \\
\hline Te-134 & $1.33 \mathrm{E}-10$ & $4.92 \mathrm{E}+05$ & $4.92 \mathrm{E}-07$ \\
\hline $\mathrm{I}-118$ & $2.76 \mathrm{E}-10$ & $1.02 \mathrm{E}+06$ & $1.02 \mathrm{E}-06$ \\
\hline $\mathrm{I}-119$ & $5.75 \mathrm{E}-11$ & $2.13 \mathrm{E}+05$ & $2.13 \mathrm{E}-07$ \\
\hline $\mathrm{I}-120$ & $4.10 \mathrm{E}-10$ & $1.52 \mathrm{E}+06$ & $1.52 \mathrm{E}-06$ \\
\hline $\mathrm{I}-120 \mathrm{~m}$ & $2.10 \mathrm{E}-10$ & $7.77 \mathrm{E}+05$ & $7.77 \mathrm{E}-07$ \\
\hline $\mathrm{I}-121$ & $1.00 \mathrm{E}-10$ & $3.70 \mathrm{E}+05$ & $3.70 \mathrm{E}-07$ \\
\hline $\mathrm{I}-123$ & $3.24 \mathrm{E}-10$ & $1.20 \mathrm{E}+06$ & $1.20 \mathrm{E}-06$ \\
\hline $\mathrm{I}-124$ & $1.90 \mathrm{E}-08$ & $7.03 \mathrm{E}+07$ & $7.03 \mathrm{E}-05$ \\
\hline $\mathrm{I}-125$ & $1.89 \mathrm{E}-08$ & $6.99 \mathrm{E}+07$ & $6.99 \mathrm{E}-05$ \\
\hline $\mathrm{I}-126$ & $4.09 \mathrm{E}-08$ & $1.51 \mathrm{E}+08$ & $1.51 \mathrm{E}-04$ \\
\hline $\mathrm{I}-128$ & $6.23 \mathrm{E}-11$ & $2.31 \mathrm{E}+05$ & $2.31 \mathrm{E}-07$ \\
\hline $\mathrm{I}-129$ & $1.21 \mathrm{E}-07$ & $4.48 \mathrm{E}+08$ & $4.48 \mathrm{E}-04$ \\
\hline $\mathrm{I}-130$ & $2.76 \mathrm{E}-09$ & $1.02 \mathrm{E}+07$ & $1.02 \mathrm{E}-05$ \\
\hline $\mathrm{I}-131$ & $3.13 \mathrm{E}-08$ & $1.16 \mathrm{E}+08$ & $1.16 \mathrm{E}-04$ \\
\hline $\mathrm{I}-132$ & $4.05 \mathrm{E}-10$ & $1.50 \mathrm{E}+06$ & $1.50 \mathrm{E}-06$ \\
\hline $\mathrm{I}-132 m$ & $2.89 \mathrm{E}-10$ & $1.07 \mathrm{E}+06$ & $1.07 \mathrm{E}-06$ \\
\hline $\mathrm{I}-133$ & $6.60 \mathrm{E}-09$ & $2.44 \mathrm{E}+07$ & $2.44 \mathrm{E}-05$ \\
\hline $\mathrm{I}-134$ & $1.37 \mathrm{E}-10$ & $5.07 \mathrm{E}+05$ & $5.07 \mathrm{E}-07$ \\
\hline $\mathrm{I}-135$ & $1.31 \mathrm{E}-09$ & $4.85 \mathrm{E}+06$ & $4.85 \mathrm{E}-06$ \\
\hline Cs-125 & $4.56 \mathrm{E}-11$ & $1.69 \mathrm{E}+05$ & $1.69 \mathrm{E}-07$ \\
\hline Cs-127 & $3.09 \mathrm{E}-11$ & $1.14 \mathrm{E}+05$ & $1.14 \mathrm{E}-07$ \\
\hline Cs-129 & $7.50 \mathrm{E}-11$ & $2.78 \mathrm{E}+05$ & $2.78 \mathrm{E}-07$ \\
\hline Cs-130 & $3.56 \mathrm{E}-11$ & $1.32 \mathrm{E}+05$ & $1.32 \mathrm{E}-07$ \\
\hline Cs-131 & $7.22 \mathrm{E}-11$ & $2.67 \mathrm{E}+05$ & $2.67 \mathrm{E}-07$ \\
\hline Cs-132 & $6.00 \mathrm{E}-10$ & $2.22 \mathrm{E}+06$ & $2.22 \mathrm{E}-06$ \\
\hline
\end{tabular}




\begin{tabular}{|c|c|c|c|}
\hline Nuclide & $\begin{array}{l}\text { Ingestion } \\
\text { Dose } \\
\text { Coefficient } \\
\text { (Sv/Bq) }\end{array}$ & $\begin{array}{l}\text { Ingestion } \\
\text { Dose } \\
\text { Coefficient } \\
\text { (mrem/Ci) }\end{array}$ & $\begin{array}{c}\text { Ingestion } \\
\text { Dose } \\
\text { Coefficient } \\
\text { (mrem/pCi) }\end{array}$ \\
\hline Cs-134 & $1.87 \mathrm{E}-08$ & $6.92 \mathrm{E}+07$ & $6.92 \mathrm{E}-05$ \\
\hline Cs-134m & $2.61 \mathrm{E}-11$ & $9.66 \mathrm{E}+04$ & $9.66 \mathrm{E}-08$ \\
\hline Cs-135 & 2.64E-09 & $9.77 \mathrm{E}+06$ & $9.77 \mathrm{E}-06$ \\
\hline Cs- $135 \mathrm{~m}$ & $2.37 \mathrm{E}-11$ & $8.77 \mathrm{E}+04$ & $8.77 \mathrm{E}-08$ \\
\hline Cs-136 & $3.45 \mathrm{E}-09$ & $1.28 \mathrm{E}+07$ & $1.28 \mathrm{E}-05$ \\
\hline Cs-137 & $1.33 \mathrm{E}-08$ & $4.92 \mathrm{E}+07$ & $4.92 \mathrm{E}-05$ \\
\hline Cs-138 & $1.28 \mathrm{E}-10$ & $4.74 \mathrm{E}+05$ & $4.74 \mathrm{E}-07$ \\
\hline Ba-124 & $9.23 \mathrm{E}-11$ & $3.42 \mathrm{E}+05$ & $3.42 \mathrm{E}-07$ \\
\hline Ba-126 & $3.36 \mathrm{E}-10$ & $1.24 \mathrm{E}+06$ & $1.24 \mathrm{E}-06$ \\
\hline Ba-127 & $3.28 \mathrm{E}-11$ & $1.21 \mathrm{E}+05$ & $1.21 \mathrm{E}-07$ \\
\hline Ba-128 & $3.56 \mathrm{E}-09$ & $1.32 \mathrm{E}+07$ & $1.32 \mathrm{E}-05$ \\
\hline Ba-129 & $6.26 \mathrm{E}-11$ & $2.32 \mathrm{E}+05$ & $2.32 \mathrm{E}-07$ \\
\hline Ba-129m & $8.81 \mathrm{E}-11$ & $3.26 \mathrm{E}+05$ & $3.26 \mathrm{E}-07$ \\
\hline Ba-131 & $6.08 \mathrm{E}-10$ & $2.25 \mathrm{E}+06$ & $2.25 \mathrm{E}-06$ \\
\hline Ba-131m & $6.65 \mathrm{E}-12$ & $2.46 \mathrm{E}+04$ & $2.46 \mathrm{E}-08$ \\
\hline Ba-133 & $2.44 \mathrm{E}-09$ & $9.03 \mathrm{E}+06$ & $9.03 \mathrm{E}-06$ \\
\hline Ba-133m & 7.19E-10 & $2.66 \mathrm{E}+06$ & $2.66 \mathrm{E}-06$ \\
\hline Ba-135m & $5.66 \mathrm{E}-10$ & $2.09 \mathrm{E}+06$ & $2.09 \mathrm{E}-06$ \\
\hline Ba-139 & $1.64 \mathrm{E}-10$ & $6.07 \mathrm{E}+05$ & $6.07 \mathrm{E}-07$ \\
\hline Ba-140 & $3.63 \mathrm{E}-09$ & $1.34 \mathrm{E}+07$ & $1.34 \mathrm{E}-05$ \\
\hline Ba-141 & $9.85 \mathrm{E}-11$ & $3.64 \mathrm{E}+05$ & $3.64 \mathrm{E}-07$ \\
\hline Ba-142 & $4.46 \mathrm{E}-11$ & $1.65 \mathrm{E}+05$ & $1.65 \mathrm{E}-07$ \\
\hline La-129 & $3.53 \mathrm{E}-11$ & $1.31 \mathrm{E}+05$ & $1.31 \mathrm{E}-07$ \\
\hline La-131 & $4.39 \mathrm{E}-11$ & $1.62 \mathrm{E}+05$ & $1.62 \mathrm{E}-07$ \\
\hline La-132 & $5.41 \mathrm{E}-10$ & $2.00 \mathrm{E}+06$ & $2.00 \mathrm{E}-06$ \\
\hline La-132m & $4.83 \mathrm{E}-11$ & $1.79 \mathrm{E}+05$ & $1.79 \mathrm{E}-07$ \\
\hline La-133 & $4.21 \mathrm{E}-11$ & $1.56 \mathrm{E}+05$ & $1.56 \mathrm{E}-07$ \\
\hline La-135 & $4.12 \mathrm{E}-11$ & $1.52 \mathrm{E}+05$ & $1.52 \mathrm{E}-07$ \\
\hline La-137 & $1.11 \mathrm{E}-10$ & $4.11 \mathrm{E}+05$ & $4.11 \mathrm{E}-07$ \\
\hline La-138 & $1.37 \mathrm{E}-09$ & $5.07 \mathrm{E}+06$ & $5.07 \mathrm{E}-06$ \\
\hline La-140 & 2.67E-09 & $9.88 \mathrm{E}+06$ & $9.88 \mathrm{E}-06$ \\
\hline La-141 & $5.10 \mathrm{E}-10$ & $1.89 \mathrm{E}+06$ & $1.89 \mathrm{E}-06$ \\
\hline La-142 & $2.31 \mathrm{E}-10$ & $8.55 \mathrm{E}+05$ & $8.55 \mathrm{E}-07$ \\
\hline La-143 & $7.51 \mathrm{E}-11$ & $2.78 \mathrm{E}+05$ & $2.78 \mathrm{E}-07$ \\
\hline Ce-130 & $9.37 \mathrm{E}-11$ & $3.47 \mathrm{E}+05$ & $3.47 \mathrm{E}-07$ \\
\hline Ce-131 & $3.62 \mathrm{E}-11$ & $1.34 \mathrm{E}+05$ & $1.34 \mathrm{E}-07$ \\
\hline Ce-132 & $4.42 \mathrm{E}-10$ & $1.64 \mathrm{E}+06$ & $1.64 \mathrm{E}-06$ \\
\hline Ce-133 & $1.21 \mathrm{E}-10$ & $4.48 \mathrm{E}+05$ & 4.48E-07 \\
\hline $\mathrm{Ce}-133 \mathrm{~m}$ & $2.69 \mathrm{E}-10$ & $9.95 \mathrm{E}+05$ & $9.95 \mathrm{E}-07$ \\
\hline Ce-134 & $3.63 \mathrm{E}-09$ & $1.34 \mathrm{E}+07$ & $1.34 \mathrm{E}-05$ \\
\hline
\end{tabular}




\begin{tabular}{|c|c|c|c|}
\hline Nuclide & $\begin{array}{c}\text { Ingestion } \\
\text { Dose } \\
\text { Coefficient } \\
(\mathbf{S v} / \mathbf{B q})\end{array}$ & $\begin{array}{c}\text { Ingestion } \\
\text { Dose } \\
\text { Coefficient } \\
\text { (mrem/Ci) }\end{array}$ & $\begin{array}{c}\text { Ingestion } \\
\text { Dose } \\
\text { Coefficient } \\
\text { (mrem/pCi) }\end{array}$ \\
\hline Ce-135 & $3.35 \mathrm{E}-10$ & $1.24 \mathrm{E}+06$ & $1.24 \mathrm{E}-06$ \\
\hline Ce-137 & $3.57 \mathrm{E}-11$ & $1.32 \mathrm{E}+05$ & $1.32 \mathrm{E}-07$ \\
\hline $\mathrm{Ce}-137 \mathrm{~m}$ & $7.59 \mathrm{E}-10$ & $2.81 \mathrm{E}+06$ & $2.81 \mathrm{E}-06$ \\
\hline Ce-139 & $3.50 \mathrm{E}-10$ & $1.30 \mathrm{E}+06$ & $1.30 \mathrm{E}-06$ \\
\hline Ce-141 & $9.78 \mathrm{E}-10$ & $3.62 \mathrm{E}+06$ & $3.62 \mathrm{E}-06$ \\
\hline Ce-143 & $1.54 \mathrm{E}-09$ & $5.70 \mathrm{E}+06$ & $5.70 \mathrm{E}-06$ \\
\hline Ce-144 & $7.25 \mathrm{E}-09$ & $2.68 \mathrm{E}+07$ & $2.68 \mathrm{E}-05$ \\
\hline Pr-134 & $6.46 \mathrm{E}-11$ & $2.39 \mathrm{E}+05$ & $2.39 \mathrm{E}-07$ \\
\hline Pr-134m & $1.12 \mathrm{E}-10$ & $4.14 \mathrm{E}+05$ & $4.14 \mathrm{E}-07$ \\
\hline Pr-135 & $5.73 \mathrm{E}-11$ & $2.12 \mathrm{E}+05$ & $2.12 \mathrm{E}-07$ \\
\hline Pr-136 & $4.47 \mathrm{E}-11$ & $1.65 \mathrm{E}+05$ & $1.65 \mathrm{E}-07$ \\
\hline Pr-137 & $4.87 \mathrm{E}-11$ & $1.80 \mathrm{E}+05$ & $1.80 \mathrm{E}-07$ \\
\hline Pr-138m & $1.67 \mathrm{E}-10$ & $6.18 \mathrm{E}+05$ & $6.18 \mathrm{E}-07$ \\
\hline Pr-139 & $4.34 \mathrm{E}-11$ & $1.61 \mathrm{E}+05$ & $1.61 \mathrm{E}-07$ \\
\hline Pr-142 & $1.82 \mathrm{E}-09$ & $6.73 \mathrm{E}+06$ & $6.73 \mathrm{E}-06$ \\
\hline Pr-142m & $2.32 \mathrm{E}-11$ & $8.58 \mathrm{E}+04$ & $8.58 \mathrm{E}-08$ \\
\hline Pr-143 & $1.62 \mathrm{E}-09$ & $5.99 \mathrm{E}+06$ & $5.99 \mathrm{E}-06$ \\
\hline Pr-144 & $6.82 \mathrm{E}-11$ & $2.52 \mathrm{E}+05$ & $2.52 \mathrm{E}-07$ \\
\hline Pr-145 & $5.44 \mathrm{E}-10$ & $2.01 \mathrm{E}+06$ & $2.01 \mathrm{E}-06$ \\
\hline Pr-146 & $1.04 \mathrm{E}-10$ & $3.85 \mathrm{E}+05$ & $3.85 \mathrm{E}-07$ \\
\hline Pr-147 & $4.52 \mathrm{E}-11$ & $1.67 \mathrm{E}+05$ & $1.67 \mathrm{E}-07$ \\
\hline $\mathrm{Nd}-135$ & $7.77 \mathrm{E}-11$ & $2.87 \mathrm{E}+05$ & $2.87 \mathrm{E}-07$ \\
\hline Nd-136 & $1.30 \mathrm{E}-10$ & $4.81 \mathrm{E}+05$ & 4.81E-07 \\
\hline Nd-137 & $7.03 \mathrm{E}-11$ & $2.60 \mathrm{E}+05$ & $2.60 \mathrm{E}-07$ \\
\hline Nd-138 & $8.71 \mathrm{E}-10$ & $3.22 \mathrm{E}+06$ & $3.22 \mathrm{E}-06$ \\
\hline Nd-139 & $2.73 \mathrm{E}-11$ & $1.01 \mathrm{E}+05$ & $1.01 \mathrm{E}-07$ \\
\hline Nd-139m & $3.10 \mathrm{E}-10$ & $1.15 \mathrm{E}+06$ & $1.15 \mathrm{E}-06$ \\
\hline Nd-140 & $2.71 \mathrm{E}-09$ & $1.00 \mathrm{E}+07$ & $1.00 \mathrm{E}-05$ \\
\hline Nd-141 & $1.13 \mathrm{E}-11$ & $4.18 \mathrm{E}+04$ & $4.18 \mathrm{E}-08$ \\
\hline Nd-144 & $5.28 \mathrm{E}-08$ & $1.95 \mathrm{E}+08$ & $1.95 \mathrm{E}-04$ \\
\hline Nd-147 & $1.48 \mathrm{E}-09$ & $5.48 \mathrm{E}+06$ & $5.48 \mathrm{E}-06$ \\
\hline Nd-149 & $1.69 \mathrm{E}-10$ & $6.25 \mathrm{E}+05$ & $6.25 \mathrm{E}-07$ \\
\hline Nd-151 & $3.82 \mathrm{E}-11$ & $1.41 \mathrm{E}+05$ & $1.41 \mathrm{E}-07$ \\
\hline Nd-152 & $6.50 \mathrm{E}-11$ & $2.41 \mathrm{E}+05$ & $2.41 \mathrm{E}-07$ \\
\hline Pm-141 & $4.66 \mathrm{E}-11$ & $1.72 \mathrm{E}+05$ & $1.72 \mathrm{E}-07$ \\
\hline Pm-143 & $3.00 \mathrm{E}-10$ & $1.11 \mathrm{E}+06$ & $1.11 \mathrm{E}-06$ \\
\hline Pm-144 & $1.24 \mathrm{E}-09$ & $4.59 \mathrm{E}+06$ & 4.59E-06 \\
\hline Pm-145 & $1.48 \mathrm{E}-10$ & $5.48 \mathrm{E}+05$ & $5.48 \mathrm{E}-07$ \\
\hline Pm-146 & $1.18 \mathrm{E}-09$ & $4.37 \mathrm{E}+06$ & 4.37E-06 \\
\hline Pm-147 & $3.63 \mathrm{E}-10$ & $1.34 \mathrm{E}+06$ & $1.34 \mathrm{E}-06$ \\
\hline
\end{tabular}




\begin{tabular}{|c|c|c|c|}
\hline Nuclide & $\begin{array}{c}\text { Ingestion } \\
\text { Dose } \\
\text { Coefficient } \\
\text { (Sv/Bq) }\end{array}$ & $\begin{array}{c}\text { Ingestion } \\
\text { Dose } \\
\text { Coefficient } \\
\text { (mrem/Ci) }\end{array}$ & $\begin{array}{c}\text { Ingestion } \\
\text { Dose } \\
\text { Coefficient } \\
\text { (mrem/pCi) }\end{array}$ \\
\hline Pm-148 & $3.68 \mathrm{E}-09$ & $1.36 \mathrm{E}+07$ & $1.36 \mathrm{E}-05$ \\
\hline Pm-148m & $2.28 \mathrm{E}-09$ & $8.44 \mathrm{E}+06$ & $8.44 \mathrm{E}-06$ \\
\hline Pm-149 & $1.37 \mathrm{E}-09$ & $5.07 \mathrm{E}+06$ & $5.07 \mathrm{E}-06$ \\
\hline Pm-150 & $3.49 \mathrm{E}-10$ & $1.29 \mathrm{E}+06$ & $1.29 \mathrm{E}-06$ \\
\hline Pm-151 & $9.97 \mathrm{E}-10$ & $3.69 \mathrm{E}+06$ & $3.69 \mathrm{E}-06$ \\
\hline Sm-140 & $1.29 \mathrm{E}-10$ & $4.77 \mathrm{E}+05$ & $4.77 \mathrm{E}-07$ \\
\hline Sm-141 & $5.14 \mathrm{E}-11$ & $1.90 \mathrm{E}+05$ & $1.90 \mathrm{E}-07$ \\
\hline Sm-141m & $8.18 \mathrm{E}-11$ & $3.03 \mathrm{E}+05$ & $3.03 \mathrm{E}-07$ \\
\hline Sm-142 & $2.43 \mathrm{E}-10$ & $8.99 \mathrm{E}+05$ & $8.99 \mathrm{E}-07$ \\
\hline $\mathrm{Sm}-145$ & $2.86 \mathrm{E}-10$ & $1.06 \mathrm{E}+06$ & $1.06 \mathrm{E}-06$ \\
\hline $\mathrm{Sm}-146$ & $7.01 \mathrm{E}-08$ & $2.59 \mathrm{E}+08$ & $2.59 \mathrm{E}-04$ \\
\hline $\mathrm{Sm}-147$ & $6.40 \mathrm{E}-08$ & $2.37 \mathrm{E}+08$ & $2.37 \mathrm{E}-04$ \\
\hline $\mathrm{Sm}-148$ & $5.50 \mathrm{E}-08$ & $2.04 \mathrm{E}+08$ & $2.04 \mathrm{E}-04$ \\
\hline $\mathrm{Sm}-151$ & $1.35 \mathrm{E}-10$ & $5.00 \mathrm{E}+05$ & $5.00 \mathrm{E}-07$ \\
\hline $\mathrm{Sm}-153$ & $1.01 \mathrm{E}-09$ & $3.74 \mathrm{E}+06$ & $3.74 \mathrm{E}-06$ \\
\hline $\mathrm{Sm}-155$ & $3.96 \mathrm{E}-11$ & $1.47 \mathrm{E}+05$ & $1.47 \mathrm{E}-07$ \\
\hline $\mathrm{Sm}-156$ & $3.48 \mathrm{E}-10$ & $1.29 \mathrm{E}+06$ & $1.29 \mathrm{E}-06$ \\
\hline $\mathrm{Eu}-145$ & $8.36 \mathrm{E}-10$ & $3.09 \mathrm{E}+06$ & $3.09 \mathrm{E}-06$ \\
\hline $\mathrm{Eu}-146$ & $1.51 \mathrm{E}-09$ & $5.59 \mathrm{E}+06$ & $5.59 \mathrm{E}-06$ \\
\hline $\mathrm{Eu}-147$ & $5.74 \mathrm{E}-10$ & $2.12 \mathrm{E}+06$ & $2.12 \mathrm{E}-06$ \\
\hline $\mathrm{Eu}-148$ & $1.62 \mathrm{E}-09$ & $5.99 \mathrm{E}+06$ & $5.99 \mathrm{E}-06$ \\
\hline $\mathrm{Eu}-149$ & $2.15 \mathrm{E}-10$ & $7.96 \mathrm{E}+05$ & $7.96 \mathrm{E}-07$ \\
\hline $\mathrm{Eu}-150$ & $1.57 \mathrm{E}-09$ & $5.81 \mathrm{E}+06$ & $5.81 \mathrm{E}-06$ \\
\hline $\mathrm{Eu}-150 \mathrm{~m}$ & $5.27 \mathrm{E}-10$ & $1.95 \mathrm{E}+06$ & $1.95 \mathrm{E}-06$ \\
\hline $\mathrm{Eu}-152$ & $1.74 \mathrm{E}-09$ & $6.44 \mathrm{E}+06$ & $6.44 \mathrm{E}-06$ \\
\hline $\mathrm{Eu}-152 \mathrm{~m}$ & $6.82 \mathrm{E}-10$ & $2.52 \mathrm{E}+06$ & $2.52 \mathrm{E}-06$ \\
\hline $\mathrm{Eu}-152 \mathrm{n}$ & $1.76 \mathrm{E}-11$ & $6.51 \mathrm{E}+04$ & $6.51 \mathrm{E}-08$ \\
\hline $\mathrm{Eu}-154$ & $2.61 \mathrm{E}-09$ & $9.66 \mathrm{E}+06$ & $9.66 \mathrm{E}-06$ \\
\hline $\mathrm{Eu}-154 \mathrm{~m}$ & $1.06 \mathrm{E}-11$ & $3.92 \mathrm{E}+04$ & $3.92 \mathrm{E}-08$ \\
\hline $\mathrm{Eu}-155$ & $4.52 \mathrm{E}-10$ & $1.67 \mathrm{E}+06$ & $1.67 \mathrm{E}-06$ \\
\hline $\mathrm{Eu}-156$ & $3.07 \mathrm{E}-09$ & $1.14 \mathrm{E}+07$ & $1.14 \mathrm{E}-05$ \\
\hline $\mathrm{Eu}-157$ & $8.37 \mathrm{E}-10$ & $3.10 \mathrm{E}+06$ & $3.10 \mathrm{E}-06$ \\
\hline $\mathrm{Eu}-158$ & $1.21 \mathrm{E}-10$ & $4.48 \mathrm{E}+05$ & $4.48 \mathrm{E}-07$ \\
\hline $\mathrm{Eu}-159$ & $6.57 \mathrm{E}-11$ & $2.43 \mathrm{E}+05$ & $2.43 \mathrm{E}-07$ \\
\hline $\mathrm{Gd}-145$ & $4.40 \mathrm{E}-11$ & $1.63 \mathrm{E}+05$ & $1.63 \mathrm{E}-07$ \\
\hline $\mathrm{Gd}-146$ & $1.26 \mathrm{E}-09$ & $4.66 \mathrm{E}+06$ & $4.66 \mathrm{E}-06$ \\
\hline $\mathrm{Gd}-147$ & $8.15 \mathrm{E}-10$ & $3.02 \mathrm{E}+06$ & $3.02 \mathrm{E}-06$ \\
\hline $\mathrm{Gd}-148$ & $7.19 \mathrm{E}-08$ & $2.66 \mathrm{E}+08$ & $2.66 \mathrm{E}-04$ \\
\hline $\mathrm{Gd}-149$ & $7.15 \mathrm{E}-10$ & $2.65 \mathrm{E}+06$ & $2.65 \mathrm{E}-06$ \\
\hline $\mathrm{Gd}-150$ & $6.78 \mathrm{E}-08$ & $2.51 \mathrm{E}+08$ & $2.51 \mathrm{E}-04$ \\
\hline
\end{tabular}




\begin{tabular}{|c|c|c|c|}
\hline Nuclide & $\begin{array}{c}\text { Ingestion } \\
\text { Dose } \\
\text { Coefficient } \\
(\mathbf{S v} / \mathbf{B q})\end{array}$ & $\begin{array}{l}\text { Ingestion } \\
\text { Dose } \\
\text { Coefficient } \\
\text { (mrem } / \mathbf{C i})\end{array}$ & $\begin{array}{c}\text { Ingestion } \\
\text { Dose } \\
\text { Coefficient } \\
\text { (mrem/pCi) }\end{array}$ \\
\hline Gd-151 & $3.07 \mathrm{E}-10$ & $1.14 \mathrm{E}+06$ & $1.14 \mathrm{E}-06$ \\
\hline Gd-152 & $5.32 \mathrm{E}-08$ & $1.97 \mathrm{E}+08$ & $1.97 \mathrm{E}-04$ \\
\hline Gd-153 & $3.74 \mathrm{E}-10$ & $1.38 \mathrm{E}+06$ & $1.38 \mathrm{E}-06$ \\
\hline Gd-159 & $6.95 \mathrm{E}-10$ & $2.57 \mathrm{E}+06$ & $2.57 \mathrm{E}-06$ \\
\hline Tb-147 & $1.62 \mathrm{E}-10$ & $5.99 \mathrm{E}+05$ & 5.99E-07 \\
\hline Tb-148 & $1.63 \mathrm{E}-10$ & $6.03 \mathrm{E}+05$ & $6.03 \mathrm{E}-07$ \\
\hline Tb-149 & $2.40 \mathrm{E}-10$ & $8.88 \mathrm{E}+05$ & $8.88 \mathrm{E}-07$ \\
\hline Tb-150 & $2.81 \mathrm{E}-10$ & $1.04 \mathrm{E}+06$ & $1.04 \mathrm{E}-06$ \\
\hline Tb-151 & $4.66 \mathrm{E}-10$ & $1.72 \mathrm{E}+06$ & $1.72 \mathrm{E}-06$ \\
\hline Tb-152 & $9.18 \mathrm{E}-10$ & $3.40 \mathrm{E}+06$ & $3.40 \mathrm{E}-06$ \\
\hline Tb-153 & $3.78 \mathrm{E}-10$ & $1.40 \mathrm{E}+06$ & $1.40 \mathrm{E}-06$ \\
\hline Tb-154 & 7.92E-10 & $2.93 \mathrm{E}+06$ & $2.93 \mathrm{E}-06$ \\
\hline Tb-155 & $3.51 \mathrm{E}-10$ & $1.30 \mathrm{E}+06$ & $1.30 \mathrm{E}-06$ \\
\hline Tb-156 & $1.48 \mathrm{E}-09$ & $5.48 \mathrm{E}+06$ & $5.48 \mathrm{E}-06$ \\
\hline Tb-156m & $2.13 \mathrm{E}-10$ & $7.88 \mathrm{E}+05$ & $7.88 \mathrm{E}-07$ \\
\hline Tb-156n & $1.16 \mathrm{E}-10$ & $4.29 \mathrm{E}+05$ & $4.29 \mathrm{E}-07$ \\
\hline Tb-157 & $5.27 \mathrm{E}-11$ & $1.95 \mathrm{E}+05$ & $1.95 \mathrm{E}-07$ \\
\hline Tb-158 & $1.45 \mathrm{E}-09$ & $5.37 \mathrm{E}+06$ & $5.37 \mathrm{E}-06$ \\
\hline Tb-160 & $2.15 \mathrm{E}-09$ & $7.96 \mathrm{E}+06$ & 7.96E-06 \\
\hline Tb-161 & $1.02 \mathrm{E}-09$ & $3.77 \mathrm{E}+06$ & $3.77 \mathrm{E}-06$ \\
\hline Tb-163 & $2.81 \mathrm{E}-11$ & $1.04 \mathrm{E}+05$ & $1.04 \mathrm{E}-07$ \\
\hline Dy-151 & $2.52 \mathrm{E}-11$ & $9.32 \mathrm{E}+04$ & $9.32 \mathrm{E}-08$ \\
\hline Dy-152 & $1.42 \mathrm{E}-10$ & $5.25 \mathrm{E}+05$ & $5.25 \mathrm{E}-07$ \\
\hline Dy-153 & $2.32 \mathrm{E}-10$ & $8.58 \mathrm{E}+05$ & $8.58 \mathrm{E}-07$ \\
\hline Dy-154 & 7.27E-08 & $2.69 \mathrm{E}+08$ & $2.69 \mathrm{E}-04$ \\
\hline Dy-155 & $1.84 \mathrm{E}-10$ & $6.81 \mathrm{E}+05$ & $6.81 \mathrm{E}-07$ \\
\hline Dy-157 & $7.76 \mathrm{E}-11$ & $2.87 \mathrm{E}+05$ & $2.87 \mathrm{E}-07$ \\
\hline Dy-159 & $1.41 \mathrm{E}-10$ & $5.22 \mathrm{E}+05$ & $5.22 \mathrm{E}-07$ \\
\hline Dy-165 & $1.49 \mathrm{E}-10$ & $5.51 \mathrm{E}+05$ & $5.51 \mathrm{E}-07$ \\
\hline Dy-166 & $2.29 \mathrm{E}-09$ & $8.47 \mathrm{E}+06$ & $8.47 \mathrm{E}-06$ \\
\hline Ho-154 & $5.40 \mathrm{E}-11$ & $2.00 \mathrm{E}+05$ & $2.00 \mathrm{E}-07$ \\
\hline Ho-155 & $5.13 \mathrm{E}-11$ & $1.90 \mathrm{E}+05$ & $1.90 \mathrm{E}-07$ \\
\hline Ho-156 & $1.27 \mathrm{E}-10$ & $4.70 \mathrm{E}+05$ & $4.70 \mathrm{E}-07$ \\
\hline Ho-157 & $9.32 \mathrm{E}-12$ & $3.45 \mathrm{E}+04$ & $3.45 \mathrm{E}-08$ \\
\hline Ho-159 & $1.09 \mathrm{E}-11$ & $4.03 \mathrm{E}+04$ & $4.03 \mathrm{E}-08$ \\
\hline Ho-160 & $2.13 \mathrm{E}-11$ & $7.88 \mathrm{E}+04$ & $7.88 \mathrm{E}-08$ \\
\hline Ho-161 & $1.71 \mathrm{E}-11$ & $6.33 \mathrm{E}+04$ & $6.33 \mathrm{E}-08$ \\
\hline Ho-162 & $4.24 \mathrm{E}-12$ & $1.57 \mathrm{E}+04$ & $1.57 \mathrm{E}-08$ \\
\hline Ho-162m & $3.27 \mathrm{E}-11$ & $1.21 \mathrm{E}+05$ & $1.21 \mathrm{E}-07$ \\
\hline Ho-163 & $4.00 \mathrm{E}-12$ & $1.48 \mathrm{E}+04$ & $1.48 \mathrm{E}-08$ \\
\hline
\end{tabular}




\begin{tabular}{|c|c|c|c|}
\hline Nuclide & $\begin{array}{l}\text { Ingestion } \\
\text { Dose } \\
\text { Coefficient } \\
(\mathrm{Sv} / \mathrm{Bq})\end{array}$ & $\begin{array}{l}\text { Ingestion } \\
\text { Dose } \\
\text { Coefficient } \\
\text { (mrem/Ci) }\end{array}$ & $\begin{array}{c}\text { Ingestion } \\
\text { Dose } \\
\text { Coefficient } \\
\text { (mrem/pCi) }\end{array}$ \\
\hline Ho-164 & $1.27 \mathrm{E}-11$ & $4.70 \mathrm{E}+04$ & $4.70 \mathrm{E}-08$ \\
\hline Ho-164m & $2.23 \mathrm{E}-11$ & $8.25 \mathrm{E}+04$ & $8.25 \mathrm{E}-08$ \\
\hline Но-166 & $1.93 \mathrm{E}-09$ & $7.14 \mathrm{E}+06$ & $7.14 \mathrm{E}-06$ \\
\hline Ho-166m & $2.55 \mathrm{E}-09$ & $9.44 \mathrm{E}+06$ & $9.44 \mathrm{E}-06$ \\
\hline Ho-167 & $1.17 \mathrm{E}-10$ & $4.33 \mathrm{E}+05$ & $4.33 \mathrm{E}-07$ \\
\hline Er-156 & $4.52 \mathrm{E}-11$ & $1.67 \mathrm{E}+05$ & $1.67 \mathrm{E}-07$ \\
\hline Er-159 & $2.92 \mathrm{E}-11$ & $1.08 \mathrm{E}+05$ & $1.08 \mathrm{E}-07$ \\
\hline Er-161 & $1.08 \mathrm{E}-10$ & $4.00 \mathrm{E}+05$ & $4.00 \mathrm{E}-07$ \\
\hline Er-163 & $3.39 \mathrm{E}-12$ & $1.25 \mathrm{E}+04$ & $1.25 \mathrm{E}-08$ \\
\hline Er-165 & $2.57 \mathrm{E}-11$ & $9.51 \mathrm{E}+04$ & $9.51 \mathrm{E}-08$ \\
\hline Er-169 & $5.16 \mathrm{E}-10$ & $1.91 \mathrm{E}+06$ & $1.91 \mathrm{E}-06$ \\
\hline Er-171 & $4.85 \mathrm{E}-10$ & $1.79 \mathrm{E}+06$ & $1.79 \mathrm{E}-06$ \\
\hline Er-172 & $1.40 \mathrm{E}-09$ & $5.18 \mathrm{E}+06$ & $5.18 \mathrm{E}-06$ \\
\hline Tm-161 & $5.05 \mathrm{E}-11$ & $1.87 \mathrm{E}+05$ & $1.87 \mathrm{E}-07$ \\
\hline Tm-162 & $5.17 \mathrm{E}-11$ & $1.91 \mathrm{E}+05$ & $1.91 \mathrm{E}-07$ \\
\hline Tm-163 & $6.97 \mathrm{E}-11$ & $2.58 \mathrm{E}+05$ & $2.58 \mathrm{E}-07$ \\
\hline Tm-165 & $4.65 \mathrm{E}-10$ & $1.72 \mathrm{E}+06$ & $1.72 \mathrm{E}-06$ \\
\hline Tm-166 & $3.62 \mathrm{E}-10$ & $1.34 \mathrm{E}+06$ & $1.34 \mathrm{E}-06$ \\
\hline Tm-167 & $7.91 \mathrm{E}-10$ & $2.93 \mathrm{E}+06$ & $2.93 \mathrm{E}-06$ \\
\hline Tm-168 & $1.34 \mathrm{E}-09$ & $4.96 \mathrm{E}+06$ & $4.96 \mathrm{E}-06$ \\
\hline Tm-170 & $1.81 \mathrm{E}-09$ & $6.70 \mathrm{E}+06$ & $6.70 \mathrm{E}-06$ \\
\hline Tm-171 & $1.48 \mathrm{E}-10$ & $5.48 \mathrm{E}+05$ & $5.48 \mathrm{E}-07$ \\
\hline Tm-172 & 2.32E-09 & $8.58 \mathrm{E}+06$ & $8.58 \mathrm{E}-06$ \\
\hline Tm-173 & $4.07 \mathrm{E}-10$ & $1.51 \mathrm{E}+06$ & $1.51 \mathrm{E}-06$ \\
\hline Tm-175 & $3.42 \mathrm{E}-11$ & $1.27 \mathrm{E}+05$ & $1.27 \mathrm{E}-07$ \\
\hline Yb-162 & $4.05 \mathrm{E}-11$ & $1.50 \mathrm{E}+05$ & $1.50 \mathrm{E}-07$ \\
\hline Yb-163 & $2.07 \mathrm{E}-11$ & $7.66 \mathrm{E}+04$ & 7.66E-08 \\
\hline Yb-164 & $1.22 \mathrm{E}-10$ & $4.51 \mathrm{E}+05$ & $4.51 \mathrm{E}-07$ \\
\hline Yb-166 & $1.23 \mathrm{E}-09$ & $4.55 \mathrm{E}+06$ & $4.55 \mathrm{E}-06$ \\
\hline Yb-167 & $9.01 \mathrm{E}-12$ & $3.33 \mathrm{E}+04$ & $3.33 \mathrm{E}-08$ \\
\hline Yb-169 & $1.10 \mathrm{E}-09$ & $4.07 \mathrm{E}+06$ & $4.07 \mathrm{E}-06$ \\
\hline Yb-175 & $6.03 \mathrm{E}-10$ & $2.23 \mathrm{E}+06$ & $2.23 \mathrm{E}-06$ \\
\hline Yb-177 & $1.25 \mathrm{E}-10$ & $4.63 \mathrm{E}+05$ & $4.63 \mathrm{E}-07$ \\
\hline Yb-178 & $1.58 \mathrm{E}-10$ & $5.85 \mathrm{E}+05$ & $5.85 \mathrm{E}-07$ \\
\hline Lu-165 & $2.85 \mathrm{E}-11$ & $1.05 \mathrm{E}+05$ & $1.05 \mathrm{E}-07$ \\
\hline Lu-167 & $6.14 \mathrm{E}-11$ & $2.27 \mathrm{E}+05$ & $2.27 \mathrm{E}-07$ \\
\hline Lu-169 & $6.77 \mathrm{E}-10$ & $2.50 \mathrm{E}+06$ & $2.50 \mathrm{E}-06$ \\
\hline Lu-170 & $1.22 \mathrm{E}-09$ & $4.51 \mathrm{E}+06$ & $4.51 \mathrm{E}-06$ \\
\hline Lu-171 & $9.05 \mathrm{E}-10$ & $3.35 \mathrm{E}+06$ & $3.35 \mathrm{E}-06$ \\
\hline Lu-172 & $1.69 \mathrm{E}-09$ & $6.25 \mathrm{E}+06$ & $6.25 \mathrm{E}-06$ \\
\hline
\end{tabular}




\begin{tabular}{|c|c|c|c|}
\hline Nuclide & $\begin{array}{l}\text { Ingestion } \\
\text { Dose } \\
\text { Coefficient } \\
\text { (Sv/Bq) }\end{array}$ & $\begin{array}{c}\text { Ingestion } \\
\text { Dose } \\
\text { Coefficient } \\
\text { (mrem/Ci) }\end{array}$ & $\begin{array}{c}\text { Ingestion } \\
\text { Dose } \\
\text { Coefficient } \\
\text { (mrem/pCi) }\end{array}$ \\
\hline Lu-173 & $4.87 \mathrm{E}-10$ & $1.80 \mathrm{E}+06$ & $1.80 \mathrm{E}-06$ \\
\hline Lu-174 & $3.83 \mathrm{E}-10$ & $1.42 \mathrm{E}+06$ & $1.42 \mathrm{E}-06$ \\
\hline Lu-174m & $7.47 \mathrm{E}-10$ & $2.76 \mathrm{E}+06$ & $2.76 \mathrm{E}-06$ \\
\hline Lu-176 & $2.42 \mathrm{E}-09$ & $8.95 \mathrm{E}+06$ & $8.95 \mathrm{E}-06$ \\
\hline Lu-176m & $2.27 \mathrm{E}-10$ & $8.40 \mathrm{E}+05$ & $8.40 \mathrm{E}-07$ \\
\hline Lu-177 & $7.32 \mathrm{E}-10$ & $2.71 \mathrm{E}+06$ & $2.71 \mathrm{E}-06$ \\
\hline Lu-177m & $2.27 \mathrm{E}-09$ & $8.40 \mathrm{E}+06$ & $8.40 \mathrm{E}-06$ \\
\hline $\begin{array}{l}\text { Lu-178 } \\
\end{array}$ & $6.25 \mathrm{E}-11$ & $2.31 \mathrm{E}+05$ & $2.31 \mathrm{E}-07$ \\
\hline Lu-178m & $4.39 \mathrm{E}-11$ & $1.62 \mathrm{E}+05$ & $1.62 \mathrm{E}-07$ \\
\hline Lu-179 & $2.97 \mathrm{E}-10$ & $1.10 \mathrm{E}+06$ & $1.10 \mathrm{E}-06$ \\
\hline Hf-170 & $5.30 \mathrm{E}-10$ & $1.96 \mathrm{E}+06$ & $1.96 \mathrm{E}-06$ \\
\hline Hf-172 & $1.46 \mathrm{E}-09$ & $5.40 \mathrm{E}+06$ & $5.40 \mathrm{E}-06$ \\
\hline Hf-173 & $2.90 \mathrm{E}-10$ & $1.07 \mathrm{E}+06$ & $1.07 \mathrm{E}-06$ \\
\hline Hf-174 & $3.15 \mathrm{E}-07$ & $1.17 \mathrm{E}+09$ & $1.17 \mathrm{E}-03$ \\
\hline Hf-175 & $5.28 \mathrm{E}-10$ & $1.95 \mathrm{E}+06$ & $1.95 \mathrm{E}-06$ \\
\hline Hf- $177 \mathrm{~m}$ & $1.08 \mathrm{E}-10$ & $4.00 \mathrm{E}+05$ & $4.00 \mathrm{E}-07$ \\
\hline Hf- $178 \mathrm{~m}$ & 4.97E-09 & $1.84 \mathrm{E}+07$ & $1.84 \mathrm{E}-05$ \\
\hline Hf-179m & $1.68 \mathrm{E}-09$ & $6.22 \mathrm{E}+06$ & $6.22 \mathrm{E}-06$ \\
\hline Hf- $180 \mathrm{~m}$ & $2.21 \mathrm{E}-10$ & $8.18 \mathrm{E}+05$ & $8.18 \mathrm{E}-07$ \\
\hline Hf-181 & $1.50 \mathrm{E}-09$ & $5.55 \mathrm{E}+06$ & $5.55 \mathrm{E}-06$ \\
\hline Hf-182 & $3.43 \mathrm{E}-09$ & $1.27 \mathrm{E}+07$ & $1.27 \mathrm{E}-05$ \\
\hline Hf- $182 \mathrm{~m}$ & $5.94 \mathrm{E}-11$ & $2.20 \mathrm{E}+05$ & $2.20 \mathrm{E}-07$ \\
\hline Hf-183 & $9.87 \mathrm{E}-11$ & $3.65 \mathrm{E}+05$ & $3.65 \mathrm{E}-07$ \\
\hline Hf-184 & $7.06 \mathrm{E}-10$ & $2.61 \mathrm{E}+06$ & $2.61 \mathrm{E}-06$ \\
\hline Ta-172 & $7.55 \mathrm{E}-11$ & $2.79 \mathrm{E}+05$ & $2.79 \mathrm{E}-07$ \\
\hline Ta-173 & $1.44 \mathrm{E}-10$ & $5.33 \mathrm{E}+05$ & $5.33 \mathrm{E}-07$ \\
\hline Тa-174 & $9.51 \mathrm{E}-11$ & $3.52 \mathrm{E}+05$ & $3.52 \mathrm{E}-07$ \\
\hline Тa-175 & $3.04 \mathrm{E}-10$ & $1.12 \mathrm{E}+06$ & $1.12 \mathrm{E}-06$ \\
\hline Тa-176 & $4.00 \mathrm{E}-10$ & $1.48 \mathrm{E}+06$ & $1.48 \mathrm{E}-06$ \\
\hline Тa-177 & $1.46 \mathrm{E}-10$ & $5.40 \mathrm{E}+05$ & $5.40 \mathrm{E}-07$ \\
\hline Ta-178m & $1.11 \mathrm{E}-10$ & $4.11 \mathrm{E}+05$ & $4.11 \mathrm{E}-07$ \\
\hline Ta-179 & 7.97E-11 & $2.95 \mathrm{E}+05$ & $2.95 \mathrm{E}-07$ \\
\hline Тa-180 & $7.56 \mathrm{E}-11$ & $2.80 \mathrm{E}+05$ & $2.80 \mathrm{E}-07$ \\
\hline Ta-182 & $1.99 \mathrm{E}-09$ & $7.36 \mathrm{E}+06$ & 7.36E-06 \\
\hline Ta- $182 \mathrm{~m}$ & $1.63 \mathrm{E}-11$ & $6.03 \mathrm{E}+04$ & $6.03 \mathrm{E}-08$ \\
\hline Ta-183 & $1.84 \mathrm{E}-09$ & $6.81 \mathrm{E}+06$ & $6.81 \mathrm{E}-06$ \\
\hline Ta-184 & $8.95 \mathrm{E}-10$ & $3.31 \mathrm{E}+06$ & $3.31 \mathrm{E}-06$ \\
\hline Ta-185 & $9.30 \mathrm{E}-11$ & $3.44 \mathrm{E}+05$ & $3.44 \mathrm{E}-07$ \\
\hline Тa-186 & $4.66 \mathrm{E}-11$ & $1.72 \mathrm{E}+05$ & $1.72 \mathrm{E}-07$ \\
\hline W-177 & 7.19E-11 & $2.66 \mathrm{E}+05$ & $2.66 \mathrm{E}-07$ \\
\hline
\end{tabular}




\begin{tabular}{|c|c|c|c|}
\hline Nuclide & $\begin{array}{l}\text { Ingestion } \\
\text { Dose } \\
\text { Coefficient } \\
\text { (Sv/Bq) }\end{array}$ & $\begin{array}{c}\text { Ingestion } \\
\text { Dose } \\
\text { Coefficient } \\
\text { (mrem/Ci) }\end{array}$ & $\begin{array}{c}\text { Ingestion } \\
\text { Dose } \\
\text { Coefficient } \\
\text { (mrem/pCi) }\end{array}$ \\
\hline W-178 & $3.28 \mathrm{E}-10$ & $1.21 \mathrm{E}+06$ & $1.21 \mathrm{E}-06$ \\
\hline W-179 & $4.72 \mathrm{E}-12$ & $1.75 \mathrm{E}+04$ & $1.75 \mathrm{E}-08$ \\
\hline W-181 & $1.13 \mathrm{E}-10$ & $4.18 \mathrm{E}+05$ & $4.18 \mathrm{E}-07$ \\
\hline W-185 & $6.06 \mathrm{E}-10$ & $2.24 \mathrm{E}+06$ & $2.24 \mathrm{E}-06$ \\
\hline W-187 & $7.98 \mathrm{E}-10$ & $2.95 \mathrm{E}+06$ & $2.95 \mathrm{E}-06$ \\
\hline W-188 & $2.85 \mathrm{E}-09$ & $1.05 \mathrm{E}+07$ & $1.05 \mathrm{E}-05$ \\
\hline W-190 & $1.11 \mathrm{E}-10$ & $4.11 \mathrm{E}+05$ & $4.11 \mathrm{E}-07$ \\
\hline Re-178 & $3.68 \mathrm{E}-11$ & $1.36 \mathrm{E}+05$ & $1.36 \mathrm{E}-07$ \\
\hline Re-179 & $1.68 \mathrm{E}-11$ & $6.22 \mathrm{E}+04$ & $6.22 \mathrm{E}-08$ \\
\hline Re-181 & $5.68 \mathrm{E}-10$ & $2.10 \mathrm{E}+06$ & $2.10 \mathrm{E}-06$ \\
\hline Re-182 & $1.87 \mathrm{E}-09$ & $6.92 \mathrm{E}+06$ & $6.92 \mathrm{E}-06$ \\
\hline Re-182m & $3.76 \mathrm{E}-10$ & $1.39 \mathrm{E}+06$ & $1.39 \mathrm{E}-06$ \\
\hline Re-183 & $1.31 \mathrm{E}-09$ & $4.85 \mathrm{E}+06$ & $4.85 \mathrm{E}-06$ \\
\hline Re-184 & $1.29 \mathrm{E}-09$ & $4.77 \mathrm{E}+06$ & 4.77E-06 \\
\hline Re-184m & $1.98 \mathrm{E}-09$ & $7.33 \mathrm{E}+06$ & $7.33 \mathrm{E}-06$ \\
\hline Re-186 & $1.99 \mathrm{E}-09$ & $7.36 \mathrm{E}+06$ & $7.36 \mathrm{E}-06$ \\
\hline Re-186m & 3.04E-09 & $1.12 \mathrm{E}+07$ & $1.12 \mathrm{E}-05$ \\
\hline Re-187 & $6.59 \mathrm{E}-12$ & $2.44 \mathrm{E}+04$ & $2.44 \mathrm{E}-08$ \\
\hline Re-188 & $1.92 \mathrm{E}-09$ & $7.10 \mathrm{E}+06$ & $7.10 \mathrm{E}-06$ \\
\hline Re-188m & $4.16 \mathrm{E}-11$ & $1.54 \mathrm{E}+05$ & $1.54 \mathrm{E}-07$ \\
\hline Re-189 & $1.04 \mathrm{E}-09$ & $3.85 \mathrm{E}+06$ & $3.85 \mathrm{E}-06$ \\
\hline Re-190m & $5.04 \mathrm{E}-10$ & $1.86 \mathrm{E}+06$ & $1.86 \mathrm{E}-06$ \\
\hline Os-180 & $2.39 \mathrm{E}-11$ & $8.84 \mathrm{E}+04$ & $8.84 \mathrm{E}-08$ \\
\hline Os-181 & $1.18 \mathrm{E}-10$ & $4.37 \mathrm{E}+05$ & 4.37E-07 \\
\hline Os-182 & $7.50 \mathrm{E}-10$ & $2.78 \mathrm{E}+06$ & $2.78 \mathrm{E}-06$ \\
\hline Os-183 & $2.95 \mathrm{E}-10$ & $1.09 \mathrm{E}+06$ & $1.09 \mathrm{E}-06$ \\
\hline Os-183m & $2.64 \mathrm{E}-10$ & $9.77 \mathrm{E}+05$ & $9.77 \mathrm{E}-07$ \\
\hline Os-185 & $6.35 \mathrm{E}-10$ & $2.35 \mathrm{E}+06$ & $2.35 \mathrm{E}-06$ \\
\hline Os-186 & $4.29 \mathrm{E}-08$ & $1.59 \mathrm{E}+08$ & $1.59 \mathrm{E}-04$ \\
\hline Os-189m & $2.36 \mathrm{E}-11$ & $8.73 \mathrm{E}+04$ & $8.73 \mathrm{E}-08$ \\
\hline Os-191 & 7.94E-10 & $2.94 \mathrm{E}+06$ & $2.94 \mathrm{E}-06$ \\
\hline Os-191m & $1.36 \mathrm{E}-10$ & $5.03 \mathrm{E}+05$ & $5.03 \mathrm{E}-07$ \\
\hline Os-193 & $1.14 \mathrm{E}-09$ & $4.22 \mathrm{E}+06$ & $4.22 \mathrm{E}-06$ \\
\hline Os-194 & $3.40 \mathrm{E}-09$ & $1.26 \mathrm{E}+07$ & $1.26 \mathrm{E}-05$ \\
\hline Os-196 & $1.48 \mathrm{E}-10$ & $5.48 \mathrm{E}+05$ & $5.48 \mathrm{E}-07$ \\
\hline Ir-182 & $6.95 \mathrm{E}-11$ & $2.57 \mathrm{E}+05$ & $2.57 \mathrm{E}-07$ \\
\hline Ir-183 & $6.81 \mathrm{E}-11$ & $2.52 \mathrm{E}+05$ & $2.52 \mathrm{E}-07$ \\
\hline Ir-184 & $2.44 \mathrm{E}-10$ & $9.03 \mathrm{E}+05$ & $9.03 \mathrm{E}-07$ \\
\hline Ir-185 & $4.28 \mathrm{E}-10$ & $1.58 \mathrm{E}+06$ & $1.58 \mathrm{E}-06$ \\
\hline Ir-186 & 7.27E-10 & $2.69 \mathrm{E}+06$ & $2.69 \mathrm{E}-06$ \\
\hline
\end{tabular}




\begin{tabular}{|c|c|c|c|}
\hline Nuclide & $\begin{array}{c}\text { Ingestion } \\
\text { Dose } \\
\text { Coefficient } \\
(\mathbf{S v} / \mathbf{B q})\end{array}$ & $\begin{array}{l}\text { Ingestion } \\
\text { Dose } \\
\text { Coefficient } \\
\text { (mrem } / \mathbf{C i})\end{array}$ & $\begin{array}{c}\text { Ingestion } \\
\text { Dose } \\
\text { Coefficient } \\
\text { (mrem/pCi) }\end{array}$ \\
\hline Ir-186m & $9.38 \mathrm{E}-11$ & $3.47 \mathrm{E}+05$ & $3.47 \mathrm{E}-07$ \\
\hline Ir-187 & $1.52 \mathrm{E}-10$ & $5.62 \mathrm{E}+05$ & $5.62 \mathrm{E}-07$ \\
\hline Ir-188 & $9.62 \mathrm{E}-10$ & $3.56 \mathrm{E}+06$ & $3.56 \mathrm{E}-06$ \\
\hline Ir-189 & $3.27 \mathrm{E}-10$ & $1.21 \mathrm{E}+06$ & $1.21 \mathrm{E}-06$ \\
\hline Ir-190 & $1.36 \mathrm{E}-09$ & $5.03 \mathrm{E}+06$ & $5.03 \mathrm{E}-06$ \\
\hline Ir-190m & $9.18 \mathrm{E}-12$ & $3.40 \mathrm{E}+04$ & $3.40 \mathrm{E}-08$ \\
\hline Ir-190n & $1.51 \mathrm{E}-10$ & $5.59 \mathrm{E}+05$ & $5.59 \mathrm{E}-07$ \\
\hline Ir-192 & $1.82 \mathrm{E}-09$ & $6.73 \mathrm{E}+06$ & $6.73 \mathrm{E}-06$ \\
\hline Ir-192n & $1.24 \mathrm{E}-09$ & $4.59 \mathrm{E}+06$ & $4.59 \mathrm{E}-06$ \\
\hline Ir-193m & $4.00 \mathrm{E}-10$ & $1.48 \mathrm{E}+06$ & $1.48 \mathrm{E}-06$ \\
\hline Ir-194 & $1.84 \mathrm{E}-09$ & $6.81 \mathrm{E}+06$ & $6.81 \mathrm{E}-06$ \\
\hline Ir-194m & $2.63 \mathrm{E}-09$ & $9.73 \mathrm{E}+06$ & $9.73 \mathrm{E}-06$ \\
\hline Ir-195 & $1.38 \mathrm{E}-10$ & $5.11 \mathrm{E}+05$ & $5.11 \mathrm{E}-07$ \\
\hline Ir-195m & $1.83 \mathrm{E}-10$ & $6.77 \mathrm{E}+05$ & $6.77 \mathrm{E}-07$ \\
\hline Ir-196m & $1.43 \mathrm{E}-10$ & $5.29 \mathrm{E}+05$ & $5.29 \mathrm{E}-07$ \\
\hline Pt-184 & $3.89 \mathrm{E}-11$ & $1.44 \mathrm{E}+05$ & $1.44 \mathrm{E}-07$ \\
\hline Pt-186 & $1.40 \mathrm{E}-10$ & $5.18 \mathrm{E}+05$ & $5.18 \mathrm{E}-07$ \\
\hline Pt-187 & $1.12 \mathrm{E}-10$ & $4.14 \mathrm{E}+05$ & $4.14 \mathrm{E}-07$ \\
\hline Pt-188 & $1.10 \mathrm{E}-09$ & $4.07 \mathrm{E}+06$ & $4.07 \mathrm{E}-06$ \\
\hline Pt-189 & $2.59 \mathrm{E}-10$ & $9.58 \mathrm{E}+05$ & $9.58 \mathrm{E}-07$ \\
\hline Pt-190 & $9.38 \mathrm{E}-09$ & $3.47 \mathrm{E}+07$ & $3.47 \mathrm{E}-05$ \\
\hline Pt-191 & $4.88 \mathrm{E}-10$ & $1.81 \mathrm{E}+06$ & $1.81 \mathrm{E}-06$ \\
\hline Pt-193 & $4.93 \mathrm{E}-11$ & $1.82 \mathrm{E}+05$ & $1.82 \mathrm{E}-07$ \\
\hline Pt-193m & $6.30 \mathrm{E}-10$ & $2.33 \mathrm{E}+06$ & $2.33 \mathrm{E}-06$ \\
\hline Pt-195m & $8.83 \mathrm{E}-10$ & $3.27 \mathrm{E}+06$ & $3.27 \mathrm{E}-06$ \\
\hline Pt-197 & $5.95 \mathrm{E}-10$ & $2.20 \mathrm{E}+06$ & $2.20 \mathrm{E}-06$ \\
\hline Pt-197m & $1.16 \mathrm{E}-10$ & $4.29 \mathrm{E}+05$ & $4.29 \mathrm{E}-07$ \\
\hline Pt-199 & $5.35 \mathrm{E}-11$ & $1.98 \mathrm{E}+05$ & $1.98 \mathrm{E}-07$ \\
\hline Pt-200 & $1.61 \mathrm{E}-09$ & $5.96 \mathrm{E}+06$ & $5.96 \mathrm{E}-06$ \\
\hline Pt-202 & $6.07 \mathrm{E}-09$ & $2.25 \mathrm{E}+07$ & $2.25 \mathrm{E}-05$ \\
\hline Au-186 & $5.91 \mathrm{E}-11$ & $2.19 \mathrm{E}+05$ & $2.19 \mathrm{E}-07$ \\
\hline $\mathrm{Au}-190$ & $5.41 \mathrm{E}-11$ & $2.00 \mathrm{E}+05$ & $2.00 \mathrm{E}-07$ \\
\hline $\mathrm{Au}-191$ & $9.47 \mathrm{E}-11$ & $3.50 \mathrm{E}+05$ & $3.50 \mathrm{E}-07$ \\
\hline $\mathrm{Au}-192$ & $2.19 \mathrm{E}-10$ & $8.10 \mathrm{E}+05$ & $8.10 \mathrm{E}-07$ \\
\hline $\mathrm{Au}-193$ & $1.71 \mathrm{E}-10$ & $6.33 \mathrm{E}+05$ & $6.33 \mathrm{E}-07$ \\
\hline $\mathrm{Au}-194$ & $5.16 \mathrm{E}-10$ & $1.91 \mathrm{E}+06$ & $1.91 \mathrm{E}-06$ \\
\hline $\mathrm{Au}-195$ & $3.55 \mathrm{E}-10$ & $1.31 \mathrm{E}+06$ & $1.31 \mathrm{E}-06$ \\
\hline $\mathrm{Au}-196$ & $4.54 \mathrm{E}-10$ & $1.68 \mathrm{E}+06$ & $1.68 \mathrm{E}-06$ \\
\hline $\mathrm{Au}-196 \mathrm{~m}$ & $5.28 \mathrm{E}-10$ & $1.95 \mathrm{E}+06$ & $1.95 \mathrm{E}-06$ \\
\hline Au-198 & 1.39E-09 & $5.14 \mathrm{E}+06$ & $5.14 \mathrm{E}-06$ \\
\hline
\end{tabular}




\begin{tabular}{|c|c|c|c|}
\hline Nuclide & $\begin{array}{c}\text { Ingestion } \\
\text { Dose } \\
\text { Coefficient } \\
\text { (Sv/Bq) }\end{array}$ & $\begin{array}{c}\text { Ingestion } \\
\text { Dose } \\
\text { Coefficient } \\
\text { (mrem/Ci) }\end{array}$ & $\begin{array}{c}\text { Ingestion } \\
\text { Dose } \\
\text { Coefficient } \\
\text { (mrem/pCi) }\end{array}$ \\
\hline $\mathrm{Au}-198 \mathrm{~m}$ & $1.61 \mathrm{E}-09$ & $5.96 \mathrm{E}+06$ & $5.96 \mathrm{E}-06$ \\
\hline $\mathrm{Au}-199$ & $6.08 \mathrm{E}-10$ & $2.25 \mathrm{E}+06$ & $2.25 \mathrm{E}-06$ \\
\hline $\mathrm{Au}-200$ & $9.08 \mathrm{E}-11$ & $3.36 \mathrm{E}+05$ & $3.36 \mathrm{E}-07$ \\
\hline $\mathrm{Au}-200 \mathrm{~m}$ & $1.32 \mathrm{E}-09$ & $4.88 \mathrm{E}+06$ & $4.88 \mathrm{E}-06$ \\
\hline $\mathrm{Au}-201$ & $3.27 \mathrm{E}-11$ & $1.21 \mathrm{E}+05$ & $1.21 \mathrm{E}-07$ \\
\hline $\mathrm{Hg}-190$ & $2.78 \mathrm{E}-11$ & $1.03 \mathrm{E}+05$ & $1.03 \mathrm{E}-07$ \\
\hline $\mathrm{Hg}-191 \mathrm{~m}$ & $6.88 \mathrm{E}-11$ & $2.55 \mathrm{E}+05$ & $2.55 \mathrm{E}-07$ \\
\hline $\mathrm{Hg}-192$ & $2.91 \mathrm{E}-10$ & $1.08 \mathrm{E}+06$ & $1.08 \mathrm{E}-06$ \\
\hline $\mathrm{Hg}-193$ & $1.39 \mathrm{E}-10$ & $5.14 \mathrm{E}+05$ & $5.14 \mathrm{E}-07$ \\
\hline $\mathrm{Hg}-193 \mathrm{~m}$ & $5.62 \mathrm{E}-10$ & $2.08 \mathrm{E}+06$ & $2.08 \mathrm{E}-06$ \\
\hline $\mathrm{Hg}-194$ & $1.56 \mathrm{E}-09$ & $5.77 \mathrm{E}+06$ & $5.77 \mathrm{E}-06$ \\
\hline $\mathrm{Hg}-195$ & $1.37 \mathrm{E}-10$ & $5.07 \mathrm{E}+05$ & $5.07 \mathrm{E}-07$ \\
\hline $\mathrm{Hg}-195 \mathrm{~m}$ & $7.47 \mathrm{E}-10$ & $2.76 \mathrm{E}+06$ & $2.76 \mathrm{E}-06$ \\
\hline $\mathrm{Hg}-197$ & $3.39 \mathrm{E}-10$ & $1.25 \mathrm{E}+06$ & $1.25 \mathrm{E}-06$ \\
\hline $\mathrm{Hg}-197 \mathrm{~m}$ & $6.58 \mathrm{E}-10$ & $2.43 \mathrm{E}+06$ & $2.43 \mathrm{E}-06$ \\
\hline $\mathrm{Hg}-199 \mathrm{~m}$ & $4.11 \mathrm{E}-11$ & $1.52 \mathrm{E}+05$ & $1.52 \mathrm{E}-07$ \\
\hline $\mathrm{Hg}-203$ & $7.23 \mathrm{E}-10$ & $2.68 \mathrm{E}+06$ & $2.68 \mathrm{E}-06$ \\
\hline $\mathrm{Tl}-194$ & $6.05 \mathrm{E}-11$ & $2.24 \mathrm{E}+05$ & $2.24 \mathrm{E}-07$ \\
\hline $\mathrm{Tl}-194 \mathrm{~m}$ & $4.95 \mathrm{E}-11$ & $1.83 \mathrm{E}+05$ & $1.83 \mathrm{E}-07$ \\
\hline $\mathrm{Tl}-195$ & $3.10 \mathrm{E}-11$ & $1.15 \mathrm{E}+05$ & $1.15 \mathrm{E}-07$ \\
\hline $\mathrm{Tl}-196$ & $6.42 \mathrm{E}-11$ & $2.38 \mathrm{E}+05$ & $2.38 \mathrm{E}-07$ \\
\hline $\mathrm{Tl}-197$ & $2.96 \mathrm{E}-11$ & $1.10 \mathrm{E}+05$ & $1.10 \mathrm{E}-07$ \\
\hline $\mathrm{Tl}-198$ & $9.03 \mathrm{E}-11$ & $3.34 \mathrm{E}+05$ & $3.34 \mathrm{E}-07$ \\
\hline $\mathrm{Tl}-198 \mathrm{~m}$ & $7.00 \mathrm{E}-11$ & $2.59 \mathrm{E}+05$ & $2.59 \mathrm{E}-07$ \\
\hline $\mathrm{Tl}-199$ & $3.48 \mathrm{E}-11$ & $1.29 \mathrm{E}+05$ & $1.29 \mathrm{E}-07$ \\
\hline $\mathrm{Tl}-200$ & $2.47 \mathrm{E}-10$ & $9.14 \mathrm{E}+05$ & $9.14 \mathrm{E}-07$ \\
\hline $\mathrm{Tl}-201$ & $1.27 \mathrm{E}-10$ & $4.70 \mathrm{E}+05$ & $4.70 \mathrm{E}-07$ \\
\hline $\mathrm{Tl}-202$ & $5.60 \mathrm{E}-10$ & $2.07 \mathrm{E}+06$ & $2.07 \mathrm{E}-06$ \\
\hline $\mathrm{Tl}-204$ & $1.61 \mathrm{E}-09$ & $5.96 \mathrm{E}+06$ & $5.96 \mathrm{E}-06$ \\
\hline $\mathrm{Pb}-194$ & $2.66 \mathrm{E}-11$ & $9.84 \mathrm{E}+04$ & $9.84 \mathrm{E}-08$ \\
\hline $\mathrm{Pb}-195 \mathrm{~m}$ & $3.20 \mathrm{E}-11$ & $1.18 \mathrm{E}+05$ & $1.18 \mathrm{E}-07$ \\
\hline $\mathrm{Pb}-196$ & $3.45 \mathrm{E}-11$ & $1.28 \mathrm{E}+05$ & $1.28 \mathrm{E}-07$ \\
\hline $\mathrm{Pb}-197 \mathrm{~m}$ & $5.73 \mathrm{E}-11$ & $2.12 \mathrm{E}+05$ & $2.12 \mathrm{E}-07$ \\
\hline $\mathrm{Pb}-198$ & $9.71 \mathrm{E}-11$ & $3.59 \mathrm{E}+05$ & $3.59 \mathrm{E}-07$ \\
\hline $\mathrm{Pb}-199$ & $4.89 \mathrm{E}-11$ & $1.81 \mathrm{E}+05$ & $1.81 \mathrm{E}-07$ \\
\hline $\mathrm{Pb}-200$ & $4.76 \mathrm{E}-10$ & $1.76 \mathrm{E}+06$ & $1.76 \mathrm{E}-06$ \\
\hline $\mathrm{Pb}-201$ & $1.97 \mathrm{E}-10$ & $7.29 \mathrm{E}+05$ & $7.29 \mathrm{E}-07$ \\
\hline $\mathrm{Pb}-202$ & $2.09 \mathrm{E}-08$ & $7.73 \mathrm{E}+07$ & $7.73 \mathrm{E}-05$ \\
\hline $\mathrm{Pb}-202 \mathrm{~m}$ & $1.80 \mathrm{E}-10$ & $6.66 \mathrm{E}+05$ & $6.66 \mathrm{E}-07$ \\
\hline $\mathrm{Pb}-203$ & $3.07 \mathrm{E}-10$ & $1.14 \mathrm{E}+06$ & $1.14 \mathrm{E}-06$ \\
\hline
\end{tabular}




\begin{tabular}{|c|c|c|c|}
\hline Nuclide & $\begin{array}{l}\text { Ingestion } \\
\text { Dose } \\
\text { Coefficient } \\
\text { (Sv/Bq) }\end{array}$ & $\begin{array}{l}\text { Ingestion } \\
\text { Dose } \\
\text { Coefficient } \\
\text { (mrem/Ci) }\end{array}$ & $\begin{array}{c}\text { Ingestion } \\
\text { Dose } \\
\text { Coefficient } \\
\text { (mrem } / p C i)\end{array}$ \\
\hline $\mathrm{Pb}-204 \mathrm{~m}$ & $6.01 \mathrm{E}-11$ & $2.22 \mathrm{E}+05$ & $2.22 \mathrm{E}-07$ \\
\hline $\mathrm{Pb}-205$ & $3.52 \mathrm{E}-10$ & $1.30 \mathrm{E}+06$ & $1.30 \mathrm{E}-06$ \\
\hline $\mathrm{Pb}-209$ & $7.47 \mathrm{E}-11$ & $2.76 \mathrm{E}+05$ & $2.76 \mathrm{E}-07$ \\
\hline $\mathrm{Pb}-210$ & $1.02 \mathrm{E}-06$ & $3.77 \mathrm{E}+09$ & $3.77 \mathrm{E}-03$ \\
\hline $\mathrm{Pb}-211$ & $2.62 \mathrm{E}-10$ & $9.69 \mathrm{E}+05$ & $9.69 \mathrm{E}-07$ \\
\hline $\mathrm{Pb}-212$ & $1.03 \mathrm{E}-08$ & $3.81 \mathrm{E}+07$ & $3.81 \mathrm{E}-05$ \\
\hline $\mathrm{Pb}-214$ & $1.99 \mathrm{E}-10$ & $7.36 \mathrm{E}+05$ & 7.36E-07 \\
\hline Bi-200 & $7.16 \mathrm{E}-11$ & $2.65 \mathrm{E}+05$ & $2.65 \mathrm{E}-07$ \\
\hline Bi-201 & $1.49 \mathrm{E}-10$ & $5.51 \mathrm{E}+05$ & $5.51 \mathrm{E}-07$ \\
\hline Bi-202 & $1.26 \mathrm{E}-10$ & $4.66 \mathrm{E}+05$ & $4.66 \mathrm{E}-07$ \\
\hline Bi-203 & $6.26 \mathrm{E}-10$ & $2.32 \mathrm{E}+06$ & $2.32 \mathrm{E}-06$ \\
\hline Bi-204 & $7.22 \mathrm{E}-10$ & $2.67 \mathrm{E}+06$ & $2.67 \mathrm{E}-06$ \\
\hline Bi-205 & $1.15 \mathrm{E}-09$ & $4.26 \mathrm{E}+06$ & $4.26 \mathrm{E}-06$ \\
\hline Bi-206 & $2.47 \mathrm{E}-09$ & $9.14 \mathrm{E}+06$ & $9.14 \mathrm{E}-06$ \\
\hline Bi-207 & $1.65 \mathrm{E}-09$ & $6.11 \mathrm{E}+06$ & $6.11 \mathrm{E}-06$ \\
\hline Bi-208 & $1.44 \mathrm{E}-09$ & $5.33 \mathrm{E}+06$ & $5.33 \mathrm{E}-06$ \\
\hline Bi-210 & $1.80 \mathrm{E}-09$ & $6.66 \mathrm{E}+06$ & $6.66 \mathrm{E}-06$ \\
\hline Bi-210m & $2.01 \mathrm{E}-08$ & $7.44 \mathrm{E}+07$ & 7.44E-05 \\
\hline Bi-212 & $3.52 \mathrm{E}-10$ & $1.30 \mathrm{E}+06$ & $1.30 \mathrm{E}-06$ \\
\hline Bi-213 & $2.68 \mathrm{E}-10$ & $9.92 \mathrm{E}+05$ & $9.92 \mathrm{E}-07$ \\
\hline Bi-214 & $1.49 \mathrm{E}-10$ & $5.51 \mathrm{E}+05$ & $5.51 \mathrm{E}-07$ \\
\hline Po-203 & $6.79 \mathrm{E}-11$ & $2.51 \mathrm{E}+05$ & $2.51 \mathrm{E}-07$ \\
\hline Po-204 & $3.77 \mathrm{E}-10$ & $1.39 \mathrm{E}+06$ & $1.39 \mathrm{E}-06$ \\
\hline Po-205 & $7.06 \mathrm{E}-11$ & $2.61 \mathrm{E}+05$ & $2.61 \mathrm{E}-07$ \\
\hline Po-206 & $6.50 \mathrm{E}-09$ & $2.41 \mathrm{E}+07$ & $2.41 \mathrm{E}-05$ \\
\hline Po-207 & $1.74 \mathrm{E}-10$ & $6.44 \mathrm{E}+05$ & $6.44 \mathrm{E}-07$ \\
\hline Po-208 & $4.43 \mathrm{E}-07$ & $1.64 \mathrm{E}+09$ & $1.64 \mathrm{E}-03$ \\
\hline Po-209 & $4.40 \mathrm{E}-07$ & $1.63 \mathrm{E}+09$ & $1.63 \mathrm{E}-03$ \\
\hline Po-210 & $3.56 \mathrm{E}-07$ & $1.32 \mathrm{E}+09$ & $1.32 \mathrm{E}-03$ \\
\hline At-205 & $7.85 \mathrm{E}-11$ & $2.90 \mathrm{E}+05$ & $2.90 \mathrm{E}-07$ \\
\hline At-206 & $8.70 \mathrm{E}-11$ & $3.22 \mathrm{E}+05$ & $3.22 \mathrm{E}-07$ \\
\hline At-207 & $3.02 \mathrm{E}-10$ & $1.12 \mathrm{E}+06$ & $1.12 \mathrm{E}-06$ \\
\hline At-208 & $1.18 \mathrm{E}-10$ & $4.37 \mathrm{E}+05$ & $4.37 \mathrm{E}-07$ \\
\hline At-209 & $5.04 \mathrm{E}-10$ & $1.86 \mathrm{E}+06$ & $1.86 \mathrm{E}-06$ \\
\hline At-210 & $1.17 \mathrm{E}-09$ & $4.33 \mathrm{E}+06$ & 4.33E-06 \\
\hline At-211 & $1.48 \mathrm{E}-08$ & $5.48 \mathrm{E}+07$ & $5.48 \mathrm{E}-05$ \\
\hline Fr-212 & $8.82 \mathrm{E}-10$ & $3.26 \mathrm{E}+06$ & $3.26 \mathrm{E}-06$ \\
\hline Fr-222 & $8.94 \mathrm{E}-10$ & $3.31 \mathrm{E}+06$ & $3.31 \mathrm{E}-06$ \\
\hline Fr-223 & 3.23E-09 & $1.20 \mathrm{E}+07$ & $1.20 \mathrm{E}-05$ \\
\hline Ra-223 & $2.17 \mathrm{E}-07$ & $8.03 \mathrm{E}+08$ & $8.03 \mathrm{E}-04$ \\
\hline
\end{tabular}




\begin{tabular}{|c|c|c|c|}
\hline Nuclide & $\begin{array}{l}\text { Ingestion } \\
\text { Dose } \\
\text { Coefficient } \\
\text { (Sv/Bq) }\end{array}$ & $\begin{array}{l}\text { Ingestion } \\
\text { Dose } \\
\text { Coefficient } \\
\text { (mrem/Ci) }\end{array}$ & $\begin{array}{c}\text { Ingestion } \\
\text { Dose } \\
\text { Coefficient } \\
\text { (mrem/pCi) }\end{array}$ \\
\hline Ra-224 & $1.26 \mathrm{E}-07$ & $4.66 \mathrm{E}+08$ & $4.66 \mathrm{E}-04$ \\
\hline Ra-225 & $2.38 \mathrm{E}-07$ & $8.81 \mathrm{E}+08$ & $8.81 \mathrm{E}-04$ \\
\hline Ra-226 & $4.53 \mathrm{E}-07$ & $1.68 \mathrm{E}+09$ & $1.68 \mathrm{E}-03$ \\
\hline Ra-227 & $1.11 \mathrm{E}-10$ & $4.11 \mathrm{E}+05$ & $4.11 \mathrm{E}-07$ \\
\hline Ra-228 & $1.60 \mathrm{E}-06$ & $5.92 \mathrm{E}+09$ & $5.92 \mathrm{E}-03$ \\
\hline Ra-230 & $2.41 \mathrm{E}-10$ & $8.92 \mathrm{E}+05$ & $8.92 \mathrm{E}-07$ \\
\hline Ac-224 & $2.78 \mathrm{E}-09$ & $1.03 \mathrm{E}+07$ & $1.03 \mathrm{E}-05$ \\
\hline Ac-225 & $5.23 \mathrm{E}-08$ & $1.94 \mathrm{E}+08$ & $1.94 \mathrm{E}-04$ \\
\hline Ac-226 & $1.45 \mathrm{E}-08$ & $5.37 \mathrm{E}+07$ & $5.37 \mathrm{E}-05$ \\
\hline Ac-227 & $3.92 \mathrm{E}-07$ & $1.45 \mathrm{E}+09$ & $1.45 \mathrm{E}-03$ \\
\hline Ac-228 & $5.14 \mathrm{E}-10$ & $1.90 \mathrm{E}+06$ & $1.90 \mathrm{E}-06$ \\
\hline Th-226 & $4.76 \mathrm{E}-10$ & $1.76 \mathrm{E}+06$ & $1.76 \mathrm{E}-06$ \\
\hline Th-227 & $1.47 \mathrm{E}-08$ & $5.44 \mathrm{E}+07$ & $5.44 \mathrm{E}-05$ \\
\hline Th-228 & $1.16 \mathrm{E}-07$ & $4.29 \mathrm{E}+08$ & $4.29 \mathrm{E}-04$ \\
\hline Th-229 & $6.09 \mathrm{E}-07$ & $2.25 \mathrm{E}+09$ & $2.25 \mathrm{E}-03$ \\
\hline Th-230 & $2.53 \mathrm{E}-07$ & $9.36 \mathrm{E}+08$ & $9.36 \mathrm{E}-04$ \\
\hline Th-231 & $4.61 \mathrm{E}-10$ & $1.71 \mathrm{E}+06$ & $1.71 \mathrm{E}-06$ \\
\hline Th-232 & $2.78 \mathrm{E}-07$ & $1.03 \mathrm{E}+09$ & $1.03 \mathrm{E}-03$ \\
\hline Th-233 & $2.93 \mathrm{E}-11$ & $1.08 \mathrm{E}+05$ & $1.08 \mathrm{E}-07$ \\
\hline Th-234 & $4.68 \mathrm{E}-09$ & $1.73 \mathrm{E}+07$ & $1.73 \mathrm{E}-05$ \\
\hline Th-236 & $1.17 \mathrm{E}-10$ & $4.33 \mathrm{E}+05$ & $4.33 \mathrm{E}-07$ \\
\hline $\mathrm{Pa}-227$ & $6.17 \mathrm{E}-10$ & $2.28 \mathrm{E}+06$ & $2.28 \mathrm{E}-06$ \\
\hline $\mathrm{Pa}-228$ & $1.08 \mathrm{E}-09$ & $4.00 \mathrm{E}+06$ & $4.00 \mathrm{E}-06$ \\
\hline $\mathrm{Pa}-229$ & $1.13 \mathrm{E}-10$ & $4.18 \mathrm{E}+05$ & $4.18 \mathrm{E}-07$ \\
\hline $\mathrm{Pa}-230$ & $1.21 \mathrm{E}-09$ & $4.48 \mathrm{E}+06$ & $4.48 \mathrm{E}-06$ \\
\hline $\mathrm{Pa}-231$ & $5.59 \mathrm{E}-07$ & $2.07 \mathrm{E}+09$ & $2.07 \mathrm{E}-03$ \\
\hline $\mathrm{Pa}-232$ & $8.87 \mathrm{E}-10$ & $3.28 \mathrm{E}+06$ & $3.28 \mathrm{E}-06$ \\
\hline $\mathrm{Pa}-233$ & $1.32 \mathrm{E}-09$ & $4.88 \mathrm{E}+06$ & $4.88 \mathrm{E}-06$ \\
\hline $\mathrm{Pa}-234$ & $5.56 \mathrm{E}-10$ & $2.06 \mathrm{E}+06$ & $2.06 \mathrm{E}-06$ \\
\hline $\mathrm{Pa}-235$ & $3.57 \mathrm{E}-11$ & $1.32 \mathrm{E}+05$ & $1.32 \mathrm{E}-07$ \\
\hline U-230 & $7.21 \mathrm{E}-08$ & $2.67 \mathrm{E}+08$ & $2.67 \mathrm{E}-04$ \\
\hline U-231 & $4.63 \mathrm{E}-10$ & $1.71 \mathrm{E}+06$ & $1.71 \mathrm{E}-06$ \\
\hline U-232 & $4.04 \mathrm{E}-07$ & $1.49 \mathrm{E}+09$ & $1.49 \mathrm{E}-03$ \\
\hline U-233 & $6.02 \mathrm{E}-08$ & $2.23 \mathrm{E}+08$ & $2.23 \mathrm{E}-04$ \\
\hline U-234 & $5.81 \mathrm{E}-08$ & $2.15 \mathrm{E}+08$ & $2.15 \mathrm{E}-04$ \\
\hline U-235 & $5.49 \mathrm{E}-08$ & $2.03 \mathrm{E}+08$ & $2.03 \mathrm{E}-04$ \\
\hline $\mathrm{U}-235 \mathrm{~m}$ & $5.82 \mathrm{E}-15$ & $2.15 \mathrm{E}+01$ & $2.15 \mathrm{E}-11$ \\
\hline U-236 & $5.47 \mathrm{E}-08$ & $2.02 \mathrm{E}+08$ & $2.02 \mathrm{E}-04$ \\
\hline U-237 & $1.06 \mathrm{E}-09$ & $3.92 \mathrm{E}+06$ & $3.92 \mathrm{E}-06$ \\
\hline U-238 & 5.24E-08 & $1.94 \mathrm{E}+08$ & $1.94 \mathrm{E}-04$ \\
\hline
\end{tabular}




\begin{tabular}{|c|c|c|c|}
\hline Nuclide & $\begin{array}{c}\text { Ingestion } \\
\text { Dose } \\
\text { Coefficient } \\
\text { (Sv/Bq) }\end{array}$ & $\begin{array}{c}\text { Ingestion } \\
\text { Dose } \\
\text { Coefficient } \\
\text { (mrem/Ci) }\end{array}$ & $\begin{array}{c}\text { Ingestion } \\
\text { Dose } \\
\text { Coefficient } \\
\text { (mrem/pCi) }\end{array}$ \\
\hline $\mathrm{U}-239$ & $3.73 \mathrm{E}-11$ & $1.38 \mathrm{E}+05$ & $1.38 \mathrm{E}-07$ \\
\hline $\mathrm{U}-240$ & $1.50 \mathrm{E}-09$ & $5.55 \mathrm{E}+06$ & $5.55 \mathrm{E}-06$ \\
\hline $\mathrm{U}-242$ & $7.07 \mathrm{E}-11$ & $2.62 \mathrm{E}+05$ & $2.62 \mathrm{E}-07$ \\
\hline $\mathrm{Np}-232$ & $1.24 \mathrm{E}-11$ & $4.59 \mathrm{E}+04$ & $4.59 \mathrm{E}-08$ \\
\hline $\mathrm{Np}-233$ & $2.94 \mathrm{E}-12$ & $1.09 \mathrm{E}+04$ & $1.09 \mathrm{E}-08$ \\
\hline $\mathrm{Np}-234$ & $8.26 \mathrm{E}-10$ & $3.06 \mathrm{E}+06$ & $3.06 \mathrm{E}-06$ \\
\hline $\mathrm{Np}-235$ & $7.96 \mathrm{E}-11$ & $2.95 \mathrm{E}+05$ & $2.95 \mathrm{E}-07$ \\
\hline $\mathrm{Np}-236$ & $2.70 \mathrm{E}-08$ & $9.99 \mathrm{E}+07$ & $9.99 \mathrm{E}-05$ \\
\hline $\mathrm{Np}-236 \mathrm{~m}$ & $2.67 \mathrm{E}-10$ & $9.88 \mathrm{E}+05$ & $9.88 \mathrm{E}-07$ \\
\hline $\mathrm{Np}-237$ & $1.25 \mathrm{E}-07$ & $4.63 \mathrm{E}+08$ & $4.63 \mathrm{E}-04$ \\
\hline $\mathrm{Np}-238$ & $1.20 \mathrm{E}-09$ & $4.44 \mathrm{E}+06$ & $4.44 \mathrm{E}-06$ \\
\hline $\mathrm{Np}-239$ & $1.11 \mathrm{E}-09$ & $4.11 \mathrm{E}+06$ & $4.11 \mathrm{E}-06$ \\
\hline $\mathrm{Np}-240$ & $9.59 \mathrm{E}-11$ & $3.55 \mathrm{E}+05$ & $3.55 \mathrm{E}-07$ \\
\hline $\mathrm{Np}-241$ & $2.09 \mathrm{E}-11$ & $7.73 \mathrm{E}+04$ & $7.73 \mathrm{E}-08$ \\
\hline $\mathrm{Pu}-232$ & $1.84 \mathrm{E}-10$ & $6.81 \mathrm{E}+05$ & $6.81 \mathrm{E}-07$ \\
\hline $\mathrm{Pu}-234$ & $2.08 \mathrm{E}-10$ & $7.70 \mathrm{E}+05$ & $7.70 \mathrm{E}-07$ \\
\hline $\mathrm{Pu}-235$ & $2.95 \mathrm{E}-12$ & $1.09 \mathrm{E}+04$ & $1.09 \mathrm{E}-08$ \\
\hline $\mathrm{Pu}-236$ & $1.10 \mathrm{E}-07$ & $4.07 \mathrm{E}+08$ & $4.07 \mathrm{E}-04$ \\
\hline $\mathrm{Pu}-237$ & $1.51 \mathrm{E}-10$ & $5.59 \mathrm{E}+05$ & $5.59 \mathrm{E}-07$ \\
\hline $\mathrm{Pu}-238$ & $2.63 \mathrm{E}-07$ & $9.73 \mathrm{E}+08$ & $9.73 \mathrm{E}-04$ \\
\hline $\mathrm{Pu}-239$ & $2.88 \mathrm{E}-07$ & $1.07 \mathrm{E}+09$ & $1.07 \mathrm{E}-03$ \\
\hline $\mathrm{Pu}-240$ & $2.88 \mathrm{E}-07$ & $1.07 \mathrm{E}+09$ & $1.07 \mathrm{E}-03$ \\
\hline $\mathrm{Pu}-241$ & $5.21 \mathrm{E}-09$ & $1.93 \mathrm{E}+07$ & $1.93 \mathrm{E}-05$ \\
\hline $\mathrm{Pu}-242$ & $2.74 \mathrm{E}-07$ & $1.01 \mathrm{E}+09$ & $1.01 \mathrm{E}-03$ \\
\hline $\mathrm{Pu}-243$ & $1.17 \mathrm{E}-10$ & $4.33 \mathrm{E}+05$ & $4.33 \mathrm{E}-07$ \\
\hline $\mathrm{Pu}-244$ & $2.73 \mathrm{E}-07$ & $1.01 \mathrm{E}+09$ & $1.01 \mathrm{E}-03$ \\
\hline $\mathrm{Pu}-245$ & $9.34 \mathrm{E}-10$ & $3.46 \mathrm{E}+06$ & $3.46 \mathrm{E}-06$ \\
\hline $\mathrm{Pu}-246$ & $3.80 \mathrm{E}-09$ & $1.41 \mathrm{E}+07$ & $1.41 \mathrm{E}-05$ \\
\hline $\mathrm{Am}-237$ & $2.38 \mathrm{E}-11$ & $8.81 \mathrm{E}+04$ & $8.81 \mathrm{E}-08$ \\
\hline $\mathrm{Am}-238$ & $4.05 \mathrm{E}-11$ & $1.50 \mathrm{E}+05$ & $1.50 \mathrm{E}-07$ \\
\hline $\mathrm{Am}-239$ & $3.32 \mathrm{E}-10$ & $1.23 \mathrm{E}+06$ & $1.23 \mathrm{E}-06$ \\
\hline $\mathrm{Am}-240$ & $7.63 \mathrm{E}-10$ & $2.82 \mathrm{E}+06$ & $2.82 \mathrm{E}-06$ \\
\hline $\mathrm{Am}-241$ & $2.38 \mathrm{E}-07$ & $8.81 \mathrm{E}+08$ & $8.81 \mathrm{E}-04$ \\
\hline $\mathrm{Am}-242$ & $4.22 \mathrm{E}-10$ & $1.56 \mathrm{E}+06$ & $1.56 \mathrm{E}-06$ \\
\hline $\mathrm{Am}-242 \mathrm{~m}$ & $2.16 \mathrm{E}-07$ & $7.99 \mathrm{E}+08$ & $7.99 \mathrm{E}-04$ \\
\hline $\mathrm{Am}-243$ & $2.36 \mathrm{E}-07$ & $8.73 \mathrm{E}+08$ & $8.73 \mathrm{E}-04$ \\
\hline $\mathrm{Am}-244$ & $6.14 \mathrm{E}-10$ & $2.27 \mathrm{E}+06$ & $2.27 \mathrm{E}-06$ \\
\hline $\mathrm{Am}-244 \mathrm{~m}$ & $4.01 \mathrm{E}-11$ & $1.48 \mathrm{E}+05$ & $1.48 \mathrm{E}-07$ \\
\hline $\mathrm{Am}-245$ & $8.45 \mathrm{E}-11$ & $3.13 \mathrm{E}+05$ & $3.13 \mathrm{E}-07$ \\
\hline $\mathrm{Am}-246$ & $8.51 \mathrm{E}-11$ & $3.15 \mathrm{E}+05$ & $3.15 \mathrm{E}-07$ \\
\hline
\end{tabular}




\begin{tabular}{|c|c|c|c|}
\hline Nuclide & $\begin{array}{l}\text { Ingestion } \\
\text { Dose } \\
\text { Coefficient } \\
(\mathrm{Sv} / \mathrm{Bq})\end{array}$ & $\begin{array}{l}\text { Ingestion } \\
\text { Dose } \\
\text { Coefficient } \\
\text { (mrem/Ci) }\end{array}$ & $\begin{array}{c}\text { Ingestion } \\
\text { Dose } \\
\text { Coefficient } \\
\text { (mrem/pCi) }\end{array}$ \\
\hline Am-246m & $4.58 \mathrm{E}-11$ & $1.69 \mathrm{E}+05$ & $1.69 \mathrm{E}-07$ \\
\hline Am-247 & $4.09 \mathrm{E}-11$ & $1.51 \mathrm{E}+05$ & $1.51 \mathrm{E}-07$ \\
\hline $\mathrm{Cm}-238$ & $8.17 \mathrm{E}-11$ & $3.02 \mathrm{E}+05$ & $3.02 \mathrm{E}-07$ \\
\hline $\mathrm{Cm}-239$ & $1.09 \mathrm{E}-10$ & $4.03 \mathrm{E}+05$ & 4.03E-07 \\
\hline $\mathrm{Cm}-240$ & $1.12 \mathrm{E}-08$ & $4.14 \mathrm{E}+07$ & $4.14 \mathrm{E}-05$ \\
\hline $\mathrm{Cm}-241$ & $1.24 \mathrm{E}-09$ & $4.59 \mathrm{E}+06$ & $4.59 \mathrm{E}-06$ \\
\hline Cm-242 & $1.92 \mathrm{E}-08$ & $7.10 \mathrm{E}+07$ & $7.10 \mathrm{E}-05$ \\
\hline $\mathrm{Cm}-243$ & $1.80 \mathrm{E}-07$ & $6.66 \mathrm{E}+08$ & $6.66 \mathrm{E}-04$ \\
\hline $\mathrm{Cm}-244$ & $1.51 \mathrm{E}-07$ & $5.59 \mathrm{E}+08$ & $5.59 \mathrm{E}-04$ \\
\hline $\mathrm{Cm}-245$ & $2.42 \mathrm{E}-07$ & $8.95 \mathrm{E}+08$ & $8.95 \mathrm{E}-04$ \\
\hline $\mathrm{Cm}-246$ & $2.41 \mathrm{E}-07$ & $8.92 \mathrm{E}+08$ & $8.92 \mathrm{E}-04$ \\
\hline $\mathrm{Cm}-247$ & $2.22 \mathrm{E}-07$ & $8.21 \mathrm{E}+08$ & $8.21 \mathrm{E}-04$ \\
\hline $\mathrm{Cm}-248$ & $9.03 \mathrm{E}-07$ & $3.34 \mathrm{E}+09$ & $3.34 \mathrm{E}-03$ \\
\hline $\mathrm{Cm}-249$ & $4.23 \mathrm{E}-11$ & $1.57 \mathrm{E}+05$ & $1.57 \mathrm{E}-07$ \\
\hline $\mathrm{Cm}-250$ & $6.18 \mathrm{E}-06$ & $2.29 \mathrm{E}+10$ & $2.29 \mathrm{E}-02$ \\
\hline $\mathrm{Cm}-251$ & $3.87 \mathrm{E}-11$ & $1.43 \mathrm{E}+05$ & $1.43 \mathrm{E}-07$ \\
\hline Bk-245 & $7.83 \mathrm{E}-10$ & $2.90 \mathrm{E}+06$ & $2.90 \mathrm{E}-06$ \\
\hline Bk-246 & $5.82 \mathrm{E}-10$ & $2.15 \mathrm{E}+06$ & $2.15 \mathrm{E}-06$ \\
\hline Bk-247 & $4.43 \mathrm{E}-07$ & $1.64 \mathrm{E}+09$ & $1.64 \mathrm{E}-03$ \\
\hline Bk-248m & $6.05 \mathrm{E}-10$ & $2.24 \mathrm{E}+06$ & $2.24 \mathrm{E}-06$ \\
\hline Bk-249 & $1.25 \mathrm{E}-09$ & $4.63 \mathrm{E}+06$ & $4.63 \mathrm{E}-06$ \\
\hline Bk-250 & $1.83 \mathrm{E}-10$ & $6.77 \mathrm{E}+05$ & $6.77 \mathrm{E}-07$ \\
\hline Bk-251 & $5.11 \mathrm{E}-11$ & $1.89 \mathrm{E}+05$ & $1.89 \mathrm{E}-07$ \\
\hline Cf-244 & $9.55 \mathrm{E}-11$ & $3.53 \mathrm{E}+05$ & $3.53 \mathrm{E}-07$ \\
\hline Cf-246 & $4.63 \mathrm{E}-09$ & $1.71 \mathrm{E}+07$ & $1.71 \mathrm{E}-05$ \\
\hline Cf-247 & $2.90 \mathrm{E}-11$ & $1.07 \mathrm{E}+05$ & $1.07 \mathrm{E}-07$ \\
\hline Cf-248 & $4.68 \mathrm{E}-08$ & $1.73 \mathrm{E}+08$ & $1.73 \mathrm{E}-04$ \\
\hline Cf-249 & $4.46 \mathrm{E}-07$ & $1.65 \mathrm{E}+09$ & $1.65 \mathrm{E}-03$ \\
\hline Cf-250 & $2.22 \mathrm{E}-07$ & $8.21 \mathrm{E}+08$ & $8.21 \mathrm{E}-04$ \\
\hline Cf-251 & $4.55 \mathrm{E}-07$ & $1.68 \mathrm{E}+09$ & $1.68 \mathrm{E}-03$ \\
\hline Cf-252 & $1.51 \mathrm{E}-07$ & $5.59 \mathrm{E}+08$ & $5.59 \mathrm{E}-04$ \\
\hline Cf-253 & $2.76 \mathrm{E}-09$ & $1.02 \mathrm{E}+07$ & $1.02 \mathrm{E}-05$ \\
\hline Cf-254 & $6.02 \mathrm{E}-07$ & $2.23 \mathrm{E}+09$ & $2.23 \mathrm{E}-03$ \\
\hline Cf- 255 & $5.46 \mathrm{E}-11$ & $2.02 \mathrm{E}+05$ & $2.02 \mathrm{E}-07$ \\
\hline Es-249 & $2.73 \mathrm{E}-11$ & $1.01 \mathrm{E}+05$ & $1.01 \mathrm{E}-07$ \\
\hline Es-250 & $6.20 \mathrm{E}-10$ & $2.29 \mathrm{E}+06$ & $2.29 \mathrm{E}-06$ \\
\hline Es- $250 \mathrm{~m}$ & $3.93 \mathrm{E}-11$ & $1.45 \mathrm{E}+05$ & $1.45 \mathrm{E}-07$ \\
\hline Es-251 & $2.45 \mathrm{E}-10$ & $9.07 \mathrm{E}+05$ & $9.07 \mathrm{E}-07$ \\
\hline Es-253 & $9.21 \mathrm{E}-09$ & $3.41 \mathrm{E}+07$ & $3.41 \mathrm{E}-05$ \\
\hline Es-254 & $4.59 \mathrm{E}-08$ & $1.70 \mathrm{E}+08$ & $1.70 \mathrm{E}-04$ \\
\hline
\end{tabular}




\begin{tabular}{|c|c|c|c|}
\hline Nuclide & $\begin{array}{c}\text { Ingestion } \\
\text { Dose } \\
\text { Coefficient } \\
\text { (Sv/Bq) }\end{array}$ & $\begin{array}{c}\text { Ingestion } \\
\text { Dose } \\
\text { Coefficient } \\
\text { (mrem/Ci) }\end{array}$ & $\begin{array}{c}\text { Ingestion } \\
\text { Dose } \\
\text { Coefficient } \\
\text { (mrem/pCi) }\end{array}$ \\
\hline Es-254m & $5.99 \mathrm{E}-09$ & $2.22 \mathrm{E}+07$ & $2.22 \mathrm{E}-05$ \\
\hline Es-255 & $9.91 \mathrm{E}-09$ & $3.67 \mathrm{E}+07$ & $3.67 \mathrm{E}-05$ \\
\hline Es-256 & $3.87 \mathrm{E}-09$ & $1.43 \mathrm{E}+07$ & $1.43 \mathrm{E}-05$ \\
\hline Fm-251 & $9.83 \mathrm{E}-11$ & $3.64 \mathrm{E}+05$ & $3.64 \mathrm{E}-07$ \\
\hline Fm-252 & $4.01 \mathrm{E}-09$ & $1.48 \mathrm{E}+07$ & $1.48 \mathrm{E}-05$ \\
\hline Fm-253 & $1.74 \mathrm{E}-09$ & $6.44 \mathrm{E}+06$ & $6.44 \mathrm{E}-06$ \\
\hline Fm-254 & $6.29 \mathrm{E}-10$ & $2.33 \mathrm{E}+06$ & $2.33 \mathrm{E}-06$ \\
\hline Fm-255 & $3.55 \mathrm{E}-09$ & $1.31 \mathrm{E}+07$ & $1.31 \mathrm{E}-05$ \\
\hline Fm-256 & $2.50 \mathrm{E}-08$ & $9.25 \mathrm{E}+07$ & $9.25 \mathrm{E}-05$ \\
\hline Fm-257 & $2.94 \mathrm{E}-08$ & $1.09 \mathrm{E}+08$ & $1.09 \mathrm{E}-04$ \\
\hline
\end{tabular}




\section{APPENDIX B \\ REFERENCE PERSON INHALATION DOSE COEFFICIENTS}

Appendix B tabulates the inhalation dose coefficients used in calculating the DCS. The dose coefficients are from DOE Standard (DOE-STD-1196-2011): Derived Concentration

Technical Standard. 
Table B-1 Inhalation Dose Coefficients

\begin{tabular}{|c|c|c|}
\hline Nuclide & $\begin{array}{c}\text { Inhalation } \\
\text { Dose } \\
\text { Coefficient } \\
\text { (Sv/Bq) }\end{array}$ & $\begin{array}{c}\text { Inhalation } \\
\text { Dose } \\
\text { Coefficient } \\
\text { (mrem/pCi) }\end{array}$ \\
\hline H-3 & $1.93 \mathrm{E}-11$ & $7.14 \mathrm{E}-08$ \\
\hline Be-7 & $6.40 \mathrm{E}-11$ & $2.37 \mathrm{E}-07$ \\
\hline Be-10 & $3.66 \mathrm{E}-08$ & $1.35 \mathrm{E}-04$ \\
\hline $\mathrm{C}-11$ & $2.56 \mathrm{E}-12$ & $9.47 \mathrm{E}-09$ \\
\hline $\mathrm{C}-14$ & $6.70 \mathrm{E}-12$ & $2.48 \mathrm{E}-08$ \\
\hline $\mathrm{F}-18$ & $6.64 \mathrm{E}-11$ & $2.46 \mathrm{E}-07$ \\
\hline $\mathrm{Na}-22$ & $3.15 \mathrm{E}-08$ & $1.17 \mathrm{E}-04$ \\
\hline $\mathrm{Na}-24$ & $5.83 \mathrm{E}-10$ & $2.16 \mathrm{E}-06$ \\
\hline $\mathrm{Mg}-28$ & $1.50 \mathrm{E}-09$ & $5.55 \mathrm{E}-06$ \\
\hline $\mathrm{Al}-26$ & $1.14 \mathrm{E}-07$ & $4.22 \mathrm{E}-04$ \\
\hline $\mathrm{Si}-31$ & $9.30 \mathrm{E}-11$ & $3.44 \mathrm{E}-07$ \\
\hline $\mathrm{Si}-32$ & $1.16 \mathrm{E}-07$ & $4.29 \mathrm{E}-04$ \\
\hline $\mathrm{P}-32$ & $4.38 \mathrm{E}-09$ & $1.62 \mathrm{E}-05$ \\
\hline $\mathrm{P}-33$ & $1.96 \mathrm{E}-09$ & $7.25 \mathrm{E}-06$ \\
\hline $\mathrm{S}-35$ & $1.56 \mathrm{E}-09$ & $5.77 \mathrm{E}-06$ \\
\hline $\mathrm{S}-38$ & $3.33 \mathrm{E}-10$ & $1.23 \mathrm{E}-06$ \\
\hline $\mathrm{Cl}-34 \mathrm{~m}$ & $5.50 \mathrm{E}-11$ & $2.04 \mathrm{E}-07$ \\
\hline $\mathrm{Cl}-36$ & $3.99 \mathrm{E}-08$ & $1.48 \mathrm{E}-04$ \\
\hline $\mathrm{Cl}-38$ & $5.70 \mathrm{E}-11$ & $2.11 \mathrm{E}-07$ \\
\hline $\mathrm{Cl}-39$ & $5.79 \mathrm{E}-11$ & $2.14 \mathrm{E}-07$ \\
\hline $\mathrm{K}-40$ & $8.87 \mathrm{E}-08$ & $3.28 \mathrm{E}-04$ \\
\hline $\mathrm{K}-42$ & $4.08 \mathrm{E}-10$ & $1.51 \mathrm{E}-06$ \\
\hline $\mathrm{K}-43$ & $4.32 \mathrm{E}-10$ & $1.60 \mathrm{E}-06$ \\
\hline $\mathrm{K}-44$ & $3.99 \mathrm{E}-11$ & $1.48 \mathrm{E}-07$ \\
\hline $\mathrm{K}-45$ & $2.63 \mathrm{E}-11$ & $9.73 \mathrm{E}-08$ \\
\hline $\mathrm{Ca}-41$ & $1.27 \mathrm{E}-10$ & $4.70 \mathrm{E}-07$ \\
\hline $\mathrm{Ca}-45$ & $2.99 \mathrm{E}-09$ & $1.11 \mathrm{E}-05$ \\
\hline $\mathrm{Ca}-47$ & $2.14 \mathrm{E}-09$ & $7.92 \mathrm{E}-06$ \\
\hline $\mathrm{Sc}-43$ & $1.49 \mathrm{E}-10$ & $5.51 \mathrm{E}-07$ \\
\hline Sc-44 & $2.23 \mathrm{E}-10$ & $8.25 \mathrm{E}-07$ \\
\hline $\mathrm{Sc}-44 \mathrm{~m}$ & $1.69 \mathrm{E}-09$ & $6.25 \mathrm{E}-06$ \\
\hline $\mathrm{Sc}-46$ & $7.60 \mathrm{E}-09$ & $2.81 \mathrm{E}-05$ \\
\hline $\mathrm{Sc}-47$ & $8.12 \mathrm{E}-10$ & $3.00 \mathrm{E}-06$ \\
\hline
\end{tabular}




\begin{tabular}{|c|c|c|}
\hline Nuclide & $\begin{array}{c}\text { Inhalation } \\
\text { Dose } \\
\text { Coefficient } \\
\text { (Sv/Bq) } \\
\end{array}$ & $\begin{array}{c}\text { Inhalation } \\
\text { Dose } \\
\text { Coefficient } \\
\text { (mrem/pCi) }\end{array}$ \\
\hline Sc-48 & 1.30E-09 & 4.81E-06 \\
\hline Sc-49 & $4.67 \mathrm{E}-11$ & $1.73 \mathrm{E}-07$ \\
\hline $\mathrm{Ti}-44$ & $1.33 \mathrm{E}-07$ & $4.92 \mathrm{E}-04$ \\
\hline $\mathrm{Ti}-45$ & $1.11 \mathrm{E}-10$ & $4.11 \mathrm{E}-07$ \\
\hline $\mathrm{V}-47$ & $3.66 \mathrm{E}-11$ & $1.35 \mathrm{E}-07$ \\
\hline $\mathrm{V}-48$ & 3.09E-09 & $1.14 \mathrm{E}-05$ \\
\hline $\mathrm{V}-49$ & $7.97 \mathrm{E}-11$ & $2.95 \mathrm{E}-07$ \\
\hline $\mathrm{V}-50$ & 6.69E-08 & $2.48 \mathrm{E}-04$ \\
\hline $\mathrm{Cr}-48$ & $2.43 \mathrm{E}-10$ & 8.99E-07 \\
\hline Cr-49 & $4.18 \mathrm{E}-11$ & $1.55 \mathrm{E}-07$ \\
\hline Cr-51 & 4.34E-11 & $1.61 \mathrm{E}-07$ \\
\hline Mn-51 & $5.17 \mathrm{E}-11$ & $1.91 \mathrm{E}-07$ \\
\hline $\mathrm{Mn}-52$ & $1.66 \mathrm{E}-09$ & $6.14 \mathrm{E}-06$ \\
\hline $\mathrm{Mn}-52 \mathrm{~m}$ & $3.55 \mathrm{E}-11$ & $1.31 \mathrm{E}-07$ \\
\hline Mn-53 & $3.65 \mathrm{E}-10$ & $1.35 \mathrm{E}-06$ \\
\hline $\mathrm{Mn}-54$ & $3.60 \mathrm{E}-09$ & $1.33 \mathrm{E}-05$ \\
\hline Mn-56 & $1.54 \mathrm{E}-10$ & $5.70 \mathrm{E}-07$ \\
\hline $\mathrm{Fe}-52$ & 7.33E-10 & $2.71 \mathrm{E}-06$ \\
\hline $\mathrm{Fe}-55$ & 4.37E-10 & $1.62 \mathrm{E}-06$ \\
\hline $\mathrm{Fe}-59$ & 4.12E-09 & $1.52 \mathrm{E}-05$ \\
\hline $\mathrm{Fe}-60$ & $1.45 \mathrm{E}-07$ & $5.37 \mathrm{E}-04$ \\
\hline Co-55 & $5.99 \mathrm{E}-10$ & $2.22 \mathrm{E}-06$ \\
\hline Co-56 & $5.40 \mathrm{E}-09$ & $2.00 \mathrm{E}-05$ \\
\hline Co-57 & $6.18 \mathrm{E}-10$ & $2.29 \mathrm{E}-06$ \\
\hline Co-58 & $1.78 \mathrm{E}-09$ & $6.59 \mathrm{E}-06$ \\
\hline Co-58m & $1.56 \mathrm{E}-11$ & $5.77 \mathrm{E}-08$ \\
\hline Co-60 & $1.12 \mathrm{E}-08$ & 4.14E-05 \\
\hline Co-60m & $1.36 \mathrm{E}-12$ & $5.03 \mathrm{E}-09$ \\
\hline Co-61 & $5.58 \mathrm{E}-11$ & $2.06 \mathrm{E}-07$ \\
\hline Co-62m & $2.44 \mathrm{E}-11$ & $9.03 \mathrm{E}-08$ \\
\hline $\mathrm{Ni}-56$ & $1.01 \mathrm{E}-09$ & $3.74 \mathrm{E}-06$ \\
\hline $\mathrm{Ni}-57$ & $6.13 \mathrm{E}-10$ & $2.27 \mathrm{E}-06$ \\
\hline $\mathrm{Ni}-59$ & $1.48 \mathrm{E}-10$ & $5.48 \mathrm{E}-07$ \\
\hline $\mathrm{Ni}-63$ & $5.43 \mathrm{E}-10$ & $2.01 \mathrm{E}-06$ \\
\hline $\mathrm{Ni}-65$ & $1.01 \mathrm{E}-10$ & $3.74 \mathrm{E}-07$ \\
\hline Ni-66 & $1.94 \mathrm{E}-09$ & 7.18E-06 \\
\hline $\mathrm{Cu}-60$ & $4.22 \mathrm{E}-11$ & $1.56 \mathrm{E}-07$ \\
\hline
\end{tabular}




\begin{tabular}{|c|c|c|}
\hline Nuclide & $\begin{array}{l}\text { Inhalation } \\
\text { Dose } \\
\text { Coefficient } \\
\text { (Sv/Bq) } \\
\end{array}$ & $\begin{array}{c}\text { Inhalation } \\
\text { Dose } \\
\text { Coefficient } \\
\text { (mrem/pCi) }\end{array}$ \\
\hline $\mathrm{Cu}-61$ & $9.02 \mathrm{E}-11$ & $3.34 \mathrm{E}-07$ \\
\hline $\mathrm{Cu}-64$ & $1.36 \mathrm{E}-10$ & $5.03 \mathrm{E}-07$ \\
\hline $\mathrm{Cu}-67$ & $6.61 \mathrm{E}-10$ & $2.45 \mathrm{E}-06$ \\
\hline $\mathrm{Zn}-62$ & $6.08 \mathrm{E}-10$ & $2.25 \mathrm{E}-06$ \\
\hline $\mathrm{Zn}-63$ & $4.23 \mathrm{E}-11$ & $1.57 \mathrm{E}-07$ \\
\hline $\mathrm{Zn}-65$ & $1.80 \mathrm{E}-09$ & $6.66 \mathrm{E}-06$ \\
\hline Zn-69 & $3.03 \mathrm{E}-11$ & $1.12 \mathrm{E}-07$ \\
\hline $\mathrm{Zn}-69 \mathrm{~m}$ & $2.93 \mathrm{E}-10$ & $1.08 \mathrm{E}-06$ \\
\hline $\mathrm{Zn}-71 \mathrm{~m}$ & $1.84 \mathrm{E}-10$ & $6.81 \mathrm{E}-07$ \\
\hline $\mathrm{Zn}-72$ & $1.42 \mathrm{E}-09$ & $5.25 \mathrm{E}-06$ \\
\hline Ga-65 & $2.06 \mathrm{E}-11$ & $7.62 \mathrm{E}-08$ \\
\hline Ga-66 & $5.70 \mathrm{E}-10$ & $2.11 \mathrm{E}-06$ \\
\hline Ga-67 & $3.01 \mathrm{E}-10$ & $1.11 \mathrm{E}-06$ \\
\hline Ga-68 & $6.14 \mathrm{E}-11$ & $2.27 \mathrm{E}-07$ \\
\hline Ga-70 & $1.95 \mathrm{E}-11$ & 7.22E-08 \\
\hline Ga-72 & $6.72 \mathrm{E}-10$ & $2.49 \mathrm{E}-06$ \\
\hline Ga-73 & $1.83 \mathrm{E}-10$ & $6.77 \mathrm{E}-07$ \\
\hline Ge-66 & $1.10 \mathrm{E}-10$ & $4.07 \mathrm{E}-07$ \\
\hline Ge-67 & $2.97 \mathrm{E}-11$ & $1.10 \mathrm{E}-07$ \\
\hline Ge-68 & $3.35 \mathrm{E}-08$ & $1.24 \mathrm{E}-04$ \\
\hline Ge-69 & $2.93 \mathrm{E}-10$ & $1.08 \mathrm{E}-06$ \\
\hline Ge-71 & $1.44 \mathrm{E}-11$ & $5.33 \mathrm{E}-08$ \\
\hline Ge-75 & $4.48 \mathrm{E}-11$ & $1.66 \mathrm{E}-07$ \\
\hline Ge-77 & $4.50 \mathrm{E}-10$ & $1.67 \mathrm{E}-06$ \\
\hline Ge-78 & $1.15 \mathrm{E}-10$ & $4.26 \mathrm{E}-07$ \\
\hline As-69 & $2.57 \mathrm{E}-11$ & $9.51 \mathrm{E}-08$ \\
\hline As-70 & $8.64 \mathrm{E}-11$ & $3.20 \mathrm{E}-07$ \\
\hline As-71 & $4.54 \mathrm{E}-10$ & $1.68 \mathrm{E}-06$ \\
\hline As- 72 & $1.14 \mathrm{E}-09$ & $4.22 \mathrm{E}-06$ \\
\hline As-73 & $1.52 \mathrm{E}-09$ & $5.62 \mathrm{E}-06$ \\
\hline As-74 & $2.78 \mathrm{E}-09$ & $1.03 \mathrm{E}-05$ \\
\hline As-76 & $9.12 \mathrm{E}-10$ & $3.37 \mathrm{E}-06$ \\
\hline As-77 & $4.83 \mathrm{E}-10$ & $1.79 \mathrm{E}-06$ \\
\hline As-78 & $1.08 \mathrm{E}-10$ & $4.00 \mathrm{E}-07$ \\
\hline $\mathrm{Se}-70$ & $4.65 \mathrm{E}-11$ & $1.72 \mathrm{E}-07$ \\
\hline Se-72 & 2.84E-09 & $1.05 \mathrm{E}-05$ \\
\hline $\mathrm{Se}-73$ & $1.06 \mathrm{E}-10$ & $3.92 \mathrm{E}-07$ \\
\hline
\end{tabular}




\begin{tabular}{|c|c|c|}
\hline Nuclide & $\begin{array}{l}\text { Inhalation } \\
\text { Dose } \\
\text { Coefficient } \\
\text { (Sv/Bq) } \\
\end{array}$ & $\begin{array}{c}\text { Inhalation } \\
\text { Dose } \\
\text { Coefficient } \\
\text { (mrem/pCi) }\end{array}$ \\
\hline $\mathrm{Se}-73 \mathrm{~m}$ & $1.20 \mathrm{E}-11$ & $4.44 \mathrm{E}-08$ \\
\hline $\mathrm{Se}-75$ & $1.24 \mathrm{E}-09$ & $4.59 \mathrm{E}-06$ \\
\hline Se-79 & $1.68 \mathrm{E}-09$ & $6.22 \mathrm{E}-06$ \\
\hline $\mathrm{Se}-81$ & $9.61 \mathrm{E}-12$ & $3.56 \mathrm{E}-08$ \\
\hline $\mathrm{Se}-81 \mathrm{~m}$ & $2.03 \mathrm{E}-11$ & $7.51 \mathrm{E}-08$ \\
\hline $\mathrm{Se}-83$ & $2.21 \mathrm{E}-11$ & $8.18 \mathrm{E}-08$ \\
\hline $\mathrm{Br}-74$ & $4.75 \mathrm{E}-11$ & $1.76 \mathrm{E}-07$ \\
\hline $\mathrm{Br}-74 \mathrm{~m}$ & $8.06 \mathrm{E}-11$ & $2.98 \mathrm{E}-07$ \\
\hline $\mathrm{Br}-75$ & $6.63 \mathrm{E}-11$ & $2.45 \mathrm{E}-07$ \\
\hline $\mathrm{Br}-76$ & $5.09 \mathrm{E}-10$ & $1.88 \mathrm{E}-06$ \\
\hline $\mathrm{Br}-77$ & $1.07 \mathrm{E}-10$ & $3.96 \mathrm{E}-07$ \\
\hline $\mathrm{Br}-80$ & $1.73 \mathrm{E}-11$ & $6.40 \mathrm{E}-08$ \\
\hline $\mathrm{Br}-80 \mathrm{~m}$ & $1.25 \mathrm{E}-10$ & $4.63 \mathrm{E}-07$ \\
\hline Br-82 & $7.72 \mathrm{E}-10$ & $2.86 \mathrm{E}-06$ \\
\hline $\mathrm{Br}-83$ & $6.04 \mathrm{E}-11$ & $2.23 \mathrm{E}-07$ \\
\hline $\mathrm{Br}-84$ & $4.62 \mathrm{E}-11$ & $1.71 \mathrm{E}-07$ \\
\hline $\mathrm{Rb}-78$ & $3.55 \mathrm{E}-11$ & $1.31 \mathrm{E}-07$ \\
\hline $\mathrm{Rb}-79$ & $3.42 \mathrm{E}-11$ & $1.27 \mathrm{E}-07$ \\
\hline $\mathrm{Rb}-81$ & $8.35 \mathrm{E}-11$ & $3.09 \mathrm{E}-07$ \\
\hline $\mathrm{Rb}-81 \mathrm{~m}$ & $1.92 \mathrm{E}-11$ & $7.10 \mathrm{E}-08$ \\
\hline $\mathrm{Rb}-82 \mathrm{~m}$ & $1.78 \mathrm{E}-10$ & $6.59 \mathrm{E}-07$ \\
\hline $\mathrm{Rb}-83$ & $1.56 \mathrm{E}-09$ & $5.77 \mathrm{E}-06$ \\
\hline $\mathrm{Rb}-84$ & $3.24 \mathrm{E}-09$ & $1.20 \mathrm{E}-05$ \\
\hline $\mathrm{Rb}-84 \mathrm{~m}$ & $1.01 \mathrm{E}-11$ & $3.74 \mathrm{E}-08$ \\
\hline $\mathrm{Rb}-86$ & $5.27 \mathrm{E}-09$ & $1.95 \mathrm{E}-05$ \\
\hline $\mathrm{Rb}-87$ & $1.68 \mathrm{E}-08$ & $6.22 \mathrm{E}-05$ \\
\hline $\mathrm{Rb}-88$ & $3.33 \mathrm{E}-11$ & $1.23 \mathrm{E}-07$ \\
\hline $\mathrm{Rb}-89$ & $2.64 \mathrm{E}-11$ & $9.77 \mathrm{E}-08$ \\
\hline Sr-80 & $1.81 \mathrm{E}-10$ & $6.70 \mathrm{E}-07$ \\
\hline Sr-81 & $3.72 \mathrm{E}-11$ & $1.38 \mathrm{E}-07$ \\
\hline Sr-82 & $1.02 \mathrm{E}-08$ & $3.77 \mathrm{E}-05$ \\
\hline Sr-83 & $3.93 \mathrm{E}-10$ & $1.45 \mathrm{E}-06$ \\
\hline Sr-85 & $7.45 \mathrm{E}-10$ & $2.76 \mathrm{E}-06$ \\
\hline Sr- $85 \mathrm{~m}$ & $4.96 \mathrm{E}-12$ & $1.84 \mathrm{E}-08$ \\
\hline $\mathrm{Sr}-87 \mathrm{~m}$ & $2.40 \mathrm{E}-11$ & $8.88 \mathrm{E}-08$ \\
\hline Sr-89 & $6.83 \mathrm{E}-09$ & $2.53 \mathrm{E}-05$ \\
\hline Sr-90 & $3.92 \mathrm{E}-08$ & $1.45 \mathrm{E}-04$ \\
\hline
\end{tabular}




\begin{tabular}{|c|c|c|}
\hline Nuclide & $\begin{array}{l}\text { Inhalation } \\
\text { Dose } \\
\text { Coefficient } \\
\text { (Sv/Bq) } \\
\end{array}$ & $\begin{array}{c}\text { Inhalation } \\
\text { Dose } \\
\text { Coefficient } \\
\text { (mrem/pCi) }\end{array}$ \\
\hline Sr-91 & $4.44 \mathrm{E}-10$ & $1.64 \mathrm{E}-06$ \\
\hline Sr-92 & $2.50 \mathrm{E}-10$ & $9.25 \mathrm{E}-07$ \\
\hline Y-84m & $7.50 \mathrm{E}-11$ & $2.78 \mathrm{E}-07$ \\
\hline$Y-85$ & $1.16 \mathrm{E}-10$ & $4.29 \mathrm{E}-07$ \\
\hline$Y-85 m$ & $2.04 \mathrm{E}-10$ & $7.55 \mathrm{E}-07$ \\
\hline $\mathrm{Y}-86$ & $5.69 \mathrm{E}-10$ & $2.11 \mathrm{E}-06$ \\
\hline $\mathrm{Y}-86 \mathrm{~m}$ & $3.42 \mathrm{E}-11$ & $1.27 \mathrm{E}-07$ \\
\hline $\mathrm{Y}-87$ & $4.64 \mathrm{E}-10$ & $1.72 \mathrm{E}-06$ \\
\hline $\mathrm{Y}-87 \mathrm{~m}$ & $1.66 \mathrm{E}-10$ & $6.14 \mathrm{E}-07$ \\
\hline $\mathrm{Y}-88$ & $6.89 \mathrm{E}-09$ & $2.55 \mathrm{E}-05$ \\
\hline$Y-90$ & $1.77 \mathrm{E}-09$ & $6.55 \mathrm{E}-06$ \\
\hline Y-90m & $1.20 \mathrm{E}-10$ & $4.44 \mathrm{E}-07$ \\
\hline Y-91 & 9.91E-09 & $3.67 \mathrm{E}-05$ \\
\hline Y-91m & $1.33 \mathrm{E}-11$ & 4.92E-08 \\
\hline $\mathrm{Y}-92$ & $2.15 \mathrm{E}-10$ & $7.96 \mathrm{E}-07$ \\
\hline$Y-93$ & $5.20 \mathrm{E}-10$ & $1.92 \mathrm{E}-06$ \\
\hline$Y-94$ & $3.29 \mathrm{E}-11$ & $1.22 \mathrm{E}-07$ \\
\hline$Y-95$ & $1.81 \mathrm{E}-11$ & $6.70 \mathrm{E}-08$ \\
\hline $\mathrm{Zr}-86$ & $5.07 \mathrm{E}-10$ & $1.88 \mathrm{E}-06$ \\
\hline $\mathrm{Zr}-87$ & $1.02 \mathrm{E}-10$ & $3.77 \mathrm{E}-07$ \\
\hline $\mathrm{Zr}-88$ & $2.86 \mathrm{E}-09$ & $1.06 \mathrm{E}-05$ \\
\hline Zr-89 & $6.15 \mathrm{E}-10$ & $2.28 \mathrm{E}-06$ \\
\hline Zr-93 & $9.02 \mathrm{E}-09$ & $3.34 \mathrm{E}-05$ \\
\hline $\mathrm{Zr}-95$ & $5.29 \mathrm{E}-09$ & $1.96 \mathrm{E}-05$ \\
\hline Zr-97 & $1.10 \mathrm{E}-09$ & $4.07 \mathrm{E}-06$ \\
\hline $\mathrm{Nb}-88$ & $3.54 \mathrm{E}-11$ & $1.31 \mathrm{E}-07$ \\
\hline $\mathrm{Nb}-89$ & $1.37 \mathrm{E}-10$ & $5.07 \mathrm{E}-07$ \\
\hline $\mathrm{Nb}-89 \mathrm{~m}$ & $8.03 \mathrm{E}-11$ & $2.97 \mathrm{E}-07$ \\
\hline $\mathrm{Nb}-90$ & $7.63 \mathrm{E}-10$ & $2.82 \mathrm{E}-06$ \\
\hline $\mathrm{Nb}-91$ & $3.03 \mathrm{E}-10$ & $1.12 \mathrm{E}-06$ \\
\hline $\mathrm{Nb}-91 \mathrm{~m}$ & $3.65 \mathrm{E}-09$ & $1.35 \mathrm{E}-05$ \\
\hline $\mathrm{Nb}-92$ & $5.46 \mathrm{E}-09$ & $2.02 \mathrm{E}-05$ \\
\hline $\mathrm{Nb}-92 \mathrm{~m}$ & $5.00 \mathrm{E}-10$ & $1.85 \mathrm{E}-06$ \\
\hline $\mathrm{Nb}-93 \mathrm{~m}$ & $6.11 \mathrm{E}-10$ & $2.26 \mathrm{E}-06$ \\
\hline $\mathrm{Nb}-94$ & $1.18 \mathrm{E}-08$ & $4.37 \mathrm{E}-05$ \\
\hline $\mathrm{Nb}-95$ & $1.64 \mathrm{E}-09$ & $6.07 \mathrm{E}-06$ \\
\hline $\mathrm{Nb}-95 \mathrm{~m}$ & $8.97 \mathrm{E}-10$ & $3.32 \mathrm{E}-06$ \\
\hline
\end{tabular}




\begin{tabular}{|c|c|c|}
\hline Nuclide & $\begin{array}{c}\text { Inhalation } \\
\text { Dose } \\
\text { Coefficient } \\
\text { (Sv/Bq) }\end{array}$ & $\begin{array}{c}\text { Inhalation } \\
\text { Dose } \\
\text { Coefficient } \\
\text { (mrem/pCi) }\end{array}$ \\
\hline Nb-96 & $7.48 \mathrm{E}-10$ & $2.77 \mathrm{E}-06$ \\
\hline Nb-97 & $5.06 \mathrm{E}-11$ & $1.87 \mathrm{E}-07$ \\
\hline Nb-98m & $6.78 \mathrm{E}-11$ & $2.51 \mathrm{E}-07$ \\
\hline Mo-90 & $4.00 \mathrm{E}-10$ & $1.48 \mathrm{E}-06$ \\
\hline Mo-91 & $2.39 \mathrm{E}-11$ & $8.84 \mathrm{E}-08$ \\
\hline Mo-93 & $6.06 \mathrm{E}-10$ & $2.24 \mathrm{E}-06$ \\
\hline Mo-93m & $2.05 \mathrm{E}-10$ & $7.59 \mathrm{E}-07$ \\
\hline Mo-99 & $1.03 \mathrm{E}-09$ & $3.81 \mathrm{E}-06$ \\
\hline Mo-101 & $2.96 \mathrm{E}-11$ & $1.10 \mathrm{E}-07$ \\
\hline Mo-102 & $2.89 \mathrm{E}-11$ & $1.07 \mathrm{E}-07$ \\
\hline Tc-93 & $5.53 \mathrm{E}-11$ & $2.05 \mathrm{E}-07$ \\
\hline Tc-93m & $2.48 \mathrm{E}-11$ & $9.18 \mathrm{E}-08$ \\
\hline Tc-94 & $1.53 \mathrm{E}-10$ & $5.66 \mathrm{E}-07$ \\
\hline Tc-94m & $5.58 \mathrm{E}-11$ & $2.06 \mathrm{E}-07$ \\
\hline Tc-95 & $1.31 \mathrm{E}-10$ & $4.85 \mathrm{E}-07$ \\
\hline Tc-95m & $1.04 \mathrm{E}-09$ & $3.85 \mathrm{E}-06$ \\
\hline Tc-96 & $8.29 \mathrm{E}-10$ & $3.07 \mathrm{E}-06$ \\
\hline Tc-96m & $8.89 \mathrm{E}-12$ & $3.29 \mathrm{E}-08$ \\
\hline Tc-97 & $2.52 \mathrm{E}-10$ & $9.32 \mathrm{E}-07$ \\
\hline Tc-97m & $3.55 \mathrm{E}-09$ & $1.31 \mathrm{E}-05$ \\
\hline Tc-98 & $8.70 \mathrm{E}-09$ & $3.22 \mathrm{E}-05$ \\
\hline Tc-99 & $4.42 \mathrm{E}-09$ & $1.64 \mathrm{E}-05$ \\
\hline Tc-99m & $2.24 \mathrm{E}-11$ & $8.29 \mathrm{E}-08$ \\
\hline Tc-101 & $1.40 \mathrm{E}-11$ & $5.18 \mathrm{E}-08$ \\
\hline Tc-104 & $3.49 \mathrm{E}-11$ & $1.29 \mathrm{E}-07$ \\
\hline Ru-94 & $5.21 \mathrm{E}-11$ & $1.93 \mathrm{E}-07$ \\
\hline Ru-95 & $4.29 \mathrm{E}-11$ & $1.59 \mathrm{E}-07$ \\
\hline Ru-97 & $1.24 \mathrm{E}-10$ & $4.59 \mathrm{E}-07$ \\
\hline Ru-103 & $2.61 \mathrm{E}-09$ & $9.66 \mathrm{E}-06$ \\
\hline Ru-105 & $2.18 \mathrm{E}-10$ & $8.07 \mathrm{E}-07$ \\
\hline Ru-106 & $3.10 \mathrm{E}-08$ & $1.15 \mathrm{E}-04$ \\
\hline Rh-97 & $2.84 \mathrm{E}-11$ & $1.05 \mathrm{E}-07$ \\
\hline Rh-97m & $3.30 \mathrm{E}-11$ & $1.22 \mathrm{E}-07$ \\
\hline Rh-99 & $1.41 \mathrm{E}-09$ & $5.22 \mathrm{E}-06$ \\
\hline Rh-101 & $4.91 \mathrm{E}-11$ & $1.82 \mathrm{E}-07$ \\
\hline 5.56E-09 & $2.06 \mathrm{E}-05$ \\
\hline
\end{tabular}




\begin{tabular}{|c|c|c|}
\hline Nuclide & $\begin{array}{c}\text { Inhalation } \\
\text { Dose } \\
\text { Coefficient } \\
\text { (Sv/Bq) }\end{array}$ & $\begin{array}{c}\text { Inhalation } \\
\text { Dose } \\
\text { Coefficient } \\
\text { (mrem/pCi) }\end{array}$ \\
\hline Rh-101m & $2.38 \mathrm{E}-10$ & $8.81 \mathrm{E}-07$ \\
\hline $\mathrm{Rh}-102$ & $8.01 \mathrm{E}-09$ & $2.96 \mathrm{E}-05$ \\
\hline $\mathrm{Rh}-102 \mathrm{~m}$ & $2.13 \mathrm{E}-08$ & $7.88 \mathrm{E}-05$ \\
\hline $\mathrm{Rh}-103 \mathrm{~m}$ & $3.10 \mathrm{E}-12$ & $1.15 \mathrm{E}-08$ \\
\hline $\mathrm{Rh}-105$ & $4.02 \mathrm{E}-10$ & $1.49 \mathrm{E}-06$ \\
\hline $\mathrm{Rh}-106 \mathrm{~m}$ & $1.32 \mathrm{E}-10$ & $4.88 \mathrm{E}-07$ \\
\hline $\mathrm{Rh}-107$ & $1.95 \mathrm{E}-11$ & $7.22 \mathrm{E}-08$ \\
\hline $\mathrm{Pd}-98$ & $3.35 \mathrm{E}-11$ & $1.24 \mathrm{E}-07$ \\
\hline $\mathrm{Pd}-99$ & $2.28 \mathrm{E}-11$ & $8.44 \mathrm{E}-08$ \\
\hline $\mathrm{Pd}-100$ & $9.38 \mathrm{E}-10$ & $3.47 \mathrm{E}-06$ \\
\hline $\mathrm{Pd}-101$ & $7.37 \mathrm{E}-11$ & $2.73 \mathrm{E}-07$ \\
\hline $\mathrm{Pd}-103$ & $5.00 \mathrm{E}-10$ & $1.85 \mathrm{E}-06$ \\
\hline $\mathrm{Pd}-107$ & $6.59 \mathrm{E}-10$ & $2.44 \mathrm{E}-06$ \\
\hline $\mathrm{Pd}-109$ & $4.40 \mathrm{E}-10$ & $1.63 \mathrm{E}-06$ \\
\hline $\mathrm{Pd}-111$ & $3.14 \mathrm{E}-11$ & $1.16 \mathrm{E}-07$ \\
\hline $\mathrm{Pd}-112$ & $1.32 \mathrm{E}-09$ & $4.88 \mathrm{E}-06$ \\
\hline $\mathrm{Ag}-101$ & $1.60 \mathrm{E}-11$ & $5.92 \mathrm{E}-08$ \\
\hline $\mathrm{Ag}-102$ & $2.15 \mathrm{E}-11$ & $7.96 \mathrm{E}-08$ \\
\hline $\mathrm{Ag}-103$ & $2.93 \mathrm{E}-11$ & $1.08 \mathrm{E}-07$ \\
\hline $\mathrm{Ag}-104$ & $4.45 \mathrm{E}-11$ & $1.65 \mathrm{E}-07$ \\
\hline $\mathrm{Ag}-104 \mathrm{~m}$ & $3.46 \mathrm{E}-11$ & $1.28 \mathrm{E}-07$ \\
\hline $\mathrm{Ag}-105$ & $8.50 \mathrm{E}-10$ & $3.15 \mathrm{E}-06$ \\
\hline $\mathrm{Ag}-106$ & $1.75 \mathrm{E}-11$ & $6.48 \mathrm{E}-08$ \\
\hline $\mathrm{Ag}-108 \mathrm{~m}$ & $8.30 \mathrm{E}-09$ & $3.07 \mathrm{E}-05$ \\
\hline $\mathrm{Ag}-110 \mathrm{~m}$ & $8.68 \mathrm{E}-09$ & $3.21 \mathrm{E}-05$ \\
\hline $\mathrm{Ag}-111$ & $1.75 \mathrm{E}-09$ & $6.48 \mathrm{E}-06$ \\
\hline $\mathrm{Ag}-112$ & $1.97 \mathrm{E}-10$ & $7.29 \mathrm{E}-07$ \\
\hline $\mathrm{Ag}-113$ & $1.83 \mathrm{E}-10$ & $6.77 \mathrm{E}-07$ \\
\hline $\mathrm{Ag}-115$ & $3.20 \mathrm{E}-11$ & $1.18 \mathrm{E}-07$ \\
\hline $\mathrm{Cd}-104$ & $5.86 \mathrm{E}-11$ & $2.17 \mathrm{E}-07$ \\
\hline $\mathrm{Cd}-105$ & $2.86 \mathrm{E}-11$ & $1.06 \mathrm{E}-07$ \\
\hline $\mathrm{Cd}-107$ & $9.49 \mathrm{E}-11$ & $3.51 \mathrm{E}-07$ \\
\hline $\mathrm{Cd}-109$ & $9.25 \mathrm{E}-09$ & $3.42 \mathrm{E}-05$ \\
\hline $\mathrm{Cd}-111 \mathrm{~m}$ & $2.66 \mathrm{E}-11$ & $9.84 \mathrm{E}-08$ \\
\hline $\mathrm{Cd}-113 \mathrm{~m}$ & $1.17 \mathrm{E}-07$ & $4.33 \mathrm{E}-04$ \\
\hline $\mathrm{Cd}-115$ & $1.25 \mathrm{E}-09$ & $4.63 \mathrm{E}-06$ \\
\hline
\end{tabular}




\begin{tabular}{|c|c|c|}
\hline Nuclide & $\begin{array}{c}\text { Inhalation } \\
\text { Dose } \\
\text { Coefficient } \\
\text { (Sv/Bq) }\end{array}$ & $\begin{array}{c}\text { Inhalation } \\
\text { Dose } \\
\text { Coefficient } \\
\text { (mrem/pCi) }\end{array}$ \\
\hline Cd-115m & $8.50 \mathrm{E}-09$ & $3.15 \mathrm{E}-05$ \\
\hline Cd-117 & $2.02 \mathrm{E}-10$ & $7.47 \mathrm{E}-07$ \\
\hline Cd-117m & $2.44 \mathrm{E}-10$ & $9.03 \mathrm{E}-07$ \\
\hline Cd-118 & $9.60 \mathrm{E}-11$ & $3.55 \mathrm{E}-07$ \\
\hline In-107 & $3.06 \mathrm{E}-11$ & $1.13 \mathrm{E}-07$ \\
\hline In-108 & $5.71 \mathrm{E}-11$ & $2.11 \mathrm{E}-07$ \\
\hline In-108m & $4.53 \mathrm{E}-11$ & $1.68 \mathrm{E}-07$ \\
\hline In-109 & $4.69 \mathrm{E}-11$ & $1.74 \mathrm{E}-07$ \\
\hline In-110 & $1.64 \mathrm{E}-10$ & $6.07 \mathrm{E}-07$ \\
\hline In-110m & $6.01 \mathrm{E}-11$ & $2.22 \mathrm{E}-07$ \\
\hline In-111 & $2.87 \mathrm{E}-10$ & $1.06 \mathrm{E}-06$ \\
\hline In-112 & $9.42 \mathrm{E}-12$ & $3.49 \mathrm{E}-08$ \\
\hline In-112m & $2.60 \mathrm{E}-11$ & $9.62 \mathrm{E}-08$ \\
\hline In-113m & $2.50 \mathrm{E}-11$ & $9.25 \mathrm{E}-08$ \\
\hline In-114m & $1.50 \mathrm{E}-08$ & $5.55 \mathrm{E}-05$ \\
\hline In-115 & $4.09 \mathrm{E}-07$ & $1.51 \mathrm{E}-03$ \\
\hline In-115m & $7.41 \mathrm{E}-11$ & $2.74 \mathrm{E}-07$ \\
\hline In-116m & $5.65 \mathrm{E}-11$ & $2.09 \mathrm{E}-07$ \\
\hline In-117 & $3.53 \mathrm{E}-11$ & $1.31 \mathrm{E}-07$ \\
\hline In-117m & $8.94 \mathrm{E}-11$ & $3.31 \mathrm{E}-07$ \\
\hline In-119m & $2.19 \mathrm{E}-11$ & $8.10 \mathrm{E}-08$ \\
\hline Sn-108 & $1.40 \mathrm{E}-11$ & $5.18 \mathrm{E}-08$ \\
\hline Sn-109 & $1.44 \mathrm{E}-11$ & $5.33 \mathrm{E}-08$ \\
\hline Sn-110 & $2.04 \mathrm{E}-10$ & $7.55 \mathrm{E}-07$ \\
\hline Sn-111 & $1.53 \mathrm{E}-11$ & $5.66 \mathrm{E}-08$ \\
\hline Sn-113 & $4.44 \mathrm{E}-09$ & $1.64 \mathrm{E}-05$ \\
\hline Sn-113m & $5.19 \mathrm{E}-12$ & $1.92 \mathrm{E}-08$ \\
\hline Sn-117m & $3.10 \mathrm{E}-09$ & $1.15 \mathrm{E}-05$ \\
\hline Sn-119m & $3.79 \mathrm{E}-09$ & $1.40 \mathrm{E}-05$ \\
\hline Sn-121 & $2.84 \mathrm{E}-10$ & $1.05 \mathrm{E}-06$ \\
\hline Sn-121m & $1.59 \mathrm{E}-08$ & $5.88 \mathrm{E}-05$ \\
\hline Sn-123 & $1.42 \mathrm{E}-08$ & $5.25 \mathrm{E}-05$ \\
\hline Sn-123m & $3.33 \mathrm{E}-11$ & $1.23 \mathrm{E}-07$ \\
\hline Sn-125 & $3.97 \mathrm{E}-09$ & $1.47 \mathrm{E}-05$ \\
\hline Sn-128 & $1.66 \mathrm{E}-07$ & $6.14 \mathrm{E}-04$ \\
\hline & $1.66 \mathrm{E}-10$ & $6.14 \mathrm{E}-07$ \\
\hline
\end{tabular}




\begin{tabular}{|c|c|c|}
\hline Nuclide & $\begin{array}{c}\text { Inhalation } \\
\text { Dose } \\
\text { Coefficient } \\
\text { (Sv/Bq) }\end{array}$ & $\begin{array}{c}\text { Inhalation } \\
\text { Dose } \\
\text { Coefficient } \\
\text { (mrem/pCi) }\end{array}$ \\
\hline Sb-115 & $1.57 \mathrm{E}-11$ & $5.81 \mathrm{E}-08$ \\
\hline Sb-116 & $1.70 \mathrm{E}-11$ & $6.29 \mathrm{E}-08$ \\
\hline $\mathrm{Sb}-116 \mathrm{~m}$ & $5.47 \mathrm{E}-11$ & $2.02 \mathrm{E}-07$ \\
\hline $\mathrm{Sb}-117$ & $1.99 \mathrm{E}-11$ & $7.36 \mathrm{E}-08$ \\
\hline $\mathrm{Sb}-118 \mathrm{~m}$ & $1.47 \mathrm{E}-10$ & $5.44 \mathrm{E}-07$ \\
\hline $\mathrm{Sb}-119$ & $4.61 \mathrm{E}-11$ & $1.71 \mathrm{E}-07$ \\
\hline $\mathrm{Sb}-120$ & $8.39 \mathrm{E}-12$ & $3.10 \mathrm{E}-08$ \\
\hline $\mathrm{Sb}-120 \mathrm{~m}$ & $1.20 \mathrm{E}-09$ & $4.44 \mathrm{E}-06$ \\
\hline $\mathrm{Sb}-122$ & $1.22 \mathrm{E}-09$ & $4.51 \mathrm{E}-06$ \\
\hline $\mathrm{Sb}-124$ & $7.15 \mathrm{E}-09$ & $2.65 \mathrm{E}-05$ \\
\hline $\mathrm{Sb}-124 \mathrm{n}$ & $7.48 \mathrm{E}-12$ & $2.77 \mathrm{E}-08$ \\
\hline $\mathrm{Sb}-125$ & $5.33 \mathrm{E}-09$ & $1.97 \mathrm{E}-05$ \\
\hline $\mathrm{Sb}-126$ & $3.54 \mathrm{E}-09$ & $1.31 \mathrm{E}-05$ \\
\hline $\mathrm{Sb}-126 \mathrm{~m}$ & $2.30 \mathrm{E}-11$ & $8.51 \mathrm{E}-08$ \\
\hline $\mathrm{Sb}-127$ & $1.92 \mathrm{E}-09$ & $7.10 \mathrm{E}-06$ \\
\hline $\mathrm{Sb}-128$ & $5.04 \mathrm{E}-10$ & $1.86 \mathrm{E}-06$ \\
\hline $\mathrm{Sb}-128 \mathrm{~m}$ & $1.72 \mathrm{E}-11$ & $6.36 \mathrm{E}-08$ \\
\hline $\mathrm{Sb}-129$ & $2.83 \mathrm{E}-10$ & $1.05 \mathrm{E}-06$ \\
\hline $\mathrm{Sb}-130$ & $6.20 \mathrm{E}-11$ & $2.29 \mathrm{E}-07$ \\
\hline $\mathrm{Te}-114$ & $3.34 \mathrm{E}-11$ & $1.24 \mathrm{E}-07$ \\
\hline $\mathrm{Te}-116$ & $1.43 \mathrm{E}-10$ & $5.29 \mathrm{E}-07$ \\
\hline $\mathrm{Te}-117$ & $3.76 \mathrm{E}-11$ & $1.39 \mathrm{E}-07$ \\
\hline $\mathrm{Te}-118$ & $2.68 \mathrm{E}-09$ & $9.92 \mathrm{E}-06$ \\
\hline $\mathrm{Te}-119$ & $1.26 \mathrm{E}-10$ & $4.66 \mathrm{E}-07$ \\
\hline $\mathrm{Te}-119 \mathrm{~m}$ & $6.12 \mathrm{E}-10$ & $2.26 \mathrm{E}-06$ \\
\hline $\mathrm{Te}-121$ & $4.78 \mathrm{E}-10$ & $1.77 \mathrm{E}-06$ \\
\hline $\mathrm{Te}-121 \mathrm{~m}$ & $4.73 \mathrm{E}-09$ & $1.75 \mathrm{E}-05$ \\
\hline $\mathrm{Te}-123$ & $5.83 \mathrm{E}-10$ & $2.16 \mathrm{E}-06$ \\
\hline $\mathrm{Te}-123 \mathrm{~m}$ & $4.39 \mathrm{E}-09$ & $1.62 \mathrm{E}-05$ \\
\hline $\mathrm{Te}-125 \mathrm{~m}$ & $3.70 \mathrm{E}-09$ & $1.37 \mathrm{E}-05$ \\
\hline $\mathrm{Te}-127$ & $1.51 \mathrm{E}-10$ & $5.59 \mathrm{E}-07$ \\
\hline $\mathrm{Te}-127 \mathrm{~m}$ & $8.23 \mathrm{E}-09$ & $3.05 \mathrm{E}-05$ \\
\hline $\mathrm{Te}-129$ & $4.35 \mathrm{E}-11$ & $1.61 \mathrm{E}-07$ \\
\hline $\mathrm{Te}-131 \mathrm{~m}$ & $7.26 \mathrm{E}-09$ & $2.69 \mathrm{E}-05$ \\
\hline & $3.38 \mathrm{E}-11$ & $1.25 \mathrm{E}-07$ \\
\hline $\mathrm{Te}-132$ & $2.48 \mathrm{E}-09$ & $9.18 \mathrm{E}-06$ \\
\hline
\end{tabular}




\begin{tabular}{|c|c|c|}
\hline Nuclide & $\begin{array}{c}\text { Inhalation } \\
\text { Dose } \\
\text { Coefficient } \\
\text { (Sv/Bq) }\end{array}$ & $\begin{array}{c}\text { Inhalation } \\
\text { Dose } \\
\text { Coefficient } \\
\text { (mrem/pCi) }\end{array}$ \\
\hline Te-133 & $2.35 \mathrm{E}-11$ & $8.70 \mathrm{E}-08$ \\
\hline Te-133m & $9.37 \mathrm{E}-11$ & $3.47 \mathrm{E}-07$ \\
\hline Te-134 & $7.56 \mathrm{E}-11$ & $2.80 \mathrm{E}-07$ \\
\hline $\mathrm{I}-118$ & $2.03 \mathrm{E}-10$ & $7.51 \mathrm{E}-07$ \\
\hline $\mathrm{I}-119$ & $5.66 \mathrm{E}-11$ & $2.09 \mathrm{E}-07$ \\
\hline $\mathrm{I}-120$ & $3.24 \mathrm{E}-10$ & $1.20 \mathrm{E}-06$ \\
\hline $\mathrm{I}-120 \mathrm{~m}$ & $1.62 \mathrm{E}-10$ & $5.99 \mathrm{E}-07$ \\
\hline $\mathrm{I}-121$ & $9.60 \mathrm{E}-11$ & $3.55 \mathrm{E}-07$ \\
\hline $\mathrm{I}-123$ & $2.85 \mathrm{E}-10$ & $1.05 \mathrm{E}-06$ \\
\hline $\mathrm{I}-124$ & $1.57 \mathrm{E}-08$ & $5.81 \mathrm{E}-05$ \\
\hline $\mathrm{I}-125$ & $1.65 \mathrm{E}-08$ & $6.11 \mathrm{E}-05$ \\
\hline $\mathrm{I}-126$ & $3.44 \mathrm{E}-08$ & $1.27 \mathrm{E}-04$ \\
\hline $\mathrm{I}-129$ & $1.08 \mathrm{E}-07$ & $4.00 \mathrm{E}-04$ \\
\hline $\mathrm{I}-130$ & $2.36 \mathrm{E}-09$ & $8.73 \mathrm{E}-06$ \\
\hline $\mathrm{I}-131$ & $2.61 \mathrm{E}-08$ & $9.66 \mathrm{E}-05$ \\
\hline $\mathrm{I}-132$ & $3.87 \mathrm{E}-10$ & $1.43 \mathrm{E}-06$ \\
\hline $\mathrm{I}-132 \mathrm{~m}$ & $2.96 \mathrm{E}-10$ & $1.10 \mathrm{E}-06$ \\
\hline $\mathrm{I}-133$ & $5.48 \mathrm{E}-09$ & $2.03 \mathrm{E}-05$ \\
\hline $\mathrm{I}-134$ & $1.62 \mathrm{E}-10$ & $5.99 \mathrm{E}-07$ \\
\hline $\mathrm{I}-135$ & $1.16 \mathrm{E}-09$ & $4.29 \mathrm{E}-06$ \\
\hline Cs-125 & $1.35 \mathrm{E}-11$ & $5.00 \mathrm{E}-08$ \\
\hline Cs-127 & $2.49 \mathrm{E}-11$ & $9.21 \mathrm{E}-08$ \\
\hline Cs-129 & $5.16 \mathrm{E}-11$ & $1.91 \mathrm{E}-07$ \\
\hline Cs-130 & $9.21 \mathrm{E}-12$ & $3.41 \mathrm{E}-08$ \\
\hline Cs-131 & $3.30 \mathrm{E}-11$ & $1.22 \mathrm{E}-07$ \\
\hline Cs-132 & $2.74 \mathrm{E}-10$ & $1.01 \mathrm{E}-06$ \\
\hline Cs-134 & $6.56 \mathrm{E}-09$ & $2.43 \mathrm{E}-05$ \\
\hline Cs-134m & $1.69 \mathrm{E}-11$ & $6.25 \mathrm{E}-08$ \\
\hline Cs-135 & $9.13 \mathrm{E}-10$ & $3.38 \mathrm{E}-06$ \\
\hline Cs-135m & $1.45 \mathrm{E}-11$ & $5.37 \mathrm{E}-08$ \\
\hline Cs-136 & $1.38 \mathrm{E}-09$ & $5.11 \mathrm{E}-06$ \\
\hline Cs-137 & $4.60 \mathrm{E}-09$ & $1.70 \mathrm{E}-05$ \\
\hline Cs-138 & $3.13 \mathrm{E}-11$ & $1.16 \mathrm{E}-07$ \\
\hline Ba-124 & $2.63 \mathrm{E}-11$ & $9.73 \mathrm{E}-08$ \\
\hline Ba-127 & $1.29 \mathrm{E}-10$ & $4.77 \mathrm{E}-07$ \\
\hline Ba-128 & $1.30 \mathrm{E}-11$ & $4.81 \mathrm{E}-08$ \\
\hline & & $5.77 \mathrm{E}-06$ \\
\hline
\end{tabular}




\begin{tabular}{|c|c|c|}
\hline Nuclide & $\begin{array}{c}\text { Inhalation } \\
\text { Dose } \\
\text { Coefficient } \\
\text { (Sv/Bq) } \\
\end{array}$ & $\begin{array}{c}\text { Inhalation } \\
\text { Dose } \\
\text { Coefficient } \\
\text { (mrem/pCi) }\end{array}$ \\
\hline Ba-129 & $3.31 \mathrm{E}-11$ & 1.22E-07 \\
\hline Ba-129m & $5.44 \mathrm{E}-11$ & $2.01 \mathrm{E}-07$ \\
\hline Ba-131 & $8.46 \mathrm{E}-10$ & $3.13 \mathrm{E}-06$ \\
\hline Ba-131m & $8.48 \mathrm{E}-12$ & $3.14 \mathrm{E}-08$ \\
\hline Ba-133 & 3.68E-09 & $1.36 \mathrm{E}-05$ \\
\hline Ba-133m & $4.98 \mathrm{E}-10$ & $1.84 \mathrm{E}-06$ \\
\hline $\mathrm{Ba}-135 \mathrm{~m}$ & $3.86 \mathrm{E}-10$ & $1.43 \mathrm{E}-06$ \\
\hline Ba-139 & $6.70 \mathrm{E}-11$ & $2.48 \mathrm{E}-07$ \\
\hline Ba-140 & $5.70 \mathrm{E}-09$ & $2.11 \mathrm{E}-05$ \\
\hline Ba-141 & $3.98 \mathrm{E}-11$ & $1.47 \mathrm{E}-07$ \\
\hline Ba-142 & $2.45 \mathrm{E}-11$ & $9.07 \mathrm{E}-08$ \\
\hline La-129 & $1.48 \mathrm{E}-11$ & $5.48 \mathrm{E}-08$ \\
\hline La-131 & $2.80 \mathrm{E}-11$ & $1.04 \mathrm{E}-07$ \\
\hline La-132 & $2.19 \mathrm{E}-10$ & $8.10 \mathrm{E}-07$ \\
\hline La-132m & $2.51 \mathrm{E}-11$ & 9.29E-08 \\
\hline La-133 & $2.08 \mathrm{E}-11$ & 7.70E-08 \\
\hline La-135 & $1.84 \mathrm{E}-11$ & $6.81 \mathrm{E}-08$ \\
\hline La-137 & 9.43E-09 & $3.49 \mathrm{E}-05$ \\
\hline La-138 & $1.62 \mathrm{E}-07$ & $5.99 \mathrm{E}-04$ \\
\hline La-140 & $1.35 \mathrm{E}-09$ & $5.00 \mathrm{E}-06$ \\
\hline La-141 & $1.93 \mathrm{E}-10$ & $7.14 \mathrm{E}-07$ \\
\hline La-142 & $1.07 \mathrm{E}-10$ & $3.96 \mathrm{E}-07$ \\
\hline La-143 & $2.71 \mathrm{E}-11$ & $1.00 \mathrm{E}-07$ \\
\hline Ce-130 & 4.09E-11 & $1.51 \mathrm{E}-07$ \\
\hline $\mathrm{Ce}-131$ & $1.62 \mathrm{E}-11$ & 5.99E-08 \\
\hline $\mathrm{Ce}-132$ & $1.65 \mathrm{E}-10$ & $6.11 \mathrm{E}-07$ \\
\hline $\mathrm{Ce}-133$ & $5.90 \mathrm{E}-11$ & $2.18 \mathrm{E}-07$ \\
\hline Ce-133m & $1.38 \mathrm{E}-10$ & $5.11 \mathrm{E}-07$ \\
\hline Ce-134 & $1.60 \mathrm{E}-09$ & $5.92 \mathrm{E}-06$ \\
\hline Ce-135 & $1.63 \mathrm{E}-10$ & $6.03 \mathrm{E}-07$ \\
\hline Ce-137 & $1.31 \mathrm{E}-11$ & $4.85 \mathrm{E}-08$ \\
\hline $\mathrm{Ce}-137 \mathrm{~m}$ & $4.82 \mathrm{E}-10$ & $1.78 \mathrm{E}-06$ \\
\hline Ce-139 & 1.89E-09 & 6.99E-06 \\
\hline $\mathrm{Ce}-141$ & 3.54E-09 & $1.31 \mathrm{E}-05$ \\
\hline $\mathrm{Ce}-143$ & $8.83 \mathrm{E}-10$ & $3.27 \mathrm{E}-06$ \\
\hline Ce-144 & $4.05 \mathrm{E}-08$ & $1.50 \mathrm{E}-04$ \\
\hline Pr-134 & $2.52 \mathrm{E}-11$ & 9.32E-08 \\
\hline
\end{tabular}




\begin{tabular}{|c|c|c|}
\hline Nuclide & $\begin{array}{c}\text { Inhalation } \\
\text { Dose } \\
\text { Coefficient } \\
\text { (Sv/Bq) }\end{array}$ & $\begin{array}{c}\text { Inhalation } \\
\text { Dose } \\
\text { Coefficient } \\
\text { (mrem/pCi) }\end{array}$ \\
\hline Pr-134m & $3.83 \mathrm{E}-11$ & $1.42 \mathrm{E}-07$ \\
\hline Pr-135 & $2.45 \mathrm{E}-11$ & $9.07 \mathrm{E}-08$ \\
\hline Pr-136 & $1.68 \mathrm{E}-11$ & $6.22 \mathrm{E}-08$ \\
\hline Pr-137 & $2.30 \mathrm{E}-11$ & $8.51 \mathrm{E}-08$ \\
\hline Pr-138m & $9.08 \mathrm{E}-11$ & $3.36 \mathrm{E}-07$ \\
\hline Pr-139 & $2.41 \mathrm{E}-11$ & $8.92 \mathrm{E}-08$ \\
\hline Pr-142 & $6.67 \mathrm{E}-10$ & $2.47 \mathrm{E}-06$ \\
\hline Pr-142m & $8.49 \mathrm{E}-12$ & $3.14 \mathrm{E}-08$ \\
\hline Pr-143 & $2.72 \mathrm{E}-09$ & $1.01 \mathrm{E}-05$ \\
\hline Pr-144 & $2.18 \mathrm{E}-11$ & $8.07 \mathrm{E}-08$ \\
\hline Pr-145 & $2.01 \mathrm{E}-10$ & $7.44 \mathrm{E}-07$ \\
\hline Pr-146 & $3.58 \mathrm{E}-11$ & $1.32 \mathrm{E}-07$ \\
\hline Pr-147 & $2.12 \mathrm{E}-11$ & $7.84 \mathrm{E}-08$ \\
\hline $\mathrm{Nd}-135$ & $3.18 \mathrm{E}-11$ & $1.18 \mathrm{E}-07$ \\
\hline $\mathrm{Nd}-136$ & $6.11 \mathrm{E}-11$ & $2.26 \mathrm{E}-07$ \\
\hline $\mathrm{Nd}-136$ & $6.38 \mathrm{E}-11$ & $2.36 \mathrm{E}-07$ \\
\hline $\mathrm{Nd}-137$ & $2.02 \mathrm{E}-11$ & $7.47 \mathrm{E}-08$ \\
\hline $\mathrm{Nd}-137$ & $3.39 \mathrm{E}-11$ & $1.25 \mathrm{E}-07$ \\
\hline $\mathrm{Nd}-138$ & $3.03 \mathrm{E}-10$ & $1.12 \mathrm{E}-06$ \\
\hline $\mathrm{Nd}-139$ & $1.25 \mathrm{E}-11$ & $4.63 \mathrm{E}-08$ \\
\hline $\mathrm{Nd}-139$ & $1.31 \mathrm{E}-11$ & $4.85 \mathrm{E}-08$ \\
\hline $\mathrm{Nd}-139 \mathrm{~m}$ & $1.11 \mathrm{E}-10$ & $4.11 \mathrm{E}-07$ \\
\hline $\mathrm{Nd}-139 \mathrm{~m}$ & $1.81 \mathrm{E}-10$ & $6.70 \mathrm{E}-07$ \\
\hline $\mathrm{Nd}-140$ & $1.33 \mathrm{E}-09$ & $4.92 \mathrm{E}-06$ \\
\hline $\mathrm{Nd}-141$ & $6.15 \mathrm{E}-12$ & $2.28 \mathrm{E}-08$ \\
\hline $\mathrm{Nd}-141$ & $6.38 \mathrm{E}-12$ & $2.36 \mathrm{E}-08$ \\
\hline $\mathrm{Nd}-144$ & $2.01 \mathrm{E}-05$ & $7.44 \mathrm{E}-02$ \\
\hline $\mathrm{Nd}-144$ & $8.12 \mathrm{E}-06$ & $3.00 \mathrm{E}-02$ \\
\hline $\mathrm{Nd}-147$ & $2.67 \mathrm{E}-09$ & $9.88 \mathrm{E}-06$ \\
\hline $\mathrm{Nd}-149$ & $1.06 \mathrm{E}-10$ & $3.92 \mathrm{E}-07$ \\
\hline $\mathrm{Nd}-151$ & $2.05 \mathrm{E}-11$ & $7.59 \mathrm{E}-08$ \\
\hline $\mathrm{Nd}-152$ & $2.80 \mathrm{E}-11$ & $1.04 \mathrm{E}-07$ \\
\hline Pm-141 & $1.73 \mathrm{E}-11$ & $6.40 \mathrm{E}-08$ \\
\hline Pm-143 & $3.27 \mathrm{E}-09$ & $1.21 \mathrm{E}-05$ \\
\hline & $1.91 \mathrm{E}-08$ & $7.07 \mathrm{E}-05$ \\
\hline Pm-145 & $8.62 \mathrm{E}-09$ & $3.19 \mathrm{E}-05$ \\
\hline & $4.78 \mathrm{E}-08$ & $1.77 \mathrm{E}-04$ \\
\hline
\end{tabular}




\begin{tabular}{|c|c|c|}
\hline Nuclide & $\begin{array}{c}\text { Inhalation } \\
\text { Dose } \\
\text { Coefficient } \\
\text { (Sv/Bq) }\end{array}$ & $\begin{array}{c}\text { Inhalation } \\
\text { Dose } \\
\text { Coefficient } \\
\text { (mrem/pCi) }\end{array}$ \\
\hline Pm-147 & $7.97 \mathrm{E}-09$ & $2.95 \mathrm{E}-05$ \\
\hline $\mathrm{Pm}-148$ & $2.51 \mathrm{E}-09$ & $9.29 \mathrm{E}-06$ \\
\hline $\mathrm{Pm}-148 \mathrm{~m}$ & $6.29 \mathrm{E}-09$ & $2.33 \mathrm{E}-05$ \\
\hline $\mathrm{Pm}-149$ & $8.38 \mathrm{E}-10$ & $3.10 \mathrm{E}-06$ \\
\hline $\mathrm{Pm}-150$ & $1.56 \mathrm{E}-10$ & $5.77 \mathrm{E}-07$ \\
\hline $\mathrm{Pm}-151$ & $5.55 \mathrm{E}-10$ & $2.05 \mathrm{E}-06$ \\
\hline $\mathrm{Sm}-140$ & $3.92 \mathrm{E}-11$ & $1.45 \mathrm{E}-07$ \\
\hline $\mathrm{Sm}-141$ & $1.90 \mathrm{E}-11$ & $7.03 \mathrm{E}-08$ \\
\hline $\mathrm{Sm}-141 \mathrm{~m}$ & $3.89 \mathrm{E}-11$ & $1.44 \mathrm{E}-07$ \\
\hline $\mathrm{Sm}-142$ & $8.87 \mathrm{E}-11$ & $3.28 \mathrm{E}-07$ \\
\hline $\mathrm{Sm}-145$ & $3.30 \mathrm{E}-09$ & $1.22 \mathrm{E}-05$ \\
\hline $\mathrm{Sm}-146$ & $2.67 \mathrm{E}-05$ & $9.88 \mathrm{E}-02$ \\
\hline $\mathrm{Sm}-147$ & $2.44 \mathrm{E}-05$ & $9.03 \mathrm{E}-02$ \\
\hline $\mathrm{Sm}-148$ & $2.10 \mathrm{E}-05$ & $7.77 \mathrm{E}-02$ \\
\hline $\mathrm{Sm}-151$ & $9.84 \mathrm{E}-09$ & $3.64 \mathrm{E}-05$ \\
\hline $\mathrm{Sm}-153$ & $7.90 \mathrm{E}-10$ & $2.92 \mathrm{E}-06$ \\
\hline $\mathrm{Sm}-155$ & $2.10 \mathrm{E}-11$ & $7.77 \mathrm{E}-08$ \\
\hline $\mathrm{Sm}-156$ & $2.78 \mathrm{E}-10$ & $1.03 \mathrm{E}-06$ \\
\hline $\mathrm{Eu}-145$ & $6.09 \mathrm{E}-10$ & $2.25 \mathrm{E}-06$ \\
\hline $\mathrm{Eu}-146$ & $9.31 \mathrm{E}-10$ & $3.44 \mathrm{E}-06$ \\
\hline $\mathrm{Eu}-147$ & $1.31 \mathrm{E}-09$ & $4.85 \mathrm{E}-06$ \\
\hline $\mathrm{Eu}-148$ & $4.14 \mathrm{E}-09$ & $1.53 \mathrm{E}-05$ \\
\hline $\mathrm{Eu}-149$ & $6.40 \mathrm{E}-10$ & $2.37 \mathrm{E}-06$ \\
\hline $\mathrm{Eu}-150$ & $1.34 \mathrm{E}-07$ & $4.96 \mathrm{E}-04$ \\
\hline $\mathrm{Eu}-150 \mathrm{~m}$ & $2.44 \mathrm{E}-10$ & $9.03 \mathrm{E}-07$ \\
\hline $\mathrm{Eu}-152$ & $9.93 \mathrm{E}-08$ & $3.67 \mathrm{E}-04$ \\
\hline $\mathrm{Eu}-152 \mathrm{~m}$ & $2.58 \mathrm{E}-10$ & $9.55 \mathrm{E}-07$ \\
\hline $\mathrm{Eu}-152 \mathrm{n}$ & $1.01 \mathrm{E}-11$ & $3.74 \mathrm{E}-08$ \\
\hline $\mathrm{Eu}-154$ & $1.15 \mathrm{E}-07$ & $4.26 \mathrm{E}-04$ \\
\hline $\mathrm{Eu}-154 \mathrm{~m}$ & $4.76 \mathrm{E}-12$ & $1.76 \mathrm{E}-08$ \\
\hline $\mathrm{Eu}-155$ & $1.38 \mathrm{E}-08$ & $5.11 \mathrm{E}-05$ \\
\hline $\mathrm{Eu}-156$ & $4.41 \mathrm{E}-09$ & $1.63 \mathrm{E}-05$ \\
\hline $\mathrm{Eu}-157$ & $3.80 \mathrm{E}-10$ & $1.41 \mathrm{E}-06$ \\
\hline $\mathrm{Eu}-158$ & $5.84 \mathrm{E}-11$ & $2.16 \mathrm{E}-07$ \\
\hline $\mathrm{Gu}-159$ & $2.85 \mathrm{E}-11$ & $1.05 \mathrm{E}-07$ \\
\hline $\mathrm{Gd}-146$ & $2.11 \mathrm{E}-11$ & $7.81 \mathrm{E}-08$ \\
\hline & $7.79 \mathrm{E}-09$ & $2.88 \mathrm{E}-05$ \\
\hline
\end{tabular}




\begin{tabular}{|c|c|c|}
\hline Nuclide & $\begin{array}{c}\text { Inhalation } \\
\text { Dose } \\
\text { Coefficient } \\
\text { (Sv/Bq) }\end{array}$ & $\begin{array}{c}\text { Inhalation } \\
\text { Dose } \\
\text { Coefficient } \\
\text { (mrem/pCi) }\end{array}$ \\
\hline Gd-147 & $5.20 \mathrm{E}-10$ & $1.92 \mathrm{E}-06$ \\
\hline Gd-148 & $2.68 \mathrm{E}-05$ & $9.92 \mathrm{E}-02$ \\
\hline Gd-149 & $1.05 \mathrm{E}-09$ & $3.89 \mathrm{E}-06$ \\
\hline Gd-150 & $2.56 \mathrm{E}-05$ & $9.47 \mathrm{E}-02$ \\
\hline Gd-151 & $1.31 \mathrm{E}-09$ & $4.85 \mathrm{E}-06$ \\
\hline Gd-152 & $2.01 \mathrm{E}-05$ & $7.44 \mathrm{E}-02$ \\
\hline Gd-153 & $2.65 \mathrm{E}-09$ & $9.81 \mathrm{E}-06$ \\
\hline Gd-159 & $3.51 \mathrm{E}-10$ & $1.30 \mathrm{E}-06$ \\
\hline Tb-147 & $8.82 \mathrm{E}-11$ & $3.26 \mathrm{E}-07$ \\
\hline Tb-148 & $8.28 \mathrm{E}-11$ & $3.06 \mathrm{E}-07$ \\
\hline Tb-149 & $4.87 \mathrm{E}-09$ & $1.80 \mathrm{E}-05$ \\
\hline Tb-150 & $1.29 \mathrm{E}-10$ & $4.77 \mathrm{E}-07$ \\
\hline Tb-151 & $2.96 \mathrm{E}-10$ & $1.10 \mathrm{E}-06$ \\
\hline Tb-152 & $3.82 \mathrm{E}-10$ & $1.41 \mathrm{E}-06$ \\
\hline Tb-153 & $2.72 \mathrm{E}-10$ & $1.01 \mathrm{E}-06$ \\
\hline Tb-154 & $4.30 \mathrm{E}-10$ & $1.59 \mathrm{E}-06$ \\
\hline Tb-155 & $3.50 \mathrm{E}-10$ & $1.30 \mathrm{E}-06$ \\
\hline Tb-156 & $1.44 \mathrm{E}-09$ & $5.33 \mathrm{E}-06$ \\
\hline Tb-156m & $2.33 \mathrm{E}-10$ & $8.62 \mathrm{E}-07$ \\
\hline Tb-156n & $1.28 \mathrm{E}-10$ & $4.74 \mathrm{E}-07$ \\
\hline Tb-157 & $3.42 \mathrm{E}-09$ & $1.27 \mathrm{E}-05$ \\
\hline Tb-158 & $1.10 \mathrm{E}-07$ & $4.07 \mathrm{E}-04$ \\
\hline Tb-160 & $9.17 \mathrm{E}-09$ & $3.39 \mathrm{E}-05$ \\
\hline Tb-161 & $1.59 \mathrm{E}-09$ & $5.88 \mathrm{E}-06$ \\
\hline Tb-163 & $2.03 \mathrm{E}-11$ & $7.51 \mathrm{E}-08$ \\
\hline Dy-151 & $1.51 \mathrm{E}-10$ & $5.59 \mathrm{E}-07$ \\
\hline Dy-152 & $7.46 \mathrm{E}-11$ & $2.76 \mathrm{E}-07$ \\
\hline Dy-153 & $1.51 \mathrm{E}-10$ & $5.59 \mathrm{E}-07$ \\
\hline Dy-154 & $2.74 \mathrm{E}-05$ & $1.01 \mathrm{E}-01$ \\
\hline Dy-155 & $1.09 \mathrm{E}-10$ & $4.03 \mathrm{E}-07$ \\
\hline Dy-157 & $3.77 \mathrm{E}-11$ & $1.39 \mathrm{E}-07$ \\
\hline Dy-159 & $5.38 \mathrm{E}-10$ & $1.99 \mathrm{E}-06$ \\
\hline Dy-165 & $7.52 \mathrm{E}-11$ & $2.78 \mathrm{E}-07$ \\
\hline Dy-166 & $2.40 \mathrm{E}-09$ & $8.88 \mathrm{E}-06$ \\
\hline Ho-155 & $1.82 \mathrm{E}-11$ & $6.73 \mathrm{E}-08$ \\
\hline & $2.87 \mathrm{E}-11$ & $1.06 \mathrm{E}-07$ \\
\hline
\end{tabular}




\begin{tabular}{|c|c|c|}
\hline Nuclide & $\begin{array}{c}\text { Inhalation } \\
\text { Dose } \\
\text { Coefficient } \\
\text { (Sv/Bq) }\end{array}$ & $\begin{array}{c}\text { Inhalation } \\
\text { Dose } \\
\text { Coefficient } \\
\text { (mrem/pCi) }\end{array}$ \\
\hline Ho-157 & $5.87 \mathrm{E}-12$ & $2.17 \mathrm{E}-08$ \\
\hline Ho-159 & $7.95 \mathrm{E}-12$ & $2.94 \mathrm{E}-08$ \\
\hline Ho-160 & $1.63 \mathrm{E}-11$ & $6.03 \mathrm{E}-08$ \\
\hline Ho-161 & $8.98 \mathrm{E}-12$ & $3.32 \mathrm{E}-08$ \\
\hline Ho-162 & $3.43 \mathrm{E}-12$ & $1.27 \mathrm{E}-08$ \\
\hline Ho-162m & $2.51 \mathrm{E}-11$ & $9.29 \mathrm{E}-08$ \\
\hline Ho-163 & $2.82 \mathrm{E}-10$ & $1.04 \mathrm{E}-06$ \\
\hline Ho-164 & $1.02 \mathrm{E}-11$ & $3.77 \mathrm{E}-08$ \\
\hline Ho-164m & $1.39 \mathrm{E}-11$ & $5.14 \mathrm{E}-08$ \\
\hline Ho-166 & $8.42 \mathrm{E}-10$ & $3.12 \mathrm{E}-06$ \\
\hline Ho-166m & $2.96 \mathrm{E}-07$ & $1.10 \mathrm{E}-03$ \\
\hline Ho-167 & $9.12 \mathrm{E}-11$ & $3.37 \mathrm{E}-07$ \\
\hline Er-156 & $2.38 \mathrm{E}-11$ & $8.81 \mathrm{E}-08$ \\
\hline Er-159 & $2.07 \mathrm{E}-11$ & $7.66 \mathrm{E}-08$ \\
\hline Er-161 & $6.43 \mathrm{E}-11$ & $2.38 \mathrm{E}-07$ \\
\hline Er-163 & $1.56 \mathrm{E}-12$ & $5.77 \mathrm{E}-09$ \\
\hline Er-165 & $1.04 \mathrm{E}-11$ & $3.85 \mathrm{E}-08$ \\
\hline Er-169 & $1.28 \mathrm{E}-09$ & $4.74 \mathrm{E}-06$ \\
\hline Er-171 & $2.70 \mathrm{E}-10$ & $9.99 \mathrm{E}-07$ \\
\hline Er-172 & $1.39 \mathrm{E}-09$ & $5.14 \mathrm{E}-06$ \\
\hline Tm-161 & $3.06 \mathrm{E}-11$ & $1.13 \mathrm{E}-07$ \\
\hline Tm-162 & $2.40 \mathrm{E}-11$ & $8.88 \mathrm{E}-08$ \\
\hline Tm-163 & $4.53 \mathrm{E}-11$ & $1.68 \mathrm{E}-07$ \\
\hline Tm-165 & $2.74 \mathrm{E}-10$ & $1.01 \mathrm{E}-06$ \\
\hline Tm-166 & $2.18 \mathrm{E}-10$ & $8.07 \mathrm{E}-07$ \\
\hline Tm-167 & $1.47 \mathrm{E}-09$ & $5.44 \mathrm{E}-06$ \\
\hline Tm-168 & $5.60 \mathrm{E}-09$ & $2.07 \mathrm{E}-05$ \\
\hline Tm-170 & $1.01 \mathrm{E}-08$ & $3.74 \mathrm{E}-05$ \\
\hline Tm-171 & $2.48 \mathrm{E}-09$ & $9.18 \mathrm{E}-06$ \\
\hline Tm-172 & $1.42 \mathrm{E}-09$ & $5.25 \mathrm{E}-06$ \\
\hline Tm-173 & $2.25 \mathrm{E}-10$ & $8.33 \mathrm{E}-07$ \\
\hline Tm-175 & $2.26 \mathrm{E}-11$ & $8.36 \mathrm{E}-08$ \\
\hline Yb-162 & $2.09 \mathrm{E}-11$ & $7.73 \mathrm{E}-08$ \\
\hline Yb-163 & $1.02 \mathrm{E}-11$ & $3.77 \mathrm{E}-08$ \\
\hline $5.31 \mathrm{E}-11$ & $1.96 \mathrm{E}-07$ \\
\hline Yb-167 & $8.09 \mathrm{E}-12$ & $2.99 \mathrm{E}-08$ \\
\hline
\end{tabular}




\begin{tabular}{|c|c|c|}
\hline Nuclide & $\begin{array}{c}\text { Inhalation } \\
\text { Dose } \\
\text { Coefficient } \\
\text { (Sv/Bq) } \\
\end{array}$ & $\begin{array}{c}\text { Inhalation } \\
\text { Dose } \\
\text { Coefficient } \\
\text { (mrem/pCi) }\end{array}$ \\
\hline Yb-169 & 3.76E-09 & 1.39E-05 \\
\hline Yb-175 & $8.11 \mathrm{E}-10$ & $3.00 \mathrm{E}-06$ \\
\hline Yb-177 & $8.45 \mathrm{E}-11$ & $3.13 \mathrm{E}-07$ \\
\hline Yb-178 & $8.63 \mathrm{E}-11$ & $3.19 \mathrm{E}-07$ \\
\hline Lu-165 & $1.63 \mathrm{E}-11$ & $6.03 \mathrm{E}-08$ \\
\hline Lu-167 & $4.56 \mathrm{E}-11$ & $1.69 \mathrm{E}-07$ \\
\hline Lu-169 & $5.16 \mathrm{E}-10$ & $1.91 \mathrm{E}-06$ \\
\hline Lu-170 & 7.57E-10 & $2.80 \mathrm{E}-06$ \\
\hline Lu-171 & $1.11 \mathrm{E}-09$ & 4.11E-06 \\
\hline Lu-172 & $1.80 \mathrm{E}-09$ & $6.66 \mathrm{E}-06$ \\
\hline Lu-173 & 5.27E-09 & $1.95 \mathrm{E}-05$ \\
\hline Lu-174 & $8.10 \mathrm{E}-09$ & $3.00 \mathrm{E}-05$ \\
\hline Lu-174m & 4.86E-09 & $1.80 \mathrm{E}-05$ \\
\hline Lu-176 & $1.61 \mathrm{E}-07$ & $5.96 \mathrm{E}-04$ \\
\hline Lu-176m & $1.32 \mathrm{E}-10$ & 4.88E-07 \\
\hline Lu-177 & $1.32 \mathrm{E}-09$ & $4.88 \mathrm{E}-06$ \\
\hline Lu-177m & $1.76 \mathrm{E}-08$ & $6.51 \mathrm{E}-05$ \\
\hline Lu-178 & 2.95E-11 & $1.09 \mathrm{E}-07$ \\
\hline Lu-178m & $3.71 \mathrm{E}-11$ & $1.37 \mathrm{E}-07$ \\
\hline Lu-179 & $1.32 \mathrm{E}-10$ & $4.88 \mathrm{E}-07$ \\
\hline Hf-170 & $3.41 \mathrm{E}-10$ & $1.26 \mathrm{E}-06$ \\
\hline Hf-172 & $3.70 \mathrm{E}-08$ & $1.37 \mathrm{E}-04$ \\
\hline Hf-173 & $2.03 \mathrm{E}-10$ & $7.51 \mathrm{E}-07$ \\
\hline Hf-174 & $3.20 \mathrm{E}-05$ & $1.18 \mathrm{E}-01$ \\
\hline Hf-175 & $1.55 \mathrm{E}-09$ & $5.74 \mathrm{E}-06$ \\
\hline Hf-177m & $1.11 \mathrm{E}-10$ & 4.11E-07 \\
\hline Hf-178m & $2.30 \mathrm{E}-07$ & $8.51 \mathrm{E}-04$ \\
\hline Hf-179m & 4.90E-09 & $1.81 \mathrm{E}-05$ \\
\hline Hf-180m & $1.67 \mathrm{E}-10$ & $6.18 \mathrm{E}-07$ \\
\hline Hf-181 & $6.52 \mathrm{E}-09$ & $2.41 \mathrm{E}-05$ \\
\hline Hf-182 & $3.04 \mathrm{E}-07$ & $1.12 \mathrm{E}-03$ \\
\hline Hf- $182 \mathrm{~m}$ & $6.11 \mathrm{E}-11$ & $2.26 \mathrm{E}-07$ \\
\hline Hf-183 & $7.30 \mathrm{E}-11$ & $2.70 \mathrm{E}-07$ \\
\hline Hf-184 & $4.08 \mathrm{E}-10$ & $1.51 \mathrm{E}-06$ \\
\hline Ta-172 & $4.30 \mathrm{E}-11$ & $1.59 \mathrm{E}-07$ \\
\hline Ta-173 & $9.37 \mathrm{E}-11$ & $3.47 \mathrm{E}-07$ \\
\hline Ta-174 & $5.71 \mathrm{E}-11$ & $2.11 \mathrm{E}-07$ \\
\hline
\end{tabular}




\begin{tabular}{|c|c|c|}
\hline Nuclide & $\begin{array}{c}\text { Inhalation } \\
\text { Dose } \\
\text { Coefficient } \\
\text { (Sv/Bq) }\end{array}$ & $\begin{array}{c}\text { Inhalation } \\
\text { Dose } \\
\text { Coefficient } \\
\text { (mrem/pCi) }\end{array}$ \\
\hline Ta-175 & $1.79 \mathrm{E}-10$ & $6.62 \mathrm{E}-07$ \\
\hline Ta-176 & $2.41 \mathrm{E}-10$ & $8.92 \mathrm{E}-07$ \\
\hline Ta-177 & $1.22 \mathrm{E}-10$ & $4.51 \mathrm{E}-07$ \\
\hline Ta-178m & $8.96 \mathrm{E}-11$ & $3.32 \mathrm{E}-07$ \\
\hline Ta-179 & $5.46 \mathrm{E}-10$ & $2.02 \mathrm{E}-06$ \\
\hline Ta-180 & $5.18 \mathrm{E}-11$ & $1.92 \mathrm{E}-07$ \\
\hline Ta-182 & $1.14 \mathrm{E}-08$ & $4.22 \mathrm{E}-05$ \\
\hline Ta-182m & $2.60 \mathrm{E}-11$ & $9.62 \mathrm{E}-08$ \\
\hline Ta-183 & $2.50 \mathrm{E}-09$ & $9.25 \mathrm{E}-06$ \\
\hline Ta-184 & $5.10 \mathrm{E}-10$ & $1.89 \mathrm{E}-06$ \\
\hline Ta-185 & $5.80 \mathrm{E}-11$ & $2.15 \mathrm{E}-07$ \\
\hline Ta-186 & $2.14 \mathrm{E}-11$ & $7.92 \mathrm{E}-08$ \\
\hline $\mathrm{W}-177$ & $5.22 \mathrm{E}-11$ & $1.93 \mathrm{E}-07$ \\
\hline $\mathrm{W}-178$ & $9.29 \mathrm{E}-10$ & $3.44 \mathrm{E}-06$ \\
\hline $\mathrm{W}-179$ & $1.62 \mathrm{E}-12$ & $5.99 \mathrm{E}-09$ \\
\hline $\mathrm{W}-181$ & $3.25 \mathrm{E}-10$ & $1.20 \mathrm{E}-06$ \\
\hline W-185 & $4.24 \mathrm{E}-09$ & $1.57 \mathrm{E}-05$ \\
\hline W-187 & $4.83 \mathrm{E}-10$ & $1.79 \mathrm{E}-06$ \\
\hline $\mathrm{W}-188$ & $1.72 \mathrm{E}-08$ & $6.36 \mathrm{E}-05$ \\
\hline $\mathrm{W}-190$ & $8.54 \mathrm{E}-11$ & $3.16 \mathrm{E}-07$ \\
\hline Re-178 & $1.90 \mathrm{E}-11$ & $7.03 \mathrm{E}-08$ \\
\hline Re-179 & $1.32 \mathrm{E}-11$ & $4.88 \mathrm{E}-08$ \\
\hline Re-181 & $3.14 \mathrm{E}-10$ & $1.16 \mathrm{E}-06$ \\
\hline Re-182 & $1.48 \mathrm{E}-09$ & $5.48 \mathrm{E}-06$ \\
\hline Re-182m & $2.56 \mathrm{E}-10$ & $9.47 \mathrm{E}-07$ \\
\hline Re-183 & $3.94 \mathrm{E}-09$ & $1.46 \mathrm{E}-05$ \\
\hline Re-184 & $2.57 \mathrm{E}-09$ & $9.51 \mathrm{E}-06$ \\
\hline Re-184m & $1.12 \mathrm{E}-08$ & $4.14 \mathrm{E}-05$ \\
\hline Re-186 & $1.31 \mathrm{E}-09$ & $4.85 \mathrm{E}-06$ \\
\hline Re-186m & $6.43 \mathrm{E}-08$ & $2.38 \mathrm{E}-04$ \\
\hline Re-187 & $4.26 \mathrm{E}-11$ & $1.58 \mathrm{E}-07$ \\
\hline Re-188 & $6.68 \mathrm{E}-10$ & $2.47 \mathrm{E}-06$ \\
\hline Re-188m & $1.77 \mathrm{E}-11$ & $6.55 \mathrm{E}-08$ \\
\hline Re-189 & $5.06 \mathrm{E}-10$ & $1.87 \mathrm{E}-06$ \\
\hline Os-180 & $2.44 \mathrm{E}-10$ & $9.03 \mathrm{E}-07$ \\
\hline Os-181 & $7.77 \mathrm{E}-11$ & $6.55 \mathrm{E}-08$ \\
\hline
\end{tabular}




\begin{tabular}{|c|c|c|}
\hline Nuclide & $\begin{array}{c}\text { Inhalation } \\
\text { Dose } \\
\text { Coefficient } \\
\text { (Sv/Bq) }\end{array}$ & $\begin{array}{c}\text { Inhalation } \\
\text { Dose } \\
\text { Coefficient } \\
\text { (mrem/pCi) }\end{array}$ \\
\hline Os-182 & $4.69 \mathrm{E}-10$ & $1.74 \mathrm{E}-06$ \\
\hline Os-183 & $2.20 \mathrm{E}-10$ & $8.14 \mathrm{E}-07$ \\
\hline Os-183m & $1.74 \mathrm{E}-10$ & $6.44 \mathrm{E}-07$ \\
\hline Os-185 & $1.73 \mathrm{E}-09$ & $6.40 \mathrm{E}-06$ \\
\hline Os-186 & $4.51 \mathrm{E}-06$ & $1.67 \mathrm{E}-02$ \\
\hline Os-189m & $6.42 \mathrm{E}-12$ & $2.38 \mathrm{E}-08$ \\
\hline Os-191 & $2.21 \mathrm{E}-09$ & $8.18 \mathrm{E}-06$ \\
\hline Os-191m & $1.84 \mathrm{E}-10$ & $6.81 \mathrm{E}-07$ \\
\hline Os-193 & $6.32 \mathrm{E}-10$ & $2.34 \mathrm{E}-06$ \\
\hline Os-194 & $9.09 \mathrm{E}-08$ & $3.36 \mathrm{E}-04$ \\
\hline Os-196 & $6.64 \mathrm{E}-11$ & $2.46 \mathrm{E}-07$ \\
\hline Ir-182 & $3.07 \mathrm{E}-11$ & $1.14 \mathrm{E}-07$ \\
\hline Ir-183 & $4.71 \mathrm{E}-11$ & $1.74 \mathrm{E}-07$ \\
\hline Ir-184 & $1.49 \mathrm{E}-10$ & $5.51 \mathrm{E}-07$ \\
\hline Ir-185 & $2.76 \mathrm{E}-10$ & $1.02 \mathrm{E}-06$ \\
\hline Ir-186 & $4.21 \mathrm{E}-10$ & $1.56 \mathrm{E}-06$ \\
\hline Ir-186m & $5.97 \mathrm{E}-11$ & $2.21 \mathrm{E}-07$ \\
\hline Ir-187 & $9.10 \mathrm{E}-11$ & $3.37 \mathrm{E}-07$ \\
\hline Ir-188 & $5.71 \mathrm{E}-10$ & $2.11 \mathrm{E}-06$ \\
\hline Ir-189 & $6.26 \mathrm{E}-10$ & $2.32 \mathrm{E}-06$ \\
\hline Ir-190 & $1.56 \mathrm{E}-09$ & $5.77 \mathrm{E}-06$ \\
\hline Ir-190m & $6.98 \mathrm{E}-12$ & $2.58 \mathrm{E}-08$ \\
\hline Ir-190n & $9.93 \mathrm{E}-11$ & $3.67 \mathrm{E}-07$ \\
\hline Ir-192 & $7.30 \mathrm{E}-09$ & $2.70 \mathrm{E}-05$ \\
\hline Ir-192n & $6.12 \mathrm{E}-08$ & $2.26 \mathrm{E}-04$ \\
\hline Ir-193m & $1.35 \mathrm{E}-09$ & $5.00 \mathrm{E}-06$ \\
\hline Ir-194 & $6.75 \mathrm{E}-10$ & $2.50 \mathrm{E}-06$ \\
\hline Ir-194m & $1.33 \mathrm{E}-08$ & $4.92 \mathrm{E}-05$ \\
\hline Ir-195 & $8.32 \mathrm{E}-11$ & $3.08 \mathrm{E}-07$ \\
\hline Ir-195m & $1.47 \mathrm{E}-10$ & $5.44 \mathrm{E}-07$ \\
\hline Pt-184 & $3.06 \mathrm{E}-11$ & $1.13 \mathrm{E}-07$ \\
\hline Pt-186 & $8.46 \mathrm{E}-11$ & $3.13 \mathrm{E}-07$ \\
\hline Pt-187 & $8.29 \mathrm{E}-11$ & $3.07 \mathrm{E}-07$ \\
\hline Pt-188 & $2.28 \mathrm{E}-09$ & $8.44 \mathrm{E}-06$ \\
\hline Pt-189 & $2.06 \mathrm{E}-10$ & $7.62 \mathrm{E}-07$ \\
\hline Pt-191 & $4.21 \mathrm{E}-10$ & $1.56 \mathrm{E}-06$ \\
\hline
\end{tabular}




\begin{tabular}{|c|c|c|}
\hline Nuclide & $\begin{array}{c}\text { Inhalation } \\
\text { Dose } \\
\text { Coefficient } \\
\text { (Sv/Bq) }\end{array}$ & $\begin{array}{c}\text { Inhalation } \\
\text { Dose } \\
\text { Coefficient } \\
\text { (mrem/pCi) }\end{array}$ \\
\hline $\mathrm{Pt}-193$ & $7.27 \mathrm{E}-10$ & $2.69 \mathrm{E}-06$ \\
\hline $\mathrm{Pt}-193 \mathrm{~m}$ & $1.08 \mathrm{E}-09$ & $4.00 \mathrm{E}-06$ \\
\hline $\mathrm{Pt}-195 \mathrm{~m}$ & $1.29 \mathrm{E}-09$ & $4.77 \mathrm{E}-06$ \\
\hline $\mathrm{Pt}-197$ & $4.31 \mathrm{E}-10$ & $1.59 \mathrm{E}-06$ \\
\hline $\mathrm{Pt}-197 \mathrm{~m}$ & $9.20 \mathrm{E}-11$ & $3.40 \mathrm{E}-07$ \\
\hline $\mathrm{Pt}-199$ & $3.38 \mathrm{E}-11$ & $1.25 \mathrm{E}-07$ \\
\hline $\mathrm{Pt}-202$ & $2.61 \mathrm{E}-09$ & $9.66 \mathrm{E}-06$ \\
\hline $\mathrm{Au}-186$ & $2.43 \mathrm{E}-11$ & $8.99 \mathrm{E}-08$ \\
\hline $\mathrm{Au}-190$ & $2.90 \mathrm{E}-11$ & $1.07 \mathrm{E}-07$ \\
\hline $\mathrm{Au}-191$ & $7.56 \mathrm{E}-11$ & $2.80 \mathrm{E}-07$ \\
\hline $\mathrm{Au}-192$ & $1.15 \mathrm{E}-10$ & $4.26 \mathrm{E}-07$ \\
\hline $\mathrm{Au}-193$ & $1.23 \mathrm{E}-10$ & $4.55 \mathrm{E}-07$ \\
\hline $\mathrm{Au}-194$ & $2.79 \mathrm{E}-10$ & $1.03 \mathrm{E}-06$ \\
\hline $\mathrm{Au}-195$ & $2.00 \mathrm{E}-09$ & $7.40 \mathrm{E}-06$ \\
\hline $\mathrm{Au}-196$ & $3.78 \mathrm{E}-10$ & $1.40 \mathrm{E}-06$ \\
\hline $\mathrm{Au}-196 \mathrm{~m}$ & $5.00 \mathrm{E}-10$ & $1.85 \mathrm{E}-06$ \\
\hline $\mathrm{Au}-198$ & $9.84 \mathrm{E}-10$ & $3.64 \mathrm{E}-06$ \\
\hline $\mathrm{Au}-198 \mathrm{~m}$ & $2.05 \mathrm{E}-09$ & $7.59 \mathrm{E}-06$ \\
\hline $\mathrm{Au}-199$ & $8.99 \mathrm{E}-10$ & $3.33 \mathrm{E}-06$ \\
\hline $\mathrm{Au}-200$ & $4.14 \mathrm{E}-11$ & $1.53 \mathrm{E}-07$ \\
\hline $\mathrm{Au}-200 \mathrm{~m}$ & $7.95 \mathrm{E}-10$ & $2.94 \mathrm{E}-06$ \\
\hline $\mathrm{Au}-201$ & $2.03 \mathrm{E}-11$ & $7.51 \mathrm{E}-08$ \\
\hline $\mathrm{Hg}-190$ & $8.33 \mathrm{E}-11$ & $3.08 \mathrm{E}-07$ \\
\hline $\mathrm{Hg}-191 \mathrm{~m}$ & $3.47 \mathrm{E}-10$ & $1.28 \mathrm{E}-06$ \\
\hline $\mathrm{Hg}-192$ & $1.08 \mathrm{E}-09$ & $4.00 \mathrm{E}-06$ \\
\hline $\mathrm{Hg}-193$ & $9.44 \mathrm{E}-10$ & $3.49 \mathrm{E}-06$ \\
\hline $\mathrm{Hg}-193 \mathrm{~m}$ & $3.25 \mathrm{E}-09$ & $1.20 \mathrm{E}-05$ \\
\hline $\mathrm{Hg}-194$ & $4.25 \mathrm{E}-08$ & $1.57 \mathrm{E}-04$ \\
\hline $\mathrm{Hg}-195$ & $1.63 \mathrm{E}-09$ & $6.03 \mathrm{E}-06$ \\
\hline $\mathrm{Hg}-195 \mathrm{~m}$ & $8.80 \mathrm{E}-09$ & $3.26 \mathrm{E}-05$ \\
\hline $\mathrm{Hg}-197$ & $5.06 \mathrm{E}-09$ & $1.87 \mathrm{E}-05$ \\
\hline $\mathrm{Hg}-197 \mathrm{~m}$ & $6.38 \mathrm{E}-09$ & $2.36 \mathrm{E}-05$ \\
\hline $\mathrm{Hg}-199 \mathrm{~m}$ & $1.91 \mathrm{E}-10$ & $7.07 \mathrm{E}-07$ \\
\hline $\mathrm{Tl}-193$ & $7.71 \mathrm{E}-09$ & $2.85 \mathrm{E}-05$ \\
\hline & $2.30 \mathrm{E}-11$ & $8.51 \mathrm{E}-08$ \\
\hline Tl-195 & $2.98 \mathrm{E}-11$ & $1.10 \mathrm{E}-07$ \\
\hline
\end{tabular}




\begin{tabular}{|c|c|c|}
\hline Nuclide & $\begin{array}{c}\text { Inhalation } \\
\text { Dose } \\
\text { Coefficient } \\
\text { (Sv/Bq) }\end{array}$ & $\begin{array}{c}\text { Inhalation } \\
\text { Dose } \\
\text { Coefficient } \\
\text { (mrem/pCi) }\end{array}$ \\
\hline $\mathrm{Tl}-196$ & $4.70 \mathrm{E}-11$ & $1.74 \mathrm{E}-07$ \\
\hline $\mathrm{Tl}-197$ & $4.50 \mathrm{E}-11$ & $1.67 \mathrm{E}-07$ \\
\hline $\mathrm{Tl}-198$ & $9.13 \mathrm{E}-11$ & $3.38 \mathrm{E}-07$ \\
\hline $\mathrm{Tl}-198 \mathrm{~m}$ & $8.09 \mathrm{E}-11$ & $2.99 \mathrm{E}-07$ \\
\hline $\mathrm{Tl}-199$ & $5.81 \mathrm{E}-11$ & $2.15 \mathrm{E}-07$ \\
\hline $\mathrm{Tl}-200$ & $2.14 \mathrm{E}-10$ & $7.92 \mathrm{E}-07$ \\
\hline $\mathrm{Tl}-201$ & $2.20 \mathrm{E}-10$ & $8.14 \mathrm{E}-07$ \\
\hline $\mathrm{Tl}-202$ & $4.59 \mathrm{E}-10$ & $1.70 \mathrm{E}-06$ \\
\hline $\mathrm{Tl}-204$ & $2.05 \mathrm{E}-08$ & $7.59 \mathrm{E}-05$ \\
\hline $\mathrm{Pb}-194$ & $1.51 \mathrm{E}-11$ & $5.59 \mathrm{E}-08$ \\
\hline $\mathrm{Pb}-195 \mathrm{~m}$ & $2.62 \mathrm{E}-11$ & $9.69 \mathrm{E}-08$ \\
\hline $\mathrm{Pb}-196$ & $2.88 \mathrm{E}-11$ & $1.07 \mathrm{E}-07$ \\
\hline $\mathrm{Pb}-197 \mathrm{~m}$ & $5.27 \mathrm{E}-11$ & $1.95 \mathrm{E}-07$ \\
\hline $\mathrm{Pb}-198$ & $7.37 \mathrm{E}-11$ & $2.73 \mathrm{E}-07$ \\
\hline $\mathrm{Pb}-199$ & $3.47 \mathrm{E}-11$ & $1.28 \mathrm{E}-07$ \\
\hline $\mathrm{Pb}-200$ & $3.77 \mathrm{E}-10$ & $1.39 \mathrm{E}-06$ \\
\hline $\mathrm{Pb}-201$ & $1.33 \mathrm{E}-10$ & $4.92 \mathrm{E}-07$ \\
\hline $\mathrm{Pb}-202$ & $1.97 \mathrm{E}-08$ & $7.29 \mathrm{E}-05$ \\
\hline $\mathrm{Pb}-202 \mathrm{~m}$ & $1.18 \mathrm{E}-10$ & $4.37 \mathrm{E}-07$ \\
\hline $\mathrm{Pb}-203$ & $2.39 \mathrm{E}-10$ & $8.84 \mathrm{E}-07$ \\
\hline $\mathrm{Pb}-204 \mathrm{~m}$ & $3.44 \mathrm{E}-11$ & $1.27 \mathrm{E}-07$ \\
\hline $\mathrm{Pb}-205$ & $2.58 \mathrm{E}-10$ & $9.55 \mathrm{E}-07$ \\
\hline $\mathrm{Pb}-209$ & $6.46 \mathrm{E}-11$ & $2.39 \mathrm{E}-07$ \\
\hline $\mathrm{Pb}-210$ & $1.21 \mathrm{E}-06$ & $4.48 \mathrm{E}-03$ \\
\hline $\mathrm{Pb}-211$ & $1.26 \mathrm{E}-08$ & $4.66 \mathrm{E}-05$ \\
\hline $\mathrm{Pb}-212$ & $1.86 \mathrm{E}-07$ & $6.88 \mathrm{E}-04$ \\
\hline $\mathrm{Pb}-214$ & $1.47 \mathrm{E}-08$ & $5.44 \mathrm{E}-05$ \\
\hline $\mathrm{Bi}-200$ & $4.39 \mathrm{E}-11$ & $1.62 \mathrm{E}-07$ \\
\hline $\mathrm{Bi}-201$ & $8.98 \mathrm{E}-11$ & $3.32 \mathrm{E}-07$ \\
\hline $\mathrm{Bi}-202$ & $7.53 \mathrm{E}-11$ & $2.79 \mathrm{E}-07$ \\
\hline $\mathrm{Bi}-203$ & $3.42 \mathrm{E}-10$ & $1.27 \mathrm{E}-06$ \\
\hline $\mathrm{Bi}-204$ & $3.81 \mathrm{E}-10$ & $1.41 \mathrm{E}-06$ \\
\hline $\mathrm{Bi}-205$ & $1.23 \mathrm{E}-09$ & $4.55 \mathrm{E}-06$ \\
\hline $\mathrm{Bi}-206$ & $2.15 \mathrm{E}-09$ & $7.96 \mathrm{E}-06$ \\
\hline & $4.08 \mathrm{E}-08$ & $1.51 \mathrm{E}-04$ \\
\hline & $3.83 \mathrm{E}-08$ & $1.42 \mathrm{E}-04$ \\
\hline
\end{tabular}




\begin{tabular}{|c|c|c|}
\hline Nuclide & $\begin{array}{c}\text { Inhalation } \\
\text { Dose } \\
\text { Coefficient } \\
\text { (Sv/Bq) } \\
\end{array}$ & $\begin{array}{c}\text { Inhalation } \\
\text { Dose } \\
\text { Coefficient } \\
\text { (mrem/pCi) }\end{array}$ \\
\hline $\mathrm{Bi}-210 \mathrm{~m}$ & $1.07 \mathrm{E}-05$ & 3.96E-02 \\
\hline Bi-212 & $3.67 \mathrm{E}-08$ & $1.36 \mathrm{E}-04$ \\
\hline $\mathrm{Bi}-213$ & $3.55 \mathrm{E}-08$ & $1.31 \mathrm{E}-04$ \\
\hline $\mathrm{Bi}-214$ & $1.72 \mathrm{E}-08$ & $6.36 \mathrm{E}-05$ \\
\hline Po-203 & $5.16 \mathrm{E}-11$ & $1.91 \mathrm{E}-07$ \\
\hline Po-204 & $4.24 \mathrm{E}-10$ & $1.57 \mathrm{E}-06$ \\
\hline Po-205 & $5.21 \mathrm{E}-11$ & $1.93 \mathrm{E}-07$ \\
\hline Рo-206 & $6.27 \mathrm{E}-08$ & $2.32 \mathrm{E}-04$ \\
\hline Po-207 & $1.05 \mathrm{E}-10$ & $3.89 \mathrm{E}-07$ \\
\hline Po-208 & 4.16E-06 & $1.54 \mathrm{E}-02$ \\
\hline Po-209 & 4.02E-06 & $1.49 \mathrm{E}-02$ \\
\hline Po-210 & $3.60 \mathrm{E}-06$ & $1.33 \mathrm{E}-02$ \\
\hline At-205 & $7.83 \mathrm{E}-10$ & $2.90 \mathrm{E}-06$ \\
\hline At-206 & $2.62 \mathrm{E}-10$ & $9.69 \mathrm{E}-07$ \\
\hline At-207 & $2.41 \mathrm{E}-09$ & $8.92 \mathrm{E}-06$ \\
\hline At-208 & $6.56 \mathrm{E}-10$ & $2.43 \mathrm{E}-06$ \\
\hline At-209 & $3.04 \mathrm{E}-09$ & $1.12 \mathrm{E}-05$ \\
\hline At-210 & $1.17 \mathrm{E}-08$ & 4.33E-05 \\
\hline At-211 & $1.30 \mathrm{E}-07$ & $4.81 \mathrm{E}-04$ \\
\hline Fr-212 & $6.98 \mathrm{E}-09$ & $2.58 \mathrm{E}-05$ \\
\hline Fr-222 & $3.08 \mathrm{E}-08$ & $1.14 \mathrm{E}-04$ \\
\hline Fr-223 & $1.33 \mathrm{E}-08$ & $4.92 \mathrm{E}-05$ \\
\hline Ra-223 & $8.05 \mathrm{E}-06$ & $2.98 \mathrm{E}-02$ \\
\hline $\mathrm{Ra}-224$ & $3.22 \mathrm{E}-06$ & $1.19 \mathrm{E}-02$ \\
\hline Ra-225 & $6.83 \mathrm{E}-06$ & $2.53 \mathrm{E}-02$ \\
\hline Ra-226 & $3.82 \mathrm{E}-06$ & $1.41 \mathrm{E}-02$ \\
\hline Ra-227 & $2.87 \mathrm{E}-10$ & $1.06 \mathrm{E}-06$ \\
\hline Ra-228 & $3.08 \mathrm{E}-06$ & $1.14 \mathrm{E}-02$ \\
\hline Ra-230 & $1.29 \mathrm{E}-10$ & 4.77E-07 \\
\hline Ac-224 & $1.24 \mathrm{E}-07$ & 4.59E-04 \\
\hline Ac- 225 & $9.18 \mathrm{E}-06$ & $3.40 \mathrm{E}-02$ \\
\hline Ac-226 & $1.41 \mathrm{E}-06$ & $5.22 \mathrm{E}-03$ \\
\hline Ac-227 & $1.61 \mathrm{E}-04$ & $5.96 \mathrm{E}-01$ \\
\hline Ac- 228 & $1.61 \mathrm{E}-08$ & $5.96 \mathrm{E}-05$ \\
\hline Th-228 & 4.35E-05 & $1.61 \mathrm{E}-01$ \\
\hline Th-229 & 7.55E-05 & $2.79 \mathrm{E}-01$ \\
\hline Th-230 & $1.47 \mathrm{E}-05$ & $5.44 \mathrm{E}-02$ \\
\hline
\end{tabular}




\begin{tabular}{|c|c|c|}
\hline Nuclide & $\begin{array}{c}\text { Inhalation } \\
\text { Dose } \\
\text { Coefficient } \\
\text { (Sv/Bq) }\end{array}$ & $\begin{array}{c}\text { Inhalation } \\
\text { Dose } \\
\text { Coefficient } \\
\text { (mrem/pCi) }\end{array}$ \\
\hline Th-232 & $2.56 \mathrm{E}-05$ & $9.47 \mathrm{E}-02$ \\
\hline $\mathrm{Th}-234$ & $8.60 \mathrm{E}-09$ & $3.18 \mathrm{E}-05$ \\
\hline $\mathrm{Pa}-227$ & $8.57 \mathrm{E}-08$ & $3.17 \mathrm{E}-04$ \\
\hline $\mathrm{Pa}-228$ & $7.78 \mathrm{E}-08$ & $2.88 \mathrm{E}-04$ \\
\hline $\mathrm{Pa}-229$ & $7.78 \mathrm{E}-09$ & $2.88 \mathrm{E}-05$ \\
\hline $\mathrm{Pa}-230$ & $7.34 \mathrm{E}-07$ & $2.72 \mathrm{E}-03$ \\
\hline $\mathrm{Pa}-231$ & $2.37 \mathrm{E}-04$ & $8.77 \mathrm{E}-01$ \\
\hline $\mathrm{Pa}-232$ & $2.60 \mathrm{E}-09$ & $9.62 \mathrm{E}-06$ \\
\hline $\mathrm{Pa}-233$ & $4.56 \mathrm{E}-09$ & $1.69 \mathrm{E}-05$ \\
\hline $\mathrm{Pa}-234$ & $3.98 \mathrm{E}-10$ & $1.47 \mathrm{E}-06$ \\
\hline $\mathrm{Pa}-235$ & $1.98 \mathrm{E}-11$ & $7.33 \mathrm{E}-08$ \\
\hline $\mathrm{U}-230$ & $1.47 \mathrm{E}-05$ & $5.44 \mathrm{E}-02$ \\
\hline $\mathrm{U}-231$ & $5.30 \mathrm{E}-10$ & $1.96 \mathrm{E}-06$ \\
\hline $\mathrm{U}-232$ & $8.62 \mathrm{E}-06$ & $3.19 \mathrm{E}-02$ \\
\hline $\mathrm{U}-233$ & $3.89 \mathrm{E}-06$ & $1.44 \mathrm{E}-02$ \\
\hline $\mathrm{U}-234$ & $3.81 \mathrm{E}-06$ & $1.41 \mathrm{E}-02$ \\
\hline $\mathrm{U}-235$ & $3.38 \mathrm{E}-06$ & $1.25 \mathrm{E}-02$ \\
\hline $\mathrm{U}-235 \mathrm{~m}$ & $9.01 \mathrm{E}-16$ & $3.33 \mathrm{E}-12$ \\
\hline $\mathrm{U}-236$ & $3.49 \mathrm{E}-06$ & $1.29 \mathrm{E}-02$ \\
\hline $\mathrm{U}-237$ & $1.84 \mathrm{E}-09$ & $6.81 \mathrm{E}-06$ \\
\hline $\mathrm{U}-238$ & $3.14 \mathrm{E}-06$ & $1.16 \mathrm{E}-02$ \\
\hline $\mathrm{U}-239$ & $2.62 \mathrm{E}-11$ & $9.69 \mathrm{E}-08$ \\
\hline $\mathrm{U}-240$ & $6.17 \mathrm{E}-10$ & $2.28 \mathrm{E}-06$ \\
\hline $\mathrm{U}-242$ & $3.21 \mathrm{E}-11$ & $1.19 \mathrm{E}-07$ \\
\hline $\mathrm{Np}-232$ & $5.20 \mathrm{E}-11$ & $1.92 \mathrm{E}-07$ \\
\hline $\mathrm{Np}-233$ & $2.20 \mathrm{E}-12$ & $8.14 \mathrm{E}-09$ \\
\hline $\mathrm{Np}-234$ & $5.09 \mathrm{E}-10$ & $1.88 \mathrm{E}-06$ \\
\hline $\mathrm{Np}-235$ & $5.41 \mathrm{E}-10$ & $2.00 \mathrm{E}-06$ \\
\hline $\mathrm{Np}-236$ & $4.51 \mathrm{E}-06$ & $1.67 \mathrm{E}-02$ \\
\hline $\mathrm{Np}-236 \mathrm{~m}$ & $5.68 \mathrm{E}-09$ & $2.10 \mathrm{E}-05$ \\
\hline $\mathrm{Np}-237$ & $2.30 \mathrm{E}-05$ & $8.51 \mathrm{E}-02$ \\
\hline $\mathrm{Np}-238$ & $2.24 \mathrm{E}-09$ & $8.29 \mathrm{E}-06$ \\
\hline $\mathrm{Np}-239$ & $1.08 \mathrm{E}-09$ & $4.00 \mathrm{E}-06$ \\
\hline $\mathrm{Np}-240$ & $8.03 \mathrm{E}-11$ & $2.97 \mathrm{E}-07$ \\
\hline $\mathrm{Pu}-232$ & $1.40 \mathrm{E}-11$ & $5.18 \mathrm{E}-08$ \\
\hline & $2.44 \mathrm{E}-08$ & $9.03 \mathrm{E}-05$ \\
\hline & $2.24 \mathrm{E}-08$ & $8.29 \mathrm{E}-05$ \\
\hline
\end{tabular}




\begin{tabular}{|c|c|c|}
\hline Nuclide & $\begin{array}{c}\text { Inhalation } \\
\text { Dose } \\
\text { Coefficient } \\
\text { (Sv/Bq) }\end{array}$ & $\begin{array}{c}\text { Inhalation } \\
\text { Dose } \\
\text { Coefficient } \\
\text { (mrem/pCi) }\end{array}$ \\
\hline $\mathrm{Pu}-235$ & $1.88 \mathrm{E}-12$ & $6.96 \mathrm{E}-09$ \\
\hline $\mathrm{Pu}-236$ & $2.05 \mathrm{E}-05$ & $7.59 \mathrm{E}-02$ \\
\hline $\mathrm{Pu}-237$ & $3.99 \mathrm{E}-10$ & $1.48 \mathrm{E}-06$ \\
\hline $\mathrm{Pu}-238$ & $4.65 \mathrm{E}-05$ & $1.72 \mathrm{E}-01$ \\
\hline $\mathrm{Pu}-239$ & $5.04 \mathrm{E}-05$ & $1.86 \mathrm{E}-01$ \\
\hline $\mathrm{Pu}-240$ & $5.04 \mathrm{E}-05$ & $1.86 \mathrm{E}-01$ \\
\hline $\mathrm{Pu}-241$ & $8.94 \mathrm{E}-07$ & $3.31 \mathrm{E}-03$ \\
\hline $\mathrm{Pu}-242$ & $4.79 \mathrm{E}-05$ & $1.77 \mathrm{E}-01$ \\
\hline $\mathrm{Pu}-243$ & $9.38 \mathrm{E}-11$ & $3.47 \mathrm{E}-07$ \\
\hline $\mathrm{Pu}-244$ & $4.70 \mathrm{E}-05$ & $1.74 \mathrm{E}-01$ \\
\hline $\mathrm{Pu}-245$ & $4.74 \mathrm{E}-10$ & $1.75 \mathrm{E}-06$ \\
\hline $\mathrm{Pu}-246$ & $4.91 \mathrm{E}-09$ & $1.82 \mathrm{E}-05$ \\
\hline $\mathrm{Am}-237$ & $2.88 \mathrm{E}-11$ & $1.07 \mathrm{E}-07$ \\
\hline $\mathrm{Am}-238$ & $9.33 \mathrm{E}-11$ & $3.45 \mathrm{E}-07$ \\
\hline $\mathrm{Am}-239$ & $2.62 \mathrm{E}-10$ & $9.69 \mathrm{E}-07$ \\
\hline $\mathrm{Am}-240$ & $5.10 \mathrm{E}-10$ & $1.89 \mathrm{E}-06$ \\
\hline $\mathrm{Am}-241$ & $4.21 \mathrm{E}-05$ & $1.56 \mathrm{E}-01$ \\
\hline $\mathrm{Am}-242$ & $1.91 \mathrm{E}-08$ & $7.07 \mathrm{E}-05$ \\
\hline $\mathrm{Am}-242 \mathrm{~m}$ & $3.67 \mathrm{E}-05$ & $1.36 \mathrm{E}-01$ \\
\hline $\mathrm{Am}-243$ & $4.16 \mathrm{E}-05$ & $1.54 \mathrm{E}-01$ \\
\hline $\mathrm{Am}-244$ & $2.10 \mathrm{E}-09$ & $7.77 \mathrm{E}-06$ \\
\hline $\mathrm{Am}-244 \mathrm{~m}$ & $8.75 \mathrm{E}-11$ & $3.24 \mathrm{E}-07$ \\
\hline $\mathrm{Am}-245$ & $6.12 \mathrm{E}-11$ & $2.26 \mathrm{E}-07$ \\
\hline $\mathrm{Am}-246$ & $8.43 \mathrm{E}-11$ & $3.12 \mathrm{E}-07$ \\
\hline $\mathrm{Am}-246 \mathrm{~m}$ & $2.67 \mathrm{E}-11$ & $9.88 \mathrm{E}-08$ \\
\hline $\mathrm{Am}-247$ & $3.02 \mathrm{E}-11$ & $1.12 \mathrm{E}-07$ \\
\hline $\mathrm{Cm}-238$ & $1.95 \mathrm{E}-09$ & $7.22 \mathrm{E}-06$ \\
\hline $\mathrm{Cm}-239$ & $7.59 \mathrm{E}-11$ & $2.81 \mathrm{E}-07$ \\
\hline $\mathrm{Cm}-240$ & $3.43 \mathrm{E}-06$ & $1.27 \mathrm{E}-02$ \\
\hline $\mathrm{Cm}-241$ & $4.00 \mathrm{E}-08$ & $1.48 \mathrm{E}-04$ \\
\hline $\mathrm{Cm}-242$ & $5.72 \mathrm{E}-06$ & $2.12 \mathrm{E}-02$ \\
\hline $\mathrm{Cm}-243$ & $3.23 \mathrm{E}-05$ & $1.20 \mathrm{E}-01$ \\
\hline $\mathrm{Bm}-244$ & $2.73 \mathrm{E}-05$ & $1.01 \mathrm{E}-01$ \\
\hline $\mathrm{Bk}-246$ & $4.27 \mathrm{E}-05$ & $1.58 \mathrm{E}-01$ \\
\hline & $1.50 \mathrm{E}-04$ & $5.55 \mathrm{E}-01$ \\
\hline $\mathrm{Bm}-245$ & $2.48 \mathrm{E}-09$ & $9.18 \mathrm{E}-06$ \\
\hline $\mathrm{Am}-10$ & $1.24 \mathrm{E}-06$ \\
\hline
\end{tabular}




\begin{tabular}{|c|c|c|}
\hline Nuclide & $\begin{array}{c}\text { Inhalation } \\
\text { Dose } \\
\text { Coefficient } \\
\text { (Sv/Bq) }\end{array}$ & $\begin{array}{c}\text { Inhalation } \\
\text { Dose } \\
\text { Coefficient } \\
\text { (mrem/pCi) }\end{array}$ \\
\hline Bk-247 & $1.77 \mathrm{E}-04$ & $6.55 \mathrm{E}-01$ \\
\hline Bk-248m & $2.81 \mathrm{E}-08$ & $1.04 \mathrm{E}-04$ \\
\hline Bk-249 & $4.40 \mathrm{E}-07$ & $1.63 \mathrm{E}-03$ \\
\hline Bk-250 & $2.33 \mathrm{E}-09$ & $8.62 \mathrm{E}-06$ \\
\hline Bk-251 & $5.09 \mathrm{E}-11$ & $1.88 \mathrm{E}-07$ \\
\hline Cf-244 & $1.50 \mathrm{E}-08$ & $5.55 \mathrm{E}-05$ \\
\hline Cf-246 & $4.92 \mathrm{E}-07$ & $1.82 \mathrm{E}-03$ \\
\hline Cf-247 & $4.62 \mathrm{E}-11$ & $1.71 \mathrm{E}-07$ \\
\hline Cf-248 & $9.79 \mathrm{E}-06$ & $3.62 \mathrm{E}-02$ \\
\hline Cf-249 & $7.29 \mathrm{E}-05$ & $2.70 \mathrm{E}-01$ \\
\hline Cf-250 & $3.61 \mathrm{E}-05$ & $1.34 \mathrm{E}-01$ \\
\hline Cf-251 & $7.41 \mathrm{E}-05$ & $2.74 \mathrm{E}-01$ \\
\hline Cf-252 & $2.25 \mathrm{E}-05$ & $8.33 \mathrm{E}-02$ \\
\hline Cf-253 & $1.46 \mathrm{E}-06$ & $5.40 \mathrm{E}-03$ \\
\hline Cf-254 & $4.68 \mathrm{E}-05$ & $1.73 \mathrm{E}-01$ \\
\hline Cf-255 & $6.24 \mathrm{E}-09$ & $2.31 \mathrm{E}-05$ \\
\hline Es-249 & $2.49 \mathrm{E}-10$ & $9.21 \mathrm{E}-07$ \\
\hline Es-250 & $6.16 \mathrm{E}-09$ & $2.28 \mathrm{E}-05$ \\
\hline Es-250m & $1.59 \mathrm{E}-09$ & $5.88 \mathrm{E}-06$ \\
\hline Es-251 & $2.32 \mathrm{E}-09$ & $8.58 \mathrm{E}-06$ \\
\hline Es-253 & $3.43 \mathrm{E}-06$ & $1.27 \mathrm{E}-02$ \\
\hline Es-254 & $1.23 \mathrm{E}-05$ & $4.55 \mathrm{E}-02$ \\
\hline Es-254m & $5.64 \mathrm{E}-07$ & $2.09 \mathrm{E}-03$ \\
\hline Es-255 & $4.95 \mathrm{E}-06$ & $1.83 \mathrm{E}-02$ \\
\hline Es-256 & $4.55 \mathrm{E}-08$ & $1.68 \mathrm{E}-04$ \\
\hline Fm-251 & $2.16 \mathrm{E}-09$ & $7.99 \mathrm{E}-06$ \\
\hline Fm-252 & $4.17 \mathrm{E}-07$ & $1.54 \mathrm{E}-03$ \\
\hline Fm-253 & $4.98 \mathrm{E}-07$ & $1.84 \mathrm{E}-03$ \\
\hline Fm-254 & $7.44 \mathrm{E}-08$ & $2.75 \mathrm{E}-04$ \\
\hline Fm-255 & $3.19 \mathrm{E}-07$ & $1.18 \mathrm{E}-03$ \\
\hline Fm-256 & $2.85 \mathrm{E}-07$ & $1.05 \mathrm{E}-03$ \\
\hline Fm-257 & $9.15 \mathrm{E}-06$ & $3.39 \mathrm{E}-02$ \\
\hline
\end{tabular}




\section{APPENDIX C \\ REFERENCE PERSON \\ EXTERNAL DOSE COEFFICIENTS}

Appendix $\mathrm{C}$ tabulates the dose rate coefficients for air immersion, water submersion, and ground shine. These dose coefficients are from the DC_PACK3 toolbox. 
Table C-1 Air Immersion and Water Submersion Dose Coefficients

\begin{tabular}{|c|c|c|c|c|c|c|c|c|}
\hline Nuclide & Half-life & Class & $\begin{array}{c}\text { Air } \\
\text { Immersion } \\
\text { Dose Rate } \\
\text { Coefficient } \\
\text { (Sv/s per } \\
\left.\text { Bq/ } \mathbf{m}^{3}\right)\end{array}$ & $\begin{array}{c}\text { Air } \\
\text { Immersion } \\
\text { Dose Rate } \\
\text { Coefficient } \\
\text { (mrem- } \\
\left.\text { m }^{3} / \mathrm{pCi}-\mathrm{yr}\right)\end{array}$ & $\begin{array}{c}\text { Air } \\
\text { Immersion } \\
\text { Effective } \\
\text { Dose Rate } \\
(\text { mrem } / \mathrm{sec} \\
\text { per } \\
\left.\mu \mathrm{Ci} / \mathrm{cm}^{3}\right)\end{array}$ & $\begin{array}{c}\text { Water } \\
\text { Submersion } \\
\text { Dose Rate } \\
\text { Coefficient } \\
\left(\mathrm{Sv}-\mathrm{m}^{3} / \mathrm{Bq}-\right. \\
\text { s) }\end{array}$ & $\begin{array}{c}\text { Water } \\
\text { Submersion } \\
\text { Dose Rate } \\
\text { Coefficient } \\
\text { (mrem- } \\
\text { m³Ci-h) }^{\mathbf{3} / p C i}\end{array}$ & $\begin{array}{c}\text { Water } \\
\text { Submersion } \\
\text { Dose Rate } \\
\text { Coefficient } \\
(\mathbf{m r e m -} \\
\left.\mathbf{m}^{\mathbf{3}} / \mu \mathrm{Ci}-\mathrm{y}\right)\end{array}$ \\
\hline H-3 & 12.32 & $\mathrm{y}$ & $0.00 \mathrm{E}+00$ & $0.00 \mathrm{E}+00$ & $0.00 \mathrm{E}+00$ & $0.00 \mathrm{E}+00$ & $0.00 \mathrm{E}+00$ & $0.00 \mathrm{E}+00$ \\
\hline $\mathrm{Be}-7$ & 53.22 & $\mathrm{~d}$ & $2.21 \mathrm{E}-15$ & $2.58 \mathrm{E}-04$ & $8.18 \mathrm{E}+00$ & $4.81 \mathrm{E}-18$ & $6.41 \mathrm{E}-11$ & $5.61 \mathrm{E}-13$ \\
\hline Be-10 & $1.51 \mathrm{E}+06$ & $\mathrm{y}$ & $1.39 \mathrm{E}-16$ & $1.62 \mathrm{E}-05$ & $5.14 \mathrm{E}-01$ & $1.55 \mathrm{E}-19$ & $2.06 \mathrm{E}-12$ & $1.81 \mathrm{E}-14$ \\
\hline $\mathrm{C}-10$ & 19.255 & $\mathrm{~S}$ & $7.90 \mathrm{E}-14$ & $9.22 \mathrm{E}-03$ & $2.92 \mathrm{E}+02$ & $1.71 \mathrm{E}-16$ & $2.28 \mathrm{E}-09$ & $2.00 \mathrm{E}-11$ \\
\hline C-11 & 20.39 & $\mathrm{~m}$ & $4.56 \mathrm{E}-14$ & $5.32 \mathrm{E}-03$ & $1.69 \mathrm{E}+02$ & $9.90 \mathrm{E}-17$ & $1.32 \mathrm{E}-09$ & $1.16 \mathrm{E}-11$ \\
\hline C-14 & $5.70 \mathrm{E}+03$ & $\mathrm{y}$ & $2.60 \mathrm{E}-18$ & $3.04 \mathrm{E}-07$ & $9.62 \mathrm{E}-03$ & $2.89 \mathrm{E}-21$ & $3.85 \mathrm{E}-14$ & $3.37 \mathrm{E}-16$ \\
\hline $\mathrm{N}-13$ & 9.965 & $\mathrm{~m}$ & $4.57 \mathrm{E}-14$ & $5.34 \mathrm{E}-03$ & $1.69 \mathrm{E}+02$ & $9.91 \mathrm{E}-17$ & $1.32 \mathrm{E}-09$ & $1.16 \mathrm{E}-11$ \\
\hline $\mathrm{N}-16$ & 7.13 & $\mathrm{~S}$ & $2.59 \mathrm{E}-13$ & $3.02 \mathrm{E}-02$ & $9.58 \mathrm{E}+02$ & $5.63 \mathrm{E}-16$ & $7.50 \mathrm{E}-09$ & $6.57 \mathrm{E}-11$ \\
\hline O-14 & 70.606 & $\mathrm{~S}$ & $1.63 \mathrm{E}-13$ & $1.90 \mathrm{E}-02$ & $6.03 \mathrm{E}+02$ & $3.52 \mathrm{E}-16$ & 4.69E-09 & $4.11 \mathrm{E}-11$ \\
\hline O-15 & 122.24 & $\mathrm{~S}$ & $4.60 \mathrm{E}-14$ & $5.37 \mathrm{E}-03$ & $1.70 \mathrm{E}+02$ & $9.95 \mathrm{E}-17$ & $1.33 \mathrm{E}-09$ & $1.16 \mathrm{E}-11$ \\
\hline O-19 & 26.464 & $\mathrm{~S}$ & $4.60 \mathrm{E}-14$ & $5.37 \mathrm{E}-03$ & $1.70 \mathrm{E}+02$ & $9.83 \mathrm{E}-17$ & $1.31 \mathrm{E}-09$ & $1.15 \mathrm{E}-11$ \\
\hline F-17 & 64.49 & $\mathrm{~s}$ & $4.60 \mathrm{E}-14$ & $5.37 \mathrm{E}-03$ & $1.70 \mathrm{E}+02$ & $9.95 \mathrm{E}-17$ & $1.33 \mathrm{E}-09$ & $1.16 \mathrm{E}-11$ \\
\hline F-18 & 109.77 & $\mathrm{~m}$ & $4.41 \mathrm{E}-14$ & $5.15 \mathrm{E}-03$ & $1.63 \mathrm{E}+02$ & $9.58 \mathrm{E}-17$ & $1.28 \mathrm{E}-09$ & $1.12 \mathrm{E}-11$ \\
\hline $\mathrm{Ne}-19$ & 17.22 & $\mathrm{~s}$ & $4.63 \mathrm{E}-14$ & $5.41 \mathrm{E}-03$ & $1.71 \mathrm{E}+02$ & $9.98 \mathrm{E}-17$ & $1.33 \mathrm{E}-09$ & $1.16 \mathrm{E}-11$ \\
\hline $\mathrm{Ne}-24$ & 3.38 & $\mathrm{~m}$ & $2.48 \mathrm{E}-14$ & $2.90 \mathrm{E}-03$ & $9.18 \mathrm{E}+01$ & $5.33 \mathrm{E}-17$ & $7.10 \mathrm{E}-10$ & $6.22 \mathrm{E}-12$ \\
\hline $\mathrm{Na}-22$ & 2.6019 & $\mathrm{y}$ & $1.02 \mathrm{E}-13$ & $1.19 \mathrm{E}-02$ & $3.77 \mathrm{E}+02$ & $2.20 \mathrm{E}-16$ & $2.93 \mathrm{E}-09$ & $2.57 \mathrm{E}-11$ \\
\hline $\mathrm{Na}-24$ & 14.959 & $\mathrm{~h}$ & $2.08 \mathrm{E}-13$ & $2.43 \mathrm{E}-02$ & $7.70 \mathrm{E}+02$ & $4.51 \mathrm{E}-16$ & $6.01 \mathrm{E}-09$ & $5.26 \mathrm{E}-11$ \\
\hline Mg-27 & 9.458 & $\mathrm{~m}$ & $4.16 \mathrm{E}-14$ & $4.86 \mathrm{E}-03$ & $1.54 \mathrm{E}+02$ & $8.98 \mathrm{E}-17$ & $1.20 \mathrm{E}-09$ & $1.05 \mathrm{E}-11$ \\
\hline $\mathrm{Mg}-28$ & 20.915 & $\mathrm{~h}$ & $6.38 \mathrm{E}-14$ & $7.45 \mathrm{E}-03$ & $2.36 \mathrm{E}+02$ & $1.38 \mathrm{E}-16$ & $1.84 \mathrm{E}-09$ & $1.61 \mathrm{E}-11$ \\
\hline Al-26 & $7.17 \mathrm{E}+05$ & $\mathrm{y}$ & $1.28 \mathrm{E}-13$ & $1.49 \mathrm{E}-02$ & $4.74 \mathrm{E}+02$ & $2.78 \mathrm{E}-16$ & $3.70 \mathrm{E}-09$ & $3.24 \mathrm{E}-11$ \\
\hline Al-28 & 2.2414 & $\mathrm{~m}$ & $8.88 \mathrm{E}-14$ & $1.04 \mathrm{E}-02$ & $3.29 \mathrm{E}+02$ & $1.91 \mathrm{E}-16$ & $2.54 \mathrm{E}-09$ & $2.23 \mathrm{E}-11$ \\
\hline
\end{tabular}




\begin{tabular}{|c|c|c|c|c|c|c|c|c|}
\hline Nuclide & Half-life & Class & $\begin{array}{c}\text { Air } \\
\text { Immersion } \\
\text { Dose Rate } \\
\text { Coefficient } \\
\left(\mathrm{Sv} / \mathbf{s ~ p e r ~}^{\mathbf{3}}\right. \\
\left.\mathrm{Bq} / \mathbf{m}^{\mathbf{3}}\right)\end{array}$ & $\begin{array}{c}\text { Air } \\
\text { Immersion } \\
\text { Dose Rate } \\
\text { Coefficient } \\
\text { (mrem- } \\
\left.\text { m³ }^{3} / \mathrm{pCi}-\mathrm{yr}\right)\end{array}$ & $\begin{array}{c}\text { Air } \\
\text { Immersion } \\
\text { Effective } \\
\text { Dose Rate } \\
\text { (mrem/sec } \\
\text { per } \\
\mu \mathrm{Ci} / \mathbf{c m}^{3} \text { ) }\end{array}$ & $\begin{array}{c}\text { Water } \\
\text { Submersion } \\
\text { Dose Rate } \\
\text { Coefficient } \\
\left(\mathbf{S v}-\mathbf{m}^{3} / \mathbf{B q}-\right. \\
\text { s) }\end{array}$ & $\begin{array}{c}\text { Water } \\
\text { Submersion } \\
\text { Dose Rate } \\
\text { Coefficient } \\
\text { (mrem- } \\
\left.\text { m³ }^{\mathbf{3}} / \mathbf{p C i}-\mathbf{h}\right)\end{array}$ & $\begin{array}{c}\text { Water } \\
\text { Submersion } \\
\text { Dose Rate } \\
\text { Coefficient } \\
(\text { mrem- } \\
\left.\text { m³ }^{3} / \mu \mathrm{Ci}-\mathrm{y}\right)\end{array}$ \\
\hline Al-29 & 6.56 & $\mathrm{~m}$ & $6.71 \mathrm{E}-14$ & $7.83 \mathrm{E}-03$ & $2.48 \mathrm{E}+02$ & $1.44 \mathrm{E}-16$ & $1.92 \mathrm{E}-09$ & $1.68 \mathrm{E}-11$ \\
\hline Si-31 & 157.3 & $\mathrm{~m}$ & $4.83 \mathrm{E}-16$ & $5.64 \mathrm{E}-05$ & $1.79 \mathrm{E}+00$ & $6.14 \mathrm{E}-19$ & $8.18 \mathrm{E}-12$ & $7.16 \mathrm{E}-14$ \\
\hline Si-32 & 132 & $\mathrm{y}$ & $1.05 \mathrm{E}-17$ & $1.23 \mathrm{E}-06$ & $3.89 \mathrm{E}-02$ & $1.14 \mathrm{E}-20$ & $1.52 \mathrm{E}-13$ & $1.33 \mathrm{E}-15$ \\
\hline $\mathrm{P}-30$ & 2.498 & $\mathrm{~m}$ & $4.69 \mathrm{E}-14$ & $5.48 \mathrm{E}-03$ & $1.74 \mathrm{E}+02$ & $1.01 \mathrm{E}-16$ & $1.35 \mathrm{E}-09$ & $1.18 \mathrm{E}-11$ \\
\hline $\mathrm{P}-32$ & 14.263 & $\mathrm{~d}$ & $5.36 \mathrm{E}-16$ & $6.26 \mathrm{E}-05$ & $1.98 \mathrm{E}+00$ & $6.45 \mathrm{E}-19$ & $8.59 \mathrm{E}-12$ & $7.53 \mathrm{E}-14$ \\
\hline $\mathrm{P}-33$ & 25.34 & $\mathrm{~d}$ & $1.44 \mathrm{E}-17$ & $1.68 \mathrm{E}-06$ & $5.33 \mathrm{E}-02$ & $1.57 \mathrm{E}-20$ & $2.09 \mathrm{E}-13$ & $1.83 \mathrm{E}-15$ \\
\hline $\mathrm{S}-35$ & 87.51 & $\mathrm{~d}$ & $3.07 \mathrm{E}-18$ & $3.58 \mathrm{E}-07$ & $1.14 \mathrm{E}-02$ & $3.40 \mathrm{E}-21$ & $4.53 \mathrm{E}-14$ & $3.97 \mathrm{E}-16$ \\
\hline S-37 & 5.05 & $\mathrm{~m}$ & $1.54 \mathrm{E}-13$ & $1.80 \mathrm{E}-02$ & $5.70 \mathrm{E}+02$ & $3.34 \mathrm{E}-16$ & $4.45 \mathrm{E}-09$ & $3.90 \mathrm{E}-11$ \\
\hline $\mathrm{S}-38$ & 170.3 & $\mathrm{~m}$ & $8.47 \mathrm{E}-14$ & $9.89 \mathrm{E}-03$ & $3.13 \mathrm{E}+02$ & $1.83 \mathrm{E}-16$ & $2.44 \mathrm{E}-09$ & $2.14 \mathrm{E}-11$ \\
\hline Cl-34 & 1.5264 & $\mathrm{~s}$ & $4.77 \mathrm{E}-14$ & $5.57 \mathrm{E}-03$ & $1.76 \mathrm{E}+02$ & $1.02 \mathrm{E}-16$ & $1.36 \mathrm{E}-09$ & $1.19 \mathrm{E}-11$ \\
\hline $\mathrm{Cl}-34 \mathrm{~m}$ & 32 & $\mathrm{~m}$ & $1.03 \mathrm{E}-13$ & $1.20 \mathrm{E}-02$ & $3.81 \mathrm{E}+02$ & $2.24 \mathrm{E}-16$ & $2.98 \mathrm{E}-09$ & $2.61 \mathrm{E}-11$ \\
\hline Cl-36 & $3.01 \mathrm{E}+05$ & $\mathrm{y}$ & $1.66 \mathrm{E}-16$ & $1.94 \mathrm{E}-05$ & $6.14 \mathrm{E}-01$ & $1.94 \mathrm{E}-19$ & $2.58 \mathrm{E}-12$ & $2.26 \mathrm{E}-14$ \\
\hline Cl-38 & 37.24 & $\mathrm{~m}$ & $7.36 \mathrm{E}-14$ & $8.59 \mathrm{E}-03$ & $2.72 \mathrm{E}+02$ & $1.58 \mathrm{E}-16$ & $2.10 \mathrm{E}-09$ & $1.84 \mathrm{E}-11$ \\
\hline Cl-39 & 55.6 & $\mathrm{~m}$ & $6.97 \mathrm{E}-14$ & $8.14 \mathrm{E}-03$ & $2.58 \mathrm{E}+02$ & $1.50 \mathrm{E}-16$ & $2.00 \mathrm{E}-09$ & $1.75 \mathrm{E}-11$ \\
\hline Cl-40 & 1.35 & $\mathrm{~m}$ & $2.09 \mathrm{E}-13$ & $2.44 \mathrm{E}-02$ & $7.73 \mathrm{E}+02$ & $4.53 \mathrm{E}-16$ & $6.03 \mathrm{E}-09$ & $5.29 \mathrm{E}-11$ \\
\hline Ar-37 & 35.04 & $\mathrm{~d}$ & $6.12 \mathrm{E}-19$ & $7.15 \mathrm{E}-08$ & $2.26 \mathrm{E}-03$ & $0.00 \mathrm{E}+00$ & $0.00 \mathrm{E}+00$ & $0.00 \mathrm{E}+00$ \\
\hline Ar-39 & 269 & $\mathrm{y}$ & $1.15 \mathrm{E}-16$ & $1.34 \mathrm{E}-05$ & $4.26 \mathrm{E}-01$ & $1.28 \mathrm{E}-19$ & $1.70 \mathrm{E}-12$ & $1.49 \mathrm{E}-14$ \\
\hline Ar-41 & 109.61 & $\mathrm{~m}$ & $6.15 \mathrm{E}-14$ & $7.18 \mathrm{E}-03$ & $2.28 \mathrm{E}+02$ & $1.33 \mathrm{E}-16$ & $1.77 \mathrm{E}-09$ & $1.55 \mathrm{E}-11$ \\
\hline Ar-42 & 32.9 & $\mathrm{y}$ & $1.26 \mathrm{E}-16$ & $1.47 \mathrm{E}-05$ & $4.66 \mathrm{E}-01$ & $1.41 \mathrm{E}-19$ & $1.88 \mathrm{E}-12$ & $1.65 \mathrm{E}-14$ \\
\hline Ar-43 & 5.37 & $\mathrm{~m}$ & $7.55 \mathrm{E}-14$ & $8.82 \mathrm{E}-03$ & $2.79 \mathrm{E}+02$ & $1.62 \mathrm{E}-16$ & $2.16 \mathrm{E}-09$ & $1.89 \mathrm{E}-11$ \\
\hline Ar-44 & 11.87 & $\mathrm{~m}$ & $9.46 \mathrm{E}-14$ & $1.10 \mathrm{E}-02$ & $3.50 \mathrm{E}+02$ & $2.04 \mathrm{E}-16$ & $2.72 \mathrm{E}-09$ & $2.38 \mathrm{E}-11$ \\
\hline K-38 & 7.636 & $\mathrm{~m}$ & $1.56 \mathrm{E}-13$ & $1.82 \mathrm{E}-02$ & $5.77 \mathrm{E}+02$ & $3.36 \mathrm{E}-16$ & $4.48 \mathrm{E}-09$ & $3.92 \mathrm{E}-11$ \\
\hline $\mathrm{K}-40$ & $1.25 \mathrm{E}+09$ & $\mathrm{y}$ & $7.94 \mathrm{E}-15$ & $9.27 \mathrm{E}-04$ & $2.94 \mathrm{E}+01$ & $1.68 \mathrm{E}-17$ & $2.24 \mathrm{E}-10$ & $1.96 \mathrm{E}-12$ \\
\hline
\end{tabular}




\begin{tabular}{|c|c|c|c|c|c|c|c|c|}
\hline Nuclide & Half-life & Class & $\begin{array}{c}\text { Air } \\
\text { Immersion } \\
\text { Dose Rate } \\
\text { Coefficient } \\
\left(\mathrm{Sv} / \mathbf{s ~ p e r ~}^{\mathbf{3}}\right. \\
\left.\mathrm{Bq} / \mathbf{m}^{\mathbf{3}}\right)\end{array}$ & $\begin{array}{c}\text { Air } \\
\text { Immersion } \\
\text { Dose Rate } \\
\text { Coefficient } \\
\text { (mrem- } \\
\left.\text { m³ }^{3} / \mathrm{pCi}-\mathrm{yr}\right)\end{array}$ & $\begin{array}{c}\text { Air } \\
\text { Immersion } \\
\text { Effective } \\
\text { Dose Rate } \\
\text { (mrem/sec } \\
\text { per } \\
\mu \mathrm{Ci} / \mathbf{c m}^{3} \text { ) }\end{array}$ & $\begin{array}{c}\text { Water } \\
\text { Submersion } \\
\text { Dose Rate } \\
\text { Coefficient } \\
\left(\mathbf{S v}-\mathbf{m}^{3} / \mathbf{B q}-\right. \\
\text { s) }\end{array}$ & $\begin{array}{c}\text { Water } \\
\text { Submersion } \\
\text { Dose Rate } \\
\text { Coefficient } \\
\text { (mrem- } \\
\left.\text { m³ }^{\mathbf{3}} / \mathbf{p C i}-\mathbf{h}\right)\end{array}$ & $\begin{array}{c}\text { Water } \\
\text { Submersion } \\
\text { Dose Rate } \\
\text { Coefficient } \\
(\text { mrem- } \\
\left.\mathbf{m}^{3} / \mu \mathrm{Ci}-\mathrm{y}\right)\end{array}$ \\
\hline $\mathrm{K}-42$ & 12.36 & $\mathrm{~h}$ & $1.49 \mathrm{E}-14$ & $1.74 \mathrm{E}-03$ & $5.51 \mathrm{E}+01$ & $3.11 \mathrm{E}-17$ & $4.14 \mathrm{E}-10$ & $3.63 \mathrm{E}-12$ \\
\hline $\mathrm{K}-43$ & 22.3 & $\mathrm{~h}$ & $4.33 \mathrm{E}-14$ & $5.06 \mathrm{E}-03$ & $1.60 \mathrm{E}+02$ & $9.39 \mathrm{E}-17$ & $1.25 \mathrm{E}-09$ & $1.10 \mathrm{E}-11$ \\
\hline K-44 & 22.13 & $\mathrm{~m}$ & $1.19 \mathrm{E}-13$ & $1.39 \mathrm{E}-02$ & $4.40 \mathrm{E}+02$ & $2.57 \mathrm{E}-16$ & $3.42 \mathrm{E}-09$ & $3.00 \mathrm{E}-11$ \\
\hline $\mathrm{K}-45$ & 17.3 & $\mathrm{~m}$ & $9.05 \mathrm{E}-14$ & $1.06 \mathrm{E}-02$ & $3.35 \mathrm{E}+02$ & $1.95 \mathrm{E}-16$ & $2.60 \mathrm{E}-09$ & $2.28 \mathrm{E}-11$ \\
\hline K-46 & 105 & $\mathrm{~s}$ & $1.47 \mathrm{E}-13$ & $1.72 \mathrm{E}-02$ & $5.44 \mathrm{E}+02$ & $3.17 \mathrm{E}-16$ & $4.22 \mathrm{E}-09$ & $3.70 \mathrm{E}-11$ \\
\hline $\mathrm{Ca}-41$ & $1.02 \mathrm{E}+05$ & $\mathrm{y}$ & $0.00 \mathrm{E}+00$ & $0.00 \mathrm{E}+00$ & $0.00 \mathrm{E}+00$ & $0.00 \mathrm{E}+00$ & $0.00 \mathrm{E}+00$ & $0.00 \mathrm{E}+00$ \\
\hline $\mathrm{Ca}-45$ & 162.67 & $\mathrm{~d}$ & $1.52 \mathrm{E}-17$ & $1.77 \mathrm{E}-06$ & $5.62 \mathrm{E}-02$ & $1.66 \mathrm{E}-20$ & $2.21 \mathrm{E}-13$ & $1.94 \mathrm{E}-15$ \\
\hline $\mathrm{Ca}-47$ & 4.536 & $\mathrm{~d}$ & $5.02 \mathrm{E}-14$ & $5.86 \mathrm{E}-03$ & $1.86 \mathrm{E}+02$ & $1.08 \mathrm{E}-16$ & $1.44 \mathrm{E}-09$ & $1.26 \mathrm{E}-11$ \\
\hline $\mathrm{Ca}-49$ & 8.718 & $\mathrm{~m}$ & $1.67 \mathrm{E}-13$ & $1.95 \mathrm{E}-02$ & $6.18 \mathrm{E}+02$ & $3.61 \mathrm{E}-16$ & $4.81 \mathrm{E}-09$ & $4.21 \mathrm{E}-11$ \\
\hline Sc- $42 \mathrm{~m}$ & 62 & $\mathrm{~s}$ & $1.98 \mathrm{E}-13$ & $2.31 \mathrm{E}-02$ & $7.33 \mathrm{E}+02$ & $4.28 \mathrm{E}-16$ & $5.70 \mathrm{E}-09$ & $4.99 \mathrm{E}-11$ \\
\hline Sc-43 & 3.891 & $\mathrm{~h}$ & $4.41 \mathrm{E}-14$ & $5.15 \mathrm{E}-03$ & $1.63 \mathrm{E}+02$ & $9.55 \mathrm{E}-17$ & $1.27 \mathrm{E}-09$ & $1.11 \mathrm{E}-11$ \\
\hline Sc-44 & 3.97 & $\mathrm{~h}$ & $9.87 \mathrm{E}-14$ & $1.15 \mathrm{E}-02$ & $3.65 \mathrm{E}+02$ & $2.14 \mathrm{E}-16$ & $2.85 \mathrm{E}-09$ & $2.50 \mathrm{E}-11$ \\
\hline Sc- $44 m$ & 58.61 & $\mathrm{~h}$ & $1.22 \mathrm{E}-14$ & $1.42 \mathrm{E}-03$ & $4.51 \mathrm{E}+01$ & $2.66 \mathrm{E}-17$ & $3.54 \mathrm{E}-10$ & $3.10 \mathrm{E}-12$ \\
\hline Sc-46 & 83.79 & $\mathrm{~d}$ & $9.37 \mathrm{E}-14$ & $1.09 \mathrm{E}-02$ & $3.47 \mathrm{E}+02$ & $2.03 \mathrm{E}-16$ & $2.70 \mathrm{E}-09$ & $2.37 \mathrm{E}-11$ \\
\hline Sc-47 & 3.3492 & $\mathrm{~d}$ & $4.70 \mathrm{E}-15$ & $5.49 \mathrm{E}-04$ & $1.74 \mathrm{E}+01$ & $1.03 \mathrm{E}-17$ & $1.37 \mathrm{E}-10$ & $1.20 \mathrm{E}-12$ \\
\hline Sc-48 & 43.67 & $\mathrm{~h}$ & $1.58 \mathrm{E}-13$ & $1.84 \mathrm{E}-02$ & $5.85 \mathrm{E}+02$ & $3.42 \mathrm{E}-16$ & $4.56 \mathrm{E}-09$ & $3.99 \mathrm{E}-11$ \\
\hline Sc-49 & 57.2 & $\mathrm{~m}$ & $7.13 \mathrm{E}-16$ & $8.33 \mathrm{E}-05$ & $2.64 \mathrm{E}+00$ & $9.20 \mathrm{E}-19$ & $1.23 \mathrm{E}-11$ & $1.07 \mathrm{E}-13$ \\
\hline Sc-50 & 102.5 & $\mathrm{~s}$ & $1.54 \mathrm{E}-13$ & $1.80 \mathrm{E}-02$ & $5.70 \mathrm{E}+02$ & $3.32 \mathrm{E}-16$ & $4.42 \mathrm{E}-09$ & $3.87 \mathrm{E}-11$ \\
\hline Ti-44 & 60 & $\mathrm{y}$ & $4.88 \mathrm{E}-15$ & $5.70 \mathrm{E}-04$ & $1.81 \mathrm{E}+01$ & $1.10 \mathrm{E}-17$ & $1.47 \mathrm{E}-10$ & $1.28 \mathrm{E}-12$ \\
\hline Ti-45 & 184.8 & $\mathrm{~m}$ & $3.90 \mathrm{E}-14$ & $4.55 \mathrm{E}-03$ & $1.44 \mathrm{E}+02$ & $8.45 \mathrm{E}-17$ & $1.13 \mathrm{E}-09$ & $9.86 \mathrm{E}-12$ \\
\hline Ti-51 & 5.76 & $\mathrm{~m}$ & $1.71 \mathrm{E}-14$ & $2.00 \mathrm{E}-03$ & $6.33 \mathrm{E}+01$ & $3.66 \mathrm{E}-17$ & $4.88 \mathrm{E}-10$ & $4.27 \mathrm{E}-12$ \\
\hline Ti-52 & 1.7 & $\mathrm{~m}$ & $5.75 \mathrm{E}-15$ & $6.71 \mathrm{E}-04$ & $2.13 \mathrm{E}+01$ & $1.22 \mathrm{E}-17$ & $1.63 \mathrm{E}-10$ & $1.42 \mathrm{E}-12$ \\
\hline $\mathrm{V}-47$ & 32.6 & $\mathrm{~m}$ & $4.50 \mathrm{E}-14$ & $5.25 \mathrm{E}-03$ & $1.67 \mathrm{E}+02$ & $9.72 \mathrm{E}-17$ & $1.29 \mathrm{E}-09$ & $1.13 \mathrm{E}-11$ \\
\hline
\end{tabular}




\begin{tabular}{|c|c|c|c|c|c|c|c|c|}
\hline Nuclide & Half-life & Class & $\begin{array}{c}\text { Air } \\
\text { Immersion } \\
\text { Dose Rate } \\
\text { Coefficient } \\
\text { (Sv/s per } \\
\left.\text { Bq/m } \mathbf{m}^{3}\right)\end{array}$ & $\begin{array}{c}\text { Air } \\
\text { Immersion } \\
\text { Dose Rate } \\
\text { Coefficient } \\
\text { (mrem- } \\
\left.\mathrm{m}^{3} / \mathrm{pCi}-\mathrm{yr}\right)\end{array}$ & $\begin{array}{c}\text { Air } \\
\text { Immersion } \\
\text { Effective } \\
\text { Dose Rate } \\
(\text { mrem } / \mathrm{sec} \\
\text { per } \\
\left.\mu \mathrm{Ci} / \mathrm{cm}^{3}\right)\end{array}$ & $\begin{array}{c}\text { Water } \\
\text { Submersion } \\
\text { Dose Rate } \\
\text { Coefficient } \\
\left(\mathrm{Sv}-\mathrm{m}^{3} / \mathrm{Bq}-\right. \\
\text { s) }\end{array}$ & $\begin{array}{c}\text { Water } \\
\text { Submersion } \\
\text { Dose Rate } \\
\text { Coefficient } \\
\text { (mrem- } \\
\text { m³Ci-h) }^{\mathbf{3} / p C i}\end{array}$ & $\begin{array}{c}\text { Water } \\
\text { Submersion } \\
\text { Dose Rate } \\
\text { Coefficient } \\
\text { (mrem- } \\
\left.\text { m³ }^{3} / \mu \mathrm{Ci}-\mathrm{y}\right)\end{array}$ \\
\hline $\mathrm{V}-48$ & 15.9735 & $\mathrm{~d}$ & $1.36 \mathrm{E}-13$ & $1.59 \mathrm{E}-02$ & $5.03 \mathrm{E}+02$ & $2.95 \mathrm{E}-16$ & $3.93 \mathrm{E}-09$ & $3.44 \mathrm{E}-11$ \\
\hline $\mathrm{V}-49$ & 330 & $\mathrm{~d}$ & $0.00 \mathrm{E}+00$ & $0.00 \mathrm{E}+00$ & $0.00 \mathrm{E}+00$ & $0.00 \mathrm{E}+00$ & $0.00 \mathrm{E}+00$ & $0.00 \mathrm{E}+00$ \\
\hline $\mathrm{V}-50$ & $1.50 \mathrm{E}+17$ & $\mathrm{y}$ & $6.87 \mathrm{E}-14$ & $8.02 \mathrm{E}-03$ & $2.54 \mathrm{E}+02$ & $1.49 \mathrm{E}-16$ & $1.98 \mathrm{E}-09$ & $1.74 \mathrm{E}-11$ \\
\hline $\mathrm{V}-52$ & 3.743 & $\mathrm{~m}$ & $7.05 \mathrm{E}-14$ & $8.23 \mathrm{E}-03$ & $2.61 \mathrm{E}+02$ & $1.52 \mathrm{E}-16$ & $2.02 \mathrm{E}-09$ & $1.77 \mathrm{E}-11$ \\
\hline $\mathrm{V}-53$ & 1.61 & $\mathrm{~m}$ & $4.93 \mathrm{E}-14$ & $5.76 \mathrm{E}-03$ & $1.82 \mathrm{E}+02$ & $1.06 \mathrm{E}-16$ & $1.41 \mathrm{E}-09$ & $1.24 \mathrm{E}-11$ \\
\hline Cr-48 & 21.56 & $\mathrm{~h}$ & $1.88 \mathrm{E}-14$ & $2.20 \mathrm{E}-03$ & $6.96 \mathrm{E}+01$ & $4.12 \mathrm{E}-17$ & $5.49 \mathrm{E}-10$ & $4.81 \mathrm{E}-12$ \\
\hline Cr-49 & 42.3 & $\mathrm{~m}$ & $4.68 \mathrm{E}-14$ & $5.46 \mathrm{E}-03$ & $1.73 \mathrm{E}+02$ & $1.02 \mathrm{E}-16$ & $1.36 \mathrm{E}-09$ & $1.19 \mathrm{E}-11$ \\
\hline $\mathrm{Cr}-51$ & 27.7025 & $\mathrm{~d}$ & $1.40 \mathrm{E}-15$ & $1.63 \mathrm{E}-04$ & $5.18 \mathrm{E}+00$ & $3.05 \mathrm{E}-18$ & $4.06 \mathrm{E}-11$ & $3.56 \mathrm{E}-13$ \\
\hline Cr-55 & 3.497 & $\mathrm{~m}$ & $1.00 \mathrm{E}-15$ & $1.17 \mathrm{E}-04$ & $3.70 \mathrm{E}+00$ & $1.30 \mathrm{E}-18$ & $1.73 \mathrm{E}-11$ & $1.52 \mathrm{E}-13$ \\
\hline Cr-56 & 5.94 & $\mathrm{~m}$ & $3.47 \mathrm{E}-15$ & $4.05 \mathrm{E}-04$ & $1.28 \mathrm{E}+01$ & $7.36 \mathrm{E}-18$ & $9.80 \mathrm{E}-11$ & $8.59 \mathrm{E}-13$ \\
\hline $\mathrm{Mn}-50 \mathrm{~m}$ & 1.75 & $\mathrm{~m}$ & $2.18 \mathrm{E}-13$ & $2.55 \mathrm{E}-02$ & $8.07 \mathrm{E}+02$ & $4.71 \mathrm{E}-16$ & $6.27 \mathrm{E}-09$ & $5.50 \mathrm{E}-11$ \\
\hline $\mathrm{Mn}-51$ & 46.2 & $\mathrm{~m}$ & $4.52 \mathrm{E}-14$ & $5.28 \mathrm{E}-03$ & $1.67 \mathrm{E}+02$ & $9.76 \mathrm{E}-17$ & $1.30 \mathrm{E}-09$ & $1.14 \mathrm{E}-11$ \\
\hline $\mathrm{Mn}-52 \mathrm{~m}$ & 21.1 & $\mathrm{~m}$ & $1.13 \mathrm{E}-13$ & $1.32 \mathrm{E}-02$ & $4.18 \mathrm{E}+02$ & $2.45 \mathrm{E}-16$ & $3.26 \mathrm{E}-09$ & $2.86 \mathrm{E}-11$ \\
\hline $\mathrm{Mn}-52$ & 5.591 & $\mathrm{~d}$ & $1.62 \mathrm{E}-13$ & $1.89 \mathrm{E}-02$ & $5.99 \mathrm{E}+02$ & $3.51 \mathrm{E}-16$ & $4.68 \mathrm{E}-09$ & $4.10 \mathrm{E}-11$ \\
\hline $\mathrm{Mn}-53$ & $3.70 \mathrm{E}+06$ & $\mathrm{y}$ & $0.00 \mathrm{E}+00$ & $0.00 \mathrm{E}+00$ & $0.00 \mathrm{E}+00$ & $0.00 \mathrm{E}+00$ & $0.00 \mathrm{E}+00$ & $0.00 \mathrm{E}+00$ \\
\hline $\mathrm{Mn}-54$ & 312.12 & $\mathrm{~d}$ & $3.83 \mathrm{E}-14$ & $4.47 \mathrm{E}-03$ & $1.42 \mathrm{E}+02$ & $8.30 \mathrm{E}-17$ & $1.11 \mathrm{E}-09$ & $9.68 \mathrm{E}-12$ \\
\hline Mn-56 & 2.5789 & $\mathrm{~h}$ & $8.17 \mathrm{E}-14$ & $9.54 \mathrm{E}-03$ & $3.02 \mathrm{E}+02$ & $1.76 \mathrm{E}-16$ & $2.34 \mathrm{E}-09$ & $2.05 \mathrm{E}-11$ \\
\hline $\mathrm{Mn}-57$ & 85.4 & $\mathrm{~s}$ & $5.30 \mathrm{E}-15$ & $6.19 \mathrm{E}-04$ & $1.96 \mathrm{E}+01$ & $1.07 \mathrm{E}-17$ & $1.43 \mathrm{E}-10$ & $1.25 \mathrm{E}-12$ \\
\hline $\mathrm{Mn}-58 \mathrm{~m}$ & 65.2 & $\mathrm{~s}$ & $1.15 \mathrm{E}-13$ & $1.34 \mathrm{E}-02$ & $4.26 \mathrm{E}+02$ & $2.47 \mathrm{E}-16$ & $3.29 \mathrm{E}-09$ & $2.88 \mathrm{E}-11$ \\
\hline $\mathrm{Fe}-52$ & 8.275 & $\mathrm{~h}$ & $3.28 \mathrm{E}-14$ & $3.83 \mathrm{E}-03$ & $1.21 \mathrm{E}+02$ & $7.13 \mathrm{E}-17$ & $9.50 \mathrm{E}-10$ & $8.32 \mathrm{E}-12$ \\
\hline $\mathrm{Fe}-53$ & 8.51 & $\mathrm{~m}$ & $5.35 \mathrm{E}-14$ & $6.25 \mathrm{E}-03$ & $1.98 \mathrm{E}+02$ & $1.15 \mathrm{E}-16$ & $1.53 \mathrm{E}-09$ & $1.34 \mathrm{E}-11$ \\
\hline Fe-53m & 2.526 & $\mathrm{~m}$ & $1.44 \mathrm{E}-13$ & $1.68 \mathrm{E}-02$ & $5.33 \mathrm{E}+02$ & $3.12 \mathrm{E}-16$ & $4.16 \mathrm{E}-09$ & $3.64 \mathrm{E}-11$ \\
\hline Fe-55 & 2.737 & $\mathrm{y}$ & $6.69 \mathrm{E}-24$ & $7.81 \mathrm{E}-13$ & $2.48 \mathrm{E}-08$ & $1.49 \mathrm{E}-26$ & $1.98 \mathrm{E}-19$ & $1.74 \mathrm{E}-21$ \\
\hline
\end{tabular}




\begin{tabular}{|c|c|c|c|c|c|c|c|c|}
\hline Nuclide & Half-life & Class & $\begin{array}{c}\text { Air } \\
\text { Immersion } \\
\text { Dose Rate } \\
\text { Coefficient } \\
\left(\mathrm{Sv} / \mathbf{s ~ p e r ~}^{\mathbf{3}}\right. \\
\left.\mathrm{Bq} / \mathbf{m}^{\mathbf{3}}\right)\end{array}$ & $\begin{array}{c}\text { Air } \\
\text { Immersion } \\
\text { Dose Rate } \\
\text { Coefficient } \\
\text { (mrem- } \\
\left.\mathrm{m}^{3} / \mathrm{pCi}-\mathrm{yr}\right)\end{array}$ & $\begin{array}{c}\text { Air } \\
\text { Immersion } \\
\text { Effective } \\
\text { Dose Rate } \\
\text { (mrem/sec } \\
\text { per } \\
\mu \mathrm{Ci} / \mathbf{c m}^{3} \text { ) }\end{array}$ & $\begin{array}{c}\text { Water } \\
\text { Submersion } \\
\text { Dose Rate } \\
\text { Coefficient } \\
\left(\mathbf{S v}-\mathbf{m}^{3} / \mathbf{B q}-\right. \\
\text { s) }\end{array}$ & $\begin{array}{c}\text { Water } \\
\text { Submersion } \\
\text { Dose Rate } \\
\text { Coefficient } \\
\text { (mrem- } \\
\left.\text { m³ }^{\mathbf{3}} / \mathbf{p C i}-\mathbf{h}\right)\end{array}$ & $\begin{array}{c}\text { Water } \\
\text { Submersion } \\
\text { Dose Rate } \\
\text { Coefficient } \\
(\text { mrem- } \\
\left.\mathbf{m}^{3} / \mu \mathrm{Ci}-\mathrm{y}\right)\end{array}$ \\
\hline Fe-59 & 44.495 & $\mathrm{~d}$ & $5.62 \mathrm{E}-14$ & $6.56 \mathrm{E}-03$ & $2.08 \mathrm{E}+02$ & $1.22 \mathrm{E}-16$ & $1.63 \mathrm{E}-09$ & $1.42 \mathrm{E}-11$ \\
\hline $\mathrm{Fe}-60$ & $1.50 \mathrm{E}+06$ & $\mathrm{y}$ & $6.93 \mathrm{E}-18$ & $8.09 \mathrm{E}-07$ & $2.56 \mathrm{E}-02$ & $7.59 \mathrm{E}-21$ & $1.01 \mathrm{E}-13$ & $8.86 \mathrm{E}-16$ \\
\hline $\mathrm{Fe}-61$ & 5.98 & $\mathrm{~m}$ & $6.68 \mathrm{E}-14$ & $7.80 \mathrm{E}-03$ & $2.47 \mathrm{E}+02$ & $1.44 \mathrm{E}-16$ & $1.92 \mathrm{E}-09$ & $1.68 \mathrm{E}-11$ \\
\hline $\mathrm{Fe}-62$ & 68 & $\mathrm{~s}$ & $2.32 \mathrm{E}-14$ & $2.71 \mathrm{E}-03$ & $8.58 \mathrm{E}+01$ & $4.98 \mathrm{E}-17$ & $6.63 \mathrm{E}-10$ & $5.81 \mathrm{E}-12$ \\
\hline Co-54m & 1.48 & $\mathrm{~m}$ & $1.85 \mathrm{E}-13$ & $2.16 \mathrm{E}-02$ & $6.85 \mathrm{E}+02$ & $3.99 \mathrm{E}-16$ & $5.31 \mathrm{E}-09$ & $4.66 \mathrm{E}-11$ \\
\hline Co-55 & 17.53 & $\mathrm{~h}$ & $9.18 \mathrm{E}-14$ & $1.07 \mathrm{E}-02$ & $3.40 \mathrm{E}+02$ & $1.99 \mathrm{E}-16$ & $2.65 \mathrm{E}-09$ & $2.32 \mathrm{E}-11$ \\
\hline Co-56 & 77.23 & $\mathrm{~d}$ & $1.76 \mathrm{E}-13$ & $2.06 \mathrm{E}-02$ & $6.51 \mathrm{E}+02$ & $3.82 \mathrm{E}-16$ & $5.09 \mathrm{E}-09$ & $4.46 \mathrm{E}-11$ \\
\hline Co-57 & 271.74 & $\mathrm{~d}$ & $4.98 \mathrm{E}-15$ & $5.81 \mathrm{E}-04$ & $1.84 \mathrm{E}+01$ & $1.11 \mathrm{E}-17$ & $1.48 \mathrm{E}-10$ & $1.30 \mathrm{E}-12$ \\
\hline Co-58 & 70.86 & $\mathrm{~d}$ & $4.44 \mathrm{E}-14$ & $5.18 \mathrm{E}-03$ & $1.64 \mathrm{E}+02$ & $9.63 \mathrm{E}-17$ & $1.28 \mathrm{E}-09$ & $1.12 \mathrm{E}-11$ \\
\hline Co-58m & 9.04 & $\mathrm{~h}$ & $6.09 \mathrm{E}-20$ & $7.11 \mathrm{E}-09$ & $2.25 \mathrm{E}-04$ & $1.43 \mathrm{E}-22$ & $1.90 \mathrm{E}-15$ & $1.67 \mathrm{E}-17$ \\
\hline Co-60 & 5.2713 & $\mathrm{y}$ & $1.19 \mathrm{E}-13$ & $1.39 \mathrm{E}-02$ & $4.40 \mathrm{E}+02$ & $2.58 \mathrm{E}-16$ & $3.44 \mathrm{E}-09$ & $3.01 \mathrm{E}-11$ \\
\hline Co-60m & 10.467 & $\mathrm{~m}$ & $1.94 \mathrm{E}-16$ & $2.27 \mathrm{E}-05$ & $7.18 \mathrm{E}-01$ & $4.23 \mathrm{E}-19$ & $5.63 \mathrm{E}-12$ & $4.94 \mathrm{E}-14$ \\
\hline Co-61 & 1.65 & $\mathrm{~h}$ & $4.06 \mathrm{E}-15$ & $4.74 \mathrm{E}-04$ & $1.50 \mathrm{E}+01$ & $8.67 \mathrm{E}-18$ & $1.15 \mathrm{E}-10$ & $1.01 \mathrm{E}-12$ \\
\hline Co-62 & 1.5 & $\mathrm{~m}$ & $7.92 \mathrm{E}-14$ & $9.25 \mathrm{E}-03$ & $2.93 \mathrm{E}+02$ & $1.70 \mathrm{E}-16$ & $2.26 \mathrm{E}-09$ & $1.98 \mathrm{E}-11$ \\
\hline Co-62m & 13.91 & $\mathrm{~m}$ & $1.30 \mathrm{E}-13$ & $1.52 \mathrm{E}-02$ & $4.81 \mathrm{E}+02$ & $2.81 \mathrm{E}-16$ & $3.74 \mathrm{E}-09$ & $3.28 \mathrm{E}-11$ \\
\hline $\mathrm{Ni}-56$ & 6.075 & $\mathrm{~d}$ & $7.82 \mathrm{E}-14$ & $9.13 \mathrm{E}-03$ & $2.89 \mathrm{E}+02$ & $1.70 \mathrm{E}-16$ & $2.26 \mathrm{E}-09$ & $1.98 \mathrm{E}-11$ \\
\hline $\mathrm{Ni}-57$ & 35.6 & $\mathrm{~h}$ & $9.19 \mathrm{E}-14$ & $1.07 \mathrm{E}-02$ & $3.40 \mathrm{E}+02$ & $1.99 \mathrm{E}-16$ & $2.65 \mathrm{E}-09$ & $2.32 \mathrm{E}-11$ \\
\hline Ni-59 & $1.01 \mathrm{E}+05$ & $\mathrm{y}$ & $6.92 \mathrm{E}-19$ & $8.08 \mathrm{E}-08$ & $2.56 \mathrm{E}-03$ & $1.50 \mathrm{E}-21$ & $2.00 \mathrm{E}-14$ & $1.75 \mathrm{E}-16$ \\
\hline Ni-63 & 100.1 & $\mathrm{y}$ & $0.00 \mathrm{E}+00$ & $0.00 \mathrm{E}+00$ & $0.00 \mathrm{E}+00$ & $0.00 \mathrm{E}+00$ & $0.00 \mathrm{E}+00$ & $0.00 \mathrm{E}+00$ \\
\hline $\mathrm{Ni}-65$ & 2.51719 & $\mathrm{~h}$ & $2.72 \mathrm{E}-14$ & $3.18 \mathrm{E}-03$ & $1.01 \mathrm{E}+02$ & $5.83 \mathrm{E}-17$ & $7.77 \mathrm{E}-10$ & $6.80 \mathrm{E}-12$ \\
\hline Ni-66 & 54.6 & $\mathrm{~h}$ & $1.36 \mathrm{E}-17$ & $1.59 \mathrm{E}-06$ & $5.03 \mathrm{E}-02$ & $1.48 \mathrm{E}-20$ & $1.97 \mathrm{E}-13$ & $1.73 \mathrm{E}-15$ \\
\hline $\mathrm{Cu}-57$ & 0.1963 & $\mathrm{~S}$ & $5.65 \mathrm{E}-14$ & $6.60 \mathrm{E}-03$ & $2.09 \mathrm{E}+02$ & $1.19 \mathrm{E}-16$ & $1.59 \mathrm{E}-09$ & $1.39 \mathrm{E}-11$ \\
\hline $\mathrm{Cu}-59$ & 81.5 & $\mathrm{~s}$ & $6.68 \mathrm{E}-14$ & $7.80 \mathrm{E}-03$ & $2.47 \mathrm{E}+02$ & $1.44 \mathrm{E}-16$ & $1.92 \mathrm{E}-09$ & $1.68 \mathrm{E}-11$ \\
\hline
\end{tabular}




\begin{tabular}{|c|c|c|c|c|c|c|c|c|}
\hline Nuclide & Half-life & Class & $\begin{array}{c}\text { Air } \\
\text { Immersion } \\
\text { Dose Rate } \\
\text { Coefficient } \\
\left(\mathrm{Sv} / \mathbf{s ~ p e r ~}^{\mathbf{3}}\right. \\
\left.\mathrm{Bq} / \mathbf{m}^{\mathbf{3}}\right)\end{array}$ & $\begin{array}{c}\text { Air } \\
\text { Immersion } \\
\text { Dose Rate } \\
\text { Coefficient } \\
\text { (mrem- } \\
\left.\text { m³ }^{3} / \mathrm{pCi}-\mathrm{yr}\right)\end{array}$ & $\begin{array}{c}\text { Air } \\
\text { Immersion } \\
\text { Effective } \\
\text { Dose Rate } \\
\text { (mrem/sec } \\
\text { per } \\
\mu \mathrm{Ci} / \mathbf{c m}^{3} \text { ) }\end{array}$ & $\begin{array}{c}\text { Water } \\
\text { Submersion } \\
\text { Dose Rate } \\
\text { Coefficient } \\
\left(\mathbf{S v}-\mathbf{m}^{3} / \mathbf{B q}-\right. \\
\text { s) }\end{array}$ & $\begin{array}{c}\text { Water } \\
\text { Submersion } \\
\text { Dose Rate } \\
\text { Coefficient } \\
\text { (mrem- } \\
\left.\text { m³ }^{\mathbf{3}} / \mathbf{p C i}-\mathbf{h}\right)\end{array}$ & $\begin{array}{c}\text { Water } \\
\text { Submersion } \\
\text { Dose Rate } \\
\text { Coefficient } \\
(\text { mrem- } \\
\left.\mathbf{m}^{\mathbf{3}} / \boldsymbol{\mu C i}-\mathrm{y}\right)\end{array}$ \\
\hline $\mathrm{Cu}-60$ & 23.7 & $\mathrm{~m}$ & $1.88 \mathrm{E}-13$ & $2.20 \mathrm{E}-02$ & $6.96 \mathrm{E}+02$ & $4.06 \mathrm{E}-16$ & $5.41 \mathrm{E}-09$ & $4.74 \mathrm{E}-11$ \\
\hline $\mathrm{Cu}-61$ & 3.333 & $\mathrm{~h}$ & $3.70 \mathrm{E}-14$ & $4.32 \mathrm{E}-03$ & $1.37 \mathrm{E}+02$ & $8.02 \mathrm{E}-17$ & $1.07 \mathrm{E}-09$ & $9.36 \mathrm{E}-12$ \\
\hline $\mathrm{Cu}-62$ & 9.673 & $\mathrm{~m}$ & $4.60 \mathrm{E}-14$ & $5.37 \mathrm{E}-03$ & $1.70 \mathrm{E}+02$ & $9.90 \mathrm{E}-17$ & $1.32 \mathrm{E}-09$ & $1.16 \mathrm{E}-11$ \\
\hline $\mathrm{Cu}-64$ & 12.7 & $\mathrm{~h}$ & $8.29 \mathrm{E}-15$ & $9.68 \mathrm{E}-04$ & $3.07 \mathrm{E}+01$ & $1.80 \mathrm{E}-17$ & $2.40 \mathrm{E}-10$ & $2.10 \mathrm{E}-12$ \\
\hline $\mathrm{Cu}-66$ & 5.12 & $\mathrm{~m}$ & $5.50 \mathrm{E}-15$ & $6.42 \mathrm{E}-04$ & $2.04 \mathrm{E}+01$ & $1.11 \mathrm{E}-17$ & $1.48 \mathrm{E}-10$ & $1.30 \mathrm{E}-12$ \\
\hline $\mathrm{Cu}-67$ & 61.83 & $\mathrm{~h}$ & $4.91 \mathrm{E}-15$ & $5.73 \mathrm{E}-04$ & $1.82 \mathrm{E}+01$ & $1.08 \mathrm{E}-17$ & $1.44 \mathrm{E}-10$ & $1.26 \mathrm{E}-12$ \\
\hline $\mathrm{Cu}-69$ & 2.85 & $\mathrm{~m}$ & $2.53 \mathrm{E}-14$ & $2.95 \mathrm{E}-03$ & $9.36 \mathrm{E}+01$ & $5.41 \mathrm{E}-17$ & $7.21 \mathrm{E}-10$ & $6.31 \mathrm{E}-12$ \\
\hline $\mathrm{Zn}-60$ & 2.38 & $\mathrm{~m}$ & $6.91 \mathrm{E}-14$ & $8.07 \mathrm{E}-03$ & $2.56 \mathrm{E}+02$ & $1.49 \mathrm{E}-16$ & $1.98 \mathrm{E}-09$ & $1.74 \mathrm{E}-11$ \\
\hline $\mathrm{Zn}-61$ & 89.1 & $\mathrm{~s}$ & $7.23 \mathrm{E}-14$ & $8.44 \mathrm{E}-03$ & $2.68 \mathrm{E}+02$ & $1.55 \mathrm{E}-16$ & $2.06 \mathrm{E}-09$ & $1.81 \mathrm{E}-11$ \\
\hline $\mathrm{Zn}-62$ & 9.186 & $\mathrm{~h}$ & $1.94 \mathrm{E}-14$ & $2.27 \mathrm{E}-03$ & $7.18 \mathrm{E}+01$ & $4.21 \mathrm{E}-17$ & $5.61 \mathrm{E}-10$ & $4.91 \mathrm{E}-12$ \\
\hline $\mathrm{Zn}-63$ & 38.47 & $\mathrm{~m}$ & $4.99 \mathrm{E}-14$ & $5.83 \mathrm{E}-03$ & $1.85 \mathrm{E}+02$ & $1.08 \mathrm{E}-16$ & $1.44 \mathrm{E}-09$ & $1.26 \mathrm{E}-11$ \\
\hline $\mathrm{Zn}-65$ & 244.06 & $\mathrm{~d}$ & $2.72 \mathrm{E}-14$ & $3.18 \mathrm{E}-03$ & $1.01 \mathrm{E}+02$ & $5.88 \mathrm{E}-17$ & $7.83 \mathrm{E}-10$ & $6.86 \mathrm{E}-12$ \\
\hline $\mathrm{Zn}-69 \mathrm{~m}$ & 13.76 & $\mathrm{~h}$ & $1.84 \mathrm{E}-14$ & $2.15 \mathrm{E}-03$ & $6.81 \mathrm{E}+01$ & $4.01 \mathrm{E}-17$ & $5.34 \mathrm{E}-10$ & $4.68 \mathrm{E}-12$ \\
\hline Zn-69 & 56.4 & $\mathrm{~m}$ & $2.00 \mathrm{E}-16$ & $2.34 \mathrm{E}-05$ & $7.40 \mathrm{E}-01$ & $2.28 \mathrm{E}-19$ & $3.04 \mathrm{E}-12$ & $2.66 \mathrm{E}-14$ \\
\hline $\mathrm{Zn}-71 \mathrm{~m}$ & 3.96 & $\mathrm{~h}$ & $7.04 \mathrm{E}-14$ & $8.22 \mathrm{E}-03$ & $2.60 \mathrm{E}+02$ & $1.53 \mathrm{E}-16$ & $2.04 \mathrm{E}-09$ & $1.79 \mathrm{E}-11$ \\
\hline $\mathrm{Zn}-71$ & 2.45 & $\mathrm{~m}$ & $1.52 \mathrm{E}-14$ & $1.77 \mathrm{E}-03$ & $5.62 \mathrm{E}+01$ & $3.22 \mathrm{E}-17$ & $4.29 \mathrm{E}-10$ & $3.76 \mathrm{E}-12$ \\
\hline $\mathrm{Zn}-72$ & 46.5 & $\mathrm{~h}$ & $6.18 \mathrm{E}-15$ & $7.22 \mathrm{E}-04$ & $2.29 \mathrm{E}+01$ & $1.37 \mathrm{E}-17$ & $1.82 \mathrm{E}-10$ & $1.60 \mathrm{E}-12$ \\
\hline Ga-64 & 2.627 & $\mathrm{~m}$ & $1.64 \mathrm{E}-13$ & $1.91 \mathrm{E}-02$ & $6.07 \mathrm{E}+02$ & $3.55 \mathrm{E}-16$ & $4.73 \mathrm{E}-09$ & $4.14 \mathrm{E}-11$ \\
\hline Ga-65 & 15.2 & $\mathrm{~m}$ & $5.23 \mathrm{E}-14$ & $6.11 \mathrm{E}-03$ & $1.94 \mathrm{E}+02$ & $1.13 \mathrm{E}-16$ & $1.51 \mathrm{E}-09$ & $1.32 \mathrm{E}-11$ \\
\hline Ga-66 & 9.49 & $\mathrm{~h}$ & $1.24 \mathrm{E}-13$ & $1.45 \mathrm{E}-02$ & $4.59 \mathrm{E}+02$ & $2.69 \mathrm{E}-16$ & $3.58 \mathrm{E}-09$ & $3.14 \mathrm{E}-11$ \\
\hline Ga-67 & 3.2612 & $\mathrm{~d}$ & $6.57 \mathrm{E}-15$ & $7.67 \mathrm{E}-04$ & $2.43 \mathrm{E}+01$ & $1.45 \mathrm{E}-17$ & $1.93 \mathrm{E}-10$ & $1.69 \mathrm{E}-12$ \\
\hline Ga-68 & 67.71 & $\mathrm{~m}$ & $4.29 \mathrm{E}-14$ & $5.01 \mathrm{E}-03$ & $1.59 \mathrm{E}+02$ & $9.27 \mathrm{E}-17$ & $1.23 \mathrm{E}-09$ & $1.08 \mathrm{E}-11$ \\
\hline Ga-70 & 21.14 & $\mathrm{~m}$ & $8.27 \mathrm{E}-16$ & $9.66 \mathrm{E}-05$ & $3.06 \mathrm{E}+00$ & $1.32 \mathrm{E}-18$ & $1.76 \mathrm{E}-11$ & $1.54 \mathrm{E}-13$ \\
\hline
\end{tabular}




\begin{tabular}{|c|c|c|c|c|c|c|c|c|}
\hline Nuclide & Half-life & Class & $\begin{array}{c}\text { Air } \\
\text { Immersion } \\
\text { Dose Rate } \\
\text { Coefficient } \\
\left(\mathrm{Sv} / \mathbf{s ~ p e r ~}^{\mathbf{3}}\right. \\
\left.\mathrm{Bq} / \mathbf{m}^{\mathbf{3}}\right)\end{array}$ & $\begin{array}{c}\text { Air } \\
\text { Immersion } \\
\text { Dose Rate } \\
\text { Coefficient } \\
\text { (mrem- } \\
\left.\text { m³ }^{3} / \mathrm{pCi}-\mathrm{yr}\right)\end{array}$ & $\begin{array}{c}\text { Air } \\
\text { Immersion } \\
\text { Effective } \\
\text { Dose Rate } \\
\text { (mrem/sec } \\
\text { per } \\
\mu \mathrm{Ci} / \mathbf{c m}^{3} \text { ) }\end{array}$ & $\begin{array}{c}\text { Water } \\
\text { Submersion } \\
\text { Dose Rate } \\
\text { Coefficient } \\
\left(\mathbf{S v}-\mathbf{m}^{3} / \mathbf{B q}-\right. \\
\text { s) }\end{array}$ & $\begin{array}{c}\text { Water } \\
\text { Submersion } \\
\text { Dose Rate } \\
\text { Coefficient } \\
\text { (mrem- } \\
\left.\text { m³ }^{\mathbf{3}} / \mathbf{p C i}-\mathbf{h}\right)\end{array}$ & $\begin{array}{c}\text { Water } \\
\text { Submersion } \\
\text { Dose Rate } \\
\text { Coefficient } \\
(\text { mrem- } \\
\left.\mathbf{m}^{3} / \mu \mathrm{Ci}-\mathrm{y}\right)\end{array}$ \\
\hline Ga-72 & 14.1 & $\mathrm{~h}$ & $1.31 \mathrm{E}-13$ & $1.53 \mathrm{E}-02$ & $4.85 \mathrm{E}+02$ & $2.83 \mathrm{E}-16$ & $3.77 \mathrm{E}-09$ & $3.30 \mathrm{E}-11$ \\
\hline Ga-73 & 4.86 & $\mathrm{~h}$ & $1.56 \mathrm{E}-14$ & $1.82 \mathrm{E}-03$ & $5.77 \mathrm{E}+01$ & $3.37 \mathrm{E}-17$ & $4.49 \mathrm{E}-10$ & $3.93 \mathrm{E}-12$ \\
\hline Ga-74 & 8.12 & $\mathrm{~m}$ & $1.55 \mathrm{E}-13$ & $1.81 \mathrm{E}-02$ & $5.74 \mathrm{E}+02$ & $3.35 \mathrm{E}-16$ & $4.46 \mathrm{E}-09$ & $3.91 \mathrm{E}-11$ \\
\hline Ge-66 & 2.26 & $\mathrm{~h}$ & $2.97 \mathrm{E}-14$ & $3.47 \mathrm{E}-03$ & $1.10 \mathrm{E}+02$ & $6.46 \mathrm{E}-17$ & $8.60 \mathrm{E}-10$ & $7.54 \mathrm{E}-12$ \\
\hline Ge-67 & 18.9 & $\mathrm{~m}$ & $6.54 \mathrm{E}-14$ & $7.64 \mathrm{E}-03$ & $2.42 \mathrm{E}+02$ & $1.41 \mathrm{E}-16$ & $1.88 \mathrm{E}-09$ & $1.65 \mathrm{E}-11$ \\
\hline Ge-68 & 270.95 & $\mathrm{~d}$ & $8.87 \mathrm{E}-20$ & $1.04 \mathrm{E}-08$ & $3.28 \mathrm{E}-04$ & $2.05 \mathrm{E}-22$ & $2.73 \mathrm{E}-15$ & $2.39 \mathrm{E}-17$ \\
\hline Ge-69 & 39.05 & $\mathrm{~h}$ & $4.38 \mathrm{E}-14$ & $5.11 \mathrm{E}-03$ & $1.62 \mathrm{E}+02$ & $9.49 \mathrm{E}-17$ & $1.26 \mathrm{E}-09$ & $1.11 \mathrm{E}-11$ \\
\hline Ge-71 & 11.43 & $\mathrm{~d}$ & $9.00 \mathrm{E}-20$ & $1.05 \mathrm{E}-08$ & $3.33 \mathrm{E}-04$ & $2.08 \mathrm{E}-22$ & $2.77 \mathrm{E}-15$ & $2.43 \mathrm{E}-17$ \\
\hline Ge-75 & 82.78 & $\mathrm{~m}$ & $1.83 \mathrm{E}-15$ & $2.14 \mathrm{E}-04$ & $6.77 \mathrm{E}+00$ & $3.71 \mathrm{E}-18$ & $4.94 \mathrm{E}-11$ & $4.33 \mathrm{E}-13$ \\
\hline Ge-77 & 11.3 & $\mathrm{~h}$ & $4.95 \mathrm{E}-14$ & $5.78 \mathrm{E}-03$ & $1.83 \mathrm{E}+02$ & $1.07 \mathrm{E}-16$ & $1.43 \mathrm{E}-09$ & $1.25 \mathrm{E}-11$ \\
\hline Ge-78 & 88 & $\mathrm{~m}$ & $1.23 \mathrm{E}-14$ & $1.44 \mathrm{E}-03$ & $4.55 \mathrm{E}+01$ & $2.68 \mathrm{E}-17$ & $3.57 \mathrm{E}-10$ & $3.13 \mathrm{E}-12$ \\
\hline As-68 & 151.6 & $\mathrm{~s}$ & $1.76 \mathrm{E}-13$ & $2.06 \mathrm{E}-02$ & $6.51 \mathrm{E}+02$ & $3.80 \mathrm{E}-16$ & $5.06 \mathrm{E}-09$ & $4.43 \mathrm{E}-11$ \\
\hline As-69 & 15.23 & $\mathrm{~m}$ & $5.25 \mathrm{E}-14$ & $6.13 \mathrm{E}-03$ & $1.94 \mathrm{E}+02$ & $1.13 \mathrm{E}-16$ & $1.51 \mathrm{E}-09$ & $1.32 \mathrm{E}-11$ \\
\hline As-70 & 52.6 & $\mathrm{~m}$ & $2.00 \mathrm{E}-13$ & $2.34 \mathrm{E}-02$ & $7.40 \mathrm{E}+02$ & $4.32 \mathrm{E}-16$ & $5.75 \mathrm{E}-09$ & $5.04 \mathrm{E}-11$ \\
\hline As-71 & 65.28 & $\mathrm{~h}$ & $2.55 \mathrm{E}-14$ & $2.98 \mathrm{E}-03$ & $9.44 \mathrm{E}+01$ & $5.56 \mathrm{E}-17$ & $7.41 \mathrm{E}-10$ & $6.49 \mathrm{E}-12$ \\
\hline As-72 & 26 & $\mathrm{~h}$ & $8.21 \mathrm{E}-14$ & $9.59 \mathrm{E}-03$ & $3.04 \mathrm{E}+02$ & $1.77 \mathrm{E}-16$ & $2.36 \mathrm{E}-09$ & $2.07 \mathrm{E}-11$ \\
\hline As-73 & 80.3 & $\mathrm{~d}$ & $1.54 \mathrm{E}-16$ & $1.80 \mathrm{E}-05$ & $5.70 \mathrm{E}-01$ & $3.55 \mathrm{E}-19$ & $4.73 \mathrm{E}-12$ & $4.14 \mathrm{E}-14$ \\
\hline As-74 & 17.77 & $\mathrm{~d}$ & $3.40 \mathrm{E}-14$ & $3.97 \mathrm{E}-03$ & $1.26 \mathrm{E}+02$ & $7.38 \mathrm{E}-17$ & $9.83 \mathrm{E}-10$ & $8.61 \mathrm{E}-12$ \\
\hline As-76 & 1.0778 & $\mathrm{~d}$ & $2.00 \mathrm{E}-14$ & $2.34 \mathrm{E}-03$ & $7.40 \mathrm{E}+01$ & $4.25 \mathrm{E}-17$ & $5.66 \mathrm{E}-10$ & $4.96 \mathrm{E}-12$ \\
\hline As-77 & 38.83 & $\mathrm{~h}$ & $4.86 \mathrm{E}-16$ & $5.67 \mathrm{E}-05$ & $1.80 \mathrm{E}+00$ & $9.34 \mathrm{E}-19$ & $1.24 \mathrm{E}-11$ & $1.09 \mathrm{E}-13$ \\
\hline As-78 & 90.7 & $\mathrm{~m}$ & $6.27 \mathrm{E}-14$ & $7.32 \mathrm{E}-03$ & $2.32 \mathrm{E}+02$ & $1.35 \mathrm{E}-16$ & $1.80 \mathrm{E}-09$ & $1.58 \mathrm{E}-11$ \\
\hline As-79 & 9.01 & $\mathrm{~m}$ & $2.25 \mathrm{E}-15$ & $2.63 \mathrm{E}-04$ & $8.33 \mathrm{E}+00$ & $4.20 \mathrm{E}-18$ & $5.59 \mathrm{E}-11$ & $4.90 \mathrm{E}-13$ \\
\hline Se-70 & 41.1 & $\mathrm{~m}$ & $3.14 \mathrm{E}-14$ & $3.67 \mathrm{E}-03$ & $1.16 \mathrm{E}+02$ & $6.83 \mathrm{E}-17$ & $9.10 \mathrm{E}-10$ & $7.97 \mathrm{E}-12$ \\
\hline
\end{tabular}




\begin{tabular}{|c|c|c|c|c|c|c|c|c|}
\hline Nuclide & Half-life & Class & $\begin{array}{c}\text { Air } \\
\text { Immersion } \\
\text { Dose Rate } \\
\text { Coefficient } \\
\text { (Sv/s per } \\
\left.\text { Bq } / \mathbf{m}^{\mathbf{3}}\right)\end{array}$ & $\begin{array}{c}\text { Air } \\
\text { Immersion } \\
\text { Dose Rate } \\
\text { Coefficient } \\
\text { (mrem- } \\
\left.\mathrm{m}^{3} / \mathrm{pCi}-\mathrm{yr}\right)\end{array}$ & $\begin{array}{c}\text { Air } \\
\text { Immersion } \\
\text { Effective } \\
\text { Dose Rate } \\
(\mathrm{mrem} / \mathrm{sec} \\
\text { per } \\
\left.\mu \mathrm{Ci} / \mathrm{cm}^{3}\right)\end{array}$ & $\begin{array}{c}\text { Water } \\
\text { Submersion } \\
\text { Dose Rate } \\
\text { Coefficient } \\
\left(\mathbf{S v}-\mathbf{m}^{3} / \mathbf{B q}-\right. \\
\text { s) }\end{array}$ & $\begin{array}{c}\text { Water } \\
\text { Submersion } \\
\text { Dose Rate } \\
\text { Coefficient } \\
\text { (mrem- } \\
\mathbf{m}^{\mathbf{3}} / \mathbf{p C i} \text {-h) }\end{array}$ & $\begin{array}{c}\text { Water } \\
\text { Submersion } \\
\text { Dose Rate } \\
\text { Coefficient } \\
(\text { mrem- } \\
\left.\text { m³ }^{3} / \mu \mathrm{Ci}-\mathrm{y}\right)\end{array}$ \\
\hline Se-71 & 4.74 & $\mathrm{~m}$ & $7.40 \mathrm{E}-14$ & $8.64 \mathrm{E}-03$ & $2.74 \mathrm{E}+02$ & $1.60 \mathrm{E}-16$ & $2.13 \mathrm{E}-09$ & $1.87 \mathrm{E}-11$ \\
\hline Se-72 & 8.4 & $\mathrm{~d}$ & $6.10 \mathrm{E}-16$ & $7.12 \mathrm{E}-05$ & $2.26 \mathrm{E}+00$ & $1.41 \mathrm{E}-18$ & $1.88 \mathrm{E}-11$ & $1.65 \mathrm{E}-13$ \\
\hline $\mathrm{Se}-73$ & 7.15 & $\mathrm{~h}$ & $4.81 \mathrm{E}-14$ & $5.62 \mathrm{E}-03$ & $1.78 \mathrm{E}+02$ & $1.05 \mathrm{E}-16$ & $1.40 \mathrm{E}-09$ & $1.23 \mathrm{E}-11$ \\
\hline Se-73m & 39.8 & $\mathrm{~m}$ & $1.18 \mathrm{E}-14$ & $1.38 \mathrm{E}-03$ & $4.37 \mathrm{E}+01$ & $2.55 \mathrm{E}-17$ & $3.40 \mathrm{E}-10$ & $2.98 \mathrm{E}-12$ \\
\hline Se-75 & 119.779 & $\mathrm{~d}$ & $1.66 \mathrm{E}-14$ & $1.94 \mathrm{E}-03$ & $6.14 \mathrm{E}+01$ & $3.64 \mathrm{E}-17$ & $4.85 \mathrm{E}-10$ & $4.25 \mathrm{E}-12$ \\
\hline $\mathrm{Se}-77 \mathrm{~m}$ & 17.36 & $\mathrm{~S}$ & $3.70 \mathrm{E}-15$ & $4.32 \mathrm{E}-04$ & $1.37 \mathrm{E}+01$ & $8.13 \mathrm{E}-18$ & $1.08 \mathrm{E}-10$ & $9.49 \mathrm{E}-13$ \\
\hline Se-79 & $2.95 \mathrm{E}+05$ & $\mathrm{y}$ & $3.05 \mathrm{E}-18$ & $3.56 \mathrm{E}-07$ & $1.13 \mathrm{E}-02$ & $3.38 \mathrm{E}-21$ & $4.50 \mathrm{E}-14$ & $3.94 \mathrm{E}-16$ \\
\hline $\mathrm{Se}-79 \mathrm{~m}$ & 3.92 & $\mathrm{~m}$ & $3.60 \mathrm{E}-16$ & $4.20 \mathrm{E}-05$ & $1.33 \mathrm{E}+00$ & $8.01 \mathrm{E}-19$ & $1.07 \mathrm{E}-11$ & $9.35 \mathrm{E}-14$ \\
\hline Se-81 & 18.45 & $\mathrm{~m}$ & $8.16 \mathrm{E}-16$ & $9.53 \mathrm{E}-05$ & $3.02 \mathrm{E}+00$ & $1.33 \mathrm{E}-18$ & $1.77 \mathrm{E}-11$ & $1.55 \mathrm{E}-13$ \\
\hline Se- $81 \mathrm{~m}$ & 57.28 & $\mathrm{~m}$ & $5.54 \mathrm{E}-16$ & $6.47 \mathrm{E}-05$ & $2.05 \mathrm{E}+00$ & $1.23 \mathrm{E}-18$ & $1.64 \mathrm{E}-11$ & $1.44 \mathrm{E}-13$ \\
\hline $\mathrm{Se}-83$ & 22.3 & $\mathrm{~m}$ & $1.24 \mathrm{E}-13$ & $1.45 \mathrm{E}-02$ & $4.59 \mathrm{E}+02$ & $2.68 \mathrm{E}-16$ & $3.57 \mathrm{E}-09$ & $3.13 \mathrm{E}-11$ \\
\hline $\mathrm{Se}-83 \mathrm{~m}$ & 70.1 & $\mathrm{~s}$ & $4.78 \mathrm{E}-14$ & $5.58 \mathrm{E}-03$ & $1.77 \mathrm{E}+02$ & $1.02 \mathrm{E}-16$ & $1.36 \mathrm{E}-09$ & $1.19 \mathrm{E}-11$ \\
\hline Se-84 & 3.1 & $\mathrm{~m}$ & $1.90 \mathrm{E}-14$ & $2.22 \mathrm{E}-03$ & $7.03 \mathrm{E}+01$ & $4.09 \mathrm{E}-17$ & $5.45 \mathrm{E}-10$ & $4.77 \mathrm{E}-12$ \\
\hline $\mathrm{Br}-72$ & 78.6 & $\mathrm{~s}$ & $1.41 \mathrm{E}-13$ & $1.65 \mathrm{E}-02$ & $5.22 \mathrm{E}+02$ & $3.03 \mathrm{E}-16$ & $4.04 \mathrm{E}-09$ & $3.54 \mathrm{E}-11$ \\
\hline $\mathrm{Br}-73$ & 3.4 & $\mathrm{~m}$ & $6.52 \mathrm{E}-14$ & $7.61 \mathrm{E}-03$ & $2.41 \mathrm{E}+02$ & $1.41 \mathrm{E}-16$ & $1.88 \mathrm{E}-09$ & $1.65 \mathrm{E}-11$ \\
\hline $\mathrm{Br}-74$ & 25.4 & $\mathrm{~m}$ & $2.30 \mathrm{E}-13$ & $2.69 \mathrm{E}-02$ & $8.51 \mathrm{E}+02$ & $4.98 \mathrm{E}-16$ & $6.63 \mathrm{E}-09$ & $5.81 \mathrm{E}-11$ \\
\hline Br-74m & 46 & $\mathrm{~m}$ & $2.00 \mathrm{E}-13$ & $2.34 \mathrm{E}-02$ & $7.40 \mathrm{E}+02$ & $4.33 \mathrm{E}-16$ & $5.77 \mathrm{E}-09$ & $5.05 \mathrm{E}-11$ \\
\hline $\mathrm{Br}-75$ & 96.7 & $\mathrm{~m}$ & $5.36 \mathrm{E}-14$ & $6.26 \mathrm{E}-03$ & $1.98 \mathrm{E}+02$ & $1.16 \mathrm{E}-16$ & $1.55 \mathrm{E}-09$ & $1.35 \mathrm{E}-11$ \\
\hline $\mathrm{Br}-76$ & 16.2 & $\mathrm{~h}$ & $1.34 \mathrm{E}-13$ & $1.56 \mathrm{E}-02$ & $4.96 \mathrm{E}+02$ & $2.91 \mathrm{E}-16$ & $3.88 \mathrm{E}-09$ & $3.40 \mathrm{E}-11$ \\
\hline $\mathrm{Br}-76 \mathrm{~m}$ & 1.31 & $\mathrm{~S}$ & $9.66 \mathrm{E}-16$ & $1.13 \mathrm{E}-04$ & $3.57 \mathrm{E}+00$ & $2.19 \mathrm{E}-18$ & $2.92 \mathrm{E}-11$ & $2.56 \mathrm{E}-13$ \\
\hline
\end{tabular}




\begin{tabular}{|c|c|c|c|c|c|c|c|c|}
\hline Nuclide & Half-life & Class & $\begin{array}{c}\text { Air } \\
\text { Immersion } \\
\text { Dose Rate } \\
\text { Coefficient } \\
\left(\mathrm{Sv} / \mathbf{s ~ p e r ~}^{\mathbf{3}}\right. \\
\left.\mathrm{Bq} / \mathbf{m}^{\mathbf{3}}\right)\end{array}$ & $\begin{array}{c}\text { Air } \\
\text { Immersion } \\
\text { Dose Rate } \\
\text { Coefficient } \\
\text { (mrem- } \\
\left.\mathrm{m}^{3} / \mathrm{pCi}-\mathrm{yr}\right)\end{array}$ & $\begin{array}{c}\text { Air } \\
\text { Immersion } \\
\text { Effective } \\
\text { Dose Rate } \\
\text { (mrem/sec } \\
\text { per } \\
\mu \mathrm{Ci} / \mathrm{cm}^{3} \text { ) } \\
\end{array}$ & $\begin{array}{c}\text { Water } \\
\text { Submersion } \\
\text { Dose Rate } \\
\text { Coefficient } \\
\left(\mathbf{S v}-\mathbf{m}^{3} / \mathbf{B q}-\right. \\
\text { s) }\end{array}$ & $\begin{array}{c}\text { Water } \\
\text { Submersion } \\
\text { Dose Rate } \\
\text { Coefficient } \\
\text { (mrem- } \\
\left.\text { m³ }^{\mathbf{3}} / \mathbf{p C i}-\mathbf{h}\right)\end{array}$ & $\begin{array}{c}\text { Water } \\
\text { Submersion } \\
\text { Dose Rate } \\
\text { Coefficient } \\
(\text { mrem- } \\
\left.\mathbf{m}^{3} / \mu \mathrm{Ci}-\mathrm{y}\right)\end{array}$ \\
\hline $\mathrm{Br}-77$ & 57.036 & $\mathrm{~h}$ & $1.40 \mathrm{E}-14$ & $1.63 \mathrm{E}-03$ & $5.18 \mathrm{E}+01$ & $3.05 \mathrm{E}-17$ & $4.06 \mathrm{E}-10$ & $3.56 \mathrm{E}-12$ \\
\hline $\mathrm{Br}-77 \mathrm{~m}$ & 4.28 & $\mathrm{~m}$ & $5.96 \mathrm{E}-16$ & $6.96 \mathrm{E}-05$ & $2.21 \mathrm{E}+00$ & $1.32 \mathrm{E}-18$ & $1.76 \mathrm{E}-11$ & $1.54 \mathrm{E}-13$ \\
\hline $\mathrm{Br}-78$ & 6.46 & $\mathrm{~m}$ & $4.69 \mathrm{E}-14$ & $5.48 \mathrm{E}-03$ & $1.74 \mathrm{E}+02$ & $1.01 \mathrm{E}-16$ & $1.35 \mathrm{E}-09$ & $1.18 \mathrm{E}-11$ \\
\hline Br-80 & 17.68 & $\mathrm{~m}$ & $3.98 \mathrm{E}-15$ & $4.65 \mathrm{E}-04$ & $1.47 \mathrm{E}+01$ & $8.09 \mathrm{E}-18$ & $1.08 \mathrm{E}-10$ & $9.44 \mathrm{E}-13$ \\
\hline $\mathrm{Br}-80 \mathrm{~m}$ & 4.4205 & $\mathrm{~h}$ & $2.38 \mathrm{E}-16$ & $2.78 \mathrm{E}-05$ & $8.81 \mathrm{E}-01$ & $5.56 \mathrm{E}-19$ & $7.41 \mathrm{E}-12$ & $6.49 \mathrm{E}-14$ \\
\hline Br-82 & 35.3 & $\mathrm{~h}$ & $1.22 \mathrm{E}-13$ & $1.42 \mathrm{E}-02$ & $4.51 \mathrm{E}+02$ & $2.64 \mathrm{E}-16$ & $3.52 \mathrm{E}-09$ & $3.08 \mathrm{E}-11$ \\
\hline $\mathrm{Br}-82 \mathrm{~m}$ & 6.13 & $\mathrm{~m}$ & $1.59 \mathrm{E}-16$ & $1.86 \mathrm{E}-05$ & $5.88 \mathrm{E}-01$ & $3.19 \mathrm{E}-19$ & $4.25 \mathrm{E}-12$ & $3.72 \mathrm{E}-14$ \\
\hline $\mathrm{Br}-83$ & 2.4 & $\mathrm{~h}$ & $5.10 \mathrm{E}-16$ & $5.95 \mathrm{E}-05$ & $1.89 \mathrm{E}+00$ & $8.98 \mathrm{E}-19$ & $1.20 \mathrm{E}-11$ & $1.05 \mathrm{E}-13$ \\
\hline $\mathrm{Br}-84$ & 31.8 & $\mathrm{~m}$ & $8.88 \mathrm{E}-14$ & $1.04 \mathrm{E}-02$ & $3.29 \mathrm{E}+02$ & $1.92 \mathrm{E}-16$ & $2.56 \mathrm{E}-09$ & $2.24 \mathrm{E}-11$ \\
\hline Br- $84 m$ & 6 & $\mathrm{~m}$ & $1.31 \mathrm{E}-13$ & $1.53 \mathrm{E}-02$ & $4.85 \mathrm{E}+02$ & $2.83 \mathrm{E}-16$ & $3.77 \mathrm{E}-09$ & $3.30 \mathrm{E}-11$ \\
\hline $\mathrm{Br}-85$ & 2.9 & $\mathrm{~m}$ & $3.98 \mathrm{E}-15$ & $4.65 \mathrm{E}-04$ & $1.47 \mathrm{E}+01$ & $7.81 \mathrm{E}-18$ & $1.04 \mathrm{E}-10$ & $9.11 \mathrm{E}-13$ \\
\hline $\mathrm{Kr}-74$ & 11.5 & $\mathrm{~m}$ & $4.70 \mathrm{E}-14$ & $5.49 \mathrm{E}-03$ & $1.74 \mathrm{E}+02$ & $1.02 \mathrm{E}-16$ & $1.36 \mathrm{E}-09$ & $1.19 \mathrm{E}-11$ \\
\hline $\mathrm{Kr}-75$ & 4.29 & $\mathrm{~m}$ & $5.85 \mathrm{E}-14$ & $6.83 \mathrm{E}-03$ & $2.16 \mathrm{E}+02$ & $1.26 \mathrm{E}-16$ & $1.68 \mathrm{E}-09$ & $1.47 \mathrm{E}-11$ \\
\hline $\mathrm{Kr}-76$ & 14.8 & $\mathrm{~h}$ & $1.83 \mathrm{E}-14$ & $2.14 \mathrm{E}-03$ & $6.77 \mathrm{E}+01$ & $4.00 \mathrm{E}-17$ & $5.33 \mathrm{E}-10$ & $4.67 \mathrm{E}-12$ \\
\hline $\mathrm{Kr}-77$ & 74.4 & $\mathrm{~m}$ & $4.63 \mathrm{E}-14$ & $5.41 \mathrm{E}-03$ & $1.71 \mathrm{E}+02$ & $1.00 \mathrm{E}-16$ & $1.33 \mathrm{E}-09$ & $1.17 \mathrm{E}-11$ \\
\hline $\mathrm{Kr}-79$ & 35.04 & $\mathrm{~h}$ & $1.11 \mathrm{E}-14$ & $1.30 \mathrm{E}-03$ & $4.11 \mathrm{E}+01$ & $2.42 \mathrm{E}-17$ & $3.22 \mathrm{E}-10$ & $2.82 \mathrm{E}-12$ \\
\hline $\mathrm{Kr}-81$ & $2.29 \mathrm{E}+05$ & $\mathrm{y}$ & $3.82 \mathrm{E}-17$ & $4.46 \mathrm{E}-06$ & $1.41 \mathrm{E}-01$ & $8.39 \mathrm{E}-20$ & $1.12 \mathrm{E}-12$ & $9.79 \mathrm{E}-15$ \\
\hline $\mathrm{Kr}-81 \mathrm{~m}$ & 13.1 & $\mathrm{~s}$ & $5.57 \mathrm{E}-15$ & $6.50 \mathrm{E}-04$ & $2.06 \mathrm{E}+01$ & $1.22 \mathrm{E}-17$ & $1.63 \mathrm{E}-10$ & $1.42 \mathrm{E}-12$ \\
\hline $\mathrm{Kr}-83 \mathrm{~m}$ & 1.83 & $\mathrm{~h}$ & $1.10 \mathrm{E}-18$ & $1.28 \mathrm{E}-07$ & $4.07 \mathrm{E}-03$ & $2.57 \mathrm{E}-21$ & $3.42 \mathrm{E}-14$ & $3.00 \mathrm{E}-16$ \\
\hline $\mathrm{Kr}-85$ & 10.756 & $\mathrm{y}$ & $2.41 \mathrm{E}-16$ & $2.81 \mathrm{E}-05$ & $8.92 \mathrm{E}-01$ & $3.75 \mathrm{E}-19$ & $5.00 \mathrm{E}-12$ & $4.38 \mathrm{E}-14$ \\
\hline $\mathrm{Kr}-85 \mathrm{~m}$ & 4.48 & $\mathrm{~h}$ & $6.85 \mathrm{E}-15$ & $8.00 \mathrm{E}-04$ & $2.53 \mathrm{E}+01$ & $1.49 \mathrm{E}-17$ & $1.98 \mathrm{E}-10$ & $1.74 \mathrm{E}-12$ \\
\hline $\mathrm{Kr}-87$ & 76.3 & $\mathrm{~m}$ & $3.97 \mathrm{E}-14$ & $4.64 \mathrm{E}-03$ & $1.47 \mathrm{E}+02$ & $8.51 \mathrm{E}-17$ & $1.13 \mathrm{E}-09$ & $9.93 \mathrm{E}-12$ \\
\hline $\mathrm{Kr}-88$ & 2.84 & $\mathrm{~h}$ & $9.71 \mathrm{E}-14$ & $1.13 \mathrm{E}-02$ & $3.59 \mathrm{E}+02$ & $2.10 \mathrm{E}-16$ & $2.80 \mathrm{E}-09$ & $2.45 \mathrm{E}-11$ \\
\hline
\end{tabular}




\begin{tabular}{|c|c|c|c|c|c|c|c|c|}
\hline Nuclide & Half-life & Class & $\begin{array}{c}\text { Air } \\
\text { Immersion } \\
\text { Dose Rate } \\
\text { Coefficient } \\
\left(\mathrm{Sv} / \mathbf{s ~ p e r ~}^{\mathbf{3}}\right. \\
\left.\mathrm{Bq} / \mathbf{m}^{\mathbf{3}}\right)\end{array}$ & $\begin{array}{c}\text { Air } \\
\text { Immersion } \\
\text { Dose Rate } \\
\text { Coefficient } \\
\text { (mrem- } \\
\left.\text { m³ }^{3} / \mathrm{pCi}-\mathrm{yr}\right)\end{array}$ & $\begin{array}{c}\text { Air } \\
\text { Immersion } \\
\text { Effective } \\
\text { Dose Rate } \\
\text { (mrem/sec } \\
\text { per } \\
\mu \mathrm{Ci} / \mathbf{c m}^{3} \text { ) }\end{array}$ & $\begin{array}{c}\text { Water } \\
\text { Submersion } \\
\text { Dose Rate } \\
\text { Coefficient } \\
\left(\mathbf{S v}-\mathbf{m}^{3} / \mathbf{B q}-\right. \\
\text { s) }\end{array}$ & $\begin{array}{c}\text { Water } \\
\text { Submersion } \\
\text { Dose Rate } \\
\text { Coefficient } \\
\text { (mrem- } \\
\left.\text { m³ }^{\mathbf{3}} / \mathbf{p C i}-\mathbf{h}\right)\end{array}$ & $\begin{array}{c}\text { Water } \\
\text { Submersion } \\
\text { Dose Rate } \\
\text { Coefficient } \\
(\text { mrem- } \\
\left.\mathbf{m}^{3} / \mu \mathrm{Ci}-\mathrm{y}\right)\end{array}$ \\
\hline $\mathrm{Kr}-89$ & 3.15 & $\mathrm{~m}$ & $9.56 \mathrm{E}-14$ & $1.12 \mathrm{E}-02$ & $3.54 \mathrm{E}+02$ & $2.06 \mathrm{E}-16$ & $2.74 \mathrm{E}-09$ & $2.40 \mathrm{E}-11$ \\
\hline Rb-77 & 3.77 & $\mathrm{~m}$ & $7.09 \mathrm{E}-14$ & $8.28 \mathrm{E}-03$ & $2.62 \mathrm{E}+02$ & $1.53 \mathrm{E}-16$ & $2.04 \mathrm{E}-09$ & $1.79 \mathrm{E}-11$ \\
\hline $\mathrm{Rb}-78$ & 17.66 & $\mathrm{~m}$ & $2.04 \mathrm{E}-13$ & $2.38 \mathrm{E}-02$ & $7.55 \mathrm{E}+02$ & $4.41 \mathrm{E}-16$ & $5.87 \mathrm{E}-09$ & $5.15 \mathrm{E}-11$ \\
\hline $\mathrm{Rb}-78 \mathrm{~m}$ & 5.74 & $\mathrm{~m}$ & $1.52 \mathrm{E}-13$ & $1.77 \mathrm{E}-02$ & $5.62 \mathrm{E}+02$ & $3.28 \mathrm{E}-16$ & $4.37 \mathrm{E}-09$ & $3.83 \mathrm{E}-11$ \\
\hline $\mathrm{Rb}-79$ & 22.9 & $\mathrm{~m}$ & $6.52 \mathrm{E}-14$ & $7.61 \mathrm{E}-03$ & $2.41 \mathrm{E}+02$ & $1.41 \mathrm{E}-16$ & $1.88 \mathrm{E}-09$ & $1.65 \mathrm{E}-11$ \\
\hline $\mathrm{Rb}-80$ & 33.4 & $\mathrm{~s}$ & $5.54 \mathrm{E}-14$ & $6.47 \mathrm{E}-03$ & $2.05 \mathrm{E}+02$ & $1.19 \mathrm{E}-16$ & $1.59 \mathrm{E}-09$ & $1.39 \mathrm{E}-11$ \\
\hline $\mathrm{Rb}-81$ & 4.576 & $\mathrm{~h}$ & $2.25 \mathrm{E}-14$ & $2.63 \mathrm{E}-03$ & $8.33 \mathrm{E}+01$ & $4.89 \mathrm{E}-17$ & $6.51 \mathrm{E}-10$ & $5.71 \mathrm{E}-12$ \\
\hline $\mathrm{Rb}-81 \mathrm{~m}$ & 30.5 & $\mathrm{~m}$ & $1.07 \mathrm{E}-15$ & $1.25 \mathrm{E}-04$ & $3.96 \mathrm{E}+00$ & $2.33 \mathrm{E}-18$ & $3.10 \mathrm{E}-11$ & $2.72 \mathrm{E}-13$ \\
\hline $\mathrm{Rb}-82$ & 1.273 & $\mathrm{~m}$ & $5.09 \mathrm{E}-14$ & $5.94 \mathrm{E}-03$ & $1.88 \mathrm{E}+02$ & $1.09 \mathrm{E}-16$ & $1.45 \mathrm{E}-09$ & $1.27 \mathrm{E}-11$ \\
\hline $\mathrm{Rb}-82 \mathrm{~m}$ & 6.472 & $\mathrm{~h}$ & $1.34 \mathrm{E}-13$ & $1.56 \mathrm{E}-02$ & $4.96 \mathrm{E}+02$ & $2.91 \mathrm{E}-16$ & $3.88 \mathrm{E}-09$ & $3.40 \mathrm{E}-11$ \\
\hline $\mathrm{Rb}-83$ & 86.2 & $\mathrm{~d}$ & $2.16 \mathrm{E}-14$ & $2.52 \mathrm{E}-03$ & $7.99 \mathrm{E}+01$ & $4.69 \mathrm{E}-17$ & $6.25 \mathrm{E}-10$ & $5.47 \mathrm{E}-12$ \\
\hline $\mathrm{Rb}-84$ & 32.77 & $\mathrm{~d}$ & $4.13 \mathrm{E}-14$ & $4.82 \mathrm{E}-03$ & $1.53 \mathrm{E}+02$ & $8.95 \mathrm{E}-17$ & $1.19 \mathrm{E}-09$ & $1.04 \mathrm{E}-11$ \\
\hline $\mathrm{Rb}-84 \mathrm{~m}$ & 20.26 & $\mathrm{~m}$ & $1.68 \mathrm{E}-14$ & $1.96 \mathrm{E}-03$ & $6.22 \mathrm{E}+01$ & $3.66 \mathrm{E}-17$ & $4.88 \mathrm{E}-10$ & $4.27 \mathrm{E}-12$ \\
\hline $\mathrm{Rb}-86$ & 18.642 & $\mathrm{~d}$ & $4.88 \mathrm{E}-15$ & $5.70 \mathrm{E}-04$ & $1.81 \mathrm{E}+01$ & $1.01 \mathrm{E}-17$ & $1.35 \mathrm{E}-10$ & $1.18 \mathrm{E}-12$ \\
\hline $\mathrm{Rb}-86 \mathrm{~m}$ & 1.017 & $\mathrm{~m}$ & $2.44 \mathrm{E}-14$ & $2.85 \mathrm{E}-03$ & $9.03 \mathrm{E}+01$ & $5.30 \mathrm{E}-17$ & $7.06 \mathrm{E}-10$ & $6.18 \mathrm{E}-12$ \\
\hline $\mathrm{Rb}-87$ & $4.92 \mathrm{E}+10$ & $\mathrm{y}$ & $3.57 \mathrm{E}-17$ & $4.17 \mathrm{E}-06$ & $1.32 \mathrm{E}-01$ & $3.90 \mathrm{E}-20$ & $5.19 \mathrm{E}-13$ & $4.55 \mathrm{E}-15$ \\
\hline Rb-88 & 17.78 & $\mathrm{~m}$ & $3.37 \mathrm{E}-14$ & $3.93 \mathrm{E}-03$ & $1.25 \mathrm{E}+02$ & $7.12 \mathrm{E}-17$ & $9.48 \mathrm{E}-10$ & $8.31 \mathrm{E}-12$ \\
\hline Rb-89 & 15.15 & $\mathrm{~m}$ & $1.09 \mathrm{E}-13$ & $1.27 \mathrm{E}-02$ & $4.03 \mathrm{E}+02$ & $2.36 \mathrm{E}-16$ & $3.14 \mathrm{E}-09$ & $2.75 \mathrm{E}-11$ \\
\hline $\mathrm{Rb}-90$ & 158 & $\mathrm{~s}$ & $1.08 \mathrm{E}-13$ & $1.26 \mathrm{E}-02$ & $4.00 \mathrm{E}+02$ & $2.33 \mathrm{E}-16$ & $3.10 \mathrm{E}-09$ & $2.72 \mathrm{E}-11$ \\
\hline $\mathrm{Rb}-90 \mathrm{~m}$ & 258 & $\mathrm{~s}$ & $1.63 \mathrm{E}-13$ & $1.90 \mathrm{E}-02$ & $6.03 \mathrm{E}+02$ & $3.51 \mathrm{E}-16$ & $4.68 \mathrm{E}-09$ & $4.10 \mathrm{E}-11$ \\
\hline Sr-79 & 2.25 & $\mathrm{~m}$ & $5.39 \mathrm{E}-14$ & $6.29 \mathrm{E}-03$ & $1.99 \mathrm{E}+02$ & $1.16 \mathrm{E}-16$ & $1.55 \mathrm{E}-09$ & $1.35 \mathrm{E}-11$ \\
\hline Sr-80 & 106.3 & $\mathrm{~m}$ & $1.92 \mathrm{E}-14$ & $2.24 \mathrm{E}-03$ & $7.10 \mathrm{E}+01$ & $4.17 \mathrm{E}-17$ & $5.55 \mathrm{E}-10$ & $4.87 \mathrm{E}-12$ \\
\hline Sr-81 & 22.3 & $\mathrm{~m}$ & $6.26 \mathrm{E}-14$ & $7.31 \mathrm{E}-03$ & $2.32 \mathrm{E}+02$ & $1.35 \mathrm{E}-16$ & $1.80 \mathrm{E}-09$ & $1.58 \mathrm{E}-11$ \\
\hline
\end{tabular}




\begin{tabular}{|c|c|c|c|c|c|c|c|c|}
\hline Nuclide & Half-life & Class & $\begin{array}{c}\text { Air } \\
\text { Immersion } \\
\text { Dose Rate } \\
\text { Coefficient } \\
\left(\mathrm{Sv} / \mathbf{s ~ p e r ~}^{\mathbf{3}}\right. \\
\left.\mathrm{Bq} / \mathbf{m}^{\mathbf{3}}\right)\end{array}$ & $\begin{array}{c}\text { Air } \\
\text { Immersion } \\
\text { Dose Rate } \\
\text { Coefficient } \\
\text { (mrem- } \\
\left.\text { m³ }^{3} / \mathrm{pCi}-\mathrm{yr}\right)\end{array}$ & $\begin{array}{c}\text { Air } \\
\text { Immersion } \\
\text { Effective } \\
\text { Dose Rate } \\
\text { (mrem/sec } \\
\text { per } \\
\mu \mathrm{Ci} / \mathbf{c m}^{3} \text { ) }\end{array}$ & $\begin{array}{c}\text { Water } \\
\text { Submersion } \\
\text { Dose Rate } \\
\text { Coefficient } \\
\left(\mathbf{S v}-\mathbf{m}^{3} / \mathbf{B q}-\right. \\
\text { s) }\end{array}$ & $\begin{array}{c}\text { Water } \\
\text { Submersion } \\
\text { Dose Rate } \\
\text { Coefficient } \\
\text { (mrem- } \\
\left.\text { m³ }^{\mathbf{3}} / \mathbf{p C i}-\mathbf{h}\right)\end{array}$ & $\begin{array}{c}\text { Water } \\
\text { Submersion } \\
\text { Dose Rate } \\
\text { Coefficient } \\
(\text { mrem- } \\
\left.\mathbf{m}^{3} / \mu \mathrm{Ci}-\mathrm{y}\right)\end{array}$ \\
\hline Sr-82 & 25.36 & $\mathrm{~d}$ & $4.73 \mathrm{E}-18$ & $5.52 \mathrm{E}-07$ & $1.75 \mathrm{E}-02$ & $1.10 \mathrm{E}-20$ & $1.47 \mathrm{E}-13$ & $1.28 \mathrm{E}-15$ \\
\hline Sr-83 & 32.41 & $\mathrm{~h}$ & $3.70 \mathrm{E}-14$ & $4.32 \mathrm{E}-03$ & $1.37 \mathrm{E}+02$ & $8.02 \mathrm{E}-17$ & $1.07 \mathrm{E}-09$ & $9.36 \mathrm{E}-12$ \\
\hline Sr- 85 & 64.84 & $\mathrm{~d}$ & $2.19 \mathrm{E}-14$ & $2.56 \mathrm{E}-03$ & $8.10 \mathrm{E}+01$ & $4.77 \mathrm{E}-17$ & $6.35 \mathrm{E}-10$ & $5.57 \mathrm{E}-12$ \\
\hline Sr- $85 \mathrm{~m}$ & 67.63 & $\mathrm{~m}$ & $9.42 \mathrm{E}-15$ & $1.10 \mathrm{E}-03$ & $3.49 \mathrm{E}+01$ & $2.07 \mathrm{E}-17$ & $2.76 \mathrm{E}-10$ & $2.42 \mathrm{E}-12$ \\
\hline Sr- $87 \mathrm{~m}$ & 2.815 & $\mathrm{~h}$ & $1.41 \mathrm{E}-14$ & $1.65 \mathrm{E}-03$ & $5.22 \mathrm{E}+01$ & $3.07 \mathrm{E}-17$ & $4.09 \mathrm{E}-10$ & $3.58 \mathrm{E}-12$ \\
\hline Sr-89 & 50.53 & $\mathrm{~d}$ & $4.39 \mathrm{E}-16$ & $5.13 \mathrm{E}-05$ & $1.62 \mathrm{E}+00$ & $5.26 \mathrm{E}-19$ & $7.01 \mathrm{E}-12$ & $6.14 \mathrm{E}-14$ \\
\hline Sr-90 & 28.79 & $\mathrm{y}$ & $9.83 \mathrm{E}-17$ & $1.15 \mathrm{E}-05$ & $3.64 \mathrm{E}-01$ & $1.09 \mathrm{E}-19$ & $1.45 \mathrm{E}-12$ & $1.27 \mathrm{E}-14$ \\
\hline Sr-91 & 9.63 & $\mathrm{~h}$ & $3.32 \mathrm{E}-14$ & $3.88 \mathrm{E}-03$ & $1.23 \mathrm{E}+02$ & $7.14 \mathrm{E}-17$ & $9.51 \mathrm{E}-10$ & $8.33 \mathrm{E}-12$ \\
\hline Sr-92 & 2.66 & $\mathrm{~h}$ & $6.41 \mathrm{E}-14$ & $7.48 \mathrm{E}-03$ & $2.37 \mathrm{E}+02$ & $1.39 \mathrm{E}-16$ & $1.85 \mathrm{E}-09$ & $1.62 \mathrm{E}-11$ \\
\hline Sr-93 & 7.423 & $\mathrm{~m}$ & $1.07 \mathrm{E}-13$ & $1.25 \mathrm{E}-02$ & $3.96 \mathrm{E}+02$ & $2.31 \mathrm{E}-16$ & $3.08 \mathrm{E}-09$ & $2.70 \mathrm{E}-11$ \\
\hline Sr-94 & 75.3 & $\mathrm{~s}$ & $6.92 \mathrm{E}-14$ & $8.08 \mathrm{E}-03$ & $2.56 \mathrm{E}+02$ & $1.49 \mathrm{E}-16$ & $1.98 \mathrm{E}-09$ & $1.74 \mathrm{E}-11$ \\
\hline Y-81 & 70.4 & $\mathrm{~s}$ & $5.35 \mathrm{E}-14$ & $6.25 \mathrm{E}-03$ & $1.98 \mathrm{E}+02$ & $1.15 \mathrm{E}-16$ & $1.53 \mathrm{E}-09$ & $1.34 \mathrm{E}-11$ \\
\hline Y-83 & 7.08 & $\mathrm{~m}$ & $6.16 \mathrm{E}-14$ & $7.19 \mathrm{E}-03$ & $2.28 \mathrm{E}+02$ & $1.33 \mathrm{E}-16$ & $1.77 \mathrm{E}-09$ & $1.55 \mathrm{E}-11$ \\
\hline $\mathrm{Y}-83 \mathrm{~m}$ & 2.85 & $\mathrm{~m}$ & $3.77 \mathrm{E}-14$ & $4.40 \mathrm{E}-03$ & $1.39 \mathrm{E}+02$ & $8.15 \mathrm{E}-17$ & $1.09 \mathrm{E}-09$ & $9.51 \mathrm{E}-12$ \\
\hline $\mathrm{Y}-84 \mathrm{~m}$ & 39.5 & $\mathrm{~m}$ & $1.84 \mathrm{E}-13$ & $2.15 \mathrm{E}-02$ & $6.81 \mathrm{E}+02$ & $3.98 \mathrm{E}-16$ & $5.30 \mathrm{E}-09$ & $4.64 \mathrm{E}-11$ \\
\hline $\mathrm{Y}-85$ & 2.68 & $\mathrm{~h}$ & $4.85 \mathrm{E}-14$ & $5.66 \mathrm{E}-03$ & $1.79 \mathrm{E}+02$ & $1.05 \mathrm{E}-16$ & $1.40 \mathrm{E}-09$ & $1.23 \mathrm{E}-11$ \\
\hline $\mathrm{Y}-85 \mathrm{~m}$ & 4.86 & $\mathrm{~h}$ & $6.18 \mathrm{E}-14$ & $7.22 \mathrm{E}-03$ & $2.29 \mathrm{E}+02$ & $1.34 \mathrm{E}-16$ & $1.78 \mathrm{E}-09$ & $1.56 \mathrm{E}-11$ \\
\hline $\mathrm{Y}-86$ & 14.74 & $\mathrm{~h}$ & $1.68 \mathrm{E}-13$ & $1.96 \mathrm{E}-02$ & $6.22 \mathrm{E}+02$ & $3.64 \mathrm{E}-16$ & $4.85 \mathrm{E}-09$ & $4.25 \mathrm{E}-11$ \\
\hline $\mathrm{Y}-86 \mathrm{~m}$ & 48 & $\mathrm{~m}$ & $9.60 \mathrm{E}-15$ & $1.12 \mathrm{E}-03$ & $3.55 \mathrm{E}+01$ & $2.10 \mathrm{E}-17$ & $2.80 \mathrm{E}-10$ & $2.45 \mathrm{E}-12$ \\
\hline $\mathrm{Y}-87$ & 79.8 & $\mathrm{~h}$ & $1.94 \mathrm{E}-14$ & $2.27 \mathrm{E}-03$ & $7.18 \mathrm{E}+01$ & $4.23 \mathrm{E}-17$ & $5.63 \mathrm{E}-10$ & $4.94 \mathrm{E}-12$ \\
\hline $\mathrm{Y}-87 \mathrm{~m}$ & 13.37 & $\mathrm{~h}$ & $1.35 \mathrm{E}-14$ & $1.58 \mathrm{E}-03$ & $5.00 \mathrm{E}+01$ & $2.94 \mathrm{E}-17$ & $3.92 \mathrm{E}-10$ & $3.43 \mathrm{E}-12$ \\
\hline $\mathrm{Y}-88$ & 106.65 & $\mathrm{~d}$ & $1.30 \mathrm{E}-13$ & $1.52 \mathrm{E}-02$ & $4.81 \mathrm{E}+02$ & $2.82 \mathrm{E}-16$ & $3.76 \mathrm{E}-09$ & $3.29 \mathrm{E}-11$ \\
\hline $\mathrm{Y}-89 \mathrm{~m}$ & 15.663 & $\mathrm{~s}$ & $4.16 \mathrm{E}-14$ & $4.86 \mathrm{E}-03$ & $1.54 \mathrm{E}+02$ & $9.02 \mathrm{E}-17$ & $1.20 \mathrm{E}-09$ & $1.05 \mathrm{E}-11$ \\
\hline
\end{tabular}




\begin{tabular}{|c|c|c|c|c|c|c|c|c|}
\hline Nuclide & Half-life & Class & $\begin{array}{c}\text { Air } \\
\text { Immersion } \\
\text { Dose Rate } \\
\text { Coefficient } \\
\left(\mathrm{Sv} / \mathbf{s ~ p e r ~}^{\mathbf{3}}\right. \\
\left.\mathrm{Bq} / \mathbf{m}^{\mathbf{3}}\right)\end{array}$ & $\begin{array}{c}\text { Air } \\
\text { Immersion } \\
\text { Dose Rate } \\
\text { Coefficient } \\
\text { (mrem- } \\
\left.\mathrm{m}^{3} / \mathrm{pCi}-\mathrm{yr}\right)\end{array}$ & $\begin{array}{c}\text { Air } \\
\text { Immersion } \\
\text { Effective } \\
\text { Dose Rate } \\
\text { (mrem/sec } \\
\text { per } \\
\mu \mathrm{Ci} / \mathbf{c m}^{3} \text { ) }\end{array}$ & $\begin{array}{c}\text { Water } \\
\text { Submersion } \\
\text { Dose Rate } \\
\text { Coefficient } \\
\left(\mathbf{S v}-\mathbf{m}^{3} / \mathbf{B q}-\right. \\
\text { s) }\end{array}$ & $\begin{array}{c}\text { Water } \\
\text { Submersion } \\
\text { Dose Rate } \\
\text { Coefficient } \\
\text { (mrem- } \\
\left.\text { m³ }^{\mathbf{3}} / \mathbf{p C i}-\mathbf{h}\right)\end{array}$ & $\begin{array}{c}\text { Water } \\
\text { Submersion } \\
\text { Dose Rate } \\
\text { Coefficient } \\
(\text { mrem- } \\
\left.\mathbf{m}^{\mathbf{3}} / \boldsymbol{\mu C i}-\mathrm{y}\right)\end{array}$ \\
\hline $\mathrm{Y}-90$ & 64.1 & $\mathrm{~h}$ & $7.91 \mathrm{E}-16$ & $9.24 \mathrm{E}-05$ & $2.93 \mathrm{E}+00$ & $9.86 \mathrm{E}-19$ & $1.31 \mathrm{E}-11$ & $1.15 \mathrm{E}-13$ \\
\hline $\mathrm{Y}-90 \mathrm{~m}$ & 3.19 & $\mathrm{~h}$ & $2.80 \mathrm{E}-14$ & $3.27 \mathrm{E}-03$ & $1.04 \mathrm{E}+02$ & $6.10 \mathrm{E}-17$ & $8.13 \mathrm{E}-10$ & $7.12 \mathrm{E}-12$ \\
\hline $\mathrm{Y}-91$ & 58.51 & $\mathrm{~d}$ & $6.01 \mathrm{E}-16$ & $7.02 \mathrm{E}-05$ & $2.22 \mathrm{E}+00$ & $8.61 \mathrm{E}-19$ & $1.15 \mathrm{E}-11$ & $1.00 \mathrm{E}-13$ \\
\hline $\mathrm{Y}-91 \mathrm{~m}$ & 49.71 & $\mathrm{~m}$ & $2.36 \mathrm{E}-14$ & $2.76 \mathrm{E}-03$ & $8.73 \mathrm{E}+01$ & $5.13 \mathrm{E}-17$ & $6.83 \mathrm{E}-10$ & $5.99 \mathrm{E}-12$ \\
\hline Y-92 & 3.54 & $\mathrm{~h}$ & $1.32 \mathrm{E}-14$ & $1.54 \mathrm{E}-03$ & $4.88 \mathrm{E}+01$ & $2.74 \mathrm{E}-17$ & $3.65 \mathrm{E}-10$ & $3.20 \mathrm{E}-12$ \\
\hline Y-93 & 10.18 & $\mathrm{~h}$ & $5.63 \mathrm{E}-15$ & $6.57 \mathrm{E}-04$ & $2.08 \mathrm{E}+01$ & $1.13 \mathrm{E}-17$ & $1.51 \mathrm{E}-10$ & $1.32 \mathrm{E}-12$ \\
\hline Y-94 & 18.7 & $\mathrm{~m}$ & $3.82 \mathrm{E}-14$ & $4.46 \mathrm{E}-03$ & $1.41 \mathrm{E}+02$ & $8.12 \mathrm{E}-17$ & $1.08 \mathrm{E}-09$ & $9.47 \mathrm{E}-12$ \\
\hline Y-95 & 10.3 & $\mathrm{~m}$ & $5.71 \mathrm{E}-14$ & $6.67 \mathrm{E}-03$ & $2.11 \mathrm{E}+02$ & $1.23 \mathrm{E}-16$ & $1.64 \mathrm{E}-09$ & $1.44 \mathrm{E}-11$ \\
\hline $\mathrm{Zr}-85$ & 7.86 & $\mathrm{~m}$ & $6.74 \mathrm{E}-14$ & $7.87 \mathrm{E}-03$ & $2.49 \mathrm{E}+02$ & $1.45 \mathrm{E}-16$ & $1.93 \mathrm{E}-09$ & $1.69 \mathrm{E}-11$ \\
\hline Zr-86 & 16.5 & $\mathrm{~h}$ & $1.20 \mathrm{E}-14$ & $1.40 \mathrm{E}-03$ & $4.44 \mathrm{E}+01$ & $2.63 \mathrm{E}-17$ & $3.50 \mathrm{E}-10$ & $3.07 \mathrm{E}-12$ \\
\hline $\mathrm{Zr}-87$ & 1.68 & $\mathrm{~h}$ & $4.22 \mathrm{E}-14$ & $4.93 \mathrm{E}-03$ & $1.56 \mathrm{E}+02$ & $9.10 \mathrm{E}-17$ & $1.21 \mathrm{E}-09$ & $1.06 \mathrm{E}-11$ \\
\hline Zr-88 & 83.4 & $\mathrm{~d}$ & $1.69 \mathrm{E}-14$ & $1.97 \mathrm{E}-03$ & $6.25 \mathrm{E}+01$ & $3.68 \mathrm{E}-17$ & $4.90 \mathrm{E}-10$ & $4.29 \mathrm{E}-12$ \\
\hline Zr-89 & 78.41 & $\mathrm{~h}$ & $5.29 \mathrm{E}-14$ & $6.18 \mathrm{E}-03$ & $1.96 \mathrm{E}+02$ & $1.15 \mathrm{E}-16$ & $1.53 \mathrm{E}-09$ & $1.34 \mathrm{E}-11$ \\
\hline $\mathrm{Zr}-89 \mathrm{~m}$ & 4.161 & $\mathrm{~m}$ & $2.88 \mathrm{E}-14$ & $3.36 \mathrm{E}-03$ & $1.07 \mathrm{E}+02$ & $6.24 \mathrm{E}-17$ & $8.31 \mathrm{E}-10$ & $7.28 \mathrm{E}-12$ \\
\hline Zr-93 & $1.53 \mathrm{E}+06$ & $\mathrm{y}$ & $6.45 \mathrm{E}-22$ & $7.53 \mathrm{E}-11$ & $2.39 \mathrm{E}-06$ & $6.75 \mathrm{E}-25$ & $8.99 \mathrm{E}-18$ & $7.88 \mathrm{E}-20$ \\
\hline Zr-95 & 64.032 & $\mathrm{~d}$ & $3.33 \mathrm{E}-14$ & $3.89 \mathrm{E}-03$ & $1.23 \mathrm{E}+02$ & $7.23 \mathrm{E}-17$ & $9.63 \mathrm{E}-10$ & $8.44 \mathrm{E}-12$ \\
\hline Zr-97 & 16.744 & $\mathrm{~h}$ & $4.07 \mathrm{E}-14$ & $4.75 \mathrm{E}-03$ & $1.51 \mathrm{E}+02$ & $8.79 \mathrm{E}-17$ & $1.17 \mathrm{E}-09$ & $1.03 \mathrm{E}-11$ \\
\hline $\mathrm{Nb}-87$ & 3.75 & $\mathrm{~m}$ & $5.54 \mathrm{E}-14$ & $6.47 \mathrm{E}-03$ & $2.05 \mathrm{E}+02$ & $1.19 \mathrm{E}-16$ & $1.59 \mathrm{E}-09$ & $1.39 \mathrm{E}-11$ \\
\hline $\mathrm{Nb}-88$ & 14.5 & $\mathrm{~m}$ & $1.94 \mathrm{E}-13$ & $2.27 \mathrm{E}-02$ & $7.18 \mathrm{E}+02$ & $4.20 \mathrm{E}-16$ & $5.59 \mathrm{E}-09$ & $4.90 \mathrm{E}-11$ \\
\hline $\mathrm{Nb}-88 \mathrm{~m}$ & 7.78 & $\mathrm{~m}$ & $1.91 \mathrm{E}-13$ & $2.23 \mathrm{E}-02$ & $7.07 \mathrm{E}+02$ & $4.13 \mathrm{E}-16$ & $5.50 \mathrm{E}-09$ & $4.82 \mathrm{E}-11$ \\
\hline $\mathrm{Nb}-89$ & 2.03 & $\mathrm{~h}$ & $6.51 \mathrm{E}-14$ & $7.60 \mathrm{E}-03$ & $2.41 \mathrm{E}+02$ & $1.40 \mathrm{E}-16$ & $1.86 \mathrm{E}-09$ & $1.63 \mathrm{E}-11$ \\
\hline $\mathrm{Nb}-89 \mathrm{~m}$ & 66 & $\mathrm{~m}$ & $5.88 \mathrm{E}-14$ & $6.87 \mathrm{E}-03$ & $2.18 \mathrm{E}+02$ & $1.27 \mathrm{E}-16$ & $1.69 \mathrm{E}-09$ & $1.48 \mathrm{E}-11$ \\
\hline $\mathrm{Nb}-90$ & 14.6 & $\mathrm{~h}$ & $2.05 \mathrm{E}-13$ & $2.39 \mathrm{E}-02$ & $7.59 \mathrm{E}+02$ & $4.44 \mathrm{E}-16$ & $5.91 \mathrm{E}-09$ & $5.18 \mathrm{E}-11$ \\
\hline
\end{tabular}




\begin{tabular}{|c|c|c|c|c|c|c|c|c|}
\hline Nuclide & Half-life & Class & $\begin{array}{c}\text { Air } \\
\text { Immersion } \\
\text { Dose Rate } \\
\text { Coefficient } \\
\left(\mathrm{Sv} / \mathrm{s} \mathrm{per}^{\mathbf{3}}\right. \\
\left.\mathrm{Bq} / \mathbf{m}^{\mathbf{3}}\right)\end{array}$ & $\begin{array}{c}\text { Air } \\
\text { Immersion } \\
\text { Dose Rate } \\
\text { Coefficient } \\
\text { (mrem- } \\
\left.\mathrm{m}^{3} / \mathrm{pCi}-\mathrm{yr}\right)\end{array}$ & $\begin{array}{c}\text { Air } \\
\text { Immersion } \\
\text { Effective } \\
\text { Dose Rate } \\
(\mathrm{mrem} / \mathrm{sec} \\
\text { per } \\
\mu \mathrm{Ci} / \mathrm{cm}^{3} \text { ) }\end{array}$ & $\begin{array}{c}\text { Water } \\
\text { Submersion } \\
\text { Dose Rate } \\
\text { Coefficient } \\
\left(\mathbf{S v}-\mathbf{m}^{3} / \mathbf{B q}-\right. \\
\text { s) }\end{array}$ & $\begin{array}{c}\text { Water } \\
\text { Submersion } \\
\text { Dose Rate } \\
\text { Coefficient } \\
\text { (mrem- } \\
\mathbf{m}^{\mathbf{3}} / \mathbf{p C i} \text {-h) }\end{array}$ & $\begin{array}{c}\text { Water } \\
\text { Submersion } \\
\text { Dose Rate } \\
\text { Coefficient } \\
(\text { mrem- } \\
\left.\text { m³ }^{3} / \mu \mathrm{Ci}-\mathrm{y}\right)\end{array}$ \\
\hline $\mathrm{Nb}-91$ & 680 & $\mathrm{y}$ & $8.45 \mathrm{E}-17$ & $9.87 \mathrm{E}-06$ & $3.13 \mathrm{E}-01$ & $1.86 \mathrm{E}-19$ & $2.48 \mathrm{E}-12$ & $2.17 \mathrm{E}-14$ \\
\hline $\mathrm{Nb}-91 \mathrm{~m}$ & 60.86 & $\mathrm{~d}$ & $1.20 \mathrm{E}-15$ & $1.40 \mathrm{E}-04$ & $4.44 \mathrm{E}+00$ & $2.59 \mathrm{E}-18$ & $3.45 \mathrm{E}-11$ & $3.02 \mathrm{E}-13$ \\
\hline $\mathrm{Nb}-92$ & $3.47 \mathrm{E}+07$ & $\mathrm{y}$ & $6.83 \mathrm{E}-14$ & $7.97 \mathrm{E}-03$ & $2.53 \mathrm{E}+02$ & $1.48 \mathrm{E}-16$ & $1.97 \mathrm{E}-09$ & $1.73 \mathrm{E}-11$ \\
\hline $\mathrm{Nb}-92 \mathrm{~m}$ & 10.15 & $\mathrm{~d}$ & $4.44 \mathrm{E}-14$ & $5.18 \mathrm{E}-03$ & $1.64 \mathrm{E}+02$ & $9.63 \mathrm{E}-17$ & $1.28 \mathrm{E}-09$ & $1.12 \mathrm{E}-11$ \\
\hline $\mathrm{Nb}-93 \mathrm{~m}$ & 16.13 & $\mathrm{y}$ & $3.04 \mathrm{E}-18$ & $3.55 \mathrm{E}-07$ & $1.12 \mathrm{E}-02$ & $7.13 \mathrm{E}-21$ & $9.50 \mathrm{E}-14$ & $8.32 \mathrm{E}-16$ \\
\hline $\mathrm{Nb}-94$ & $2.03 \mathrm{E}+04$ & $\mathrm{y}$ & $7.13 \mathrm{E}-14$ & $8.33 \mathrm{E}-03$ & $2.64 \mathrm{E}+02$ & $1.55 \mathrm{E}-16$ & $2.06 \mathrm{E}-09$ & $1.81 \mathrm{E}-11$ \\
\hline $\mathrm{Nb}-94 \mathrm{~m}$ & 6.263 & $\mathrm{~m}$ & $2.15 \mathrm{E}-16$ & $2.51 \mathrm{E}-05$ & $7.96 \mathrm{E}-01$ & $4.67 \mathrm{E}-19$ & $6.22 \mathrm{E}-12$ & $5.45 \mathrm{E}-14$ \\
\hline $\mathrm{Nb}-95$ & 34.991 & $\mathrm{~d}$ & $3.49 \mathrm{E}-14$ & $4.08 \mathrm{E}-03$ & $1.29 \mathrm{E}+02$ & $7.56 \mathrm{E}-17$ & $1.01 \mathrm{E}-09$ & $8.82 \mathrm{E}-12$ \\
\hline $\mathrm{Nb}-95 \mathrm{~m}$ & 3.61 & $\mathrm{~d}$ & $2.83 \mathrm{E}-15$ & $3.30 \mathrm{E}-04$ & $1.05 \mathrm{E}+01$ & $6.10 \mathrm{E}-18$ & $8.13 \mathrm{E}-11$ & $7.12 \mathrm{E}-13$ \\
\hline $\mathrm{Nb}-96$ & 23.35 & $\mathrm{~h}$ & $1.13 \mathrm{E}-13$ & $1.32 \mathrm{E}-02$ & $4.18 \mathrm{E}+02$ & $2.45 \mathrm{E}-16$ & $3.26 \mathrm{E}-09$ & $2.86 \mathrm{E}-11$ \\
\hline $\mathrm{Nb}-97$ & 72.1 & $\mathrm{~m}$ & $3.04 \mathrm{E}-14$ & $3.55 \mathrm{E}-03$ & $1.12 \mathrm{E}+02$ & $6.56 \mathrm{E}-17$ & $8.74 \mathrm{E}-10$ & $7.65 \mathrm{E}-12$ \\
\hline $\mathrm{Nb}-98 \mathrm{~m}$ & 51.3 & $\mathrm{~m}$ & $1.32 \mathrm{E}-13$ & $1.54 \mathrm{E}-02$ & $4.88 \mathrm{E}+02$ & $2.86 \mathrm{E}-16$ & $3.81 \mathrm{E}-09$ & $3.34 \mathrm{E}-11$ \\
\hline $\mathrm{Nb}-99$ & 15 & $\mathrm{~s}$ & $8.32 \mathrm{E}-15$ & $9.71 \mathrm{E}-04$ & $3.08 \mathrm{E}+01$ & $1.72 \mathrm{E}-17$ & $2.29 \mathrm{E}-10$ & $2.01 \mathrm{E}-12$ \\
\hline $\mathrm{Nb}-99 \mathrm{~m}$ & 2.6 & $\mathrm{~m}$ & $3.86 \mathrm{E}-14$ & $4.51 \mathrm{E}-03$ & $1.43 \mathrm{E}+02$ & $8.26 \mathrm{E}-17$ & $1.10 \mathrm{E}-09$ & $9.64 \mathrm{E}-12$ \\
\hline Mo-89 & 2.11 & $\mathrm{~m}$ & $5.70 \mathrm{E}-14$ & $6.66 \mathrm{E}-03$ & $2.11 \mathrm{E}+02$ & $1.22 \mathrm{E}-16$ & $1.63 \mathrm{E}-09$ & $1.42 \mathrm{E}-11$ \\
\hline Mo-90 & 5.56 & $\mathrm{~h}$ & $3.66 \mathrm{E}-14$ & $4.27 \mathrm{E}-03$ & $1.35 \mathrm{E}+02$ & $7.98 \mathrm{E}-17$ & $1.06 \mathrm{E}-09$ & $9.31 \mathrm{E}-12$ \\
\hline Mo-91 & 15.49 & $\mathrm{~m}$ & $4.50 \mathrm{E}-14$ & $5.25 \mathrm{E}-03$ & $1.67 \mathrm{E}+02$ & $9.66 \mathrm{E}-17$ & $1.29 \mathrm{E}-09$ & $1.13 \mathrm{E}-11$ \\
\hline Mo-91m & 64.6 & $\mathrm{~s}$ & $6.45 \mathrm{E}-14$ & $7.53 \mathrm{E}-03$ & $2.39 \mathrm{E}+02$ & $1.40 \mathrm{E}-16$ & $1.86 \mathrm{E}-09$ & $1.63 \mathrm{E}-11$ \\
\hline Mo-93 & $4.00 \mathrm{E}+03$ & $\mathrm{y}$ & $1.70 \mathrm{E}-17$ & $1.98 \mathrm{E}-06$ & $6.29 \mathrm{E}-02$ & $3.99 \mathrm{E}-20$ & $5.31 \mathrm{E}-13$ & $4.66 \mathrm{E}-15$ \\
\hline
\end{tabular}




\begin{tabular}{|c|c|c|c|c|c|c|c|c|}
\hline Nuclide & Half-life & Class & $\begin{array}{c}\text { Air } \\
\text { Immersion } \\
\text { Dose Rate } \\
\text { Coefficient } \\
(\text { Sv/s per } \\
\left.\text { Bq/ } \mathbf{m}^{3}\right)\end{array}$ & $\begin{array}{c}\text { Air } \\
\text { Immersion } \\
\text { Dose Rate } \\
\text { Coefficient } \\
\text { (mrem- } \\
\text { m³ } / \text { pi-yr) }\end{array}$ & $\begin{array}{c}\text { Air } \\
\text { Immersion } \\
\text { Effective } \\
\text { Dose Rate } \\
(\mathrm{mrem} / \mathrm{sec} \\
\mathrm{per} \\
\left.\mu \mathrm{Ci} / \mathrm{cm}^{3}\right)\end{array}$ & $\begin{array}{c}\text { Water } \\
\text { Submersion } \\
\text { Dose Rate } \\
\text { Coefficient } \\
\left(\mathrm{Sv}-\mathbf{m}^{3} / \mathbf{B q}-\right. \\
\text { s) }\end{array}$ & $\begin{array}{c}\text { Water } \\
\text { Submersion } \\
\text { Dose Rate } \\
\text { Coefficient } \\
\text { (mrem- } \\
\text { m³Ci-h) }^{\mathbf{3} / p C i}\end{array}$ & $\begin{array}{c}\text { Water } \\
\text { Submersion } \\
\text { Dose Rate } \\
\text { Coefficient } \\
(\text { mrem- } \\
\left.\text { m }^{3} / \mu \mathrm{Ci}-\mathrm{y}\right)\end{array}$ \\
\hline Mo-93m & 6.85 & $\mathrm{~h}$ & $1.09 \mathrm{E}-13$ & $1.27 \mathrm{E}-02$ & $4.03 \mathrm{E}+02$ & $2.36 \mathrm{E}-16$ & $3.14 \mathrm{E}-09$ & $2.75 \mathrm{E}-11$ \\
\hline Mo-99 & 65.94 & $\mathrm{~h}$ & $6.92 \mathrm{E}-15$ & $8.08 \mathrm{E}-04$ & $2.56 \mathrm{E}+01$ & $1.48 \mathrm{E}-17$ & $1.97 \mathrm{E}-10$ & $1.73 \mathrm{E}-12$ \\
\hline Mo-101 & 14.61 & $\mathrm{~m}$ & $6.97 \mathrm{E}-14$ & $8.14 \mathrm{E}-03$ & $2.58 \mathrm{E}+02$ & $1.51 \mathrm{E}-16$ & $2.01 \mathrm{E}-09$ & $1.76 \mathrm{E}-11$ \\
\hline Mo-102 & 11.3 & $\mathrm{~m}$ & $1.02 \mathrm{E}-15$ & 1.19E-04 & $3.77 \mathrm{E}+00$ & $2.01 \mathrm{E}-18$ & $2.68 \mathrm{E}-11$ & $2.35 \mathrm{E}-13$ \\
\hline Tc-91 & 3.14 & $\mathrm{~m}$ & $1.20 \mathrm{E}-13$ & $1.40 \mathrm{E}-02$ & $4.44 \mathrm{E}+02$ & $2.60 \mathrm{E}-16$ & $3.46 \mathrm{E}-09$ & $3.03 \mathrm{E}-11$ \\
\hline Tc-91m & 3.3 & $\mathrm{~m}$ & $6.60 \mathrm{E}-14$ & 7.71E-03 & $2.44 \mathrm{E}+02$ & $1.42 \mathrm{E}-16$ & $1.89 \mathrm{E}-09$ & $1.66 \mathrm{E}-11$ \\
\hline Tc-92 & 4.25 & $\mathrm{~m}$ & $1.79 \mathrm{E}-13$ & $2.09 \mathrm{E}-02$ & $6.62 \mathrm{E}+02$ & $3.88 \mathrm{E}-16$ & $5.17 \mathrm{E}-09$ & $4.53 \mathrm{E}-11$ \\
\hline Tc-93 & 2.75 & $\mathrm{~h}$ & $7.46 \mathrm{E}-14$ & $8.71 \mathrm{E}-03$ & $2.76 \mathrm{E}+02$ & $1.62 \mathrm{E}-16$ & $2.16 \mathrm{E}-09$ & $1.89 \mathrm{E}-11$ \\
\hline Tc-93m & 43.5 & $\mathrm{~m}$ & $4.66 \mathrm{E}-14$ & $5.44 \mathrm{E}-03$ & $1.72 \mathrm{E}+02$ & $1.01 \mathrm{E}-16$ & $1.35 \mathrm{E}-09$ & $1.18 \mathrm{E}-11$ \\
\hline Tc-94 & 293 & $\mathrm{~m}$ & $1.21 \mathrm{E}-13$ & $1.41 \mathrm{E}-02$ & $4.48 \mathrm{E}+02$ & $2.63 \mathrm{E}-16$ & $3.50 \mathrm{E}-09$ & $3.07 \mathrm{E}-11$ \\
\hline Tc-94m & 52 & $\mathrm{~m}$ & $9.11 \mathrm{E}-14$ & $1.06 \mathrm{E}-02$ & $3.37 \mathrm{E}+02$ & $1.97 \mathrm{E}-16$ & $2.62 \mathrm{E}-09$ & $2.30 \mathrm{E}-11$ \\
\hline Tc-95 & 20 & $\mathrm{~h}$ & $3.59 \mathrm{E}-14$ & 4.19E-03 & $1.33 \mathrm{E}+02$ & $7.78 \mathrm{E}-17$ & $1.04 \mathrm{E}-09$ & $9.08 \mathrm{E}-12$ \\
\hline Tc-95m & 61 & $\mathrm{~d}$ & $3.06 \mathrm{E}-14$ & $3.57 \mathrm{E}-03$ & $1.13 \mathrm{E}+02$ & $6.64 \mathrm{E}-17$ & $8.84 \mathrm{E}-10$ & $7.75 \mathrm{E}-12$ \\
\hline Tc-96 & 4.28 & $\mathrm{~d}$ & $1.14 \mathrm{E}-13$ & $1.33 \mathrm{E}-02$ & $4.22 \mathrm{E}+02$ & $2.48 \mathrm{E}-16$ & $3.30 \mathrm{E}-09$ & $2.89 \mathrm{E}-11$ \\
\hline Tc-96m & 51.5 & $\mathrm{~m}$ & $1.94 \mathrm{E}-15$ & $2.27 \mathrm{E}-04$ & $7.18 \mathrm{E}+00$ & $4.21 \mathrm{E}-18$ & $5.61 \mathrm{E}-11$ & $4.91 \mathrm{E}-13$ \\
\hline Tc-97 & $2.60 \mathrm{E}+06$ & $\mathrm{y}$ & $2.21 \mathrm{E}-17$ & $2.58 \mathrm{E}-06$ & $8.18 \mathrm{E}-02$ & $5.20 \mathrm{E}-20$ & $6.93 \mathrm{E}-13$ & $6.07 \mathrm{E}-15$ \\
\hline Tc-97m & 90.1 & $\mathrm{~d}$ & $3.68 \mathrm{E}-17$ & $4.30 \mathrm{E}-06$ & $1.36 \mathrm{E}-01$ & $8.10 \mathrm{E}-20$ & $1.08 \mathrm{E}-12$ & $9.45 \mathrm{E}-15$ \\
\hline Tc-98 & $4.20 \mathrm{E}+06$ & $\mathrm{y}$ & $6.41 \mathrm{E}-14$ & $7.48 \mathrm{E}-03$ & $2.37 \mathrm{E}+02$ & $1.39 \mathrm{E}-16$ & $1.85 \mathrm{E}-09$ & $1.62 \mathrm{E}-11$ \\
\hline Tc-99 & $2.11 \mathrm{E}+05$ & $\mathrm{y}$ & $2.88 \mathrm{E}-17$ & $3.36 \mathrm{E}-06$ & $1.07 \mathrm{E}-01$ & $3.14 \mathrm{E}-20$ & $4.18 \mathrm{E}-13$ & $3.66 \mathrm{E}-15$ \\
\hline Tc-99m & 6.015 & $\mathrm{~h}$ & $5.27 \mathrm{E}-15$ & $6.15 \mathrm{E}-04$ & $1.95 \mathrm{E}+01$ & $1.17 \mathrm{E}-17$ & $1.56 \mathrm{E}-10$ & $1.37 \mathrm{E}-12$ \\
\hline Tc-101 & 14.2 & $\mathrm{~m}$ & $1.52 \mathrm{E}-14$ & $1.77 \mathrm{E}-03$ & $5.62 \mathrm{E}+01$ & $3.28 \mathrm{E}-17$ & $4.37 \mathrm{E}-10$ & $3.83 \mathrm{E}-12$ \\
\hline Tc-102 & 5.28 & $\mathrm{~s}$ & $5.85 \mathrm{E}-15$ & $6.83 \mathrm{E}-04$ & $2.16 \mathrm{E}+01$ & $1.10 \mathrm{E}-17$ & $1.47 \mathrm{E}-10$ & $1.28 \mathrm{E}-12$ \\
\hline Tc- $102 \mathrm{~m}$ & 4.35 & $\mathrm{~m}$ & $1.18 \mathrm{E}-13$ & $1.38 \mathrm{E}-02$ & $4.37 \mathrm{E}+02$ & $2.56 \mathrm{E}-16$ & $3.41 \mathrm{E}-09$ & $2.99 \mathrm{E}-11$ \\
\hline
\end{tabular}




\begin{tabular}{|c|c|c|c|c|c|c|c|c|}
\hline Nuclide & Half-life & Class & $\begin{array}{c}\text { Air } \\
\text { Immersion } \\
\text { Dose Rate } \\
\text { Coefficient } \\
\left(\mathrm{Sv} / \mathbf{s ~ p e r ~}^{\mathbf{3}}\right. \\
\left.\mathrm{Bq} / \mathbf{m}^{\mathbf{3}}\right)\end{array}$ & $\begin{array}{c}\text { Air } \\
\text { Immersion } \\
\text { Dose Rate } \\
\text { Coefficient } \\
\text { (mrem- } \\
\left.\text { m³ }^{3} / \mathrm{pCi}-\mathrm{yr}\right)\end{array}$ & $\begin{array}{c}\text { Air } \\
\text { Immersion } \\
\text { Effective } \\
\text { Dose Rate } \\
\text { (mrem/sec } \\
\text { per } \\
\mu \mathrm{Ci} / \mathbf{c m}^{3} \text { ) }\end{array}$ & $\begin{array}{c}\text { Water } \\
\text { Submersion } \\
\text { Dose Rate } \\
\text { Coefficient } \\
\left(\mathbf{S v}-\mathbf{m}^{3} / \mathbf{B q}-\right. \\
\text { s) }\end{array}$ & $\begin{array}{c}\text { Water } \\
\text { Submersion } \\
\text { Dose Rate } \\
\text { Coefficient } \\
\text { (mrem- } \\
\left.\text { m³ }^{\mathbf{3}} / \mathbf{p C i}-\mathbf{h}\right)\end{array}$ & $\begin{array}{c}\text { Water } \\
\text { Submersion } \\
\text { Dose Rate } \\
\text { Coefficient } \\
(\text { mrem- } \\
\left.\mathbf{m}^{3} / \mu \mathrm{Ci}-\mathrm{y}\right)\end{array}$ \\
\hline Tc-104 & 18.3 & $\mathrm{~m}$ & $1.10 \mathrm{E}-13$ & $1.28 \mathrm{E}-02$ & $4.07 \mathrm{E}+02$ & $2.37 \mathrm{E}-16$ & $3.16 \mathrm{E}-09$ & $2.77 \mathrm{E}-11$ \\
\hline Tc-105 & 7.6 & $\mathrm{~m}$ & $3.77 \mathrm{E}-14$ & $4.40 \mathrm{E}-03$ & $1.39 \mathrm{E}+02$ & $8.10 \mathrm{E}-17$ & $1.08 \mathrm{E}-09$ & $9.45 \mathrm{E}-12$ \\
\hline Ru-92 & 3.65 & $\mathrm{~m}$ & $9.44 \mathrm{E}-14$ & $1.10 \mathrm{E}-02$ & $3.49 \mathrm{E}+02$ & $2.05 \mathrm{E}-16$ & $2.73 \mathrm{E}-09$ & $2.39 \mathrm{E}-11$ \\
\hline Ru-94 & 51.8 & $\mathrm{~m}$ & $2.28 \mathrm{E}-14$ & $2.66 \mathrm{E}-03$ & $8.44 \mathrm{E}+01$ & $4.96 \mathrm{E}-17$ & $6.61 \mathrm{E}-10$ & $5.79 \mathrm{E}-12$ \\
\hline Ru-95 & 1.643 & $\mathrm{~h}$ & $5.68 \mathrm{E}-14$ & $6.63 \mathrm{E}-03$ & $2.10 \mathrm{E}+02$ & $1.23 \mathrm{E}-16$ & $1.64 \mathrm{E}-09$ & $1.44 \mathrm{E}-11$ \\
\hline Ru-97 & 2.9 & $\mathrm{~d}$ & $9.96 \mathrm{E}-15$ & $1.16 \mathrm{E}-03$ & $3.69 \mathrm{E}+01$ & $2.18 \mathrm{E}-17$ & $2.90 \mathrm{E}-10$ & $2.54 \mathrm{E}-12$ \\
\hline Ru-103 & 39.26 & $\mathrm{~d}$ & $2.21 \mathrm{E}-14$ & $2.58 \mathrm{E}-03$ & $8.18 \mathrm{E}+01$ & $4.80 \mathrm{E}-17$ & $6.39 \mathrm{E}-10$ & $5.60 \mathrm{E}-12$ \\
\hline Ru-105 & 4.44 & $\mathrm{~h}$ & $3.39 \mathrm{E}-14$ & $3.96 \mathrm{E}-03$ & $1.25 \mathrm{E}+02$ & $7.34 \mathrm{E}-17$ & $9.78 \mathrm{E}-10$ & $8.56 \mathrm{E}-12$ \\
\hline Ru-106 & 373.59 & $\mathrm{~d}$ & $0.00 \mathrm{E}+00$ & $0.00 \mathrm{E}+00$ & $0.00 \mathrm{E}+00$ & $0.00 \mathrm{E}+00$ & $0.00 \mathrm{E}+00$ & $0.00 \mathrm{E}+00$ \\
\hline Ru-107 & 3.75 & $\mathrm{~m}$ & $1.69 \mathrm{E}-14$ & $1.97 \mathrm{E}-03$ & $6.25 \mathrm{E}+01$ & $3.57 \mathrm{E}-17$ & $4.76 \mathrm{E}-10$ & $4.17 \mathrm{E}-12$ \\
\hline Ru-108 & 4.55 & $\mathrm{~m}$ & $2.92 \mathrm{E}-15$ & $3.41 \mathrm{E}-04$ & $1.08 \mathrm{E}+01$ & $6.10 \mathrm{E}-18$ & $8.13 \mathrm{E}-11$ & $7.12 \mathrm{E}-13$ \\
\hline Rh-94 & 70.6 & $\mathrm{~s}$ & $1.81 \mathrm{E}-13$ & $2.11 \mathrm{E}-02$ & $6.70 \mathrm{E}+02$ & $3.89 \mathrm{E}-16$ & $5.18 \mathrm{E}-09$ & $4.54 \mathrm{E}-11$ \\
\hline Rh-95 & 5.02 & $\mathrm{~m}$ & $1.21 \mathrm{E}-13$ & $1.41 \mathrm{E}-02$ & $4.48 \mathrm{E}+02$ & $2.62 \mathrm{E}-16$ & $3.49 \mathrm{E}-09$ & $3.06 \mathrm{E}-11$ \\
\hline Rh-95m & 1.96 & $\mathrm{~m}$ & $4.29 \mathrm{E}-14$ & $5.01 \mathrm{E}-03$ & $1.59 \mathrm{E}+02$ & $9.31 \mathrm{E}-17$ & $1.24 \mathrm{E}-09$ & $1.09 \mathrm{E}-11$ \\
\hline Rh-96 & 9.9 & $\mathrm{~m}$ & $1.81 \mathrm{E}-13$ & $2.11 \mathrm{E}-02$ & $6.70 \mathrm{E}+02$ & $3.93 \mathrm{E}-16$ & $5.23 \mathrm{E}-09$ & $4.59 \mathrm{E}-11$ \\
\hline Rh-96m & 1.51 & $\mathrm{~m}$ & $6.03 \mathrm{E}-14$ & $7.04 \mathrm{E}-03$ & $2.23 \mathrm{E}+02$ & $1.30 \mathrm{E}-16$ & $1.73 \mathrm{E}-09$ & $1.52 \mathrm{E}-11$ \\
\hline Rh-97 & 30.7 & $\mathrm{~m}$ & $6.60 \mathrm{E}-14$ & $7.71 \mathrm{E}-03$ & $2.44 \mathrm{E}+02$ & $1.43 \mathrm{E}-16$ & $1.90 \mathrm{E}-09$ & $1.67 \mathrm{E}-11$ \\
\hline Rh-97m & 46.2 & $\mathrm{~m}$ & $1.06 \mathrm{E}-13$ & $1.24 \mathrm{E}-02$ & $3.92 \mathrm{E}+02$ & $2.31 \mathrm{E}-16$ & $3.08 \mathrm{E}-09$ & $2.70 \mathrm{E}-11$ \\
\hline Rh-98 & 8.7 & $\mathrm{~m}$ & $8.34 \mathrm{E}-14$ & $9.74 \mathrm{E}-03$ & $3.09 \mathrm{E}+02$ & $1.80 \mathrm{E}-16$ & $2.40 \mathrm{E}-09$ & $2.10 \mathrm{E}-11$ \\
\hline Rh-99 & 16.1 & $\mathrm{~d}$ & $2.42 \mathrm{E}-14$ & $2.83 \mathrm{E}-03$ & $8.95 \mathrm{E}+01$ & $5.28 \mathrm{E}-17$ & $7.03 \mathrm{E}-10$ & $6.16 \mathrm{E}-12$ \\
\hline Rh-99m & 4.7 & $\mathrm{~h}$ & $2.89 \mathrm{E}-14$ & $3.37 \mathrm{E}-03$ & $1.07 \mathrm{E}+02$ & $6.28 \mathrm{E}-17$ & $8.36 \mathrm{E}-10$ & $7.33 \mathrm{E}-12$ \\
\hline Rh-100 & 20.8 & $\mathrm{~h}$ & $1.32 \mathrm{E}-13$ & $1.54 \mathrm{E}-02$ & $4.88 \mathrm{E}+02$ & $2.85 \mathrm{E}-16$ & $3.80 \mathrm{E}-09$ & $3.33 \mathrm{E}-11$ \\
\hline Rh-100m & 4.6 & $\mathrm{~m}$ & $2.03 \mathrm{E}-15$ & $2.37 \mathrm{E}-04$ & $7.51 \mathrm{E}+00$ & $4.43 \mathrm{E}-18$ & $5.90 \mathrm{E}-11$ & $5.17 \mathrm{E}-13$ \\
\hline
\end{tabular}




\begin{tabular}{|c|c|c|c|c|c|c|c|c|}
\hline Nuclide & Half-life & Class & $\begin{array}{c}\text { Air } \\
\text { Immersion } \\
\text { Dose Rate } \\
\text { Coefficient } \\
(\mathrm{Sv} / \mathrm{s} \mathrm{per} \\
\left.\mathrm{Bq} / \mathbf{m}^{\mathbf{3}}\right)\end{array}$ & $\begin{array}{c}\text { Air } \\
\text { Immersion } \\
\text { Dose Rate } \\
\text { Coefficient } \\
\text { (mrem- } \\
\left.\text { m³ }^{3} / \mathrm{pCi}-\mathrm{yr}\right)\end{array}$ & $\begin{array}{c}\text { Air } \\
\text { Immersion } \\
\text { Effective } \\
\text { Dose Rate } \\
(\mathrm{mrem} / \mathrm{sec} \\
\mathrm{per} \\
\left.\mu \mathrm{Ci} / \mathrm{cm}^{3}\right)\end{array}$ & $\begin{array}{c}\text { Water } \\
\text { Submersion } \\
\text { Dose Rate } \\
\text { Coefficient } \\
\left(\mathrm{Sv}-\mathbf{m}^{3} / \mathbf{B q}-\right. \\
\text { s) }\end{array}$ & $\begin{array}{c}\text { Water } \\
\text { Submersion } \\
\text { Dose Rate } \\
\text { Coefficient } \\
\text { (mrem- } \\
\text { m³Ci-h) }^{\mathbf{3} / p C i}\end{array}$ & $\begin{array}{c}\text { Water } \\
\text { Submersion } \\
\text { Dose Rate } \\
\text { Coefficient } \\
(\text { mrem- } \\
\left.\text { m }^{3} / \mu \mathrm{Ci}-\mathrm{y}\right)\end{array}$ \\
\hline Rh-101 & 3.3 & $\mathrm{y}$ & $1.17 \mathrm{E}-14$ & $1.37 \mathrm{E}-03$ & $4.33 \mathrm{E}+01$ & $2.58 \mathrm{E}-17$ & $3.44 \mathrm{E}-10$ & $3.01 \mathrm{E}-12$ \\
\hline Rh-101m & 4.34 & $\mathrm{~d}$ & $1.21 \mathrm{E}-14$ & $1.41 \mathrm{E}-03$ & $4.48 \mathrm{E}+01$ & $2.64 \mathrm{E}-17$ & $3.52 \mathrm{E}-10$ & $3.08 \mathrm{E}-12$ \\
\hline Rh-102 & 207 & $\mathrm{~d}$ & $2.24 \mathrm{E}-14$ & $2.62 \mathrm{E}-03$ & $8.29 \mathrm{E}+01$ & $4.86 \mathrm{E}-17$ & $6.47 \mathrm{E}-10$ & $5.67 \mathrm{E}-12$ \\
\hline Rh-102m & 3.742 & $\mathrm{y}$ & $9.74 \mathrm{E}-14$ & $1.14 \mathrm{E}-02$ & $3.60 \mathrm{E}+02$ & $2.11 \mathrm{E}-16$ & $2.81 \mathrm{E}-09$ & $2.46 \mathrm{E}-11$ \\
\hline Rh-103m & 56.114 & $\mathrm{~m}$ & 5.61E-18 & $6.55 \mathrm{E}-07$ & $2.08 \mathrm{E}-02$ & $1.32 \mathrm{E}-20$ & $1.76 \mathrm{E}-13$ & $1.54 \mathrm{E}-15$ \\
\hline Rh-104 & 42.3 & $\mathrm{~s}$ & $1.40 \mathrm{E}-15$ & $1.63 \mathrm{E}-04$ & $5.18 \mathrm{E}+00$ & $2.26 \mathrm{E}-18$ & $3.01 \mathrm{E}-11$ & $2.64 \mathrm{E}-13$ \\
\hline Rh-104m & 4.34 & $\mathrm{~m}$ & $9.14 \mathrm{E}-16$ & $1.07 \mathrm{E}-04$ & $3.38 \mathrm{E}+00$ & $2.09 \mathrm{E}-18$ & $2.78 \mathrm{E}-11$ & $2.44 \mathrm{E}-13$ \\
\hline Rh-105 & 35.36 & $\mathrm{~h}$ & $3.47 \mathrm{E}-15$ & $4.05 \mathrm{E}-04$ & $1.28 \mathrm{E}+01$ & $7.49 \mathrm{E}-18$ & $9.98 \mathrm{E}-11$ & $8.74 \mathrm{E}-13$ \\
\hline Rh-106 & 29.8 & $\mathrm{~s}$ & $1.07 \mathrm{E}-14$ & $1.25 \mathrm{E}-03$ & $3.96 \mathrm{E}+01$ & $2.20 \mathrm{E}-17$ & $2.93 \mathrm{E}-10$ & $2.57 \mathrm{E}-12$ \\
\hline Rh-106m & 131 & $\mathrm{~m}$ & $1.32 \mathrm{E}-13$ & $1.54 \mathrm{E}-02$ & $4.88 \mathrm{E}+02$ & $2.86 \mathrm{E}-16$ & $3.81 \mathrm{E}-09$ & $3.34 \mathrm{E}-11$ \\
\hline Rh-107 & 21.7 & $\mathrm{~m}$ & $1.41 \mathrm{E}-14$ & $1.65 \mathrm{E}-03$ & $5.22 \mathrm{E}+01$ & $3.05 \mathrm{E}-17$ & $4.06 \mathrm{E}-10$ & $3.56 \mathrm{E}-12$ \\
\hline Rh-108 & 16.8 & $\mathrm{~s}$ & $1.61 \mathrm{E}-14$ & $1.88 \mathrm{E}-03$ & $5.96 \mathrm{E}+01$ & $3.34 \mathrm{E}-17$ & $4.45 \mathrm{E}-10$ & $3.90 \mathrm{E}-12$ \\
\hline Rh-109 & 80 & $\mathrm{~s}$ & $1.38 \mathrm{E}-14$ & $1.61 \mathrm{E}-03$ & $5.11 \mathrm{E}+01$ & $2.95 \mathrm{E}-17$ & $3.93 \mathrm{E}-10$ & $3.44 \mathrm{E}-12$ \\
\hline Pd-96 & 122 & $\mathrm{~s}$ & $6.51 \mathrm{E}-14$ & $7.60 \mathrm{E}-03$ & $2.41 \mathrm{E}+02$ & $1.41 \mathrm{E}-16$ & $1.88 \mathrm{E}-09$ & $1.65 \mathrm{E}-11$ \\
\hline Pd-97 & 3.1 & $\mathrm{~m}$ & $1.12 \mathrm{E}-13$ & $1.31 \mathrm{E}-02$ & $4.14 \mathrm{E}+02$ & $2.43 \mathrm{E}-16$ & $3.24 \mathrm{E}-09$ & $2.84 \mathrm{E}-11$ \\
\hline Pd-98 & 17.7 & $\mathrm{~m}$ & $1.76 \mathrm{E}-14$ & $2.06 \mathrm{E}-03$ & $6.51 \mathrm{E}+01$ & $3.85 \mathrm{E}-17$ & $5.13 \mathrm{E}-10$ & $4.49 \mathrm{E}-12$ \\
\hline Pd-99 & 21.4 & $\mathrm{~m}$ & $5.88 \mathrm{E}-14$ & $6.87 \mathrm{E}-03$ & $2.18 \mathrm{E}+02$ & $1.28 \mathrm{E}-16$ & $1.70 \mathrm{E}-09$ & $1.49 \mathrm{E}-11$ \\
\hline $\mathrm{Pd}-100$ & 3.63 & $\mathrm{~d}$ & $3.64 \mathrm{E}-15$ & $4.25 \mathrm{E}-04$ & $1.35 \mathrm{E}+01$ & $8.19 \mathrm{E}-18$ & $1.09 \mathrm{E}-10$ & $9.56 \mathrm{E}-13$ \\
\hline $\mathrm{Pd}-101$ & 8.47 & $\mathrm{~h}$ & $1.48 \mathrm{E}-14$ & $1.73 \mathrm{E}-03$ & $5.48 \mathrm{E}+01$ & $3.22 \mathrm{E}-17$ & $4.29 \mathrm{E}-10$ & $3.76 \mathrm{E}-12$ \\
\hline $\mathrm{Pd}-103$ & 16.991 & $\mathrm{~d}$ & $5.29 \mathrm{E}-17$ & $6.18 \mathrm{E}-06$ & $1.96 \mathrm{E}-01$ & $1.24 \mathrm{E}-19$ & $1.65 \mathrm{E}-12$ & $1.45 \mathrm{E}-14$ \\
\hline $\mathrm{Pd}-107$ & $6.50 \mathrm{E}+06$ & $\mathrm{y}$ & $0.00 \mathrm{E}+00$ & $0.00 \mathrm{E}+00$ & $0.00 \mathrm{E}+00$ & $0.00 \mathrm{E}+00$ & $0.00 \mathrm{E}+00$ & $0.00 \mathrm{E}+00$ \\
\hline Pd-109 & 13.7012 & $\mathrm{~h}$ & $4.20 \mathrm{E}-16$ & 4.90E-05 & $1.55 \mathrm{E}+00$ & $6.88 \mathrm{E}-19$ & $9.16 \mathrm{E}-12$ & $8.03 \mathrm{E}-14$ \\
\hline Pd-109m & 4.69 & $\mathrm{~m}$ & $4.59 \mathrm{E}-15$ & $5.36 \mathrm{E}-04$ & $1.70 \mathrm{E}+01$ & $1.01 \mathrm{E}-17$ & $1.35 \mathrm{E}-10$ & $1.18 \mathrm{E}-12$ \\
\hline
\end{tabular}




\begin{tabular}{|c|c|c|c|c|c|c|c|c|}
\hline Nuclide & Half-life & Class & $\begin{array}{c}\text { Air } \\
\text { Immersion } \\
\text { Dose Rate } \\
\text { Coefficient } \\
(\text { Sv/s per } \\
\left.\text { Bq/ } \mathbf{m}^{3}\right)\end{array}$ & $\begin{array}{c}\text { Air } \\
\text { Immersion } \\
\text { Dose Rate } \\
\text { Coefficient } \\
\text { (mrem- } \\
\text { m³Ci-yr) }^{3} / \mathrm{pCi}\end{array}$ & $\begin{array}{c}\text { Air } \\
\text { Immersion } \\
\text { Effective } \\
\text { Dose Rate } \\
(\mathrm{mrem} / \mathrm{sec} \\
\mathrm{per} \\
\left.\mu \mathrm{Ci} / \mathrm{cm}^{3}\right)\end{array}$ & $\begin{array}{c}\text { Water } \\
\text { Submersion } \\
\text { Dose Rate } \\
\text { Coefficient } \\
\left(\mathrm{Sv}-\mathbf{m}^{3} / \mathbf{B q}-\right. \\
\text { s) }\end{array}$ & $\begin{array}{c}\text { Water } \\
\text { Submersion } \\
\text { Dose Rate } \\
\text { Coefficient } \\
\text { (mrem- } \\
\text { m³Ci-h) }^{\mathbf{3} / p C i}\end{array}$ & $\begin{array}{c}\text { Water } \\
\text { Submersion } \\
\text { Dose Rate } \\
\text { Coefficient } \\
(\text { mrem- } \\
\left.\text { m }^{3} / \mu \mathrm{Ci}-\mathrm{y}\right)\end{array}$ \\
\hline Pd-111 & 23.4 & $\mathrm{~m}$ & $2.89 \mathrm{E}-15$ & $3.37 \mathrm{E}-04$ & $1.07 \mathrm{E}+01$ & $5.62 \mathrm{E}-18$ & $7.49 \mathrm{E}-11$ & $6.56 \mathrm{E}-13$ \\
\hline $\mathrm{Pd}-112$ & 21.03 & $\mathrm{~h}$ & $2.68 \mathrm{E}-17$ & $3.13 \mathrm{E}-06$ & $9.92 \mathrm{E}-02$ & $4.33 \mathrm{E}-20$ & $5.77 \mathrm{E}-13$ & $5.05 \mathrm{E}-15$ \\
\hline $\mathrm{Pd}-114$ & 2.42 & $\mathrm{~m}$ & $1.49 \mathrm{E}-15$ & $1.74 \mathrm{E}-04$ & $5.51 \mathrm{E}+00$ & $2.89 \mathrm{E}-18$ & $3.85 \mathrm{E}-11$ & $3.37 \mathrm{E}-13$ \\
\hline Ag-99 & 124 & $\mathrm{~s}$ & $1.08 \mathrm{E}-13$ & $1.26 \mathrm{E}-02$ & $4.00 \mathrm{E}+02$ & $2.33 \mathrm{E}-16$ & $3.10 \mathrm{E}-09$ & $2.72 \mathrm{E}-11$ \\
\hline $\mathrm{Ag}-100 \mathrm{~m}$ & 2.24 & $\mathrm{~m}$ & $1.33 \mathrm{E}-13$ & $1.55 \mathrm{E}-02$ & $4.92 \mathrm{E}+02$ & $2.87 \mathrm{E}-16$ & $3.82 \mathrm{E}-09$ & $3.35 \mathrm{E}-11$ \\
\hline Ag-101 & 11.1 & $\mathrm{~m}$ & $7.19 \mathrm{E}-14$ & $8.40 \mathrm{E}-03$ & $2.66 \mathrm{E}+02$ & $1.56 \mathrm{E}-16$ & $2.08 \mathrm{E}-09$ & $1.82 \mathrm{E}-11$ \\
\hline Ag-102 & 12.9 & $\mathrm{~m}$ & $1.60 \mathrm{E}-13$ & $1.87 \mathrm{E}-02$ & $5.92 \mathrm{E}+02$ & $3.47 \mathrm{E}-16$ & $4.62 \mathrm{E}-09$ & $4.05 \mathrm{E}-11$ \\
\hline Ag-102m & 7.7 & $\mathrm{~m}$ & $9.72 \mathrm{E}-14$ & $1.13 \mathrm{E}-02$ & $3.60 \mathrm{E}+02$ & $2.10 \mathrm{E}-16$ & $2.80 \mathrm{E}-09$ & $2.45 \mathrm{E}-11$ \\
\hline Ag-103 & 65.7 & $\mathrm{~m}$ & $3.79 \mathrm{E}-14$ & $4.43 \mathrm{E}-03$ & $1.40 \mathrm{E}+02$ & $8.23 \mathrm{E}-17$ & $1.10 \mathrm{E}-09$ & $9.60 \mathrm{E}-12$ \\
\hline Ag-104 & 69.2 & $\mathrm{~m}$ & $1.24 \mathrm{E}-13$ & $1.45 \mathrm{E}-02$ & $4.59 \mathrm{E}+02$ & $2.70 \mathrm{E}-16$ & $3.60 \mathrm{E}-09$ & $3.15 \mathrm{E}-11$ \\
\hline Ag-104m & 33.5 & $\mathrm{~m}$ & $8.45 \mathrm{E}-14$ & $9.87 \mathrm{E}-03$ & $3.13 \mathrm{E}+02$ & $1.83 \mathrm{E}-16$ & $2.44 \mathrm{E}-09$ & $2.14 \mathrm{E}-11$ \\
\hline Ag-105 & 41.29 & $\mathrm{~d}$ & $2.21 \mathrm{E}-14$ & $2.58 \mathrm{E}-03$ & $8.18 \mathrm{E}+01$ & $4.81 \mathrm{E}-17$ & $6.41 \mathrm{E}-10$ & $5.61 \mathrm{E}-12$ \\
\hline Ag-105m & 7.23 & $\mathrm{~m}$ & $4.42 \mathrm{E}-17$ & $5.16 \mathrm{E}-06$ & $1.64 \mathrm{E}-01$ & $9.63 \mathrm{E}-20$ & $1.28 \mathrm{E}-12$ & $1.12 \mathrm{E}-14$ \\
\hline Ag-106 & 23.96 & $\mathrm{~m}$ & $3.13 \mathrm{E}-14$ & $3.65 \mathrm{E}-03$ & $1.16 \mathrm{E}+02$ & $6.77 \mathrm{E}-17$ & $9.02 \mathrm{E}-10$ & $7.90 \mathrm{E}-12$ \\
\hline $\mathrm{Ag}-106 \mathrm{~m}$ & 8.28 & $\mathrm{~d}$ & $1.29 \mathrm{E}-13$ & $1.51 \mathrm{E}-02$ & $4.77 \mathrm{E}+02$ & $2.79 \mathrm{E}-16$ & $3.72 \mathrm{E}-09$ & $3.26 \mathrm{E}-11$ \\
\hline Ag-108 & 2.37 & $\mathrm{~m}$ & $1.27 \mathrm{E}-15$ & $1.48 \mathrm{E}-04$ & $4.70 \mathrm{E}+00$ & $2.32 \mathrm{E}-18$ & $3.09 \mathrm{E}-11$ & $2.71 \mathrm{E}-13$ \\
\hline Ag-108m & 418 & $\mathrm{y}$ & $7.23 \mathrm{E}-14$ & $8.44 \mathrm{E}-03$ & $2.68 \mathrm{E}+02$ & $1.57 \mathrm{E}-16$ & $2.09 \mathrm{E}-09$ & $1.83 \mathrm{E}-11$ \\
\hline Ag-109m & 39.6 & $\mathrm{~s}$ & $1.58 \mathrm{E}-16$ & $1.84 \mathrm{E}-05$ & $5.85 \mathrm{E}-01$ & $3.56 \mathrm{E}-19$ & $4.74 \mathrm{E}-12$ & $4.15 \mathrm{E}-14$ \\
\hline Ag-110 & 24.6 & $\mathrm{~s}$ & $2.46 \mathrm{E}-15$ & $2.87 \mathrm{E}-04$ & $9.10 \mathrm{E}+00$ & $4.38 \mathrm{E}-18$ & $5.83 \mathrm{E}-11$ & $5.11 \mathrm{E}-13$ \\
\hline $\mathrm{Ag}-110 \mathrm{~m}$ & 249.76 & $\mathrm{~d}$ & $1.28 \mathrm{E}-13$ & $1.49 \mathrm{E}-02$ & $4.74 \mathrm{E}+02$ & $2.77 \mathrm{E}-16$ & $3.69 \mathrm{E}-09$ & $3.23 \mathrm{E}-11$ \\
\hline Ag-111 & 7.45 & $\mathrm{~d}$ & $1.39 \mathrm{E}-15$ & $1.62 \mathrm{E}-04$ & $5.14 \mathrm{E}+00$ & $2.80 \mathrm{E}-18$ & $3.73 \mathrm{E}-11$ & $3.27 \mathrm{E}-13$ \\
\hline Ag-111m & 64.8 & $\mathrm{~s}$ & $1.68 \mathrm{E}-16$ & $1.96 \mathrm{E}-05$ & $6.22 \mathrm{E}-01$ & $3.71 \mathrm{E}-19$ & $4.94 \mathrm{E}-12$ & $4.33 \mathrm{E}-14$ \\
\hline Ag-112 & 3.13 & $\mathrm{~h}$ & $3.39 \mathrm{E}-14$ & $3.96 \mathrm{E}-03$ & $1.25 \mathrm{E}+02$ & $7.24 \mathrm{E}-17$ & $9.64 \mathrm{E}-10$ & $8.45 \mathrm{E}-12$ \\
\hline
\end{tabular}




\begin{tabular}{|c|c|c|c|c|c|c|c|c|}
\hline Nuclide & Half-life & Class & $\begin{array}{c}\text { Air } \\
\text { Immersion } \\
\text { Dose Rate } \\
\text { Coefficient } \\
\left(\mathrm{Sv} / \mathrm{s} \mathrm{per}^{\mathbf{3}}\right. \\
\left.\mathrm{Bq} / \mathbf{m}^{\mathbf{3}}\right)\end{array}$ & $\begin{array}{c}\text { Air } \\
\text { Immersion } \\
\text { Dose Rate } \\
\text { Coefficient } \\
\text { (mrem- } \\
\left.\mathrm{m}^{3} / \mathrm{pCi}-\mathrm{yr}\right)\end{array}$ & $\begin{array}{c}\text { Air } \\
\text { Immersion } \\
\text { Effective } \\
\text { Dose Rate } \\
\text { (mrem/sec } \\
\text { per } \\
\mu \mathrm{Ci} / \mathrm{cm}^{3} \text { ) }\end{array}$ & $\begin{array}{c}\text { Water } \\
\text { Submersion } \\
\text { Dose Rate } \\
\text { Coefficient } \\
\left(\mathbf{S v}-\mathbf{m}^{3} / \mathbf{B q}-\right. \\
\text { s) }\end{array}$ & $\begin{array}{c}\text { Water } \\
\text { Submersion } \\
\text { Dose Rate } \\
\text { Coefficient } \\
\text { (mrem- } \\
\mathbf{m}^{\mathbf{3}} / \mathbf{p C i} \text {-h) }\end{array}$ & $\begin{array}{c}\text { Water } \\
\text { Submersion } \\
\text { Dose Rate } \\
\text { Coefficient } \\
(\text { mrem- } \\
\left.\text { m³ }^{\mathbf{3}} / \boldsymbol{\mu C i}-\mathbf{y}\right)\end{array}$ \\
\hline Ag-113 & 5.37 & $\mathrm{~h}$ & $3.83 \mathrm{E}-15$ & $4.47 \mathrm{E}-04$ & $1.42 \mathrm{E}+01$ & $7.75 \mathrm{E}-18$ & $1.03 \mathrm{E}-10$ & $9.04 \mathrm{E}-13$ \\
\hline $\mathrm{Ag}-113 \mathrm{~m}$ & 68.7 & $\mathrm{~s}$ & $9.55 \mathrm{E}-15$ & $1.12 \mathrm{E}-03$ & $3.53 \mathrm{E}+01$ & $2.07 \mathrm{E}-17$ & $2.76 \mathrm{E}-10$ & $2.42 \mathrm{E}-12$ \\
\hline Ag-114 & 4.6 & $\mathrm{~s}$ & $1.46 \mathrm{E}-14$ & $1.70 \mathrm{E}-03$ & $5.40 \mathrm{E}+01$ & $2.97 \mathrm{E}-17$ & $3.96 \mathrm{E}-10$ & $3.47 \mathrm{E}-12$ \\
\hline Ag-115 & 20 & $\mathrm{~m}$ & $2.40 \mathrm{E}-14$ & $2.80 \mathrm{E}-03$ & $8.88 \mathrm{E}+01$ & $5.12 \mathrm{E}-17$ & $6.82 \mathrm{E}-10$ & $5.97 \mathrm{E}-12$ \\
\hline Ag-116 & 2.68 & $\mathrm{~m}$ & $1.07 \mathrm{E}-13$ & $1.25 \mathrm{E}-02$ & $3.96 \mathrm{E}+02$ & $2.29 \mathrm{E}-16$ & $3.05 \mathrm{E}-09$ & $2.67 \mathrm{E}-11$ \\
\hline Ag-117 & 73.6 & $\mathrm{~s}$ & $6.50 \mathrm{E}-14$ & $7.59 \mathrm{E}-03$ & $2.41 \mathrm{E}+02$ & $1.40 \mathrm{E}-16$ & $1.86 \mathrm{E}-09$ & $1.63 \mathrm{E}-11$ \\
\hline $\mathrm{Cd}-101$ & 1.36 & $\mathrm{~m}$ & $1.17 \mathrm{E}-13$ & $1.37 \mathrm{E}-02$ & $4.33 \mathrm{E}+02$ & $2.53 \mathrm{E}-16$ & $3.37 \mathrm{E}-09$ & $2.95 \mathrm{E}-11$ \\
\hline $\mathrm{Cd}-102$ & 5.5 & $\mathrm{~m}$ & $3.73 \mathrm{E}-14$ & $4.36 \mathrm{E}-03$ & $1.38 \mathrm{E}+02$ & $8.10 \mathrm{E}-17$ & $1.08 \mathrm{E}-09$ & $9.45 \mathrm{E}-12$ \\
\hline $\mathrm{Cd}-103$ & 7.3 & $\mathrm{~m}$ & $1.00 \mathrm{E}-13$ & $1.17 \mathrm{E}-02$ & $3.70 \mathrm{E}+02$ & $2.17 \mathrm{E}-16$ & $2.89 \mathrm{E}-09$ & $2.53 \mathrm{E}-11$ \\
\hline Cd-104 & 57.7 & $\mathrm{~m}$ & $1.01 \mathrm{E}-14$ & $1.18 \mathrm{E}-03$ & $3.74 \mathrm{E}+01$ & $2.21 \mathrm{E}-17$ & $2.94 \mathrm{E}-10$ & $2.58 \mathrm{E}-12$ \\
\hline $\mathrm{Cd}-105$ & 55.5 & $\mathrm{~m}$ & $6.13 \mathrm{E}-14$ & $7.16 \mathrm{E}-03$ & $2.27 \mathrm{E}+02$ & $1.33 \mathrm{E}-16$ & $1.77 \mathrm{E}-09$ & $1.55 \mathrm{E}-11$ \\
\hline Cd-107 & 6.5 & $\mathrm{~h}$ & $4.90 \mathrm{E}-16$ & $5.72 \mathrm{E}-05$ & $1.81 \mathrm{E}+00$ & $1.09 \mathrm{E}-18$ & $1.45 \mathrm{E}-11$ & $1.27 \mathrm{E}-13$ \\
\hline Cd-109 & 461.4 & $\mathrm{~d}$ & $2.27 \mathrm{E}-16$ & $2.65 \mathrm{E}-05$ & $8.40 \mathrm{E}-01$ & $5.20 \mathrm{E}-19$ & $6.93 \mathrm{E}-12$ & $6.07 \mathrm{E}-14$ \\
\hline $\mathrm{Cd}-111 \mathrm{~m}$ & 48.5 & $\mathrm{~m}$ & $1.20 \mathrm{E}-14$ & $1.40 \mathrm{E}-03$ & $4.44 \mathrm{E}+01$ & $2.64 \mathrm{E}-17$ & $3.52 \mathrm{E}-10$ & $3.08 \mathrm{E}-12$ \\
\hline Cd-113 & $7.70 \mathrm{E}+15$ & $\mathrm{y}$ & $2.49 \mathrm{E}-17$ & $2.91 \mathrm{E}-06$ & $9.21 \mathrm{E}-02$ & $2.72 \mathrm{E}-20$ & $3.62 \mathrm{E}-13$ & $3.17 \mathrm{E}-15$ \\
\hline Cd-113m & 14.1 & $\mathrm{y}$ & $9.28 \mathrm{E}-17$ & $1.08 \mathrm{E}-05$ & $3.43 \mathrm{E}-01$ & $1.06 \mathrm{E}-19$ & $1.41 \mathrm{E}-12$ & $1.24 \mathrm{E}-14$ \\
\hline Cd-115 & 53.46 & $\mathrm{~h}$ & $8.73 \mathrm{E}-15$ & $1.02 \mathrm{E}-03$ & $3.23 \mathrm{E}+01$ & $1.88 \mathrm{E}-17$ & $2.50 \mathrm{E}-10$ & $2.19 \mathrm{E}-12$ \\
\hline $\mathrm{Cd}-115 \mathrm{~m}$ & 44.6 & $\mathrm{~d}$ & $1.99 \mathrm{E}-15$ & $2.32 \mathrm{E}-04$ & $7.36 \mathrm{E}+00$ & $3.87 \mathrm{E}-18$ & $5.15 \mathrm{E}-11$ & $4.52 \mathrm{E}-13$ \\
\hline Cd-117 & 2.49 & $\mathrm{~h}$ & $5.11 \mathrm{E}-14$ & $5.97 \mathrm{E}-03$ & $1.89 \mathrm{E}+02$ & $1.10 \mathrm{E}-16$ & $1.47 \mathrm{E}-09$ & $1.28 \mathrm{E}-11$ \\
\hline
\end{tabular}




\begin{tabular}{|c|c|c|c|c|c|c|c|c|}
\hline Nuclide & Half-life & Class & 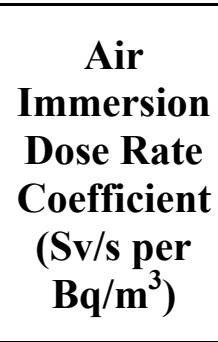 & $\begin{array}{c}\text { Air } \\
\text { Immersion } \\
\text { Dose Rate } \\
\text { Coefficient } \\
\text { (mrem- } \\
\text { m }^{3} / \text { pCi-yr) }\end{array}$ & $\begin{array}{c}\text { Air } \\
\text { Immersion } \\
\text { Effective } \\
\text { Dose Rate } \\
(\mathrm{mrem} / \mathrm{sec} \\
\mathrm{per} \\
\left.\mu \mathrm{Ci} / \mathrm{cm}^{3}\right)\end{array}$ & $\begin{array}{c}\text { Water } \\
\text { Submersion } \\
\text { Dose Rate } \\
\text { Coefficient } \\
\left(\mathrm{Sv}-\mathbf{m}^{3} / \mathbf{B q}-\right. \\
\text { s) }\end{array}$ & $\begin{array}{c}\text { Water } \\
\text { Submersion } \\
\text { Dose Rate } \\
\text { Coefficient } \\
\text { (mrem- } \\
\left.\text { m³ }^{\mathbf{3}} / \mathbf{p C i}-\mathbf{h}\right)\end{array}$ & $\begin{array}{c}\text { Water } \\
\text { Submersion } \\
\text { Dose Rate } \\
\text { Coefficient } \\
(\text { mrem- } \\
\left.\text { m }^{3} / \mu \mathrm{Ci}-\mathrm{y}\right)\end{array}$ \\
\hline $\mathrm{Cd}-117 \mathrm{~m}$ & 3.36 & $\mathrm{~h}$ & $9.88 \mathrm{E}-14$ & $1.15 \mathrm{E}-02$ & $3.66 \mathrm{E}+02$ & $2.14 \mathrm{E}-16$ & $2.85 \mathrm{E}-09$ & $2.50 \mathrm{E}-11$ \\
\hline Cd-118 & 50.3 & $\mathrm{~m}$ & $7.25 \mathrm{E}-17$ & $8.47 \mathrm{E}-06$ & $2.68 \mathrm{E}-01$ & $8.02 \mathrm{E}-20$ & $1.07 \mathrm{E}-12$ & $9.36 \mathrm{E}-15$ \\
\hline Cd-119 & 2.69 & $\mathrm{~m}$ & $7.96 \mathrm{E}-14$ & $9.29 \mathrm{E}-03$ & $2.95 \mathrm{E}+02$ & $1.72 \mathrm{E}-16$ & $2.29 \mathrm{E}-09$ & $2.01 \mathrm{E}-11$ \\
\hline Cd-119m & 2.2 & $\mathrm{~m}$ & $1.11 \mathrm{E}-13$ & $1.30 \mathrm{E}-02$ & $4.11 \mathrm{E}+02$ & $2.41 \mathrm{E}-16$ & $3.21 \mathrm{E}-09$ & $2.81 \mathrm{E}-11$ \\
\hline In-103 & 60 & $\mathrm{~s}$ & $1.30 \mathrm{E}-13$ & $1.52 \mathrm{E}-02$ & $4.81 \mathrm{E}+02$ & $2.82 \mathrm{E}-16$ & $3.76 \mathrm{E}-09$ & $3.29 \mathrm{E}-11$ \\
\hline In-105 & 5.07 & $\mathrm{~m}$ & $8.97 \mathrm{E}-14$ & $1.05 \mathrm{E}-02$ & $3.32 \mathrm{E}+02$ & $1.94 \mathrm{E}-16$ & $2.58 \mathrm{E}-09$ & $2.26 \mathrm{E}-11$ \\
\hline In-106m & 5.2 & $\mathrm{~m}$ & $1.35 \mathrm{E}-13$ & $1.58 \mathrm{E}-02$ & $5.00 \mathrm{E}+02$ & $2.92 \mathrm{E}-16$ & $3.89 \mathrm{E}-09$ & $3.41 \mathrm{E}-11$ \\
\hline In-106 & 6.2 & $\mathrm{~m}$ & $1.63 \mathrm{E}-13$ & $1.90 \mathrm{E}-02$ & $6.03 \mathrm{E}+02$ & $3.53 \mathrm{E}-16$ & $4.70 \mathrm{E}-09$ & $4.12 \mathrm{E}-11$ \\
\hline In-107 & 32.4 & $\mathrm{~m}$ & $7.18 \mathrm{E}-14$ & $8.38 \mathrm{E}-03$ & $2.66 \mathrm{E}+02$ & $1.56 \mathrm{E}-16$ & $2.08 \mathrm{E}-09$ & $1.82 \mathrm{E}-11$ \\
\hline In-108m & 39.6 & $\mathrm{~m}$ & $1.35 \mathrm{E}-13$ & $1.58 \mathrm{E}-02$ & $5.00 \mathrm{E}+02$ & $2.92 \mathrm{E}-16$ & $3.89 \mathrm{E}-09$ & $3.41 \mathrm{E}-11$ \\
\hline In-108 & 58 & $\mathrm{~m}$ & $1.81 \mathrm{E}-13$ & $2.11 \mathrm{E}-02$ & $6.70 \mathrm{E}+02$ & $3.93 \mathrm{E}-16$ & $5.23 \mathrm{E}-09$ & $4.59 \mathrm{E}-11$ \\
\hline In-109m & 1.34 & $\mathrm{~m}$ & $2.74 \mathrm{E}-14$ & $3.20 \mathrm{E}-03$ & $1.01 \mathrm{E}+02$ & $5.95 \mathrm{E}-17$ & $7.93 \mathrm{E}-10$ & $6.94 \mathrm{E}-12$ \\
\hline In-109 & 4.2 & $\mathrm{~h}$ & $2.86 \mathrm{E}-14$ & $3.34 \mathrm{E}-03$ & $1.06 \mathrm{E}+02$ & $6.23 \mathrm{E}-17$ & $8.30 \mathrm{E}-10$ & $7.27 \mathrm{E}-12$ \\
\hline In-110m & 69.1 & $\mathrm{~m}$ & $7.26 \mathrm{E}-14$ & $8.48 \mathrm{E}-03$ & $2.69 \mathrm{E}+02$ & $1.57 \mathrm{E}-16$ & $2.09 \mathrm{E}-09$ & $1.83 \mathrm{E}-11$ \\
\hline In-110 & 4.9 & $\mathrm{~h}$ & $1.41 \mathrm{E}-13$ & $1.65 \mathrm{E}-02$ & $5.22 \mathrm{E}+02$ & $3.06 \mathrm{E}-16$ & $4.08 \mathrm{E}-09$ & $3.57 \mathrm{E}-11$ \\
\hline In-111m & 7.7 & $\mathrm{~m}$ & $2.10 \mathrm{E}-14$ & $2.45 \mathrm{E}-03$ & $7.77 \mathrm{E}+01$ & $4.55 \mathrm{E}-17$ & $6.06 \mathrm{E}-10$ & $5.31 \mathrm{E}-12$ \\
\hline In-111 & 2.8047 & $\mathrm{~d}$ & $1.69 \mathrm{E}-14$ & $1.97 \mathrm{E}-03$ & $6.25 \mathrm{E}+01$ & $3.70 \mathrm{E}-17$ & $4.93 \mathrm{E}-10$ & $4.32 \mathrm{E}-12$ \\
\hline In-112m & 20.56 & $\mathrm{~m}$ & $9.86 \mathrm{E}-16$ & $1.15 \mathrm{E}-04$ & $3.65 \mathrm{E}+00$ & $2.15 \mathrm{E}-18$ & $2.86 \mathrm{E}-11$ & $2.51 \mathrm{E}-13$ \\
\hline In-112 & 14.97 & $\mathrm{~m}$ & $1.19 \mathrm{E}-14$ & $1.39 \mathrm{E}-03$ & $4.40 \mathrm{E}+01$ & $2.56 \mathrm{E}-17$ & $3.41 \mathrm{E}-10$ & $2.99 \mathrm{E}-12$ \\
\hline In-113m & 1.6579 & $\mathrm{~h}$ & $1.13 \mathrm{E}-14$ & $1.32 \mathrm{E}-03$ & $4.18 \mathrm{E}+01$ & $2.46 \mathrm{E}-17$ & $3.28 \mathrm{E}-10$ & $2.87 \mathrm{E}-12$ \\
\hline In-114m & 49.51 & $\mathrm{~d}$ & $3.26 \mathrm{E}-15$ & $3.81 \mathrm{E}-04$ & $1.21 \mathrm{E}+01$ & $7.07 \mathrm{E}-18$ & $9.42 \mathrm{E}-11$ & $8.25 \mathrm{E}-13$ \\
\hline In-114 & 71.9 & $\mathrm{~S}$ & $7.27 \mathrm{E}-16$ & $8.49 \mathrm{E}-05$ & $2.69 \mathrm{E}+00$ & $9.87 \mathrm{E}-19$ & $1.31 \mathrm{E}-11$ & $1.15 \mathrm{E}-13$ \\
\hline In-115m & 4.486 & $\mathrm{~h}$ & $6.94 \mathrm{E}-15$ & $8.10 \mathrm{E}-04$ & $2.57 \mathrm{E}+01$ & $1.50 \mathrm{E}-17$ & $2.00 \mathrm{E}-10$ & $1.75 \mathrm{E}-12$ \\
\hline
\end{tabular}




\begin{tabular}{|c|c|c|c|c|c|c|c|c|}
\hline Nuclide & Half-life & Class & $\begin{array}{c}\text { Air } \\
\text { Immersion } \\
\text { Dose Rate } \\
\text { Coefficient } \\
\left(\mathrm{Sv} / \mathbf{s ~ p e r ~}^{\mathbf{3}}\right. \\
\left.\mathrm{Bq} / \mathbf{m}^{\mathbf{3}}\right)\end{array}$ & $\begin{array}{c}\text { Air } \\
\text { Immersion } \\
\text { Dose Rate } \\
\text { Coefficient } \\
\text { (mrem- } \\
\left.\text { m³ }^{3} / \mathrm{pCi}-\mathrm{yr}\right)\end{array}$ & $\begin{array}{c}\text { Air } \\
\text { Immersion } \\
\text { Effective } \\
\text { Dose Rate } \\
\text { (mrem/sec } \\
\text { per } \\
\mu \mathrm{Ci} / \mathrm{cm}^{3} \text { ) } \\
\end{array}$ & $\begin{array}{c}\text { Water } \\
\text { Submersion } \\
\text { Dose Rate } \\
\text { Coefficient } \\
\left(\mathbf{S v}-\mathbf{m}^{3} / \mathbf{B q}-\right. \\
\text { s) }\end{array}$ & $\begin{array}{c}\text { Water } \\
\text { Submersion } \\
\text { Dose Rate } \\
\text { Coefficient } \\
\text { (mrem- } \\
\left.\text { m³ }^{\mathbf{3}} / \mathbf{p C i}-\mathbf{h}\right)\end{array}$ & $\begin{array}{c}\text { Water } \\
\text { Submersion } \\
\text { Dose Rate } \\
\text { Coefficient } \\
(\text { mrem- } \\
\left.\mathbf{m}^{3} / \mu \mathrm{Ci}-\mathrm{y}\right)\end{array}$ \\
\hline In-115 & $4.41 \mathrm{E}+14$ & $\mathrm{y}$ & $6.60 \mathrm{E}-17$ & $7.71 \mathrm{E}-06$ & $2.44 \mathrm{E}-01$ & $7.29 \mathrm{E}-20$ & $9.71 \mathrm{E}-13$ & $8.51 \mathrm{E}-15$ \\
\hline In-116m & 54.41 & $\mathrm{~m}$ & $1.18 \mathrm{E}-13$ & $1.38 \mathrm{E}-02$ & $4.37 \mathrm{E}+02$ & $2.55 \mathrm{E}-16$ & $3.40 \mathrm{E}-09$ & $2.98 \mathrm{E}-11$ \\
\hline In-117m & 116.2 & $\mathrm{~m}$ & $4.08 \mathrm{E}-15$ & $4.76 \mathrm{E}-04$ & $1.51 \mathrm{E}+01$ & $8.63 \mathrm{E}-18$ & $1.15 \mathrm{E}-10$ & $1.01 \mathrm{E}-12$ \\
\hline In-117 & 43.2 & $\mathrm{~m}$ & $3.08 \mathrm{E}-14$ & $3.60 \mathrm{E}-03$ & $1.14 \mathrm{E}+02$ & $6.69 \mathrm{E}-17$ & $8.91 \mathrm{E}-10$ & $7.81 \mathrm{E}-12$ \\
\hline In-118m & 4.364 & $\mathrm{~m}$ & $1.31 \mathrm{E}-13$ & $1.53 \mathrm{E}-02$ & $4.85 \mathrm{E}+02$ & $2.83 \mathrm{E}-16$ & $3.77 \mathrm{E}-09$ & $3.30 \mathrm{E}-11$ \\
\hline In-118 & 5 & $\mathrm{~s}$ & $5.72 \mathrm{E}-15$ & $6.68 \mathrm{E}-04$ & $2.12 \mathrm{E}+01$ & $1.08 \mathrm{E}-17$ & $1.44 \mathrm{E}-10$ & $1.26 \mathrm{E}-12$ \\
\hline In-119m & 18 & $\mathrm{~m}$ & $3.89 \mathrm{E}-15$ & $4.54 \mathrm{E}-04$ & $1.44 \mathrm{E}+01$ & $7.63 \mathrm{E}-18$ & $1.02 \mathrm{E}-10$ & $8.90 \mathrm{E}-13$ \\
\hline In-119 & 2.4 & $\mathrm{~m}$ & $3.54 \mathrm{E}-14$ & $4.13 \mathrm{E}-03$ & $1.31 \mathrm{E}+02$ & $7.63 \mathrm{E}-17$ & $1.02 \mathrm{E}-09$ & $8.90 \mathrm{E}-12$ \\
\hline In-121m & 3.88 & $\mathrm{~m}$ & $3.92 \mathrm{E}-15$ & $4.58 \mathrm{E}-04$ & $1.45 \mathrm{E}+01$ & $7.29 \mathrm{E}-18$ & $9.71 \mathrm{E}-11$ & $8.51 \mathrm{E}-13$ \\
\hline In-121 & 23.1 & $\mathrm{~s}$ & $4.36 \mathrm{E}-14$ & $5.09 \mathrm{E}-03$ & $1.61 \mathrm{E}+02$ & $9.37 \mathrm{E}-17$ & $1.25 \mathrm{E}-09$ & $1.09 \mathrm{E}-11$ \\
\hline Sn-106 & 1.92 & $\mathrm{~m}$ & $5.40 \mathrm{E}-14$ & $6.31 \mathrm{E}-03$ & $2.00 \mathrm{E}+02$ & $1.17 \mathrm{E}-16$ & $1.56 \mathrm{E}-09$ & $1.37 \mathrm{E}-11$ \\
\hline Sn-108 & 10.3 & $\mathrm{~m}$ & $2.96 \mathrm{E}-14$ & $3.46 \mathrm{E}-03$ & $1.10 \mathrm{E}+02$ & $6.45 \mathrm{E}-17$ & $8.59 \mathrm{E}-10$ & $7.53 \mathrm{E}-12$ \\
\hline Sn-109 & 18 & $\mathrm{~m}$ & $1.05 \mathrm{E}-13$ & $1.23 \mathrm{E}-02$ & $3.89 \mathrm{E}+02$ & $2.28 \mathrm{E}-16$ & $3.04 \mathrm{E}-09$ & $2.66 \mathrm{E}-11$ \\
\hline Sn-110 & 4.11 & $\mathrm{~h}$ & $1.21 \mathrm{E}-14$ & $1.41 \mathrm{E}-03$ & $4.48 \mathrm{E}+01$ & $2.65 \mathrm{E}-17$ & $3.53 \mathrm{E}-10$ & $3.09 \mathrm{E}-12$ \\
\hline $\mathrm{Sn}-111$ & 35.3 & $\mathrm{~m}$ & $2.21 \mathrm{E}-14$ & $2.58 \mathrm{E}-03$ & $8.18 \mathrm{E}+01$ & $4.79 \mathrm{E}-17$ & $6.38 \mathrm{E}-10$ & $5.59 \mathrm{E}-12$ \\
\hline $\mathrm{Sn}-113 \mathrm{~m}$ & 21.4 & $\mathrm{~m}$ & $1.02 \mathrm{E}-16$ & $1.19 \mathrm{E}-05$ & $3.77 \mathrm{E}-01$ & $2.38 \mathrm{E}-19$ & $3.17 \mathrm{E}-12$ & $2.78 \mathrm{E}-14$ \\
\hline Sn-113 & 115.09 & $\mathrm{~d}$ & $3.45 \mathrm{E}-16$ & $4.03 \mathrm{E}-05$ & $1.28 \mathrm{E}+00$ & $7.73 \mathrm{E}-19$ & $1.03 \mathrm{E}-11$ & $9.02 \mathrm{E}-14$ \\
\hline $\mathrm{Sn}-117 \mathrm{~m}$ & 13.76 & $\mathrm{~d}$ & $6.13 \mathrm{E}-15$ & $7.16 \mathrm{E}-04$ & $2.27 \mathrm{E}+01$ & $1.35 \mathrm{E}-17$ & $1.80 \mathrm{E}-10$ & $1.58 \mathrm{E}-12$ \\
\hline Sn-119m & 293.1 & $\mathrm{~d}$ & $9.23 \mathrm{E}-17$ & $1.08 \mathrm{E}-05$ & $3.42 \mathrm{E}-01$ & $2.17 \mathrm{E}-19$ & $2.89 \mathrm{E}-12$ & $2.53 \mathrm{E}-14$ \\
\hline $\mathrm{Sn}-121 \mathrm{~m}$ & 43.9 & $\mathrm{y}$ & $5.31 \mathrm{E}-17$ & $6.20 \mathrm{E}-06$ & $1.96 \mathrm{E}-01$ & $1.12 \mathrm{E}-19$ & $1.49 \mathrm{E}-12$ & $1.31 \mathrm{E}-14$ \\
\hline Sn-121 & 27.03 & $\mathrm{~h}$ & $3.98 \mathrm{E}-17$ & $4.65 \mathrm{E}-06$ & $1.47 \mathrm{E}-01$ & $4.36 \mathrm{E}-20$ & $5.81 \mathrm{E}-13$ & $5.09 \mathrm{E}-15$ \\
\hline $\mathrm{Sn}-123 \mathrm{~m}$ & 40.06 & $\mathrm{~m}$ & $6.21 \mathrm{E}-15$ & $7.25 \mathrm{E}-04$ & $2.30 \mathrm{E}+01$ & $1.34 \mathrm{E}-17$ & $1.78 \mathrm{E}-10$ & $1.56 \mathrm{E}-12$ \\
\hline Sn-123 & 129.2 & $\mathrm{~d}$ & $7.01 \mathrm{E}-16$ & $8.19 \mathrm{E}-05$ & $2.59 \mathrm{E}+00$ & $1.15 \mathrm{E}-18$ & $1.53 \mathrm{E}-11$ & $1.34 \mathrm{E}-13$ \\
\hline
\end{tabular}




\begin{tabular}{|c|c|c|c|c|c|c|c|c|}
\hline Nuclide & Half-life & Class & $\begin{array}{c}\text { Air } \\
\text { Immersion } \\
\text { Dose Rate } \\
\text { Coefficient } \\
(\mathrm{Sv} / \mathrm{s} \mathrm{per} \\
\left.\mathrm{Bq} / \mathbf{m}^{\mathbf{3}}\right)\end{array}$ & $\begin{array}{c}\text { Air } \\
\text { Immersion } \\
\text { Dose Rate } \\
\text { Coefficient } \\
\text { (mrem- } \\
\text { m }^{3} / \text { pCi-yr) }\end{array}$ & $\begin{array}{c}\text { Air } \\
\text { Immersion } \\
\text { Effective } \\
\text { Dose Rate } \\
(\mathrm{mrem} / \mathrm{sec} \\
\mathrm{per} \\
\left.\mu \mathrm{Ci} / \mathrm{cm}^{3}\right)\end{array}$ & $\begin{array}{c}\text { Water } \\
\text { Submersion } \\
\text { Dose Rate } \\
\text { Coefficient } \\
\left(\mathrm{Sv}-\mathbf{m}^{3} / \mathbf{B q}-\right. \\
\text { s) }\end{array}$ & $\begin{array}{c}\text { Water } \\
\text { Submersion } \\
\text { Dose Rate } \\
\text { Coefficient } \\
\text { (mrem- } \\
\text { m³Ci-h) }^{3} / \mathbf{p C i}\end{array}$ & $\begin{array}{c}\text { Water } \\
\text { Submersion } \\
\text { Dose Rate } \\
\text { Coefficient } \\
(\text { mrem- } \\
\left.\text { m }^{3} / \mu \mathrm{Ci}-\mathrm{y}\right)\end{array}$ \\
\hline $\mathrm{Sn}-125 \mathrm{~m}$ & 9.52 & $\mathrm{~m}$ & $1.60 \mathrm{E}-14$ & $1.87 \mathrm{E}-03$ & $5.92 \mathrm{E}+01$ & $3.42 \mathrm{E}-17$ & $4.56 \mathrm{E}-10$ & $3.99 \mathrm{E}-12$ \\
\hline $\mathrm{Sn}-125$ & 9.64 & $\mathrm{~d}$ & $1.64 \mathrm{E}-14$ & $1.91 \mathrm{E}-03$ & $6.07 \mathrm{E}+01$ & $3.49 \mathrm{E}-17$ & $4.65 \mathrm{E}-10$ & $4.07 \mathrm{E}-12$ \\
\hline Sn-126 & $2.30 \mathrm{E}+05$ & $\mathrm{y}$ & $1.82 \mathrm{E}-15$ & $2.13 \mathrm{E}-04$ & $6.73 \mathrm{E}+00$ & $4.09 \mathrm{E}-18$ & $5.45 \mathrm{E}-11$ & $4.77 \mathrm{E}-13$ \\
\hline $\mathrm{Sn}-127 \mathrm{~m}$ & 4.13 & $\mathrm{~m}$ & $2.67 \mathrm{E}-14$ & $3.12 \mathrm{E}-03$ & $9.88 \mathrm{E}+01$ & $5.72 \mathrm{E}-17$ & $7.62 \mathrm{E}-10$ & $6.67 \mathrm{E}-12$ \\
\hline Sn-127 & 2.1 & $\mathrm{~h}$ & $9.02 \mathrm{E}-14$ & $1.05 \mathrm{E}-02$ & $3.34 \mathrm{E}+02$ & $1.95 \mathrm{E}-16$ & $2.60 \mathrm{E}-09$ & $2.28 \mathrm{E}-11$ \\
\hline $\mathrm{Sn}-128$ & 59.07 & $\mathrm{~m}$ & $2.52 \mathrm{E}-14$ & 2.94E-03 & $9.32 \mathrm{E}+01$ & 5.49E-17 & $7.31 \mathrm{E}-10$ & $6.41 \mathrm{E}-12$ \\
\hline Sn-129 & 2.23 & $\mathrm{~m}$ & $4.76 \mathrm{E}-14$ & $5.56 \mathrm{E}-03$ & $1.76 \mathrm{E}+02$ & $1.02 \mathrm{E}-16$ & $1.36 \mathrm{E}-09$ & $1.19 \mathrm{E}-11$ \\
\hline $\mathrm{Sn}-130 \mathrm{~m}$ & 1.7 & $\mathrm{~m}$ & $4.20 \mathrm{E}-14$ & $4.90 \mathrm{E}-03$ & $1.55 \mathrm{E}+02$ & $8.99 \mathrm{E}-17$ & $1.20 \mathrm{E}-09$ & $1.05 \mathrm{E}-11$ \\
\hline Sn-130 & 3.72 & $\mathrm{~m}$ & $4.16 \mathrm{E}-14$ & $4.86 \mathrm{E}-03$ & $1.54 \mathrm{E}+02$ & $9.04 \mathrm{E}-17$ & $1.20 \mathrm{E}-09$ & $1.05 \mathrm{E}-11$ \\
\hline Sb-111 & 75 & $\mathrm{~s}$ & $6.76 \mathrm{E}-14$ & $7.89 \mathrm{E}-03$ & $2.50 \mathrm{E}+02$ & $1.46 \mathrm{E}-16$ & $1.94 \mathrm{E}-09$ & $1.70 \mathrm{E}-11$ \\
\hline Sb-113 & 6.67 & $\mathrm{~m}$ & $5.71 \mathrm{E}-14$ & $6.67 \mathrm{E}-03$ & $2.11 \mathrm{E}+02$ & $1.24 \mathrm{E}-16$ & $1.65 \mathrm{E}-09$ & $1.45 \mathrm{E}-11$ \\
\hline Sb-114 & 3.49 & $\mathrm{~m}$ & $1.27 \mathrm{E}-13$ & $1.48 \mathrm{E}-02$ & $4.70 \mathrm{E}+02$ & $2.74 \mathrm{E}-16$ & $3.65 \mathrm{E}-09$ & $3.20 \mathrm{E}-11$ \\
\hline Sb-115 & 32.1 & $\mathrm{~m}$ & $3.94 \mathrm{E}-14$ & $4.60 \mathrm{E}-03$ & $1.46 \mathrm{E}+02$ & $8.55 \mathrm{E}-17$ & $1.14 \mathrm{E}-09$ & $9.98 \mathrm{E}-12$ \\
\hline Sb-116 & 15.8 & $\mathrm{~m}$ & $1.08 \mathrm{E}-13$ & $1.26 \mathrm{E}-02$ & $4.00 \mathrm{E}+02$ & $2.33 \mathrm{E}-16$ & $3.10 \mathrm{E}-09$ & $2.72 \mathrm{E}-11$ \\
\hline $\mathrm{Sb}-116 \mathrm{~m}$ & 60.3 & $\mathrm{~m}$ & $1.43 \mathrm{E}-13$ & $1.67 \mathrm{E}-02$ & $5.29 \mathrm{E}+02$ & $3.11 \mathrm{E}-16$ & 4.14E-09 & $3.63 \mathrm{E}-11$ \\
\hline Sb-117 & 2.8 & $\mathrm{~h}$ & $7.23 \mathrm{E}-15$ & $8.44 \mathrm{E}-04$ & $2.68 \mathrm{E}+01$ & $1.59 \mathrm{E}-17$ & $2.12 \mathrm{E}-10$ & $1.86 \mathrm{E}-12$ \\
\hline $\mathrm{Sb}-118$ & 3.6 & $\mathrm{~m}$ & $3.65 \mathrm{E}-14$ & $4.26 \mathrm{E}-03$ & $1.35 \mathrm{E}+02$ & $7.86 \mathrm{E}-17$ & $1.05 \mathrm{E}-09$ & $9.17 \mathrm{E}-12$ \\
\hline Sb-118m & 5 & $\mathrm{~h}$ & $1.21 \mathrm{E}-13$ & $1.41 \mathrm{E}-02$ & $4.48 \mathrm{E}+02$ & $2.61 \mathrm{E}-16$ & $3.48 \mathrm{E}-09$ & $3.05 \mathrm{E}-11$ \\
\hline Sb-119 & 38.19 & $\mathrm{~h}$ & $1.51 \mathrm{E}-16$ & $1.76 \mathrm{E}-05$ & $5.59 \mathrm{E}-01$ & $3.55 \mathrm{E}-19$ & $4.73 \mathrm{E}-12$ & $4.14 \mathrm{E}-14$ \\
\hline Sb-120 & 15.89 & $\mathrm{~m}$ & $2.00 \mathrm{E}-14$ & $2.34 \mathrm{E}-03$ & $7.40 \mathrm{E}+01$ & $4.32 \mathrm{E}-17$ & $5.75 \mathrm{E}-10$ & $5.04 \mathrm{E}-12$ \\
\hline $\mathrm{Sb}-120 \mathrm{~m}$ & 5.76 & $\mathrm{~d}$ & $1.13 \mathrm{E}-13$ & $1.32 \mathrm{E}-02$ & $4.18 \mathrm{E}+02$ & $2.46 \mathrm{E}-16$ & $3.28 \mathrm{E}-09$ & $2.87 \mathrm{E}-11$ \\
\hline Sb-122 & 2.7238 & $\mathrm{~d}$ & $2.04 \mathrm{E}-14$ & $2.38 \mathrm{E}-03$ & $7.55 \mathrm{E}+01$ & $4.39 \mathrm{E}-17$ & $5.85 \mathrm{E}-10$ & $5.12 \mathrm{E}-12$ \\
\hline Sb-122m & 4.191 & $\mathrm{~m}$ & $1.77 \mathrm{E}-15$ & $2.07 \mathrm{E}-04$ & $6.55 \mathrm{E}+00$ & $4.04 \mathrm{E}-18$ & $5.38 \mathrm{E}-11$ & $4.71 \mathrm{E}-13$ \\
\hline
\end{tabular}




\begin{tabular}{|c|c|c|c|c|c|c|c|c|}
\hline Nuclide & Half-life & Class & $\begin{array}{c}\text { Air } \\
\text { Immersion } \\
\text { Dose Rate } \\
\text { Coefficient } \\
(\mathrm{Sv} / \mathrm{s} \mathrm{per} \\
\left.\mathrm{Bq} / \mathbf{m}^{3}\right)\end{array}$ & $\begin{array}{c}\text { Air } \\
\text { Immersion } \\
\text { Dose Rate } \\
\text { Coefficient } \\
\text { (mrem- } \\
\left.\text { m³ }^{3} / \mathrm{pCi}-\mathrm{yr}\right)\end{array}$ & $\begin{array}{c}\text { Air } \\
\text { Immersion } \\
\text { Effective } \\
\text { Dose Rate } \\
(\mathrm{mrem} / \mathrm{sec} \\
\mathrm{per} \\
\left.\mu \mathrm{Ci} / \mathrm{cm}^{3}\right)\end{array}$ & $\begin{array}{c}\text { Water } \\
\text { Submersion } \\
\text { Dose Rate } \\
\text { Coefficient } \\
\left(\mathrm{Sv}-\mathbf{m}^{3} / \mathbf{B q}-\right. \\
\text { s) }\end{array}$ & $\begin{array}{c}\text { Water } \\
\text { Submersion } \\
\text { Dose Rate } \\
\text { Coefficient } \\
\text { (mrem- } \\
\text { m³Ci-h) }^{\mathbf{3} / p C i}\end{array}$ & $\begin{array}{c}\text { Water } \\
\text { Submersion } \\
\text { Dose Rate } \\
\text { Coefficient } \\
\text { (mrem- } \\
\left.\text { m³ }^{3} / \mu \mathrm{Ci}-\mathrm{y}\right)\end{array}$ \\
\hline $\mathrm{Sb}-124$ & 60.2 & $\mathrm{~d}$ & $8.80 \mathrm{E}-14$ & $1.03 \mathrm{E}-02$ & $3.26 \mathrm{E}+02$ & $1.90 \mathrm{E}-16$ & $2.53 \mathrm{E}-09$ & $2.22 \mathrm{E}-11$ \\
\hline $\mathrm{Sb}-124 \mathrm{~m}$ & 93 & $\mathrm{~s}$ & $1.98 \mathrm{E}-14$ & $2.31 \mathrm{E}-03$ & $7.33 \mathrm{E}+01$ & $4.30 \mathrm{E}-17$ & $5.73 \mathrm{E}-10$ & $5.02 \mathrm{E}-12$ \\
\hline Sb-124n & 20.2 & $\mathrm{~m}$ & $5.63 \mathrm{E}-21$ & $6.57 \mathrm{E}-10$ & $2.08 \mathrm{E}-05$ & $1.32 \mathrm{E}-23$ & $1.76 \mathrm{E}-16$ & $1.54 \mathrm{E}-18$ \\
\hline $\mathrm{Sb}-125$ & 2.75856 & $\mathrm{y}$ & $1.90 \mathrm{E}-14$ & $2.22 \mathrm{E}-03$ & $7.03 \mathrm{E}+01$ & $4.12 \mathrm{E}-17$ & $5.49 \mathrm{E}-10$ & $4.81 \mathrm{E}-12$ \\
\hline $\mathrm{Sb}-126$ & 12.35 & $\mathrm{~d}$ & $1.25 \mathrm{E}-13$ & $1.46 \mathrm{E}-02$ & $4.63 \mathrm{E}+02$ & $2.71 \mathrm{E}-16$ & $3.61 \mathrm{E}-09$ & $3.16 \mathrm{E}-11$ \\
\hline $\mathrm{Sb}-126 \mathrm{~m}$ & 19.15 & $\mathrm{~m}$ & $7.02 \mathrm{E}-14$ & $8.20 \mathrm{E}-03$ & $2.60 \mathrm{E}+02$ & $1.52 \mathrm{E}-16$ & $2.02 \mathrm{E}-09$ & $1.77 \mathrm{E}-11$ \\
\hline Sb-127 & 3.85 & $\mathrm{~d}$ & $3.14 \mathrm{E}-14$ & $3.67 \mathrm{E}-03$ & $1.16 \mathrm{E}+02$ & $6.80 \mathrm{E}-17$ & $9.06 \mathrm{E}-10$ & $7.93 \mathrm{E}-12$ \\
\hline Sb-128 & 9.01 & $\mathrm{~h}$ & $1.41 \mathrm{E}-13$ & $1.65 \mathrm{E}-02$ & $5.22 \mathrm{E}+02$ & $3.05 \mathrm{E}-16$ & $4.06 \mathrm{E}-09$ & $3.56 \mathrm{E}-11$ \\
\hline Sb-128m & 10.4 & $\mathrm{~m}$ & $8.73 \mathrm{E}-14$ & $1.02 \mathrm{E}-02$ & $3.23 \mathrm{E}+02$ & $1.89 \mathrm{E}-16$ & $2.52 \mathrm{E}-09$ & $2.21 \mathrm{E}-11$ \\
\hline Sb-129 & 4.4 & $\mathrm{~h}$ & $6.83 \mathrm{E}-14$ & 7.97E-03 & $2.53 \mathrm{E}+02$ & $1.48 \mathrm{E}-16$ & $1.97 \mathrm{E}-09$ & $1.73 \mathrm{E}-11$ \\
\hline Sb-130 & 39.5 & $\mathrm{~m}$ & $1.51 \mathrm{E}-13$ & $1.76 \mathrm{E}-02$ & $5.59 \mathrm{E}+02$ & $3.26 \mathrm{E}-16$ & 4.34E-09 & $3.80 \mathrm{E}-11$ \\
\hline Sb-130m & 6.3 & $\mathrm{~m}$ & $1.26 \mathrm{E}-13$ & $1.47 \mathrm{E}-02$ & $4.66 \mathrm{E}+02$ & $2.72 \mathrm{E}-16$ & $3.62 \mathrm{E}-09$ & $3.17 \mathrm{E}-11$ \\
\hline Sb-131 & 23.03 & $\mathrm{~m}$ & $9.85 \mathrm{E}-14$ & $1.15 \mathrm{E}-02$ & $3.64 \mathrm{E}+02$ & $2.13 \mathrm{E}-16$ & $2.84 \mathrm{E}-09$ & $2.49 \mathrm{E}-11$ \\
\hline $\mathrm{Sb}-133$ & 2.5 & $\mathrm{~m}$ & $1.33 \mathrm{E}-13$ & $1.55 \mathrm{E}-02$ & $4.92 \mathrm{E}+02$ & $2.87 \mathrm{E}-16$ & $3.82 \mathrm{E}-09$ & $3.35 \mathrm{E}-11$ \\
\hline Te-113 & 1.7 & $\mathrm{~m}$ & $1.05 \mathrm{E}-13$ & $1.23 \mathrm{E}-02$ & $3.89 \mathrm{E}+02$ & $2.27 \mathrm{E}-16$ & $3.02 \mathrm{E}-09$ & $2.65 \mathrm{E}-11$ \\
\hline Te-114 & 15.2 & $\mathrm{~m}$ & $5.92 \mathrm{E}-14$ & $6.91 \mathrm{E}-03$ & $2.19 \mathrm{E}+02$ & $1.28 \mathrm{E}-16$ & $1.70 \mathrm{E}-09$ & $1.49 \mathrm{E}-11$ \\
\hline Te-115 & 5.8 & $\mathrm{~m}$ & $1.05 \mathrm{E}-13$ & $1.23 \mathrm{E}-02$ & $3.89 \mathrm{E}+02$ & $2.26 \mathrm{E}-16$ & $3.01 \mathrm{E}-09$ & $2.64 \mathrm{E}-11$ \\
\hline Te-115m & 6.7 & $\mathrm{~m}$ & $1.22 \mathrm{E}-13$ & $1.42 \mathrm{E}-02$ & $4.51 \mathrm{E}+02$ & $2.65 \mathrm{E}-16$ & $3.53 \mathrm{E}-09$ & $3.09 \mathrm{E}-11$ \\
\hline Te-116 & 2.49 & $\mathrm{~h}$ & $3.66 \mathrm{E}-15$ & $4.27 \mathrm{E}-04$ & $1.35 \mathrm{E}+01$ & $8.08 \mathrm{E}-18$ & $1.08 \mathrm{E}-10$ & $9.43 \mathrm{E}-13$ \\
\hline Te-117 & 62 & $\mathrm{~m}$ & $7.25 \mathrm{E}-14$ & $8.47 \mathrm{E}-03$ & $2.68 \mathrm{E}+02$ & $1.57 \mathrm{E}-16$ & $2.09 \mathrm{E}-09$ & $1.83 \mathrm{E}-11$ \\
\hline Te-118 & 6 & $\mathrm{~d}$ & $1.51 \mathrm{E}-16$ & $1.76 \mathrm{E}-05$ & $5.59 \mathrm{E}-01$ & $3.56 \mathrm{E}-19$ & $4.74 \mathrm{E}-12$ & $4.15 \mathrm{E}-14$ \\
\hline Te-119 & 16.05 & $\mathrm{~h}$ & $3.43 \mathrm{E}-14$ & $4.00 \mathrm{E}-03$ & $1.27 \mathrm{E}+02$ & $7.44 \mathrm{E}-17$ & $9.91 \mathrm{E}-10$ & $8.68 \mathrm{E}-12$ \\
\hline Te-119m & 4.7 & $\mathrm{~d}$ & $6.97 \mathrm{E}-14$ & $8.14 \mathrm{E}-03$ & $2.58 \mathrm{E}+02$ & $1.51 \mathrm{E}-16$ & $2.01 \mathrm{E}-09$ & $1.76 \mathrm{E}-11$ \\
\hline
\end{tabular}




\begin{tabular}{|c|c|c|c|c|c|c|c|c|}
\hline Nuclide & Half-life & Class & $\begin{array}{c}\text { Air } \\
\text { Immersion } \\
\text { Dose Rate } \\
\text { Coefficient } \\
\left(\mathrm{Sv} / \mathrm{s} \mathrm{per}^{\mathbf{3}}\right. \\
\left.\mathrm{Bq} / \mathbf{m}^{\mathbf{3}}\right)\end{array}$ & $\begin{array}{c}\text { Air } \\
\text { Immersion } \\
\text { Dose Rate } \\
\text { Coefficient } \\
\text { (mrem- } \\
\left.\text { m }^{3} / \mathrm{pCi}-\mathrm{yr}\right)\end{array}$ & $\begin{array}{c}\text { Air } \\
\text { Immersion } \\
\text { Effective } \\
\text { Dose Rate } \\
\text { (mrem/sec } \\
\text { per } \\
\mu \mathrm{Ci} / \mathrm{cm}^{3} \text { ) }\end{array}$ & $\begin{array}{c}\text { Water } \\
\text { Submersion } \\
\text { Dose Rate } \\
\text { Coefficient } \\
\left(\mathbf{S v}-\mathbf{m}^{3} / \mathbf{B q}-\right. \\
\text { s) }\end{array}$ & $\begin{array}{c}\text { Water } \\
\text { Submersion } \\
\text { Dose Rate } \\
\text { Coefficient } \\
\text { (mrem- } \\
\mathbf{m}^{\mathbf{3}} / \mathbf{p C i} \text {-h) }\end{array}$ & $\begin{array}{c}\text { Water } \\
\text { Submersion } \\
\text { Dose Rate } \\
\text { Coefficient } \\
(\text { mrem- } \\
\left.\text { m³ }^{\mathbf{3}} / \boldsymbol{\mu C i}-\mathbf{y}\right)\end{array}$ \\
\hline Te-121 & 19.16 & $\mathrm{~d}$ & $2.51 \mathrm{E}-14$ & $2.93 \mathrm{E}-03$ & $9.29 \mathrm{E}+01$ & $5.45 \mathrm{E}-17$ & $7.26 \mathrm{E}-10$ & $6.36 \mathrm{E}-12$ \\
\hline $\mathrm{Te}-121 \mathrm{~m}$ & 154 & $\mathrm{~d}$ & $9.02 \mathrm{E}-15$ & $1.05 \mathrm{E}-03$ & $3.34 \mathrm{E}+01$ & $1.98 \mathrm{E}-17$ & $2.64 \mathrm{E}-10$ & $2.31 \mathrm{E}-12$ \\
\hline Te-123 & $6.00 \mathrm{E}+14$ & $\mathrm{y}$ & $2.63 \mathrm{E}-19$ & $3.07 \mathrm{E}-08$ & $9.73 \mathrm{E}-04$ & $6.19 \mathrm{E}-22$ & $8.25 \mathrm{E}-15$ & $7.22 \mathrm{E}-17$ \\
\hline Te-123m & 119.25 & $\mathrm{~d}$ & $5.81 \mathrm{E}-15$ & $6.78 \mathrm{E}-04$ & $2.15 \mathrm{E}+01$ & $1.28 \mathrm{E}-17$ & $1.70 \mathrm{E}-10$ & $1.49 \mathrm{E}-12$ \\
\hline Te-125m & 57.4 & $\mathrm{~d}$ & $3.36 \mathrm{E}-16$ & $3.92 \mathrm{E}-05$ & $1.24 \mathrm{E}+00$ & $7.78 \mathrm{E}-19$ & $1.04 \mathrm{E}-11$ & $9.08 \mathrm{E}-14$ \\
\hline Te-127 & 9.35 & $\mathrm{~h}$ & $3.35 \mathrm{E}-16$ & $3.91 \mathrm{E}-05$ & $1.24 \mathrm{E}+00$ & $6.02 \mathrm{E}-19$ & $8.02 \mathrm{E}-12$ & $7.02 \mathrm{E}-14$ \\
\hline Te- $127 \mathrm{~m}$ & 109 & $\mathrm{~d}$ & $1.12 \mathrm{E}-16$ & $1.31 \mathrm{E}-05$ & $4.14 \mathrm{E}-01$ & $2.55 \mathrm{E}-19$ & $3.40 \mathrm{E}-12$ & $2.98 \mathrm{E}-14$ \\
\hline Te-129 & 69.6 & $\mathrm{~m}$ & $2.99 \mathrm{E}-15$ & $3.49 \mathrm{E}-04$ & $1.11 \mathrm{E}+01$ & $6.13 \mathrm{E}-18$ & $8.17 \mathrm{E}-11$ & $7.15 \mathrm{E}-13$ \\
\hline Te-129m & 33.6 & $\mathrm{~d}$ & $1.57 \mathrm{E}-15$ & $1.83 \mathrm{E}-04$ & $5.81 \mathrm{E}+00$ & $3.26 \mathrm{E}-18$ & $4.34 \mathrm{E}-11$ & $3.80 \mathrm{E}-13$ \\
\hline $\mathrm{Te}-131$ & 25 & $\mathrm{~m}$ & $1.92 \mathrm{E}-14$ & $2.24 \mathrm{E}-03$ & $7.10 \mathrm{E}+01$ & $4.14 \mathrm{E}-17$ & $5.51 \mathrm{E}-10$ & $4.83 \mathrm{E}-12$ \\
\hline Te- $131 \mathrm{~m}$ & 30 & $\mathrm{~h}$ & $6.69 \mathrm{E}-14$ & $7.81 \mathrm{E}-03$ & $2.48 \mathrm{E}+02$ & $1.45 \mathrm{E}-16$ & $1.93 \mathrm{E}-09$ & $1.69 \mathrm{E}-11$ \\
\hline Te-132 & 3.204 & $\mathrm{~d}$ & $9.35 \mathrm{E}-15$ & $1.09 \mathrm{E}-03$ & $3.46 \mathrm{E}+01$ & $2.05 \mathrm{E}-17$ & $2.73 \mathrm{E}-10$ & $2.39 \mathrm{E}-12$ \\
\hline Te-133 & 12.5 & $\mathrm{~m}$ & $5.67 \mathrm{E}-14$ & $6.62 \mathrm{E}-03$ & $2.10 \mathrm{E}+02$ & $1.23 \mathrm{E}-16$ & $1.64 \mathrm{E}-09$ & $1.44 \mathrm{E}-11$ \\
\hline Te-133m & 55.4 & $\mathrm{~m}$ & $8.64 \mathrm{E}-14$ & $1.01 \mathrm{E}-02$ & $3.20 \mathrm{E}+02$ & $1.87 \mathrm{E}-16$ & $2.49 \mathrm{E}-09$ & $2.18 \mathrm{E}-11$ \\
\hline Te-134 & 41.8 & $\mathrm{~m}$ & $3.87 \mathrm{E}-14$ & $4.52 \mathrm{E}-03$ & $1.43 \mathrm{E}+02$ & $8.42 \mathrm{E}-17$ & $1.12 \mathrm{E}-09$ & $9.82 \mathrm{E}-12$ \\
\hline $\mathrm{I}-118$ & 13.7 & $\mathrm{~m}$ & $9.38 \mathrm{E}-14$ & $1.10 \mathrm{E}-02$ & $3.47 \mathrm{E}+02$ & $2.02 \mathrm{E}-16$ & $2.69 \mathrm{E}-09$ & $2.36 \mathrm{E}-11$ \\
\hline $\mathrm{I}-118 \mathrm{~m}$ & 8.5 & $\mathrm{~m}$ & $1.71 \mathrm{E}-13$ & $2.00 \mathrm{E}-02$ & $6.33 \mathrm{E}+02$ & $3.71 \mathrm{E}-16$ & $4.94 \mathrm{E}-09$ & $4.33 \mathrm{E}-11$ \\
\hline I-119 & 19.1 & $\mathrm{~m}$ & $4.06 \mathrm{E}-14$ & $4.74 \mathrm{E}-03$ & $1.50 \mathrm{E}+02$ & $8.79 \mathrm{E}-17$ & $1.17 \mathrm{E}-09$ & $1.03 \mathrm{E}-11$ \\
\hline $\mathrm{I}-120$ & 81.6 & $\mathrm{~m}$ & $1.28 \mathrm{E}-13$ & $1.49 \mathrm{E}-02$ & $4.74 \mathrm{E}+02$ & $2.76 \mathrm{E}-16$ & $3.68 \mathrm{E}-09$ & $3.22 \mathrm{E}-11$ \\
\hline
\end{tabular}




\begin{tabular}{|c|c|c|c|c|c|c|c|c|}
\hline Nuclide & Half-life & Class & $\begin{array}{c}\text { Air } \\
\text { Immersion } \\
\text { Dose Rate } \\
\text { Coefficient } \\
\left(\mathrm{Sv} / \mathbf{s ~ p e r ~}^{\mathbf{3}}\right. \\
\left.\mathrm{Bq} / \mathbf{m}^{\mathbf{3}}\right)\end{array}$ & $\begin{array}{c}\text { Air } \\
\text { Immersion } \\
\text { Dose Rate } \\
\text { Coefficient } \\
\text { (mrem- } \\
\left.\text { m³ }^{3} / \mathrm{pCi}-\mathrm{yr}\right)\end{array}$ & $\begin{array}{c}\text { Air } \\
\text { Immersion } \\
\text { Effective } \\
\text { Dose Rate } \\
\text { (mrem/sec } \\
\text { per } \\
\mu \mathrm{Ci} / \mathbf{c m}^{3} \text { ) }\end{array}$ & $\begin{array}{c}\text { Water } \\
\text { Submersion } \\
\text { Dose Rate } \\
\text { Coefficient } \\
\left(\mathbf{S v}-\mathbf{m}^{3} / \mathbf{B q}-\right. \\
\text { s) }\end{array}$ & $\begin{array}{c}\text { Water } \\
\text { Submersion } \\
\text { Dose Rate } \\
\text { Coefficient } \\
\text { (mrem- } \\
\left.\text { m³ }^{\mathbf{3}} / \mathbf{p C i}-\mathbf{h}\right)\end{array}$ & $\begin{array}{c}\text { Water } \\
\text { Submersion } \\
\text { Dose Rate } \\
\text { Coefficient } \\
(\text { mrem- } \\
\left.\text { m³ }^{3} / \mu \mathrm{Ci}-\mathrm{y}\right)\end{array}$ \\
\hline $\mathrm{I}-120 \mathrm{~m}$ & 53 & $\mathrm{~m}$ & $1.63 \mathrm{E}-13$ & $1.90 \mathrm{E}-02$ & $6.03 \mathrm{E}+02$ & $3.53 \mathrm{E}-16$ & $4.70 \mathrm{E}-09$ & $4.12 \mathrm{E}-11$ \\
\hline $\mathrm{I}-121$ & 2.12 & $\mathrm{~h}$ & $1.69 \mathrm{E}-14$ & $1.97 \mathrm{E}-03$ & $6.25 \mathrm{E}+01$ & $3.70 \mathrm{E}-17$ & $4.93 \mathrm{E}-10$ & $4.32 \mathrm{E}-12$ \\
\hline I-122 & 3.63 & $\mathrm{~m}$ & $4.39 \mathrm{E}-14$ & $5.13 \mathrm{E}-03$ & $1.62 \mathrm{E}+02$ & $9.45 \mathrm{E}-17$ & $1.26 \mathrm{E}-09$ & $1.10 \mathrm{E}-11$ \\
\hline $\mathrm{I}-123$ & 13.27 & $\mathrm{~h}$ & $6.55 \mathrm{E}-15$ & $7.65 \mathrm{E}-04$ & $2.42 \mathrm{E}+01$ & $1.45 \mathrm{E}-17$ & $1.93 \mathrm{E}-10$ & $1.69 \mathrm{E}-12$ \\
\hline $\mathrm{I}-124$ & 4.176 & $\mathrm{~d}$ & $5.11 \mathrm{E}-14$ & $5.97 \mathrm{E}-03$ & $1.89 \mathrm{E}+02$ & $1.11 \mathrm{E}-16$ & $1.48 \mathrm{E}-09$ & $1.30 \mathrm{E}-11$ \\
\hline $\mathrm{I}-125$ & 59.4 & $\mathrm{~d}$ & $3.78 \mathrm{E}-16$ & $4.41 \mathrm{E}-05$ & $1.40 \mathrm{E}+00$ & $8.88 \mathrm{E}-19$ & $1.18 \mathrm{E}-11$ & $1.04 \mathrm{E}-13$ \\
\hline I-126 & 12.93 & $\mathrm{~d}$ & $1.92 \mathrm{E}-14$ & $2.24 \mathrm{E}-03$ & $7.10 \mathrm{E}+01$ & $4.17 \mathrm{E}-17$ & $5.55 \mathrm{E}-10$ & $4.87 \mathrm{E}-12$ \\
\hline $\mathrm{I}-128$ & 24.99 & $\mathrm{~m}$ & $3.55 \mathrm{E}-15$ & $4.15 \mathrm{E}-04$ & $1.31 \mathrm{E}+01$ & $7.16 \mathrm{E}-18$ & $9.54 \mathrm{E}-11$ & $8.35 \mathrm{E}-13$ \\
\hline I-129 & $1.57 \mathrm{E}+07$ & $\mathrm{y}$ & $2.86 \mathrm{E}-16$ & $3.34 \mathrm{E}-05$ & $1.06 \mathrm{E}+00$ & $6.68 \mathrm{E}-19$ & $8.90 \mathrm{E}-12$ & $7.79 \mathrm{E}-14$ \\
\hline $\mathrm{I}-130$ & 12.36 & $\mathrm{~h}$ & $9.68 \mathrm{E}-14$ & $1.13 \mathrm{E}-02$ & $3.58 \mathrm{E}+02$ & $2.10 \mathrm{E}-16$ & $2.80 \mathrm{E}-09$ & $2.45 \mathrm{E}-11$ \\
\hline $\mathrm{I}-130 \mathrm{~m}$ & 8.84 & $\mathrm{~m}$ & $4.88 \mathrm{E}-15$ & $5.70 \mathrm{E}-04$ & $1.81 \mathrm{E}+01$ & $1.05 \mathrm{E}-17$ & $1.40 \mathrm{E}-10$ & $1.23 \mathrm{E}-12$ \\
\hline $\mathrm{I}-131$ & 8.0207 & $\mathrm{~d}$ & $1.70 \mathrm{E}-14$ & $1.98 \mathrm{E}-03$ & $6.29 \mathrm{E}+01$ & $3.69 \mathrm{E}-17$ & $4.92 \mathrm{E}-10$ & $4.31 \mathrm{E}-12$ \\
\hline $\mathrm{I}-132$ & 2.295 & $\mathrm{~h}$ & $1.04 \mathrm{E}-13$ & $1.21 \mathrm{E}-02$ & $3.85 \mathrm{E}+02$ & $2.26 \mathrm{E}-16$ & $3.01 \mathrm{E}-09$ & $2.64 \mathrm{E}-11$ \\
\hline $\mathrm{I}-132 \mathrm{~m}$ & 1.387 & $\mathrm{~h}$ & $1.50 \mathrm{E}-14$ & $1.75 \mathrm{E}-03$ & $5.55 \mathrm{E}+01$ & $3.26 \mathrm{E}-17$ & $4.34 \mathrm{E}-10$ & $3.80 \mathrm{E}-12$ \\
\hline $\mathrm{I}-133$ & 20.8 & $\mathrm{~h}$ & $2.78 \mathrm{E}-14$ & $3.25 \mathrm{E}-03$ & $1.03 \mathrm{E}+02$ & $6.02 \mathrm{E}-17$ & $8.02 \mathrm{E}-10$ & $7.02 \mathrm{E}-12$ \\
\hline I-134 & 52.5 & $\mathrm{~m}$ & $1.21 \mathrm{E}-13$ & $1.41 \mathrm{E}-02$ & $4.48 \mathrm{E}+02$ & $2.61 \mathrm{E}-16$ & $3.48 \mathrm{E}-09$ & $3.05 \mathrm{E}-11$ \\
\hline $\mathrm{I}-134 \mathrm{~m}$ & 3.6 & $\mathrm{~m}$ & $1.19 \mathrm{E}-14$ & $1.39 \mathrm{E}-03$ & $4.40 \mathrm{E}+01$ & $2.60 \mathrm{E}-17$ & $3.46 \mathrm{E}-10$ & $3.03 \mathrm{E}-12$ \\
\hline $\mathrm{I}-135$ & 6.57 & $\mathrm{~h}$ & $7.57 \mathrm{E}-14$ & $8.84 \mathrm{E}-03$ & $2.80 \mathrm{E}+02$ & $1.64 \mathrm{E}-16$ & $2.18 \mathrm{E}-09$ & $1.91 \mathrm{E}-11$ \\
\hline $\mathrm{Xe}-120$ & 40 & $\mathrm{~m}$ & $1.65 \mathrm{E}-14$ & $1.93 \mathrm{E}-03$ & $6.11 \mathrm{E}+01$ & $3.60 \mathrm{E}-17$ & $4.80 \mathrm{E}-10$ & $4.20 \mathrm{E}-12$ \\
\hline $\mathrm{Xe}-121$ & 40.1 & $\mathrm{~m}$ & $6.95 \mathrm{E}-14$ & $8.12 \mathrm{E}-03$ & $2.57 \mathrm{E}+02$ & $1.50 \mathrm{E}-16$ & $2.00 \mathrm{E}-09$ & $1.75 \mathrm{E}-11$ \\
\hline $\mathrm{Xe}-122$ & 20.1 & $\mathrm{~h}$ & $2.19 \mathrm{E}-15$ & $2.56 \mathrm{E}-04$ & $8.10 \mathrm{E}+00$ & $4.81 \mathrm{E}-18$ & $6.41 \mathrm{E}-11$ & $5.61 \mathrm{E}-13$ \\
\hline $\mathrm{Xe}-123$ & 2.08 & $\mathrm{~h}$ & $2.85 \mathrm{E}-14$ & $3.33 \mathrm{E}-03$ & $1.05 \mathrm{E}+02$ & $6.20 \mathrm{E}-17$ & $8.26 \mathrm{E}-10$ & $7.23 \mathrm{E}-12$ \\
\hline $\mathrm{Xe}-125$ & 16.9 & $\mathrm{~h}$ & $1.08 \mathrm{E}-14$ & $1.26 \mathrm{E}-03$ & $4.00 \mathrm{E}+01$ & $2.37 \mathrm{E}-17$ & $3.16 \mathrm{E}-10$ & $2.77 \mathrm{E}-12$ \\
\hline
\end{tabular}




\begin{tabular}{|c|c|c|c|c|c|c|c|c|}
\hline Nuclide & Half-life & Class & $\begin{array}{c}\text { Air } \\
\text { Immersion } \\
\text { Dose Rate } \\
\text { Coefficient } \\
\left(\mathrm{Sv} / \mathbf{s ~ p e r ~}^{\mathbf{3}}\right. \\
\left.\mathrm{Bq} / \mathbf{m}^{\mathbf{3}}\right)\end{array}$ & $\begin{array}{c}\text { Air } \\
\text { Immersion } \\
\text { Dose Rate } \\
\text { Coefficient } \\
\text { (mrem- } \\
\left.\text { m³ }^{3} / \mathrm{pCi}-\mathrm{yr}\right)\end{array}$ & $\begin{array}{c}\text { Air } \\
\text { Immersion } \\
\text { Effective } \\
\text { Dose Rate } \\
\text { (mrem/sec } \\
\text { per } \\
\mu \mathrm{Ci} / \mathrm{cm}^{3} \text { ) } \\
\end{array}$ & $\begin{array}{c}\text { Water } \\
\text { Submersion } \\
\text { Dose Rate } \\
\text { Coefficient } \\
\left(\mathbf{S v}-\mathbf{m}^{3} / \mathbf{B q}-\right. \\
\text { s) }\end{array}$ & $\begin{array}{c}\text { Water } \\
\text { Submersion } \\
\text { Dose Rate } \\
\text { Coefficient } \\
\text { (mrem- } \\
\left.\text { m³ }^{\mathbf{3}} / \mathbf{p C i}-\mathbf{h}\right)\end{array}$ & $\begin{array}{c}\text { Water } \\
\text { Submersion } \\
\text { Dose Rate } \\
\text { Coefficient } \\
(\text { mrem- } \\
\left.\mathbf{m}^{3} / \mu \mathrm{Ci}-\mathrm{y}\right)\end{array}$ \\
\hline $\mathrm{Xe}-127$ & 36.4 & $\mathrm{~d}$ & $1.13 \mathrm{E}-14$ & $1.32 \mathrm{E}-03$ & $4.18 \mathrm{E}+01$ & $2.48 \mathrm{E}-17$ & $3.30 \mathrm{E}-10$ & $2.89 \mathrm{E}-12$ \\
\hline $\mathrm{Xe}-127 \mathrm{~m}$ & 69.2 & $\mathrm{~s}$ & $6.57 \mathrm{E}-15$ & $7.67 \mathrm{E}-04$ & $2.43 \mathrm{E}+01$ & $1.45 \mathrm{E}-17$ & $1.93 \mathrm{E}-10$ & $1.69 \mathrm{E}-12$ \\
\hline $\mathrm{Xe}-129 \mathrm{~m}$ & 8.88 & $\mathrm{~d}$ & $9.18 \mathrm{E}-16$ & $1.07 \mathrm{E}-04$ & $3.40 \mathrm{E}+00$ & $2.01 \mathrm{E}-18$ & $2.68 \mathrm{E}-11$ & $2.35 \mathrm{E}-13$ \\
\hline $\mathrm{Xe}-131 \mathrm{~m}$ & 11.84 & $\mathrm{~d}$ & $3.57 \mathrm{E}-16$ & $4.17 \mathrm{E}-05$ & $1.32 \mathrm{E}+00$ & $7.64 \mathrm{E}-19$ & $1.02 \mathrm{E}-11$ & $8.91 \mathrm{E}-14$ \\
\hline $\mathrm{Xe}-133$ & 5.243 & $\mathrm{~d}$ & $1.37 \mathrm{E}-15$ & $1.60 \mathrm{E}-04$ & $5.07 \mathrm{E}+00$ & $3.06 \mathrm{E}-18$ & $4.08 \mathrm{E}-11$ & $3.57 \mathrm{E}-13$ \\
\hline $\mathrm{Xe}-133 \mathrm{~m}$ & 2.19 & $\mathrm{~d}$ & $1.29 \mathrm{E}-15$ & $1.51 \mathrm{E}-04$ & $4.77 \mathrm{E}+00$ & $2.75 \mathrm{E}-18$ & $3.66 \mathrm{E}-11$ & $3.21 \mathrm{E}-13$ \\
\hline $\mathrm{Xe}-135$ & 9.14 & $\mathrm{~h}$ & $1.10 \mathrm{E}-14$ & $1.28 \mathrm{E}-03$ & $4.07 \mathrm{E}+01$ & $2.39 \mathrm{E}-17$ & $3.18 \mathrm{E}-10$ & $2.79 \mathrm{E}-12$ \\
\hline $\mathrm{Xe}-135 \mathrm{~m}$ & 15.29 & $\mathrm{~m}$ & $1.89 \mathrm{E}-14$ & $2.21 \mathrm{E}-03$ & $6.99 \mathrm{E}+01$ & $4.09 \mathrm{E}-17$ & $5.45 \mathrm{E}-10$ & $4.77 \mathrm{E}-12$ \\
\hline $\mathrm{Xe}-137$ & 3.818 & $\mathrm{~m}$ & $1.04 \mathrm{E}-14$ & $1.21 \mathrm{E}-03$ & $3.85 \mathrm{E}+01$ & $2.12 \mathrm{E}-17$ & $2.82 \mathrm{E}-10$ & $2.47 \mathrm{E}-12$ \\
\hline $\mathrm{Xe}-138$ & 14.08 & $\mathrm{~m}$ & $5.47 \mathrm{E}-14$ & $6.39 \mathrm{E}-03$ & $2.02 \mathrm{E}+02$ & $1.18 \mathrm{E}-16$ & $1.57 \mathrm{E}-09$ & $1.38 \mathrm{E}-11$ \\
\hline Cs-121 & 155 & $\mathrm{~s}$ & $5.41 \mathrm{E}-14$ & $6.32 \mathrm{E}-03$ & $2.00 \mathrm{E}+02$ & $1.16 \mathrm{E}-16$ & $1.55 \mathrm{E}-09$ & $1.35 \mathrm{E}-11$ \\
\hline Cs-121m & 122 & $\mathrm{~s}$ & $5.38 \mathrm{E}-14$ & $6.28 \mathrm{E}-03$ & $1.99 \mathrm{E}+02$ & $1.16 \mathrm{E}-16$ & $1.55 \mathrm{E}-09$ & $1.35 \mathrm{E}-11$ \\
\hline Cs-123 & 5.88 & $\mathrm{~m}$ & $4.89 \mathrm{E}-14$ & $5.71 \mathrm{E}-03$ & $1.81 \mathrm{E}+02$ & $1.06 \mathrm{E}-16$ & $1.41 \mathrm{E}-09$ & $1.24 \mathrm{E}-11$ \\
\hline Cs-124 & 30.8 & $\mathrm{~s}$ & $5.43 \mathrm{E}-14$ & $6.34 \mathrm{E}-03$ & $2.01 \mathrm{E}+02$ & $1.16 \mathrm{E}-16$ & $1.55 \mathrm{E}-09$ & $1.35 \mathrm{E}-11$ \\
\hline Cs-125 & 45 & $\mathrm{~m}$ & $3.37 \mathrm{E}-14$ & $3.93 \mathrm{E}-03$ & $1.25 \mathrm{E}+02$ & $7.31 \mathrm{E}-17$ & $9.74 \mathrm{E}-10$ & $8.53 \mathrm{E}-12$ \\
\hline Cs-126 & 1.64 & $\mathrm{~m}$ & $5.29 \mathrm{E}-14$ & $6.18 \mathrm{E}-03$ & $1.96 \mathrm{E}+02$ & $1.14 \mathrm{E}-16$ & $1.52 \mathrm{E}-09$ & $1.33 \mathrm{E}-11$ \\
\hline Cs-127 & 6.25 & $\mathrm{~h}$ & $1.84 \mathrm{E}-14$ & $2.15 \mathrm{E}-03$ & $6.81 \mathrm{E}+01$ & $4.01 \mathrm{E}-17$ & $5.34 \mathrm{E}-10$ & $4.68 \mathrm{E}-12$ \\
\hline Cs-128 & 3.64 & $\mathrm{~m}$ & $4.04 \mathrm{E}-14$ & $4.72 \mathrm{E}-03$ & $1.49 \mathrm{E}+02$ & $8.71 \mathrm{E}-17$ & $1.16 \mathrm{E}-09$ & $1.02 \mathrm{E}-11$ \\
\hline Cs-129 & 32.06 & $\mathrm{~h}$ & $1.12 \mathrm{E}-14$ & $1.31 \mathrm{E}-03$ & $4.14 \mathrm{E}+01$ & $2.46 \mathrm{E}-17$ & $3.28 \mathrm{E}-10$ & $2.87 \mathrm{E}-12$ \\
\hline Cs-130 & 29.21 & $\mathrm{~m}$ & $2.23 \mathrm{E}-14$ & $2.60 \mathrm{E}-03$ & $8.25 \mathrm{E}+01$ & $4.83 \mathrm{E}-17$ & $6.43 \mathrm{E}-10$ & $5.64 \mathrm{E}-12$ \\
\hline Cs-130m & 3.46 & $\mathrm{~m}$ & $2.01 \mathrm{E}-15$ & $2.35 \mathrm{E}-04$ & $7.44 \mathrm{E}+00$ & $4.53 \mathrm{E}-18$ & $6.03 \mathrm{E}-11$ & $5.29 \mathrm{E}-13$ \\
\hline Cs-131 & 9.689 & $\mathrm{~d}$ & $2.40 \mathrm{E}-16$ & $2.80 \mathrm{E}-05$ & 8.88E-01 & $5.63 \mathrm{E}-19$ & $7.50 \mathrm{E}-12$ & $6.57 \mathrm{E}-14$ \\
\hline Cs-132 & 6.479 & $\mathrm{~d}$ & $3.15 \mathrm{E}-14$ & $3.68 \mathrm{E}-03$ & $1.17 \mathrm{E}+02$ & $6.85 \mathrm{E}-17$ & $9.12 \mathrm{E}-10$ & $7.99 \mathrm{E}-12$ \\
\hline
\end{tabular}




\begin{tabular}{|c|c|c|c|c|c|c|c|c|}
\hline Nuclide & Half-life & Class & $\begin{array}{c}\text { Air } \\
\text { Immersion } \\
\text { Dose Rate } \\
\text { Coefficient } \\
(\mathrm{Sv} / \mathrm{s} \mathrm{per} \\
\left.\mathrm{Bq} / \mathbf{m}^{3}\right)\end{array}$ & $\begin{array}{c}\text { Air } \\
\text { Immersion } \\
\text { Dose Rate } \\
\text { Coefficient } \\
\text { (mrem- } \\
\left.\text { m³ }^{3} / \mathrm{pCi}-\mathrm{yr}\right)\end{array}$ & $\begin{array}{c}\text { Air } \\
\text { Immersion } \\
\text { Effective } \\
\text { Dose Rate } \\
(\mathrm{mrem} / \mathrm{sec} \\
\mathrm{per} \\
\left.\mu \mathrm{Ci} / \mathrm{cm}^{3}\right)\end{array}$ & $\begin{array}{c}\text { Water } \\
\text { Submersion } \\
\text { Dose Rate } \\
\text { Coefficient } \\
\left(\mathrm{Sv}-\mathbf{m}^{3} / \mathbf{B q}-\right. \\
\text { s) }\end{array}$ & $\begin{array}{c}\text { Water } \\
\text { Submersion } \\
\text { Dose Rate } \\
\text { Coefficient } \\
\text { (mrem- } \\
\text { m³Ci-h) }^{\mathbf{3} / p C i}\end{array}$ & $\begin{array}{c}\text { Water } \\
\text { Submersion } \\
\text { Dose Rate } \\
\text { Coefficient } \\
(\text { mrem- } \\
\left.\text { m }^{3} / \mu \mathrm{Ci}-\mathrm{y}\right)\end{array}$ \\
\hline Cs-134 & 2.0648 & $\mathrm{y}$ & $7.07 \mathrm{E}-14$ & $8.26 \mathrm{E}-03$ & $2.62 \mathrm{E}+02$ & $1.53 \mathrm{E}-16$ & $2.04 \mathrm{E}-09$ & $1.79 \mathrm{E}-11$ \\
\hline Cs-134m & 2.903 & $\mathrm{~h}$ & $8.00 \mathrm{E}-16$ & 9.34E-05 & $2.96 \mathrm{E}+00$ & $1.77 \mathrm{E}-18$ & $2.36 \mathrm{E}-11$ & $2.07 \mathrm{E}-13$ \\
\hline Cs-135 & $2.30 \mathrm{E}+06$ & $\mathrm{y}$ & $2.17 \mathrm{E}-17$ & $2.53 \mathrm{E}-06$ & $8.03 \mathrm{E}-02$ & $2.37 \mathrm{E}-20$ & $3.16 \mathrm{E}-13$ & $2.77 \mathrm{E}-15$ \\
\hline Cs-135m & 53 & $\mathrm{~m}$ & $7.31 \mathrm{E}-14$ & $8.54 \mathrm{E}-03$ & $2.70 \mathrm{E}+02$ & $1.59 \mathrm{E}-16$ & $2.12 \mathrm{E}-09$ & $1.86 \mathrm{E}-11$ \\
\hline Cs-136 & 13.16 & $\mathrm{~d}$ & $9.81 \mathrm{E}-14$ & $1.15 \mathrm{E}-02$ & $3.63 \mathrm{E}+02$ & $2.13 \mathrm{E}-16$ & $2.84 \mathrm{E}-09$ & $2.49 \mathrm{E}-11$ \\
\hline Cs-137 & 30.1671 & $\mathrm{y}$ & $9.40 \mathrm{E}-17$ & $1.10 \mathrm{E}-05$ & $3.48 \mathrm{E}-01$ & $1.05 \mathrm{E}-19$ & $1.40 \mathrm{E}-12$ & $1.23 \mathrm{E}-14$ \\
\hline Cs-138 & 33.41 & $\mathrm{~m}$ & $1.15 \mathrm{E}-13$ & $1.34 \mathrm{E}-02$ & $4.26 \mathrm{E}+02$ & $2.48 \mathrm{E}-16$ & $3.30 \mathrm{E}-09$ & $2.89 \mathrm{E}-11$ \\
\hline Cs-138m & 2.91 & $\mathrm{~m}$ & $1.92 \mathrm{E}-14$ & $2.24 \mathrm{E}-03$ & $7.10 \mathrm{E}+01$ & $4.14 \mathrm{E}-17$ & $5.51 \mathrm{E}-10$ & $4.83 \mathrm{E}-12$ \\
\hline Cs-139 & 9.27 & $\mathrm{~m}$ & $1.66 \mathrm{E}-14$ & $1.94 \mathrm{E}-03$ & $6.14 \mathrm{E}+01$ & $3.45 \mathrm{E}-17$ & $4.60 \mathrm{E}-10$ & $4.03 \mathrm{E}-12$ \\
\hline Cs-140 & 63.7 & $\mathrm{~s}$ & $8.89 \mathrm{E}-14$ & $1.04 \mathrm{E}-02$ & $3.29 \mathrm{E}+02$ & $1.91 \mathrm{E}-16$ & $2.54 \mathrm{E}-09$ & $2.23 \mathrm{E}-11$ \\
\hline Ba-124 & 11 & $\mathrm{~m}$ & $2.51 \mathrm{E}-14$ & $2.93 \mathrm{E}-03$ & $9.29 \mathrm{E}+01$ & $5.46 \mathrm{E}-17$ & $7.27 \mathrm{E}-10$ & $6.37 \mathrm{E}-12$ \\
\hline Ba-126 & 100 & $\mathrm{~m}$ & $2.56 \mathrm{E}-14$ & $2.99 \mathrm{E}-03$ & $9.47 \mathrm{E}+01$ & $5.57 \mathrm{E}-17$ & $7.42 \mathrm{E}-10$ & $6.50 \mathrm{E}-12$ \\
\hline Ba-127 & 12.7 & $\mathrm{~m}$ & $3.28 \mathrm{E}-14$ & $3.83 \mathrm{E}-03$ & $1.21 \mathrm{E}+02$ & $7.08 \mathrm{E}-17$ & $9.43 \mathrm{E}-10$ & $8.26 \mathrm{E}-12$ \\
\hline $\mathrm{Ba}-128$ & 2.43 & $\mathrm{~d}$ & $2.12 \mathrm{E}-15$ & $2.48 \mathrm{E}-04$ & $7.84 \mathrm{E}+00$ & $4.67 \mathrm{E}-18$ & $6.22 \mathrm{E}-11$ & $5.45 \mathrm{E}-13$ \\
\hline Ba-129 & 2.23 & $\mathrm{~h}$ & $1.44 \mathrm{E}-14$ & $1.68 \mathrm{E}-03$ & $5.33 \mathrm{E}+01$ & $3.13 \mathrm{E}-17$ & $4.17 \mathrm{E}-10$ & $3.65 \mathrm{E}-12$ \\
\hline Ba-129m & 2.16 & $\mathrm{~h}$ & $7.20 \mathrm{E}-14$ & $8.41 \mathrm{E}-03$ & $2.66 \mathrm{E}+02$ & $1.56 \mathrm{E}-16$ & $2.08 \mathrm{E}-09$ & $1.82 \mathrm{E}-11$ \\
\hline Ba-131 & 11.5 & $\mathrm{~d}$ & $2.00 \mathrm{E}-14$ & $2.34 \mathrm{E}-03$ & $7.40 \mathrm{E}+01$ & $4.36 \mathrm{E}-17$ & $5.81 \mathrm{E}-10$ & $5.09 \mathrm{E}-12$ \\
\hline Ba-131m & 14.6 & $\mathrm{~m}$ & $2.65 \mathrm{E}-15$ & $3.09 \mathrm{E}-04$ & $9.81 \mathrm{E}+00$ & $5.93 \mathrm{E}-18$ & $7.90 \mathrm{E}-11$ & $6.92 \mathrm{E}-13$ \\
\hline Ba-133 & 10.52 & $\mathrm{y}$ & $1.62 \mathrm{E}-14$ & $1.89 \mathrm{E}-03$ & $5.99 \mathrm{E}+01$ & $3.56 \mathrm{E}-17$ & $4.74 \mathrm{E}-10$ & $4.15 \mathrm{E}-12$ \\
\hline Ba-133m & 38.9 & $\mathrm{~h}$ & $2.49 \mathrm{E}-15$ & $2.91 \mathrm{E}-04$ & $9.21 \mathrm{E}+00$ & $5.36 \mathrm{E}-18$ & $7.14 \mathrm{E}-11$ & $6.25 \mathrm{E}-13$ \\
\hline Ba-135m & 28.7 & $\mathrm{~h}$ & $2.16 \mathrm{E}-15$ & $2.52 \mathrm{E}-04$ & $7.99 \mathrm{E}+00$ & $4.65 \mathrm{E}-18$ & $6.19 \mathrm{E}-11$ & $5.43 \mathrm{E}-13$ \\
\hline Ba-137m & 2.552 & $\mathrm{~m}$ & $2.69 \mathrm{E}-14$ & $3.14 \mathrm{E}-03$ & $9.95 \mathrm{E}+01$ & $5.83 \mathrm{E}-17$ & $7.77 \mathrm{E}-10$ & $6.80 \mathrm{E}-12$ \\
\hline Ba-139 & 83.06 & $\mathrm{~m}$ & $2.67 \mathrm{E}-15$ & $3.12 \mathrm{E}-04$ & $9.88 \mathrm{E}+00$ & $5.17 \mathrm{E}-18$ & $6.89 \mathrm{E}-11$ & $6.03 \mathrm{E}-13$ \\
\hline
\end{tabular}




\begin{tabular}{|c|c|c|c|c|c|c|c|c|}
\hline Nuclide & Half-life & Class & 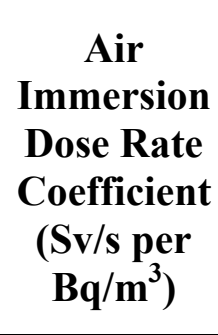 & $\begin{array}{c}\text { Air } \\
\text { Immersion } \\
\text { Dose Rate } \\
\text { Coefficient } \\
\text { (mrem- } \\
\text { m³Ci-yr) }^{3} / \mathrm{pCi}\end{array}$ & $\begin{array}{c}\text { Air } \\
\text { Immersion } \\
\text { Effective } \\
\text { Dose Rate } \\
(\mathrm{mrem} / \mathrm{sec} \\
\mathrm{per} \\
\left.\mu \mathrm{Ci} / \mathrm{cm}^{3}\right)\end{array}$ & $\begin{array}{c}\text { Water } \\
\text { Submersion } \\
\text { Dose Rate } \\
\text { Coefficient } \\
\left(\mathrm{Sv}-\mathbf{m}^{3} / \mathbf{B q}-\right. \\
\text { s) }\end{array}$ & $\begin{array}{c}\text { Water } \\
\text { Submersion } \\
\text { Dose Rate } \\
\text { Coefficient } \\
\text { (mrem- } \\
\text { m³Ci-h) }^{\mathbf{3} / p C i}\end{array}$ & $\begin{array}{c}\text { Water } \\
\text { Submersion } \\
\text { Dose Rate } \\
\text { Coefficient } \\
(\text { mrem- } \\
\left.\text { m }^{3} / \mu \mathrm{Ci}-\mathrm{y}\right)\end{array}$ \\
\hline Ba-140 & 12.752 & $\mathrm{~d}$ & $8.06 \mathrm{E}-15$ & $9.41 \mathrm{E}-04$ & $2.98 \mathrm{E}+01$ & $1.74 \mathrm{E}-17$ & $2.32 \mathrm{E}-10$ & $2.03 \mathrm{E}-12$ \\
\hline Ba-141 & 18.27 & $\mathrm{~m}$ & $4.32 \mathrm{E}-14$ & $5.04 \mathrm{E}-03$ & $1.60 \mathrm{E}+02$ & $9.32 \mathrm{E}-17$ & $1.24 \mathrm{E}-09$ & $1.09 \mathrm{E}-11$ \\
\hline Ba-142 & 10.6 & $\mathrm{~m}$ & $4.84 \mathrm{E}-14$ & $5.65 \mathrm{E}-03$ & $1.79 \mathrm{E}+02$ & $1.05 \mathrm{E}-16$ & $1.40 \mathrm{E}-09$ & $1.23 \mathrm{E}-11$ \\
\hline La-128 & 5.18 & $\mathrm{~m}$ & $1.30 \mathrm{E}-13$ & $1.52 \mathrm{E}-02$ & $4.81 \mathrm{E}+02$ & $2.82 \mathrm{E}-16$ & $3.76 \mathrm{E}-09$ & $3.29 \mathrm{E}-11$ \\
\hline La-129 & 11.6 & $\mathrm{~m}$ & $4.11 \mathrm{E}-14$ & $4.80 \mathrm{E}-03$ & $1.52 \mathrm{E}+02$ & $8.90 \mathrm{E}-17$ & $1.19 \mathrm{E}-09$ & $1.04 \mathrm{E}-11$ \\
\hline La-130 & 8.7 & $\mathrm{~m}$ & $1.03 \mathrm{E}-13$ & $1.20 \mathrm{E}-02$ & $3.81 \mathrm{E}+02$ & $2.23 \mathrm{E}-16$ & $2.97 \mathrm{E}-09$ & $2.60 \mathrm{E}-11$ \\
\hline La-131 & 59 & $\mathrm{~m}$ & $2.88 \mathrm{E}-14$ & $3.36 \mathrm{E}-03$ & $1.07 \mathrm{E}+02$ & $6.26 \mathrm{E}-17$ & $8.34 \mathrm{E}-10$ & $7.30 \mathrm{E}-12$ \\
\hline La-132 & 4.8 & $\mathrm{~h}$ & $9.34 \mathrm{E}-14$ & $1.09 \mathrm{E}-02$ & $3.46 \mathrm{E}+02$ & $2.02 \mathrm{E}-16$ & $2.69 \mathrm{E}-09$ & $2.36 \mathrm{E}-11$ \\
\hline La-132m & 24.3 & $\mathrm{~m}$ & $2.95 \mathrm{E}-14$ & $3.44 \mathrm{E}-03$ & $1.09 \mathrm{E}+02$ & $6.42 \mathrm{E}-17$ & $8.55 \mathrm{E}-10$ & $7.49 \mathrm{E}-12$ \\
\hline La-133 & 3.912 & $\mathrm{~h}$ & $6.39 \mathrm{E}-15$ & $7.46 \mathrm{E}-04$ & $2.36 \mathrm{E}+01$ & $1.39 \mathrm{E}-17$ & $1.85 \mathrm{E}-10$ & $1.62 \mathrm{E}-12$ \\
\hline La-134 & 6.45 & $\mathrm{~m}$ & $3.26 \mathrm{E}-14$ & $3.81 \mathrm{E}-03$ & $1.21 \mathrm{E}+02$ & $7.02 \mathrm{E}-17$ & $9.35 \mathrm{E}-10$ & $8.19 \mathrm{E}-12$ \\
\hline La-135 & 19.5 & $\mathrm{~h}$ & $7.73 \mathrm{E}-16$ & $9.03 \mathrm{E}-05$ & $2.86 \mathrm{E}+00$ & $1.73 \mathrm{E}-18$ & $2.30 \mathrm{E}-11$ & $2.02 \mathrm{E}-13$ \\
\hline La-136 & 9.87 & $\mathrm{~m}$ & $1.79 \mathrm{E}-14$ & $2.09 \mathrm{E}-03$ & $6.62 \mathrm{E}+01$ & $3.86 \mathrm{E}-17$ & $5.14 \mathrm{E}-10$ & $4.50 \mathrm{E}-12$ \\
\hline La-137 & $6.00 \mathrm{E}+04$ & $\mathrm{y}$ & $3.07 \mathrm{E}-16$ & $3.58 \mathrm{E}-05$ & $1.14 \mathrm{E}+00$ & $7.20 \mathrm{E}-19$ & $9.59 \mathrm{E}-12$ & $8.40 \mathrm{E}-14$ \\
\hline La-138 & $1.02 \mathrm{E}+11$ & $\mathrm{y}$ & $5.82 \mathrm{E}-14$ & $6.80 \mathrm{E}-03$ & $2.15 \mathrm{E}+02$ & $1.26 \mathrm{E}-16$ & $1.68 \mathrm{E}-09$ & $1.47 \mathrm{E}-11$ \\
\hline La-140 & 1.6781 & $\mathrm{~d}$ & $1.11 \mathrm{E}-13$ & $1.30 \mathrm{E}-02$ & $4.11 \mathrm{E}+02$ & $2.39 \mathrm{E}-16$ & $3.18 \mathrm{E}-09$ & $2.79 \mathrm{E}-11$ \\
\hline La-141 & 3.92 & $\mathrm{~h}$ & $2.14 \mathrm{E}-15$ & $2.50 \mathrm{E}-04$ & $7.92 \mathrm{E}+00$ & $3.86 \mathrm{E}-18$ & $5.14 \mathrm{E}-11$ & $4.50 \mathrm{E}-13$ \\
\hline La-142 & 91.1 & $\mathrm{~m}$ & $1.19 \mathrm{E}-13$ & $1.39 \mathrm{E}-02$ & $4.40 \mathrm{E}+02$ & $2.56 \mathrm{E}-16$ & $3.41 \mathrm{E}-09$ & $2.99 \mathrm{E}-11$ \\
\hline La-143 & 14.2 & $\mathrm{~m}$ & $1.39 \mathrm{E}-14$ & $1.62 \mathrm{E}-03$ & $5.14 \mathrm{E}+01$ & $2.91 \mathrm{E}-17$ & $3.88 \mathrm{E}-10$ & $3.40 \mathrm{E}-12$ \\
\hline Ce-130 & 22.9 & $\mathrm{~m}$ & $2.14 \mathrm{E}-14$ & $2.50 \mathrm{E}-03$ & $7.92 \mathrm{E}+01$ & $4.66 \mathrm{E}-17$ & $6.21 \mathrm{E}-10$ & $5.44 \mathrm{E}-12$ \\
\hline Ce-131 & 10.2 & $\mathrm{~m}$ & $7.43 \mathrm{E}-14$ & $8.68 \mathrm{E}-03$ & $2.75 \mathrm{E}+02$ & $1.61 \mathrm{E}-16$ & $2.14 \mathrm{E}-09$ & $1.88 \mathrm{E}-11$ \\
\hline Ce-132 & 3.51 & $\mathrm{~h}$ & $1.09 \mathrm{E}-14$ & $1.27 \mathrm{E}-03$ & $4.03 \mathrm{E}+01$ & $2.40 \mathrm{E}-17$ & $3.20 \mathrm{E}-10$ & $2.80 \mathrm{E}-12$ \\
\hline $\mathrm{Ce}-133$ & 97 & $\mathrm{~m}$ & $2.28 \mathrm{E}-14$ & $2.66 \mathrm{E}-03$ & $8.44 \mathrm{E}+01$ & $4.96 \mathrm{E}-17$ & $6.61 \mathrm{E}-10$ & $5.79 \mathrm{E}-12$ \\
\hline
\end{tabular}




\begin{tabular}{|c|c|c|c|c|c|c|c|c|}
\hline Nuclide & Half-life & Class & $\begin{array}{c}\text { Air } \\
\text { Immersion } \\
\text { Dose Rate } \\
\text { Coefficient } \\
\left(\mathrm{Sv} / \mathrm{s} \mathrm{per}^{\mathbf{3}}\right. \\
\left.\mathrm{Bq} / \mathbf{m}^{\mathbf{3}}\right)\end{array}$ & $\begin{array}{c}\text { Air } \\
\text { Immersion } \\
\text { Dose Rate } \\
\text { Coefficient } \\
\text { (mrem- } \\
\left.\mathrm{m}^{3} / \mathrm{pCi}-\mathrm{yr}\right)\end{array}$ & $\begin{array}{c}\text { Air } \\
\text { Immersion } \\
\text { Effective } \\
\text { Dose Rate } \\
\text { (mrem/sec } \\
\text { per } \\
\mu \mathrm{Ci} / \mathrm{cm}^{3} \text { ) }\end{array}$ & $\begin{array}{c}\text { Water } \\
\text { Submersion } \\
\text { Dose Rate } \\
\text { Coefficient } \\
\left(\mathbf{S v}-\mathbf{m}^{3} / \mathbf{B q}-\right. \\
\text { s) }\end{array}$ & $\begin{array}{c}\text { Water } \\
\text { Submersion } \\
\text { Dose Rate } \\
\text { Coefficient } \\
\text { (mrem- } \\
\mathbf{m}^{\mathbf{3}} / \mathbf{p C i} \text {-h) }\end{array}$ & $\begin{array}{c}\text { Water } \\
\text { Submersion } \\
\text { Dose Rate } \\
\text { Coefficient } \\
(\text { mrem- } \\
\left.\text { m³ }^{\mathbf{3}} / \boldsymbol{\mu C i}-\mathbf{y}\right)\end{array}$ \\
\hline $\mathrm{Ce}-133 \mathrm{~m}$ & 4.9 & $\mathrm{~h}$ & $7.95 \mathrm{E}-14$ & $9.28 \mathrm{E}-03$ & $2.94 \mathrm{E}+02$ & $1.73 \mathrm{E}-16$ & $2.30 \mathrm{E}-09$ & $2.02 \mathrm{E}-11$ \\
\hline $\mathrm{Ce}-134$ & 3.16 & $\mathrm{~d}$ & $4.20 \mathrm{E}-16$ & $4.90 \mathrm{E}-05$ & $1.55 \mathrm{E}+00$ & $9.72 \mathrm{E}-19$ & $1.29 \mathrm{E}-11$ & $1.13 \mathrm{E}-13$ \\
\hline $\mathrm{Ce}-135$ & 17.7 & $\mathrm{~h}$ & $3.61 \mathrm{E}-14$ & $4.22 \mathrm{E}-03$ & $1.34 \mathrm{E}+02$ & $7.85 \mathrm{E}-17$ & $1.05 \mathrm{E}-09$ & $9.16 \mathrm{E}-12$ \\
\hline $\mathrm{Ce}-137$ & 9 & $\mathrm{~h}$ & $8.48 \mathrm{E}-16$ & $9.90 \mathrm{E}-05$ & $3.14 \mathrm{E}+00$ & $1.90 \mathrm{E}-18$ & $2.53 \mathrm{E}-11$ & $2.22 \mathrm{E}-13$ \\
\hline $\mathrm{Ce}-137 \mathrm{~m}$ & 34.4 & $\mathrm{~h}$ & $1.94 \mathrm{E}-15$ & $2.27 \mathrm{E}-04$ & $7.18 \mathrm{E}+00$ & $4.18 \mathrm{E}-18$ & $5.57 \mathrm{E}-11$ & $4.88 \mathrm{E}-13$ \\
\hline $\mathrm{Ce}-139$ & 137.641 & $\mathrm{~d}$ & $5.98 \mathrm{E}-15$ & $6.98 \mathrm{E}-04$ & $2.21 \mathrm{E}+01$ & $1.32 \mathrm{E}-17$ & $1.76 \mathrm{E}-10$ & $1.54 \mathrm{E}-12$ \\
\hline Ce-141 & 32.508 & $\mathrm{~d}$ & $3.13 \mathrm{E}-15$ & $3.65 \mathrm{E}-04$ & $1.16 \mathrm{E}+01$ & $6.87 \mathrm{E}-18$ & $9.15 \mathrm{E}-11$ & $8.02 \mathrm{E}-13$ \\
\hline $\mathrm{Ce}-143$ & 33.039 & $\mathrm{~h}$ & $1.19 \mathrm{E}-14$ & $1.39 \mathrm{E}-03$ & $4.40 \mathrm{E}+01$ & $2.58 \mathrm{E}-17$ & $3.44 \mathrm{E}-10$ & $3.01 \mathrm{E}-12$ \\
\hline $\mathrm{Ce}-144$ & 284.91 & $\mathrm{~d}$ & $7.35 \mathrm{E}-16$ & $8.58 \mathrm{E}-05$ & $2.72 \mathrm{E}+00$ & $1.61 \mathrm{E}-18$ & $2.14 \mathrm{E}-11$ & $1.88 \mathrm{E}-13$ \\
\hline $\mathrm{Ce}-145$ & 3.01 & $\mathrm{~m}$ & $3.64 \mathrm{E}-14$ & $4.25 \mathrm{E}-03$ & $1.35 \mathrm{E}+02$ & $7.87 \mathrm{E}-17$ & $1.05 \mathrm{E}-09$ & $9.18 \mathrm{E}-12$ \\
\hline Pr-134 & 11 & $\mathrm{~m}$ & $1.44 \mathrm{E}-13$ & $1.68 \mathrm{E}-02$ & $5.33 \mathrm{E}+02$ & $3.12 \mathrm{E}-16$ & $4.16 \mathrm{E}-09$ & $3.64 \mathrm{E}-11$ \\
\hline Pr-134m & 17 & $\mathrm{~m}$ & $1.08 \mathrm{E}-13$ & $1.26 \mathrm{E}-02$ & $4.00 \mathrm{E}+02$ & $2.34 \mathrm{E}-16$ & $3.12 \mathrm{E}-09$ & $2.73 \mathrm{E}-11$ \\
\hline Pr-135 & 24 & $\mathrm{~m}$ & $3.89 \mathrm{E}-14$ & $4.54 \mathrm{E}-03$ & $1.44 \mathrm{E}+02$ & $8.42 \mathrm{E}-17$ & $1.12 \mathrm{E}-09$ & $9.82 \mathrm{E}-12$ \\
\hline Pr-136 & 13.1 & $\mathrm{~m}$ & $9.95 \mathrm{E}-14$ & $1.16 \mathrm{E}-02$ & $3.68 \mathrm{E}+02$ & $2.15 \mathrm{E}-16$ & $2.86 \mathrm{E}-09$ & $2.51 \mathrm{E}-11$ \\
\hline Pr-137 & 1.28 & $\mathrm{~h}$ & $1.62 \mathrm{E}-14$ & $1.89 \mathrm{E}-03$ & $5.99 \mathrm{E}+01$ & $3.50 \mathrm{E}-17$ & $4.66 \mathrm{E}-10$ & $4.08 \mathrm{E}-12$ \\
\hline Pr-138 & 1.45 & $\mathrm{~m}$ & $3.73 \mathrm{E}-14$ & $4.36 \mathrm{E}-03$ & $1.38 \mathrm{E}+02$ & $8.02 \mathrm{E}-17$ & $1.07 \mathrm{E}-09$ & $9.36 \mathrm{E}-12$ \\
\hline Pr-138m & 2.12 & $\mathrm{~h}$ & $1.13 \mathrm{E}-13$ & $1.32 \mathrm{E}-02$ & $4.18 \mathrm{E}+02$ & $2.45 \mathrm{E}-16$ & $3.26 \mathrm{E}-09$ & $2.86 \mathrm{E}-11$ \\
\hline Pr-139 & 4.41 & $\mathrm{~h}$ & $5.10 \mathrm{E}-15$ & $5.95 \mathrm{E}-04$ & $1.89 \mathrm{E}+01$ & $1.11 \mathrm{E}-17$ & $1.48 \mathrm{E}-10$ & $1.30 \mathrm{E}-12$ \\
\hline Pr-140 & 3.39 & $\mathrm{~m}$ & $2.44 \mathrm{E}-14$ & $2.85 \mathrm{E}-03$ & $9.03 \mathrm{E}+01$ & $5.27 \mathrm{E}-17$ & $7.02 \mathrm{E}-10$ & $6.15 \mathrm{E}-12$ \\
\hline
\end{tabular}




\begin{tabular}{|c|c|c|c|c|c|c|c|c|}
\hline Nuclide & Half-life & Class & 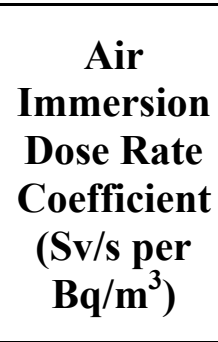 & $\begin{array}{c}\text { Air } \\
\text { Immersion } \\
\text { Dose Rate } \\
\text { Coefficient } \\
\text { (mrem- } \\
\text { m }^{3} / \text { pCi-yr) }\end{array}$ & $\begin{array}{c}\text { Air } \\
\text { Immersion } \\
\text { Effective } \\
\text { Dose Rate } \\
(\mathrm{mrem} / \mathrm{sec} \\
\mathrm{per} \\
\left.\mu \mathrm{Ci} / \mathrm{cm}^{3}\right)\end{array}$ & $\begin{array}{c}\text { Water } \\
\text { Submersion } \\
\text { Dose Rate } \\
\text { Coefficient } \\
\left(\mathrm{Sv}-\mathbf{m}^{3} / \mathbf{B q}-\right. \\
\text { s) }\end{array}$ & $\begin{array}{c}\text { Water } \\
\text { Submersion } \\
\text { Dose Rate } \\
\text { Coefficient } \\
\text { (mrem- } \\
\left.\text { m³ }^{\mathbf{3}} / \mathbf{p C i}-\mathbf{h}\right)\end{array}$ & $\begin{array}{c}\text { Water } \\
\text { Submersion } \\
\text { Dose Rate } \\
\text { Coefficient } \\
(\text { mrem- } \\
\left.\text { m }^{3} / \mu \mathrm{Ci}-\mathrm{y}\right)\end{array}$ \\
\hline Pr-142 & 19.12 & $\mathrm{~h}$ & $3.49 \mathrm{E}-15$ & $4.08 \mathrm{E}-04$ & $1.29 \mathrm{E}+01$ & $6.94 \mathrm{E}-18$ & $9.24 \mathrm{E}-11$ & $8.10 \mathrm{E}-13$ \\
\hline Pr-142m & 14.6 & $\mathrm{~m}$ & $0.00 \mathrm{E}+00$ & $0.00 \mathrm{E}+00$ & $0.00 \mathrm{E}+00$ & $0.00 \mathrm{E}+00$ & $0.00 \mathrm{E}+00$ & $0.00 \mathrm{E}+00$ \\
\hline Pr-143 & 13.57 & $\mathrm{~d}$ & $1.95 \mathrm{E}-16$ & $2.28 \mathrm{E}-05$ & $7.22 \mathrm{E}-01$ & $2.22 \mathrm{E}-19$ & $2.96 \mathrm{E}-12$ & $2.59 \mathrm{E}-14$ \\
\hline Pr-144 & 17.28 & $\mathrm{~m}$ & $2.51 \mathrm{E}-15$ & $2.93 \mathrm{E}-04$ & $9.29 \mathrm{E}+00$ & $4.45 \mathrm{E}-18$ & $5.93 \mathrm{E}-11$ & $5.19 \mathrm{E}-13$ \\
\hline Pr-144m & 7.2 & $\mathrm{~m}$ & $2.52 \mathrm{E}-16$ & $2.94 \mathrm{E}-05$ & $9.32 \mathrm{E}-01$ & $5.76 \mathrm{E}-19$ & $7.67 \mathrm{E}-12$ & $6.72 \mathrm{E}-14$ \\
\hline Pr-145 & 5.984 & $\mathrm{~h}$ & $1.37 \mathrm{E}-15$ & $1.60 \mathrm{E}-04$ & $5.07 \mathrm{E}+00$ & $2.46 \mathrm{E}-18$ & $3.28 \mathrm{E}-11$ & $2.87 \mathrm{E}-13$ \\
\hline Pr-146 & 24.15 & $\mathrm{~m}$ & $4.92 \mathrm{E}-14$ & $5.74 \mathrm{E}-03$ & $1.82 \mathrm{E}+02$ & $1.06 \mathrm{E}-16$ & $1.41 \mathrm{E}-09$ & $1.24 \mathrm{E}-11$ \\
\hline Pr-147 & 13.4 & $\mathrm{~m}$ & $2.18 \mathrm{E}-14$ & $2.55 \mathrm{E}-03$ & $8.07 \mathrm{E}+01$ & $4.69 \mathrm{E}-17$ & $6.25 \mathrm{E}-10$ & $5.47 \mathrm{E}-12$ \\
\hline Pr-148 & 2.29 & $\mathrm{~m}$ & $4.83 \mathrm{E}-14$ & $5.64 \mathrm{E}-03$ & $1.79 \mathrm{E}+02$ & $1.03 \mathrm{E}-16$ & $1.37 \mathrm{E}-09$ & $1.20 \mathrm{E}-11$ \\
\hline Pr-148m & 2.01 & $\mathrm{~m}$ & $4.37 \mathrm{E}-14$ & $5.10 \mathrm{E}-03$ & $1.62 \mathrm{E}+02$ & $9.36 \mathrm{E}-17$ & $1.25 \mathrm{E}-09$ & $1.09 \mathrm{E}-11$ \\
\hline $\mathrm{Nd}-134$ & 8.5 & $\mathrm{~m}$ & $2.32 \mathrm{E}-14$ & $2.71 \mathrm{E}-03$ & $8.58 \mathrm{E}+01$ & $5.06 \mathrm{E}-17$ & $6.74 \mathrm{E}-10$ & $5.90 \mathrm{E}-12$ \\
\hline $\mathrm{Nd}-135$ & 12.4 & $\mathrm{~m}$ & $5.63 \mathrm{E}-14$ & $6.57 \mathrm{E}-03$ & $2.08 \mathrm{E}+02$ & $1.22 \mathrm{E}-16$ & $1.63 \mathrm{E}-09$ & $1.42 \mathrm{E}-11$ \\
\hline $\mathrm{Nd}-136$ & 50.65 & $\mathrm{~m}$ & $1.10 \mathrm{E}-14$ & $1.28 \mathrm{E}-03$ & $4.07 \mathrm{E}+01$ & $2.41 \mathrm{E}-17$ & $3.21 \mathrm{E}-10$ & $2.81 \mathrm{E}-12$ \\
\hline Nd-137 & 38.5 & $\mathrm{~m}$ & $5.33 \mathrm{E}-14$ & $6.22 \mathrm{E}-03$ & $1.97 \mathrm{E}+02$ & $1.16 \mathrm{E}-16$ & $1.55 \mathrm{E}-09$ & $1.35 \mathrm{E}-11$ \\
\hline $\mathrm{Nd}-138$ & 5.04 & $\mathrm{~h}$ & $1.08 \mathrm{E}-15$ & $1.26 \mathrm{E}-04$ & $4.00 \mathrm{E}+00$ & $2.44 \mathrm{E}-18$ & $3.25 \mathrm{E}-11$ & $2.85 \mathrm{E}-13$ \\
\hline Nd-139 & 29.7 & $\mathrm{~m}$ & $1.95 \mathrm{E}-14$ & $2.28 \mathrm{E}-03$ & $7.22 \mathrm{E}+01$ & $4.22 \mathrm{E}-17$ & $5.62 \mathrm{E}-10$ & $4.92 \mathrm{E}-12$ \\
\hline $\mathrm{Nd}-139 \mathrm{~m}$ & 5.5 & $\mathrm{~h}$ & $7.19 \mathrm{E}-14$ & $8.40 \mathrm{E}-03$ & $2.66 \mathrm{E}+02$ & $1.56 \mathrm{E}-16$ & $2.08 \mathrm{E}-09$ & $1.82 \mathrm{E}-11$ \\
\hline $\mathrm{Nd}-140$ & 3.37 & $\mathrm{~d}$ & $4.44 \mathrm{E}-16$ & $5.18 \mathrm{E}-05$ & $1.64 \mathrm{E}+00$ & $1.04 \mathrm{E}-18$ & $1.39 \mathrm{E}-11$ & $1.21 \mathrm{E}-13$ \\
\hline $\mathrm{Nd}-141$ & 2.49 & $\mathrm{~h}$ & $2.64 \mathrm{E}-15$ & $3.08 \mathrm{E}-04$ & $9.77 \mathrm{E}+00$ & $5.79 \mathrm{E}-18$ & $7.71 \mathrm{E}-11$ & $6.76 \mathrm{E}-13$ \\
\hline $\mathrm{Nd}-141 \mathrm{~m}$ & 62 & $\mathrm{~S}$ & $3.16 \mathrm{E}-14$ & $3.69 \mathrm{E}-03$ & $1.17 \mathrm{E}+02$ & $6.85 \mathrm{E}-17$ & $9.12 \mathrm{E}-10$ & $7.99 \mathrm{E}-12$ \\
\hline $\mathrm{Nd}-144$ & $2.29 \mathrm{E}+15$ & $\mathrm{y}$ & $0.00 \mathrm{E}+00$ & $0.00 \mathrm{E}+00$ & $0.00 \mathrm{E}+00$ & $0.00 \mathrm{E}+00$ & $0.00 \mathrm{E}+00$ & $0.00 \mathrm{E}+00$ \\
\hline Nd-147 & 10.98 & $\mathrm{~d}$ & $5.74 \mathrm{E}-15$ & $6.70 \mathrm{E}-04$ & $2.12 \mathrm{E}+01$ & $1.25 \mathrm{E}-17$ & $1.67 \mathrm{E}-10$ & $1.46 \mathrm{E}-12$ \\
\hline Nd-149 & 1.728 & $\mathrm{~h}$ & $1.63 \mathrm{E}-14$ & $1.90 \mathrm{E}-03$ & $6.03 \mathrm{E}+01$ & $3.53 \mathrm{E}-17$ & $4.70 \mathrm{E}-10$ & $4.12 \mathrm{E}-12$ \\
\hline
\end{tabular}




\begin{tabular}{|c|c|c|c|c|c|c|c|c|}
\hline Nuclide & Half-life & Class & 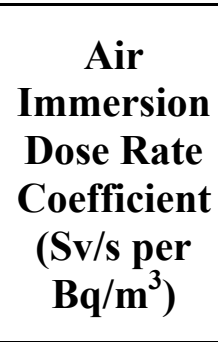 & $\begin{array}{c}\text { Air } \\
\text { Immersion } \\
\text { Dose Rate } \\
\text { Coefficient } \\
\text { (mrem- } \\
\text { m }^{3} / \text { pCi-yr) }\end{array}$ & $\begin{array}{c}\text { Air } \\
\text { Immersion } \\
\text { Effective } \\
\text { Dose Rate } \\
(\mathrm{mrem} / \mathrm{sec} \\
\mathrm{per} \\
\left.\mu \mathrm{Ci} / \mathrm{cm}^{3}\right)\end{array}$ & $\begin{array}{c}\text { Water } \\
\text { Submersion } \\
\text { Dose Rate } \\
\text { Coefficient } \\
\left(\mathrm{Sv}-\mathbf{m}^{3} / \mathbf{B q}-\right. \\
\text { s) }\end{array}$ & $\begin{array}{c}\text { Water } \\
\text { Submersion } \\
\text { Dose Rate } \\
\text { Coefficient } \\
\text { (mrem- } \\
\left.\text { m³ }^{\mathbf{3}} / \mathbf{p C i}-\mathbf{h}\right)\end{array}$ & $\begin{array}{c}\text { Water } \\
\text { Submersion } \\
\text { Dose Rate } \\
\text { Coefficient } \\
(\text { mrem- } \\
\left.\text { m }^{3} / \mu \mathrm{Ci}-\mathrm{y}\right)\end{array}$ \\
\hline $\mathrm{Nd}-151$ & 12.44 & $\mathrm{~m}$ & $3.93 \mathrm{E}-14$ & $4.59 \mathrm{E}-03$ & $1.45 \mathrm{E}+02$ & $8.49 \mathrm{E}-17$ & $1.13 \mathrm{E}-09$ & $9.91 \mathrm{E}-12$ \\
\hline $\mathrm{Nd}-152$ & 11.4 & $\mathrm{~m}$ & $7.29 \mathrm{E}-15$ & $8.51 \mathrm{E}-04$ & $2.70 \mathrm{E}+01$ & $1.57 \mathrm{E}-17$ & $2.09 \mathrm{E}-10$ & $1.83 \mathrm{E}-12$ \\
\hline Pm-136 & 107 & $\mathrm{~s}$ & $1.25 \mathrm{E}-13$ & $1.46 \mathrm{E}-02$ & $4.63 \mathrm{E}+02$ & $2.69 \mathrm{E}-16$ & $3.58 \mathrm{E}-09$ & $3.14 \mathrm{E}-11$ \\
\hline $\mathrm{Pm}-137 \mathrm{~m}$ & 2.4 & $\mathrm{~m}$ & $8.01 \mathrm{E}-14$ & $9.35 \mathrm{E}-03$ & $2.96 \mathrm{E}+02$ & $1.74 \mathrm{E}-16$ & $2.32 \mathrm{E}-09$ & $2.03 \mathrm{E}-11$ \\
\hline Pm-139 & 4.15 & $\mathrm{~m}$ & $4.29 \mathrm{E}-14$ & $5.01 \mathrm{E}-03$ & $1.59 \mathrm{E}+02$ & $9.24 \mathrm{E}-17$ & $1.23 \mathrm{E}-09$ & $1.08 \mathrm{E}-11$ \\
\hline Pm-140 & 9.2 & $\mathrm{~s}$ & $4.93 \mathrm{E}-14$ & $5.76 \mathrm{E}-03$ & $1.82 \mathrm{E}+02$ & $1.05 \mathrm{E}-16$ & 1.40E-09 & $1.23 \mathrm{E}-11$ \\
\hline Pm-140m & 5.95 & $\mathrm{~m}$ & $1.39 \mathrm{E}-13$ & $1.62 \mathrm{E}-02$ & $5.14 \mathrm{E}+02$ & $3.01 \mathrm{E}-16$ & $4.01 \mathrm{E}-09$ & $3.51 \mathrm{E}-11$ \\
\hline $\mathrm{Pm}-141$ & 20.9 & $\mathrm{~m}$ & $3.35 \mathrm{E}-14$ & $3.91 \mathrm{E}-03$ & $1.24 \mathrm{E}+02$ & $7.24 \mathrm{E}-17$ & $9.64 \mathrm{E}-10$ & $8.45 \mathrm{E}-12$ \\
\hline Pm-142 & 40.5 & $\mathrm{~s}$ & $3.95 \mathrm{E}-14$ & $4.61 \mathrm{E}-03$ & $1.46 \mathrm{E}+02$ & $8.48 \mathrm{E}-17$ & $1.13 \mathrm{E}-09$ & $9.89 \mathrm{E}-12$ \\
\hline Pm-143 & 265 & $\mathrm{~d}$ & $1.35 \mathrm{E}-14$ & $1.58 \mathrm{E}-03$ & $5.00 \mathrm{E}+01$ & $2.93 \mathrm{E}-17$ & $3.90 \mathrm{E}-10$ & $3.42 \mathrm{E}-12$ \\
\hline Pm-144 & 363 & $\mathrm{~d}$ & $6.95 \mathrm{E}-14$ & $8.12 \mathrm{E}-03$ & $2.57 \mathrm{E}+02$ & $1.51 \mathrm{E}-16$ & $2.01 \mathrm{E}-09$ & $1.76 \mathrm{E}-11$ \\
\hline Pm-145 & 17.7 & $\mathrm{y}$ & $5.50 \mathrm{E}-16$ & $6.42 \mathrm{E}-05$ & $2.04 \mathrm{E}+00$ & $1.28 \mathrm{E}-18$ & $1.70 \mathrm{E}-11$ & $1.49 \mathrm{E}-13$ \\
\hline Pm-146 & 5.53 & $\mathrm{y}$ & $3.33 \mathrm{E}-14$ & $3.89 \mathrm{E}-03$ & $1.23 \mathrm{E}+02$ & $7.23 \mathrm{E}-17$ & $9.63 \mathrm{E}-10$ & $8.44 \mathrm{E}-12$ \\
\hline Pm-147 & 2.6234 & $\mathrm{y}$ & $8.66 \mathrm{E}-18$ & $1.01 \mathrm{E}-06$ & $3.20 \mathrm{E}-02$ & $9.64 \mathrm{E}-21$ & $1.28 \mathrm{E}-13$ & $1.12 \mathrm{E}-15$ \\
\hline Pm-148 & 5.368 & $\mathrm{~d}$ & $2.76 \mathrm{E}-14$ & $3.22 \mathrm{E}-03$ & $1.02 \mathrm{E}+02$ & $5.93 \mathrm{E}-17$ & $7.90 \mathrm{E}-10$ & $6.92 \mathrm{E}-12$ \\
\hline Pm-148m & 41.29 & $\mathrm{~d}$ & $8.99 \mathrm{E}-14$ & $1.05 \mathrm{E}-02$ & $3.33 \mathrm{E}+02$ & $1.95 \mathrm{E}-16$ & $2.60 \mathrm{E}-09$ & $2.28 \mathrm{E}-11$ \\
\hline Pm-149 & 53.08 & $\mathrm{~h}$ & $7.60 \mathrm{E}-16$ & $8.87 \mathrm{E}-05$ & $2.81 \mathrm{E}+00$ & $1.41 \mathrm{E}-18$ & $1.88 \mathrm{E}-11$ & $1.65 \mathrm{E}-13$ \\
\hline Pm-150 & 2.68 & $\mathrm{~h}$ & $6.97 \mathrm{E}-14$ & $8.14 \mathrm{E}-03$ & $2.58 \mathrm{E}+02$ & $1.50 \mathrm{E}-16$ & $2.00 \mathrm{E}-09$ & $1.75 \mathrm{E}-11$ \\
\hline Pm-151 & 28.4 & $\mathrm{~h}$ & $1.44 \mathrm{E}-14$ & $1.68 \mathrm{E}-03$ & $5.33 \mathrm{E}+01$ & $3.12 \mathrm{E}-17$ & $4.16 \mathrm{E}-10$ & $3.64 \mathrm{E}-12$ \\
\hline Pm-152 & 4.12 & $\mathrm{~m}$ & $1.45 \mathrm{E}-14$ & $1.69 \mathrm{E}-03$ & $5.37 \mathrm{E}+01$ & $3.04 \mathrm{E}-17$ & $4.05 \mathrm{E}-10$ & $3.55 \mathrm{E}-12$ \\
\hline Pm-152m & 7.52 & $\mathrm{~m}$ & $7.07 \mathrm{E}-14$ & $8.26 \mathrm{E}-03$ & $2.62 \mathrm{E}+02$ & $1.53 \mathrm{E}-16$ & $2.04 \mathrm{E}-09$ & $1.79 \mathrm{E}-11$ \\
\hline Pm-153 & 5.25 & $\mathrm{~m}$ & $3.40 \mathrm{E}-15$ & $3.97 \mathrm{E}-04$ & $1.26 \mathrm{E}+01$ & $7.02 \mathrm{E}-18$ & $9.35 \mathrm{E}-11$ & $8.19 \mathrm{E}-13$ \\
\hline Pm-154 & 1.73 & $\mathrm{~m}$ & $8.73 \mathrm{E}-14$ & $1.02 \mathrm{E}-02$ & $3.23 \mathrm{E}+02$ & $1.88 \mathrm{E}-16$ & $2.50 \mathrm{E}-09$ & $2.19 \mathrm{E}-11$ \\
\hline
\end{tabular}




\begin{tabular}{|c|c|c|c|c|c|c|c|c|}
\hline Nuclide & Half-life & Class & 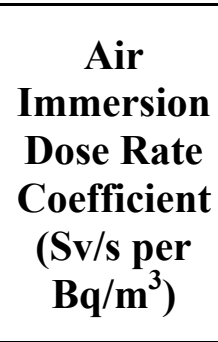 & $\begin{array}{c}\text { Air } \\
\text { Immersion } \\
\text { Dose Rate } \\
\text { Coefficient } \\
\text { (mrem- } \\
\text { m }^{3} / \text { pCi-yr) }\end{array}$ & $\begin{array}{c}\text { Air } \\
\text { Immersion } \\
\text { Effective } \\
\text { Dose Rate } \\
(\mathrm{mrem} / \mathrm{sec} \\
\mathrm{per} \\
\left.\mu \mathrm{Ci} / \mathrm{cm}^{3}\right)\end{array}$ & $\begin{array}{c}\text { Water } \\
\text { Submersion } \\
\text { Dose Rate } \\
\text { Coefficient } \\
\left(\mathrm{Sv}-\mathrm{m}^{3} / \mathrm{Bq}-\right. \\
\text { s) }\end{array}$ & $\begin{array}{c}\text { Water } \\
\text { Submersion } \\
\text { Dose Rate } \\
\text { Coefficient } \\
\text { (mrem- } \\
\left.\text { m³ }^{\mathbf{3}} / \mathbf{p C i}-\mathbf{h}\right)\end{array}$ & $\begin{array}{c}\text { Water } \\
\text { Submersion } \\
\text { Dose Rate } \\
\text { Coefficient } \\
(\text { mrem- } \\
\left.\text { m }^{3} / \mu \mathrm{Ci}-\mathrm{y}\right)\end{array}$ \\
\hline Pm-154m & 2.68 & $\mathrm{~m}$ & $8.50 \mathrm{E}-14$ & $9.92 \mathrm{E}-03$ & $3.15 \mathrm{E}+02$ & $1.84 \mathrm{E}-16$ & $2.45 \mathrm{E}-09$ & $2.15 \mathrm{E}-11$ \\
\hline Sm-139 & 2.57 & $\mathrm{~m}$ & $6.64 \mathrm{E}-14$ & $7.75 \mathrm{E}-03$ & $2.46 \mathrm{E}+02$ & $1.44 \mathrm{E}-16$ & $1.92 \mathrm{E}-09$ & $1.68 \mathrm{E}-11$ \\
\hline Sm-140 & 14.82 & $\mathrm{~m}$ & $2.53 \mathrm{E}-14$ & $2.95 \mathrm{E}-03$ & $9.36 \mathrm{E}+01$ & $5.49 \mathrm{E}-17$ & $7.31 \mathrm{E}-10$ & $6.41 \mathrm{E}-12$ \\
\hline Sm-141 & 10.2 & $\mathrm{~m}$ & $6.47 \mathrm{E}-14$ & $7.55 \mathrm{E}-03$ & $2.39 \mathrm{E}+02$ & $1.40 \mathrm{E}-16$ & $1.86 \mathrm{E}-09$ & $1.63 \mathrm{E}-11$ \\
\hline Sm-141m & 22.6 & $\mathrm{~m}$ & $8.92 \mathrm{E}-14$ & $1.04 \mathrm{E}-02$ & $3.30 \mathrm{E}+02$ & $1.93 \mathrm{E}-16$ & $2.57 \mathrm{E}-09$ & $2.25 \mathrm{E}-11$ \\
\hline Sm-142 & 72.49 & $\mathrm{~m}$ & $4.17 \mathrm{E}-15$ & $4.87 \mathrm{E}-04$ & $1.54 \mathrm{E}+01$ & $9.11 \mathrm{E}-18$ & $1.21 \mathrm{E}-10$ & $1.06 \mathrm{E}-12$ \\
\hline Sm-143 & 8.75 & $\mathrm{~m}$ & $2.36 \mathrm{E}-14$ & $2.76 \mathrm{E}-03$ & $8.73 \mathrm{E}+01$ & $5.11 \mathrm{E}-17$ & $6.81 \mathrm{E}-10$ & $5.96 \mathrm{E}-12$ \\
\hline Sm-143m & 66 & $\mathrm{~s}$ & $3.11 \mathrm{E}-14$ & $3.63 \mathrm{E}-03$ & $1.15 \mathrm{E}+02$ & $6.75 \mathrm{E}-17$ & $8.99 \mathrm{E}-10$ & $7.88 \mathrm{E}-12$ \\
\hline Sm-145 & 340 & $\mathrm{~d}$ & $1.23 \mathrm{E}-15$ & $1.44 \mathrm{E}-04$ & $4.55 \mathrm{E}+00$ & $2.86 \mathrm{E}-18$ & $3.81 \mathrm{E}-11$ & $3.34 \mathrm{E}-13$ \\
\hline Sm-146 & $1.03 \mathrm{E}+08$ & $\mathrm{y}$ & $0.00 \mathrm{E}+00$ & $0.00 \mathrm{E}+00$ & $0.00 \mathrm{E}+00$ & $0.00 \mathrm{E}+00$ & $0.00 \mathrm{E}+00$ & $0.00 \mathrm{E}+00$ \\
\hline Sm-147 & $1.06 \mathrm{E}+11$ & $\mathrm{y}$ & $0.00 \mathrm{E}+00$ & $0.00 \mathrm{E}+00$ & $0.00 \mathrm{E}+00$ & $0.00 \mathrm{E}+00$ & $0.00 \mathrm{E}+00$ & $0.00 \mathrm{E}+00$ \\
\hline Sm-148 & $7.00 \mathrm{E}+15$ & $\mathrm{y}$ & $0.00 \mathrm{E}+00$ & $0.00 \mathrm{E}+00$ & $0.00 \mathrm{E}+00$ & $0.00 \mathrm{E}+00$ & $0.00 \mathrm{E}+00$ & $0.00 \mathrm{E}+00$ \\
\hline Sm-151 & 90 & $\mathrm{y}$ & $2.65 \mathrm{E}-20$ & 3.09E-09 & $9.81 \mathrm{E}-05$ & $6.20 \mathrm{E}-23$ & $8.26 \mathrm{E}-16$ & $7.23 \mathrm{E}-18$ \\
\hline Sm-153 & 46.5 & $\mathrm{~h}$ & $2.12 \mathrm{E}-15$ & $2.48 \mathrm{E}-04$ & $7.84 \mathrm{E}+00$ & $4.65 \mathrm{E}-18$ & $6.19 \mathrm{E}-11$ & $5.43 \mathrm{E}-13$ \\
\hline Sm-155 & 22.3 & $\mathrm{~m}$ & $4.43 \mathrm{E}-15$ & $5.17 \mathrm{E}-04$ & $1.64 \mathrm{E}+01$ & $9.44 \mathrm{E}-18$ & $1.26 \mathrm{E}-10$ & $1.10 \mathrm{E}-12$ \\
\hline Sm-156 & 9.4 & $\mathrm{~h}$ & $4.73 \mathrm{E}-15$ & $5.52 \mathrm{E}-04$ & $1.75 \mathrm{E}+01$ & $1.04 \mathrm{E}-17$ & $1.39 \mathrm{E}-10$ & $1.21 \mathrm{E}-12$ \\
\hline Sm-157 & 8.03 & $\mathrm{~m}$ & $1.91 \mathrm{E}-14$ & $2.23 \mathrm{E}-03$ & $7.07 \mathrm{E}+01$ & $4.09 \mathrm{E}-17$ & $5.45 \mathrm{E}-10$ & $4.77 \mathrm{E}-12$ \\
\hline Eu-142 & 2.34 & $\mathrm{~s}$ & $5.78 \mathrm{E}-14$ & $6.75 \mathrm{E}-03$ & $2.14 \mathrm{E}+02$ & $1.23 \mathrm{E}-16$ & $1.64 \mathrm{E}-09$ & $1.44 \mathrm{E}-11$ \\
\hline Eu-142m & 1.223 & $\mathrm{~m}$ & $1.58 \mathrm{E}-13$ & $1.84 \mathrm{E}-02$ & $5.85 \mathrm{E}+02$ & $3.42 \mathrm{E}-16$ & $4.56 \mathrm{E}-09$ & $3.99 \mathrm{E}-11$ \\
\hline Eu-143 & 2.59 & $\mathrm{~m}$ & $5.26 \mathrm{E}-14$ & $6.14 \mathrm{E}-03$ & $1.95 \mathrm{E}+02$ & $1.13 \mathrm{E}-16$ & $1.51 \mathrm{E}-09$ & $1.32 \mathrm{E}-11$ \\
\hline Eu-144 & 10.2 & $\mathrm{~S}$ & $5.18 \mathrm{E}-14$ & $6.05 \mathrm{E}-03$ & $1.92 \mathrm{E}+02$ & $1.11 \mathrm{E}-16$ & $1.48 \mathrm{E}-09$ & $1.30 \mathrm{E}-11$ \\
\hline Eu-145 & 5.93 & $\mathrm{~d}$ & $5.95 \mathrm{E}-14$ & $6.95 \mathrm{E}-03$ & $2.20 \mathrm{E}+02$ & $1.29 \mathrm{E}-16$ & $1.72 \mathrm{E}-09$ & $1.51 \mathrm{E}-11$ \\
\hline Eu-146 & 4.61 & $\mathrm{~d}$ & $1.11 \mathrm{E}-13$ & $1.30 \mathrm{E}-02$ & $4.11 \mathrm{E}+02$ & $2.40 \mathrm{E}-16$ & $3.20 \mathrm{E}-09$ & $2.80 \mathrm{E}-11$ \\
\hline
\end{tabular}




\begin{tabular}{|c|c|c|c|c|c|c|c|c|}
\hline Nuclide & Half-life & Class & $\begin{array}{c}\text { Air } \\
\text { Immersion } \\
\text { Dose Rate } \\
\text { Coefficient } \\
\left(\mathrm{Sv} / \mathbf{s ~ p e r ~}^{\mathbf{3}}\right. \\
\left.\mathrm{Bq} / \mathbf{m}^{\mathbf{3}}\right)\end{array}$ & $\begin{array}{c}\text { Air } \\
\text { Immersion } \\
\text { Dose Rate } \\
\text { Coefficient } \\
\text { (mrem- } \\
\left.\mathrm{m}^{3} / \mathrm{pCi}-\mathrm{yr}\right)\end{array}$ & $\begin{array}{c}\text { Air } \\
\text { Immersion } \\
\text { Effective } \\
\text { Dose Rate } \\
\text { (mrem/sec } \\
\text { per } \\
\mu \mathrm{Ci} / \mathbf{c m}^{3} \text { ) }\end{array}$ & $\begin{array}{c}\text { Water } \\
\text { Submersion } \\
\text { Dose Rate } \\
\text { Coefficient } \\
\left(\mathbf{S v}-\mathbf{m}^{3} / \mathbf{B q}-\right. \\
\text { s) }\end{array}$ & $\begin{array}{c}\text { Water } \\
\text { Submersion } \\
\text { Dose Rate } \\
\text { Coefficient } \\
\text { (mrem- } \\
\text { m³Ci-h) }^{3} / \text { pCi }\end{array}$ & $\begin{array}{c}\text { Water } \\
\text { Submersion } \\
\text { Dose Rate } \\
\text { Coefficient } \\
(\mathbf{m r e m -} \\
\left.\mathbf{m}^{3} / \mu \mathrm{Ci}-\mathrm{y}\right)\end{array}$ \\
\hline Eu-147 & 24.1 & $\mathrm{~d}$ & $2.03 \mathrm{E}-14$ & $2.37 \mathrm{E}-03$ & $7.51 \mathrm{E}+01$ & $4.42 \mathrm{E}-17$ & $5.89 \mathrm{E}-10$ & $5.16 \mathrm{E}-12$ \\
\hline Eu-148 & 54.5 & $\mathrm{~d}$ & $1.01 \mathrm{E}-13$ & $1.18 \mathrm{E}-02$ & $3.74 \mathrm{E}+02$ & $2.19 \mathrm{E}-16$ & $2.92 \mathrm{E}-09$ & $2.56 \mathrm{E}-11$ \\
\hline Eu-149 & 93.1 & $\mathrm{~d}$ & $2.03 \mathrm{E}-15$ & $2.37 \mathrm{E}-04$ & $7.51 \mathrm{E}+00$ & $4.53 \mathrm{E}-18$ & $6.03 \mathrm{E}-11$ & $5.29 \mathrm{E}-13$ \\
\hline Eu-150 & 36.9 & $\mathrm{y}$ & $6.92 \mathrm{E}-14$ & $8.08 \mathrm{E}-03$ & $2.56 \mathrm{E}+02$ & $1.51 \mathrm{E}-16$ & $2.01 \mathrm{E}-09$ & $1.76 \mathrm{E}-11$ \\
\hline Eu-150m & 12.8 & $\mathrm{~h}$ & $2.33 \mathrm{E}-15$ & $2.72 \mathrm{E}-04$ & $8.62 \mathrm{E}+00$ & $4.88 \mathrm{E}-18$ & $6.50 \mathrm{E}-11$ & $5.69 \mathrm{E}-13$ \\
\hline Eu-152 & 13.537 & $\mathrm{y}$ & $5.38 \mathrm{E}-14$ & $6.28 \mathrm{E}-03$ & $1.99 \mathrm{E}+02$ & $1.17 \mathrm{E}-16$ & $1.56 \mathrm{E}-09$ & $1.37 \mathrm{E}-11$ \\
\hline Eu-152m & 9.3116 & $\mathrm{~h}$ & $1.37 \mathrm{E}-14$ & $1.60 \mathrm{E}-03$ & $5.07 \mathrm{E}+01$ & $2.94 \mathrm{E}-17$ & $3.92 \mathrm{E}-10$ & $3.43 \mathrm{E}-12$ \\
\hline Eu-152n & 96 & $\mathrm{~m}$ & $2.63 \mathrm{E}-15$ & $3.07 \mathrm{E}-04$ & $9.73 \mathrm{E}+00$ & $5.92 \mathrm{E}-18$ & $7.89 \mathrm{E}-11$ & $6.91 \mathrm{E}-13$ \\
\hline Eu-154 & 8.593 & $\mathrm{y}$ & $5.78 \mathrm{E}-14$ & $6.75 \mathrm{E}-03$ & $2.14 \mathrm{E}+02$ & $1.25 \mathrm{E}-16$ & $1.67 \mathrm{E}-09$ & $1.46 \mathrm{E}-11$ \\
\hline Eu-154m & 46 & $\mathrm{~m}$ & $2.15 \mathrm{E}-15$ & $2.51 \mathrm{E}-04$ & $7.96 \mathrm{E}+00$ & $4.87 \mathrm{E}-18$ & $6.49 \mathrm{E}-11$ & $5.68 \mathrm{E}-13$ \\
\hline Eu-155 & 4.7611 & $\mathrm{y}$ & $2.17 \mathrm{E}-15$ & $2.53 \mathrm{E}-04$ & $8.03 \mathrm{E}+00$ & $4.87 \mathrm{E}-18$ & $6.49 \mathrm{E}-11$ & $5.68 \mathrm{E}-13$ \\
\hline Eu-156 & 15.19 & $\mathrm{~d}$ & $5.94 \mathrm{E}-14$ & $6.94 \mathrm{E}-03$ & $2.20 \mathrm{E}+02$ & $1.28 \mathrm{E}-16$ & $1.70 \mathrm{E}-09$ & $1.49 \mathrm{E}-11$ \\
\hline Eu-157 & 15.18 & $\mathrm{~h}$ & $1.23 \mathrm{E}-14$ & $1.44 \mathrm{E}-03$ & $4.55 \mathrm{E}+01$ & $2.67 \mathrm{E}-17$ & $3.56 \mathrm{E}-10$ & $3.12 \mathrm{E}-12$ \\
\hline Eu-158 & 45.9 & $\mathrm{~m}$ & $6.14 \mathrm{E}-14$ & $7.17 \mathrm{E}-03$ & $2.27 \mathrm{E}+02$ & $1.32 \mathrm{E}-16$ & $1.76 \mathrm{E}-09$ & $1.54 \mathrm{E}-11$ \\
\hline Eu-159 & 18.1 & $\mathrm{~m}$ & $1.32 \mathrm{E}-14$ & $1.54 \mathrm{E}-03$ & $4.88 \mathrm{E}+01$ & $2.83 \mathrm{E}-17$ & $3.77 \mathrm{E}-10$ & $3.30 \mathrm{E}-12$ \\
\hline Gd-142 & 70.2 & $\mathrm{~S}$ & $4.78 \mathrm{E}-14$ & $5.58 \mathrm{E}-03$ & $1.77 \mathrm{E}+02$ & $1.03 \mathrm{E}-16$ & $1.37 \mathrm{E}-09$ & $1.20 \mathrm{E}-11$ \\
\hline Gd-143m & 110 & $\mathrm{~S}$ & $9.77 \mathrm{E}-14$ & $1.14 \mathrm{E}-02$ & $3.61 \mathrm{E}+02$ & $2.11 \mathrm{E}-16$ & $2.81 \mathrm{E}-09$ & $2.46 \mathrm{E}-11$ \\
\hline Gd-144 & 4.47 & $\mathrm{~m}$ & $4.25 \mathrm{E}-14$ & $4.96 \mathrm{E}-03$ & $1.57 \mathrm{E}+02$ & $9.19 \mathrm{E}-17$ & $1.22 \mathrm{E}-09$ & $1.07 \mathrm{E}-11$ \\
\hline Gd-145 & 23 & $\mathrm{~m}$ & $1.18 \mathrm{E}-13$ & $1.38 \mathrm{E}-02$ & $4.37 \mathrm{E}+02$ & $2.55 \mathrm{E}-16$ & $3.40 \mathrm{E}-09$ & $2.98 \mathrm{E}-11$ \\
\hline Gd-145m & 85 & $\mathrm{~s}$ & $3.08 \mathrm{E}-14$ & $3.60 \mathrm{E}-03$ & $1.14 \mathrm{E}+02$ & $6.67 \mathrm{E}-17$ & $8.88 \mathrm{E}-10$ & $7.78 \mathrm{E}-12$ \\
\hline Gd-146 & 48.27 & $\mathrm{~d}$ & $8.74 \mathrm{E}-15$ & $1.02 \mathrm{E}-03$ & $3.23 \mathrm{E}+01$ & $1.95 \mathrm{E}-17$ & $2.60 \mathrm{E}-10$ & $2.28 \mathrm{E}-12$ \\
\hline Gd-147 & 38.1 & $\mathrm{~h}$ & $6.29 \mathrm{E}-14$ & $7.34 \mathrm{E}-03$ & $2.33 \mathrm{E}+02$ & $1.37 \mathrm{E}-16$ & $1.82 \mathrm{E}-09$ & $1.60 \mathrm{E}-11$ \\
\hline Gd-148 & 74.6 & $\mathrm{y}$ & $0.00 \mathrm{E}+00$ & $0.00 \mathrm{E}+00$ & $0.00 \mathrm{E}+00$ & $0.00 \mathrm{E}+00$ & $0.00 \mathrm{E}+00$ & $0.00 \mathrm{E}+00$ \\
\hline
\end{tabular}




\begin{tabular}{|c|c|c|c|c|c|c|c|c|}
\hline Nuclide & Half-life & Class & $\begin{array}{c}\text { Air } \\
\text { Immersion } \\
\text { Dose Rate } \\
\text { Coefficient } \\
(\mathrm{Sv} / \mathrm{s} \mathrm{per} \\
\left.\mathrm{Bq} / \mathbf{m}^{3}\right)\end{array}$ & $\begin{array}{c}\text { Air } \\
\text { Immersion } \\
\text { Dose Rate } \\
\text { Coefficient } \\
\text { (mrem- } \\
\left.\text { m³ }^{3} / \mathrm{pCi}-\mathrm{yr}\right)\end{array}$ & $\begin{array}{c}\text { Air } \\
\text { Immersion } \\
\text { Effective } \\
\text { Dose Rate } \\
(\mathrm{mrem} / \mathrm{sec} \\
\mathrm{per} \\
\left.\mu \mathrm{Ci} / \mathrm{cm}^{3}\right)\end{array}$ & $\begin{array}{c}\text { Water } \\
\text { Submersion } \\
\text { Dose Rate } \\
\text { Coefficient } \\
\left(\mathrm{Sv}-\mathbf{m}^{3} / \mathbf{B q}-\right. \\
\text { s) }\end{array}$ & $\begin{array}{c}\text { Water } \\
\text { Submersion } \\
\text { Dose Rate } \\
\text { Coefficient } \\
\text { (mrem- } \\
\text { m³Ci-h) }^{\mathbf{3} / p C i}\end{array}$ & $\begin{array}{c}\text { Water } \\
\text { Submersion } \\
\text { Dose Rate } \\
\text { Coefficient } \\
(\text { mrem- } \\
\left.\text { m }^{3} / \mu \mathrm{Ci}-\mathrm{y}\right)\end{array}$ \\
\hline Gd-149 & 9.28 & $\mathrm{~d}$ & $2.24 \mathrm{E}-14$ & $2.62 \mathrm{E}-03$ & $8.29 \mathrm{E}+01$ & $4.89 \mathrm{E}-17$ & $6.51 \mathrm{E}-10$ & $5.71 \mathrm{E}-12$ \\
\hline Gd-150 & $1.79 \mathrm{E}+06$ & $\mathrm{y}$ & $0.00 \mathrm{E}+00$ & $0.00 \mathrm{E}+00$ & $0.00 \mathrm{E}+00$ & $0.00 \mathrm{E}+00$ & $0.00 \mathrm{E}+00$ & $0.00 \mathrm{E}+00$ \\
\hline Gd-151 & 124 & $\mathrm{~d}$ & $2.15 \mathrm{E}-15$ & $2.51 \mathrm{E}-04$ & $7.96 \mathrm{E}+00$ & $4.81 \mathrm{E}-18$ & $6.41 \mathrm{E}-11$ & $5.61 \mathrm{E}-13$ \\
\hline Gd-152 & $1.08 \mathrm{E}+14$ & $\mathrm{y}$ & $0.00 \mathrm{E}+00$ & $0.00 \mathrm{E}+00$ & $0.00 \mathrm{E}+00$ & $0.00 \mathrm{E}+00$ & $0.00 \mathrm{E}+00$ & $0.00 \mathrm{E}+00$ \\
\hline Gd-153 & 240.4 & $\mathrm{~d}$ & $3.11 \mathrm{E}-15$ & $3.63 \mathrm{E}-04$ & $1.15 \mathrm{E}+01$ & $7.05 \mathrm{E}-18$ & $9.39 \mathrm{E}-11$ & $8.23 \mathrm{E}-13$ \\
\hline Gd-159 & 18.479 & $\mathrm{~h}$ & $2.35 \mathrm{E}-15$ & $2.74 \mathrm{E}-04$ & $8.70 \mathrm{E}+00$ & $4.95 \mathrm{E}-18$ & $6.59 \mathrm{E}-11$ & $5.78 \mathrm{E}-13$ \\
\hline Gd-162 & 8.4 & $\mathrm{~m}$ & $1.86 \mathrm{E}-14$ & $2.17 \mathrm{E}-03$ & $6.88 \mathrm{E}+01$ & $4.02 \mathrm{E}-17$ & $5.35 \mathrm{E}-10$ & $4.69 \mathrm{E}-12$ \\
\hline Tb-146 & 23 & $\mathrm{~s}$ & $1.74 \mathrm{E}-13$ & $2.03 \mathrm{E}-02$ & $6.44 \mathrm{E}+02$ & $3.77 \mathrm{E}-16$ & $5.02 \mathrm{E}-09$ & $4.40 \mathrm{E}-11$ \\
\hline Tb-147 & 1.64 & $\mathrm{~h}$ & $1.02 \mathrm{E}-13$ & $1.19 \mathrm{E}-02$ & $3.77 \mathrm{E}+02$ & $2.20 \mathrm{E}-16$ & $2.93 \mathrm{E}-09$ & $2.57 \mathrm{E}-11$ \\
\hline Tb-147m & 1.87 & $\mathrm{~m}$ & $9.11 \mathrm{E}-14$ & $1.06 \mathrm{E}-02$ & $3.37 \mathrm{E}+02$ & $1.97 \mathrm{E}-16$ & $2.62 \mathrm{E}-09$ & $2.30 \mathrm{E}-11$ \\
\hline Tb-148 & 60 & $\mathrm{~m}$ & $1.11 \mathrm{E}-13$ & $1.30 \mathrm{E}-02$ & $4.11 \mathrm{E}+02$ & $2.40 \mathrm{E}-16$ & $3.20 \mathrm{E}-09$ & $2.80 \mathrm{E}-11$ \\
\hline Tb-148m & 2.2 & $\mathrm{~m}$ & $1.42 \mathrm{E}-13$ & $1.66 \mathrm{E}-02$ & $5.25 \mathrm{E}+02$ & $3.09 \mathrm{E}-16$ & $4.12 \mathrm{E}-09$ & $3.61 \mathrm{E}-11$ \\
\hline Tb-149 & 4.118 & $\mathrm{~h}$ & $6.28 \mathrm{E}-14$ & 7.33E-03 & $2.32 \mathrm{E}+02$ & $1.36 \mathrm{E}-16$ & $1.81 \mathrm{E}-09$ & $1.59 \mathrm{E}-11$ \\
\hline Tb-149m & 4.16 & $\mathrm{~m}$ & $6.20 \mathrm{E}-14$ & 7.24E-03 & $2.29 \mathrm{E}+02$ & $1.34 \mathrm{E}-16$ & $1.78 \mathrm{E}-09$ & $1.56 \mathrm{E}-11$ \\
\hline Tb-150 & 3.48 & $\mathrm{~h}$ & $1.17 \mathrm{E}-13$ & $1.37 \mathrm{E}-02$ & $4.33 \mathrm{E}+02$ & $2.54 \mathrm{E}-16$ & $3.38 \mathrm{E}-09$ & $2.96 \mathrm{E}-11$ \\
\hline Tb-150m & 5.8 & $\mathrm{~m}$ & $1.12 \mathrm{E}-13$ & $1.31 \mathrm{E}-02$ & $4.14 \mathrm{E}+02$ & $2.44 \mathrm{E}-16$ & $3.25 \mathrm{E}-09$ & $2.85 \mathrm{E}-11$ \\
\hline Tb-151 & 17.609 & $\mathrm{~h}$ & $4.34 \mathrm{E}-14$ & $5.07 \mathrm{E}-03$ & $1.61 \mathrm{E}+02$ & $9.47 \mathrm{E}-17$ & $1.26 \mathrm{E}-09$ & $1.10 \mathrm{E}-11$ \\
\hline Tb-151m & 25 & $\mathrm{~s}$ & $3.20 \mathrm{E}-15$ & $3.74 \mathrm{E}-04$ & $1.18 \mathrm{E}+01$ & $6.99 \mathrm{E}-18$ & $9.31 \mathrm{E}-11$ & $8.16 \mathrm{E}-13$ \\
\hline Tb-152 & 17.5 & $\mathrm{~h}$ & $6.98 \mathrm{E}-14$ & $8.15 \mathrm{E}-03$ & $2.58 \mathrm{E}+02$ & $1.51 \mathrm{E}-16$ & $2.01 \mathrm{E}-09$ & $1.76 \mathrm{E}-11$ \\
\hline Tb-152m & 4.2 & $\mathrm{~m}$ & $3.28 \mathrm{E}-14$ & $3.83 \mathrm{E}-03$ & $1.21 \mathrm{E}+02$ & $7.16 \mathrm{E}-17$ & $9.54 \mathrm{E}-10$ & $8.35 \mathrm{E}-12$ \\
\hline Tb-153 & 2.34 & $\mathrm{~d}$ & $1.35 \mathrm{E}-14$ & $1.58 \mathrm{E}-03$ & $5.00 \mathrm{E}+01$ & $2.97 \mathrm{E}-17$ & $3.96 \mathrm{E}-10$ & $3.47 \mathrm{E}-12$ \\
\hline Tb-154 & 21.5 & $\mathrm{~h}$ & $1.11 \mathrm{E}-13$ & $1.30 \mathrm{E}-02$ & $4.11 \mathrm{E}+02$ & $2.40 \mathrm{E}-16$ & $3.20 \mathrm{E}-09$ & $2.80 \mathrm{E}-11$ \\
\hline Tb-155 & 5.32 & $\mathrm{~d}$ & $6.30 \mathrm{E}-15$ & $7.36 \mathrm{E}-04$ & $2.33 \mathrm{E}+01$ & $1.40 \mathrm{E}-17$ & $1.86 \mathrm{E}-10$ & $1.63 \mathrm{E}-12$ \\
\hline
\end{tabular}




\begin{tabular}{|c|c|c|c|c|c|c|c|c|}
\hline Nuclide & Half-life & Class & $\begin{array}{c}\text { Air } \\
\text { Immersion } \\
\text { Dose Rate } \\
\text { Coefficient } \\
\left(\mathrm{Sv} / \mathbf{s ~ p e r ~}^{\mathbf{3}}\right. \\
\left.\mathrm{Bq} / \mathbf{m}^{\mathbf{3}}\right)\end{array}$ & $\begin{array}{c}\text { Air } \\
\text { Immersion } \\
\text { Dose Rate } \\
\text { Coefficient } \\
\text { (mrem- } \\
\left.\text { m³ }^{3} / \mathrm{pCi}-\mathrm{yr}\right)\end{array}$ & $\begin{array}{c}\text { Air } \\
\text { Immersion } \\
\text { Effective } \\
\text { Dose Rate } \\
\text { (mrem/sec } \\
\text { per } \\
\mu \mathrm{Ci} / \mathbf{c m}^{3} \text { ) }\end{array}$ & $\begin{array}{c}\text { Water } \\
\text { Submersion } \\
\text { Dose Rate } \\
\text { Coefficient } \\
\left(\mathbf{S v}-\mathbf{m}^{3} / \mathbf{B q}-\right. \\
\text { s) }\end{array}$ & $\begin{array}{c}\text { Water } \\
\text { Submersion } \\
\text { Dose Rate } \\
\text { Coefficient } \\
\text { (mrem- } \\
\left.\text { m³ }^{\mathbf{3}} / \mathbf{p C i}-\mathbf{h}\right)\end{array}$ & $\begin{array}{c}\text { Water } \\
\text { Submersion } \\
\text { Dose Rate } \\
\text { Coefficient } \\
(\text { mrem- } \\
\left.\mathbf{m}^{\mathbf{3}} / \boldsymbol{\mu C i}-\mathrm{y}\right)\end{array}$ \\
\hline Tb-156 & 5.35 & $\mathrm{~d}$ & $8.87 \mathrm{E}-14$ & $1.04 \mathrm{E}-02$ & $3.28 \mathrm{E}+02$ & $1.93 \mathrm{E}-16$ & $2.57 \mathrm{E}-09$ & $2.25 \mathrm{E}-11$ \\
\hline $\mathrm{Tb}-156 \mathrm{~m}$ & 24.4 & $\mathrm{~h}$ & $9.24 \mathrm{E}-16$ & $1.08 \mathrm{E}-04$ & $3.42 \mathrm{E}+00$ & $2.13 \mathrm{E}-18$ & $2.84 \mathrm{E}-11$ & $2.49 \mathrm{E}-13$ \\
\hline Tb-156n & 5.3 & $\mathrm{~h}$ & $9.97 \mathrm{E}-17$ & $1.16 \mathrm{E}-05$ & $3.69 \mathrm{E}-01$ & $2.25 \mathrm{E}-19$ & $3.00 \mathrm{E}-12$ & $2.63 \mathrm{E}-14$ \\
\hline Tb-157 & 71 & $\mathrm{y}$ & $9.83 \mathrm{E}-17$ & $1.15 \mathrm{E}-05$ & $3.64 \mathrm{E}-01$ & $2.28 \mathrm{E}-19$ & $3.04 \mathrm{E}-12$ & $2.66 \mathrm{E}-14$ \\
\hline Tb-158 & 180 & $\mathrm{y}$ & $3.61 \mathrm{E}-14$ & $4.22 \mathrm{E}-03$ & $1.34 \mathrm{E}+02$ & $7.84 \mathrm{E}-17$ & $1.04 \mathrm{E}-09$ & $9.15 \mathrm{E}-12$ \\
\hline Tb-160 & 72.3 & $\mathrm{~d}$ & $5.20 \mathrm{E}-14$ & $6.07 \mathrm{E}-03$ & $1.92 \mathrm{E}+02$ & $1.13 \mathrm{E}-16$ & $1.51 \mathrm{E}-09$ & $1.32 \mathrm{E}-11$ \\
\hline Tb-161 & 6.906 & $\mathrm{~d}$ & $9.14 \mathrm{E}-16$ & $1.07 \mathrm{E}-04$ & $3.38 \mathrm{E}+00$ & $2.01 \mathrm{E}-18$ & $2.68 \mathrm{E}-11$ & $2.35 \mathrm{E}-13$ \\
\hline Tb-162 & 7.6 & $\mathrm{~m}$ & $5.04 \mathrm{E}-14$ & $5.88 \mathrm{E}-03$ & $1.86 \mathrm{E}+02$ & $1.09 \mathrm{E}-16$ & $1.45 \mathrm{E}-09$ & $1.27 \mathrm{E}-11$ \\
\hline Tb-163 & 19.5 & $\mathrm{~m}$ & $3.51 \mathrm{E}-14$ & $4.10 \mathrm{E}-03$ & $1.30 \mathrm{E}+02$ & $7.62 \mathrm{E}-17$ & $1.01 \mathrm{E}-09$ & $8.89 \mathrm{E}-12$ \\
\hline Tb-164 & 3 & $\mathrm{~m}$ & $1.14 \mathrm{E}-13$ & $1.33 \mathrm{E}-02$ & $4.22 \mathrm{E}+02$ & $2.47 \mathrm{E}-16$ & $3.29 \mathrm{E}-09$ & $2.88 \mathrm{E}-11$ \\
\hline Tb-165 & 2.11 & $\mathrm{~m}$ & $4.04 \mathrm{E}-14$ & $4.72 \mathrm{E}-03$ & $1.49 \mathrm{E}+02$ & $8.67 \mathrm{E}-17$ & $1.15 \mathrm{E}-09$ & $1.01 \mathrm{E}-11$ \\
\hline Dy-148 & 3.3 & $\mathrm{~m}$ & $3.15 \mathrm{E}-14$ & $3.68 \mathrm{E}-03$ & $1.17 \mathrm{E}+02$ & $6.85 \mathrm{E}-17$ & $9.12 \mathrm{E}-10$ & $7.99 \mathrm{E}-12$ \\
\hline Dy-149 & 4.2 & $\mathrm{~m}$ & $7.52 \mathrm{E}-14$ & $8.78 \mathrm{E}-03$ & $2.78 \mathrm{E}+02$ & $1.63 \mathrm{E}-16$ & $2.17 \mathrm{E}-09$ & $1.90 \mathrm{E}-11$ \\
\hline Dy-150 & 7.17 & $\mathrm{~m}$ & $1.18 \mathrm{E}-14$ & $1.38 \mathrm{E}-03$ & $4.37 \mathrm{E}+01$ & $2.57 \mathrm{E}-17$ & $3.42 \mathrm{E}-10$ & $3.00 \mathrm{E}-12$ \\
\hline Dy-151 & 17.9 & $\mathrm{~m}$ & $6.28 \mathrm{E}-14$ & $7.33 \mathrm{E}-03$ & $2.32 \mathrm{E}+02$ & $1.36 \mathrm{E}-16$ & $1.81 \mathrm{E}-09$ & $1.59 \mathrm{E}-11$ \\
\hline Dy-152 & 2.38 & $\mathrm{~h}$ & $1.18 \mathrm{E}-14$ & $1.38 \mathrm{E}-03$ & $4.37 \mathrm{E}+01$ & $2.59 \mathrm{E}-17$ & $3.45 \mathrm{E}-10$ & $3.02 \mathrm{E}-12$ \\
\hline Dy-153 & 6.4 & $\mathrm{~h}$ & $3.82 \mathrm{E}-14$ & $4.46 \mathrm{E}-03$ & $1.41 \mathrm{E}+02$ & $8.34 \mathrm{E}-17$ & $1.11 \mathrm{E}-09$ & $9.73 \mathrm{E}-12$ \\
\hline Dy-154 & $3.00 \mathrm{E}+06$ & $\mathrm{y}$ & $0.00 \mathrm{E}+00$ & $0.00 \mathrm{E}+00$ & $0.00 \mathrm{E}+00$ & $0.00 \mathrm{E}+00$ & $0.00 \mathrm{E}+00$ & $0.00 \mathrm{E}+00$ \\
\hline Dy-155 & 9.9 & $\mathrm{~h}$ & $2.96 \mathrm{E}-14$ & $3.46 \mathrm{E}-03$ & $1.10 \mathrm{E}+02$ & $6.45 \mathrm{E}-17$ & $8.59 \mathrm{E}-10$ & $7.53 \mathrm{E}-12$ \\
\hline Dy-157 & 8.14 & $\mathrm{~h}$ & $1.44 \mathrm{E}-14$ & $1.68 \mathrm{E}-03$ & $5.33 \mathrm{E}+01$ & $3.15 \mathrm{E}-17$ & $4.20 \mathrm{E}-10$ & $3.68 \mathrm{E}-12$ \\
\hline Dy-159 & 144.4 & $\mathrm{~d}$ & $1.00 \mathrm{E}-15$ & $1.17 \mathrm{E}-04$ & $3.70 \mathrm{E}+00$ & $2.32 \mathrm{E}-18$ & $3.09 \mathrm{E}-11$ & $2.71 \mathrm{E}-13$ \\
\hline Dy-165 & 2.334 & $\mathrm{~h}$ & $1.39 \mathrm{E}-15$ & $1.62 \mathrm{E}-04$ & $5.14 \mathrm{E}+00$ & $2.73 \mathrm{E}-18$ & $3.64 \mathrm{E}-11$ & $3.19 \mathrm{E}-13$ \\
\hline Dy-165m & 1.257 & $\mathrm{~m}$ & $7.08 \mathrm{E}-16$ & $8.27 \mathrm{E}-05$ & $2.62 \mathrm{E}+00$ & $1.55 \mathrm{E}-18$ & $2.06 \mathrm{E}-11$ & $1.81 \mathrm{E}-13$ \\
\hline
\end{tabular}




\begin{tabular}{|c|c|c|c|c|c|c|c|c|}
\hline Nuclide & Half-life & Class & 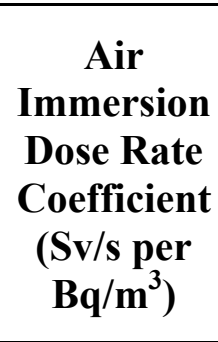 & $\begin{array}{c}\text { Air } \\
\text { Immersion } \\
\text { Dose Rate } \\
\text { Coefficient } \\
\text { (mrem- } \\
\text { m }^{3} / \text { pCi-yr) }\end{array}$ & $\begin{array}{c}\text { Air } \\
\text { Immersion } \\
\text { Effective } \\
\text { Dose Rate } \\
(\mathrm{mrem} / \mathrm{sec} \\
\mathrm{per} \\
\left.\mu \mathrm{Ci} / \mathrm{cm}^{3}\right)\end{array}$ & $\begin{array}{c}\text { Water } \\
\text { Submersion } \\
\text { Dose Rate } \\
\text { Coefficient } \\
\left(\mathrm{Sv}-\mathbf{m}^{3} / \mathbf{B q}-\right. \\
\text { s) }\end{array}$ & $\begin{array}{c}\text { Water } \\
\text { Submersion } \\
\text { Dose Rate } \\
\text { Coefficient } \\
\text { (mrem- } \\
\left.\text { m³ }^{\mathbf{3}} / \mathbf{p C i}-\mathbf{h}\right)\end{array}$ & $\begin{array}{c}\text { Water } \\
\text { Submersion } \\
\text { Dose Rate } \\
\text { Coefficient } \\
(\text { mrem- } \\
\left.\text { m }^{3} / \mu \mathrm{Ci}-\mathrm{y}\right)\end{array}$ \\
\hline Dy-166 & 81.6 & $\mathrm{~h}$ & $1.31 \mathrm{E}-15$ & $1.53 \mathrm{E}-04$ & $4.85 \mathrm{E}+00$ & $2.91 \mathrm{E}-18$ & $3.88 \mathrm{E}-11$ & $3.40 \mathrm{E}-13$ \\
\hline Dy-167 & 6.2 & $\mathrm{~m}$ & $2.40 \mathrm{E}-14$ & $2.80 \mathrm{E}-03$ & $8.88 \mathrm{E}+01$ & $5.17 \mathrm{E}-17$ & $6.89 \mathrm{E}-10$ & $6.03 \mathrm{E}-12$ \\
\hline Dy-168 & 8.7 & $\mathrm{~m}$ & $1.73 \mathrm{E}-14$ & $2.02 \mathrm{E}-03$ & $6.40 \mathrm{E}+01$ & $3.74 \mathrm{E}-17$ & $4.98 \mathrm{E}-10$ & $4.36 \mathrm{E}-12$ \\
\hline Ho-150 & 76.8 & $\mathrm{~S}$ & $8.73 \mathrm{E}-14$ & $1.02 \mathrm{E}-02$ & $3.23 \mathrm{E}+02$ & $1.88 \mathrm{E}-16$ & $2.50 \mathrm{E}-09$ & $2.19 \mathrm{E}-11$ \\
\hline Ho-153 & 2.01 & $\mathrm{~m}$ & $4.61 \mathrm{E}-14$ & $5.38 \mathrm{E}-03$ & $1.71 \mathrm{E}+02$ & $9.99 \mathrm{E}-17$ & $1.33 \mathrm{E}-09$ & $1.17 \mathrm{E}-11$ \\
\hline Ho- $153 \mathrm{~m}$ & 9.3 & $\mathrm{~m}$ & $4.72 \mathrm{E}-14$ & $5.51 \mathrm{E}-03$ & $1.75 \mathrm{E}+02$ & $1.02 \mathrm{E}-16$ & $1.36 \mathrm{E}-09$ & $1.19 \mathrm{E}-11$ \\
\hline Ho-154 & 11.76 & $\mathrm{~m}$ & $8.63 \mathrm{E}-14$ & $1.01 \mathrm{E}-02$ & $3.19 \mathrm{E}+02$ & $1.87 \mathrm{E}-16$ & $2.49 \mathrm{E}-09$ & $2.18 \mathrm{E}-11$ \\
\hline Ho- $154 \mathrm{~m}$ & 3.1 & $\mathrm{~m}$ & $1.09 \mathrm{E}-13$ & $1.27 \mathrm{E}-02$ & $4.03 \mathrm{E}+02$ & $2.37 \mathrm{E}-16$ & $3.16 \mathrm{E}-09$ & $2.77 \mathrm{E}-11$ \\
\hline Ho-155 & 48 & $\mathrm{~m}$ & $2.71 \mathrm{E}-14$ & $3.16 \mathrm{E}-03$ & $1.00 \mathrm{E}+02$ & $5.89 \mathrm{E}-17$ & $7.85 \mathrm{E}-10$ & $6.87 \mathrm{E}-12$ \\
\hline Ho-156 & 56 & $\mathrm{~m}$ & $9.78 \mathrm{E}-14$ & $1.14 \mathrm{E}-02$ & $3.62 \mathrm{E}+02$ & $2.12 \mathrm{E}-16$ & $2.82 \mathrm{E}-09$ & $2.47 \mathrm{E}-11$ \\
\hline Ho-157 & 12.6 & $\mathrm{~m}$ & $2.46 \mathrm{E}-14$ & $2.87 \mathrm{E}-03$ & $9.10 \mathrm{E}+01$ & $5.38 \mathrm{E}-17$ & $7.17 \mathrm{E}-10$ & $6.28 \mathrm{E}-12$ \\
\hline Ho-159 & 33.05 & $\mathrm{~m}$ & $1.52 \mathrm{E}-14$ & $1.77 \mathrm{E}-03$ & $5.62 \mathrm{E}+01$ & $3.36 \mathrm{E}-17$ & $4.48 \mathrm{E}-10$ & $3.92 \mathrm{E}-12$ \\
\hline Ho-160 & 25.6 & $\mathrm{~m}$ & $7.64 \mathrm{E}-14$ & $8.92 \mathrm{E}-03$ & $2.83 \mathrm{E}+02$ & $1.66 \mathrm{E}-16$ & $2.21 \mathrm{E}-09$ & $1.94 \mathrm{E}-11$ \\
\hline Ho-161 & 2.48 & $\mathrm{~h}$ & $1.33 \mathrm{E}-15$ & $1.55 \mathrm{E}-04$ & $4.92 \mathrm{E}+00$ & $3.06 \mathrm{E}-18$ & $4.08 \mathrm{E}-11$ & $3.57 \mathrm{E}-13$ \\
\hline Ho-162 & 15 & $\mathrm{~m}$ & $6.54 \mathrm{E}-15$ & 7.64E-04 & $2.42 \mathrm{E}+01$ & $1.43 \mathrm{E}-17$ & $1.90 \mathrm{E}-10$ & $1.67 \mathrm{E}-12$ \\
\hline Ho- $162 \mathrm{~m}$ & 67 & $\mathrm{~m}$ & $2.48 \mathrm{E}-14$ & $2.90 \mathrm{E}-03$ & $9.18 \mathrm{E}+01$ & $5.40 \mathrm{E}-17$ & $7.19 \mathrm{E}-10$ & $6.30 \mathrm{E}-12$ \\
\hline Ho-163 & 4570 & $\mathrm{y}$ & $0.00 \mathrm{E}+00$ & $0.00 \mathrm{E}+00$ & $0.00 \mathrm{E}+00$ & $0.00 \mathrm{E}+00$ & $0.00 \mathrm{E}+00$ & $0.00 \mathrm{E}+00$ \\
\hline Ho-164 & 29 & $\mathrm{~m}$ & $8.04 \mathrm{E}-16$ & $9.39 \mathrm{E}-05$ & $2.97 \mathrm{E}+00$ & $1.76 \mathrm{E}-18$ & $2.34 \mathrm{E}-11$ & $2.05 \mathrm{E}-13$ \\
\hline Ho- $164 \mathrm{~m}$ & 38 & $\mathrm{~m}$ & $1.06 \mathrm{E}-15$ & $1.24 \mathrm{E}-04$ & $3.92 \mathrm{E}+00$ & $2.44 \mathrm{E}-18$ & $3.25 \mathrm{E}-11$ & $2.85 \mathrm{E}-13$ \\
\hline Ho-166 & 26.8 & $\mathrm{~h}$ & $1.75 \mathrm{E}-15$ & $2.04 \mathrm{E}-04$ & $6.48 \mathrm{E}+00$ & $3.34 \mathrm{E}-18$ & $4.45 \mathrm{E}-11$ & $3.90 \mathrm{E}-13$ \\
\hline Ho-166m & $1.20 \mathrm{E}+03$ & $\mathrm{y}$ & $7.29 \mathrm{E}-14$ & $8.51 \mathrm{E}-03$ & $2.70 \mathrm{E}+02$ & $1.59 \mathrm{E}-16$ & $2.12 \mathrm{E}-09$ & $1.86 \mathrm{E}-11$ \\
\hline Ho-167 & 3.1 & $\mathrm{~h}$ & $1.60 \mathrm{E}-14$ & $1.87 \mathrm{E}-03$ & $5.92 \mathrm{E}+01$ & $3.48 \mathrm{E}-17$ & $4.64 \mathrm{E}-10$ & $4.06 \mathrm{E}-12$ \\
\hline Ho-168 & 2.99 & $\mathrm{~m}$ & $4.06 \mathrm{E}-14$ & 4.74E-03 & $1.50 \mathrm{E}+02$ & $8.74 \mathrm{E}-17$ & $1.16 \mathrm{E}-09$ & $1.02 \mathrm{E}-11$ \\
\hline
\end{tabular}




\begin{tabular}{|c|c|c|c|c|c|c|c|c|}
\hline Nuclide & Half-life & Class & $\begin{array}{c}\text { Air } \\
\text { Immersion } \\
\text { Dose Rate } \\
\text { Coefficient } \\
(\text { Sv/s per } \\
\left.\text { Bq/ } \mathbf{m}^{3}\right)\end{array}$ & $\begin{array}{c}\text { Air } \\
\text { Immersion } \\
\text { Dose Rate } \\
\text { Coefficient } \\
\text { (mrem- } \\
\text { m³Ci-yr) }^{3} / \mathrm{pCi}\end{array}$ & $\begin{array}{c}\text { Air } \\
\text { Immersion } \\
\text { Effective } \\
\text { Dose Rate } \\
(\mathrm{mrem} / \mathrm{sec} \\
\mathrm{per} \\
\left.\mu \mathrm{Ci} / \mathrm{cm}^{3}\right)\end{array}$ & $\begin{array}{c}\text { Water } \\
\text { Submersion } \\
\text { Dose Rate } \\
\text { Coefficient } \\
\left(\mathrm{Sv}-\mathbf{m}^{3} / \mathbf{B q}-\right. \\
\text { s) }\end{array}$ & $\begin{array}{c}\text { Water } \\
\text { Submersion } \\
\text { Dose Rate } \\
\text { Coefficient } \\
\text { (mrem- } \\
\text { m³Ci-h) }^{\mathbf{3} / p C i}\end{array}$ & $\begin{array}{c}\text { Water } \\
\text { Submersion } \\
\text { Dose Rate } \\
\text { Coefficient } \\
(\text { mrem- } \\
\left.\text { m }^{3} / \mu \mathrm{Ci}-\mathrm{y}\right)\end{array}$ \\
\hline Ho- $168 \mathrm{~m}$ & 132 & $\mathrm{~S}$ & $1.50 \mathrm{E}-16$ & $1.75 \mathrm{E}-05$ & 5.55E-01 & $3.46 \mathrm{E}-19$ & $4.61 \mathrm{E}-12$ & $4.04 \mathrm{E}-14$ \\
\hline Ho-170 & 2.76 & $\mathrm{~m}$ & $7.83 \mathrm{E}-14$ & 9.14E-03 & $2.90 \mathrm{E}+02$ & $1.69 \mathrm{E}-16$ & $2.25 \mathrm{E}-09$ & $1.97 \mathrm{E}-11$ \\
\hline Er-154 & 3.73 & $\mathrm{~m}$ & $2.24 \mathrm{E}-15$ & $2.62 \mathrm{E}-04$ & $8.29 \mathrm{E}+00$ & $5.00 \mathrm{E}-18$ & $6.66 \mathrm{E}-11$ & $5.83 \mathrm{E}-13$ \\
\hline Er-156 & 19.5 & $\mathrm{~m}$ & $1.72 \mathrm{E}-15$ & $2.01 \mathrm{E}-04$ & $6.36 \mathrm{E}+00$ & $3.89 \mathrm{E}-18$ & $5.18 \mathrm{E}-11$ & $4.54 \mathrm{E}-13$ \\
\hline Er-159 & 36 & $\mathrm{~m}$ & $4.36 \mathrm{E}-14$ & $5.09 \mathrm{E}-03$ & $1.61 \mathrm{E}+02$ & $9.47 \mathrm{E}-17$ & $1.26 \mathrm{E}-09$ & $1.10 \mathrm{E}-11$ \\
\hline Er-161 & 3.21 & $\mathrm{~h}$ & $4.46 \mathrm{E}-14$ & $5.21 \mathrm{E}-03$ & $1.65 \mathrm{E}+02$ & $9.70 \mathrm{E}-17$ & $1.29 \mathrm{E}-09$ & $1.13 \mathrm{E}-11$ \\
\hline Er-163 & 75 & $\mathrm{~m}$ & $9.82 \mathrm{E}-16$ & $1.15 \mathrm{E}-04$ & $3.63 \mathrm{E}+00$ & $2.26 \mathrm{E}-18$ & $3.01 \mathrm{E}-11$ & $2.64 \mathrm{E}-13$ \\
\hline Er-165 & 10.36 & $\mathrm{~h}$ & $9.01 \mathrm{E}-16$ & $1.05 \mathrm{E}-04$ & $3.33 \mathrm{E}+00$ & $2.08 \mathrm{E}-18$ & $2.77 \mathrm{E}-11$ & $2.43 \mathrm{E}-13$ \\
\hline Er-167m & 2.269 & $\mathrm{~S}$ & $4.03 \mathrm{E}-15$ & $4.71 \mathrm{E}-04$ & $1.49 \mathrm{E}+01$ & $8.83 \mathrm{E}-18$ & $1.18 \mathrm{E}-10$ & $1.03 \mathrm{E}-12$ \\
\hline Er-169 & 9.4 & $\mathrm{~d}$ & $2.95 \mathrm{E}-17$ & $3.44 \mathrm{E}-06$ & $1.09 \mathrm{E}-01$ & $3.24 \mathrm{E}-20$ & $4.32 \mathrm{E}-13$ & $3.78 \mathrm{E}-15$ \\
\hline Er-171 & 7.516 & $\mathrm{~h}$ & $1.61 \mathrm{E}-14$ & $1.88 \mathrm{E}-03$ & $5.96 \mathrm{E}+01$ & $3.50 \mathrm{E}-17$ & $4.66 \mathrm{E}-10$ & $4.08 \mathrm{E}-12$ \\
\hline Er-172 & 49.3 & $\mathrm{~h}$ & $2.24 \mathrm{E}-14$ & $2.62 \mathrm{E}-03$ & $8.29 \mathrm{E}+01$ & $4.88 \mathrm{E}-17$ & $6.50 \mathrm{E}-10$ & $5.69 \mathrm{E}-12$ \\
\hline Er-173 & 1.434 & $\mathrm{~m}$ & $3.73 \mathrm{E}-14$ & $4.36 \mathrm{E}-03$ & $1.38 \mathrm{E}+02$ & $8.08 \mathrm{E}-17$ & $1.08 \mathrm{E}-09$ & $9.43 \mathrm{E}-12$ \\
\hline Tm-161 & 30.2 & $\mathrm{~m}$ & $5.89 \mathrm{E}-14$ & $6.88 \mathrm{E}-03$ & $2.18 \mathrm{E}+02$ & $1.28 \mathrm{E}-16$ & $1.70 \mathrm{E}-09$ & $1.49 \mathrm{E}-11$ \\
\hline Tm-162 & 21.7 & $\mathrm{~m}$ & $9.14 \mathrm{E}-14$ & $1.07 \mathrm{E}-02$ & $3.38 \mathrm{E}+02$ & $1.98 \mathrm{E}-16$ & $2.64 \mathrm{E}-09$ & $2.31 \mathrm{E}-11$ \\
\hline Tm-163 & 1.81 & $\mathrm{~h}$ & $6.03 \mathrm{E}-14$ & 7.04E-03 & $2.23 \mathrm{E}+02$ & $1.31 \mathrm{E}-16$ & $1.74 \mathrm{E}-09$ & $1.53 \mathrm{E}-11$ \\
\hline Tm-164 & 2 & $\mathrm{~m}$ & $3.57 \mathrm{E}-14$ & $4.17 \mathrm{E}-03$ & $1.32 \mathrm{E}+02$ & $7.70 \mathrm{E}-17$ & $1.03 \mathrm{E}-09$ & $8.98 \mathrm{E}-12$ \\
\hline Tm-165 & 30.06 & $\mathrm{~h}$ & $2.40 \mathrm{E}-14$ & $2.80 \mathrm{E}-03$ & $8.88 \mathrm{E}+01$ & $5.23 \mathrm{E}-17$ & $6.97 \mathrm{E}-10$ & $6.10 \mathrm{E}-12$ \\
\hline Tm-166 & 7.7 & $\mathrm{~h}$ & $9.30 \mathrm{E}-14$ & $1.09 \mathrm{E}-02$ & $3.44 \mathrm{E}+02$ & $2.02 \mathrm{E}-16$ & $2.69 \mathrm{E}-09$ & $2.36 \mathrm{E}-11$ \\
\hline Tm-167 & 9.25 & $\mathrm{~d}$ & $5.49 \mathrm{E}-15$ & $6.41 \mathrm{E}-04$ & $2.03 \mathrm{E}+01$ & $1.21 \mathrm{E}-17$ & $1.61 \mathrm{E}-10$ & $1.41 \mathrm{E}-12$ \\
\hline Tm-168 & 93.1 & $\mathrm{~d}$ & $5.51 \mathrm{E}-14$ & $6.43 \mathrm{E}-03$ & $2.04 \mathrm{E}+02$ & $1.20 \mathrm{E}-16$ & $1.60 \mathrm{E}-09$ & $1.40 \mathrm{E}-11$ \\
\hline Tm-170 & 128.6 & $\mathrm{~d}$ & $3.25 \mathrm{E}-16$ & $3.79 \mathrm{E}-05$ & $1.20 \mathrm{E}+00$ & $5.14 \mathrm{E}-19$ & $6.85 \mathrm{E}-12$ & $6.00 \mathrm{E}-14$ \\
\hline Tm-171 & 1.92 & $\mathrm{y}$ & $1.70 \mathrm{E}-17$ & $1.98 \mathrm{E}-06$ & $6.29 \mathrm{E}-02$ & $3.89 \mathrm{E}-20$ & $5.18 \mathrm{E}-13$ & $4.54 \mathrm{E}-15$ \\
\hline
\end{tabular}




\begin{tabular}{|c|c|c|c|c|c|c|c|c|}
\hline Nuclide & Half-life & Class & $\begin{array}{c}\text { Air } \\
\text { Immersion } \\
\text { Dose Rate } \\
\text { Coefficient } \\
\left(\mathrm{Sv} / \mathbf{s ~ p e r ~}^{\mathbf{3}}\right. \\
\left.\mathrm{Bq} / \mathbf{m}^{\mathbf{3}}\right)\end{array}$ & $\begin{array}{c}\text { Air } \\
\text { Immersion } \\
\text { Dose Rate } \\
\text { Coefficient } \\
\text { (mrem- } \\
\left.\text { m³ }^{3} / \mathrm{pCi}-\mathrm{yr}\right)\end{array}$ & $\begin{array}{c}\text { Air } \\
\text { Immersion } \\
\text { Effective } \\
\text { Dose Rate } \\
\text { (mrem/sec } \\
\text { per } \\
\mu \mathrm{Ci} / \mathrm{cm}^{3} \text { ) } \\
\end{array}$ & $\begin{array}{c}\text { Water } \\
\text { Submersion } \\
\text { Dose Rate } \\
\text { Coefficient } \\
\left(\mathbf{S v}-\mathbf{m}^{3} / \mathbf{B q}-\right. \\
\text { s) }\end{array}$ & $\begin{array}{c}\text { Water } \\
\text { Submersion } \\
\text { Dose Rate } \\
\text { Coefficient } \\
\text { (mrem- } \\
\left.\text { m³ }^{\mathbf{3}} / \mathbf{p C i}-\mathbf{h}\right)\end{array}$ & $\begin{array}{c}\text { Water } \\
\text { Submersion } \\
\text { Dose Rate } \\
\text { Coefficient } \\
(\text { mrem- } \\
\left.\mathbf{m}^{3} / \mu \mathrm{Ci}-\mathrm{y}\right)\end{array}$ \\
\hline Tm-172 & 63.6 & $\mathrm{~h}$ & $2.29 \mathrm{E}-14$ & $2.67 \mathrm{E}-03$ & $8.47 \mathrm{E}+01$ & $4.92 \mathrm{E}-17$ & $6.55 \mathrm{E}-10$ & $5.74 \mathrm{E}-12$ \\
\hline Tm-173 & 8.24 & $\mathrm{~h}$ & $1.72 \mathrm{E}-14$ & $2.01 \mathrm{E}-03$ & $6.36 \mathrm{E}+01$ & $3.74 \mathrm{E}-17$ & $4.98 \mathrm{E}-10$ & $4.36 \mathrm{E}-12$ \\
\hline Tm-174 & 5.4 & $\mathrm{~m}$ & $8.05 \mathrm{E}-14$ & $9.40 \mathrm{E}-03$ & $2.98 \mathrm{E}+02$ & $1.75 \mathrm{E}-16$ & $2.33 \mathrm{E}-09$ & $2.04 \mathrm{E}-11$ \\
\hline Tm-175 & 15.2 & $\mathrm{~m}$ & $4.96 \mathrm{E}-14$ & $5.79 \mathrm{E}-03$ & $1.84 \mathrm{E}+02$ & $1.07 \mathrm{E}-16$ & $1.43 \mathrm{E}-09$ & $1.25 \mathrm{E}-11$ \\
\hline Tm-176 & 1.85 & $\mathrm{~m}$ & $9.39 \mathrm{E}-14$ & $1.10 \mathrm{E}-02$ & $3.47 \mathrm{E}+02$ & $2.03 \mathrm{E}-16$ & $2.70 \mathrm{E}-09$ & $2.37 \mathrm{E}-11$ \\
\hline Yb-162 & 18.87 & $\mathrm{~m}$ & $1.00 \mathrm{E}-14$ & $1.17 \mathrm{E}-03$ & $3.70 \mathrm{E}+01$ & $2.21 \mathrm{E}-17$ & $2.94 \mathrm{E}-10$ & $2.58 \mathrm{E}-12$ \\
\hline Yb-163 & 11.05 & $\mathrm{~m}$ & $3.29 \mathrm{E}-14$ & $3.84 \mathrm{E}-03$ & $1.22 \mathrm{E}+02$ & $7.13 \mathrm{E}-17$ & $9.50 \mathrm{E}-10$ & $8.32 \mathrm{E}-12$ \\
\hline Yb-164 & 75.8 & $\mathrm{~m}$ & $1.61 \mathrm{E}-15$ & $1.88 \mathrm{E}-04$ & $5.96 \mathrm{E}+00$ & $3.64 \mathrm{E}-18$ & $4.85 \mathrm{E}-11$ & $4.25 \mathrm{E}-13$ \\
\hline Yb-165 & 9.9 & $\mathrm{~m}$ & $1.37 \mathrm{E}-14$ & $1.60 \mathrm{E}-03$ & $5.07 \mathrm{E}+01$ & $3.01 \mathrm{E}-17$ & $4.01 \mathrm{E}-10$ & $3.51 \mathrm{E}-12$ \\
\hline Yb-166 & 56.7 & $\mathrm{~h}$ & $2.37 \mathrm{E}-15$ & $2.77 \mathrm{E}-04$ & $8.77 \mathrm{E}+00$ & $5.42 \mathrm{E}-18$ & $7.22 \mathrm{E}-11$ & $6.32 \mathrm{E}-13$ \\
\hline Yb-167 & 17.5 & $\mathrm{~m}$ & $9.55 \mathrm{E}-15$ & $1.12 \mathrm{E}-03$ & $3.53 \mathrm{E}+01$ & $2.14 \mathrm{E}-17$ & $2.85 \mathrm{E}-10$ & $2.50 \mathrm{E}-12$ \\
\hline Yb-169 & 32.026 & $\mathrm{~d}$ & $1.19 \mathrm{E}-14$ & $1.39 \mathrm{E}-03$ & $4.40 \mathrm{E}+01$ & $2.65 \mathrm{E}-17$ & $3.53 \mathrm{E}-10$ & $3.09 \mathrm{E}-12$ \\
\hline Yb-175 & 4.185 & $\mathrm{~d}$ & $1.73 \mathrm{E}-15$ & $2.02 \mathrm{E}-04$ & $6.40 \mathrm{E}+00$ & $3.73 \mathrm{E}-18$ & $4.97 \mathrm{E}-11$ & $4.35 \mathrm{E}-13$ \\
\hline Yb-177 & 1.911 & $\mathrm{~h}$ & $9.11 \mathrm{E}-15$ & $1.06 \mathrm{E}-03$ & $3.37 \mathrm{E}+01$ & $1.95 \mathrm{E}-17$ & $2.60 \mathrm{E}-10$ & $2.28 \mathrm{E}-12$ \\
\hline Yb-178 & 74 & $\mathrm{~m}$ & $1.76 \mathrm{E}-15$ & $2.06 \mathrm{E}-04$ & $6.51 \mathrm{E}+00$ & $3.74 \mathrm{E}-18$ & $4.98 \mathrm{E}-11$ & $4.36 \mathrm{E}-13$ \\
\hline Yb-179 & 8 & $\mathrm{~m}$ & $4.40 \mathrm{E}-14$ & $5.14 \mathrm{E}-03$ & $1.63 \mathrm{E}+02$ & $9.52 \mathrm{E}-17$ & $1.27 \mathrm{E}-09$ & $1.11 \mathrm{E}-11$ \\
\hline Lu-165 & 10.74 & $\mathrm{~m}$ & $5.02 \mathrm{E}-14$ & $5.86 \mathrm{E}-03$ & $1.86 \mathrm{E}+02$ & $1.09 \mathrm{E}-16$ & $1.45 \mathrm{E}-09$ & $1.27 \mathrm{E}-11$ \\
\hline Lu-167 & 51.5 & $\mathrm{~m}$ & $7.94 \mathrm{E}-14$ & $9.27 \mathrm{E}-03$ & $2.94 \mathrm{E}+02$ & $1.72 \mathrm{E}-16$ & $2.29 \mathrm{E}-09$ & $2.01 \mathrm{E}-11$ \\
\hline Lu-169 & 34.06 & $\mathrm{~h}$ & $6.07 \mathrm{E}-14$ & $7.09 \mathrm{E}-03$ & $2.25 \mathrm{E}+02$ & $1.32 \mathrm{E}-16$ & $1.76 \mathrm{E}-09$ & $1.54 \mathrm{E}-11$ \\
\hline Lu-169m & 160 & $\mathrm{~s}$ & $2.52 \mathrm{E}-20$ & $2.94 \mathrm{E}-09$ & $9.32 \mathrm{E}-05$ & $5.83 \mathrm{E}-23$ & $7.77 \mathrm{E}-16$ & $6.80 \mathrm{E}-18$ \\
\hline Lu-170 & 2.012 & $\mathrm{~d}$ & $1.25 \mathrm{E}-13$ & $1.46 \mathrm{E}-02$ & $4.63 \mathrm{E}+02$ & $2.71 \mathrm{E}-16$ & $3.61 \mathrm{E}-09$ & $3.16 \mathrm{E}-11$ \\
\hline Lu-171 & 8.24 & $\mathrm{~d}$ & $2.79 \mathrm{E}-14$ & $3.26 \mathrm{E}-03$ & $1.03 \mathrm{E}+02$ & $6.09 \mathrm{E}-17$ & $8.11 \mathrm{E}-10$ & $7.11 \mathrm{E}-12$ \\
\hline Lu-171m & 79 & $\mathrm{~s}$ & $9.86 \mathrm{E}-18$ & $1.15 \mathrm{E}-06$ & $3.65 \mathrm{E}-02$ & $2.24 \mathrm{E}-20$ & $2.98 \mathrm{E}-13$ & $2.61 \mathrm{E}-15$ \\
\hline
\end{tabular}




\begin{tabular}{|c|c|c|c|c|c|c|c|c|}
\hline Nuclide & Half-life & Class & $\begin{array}{c}\text { Air } \\
\text { Immersion } \\
\text { Dose Rate } \\
\text { Coefficient } \\
\left(\mathrm{Sv} / \mathbf{s ~ p e r ~}^{\mathbf{3}}\right. \\
\left.\mathrm{Bq} / \mathbf{m}^{\mathbf{3}}\right)\end{array}$ & $\begin{array}{c}\text { Air } \\
\text { Immersion } \\
\text { Dose Rate } \\
\text { Coefficient } \\
\text { (mrem- } \\
\left.\text { m³ }^{3} / \mathrm{pCi}-\mathrm{yr}\right)\end{array}$ & $\begin{array}{c}\text { Air } \\
\text { Immersion } \\
\text { Effective } \\
\text { Dose Rate } \\
\text { (mrem/sec } \\
\text { per } \\
\mu \mathrm{Ci} / \mathbf{c m}^{3} \text { ) }\end{array}$ & $\begin{array}{c}\text { Water } \\
\text { Submersion } \\
\text { Dose Rate } \\
\text { Coefficient } \\
\left(\mathbf{S v}-\mathbf{m}^{3} / \mathbf{B q}-\right. \\
\text { s) }\end{array}$ & $\begin{array}{c}\text { Water } \\
\text { Submersion } \\
\text { Dose Rate } \\
\text { Coefficient } \\
\text { (mrem- } \\
\left.\text { m³ }^{\mathbf{3}} / \mathbf{p C i}-\mathbf{h}\right)\end{array}$ & $\begin{array}{c}\text { Water } \\
\text { Submersion } \\
\text { Dose Rate } \\
\text { Coefficient } \\
(\text { mrem- } \\
\left.\mathbf{m}^{3} / \mu \mathrm{Ci}-\mathrm{y}\right)\end{array}$ \\
\hline Lu-172 & 6.7 & $\mathrm{~d}$ & $8.96 \mathrm{E}-14$ & $1.05 \mathrm{E}-02$ & $3.32 \mathrm{E}+02$ & $1.94 \mathrm{E}-16$ & $2.58 \mathrm{E}-09$ & $2.26 \mathrm{E}-11$ \\
\hline Lu-172m & 3.7 & $\mathrm{~m}$ & $3.90 \mathrm{E}-20$ & $4.55 \mathrm{E}-09$ & $1.44 \mathrm{E}-04$ & $9.04 \mathrm{E}-23$ & $1.20 \mathrm{E}-15$ & $1.05 \mathrm{E}-17$ \\
\hline Lu-173 & 1.37 & $\mathrm{y}$ & $6.47 \mathrm{E}-15$ & $7.55 \mathrm{E}-04$ & $2.39 \mathrm{E}+01$ & $1.44 \mathrm{E}-17$ & $1.92 \mathrm{E}-10$ & $1.68 \mathrm{E}-12$ \\
\hline Lu-174 & 3.31 & $\mathrm{y}$ & $4.46 \mathrm{E}-15$ & $5.21 \mathrm{E}-04$ & $1.65 \mathrm{E}+01$ & $9.84 \mathrm{E}-18$ & $1.31 \mathrm{E}-10$ & $1.15 \mathrm{E}-12$ \\
\hline Lu-174m & 142 & $\mathrm{~d}$ & $1.78 \mathrm{E}-15$ & $2.08 \mathrm{E}-04$ & $6.59 \mathrm{E}+00$ & $4.04 \mathrm{E}-18$ & $5.38 \mathrm{E}-11$ & $4.71 \mathrm{E}-13$ \\
\hline Lu-176 & $3.85 \mathrm{E}+10$ & $\mathrm{y}$ & $2.07 \mathrm{E}-14$ & $2.42 \mathrm{E}-03$ & $7.66 \mathrm{E}+01$ & $4.52 \mathrm{E}-17$ & $6.02 \mathrm{E}-10$ & $5.27 \mathrm{E}-12$ \\
\hline Lu-176m & 3.635 & $\mathrm{~h}$ & $7.71 \mathrm{E}-16$ & $9.00 \mathrm{E}-05$ & $2.85 \mathrm{E}+00$ & $1.41 \mathrm{E}-18$ & $1.88 \mathrm{E}-11$ & $1.65 \mathrm{E}-13$ \\
\hline Lu-177 & 6.647 & $\mathrm{~d}$ & $1.50 \mathrm{E}-15$ & $1.75 \mathrm{E}-04$ & $5.55 \mathrm{E}+00$ & $3.25 \mathrm{E}-18$ & $4.33 \mathrm{E}-11$ & $3.79 \mathrm{E}-13$ \\
\hline Lu-177m & 160.4 & $\mathrm{~d}$ & $4.23 \mathrm{E}-14$ & $4.94 \mathrm{E}-03$ & $1.57 \mathrm{E}+02$ & $9.29 \mathrm{E}-17$ & $1.24 \mathrm{E}-09$ & $1.08 \mathrm{E}-11$ \\
\hline Lu-178 & 28.4 & $\mathrm{~m}$ & $6.44 \mathrm{E}-15$ & $7.52 \mathrm{E}-04$ & $2.38 \mathrm{E}+01$ & $1.34 \mathrm{E}-17$ & $1.78 \mathrm{E}-10$ & $1.56 \mathrm{E}-12$ \\
\hline Lu-178m & 23.1 & $\mathrm{~m}$ & $4.53 \mathrm{E}-14$ & $5.29 \mathrm{E}-03$ & $1.68 \mathrm{E}+02$ & $9.89 \mathrm{E}-17$ & $1.32 \mathrm{E}-09$ & $1.15 \mathrm{E}-11$ \\
\hline Lu-179 & 4.59 & $\mathrm{~h}$ & $1.63 \mathrm{E}-15$ & $1.90 \mathrm{E}-04$ & $6.03 \mathrm{E}+00$ & $3.23 \mathrm{E}-18$ & $4.30 \mathrm{E}-11$ & $3.77 \mathrm{E}-13$ \\
\hline Lu-180 & 5.7 & $\mathrm{~m}$ & $7.09 \mathrm{E}-14$ & $8.28 \mathrm{E}-03$ & $2.62 \mathrm{E}+02$ & $1.53 \mathrm{E}-16$ & $2.04 \mathrm{E}-09$ & $1.79 \mathrm{E}-11$ \\
\hline Lu-181 & 3.5 & $\mathrm{~m}$ & $2.58 \mathrm{E}-14$ & $3.01 \mathrm{E}-03$ & $9.55 \mathrm{E}+01$ & $5.57 \mathrm{E}-17$ & $7.42 \mathrm{E}-10$ & $6.50 \mathrm{E}-12$ \\
\hline Hf-167 & 2.05 & $\mathrm{~m}$ & $2.73 \mathrm{E}-14$ & $3.19 \mathrm{E}-03$ & $1.01 \mathrm{E}+02$ & $5.91 \mathrm{E}-17$ & $7.87 \mathrm{E}-10$ & $6.90 \mathrm{E}-12$ \\
\hline Hf-169 & 3.24 & $\mathrm{~m}$ & $2.78 \mathrm{E}-14$ & $3.25 \mathrm{E}-03$ & $1.03 \mathrm{E}+02$ & $6.05 \mathrm{E}-17$ & $8.06 \mathrm{E}-10$ & $7.06 \mathrm{E}-12$ \\
\hline Hf-170 & 16.01 & $\mathrm{~h}$ & $1.83 \mathrm{E}-14$ & $2.14 \mathrm{E}-03$ & $6.77 \mathrm{E}+01$ & $4.01 \mathrm{E}-17$ & $5.34 \mathrm{E}-10$ & $4.68 \mathrm{E}-12$ \\
\hline Hf-172 & 1.87 & $\mathrm{y}$ & $3.09 \mathrm{E}-15$ & $3.61 \mathrm{E}-04$ & $1.14 \mathrm{E}+01$ & $7.02 \mathrm{E}-18$ & $9.35 \mathrm{E}-11$ & $8.19 \mathrm{E}-13$ \\
\hline Hf-173 & 23.6 & $\mathrm{~h}$ & $1.62 \mathrm{E}-14$ & $1.89 \mathrm{E}-03$ & $5.99 \mathrm{E}+01$ & $3.57 \mathrm{E}-17$ & $4.76 \mathrm{E}-10$ & $4.17 \mathrm{E}-12$ \\
\hline Hf-174 & $2.00 \mathrm{E}+15$ & $\mathrm{y}$ & $0.00 \mathrm{E}+00$ & $0.00 \mathrm{E}+00$ & $0.00 \mathrm{E}+00$ & $0.00 \mathrm{E}+00$ & $0.00 \mathrm{E}+00$ & $0.00 \mathrm{E}+00$ \\
\hline Hf-175 & 70 & $\mathrm{~d}$ & $1.47 \mathrm{E}-14$ & $1.72 \mathrm{E}-03$ & $5.44 \mathrm{E}+01$ & $3.22 \mathrm{E}-17$ & $4.29 \mathrm{E}-10$ & $3.76 \mathrm{E}-12$ \\
\hline Hf-177m & 51.4 & $\mathrm{~m}$ & $9.84 \mathrm{E}-14$ & $1.15 \mathrm{E}-02$ & $3.64 \mathrm{E}+02$ & $2.15 \mathrm{E}-16$ & $2.86 \mathrm{E}-09$ & $2.51 \mathrm{E}-11$ \\
\hline Hf-178m & 31 & $\mathrm{y}$ & $9.78 \mathrm{E}-14$ & $1.14 \mathrm{E}-02$ & $3.62 \mathrm{E}+02$ & $2.13 \mathrm{E}-16$ & $2.84 \mathrm{E}-09$ & $2.49 \mathrm{E}-11$ \\
\hline
\end{tabular}




\begin{tabular}{|c|c|c|c|c|c|c|c|c|}
\hline Nuclide & Half-life & Class & $\begin{array}{c}\text { Air } \\
\text { Immersion } \\
\text { Dose Rate } \\
\text { Coefficient } \\
\left(\mathrm{Sv} / \mathbf{s ~ p e r ~}^{\mathbf{3}}\right. \\
\left.\mathrm{Bq} / \mathbf{m}^{\mathbf{3}}\right)\end{array}$ & $\begin{array}{c}\text { Air } \\
\text { Immersion } \\
\text { Dose Rate } \\
\text { Coefficient } \\
\text { (mrem- } \\
\left.\mathrm{m}^{3} / \mathrm{pCi}-\mathrm{yr}\right)\end{array}$ & $\begin{array}{c}\text { Air } \\
\text { Immersion } \\
\text { Effective } \\
\text { Dose Rate } \\
\text { (mrem/sec } \\
\text { per } \\
\mu \mathrm{Ci} / \mathbf{c m}^{3} \text { ) }\end{array}$ & $\begin{array}{c}\text { Water } \\
\text { Submersion } \\
\text { Dose Rate } \\
\text { Coefficient } \\
\left(\mathbf{S v}-\mathbf{m}^{3} / \mathbf{B q}-\right. \\
\text { s) }\end{array}$ & $\begin{array}{c}\text { Water } \\
\text { Submersion } \\
\text { Dose Rate } \\
\text { Coefficient } \\
\text { (mrem- } \\
\left.\text { m³ }^{\mathbf{3}} / \mathbf{p C i}-\mathbf{h}\right)\end{array}$ & $\begin{array}{c}\text { Water } \\
\text { Submersion } \\
\text { Dose Rate } \\
\text { Coefficient } \\
(\text { mrem- } \\
\left.\mathbf{m}^{\mathbf{3}} / \boldsymbol{\mu C i}-\mathrm{y}\right)\end{array}$ \\
\hline Hf-179m & 25.05 & $\mathrm{~d}$ & $3.92 \mathrm{E}-14$ & $4.58 \mathrm{E}-03$ & $1.45 \mathrm{E}+02$ & $8.59 \mathrm{E}-17$ & $1.14 \mathrm{E}-09$ & $1.00 \mathrm{E}-11$ \\
\hline Hf-180m & 5.5 & $\mathrm{~h}$ & $4.27 \mathrm{E}-14$ & $4.99 \mathrm{E}-03$ & $1.58 \mathrm{E}+02$ & $9.33 \mathrm{E}-17$ & $1.24 \mathrm{E}-09$ & $1.09 \mathrm{E}-11$ \\
\hline Hf-181 & 42.39 & $\mathrm{~d}$ & $2.32 \mathrm{E}-14$ & $2.71 \mathrm{E}-03$ & $8.58 \mathrm{E}+01$ & $5.05 \mathrm{E}-17$ & $6.73 \mathrm{E}-10$ & $5.89 \mathrm{E}-12$ \\
\hline Hf-182 & $9.00 \mathrm{E}+06$ & $\mathrm{y}$ & $1.04 \mathrm{E}-14$ & $1.21 \mathrm{E}-03$ & $3.85 \mathrm{E}+01$ & $2.27 \mathrm{E}-17$ & $3.02 \mathrm{E}-10$ & $2.65 \mathrm{E}-12$ \\
\hline Hf-182m & 61.5 & $\mathrm{~m}$ & $3.99 \mathrm{E}-14$ & $4.66 \mathrm{E}-03$ & $1.48 \mathrm{E}+02$ & $8.69 \mathrm{E}-17$ & $1.16 \mathrm{E}-09$ & $1.01 \mathrm{E}-11$ \\
\hline Hf-183 & 1.067 & $\mathrm{~h}$ & $3.50 \mathrm{E}-14$ & $4.09 \mathrm{E}-03$ & $1.30 \mathrm{E}+02$ & $7.58 \mathrm{E}-17$ & $1.01 \mathrm{E}-09$ & $8.84 \mathrm{E}-12$ \\
\hline Hf-184 & 4.12 & $\mathrm{~h}$ & $9.88 \mathrm{E}-15$ & $1.15 \mathrm{E}-03$ & $3.66 \mathrm{E}+01$ & $2.15 \mathrm{E}-17$ & $2.86 \mathrm{E}-10$ & $2.51 \mathrm{E}-12$ \\
\hline Ta-170 & 6.76 & $\mathrm{~m}$ & $4.88 \mathrm{E}-14$ & $5.70 \mathrm{E}-03$ & $1.81 \mathrm{E}+02$ & $1.05 \mathrm{E}-16$ & $1.40 \mathrm{E}-09$ & $1.23 \mathrm{E}-11$ \\
\hline Ta-172 & 36.8 & $\mathrm{~m}$ & $7.82 \mathrm{E}-14$ & $9.13 \mathrm{E}-03$ & $2.89 \mathrm{E}+02$ & $1.69 \mathrm{E}-16$ & $2.25 \mathrm{E}-09$ & $1.97 \mathrm{E}-11$ \\
\hline Ta-173 & 3.14 & $\mathrm{~h}$ & $2.56 \mathrm{E}-14$ & $2.99 \mathrm{E}-03$ & $9.47 \mathrm{E}+01$ & $5.58 \mathrm{E}-17$ & $7.43 \mathrm{E}-10$ & $6.51 \mathrm{E}-12$ \\
\hline Ta-174 & 1.14 & $\mathrm{~h}$ & $4.46 \mathrm{E}-14$ & $5.21 \mathrm{E}-03$ & $1.65 \mathrm{E}+02$ & $9.68 \mathrm{E}-17$ & $1.29 \mathrm{E}-09$ & $1.13 \mathrm{E}-11$ \\
\hline Ta-175 & 10.5 & $\mathrm{~h}$ & $5.09 \mathrm{E}-14$ & $5.94 \mathrm{E}-03$ & $1.88 \mathrm{E}+02$ & $1.11 \mathrm{E}-16$ & $1.48 \mathrm{E}-09$ & $1.30 \mathrm{E}-11$ \\
\hline Ta-176 & 8.09 & $\mathrm{~h}$ & $1.07 \mathrm{E}-13$ & $1.25 \mathrm{E}-02$ & $3.96 \mathrm{E}+02$ & $2.32 \mathrm{E}-16$ & $3.09 \mathrm{E}-09$ & $2.71 \mathrm{E}-11$ \\
\hline Ta-177 & 56.56 & $\mathrm{~h}$ & $2.17 \mathrm{E}-15$ & $2.53 \mathrm{E}-04$ & $8.03 \mathrm{E}+00$ & $4.90 \mathrm{E}-18$ & $6.53 \mathrm{E}-11$ & $5.72 \mathrm{E}-13$ \\
\hline Ta-178 & 9.31 & $\mathrm{~m}$ & $4.72 \mathrm{E}-15$ & $5.51 \mathrm{E}-04$ & $1.75 \mathrm{E}+01$ & $1.04 \mathrm{E}-17$ & $1.39 \mathrm{E}-10$ & $1.21 \mathrm{E}-12$ \\
\hline Ta-178m & 2.36 & $\mathrm{~h}$ & $4.91 \mathrm{E}-14$ & $5.73 \mathrm{E}-03$ & $1.82 \mathrm{E}+02$ & $1.08 \mathrm{E}-16$ & $1.44 \mathrm{E}-09$ & $1.26 \mathrm{E}-11$ \\
\hline Ta-179 & 1.82 & $\mathrm{y}$ & $6.96 \mathrm{E}-16$ & $8.13 \mathrm{E}-05$ & $2.58 \mathrm{E}+00$ & $1.59 \mathrm{E}-18$ & $2.12 \mathrm{E}-11$ & $1.86 \mathrm{E}-13$ \\
\hline Ta-180 & 8.152 & $\mathrm{~h}$ & $1.42 \mathrm{E}-15$ & $1.66 \mathrm{E}-04$ & $5.25 \mathrm{E}+00$ & $3.22 \mathrm{E}-18$ & $4.29 \mathrm{E}-11$ & $3.76 \mathrm{E}-13$ \\
\hline Ta-182 & 114.43 & $\mathrm{~d}$ & $5.98 \mathrm{E}-14$ & $6.98 \mathrm{E}-03$ & $2.21 \mathrm{E}+02$ & $1.30 \mathrm{E}-16$ & $1.73 \mathrm{E}-09$ & $1.52 \mathrm{E}-11$ \\
\hline Ta-182m & 15.84 & $\mathrm{~m}$ & $1.05 \mathrm{E}-14$ & $1.23 \mathrm{E}-03$ & $3.89 \mathrm{E}+01$ & $2.31 \mathrm{E}-17$ & $3.08 \mathrm{E}-10$ & $2.70 \mathrm{E}-12$ \\
\hline
\end{tabular}




\begin{tabular}{|c|c|c|c|c|c|c|c|c|}
\hline Nuclide & Half-life & Class & $\begin{array}{c}\text { Air } \\
\text { Immersion } \\
\text { Dose Rate } \\
\text { Coefficient } \\
(\text { Sv/s per } \\
\left.\text { Bq/ } \mathbf{m}^{3}\right)\end{array}$ & $\begin{array}{c}\text { Air } \\
\text { Immersion } \\
\text { Dose Rate } \\
\text { Coefficient } \\
\text { (mrem- } \\
\text { m³Ci-yr) }^{3} / \mathrm{pCi}\end{array}$ & $\begin{array}{c}\text { Air } \\
\text { Immersion } \\
\text { Effective } \\
\text { Dose Rate } \\
(\mathrm{mrem} / \mathrm{sec} \\
\mathrm{per} \\
\left.\mu \mathrm{Ci} / \mathrm{cm}^{3}\right)\end{array}$ & $\begin{array}{c}\text { Water } \\
\text { Submersion } \\
\text { Dose Rate } \\
\text { Coefficient } \\
\left(\mathrm{Sv}-\mathbf{m}^{3} / \mathbf{B q}-\right. \\
\text { s) }\end{array}$ & $\begin{array}{c}\text { Water } \\
\text { Submersion } \\
\text { Dose Rate } \\
\text { Coefficient } \\
\text { (mrem- } \\
\text { m³Ci-h) }^{\mathbf{3} / p C i}\end{array}$ & $\begin{array}{c}\text { Water } \\
\text { Submersion } \\
\text { Dose Rate } \\
\text { Coefficient } \\
(\text { mrem- } \\
\left.\text { m }^{3} / \mu \mathrm{Ci}-\mathrm{y}\right)\end{array}$ \\
\hline Ta-183 & 5.1 & $\mathrm{~d}$ & $1.20 \mathrm{E}-14$ & $1.40 \mathrm{E}-03$ & $4.44 \mathrm{E}+01$ & $2.63 \mathrm{E}-17$ & $3.50 \mathrm{E}-10$ & $3.07 \mathrm{E}-12$ \\
\hline Ta-184 & 8.7 & $\mathrm{~h}$ & $7.07 \mathrm{E}-14$ & $8.26 \mathrm{E}-03$ & $2.62 \mathrm{E}+02$ & $1.54 \mathrm{E}-16$ & $2.05 \mathrm{E}-09$ & $1.80 \mathrm{E}-11$ \\
\hline Ta-185 & 49.4 & $\mathrm{~m}$ & $6.70 \mathrm{E}-15$ & $7.82 \mathrm{E}-04$ & $2.48 \mathrm{E}+01$ & $1.43 \mathrm{E}-17$ & $1.90 \mathrm{E}-10$ & $1.67 \mathrm{E}-12$ \\
\hline Ta-186 & 10.5 & $\mathrm{~m}$ & $6.43 \mathrm{E}-14$ & $7.51 \mathrm{E}-03$ & $2.38 \mathrm{E}+02$ & $1.39 \mathrm{E}-16$ & $1.85 \mathrm{E}-09$ & $1.62 \mathrm{E}-11$ \\
\hline W-177 & 132 & $\mathrm{~m}$ & $3.99 \mathrm{E}-14$ & $4.66 \mathrm{E}-03$ & $1.48 \mathrm{E}+02$ & $8.70 \mathrm{E}-17$ & $1.16 \mathrm{E}-09$ & $1.02 \mathrm{E}-11$ \\
\hline W-178 & 21.6 & $\mathrm{~d}$ & $4.33 \mathrm{E}-16$ & $5.06 \mathrm{E}-05$ & $1.60 \mathrm{E}+00$ & $9.89 \mathrm{E}-19$ & $1.32 \mathrm{E}-11$ & $1.15 \mathrm{E}-13$ \\
\hline W-179 & 37.05 & $\mathrm{~m}$ & $1.45 \mathrm{E}-15$ & $1.69 \mathrm{E}-04$ & $5.37 \mathrm{E}+00$ & $3.32 \mathrm{E}-18$ & $4.42 \mathrm{E}-11$ & $3.87 \mathrm{E}-13$ \\
\hline W-179m & 6.4 & $\mathrm{~m}$ & $1.99 \mathrm{E}-15$ & $2.32 \mathrm{E}-04$ & $7.36 \mathrm{E}+00$ & $4.39 \mathrm{E}-18$ & $5.85 \mathrm{E}-11$ & $5.12 \mathrm{E}-13$ \\
\hline W-181 & 121.2 & $\mathrm{~d}$ & $1.15 \mathrm{E}-15$ & $1.34 \mathrm{E}-04$ & $4.26 \mathrm{E}+00$ & $2.63 \mathrm{E}-18$ & $3.50 \mathrm{E}-11$ & $3.07 \mathrm{E}-13$ \\
\hline W-185 & 75.1 & $\mathrm{~d}$ & $4.96 \mathrm{E}-17$ & $5.79 \mathrm{E}-06$ & $1.84 \mathrm{E}-01$ & $5.67 \mathrm{E}-20$ & $7.55 \mathrm{E}-13$ & $6.62 \mathrm{E}-15$ \\
\hline $\mathrm{W}-185 \mathrm{~m}$ & 1.597 & $\mathrm{~m}$ & $9.33 \mathrm{E}-16$ & $1.09 \mathrm{E}-04$ & $3.45 \mathrm{E}+00$ & $2.05 \mathrm{E}-18$ & $2.73 \mathrm{E}-11$ & $2.39 \mathrm{E}-13$ \\
\hline W-187 & 23.72 & $\mathrm{~h}$ & $2.00 \mathrm{E}-14$ & $2.34 \mathrm{E}-03$ & $7.40 \mathrm{E}+01$ & $4.33 \mathrm{E}-17$ & $5.77 \mathrm{E}-10$ & $5.05 \mathrm{E}-12$ \\
\hline W-188 & 69.78 & $\mathrm{~d}$ & $1.10 \mathrm{E}-16$ & $1.28 \mathrm{E}-05$ & $4.07 \mathrm{E}-01$ & $2.08 \mathrm{E}-19$ & $2.77 \mathrm{E}-12$ & $2.43 \mathrm{E}-14$ \\
\hline W-190 & 30 & $\mathrm{~m}$ & $5.75 \mathrm{E}-15$ & $6.71 \mathrm{E}-04$ & $2.13 \mathrm{E}+01$ & $1.26 \mathrm{E}-17$ & $1.68 \mathrm{E}-10$ & $1.47 \mathrm{E}-12$ \\
\hline Re-178 & 13.2 & $\mathrm{~m}$ & $8.17 \mathrm{E}-14$ & $9.54 \mathrm{E}-03$ & $3.02 \mathrm{E}+02$ & $1.77 \mathrm{E}-16$ & $2.36 \mathrm{E}-09$ & $2.07 \mathrm{E}-11$ \\
\hline Re-179 & 19.5 & $\mathrm{~m}$ & $4.90 \mathrm{E}-14$ & $5.72 \mathrm{E}-03$ & $1.81 \mathrm{E}+02$ & $1.07 \mathrm{E}-16$ & $1.43 \mathrm{E}-09$ & $1.25 \mathrm{E}-11$ \\
\hline Re-180 & 2.44 & $\mathrm{~m}$ & $5.44 \mathrm{E}-14$ & $6.35 \mathrm{E}-03$ & $2.01 \mathrm{E}+02$ & $1.18 \mathrm{E}-16$ & $1.57 \mathrm{E}-09$ & $1.38 \mathrm{E}-11$ \\
\hline Re-181 & 19.9 & $\mathrm{~h}$ & $3.52 \mathrm{E}-14$ & $4.11 \mathrm{E}-03$ & $1.30 \mathrm{E}+02$ & $7.66 \mathrm{E}-17$ & $1.02 \mathrm{E}-09$ & $8.94 \mathrm{E}-12$ \\
\hline Re-182 & 64 & $\mathrm{~h}$ & $8.10 \mathrm{E}-14$ & $9.46 \mathrm{E}-03$ & $3.00 \mathrm{E}+02$ & $1.76 \mathrm{E}-16$ & $2.34 \mathrm{E}-09$ & $2.05 \mathrm{E}-11$ \\
\hline Re-182m & 12.7 & $\mathrm{~h}$ & $5.59 \mathrm{E}-14$ & $6.53 \mathrm{E}-03$ & $2.07 \mathrm{E}+02$ & $1.22 \mathrm{E}-16$ & $1.63 \mathrm{E}-09$ & $1.42 \mathrm{E}-11$ \\
\hline Re-183 & 70 & $\mathrm{~d}$ & $5.53 \mathrm{E}-15$ & $6.46 \mathrm{E}-04$ & $2.05 \mathrm{E}+01$ & $1.24 \mathrm{E}-17$ & $1.65 \mathrm{E}-10$ & $1.45 \mathrm{E}-12$ \\
\hline Re-184 & 38 & $\mathrm{~d}$ & $3.99 \mathrm{E}-14$ & $4.66 \mathrm{E}-03$ & $1.48 \mathrm{E}+02$ & $8.68 \mathrm{E}-17$ & $1.16 \mathrm{E}-09$ & $1.01 \mathrm{E}-11$ \\
\hline Re-184m & 169 & $\mathrm{~d}$ & $1.64 \mathrm{E}-14$ & $1.91 \mathrm{E}-03$ & $6.07 \mathrm{E}+01$ & $3.58 \mathrm{E}-17$ & $4.77 \mathrm{E}-10$ & $4.18 \mathrm{E}-12$ \\
\hline
\end{tabular}




\begin{tabular}{|c|c|c|c|c|c|c|c|c|}
\hline Nuclide & Half-life & Class & $\begin{array}{c}\text { Air } \\
\text { Immersion } \\
\text { Dose Rate } \\
\text { Coefficient } \\
(\mathrm{Sv} / \mathrm{s} \mathrm{per} \\
\left.\mathrm{Bq} / \mathbf{m}^{\mathbf{3}}\right)\end{array}$ & $\begin{array}{c}\text { Air } \\
\text { Immersion } \\
\text { Dose Rate } \\
\text { Coefficient } \\
\text { (mrem- } \\
\left.\text { m³ }^{3} / \mathrm{pCi}-\mathrm{yr}\right)\end{array}$ & $\begin{array}{c}\text { Air } \\
\text { Immersion } \\
\text { Effective } \\
\text { Dose Rate } \\
(\mathrm{mrem} / \mathrm{sec} \\
\mathrm{per} \\
\left.\mu \mathrm{Ci} / \mathrm{cm}^{3}\right)\end{array}$ & $\begin{array}{c}\text { Water } \\
\text { Submersion } \\
\text { Dose Rate } \\
\text { Coefficient } \\
\left(\mathrm{Sv}-\mathbf{m}^{3} / \mathbf{B q}-\right. \\
\text { s) }\end{array}$ & $\begin{array}{c}\text { Water } \\
\text { Submersion } \\
\text { Dose Rate } \\
\text { Coefficient } \\
\text { (mrem- } \\
\text { m³Ci-h) }^{\mathbf{3} / p C i}\end{array}$ & $\begin{array}{c}\text { Water } \\
\text { Submersion } \\
\text { Dose Rate } \\
\text { Coefficient } \\
(\text { mrem- } \\
\left.\text { m }^{3} / \mu \mathrm{Ci}-\mathrm{y}\right)\end{array}$ \\
\hline Re-186 & 3.7183 & $\mathrm{~d}$ & $9.95 \mathrm{E}-16$ & $1.16 \mathrm{E}-04$ & $3.68 \mathrm{E}+00$ & $1.99 \mathrm{E}-18$ & $2.65 \mathrm{E}-11$ & $2.32 \mathrm{E}-13$ \\
\hline Re-186m & $2.00 \mathrm{E}+05$ & $\mathrm{y}$ & $4.26 \mathrm{E}-16$ & 4.97E-05 & $1.58 \mathrm{E}+00$ & $9.72 \mathrm{E}-19$ & $1.29 \mathrm{E}-11$ & $1.13 \mathrm{E}-13$ \\
\hline $\mathrm{Re}-187$ & $4.12 \mathrm{E}+10$ & $\mathrm{y}$ & $0.00 \mathrm{E}+00$ & $0.00 \mathrm{E}+00$ & $0.00 \mathrm{E}+00$ & $0.00 \mathrm{E}+00$ & $0.00 \mathrm{E}+00$ & $0.00 \mathrm{E}+00$ \\
\hline Re-188 & 17.004 & $\mathrm{~h}$ & $3.29 \mathrm{E}-15$ & $3.84 \mathrm{E}-04$ & $1.22 \mathrm{E}+01$ & $6.60 \mathrm{E}-18$ & $8.79 \mathrm{E}-11$ & $7.70 \mathrm{E}-13$ \\
\hline Re-188m & 18.59 & $\mathrm{~m}$ & $2.26 \mathrm{E}-15$ & $2.64 \mathrm{E}-04$ & $8.36 \mathrm{E}+00$ & $5.12 \mathrm{E}-18$ & $6.82 \mathrm{E}-11$ & $5.97 \mathrm{E}-13$ \\
\hline Re-189 & 24.3 & $\mathrm{~h}$ & $2.55 \mathrm{E}-15$ & $2.98 \mathrm{E}-04$ & $9.44 \mathrm{E}+00$ & $5.40 \mathrm{E}-18$ & $7.19 \mathrm{E}-11$ & $6.30 \mathrm{E}-13$ \\
\hline Re-190 & 3.1 & $\mathrm{~m}$ & $6.04 \mathrm{E}-14$ & $7.05 \mathrm{E}-03$ & $2.23 \mathrm{E}+02$ & $1.31 \mathrm{E}-16$ & $1.74 \mathrm{E}-09$ & $1.53 \mathrm{E}-11$ \\
\hline Re-190m & 3.2 & $\mathrm{~h}$ & $4.13 \mathrm{E}-14$ & $4.82 \mathrm{E}-03$ & $1.53 \mathrm{E}+02$ & $8.96 \mathrm{E}-17$ & $1.19 \mathrm{E}-09$ & $1.05 \mathrm{E}-11$ \\
\hline Os-180 & 21.5 & $\mathrm{~m}$ & $4.67 \mathrm{E}-15$ & $5.45 \mathrm{E}-04$ & $1.73 \mathrm{E}+01$ & $1.03 \mathrm{E}-17$ & $1.37 \mathrm{E}-10$ & $1.20 \mathrm{E}-12$ \\
\hline Os-181 & 105 & $\mathrm{~m}$ & $6.31 \mathrm{E}-14$ & 7.37E-03 & $2.33 \mathrm{E}+02$ & $1.37 \mathrm{E}-16$ & $1.82 \mathrm{E}-09$ & $1.60 \mathrm{E}-11$ \\
\hline Os-182 & 22.1 & $\mathrm{~h}$ & $1.82 \mathrm{E}-14$ & $2.13 \mathrm{E}-03$ & $6.73 \mathrm{E}+01$ & $3.98 \mathrm{E}-17$ & $5.30 \mathrm{E}-10$ & $4.64 \mathrm{E}-12$ \\
\hline Os-183 & 13 & $\mathrm{~h}$ & $2.66 \mathrm{E}-14$ & $3.11 \mathrm{E}-03$ & $9.84 \mathrm{E}+01$ & $5.83 \mathrm{E}-17$ & $7.77 \mathrm{E}-10$ & $6.80 \mathrm{E}-12$ \\
\hline Os-183m & 9.9 & $\mathrm{~h}$ & $4.63 \mathrm{E}-14$ & $5.41 \mathrm{E}-03$ & $1.71 \mathrm{E}+02$ & $1.00 \mathrm{E}-16$ & $1.33 \mathrm{E}-09$ & $1.17 \mathrm{E}-11$ \\
\hline Os-185 & 93.6 & $\mathrm{~d}$ & $3.06 \mathrm{E}-14$ & $3.57 \mathrm{E}-03$ & $1.13 \mathrm{E}+02$ & $6.65 \mathrm{E}-17$ & $8.86 \mathrm{E}-10$ & $7.76 \mathrm{E}-12$ \\
\hline Os-186 & $2.00 \mathrm{E}+15$ & $\mathrm{y}$ & $0.00 \mathrm{E}+00$ & $0.00 \mathrm{E}+00$ & $0.00 \mathrm{E}+00$ & $0.00 \mathrm{E}+00$ & $0.00 \mathrm{E}+00$ & $0.00 \mathrm{E}+00$ \\
\hline Os-189m & 5.8 & $\mathrm{~h}$ & $1.03 \mathrm{E}-19$ & $1.20 \mathrm{E}-08$ & $3.81 \mathrm{E}-04$ & $2.38 \mathrm{E}-22$ & $3.17 \mathrm{E}-15$ & $2.78 \mathrm{E}-17$ \\
\hline Os-190m & 9.9 & $\mathrm{~m}$ & $7.04 \mathrm{E}-14$ & $8.22 \mathrm{E}-03$ & $2.60 \mathrm{E}+02$ & $1.53 \mathrm{E}-16$ & $2.04 \mathrm{E}-09$ & $1.79 \mathrm{E}-11$ \\
\hline Os-191 & 15.4 & $\mathrm{~d}$ & $2.95 \mathrm{E}-15$ & $3.44 \mathrm{E}-04$ & $1.09 \mathrm{E}+01$ & $6.62 \mathrm{E}-18$ & $8.82 \mathrm{E}-11$ & $7.72 \mathrm{E}-13$ \\
\hline Os-191m & 13.1 & $\mathrm{~h}$ & $1.82 \mathrm{E}-16$ & $2.13 \mathrm{E}-05$ & $6.73 \mathrm{E}-01$ & $4.15 \mathrm{E}-19$ & $5.53 \mathrm{E}-12$ & 4.84E-14 \\
\hline Os-193 & 30.11 & $\mathrm{~h}$ & $3.03 \mathrm{E}-15$ & $3.54 \mathrm{E}-04$ & $1.12 \mathrm{E}+01$ & $6.41 \mathrm{E}-18$ & $8.54 \mathrm{E}-11$ & $7.48 \mathrm{E}-13$ \\
\hline Os-194 & 6 & $\mathrm{y}$ & $5.09 \mathrm{E}-17$ & $5.94 \mathrm{E}-06$ & $1.88 \mathrm{E}-01$ & $1.18 \mathrm{E}-19$ & $1.57 \mathrm{E}-12$ & $1.38 \mathrm{E}-14$ \\
\hline Os-196 & 34.9 & $\mathrm{~m}$ & $3.64 \mathrm{E}-15$ & $4.25 \mathrm{E}-04$ & $1.35 \mathrm{E}+01$ & 7.73E-18 & $1.03 \mathrm{E}-10$ & $9.02 \mathrm{E}-13$ \\
\hline Ir-180 & 1.5 & $\mathrm{~m}$ & $7.24 \mathrm{E}-14$ & $8.45 \mathrm{E}-03$ & $2.68 \mathrm{E}+02$ & $1.56 \mathrm{E}-16$ & $2.08 \mathrm{E}-09$ & $1.82 \mathrm{E}-11$ \\
\hline
\end{tabular}




\begin{tabular}{|c|c|c|c|c|c|c|c|c|}
\hline Nuclide & Half-life & Class & $\begin{array}{c}\text { Air } \\
\text { Immersion } \\
\text { Dose Rate } \\
\text { Coefficient } \\
\left(\mathrm{Sv} / \mathbf{s ~ p e r ~}^{\mathbf{3}}\right. \\
\left.\mathrm{Bq} / \mathbf{m}^{\mathbf{3}}\right)\end{array}$ & $\begin{array}{c}\text { Air } \\
\text { Immersion } \\
\text { Dose Rate } \\
\text { Coefficient } \\
\text { (mrem- } \\
\left.\text { m³ }^{3} / \mathrm{pCi}-\mathrm{yr}\right)\end{array}$ & $\begin{array}{c}\text { Air } \\
\text { Immersion } \\
\text { Effective } \\
\text { Dose Rate } \\
\text { (mrem/sec } \\
\text { per } \\
\mu \mathrm{Ci} / \mathrm{cm}^{3} \text { ) } \\
\end{array}$ & $\begin{array}{c}\text { Water } \\
\text { Submersion } \\
\text { Dose Rate } \\
\text { Coefficient } \\
\left(\mathbf{S v}-\mathbf{m}^{3} / \mathbf{B q}-\right. \\
\text { s) }\end{array}$ & $\begin{array}{c}\text { Water } \\
\text { Submersion } \\
\text { Dose Rate } \\
\text { Coefficient } \\
\text { (mrem- } \\
\left.\text { m³ }^{\mathbf{3}} / \mathbf{p C i}-\mathbf{h}\right)\end{array}$ & $\begin{array}{c}\text { Water } \\
\text { Submersion } \\
\text { Dose Rate } \\
\text { Coefficient } \\
(\text { mrem- } \\
\left.\mathbf{m}^{3} / \mu \mathrm{Ci}-\mathrm{y}\right)\end{array}$ \\
\hline Ir-182 & 15 & $\mathrm{~m}$ & $6.42 \mathrm{E}-14$ & $7.50 \mathrm{E}-03$ & $2.38 \mathrm{E}+02$ & $1.39 \mathrm{E}-16$ & $1.85 \mathrm{E}-09$ & $1.62 \mathrm{E}-11$ \\
\hline Ir-183 & 58 & $\mathrm{~m}$ & $5.48 \mathrm{E}-14$ & $6.40 \mathrm{E}-03$ & $2.03 \mathrm{E}+02$ & $1.19 \mathrm{E}-16$ & $1.59 \mathrm{E}-09$ & $1.39 \mathrm{E}-11$ \\
\hline Ir-184 & 3.09 & $\mathrm{~h}$ & $9.01 \mathrm{E}-14$ & $1.05 \mathrm{E}-02$ & $3.33 \mathrm{E}+02$ & $1.96 \mathrm{E}-16$ & $2.61 \mathrm{E}-09$ & $2.29 \mathrm{E}-11$ \\
\hline Ir-185 & 14.4 & $\mathrm{~h}$ & $3.95 \mathrm{E}-14$ & $4.61 \mathrm{E}-03$ & $1.46 \mathrm{E}+02$ & $8.59 \mathrm{E}-17$ & $1.14 \mathrm{E}-09$ & $1.00 \mathrm{E}-11$ \\
\hline Ir-186 & 16.64 & $\mathrm{~h}$ & $7.63 \mathrm{E}-14$ & $8.91 \mathrm{E}-03$ & $2.82 \mathrm{E}+02$ & $1.66 \mathrm{E}-16$ & $2.21 \mathrm{E}-09$ & $1.94 \mathrm{E}-11$ \\
\hline Ir-186m & 1.92 & $\mathrm{~h}$ & $5.81 \mathrm{E}-14$ & $6.78 \mathrm{E}-03$ & $2.15 \mathrm{E}+02$ & $1.26 \mathrm{E}-16$ & $1.68 \mathrm{E}-09$ & $1.47 \mathrm{E}-11$ \\
\hline Ir-187 & 10.5 & $\mathrm{~h}$ & $1.40 \mathrm{E}-14$ & $1.63 \mathrm{E}-03$ & $5.18 \mathrm{E}+01$ & $3.07 \mathrm{E}-17$ & $4.09 \mathrm{E}-10$ & $3.58 \mathrm{E}-12$ \\
\hline Ir-188 & 41.5 & $\mathrm{~h}$ & $1.01 \mathrm{E}-13$ & $1.18 \mathrm{E}-02$ & $3.74 \mathrm{E}+02$ & $2.19 \mathrm{E}-16$ & $2.92 \mathrm{E}-09$ & $2.56 \mathrm{E}-11$ \\
\hline Ir-189 & 13.2 & $\mathrm{~d}$ & $2.67 \mathrm{E}-15$ & $3.12 \mathrm{E}-04$ & $9.88 \mathrm{E}+00$ & $5.99 \mathrm{E}-18$ & $7.98 \mathrm{E}-11$ & $6.99 \mathrm{E}-13$ \\
\hline Ir-190 & 11.78 & $\mathrm{~d}$ & $6.51 \mathrm{E}-14$ & $7.60 \mathrm{E}-03$ & $2.41 \mathrm{E}+02$ & $1.42 \mathrm{E}-16$ & $1.89 \mathrm{E}-09$ & $1.66 \mathrm{E}-11$ \\
\hline Ir-190m & 1.12 & $\mathrm{~h}$ & $1.14 \mathrm{E}-19$ & $1.33 \mathrm{E}-08$ & $4.22 \mathrm{E}-04$ & $2.65 \mathrm{E}-22$ & $3.53 \mathrm{E}-15$ & $3.09 \mathrm{E}-17$ \\
\hline Ir-190n & 3.087 & $\mathrm{~h}$ & $1.82 \mathrm{E}-15$ & $2.13 \mathrm{E}-04$ & $6.73 \mathrm{E}+00$ & $4.12 \mathrm{E}-18$ & $5.49 \mathrm{E}-11$ & $4.81 \mathrm{E}-13$ \\
\hline Ir-191m & 4.94 & $\mathrm{~s}$ & $2.66 \mathrm{E}-15$ & $3.11 \mathrm{E}-04$ & $9.84 \mathrm{E}+00$ & $5.96 \mathrm{E}-18$ & $7.94 \mathrm{E}-11$ & $6.95 \mathrm{E}-13$ \\
\hline Ir-192 & 73.827 & $\mathrm{~d}$ & $3.61 \mathrm{E}-14$ & $4.22 \mathrm{E}-03$ & $1.34 \mathrm{E}+02$ & $7.86 \mathrm{E}-17$ & $1.05 \mathrm{E}-09$ & $9.17 \mathrm{E}-12$ \\
\hline Ir-192m & 1.45 & $\mathrm{~m}$ & $2.68 \mathrm{E}-18$ & $3.13 \mathrm{E}-07$ & $9.92 \mathrm{E}-03$ & $5.92 \mathrm{E}-21$ & $7.89 \mathrm{E}-14$ & $6.91 \mathrm{E}-16$ \\
\hline Ir-192n & 241 & $\mathrm{y}$ & $6.65 \mathrm{E}-17$ & $7.76 \mathrm{E}-06$ & $2.46 \mathrm{E}-01$ & $9.60 \mathrm{E}-20$ & $1.28 \mathrm{E}-12$ & $1.12 \mathrm{E}-14$ \\
\hline Ir-193m & 10.53 & $\mathrm{~d}$ & $1.04 \mathrm{E}-17$ & $1.21 \mathrm{E}-06$ & $3.85 \mathrm{E}-02$ & $2.32 \mathrm{E}-20$ & $3.09 \mathrm{E}-13$ & $2.71 \mathrm{E}-15$ \\
\hline Ir-194 & 19.28 & $\mathrm{~h}$ & $4.77 \mathrm{E}-15$ & $5.57 \mathrm{E}-04$ & $1.76 \mathrm{E}+01$ & $9.74 \mathrm{E}-18$ & $1.30 \mathrm{E}-10$ & $1.14 \mathrm{E}-12$ \\
\hline Ir-194m & 171 & $\mathrm{~d}$ & $1.04 \mathrm{E}-13$ & $1.21 \mathrm{E}-02$ & $3.85 \mathrm{E}+02$ & $2.26 \mathrm{E}-16$ & $3.01 \mathrm{E}-09$ & $2.64 \mathrm{E}-11$ \\
\hline Ir-195 & 2.5 & $\mathrm{~h}$ & $2.20 \mathrm{E}-15$ & $2.57 \mathrm{E}-04$ & $8.14 \mathrm{E}+00$ & $4.72 \mathrm{E}-18$ & $6.29 \mathrm{E}-11$ & $5.51 \mathrm{E}-13$ \\
\hline Ir-195m & 3.8 & $\mathrm{~h}$ & $1.63 \mathrm{E}-14$ & $1.90 \mathrm{E}-03$ & $6.03 \mathrm{E}+01$ & $3.56 \mathrm{E}-17$ & $4.74 \mathrm{E}-10$ & $4.15 \mathrm{E}-12$ \\
\hline Ir-196 & 52 & $\mathrm{~S}$ & $1.16 \mathrm{E}-14$ & $1.35 \mathrm{E}-03$ & $4.29 \mathrm{E}+01$ & $2.42 \mathrm{E}-17$ & $3.22 \mathrm{E}-10$ & $2.82 \mathrm{E}-12$ \\
\hline Ir-196m & 1.4 & $\mathrm{~h}$ & $1.10 \mathrm{E}-13$ & $1.28 \mathrm{E}-02$ & $4.07 \mathrm{E}+02$ & $2.39 \mathrm{E}-16$ & $3.18 \mathrm{E}-09$ & $2.79 \mathrm{E}-11$ \\
\hline
\end{tabular}




\begin{tabular}{|c|c|c|c|c|c|c|c|c|}
\hline Nuclide & Half-life & Class & $\begin{array}{c}\text { Air } \\
\text { Immersion } \\
\text { Dose Rate } \\
\text { Coefficient } \\
(\text { Sv/s per } \\
\left.\text { Bq/ } \mathbf{m}^{3}\right)\end{array}$ & $\begin{array}{c}\text { Air } \\
\text { Immersion } \\
\text { Dose Rate } \\
\text { Coefficient } \\
\text { (mrem- } \\
\text { m³Ci-yr) }^{3} / \mathrm{pCi}\end{array}$ & $\begin{array}{c}\text { Air } \\
\text { Immersion } \\
\text { Effective } \\
\text { Dose Rate } \\
(\mathrm{mrem} / \mathrm{sec} \\
\mathrm{per} \\
\left.\mu \mathrm{Ci} / \mathrm{cm}^{3}\right)\end{array}$ & $\begin{array}{c}\text { Water } \\
\text { Submersion } \\
\text { Dose Rate } \\
\text { Coefficient } \\
\left(\mathrm{Sv}-\mathbf{m}^{3} / \mathbf{B q}-\right. \\
\text { s) }\end{array}$ & $\begin{array}{c}\text { Water } \\
\text { Submersion } \\
\text { Dose Rate } \\
\text { Coefficient } \\
\text { (mrem- } \\
\text { m³Ci-h) }^{\mathbf{3} / p C i}\end{array}$ & $\begin{array}{c}\text { Water } \\
\text { Submersion } \\
\text { Dose Rate } \\
\text { Coefficient } \\
(\text { mrem- } \\
\left.\text { m }^{3} / \mu \mathrm{Ci}-\mathrm{y}\right)\end{array}$ \\
\hline Pt-184 & 17.3 & $\mathrm{~m}$ & $3.02 \mathrm{E}-14$ & $3.53 \mathrm{E}-03$ & $1.12 \mathrm{E}+02$ & $6.63 \mathrm{E}-17$ & $8.83 \mathrm{E}-10$ & $7.74 \mathrm{E}-12$ \\
\hline Pt-186 & 2.08 & $\mathrm{~h}$ & $2.99 \mathrm{E}-14$ & $3.49 \mathrm{E}-03$ & $1.11 \mathrm{E}+02$ & $6.51 \mathrm{E}-17$ & $8.67 \mathrm{E}-10$ & $7.60 \mathrm{E}-12$ \\
\hline Pt-187 & 2.35 & $\mathrm{~h}$ & $2.66 \mathrm{E}-14$ & $3.11 \mathrm{E}-03$ & $9.84 \mathrm{E}+01$ & $5.81 \mathrm{E}-17$ & $7.74 \mathrm{E}-10$ & $6.78 \mathrm{E}-12$ \\
\hline Pt-188 & 10.2 & $\mathrm{~d}$ & $8.04 \mathrm{E}-15$ & 9.39E-04 & $2.97 \mathrm{E}+01$ & $1.78 \mathrm{E}-17$ & $2.37 \mathrm{E}-10$ & $2.08 \mathrm{E}-12$ \\
\hline Pt-189 & 10.87 & $\mathrm{~h}$ & $2.06 \mathrm{E}-14$ & $2.41 \mathrm{E}-03$ & $7.62 \mathrm{E}+01$ & $4.51 \mathrm{E}-17$ & $6.01 \mathrm{E}-10$ & $5.26 \mathrm{E}-12$ \\
\hline Pt-190 & $6.50 \mathrm{E}+11$ & $\mathrm{y}$ & $0.00 \mathrm{E}+00$ & $0.00 \mathrm{E}+00$ & $0.00 \mathrm{E}+00$ & $0.00 \mathrm{E}+00$ & $0.00 \mathrm{E}+00$ & $0.00 \mathrm{E}+00$ \\
\hline Pt-191 & 2.802 & $\mathrm{~d}$ & $1.18 \mathrm{E}-14$ & $1.38 \mathrm{E}-03$ & $4.37 \mathrm{E}+01$ & $2.60 \mathrm{E}-17$ & $3.46 \mathrm{E}-10$ & $3.03 \mathrm{E}-12$ \\
\hline Pt-193 & 50 & $\mathrm{y}$ & $2.83 \mathrm{E}-19$ & $3.30 \mathrm{E}-08$ & $1.05 \mathrm{E}-03$ & $6.57 \mathrm{E}-22$ & $8.75 \mathrm{E}-15$ & $7.67 \mathrm{E}-17$ \\
\hline Pt-193m & 4.33 & $\mathrm{~d}$ & $3.68 \mathrm{E}-16$ & $4.30 \mathrm{E}-05$ & $1.36 \mathrm{E}+00$ & $8.04 \mathrm{E}-19$ & $1.07 \mathrm{E}-11$ & $9.38 \mathrm{E}-14$ \\
\hline Pt-195m & 4.02 & $\mathrm{~d}$ & $2.45 \mathrm{E}-15$ & $2.86 \mathrm{E}-04$ & $9.07 \mathrm{E}+00$ & $5.52 \mathrm{E}-18$ & $7.35 \mathrm{E}-11$ & $6.44 \mathrm{E}-13$ \\
\hline Pt-197 & 19.8915 & $\mathrm{~h}$ & $9.91 \mathrm{E}-16$ & $1.16 \mathrm{E}-04$ & $3.67 \mathrm{E}+00$ & $2.10 \mathrm{E}-18$ & $2.80 \mathrm{E}-11$ & $2.45 \mathrm{E}-13$ \\
\hline Pt-197m & 95.41 & $\mathrm{~m}$ & $3.26 \mathrm{E}-15$ & $3.81 \mathrm{E}-04$ & $1.21 \mathrm{E}+01$ & $7.05 \mathrm{E}-18$ & $9.39 \mathrm{E}-11$ & $8.23 \mathrm{E}-13$ \\
\hline Pt-199 & 30.8 & $\mathrm{~m}$ & $9.23 \mathrm{E}-15$ & $1.08 \mathrm{E}-03$ & $3.42 \mathrm{E}+01$ & $1.97 \mathrm{E}-17$ & $2.62 \mathrm{E}-10$ & $2.30 \mathrm{E}-12$ \\
\hline Pt-200 & 12.5 & $\mathrm{~h}$ & $2.33 \mathrm{E}-15$ & $2.72 \mathrm{E}-04$ & $8.62 \mathrm{E}+00$ & $5.09 \mathrm{E}-18$ & $6.78 \mathrm{E}-11$ & $5.94 \mathrm{E}-13$ \\
\hline Pt-202 & 44 & $\mathrm{~h}$ & $5.03 \mathrm{E}-16$ & $5.87 \mathrm{E}-05$ & $1.86 \mathrm{E}+00$ & $6.05 \mathrm{E}-19$ & $8.06 \mathrm{E}-12$ & $7.06 \mathrm{E}-14$ \\
\hline $\mathrm{Au}-186$ & 10.7 & $\mathrm{~m}$ & $6.87 \mathrm{E}-14$ & $8.02 \mathrm{E}-03$ & $2.54 \mathrm{E}+02$ & $1.49 \mathrm{E}-16$ & $1.98 \mathrm{E}-09$ & $1.74 \mathrm{E}-11$ \\
\hline $\mathrm{Au}-187$ & 8.4 & $\mathrm{~m}$ & $4.96 \mathrm{E}-14$ & $5.79 \mathrm{E}-03$ & $1.84 \mathrm{E}+02$ & $1.08 \mathrm{E}-16$ & $1.44 \mathrm{E}-09$ & $1.26 \mathrm{E}-11$ \\
\hline $\mathrm{Au}-190$ & 42.8 & $\mathrm{~m}$ & $1.16 \mathrm{E}-13$ & $1.35 \mathrm{E}-02$ & $4.29 \mathrm{E}+02$ & $2.51 \mathrm{E}-16$ & $3.34 \mathrm{E}-09$ & $2.93 \mathrm{E}-11$ \\
\hline $\mathrm{Au}-191$ & 3.18 & $\mathrm{~h}$ & $2.55 \mathrm{E}-14$ & $2.98 \mathrm{E}-03$ & $9.44 \mathrm{E}+01$ & $5.58 \mathrm{E}-17$ & $7.43 \mathrm{E}-10$ & $6.51 \mathrm{E}-12$ \\
\hline $\mathrm{Au}-192$ & 4.94 & $\mathrm{~h}$ & $9.29 \mathrm{E}-14$ & $1.08 \mathrm{E}-02$ & $3.44 \mathrm{E}+02$ & $2.02 \mathrm{E}-16$ & $2.69 \mathrm{E}-09$ & $2.36 \mathrm{E}-11$ \\
\hline Au-193 & 17.65 & $\mathrm{~h}$ & $6.41 \mathrm{E}-15$ & $7.48 \mathrm{E}-04$ & $2.37 \mathrm{E}+01$ & $1.42 \mathrm{E}-17$ & $1.89 \mathrm{E}-10$ & $1.66 \mathrm{E}-12$ \\
\hline $\mathrm{Au}-193 \mathrm{~m}$ & 3.9 & $\mathrm{~s}$ & $8.37 \mathrm{E}-15$ & $9.77 \mathrm{E}-04$ & $3.10 \mathrm{E}+01$ & $1.83 \mathrm{E}-17$ & $2.44 \mathrm{E}-10$ & $2.14 \mathrm{E}-12$ \\
\hline Au-194 & 38.02 & $\mathrm{~h}$ & $4.81 \mathrm{E}-14$ & $5.62 \mathrm{E}-03$ & $1.78 \mathrm{E}+02$ & $1.05 \mathrm{E}-16$ & $1.40 \mathrm{E}-09$ & $1.23 \mathrm{E}-11$ \\
\hline
\end{tabular}




\begin{tabular}{|c|c|c|c|c|c|c|c|c|}
\hline Nuclide & Half-life & Class & $\begin{array}{c}\text { Air } \\
\text { Immersion } \\
\text { Dose Rate } \\
\text { Coefficient } \\
(\mathrm{Sv} / \mathrm{s} \mathrm{per} \\
\left.\mathrm{Bq} / \mathbf{m}^{3}\right)\end{array}$ & $\begin{array}{c}\text { Air } \\
\text { Immersion } \\
\text { Dose Rate } \\
\text { Coefficient } \\
\text { (mrem- } \\
\text { m³Ci-yr) }^{3} / \mathrm{pCi}\end{array}$ & $\begin{array}{c}\text { Air } \\
\text { Immersion } \\
\text { Effective } \\
\text { Dose Rate } \\
(\mathrm{mrem} / \mathrm{sec} \\
\mathrm{per} \\
\left.\mu \mathrm{Ci} / \mathrm{cm}^{3}\right)\end{array}$ & $\begin{array}{c}\text { Water } \\
\text { Submersion } \\
\text { Dose Rate } \\
\text { Coefficient } \\
\left(\mathrm{Sv}-\mathbf{m}^{3} / \mathbf{B q}-\right. \\
\text { s) }\end{array}$ & $\begin{array}{c}\text { Water } \\
\text { Submersion } \\
\text { Dose Rate } \\
\text { Coefficient } \\
\text { (mrem- } \\
\left.\text { m³ }^{3} / \mathbf{p C i}-\mathbf{h}\right)\end{array}$ & $\begin{array}{c}\text { Water } \\
\text { Submersion } \\
\text { Dose Rate } \\
\text { Coefficient } \\
(\text { mrem- } \\
\left.\text { m }^{3} / \mu \mathrm{Ci}-\mathrm{y}\right)\end{array}$ \\
\hline $\mathrm{Au}-195$ & 186.098 & $\mathrm{~d}$ & $2.70 \mathrm{E}-15$ & $3.15 \mathrm{E}-04$ & $9.99 \mathrm{E}+00$ & $6.10 \mathrm{E}-18$ & $8.13 \mathrm{E}-11$ & $7.12 \mathrm{E}-13$ \\
\hline $\mathrm{Au}-195 \mathrm{~m}$ & 30.5 & $\mathrm{~s}$ & $8.52 \mathrm{E}-15$ & $9.95 \mathrm{E}-04$ & $3.15 \mathrm{E}+01$ & $1.87 \mathrm{E}-17$ & $2.49 \mathrm{E}-10$ & $2.18 \mathrm{E}-12$ \\
\hline Au-196 & 6.183 & $\mathrm{~d}$ & $2.02 \mathrm{E}-14$ & $2.36 \mathrm{E}-03$ & $7.47 \mathrm{E}+01$ & $4.42 \mathrm{E}-17$ & $5.89 \mathrm{E}-10$ & $5.16 \mathrm{E}-12$ \\
\hline $\mathrm{Au}-196 \mathrm{~m}$ & 9.6 & $\mathrm{~h}$ & $9.70 \mathrm{E}-15$ & $1.13 \mathrm{E}-03$ & $3.59 \mathrm{E}+01$ & $2.14 \mathrm{E}-17$ & $2.85 \mathrm{E}-10$ & $2.50 \mathrm{E}-12$ \\
\hline Au-198 & 2.69517 & $\mathrm{~d}$ & $1.80 \mathrm{E}-14$ & $2.10 \mathrm{E}-03$ & $6.66 \mathrm{E}+01$ & $3.90 \mathrm{E}-17$ & $5.19 \mathrm{E}-10$ & $4.55 \mathrm{E}-12$ \\
\hline $\mathrm{Au}-198 \mathrm{~m}$ & 2.27 & $\mathrm{~d}$ & $2.21 \mathrm{E}-14$ & $2.58 \mathrm{E}-03$ & $8.18 \mathrm{E}+01$ & $4.87 \mathrm{E}-17$ & $6.49 \mathrm{E}-10$ & $5.68 \mathrm{E}-12$ \\
\hline Au-199 & 3.139 & $\mathrm{~d}$ & $3.97 \mathrm{E}-15$ & 4.64E-04 & $1.47 \mathrm{E}+01$ & $8.74 \mathrm{E}-18$ & $1.16 \mathrm{E}-10$ & $1.02 \mathrm{E}-12$ \\
\hline $\mathrm{Au}-200$ & 48.4 & $\mathrm{~m}$ & $1.33 \mathrm{E}-14$ & $1.55 \mathrm{E}-03$ & $4.92 \mathrm{E}+01$ & $2.84 \mathrm{E}-17$ & $3.78 \mathrm{E}-10$ & $3.31 \mathrm{E}-12$ \\
\hline $\mathrm{Au}-200 \mathrm{~m}$ & 18.7 & $\mathrm{~h}$ & $8.84 \mathrm{E}-14$ & $1.03 \mathrm{E}-02$ & $3.27 \mathrm{E}+02$ & $1.92 \mathrm{E}-16$ & $2.56 \mathrm{E}-09$ & $2.24 \mathrm{E}-11$ \\
\hline $\mathrm{Au}-201$ & 26 & $\mathrm{~m}$ & $1.79 \mathrm{E}-15$ & $2.09 \mathrm{E}-04$ & $6.62 \mathrm{E}+00$ & $3.62 \mathrm{E}-18$ & $4.82 \mathrm{E}-11$ & $4.22 \mathrm{E}-13$ \\
\hline $\mathrm{Au}-202$ & 28.8 & $\mathrm{~s}$ & $8.90 \mathrm{E}-15$ & $1.04 \mathrm{E}-03$ & $3.29 \mathrm{E}+01$ & $1.85 \mathrm{E}-17$ & $2.46 \mathrm{E}-10$ & $2.16 \mathrm{E}-12$ \\
\hline $\mathrm{Hg}-190$ & 20 & $\mathrm{~m}$ & $7.74 \mathrm{E}-15$ & 9.04E-04 & $2.86 \mathrm{E}+01$ & $1.72 \mathrm{E}-17$ & $2.29 \mathrm{E}-10$ & $2.01 \mathrm{E}-12$ \\
\hline $\mathrm{Hg}-191 \mathrm{~m}$ & 50.8 & $\mathrm{~m}$ & $6.73 \mathrm{E}-14$ & $7.86 \mathrm{E}-03$ & $2.49 \mathrm{E}+02$ & $1.46 \mathrm{E}-16$ & $1.94 \mathrm{E}-09$ & $1.70 \mathrm{E}-11$ \\
\hline Hg-192 & 4.85 & $\mathrm{~h}$ & $1.10 \mathrm{E}-14$ & $1.28 \mathrm{E}-03$ & $4.07 \mathrm{E}+01$ & $2.44 \mathrm{E}-17$ & $3.25 \mathrm{E}-10$ & $2.85 \mathrm{E}-12$ \\
\hline $\mathrm{Hg}-193$ & 3.8 & $\mathrm{~h}$ & $3.81 \mathrm{E}-14$ & $4.45 \mathrm{E}-03$ & $1.41 \mathrm{E}+02$ & $8.29 \mathrm{E}-17$ & $1.10 \mathrm{E}-09$ & $9.67 \mathrm{E}-12$ \\
\hline $\mathrm{Hg}-193 \mathrm{~m}$ & 11.8 & $\mathrm{~h}$ & $4.67 \mathrm{E}-14$ & $5.45 \mathrm{E}-03$ & $1.73 \mathrm{E}+02$ & $1.01 \mathrm{E}-16$ & $1.35 \mathrm{E}-09$ & $1.18 \mathrm{E}-11$ \\
\hline Hg-194 & 440 & $\mathrm{y}$ & $4.49 \mathrm{E}-19$ & $5.24 \mathrm{E}-08$ & $1.66 \mathrm{E}-03$ & $1.04 \mathrm{E}-21$ & $1.39 \mathrm{E}-14$ & $1.21 \mathrm{E}-16$ \\
\hline Hg-195 & 10.53 & $\mathrm{~h}$ & $8.23 \mathrm{E}-15$ & $9.61 \mathrm{E}-04$ & $3.05 \mathrm{E}+01$ & $1.81 \mathrm{E}-17$ & $2.41 \mathrm{E}-10$ & $2.11 \mathrm{E}-12$ \\
\hline $\mathrm{Hg}-195 \mathrm{~m}$ & 41.6 & $\mathrm{~h}$ & $8.37 \mathrm{E}-15$ & $9.77 \mathrm{E}-04$ & $3.10 \mathrm{E}+01$ & $1.83 \mathrm{E}-17$ & $2.44 \mathrm{E}-10$ & $2.14 \mathrm{E}-12$ \\
\hline Hg-197 & 64.94 & $\mathrm{~h}$ & $2.39 \mathrm{E}-15$ & $2.79 \mathrm{E}-04$ & $8.84 \mathrm{E}+00$ & $5.41 \mathrm{E}-18$ & $7.21 \mathrm{E}-11$ & $6.31 \mathrm{E}-13$ \\
\hline $\mathrm{Hg}-197 \mathrm{~m}$ & 23.8 & $\mathrm{~h}$ & $3.75 \mathrm{E}-15$ & $4.38 \mathrm{E}-04$ & $1.39 \mathrm{E}+01$ & $8.28 \mathrm{E}-18$ & $1.10 \mathrm{E}-10$ & $9.66 \mathrm{E}-13$ \\
\hline $\mathrm{Hg}-199 \mathrm{~m}$ & 42.66 & $\mathrm{~m}$ & $7.53 \mathrm{E}-15$ & 8.79E-04 & $2.79 \mathrm{E}+01$ & $1.65 \mathrm{E}-17$ & $2.20 \mathrm{E}-10$ & $1.93 \mathrm{E}-12$ \\
\hline $\mathrm{Hg}-203$ & 46.612 & $\mathrm{~d}$ & $1.04 \mathrm{E}-14$ & $1.21 \mathrm{E}-03$ & $3.85 \mathrm{E}+01$ & $2.27 \mathrm{E}-17$ & $3.02 \mathrm{E}-10$ & $2.65 \mathrm{E}-12$ \\
\hline
\end{tabular}




\begin{tabular}{|c|c|c|c|c|c|c|c|c|}
\hline Nuclide & Half-life & Class & $\begin{array}{c}\text { Air } \\
\text { Immersion } \\
\text { Dose Rate } \\
\text { Coefficient } \\
\left(\mathrm{Sv} / \mathbf{s ~ p e r ~}^{\mathbf{3}}\right. \\
\left.\mathrm{Bq} / \mathbf{m}^{\mathbf{3}}\right)\end{array}$ & $\begin{array}{c}\text { Air } \\
\text { Immersion } \\
\text { Dose Rate } \\
\text { Coefficient } \\
\text { (mrem- } \\
\left.\text { m³ }^{3} / \mathrm{pCi}-\mathrm{yr}\right)\end{array}$ & $\begin{array}{c}\text { Air } \\
\text { Immersion } \\
\text { Effective } \\
\text { Dose Rate } \\
\text { (mrem/sec } \\
\text { per } \\
\mu \mathrm{Ci} / \mathbf{c m}^{3} \text { ) }\end{array}$ & $\begin{array}{c}\text { Water } \\
\text { Submersion } \\
\text { Dose Rate } \\
\text { Coefficient } \\
\left(\mathbf{S v}-\mathbf{m}^{3} / \mathbf{B q}-\right. \\
\text { s) }\end{array}$ & $\begin{array}{c}\text { Water } \\
\text { Submersion } \\
\text { Dose Rate } \\
\text { Coefficient } \\
\text { (mrem- } \\
\left.\text { m³ }^{\mathbf{3}} / \mathbf{p C i}-\mathbf{h}\right)\end{array}$ & $\begin{array}{c}\text { Water } \\
\text { Submersion } \\
\text { Dose Rate } \\
\text { Coefficient } \\
(\text { mrem- } \\
\left.\text { m³ }^{3} / \mu \mathrm{Ci}-\mathrm{y}\right)\end{array}$ \\
\hline $\mathrm{Hg}-205$ & 5.2 & $\mathrm{~m}$ & $6.19 \mathrm{E}-16$ & $7.23 \mathrm{E}-05$ & $2.29 \mathrm{E}+00$ & $9.63 \mathrm{E}-19$ & $1.28 \mathrm{E}-11$ & $1.12 \mathrm{E}-13$ \\
\hline $\mathrm{Hg}-206$ & 8.15 & $\mathrm{~m}$ & $5.56 \mathrm{E}-15$ & $6.49 \mathrm{E}-04$ & $2.06 \mathrm{E}+01$ & $1.19 \mathrm{E}-17$ & $1.59 \mathrm{E}-10$ & $1.39 \mathrm{E}-12$ \\
\hline $\mathrm{Hg}-207$ & 2.9 & $\mathrm{~m}$ & $1.28 \mathrm{E}-13$ & $1.49 \mathrm{E}-02$ & $4.74 \mathrm{E}+02$ & $2.77 \mathrm{E}-16$ & $3.69 \mathrm{E}-09$ & $3.23 \mathrm{E}-11$ \\
\hline Tl-190 & 2.6 & $\mathrm{~m}$ & $5.94 \mathrm{E}-14$ & $6.94 \mathrm{E}-03$ & $2.20 \mathrm{E}+02$ & $1.28 \mathrm{E}-16$ & $1.70 \mathrm{E}-09$ & $1.49 \mathrm{E}-11$ \\
\hline Tl-190m & 3.7 & $\mathrm{~m}$ & $1.11 \mathrm{E}-13$ & $1.30 \mathrm{E}-02$ & $4.11 \mathrm{E}+02$ & $2.41 \mathrm{E}-16$ & $3.21 \mathrm{E}-09$ & $2.81 \mathrm{E}-11$ \\
\hline Tl-194 & 33 & $\mathrm{~m}$ & $4.10 \mathrm{E}-14$ & 4.79E-03 & $1.52 \mathrm{E}+02$ & $8.87 \mathrm{E}-17$ & $1.18 \mathrm{E}-09$ & $1.03 \mathrm{E}-11$ \\
\hline T1-194m & 32.8 & $\mathrm{~m}$ & $1.13 \mathrm{E}-13$ & $1.32 \mathrm{E}-02$ & $4.18 \mathrm{E}+02$ & $2.46 \mathrm{E}-16$ & $3.28 \mathrm{E}-09$ & $2.87 \mathrm{E}-11$ \\
\hline T1-195 & 1.16 & $\mathrm{~h}$ & $5.74 \mathrm{E}-14$ & $6.70 \mathrm{E}-03$ & $2.12 \mathrm{E}+02$ & $1.25 \mathrm{E}-16$ & $1.67 \mathrm{E}-09$ & $1.46 \mathrm{E}-11$ \\
\hline Tl-196 & 1.84 & $\mathrm{~h}$ & $8.80 \mathrm{E}-14$ & $1.03 \mathrm{E}-02$ & $3.26 \mathrm{E}+02$ & $1.91 \mathrm{E}-16$ & $2.54 \mathrm{E}-09$ & $2.23 \mathrm{E}-11$ \\
\hline Tl-197 & 2.84 & $\mathrm{~h}$ & $2.02 \mathrm{E}-14$ & $2.36 \mathrm{E}-03$ & $7.47 \mathrm{E}+01$ & $4.40 \mathrm{E}-17$ & $5.86 \mathrm{E}-10$ & $5.13 \mathrm{E}-12$ \\
\hline Tl-198 & 5.3 & $\mathrm{~h}$ & $9.50 \mathrm{E}-14$ & $1.11 \mathrm{E}-02$ & $3.52 \mathrm{E}+02$ & $2.06 \mathrm{E}-16$ & $2.74 \mathrm{E}-09$ & $2.40 \mathrm{E}-11$ \\
\hline Tl-198m & 1.87 & $\mathrm{~h}$ & $5.36 \mathrm{E}-14$ & $6.26 \mathrm{E}-03$ & $1.98 \mathrm{E}+02$ & $1.17 \mathrm{E}-16$ & $1.56 \mathrm{E}-09$ & $1.37 \mathrm{E}-11$ \\
\hline T1-199 & 7.42 & $\mathrm{~h}$ & $1.03 \mathrm{E}-14$ & $1.20 \mathrm{E}-03$ & $3.81 \mathrm{E}+01$ & $2.27 \mathrm{E}-17$ & $3.02 \mathrm{E}-10$ & $2.65 \mathrm{E}-12$ \\
\hline Tl-200 & 26.1 & $\mathrm{~h}$ & $5.98 \mathrm{E}-14$ & $6.98 \mathrm{E}-03$ & $2.21 \mathrm{E}+02$ & $1.30 \mathrm{E}-16$ & $1.73 \mathrm{E}-09$ & $1.52 \mathrm{E}-11$ \\
\hline Tl-201 & 72.912 & $\mathrm{~h}$ & $3.26 \mathrm{E}-15$ & $3.81 \mathrm{E}-04$ & $1.21 \mathrm{E}+01$ & $7.32 \mathrm{E}-18$ & $9.75 \mathrm{E}-11$ & $8.54 \mathrm{E}-13$ \\
\hline Tl-202 & 12.23 & $\mathrm{~d}$ & $1.99 \mathrm{E}-14$ & $2.32 \mathrm{E}-03$ & $7.36 \mathrm{E}+01$ & $4.36 \mathrm{E}-17$ & $5.81 \mathrm{E}-10$ & $5.09 \mathrm{E}-12$ \\
\hline Tl-204 & 3.78 & $\mathrm{y}$ & $1.75 \mathrm{E}-16$ & $2.04 \mathrm{E}-05$ & $6.48 \mathrm{E}-01$ & $2.44 \mathrm{E}-19$ & $3.25 \mathrm{E}-12$ & $2.85 \mathrm{E}-14$ \\
\hline Tl-206 & 4.2 & $\mathrm{~m}$ & $3.97 \mathrm{E}-16$ & $4.64 \mathrm{E}-05$ & $1.47 \mathrm{E}+00$ & $4.75 \mathrm{E}-19$ & $6.33 \mathrm{E}-12$ & $5.54 \mathrm{E}-14$ \\
\hline Tl-206m & 3.74 & $\mathrm{~m}$ & $1.09 \mathrm{E}-13$ & $1.27 \mathrm{E}-02$ & $4.03 \mathrm{E}+02$ & $2.37 \mathrm{E}-16$ & $3.16 \mathrm{E}-09$ & $2.77 \mathrm{E}-11$ \\
\hline T1-207 & 4.77 & $\mathrm{~m}$ & $4.61 \mathrm{E}-16$ & $5.38 \mathrm{E}-05$ & $1.71 \mathrm{E}+00$ & $6.50 \mathrm{E}-19$ & $8.66 \mathrm{E}-12$ & $7.58 \mathrm{E}-14$ \\
\hline Tl-208 & 3.053 & $\mathrm{~m}$ & $1.68 \mathrm{E}-13$ & $1.96 \mathrm{E}-02$ & $6.22 \mathrm{E}+02$ & $3.64 \mathrm{E}-16$ & $4.85 \mathrm{E}-09$ & $4.25 \mathrm{E}-11$ \\
\hline Tl-209 & 2.161 & $\mathrm{~m}$ & $1.02 \mathrm{E}-13$ & $1.19 \mathrm{E}-02$ & $3.77 \mathrm{E}+02$ & $2.20 \mathrm{E}-16$ & $2.93 \mathrm{E}-09$ & $2.57 \mathrm{E}-11$ \\
\hline Tl-210 & 1.3 & $\mathrm{~m}$ & $1.32 \mathrm{E}-13$ & $1.54 \mathrm{E}-02$ & $4.88 \mathrm{E}+02$ & $2.85 \mathrm{E}-16$ & $3.80 \mathrm{E}-09$ & $3.33 \mathrm{E}-11$ \\
\hline
\end{tabular}




\begin{tabular}{|c|c|c|c|c|c|c|c|c|}
\hline Nuclide & Half-life & Class & $\begin{array}{c}\text { Air } \\
\text { Immersion } \\
\text { Dose Rate } \\
\text { Coefficient } \\
(\mathrm{Sv} / \mathrm{s} \mathrm{per} \\
\left.\mathrm{Bq} / \mathbf{m}^{3}\right)\end{array}$ & $\begin{array}{c}\text { Air } \\
\text { Immersion } \\
\text { Dose Rate } \\
\text { Coefficient } \\
\text { (mrem- } \\
\left.\text { m³ }^{3} / \mathrm{pCi}-\mathrm{yr}\right)\end{array}$ & $\begin{array}{c}\text { Air } \\
\text { Immersion } \\
\text { Effective } \\
\text { Dose Rate } \\
(\mathrm{mrem} / \mathrm{sec} \\
\mathrm{per} \\
\left.\mu \mathrm{Ci} / \mathrm{cm}^{3}\right)\end{array}$ & $\begin{array}{c}\text { Water } \\
\text { Submersion } \\
\text { Dose Rate } \\
\text { Coefficient } \\
\left(\mathrm{Sv}-\mathbf{m}^{3} / \mathbf{B q}-\right. \\
\text { s) }\end{array}$ & $\begin{array}{c}\text { Water } \\
\text { Submersion } \\
\text { Dose Rate } \\
\text { Coefficient } \\
\text { (mrem- } \\
\left.\text { m³ }^{3} / \mathbf{p C i}-\mathbf{h}\right)\end{array}$ & $\begin{array}{c}\text { Water } \\
\text { Submersion } \\
\text { Dose Rate } \\
\text { Coefficient } \\
(\text { mrem- } \\
\left.\text { m }^{3} / \mu \mathrm{Ci}-\mathrm{y}\right)\end{array}$ \\
\hline $\mathrm{Pb}-194$ & 12 & $\mathrm{~m}$ & $4.94 \mathrm{E}-14$ & $5.77 \mathrm{E}-03$ & $1.83 \mathrm{E}+02$ & $1.07 \mathrm{E}-16$ & $1.43 \mathrm{E}-09$ & $1.25 \mathrm{E}-11$ \\
\hline $\mathrm{Pb}-195 \mathrm{~m}$ & 15 & $\mathrm{~m}$ & $7.40 \mathrm{E}-14$ & 8.64E-03 & $2.74 \mathrm{E}+02$ & $1.61 \mathrm{E}-16$ & $2.14 \mathrm{E}-09$ & $1.88 \mathrm{E}-11$ \\
\hline $\mathrm{Pb}-196$ & 37 & $\mathrm{~m}$ & $2.10 \mathrm{E}-14$ & $2.45 \mathrm{E}-03$ & $7.77 \mathrm{E}+01$ & $4.60 \mathrm{E}-17$ & $6.13 \mathrm{E}-10$ & $5.37 \mathrm{E}-12$ \\
\hline $\mathrm{Pb}-197$ & 8 & $\mathrm{~m}$ & $7.14 \mathrm{E}-14$ & $8.34 \mathrm{E}-03$ & $2.64 \mathrm{E}+02$ & $1.55 \mathrm{E}-16$ & $2.06 \mathrm{E}-09$ & $1.81 \mathrm{E}-11$ \\
\hline $\mathrm{Pb}-197 \mathrm{~m}$ & 43 & $\mathrm{~m}$ & $5.22 \mathrm{E}-14$ & $6.10 \mathrm{E}-03$ & $1.93 \mathrm{E}+02$ & $1.14 \mathrm{E}-16$ & $1.52 \mathrm{E}-09$ & $1.33 \mathrm{E}-11$ \\
\hline $\mathrm{Pb}-198$ & 2.4 & $\mathrm{~h}$ & $1.86 \mathrm{E}-14$ & $2.17 \mathrm{E}-03$ & $6.88 \mathrm{E}+01$ & $4.07 \mathrm{E}-17$ & $5.42 \mathrm{E}-10$ & $4.75 \mathrm{E}-12$ \\
\hline Pb-199 & 90 & $\mathrm{~m}$ & $4.79 \mathrm{E}-14$ & $5.59 \mathrm{E}-03$ & $1.77 \mathrm{E}+02$ & $1.04 \mathrm{E}-16$ & $1.39 \mathrm{E}-09$ & $1.21 \mathrm{E}-11$ \\
\hline $\mathrm{Pb}-200$ & 21.5 & $\mathrm{~h}$ & $8.16 \mathrm{E}-15$ & $9.53 \mathrm{E}-04$ & $3.02 \mathrm{E}+01$ & $1.81 \mathrm{E}-17$ & $2.41 \mathrm{E}-10$ & $2.11 \mathrm{E}-12$ \\
\hline $\mathrm{Pb}-201$ & 9.33 & $\mathrm{~h}$ & $3.34 \mathrm{E}-14$ & $3.90 \mathrm{E}-03$ & $1.24 \mathrm{E}+02$ & $7.28 \mathrm{E}-17$ & $9.70 \mathrm{E}-10$ & $8.49 \mathrm{E}-12$ \\
\hline $\mathrm{Pb}-201 \mathrm{~m}$ & 61 & $\mathrm{~s}$ & $1.63 \mathrm{E}-14$ & $1.90 \mathrm{E}-03$ & $6.03 \mathrm{E}+01$ & $3.54 \mathrm{E}-17$ & $4.72 \mathrm{E}-10$ & $4.13 \mathrm{E}-12$ \\
\hline $\mathrm{Pb}-202$ & $5.25 \mathrm{E}+04$ & $\mathrm{y}$ & $4.85 \mathrm{E}-19$ & $5.66 \mathrm{E}-08$ & $1.79 \mathrm{E}-03$ & $1.12 \mathrm{E}-21$ & $1.49 \mathrm{E}-14$ & $1.31 \mathrm{E}-16$ \\
\hline $\mathrm{Pb}-202 \mathrm{~m}$ & 3.53 & $\mathrm{~h}$ & $9.06 \mathrm{E}-14$ & $1.06 \mathrm{E}-02$ & $3.35 \mathrm{E}+02$ & $1.97 \mathrm{E}-16$ & $2.62 \mathrm{E}-09$ & $2.30 \mathrm{E}-11$ \\
\hline $\mathrm{Pb}-203$ & 51.873 & $\mathrm{~h}$ & $1.31 \mathrm{E}-14$ & $1.53 \mathrm{E}-03$ & $4.85 \mathrm{E}+01$ & $2.88 \mathrm{E}-17$ & $3.84 \mathrm{E}-10$ & $3.36 \mathrm{E}-12$ \\
\hline $\mathrm{Pb}-204 \mathrm{~m}$ & 67.2 & $\mathrm{~m}$ & $9.45 \mathrm{E}-14$ & $1.10 \mathrm{E}-02$ & $3.50 \mathrm{E}+02$ & $2.05 \mathrm{E}-16$ & $2.73 \mathrm{E}-09$ & $2.39 \mathrm{E}-11$ \\
\hline $\mathrm{Pb}-205$ & $1.53 \mathrm{E}+07$ & $\mathrm{y}$ & $4.92 \mathrm{E}-19$ & $5.74 \mathrm{E}-08$ & $1.82 \mathrm{E}-03$ & $1.14 \mathrm{E}-21$ & $1.52 \mathrm{E}-14$ & $1.33 \mathrm{E}-16$ \\
\hline $\mathrm{Pb}-209$ & 3.253 & $\mathrm{~h}$ & $1.00 \mathrm{E}-16$ & $1.17 \mathrm{E}-05$ & $3.70 \mathrm{E}-01$ & $1.12 \mathrm{E}-19$ & $1.49 \mathrm{E}-12$ & $1.31 \mathrm{E}-14$ \\
\hline $\mathrm{Pb}-210$ & 22.2 & $\mathrm{y}$ & $4.71 \mathrm{E}-17$ & $5.50 \mathrm{E}-06$ & $1.74 \mathrm{E}-01$ & $1.09 \mathrm{E}-19$ & $1.45 \mathrm{E}-12$ & $1.27 \mathrm{E}-14$ \\
\hline $\mathrm{Pb}-211$ & 36.1 & $\mathrm{~m}$ & $3.22 \mathrm{E}-15$ & $3.76 \mathrm{E}-04$ & $1.19 \mathrm{E}+01$ & $6.68 \mathrm{E}-18$ & $8.90 \mathrm{E}-11$ & $7.79 \mathrm{E}-13$ \\
\hline $\mathrm{Pb}-212$ & 10.64 & $\mathrm{~h}$ & $6.11 \mathrm{E}-15$ & 7.13E-04 & $2.26 \mathrm{E}+01$ & $1.34 \mathrm{E}-17$ & $1.78 \mathrm{E}-10$ & $1.56 \mathrm{E}-12$ \\
\hline $\mathrm{Pb}-214$ & 26.8 & $\mathrm{~m}$ & $1.11 \mathrm{E}-14$ & $1.30 \mathrm{E}-03$ & $4.11 \mathrm{E}+01$ & $2.41 \mathrm{E}-17$ & $3.21 \mathrm{E}-10$ & $2.81 \mathrm{E}-12$ \\
\hline Bi-197 & 9.3 & $\mathrm{~m}$ & $7.85 \mathrm{E}-14$ & $9.17 \mathrm{E}-03$ & $2.90 \mathrm{E}+02$ & $1.70 \mathrm{E}-16$ & $2.26 \mathrm{E}-09$ & $1.98 \mathrm{E}-11$ \\
\hline Bi-200 & 36.4 & $\mathrm{~m}$ & $1.10 \mathrm{E}-13$ & $1.28 \mathrm{E}-02$ & $4.07 \mathrm{E}+02$ & $2.39 \mathrm{E}-16$ & $3.18 \mathrm{E}-09$ & $2.79 \mathrm{E}-11$ \\
\hline Bi-201 & 108 & $\mathrm{~m}$ & $8.11 \mathrm{E}-14$ & $9.47 \mathrm{E}-03$ & $3.00 \mathrm{E}+02$ & $1.76 \mathrm{E}-16$ & $2.34 \mathrm{E}-09$ & $2.05 \mathrm{E}-11$ \\
\hline
\end{tabular}




\begin{tabular}{|c|c|c|c|c|c|c|c|c|}
\hline Nuclide & Half-life & Class & $\begin{array}{c}\text { Air } \\
\text { Immersion } \\
\text { Dose Rate } \\
\text { Coefficient } \\
\left(\mathrm{Sv} / \mathbf{s ~ p e r ~}^{\mathbf{3}}\right. \\
\left.\mathrm{Bq} / \mathbf{m}^{\mathbf{3}}\right)\end{array}$ & $\begin{array}{c}\text { Air } \\
\text { Immersion } \\
\text { Dose Rate } \\
\text { Coefficient } \\
\text { (mrem- } \\
\left.\text { m³ }^{3} / \mathrm{pCi}-\mathrm{yr}\right)\end{array}$ & $\begin{array}{c}\text { Air } \\
\text { Immersion } \\
\text { Effective } \\
\text { Dose Rate } \\
\text { (mrem/sec } \\
\text { per } \\
\mu \mathrm{Ci} / \mathbf{c m}^{3} \text { ) }\end{array}$ & $\begin{array}{c}\text { Water } \\
\text { Submersion } \\
\text { Dose Rate } \\
\text { Coefficient } \\
\left(\mathbf{S v}-\mathbf{m}^{3} / \mathbf{B q}-\right. \\
\text { s) }\end{array}$ & $\begin{array}{c}\text { Water } \\
\text { Submersion } \\
\text { Dose Rate } \\
\text { Coefficient } \\
\text { (mrem- } \\
\left.\text { m³ }^{\mathbf{3}} / \mathbf{p C i}-\mathbf{h}\right)\end{array}$ & $\begin{array}{c}\text { Water } \\
\text { Submersion } \\
\text { Dose Rate } \\
\text { Coefficient } \\
(\text { mrem- } \\
\left.\mathbf{m}^{\mathbf{3}} / \boldsymbol{\mu C i}-\mathrm{y}\right)\end{array}$ \\
\hline Bi-202 & 1.72 & $\mathrm{~h}$ & $1.26 \mathrm{E}-13$ & $1.47 \mathrm{E}-02$ & $4.66 \mathrm{E}+02$ & $2.74 \mathrm{E}-16$ & $3.65 \mathrm{E}-09$ & $3.20 \mathrm{E}-11$ \\
\hline Bi-203 & 11.76 & $\mathrm{~h}$ & $1.13 \mathrm{E}-13$ & $1.32 \mathrm{E}-02$ & $4.18 \mathrm{E}+02$ & $2.44 \mathrm{E}-16$ & $3.25 \mathrm{E}-09$ & $2.85 \mathrm{E}-11$ \\
\hline Bi-204 & 11.22 & $\mathrm{~h}$ & $1.35 \mathrm{E}-13$ & $1.58 \mathrm{E}-02$ & $5.00 \mathrm{E}+02$ & $2.92 \mathrm{E}-16$ & $3.89 \mathrm{E}-09$ & $3.41 \mathrm{E}-11$ \\
\hline Bi-205 & 15.31 & $\mathrm{~d}$ & $7.99 \mathrm{E}-14$ & $9.33 \mathrm{E}-03$ & $2.96 \mathrm{E}+02$ & $1.73 \mathrm{E}-16$ & $2.30 \mathrm{E}-09$ & $2.02 \mathrm{E}-11$ \\
\hline Bi-206 & 6.243 & $\mathrm{~d}$ & $1.51 \mathrm{E}-13$ & $1.76 \mathrm{E}-02$ & $5.59 \mathrm{E}+02$ & $3.28 \mathrm{E}-16$ & $4.37 \mathrm{E}-09$ & $3.83 \mathrm{E}-11$ \\
\hline Bi-207 & 32.9 & $\mathrm{y}$ & $7.03 \mathrm{E}-14$ & $8.21 \mathrm{E}-03$ & $2.60 \mathrm{E}+02$ & $1.53 \mathrm{E}-16$ & $2.04 \mathrm{E}-09$ & $1.79 \mathrm{E}-11$ \\
\hline Bi-208 & $3.68 \mathrm{E}+05$ & $\mathrm{y}$ & $1.35 \mathrm{E}-13$ & $1.58 \mathrm{E}-02$ & $5.00 \mathrm{E}+02$ & $2.93 \mathrm{E}-16$ & $3.90 \mathrm{E}-09$ & $3.42 \mathrm{E}-11$ \\
\hline Bi-210 & 5.013 & $\mathrm{~d}$ & $2.58 \mathrm{E}-16$ & $3.01 \mathrm{E}-05$ & $9.55 \mathrm{E}-01$ & $2.98 \mathrm{E}-19$ & $3.97 \mathrm{E}-12$ & $3.48 \mathrm{E}-14$ \\
\hline $\mathrm{Bi}-210 \mathrm{~m}$ & $3.04 \mathrm{E}+06$ & $\mathrm{y}$ & $1.14 \mathrm{E}-14$ & $1.33 \mathrm{E}-03$ & $4.22 \mathrm{E}+01$ & $2.49 \mathrm{E}-17$ & $3.32 \mathrm{E}-10$ & $2.91 \mathrm{E}-12$ \\
\hline $\mathrm{Bi}-211$ & 2.14 & $\mathrm{~m}$ & $2.07 \mathrm{E}-15$ & $2.42 \mathrm{E}-04$ & $7.66 \mathrm{E}+00$ & $4.51 \mathrm{E}-18$ & $6.01 \mathrm{E}-11$ & $5.26 \mathrm{E}-13$ \\
\hline Bi-212 & 60.55 & $\mathrm{~m}$ & $5.17 \mathrm{E}-15$ & $6.04 \mathrm{E}-04$ & $1.91 \mathrm{E}+01$ & $1.08 \mathrm{E}-17$ & $1.44 \mathrm{E}-10$ & $1.26 \mathrm{E}-12$ \\
\hline Bi-212n & 7 & $\mathrm{~m}$ & $3.89 \mathrm{E}-16$ & $4.54 \mathrm{E}-05$ & $1.44 \mathrm{E}+00$ & $4.61 \mathrm{E}-19$ & $6.14 \mathrm{E}-12$ & $5.38 \mathrm{E}-14$ \\
\hline Bi-213 & 45.59 & $\mathrm{~m}$ & $5.94 \mathrm{E}-15$ & $6.94 \mathrm{E}-04$ & $2.20 \mathrm{E}+01$ & $1.26 \mathrm{E}-17$ & $1.68 \mathrm{E}-10$ & $1.47 \mathrm{E}-12$ \\
\hline Bi-214 & 19.9 & $\mathrm{~m}$ & $7.11 \mathrm{E}-14$ & $8.30 \mathrm{E}-03$ & $2.63 \mathrm{E}+02$ & $1.54 \mathrm{E}-16$ & $2.05 \mathrm{E}-09$ & $1.80 \mathrm{E}-11$ \\
\hline Bi-215 & 7.6 & $\mathrm{~m}$ & $1.18 \mathrm{E}-14$ & $1.38 \mathrm{E}-03$ & $4.37 \mathrm{E}+01$ & $2.52 \mathrm{E}-17$ & $3.36 \mathrm{E}-10$ & $2.94 \mathrm{E}-12$ \\
\hline Bi-216 & 2.17 & $\mathrm{~m}$ & $3.41 \mathrm{E}-14$ & $3.98 \mathrm{E}-03$ & $1.26 \mathrm{E}+02$ & $7.31 \mathrm{E}-17$ & $9.74 \mathrm{E}-10$ & $8.53 \mathrm{E}-12$ \\
\hline Po-203 & 36.7 & $\mathrm{~m}$ & $7.55 \mathrm{E}-14$ & $8.82 \mathrm{E}-03$ & $2.79 \mathrm{E}+02$ & $1.64 \mathrm{E}-16$ & $2.18 \mathrm{E}-09$ & $1.91 \mathrm{E}-11$ \\
\hline Po-204 & 3.53 & $\mathrm{~h}$ & $5.16 \mathrm{E}-14$ & $6.02 \mathrm{E}-03$ & $1.91 \mathrm{E}+02$ & $1.12 \mathrm{E}-16$ & $1.49 \mathrm{E}-09$ & $1.31 \mathrm{E}-11$ \\
\hline Po-205 & 1.66 & $\mathrm{~h}$ & $7.31 \mathrm{E}-14$ & $8.54 \mathrm{E}-03$ & $2.70 \mathrm{E}+02$ & $1.59 \mathrm{E}-16$ & $2.12 \mathrm{E}-09$ & $1.86 \mathrm{E}-11$ \\
\hline Po-206 & 8.8 & $\mathrm{~d}$ & $5.33 \mathrm{E}-14$ & $6.22 \mathrm{E}-03$ & $1.97 \mathrm{E}+02$ & $1.16 \mathrm{E}-16$ & $1.55 \mathrm{E}-09$ & $1.35 \mathrm{E}-11$ \\
\hline Po-207 & 5.8 & $\mathrm{~h}$ & $5.86 \mathrm{E}-14$ & $6.84 \mathrm{E}-03$ & $2.17 \mathrm{E}+02$ & $1.27 \mathrm{E}-16$ & $1.69 \mathrm{E}-09$ & $1.48 \mathrm{E}-11$ \\
\hline Po-208 & 2.898 & $\mathrm{y}$ & $9.36 \mathrm{E}-19$ & $1.09 \mathrm{E}-07$ & $3.46 \mathrm{E}-03$ & $2.04 \mathrm{E}-21$ & $2.72 \mathrm{E}-14$ & $2.38 \mathrm{E}-16$ \\
\hline Po-209 & 102 & $\mathrm{y}$ & $2.76 \mathrm{E}-16$ & $3.22 \mathrm{E}-05$ & $1.02 \mathrm{E}+00$ & $6.01 \mathrm{E}-19$ & $8.01 \mathrm{E}-12$ & $7.01 \mathrm{E}-14$ \\
\hline
\end{tabular}




\begin{tabular}{|c|c|c|c|c|c|c|c|c|}
\hline Nuclide & Half-life & Class & $\begin{array}{c}\text { Air } \\
\text { Immersion } \\
\text { Dose Rate } \\
\text { Coefficient } \\
\left(\mathrm{Sv} / \mathbf{s ~ p e r ~}^{\mathbf{3}}\right. \\
\left.\mathrm{Bq} / \mathbf{m}^{\mathbf{3}}\right)\end{array}$ & $\begin{array}{c}\text { Air } \\
\text { Immersion } \\
\text { Dose Rate } \\
\text { Coefficient } \\
\text { (mrem- } \\
\left.\text { m }^{3} / \mathrm{pCi}-\mathrm{yr}\right)\end{array}$ & $\begin{array}{c}\text { Air } \\
\text { Immersion } \\
\text { Effective } \\
\text { Dose Rate } \\
\text { (mrem/sec } \\
\text { per } \\
\mu \mathrm{Ci} / \mathrm{cm}^{3} \text { ) } \\
\end{array}$ & $\begin{array}{c}\text { Water } \\
\text { Submersion } \\
\text { Dose Rate } \\
\text { Coefficient } \\
\left(\mathbf{S v}-\mathbf{m}^{3} / \mathbf{B q}-\right. \\
\text { s) }\end{array}$ & $\begin{array}{c}\text { Water } \\
\text { Submersion } \\
\text { Dose Rate } \\
\text { Coefficient } \\
\text { (mrem- } \\
\left.\text { m³ }^{\mathbf{3}} / \mathbf{p C i}-\mathbf{h}\right)\end{array}$ & $\begin{array}{c}\text { Water } \\
\text { Submersion } \\
\text { Dose Rate } \\
\text { Coefficient } \\
(\text { mrem- } \\
\left.\text { m³ }^{3} / \mu \mathrm{Ci}-\mathrm{y}\right)\end{array}$ \\
\hline Po-210 & 138.376 & $\mathrm{~d}$ & $4.45 \mathrm{E}-19$ & $5.20 \mathrm{E}-08$ & $1.65 \mathrm{E}-03$ & $9.65 \mathrm{E}-22$ & $1.29 \mathrm{E}-14$ & $1.13 \mathrm{E}-16$ \\
\hline Po-211 & 0.516 & $\mathrm{~s}$ & $3.73 \mathrm{E}-16$ & $4.36 \mathrm{E}-05$ & $1.38 \mathrm{E}+00$ & $8.08 \mathrm{E}-19$ & $1.08 \mathrm{E}-11$ & $9.43 \mathrm{E}-14$ \\
\hline Po-212 & $2.99 \mathrm{E}-07$ & $\mathrm{~s}$ & $0.00 \mathrm{E}+00$ & $0.00 \mathrm{E}+00$ & $0.00 \mathrm{E}+00$ & $0.00 \mathrm{E}+00$ & $0.00 \mathrm{E}+00$ & $0.00 \mathrm{E}+00$ \\
\hline Po-212m & 45.1 & $\mathrm{~S}$ & $3.99 \mathrm{E}-15$ & $4.66 \mathrm{E}-04$ & $1.48 \mathrm{E}+01$ & $8.65 \mathrm{E}-18$ & $1.15 \mathrm{E}-10$ & $1.01 \mathrm{E}-12$ \\
\hline Po-213 & $4.20 \mathrm{E}-06$ & $\mathrm{~s}$ & $1.71 \mathrm{E}-18$ & $2.00 \mathrm{E}-07$ & $6.33 \mathrm{E}-03$ & $3.71 \mathrm{E}-21$ & $4.94 \mathrm{E}-14$ & $4.33 \mathrm{E}-16$ \\
\hline Po-214 & $1.64 \mathrm{E}-04$ & $\mathrm{~s}$ & $3.80 \mathrm{E}-18$ & $4.44 \mathrm{E}-07$ & $1.41 \mathrm{E}-02$ & $8.23 \mathrm{E}-21$ & $1.10 \mathrm{E}-13$ & $9.60 \mathrm{E}-16$ \\
\hline Po-215 & $1.78 \mathrm{E}-03$ & $\mathrm{~s}$ & $7.80 \mathrm{E}-18$ & $9.11 \mathrm{E}-07$ & $2.89 \mathrm{E}-02$ & $1.70 \mathrm{E}-20$ & $2.26 \mathrm{E}-13$ & $1.98 \mathrm{E}-15$ \\
\hline Po-216 & 0.145 & $\mathrm{~s}$ & $7.00 \mathrm{E}-19$ & $8.17 \mathrm{E}-08$ & $2.59 \mathrm{E}-03$ & $1.52 \mathrm{E}-21$ & $2.02 \mathrm{E}-14$ & $1.77 \mathrm{E}-16$ \\
\hline Po-218 & 3.1 & $\mathrm{~m}$ & $2.62 \mathrm{E}-21$ & $3.06 \mathrm{E}-10$ & $9.69 \mathrm{E}-06$ & $2.86 \mathrm{E}-24$ & $3.81 \mathrm{E}-17$ & $3.34 \mathrm{E}-19$ \\
\hline At-204 & 9.2 & $\mathrm{~m}$ & $1.04 \mathrm{E}-13$ & $1.21 \mathrm{E}-02$ & $3.85 \mathrm{E}+02$ & $2.26 \mathrm{E}-16$ & $3.01 \mathrm{E}-09$ & $2.64 \mathrm{E}-11$ \\
\hline At-205 & 26.2 & $\mathrm{~m}$ & $5.22 \mathrm{E}-14$ & $6.10 \mathrm{E}-03$ & $1.93 \mathrm{E}+02$ & $1.13 \mathrm{E}-16$ & $1.51 \mathrm{E}-09$ & $1.32 \mathrm{E}-11$ \\
\hline At-206 & 30.6 & $\mathrm{~m}$ & $1.12 \mathrm{E}-13$ & $1.31 \mathrm{E}-02$ & $4.14 \mathrm{E}+02$ & $2.44 \mathrm{E}-16$ & $3.25 \mathrm{E}-09$ & $2.85 \mathrm{E}-11$ \\
\hline At-207 & 1.8 & $\mathrm{~h}$ & $9.34 \mathrm{E}-14$ & $1.09 \mathrm{E}-02$ & $3.46 \mathrm{E}+02$ & $2.03 \mathrm{E}-16$ & $2.70 \mathrm{E}-09$ & $2.37 \mathrm{E}-11$ \\
\hline At-208 & 1.63 & $\mathrm{~h}$ & $1.40 \mathrm{E}-13$ & $1.63 \mathrm{E}-02$ & $5.18 \mathrm{E}+02$ & $3.03 \mathrm{E}-16$ & $4.04 \mathrm{E}-09$ & $3.54 \mathrm{E}-11$ \\
\hline At-209 & 5.41 & $\mathrm{~h}$ & $1.03 \mathrm{E}-13$ & $1.20 \mathrm{E}-02$ & $3.81 \mathrm{E}+02$ & $2.24 \mathrm{E}-16$ & $2.98 \mathrm{E}-09$ & $2.61 \mathrm{E}-11$ \\
\hline At-210 & 8.1 & $\mathrm{~h}$ & $1.40 \mathrm{E}-13$ & $1.63 \mathrm{E}-02$ & $5.18 \mathrm{E}+02$ & $3.03 \mathrm{E}-16$ & $4.04 \mathrm{E}-09$ & $3.54 \mathrm{E}-11$ \\
\hline At-211 & 7.214 & $\mathrm{~h}$ & $1.27 \mathrm{E}-15$ & $1.48 \mathrm{E}-04$ & $4.70 \mathrm{E}+00$ & $2.86 \mathrm{E}-18$ & $3.81 \mathrm{E}-11$ & $3.34 \mathrm{E}-13$ \\
\hline At-215 & $1.00 \mathrm{E}-04$ & $\mathrm{~s}$ & $7.53 \mathrm{E}-18$ & $8.79 \mathrm{E}-07$ & $2.79 \mathrm{E}-02$ & $1.64 \mathrm{E}-20$ & $2.18 \mathrm{E}-13$ & $1.91 \mathrm{E}-15$ \\
\hline At-216 & $3.00 \mathrm{E}-04$ & $\mathrm{~s}$ & $9.52 \mathrm{E}-17$ & $1.11 \mathrm{E}-05$ & $3.52 \mathrm{E}-01$ & $2.12 \mathrm{E}-19$ & $2.82 \mathrm{E}-12$ & $2.47 \mathrm{E}-14$ \\
\hline At-217 & $3.23 \mathrm{E}-02$ & $\mathrm{~s}$ & $1.06 \mathrm{E}-17$ & $1.24 \mathrm{E}-06$ & $3.92 \mathrm{E}-02$ & $2.31 \mathrm{E}-20$ & $3.08 \mathrm{E}-13$ & $2.70 \mathrm{E}-15$ \\
\hline At-218 & 1.5 & $\mathrm{~s}$ & $9.80 \mathrm{E}-19$ & $1.14 \mathrm{E}-07$ & $3.63 \mathrm{E}-03$ & $1.25 \mathrm{E}-21$ & $1.67 \mathrm{E}-14$ & $1.46 \mathrm{E}-16$ \\
\hline At-219 & 56 & $\mathrm{~s}$ & $0.00 \mathrm{E}+00$ & $0.00 \mathrm{E}+00$ & $0.00 \mathrm{E}+00$ & $0.00 \mathrm{E}+00$ & $0.00 \mathrm{E}+00$ & $0.00 \mathrm{E}+00$ \\
\hline At-220 & 3.71 & $\mathrm{~m}$ & $2.09 \mathrm{E}-14$ & $2.44 \mathrm{E}-03$ & $7.73 \mathrm{E}+01$ & $4.46 \mathrm{E}-17$ & $5.94 \mathrm{E}-10$ & $5.20 \mathrm{E}-12$ \\
\hline
\end{tabular}




\begin{tabular}{|c|c|c|c|c|c|c|c|c|}
\hline Nuclide & Half-life & Class & $\begin{array}{c}\text { Air } \\
\text { Immersion } \\
\text { Dose Rate } \\
\text { Coefficient } \\
(\text { Sv/s per } \\
\left.\text { Bq/ } \mathbf{m}^{3}\right)\end{array}$ & $\begin{array}{c}\text { Air } \\
\text { Immersion } \\
\text { Dose Rate } \\
\text { Coefficient } \\
\text { (mrem- } \\
\left.\text { m³ }^{3} / \mathrm{pCi}-\mathrm{yr}\right)\end{array}$ & $\begin{array}{c}\text { Air } \\
\text { Immersion } \\
\text { Effective } \\
\text { Dose Rate } \\
(\mathrm{mrem} / \mathrm{sec} \\
\mathrm{per} \\
\left.\mu \mathrm{Ci} / \mathrm{cm}^{3}\right)\end{array}$ & $\begin{array}{c}\text { Water } \\
\text { Submersion } \\
\text { Dose Rate } \\
\text { Coefficient } \\
\left(\mathrm{Sv}-\mathbf{m}^{3} / \mathbf{B q}-\right. \\
\text { s) }\end{array}$ & $\begin{array}{c}\text { Water } \\
\text { Submersion } \\
\text { Dose Rate } \\
\text { Coefficient } \\
\text { (mrem- } \\
\text { m³Ci-h) }^{\mathbf{3} / p C i}\end{array}$ & $\begin{array}{c}\text { Water } \\
\text { Submersion } \\
\text { Dose Rate } \\
\text { Coefficient } \\
(\text { mrem- } \\
\left.\text { m }^{3} / \mu \mathrm{Ci}-\mathrm{y}\right)\end{array}$ \\
\hline Rn-207 & 9.25 & $\mathrm{~m}$ & $4.41 \mathrm{E}-14$ & $5.15 \mathrm{E}-03$ & $1.63 \mathrm{E}+02$ & $9.59 \mathrm{E}-17$ & $1.28 \mathrm{E}-09$ & $1.12 \mathrm{E}-11$ \\
\hline Rn-209 & 28.5 & $\mathrm{~m}$ & $5.47 \mathrm{E}-14$ & $6.39 \mathrm{E}-03$ & $2.02 \mathrm{E}+02$ & $1.19 \mathrm{E}-16$ & $1.59 \mathrm{E}-09$ & $1.39 \mathrm{E}-11$ \\
\hline $\mathrm{Rn}-210$ & 2.4 & $\mathrm{~h}$ & $1.27 \mathrm{E}-14$ & $1.48 \mathrm{E}-03$ & $4.70 \mathrm{E}+01$ & 5.89E-18 & $7.85 \mathrm{E}-11$ & $6.87 \mathrm{E}-13$ \\
\hline Rn-211 & 14.6 & $\mathrm{~h}$ & $8.62 \mathrm{E}-14$ & $1.01 \mathrm{E}-02$ & $3.19 \mathrm{E}+02$ & $1.87 \mathrm{E}-16$ & $2.49 \mathrm{E}-09$ & $2.18 \mathrm{E}-11$ \\
\hline $\mathrm{Rn}-212$ & 23.9 & $\mathrm{~m}$ & $1.38 \mathrm{E}-14$ & $1.61 \mathrm{E}-03$ & $5.11 \mathrm{E}+01$ & $3.32 \mathrm{E}-20$ & $4.42 \mathrm{E}-13$ & $3.87 \mathrm{E}-15$ \\
\hline $\mathrm{Rn}-215$ & 2.3 & us & $1.90 \mathrm{E}-14$ & $2.22 \mathrm{E}-03$ & $7.03 \mathrm{E}+01$ & $0.00 \mathrm{E}+00$ & $0.00 \mathrm{E}+00$ & $0.00 \mathrm{E}+00$ \\
\hline $\mathrm{Rn}-216$ & $4.50 \mathrm{E}-05$ & $\mathrm{~s}$ & $1.77 \mathrm{E}-14$ & $2.07 \mathrm{E}-03$ & $6.55 \mathrm{E}+01$ & $0.00 \mathrm{E}+00$ & $0.00 \mathrm{E}+00$ & $0.00 \mathrm{E}+00$ \\
\hline $\mathrm{Rn}-217$ & $5.40 \mathrm{E}-04$ & $\mathrm{~s}$ & $1.70 \mathrm{E}-14$ & $1.98 \mathrm{E}-03$ & $6.29 \mathrm{E}+01$ & $0.00 \mathrm{E}+00$ & $0.00 \mathrm{E}+00$ & $0.00 \mathrm{E}+00$ \\
\hline $\mathrm{Rn}-218$ & $3.50 \mathrm{E}-02$ & $\mathrm{~S}$ & $1.58 \mathrm{E}-14$ & $1.84 \mathrm{E}-03$ & $5.85 \mathrm{E}+01$ & $7.39 \mathrm{E}-20$ & $9.84 \mathrm{E}-13$ & $8.62 \mathrm{E}-15$ \\
\hline Rn-219 & 3.96 & $\mathrm{~s}$ & $1.48 \mathrm{E}-14$ & $1.73 \mathrm{E}-03$ & $5.48 \mathrm{E}+01$ & $5.60 \mathrm{E}-18$ & $7.46 \mathrm{E}-11$ & $6.53 \mathrm{E}-13$ \\
\hline $\mathrm{Rn}-220$ & 55.6 & $\mathrm{~S}$ & $1.38 \mathrm{E}-14$ & $1.61 \mathrm{E}-03$ & $5.11 \mathrm{E}+01$ & $6.10 \mathrm{E}-20$ & $8.13 \mathrm{E}-13$ & $7.12 \mathrm{E}-15$ \\
\hline $\mathrm{Rn}-222$ & 3.8235 & $\mathrm{~d}$ & $1.20 \mathrm{E}-14$ & $1.40 \mathrm{E}-03$ & $4.44 \mathrm{E}+01$ & $3.76 \mathrm{E}-20$ & $5.01 \mathrm{E}-13$ & $4.39 \mathrm{E}-15$ \\
\hline Rn-223 & 24.3 & $\mathrm{~m}$ & $1.56 \mathrm{E}-14$ & $1.82 \mathrm{E}-03$ & $5.77 \mathrm{E}+01$ & $3.35 \mathrm{E}-17$ & $4.46 \mathrm{E}-10$ & $3.91 \mathrm{E}-12$ \\
\hline Fr-212 & 20 & $\mathrm{~m}$ & $5.26 \mathrm{E}-14$ & $6.14 \mathrm{E}-03$ & $1.95 \mathrm{E}+02$ & $1.14 \mathrm{E}-16$ & $1.52 \mathrm{E}-09$ & $1.33 \mathrm{E}-11$ \\
\hline Fr-219 & $2.00 \mathrm{E}-02$ & $\mathrm{~s}$ & $1.57 \mathrm{E}-16$ & $1.83 \mathrm{E}-05$ & $5.81 \mathrm{E}-01$ & $3.41 \mathrm{E}-19$ & $4.54 \mathrm{E}-12$ & $3.98 \mathrm{E}-14$ \\
\hline Fr-220 & 27.4 & $\mathrm{~S}$ & $3.52 \mathrm{E}-16$ & $4.11 \mathrm{E}-05$ & $1.30 \mathrm{E}+00$ & $7.85 \mathrm{E}-19$ & $1.05 \mathrm{E}-11$ & $9.16 \mathrm{E}-14$ \\
\hline Fr-221 & 4.9 & $\mathrm{~m}$ & $1.25 \mathrm{E}-15$ & $1.46 \mathrm{E}-04$ & $4.63 \mathrm{E}+00$ & $2.75 \mathrm{E}-18$ & $3.66 \mathrm{E}-11$ & $3.21 \mathrm{E}-13$ \\
\hline Fr-222 & 14.2 & $\mathrm{~m}$ & $8.17 \mathrm{E}-15$ & $9.54 \mathrm{E}-04$ & $3.02 \mathrm{E}+01$ & $1.74 \mathrm{E}-17$ & $2.32 \mathrm{E}-10$ & $2.03 \mathrm{E}-12$ \\
\hline Fr-223 & 22 & $\mathrm{~m}$ & $2.15 \mathrm{E}-15$ & $2.51 \mathrm{E}-04$ & $7.96 \mathrm{E}+00$ & $4.55 \mathrm{E}-18$ & $6.06 \mathrm{E}-11$ & $5.31 \mathrm{E}-13$ \\
\hline Fr-224 & 3.33 & $\mathrm{~m}$ & $2.62 \mathrm{E}-14$ & $3.06 \mathrm{E}-03$ & $9.69 \mathrm{E}+01$ & $5.63 \mathrm{E}-17$ & $7.50 \mathrm{E}-10$ & $6.57 \mathrm{E}-12$ \\
\hline Fr-227 & 2.47 & $\mathrm{~m}$ & $2.00 \mathrm{E}-14$ & $2.34 \mathrm{E}-03$ & $7.40 \mathrm{E}+01$ & $4.32 \mathrm{E}-17$ & $5.75 \mathrm{E}-10$ & $5.04 \mathrm{E}-12$ \\
\hline Ra-219 & 10 & $\mathrm{~ms}$ & $7.37 \mathrm{E}-15$ & $8.61 \mathrm{E}-04$ & $2.73 \mathrm{E}+01$ & $1.61 \mathrm{E}-17$ & $2.14 \mathrm{E}-10$ & $1.88 \mathrm{E}-12$ \\
\hline Ra-220 & $1.79 \mathrm{E}-02$ & $\mathrm{~s}$ & $2.07 \mathrm{E}-16$ & $2.42 \mathrm{E}-05$ & $7.66 \mathrm{E}-01$ & $4.51 \mathrm{E}-19$ & $6.01 \mathrm{E}-12$ & $5.26 \mathrm{E}-14$ \\
\hline
\end{tabular}




\begin{tabular}{|c|c|c|c|c|c|c|c|c|}
\hline Nuclide & Half-life & Class & $\begin{array}{c}\text { Air } \\
\text { Immersion } \\
\text { Dose Rate } \\
\text { Coefficient } \\
\left(\mathrm{Sv} / \mathbf{s ~ p e r ~}^{\mathbf{3}}\right. \\
\left.\mathrm{Bq} / \mathbf{m}^{\mathbf{3}}\right)\end{array}$ & $\begin{array}{c}\text { Air } \\
\text { Immersion } \\
\text { Dose Rate } \\
\text { Coefficient } \\
\text { (mrem- } \\
\left.\text { m³ }^{3} / \mathrm{pCi}-\mathrm{yr}\right)\end{array}$ & $\begin{array}{c}\text { Air } \\
\text { Immersion } \\
\text { Effective } \\
\text { Dose Rate } \\
\text { (mrem/sec } \\
\text { per } \\
\mu \mathrm{Ci} / \mathrm{cm}^{3} \text { ) } \\
\end{array}$ & $\begin{array}{c}\text { Water } \\
\text { Submersion } \\
\text { Dose Rate } \\
\text { Coefficient } \\
\left(\mathbf{S v}-\mathbf{m}^{3} / \mathbf{B q}-\right. \\
\text { s) }\end{array}$ & $\begin{array}{c}\text { Water } \\
\text { Submersion } \\
\text { Dose Rate } \\
\text { Coefficient } \\
\text { (mrem- } \\
\left.\text { m³ }^{\mathbf{3}} / \mathbf{p C i}-\mathbf{h}\right)\end{array}$ & $\begin{array}{c}\text { Water } \\
\text { Submersion } \\
\text { Dose Rate } \\
\text { Coefficient } \\
(\text { mrem- } \\
\left.\text { m³ }^{3} / \mu \mathrm{Ci}-\mathrm{y}\right)\end{array}$ \\
\hline Ra-221 & 28 & $\mathrm{~S}$ & $1.47 \mathrm{E}-15$ & $1.72 \mathrm{E}-04$ & $5.44 \mathrm{E}+00$ & $3.24 \mathrm{E}-18$ & $4.32 \mathrm{E}-11$ & $3.78 \mathrm{E}-13$ \\
\hline Ra-222 & 38 & $\mathrm{~s}$ & $4.04 \mathrm{E}-16$ & $4.72 \mathrm{E}-05$ & $1.49 \mathrm{E}+00$ & $8.81 \mathrm{E}-19$ & $1.17 \mathrm{E}-11$ & $1.03 \mathrm{E}-13$ \\
\hline Ra-223 & 11.43 & $\mathrm{~d}$ & $5.74 \mathrm{E}-15$ & $6.70 \mathrm{E}-04$ & $2.12 \mathrm{E}+01$ & $1.27 \mathrm{E}-17$ & $1.69 \mathrm{E}-10$ & $1.48 \mathrm{E}-12$ \\
\hline Ra-224 & 3.66 & $\mathrm{~d}$ & $4.51 \mathrm{E}-16$ & $5.27 \mathrm{E}-05$ & $1.67 \mathrm{E}+00$ & $9.87 \mathrm{E}-19$ & $1.31 \mathrm{E}-11$ & $1.15 \mathrm{E}-13$ \\
\hline Ra-225 & 14.9 & $\mathrm{~d}$ & $2.47 \mathrm{E}-16$ & $2.88 \mathrm{E}-05$ & $9.14 \mathrm{E}-01$ & $5.43 \mathrm{E}-19$ & $7.23 \mathrm{E}-12$ & $6.34 \mathrm{E}-14$ \\
\hline Ra-226 & 1600 & $\mathrm{y}$ & $3.11 \mathrm{E}-16$ & $3.63 \mathrm{E}-05$ & $1.15 \mathrm{E}+00$ & $6.84 \mathrm{E}-19$ & $9.11 \mathrm{E}-12$ & $7.98 \mathrm{E}-14$ \\
\hline Ra-227 & 42.2 & $\mathrm{~m}$ & $6.37 \mathrm{E}-15$ & 7.44E-04 & $2.36 \mathrm{E}+01$ & $1.37 \mathrm{E}-17$ & $1.82 \mathrm{E}-10$ & $1.60 \mathrm{E}-12$ \\
\hline Ra-228 & 5.75 & $\mathrm{y}$ & $2.89 \mathrm{E}-18$ & $3.37 \mathrm{E}-07$ & $1.07 \mathrm{E}-02$ & $6.78 \mathrm{E}-21$ & $9.03 \mathrm{E}-14$ & $7.91 \mathrm{E}-16$ \\
\hline Ra-230 & 93 & $\mathrm{~m}$ & $3.33 \mathrm{E}-15$ & $3.89 \mathrm{E}-04$ & $1.23 \mathrm{E}+01$ & $7.23 \mathrm{E}-18$ & $9.63 \mathrm{E}-11$ & $8.44 \mathrm{E}-13$ \\
\hline Ac-223 & 2.1 & $\mathrm{~m}$ & $7.28 \mathrm{E}-16$ & $8.50 \mathrm{E}-05$ & $2.69 \mathrm{E}+00$ & $1.60 \mathrm{E}-18$ & $2.13 \mathrm{E}-11$ & $1.87 \mathrm{E}-13$ \\
\hline Ac-224 & 2.78 & $\mathrm{~h}$ & $9.33 \mathrm{E}-15$ & $1.09 \mathrm{E}-03$ & $3.45 \mathrm{E}+01$ & $2.06 \mathrm{E}-17$ & $2.74 \mathrm{E}-10$ & $2.40 \mathrm{E}-12$ \\
\hline Ac- 225 & 10 & $\mathrm{~d}$ & $5.66 \mathrm{E}-16$ & $6.61 \mathrm{E}-05$ & $2.09 \mathrm{E}+00$ & $1.26 \mathrm{E}-18$ & $1.68 \mathrm{E}-11$ & $1.47 \mathrm{E}-13$ \\
\hline Ac-226 & 29.37 & $\mathrm{~h}$ & $5.68 \mathrm{E}-15$ & $6.63 \mathrm{E}-04$ & $2.10 \mathrm{E}+01$ & $1.23 \mathrm{E}-17$ & $1.64 \mathrm{E}-10$ & $1.44 \mathrm{E}-12$ \\
\hline Ac-227 & 21.772 & $\mathrm{y}$ & $3.65 \mathrm{E}-18$ & $4.26 \mathrm{E}-07$ & $1.35 \mathrm{E}-02$ & $8.25 \mathrm{E}-21$ & $1.10 \mathrm{E}-13$ & $9.63 \mathrm{E}-16$ \\
\hline Ac-228 & 6.15 & $\mathrm{~h}$ & $4.01 \mathrm{E}-14$ & $4.68 \mathrm{E}-03$ & $1.48 \mathrm{E}+02$ & $8.67 \mathrm{E}-17$ & $1.15 \mathrm{E}-09$ & $1.01 \mathrm{E}-11$ \\
\hline Ac-230 & 122 & $\mathrm{~s}$ & $2.66 \mathrm{E}-14$ & $3.11 \mathrm{E}-03$ & $9.84 \mathrm{E}+01$ & $5.70 \mathrm{E}-17$ & $7.59 \mathrm{E}-10$ & $6.65 \mathrm{E}-12$ \\
\hline Ac-231 & 7.5 & $\mathrm{~m}$ & $1.84 \mathrm{E}-14$ & $2.15 \mathrm{E}-03$ & $6.81 \mathrm{E}+01$ & $3.98 \mathrm{E}-17$ & $5.30 \mathrm{E}-10$ & $4.64 \mathrm{E}-12$ \\
\hline Ac-232 & 119 & $\mathrm{~s}$ & $5.65 \mathrm{E}-14$ & $6.60 \mathrm{E}-03$ & $2.09 \mathrm{E}+02$ & $1.22 \mathrm{E}-16$ & $1.63 \mathrm{E}-09$ & $1.42 \mathrm{E}-11$ \\
\hline Ac-233 & 145 & $\mathrm{~s}$ & $2.29 \mathrm{E}-14$ & $2.67 \mathrm{E}-03$ & $8.47 \mathrm{E}+01$ & $4.92 \mathrm{E}-17$ & $6.55 \mathrm{E}-10$ & $5.74 \mathrm{E}-12$ \\
\hline Th-223 & 0.6 & $\mathrm{~s}$ & $2.78 \mathrm{E}-15$ & $3.25 \mathrm{E}-04$ & $1.03 \mathrm{E}+01$ & $6.18 \mathrm{E}-18$ & $8.23 \mathrm{E}-11$ & $7.21 \mathrm{E}-13$ \\
\hline Th-224 & 1.05 & $\mathrm{~s}$ & $9.75 \mathrm{E}-16$ & $1.14 \mathrm{E}-04$ & $3.61 \mathrm{E}+00$ & $2.14 \mathrm{E}-18$ & $2.85 \mathrm{E}-11$ & $2.50 \mathrm{E}-13$ \\
\hline Th-226 & 30.57 & $\mathrm{~m}$ & $3.21 \mathrm{E}-16$ & $3.75 \mathrm{E}-05$ & $1.19 \mathrm{E}+00$ & $7.08 \mathrm{E}-19$ & $9.43 \mathrm{E}-12$ & $8.26 \mathrm{E}-14$ \\
\hline Th-227 & 18.68 & $\mathrm{~d}$ & $5.22 \mathrm{E}-15$ & $6.10 \mathrm{E}-04$ & $1.93 \mathrm{E}+01$ & $1.15 \mathrm{E}-17$ & $1.53 \mathrm{E}-10$ & $1.34 \mathrm{E}-12$ \\
\hline
\end{tabular}




\begin{tabular}{|c|c|c|c|c|c|c|c|c|}
\hline Nuclide & Half-life & Class & $\begin{array}{c}\text { Air } \\
\text { Immersion } \\
\text { Dose Rate } \\
\text { Coefficient } \\
\left(\mathrm{Sv} / \mathbf{s ~ p e r ~}^{\mathbf{3}}\right. \\
\left.\mathrm{Bq} / \mathbf{m}^{\mathbf{3}}\right)\end{array}$ & $\begin{array}{c}\text { Air } \\
\text { Immersion } \\
\text { Dose Rate } \\
\text { Coefficient } \\
\text { (mrem- } \\
\left.\text { m³ }^{3} / \mathrm{pCi}-\mathrm{yr}\right)\end{array}$ & $\begin{array}{c}\text { Air } \\
\text { Immersion } \\
\text { Effective } \\
\text { Dose Rate } \\
\text { (mrem/sec } \\
\text { per } \\
\mu \mathrm{Ci} / \mathrm{cm}^{3} \text { ) } \\
\end{array}$ & $\begin{array}{c}\text { Water } \\
\text { Submersion } \\
\text { Dose Rate } \\
\text { Coefficient } \\
\left(\mathbf{S v}-\mathbf{m}^{3} / \mathbf{B q}-\right. \\
\text { s) }\end{array}$ & $\begin{array}{c}\text { Water } \\
\text { Submersion } \\
\text { Dose Rate } \\
\text { Coefficient } \\
\text { (mrem- } \\
\left.\text { m³ }^{\mathbf{3}} / \mathbf{p C i}-\mathbf{h}\right)\end{array}$ & $\begin{array}{c}\text { Water } \\
\text { Submersion } \\
\text { Dose Rate } \\
\text { Coefficient } \\
(\mathbf{m r e m -} \\
\left.\mathbf{m}^{\mathbf{3}} / \boldsymbol{\mu C i}-\mathbf{y}\right)\end{array}$ \\
\hline Th-228 & 1.9116 & $\mathrm{y}$ & $8.25 \mathrm{E}-17$ & $9.63 \mathrm{E}-06$ & $3.05 \mathrm{E}-01$ & $1.84 \mathrm{E}-19$ & $2.45 \mathrm{E}-12$ & $2.15 \mathrm{E}-14$ \\
\hline Th-229 & $7.34 \mathrm{E}+03$ & $\mathrm{y}$ & $3.32 \mathrm{E}-15$ & $3.88 \mathrm{E}-04$ & $1.23 \mathrm{E}+01$ & $7.40 \mathrm{E}-18$ & $9.86 \mathrm{E}-11$ & $8.63 \mathrm{E}-13$ \\
\hline Th-230 & $7.54 \mathrm{E}+04$ & $\mathrm{y}$ & $1.52 \mathrm{E}-17$ & $1.77 \mathrm{E}-06$ & $5.62 \mathrm{E}-02$ & $3.42 \mathrm{E}-20$ & $4.56 \mathrm{E}-13$ & $3.99 \mathrm{E}-15$ \\
\hline Th-231 & 25.52 & $\mathrm{~h}$ & $4.63 \mathrm{E}-16$ & $5.41 \mathrm{E}-05$ & $1.71 \mathrm{E}+00$ & $1.02 \mathrm{E}-18$ & $1.36 \mathrm{E}-11$ & $1.19 \mathrm{E}-13$ \\
\hline Th-232 & $1.41 \mathrm{E}+10$ & $\mathrm{y}$ & $7.90 \mathrm{E}-18$ & $9.22 \mathrm{E}-07$ & $2.92 \mathrm{E}-02$ & $1.80 \mathrm{E}-20$ & $2.40 \mathrm{E}-13$ & $2.10 \mathrm{E}-15$ \\
\hline Th-233 & 22.3 & $\mathrm{~m}$ & $1.79 \mathrm{E}-15$ & $2.09 \mathrm{E}-04$ & $6.62 \mathrm{E}+00$ & $3.63 \mathrm{E}-18$ & $4.84 \mathrm{E}-11$ & $4.24 \mathrm{E}-13$ \\
\hline Th-234 & 24.1 & $\mathrm{~d}$ & $3.22 \mathrm{E}-16$ & $3.76 \mathrm{E}-05$ & $1.19 \mathrm{E}+00$ & $7.22 \mathrm{E}-19$ & $9.62 \mathrm{E}-12$ & $8.42 \mathrm{E}-14$ \\
\hline Th-235 & 7.1 & $\mathrm{~m}$ & $2.93 \mathrm{E}-15$ & $3.42 \mathrm{E}-04$ & $1.08 \mathrm{E}+01$ & $5.86 \mathrm{E}-18$ & $7.81 \mathrm{E}-11$ & $6.84 \mathrm{E}-13$ \\
\hline Th-236 & 37.5 & $\mathrm{~m}$ & $1.68 \mathrm{E}-15$ & $1.96 \mathrm{E}-04$ & $6.22 \mathrm{E}+00$ & $3.44 \mathrm{E}-18$ & $4.58 \mathrm{E}-11$ & $4.01 \mathrm{E}-13$ \\
\hline $\mathrm{Pa}-227$ & 38.3 & $\mathrm{~m}$ & $7.18 \mathrm{E}-16$ & $8.38 \mathrm{E}-05$ & $2.66 \mathrm{E}+00$ & $1.61 \mathrm{E}-18$ & $2.14 \mathrm{E}-11$ & $1.88 \mathrm{E}-13$ \\
\hline $\mathrm{Pa}-228$ & 22 & $\mathrm{~h}$ & $6.21 \mathrm{E}-14$ & $7.25 \mathrm{E}-03$ & $2.30 \mathrm{E}+02$ & $1.35 \mathrm{E}-16$ & $1.80 \mathrm{E}-09$ & $1.58 \mathrm{E}-11$ \\
\hline Pa-229 & 1.5 & $\mathrm{~d}$ & $2.33 \mathrm{E}-15$ & $2.72 \mathrm{E}-04$ & $8.62 \mathrm{E}+00$ & $5.22 \mathrm{E}-18$ & $6.95 \mathrm{E}-11$ & $6.09 \mathrm{E}-13$ \\
\hline $\mathrm{Pa}-230$ & 17.4 & $\mathrm{~d}$ & $2.99 \mathrm{E}-14$ & $3.49 \mathrm{E}-03$ & $1.11 \mathrm{E}+02$ & $6.50 \mathrm{E}-17$ & $8.66 \mathrm{E}-10$ & $7.58 \mathrm{E}-12$ \\
\hline $\mathrm{Pa}-231$ & $3.28 \mathrm{E}+04$ & $\mathrm{y}$ & $1.45 \mathrm{E}-15$ & $1.69 \mathrm{E}-04$ & $5.37 \mathrm{E}+00$ & $3.18 \mathrm{E}-18$ & $4.24 \mathrm{E}-11$ & $3.71 \mathrm{E}-13$ \\
\hline $\mathrm{Pa}-232$ & 1.31 & $\mathrm{~d}$ & $4.26 \mathrm{E}-14$ & $4.97 \mathrm{E}-03$ & $1.58 \mathrm{E}+02$ & $9.25 \mathrm{E}-17$ & $1.23 \mathrm{E}-09$ & $1.08 \mathrm{E}-11$ \\
\hline $\mathrm{Pa}-233$ & 26.967 & $\mathrm{~d}$ & $9.27 \mathrm{E}-15$ & $1.08 \mathrm{E}-03$ & $3.43 \mathrm{E}+01$ & $2.03 \mathrm{E}-17$ & $2.70 \mathrm{E}-10$ & $2.37 \mathrm{E}-12$ \\
\hline $\mathrm{Pa}-234$ & 6.7 & $\mathrm{~h}$ & $6.67 \mathrm{E}-14$ & $7.79 \mathrm{E}-03$ & $2.47 \mathrm{E}+02$ & $1.45 \mathrm{E}-16$ & $1.93 \mathrm{E}-09$ & $1.69 \mathrm{E}-11$ \\
\hline $\mathrm{Pa}-234 \mathrm{~m}$ & 1.17 & $\mathrm{~m}$ & $1.42 \mathrm{E}-15$ & $1.66 \mathrm{E}-04$ & $5.25 \mathrm{E}+00$ & $2.45 \mathrm{E}-18$ & $3.26 \mathrm{E}-11$ & $2.86 \mathrm{E}-13$ \\
\hline $\mathrm{Pa}-235$ & 24.5 & $\mathrm{~m}$ & $3.35 \mathrm{E}-16$ & $3.91 \mathrm{E}-05$ & $1.24 \mathrm{E}+00$ & $3.93 \mathrm{E}-19$ & $5.23 \mathrm{E}-12$ & $4.59 \mathrm{E}-14$ \\
\hline $\mathrm{Pa}-236$ & 9.1 & $\mathrm{~m}$ & $4.36 \mathrm{E}-14$ & $5.09 \mathrm{E}-03$ & $1.61 \mathrm{E}+02$ & $9.38 \mathrm{E}-17$ & $1.25 \mathrm{E}-09$ & $1.09 \mathrm{E}-11$ \\
\hline $\mathrm{Pa}-237$ & 8.7 & $\mathrm{~m}$ & $2.82 \mathrm{E}-14$ & $3.29 \mathrm{E}-03$ & $1.04 \mathrm{E}+02$ & $6.07 \mathrm{E}-17$ & $8.09 \mathrm{E}-10$ & $7.08 \mathrm{E}-12$ \\
\hline U-227 & 1.1 & $\mathrm{~m}$ & $4.85 \mathrm{E}-15$ & $5.66 \mathrm{E}-04$ & $1.79 \mathrm{E}+01$ & $1.07 \mathrm{E}-17$ & $1.43 \mathrm{E}-10$ & $1.25 \mathrm{E}-12$ \\
\hline U-228 & 9.1 & $\mathrm{~m}$ & $1.59 \mathrm{E}-16$ & $1.86 \mathrm{E}-05$ & $5.88 \mathrm{E}-01$ & $3.53 \mathrm{E}-19$ & $4.70 \mathrm{E}-12$ & $4.12 \mathrm{E}-14$ \\
\hline
\end{tabular}




\begin{tabular}{|c|c|c|c|c|c|c|c|c|}
\hline Nuclide & Half-life & Class & $\begin{array}{c}\text { Air } \\
\text { Immersion } \\
\text { Dose Rate } \\
\text { Coefficient } \\
\left(\mathrm{Sv} / \mathbf{s ~ p e r ~}^{\mathbf{3}}\right. \\
\left.\mathrm{Bq} / \mathbf{m}^{\mathbf{3}}\right)\end{array}$ & $\begin{array}{c}\text { Air } \\
\text { Immersion } \\
\text { Dose Rate } \\
\text { Coefficient } \\
\text { (mrem- } \\
\left.\text { m³ }^{3} / \mathrm{pCi}-\mathrm{yr}\right)\end{array}$ & $\begin{array}{c}\text { Air } \\
\text { Immersion } \\
\text { Effective } \\
\text { Dose Rate } \\
\text { (mrem/sec } \\
\text { per } \\
\mu \mathrm{Ci} / \mathbf{c m}^{3} \text { ) }\end{array}$ & $\begin{array}{c}\text { Water } \\
\text { Submersion } \\
\text { Dose Rate } \\
\text { Coefficient } \\
\left(\mathbf{S v}-\mathbf{m}^{3} / \mathbf{B q}-\right. \\
\text { s) }\end{array}$ & $\begin{array}{c}\text { Water } \\
\text { Submersion } \\
\text { Dose Rate } \\
\text { Coefficient } \\
\text { (mrem- } \\
\left.\text { m³ }^{\mathbf{3}} / \mathbf{p C i}-\mathbf{h}\right)\end{array}$ & $\begin{array}{c}\text { Water } \\
\text { Submersion } \\
\text { Dose Rate } \\
\text { Coefficient } \\
(\text { mrem- } \\
\left.\mathbf{m}^{3} / \mu \mathrm{Ci}-\mathrm{y}\right)\end{array}$ \\
\hline U-230 & 20.8 & $\mathrm{~d}$ & $4.56 \mathrm{E}-17$ & $5.32 \mathrm{E}-06$ & $1.69 \mathrm{E}-01$ & $1.02 \mathrm{E}-19$ & $1.36 \mathrm{E}-12$ & $1.19 \mathrm{E}-14$ \\
\hline U-231 & 4.2 & $\mathrm{~d}$ & $2.66 \mathrm{E}-15$ & $3.11 \mathrm{E}-04$ & $9.84 \mathrm{E}+00$ & $5.96 \mathrm{E}-18$ & $7.94 \mathrm{E}-11$ & $6.95 \mathrm{E}-13$ \\
\hline U-232 & 68.9 & $\mathrm{y}$ & $1.08 \mathrm{E}-17$ & $1.26 \mathrm{E}-06$ & $4.00 \mathrm{E}-02$ & $2.43 \mathrm{E}-20$ & $3.24 \mathrm{E}-13$ & $2.84 \mathrm{E}-15$ \\
\hline U-233 & $1.59 \mathrm{E}+05$ & $\mathrm{y}$ & $1.06 \mathrm{E}-17$ & $1.24 \mathrm{E}-06$ & $3.92 \mathrm{E}-02$ & $2.35 \mathrm{E}-20$ & $3.13 \mathrm{E}-13$ & $2.74 \mathrm{E}-15$ \\
\hline U-234 & $2.46 \mathrm{E}+05$ & $\mathrm{y}$ & $6.14 \mathrm{E}-18$ & $7.17 \mathrm{E}-07$ & $2.27 \mathrm{E}-02$ & $1.40 \mathrm{E}-20$ & $1.86 \mathrm{E}-13$ & $1.63 \mathrm{E}-15$ \\
\hline U-235 & $7.04 \mathrm{E}+08$ & $\mathrm{y}$ & $6.87 \mathrm{E}-15$ & $8.02 \mathrm{E}-04$ & $2.54 \mathrm{E}+01$ & $1.51 \mathrm{E}-17$ & $2.01 \mathrm{E}-10$ & $1.76 \mathrm{E}-12$ \\
\hline U-235m & 26 & $\mathrm{~m}$ & $0.00 \mathrm{E}+00$ & $0.00 \mathrm{E}+00$ & $0.00 \mathrm{E}+00$ & $0.00 \mathrm{E}+00$ & $0.00 \mathrm{E}+00$ & $0.00 \mathrm{E}+00$ \\
\hline U-236 & $2.34 \mathrm{E}+07$ & $\mathrm{y}$ & $3.78 \mathrm{E}-18$ & $4.41 \mathrm{E}-07$ & $1.40 \mathrm{E}-02$ & $8.69 \mathrm{E}-21$ & $1.16 \mathrm{E}-13$ & $1.01 \mathrm{E}-15$ \\
\hline U-237 & 6.75 & $\mathrm{~d}$ & $5.28 \mathrm{E}-15$ & $6.17 \mathrm{E}-04$ & $1.95 \mathrm{E}+01$ & $1.17 \mathrm{E}-17$ & $1.56 \mathrm{E}-10$ & $1.37 \mathrm{E}-12$ \\
\hline U-238 & $4.47 \mathrm{E}+09$ & $\mathrm{y}$ & $3.20 \mathrm{E}-18$ & $3.74 \mathrm{E}-07$ & $1.18 \mathrm{E}-02$ & $7.32 \mathrm{E}-21$ & $9.75 \mathrm{E}-14$ & $8.54 \mathrm{E}-16$ \\
\hline U-239 & 23.45 & $\mathrm{~m}$ & $2.10 \mathrm{E}-15$ & $2.45 \mathrm{E}-04$ & $7.77 \mathrm{E}+00$ & $4.41 \mathrm{E}-18$ & $5.87 \mathrm{E}-11$ & $5.15 \mathrm{E}-13$ \\
\hline U-240 & 14.1 & $\mathrm{~h}$ & $2.07 \mathrm{E}-16$ & $2.42 \mathrm{E}-05$ & $7.66 \mathrm{E}-01$ & $4.30 \mathrm{E}-19$ & $5.73 \mathrm{E}-12$ & $5.02 \mathrm{E}-14$ \\
\hline U-242 & 16.8 & $\mathrm{~m}$ & $1.96 \mathrm{E}-15$ & $2.29 \mathrm{E}-04$ & $7.25 \mathrm{E}+00$ & $4.03 \mathrm{E}-18$ & $5.37 \mathrm{E}-11$ & $4.70 \mathrm{E}-13$ \\
\hline $\mathrm{Np}-232$ & 14.7 & $\mathrm{~m}$ & $5.35 \mathrm{E}-14$ & $6.25 \mathrm{E}-03$ & $1.98 \mathrm{E}+02$ & $1.16 \mathrm{E}-16$ & $1.55 \mathrm{E}-09$ & $1.35 \mathrm{E}-11$ \\
\hline $\mathrm{Np}-233$ & 36.2 & $\mathrm{~m}$ & $3.38 \mathrm{E}-15$ & $3.95 \mathrm{E}-04$ & $1.25 \mathrm{E}+01$ & $7.52 \mathrm{E}-18$ & $1.00 \mathrm{E}-10$ & $8.77 \mathrm{E}-13$ \\
\hline $\mathrm{Np}-234$ & 4.4 & $\mathrm{~d}$ & $5.23 \mathrm{E}-14$ & $6.11 \mathrm{E}-03$ & $1.94 \mathrm{E}+02$ & $1.13 \mathrm{E}-16$ & $1.51 \mathrm{E}-09$ & $1.32 \mathrm{E}-11$ \\
\hline $\mathrm{Np}-235$ & 396.1 & $\mathrm{~d}$ & $2.84 \mathrm{E}-17$ & $3.32 \mathrm{E}-06$ & $1.05 \mathrm{E}-01$ & $6.43 \mathrm{E}-20$ & $8.56 \mathrm{E}-13$ & $7.50 \mathrm{E}-15$ \\
\hline $\mathrm{Np}-236$ & $1.54 \mathrm{E}+05$ & $\mathrm{y}$ & $5.57 \mathrm{E}-15$ & $6.50 \mathrm{E}-04$ & $2.06 \mathrm{E}+01$ & $1.23 \mathrm{E}-17$ & $1.64 \mathrm{E}-10$ & $1.44 \mathrm{E}-12$ \\
\hline $\mathrm{Np}-236 \mathrm{~m}$ & 22.5 & $\mathrm{~h}$ & $1.90 \mathrm{E}-15$ & $2.22 \mathrm{E}-04$ & $7.03 \mathrm{E}+00$ & $4.18 \mathrm{E}-18$ & $5.57 \mathrm{E}-11$ & $4.88 \mathrm{E}-13$ \\
\hline $\mathrm{Np}-237$ & $2.14 \mathrm{E}+06$ & $\mathrm{y}$ & $8.60 \mathrm{E}-16$ & $1.00 \mathrm{E}-04$ & $3.18 \mathrm{E}+00$ & $1.93 \mathrm{E}-18$ & $2.57 \mathrm{E}-11$ & $2.25 \mathrm{E}-13$ \\
\hline $\mathrm{Np}-238$ & 2.117 & $\mathrm{~d}$ & $2.72 \mathrm{E}-14$ & $3.18 \mathrm{E}-03$ & $1.01 \mathrm{E}+02$ & $5.88 \mathrm{E}-17$ & $7.83 \mathrm{E}-10$ & $6.86 \mathrm{E}-12$ \\
\hline $\mathrm{Np}-239$ & 2.3565 & $\mathrm{~d}$ & $7.35 \mathrm{E}-15$ & $8.58 \mathrm{E}-04$ & $2.72 \mathrm{E}+01$ & $1.62 \mathrm{E}-17$ & $2.16 \mathrm{E}-10$ & $1.89 \mathrm{E}-12$ \\
\hline $\mathrm{Np}-240$ & 61.9 & $\mathrm{~m}$ & $4.73 \mathrm{E}-14$ & $5.52 \mathrm{E}-03$ & $1.75 \mathrm{E}+02$ & $1.03 \mathrm{E}-16$ & $1.37 \mathrm{E}-09$ & $1.20 \mathrm{E}-11$ \\
\hline
\end{tabular}




\begin{tabular}{|c|c|c|c|c|c|c|c|c|}
\hline Nuclide & Half-life & Class & $\begin{array}{c}\text { Air } \\
\text { Immersion } \\
\text { Dose Rate } \\
\text { Coefficient } \\
(\text { Sv/s per } \\
\left.\text { Bq/ } \mathbf{m}^{3}\right)\end{array}$ & $\begin{array}{c}\text { Air } \\
\text { Immersion } \\
\text { Dose Rate } \\
\text { Coefficient } \\
\text { (mrem- } \\
\text { m³Ci-yr) }^{3} / \mathrm{pCi}\end{array}$ & $\begin{array}{c}\text { Air } \\
\text { Immersion } \\
\text { Effective } \\
\text { Dose Rate } \\
(\mathrm{mrem} / \mathrm{sec} \\
\mathrm{per} \\
\left.\mu \mathrm{Ci} / \mathrm{cm}^{3}\right)\end{array}$ & $\begin{array}{c}\text { Water } \\
\text { Submersion } \\
\text { Dose Rate } \\
\text { Coefficient } \\
\left(\mathrm{Sv}-\mathbf{m}^{3} / \mathbf{B q}-\right. \\
\text { s) }\end{array}$ & $\begin{array}{c}\text { Water } \\
\text { Submersion } \\
\text { Dose Rate } \\
\text { Coefficient } \\
\text { (mrem- } \\
\text { m³Ci-h) }^{\mathbf{3} / p C i}\end{array}$ & $\begin{array}{c}\text { Water } \\
\text { Submersion } \\
\text { Dose Rate } \\
\text { Coefficient } \\
(\text { mrem- } \\
\left.\text { m }^{3} / \mu \mathrm{Ci}-\mathrm{y}\right)\end{array}$ \\
\hline $\mathrm{Np}-240 \mathrm{~m}$ & 7.22 & $\mathrm{~m}$ & $1.49 \mathrm{E}-14$ & $1.74 \mathrm{E}-03$ & $5.51 \mathrm{E}+01$ & $3.20 \mathrm{E}-17$ & $4.26 \mathrm{E}-10$ & $3.73 \mathrm{E}-12$ \\
\hline $\mathrm{Np}-241$ & 13.9 & $\mathrm{~m}$ & $1.79 \mathrm{E}-15$ & $2.09 \mathrm{E}-04$ & $6.62 \mathrm{E}+00$ & $3.67 \mathrm{E}-18$ & $4.89 \mathrm{E}-11$ & $4.28 \mathrm{E}-13$ \\
\hline $\mathrm{Np}-242$ & 2.2 & $\mathrm{~m}$ & $1.33 \mathrm{E}-14$ & $1.55 \mathrm{E}-03$ & $4.92 \mathrm{E}+01$ & $2.82 \mathrm{E}-17$ & $3.76 \mathrm{E}-10$ & $3.29 \mathrm{E}-12$ \\
\hline $\mathrm{Np}-242 \mathrm{~m}$ & 5.5 & $\mathrm{~m}$ & $4.16 \mathrm{E}-14$ & $4.86 \mathrm{E}-03$ & $1.54 \mathrm{E}+02$ & $8.99 \mathrm{E}-17$ & $1.20 \mathrm{E}-09$ & $1.05 \mathrm{E}-11$ \\
\hline $\mathrm{Pu}-232$ & 33.7 & $\mathrm{~m}$ & $2.31 \mathrm{E}-15$ & $2.70 \mathrm{E}-04$ & $8.55 \mathrm{E}+00$ & $5.16 \mathrm{E}-18$ & $6.87 \mathrm{E}-11$ & $6.02 \mathrm{E}-13$ \\
\hline $\mathrm{Pu}-234$ & 8.8 & $\mathrm{~h}$ & $2.50 \mathrm{E}-15$ & $2.92 \mathrm{E}-04$ & $9.25 \mathrm{E}+00$ & $5.58 \mathrm{E}-18$ & $7.43 \mathrm{E}-11$ & $6.51 \mathrm{E}-13$ \\
\hline $\mathrm{Pu}-235$ & 25.3 & $\mathrm{~m}$ & $3.46 \mathrm{E}-15$ & $4.04 \mathrm{E}-04$ & $1.28 \mathrm{E}+01$ & $7.70 \mathrm{E}-18$ & $1.03 \mathrm{E}-10$ & $8.98 \mathrm{E}-13$ \\
\hline $\mathrm{Pu}-236$ & 2.858 & $\mathrm{y}$ & $4.33 \mathrm{E}-18$ & $5.06 \mathrm{E}-07$ & $1.60 \mathrm{E}-02$ & $1.00 \mathrm{E}-20$ & $1.33 \mathrm{E}-13$ & $1.17 \mathrm{E}-15$ \\
\hline $\mathrm{Pu}-237$ & 45.2 & $\mathrm{~d}$ & $1.79 \mathrm{E}-15$ & $2.09 \mathrm{E}-04$ & $6.62 \mathrm{E}+00$ & $4.00 \mathrm{E}-18$ & $5.33 \mathrm{E}-11$ & $4.67 \mathrm{E}-13$ \\
\hline $\mathrm{Pu}-238$ & 87.7 & $\mathrm{y}$ & $3.36 \mathrm{E}-18$ & $3.92 \mathrm{E}-07$ & $1.24 \mathrm{E}-02$ & $7.81 \mathrm{E}-21$ & $1.04 \mathrm{E}-13$ & $9.11 \mathrm{E}-16$ \\
\hline $\mathrm{Pu}-239$ & $2.41 \mathrm{E}+04$ & $\mathrm{y}$ & $3.77 \mathrm{E}-18$ & $4.40 \mathrm{E}-07$ & $1.39 \mathrm{E}-02$ & $8.51 \mathrm{E}-21$ & $1.13 \mathrm{E}-13$ & $9.93 \mathrm{E}-16$ \\
\hline $\mathrm{Pu}-240$ & 6564 & $\mathrm{y}$ & $3.29 \mathrm{E}-18$ & $3.84 \mathrm{E}-07$ & $1.22 \mathrm{E}-02$ & $7.66 \mathrm{E}-21$ & $1.02 \mathrm{E}-13$ & $8.94 \mathrm{E}-16$ \\
\hline $\mathrm{Pu}-241$ & 14.35 & $\mathrm{y}$ & $6.15 \mathrm{E}-20$ & $7.18 \mathrm{E}-09$ & $2.28 \mathrm{E}-04$ & $1.37 \mathrm{E}-22$ & $1.82 \mathrm{E}-15$ & $1.60 \mathrm{E}-17$ \\
\hline $\mathrm{Pu}-242$ & $3.75 \mathrm{E}+05$ & $\mathrm{y}$ & $6.43 \mathrm{E}-18$ & $7.51 \mathrm{E}-07$ & $2.38 \mathrm{E}-02$ & $1.43 \mathrm{E}-20$ & $1.90 \mathrm{E}-13$ & $1.67 \mathrm{E}-15$ \\
\hline $\mathrm{Pu}-243$ & 4.956 & $\mathrm{~h}$ & $9.65 \mathrm{E}-16$ & $1.13 \mathrm{E}-04$ & $3.57 \mathrm{E}+00$ & $2.08 \mathrm{E}-18$ & $2.77 \mathrm{E}-11$ & $2.43 \mathrm{E}-13$ \\
\hline $\mathrm{Pu}-244$ & $8.00 \mathrm{E}+07$ & $\mathrm{y}$ & $9.70 \mathrm{E}-16$ & $1.13 \mathrm{E}-04$ & $3.59 \mathrm{E}+00$ & $2.09 \mathrm{E}-18$ & $2.78 \mathrm{E}-11$ & $2.44 \mathrm{E}-13$ \\
\hline $\mathrm{Pu}-245$ & 10.5 & $\mathrm{~h}$ & $1.81 \mathrm{E}-14$ & $2.11 \mathrm{E}-03$ & $6.70 \mathrm{E}+01$ & $3.92 \mathrm{E}-17$ & $5.22 \mathrm{E}-10$ & $4.57 \mathrm{E}-12$ \\
\hline $\mathrm{Pu}-246$ & 10.84 & $\mathrm{~d}$ & $5.43 \mathrm{E}-15$ & $6.34 \mathrm{E}-04$ & $2.01 \mathrm{E}+01$ & $1.20 \mathrm{E}-17$ & $1.60 \mathrm{E}-10$ & $1.40 \mathrm{E}-12$ \\
\hline Am-237 & 73 & $\mathrm{~m}$ & $1.55 \mathrm{E}-14$ & $1.81 \mathrm{E}-03$ & $5.74 \mathrm{E}+01$ & $3.41 \mathrm{E}-17$ & $4.54 \mathrm{E}-10$ & $3.98 \mathrm{E}-12$ \\
\hline Am-238 & 98 & $\mathrm{~m}$ & $4.09 \mathrm{E}-14$ & $4.78 \mathrm{E}-03$ & $1.51 \mathrm{E}+02$ & $8.88 \mathrm{E}-17$ & $1.18 \mathrm{E}-09$ & $1.04 \mathrm{E}-11$ \\
\hline Am-239 & 11.9 & $\mathrm{~h}$ & $9.37 \mathrm{E}-15$ & $1.09 \mathrm{E}-03$ & $3.47 \mathrm{E}+01$ & $2.07 \mathrm{E}-17$ & $2.76 \mathrm{E}-10$ & $2.42 \mathrm{E}-12$ \\
\hline Am-240 & 50.8 & $\mathrm{~h}$ & $4.69 \mathrm{E}-14$ & $5.48 \mathrm{E}-03$ & $1.74 \mathrm{E}+02$ & $1.02 \mathrm{E}-16$ & $1.36 \mathrm{E}-09$ & $1.19 \mathrm{E}-11$ \\
\hline Am-241 & 432.2 & $\mathrm{y}$ & $6.72 \mathrm{E}-16$ & $7.85 \mathrm{E}-05$ & $2.49 \mathrm{E}+00$ & $1.54 \mathrm{E}-18$ & $2.05 \mathrm{E}-11$ & $1.80 \mathrm{E}-13$ \\
\hline
\end{tabular}




\begin{tabular}{|c|c|c|c|c|c|c|c|c|}
\hline Nuclide & Half-life & Class & $\begin{array}{c}\text { Air } \\
\text { Immersion } \\
\text { Dose Rate } \\
\text { Coefficient } \\
\left(\mathrm{Sv} / \mathbf{s ~ p e r ~}^{\mathbf{3}}\right. \\
\left.\mathrm{Bq} / \mathbf{m}^{\mathbf{3}}\right)\end{array}$ & $\begin{array}{c}\text { Air } \\
\text { Immersion } \\
\text { Dose Rate } \\
\text { Coefficient } \\
\text { (mrem- } \\
\left.\text { m³ }^{3} / \mathrm{pCi}-\mathrm{yr}\right)\end{array}$ & $\begin{array}{c}\text { Air } \\
\text { Immersion } \\
\text { Effective } \\
\text { Dose Rate } \\
\text { (mrem/sec } \\
\text { per } \\
\mu \mathrm{Ci} / \mathrm{cm}^{3} \text { ) } \\
\end{array}$ & $\begin{array}{c}\text { Water } \\
\text { Submersion } \\
\text { Dose Rate } \\
\text { Coefficient } \\
\left(\mathbf{S v}-\mathbf{m}^{3} / \mathbf{B q}-\right. \\
\text { s) }\end{array}$ & $\begin{array}{c}\text { Water } \\
\text { Submersion } \\
\text { Dose Rate } \\
\text { Coefficient } \\
\text { (mrem- } \\
\left.\text { m³ }^{\mathbf{3}} / \mathbf{p C i}-\mathbf{h}\right)\end{array}$ & $\begin{array}{c}\text { Water } \\
\text { Submersion } \\
\text { Dose Rate } \\
\text { Coefficient } \\
(\text { mrem- } \\
\left.\mathbf{m}^{3} / \mu \mathrm{Ci}-\mathrm{y}\right)\end{array}$ \\
\hline Am-242 & 16.02 & $\mathrm{~h}$ & $6.11 \mathrm{E}-16$ & $7.13 \mathrm{E}-05$ & $2.26 \mathrm{E}+00$ & $1.27 \mathrm{E}-18$ & $1.69 \mathrm{E}-11$ & $1.48 \mathrm{E}-13$ \\
\hline Am-242m & 141 & $\mathrm{y}$ & $1.98 \mathrm{E}-17$ & $2.31 \mathrm{E}-06$ & $7.33 \mathrm{E}-02$ & $4.53 \mathrm{E}-20$ & $6.03 \mathrm{E}-13$ & $5.29 \mathrm{E}-15$ \\
\hline Am-243 & $7.37 \mathrm{E}+03$ & $\mathrm{y}$ & $1.92 \mathrm{E}-15$ & $2.24 \mathrm{E}-04$ & $7.10 \mathrm{E}+00$ & $4.34 \mathrm{E}-18$ & $5.78 \mathrm{E}-11$ & $5.06 \mathrm{E}-13$ \\
\hline Am-244 & 10.1 & $\mathrm{~h}$ & $3.58 \mathrm{E}-14$ & $4.18 \mathrm{E}-03$ & $1.32 \mathrm{E}+02$ & $7.77 \mathrm{E}-17$ & $1.03 \mathrm{E}-09$ & $9.07 \mathrm{E}-12$ \\
\hline Am-244m & 26 & $\mathrm{~m}$ & $1.04 \mathrm{E}-15$ & $1.21 \mathrm{E}-04$ & $3.85 \mathrm{E}+00$ & $1.89 \mathrm{E}-18$ & $2.52 \mathrm{E}-11$ & $2.21 \mathrm{E}-13$ \\
\hline Am-245 & 2.05 & $\mathrm{~h}$ & $1.45 \mathrm{E}-15$ & $1.69 \mathrm{E}-04$ & $5.37 \mathrm{E}+00$ & $3.03 \mathrm{E}-18$ & $4.04 \mathrm{E}-11$ & $3.54 \mathrm{E}-13$ \\
\hline Am-246 & 39 & $\mathrm{~m}$ & $3.27 \mathrm{E}-14$ & $3.82 \mathrm{E}-03$ & $1.21 \mathrm{E}+02$ & $7.09 \mathrm{E}-17$ & $9.44 \mathrm{E}-10$ & $8.27 \mathrm{E}-12$ \\
\hline Am-246m & 25 & $\mathrm{~m}$ & $4.57 \mathrm{E}-14$ & $5.34 \mathrm{E}-03$ & $1.69 \mathrm{E}+02$ & $9.86 \mathrm{E}-17$ & $1.31 \mathrm{E}-09$ & $1.15 \mathrm{E}-11$ \\
\hline Am-247 & 23 & $\mathrm{~m}$ & $5.85 \mathrm{E}-15$ & $6.83 \mathrm{E}-04$ & $2.16 \mathrm{E}+01$ & $1.25 \mathrm{E}-17$ & $1.67 \mathrm{E}-10$ & $1.46 \mathrm{E}-12$ \\
\hline $\mathrm{Cm}-238$ & 2.4 & $\mathrm{~h}$ & $3.05 \mathrm{E}-15$ & $3.56 \mathrm{E}-04$ & $1.13 \mathrm{E}+01$ & $6.80 \mathrm{E}-18$ & $9.06 \mathrm{E}-11$ & $7.93 \mathrm{E}-13$ \\
\hline Cm-239 & 2.9 & $\mathrm{~h}$ & $1.05 \mathrm{E}-14$ & $1.23 \mathrm{E}-03$ & $3.89 \mathrm{E}+01$ & $2.32 \mathrm{E}-17$ & $3.09 \mathrm{E}-10$ & $2.71 \mathrm{E}-12$ \\
\hline $\mathrm{Cm}-240$ & 27 & $\mathrm{~d}$ & $4.54 \mathrm{E}-18$ & $5.30 \mathrm{E}-07$ & $1.68 \mathrm{E}-02$ & $1.06 \mathrm{E}-20$ & $1.41 \mathrm{E}-13$ & $1.24 \mathrm{E}-15$ \\
\hline $\mathrm{Cm}-241$ & 32.8 & $\mathrm{~d}$ & $2.12 \mathrm{E}-14$ & $2.48 \mathrm{E}-03$ & $7.84 \mathrm{E}+01$ & $4.63 \mathrm{E}-17$ & $6.17 \mathrm{E}-10$ & $5.40 \mathrm{E}-12$ \\
\hline $\mathrm{Cm}-242$ & 162.8 & $\mathrm{~d}$ & $3.90 \mathrm{E}-18$ & $4.55 \mathrm{E}-07$ & $1.44 \mathrm{E}-02$ & $9.09 \mathrm{E}-21$ & $1.21 \mathrm{E}-13$ & $1.06 \mathrm{E}-15$ \\
\hline $\mathrm{Cm}-243$ & 29.1 & $\mathrm{y}$ & $5.33 \mathrm{E}-15$ & $6.22 \mathrm{E}-04$ & $1.97 \mathrm{E}+01$ & $1.17 \mathrm{E}-17$ & $1.56 \mathrm{E}-10$ & $1.37 \mathrm{E}-12$ \\
\hline $\mathrm{Cm}-244$ & 18.1 & $\mathrm{y}$ & $4.00 \mathrm{E}-18$ & $4.67 \mathrm{E}-07$ & $1.48 \mathrm{E}-02$ & $9.22 \mathrm{E}-21$ & $1.23 \mathrm{E}-13$ & $1.08 \mathrm{E}-15$ \\
\hline Cm-245 & $8.50 \mathrm{E}+03$ & $\mathrm{y}$ & $4.00 \mathrm{E}-15$ & $4.67 \mathrm{E}-04$ & $1.48 \mathrm{E}+01$ & $8.90 \mathrm{E}-18$ & $1.19 \mathrm{E}-10$ & $1.04 \mathrm{E}-12$ \\
\hline $\mathrm{Cm}-246$ & $4.76 \mathrm{E}+03$ & $\mathrm{y}$ & $1.79 \mathrm{E}-16$ & $2.09 \mathrm{E}-05$ & $6.62 \mathrm{E}-01$ & $3.87 \mathrm{E}-19$ & $5.15 \mathrm{E}-12$ & $4.52 \mathrm{E}-14$ \\
\hline $\mathrm{Cm}-247$ & $1.56 \mathrm{E}+07$ & $\mathrm{y}$ & $1.38 \mathrm{E}-14$ & $1.61 \mathrm{E}-03$ & $5.11 \mathrm{E}+01$ & $3.01 \mathrm{E}-17$ & $4.01 \mathrm{E}-10$ & $3.51 \mathrm{E}-12$ \\
\hline $\mathrm{Cm}-248$ & $3.48 \mathrm{E}+05$ & $\mathrm{y}$ & $6.41 \mathrm{E}-14$ & $7.48 \mathrm{E}-03$ & $2.37 \mathrm{E}+02$ & $1.39 \mathrm{E}-16$ & $1.85 \mathrm{E}-09$ & $1.62 \mathrm{E}-11$ \\
\hline Cm-249 & 64.15 & $\mathrm{~m}$ & $1.02 \mathrm{E}-15$ & $1.19 \mathrm{E}-04$ & $3.77 \mathrm{E}+00$ & $2.04 \mathrm{E}-18$ & $2.72 \mathrm{E}-11$ & $2.38 \mathrm{E}-13$ \\
\hline $\mathrm{Cm}-250$ & 8300 & $\mathrm{y}$ & $6.52 \mathrm{E}-13$ & $7.61 \mathrm{E}-02$ & $2.41 \mathrm{E}+03$ & $1.41 \mathrm{E}-15$ & $1.88 \mathrm{E}-08$ & $1.65 \mathrm{E}-10$ \\
\hline $\mathrm{Cm}-251$ & 16.8 & $\mathrm{~m}$ & $5.20 \mathrm{E}-15$ & $6.07 \mathrm{E}-04$ & $1.92 \mathrm{E}+01$ & $1.10 \mathrm{E}-17$ & $1.47 \mathrm{E}-10$ & $1.28 \mathrm{E}-12$ \\
\hline
\end{tabular}




\begin{tabular}{|c|c|c|c|c|c|c|c|c|}
\hline Nuclide & Half-life & Class & $\begin{array}{c}\text { Air } \\
\text { Immersion } \\
\text { Dose Rate } \\
\text { Coefficient } \\
\text { (Sv/s per } \\
\left.\text { Bq } / \mathbf{m}^{\mathbf{3}}\right)\end{array}$ & $\begin{array}{c}\text { Air } \\
\text { Immersion } \\
\text { Dose Rate } \\
\text { Coefficient } \\
\text { (mrem- } \\
\left.\mathrm{m}^{3} / \mathrm{pCi}-\mathrm{yr}\right)\end{array}$ & $\begin{array}{c}\text { Air } \\
\text { Immersion } \\
\text { Effective } \\
\text { Dose Rate } \\
(\mathrm{mrem} / \mathrm{sec} \\
\text { per } \\
\left.\mu \mathrm{Ci} / \mathrm{cm}^{3}\right)\end{array}$ & $\begin{array}{c}\text { Water } \\
\text { Submersion } \\
\text { Dose Rate } \\
\text { Coefficient } \\
\left(\mathbf{S v}-\mathbf{m}^{3} / \mathbf{B q}-\right. \\
\text { s) }\end{array}$ & $\begin{array}{c}\text { Water } \\
\text { Submersion } \\
\text { Dose Rate } \\
\text { Coefficient } \\
\text { (mrem- } \\
\mathbf{m}^{\mathbf{3}} / \mathbf{p C i} \text {-h) }\end{array}$ & $\begin{array}{c}\text { Water } \\
\text { Submersion } \\
\text { Dose Rate } \\
\text { Coefficient } \\
(\text { mrem- } \\
\left.\text { m³ }^{3} / \mu \mathrm{Ci}-\mathrm{y}\right)\end{array}$ \\
\hline Bk-245 & 4.94 & $\mathrm{~d}$ & $9.29 \mathrm{E}-15$ & $1.08 \mathrm{E}-03$ & $3.44 \mathrm{E}+01$ & $2.05 \mathrm{E}-17$ & $2.73 \mathrm{E}-10$ & $2.39 \mathrm{E}-12$ \\
\hline Bk-246 & 1.8 & $\mathrm{~d}$ & $3.82 \mathrm{E}-14$ & $4.46 \mathrm{E}-03$ & $1.41 \mathrm{E}+02$ & $8.30 \mathrm{E}-17$ & $1.11 \mathrm{E}-09$ & $9.68 \mathrm{E}-12$ \\
\hline Bk-247 & $1.38 \mathrm{E}+03$ & $\mathrm{y}$ & $5.99 \mathrm{E}-15$ & $6.99 \mathrm{E}-04$ & $2.22 \mathrm{E}+01$ & $1.32 \mathrm{E}-17$ & $1.76 \mathrm{E}-10$ & $1.54 \mathrm{E}-12$ \\
\hline Bk-248m & 23.7 & $\mathrm{~h}$ & $2.28 \mathrm{E}-15$ & $2.66 \mathrm{E}-04$ & $8.44 \mathrm{E}+00$ & $4.91 \mathrm{E}-18$ & $6.54 \mathrm{E}-11$ & $5.73 \mathrm{E}-13$ \\
\hline Bk-249 & 330 & $\mathrm{~d}$ & $4.37 \mathrm{E}-19$ & $5.10 \mathrm{E}-08$ & $1.62 \mathrm{E}-03$ & $5.42 \mathrm{E}-22$ & $7.22 \mathrm{E}-15$ & $6.32 \mathrm{E}-17$ \\
\hline Bk-250 & 3.212 & $\mathrm{~h}$ & $4.18 \mathrm{E}-14$ & $4.88 \mathrm{E}-03$ & $1.55 \mathrm{E}+02$ & $9.03 \mathrm{E}-17$ & $1.20 \mathrm{E}-09$ & $1.05 \mathrm{E}-11$ \\
\hline Bk-251 & 55.6 & $\mathrm{~m}$ & $3.56 \mathrm{E}-15$ & $4.16 \mathrm{E}-04$ & $1.32 \mathrm{E}+01$ & $7.70 \mathrm{E}-18$ & $1.03 \mathrm{E}-10$ & $8.98 \mathrm{E}-13$ \\
\hline Cf-244 & 19.4 & $\mathrm{~m}$ & $4.32 \mathrm{E}-18$ & $5.04 \mathrm{E}-07$ & $1.60 \mathrm{E}-02$ & $1.01 \mathrm{E}-20$ & $1.35 \mathrm{E}-13$ & $1.18 \mathrm{E}-15$ \\
\hline Cf-246 & 35.7 & $\mathrm{~h}$ & $4.90 \mathrm{E}-18$ & $5.72 \mathrm{E}-07$ & $1.81 \mathrm{E}-02$ & $1.12 \mathrm{E}-20$ & $1.49 \mathrm{E}-13$ & $1.31 \mathrm{E}-15$ \\
\hline Cf-247 & 3.11 & $\mathrm{~h}$ & $3.58 \mathrm{E}-15$ & $4.18 \mathrm{E}-04$ & $1.32 \mathrm{E}+01$ & $7.95 \mathrm{E}-18$ & $1.06 \mathrm{E}-10$ & $9.28 \mathrm{E}-13$ \\
\hline Cf-248 & 334 & $\mathrm{~d}$ & $2.00 \mathrm{E}-17$ & $2.34 \mathrm{E}-06$ & $7.40 \mathrm{E}-02$ & $4.40 \mathrm{E}-20$ & $5.86 \mathrm{E}-13$ & $5.13 \mathrm{E}-15$ \\
\hline Cf-249 & 351 & $\mathrm{y}$ & $1.43 \mathrm{E}-14$ & $1.67 \mathrm{E}-03$ & $5.29 \mathrm{E}+01$ & $3.11 \mathrm{E}-17$ & $4.14 \mathrm{E}-10$ & $3.63 \mathrm{E}-12$ \\
\hline Cf-250 & 13.08 & $\mathrm{y}$ & $4.82 \mathrm{E}-16$ & $5.63 \mathrm{E}-05$ & $1.78 \mathrm{E}+00$ & $1.04 \mathrm{E}-18$ & $1.39 \mathrm{E}-11$ & $1.21 \mathrm{E}-13$ \\
\hline Cf-251 & 900 & $\mathrm{y}$ & $4.84 \mathrm{E}-15$ & $5.65 \mathrm{E}-04$ & $1.79 \mathrm{E}+01$ & $1.07 \mathrm{E}-17$ & $1.43 \mathrm{E}-10$ & $1.25 \mathrm{E}-12$ \\
\hline Cf-252 & 2.645 & $\mathrm{y}$ & $2.23 \mathrm{E}-14$ & $2.60 \mathrm{E}-03$ & $8.25 \mathrm{E}+01$ & $4.81 \mathrm{E}-17$ & $6.41 \mathrm{E}-10$ & $5.61 \mathrm{E}-12$ \\
\hline Cf-253 & 17.81 & $\mathrm{~d}$ & $3.15 \mathrm{E}-17$ & $3.68 \mathrm{E}-06$ & $1.17 \mathrm{E}-01$ & $5.64 \mathrm{E}-20$ & $7.51 \mathrm{E}-13$ & $6.58 \mathrm{E}-15$ \\
\hline Cf-254 & 60.5 & $\mathrm{~d}$ & $8.24 \mathrm{E}-13$ & $9.62 \mathrm{E}-02$ & $3.05 \mathrm{E}+03$ & $1.78 \mathrm{E}-15$ & $2.37 \mathrm{E}-08$ & $2.08 \mathrm{E}-10$ \\
\hline Cf-255 & 85 & $\mathrm{~m}$ & $1.16 \mathrm{E}-16$ & $1.35 \mathrm{E}-05$ & $4.29 \mathrm{E}-01$ & $1.30 \mathrm{E}-19$ & $1.73 \mathrm{E}-12$ & $1.52 \mathrm{E}-14$ \\
\hline Es-249 & 102.2 & $\mathrm{~m}$ & $1.77 \mathrm{E}-14$ & $2.07 \mathrm{E}-03$ & $6.55 \mathrm{E}+01$ & $3.86 \mathrm{E}-17$ & $5.14 \mathrm{E}-10$ & $4.50 \mathrm{E}-12$ \\
\hline Es-250 & 8.6 & $\mathrm{~h}$ & $5.25 \mathrm{E}-14$ & $6.13 \mathrm{E}-03$ & $1.94 \mathrm{E}+02$ & $1.14 \mathrm{E}-16$ & $1.52 \mathrm{E}-09$ & $1.33 \mathrm{E}-11$ \\
\hline Es-250m & 2.22 & $\mathrm{~h}$ & $2.49 \mathrm{E}-14$ & $2.91 \mathrm{E}-03$ & $9.21 \mathrm{E}+01$ & $5.42 \mathrm{E}-17$ & $7.22 \mathrm{E}-10$ & $6.32 \mathrm{E}-12$ \\
\hline Es-251 & 33 & $\mathrm{~h}$ & $3.58 \mathrm{E}-15$ & $4.18 \mathrm{E}-04$ & $1.32 \mathrm{E}+01$ & $7.96 \mathrm{E}-18$ & $1.06 \mathrm{E}-10$ & $9.29 \mathrm{E}-13$ \\
\hline
\end{tabular}




\begin{tabular}{|c|c|c|c|c|c|c|c|c|}
\hline Nuclide & Half-life & Class & $\begin{array}{c}\text { Air } \\
\text { Immersion } \\
\text { Dose Rate } \\
\text { Coefficient } \\
\text { (Sv/s per } \\
\left.\text { Bq } / \mathbf{m}^{\mathbf{3}}\right)\end{array}$ & $\begin{array}{c}\text { Air } \\
\text { Immersion } \\
\text { Dose Rate } \\
\text { Coefficient } \\
\text { (mrem- } \\
\left.\mathrm{m}^{3} / \mathrm{pCi}-\mathrm{yr}\right)\end{array}$ & $\begin{array}{c}\text { Air } \\
\text { Immersion } \\
\text { Effective } \\
\text { Dose Rate } \\
\text { (mrem/sec } \\
\text { per } \\
\mu \mathrm{Ci} / \mathrm{cm}^{3} \text { ) }\end{array}$ & $\begin{array}{c}\text { Water } \\
\text { Submersion } \\
\text { Dose Rate } \\
\text { Coefficient } \\
\left(\mathbf{S v}-\mathbf{m}^{3} / \mathbf{B q}-\right. \\
\text { s) }\end{array}$ & $\begin{array}{c}\text { Water } \\
\text { Submersion } \\
\text { Dose Rate } \\
\text { Coefficient } \\
\text { (mrem- } \\
\left.\text { m }^{\mathbf{3}} / \mathbf{p C i}-\mathbf{h}\right)\end{array}$ & $\begin{array}{c}\text { Water } \\
\text { Submersion } \\
\text { Dose Rate } \\
\text { Coefficient } \\
\text { (mrem- } \\
\left.\mathbf{m}^{\mathbf{3}} / \mu \mathrm{Ci}-\mathrm{y}\right)\end{array}$ \\
\hline Es-253 & 20.47 & $\mathrm{~d}$ & $1.50 \mathrm{E}-17$ & $1.75 \mathrm{E}-06$ & $5.55 \mathrm{E}-02$ & $3.30 \mathrm{E}-20$ & $4.40 \mathrm{E}-13$ & $3.85 \mathrm{E}-15$ \\
\hline Es-254 & 275.7 & $\mathrm{~d}$ & $1.47 \mathrm{E}-16$ & $1.72 \mathrm{E}-05$ & $5.44 \mathrm{E}-01$ & $3.33 \mathrm{E}-19$ & $4.44 \mathrm{E}-12$ & $3.89 \mathrm{E}-14$ \\
\hline Es-254m & 39.3 & $\mathrm{~h}$ & $2.13 \mathrm{E}-14$ & $2.49 \mathrm{E}-03$ & $7.88 \mathrm{E}+01$ & $4.61 \mathrm{E}-17$ & $6.14 \mathrm{E}-10$ & $5.38 \mathrm{E}-12$ \\
\hline Es-255 & 39.8 & $\mathrm{~d}$ & $4.95 \mathrm{E}-17$ & $5.78 \mathrm{E}-06$ & $1.83 \mathrm{E}-01$ & $8.96 \mathrm{E}-20$ & $1.19 \mathrm{E}-12$ & $1.05 \mathrm{E}-14$ \\
\hline Es-256 & 25.4 & $\mathrm{~m}$ & $4.33 \mathrm{E}-16$ & $5.06 \mathrm{E}-05$ & $1.60 \mathrm{E}+00$ & 5.29E-19 & $7.05 \mathrm{E}-12$ & $6.17 \mathrm{E}-14$ \\
\hline Fm-251 & 5.3 & $\mathrm{~h}$ & $6.35 \mathrm{E}-15$ & $7.41 \mathrm{E}-04$ & $2.35 \mathrm{E}+01$ & $1.40 \mathrm{E}-17$ & $1.86 \mathrm{E}-10$ & $1.63 \mathrm{E}-12$ \\
\hline Fm-252 & 25.39 & $\mathrm{~h}$ & $1.71 \mathrm{E}-17$ & $2.00 \mathrm{E}-06$ & $6.33 \mathrm{E}-02$ & $3.78 \mathrm{E}-20$ & $5.03 \mathrm{E}-13$ & $4.41 \mathrm{E}-15$ \\
\hline Fm-253 & 3 & $\mathrm{~d}$ & $2.32 \mathrm{E}-15$ & $2.71 \mathrm{E}-04$ & $8.58 \mathrm{E}+00$ & $5.15 \mathrm{E}-18$ & $6.86 \mathrm{E}-11$ & $6.01 \mathrm{E}-13$ \\
\hline Fm-254 & 3.24 & $\mathrm{~h}$ & $3.47 \mathrm{E}-16$ & $4.05 \mathrm{E}-05$ & $1.28 \mathrm{E}+00$ & $7.50 \mathrm{E}-19$ & $9.99 \mathrm{E}-12$ & $8.75 \mathrm{E}-14$ \\
\hline Fm-255 & 20.07 & $\mathrm{~h}$ & $9.47 \mathrm{E}-17$ & $1.11 \mathrm{E}-05$ & $3.50 \mathrm{E}-01$ & $2.16 \mathrm{E}-19$ & $2.88 \mathrm{E}-12$ & $2.52 \mathrm{E}-14$ \\
\hline Fm-256 & 157.6 & $\mathrm{~m}$ & $6.07 \mathrm{E}-13$ & $7.09 \mathrm{E}-02$ & $2.25 \mathrm{E}+03$ & $1.31 \mathrm{E}-15$ & $1.74 \mathrm{E}-08$ & $1.53 \mathrm{E}-10$ \\
\hline Fm-257 & 100.5 & $\mathrm{~d}$ & $6.02 \mathrm{E}-15$ & $7.03 \mathrm{E}-04$ & $2.23 \mathrm{E}+01$ & $1.32 \mathrm{E}-17$ & $1.76 \mathrm{E}-10$ & $1.54 \mathrm{E}-12$ \\
\hline
\end{tabular}


Table C-2 Ground Shine Dose Rate Coefficients

\begin{tabular}{|c|c|c|c|}
\hline Nuclide & $\begin{array}{c}\text { Ground } \\
\text { Shine Dose } \\
\text { Rate } \\
\text { Coefficient } \\
\left(\mathrm{Sv}-\mathbf{m}^{2} / \mathrm{Bq}-\mathrm{s}\right) \\
\end{array}$ & $\begin{array}{c}\text { Ground Shine } \\
\text { Dose Rate } \\
\text { Coefficient } \\
\text { mrem-m }{ }^{2} / p C i-h\end{array}$ & $\begin{array}{l}\text { Ground Shine } \\
\text { Dose Rate } \\
\text { Coefficient } \\
\text { mrem-m } / \mu \mathrm{Ci}-\mathrm{y}\end{array}$ \\
\hline $\mathrm{H}-3$ & $0.00 \mathrm{E}+00$ & $0.00 \mathrm{E}+00$ & $0.00 \mathrm{E}+00$ \\
\hline $\mathrm{Be}-7$ & $4.76 \mathrm{E}-17$ & $6.34 \mathrm{E}-10$ & $5.55 \mathrm{E}+00$ \\
\hline Be-10 & $3.44 \mathrm{E}-18$ & $4.58 \mathrm{E}-11$ & $4.01 \mathrm{E}-01$ \\
\hline $\mathrm{C}-10$ & $1.76 \mathrm{E}-15$ & 2.34E-08 & $2.05 \mathrm{E}+02$ \\
\hline $\mathrm{C}-11$ & $1.00 \mathrm{E}-15$ & $1.33 \mathrm{E}-08$ & $1.17 \mathrm{E}+02$ \\
\hline C-14 & $1.28 \mathrm{E}-20$ & $1.70 \mathrm{E}-13$ & $1.49 \mathrm{E}-03$ \\
\hline $\mathrm{N}-13$ & $1.03 \mathrm{E}-15$ & $1.37 \mathrm{E}-08$ & $1.20 \mathrm{E}+02$ \\
\hline $\mathrm{N}-16$ & $3.44 \mathrm{E}-15$ & $4.58 \mathrm{E}-08$ & $4.01 \mathrm{E}+02$ \\
\hline O-14 & $3.03 \mathrm{E}-15$ & 4.04E-08 & $3.54 \mathrm{E}+02$ \\
\hline $\mathrm{O}-15$ & $1.07 \mathrm{E}-15$ & $1.43 \mathrm{E}-08$ & $1.25 \mathrm{E}+02$ \\
\hline O-19 & $1.03 \mathrm{E}-15$ & $1.37 \mathrm{E}-08$ & $1.20 \mathrm{E}+02$ \\
\hline $\mathrm{F}-17$ & $1.07 \mathrm{E}-15$ & $1.43 \mathrm{E}-08$ & $1.25 \mathrm{E}+02$ \\
\hline F-18 & $9.49 \mathrm{E}-16$ & $1.26 \mathrm{E}-08$ & $1.11 \mathrm{E}+02$ \\
\hline $\mathrm{Ne}-19$ & $1.09 \mathrm{E}-15$ & $1.45 \mathrm{E}-08$ & $1.27 \mathrm{E}+02$ \\
\hline $\mathrm{Ne}-24$ & $6.15 \mathrm{E}-16$ & 8.19E-09 & $7.18 \mathrm{E}+01$ \\
\hline $\mathrm{Na}-22$ & $2.05 \mathrm{E}-15$ & $2.73 \mathrm{E}-08$ & $2.39 \mathrm{E}+02$ \\
\hline $\mathrm{Na}-24$ & $3.59 \mathrm{E}-15$ & $4.78 \mathrm{E}-08$ & $4.19 \mathrm{E}+02$ \\
\hline $\mathrm{Mg}-27$ & $9.25 \mathrm{E}-16$ & $1.23 \mathrm{E}-08$ & $1.08 \mathrm{E}+02$ \\
\hline $\mathrm{Mg}-28$ & $1.26 \mathrm{E}-15$ & $1.68 \mathrm{E}-08$ & $1.47 \mathrm{E}+02$ \\
\hline Al-26 & $2.47 \mathrm{E}-15$ & 3.29E-08 & $2.88 \mathrm{E}+02$ \\
\hline Al-28 & $1.71 \mathrm{E}-15$ & $2.28 \mathrm{E}-08$ & $2.00 \mathrm{E}+02$ \\
\hline Al-29 & $1.37 \mathrm{E}-15$ & $1.82 \mathrm{E}-08$ & $1.60 \mathrm{E}+02$ \\
\hline $\mathrm{Si}-31$ & $7.13 \mathrm{E}-17$ & $9.50 \mathrm{E}-10$ & $8.32 \mathrm{E}+00$ \\
\hline $\mathrm{Si}-32$ & $2.87 \mathrm{E}-20$ & $3.82 \mathrm{E}-13$ & $3.35 \mathrm{E}-03$ \\
\hline P-30 & $1.13 \mathrm{E}-15$ & $1.51 \mathrm{E}-08$ & $1.32 \mathrm{E}+02$ \\
\hline $\mathrm{P}-32$ & $8.52 \mathrm{E}-17$ & $1.13 \mathrm{E}-09$ & $9.94 \mathrm{E}+00$ \\
\hline $\mathrm{P}-33$ & $3.64 \mathrm{E}-20$ & $4.85 \mathrm{E}-13$ & $4.25 \mathrm{E}-03$ \\
\hline $\mathrm{S}-35$ & $1.33 \mathrm{E}-20$ & $1.77 \mathrm{E}-13$ & $1.55 \mathrm{E}-03$ \\
\hline S-37 & $2.48 \mathrm{E}-15$ & $3.30 \mathrm{E}-08$ & $2.89 \mathrm{E}+02$ \\
\hline S-38 & $1.52 \mathrm{E}-15$ & $2.02 \mathrm{E}-08$ & $1.77 \mathrm{E}+02$ \\
\hline $\mathrm{Cl}-34$ & $1.17 \mathrm{E}-15$ & $1.56 \mathrm{E}-08$ & $1.37 \mathrm{E}+02$ \\
\hline $\mathrm{Cl}-34 \mathrm{~m}$ & $1.91 \mathrm{E}-15$ & $2.54 \mathrm{E}-08$ & $2.23 \mathrm{E}+02$ \\
\hline Cl-36 & $1.11 \mathrm{E}-17$ & $1.48 \mathrm{E}-10$ & $1.30 \mathrm{E}+00$ \\
\hline Cl-38 & $1.40 \mathrm{E}-15$ & $1.86 \mathrm{E}-08$ & $1.63 \mathrm{E}+02$ \\
\hline Cl-39 & $1.42 \mathrm{E}-15$ & $1.89 \mathrm{E}-08$ & $1.66 \mathrm{E}+02$ \\
\hline $\mathrm{Cl}-40$ & $3.60 \mathrm{E}-15$ & $4.80 \mathrm{E}-08$ & $4.20 \mathrm{E}+02$ \\
\hline
\end{tabular}


SRNL-STI-2013-00115

\begin{tabular}{|c|c|c|c|}
\hline Nuclide & $\begin{array}{c}\text { Ground } \\
\text { Shine Dose } \\
\text { Rate } \\
\text { Coefficient } \\
\text { (Sv-m } / \mathbf{B q}-\mathbf{s})\end{array}$ & $\begin{array}{l}\text { Ground Shine } \\
\text { Dose Rate } \\
\text { Coefficient } \\
\text { mrem-m }{ }^{2} / p C i-h\end{array}$ & $\begin{array}{c}\text { Ground Shine } \\
\text { Dose Rate } \\
\text { Coefficient } \\
\text { mrem-m }{ }^{2} / \mu \mathrm{Ci}-\mathrm{y}\end{array}$ \\
\hline Ar-37 & $0.00 \mathrm{E}+00$ & $0.00 \mathrm{E}+00$ & $0.00 \mathrm{E}+00$ \\
\hline Ar-39 & $2.52 \mathrm{E}-18$ & $3.36 \mathrm{E}-11$ & $2.94 \mathrm{E}-01$ \\
\hline Ar-41 & $1.22 \mathrm{E}-15$ & $1.63 \mathrm{E}-08$ & $1.42 \mathrm{E}+02$ \\
\hline Ar-42 & $4.08 \mathrm{E}-18$ & $5.43 \mathrm{E}-11$ & $4.76 \mathrm{E}-01$ \\
\hline Ar-43 & $1.52 \mathrm{E}-15$ & $2.02 \mathrm{E}-08$ & $1.77 \mathrm{E}+02$ \\
\hline Ar-44 & $1.78 \mathrm{E}-15$ & $2.37 \mathrm{E}-08$ & $2.08 \mathrm{E}+02$ \\
\hline $\mathrm{K}-38$ & $2.97 \mathrm{E}-15$ & $3.96 \mathrm{E}-08$ & $3.47 \mathrm{E}+02$ \\
\hline $\mathrm{K}-40$ & $2.04 \mathrm{E}-16$ & 2.72E-09 & $2.38 \mathrm{E}+01$ \\
\hline $\mathrm{K}-42$ & $4.00 \mathrm{E}-16$ & 5.33E-09 & $4.67 \mathrm{E}+01$ \\
\hline $\mathrm{K}-43$ & $9.35 \mathrm{E}-16$ & $1.25 \mathrm{E}-08$ & $1.09 \mathrm{E}+02$ \\
\hline $\mathrm{K}-44$ & $2.23 \mathrm{E}-15$ & $2.97 \mathrm{E}-08$ & $2.60 \mathrm{E}+02$ \\
\hline $\mathrm{K}-45$ & $1.74 \mathrm{E}-15$ & $2.32 \mathrm{E}-08$ & $2.03 \mathrm{E}+02$ \\
\hline $\mathrm{K}-46$ & $2.66 \mathrm{E}-15$ & $3.54 \mathrm{E}-08$ & $3.10 \mathrm{E}+02$ \\
\hline $\mathrm{Ca}-41$ & $0.00 \mathrm{E}+00$ & $0.00 \mathrm{E}+00$ & $0.00 \mathrm{E}+00$ \\
\hline $\mathrm{Ca}-45$ & $3.78 \mathrm{E}-20$ & $5.03 \mathrm{E}-13$ & $4.41 \mathrm{E}-03$ \\
\hline $\mathrm{Ca}-47$ & $9.90 \mathrm{E}-16$ & $1.32 \mathrm{E}-08$ & $1.16 \mathrm{E}+02$ \\
\hline Ca-49 & $2.67 \mathrm{E}-15$ & $3.56 \mathrm{E}-08$ & $3.12 \mathrm{E}+02$ \\
\hline Sc- $42 \mathrm{~m}$ & $4.03 \mathrm{E}-15$ & $5.37 \mathrm{E}-08$ & $4.70 \mathrm{E}+02$ \\
\hline Sc-43 & $9.82 \mathrm{E}-16$ & $1.31 \mathrm{E}-08$ & $1.15 \mathrm{E}+02$ \\
\hline Sc-44 & $2.08 \mathrm{E}-15$ & $2.77 \mathrm{E}-08$ & $2.43 \mathrm{E}+02$ \\
\hline Sc- $44 m$ & $2.56 \mathrm{E}-16$ & $3.41 \mathrm{E}-09$ & $2.99 \mathrm{E}+01$ \\
\hline Sc-46 & $1.88 \mathrm{E}-15$ & $2.50 \mathrm{E}-08$ & $2.19 \mathrm{E}+02$ \\
\hline Sc-47 & $1.00 \mathrm{E}-16$ & $1.33 \mathrm{E}-09$ & $1.17 \mathrm{E}+01$ \\
\hline Sc-48 & $3.11 \mathrm{E}-15$ & $4.14 \mathrm{E}-08$ & $3.63 \mathrm{E}+02$ \\
\hline Sc-49 & $1.01 \mathrm{E}-16$ & $1.35 \mathrm{E}-09$ & $1.18 \mathrm{E}+01$ \\
\hline Sc-50 & $3.10 \mathrm{E}-15$ & 4.13E-08 & $3.62 \mathrm{E}+02$ \\
\hline Ti-44 & $1.23 \mathrm{E}-16$ & $1.64 \mathrm{E}-09$ & $1.44 \mathrm{E}+01$ \\
\hline Ti-45 & $8.66 \mathrm{E}-16$ & $1.15 \mathrm{E}-08$ & $1.01 \mathrm{E}+02$ \\
\hline Ti-51 & $4.54 \mathrm{E}-16$ & $6.05 \mathrm{E}-09$ & $5.30 \mathrm{E}+01$ \\
\hline Ti-52 & $2.05 \mathrm{E}-16$ & $2.73 \mathrm{E}-09$ & $2.39 \mathrm{E}+01$ \\
\hline $\mathrm{V}-47$ & $1.05 \mathrm{E}-15$ & $1.40 \mathrm{E}-08$ & $1.23 \mathrm{E}+02$ \\
\hline $\mathrm{V}-48$ & $2.71 \mathrm{E}-15$ & $3.61 \mathrm{E}-08$ & $3.16 \mathrm{E}+02$ \\
\hline $\mathrm{V}-49$ & $0.00 \mathrm{E}+00$ & $0.00 \mathrm{E}+00$ & $0.00 \mathrm{E}+00$ \\
\hline $\mathrm{V}-50$ & $1.28 \mathrm{E}-15$ & $1.70 \mathrm{E}-08$ & $1.49 \mathrm{E}+02$ \\
\hline V-52 & $1.43 \mathrm{E}-15$ & $1.90 \mathrm{E}-08$ & $1.67 \mathrm{E}+02$ \\
\hline $\mathrm{V}-53$ & $1.09 \mathrm{E}-15$ & $1.45 \mathrm{E}-08$ & $1.27 \mathrm{E}+02$ \\
\hline Cr-48 & $4.05 \mathrm{E}-16$ & 5.39E-09 & $4.73 \mathrm{E}+01$ \\
\hline Cr-49 & $1.07 \mathrm{E}-15$ & $1.43 \mathrm{E}-08$ & $1.25 \mathrm{E}+02$ \\
\hline Cr-51 & $2.99 \mathrm{E}-17$ & $3.98 \mathrm{E}-10$ & $3.49 \mathrm{E}+00$ \\
\hline
\end{tabular}


SRNL-STI-2013-00115

\begin{tabular}{|c|c|c|c|}
\hline Nuclide & $\begin{array}{c}\text { Ground } \\
\text { Shine Dose } \\
\text { Rate } \\
\text { Coefficient } \\
\text { (Sv-m } / \mathbf{B q}-\mathbf{s})\end{array}$ & $\begin{array}{l}\text { Ground Shine } \\
\text { Dose Rate } \\
\text { Coefficient } \\
\text { mrem-m }{ }^{2} / p C i-h\end{array}$ & $\begin{array}{c}\text { Ground Shine } \\
\text { Dose Rate } \\
\text { Coefficient } \\
\text { mrem-m }{ }^{2} / \mu \mathrm{Ci}-\mathrm{y}\end{array}$ \\
\hline $\mathrm{Cr}-55$ & $1.29 \mathrm{E}-16$ & $1.72 \mathrm{E}-09$ & $1.51 \mathrm{E}+01$ \\
\hline Cr-56 & $1.48 \mathrm{E}-16$ & $1.97 \mathrm{E}-09$ & $1.73 \mathrm{E}+01$ \\
\hline $\mathrm{Mn}-50 \mathrm{~m}$ & $4.47 \mathrm{E}-15$ & $5.95 \mathrm{E}-08$ & $5.22 \mathrm{E}+02$ \\
\hline $\mathrm{Mn}-51$ & $1.07 \mathrm{E}-15$ & $1.43 \mathrm{E}-08$ & $1.25 \mathrm{E}+02$ \\
\hline $\mathrm{Mn}-52 \mathrm{~m}$ & $2.36 \mathrm{E}-15$ & $3.14 \mathrm{E}-08$ & $2.75 \mathrm{E}+02$ \\
\hline Mn-52 & $3.21 \mathrm{E}-15$ & $4.28 \mathrm{E}-08$ & $3.75 \mathrm{E}+02$ \\
\hline Mn-53 & $0.00 \mathrm{E}+00$ & $0.00 \mathrm{E}+00$ & $0.00 \mathrm{E}+00$ \\
\hline Mn-54 & 7.89E-16 & $1.05 \mathrm{E}-08$ & $9.21 \mathrm{E}+01$ \\
\hline $\mathrm{Mn}-56$ & $1.62 \mathrm{E}-15$ & $2.16 \mathrm{E}-08$ & $1.89 \mathrm{E}+02$ \\
\hline Mn-57 & $2.17 \mathrm{E}-16$ & $2.89 \mathrm{E}-09$ & $2.53 \mathrm{E}+01$ \\
\hline $\mathrm{Mn}-58 \mathrm{~m}$ & $2.36 \mathrm{E}-15$ & $3.14 \mathrm{E}-08$ & $2.75 \mathrm{E}+02$ \\
\hline $\mathrm{Fe}-52$ & $7.10 \mathrm{E}-16$ & $9.46 \mathrm{E}-09$ & $8.28 \mathrm{E}+01$ \\
\hline $\mathrm{Fe}-53$ & $1.25 \mathrm{E}-15$ & $1.67 \mathrm{E}-08$ & $1.46 \mathrm{E}+02$ \\
\hline $\mathrm{Fe}-53 \mathrm{~m}$ & $2.81 \mathrm{E}-15$ & $3.74 \mathrm{E}-08$ & $3.28 \mathrm{E}+02$ \\
\hline $\mathrm{Fe}-55$ & $1.45 \mathrm{E}-25$ & $1.93 \mathrm{E}-18$ & $1.69 \mathrm{E}-08$ \\
\hline $\mathrm{Fe}-59$ & $1.10 \mathrm{E}-15$ & $1.47 \mathrm{E}-08$ & $1.28 \mathrm{E}+02$ \\
\hline $\mathrm{Fe}-60$ & $2.29 \mathrm{E}-20$ & $3.05 \mathrm{E}-13$ & $2.67 \mathrm{E}-03$ \\
\hline Fe-61 & $1.40 \mathrm{E}-15$ & $1.86 \mathrm{E}-08$ & $1.63 \mathrm{E}+02$ \\
\hline Fe-62 & $5.85 \mathrm{E}-16$ & 7.79E-09 & $6.83 \mathrm{E}+01$ \\
\hline Co-54m & $3.85 \mathrm{E}-15$ & $5.13 \mathrm{E}-08$ & $4.49 \mathrm{E}+02$ \\
\hline Co-55 & $1.92 \mathrm{E}-15$ & $2.56 \mathrm{E}-08$ & $2.24 \mathrm{E}+02$ \\
\hline Co-56 & $3.27 \mathrm{E}-15$ & $4.36 \mathrm{E}-08$ & $3.82 \mathrm{E}+02$ \\
\hline Co-57 & $1.09 \mathrm{E}-16$ & $1.45 \mathrm{E}-09$ & $1.27 \mathrm{E}+01$ \\
\hline Co-58 & $9.22 \mathrm{E}-16$ & $1.23 \mathrm{E}-08$ & $1.08 \mathrm{E}+02$ \\
\hline Co-58m & $6.68 \mathrm{E}-21$ & $8.90 \mathrm{E}-14$ & 7.79E-04 \\
\hline Co-60 & $2.30 \mathrm{E}-15$ & $3.06 \mathrm{E}-08$ & $2.68 \mathrm{E}+02$ \\
\hline Co-60m & $4.24 \mathrm{E}-18$ & $5.65 \mathrm{E}-11$ & $4.95 \mathrm{E}-01$ \\
\hline Co-61 & $1.36 \mathrm{E}-16$ & $1.81 \mathrm{E}-09$ & $1.59 \mathrm{E}+01$ \\
\hline Co-62 & $1.61 \mathrm{E}-15$ & $2.14 \mathrm{E}-08$ & $1.88 \mathrm{E}+02$ \\
\hline Co-62m & $2.57 \mathrm{E}-15$ & $3.42 \mathrm{E}-08$ & $3.00 \mathrm{E}+02$ \\
\hline Ni-56 & $1.61 \mathrm{E}-15$ & $2.14 \mathrm{E}-08$ & $1.88 \mathrm{E}+02$ \\
\hline $\mathrm{Ni}-57$ & $1.78 \mathrm{E}-15$ & $2.37 \mathrm{E}-08$ & $2.08 \mathrm{E}+02$ \\
\hline Ni-59 & $1.48 \mathrm{E}-20$ & $1.97 \mathrm{E}-13$ & $1.73 \mathrm{E}-03$ \\
\hline Ni-63 & $0.00 \mathrm{E}+00$ & $0.00 \mathrm{E}+00$ & $0.00 \mathrm{E}+00$ \\
\hline $\mathrm{Ni}-65$ & $5.77 \mathrm{E}-16$ & 7.69E-09 & $6.73 \mathrm{E}+01$ \\
\hline Ni-66 & $3.45 \mathrm{E}-20$ & $4.60 \mathrm{E}-13$ & $4.03 \mathrm{E}-03$ \\
\hline $\mathrm{Cu}-57$ & $1.36 \mathrm{E}-15$ & $1.81 \mathrm{E}-08$ & $1.59 \mathrm{E}+02$ \\
\hline $\mathrm{Cu}-59$ & $1.52 \mathrm{E}-15$ & $2.02 \mathrm{E}-08$ & $1.77 \mathrm{E}+02$ \\
\hline $\mathrm{Cu}-60$ & $3.65 \mathrm{E}-15$ & $4.86 \mathrm{E}-08$ & $4.26 \mathrm{E}+02$ \\
\hline
\end{tabular}


SRNL-STI-2013-00115

\begin{tabular}{|c|c|c|c|}
\hline Nuclide & $\begin{array}{c}\text { Ground } \\
\text { Shine Dose } \\
\text { Rate } \\
\text { Coefficient } \\
\left(\mathrm{Sv}-\mathbf{m}^{2} / \mathbf{B q}-\mathbf{s}\right)\end{array}$ & $\begin{array}{l}\text { Ground Shine } \\
\text { Dose Rate } \\
\text { Coefficient } \\
\text { mrem-m }{ }^{2} / p C i-h\end{array}$ & $\begin{array}{c}\text { Ground Shine } \\
\text { Dose Rate } \\
\text { Coefficient } \\
\text { mrem-m }{ }^{2} / \mu \mathrm{Ci}-\mathrm{y}\end{array}$ \\
\hline $\mathrm{Cu}-61$ & $8.15 \mathrm{E}-16$ & $1.09 \mathrm{E}-08$ & $9.51 \mathrm{E}+01$ \\
\hline $\mathrm{Cu}-62$ & $1.11 \mathrm{E}-15$ & $1.48 \mathrm{E}-08$ & $1.30 \mathrm{E}+02$ \\
\hline $\mathrm{Cu}-64$ & $1.78 \mathrm{E}-16$ & 2.37E-09 & $2.08 \mathrm{E}+01$ \\
\hline $\mathrm{Cu}-66$ & $2.15 \mathrm{E}-16$ & $2.86 \mathrm{E}-09$ & $2.51 \mathrm{E}+01$ \\
\hline $\mathrm{Cu}-67$ & $1.05 \mathrm{E}-16$ & $1.40 \mathrm{E}-09$ & $1.23 \mathrm{E}+01$ \\
\hline $\mathrm{Cu}-69$ & $5.97 \mathrm{E}-16$ & 7.95E-09 & $6.97 \mathrm{E}+01$ \\
\hline Zn-60 & $1.59 \mathrm{E}-15$ & 2.12E-08 & $1.86 \mathrm{E}+02$ \\
\hline Zn-61 & $1.61 \mathrm{E}-15$ & $2.14 \mathrm{E}-08$ & $1.88 \mathrm{E}+02$ \\
\hline Zn-62 & $4.19 \mathrm{E}-16$ & $5.58 \mathrm{E}-09$ & $4.89 \mathrm{E}+01$ \\
\hline Zn-63 & $1.16 \mathrm{E}-15$ & $1.55 \mathrm{E}-08$ & $1.35 \mathrm{E}+02$ \\
\hline Zn-65 & $5.37 \mathrm{E}-16$ & $7.15 \mathrm{E}-09$ & $6.27 \mathrm{E}+01$ \\
\hline $\mathrm{Zn}-69 \mathrm{~m}$ & $3.98 \mathrm{E}-16$ & 5.30E-09 & $4.64 \mathrm{E}+01$ \\
\hline Zn-69 & $2.09 \mathrm{E}-17$ & $2.78 \mathrm{E}-10$ & $2.44 \mathrm{E}+00$ \\
\hline $\mathrm{Zn}-71 \mathrm{~m}$ & $1.54 \mathrm{E}-15$ & $2.05 \mathrm{E}-08$ & $1.80 \mathrm{E}+02$ \\
\hline Zn-71 & $4.19 \mathrm{E}-16$ & $5.58 \mathrm{E}-09$ & $4.89 \mathrm{E}+01$ \\
\hline $\mathrm{Zn}-72$ & $1.33 \mathrm{E}-16$ & $1.77 \mathrm{E}-09$ & $1.55 \mathrm{E}+01$ \\
\hline Ga-64 & $3.18 \mathrm{E}-15$ & $4.24 \mathrm{E}-08$ & $3.71 \mathrm{E}+02$ \\
\hline Ga-65 & $1.20 \mathrm{E}-15$ & $1.60 \mathrm{E}-08$ & $1.40 \mathrm{E}+02$ \\
\hline Ga-66 & $2.26 \mathrm{E}-15$ & $3.01 \mathrm{E}-08$ & $2.64 \mathrm{E}+02$ \\
\hline Ga-67 & $1.43 \mathrm{E}-16$ & $1.90 \mathrm{E}-09$ & $1.67 \mathrm{E}+01$ \\
\hline Ga-68 & $9.97 \mathrm{E}-16$ & $1.33 \mathrm{E}-08$ & $1.16 \mathrm{E}+02$ \\
\hline Ga-70 & $8.45 \mathrm{E}-17$ & 1.13E-09 & $9.86 \mathrm{E}+00$ \\
\hline Ga-72 & $2.48 \mathrm{E}-15$ & $3.30 \mathrm{E}-08$ & $2.89 \mathrm{E}+02$ \\
\hline Ga-73 & $3.71 \mathrm{E}-16$ & 4.94E-09 & $4.33 \mathrm{E}+01$ \\
\hline Ga-74 & $2.90 \mathrm{E}-15$ & $3.86 \mathrm{E}-08$ & $3.38 \mathrm{E}+02$ \\
\hline Ge-66 & $6.41 \mathrm{E}-16$ & $8.54 \mathrm{E}-09$ & $7.48 \mathrm{E}+01$ \\
\hline Ge-67 & $1.47 \mathrm{E}-15$ & $1.96 \mathrm{E}-08$ & $1.72 \mathrm{E}+02$ \\
\hline Ge-68 & $3.61 \mathrm{E}-20$ & $4.81 \mathrm{E}-13$ & $4.21 \mathrm{E}-03$ \\
\hline Ge-69 & $8.99 \mathrm{E}-16$ & $1.20 \mathrm{E}-08$ & $1.05 \mathrm{E}+02$ \\
\hline Ge-71 & $3.66 \mathrm{E}-20$ & $4.88 \mathrm{E}-13$ & $4.27 \mathrm{E}-03$ \\
\hline Ge-75 & $7.30 \mathrm{E}-17$ & $9.72 \mathrm{E}-10$ & $8.52 \mathrm{E}+00$ \\
\hline Ge-77 & $1.08 \mathrm{E}-15$ & $1.44 \mathrm{E}-08$ & $1.26 \mathrm{E}+02$ \\
\hline Ge-78 & $2.66 \mathrm{E}-16$ & $3.54 \mathrm{E}-09$ & $3.10 \mathrm{E}+01$ \\
\hline As-68 & $3.61 \mathrm{E}-15$ & 4.81E-08 & $4.21 \mathrm{E}+02$ \\
\hline As-69 & $1.22 \mathrm{E}-15$ & $1.63 \mathrm{E}-08$ & $1.42 \mathrm{E}+02$ \\
\hline As-70 & $4.04 \mathrm{E}-15$ & $5.38 \mathrm{E}-08$ & $4.71 \mathrm{E}+02$ \\
\hline As-71 & $5.44 \mathrm{E}-16$ & 7.25E-09 & $6.35 \mathrm{E}+01$ \\
\hline As-72 & $1.80 \mathrm{E}-15$ & $2.40 \mathrm{E}-08$ & $2.10 \mathrm{E}+02$ \\
\hline As-73 & $5.14 \mathrm{E}-18$ & $6.85 \mathrm{E}-11$ & $6.00 \mathrm{E}-01$ \\
\hline
\end{tabular}


SRNL-STI-2013-00115

\begin{tabular}{|c|c|c|c|}
\hline Nuclide & $\begin{array}{c}\text { Ground } \\
\text { Shine Dose } \\
\text { Rate } \\
\text { Coefficient } \\
\text { (Sv-m } / \mathbf{B q}-\mathbf{s})\end{array}$ & $\begin{array}{l}\text { Ground Shine } \\
\text { Dose Rate } \\
\text { Coefficient } \\
\text { mrem-m }{ }^{2} / p C i-h\end{array}$ & $\begin{array}{c}\text { Ground Shine } \\
\text { Dose Rate } \\
\text { Coefficient } \\
\text { mrem-m }{ }^{2} / \mu \mathrm{Ci}-\mathrm{y}\end{array}$ \\
\hline As-74 & $7.46 \mathrm{E}-16$ & $9.94 \mathrm{E}-09$ & $8.70 \mathrm{E}+01$ \\
\hline As-76 & $5.13 \mathrm{E}-16$ & $6.83 \mathrm{E}-09$ & $5.99 \mathrm{E}+01$ \\
\hline As-77 & $1.33 \mathrm{E}-17$ & $1.77 \mathrm{E}-10$ & $1.55 \mathrm{E}+00$ \\
\hline As-78 & $1.33 \mathrm{E}-15$ & $1.77 \mathrm{E}-08$ & $1.55 \mathrm{E}+02$ \\
\hline As-79 & $1.38 \mathrm{E}-16$ & $1.84 \mathrm{E}-09$ & $1.61 \mathrm{E}+01$ \\
\hline Se-70 & $6.96 \mathrm{E}-16$ & 9.27E-09 & $8.12 \mathrm{E}+01$ \\
\hline Se-71 & $1.66 \mathrm{E}-15$ & $2.21 \mathrm{E}-08$ & $1.94 \mathrm{E}+02$ \\
\hline Se-72 & $2.43 \mathrm{E}-17$ & $3.24 \mathrm{E}-10$ & $2.84 \mathrm{E}+00$ \\
\hline $\mathrm{Se}-73$ & $1.08 \mathrm{E}-15$ & $1.44 \mathrm{E}-08$ & $1.26 \mathrm{E}+02$ \\
\hline $\mathrm{Se}-73 \mathrm{~m}$ & $2.67 \mathrm{E}-16$ & $3.56 \mathrm{E}-09$ & $3.12 \mathrm{E}+01$ \\
\hline Se-75 & $3.56 \mathrm{E}-16$ & 4.74E-09 & $4.15 \mathrm{E}+01$ \\
\hline Se- $77 \mathrm{~m}$ & $7.88 \mathrm{E}-17$ & $1.05 \mathrm{E}-09$ & $9.19 \mathrm{E}+00$ \\
\hline Se-79 & $1.45 \mathrm{E}-20$ & $1.93 \mathrm{E}-13$ & $1.69 \mathrm{E}-03$ \\
\hline Se-79m & $8.56 \mathrm{E}-18$ & $1.14 \mathrm{E}-10$ & 9.99E-01 \\
\hline Se-81 & $8.02 \mathrm{E}-17$ & $1.07 \mathrm{E}-09$ & $9.36 \mathrm{E}+00$ \\
\hline Se- $81 \mathrm{~m}$ & $1.27 \mathrm{E}-17$ & $1.69 \mathrm{E}-10$ & $1.48 \mathrm{E}+00$ \\
\hline $\mathrm{Se}-83$ & $2.45 \mathrm{E}-15$ & $3.26 \mathrm{E}-08$ & $2.86 \mathrm{E}+02$ \\
\hline Se-83m & $1.04 \mathrm{E}-15$ & $1.39 \mathrm{E}-08$ & $1.21 \mathrm{E}+02$ \\
\hline $\mathrm{Se}-84$ & $4.63 \mathrm{E}-16$ & $6.17 \mathrm{E}-09$ & $5.40 \mathrm{E}+01$ \\
\hline $\mathrm{Br}-72$ & $2.99 \mathrm{E}-15$ & 3.98E-08 & $3.49 \mathrm{E}+02$ \\
\hline Br-73 & $1.51 \mathrm{E}-15$ & $2.01 \mathrm{E}-08$ & $1.76 \mathrm{E}+02$ \\
\hline $\mathrm{Br}-74$ & $4.10 \mathrm{E}-15$ & $5.46 \mathrm{E}-08$ & $4.78 \mathrm{E}+02$ \\
\hline $\mathrm{Br}-74 \mathrm{~m}$ & $3.85 \mathrm{E}-15$ & 5.13E-08 & $4.49 \mathrm{E}+02$ \\
\hline $\mathrm{Br}-75$ & $1.20 \mathrm{E}-15$ & $1.60 \mathrm{E}-08$ & $1.40 \mathrm{E}+02$ \\
\hline $\mathrm{Br}-76$ & $2.58 \mathrm{E}-15$ & $3.44 \mathrm{E}-08$ & $3.01 \mathrm{E}+02$ \\
\hline $\mathrm{Br}-76 \mathrm{~m}$ & $3.23 \mathrm{E}-17$ & $4.30 \mathrm{E}-10$ & $3.77 \mathrm{E}+00$ \\
\hline $\mathrm{Br}-77$ & $2.99 \mathrm{E}-16$ & 3.98E-09 & $3.49 \mathrm{E}+01$ \\
\hline $\mathrm{Br}-77 \mathrm{~m}$ & $1.37 \mathrm{E}-17$ & $1.82 \mathrm{E}-10$ & $1.60 \mathrm{E}+00$ \\
\hline Br-78 & $1.11 \mathrm{E}-15$ & $1.48 \mathrm{E}-08$ & $1.30 \mathrm{E}+02$ \\
\hline Br-80 & $1.60 \mathrm{E}-16$ & $2.13 \mathrm{E}-09$ & $1.87 \mathrm{E}+01$ \\
\hline Br-80m & $1.38 \mathrm{E}-17$ & $1.84 \mathrm{E}-10$ & $1.61 \mathrm{E}+00$ \\
\hline Br-82 & $2.48 \mathrm{E}-15$ & $3.30 \mathrm{E}-08$ & $2.89 \mathrm{E}+02$ \\
\hline $\mathrm{Br}-82 \mathrm{~m}$ & $6.67 \mathrm{E}-18$ & $8.88 \mathrm{E}-11$ & $7.78 \mathrm{E}-01$ \\
\hline $\mathrm{Br}-83$ & $2.88 \mathrm{E}-17$ & $3.84 \mathrm{E}-10$ & $3.36 \mathrm{E}+00$ \\
\hline Br-84 & $1.65 \mathrm{E}-15$ & $2.20 \mathrm{E}-08$ & $1.93 \mathrm{E}+02$ \\
\hline Br- $84 m$ & $2.66 \mathrm{E}-15$ & $3.54 \mathrm{E}-08$ & $3.10 \mathrm{E}+02$ \\
\hline $\mathrm{Br}-85$ & $1.83 \mathrm{E}-16$ & 2.44E-09 & $2.14 \mathrm{E}+01$ \\
\hline $\mathrm{Kr}-74$ & $1.07 \mathrm{E}-15$ & $1.43 \mathrm{E}-08$ & $1.25 \mathrm{E}+02$ \\
\hline $\mathrm{Kr}-75$ & $1.37 \mathrm{E}-15$ & $1.82 \mathrm{E}-08$ & $1.60 \mathrm{E}+02$ \\
\hline
\end{tabular}


SRNL-STI-2013-00115

\begin{tabular}{|c|c|c|c|}
\hline Nuclide & $\begin{array}{c}\text { Ground } \\
\text { Shine Dose } \\
\text { Rate } \\
\text { Coefficient } \\
\text { (Sv-m } / \mathbf{B q}-\mathbf{s})\end{array}$ & $\begin{array}{l}\text { Ground Shine } \\
\text { Dose Rate } \\
\text { Coefficient } \\
\text { mrem-m }{ }^{2} / p C i-h\end{array}$ & $\begin{array}{l}\text { Ground Shine } \\
\text { Dose Rate } \\
\text { Coefficient } \\
\text { mrem-m }{ }^{2} / \mu \mathrm{Ci}-\mathrm{y}\end{array}$ \\
\hline $\mathrm{Kr}-76$ & $3.96 \mathrm{E}-16$ & $5.27 \mathrm{E}-09$ & $4.62 \mathrm{E}+01$ \\
\hline $\mathrm{Kr}-77$ & $1.06 \mathrm{E}-15$ & $1.41 \mathrm{E}-08$ & $1.24 \mathrm{E}+02$ \\
\hline $\mathrm{Kr}-79$ & $2.37 \mathrm{E}-16$ & $3.16 \mathrm{E}-09$ & $2.77 \mathrm{E}+01$ \\
\hline $\mathrm{Kr}-81$ & $1.57 \mathrm{E}-18$ & $2.09 \mathrm{E}-11$ & $1.83 \mathrm{E}-01$ \\
\hline $\mathrm{Kr}-81 \mathrm{~m}$ & $1.18 \mathrm{E}-16$ & $1.57 \mathrm{E}-09$ & $1.38 \mathrm{E}+01$ \\
\hline $\mathrm{Kr}-83 \mathrm{~m}$ & $3.26 \mathrm{E}-19$ & $4.34 \mathrm{E}-12$ & $3.80 \mathrm{E}-02$ \\
\hline $\mathrm{Kr}-85$ & $1.05 \mathrm{E}-17$ & $1.40 \mathrm{E}-10$ & $1.23 \mathrm{E}+00$ \\
\hline $\mathrm{Kr}-85 \mathrm{~m}$ & $1.56 \mathrm{E}-16$ & $2.08 \mathrm{E}-09$ & $1.82 \mathrm{E}+01$ \\
\hline $\mathrm{Kr}-87$ & $8.38 \mathrm{E}-16$ & $1.12 \mathrm{E}-08$ & $9.78 \mathrm{E}+01$ \\
\hline $\mathrm{Kr}-88$ & $1.72 \mathrm{E}-15$ & $2.29 \mathrm{E}-08$ & $2.01 \mathrm{E}+02$ \\
\hline $\mathrm{Kr}-89$ & $1.84 \mathrm{E}-15$ & $2.45 \mathrm{E}-08$ & $2.15 \mathrm{E}+02$ \\
\hline $\mathrm{Rb}-77$ & $1.62 \mathrm{E}-15$ & $2.16 \mathrm{E}-08$ & $1.89 \mathrm{E}+02$ \\
\hline $\mathrm{Rb}-78$ & $3.67 \mathrm{E}-15$ & $4.89 \mathrm{E}-08$ & $4.28 \mathrm{E}+02$ \\
\hline $\mathrm{Rb}-78 \mathrm{~m}$ & $3.11 \mathrm{E}-15$ & 4.14E-08 & $3.63 \mathrm{E}+02$ \\
\hline $\mathrm{Rb}-79$ & $1.46 \mathrm{E}-15$ & $1.94 \mathrm{E}-08$ & $1.70 \mathrm{E}+02$ \\
\hline $\mathrm{Rb}-80$ & $1.33 \mathrm{E}-15$ & $1.77 \mathrm{E}-08$ & $1.55 \mathrm{E}+02$ \\
\hline $\mathrm{Rb}-81$ & $4.90 \mathrm{E}-16$ & $6.53 \mathrm{E}-09$ & $5.72 \mathrm{E}+01$ \\
\hline $\mathrm{Rb}-81 \mathrm{~m}$ & $2.43 \mathrm{E}-17$ & $3.24 \mathrm{E}-10$ & $2.84 \mathrm{E}+00$ \\
\hline $\mathrm{Rb}-82$ & $1.21 \mathrm{E}-15$ & $1.61 \mathrm{E}-08$ & $1.41 \mathrm{E}+02$ \\
\hline $\mathrm{Rb}-82 \mathrm{~m}$ & $2.74 \mathrm{E}-15$ & $3.65 \mathrm{E}-08$ & $3.20 \mathrm{E}+02$ \\
\hline $\mathrm{Rb}-83$ & $4.64 \mathrm{E}-16$ & $6.18 \mathrm{E}-09$ & $5.41 \mathrm{E}+01$ \\
\hline $\mathrm{Rb}-84$ & $8.70 \mathrm{E}-16$ & $1.16 \mathrm{E}-08$ & $1.02 \mathrm{E}+02$ \\
\hline $\mathrm{Rb}-84 \mathrm{~m}$ & $3.58 \mathrm{E}-16$ & 4.77E-09 & $4.18 \mathrm{E}+01$ \\
\hline $\mathrm{Rb}-86$ & $1.65 \mathrm{E}-16$ & $2.20 \mathrm{E}-09$ & $1.93 \mathrm{E}+01$ \\
\hline $\mathrm{Rb}-86 \mathrm{~m}$ & $5.23 \mathrm{E}-16$ & $6.97 \mathrm{E}-09$ & $6.10 \mathrm{E}+01$ \\
\hline $\mathrm{Rb}-87$ & 7.89E-20 & $1.05 \mathrm{E}-12$ & $9.21 \mathrm{E}-03$ \\
\hline $\mathrm{Rb}-88$ & $7.48 \mathrm{E}-16$ & $9.96 \mathrm{E}-09$ & $8.73 \mathrm{E}+01$ \\
\hline $\mathrm{Rb}-89$ & $2.12 \mathrm{E}-15$ & $2.82 \mathrm{E}-08$ & $2.47 \mathrm{E}+02$ \\
\hline $\mathrm{Rb}-90$ & $1.84 \mathrm{E}-15$ & $2.45 \mathrm{E}-08$ & $2.15 \mathrm{E}+02$ \\
\hline $\mathrm{Rb}-90 \mathrm{~m}$ & $2.95 \mathrm{E}-15$ & $3.93 \mathrm{E}-08$ & $3.44 \mathrm{E}+02$ \\
\hline Sr-79 & $1.30 \mathrm{E}-15$ & $1.73 \mathrm{E}-08$ & $1.52 \mathrm{E}+02$ \\
\hline Sr- 80 & $4.13 \mathrm{E}-16$ & $5.50 \mathrm{E}-09$ & $4.82 \mathrm{E}+01$ \\
\hline Sr-81 & $1.43 \mathrm{E}-15$ & $1.90 \mathrm{E}-08$ & $1.67 \mathrm{E}+02$ \\
\hline Sr-82 & $1.52 \mathrm{E}-18$ & $2.02 \mathrm{E}-11$ & $1.77 \mathrm{E}-01$ \\
\hline Sr-83 & $7.80 \mathrm{E}-16$ & $1.04 \mathrm{E}-08$ & $9.10 \mathrm{E}+01$ \\
\hline Sr-85 & $4.72 \mathrm{E}-16$ & $6.29 \mathrm{E}-09$ & $5.51 \mathrm{E}+01$ \\
\hline Sr- $85 \mathrm{~m}$ & $2.00 \mathrm{E}-16$ & $2.66 \mathrm{E}-09$ & $2.33 \mathrm{E}+01$ \\
\hline Sr- $87 \mathrm{~m}$ & $3.03 \mathrm{E}-16$ & $4.04 \mathrm{E}-09$ & $3.54 \mathrm{E}+01$ \\
\hline Sr-89 & $6.88 \mathrm{E}-17$ & $9.16 \mathrm{E}-10$ & $8.03 \mathrm{E}+00$ \\
\hline
\end{tabular}


SRNL-STI-2013-00115

\begin{tabular}{|c|c|c|c|}
\hline Nuclide & $\begin{array}{c}\text { Ground } \\
\text { Shine Dose } \\
\text { Rate } \\
\text { Coefficient } \\
\left(\mathrm{Sv}-\mathbf{m}^{2} / \mathbf{B q}-\mathbf{s}\right)\end{array}$ & $\begin{array}{l}\text { Ground Shine } \\
\text { Dose Rate } \\
\text { Coefficient } \\
\text { mrem-m²/pCi-h }\end{array}$ & $\begin{array}{c}\text { Ground Shine } \\
\text { Dose Rate } \\
\text { Coefficient } \\
\text { mrem-m }{ }^{2} / \mu \mathrm{Ci}-\mathrm{y}\end{array}$ \\
\hline Sr-90 & $1.64 \mathrm{E}-18$ & $2.18 \mathrm{E}-11$ & $1.91 \mathrm{E}-01$ \\
\hline Sr-91 & $7.35 \mathrm{E}-16$ & $9.79 \mathrm{E}-09$ & $8.58 \mathrm{E}+01$ \\
\hline Sr-92 & $1.22 \mathrm{E}-15$ & $1.63 \mathrm{E}-08$ & $1.42 \mathrm{E}+02$ \\
\hline Sr-93 & $2.17 \mathrm{E}-15$ & $2.89 \mathrm{E}-08$ & $2.53 \mathrm{E}+02$ \\
\hline Sr-94 & $1.40 \mathrm{E}-15$ & $1.86 \mathrm{E}-08$ & $1.63 \mathrm{E}+02$ \\
\hline $\mathrm{Y}-81$ & $1.29 \mathrm{E}-15$ & $1.72 \mathrm{E}-08$ & $1.51 \mathrm{E}+02$ \\
\hline Y-83 & $1.41 \mathrm{E}-15$ & $1.88 \mathrm{E}-08$ & $1.65 \mathrm{E}+02$ \\
\hline $\mathrm{Y}-83 \mathrm{~m}$ & $8.80 \mathrm{E}-16$ & $1.17 \mathrm{E}-08$ & $1.03 \mathrm{E}+02$ \\
\hline $\mathrm{Y}-84 \mathrm{~m}$ & $3.86 \mathrm{E}-15$ & 5.14E-08 & $4.50 \mathrm{E}+02$ \\
\hline Y-85 & $1.09 \mathrm{E}-15$ & $1.45 \mathrm{E}-08$ & $1.27 \mathrm{E}+02$ \\
\hline $\mathrm{Y}-85 \mathrm{~m}$ & $1.29 \mathrm{E}-15$ & $1.72 \mathrm{E}-08$ & $1.51 \mathrm{E}+02$ \\
\hline Y-86 & $3.31 \mathrm{E}-15$ & 4.41E-08 & $3.86 \mathrm{E}+02$ \\
\hline $\mathrm{Y}-86 \mathrm{~m}$ & $2.03 \mathrm{E}-16$ & $2.70 \mathrm{E}-09$ & $2.37 \mathrm{E}+01$ \\
\hline Y-87 & $4.20 \mathrm{E}-16$ & 5.59E-09 & $4.90 \mathrm{E}+01$ \\
\hline $\mathrm{Y}-87 \mathrm{~m}$ & $2.91 \mathrm{E}-16$ & $3.88 \mathrm{E}-09$ & $3.40 \mathrm{E}+01$ \\
\hline $\mathrm{Y}-88$ & $2.41 \mathrm{E}-15$ & $3.21 \mathrm{E}-08$ & $2.81 \mathrm{E}+02$ \\
\hline Y-89m & $8.49 \mathrm{E}-16$ & $1.13 \mathrm{E}-08$ & $9.91 \mathrm{E}+01$ \\
\hline Y-90 & $1.10 \mathrm{E}-16$ & $1.47 \mathrm{E}-09$ & $1.28 \mathrm{E}+01$ \\
\hline $\mathrm{Y}-90 \mathrm{~m}$ & $6.02 \mathrm{E}-16$ & 8.02E-09 & $7.02 \mathrm{E}+01$ \\
\hline Y-91 & $7.43 \mathrm{E}-17$ & $9.90 \mathrm{E}-10$ & $8.67 \mathrm{E}+00$ \\
\hline Y-91m & $5.08 \mathrm{E}-16$ & $6.77 \mathrm{E}-09$ & $5.93 \mathrm{E}+01$ \\
\hline $\mathrm{Y}-92$ & $3.83 \mathrm{E}-16$ & $5.10 \mathrm{E}-09$ & $4.47 \mathrm{E}+01$ \\
\hline Y-93 & $2.17 \mathrm{E}-16$ & 2.89E-09 & $2.53 \mathrm{E}+01$ \\
\hline Y-94 & $8.90 \mathrm{E}-16$ & 1.19E-08 & $1.04 \mathrm{E}+02$ \\
\hline $\mathrm{Y}-95$ & $1.09 \mathrm{E}-15$ & $1.45 \mathrm{E}-08$ & $1.27 \mathrm{E}+02$ \\
\hline $\mathrm{Zr}-85$ & $1.54 \mathrm{E}-15$ & $2.05 \mathrm{E}-08$ & $1.80 \mathrm{E}+02$ \\
\hline Zr-86 & $2.63 \mathrm{E}-16$ & $3.50 \mathrm{E}-09$ & $3.07 \mathrm{E}+01$ \\
\hline Zr-87 & $9.81 \mathrm{E}-16$ & $1.31 \mathrm{E}-08$ & $1.14 \mathrm{E}+02$ \\
\hline Zr-88 & $3.66 \mathrm{E}-16$ & $4.88 \mathrm{E}-09$ & $4.27 \mathrm{E}+01$ \\
\hline Zr-89 & $1.10 \mathrm{E}-15$ & $1.47 \mathrm{E}-08$ & $1.28 \mathrm{E}+02$ \\
\hline Zr-89m & $6.04 \mathrm{E}-16$ & $8.05 \mathrm{E}-09$ & $7.05 \mathrm{E}+01$ \\
\hline Zr-93 & $0.00 \mathrm{E}+00$ & $0.00 \mathrm{E}+00$ & $0.00 \mathrm{E}+00$ \\
\hline Zr-95 & $6.96 \mathrm{E}-16$ & $9.27 \mathrm{E}-09$ & $8.12 \mathrm{E}+01$ \\
\hline Zr-97 & $9.18 \mathrm{E}-16$ & $1.22 \mathrm{E}-08$ & $1.07 \mathrm{E}+02$ \\
\hline $\mathrm{Nb}-87$ & $1.32 \mathrm{E}-15$ & $1.76 \mathrm{E}-08$ & $1.54 \mathrm{E}+02$ \\
\hline $\mathrm{Nb}-88$ & $4.11 \mathrm{E}-15$ & $5.47 \mathrm{E}-08$ & $4.80 \mathrm{E}+02$ \\
\hline $\mathrm{Nb}-88 \mathrm{~m}$ & 3.99E-15 & 5.31E-08 & $4.66 \mathrm{E}+02$ \\
\hline $\mathrm{Nb}-89$ & $1.37 \mathrm{E}-15$ & $1.82 \mathrm{E}-08$ & $1.60 \mathrm{E}+02$ \\
\hline $\mathrm{Nb}-89 \mathrm{~m}$ & $1.34 \mathrm{E}-15$ & $1.78 \mathrm{E}-08$ & $1.56 \mathrm{E}+02$ \\
\hline
\end{tabular}


SRNL-STI-2013-00115

\begin{tabular}{|c|c|c|c|}
\hline Nuclide & $\begin{array}{c}\text { Ground } \\
\text { Shine Dose } \\
\text { Rate } \\
\text { Coefficient } \\
\text { (Sv-m } / \mathbf{B q}-\mathbf{s})\end{array}$ & $\begin{array}{l}\text { Ground Shine } \\
\text { Dose Rate } \\
\text { Coefficient } \\
\text { mrem-m }{ }^{2} / p C i-h\end{array}$ & $\begin{array}{c}\text { Ground Shine } \\
\text { Dose Rate } \\
\text { Coefficient } \\
\text { mrem-m }{ }^{2} / \mu \mathrm{Ci}-\mathrm{y}\end{array}$ \\
\hline $\mathrm{Nb}-90$ & $3.78 \mathrm{E}-15$ & $5.03 \mathrm{E}-08$ & $4.41 \mathrm{E}+02$ \\
\hline $\mathrm{Nb}-91$ & $4.74 \mathrm{E}-18$ & $6.31 \mathrm{E}-11$ & $5.53 \mathrm{E}-01$ \\
\hline $\mathrm{Nb}-91 \mathrm{~m}$ & $2.62 \mathrm{E}-17$ & $3.49 \mathrm{E}-10$ & $3.06 \mathrm{E}+00$ \\
\hline $\mathrm{Nb}-92$ & $1.42 \mathrm{E}-15$ & $1.89 \mathrm{E}-08$ & $1.66 \mathrm{E}+02$ \\
\hline $\mathrm{Nb}-92 \mathrm{~m}$ & $9.03 \mathrm{E}-16$ & $1.20 \mathrm{E}-08$ & $1.05 \mathrm{E}+02$ \\
\hline $\mathrm{Nb}-93 \mathrm{~m}$ & $6.83 \mathrm{E}-19$ & $9.10 \mathrm{E}-12$ & $7.97 \mathrm{E}-02$ \\
\hline $\mathrm{Nb}-94$ & $1.48 \mathrm{E}-15$ & $1.97 \mathrm{E}-08$ & $1.73 \mathrm{E}+02$ \\
\hline $\mathrm{Nb}-94 \mathrm{~m}$ & $6.97 \mathrm{E}-18$ & $9.28 \mathrm{E}-11$ & $8.13 \mathrm{E}-01$ \\
\hline $\mathrm{Nb}-95$ & $7.26 \mathrm{E}-16$ & $9.67 \mathrm{E}-09$ & $8.47 \mathrm{E}+01$ \\
\hline $\mathrm{Nb}-95 \mathrm{~m}$ & $6.25 \mathrm{E}-17$ & $8.33 \mathrm{E}-10$ & $7.29 \mathrm{E}+00$ \\
\hline $\mathrm{Nb}-96$ & $2.33 \mathrm{E}-15$ & $3.10 \mathrm{E}-08$ & $2.72 \mathrm{E}+02$ \\
\hline $\mathrm{Nb}-97$ & $6.82 \mathrm{E}-16$ & $9.08 \mathrm{E}-09$ & $7.96 \mathrm{E}+01$ \\
\hline $\mathrm{Nb}-98 \mathrm{~m}$ & $2.71 \mathrm{E}-15$ & $3.61 \mathrm{E}-08$ & $3.16 \mathrm{E}+02$ \\
\hline $\mathrm{Nb}-99$ & $3.08 \mathrm{E}-16$ & $4.10 \mathrm{E}-09$ & $3.59 \mathrm{E}+01$ \\
\hline $\mathrm{Nb}-99 \mathrm{~m}$ & $8.03 \mathrm{E}-16$ & $1.07 \mathrm{E}-08$ & $9.37 \mathrm{E}+01$ \\
\hline Mo-89 & $1.34 \mathrm{E}-15$ & $1.78 \mathrm{E}-08$ & $1.56 \mathrm{E}+02$ \\
\hline Mo-90 & $7.83 \mathrm{E}-16$ & $1.04 \mathrm{E}-08$ & $9.14 \mathrm{E}+01$ \\
\hline Mo-91 & $1.08 \mathrm{E}-15$ & $1.44 \mathrm{E}-08$ & $1.26 \mathrm{E}+02$ \\
\hline Mo-91m & $1.36 \mathrm{E}-15$ & $1.81 \mathrm{E}-08$ & $1.59 \mathrm{E}+02$ \\
\hline Мo-93 & $3.83 \mathrm{E}-18$ & $5.10 \mathrm{E}-11$ & $4.47 \mathrm{E}-01$ \\
\hline Mo-93m & $2.13 \mathrm{E}-15$ & $2.84 \mathrm{E}-08$ & $2.49 \mathrm{E}+02$ \\
\hline Mo-99 & $1.77 \mathrm{E}-16$ & $2.36 \mathrm{E}-09$ & $2.07 \mathrm{E}+01$ \\
\hline Mo-101 & $1.40 \mathrm{E}-15$ & $1.86 \mathrm{E}-08$ & $1.63 \mathrm{E}+02$ \\
\hline Mo-102 & $4.41 \mathrm{E}-17$ & $5.87 \mathrm{E}-10$ & $5.15 \mathrm{E}+00$ \\
\hline Tc-91 & $2.41 \mathrm{E}-15$ & $3.21 \mathrm{E}-08$ & $2.81 \mathrm{E}+02$ \\
\hline Tc-91m & $1.53 \mathrm{E}-15$ & $2.04 \mathrm{E}-08$ & $1.79 \mathrm{E}+02$ \\
\hline Tc-92 & $3.71 \mathrm{E}-15$ & 4.94E-08 & $4.33 \mathrm{E}+02$ \\
\hline Tc-93 & $1.42 \mathrm{E}-15$ & $1.89 \mathrm{E}-08$ & $1.66 \mathrm{E}+02$ \\
\hline Tc-93m & $8.31 \mathrm{E}-16$ & $1.11 \mathrm{E}-08$ & $9.70 \mathrm{E}+01$ \\
\hline Tc-94 & $2.51 \mathrm{E}-15$ & $3.34 \mathrm{E}-08$ & $2.93 \mathrm{E}+02$ \\
\hline Tc-94m & $1.91 \mathrm{E}-15$ & $2.54 \mathrm{E}-08$ & $2.23 \mathrm{E}+02$ \\
\hline Tc-95 & $7.48 \mathrm{E}-16$ & $9.96 \mathrm{E}-09$ & $8.73 \mathrm{E}+01$ \\
\hline Tc-95m & $6.43 \mathrm{E}-16$ & $8.56 \mathrm{E}-09$ & $7.50 \mathrm{E}+01$ \\
\hline Tc-96 & $2.36 \mathrm{E}-15$ & $3.14 \mathrm{E}-08$ & $2.75 \mathrm{E}+02$ \\
\hline Tc-96m & $4.16 \mathrm{E}-17$ & $5.54 \mathrm{E}-10$ & $4.85 \mathrm{E}+00$ \\
\hline Tc-97 & $4.56 \mathrm{E}-18$ & $6.07 \mathrm{E}-11$ & 5.32E-01 \\
\hline Tc-97m & $4.37 \mathrm{E}-18$ & $5.82 \mathrm{E}-11$ & $5.10 \mathrm{E}-01$ \\
\hline Tc-98 & $1.34 \mathrm{E}-15$ & $1.78 \mathrm{E}-08$ & $1.56 \mathrm{E}+02$ \\
\hline Tc-99 & $6.55 \mathrm{E}-20$ & $8.72 \mathrm{E}-13$ & 7.64E-03 \\
\hline
\end{tabular}


SRNL-STI-2013-00115

\begin{tabular}{|c|c|c|c|}
\hline Nuclide & $\begin{array}{c}\text { Ground } \\
\text { Shine Dose } \\
\text { Rate } \\
\text { Coefficient } \\
\left(\mathrm{Sv}-\mathrm{m}^{2} / \mathbf{B q}-\mathbf{s}\right)\end{array}$ & $\begin{array}{l}\text { Ground Shine } \\
\text { Dose Rate } \\
\text { Coefficient } \\
\text { mrem-m }{ }^{2} / \mathbf{p C i}-\mathrm{h}\end{array}$ & $\begin{array}{l}\text { Ground Shine } \\
\text { Dose Rate } \\
\text { Coefficient } \\
\text { mrem-m } / \mu \mathrm{Ci}-\mathrm{y}\end{array}$ \\
\hline Tc-99m & $1.14 \mathrm{E}-16$ & $1.52 \mathrm{E}-09$ & $1.33 \mathrm{E}+01$ \\
\hline Tc-101 & $3.66 \mathrm{E}-16$ & $4.88 \mathrm{E}-09$ & $4.27 \mathrm{E}+01$ \\
\hline Tc-102 & $2.59 \mathrm{E}-16$ & $3.45 \mathrm{E}-09$ & $3.02 \mathrm{E}+01$ \\
\hline Tc-102m & $2.34 \mathrm{E}-15$ & $3.12 \mathrm{E}-08$ & $2.73 \mathrm{E}+02$ \\
\hline Tc-104 & $2.17 \mathrm{E}-15$ & $2.89 \mathrm{E}-08$ & $2.53 \mathrm{E}+02$ \\
\hline Tc-105 & $8.68 \mathrm{E}-16$ & $1.16 \mathrm{E}-08$ & $1.01 \mathrm{E}+02$ \\
\hline Ru-92 & $2.00 \mathrm{E}-15$ & $2.66 \mathrm{E}-08$ & $2.33 \mathrm{E}+02$ \\
\hline Ru-94 & $4.85 \mathrm{E}-16$ & $6.46 \mathrm{E}-09$ & $5.66 \mathrm{E}+01$ \\
\hline $\mathrm{Ru}-95$ & $1.16 \mathrm{E}-15$ & $1.55 \mathrm{E}-08$ & $1.35 \mathrm{E}+02$ \\
\hline $\mathrm{Ru}-97$ & $2.17 \mathrm{E}-16$ & 2.89E-09 & $2.53 \mathrm{E}+01$ \\
\hline $\mathrm{Ru}-103$ & $4.74 \mathrm{E}-16$ & $6.31 \mathrm{E}-09$ & $5.53 \mathrm{E}+01$ \\
\hline $\mathrm{Ru}-105$ & $7.48 \mathrm{E}-16$ & $9.96 \mathrm{E}-09$ & $8.73 \mathrm{E}+01$ \\
\hline $\mathrm{Ru}-106$ & $0.00 \mathrm{E}+00$ & $0.00 \mathrm{E}+00$ & $0.00 \mathrm{E}+00$ \\
\hline $\mathrm{Ru}-107$ & $4.42 \mathrm{E}-16$ & 5.89E-09 & $5.16 \mathrm{E}+01$ \\
\hline $\mathrm{Ru}-108$ & $1.05 \mathrm{E}-16$ & $1.40 \mathrm{E}-09$ & $1.23 \mathrm{E}+01$ \\
\hline Rh-94 & $3.70 \mathrm{E}-15$ & $4.93 \mathrm{E}-08$ & $4.32 \mathrm{E}+02$ \\
\hline Rh-95 & $2.45 \mathrm{E}-15$ & $3.26 \mathrm{E}-08$ & $2.86 \mathrm{E}+02$ \\
\hline Rh-95m & $8.24 \mathrm{E}-16$ & $1.10 \mathrm{E}-08$ & $9.61 \mathrm{E}+01$ \\
\hline Rh-96 & $3.77 \mathrm{E}-15$ & 5.02E-08 & $4.40 \mathrm{E}+02$ \\
\hline Rh-96m & $1.24 \mathrm{E}-15$ & $1.65 \mathrm{E}-08$ & $1.45 \mathrm{E}+02$ \\
\hline Rh-97 & $1.42 \mathrm{E}-15$ & $1.89 \mathrm{E}-08$ & $1.66 \mathrm{E}+02$ \\
\hline Rh-97m & $1.97 \mathrm{E}-15$ & $2.62 \mathrm{E}-08$ & $2.30 \mathrm{E}+02$ \\
\hline Rh-98 & $1.85 \mathrm{E}-15$ & $2.46 \mathrm{E}-08$ & $2.16 \mathrm{E}+02$ \\
\hline Rh-99 & $5.23 \mathrm{E}-16$ & $6.97 \mathrm{E}-09$ & $6.10 \mathrm{E}+01$ \\
\hline Rh-99m & $6.08 \mathrm{E}-16$ & $8.10 \mathrm{E}-09$ & $7.09 \mathrm{E}+01$ \\
\hline Rh-100 & $2.46 \mathrm{E}-15$ & $3.28 \mathrm{E}-08$ & $2.87 \mathrm{E}+02$ \\
\hline Rh-100m & $5.15 \mathrm{E}-17$ & $6.86 \mathrm{E}-10$ & $6.01 \mathrm{E}+00$ \\
\hline Rh-101 & $2.57 \mathrm{E}-16$ & $3.42 \mathrm{E}-09$ & $3.00 \mathrm{E}+01$ \\
\hline Rh-101m & $2.65 \mathrm{E}-16$ & $3.53 \mathrm{E}-09$ & $3.09 \mathrm{E}+01$ \\
\hline Rh-102 & $4.94 \mathrm{E}-16$ & $6.58 \mathrm{E}-09$ & $5.76 \mathrm{E}+01$ \\
\hline $\mathrm{Rh}-102 \mathrm{~m}$ & $2.03 \mathrm{E}-15$ & $2.70 \mathrm{E}-08$ & $2.37 \mathrm{E}+02$ \\
\hline $\mathrm{Rh}-103 \mathrm{~m}$ & 8.26E-19 & $1.10 \mathrm{E}-11$ & $9.64 \mathrm{E}-02$ \\
\hline Rh-104 & $1.28 \mathrm{E}-16$ & $1.70 \mathrm{E}-09$ & $1.49 \mathrm{E}+01$ \\
\hline Rh-104m & $3.43 \mathrm{E}-17$ & $4.57 \mathrm{E}-10$ & $4.00 \mathrm{E}+00$ \\
\hline Rh-105 & $7.38 \mathrm{E}-17$ & $9.83 \mathrm{E}-10$ & $8.61 \mathrm{E}+00$ \\
\hline Rh-106 & $3.46 \mathrm{E}-16$ & $4.61 \mathrm{E}-09$ & $4.04 \mathrm{E}+01$ \\
\hline Rh-106m & $2.69 \mathrm{E}-15$ & $3.58 \mathrm{E}-08$ & $3.14 \mathrm{E}+02$ \\
\hline Rh-107 & $3.38 \mathrm{E}-16$ & $4.50 \mathrm{E}-09$ & $3.94 \mathrm{E}+01$ \\
\hline Rh-108 & $4.79 \mathrm{E}-16$ & $6.38 \mathrm{E}-09$ & $5.59 \mathrm{E}+01$ \\
\hline
\end{tabular}


SRNL-STI-2013-00115

\begin{tabular}{|c|c|c|c|}
\hline Nuclide & $\begin{array}{c}\text { Ground } \\
\text { Shine Dose } \\
\text { Rate } \\
\text { Coefficient } \\
\text { (Sv-m } / \mathbf{B q}-\mathbf{s})\end{array}$ & $\begin{array}{l}\text { Ground Shine } \\
\text { Dose Rate } \\
\text { Coefficient } \\
\text { mrem-m }{ }^{2} / p C i-h\end{array}$ & $\begin{array}{c}\text { Ground Shine } \\
\text { Dose Rate } \\
\text { Coefficient } \\
\text { mrem-m }{ }^{2} / \mu \mathrm{Ci}-\mathrm{y}\end{array}$ \\
\hline Rh-109 & $3.91 \mathrm{E}-16$ & $5.21 \mathrm{E}-09$ & $4.56 \mathrm{E}+01$ \\
\hline Pd-96 & $1.38 \mathrm{E}-15$ & $1.84 \mathrm{E}-08$ & $1.61 \mathrm{E}+02$ \\
\hline Pd-97 & $2.26 \mathrm{E}-15$ & $3.01 \mathrm{E}-08$ & $2.64 \mathrm{E}+02$ \\
\hline Pd-98 & $3.82 \mathrm{E}-16$ & $5.09 \mathrm{E}-09$ & $4.46 \mathrm{E}+01$ \\
\hline Pd-99 & $1.24 \mathrm{E}-15$ & $1.65 \mathrm{E}-08$ & $1.45 \mathrm{E}+02$ \\
\hline Pd-100 & $9.98 \mathrm{E}-17$ & 1.33E-09 & $1.16 \mathrm{E}+01$ \\
\hline Pd-101 & $3.21 \mathrm{E}-16$ & $4.28 \mathrm{E}-09$ & $3.75 \mathrm{E}+01$ \\
\hline Pd-103 & $7.66 \mathrm{E}-18$ & $1.02 \mathrm{E}-10$ & $8.94 \mathrm{E}-01$ \\
\hline Pd-107 & $0.00 \mathrm{E}+00$ & $0.00 \mathrm{E}+00$ & $0.00 \mathrm{E}+00$ \\
\hline Pd-109 & $3.72 \mathrm{E}-17$ & $4.96 \mathrm{E}-10$ & $4.34 \mathrm{E}+00$ \\
\hline Pd-109m & $1.00 \mathrm{E}-16$ & $1.33 \mathrm{E}-09$ & $1.17 \mathrm{E}+01$ \\
\hline Pd-111 & $1.46 \mathrm{E}-16$ & 1.94E-09 & $1.70 \mathrm{E}+01$ \\
\hline Pd-112 & 2.22E-18 & $2.96 \mathrm{E}-11$ & $2.59 \mathrm{E}-01$ \\
\hline Pd-114 & $8.36 \mathrm{E}-17$ & $1.11 \mathrm{E}-09$ & $9.75 \mathrm{E}+00$ \\
\hline Ag-99 & $2.28 \mathrm{E}-15$ & $3.04 \mathrm{E}-08$ & $2.66 \mathrm{E}+02$ \\
\hline Ag-100m & $2.81 \mathrm{E}-15$ & $3.74 \mathrm{E}-08$ & $3.28 \mathrm{E}+02$ \\
\hline Ag-101 & $1.57 \mathrm{E}-15$ & $2.09 \mathrm{E}-08$ & $1.83 \mathrm{E}+02$ \\
\hline Ag-102 & $3.24 \mathrm{E}-15$ & $4.32 \mathrm{E}-08$ & $3.78 \mathrm{E}+02$ \\
\hline Ag-102m & $1.81 \mathrm{E}-15$ & $2.41 \mathrm{E}-08$ & $2.11 \mathrm{E}+02$ \\
\hline Ag-103 & $8.05 \mathrm{E}-16$ & $1.07 \mathrm{E}-08$ & $9.39 \mathrm{E}+01$ \\
\hline Ag-104 & $2.54 \mathrm{E}-15$ & $3.38 \mathrm{E}-08$ & $2.96 \mathrm{E}+02$ \\
\hline Ag-104m & $1.74 \mathrm{E}-15$ & 2.32E-08 & $2.03 \mathrm{E}+02$ \\
\hline Ag-105 & $4.78 \mathrm{E}-16$ & $6.37 \mathrm{E}-09$ & $5.58 \mathrm{E}+01$ \\
\hline Ag-105m & $9.60 \mathrm{E}-19$ & $1.28 \mathrm{E}-11$ & $1.12 \mathrm{E}-01$ \\
\hline Ag-106 & $7.28 \mathrm{E}-16$ & $9.70 \mathrm{E}-09$ & $8.49 \mathrm{E}+01$ \\
\hline Ag-106m & $2.63 \mathrm{E}-15$ & $3.50 \mathrm{E}-08$ & $3.07 \mathrm{E}+02$ \\
\hline Ag-108 & $8.98 \mathrm{E}-17$ & $1.20 \mathrm{E}-09$ & $1.05 \mathrm{E}+01$ \\
\hline Ag-108m & $1.54 \mathrm{E}-15$ & $2.05 \mathrm{E}-08$ & $1.80 \mathrm{E}+02$ \\
\hline Ag-109m & $7.41 \mathrm{E}-18$ & $9.87 \mathrm{E}-11$ & $8.65 \mathrm{E}-01$ \\
\hline Ag-110 & $1.63 \mathrm{E}-16$ & $2.17 \mathrm{E}-09$ & $1.90 \mathrm{E}+01$ \\
\hline $\mathrm{Ag}-110 \mathrm{~m}$ & $2.59 \mathrm{E}-15$ & $3.45 \mathrm{E}-08$ & $3.02 \mathrm{E}+02$ \\
\hline Ag-111 & $5.29 \mathrm{E}-17$ & $7.05 \mathrm{E}-10$ & $6.17 \mathrm{E}+00$ \\
\hline Ag-111m & $5.79 \mathrm{E}-18$ & $7.71 \mathrm{E}-11$ & $6.76 \mathrm{E}-01$ \\
\hline Ag-112 & 7.72E-16 & $1.03 \mathrm{E}-08$ & $9.01 \mathrm{E}+01$ \\
\hline Ag-113 & $1.59 \mathrm{E}-16$ & $2.12 \mathrm{E}-09$ & $1.86 \mathrm{E}+01$ \\
\hline Ag-113m & $2.23 \mathrm{E}-16$ & $2.97 \mathrm{E}-09$ & $2.60 \mathrm{E}+01$ \\
\hline Ag-114 & $4.31 \mathrm{E}-16$ & $5.74 \mathrm{E}-09$ & $5.03 \mathrm{E}+01$ \\
\hline Ag-115 & $5.54 \mathrm{E}-16$ & 7.38E-09 & $6.46 \mathrm{E}+01$ \\
\hline Ag-116 & $2.07 \mathrm{E}-15$ & $2.76 \mathrm{E}-08$ & $2.42 \mathrm{E}+02$ \\
\hline
\end{tabular}


SRNL-STI-2013-00115

\begin{tabular}{|c|c|c|c|}
\hline Nuclide & $\begin{array}{c}\text { Ground } \\
\text { Shine Dose } \\
\text { Rate } \\
\text { Coefficient } \\
\left(\mathrm{Sv}-\mathbf{m}^{2} / \mathbf{B q}-\mathbf{s}\right)\end{array}$ & $\begin{array}{l}\text { Ground Shine } \\
\text { Dose Rate } \\
\text { Coefficient } \\
\text { mrem-m²/pCi-h }\end{array}$ & $\begin{array}{c}\text { Ground Shine } \\
\text { Dose Rate } \\
\text { Coefficient } \\
\text { mrem-m }{ }^{2} / \mu \mathrm{Ci}-\mathrm{y}\end{array}$ \\
\hline Ag-117 & $1.28 \mathrm{E}-15$ & $1.70 \mathrm{E}-08$ & $1.49 \mathrm{E}+02$ \\
\hline Cd-101 & $2.39 \mathrm{E}-15$ & $3.18 \mathrm{E}-08$ & $2.79 \mathrm{E}+02$ \\
\hline Cd-102 & $7.91 \mathrm{E}-16$ & $1.05 \mathrm{E}-08$ & $9.23 \mathrm{E}+01$ \\
\hline $\mathrm{Cd}-103$ & $1.91 \mathrm{E}-15$ & $2.54 \mathrm{E}-08$ & $2.23 \mathrm{E}+02$ \\
\hline Cd-104 & $2.29 \mathrm{E}-16$ & $3.05 \mathrm{E}-09$ & $2.67 \mathrm{E}+01$ \\
\hline Cd-105 & $1.20 \mathrm{E}-15$ & $1.60 \mathrm{E}-08$ & $1.40 \mathrm{E}+02$ \\
\hline Cd-107 & $2.28 \mathrm{E}-17$ & $3.04 \mathrm{E}-10$ & $2.66 \mathrm{E}+00$ \\
\hline Cd-109 & $1.65 \mathrm{E}-17$ & $2.20 \mathrm{E}-10$ & $1.93 \mathrm{E}+00$ \\
\hline $\mathrm{Cd}-111 \mathrm{~m}$ & $2.60 \mathrm{E}-16$ & $3.46 \mathrm{E}-09$ & $3.03 \mathrm{E}+01$ \\
\hline Cd-113 & $5.74 \mathrm{E}-20$ & $7.65 \mathrm{E}-13$ & $6.70 \mathrm{E}-03$ \\
\hline $\mathrm{Cd}-113 \mathrm{~m}$ & $1.78 \mathrm{E}-18$ & $2.37 \mathrm{E}-11$ & $2.08 \mathrm{E}-01$ \\
\hline $\mathrm{Cd}-115$ & $2.07 \mathrm{E}-16$ & $2.76 \mathrm{E}-09$ & $2.42 \mathrm{E}+01$ \\
\hline $\mathrm{Cd}-115 \mathrm{~m}$ & $1.02 \mathrm{E}-16$ & $1.36 \mathrm{E}-09$ & $1.19 \mathrm{E}+01$ \\
\hline $\mathrm{Cd}-117$ & $1.03 \mathrm{E}-15$ & $1.37 \mathrm{E}-08$ & $1.20 \mathrm{E}+02$ \\
\hline $\mathrm{Cd}-117 \mathrm{~m}$ & $1.84 \mathrm{E}-15$ & $2.45 \mathrm{E}-08$ & $2.15 \mathrm{E}+02$ \\
\hline $\mathrm{Cd}-118$ & $5.80 \mathrm{E}-19$ & 7.73E-12 & $6.77 \mathrm{E}-02$ \\
\hline Cd-119 & $1.56 \mathrm{E}-15$ & $2.08 \mathrm{E}-08$ & $1.82 \mathrm{E}+02$ \\
\hline $\mathrm{Cd}-119 \mathrm{~m}$ & $2.15 \mathrm{E}-15$ & $2.86 \mathrm{E}-08$ & $2.51 \mathrm{E}+02$ \\
\hline In-103 & $2.67 \mathrm{E}-15$ & $3.56 \mathrm{E}-08$ & $3.12 \mathrm{E}+02$ \\
\hline In-105 & $1.90 \mathrm{E}-15$ & $2.53 \mathrm{E}-08$ & $2.22 \mathrm{E}+02$ \\
\hline In-106m & $2.73 \mathrm{E}-15$ & $3.64 \mathrm{E}-08$ & $3.19 \mathrm{E}+02$ \\
\hline In-106 & $3.47 \mathrm{E}-15$ & 4.62E-08 & $4.05 \mathrm{E}+02$ \\
\hline In-107 & $1.43 \mathrm{E}-15$ & $1.90 \mathrm{E}-08$ & $1.67 \mathrm{E}+02$ \\
\hline In-108m & $2.52 \mathrm{E}-15$ & $3.36 \mathrm{E}-08$ & $2.94 \mathrm{E}+02$ \\
\hline In-108 & $3.66 \mathrm{E}-15$ & $4.88 \mathrm{E}-08$ & $4.27 \mathrm{E}+02$ \\
\hline In-109m & $5.86 \mathrm{E}-16$ & 7.81E-09 & $6.84 \mathrm{E}+01$ \\
\hline In-109 & 5.94E-16 & 7.91E-09 & $6.93 \mathrm{E}+01$ \\
\hline In-110m & $1.55 \mathrm{E}-15$ & $2.06 \mathrm{E}-08$ & $1.81 \mathrm{E}+02$ \\
\hline In-110 & $2.92 \mathrm{E}-15$ & $3.89 \mathrm{E}-08$ & $3.41 \mathrm{E}+02$ \\
\hline In-111m & $4.56 \mathrm{E}-16$ & $6.07 \mathrm{E}-09$ & $5.32 \mathrm{E}+01$ \\
\hline In-111 & $3.69 \mathrm{E}-16$ & 4.92E-09 & $4.31 \mathrm{E}+01$ \\
\hline In-112m & $2.80 \mathrm{E}-17$ & $3.73 \mathrm{E}-10$ & $3.27 \mathrm{E}+00$ \\
\hline In-112 & $2.74 \mathrm{E}-16$ & $3.65 \mathrm{E}-09$ & $3.20 \mathrm{E}+01$ \\
\hline In-113m & $2.46 \mathrm{E}-16$ & $3.28 \mathrm{E}-09$ & $2.87 \mathrm{E}+01$ \\
\hline In-114m & $7.28 \mathrm{E}-17$ & $9.70 \mathrm{E}-10$ & $8.49 \mathrm{E}+00$ \\
\hline In-114 & $9.64 \mathrm{E}-17$ & $1.28 \mathrm{E}-09$ & $1.12 \mathrm{E}+01$ \\
\hline In-115m & $1.52 \mathrm{E}-16$ & $2.02 \mathrm{E}-09$ & $1.77 \mathrm{E}+01$ \\
\hline In-115 & $3.74 \mathrm{E}-19$ & $4.98 \mathrm{E}-12$ & $4.36 \mathrm{E}-02$ \\
\hline In-116m & $2.27 \mathrm{E}-15$ & $3.02 \mathrm{E}-08$ & $2.65 \mathrm{E}+02$ \\
\hline
\end{tabular}


SRNL-STI-2013-00115

\begin{tabular}{|c|c|c|c|}
\hline Nuclide & $\begin{array}{c}\text { Ground } \\
\text { Shine Dose } \\
\text { Rate } \\
\text { Coefficient } \\
\left(\mathrm{Sv}-\mathbf{m}^{2} / \mathbf{B q}-\mathbf{s}\right)\end{array}$ & $\begin{array}{l}\text { Ground Shine } \\
\text { Dose Rate } \\
\text { Coefficient } \\
\text { mrem-m } / \mathbf{p C i} \text {-h }\end{array}$ & $\begin{array}{l}\text { Ground Shine } \\
\text { Dose Rate } \\
\text { Coefficient } \\
\text { mrem-m }{ }^{2} / \mu \mathrm{Ci}-\mathrm{y}\end{array}$ \\
\hline In-117m & $1.25 \mathrm{E}-16$ & $1.67 \mathrm{E}-09$ & $1.46 \mathrm{E}+01$ \\
\hline In-117 & $6.65 \mathrm{E}-16$ & $8.86 \mathrm{E}-09$ & $7.76 \mathrm{E}+01$ \\
\hline In-118m & $2.65 \mathrm{E}-15$ & $3.53 \mathrm{E}-08$ & $3.09 \mathrm{E}+02$ \\
\hline In-118 & $2.52 \mathrm{E}-16$ & $3.36 \mathrm{E}-09$ & $2.94 \mathrm{E}+01$ \\
\hline In-119m & $1.77 \mathrm{E}-16$ & $2.36 \mathrm{E}-09$ & $2.07 \mathrm{E}+01$ \\
\hline In-119 & 7.99E-16 & $1.06 \mathrm{E}-08$ & $9.32 \mathrm{E}+01$ \\
\hline In- $121 \mathrm{~m}$ & $2.12 \mathrm{E}-16$ & 2.82E-09 & $2.47 \mathrm{E}+01$ \\
\hline In-121 & $9.89 \mathrm{E}-16$ & $1.32 \mathrm{E}-08$ & $1.15 \mathrm{E}+02$ \\
\hline Sn-106 & $1.14 \mathrm{E}-15$ & $1.52 \mathrm{E}-08$ & $1.33 \mathrm{E}+02$ \\
\hline Sn-108 & $6.40 \mathrm{E}-16$ & $8.52 \mathrm{E}-09$ & $7.47 \mathrm{E}+01$ \\
\hline Sn-109 & $1.99 \mathrm{E}-15$ & $2.65 \mathrm{E}-08$ & $2.32 \mathrm{E}+02$ \\
\hline Sn-110 & $2.68 \mathrm{E}-16$ & 3.57E-09 & $3.13 \mathrm{E}+01$ \\
\hline Sn-111 & $4.78 \mathrm{E}-16$ & $6.37 \mathrm{E}-09$ & $5.58 \mathrm{E}+01$ \\
\hline Sn-113m & $9.32 \mathrm{E}-18$ & $1.24 \mathrm{E}-10$ & $1.09 \mathrm{E}+00$ \\
\hline Sn-113 & $1.70 \mathrm{E}-17$ & $2.26 \mathrm{E}-10$ & $1.98 \mathrm{E}+00$ \\
\hline Sn-117m & $1.40 \mathrm{E}-16$ & $1.86 \mathrm{E}-09$ & $1.63 \mathrm{E}+01$ \\
\hline Sn-119m & $9.81 \mathrm{E}-18$ & $1.31 \mathrm{E}-10$ & $1.14 \mathrm{E}+00$ \\
\hline Sn-121m & $3.65 \mathrm{E}-18$ & $4.86 \mathrm{E}-11$ & $4.26 \mathrm{E}-01$ \\
\hline Sn-121 & $9.05 \mathrm{E}-20$ & $1.21 \mathrm{E}-12$ & $1.06 \mathrm{E}-02$ \\
\hline Sn-123m & $1.75 \mathrm{E}-16$ & 2.33E-09 & $2.04 \mathrm{E}+01$ \\
\hline Sn-123 & $6.55 \mathrm{E}-17$ & $8.72 \mathrm{E}-10$ & $7.64 \mathrm{E}+00$ \\
\hline $\mathrm{Sn}-125 \mathrm{~m}$ & 4.23E-16 & $5.63 \mathrm{E}-09$ & $4.94 \mathrm{E}+01$ \\
\hline Sn-125 & $4.01 \mathrm{E}-16$ & $5.34 \mathrm{E}-09$ & $4.68 \mathrm{E}+01$ \\
\hline Sn-126 & $4.82 \mathrm{E}-17$ & $6.42 \mathrm{E}-10$ & $5.62 \mathrm{E}+00$ \\
\hline $\mathrm{Sn}-127 \mathrm{~m}$ & $6.65 \mathrm{E}-16$ & $8.86 \mathrm{E}-09$ & $7.76 \mathrm{E}+01$ \\
\hline Sn-127 & $1.80 \mathrm{E}-15$ & $2.40 \mathrm{E}-08$ & $2.10 \mathrm{E}+02$ \\
\hline Sn-128 & $5.67 \mathrm{E}-16$ & 7.55E-09 & $6.62 \mathrm{E}+01$ \\
\hline Sn-129 & $1.08 \mathrm{E}-15$ & $1.44 \mathrm{E}-08$ & $1.26 \mathrm{E}+02$ \\
\hline Sn-130m & $9.64 \mathrm{E}-16$ & $1.28 \mathrm{E}-08$ & $1.12 \mathrm{E}+02$ \\
\hline Sn-130 & $9.19 \mathrm{E}-16$ & $1.22 \mathrm{E}-08$ & $1.07 \mathrm{E}+02$ \\
\hline $\mathrm{Sb}-111$ & $1.55 \mathrm{E}-15$ & $2.06 \mathrm{E}-08$ & $1.81 \mathrm{E}+02$ \\
\hline Sb-113 & $1.29 \mathrm{E}-15$ & $1.72 \mathrm{E}-08$ & $1.51 \mathrm{E}+02$ \\
\hline Sb-114 & $2.61 \mathrm{E}-15$ & $3.48 \mathrm{E}-08$ & $3.05 \mathrm{E}+02$ \\
\hline Sb-115 & $8.72 \mathrm{E}-16$ & $1.16 \mathrm{E}-08$ & $1.02 \mathrm{E}+02$ \\
\hline Sb-116 & $2.15 \mathrm{E}-15$ & $2.86 \mathrm{E}-08$ & $2.51 \mathrm{E}+02$ \\
\hline Sb-116m & $2.89 \mathrm{E}-15$ & $3.85 \mathrm{E}-08$ & $3.37 \mathrm{E}+02$ \\
\hline Sb-117 & $1.66 \mathrm{E}-16$ & $2.21 \mathrm{E}-09$ & $1.94 \mathrm{E}+01$ \\
\hline Sb-118 & $8.67 \mathrm{E}-16$ & $1.15 \mathrm{E}-08$ & $1.01 \mathrm{E}+02$ \\
\hline Sb-118m & $2.41 \mathrm{E}-15$ & $3.21 \mathrm{E}-08$ & $2.81 \mathrm{E}+02$ \\
\hline
\end{tabular}


SRNL-STI-2013-00115

\begin{tabular}{|c|c|c|c|}
\hline Nuclide & $\begin{array}{c}\text { Ground } \\
\text { Shine Dose } \\
\text { Rate } \\
\text { Coefficient } \\
\text { (Sv-m } / \mathbf{B q}-\mathbf{s})\end{array}$ & $\begin{array}{l}\text { Ground Shine } \\
\text { Dose Rate } \\
\text { Coefficient } \\
\text { mrem-m²/pCi-h }\end{array}$ & $\begin{array}{c}\text { Ground Shine } \\
\text { Dose Rate } \\
\text { Coefficient } \\
\text { mrem-m }{ }^{2} / \mu \mathrm{Ci}-\mathrm{y}\end{array}$ \\
\hline Sb-119 & $1.56 \mathrm{E}-17$ & $2.08 \mathrm{E}-10$ & $1.82 \mathrm{E}+00$ \\
\hline Sb-120 & $4.67 \mathrm{E}-16$ & $6.22 \mathrm{E}-09$ & $5.45 \mathrm{E}+01$ \\
\hline $\mathrm{Sb}-120 \mathrm{~m}$ & $2.28 \mathrm{E}-15$ & $3.04 \mathrm{E}-08$ & $2.66 \mathrm{E}+02$ \\
\hline $\mathrm{Sb}-122$ & $4.88 \mathrm{E}-16$ & $6.50 \mathrm{E}-09$ & $5.69 \mathrm{E}+01$ \\
\hline Sb-122m & $5.88 \mathrm{E}-17$ & $7.83 \mathrm{E}-10$ & $6.86 \mathrm{E}+00$ \\
\hline Sb-124 & $1.73 \mathrm{E}-15$ & $2.30 \mathrm{E}-08$ & $2.02 \mathrm{E}+02$ \\
\hline Sb-124m & $4.30 \mathrm{E}-16$ & 5.73E-09 & $5.02 \mathrm{E}+01$ \\
\hline $\mathrm{Sb}-124 \mathrm{n}$ & $5.69 \mathrm{E}-22$ & $7.58 \mathrm{E}-15$ & $6.64 \mathrm{E}-05$ \\
\hline $\mathrm{Sb}-125$ & $4.14 \mathrm{E}-16$ & 5.51E-09 & $4.83 \mathrm{E}+01$ \\
\hline Sb-126 & $2.65 \mathrm{E}-15$ & $3.53 \mathrm{E}-08$ & $3.09 \mathrm{E}+02$ \\
\hline $\mathrm{Sb}-126 \mathrm{~m}$ & $1.55 \mathrm{E}-15$ & $2.06 \mathrm{E}-08$ & $1.81 \mathrm{E}+02$ \\
\hline Sb-127 & $6.80 \mathrm{E}-16$ & $9.06 \mathrm{E}-09$ & $7.93 \mathrm{E}+01$ \\
\hline Sb-128 & $2.98 \mathrm{E}-15$ & $3.97 \mathrm{E}-08$ & $3.48 \mathrm{E}+02$ \\
\hline $\mathrm{Sb}-128 \mathrm{~m}$ & $1.92 \mathrm{E}-15$ & $2.56 \mathrm{E}-08$ & $2.24 \mathrm{E}+02$ \\
\hline Sb-129 & $1.39 \mathrm{E}-15$ & $1.85 \mathrm{E}-08$ & $1.62 \mathrm{E}+02$ \\
\hline Sb-130 & $3.15 \mathrm{E}-15$ & 4.20E-08 & $3.68 \mathrm{E}+02$ \\
\hline $\mathrm{Sb}-130 \mathrm{~m}$ & $2.66 \mathrm{E}-15$ & $3.54 \mathrm{E}-08$ & $3.10 \mathrm{E}+02$ \\
\hline $\mathrm{Sb}-131$ & $1.96 \mathrm{E}-15$ & $2.61 \mathrm{E}-08$ & $2.29 \mathrm{E}+02$ \\
\hline Sb-133 & $2.54 \mathrm{E}-15$ & $3.38 \mathrm{E}-08$ & $2.96 \mathrm{E}+02$ \\
\hline Te-113 & $2.22 \mathrm{E}-15$ & $2.96 \mathrm{E}-08$ & $2.59 \mathrm{E}+02$ \\
\hline Te-114 & $1.18 \mathrm{E}-15$ & $1.57 \mathrm{E}-08$ & $1.38 \mathrm{E}+02$ \\
\hline Te-115 & $2.18 \mathrm{E}-15$ & $2.90 \mathrm{E}-08$ & $2.54 \mathrm{E}+02$ \\
\hline Te-115m & $2.48 \mathrm{E}-15$ & 3.30E-08 & $2.89 \mathrm{E}+02$ \\
\hline Te-116 & $9.70 \mathrm{E}-17$ & $1.29 \mathrm{E}-09$ & $1.13 \mathrm{E}+01$ \\
\hline Te-117 & $1.45 \mathrm{E}-15$ & $1.93 \mathrm{E}-08$ & $1.69 \mathrm{E}+02$ \\
\hline Te-118 & $1.42 \mathrm{E}-17$ & $1.89 \mathrm{E}-10$ & $1.66 \mathrm{E}+00$ \\
\hline Te-119 & $7.21 \mathrm{E}-16$ & $9.60 \mathrm{E}-09$ & $8.41 \mathrm{E}+01$ \\
\hline Te-119m & $1.38 \mathrm{E}-15$ & $1.84 \mathrm{E}-08$ & $1.61 \mathrm{E}+02$ \\
\hline Te-121 & $5.47 \mathrm{E}-16$ & 7.29E-09 & $6.38 \mathrm{E}+01$ \\
\hline Te-121m & $1.98 \mathrm{E}-16$ & 2.64E-09 & $2.31 \mathrm{E}+01$ \\
\hline Te-123 & $2.47 \mathrm{E}-20$ & $3.29 \mathrm{E}-13$ & $2.88 \mathrm{E}-03$ \\
\hline $\mathrm{Te}-123 \mathrm{~m}$ & $1.32 \mathrm{E}-16$ & $1.76 \mathrm{E}-09$ & $1.54 \mathrm{E}+01$ \\
\hline Te-125m & $2.68 \mathrm{E}-17$ & $3.57 \mathrm{E}-10$ & $3.13 \mathrm{E}+00$ \\
\hline Te-127 & $1.05 \mathrm{E}-17$ & $1.40 \mathrm{E}-10$ & $1.23 \mathrm{E}+00$ \\
\hline $\mathrm{Te}-127 \mathrm{~m}$ & $8.54 \mathrm{E}-18$ & $1.14 \mathrm{E}-10$ & $9.96 \mathrm{E}-01$ \\
\hline Te-129 & $1.16 \mathrm{E}-16$ & $1.55 \mathrm{E}-09$ & $1.35 \mathrm{E}+01$ \\
\hline Te-129m & 5.83E-17 & $7.77 \mathrm{E}-10$ & $6.80 \mathrm{E}+00$ \\
\hline Te-131 & $4.72 \mathrm{E}-16$ & $6.29 \mathrm{E}-09$ & $5.51 \mathrm{E}+01$ \\
\hline Te-131m & $1.36 \mathrm{E}-15$ & $1.81 \mathrm{E}-08$ & $1.59 \mathrm{E}+02$ \\
\hline
\end{tabular}


SRNL-STI-2013-00115

\begin{tabular}{|c|c|c|c|}
\hline Nuclide & $\begin{array}{c}\text { Ground } \\
\text { Shine Dose } \\
\text { Rate } \\
\text { Coefficient } \\
\left(\mathrm{Sv}-\mathbf{m}^{2} / \mathbf{B q}-\mathbf{s}\right)\end{array}$ & $\begin{array}{l}\text { Ground Shine } \\
\text { Dose Rate } \\
\text { Coefficient } \\
\text { mrem-m²/pCi-h }\end{array}$ & $\begin{array}{c}\text { Ground Shine } \\
\text { Dose Rate } \\
\text { Coefficient } \\
\text { mrem-m }{ }^{2} / \mu \mathrm{Ci}-\mathrm{y}\end{array}$ \\
\hline Te-132 & $2.13 \mathrm{E}-16$ & $2.84 \mathrm{E}-09$ & $2.49 \mathrm{E}+01$ \\
\hline Te-133 & $1.18 \mathrm{E}-15$ & $1.57 \mathrm{E}-08$ & $1.38 \mathrm{E}+02$ \\
\hline Te-133m & $1.76 \mathrm{E}-15$ & 2.34E-08 & $2.05 \mathrm{E}+02$ \\
\hline Te-134 & $8.25 \mathrm{E}-16$ & $1.10 \mathrm{E}-08$ & $9.63 \mathrm{E}+01$ \\
\hline $\mathrm{I}-118$ & $2.08 \mathrm{E}-15$ & $2.77 \mathrm{E}-08$ & $2.43 \mathrm{E}+02$ \\
\hline $\mathrm{I}-118 \mathrm{~m}$ & $3.67 \mathrm{E}-15$ & $4.89 \mathrm{E}-08$ & $4.28 \mathrm{E}+02$ \\
\hline $\mathrm{I}-119$ & $9.21 \mathrm{E}-16$ & $1.23 \mathrm{E}-08$ & $1.07 \mathrm{E}+02$ \\
\hline $\mathrm{I}-120$ & $2.52 \mathrm{E}-15$ & $3.36 \mathrm{E}-08$ & $2.94 \mathrm{E}+02$ \\
\hline $\mathrm{I}-120 \mathrm{~m}$ & $3.39 \mathrm{E}-15$ & $4.52 \mathrm{E}-08$ & $3.96 \mathrm{E}+02$ \\
\hline $\mathrm{I}-121$ & $3.75 \mathrm{E}-16$ & $5.00 \mathrm{E}-09$ & $4.38 \mathrm{E}+01$ \\
\hline $\mathrm{I}-122$ & $1.04 \mathrm{E}-15$ & $1.39 \mathrm{E}-08$ & $1.21 \mathrm{E}+02$ \\
\hline $\mathrm{I}-123$ & $1.54 \mathrm{E}-16$ & $2.05 \mathrm{E}-09$ & $1.80 \mathrm{E}+01$ \\
\hline $\mathrm{I}-124$ & $1.05 \mathrm{E}-15$ & $1.40 \mathrm{E}-08$ & $1.23 \mathrm{E}+02$ \\
\hline $\mathrm{I}-125$ & $3.19 \mathrm{E}-17$ & $4.25 \mathrm{E}-10$ & $3.72 \mathrm{E}+00$ \\
\hline $\mathrm{I}-126$ & $4.23 \mathrm{E}-16$ & $5.63 \mathrm{E}-09$ & $4.94 \mathrm{E}+01$ \\
\hline $\mathrm{I}-128$ & $1.54 \mathrm{E}-16$ & 2.05E-09 & $1.80 \mathrm{E}+01$ \\
\hline $\mathrm{I}-129$ & $1.99 \mathrm{E}-17$ & $2.65 \mathrm{E}-10$ & $2.32 \mathrm{E}+00$ \\
\hline $\mathrm{I}-130$ & $2.05 \mathrm{E}-15$ & $2.73 \mathrm{E}-08$ & $2.39 \mathrm{E}+02$ \\
\hline $\mathrm{I}-130 \mathrm{~m}$ & $1.20 \mathrm{E}-16$ & $1.60 \mathrm{E}-09$ & $1.40 \mathrm{E}+01$ \\
\hline $\mathrm{I}-131$ & $3.65 \mathrm{E}-16$ & $4.86 \mathrm{E}-09$ & $4.26 \mathrm{E}+01$ \\
\hline $\mathrm{I}-132$ & $2.18 \mathrm{E}-15$ & $2.90 \mathrm{E}-08$ & $2.54 \mathrm{E}+02$ \\
\hline $\mathrm{I}-132 \mathrm{~m}$ & $3.27 \mathrm{E}-16$ & $4.36 \mathrm{E}-09$ & $3.82 \mathrm{E}+01$ \\
\hline $\mathrm{I}-133$ & $6.22 \mathrm{E}-16$ & $8.29 \mathrm{E}-09$ & $7.26 \mathrm{E}+01$ \\
\hline $\mathrm{I}-134$ & $2.49 \mathrm{E}-15$ & $3.32 \mathrm{E}-08$ & $2.91 \mathrm{E}+02$ \\
\hline $\mathrm{I}-134 \mathrm{~m}$ & $2.69 \mathrm{E}-16$ & $3.58 \mathrm{E}-09$ & $3.14 \mathrm{E}+01$ \\
\hline $\mathrm{I}-135$ & $1.47 \mathrm{E}-15$ & $1.96 \mathrm{E}-08$ & $1.72 \mathrm{E}+02$ \\
\hline Xe-120 & $3.71 \mathrm{E}-16$ & 4.94E-09 & $4.33 \mathrm{E}+01$ \\
\hline Xe-121 & $1.40 \mathrm{E}-15$ & $1.86 \mathrm{E}-08$ & $1.63 \mathrm{E}+02$ \\
\hline Xe-122 & $6.01 \mathrm{E}-17$ & $8.01 \mathrm{E}-10$ & $7.01 \mathrm{E}+00$ \\
\hline Xe-123 & $6.09 \mathrm{E}-16$ & $8.11 \mathrm{E}-09$ & $7.11 \mathrm{E}+01$ \\
\hline Xe-125 & $2.47 \mathrm{E}-16$ & $3.29 \mathrm{E}-09$ & $2.88 \mathrm{E}+01$ \\
\hline $\mathrm{Xe}-127$ & $2.56 \mathrm{E}-16$ & $3.41 \mathrm{E}-09$ & $2.99 \mathrm{E}+01$ \\
\hline $\mathrm{Xe}-127 \mathrm{~m}$ & $1.51 \mathrm{E}-16$ & $2.01 \mathrm{E}-09$ & $1.76 \mathrm{E}+01$ \\
\hline $\mathrm{Xe}-129 \mathrm{~m}$ & $4.17 \mathrm{E}-17$ & $5.55 \mathrm{E}-10$ & $4.87 \mathrm{E}+00$ \\
\hline $\mathrm{Xe}-131 \mathrm{~m}$ & $1.63 \mathrm{E}-17$ & $2.17 \mathrm{E}-10$ & $1.90 \mathrm{E}+00$ \\
\hline Xe-133 & $4.06 \mathrm{E}-17$ & $5.41 \mathrm{E}-10$ & $4.74 \mathrm{E}+00$ \\
\hline Xe-133m & $3.53 \mathrm{E}-17$ & $4.70 \mathrm{E}-10$ & $4.12 \mathrm{E}+00$ \\
\hline $\mathrm{Xe}-135$ & $2.50 \mathrm{E}-16$ & $3.33 \mathrm{E}-09$ & $2.92 \mathrm{E}+01$ \\
\hline $\mathrm{Xe}-135 \mathrm{~m}$ & $4.15 \mathrm{E}-16$ & $5.53 \mathrm{E}-09$ & $4.84 \mathrm{E}+01$ \\
\hline
\end{tabular}


SRNL-STI-2013-00115

\begin{tabular}{|c|c|c|c|}
\hline Nuclide & $\begin{array}{c}\text { Ground } \\
\text { Shine Dose } \\
\text { Rate } \\
\text { Coefficient } \\
\left(\mathrm{Sv}-\mathbf{m}^{2} / \mathbf{B q}-\mathbf{s}\right)\end{array}$ & $\begin{array}{l}\text { Ground Shine } \\
\text { Dose Rate } \\
\text { Coefficient } \\
\text { mrem-m²/pCi-h }\end{array}$ & $\begin{array}{l}\text { Ground Shine } \\
\text { Dose Rate } \\
\text { Coefficient } \\
\text { mrem-m } / \mu \mathrm{Ci}-\mathrm{y}\end{array}$ \\
\hline $\mathrm{Xe}-137$ & $3.48 \mathrm{E}-16$ & 4.64E-09 & $4.06 \mathrm{E}+01$ \\
\hline Xe-138 & $1.07 \mathrm{E}-15$ & $1.43 \mathrm{E}-08$ & $1.25 \mathrm{E}+02$ \\
\hline Cs-121 & $1.28 \mathrm{E}-15$ & $1.70 \mathrm{E}-08$ & $1.49 \mathrm{E}+02$ \\
\hline Cs-121m & $1.25 \mathrm{E}-15$ & $1.67 \mathrm{E}-08$ & $1.46 \mathrm{E}+02$ \\
\hline Cs-123 & $1.13 \mathrm{E}-15$ & $1.51 \mathrm{E}-08$ & $1.32 \mathrm{E}+02$ \\
\hline Cs-124 & $1.28 \mathrm{E}-15$ & $1.70 \mathrm{E}-08$ & $1.49 \mathrm{E}+02$ \\
\hline Cs-125 & $7.54 \mathrm{E}-16$ & $1.00 \mathrm{E}-08$ & $8.80 \mathrm{E}+01$ \\
\hline Cs-126 & $1.23 \mathrm{E}-15$ & $1.64 \mathrm{E}-08$ & $1.44 \mathrm{E}+02$ \\
\hline Cs-127 & $4.08 \mathrm{E}-16$ & 5.43E-09 & $4.76 \mathrm{E}+01$ \\
\hline Cs-128 & $9.47 \mathrm{E}-16$ & $1.26 \mathrm{E}-08$ & $1.10 \mathrm{E}+02$ \\
\hline Cs-129 & $2.60 \mathrm{E}-16$ & $3.46 \mathrm{E}-09$ & $3.03 \mathrm{E}+01$ \\
\hline Cs-130 & $5.25 \mathrm{E}-16$ & 6.99E-09 & $6.13 \mathrm{E}+01$ \\
\hline Cs-130m & $6.26 \mathrm{E}-17$ & $8.34 \mathrm{E}-10$ & $7.30 \mathrm{E}+00$ \\
\hline Cs-131 & $1.80 \mathrm{E}-17$ & $2.40 \mathrm{E}-10$ & $2.10 \mathrm{E}+00$ \\
\hline Cs-132 & $6.77 \mathrm{E}-16$ & $9.02 \mathrm{E}-09$ & $7.90 \mathrm{E}+01$ \\
\hline Cs-134 & $1.48 \mathrm{E}-15$ & $1.97 \mathrm{E}-08$ & $1.73 \mathrm{E}+02$ \\
\hline Cs- $134 \mathrm{~m}$ & $2.25 \mathrm{E}-17$ & $3.00 \mathrm{E}-10$ & $2.63 \mathrm{E}+00$ \\
\hline Cs-135 & $5.06 \mathrm{E}-20$ & $6.74 \mathrm{E}-13$ & $5.90 \mathrm{E}-03$ \\
\hline Cs- $135 \mathrm{~m}$ & $1.52 \mathrm{E}-15$ & $2.02 \mathrm{E}-08$ & $1.77 \mathrm{E}+02$ \\
\hline Cs-136 & $1.99 \mathrm{E}-15$ & $2.65 \mathrm{E}-08$ & $2.32 \mathrm{E}+02$ \\
\hline Cs-137 & $3.13 \mathrm{E}-18$ & $4.17 \mathrm{E}-11$ & $3.65 \mathrm{E}-01$ \\
\hline Cs-138 & $2.26 \mathrm{E}-15$ & 3.01E-08 & $2.64 \mathrm{E}+02$ \\
\hline Cs-138m & $4.07 \mathrm{E}-16$ & 5.42E-09 & $4.75 \mathrm{E}+01$ \\
\hline Cs-139 & $4.33 \mathrm{E}-16$ & $5.77 \mathrm{E}-09$ & $5.05 \mathrm{E}+01$ \\
\hline Cs-140 & $1.74 \mathrm{E}-15$ & 2.32E-08 & $2.03 \mathrm{E}+02$ \\
\hline Ba-124 & $5.53 \mathrm{E}-16$ & 7.37E-09 & $6.45 \mathrm{E}+01$ \\
\hline $\mathrm{Ba}-126$ & $5.40 \mathrm{E}-16$ & 7.19E-09 & $6.30 \mathrm{E}+01$ \\
\hline Ba-127 & $7.54 \mathrm{E}-16$ & $1.00 \mathrm{E}-08$ & $8.80 \mathrm{E}+01$ \\
\hline Ba-128 & $5.86 \mathrm{E}-17$ & $7.81 \mathrm{E}-10$ & $6.84 \mathrm{E}+00$ \\
\hline Ba-129 & $3.23 \mathrm{E}-16$ & 4.30E-09 & $3.77 \mathrm{E}+01$ \\
\hline Ba-129m & $1.47 \mathrm{E}-15$ & $1.96 \mathrm{E}-08$ & $1.72 \mathrm{E}+02$ \\
\hline Ba-131 & $4.45 \mathrm{E}-16$ & $5.93 \mathrm{E}-09$ & $5.19 \mathrm{E}+01$ \\
\hline $\mathrm{Ba}-131 \mathrm{~m}$ & $6.73 \mathrm{E}-17$ & $8.96 \mathrm{E}-10$ & $7.85 \mathrm{E}+00$ \\
\hline Ba-133 & $3.73 \mathrm{E}-16$ & 4.97E-09 & $4.35 \mathrm{E}+01$ \\
\hline Ba-133m & $6.08 \mathrm{E}-17$ & $8.10 \mathrm{E}-10$ & $7.09 \mathrm{E}+00$ \\
\hline $\mathrm{Ba}-135 \mathrm{~m}$ & $5.38 \mathrm{E}-17$ & $7.17 \mathrm{E}-10$ & $6.28 \mathrm{E}+00$ \\
\hline Ba-137m & $5.77 \mathrm{E}-16$ & 7.69E-09 & $6.73 \mathrm{E}+01$ \\
\hline Ba-139 & $1.49 \mathrm{E}-16$ & $1.98 \mathrm{E}-09$ & $1.74 \mathrm{E}+01$ \\
\hline $\mathrm{Ba}-140$ & $1.91 \mathrm{E}-16$ & $2.54 \mathrm{E}-09$ & $2.23 \mathrm{E}+01$ \\
\hline
\end{tabular}


SRNL-STI-2013-00115

\begin{tabular}{|c|c|c|c|}
\hline Nuclide & $\begin{array}{c}\text { Ground } \\
\text { Shine Dose } \\
\text { Rate } \\
\text { Coefficient } \\
\left(\mathrm{Sv}-\mathbf{m}^{2} / \mathbf{B q}-\mathbf{s}\right)\end{array}$ & $\begin{array}{l}\text { Ground Shine } \\
\text { Dose Rate } \\
\text { Coefficient } \\
\text { mrem-m²/pCi-h }\end{array}$ & $\begin{array}{c}\text { Ground Shine } \\
\text { Dose Rate } \\
\text { Coefficient } \\
\text { mrem-m }{ }^{2} / \mu \mathrm{Ci}-\mathrm{y}\end{array}$ \\
\hline Ba-141 & $9.67 \mathrm{E}-16$ & $1.29 \mathrm{E}-08$ & $1.13 \mathrm{E}+02$ \\
\hline Ba-142 & $1.01 \mathrm{E}-15$ & $1.35 \mathrm{E}-08$ & $1.18 \mathrm{E}+02$ \\
\hline La-128 & $2.79 \mathrm{E}-15$ & 3.72E-08 & $3.26 \mathrm{E}+02$ \\
\hline La-129 & $9.41 \mathrm{E}-16$ & $1.25 \mathrm{E}-08$ & $1.10 \mathrm{E}+02$ \\
\hline La-130 & $2.20 \mathrm{E}-15$ & $2.93 \mathrm{E}-08$ & $2.57 \mathrm{E}+02$ \\
\hline La-131 & $6.44 \mathrm{E}-16$ & $8.58 \mathrm{E}-09$ & $7.51 \mathrm{E}+01$ \\
\hline La-132 & $1.89 \mathrm{E}-15$ & $2.52 \mathrm{E}-08$ & $2.21 \mathrm{E}+02$ \\
\hline La-132m & $6.36 \mathrm{E}-16$ & $8.47 \mathrm{E}-09$ & $7.42 \mathrm{E}+01$ \\
\hline La-133 & $1.51 \mathrm{E}-16$ & $2.01 \mathrm{E}-09$ & $1.76 \mathrm{E}+01$ \\
\hline La-134 & $7.73 \mathrm{E}-16$ & $1.03 \mathrm{E}-08$ & $9.02 \mathrm{E}+01$ \\
\hline La-135 & $3.05 \mathrm{E}-17$ & $4.06 \mathrm{E}-10$ & $3.56 \mathrm{E}+00$ \\
\hline La-136 & $4.22 \mathrm{E}-16$ & 5.62E-09 & $4.92 \mathrm{E}+01$ \\
\hline La-137 & $2.01 \mathrm{E}-17$ & $2.68 \mathrm{E}-10$ & $2.35 \mathrm{E}+00$ \\
\hline La-138 & $1.12 \mathrm{E}-15$ & 1.49E-08 & $1.31 \mathrm{E}+02$ \\
\hline La-140 & $2.15 \mathrm{E}-15$ & $2.86 \mathrm{E}-08$ & $2.51 \mathrm{E}+02$ \\
\hline La-141 & $1.40 \mathrm{E}-16$ & $1.86 \mathrm{E}-09$ & $1.63 \mathrm{E}+01$ \\
\hline La-142 & $2.16 \mathrm{E}-15$ & $2.88 \mathrm{E}-08$ & $2.52 \mathrm{E}+02$ \\
\hline La-143 & $3.76 \mathrm{E}-16$ & $5.01 \mathrm{E}-09$ & $4.39 \mathrm{E}+01$ \\
\hline Ce-130 & $4.65 \mathrm{E}-16$ & 6.19E-09 & $5.43 \mathrm{E}+01$ \\
\hline Ce-131 & $1.58 \mathrm{E}-15$ & $2.10 \mathrm{E}-08$ & $1.84 \mathrm{E}+02$ \\
\hline Ce-132 & $2.49 \mathrm{E}-16$ & $3.32 \mathrm{E}-09$ & $2.91 \mathrm{E}+01$ \\
\hline $\mathrm{Ce}-133$ & $5.49 \mathrm{E}-16$ & 7.31E-09 & $6.41 \mathrm{E}+01$ \\
\hline Ce-133m & $1.61 \mathrm{E}-15$ & $2.14 \mathrm{E}-08$ & $1.88 \mathrm{E}+02$ \\
\hline $\mathrm{Ce}-134$ & $2.30 \mathrm{E}-17$ & $3.06 \mathrm{E}-10$ & $2.68 \mathrm{E}+00$ \\
\hline Ce-135 & $7.75 \mathrm{E}-16$ & $1.03 \mathrm{E}-08$ & $9.04 \mathrm{E}+01$ \\
\hline $\mathrm{Ce}-137$ & $3.25 \mathrm{E}-17$ & $4.33 \mathrm{E}-10$ & $3.79 \mathrm{E}+00$ \\
\hline $\mathrm{Ce}-137 \mathrm{~m}$ & $4.97 \mathrm{E}-17$ & $6.62 \mathrm{E}-10$ & $5.80 \mathrm{E}+00$ \\
\hline Ce-139 & $1.43 \mathrm{E}-16$ & $1.90 \mathrm{E}-09$ & $1.67 \mathrm{E}+01$ \\
\hline Ce-141 & $6.97 \mathrm{E}-17$ & $9.28 \mathrm{E}-10$ & $8.13 \mathrm{E}+00$ \\
\hline Ce-143 & $2.99 \mathrm{E}-16$ & $3.98 \mathrm{E}-09$ & $3.49 \mathrm{E}+01$ \\
\hline Ce-144 & $1.73 \mathrm{E}-17$ & $2.30 \mathrm{E}-10$ & $2.02 \mathrm{E}+00$ \\
\hline Ce-145 & $8.40 \mathrm{E}-16$ & $1.12 \mathrm{E}-08$ & $9.80 \mathrm{E}+01$ \\
\hline Pr-134 & $3.08 \mathrm{E}-15$ & $4.10 \mathrm{E}-08$ & $3.59 \mathrm{E}+02$ \\
\hline Pr-134m & $2.29 \mathrm{E}-15$ & $3.05 \mathrm{E}-08$ & $2.67 \mathrm{E}+02$ \\
\hline Pr-135 & $8.87 \mathrm{E}-16$ & $1.18 \mathrm{E}-08$ & $1.03 \mathrm{E}+02$ \\
\hline Pr-136 & $2.08 \mathrm{E}-15$ & $2.77 \mathrm{E}-08$ & $2.43 \mathrm{E}+02$ \\
\hline Pr-137 & $3.71 \mathrm{E}-16$ & 4.94E-09 & $4.33 \mathrm{E}+01$ \\
\hline Pr-138 & $9.00 \mathrm{E}-16$ & $1.20 \mathrm{E}-08$ & $1.05 \mathrm{E}+02$ \\
\hline Pr-138m & $2.35 \mathrm{E}-15$ & $3.13 \mathrm{E}-08$ & $2.74 \mathrm{E}+02$ \\
\hline
\end{tabular}


SRNL-STI-2013-00115

\begin{tabular}{|c|c|c|c|}
\hline Nuclide & $\begin{array}{c}\text { Ground } \\
\text { Shine Dose } \\
\text { Rate } \\
\text { Coefficient } \\
\left(\mathrm{Sv}-\mathbf{m}^{2} / \mathbf{B q}-\mathbf{s}\right)\end{array}$ & $\begin{array}{l}\text { Ground Shine } \\
\text { Dose Rate } \\
\text { Coefficient } \\
\text { mrem-m } / \mathbf{p C i} \text {-h }\end{array}$ & $\begin{array}{l}\text { Ground Shine } \\
\text { Dose Rate } \\
\text { Coefficient } \\
\text { mrem-m } / \mu \mathrm{Ci}-\mathrm{y}\end{array}$ \\
\hline Pr-139 & $1.24 \mathrm{E}-16$ & $1.65 \mathrm{E}-09$ & $1.45 \mathrm{E}+01$ \\
\hline Pr-140 & $5.86 \mathrm{E}-16$ & 7.81E-09 & $6.84 \mathrm{E}+01$ \\
\hline Pr-142 & $1.47 \mathrm{E}-16$ & $1.96 \mathrm{E}-09$ & $1.72 \mathrm{E}+01$ \\
\hline $\operatorname{Pr}-142 \mathrm{~m}$ & $0.00 \mathrm{E}+00$ & $0.00 \mathrm{E}+00$ & $0.00 \mathrm{E}+00$ \\
\hline Pr-143 & $2.08 \mathrm{E}-17$ & $2.77 \mathrm{E}-10$ & $2.43 \mathrm{E}+00$ \\
\hline Pr-144 & $1.61 \mathrm{E}-16$ & $2.14 \mathrm{E}-09$ & $1.88 \mathrm{E}+01$ \\
\hline Pr-144m & $1.09 \mathrm{E}-17$ & $1.45 \mathrm{E}-10$ & $1.27 \mathrm{E}+00$ \\
\hline $\operatorname{Pr}-145$ & $9.87 \mathrm{E}-17$ & $1.31 \mathrm{E}-09$ & $1.15 \mathrm{E}+01$ \\
\hline Pr-146 & $1.06 \mathrm{E}-15$ & $1.41 \mathrm{E}-08$ & $1.24 \mathrm{E}+02$ \\
\hline Pr-147 & $5.50 \mathrm{E}-16$ & 7.33E-09 & $6.42 \mathrm{E}+01$ \\
\hline Pr-148 & $1.07 \mathrm{E}-15$ & $1.43 \mathrm{E}-08$ & $1.25 \mathrm{E}+02$ \\
\hline Pr-148m & $1.05 \mathrm{E}-15$ & $1.40 \mathrm{E}-08$ & $1.23 \mathrm{E}+02$ \\
\hline $\mathrm{Nd}-134$ & $5.25 \mathrm{E}-16$ & $6.99 \mathrm{E}-09$ & $6.13 \mathrm{E}+01$ \\
\hline $\mathrm{Nd}-135$ & $1.30 \mathrm{E}-15$ & $1.73 \mathrm{E}-08$ & $1.52 \mathrm{E}+02$ \\
\hline $\mathrm{Nd}-136$ & $2.59 \mathrm{E}-16$ & $3.45 \mathrm{E}-09$ & $3.02 \mathrm{E}+01$ \\
\hline $\mathrm{Nd}-137$ & $1.13 \mathrm{E}-15$ & $1.51 \mathrm{E}-08$ & $1.32 \mathrm{E}+02$ \\
\hline $\mathrm{Nd}-138$ & $3.80 \mathrm{E}-17$ & $5.06 \mathrm{E}-10$ & $4.43 \mathrm{E}+00$ \\
\hline Nd-139 & $4.43 \mathrm{E}-16$ & $5.90 \mathrm{E}-09$ & $5.17 \mathrm{E}+01$ \\
\hline $\mathrm{Nd}-139 \mathrm{~m}$ & $1.48 \mathrm{E}-15$ & $1.97 \mathrm{E}-08$ & $1.73 \mathrm{E}+02$ \\
\hline Nd-140 & $2.39 \mathrm{E}-17$ & $3.18 \mathrm{E}-10$ & $2.79 \mathrm{E}+00$ \\
\hline Nd-141 & $6.94 \mathrm{E}-17$ & $9.24 \mathrm{E}-10$ & $8.10 \mathrm{E}+00$ \\
\hline $\mathrm{Nd}-141 \mathrm{~m}$ & $6.68 \mathrm{E}-16$ & 8.90E-09 & $7.79 \mathrm{E}+01$ \\
\hline Nd-144 & $0.00 \mathrm{E}+00$ & $0.00 \mathrm{E}+00$ & $0.00 \mathrm{E}+00$ \\
\hline $\mathrm{Nd}-147$ & $1.40 \mathrm{E}-16$ & $1.86 \mathrm{E}-09$ & $1.63 \mathrm{E}+01$ \\
\hline Nd-149 & $3.94 \mathrm{E}-16$ & $5.25 \mathrm{E}-09$ & $4.60 \mathrm{E}+01$ \\
\hline $\mathrm{Nd}-151$ & $8.56 \mathrm{E}-16$ & $1.14 \mathrm{E}-08$ & $9.99 \mathrm{E}+01$ \\
\hline $\mathrm{Nd}-152$ & $1.75 \mathrm{E}-16$ & 2.33E-09 & $2.04 \mathrm{E}+01$ \\
\hline Pm-136 & $2.77 \mathrm{E}-15$ & 3.69E-08 & $3.23 \mathrm{E}+02$ \\
\hline Pm-137m & $1.79 \mathrm{E}-15$ & $2.38 \mathrm{E}-08$ & $2.09 \mathrm{E}+02$ \\
\hline Pm-139 & $9.99 \mathrm{E}-16$ & $1.33 \mathrm{E}-08$ & $1.17 \mathrm{E}+02$ \\
\hline Pm-140 & $1.18 \mathrm{E}-15$ & $1.57 \mathrm{E}-08$ & $1.38 \mathrm{E}+02$ \\
\hline Pm-140m & $2.97 \mathrm{E}-15$ & $3.96 \mathrm{E}-08$ & $3.47 \mathrm{E}+02$ \\
\hline Pm-141 & $7.60 \mathrm{E}-16$ & $1.01 \mathrm{E}-08$ & $8.87 \mathrm{E}+01$ \\
\hline Pm-142 & $9.46 \mathrm{E}-16$ & $1.26 \mathrm{E}-08$ & $1.10 \mathrm{E}+02$ \\
\hline Pm-143 & $2.97 \mathrm{E}-16$ & $3.96 \mathrm{E}-09$ & $3.47 \mathrm{E}+01$ \\
\hline Pm-144 & $1.49 \mathrm{E}-15$ & $1.98 \mathrm{E}-08$ & $1.74 \mathrm{E}+02$ \\
\hline Pm-145 & $2.64 \mathrm{E}-17$ & $3.52 \mathrm{E}-10$ & $3.08 \mathrm{E}+00$ \\
\hline Pm-146 & 7.17E-16 & $9.55 \mathrm{E}-09$ & $8.37 \mathrm{E}+01$ \\
\hline Pm-147 & $2.81 \mathrm{E}-20$ & $3.74 \mathrm{E}-13$ & $3.28 \mathrm{E}-03$ \\
\hline
\end{tabular}


SRNL-STI-2013-00115

\begin{tabular}{|c|c|c|c|}
\hline Nuclide & $\begin{array}{c}\text { Ground } \\
\text { Shine Dose } \\
\text { Rate } \\
\text { Coefficient } \\
\left(\mathrm{Sv}-\mathbf{m}^{2} / \mathbf{B q}-\mathbf{s}\right) \\
\end{array}$ & $\begin{array}{l}\text { Ground Shine } \\
\text { Dose Rate } \\
\text { Coefficient } \\
\text { mrem-m } / \mathbf{p C i} \text {-h }\end{array}$ & $\begin{array}{l}\text { Ground Shine } \\
\text { Dose Rate } \\
\text { Coefficient } \\
\text { mrem-m } / \mu \mathrm{Ci}-\mathrm{y}\end{array}$ \\
\hline Pm-148 & $6.11 \mathrm{E}-16$ & $8.14 \mathrm{E}-09$ & $7.13 \mathrm{E}+01$ \\
\hline Pm-148m & $1.90 \mathrm{E}-15$ & $2.53 \mathrm{E}-08$ & $2.22 \mathrm{E}+02$ \\
\hline Pm-149 & $4.14 \mathrm{E}-17$ & $5.51 \mathrm{E}-10$ & $4.83 \mathrm{E}+00$ \\
\hline Pm-150 & $1.44 \mathrm{E}-15$ & $1.92 \mathrm{E}-08$ & $1.68 \mathrm{E}+02$ \\
\hline Pm-151 & $3.25 \mathrm{E}-16$ & $4.33 \mathrm{E}-09$ & $3.79 \mathrm{E}+01$ \\
\hline Pm-152 & $4.04 \mathrm{E}-16$ & 5.38E-09 & $4.71 \mathrm{E}+01$ \\
\hline Pm-152m & $1.50 \mathrm{E}-15$ & $2.00 \mathrm{E}-08$ & $1.75 \mathrm{E}+02$ \\
\hline Pm-153 & $1.48 \mathrm{E}-16$ & $1.97 \mathrm{E}-09$ & $1.73 \mathrm{E}+01$ \\
\hline Pm-154 & $1.70 \mathrm{E}-15$ & $2.26 \mathrm{E}-08$ & $1.98 \mathrm{E}+02$ \\
\hline Pm-154m & $1.75 \mathrm{E}-15$ & 2.33E-08 & $2.04 \mathrm{E}+02$ \\
\hline Sm-139 & $1.48 \mathrm{E}-15$ & $1.97 \mathrm{E}-08$ & $1.73 \mathrm{E}+02$ \\
\hline Sm-140 & $5.47 \mathrm{E}-16$ & 7.29E-09 & $6.38 \mathrm{E}+01$ \\
\hline Sm-141 & $1.40 \mathrm{E}-15$ & $1.86 \mathrm{E}-08$ & $1.63 \mathrm{E}+02$ \\
\hline Sm-141m & $1.86 \mathrm{E}-15$ & $2.48 \mathrm{E}-08$ & $2.17 \mathrm{E}+02$ \\
\hline Sm-142 & $1.06 \mathrm{E}-16$ & $1.41 \mathrm{E}-09$ & $1.24 \mathrm{E}+01$ \\
\hline Sm-143 & $5.61 \mathrm{E}-16$ & 7.47E-09 & $6.55 \mathrm{E}+01$ \\
\hline Sm-143m & $6.60 \mathrm{E}-16$ & 8.79E-09 & $7.70 \mathrm{E}+01$ \\
\hline Sm-145 & $5.46 \mathrm{E}-17$ & $7.27 \mathrm{E}-10$ & $6.37 \mathrm{E}+00$ \\
\hline Sm-146 & $0.00 \mathrm{E}+00$ & $0.00 \mathrm{E}+00$ & $0.00 \mathrm{E}+00$ \\
\hline Sm-147 & $0.00 \mathrm{E}+00$ & $0.00 \mathrm{E}+00$ & $0.00 \mathrm{E}+00$ \\
\hline Sm-148 & $0.00 \mathrm{E}+00$ & $0.00 \mathrm{E}+00$ & $0.00 \mathrm{E}+00$ \\
\hline Sm-151 & $3.81 \mathrm{E}-21$ & $5.07 \mathrm{E}-14$ & $4.45 \mathrm{E}-04$ \\
\hline Sm-153 & $6.25 \mathrm{E}-17$ & $8.33 \mathrm{E}-10$ & $7.29 \mathrm{E}+00$ \\
\hline Sm-155 & $1.55 \mathrm{E}-16$ & $2.06 \mathrm{E}-09$ & $1.81 \mathrm{E}+01$ \\
\hline Sm-156 & $1.07 \mathrm{E}-16$ & $1.43 \mathrm{E}-09$ & $1.25 \mathrm{E}+01$ \\
\hline Sm-157 & $4.83 \mathrm{E}-16$ & $6.43 \mathrm{E}-09$ & $5.64 \mathrm{E}+01$ \\
\hline Eu-142 & $1.36 \mathrm{E}-15$ & $1.81 \mathrm{E}-08$ & $1.59 \mathrm{E}+02$ \\
\hline Eu-142m & $3.40 \mathrm{E}-15$ & $4.53 \mathrm{E}-08$ & $3.97 \mathrm{E}+02$ \\
\hline Eu-143 & $1.18 \mathrm{E}-15$ & $1.57 \mathrm{E}-08$ & $1.38 \mathrm{E}+02$ \\
\hline Eu-144 & $1.21 \mathrm{E}-15$ & $1.61 \mathrm{E}-08$ & $1.41 \mathrm{E}+02$ \\
\hline Eu-145 & $1.18 \mathrm{E}-15$ & $1.57 \mathrm{E}-08$ & $1.38 \mathrm{E}+02$ \\
\hline Eu-146 & $2.24 \mathrm{E}-15$ & $2.98 \mathrm{E}-08$ & $2.61 \mathrm{E}+02$ \\
\hline Eu-147 & $4.38 \mathrm{E}-16$ & $5.83 \mathrm{E}-09$ & $5.11 \mathrm{E}+01$ \\
\hline Eu-148 & $2.10 \mathrm{E}-15$ & $2.80 \mathrm{E}-08$ & $2.45 \mathrm{E}+02$ \\
\hline Eu-149 & $5.85 \mathrm{E}-17$ & $7.79 \mathrm{E}-10$ & $6.83 \mathrm{E}+00$ \\
\hline Eu-150 & $1.47 \mathrm{E}-15$ & $1.96 \mathrm{E}-08$ & $1.72 \mathrm{E}+02$ \\
\hline Eu-150m & $7.01 \mathrm{E}-17$ & $9.34 \mathrm{E}-10$ & $8.18 \mathrm{E}+00$ \\
\hline Eu-152 & $1.09 \mathrm{E}-15$ & $1.45 \mathrm{E}-08$ & $1.27 \mathrm{E}+02$ \\
\hline $\mathrm{Eu}-152 \mathrm{~m}$ & $3.36 \mathrm{E}-16$ & $4.48 \mathrm{E}-09$ & $3.92 \mathrm{E}+01$ \\
\hline
\end{tabular}


SRNL-STI-2013-00115

\begin{tabular}{|c|c|c|c|}
\hline Nuclide & $\begin{array}{c}\text { Ground } \\
\text { Shine Dose } \\
\text { Rate } \\
\text { Coefficient } \\
\left(\mathrm{Sv}-\mathrm{m}^{2} / \mathbf{B q}-\mathbf{s}\right)\end{array}$ & $\begin{array}{l}\text { Ground Shine } \\
\text { Dose Rate } \\
\text { Coefficient } \\
\text { mrem-m }{ }^{2} / \mathbf{p C i}-\mathrm{h}\end{array}$ & $\begin{array}{l}\text { Ground Shine } \\
\text { Dose Rate } \\
\text { Coefficient } \\
\text { mrem-m } / \mu \mathrm{Ci}-\mathrm{y}\end{array}$ \\
\hline Eu-152n & $6.50 \mathrm{E}-17$ & $8.66 \mathrm{E}-10$ & $7.58 \mathrm{E}+00$ \\
\hline Eu-154 & $1.17 \mathrm{E}-15$ & $1.56 \mathrm{E}-08$ & $1.37 \mathrm{E}+02$ \\
\hline $\mathrm{Eu}-154 \mathrm{~m}$ & $5.98 \mathrm{E}-17$ & $7.97 \mathrm{E}-10$ & $6.98 \mathrm{E}+00$ \\
\hline Eu-155 & 5.39E-17 & $7.18 \mathrm{E}-10$ & $6.29 \mathrm{E}+00$ \\
\hline Eu-156 & $1.16 \mathrm{E}-15$ & $1.55 \mathrm{E}-08$ & $1.35 \mathrm{E}+02$ \\
\hline $\mathrm{Eu}-157$ & $3.05 \mathrm{E}-16$ & $4.06 \mathrm{E}-09$ & $3.56 \mathrm{E}+01$ \\
\hline Eu-158 & $1.30 \mathrm{E}-15$ & $1.73 \mathrm{E}-08$ & $1.52 \mathrm{E}+02$ \\
\hline Eu-159 & $3.80 \mathrm{E}-16$ & $5.06 \mathrm{E}-09$ & $4.43 \mathrm{E}+01$ \\
\hline Gd-142 & $1.06 \mathrm{E}-15$ & $1.41 \mathrm{E}-08$ & $1.24 \mathrm{E}+02$ \\
\hline Gd-143m & $2.10 \mathrm{E}-15$ & $2.80 \mathrm{E}-08$ & $2.45 \mathrm{E}+02$ \\
\hline Gd-144 & $8.98 \mathrm{E}-16$ & $1.20 \mathrm{E}-08$ & $1.05 \mathrm{E}+02$ \\
\hline Gd-145 & $2.19 \mathrm{E}-15$ & $2.92 \mathrm{E}-08$ & $2.56 \mathrm{E}+02$ \\
\hline Gd-145m & $6.67 \mathrm{E}-16$ & $8.88 \mathrm{E}-09$ & $7.78 \mathrm{E}+01$ \\
\hline Gd-146 & $2.24 \mathrm{E}-16$ & $2.98 \mathrm{E}-09$ & $2.61 \mathrm{E}+01$ \\
\hline Gd-147 & $1.31 \mathrm{E}-15$ & $1.74 \mathrm{E}-08$ & $1.53 \mathrm{E}+02$ \\
\hline Gd-148 & $0.00 \mathrm{E}+00$ & $0.00 \mathrm{E}+00$ & $0.00 \mathrm{E}+00$ \\
\hline Gd-149 & $4.94 \mathrm{E}-16$ & $6.58 \mathrm{E}-09$ & $5.76 \mathrm{E}+01$ \\
\hline Gd-150 & $0.00 \mathrm{E}+00$ & $0.00 \mathrm{E}+00$ & $0.00 \mathrm{E}+00$ \\
\hline Gd-151 & $6.17 \mathrm{E}-17$ & $8.22 \mathrm{E}-10$ & $7.20 \mathrm{E}+00$ \\
\hline Gd-152 & $0.00 \mathrm{E}+00$ & $0.00 \mathrm{E}+00$ & $0.00 \mathrm{E}+00$ \\
\hline Gd-153 & $9.21 \mathrm{E}-17$ & $1.23 \mathrm{E}-09$ & $1.07 \mathrm{E}+01$ \\
\hline Gd-159 & $6.97 \mathrm{E}-17$ & $9.28 \mathrm{E}-10$ & $8.13 \mathrm{E}+00$ \\
\hline Gd-162 & $4.18 \mathrm{E}-16$ & $5.57 \mathrm{E}-09$ & $4.88 \mathrm{E}+01$ \\
\hline Tb-146 & $3.42 \mathrm{E}-15$ & $4.56 \mathrm{E}-08$ & $3.99 \mathrm{E}+02$ \\
\hline Tb-147 & $2.05 \mathrm{E}-15$ & $2.73 \mathrm{E}-08$ & $2.39 \mathrm{E}+02$ \\
\hline Tb-147m & $1.77 \mathrm{E}-15$ & $2.36 \mathrm{E}-08$ & $2.07 \mathrm{E}+02$ \\
\hline Tb-148 & $2.26 \mathrm{E}-15$ & $3.01 \mathrm{E}-08$ & $2.64 \mathrm{E}+02$ \\
\hline $\mathrm{Tb}-148 \mathrm{~m}$ & $3.00 \mathrm{E}-15$ & $4.00 \mathrm{E}-08$ & $3.50 \mathrm{E}+02$ \\
\hline Tb-149 & $1.26 \mathrm{E}-15$ & $1.68 \mathrm{E}-08$ & $1.47 \mathrm{E}+02$ \\
\hline Tb-149m & $1.33 \mathrm{E}-15$ & $1.77 \mathrm{E}-08$ & $1.55 \mathrm{E}+02$ \\
\hline Tb-150 & $2.21 \mathrm{E}-15$ & $2.94 \mathrm{E}-08$ & $2.58 \mathrm{E}+02$ \\
\hline Tb-150m & $2.41 \mathrm{E}-15$ & $3.21 \mathrm{E}-08$ & $2.81 \mathrm{E}+02$ \\
\hline Tb-151 & $9.31 \mathrm{E}-16$ & $1.24 \mathrm{E}-08$ & $1.09 \mathrm{E}+02$ \\
\hline Tb-151m & 7.30E-17 & $9.72 \mathrm{E}-10$ & $8.52 \mathrm{E}+00$ \\
\hline Tb-152 & $1.39 \mathrm{E}-15$ & $1.85 \mathrm{E}-08$ & $1.62 \mathrm{E}+02$ \\
\hline Tb-152m & $7.14 \mathrm{E}-16$ & $9.51 \mathrm{E}-09$ & $8.33 \mathrm{E}+01$ \\
\hline Tb-153 & $3.05 \mathrm{E}-16$ & 4.06E-09 & $3.56 \mathrm{E}+01$ \\
\hline Tb-154 & $2.02 \mathrm{E}-15$ & $2.69 \mathrm{E}-08$ & $2.36 \mathrm{E}+02$ \\
\hline Tb-155 & $1.59 \mathrm{E}-16$ & $2.12 \mathrm{E}-09$ & $1.86 \mathrm{E}+01$ \\
\hline
\end{tabular}


SRNL-STI-2013-00115

\begin{tabular}{|c|c|c|c|}
\hline Nuclide & $\begin{array}{c}\text { Ground } \\
\text { Shine Dose } \\
\text { Rate } \\
\text { Coefficient } \\
\left(\mathrm{Sv}-\mathbf{m}^{2} / \mathbf{B q}-\mathbf{s}\right)\end{array}$ & $\begin{array}{l}\text { Ground Shine } \\
\text { Dose Rate } \\
\text { Coefficient } \\
\text { mrem-m } / \mathbf{p C i} \text {-h }\end{array}$ & $\begin{array}{l}\text { Ground Shine } \\
\text { Dose Rate } \\
\text { Coefficient } \\
\text { mrem-m } / \mu \mathrm{Ci}-\mathrm{y}\end{array}$ \\
\hline Tb-156 & $1.79 \mathrm{E}-15$ & $2.38 \mathrm{E}-08$ & $2.09 \mathrm{E}+02$ \\
\hline Tb-156m & $3.27 \mathrm{E}-17$ & $4.36 \mathrm{E}-10$ & $3.82 \mathrm{E}+00$ \\
\hline $\mathrm{Tb}-156 \mathrm{n}$ & $3.21 \mathrm{E}-18$ & $4.28 \mathrm{E}-11$ & $3.75 \mathrm{E}-01$ \\
\hline Tb-157 & 4.04E-18 & $5.38 \mathrm{E}-11$ & 4.71E-01 \\
\hline Tb-158 & $7.53 \mathrm{E}-16$ & $1.00 \mathrm{E}-08$ & $8.79 \mathrm{E}+01$ \\
\hline Tb-160 & $1.06 \mathrm{E}-15$ & $1.41 \mathrm{E}-08$ & $1.24 \mathrm{E}+02$ \\
\hline Tb-161 & $3.03 \mathrm{E}-17$ & $4.04 \mathrm{E}-10$ & $3.54 \mathrm{E}+00$ \\
\hline Tb-162 & $1.09 \mathrm{E}-15$ & $1.45 \mathrm{E}-08$ & $1.27 \mathrm{E}+02$ \\
\hline Tb-163 & $7.74 \mathrm{E}-16$ & $1.03 \mathrm{E}-08$ & $9.03 \mathrm{E}+01$ \\
\hline Tb-164 & $2.35 \mathrm{E}-15$ & $3.13 \mathrm{E}-08$ & $2.74 \mathrm{E}+02$ \\
\hline Tb-165 & $8.64 \mathrm{E}-16$ & $1.15 \mathrm{E}-08$ & $1.01 \mathrm{E}+02$ \\
\hline Dy-148 & $6.81 \mathrm{E}-16$ & 9.07E-09 & $7.95 \mathrm{E}+01$ \\
\hline Dy-149 & $1.49 \mathrm{E}-15$ & $1.98 \mathrm{E}-08$ & $1.74 \mathrm{E}+02$ \\
\hline Dy-150 & $2.63 \mathrm{E}-16$ & $3.50 \mathrm{E}-09$ & $3.07 \mathrm{E}+01$ \\
\hline Dy-151 & $1.27 \mathrm{E}-15$ & $1.69 \mathrm{E}-08$ & $1.48 \mathrm{E}+02$ \\
\hline Dy-152 & $2.64 \mathrm{E}-16$ & $3.52 \mathrm{E}-09$ & $3.08 \mathrm{E}+01$ \\
\hline Dy-153 & $8.05 \mathrm{E}-16$ & $1.07 \mathrm{E}-08$ & $9.39 \mathrm{E}+01$ \\
\hline Dy-154 & $0.00 \mathrm{E}+00$ & $0.00 \mathrm{E}+00$ & $0.00 \mathrm{E}+00$ \\
\hline Dy-155 & $6.18 \mathrm{E}-16$ & 8.23E-09 & $7.21 \mathrm{E}+01$ \\
\hline Dy-157 & $3.24 \mathrm{E}-16$ & 4.32E-09 & $3.78 \mathrm{E}+01$ \\
\hline Dy-159 & $3.91 \mathrm{E}-17$ & $5.21 \mathrm{E}-10$ & $4.56 \mathrm{E}+00$ \\
\hline Dy-165 & $6.94 \mathrm{E}-17$ & $9.24 \mathrm{E}-10$ & $8.10 \mathrm{E}+00$ \\
\hline Dy-165m & $1.71 \mathrm{E}-17$ & $2.28 \mathrm{E}-10$ & $2.00 \mathrm{E}+00$ \\
\hline Dy-166 & $3.76 \mathrm{E}-17$ & $5.01 \mathrm{E}-10$ & $4.39 \mathrm{E}+00$ \\
\hline Dy-167 & $5.81 \mathrm{E}-16$ & 7.74E-09 & $6.78 \mathrm{E}+01$ \\
\hline Dy-168 & 4.01E-16 & 5.34E-09 & $4.68 \mathrm{E}+01$ \\
\hline Но-150 & $1.97 \mathrm{E}-15$ & 2.62E-08 & $2.30 \mathrm{E}+02$ \\
\hline Ho-153 & $1.03 \mathrm{E}-15$ & $1.37 \mathrm{E}-08$ & $1.20 \mathrm{E}+02$ \\
\hline Нo-153m & $1.07 \mathrm{E}-15$ & $1.43 \mathrm{E}-08$ & $1.25 \mathrm{E}+02$ \\
\hline Ho-154 & $1.88 \mathrm{E}-15$ & $2.50 \mathrm{E}-08$ & $2.19 \mathrm{E}+02$ \\
\hline Ho- $154 \mathrm{~m}$ & $2.37 \mathrm{E}-15$ & $3.16 \mathrm{E}-08$ & $2.77 \mathrm{E}+02$ \\
\hline Ho-155 & $5.89 \mathrm{E}-16$ & 7.85E-09 & $6.87 \mathrm{E}+01$ \\
\hline Но-156 & $2.00 \mathrm{E}-15$ & $2.66 \mathrm{E}-08$ & $2.33 \mathrm{E}+02$ \\
\hline Ho-157 & $5.45 \mathrm{E}-16$ & 7.26E-09 & $6.36 \mathrm{E}+01$ \\
\hline Но-159 & $3.52 \mathrm{E}-16$ & 4.69E-09 & $4.11 \mathrm{E}+01$ \\
\hline Но-160 & $1.59 \mathrm{E}-15$ & 2.12E-08 & $1.86 \mathrm{E}+02$ \\
\hline Но-161 & 4.84E-17 & $6.45 \mathrm{E}-10$ & $5.65 \mathrm{E}+00$ \\
\hline Но-162 & $1.51 \mathrm{E}-16$ & 2.01E-09 & $1.76 \mathrm{E}+01$ \\
\hline Нo-162m & $5.15 \mathrm{E}-16$ & $6.86 \mathrm{E}-09$ & $6.01 \mathrm{E}+01$ \\
\hline
\end{tabular}


SRNL-STI-2013-00115

\begin{tabular}{|c|c|c|c|}
\hline Nuclide & $\begin{array}{c}\text { Ground } \\
\text { Shine Dose } \\
\text { Rate } \\
\text { Coefficient } \\
\left(\mathrm{Sv}-\mathbf{m}^{2} / \mathbf{B q}-\mathbf{s}\right) \\
\end{array}$ & $\begin{array}{l}\text { Ground Shine } \\
\text { Dose Rate } \\
\text { Coefficient } \\
\text { mrem-m } / \mathbf{p C i} \text {-h }\end{array}$ & $\begin{array}{l}\text { Ground Shine } \\
\text { Dose Rate } \\
\text { Coefficient } \\
\text { mrem-m } / \mu \mathrm{Ci}-\mathrm{y}\end{array}$ \\
\hline Но-163 & $0.00 \mathrm{E}+00$ & $0.00 \mathrm{E}+00$ & $0.00 \mathrm{E}+00$ \\
\hline Ho-164 & $3.36 \mathrm{E}-17$ & $4.48 \mathrm{E}-10$ & $3.92 \mathrm{E}+00$ \\
\hline Ho- $164 \mathrm{~m}$ & $3.86 \mathrm{E}-17$ & $5.14 \mathrm{E}-10$ & $4.50 \mathrm{E}+00$ \\
\hline Но-166 & $1.06 \mathrm{E}-16$ & $1.41 \mathrm{E}-09$ & $1.24 \mathrm{E}+01$ \\
\hline Ho-166m & $1.53 \mathrm{E}-15$ & $2.04 \mathrm{E}-08$ & $1.79 \mathrm{E}+02$ \\
\hline Но-167 & $3.52 \mathrm{E}-16$ & 4.69E-09 & $4.11 \mathrm{E}+01$ \\
\hline Нo-168 & $9.17 \mathrm{E}-16$ & $1.22 \mathrm{E}-08$ & $1.07 \mathrm{E}+02$ \\
\hline Ho- $168 \mathrm{~m}$ & $5.42 \mathrm{E}-18$ & $7.22 \mathrm{E}-11$ & $6.32 \mathrm{E}-01$ \\
\hline Но-170 & $1.68 \mathrm{E}-15$ & 2.24E-08 & $1.96 \mathrm{E}+02$ \\
\hline Er-154 & $6.72 \mathrm{E}-17$ & $8.95 \mathrm{E}-10$ & $7.84 \mathrm{E}+00$ \\
\hline Er-156 & $5.67 \mathrm{E}-17$ & $7.55 \mathrm{E}-10$ & $6.62 \mathrm{E}+00$ \\
\hline Er-159 & $8.98 \mathrm{E}-16$ & $1.20 \mathrm{E}-08$ & $1.05 \mathrm{E}+02$ \\
\hline Er-161 & $9.25 \mathrm{E}-16$ & $1.23 \mathrm{E}-08$ & $1.08 \mathrm{E}+02$ \\
\hline Er-163 & $3.47 \mathrm{E}-17$ & $4.62 \mathrm{E}-10$ & $4.05 \mathrm{E}+00$ \\
\hline Er-165 & $3.25 \mathrm{E}-17$ & $4.33 \mathrm{E}-10$ & $3.79 \mathrm{E}+00$ \\
\hline Er- $167 \mathrm{~m}$ & $8.81 \mathrm{E}-17$ & $1.17 \mathrm{E}-09$ & $1.03 \mathrm{E}+01$ \\
\hline Er-169 & $6.98 \mathrm{E}-20$ & $9.30 \mathrm{E}-13$ & $8.14 \mathrm{E}-03$ \\
\hline Er-171 & $3.77 \mathrm{E}-16$ & $5.02 \mathrm{E}-09$ & $4.40 \mathrm{E}+01$ \\
\hline Er-172 & $4.90 \mathrm{E}-16$ & $6.53 \mathrm{E}-09$ & $5.72 \mathrm{E}+01$ \\
\hline Er-173 & $8.47 \mathrm{E}-16$ & $1.13 \mathrm{E}-08$ & $9.88 \mathrm{E}+01$ \\
\hline Tm-161 & $1.19 \mathrm{E}-15$ & $1.59 \mathrm{E}-08$ & $1.39 \mathrm{E}+02$ \\
\hline Tm-162 & $1.78 \mathrm{E}-15$ & $2.37 \mathrm{E}-08$ & $2.08 \mathrm{E}+02$ \\
\hline Tm-163 & $1.20 \mathrm{E}-15$ & $1.60 \mathrm{E}-08$ & $1.40 \mathrm{E}+02$ \\
\hline Tm-164 & $7.84 \mathrm{E}-16$ & $1.04 \mathrm{E}-08$ & $9.15 \mathrm{E}+01$ \\
\hline Tm-165 & $5.23 \mathrm{E}-16$ & $6.97 \mathrm{E}-09$ & $6.10 \mathrm{E}+01$ \\
\hline Tm-166 & $1.79 \mathrm{E}-15$ & $2.38 \mathrm{E}-08$ & $2.09 \mathrm{E}+02$ \\
\hline Tm-167 & $1.33 \mathrm{E}-16$ & $1.77 \mathrm{E}-09$ & $1.55 \mathrm{E}+01$ \\
\hline Tm-168 & $1.17 \mathrm{E}-15$ & $1.56 \mathrm{E}-08$ & $1.37 \mathrm{E}+02$ \\
\hline Tm-170 & $2.49 \mathrm{E}-17$ & $3.32 \mathrm{E}-10$ & $2.91 \mathrm{E}+00$ \\
\hline Tm-171 & $5.31 \mathrm{E}-19$ & $7.07 \mathrm{E}-12$ & $6.20 \mathrm{E}-02$ \\
\hline Tm-172 & $4.84 \mathrm{E}-16$ & $6.45 \mathrm{E}-09$ & $5.65 \mathrm{E}+01$ \\
\hline Tm-173 & $3.86 \mathrm{E}-16$ & 5.14E-09 & $4.50 \mathrm{E}+01$ \\
\hline Tm-174 & $1.70 \mathrm{E}-15$ & $2.26 \mathrm{E}-08$ & $1.98 \mathrm{E}+02$ \\
\hline Tm-175 & $1.07 \mathrm{E}-15$ & $1.43 \mathrm{E}-08$ & $1.25 \mathrm{E}+02$ \\
\hline Tm-176 & $1.87 \mathrm{E}-15$ & 2.49E-08 & $2.18 \mathrm{E}+02$ \\
\hline Yb-162 & $2.30 \mathrm{E}-16$ & $3.06 \mathrm{E}-09$ & $2.68 \mathrm{E}+01$ \\
\hline Yb-163 & $7.04 \mathrm{E}-16$ & $9.38 \mathrm{E}-09$ & $8.21 \mathrm{E}+01$ \\
\hline Yb-164 & 4.77E-17 & $6.35 \mathrm{E}-10$ & $5.57 \mathrm{E}+00$ \\
\hline Yb-165 & $3.13 \mathrm{E}-16$ & 4.17E-09 & $3.65 \mathrm{E}+01$ \\
\hline
\end{tabular}


SRNL-STI-2013-00115

\begin{tabular}{|c|c|c|c|}
\hline Nuclide & $\begin{array}{c}\text { Ground } \\
\text { Shine Dose } \\
\text { Rate } \\
\text { Coefficient } \\
\left(\mathrm{Sv}-\mathbf{m}^{2} / \mathbf{B q}-\mathbf{s}\right) \\
\end{array}$ & $\begin{array}{l}\text { Ground Shine } \\
\text { Dose Rate } \\
\text { Coefficient } \\
\text { mrem-m } / \mathbf{p C i} \text {-h }\end{array}$ & $\begin{array}{l}\text { Ground Shine } \\
\text { Dose Rate } \\
\text { Coefficient } \\
\text { mrem-m } / \mu \mathrm{Ci}-\mathrm{y}\end{array}$ \\
\hline Yb-166 & 7.49E-17 & $9.98 \mathrm{E}-10$ & $8.74 \mathrm{E}+00$ \\
\hline Yb-167 & $2.38 \mathrm{E}-16$ & $3.17 \mathrm{E}-09$ & $2.78 \mathrm{E}+01$ \\
\hline Yb-169 & $2.95 \mathrm{E}-16$ & 3.93E-09 & $3.44 \mathrm{E}+01$ \\
\hline Yb-175 & $3.69 \mathrm{E}-17$ & $4.92 \mathrm{E}-10$ & $4.31 \mathrm{E}+00$ \\
\hline Yb-177 & $2.23 \mathrm{E}-16$ & $2.97 \mathrm{E}-09$ & $2.60 \mathrm{E}+01$ \\
\hline Yb-178 & $3.91 \mathrm{E}-17$ & $5.21 \mathrm{E}-10$ & $4.56 \mathrm{E}+00$ \\
\hline Yb-179 & $1.01 \mathrm{E}-15$ & $1.35 \mathrm{E}-08$ & $1.18 \mathrm{E}+02$ \\
\hline Lu-165 & $1.06 \mathrm{E}-15$ & $1.41 \mathrm{E}-08$ & $1.24 \mathrm{E}+02$ \\
\hline Lu-167 & $1.53 \mathrm{E}-15$ & $2.04 \mathrm{E}-08$ & $1.79 \mathrm{E}+02$ \\
\hline Lu-169 & $1.20 \mathrm{E}-15$ & $1.60 \mathrm{E}-08$ & $1.40 \mathrm{E}+02$ \\
\hline Lu-169m & $9.37 \mathrm{E}-21$ & $1.25 \mathrm{E}-13$ & $1.09 \mathrm{E}-03$ \\
\hline Lu-170 & $2.26 \mathrm{E}-15$ & 3.01E-08 & $2.64 \mathrm{E}+02$ \\
\hline Lu-171 & $6.07 \mathrm{E}-16$ & 8.09E-09 & $7.08 \mathrm{E}+01$ \\
\hline $\mathrm{Lu}-171 \mathrm{~m}$ & $2.89 \mathrm{E}-19$ & $3.85 \mathrm{E}-12$ & $3.37 \mathrm{E}-02$ \\
\hline Lu-172 & $1.82 \mathrm{E}-15$ & $2.42 \mathrm{E}-08$ & $2.12 \mathrm{E}+02$ \\
\hline Lu-172m & 4.55E-21 & $6.06 \mathrm{E}-14$ & $5.31 \mathrm{E}-04$ \\
\hline Lu-173 & $1.64 \mathrm{E}-16$ & 2.18E-09 & $1.91 \mathrm{E}+01$ \\
\hline Lu-174 & $1.03 \mathrm{E}-16$ & $1.37 \mathrm{E}-09$ & $1.20 \mathrm{E}+01$ \\
\hline Lu-174m & $5.20 \mathrm{E}-17$ & $6.93 \mathrm{E}-10$ & $6.07 \mathrm{E}+00$ \\
\hline Lu-176 & $4.47 \mathrm{E}-16$ & $5.95 \mathrm{E}-09$ & $5.22 \mathrm{E}+01$ \\
\hline Lu-176m & $5.62 \mathrm{E}-17$ & 7.49E-10 & $6.56 \mathrm{E}+00$ \\
\hline Lu-177 & $3.21 \mathrm{E}-17$ & $4.28 \mathrm{E}-10$ & $3.75 \mathrm{E}+00$ \\
\hline Lu-177m & $9.27 \mathrm{E}-16$ & $1.23 \mathrm{E}-08$ & $1.08 \mathrm{E}+02$ \\
\hline Lu-178 & $2.00 \mathrm{E}-16$ & $2.66 \mathrm{E}-09$ & $2.33 \mathrm{E}+01$ \\
\hline Lu-178m & $1.01 \mathrm{E}-15$ & $1.35 \mathrm{E}-08$ & $1.18 \mathrm{E}+02$ \\
\hline Lu-179 & $7.96 \mathrm{E}-17$ & $1.06 \mathrm{E}-09$ & $9.29 \mathrm{E}+00$ \\
\hline Lu-180 & $1.46 \mathrm{E}-15$ & $1.94 \mathrm{E}-08$ & $1.70 \mathrm{E}+02$ \\
\hline Lu-181 & $6.29 \mathrm{E}-16$ & 8.38E-09 & $7.34 \mathrm{E}+01$ \\
\hline Hf-167 & $6.39 \mathrm{E}-16$ & 8.51E-09 & $7.46 \mathrm{E}+01$ \\
\hline Hf-169 & $6.22 \mathrm{E}-16$ & $8.29 \mathrm{E}-09$ & $7.26 \mathrm{E}+01$ \\
\hline Hf-170 & $4.09 \mathrm{E}-16$ & $5.45 \mathrm{E}-09$ & $4.77 \mathrm{E}+01$ \\
\hline Hf-172 & 8.91E-17 & 1.19E-09 & $1.04 \mathrm{E}+01$ \\
\hline Hf-173 & $3.63 \mathrm{E}-16$ & 4.84E-09 & $4.24 \mathrm{E}+01$ \\
\hline Hf-174 & $0.00 \mathrm{E}+00$ & $0.00 \mathrm{E}+00$ & $0.00 \mathrm{E}+00$ \\
\hline Hf-175 & $3.29 \mathrm{E}-16$ & $4.38 \mathrm{E}-09$ & $3.84 \mathrm{E}+01$ \\
\hline Hf- $177 \mathrm{~m}$ & $2.13 \mathrm{E}-15$ & 2.84E-08 & $2.49 \mathrm{E}+02$ \\
\hline Hf-178m & $2.11 \mathrm{E}-15$ & $2.81 \mathrm{E}-08$ & $2.46 \mathrm{E}+02$ \\
\hline Hf-179m & $8.60 \mathrm{E}-16$ & $1.15 \mathrm{E}-08$ & $1.00 \mathrm{E}+02$ \\
\hline Hf-180m & $9.29 \mathrm{E}-16$ & $1.24 \mathrm{E}-08$ & $1.08 \mathrm{E}+02$ \\
\hline
\end{tabular}


SRNL-STI-2013-00115

\begin{tabular}{|c|c|c|c|}
\hline Nuclide & $\begin{array}{c}\text { Ground } \\
\text { Shine Dose } \\
\text { Rate } \\
\text { Coefficient } \\
\left(\mathrm{Sv}-\mathbf{m}^{2} / \mathbf{B q}-\mathbf{s}\right)\end{array}$ & $\begin{array}{l}\text { Ground Shine } \\
\text { Dose Rate } \\
\text { Coefficient } \\
\text { mrem-m²/pCi-h }\end{array}$ & $\begin{array}{c}\text { Ground Shine } \\
\text { Dose Rate } \\
\text { Coefficient } \\
\text { mrem-m }{ }^{2} / \mu \mathrm{Ci}-\mathrm{y}\end{array}$ \\
\hline Hf-181 & $5.02 \mathrm{E}-16$ & $6.69 \mathrm{E}-09$ & $5.86 \mathrm{E}+01$ \\
\hline Hf-182 & $2.23 \mathrm{E}-16$ & $2.97 \mathrm{E}-09$ & $2.60 \mathrm{E}+01$ \\
\hline Hf- $182 \mathrm{~m}$ & $8.59 \mathrm{E}-16$ & $1.14 \mathrm{E}-08$ & $1.00 \mathrm{E}+02$ \\
\hline Hf-183 & $7.73 \mathrm{E}-16$ & $1.03 \mathrm{E}-08$ & $9.02 \mathrm{E}+01$ \\
\hline Hf-184 & $2.36 \mathrm{E}-16$ & $3.14 \mathrm{E}-09$ & $2.75 \mathrm{E}+01$ \\
\hline Ta-170 & $1.14 \mathrm{E}-15$ & $1.52 \mathrm{E}-08$ & $1.33 \mathrm{E}+02$ \\
\hline Ta-172 & $1.62 \mathrm{E}-15$ & $2.16 \mathrm{E}-08$ & $1.89 \mathrm{E}+02$ \\
\hline Ta-173 & $5.42 \mathrm{E}-16$ & 7.22E-09 & $6.32 \mathrm{E}+01$ \\
\hline Ta-174 & $9.37 \mathrm{E}-16$ & $1.25 \mathrm{E}-08$ & $1.09 \mathrm{E}+02$ \\
\hline Ta-175 & $1.01 \mathrm{E}-15$ & $1.35 \mathrm{E}-08$ & $1.18 \mathrm{E}+02$ \\
\hline Ta-176 & $2.00 \mathrm{E}-15$ & $2.66 \mathrm{E}-08$ & $2.33 \mathrm{E}+02$ \\
\hline Ta-177 & 5.89E-17 & $7.85 \mathrm{E}-10$ & $6.87 \mathrm{E}+00$ \\
\hline Ta-178 & $1.09 \mathrm{E}-16$ & $1.45 \mathrm{E}-09$ & $1.27 \mathrm{E}+01$ \\
\hline Ta-178m & $1.08 \mathrm{E}-15$ & $1.44 \mathrm{E}-08$ & $1.26 \mathrm{E}+02$ \\
\hline Ta-179 & $2.12 \mathrm{E}-17$ & $2.82 \mathrm{E}-10$ & $2.47 \mathrm{E}+00$ \\
\hline Ta-180 & $4.18 \mathrm{E}-17$ & $5.57 \mathrm{E}-10$ & $4.88 \mathrm{E}+00$ \\
\hline Ta-182 & $1.19 \mathrm{E}-15$ & $1.59 \mathrm{E}-08$ & $1.39 \mathrm{E}+02$ \\
\hline Ta-182m & $2.37 \mathrm{E}-16$ & $3.16 \mathrm{E}-09$ & $2.77 \mathrm{E}+01$ \\
\hline Ta-183 & $2.70 \mathrm{E}-16$ & 3.60E-09 & $3.15 \mathrm{E}+01$ \\
\hline Ta-184 & $1.51 \mathrm{E}-15$ & $2.01 \mathrm{E}-08$ & $1.76 \mathrm{E}+02$ \\
\hline Ta-185 & $2.15 \mathrm{E}-16$ & $2.86 \mathrm{E}-09$ & $2.51 \mathrm{E}+01$ \\
\hline Ta-186 & $1.45 \mathrm{E}-15$ & $1.93 \mathrm{E}-08$ & $1.69 \mathrm{E}+02$ \\
\hline W-177 & $8.50 \mathrm{E}-16$ & $1.13 \mathrm{E}-08$ & $9.92 \mathrm{E}+01$ \\
\hline W-178 & $1.28 \mathrm{E}-17$ & $1.70 \mathrm{E}-10$ & $1.49 \mathrm{E}+00$ \\
\hline W-179 & $4.54 \mathrm{E}-17$ & $6.05 \mathrm{E}-10$ & $5.30 \mathrm{E}+00$ \\
\hline W-179m & $4.88 \mathrm{E}-17$ & $6.50 \mathrm{E}-10$ & $5.69 \mathrm{E}+00$ \\
\hline W-181 & $3.40 \mathrm{E}-17$ & $4.53 \mathrm{E}-10$ & $3.97 \mathrm{E}+00$ \\
\hline W-185 & $1.67 \mathrm{E}-19$ & $2.22 \mathrm{E}-12$ & $1.95 \mathrm{E}-02$ \\
\hline $\mathrm{W}-185 \mathrm{~m}$ & $2.15 \mathrm{E}-17$ & $2.86 \mathrm{E}-10$ & $2.51 \mathrm{E}+00$ \\
\hline W-187 & $4.41 \mathrm{E}-16$ & $5.87 \mathrm{E}-09$ & $5.15 \mathrm{E}+01$ \\
\hline W-188 & $1.81 \mathrm{E}-18$ & $2.41 \mathrm{E}-11$ & $2.11 \mathrm{E}-01$ \\
\hline W-190 & $1.54 \mathrm{E}-16$ & $2.05 \mathrm{E}-09$ & $1.80 \mathrm{E}+01$ \\
\hline Re-178 & $1.58 \mathrm{E}-15$ & $2.10 \mathrm{E}-08$ & $1.84 \mathrm{E}+02$ \\
\hline Re-179 & $9.99 \mathrm{E}-16$ & $1.33 \mathrm{E}-08$ & $1.17 \mathrm{E}+02$ \\
\hline Re-180 & $1.13 \mathrm{E}-15$ & $1.51 \mathrm{E}-08$ & $1.32 \mathrm{E}+02$ \\
\hline Re-181 & $7.50 \mathrm{E}-16$ & 9.99E-09 & $8.75 \mathrm{E}+01$ \\
\hline Re-182 & $1.65 \mathrm{E}-15$ & $2.20 \mathrm{E}-08$ & $1.93 \mathrm{E}+02$ \\
\hline $\mathrm{Re}-182 \mathrm{~m}$ & $1.12 \mathrm{E}-15$ & $1.49 \mathrm{E}-08$ & $1.31 \mathrm{E}+02$ \\
\hline Re-183 & $1.37 \mathrm{E}-16$ & $1.82 \mathrm{E}-09$ & $1.60 \mathrm{E}+01$ \\
\hline
\end{tabular}


SRNL-STI-2013-00115

\begin{tabular}{|c|c|c|c|}
\hline Nuclide & $\begin{array}{c}\text { Ground } \\
\text { Shine Dose } \\
\text { Rate } \\
\text { Coefficient } \\
\left(\mathrm{Sv}-\mathbf{m}^{2} / \mathbf{B q}-\mathbf{s}\right)\end{array}$ & $\begin{array}{l}\text { Ground Shine } \\
\text { Dose Rate } \\
\text { Coefficient } \\
\text { mrem-m²/pCi-h }\end{array}$ & $\begin{array}{c}\text { Ground Shine } \\
\text { Dose Rate } \\
\text { Coefficient } \\
\text { mrem-m }{ }^{2} / \mu \mathrm{Ci}-\mathrm{y}\end{array}$ \\
\hline Re-184 & $8.35 \mathrm{E}-16$ & $1.11 \mathrm{E}-08$ & $9.74 \mathrm{E}+01$ \\
\hline Re-184m & $3.52 \mathrm{E}-16$ & $4.69 \mathrm{E}-09$ & $4.11 \mathrm{E}+01$ \\
\hline Re-186 & $4.34 \mathrm{E}-17$ & $5.78 \mathrm{E}-10$ & $5.06 \mathrm{E}+00$ \\
\hline Re-186m & $1.31 \mathrm{E}-17$ & $1.74 \mathrm{E}-10$ & $1.53 \mathrm{E}+00$ \\
\hline Re-187 & $0.00 \mathrm{E}+00$ & $0.00 \mathrm{E}+00$ & $0.00 \mathrm{E}+00$ \\
\hline Re-188 & $1.48 \mathrm{E}-16$ & $1.97 \mathrm{E}-09$ & $1.73 \mathrm{E}+01$ \\
\hline Re-188m & $5.94 \mathrm{E}-17$ & 7.91E-10 & $6.93 \mathrm{E}+00$ \\
\hline Re-189 & $7.14 \mathrm{E}-17$ & $9.51 \mathrm{E}-10$ & $8.33 \mathrm{E}+00$ \\
\hline Re-190 & $1.34 \mathrm{E}-15$ & $1.78 \mathrm{E}-08$ & $1.56 \mathrm{E}+02$ \\
\hline Re-190m & $9.13 \mathrm{E}-16$ & $1.22 \mathrm{E}-08$ & $1.07 \mathrm{E}+02$ \\
\hline Os-180 & $1.12 \mathrm{E}-16$ & 1.49E-09 & $1.31 \mathrm{E}+01$ \\
\hline Os-181 & $1.27 \mathrm{E}-15$ & 1.69E-08 & $1.48 \mathrm{E}+02$ \\
\hline Os-182 & $4.02 \mathrm{E}-16$ & $5.35 \mathrm{E}-09$ & $4.69 \mathrm{E}+01$ \\
\hline Os-183 & $5.83 \mathrm{E}-16$ & 7.77E-09 & $6.80 \mathrm{E}+01$ \\
\hline Os-183m & $9.31 \mathrm{E}-16$ & $1.24 \mathrm{E}-08$ & $1.09 \mathrm{E}+02$ \\
\hline Os-185 & $6.54 \mathrm{E}-16$ & 8.71E-09 & $7.63 \mathrm{E}+01$ \\
\hline Os-186 & $0.00 \mathrm{E}+00$ & $0.00 \mathrm{E}+00$ & $0.00 \mathrm{E}+00$ \\
\hline Os-189m & $4.03 \mathrm{E}-20$ & $5.37 \mathrm{E}-13$ & $4.70 \mathrm{E}-03$ \\
\hline Os-190m & $1.51 \mathrm{E}-15$ & $2.01 \mathrm{E}-08$ & $1.76 \mathrm{E}+02$ \\
\hline Os-191 & $7.11 \mathrm{E}-17$ & $9.47 \mathrm{E}-10$ & $8.30 \mathrm{E}+00$ \\
\hline Os-191m & $5.04 \mathrm{E}-18$ & $6.71 \mathrm{E}-11$ & $5.88 \mathrm{E}-01$ \\
\hline Os-193 & $9.02 \mathrm{E}-17$ & $1.20 \mathrm{E}-09$ & $1.05 \mathrm{E}+01$ \\
\hline Os-194 & $2.21 \mathrm{E}-18$ & $2.94 \mathrm{E}-11$ & $2.58 \mathrm{E}-01$ \\
\hline Os-196 & $1.05 \mathrm{E}-16$ & $1.40 \mathrm{E}-09$ & $1.23 \mathrm{E}+01$ \\
\hline Ir-180 & $1.62 \mathrm{E}-15$ & $2.16 \mathrm{E}-08$ & $1.89 \mathrm{E}+02$ \\
\hline Ir-182 & $1.42 \mathrm{E}-15$ & $1.89 \mathrm{E}-08$ & $1.66 \mathrm{E}+02$ \\
\hline Ir-183 & $1.09 \mathrm{E}-15$ & $1.45 \mathrm{E}-08$ & $1.27 \mathrm{E}+02$ \\
\hline Ir-184 & $1.84 \mathrm{E}-15$ & $2.45 \mathrm{E}-08$ & $2.15 \mathrm{E}+02$ \\
\hline Ir-185 & $7.67 \mathrm{E}-16$ & $1.02 \mathrm{E}-08$ & $8.95 \mathrm{E}+01$ \\
\hline Ir-186 & $1.53 \mathrm{E}-15$ & $2.04 \mathrm{E}-08$ & $1.79 \mathrm{E}+02$ \\
\hline Ir-186m & $1.15 \mathrm{E}-15$ & $1.53 \mathrm{E}-08$ & $1.34 \mathrm{E}+02$ \\
\hline Ir-187 & $3.06 \mathrm{E}-16$ & $4.08 \mathrm{E}-09$ & $3.57 \mathrm{E}+01$ \\
\hline Ir-188 & $1.86 \mathrm{E}-15$ & $2.48 \mathrm{E}-08$ & $2.17 \mathrm{E}+02$ \\
\hline Ir-189 & $6.74 \mathrm{E}-17$ & $8.98 \mathrm{E}-10$ & $7.86 \mathrm{E}+00$ \\
\hline Ir-190 & $1.40 \mathrm{E}-15$ & $1.86 \mathrm{E}-08$ & $1.63 \mathrm{E}+02$ \\
\hline Ir-190m & $4.43 \mathrm{E}-20$ & $5.90 \mathrm{E}-13$ & $5.17 \mathrm{E}-03$ \\
\hline Ir-190n & $4.87 \mathrm{E}-17$ & $6.49 \mathrm{E}-10$ & $5.68 \mathrm{E}+00$ \\
\hline Ir-191m & $6.40 \mathrm{E}-17$ & $8.52 \mathrm{E}-10$ & $7.47 \mathrm{E}+00$ \\
\hline Ir-192 & $7.75 \mathrm{E}-16$ & $1.03 \mathrm{E}-08$ & $9.04 \mathrm{E}+01$ \\
\hline
\end{tabular}


SRNL-STI-2013-00115

\begin{tabular}{|c|c|c|c|}
\hline Nuclide & $\begin{array}{c}\text { Ground } \\
\text { Shine Dose } \\
\text { Rate } \\
\text { Coefficient } \\
\left(\mathrm{Sv}-\mathbf{m}^{2} / \mathbf{B q}-\mathbf{s}\right)\end{array}$ & $\begin{array}{l}\text { Ground Shine } \\
\text { Dose Rate } \\
\text { Coefficient } \\
\text { mrem-m }{ }^{2} / p C i-h\end{array}$ & $\begin{array}{l}\text { Ground Shine } \\
\text { Dose Rate } \\
\text { Coefficient } \\
\text { mrem-m } / \mu \mathrm{Ci}-\mathrm{y}\end{array}$ \\
\hline Ir-192m & $2.13 \mathrm{E}-19$ & $2.84 \mathrm{E}-12$ & $2.49 \mathrm{E}-02$ \\
\hline Ir-192n & $8.49 \mathrm{E}-19$ & $1.13 \mathrm{E}-11$ & $9.91 \mathrm{E}-02$ \\
\hline Ir-193m & $3.18 \mathrm{E}-19$ & $4.24 \mathrm{E}-12$ & $3.71 \mathrm{E}-02$ \\
\hline Ir-194 & $1.81 \mathrm{E}-16$ & $2.41 \mathrm{E}-09$ & $2.11 \mathrm{E}+01$ \\
\hline Ir-194m & $2.22 \mathrm{E}-15$ & $2.96 \mathrm{E}-08$ & $2.59 \mathrm{E}+02$ \\
\hline Ir-195 & $7.38 \mathrm{E}-17$ & $9.83 \mathrm{E}-10$ & $8.61 \mathrm{E}+00$ \\
\hline Ir-195m & $3.60 \mathrm{E}-16$ & 4.80E-09 & $4.20 \mathrm{E}+01$ \\
\hline Ir-196 & $3.48 \mathrm{E}-16$ & 4.64E-09 & $4.06 \mathrm{E}+01$ \\
\hline Ir-196m & $2.36 \mathrm{E}-15$ & $3.14 \mathrm{E}-08$ & $2.75 \mathrm{E}+02$ \\
\hline Pt-184 & $6.68 \mathrm{E}-16$ & 8.90E-09 & $7.79 \mathrm{E}+01$ \\
\hline Pt-186 & $6.43 \mathrm{E}-16$ & $8.56 \mathrm{E}-09$ & $7.50 \mathrm{E}+01$ \\
\hline Pt-187 & $5.77 \mathrm{E}-16$ & 7.69E-09 & $6.73 \mathrm{E}+01$ \\
\hline Pt-188 & $1.84 \mathrm{E}-16$ & $2.45 \mathrm{E}-09$ & $2.15 \mathrm{E}+01$ \\
\hline Pt-189 & $4.49 \mathrm{E}-16$ & 5.98E-09 & $5.24 \mathrm{E}+01$ \\
\hline Pt-190 & $0.00 \mathrm{E}+00$ & $0.00 \mathrm{E}+00$ & $0.00 \mathrm{E}+00$ \\
\hline Pt-191 & $2.70 \mathrm{E}-16$ & 3.60E-09 & $3.15 \mathrm{E}+01$ \\
\hline Pt-193 & $1.07 \mathrm{E}-19$ & $1.43 \mathrm{E}-12$ & $1.25 \mathrm{E}-02$ \\
\hline Pt-193m & $9.06 \mathrm{E}-18$ & $1.21 \mathrm{E}-10$ & $1.06 \mathrm{E}+00$ \\
\hline Pt-195m & $6.20 \mathrm{E}-17$ & $8.26 \mathrm{E}-10$ & $7.23 \mathrm{E}+00$ \\
\hline Pt-197 & $2.42 \mathrm{E}-17$ & $3.22 \mathrm{E}-10$ & $2.82 \mathrm{E}+00$ \\
\hline Pt-197m & $7.27 \mathrm{E}-17$ & $9.68 \mathrm{E}-10$ & $8.48 \mathrm{E}+00$ \\
\hline Pt-199 & $2.46 \mathrm{E}-16$ & $3.28 \mathrm{E}-09$ & $2.87 \mathrm{E}+01$ \\
\hline Pt-200 & $5.40 \mathrm{E}-17$ & 7.19E-10 & $6.30 \mathrm{E}+00$ \\
\hline Pt-202 & $7.79 \mathrm{E}-17$ & $1.04 \mathrm{E}-09$ & $9.09 \mathrm{E}+00$ \\
\hline $\mathrm{Au}-186$ & $1.50 \mathrm{E}-15$ & $2.00 \mathrm{E}-08$ & $1.75 \mathrm{E}+02$ \\
\hline $\mathrm{Au}-187$ & $9.75 \mathrm{E}-16$ & $1.30 \mathrm{E}-08$ & $1.14 \mathrm{E}+02$ \\
\hline $\mathrm{Au}-190$ & $2.11 \mathrm{E}-15$ & $2.81 \mathrm{E}-08$ & $2.46 \mathrm{E}+02$ \\
\hline $\mathrm{Au}-191$ & $5.54 \mathrm{E}-16$ & 7.38E-09 & $6.46 \mathrm{E}+01$ \\
\hline $\mathrm{Au}-192$ & $1.73 \mathrm{E}-15$ & $2.30 \mathrm{E}-08$ & $2.02 \mathrm{E}+02$ \\
\hline Au-193 & $1.49 \mathrm{E}-16$ & $1.98 \mathrm{E}-09$ & $1.74 \mathrm{E}+01$ \\
\hline $\mathrm{Au}-193 \mathrm{~m}$ & $1.80 \mathrm{E}-16$ & 2.40E-09 & $2.10 \mathrm{E}+01$ \\
\hline $\mathrm{Au}-194$ & $9.45 \mathrm{E}-16$ & $1.26 \mathrm{E}-08$ & $1.10 \mathrm{E}+02$ \\
\hline $\mathrm{Au}-195$ & $6.93 \mathrm{E}-17$ & $9.23 \mathrm{E}-10$ & $8.09 \mathrm{E}+00$ \\
\hline $\mathrm{Au}-195 \mathrm{~m}$ & $1.84 \mathrm{E}-16$ & $2.45 \mathrm{E}-09$ & $2.15 \mathrm{E}+01$ \\
\hline Au-196 & $4.43 \mathrm{E}-16$ & $5.90 \mathrm{E}-09$ & $5.17 \mathrm{E}+01$ \\
\hline $\mathrm{Au}-196 \mathrm{~m}$ & $2.16 \mathrm{E}-16$ & $2.88 \mathrm{E}-09$ & $2.52 \mathrm{E}+01$ \\
\hline $\mathrm{Au}-198$ & $4.05 \mathrm{E}-16$ & 5.39E-09 & $4.73 \mathrm{E}+01$ \\
\hline $\mathrm{Au}-198 \mathrm{~m}$ & $4.82 \mathrm{E}-16$ & 6.42E-09 & $5.62 \mathrm{E}+01$ \\
\hline $\mathrm{Au}-199$ & $8.61 \mathrm{E}-17$ & $1.15 \mathrm{E}-09$ & $1.00 \mathrm{E}+01$ \\
\hline
\end{tabular}


SRNL-STI-2013-00115

\begin{tabular}{|c|c|c|c|}
\hline Nuclide & $\begin{array}{c}\text { Ground } \\
\text { Shine Dose } \\
\text { Rate } \\
\text { Coefficient } \\
\left(\mathrm{Sv}-\mathbf{m}^{2} / \mathbf{B q}-\mathbf{s}\right) \\
\end{array}$ & $\begin{array}{l}\text { Ground Shine } \\
\text { Dose Rate } \\
\text { Coefficient } \\
\text { mrem-m } / \mathbf{p C i} \text {-h }\end{array}$ & $\begin{array}{l}\text { Ground Shine } \\
\text { Dose Rate } \\
\text { Coefficient } \\
\text { mrem-m } / \mu \mathrm{Ci}-\mathrm{y}\end{array}$ \\
\hline Au-200 & $3.37 \mathrm{E}-16$ & 4.49E-09 & $3.93 \mathrm{E}+01$ \\
\hline $\mathrm{Au}-200 \mathrm{~m}$ & $1.88 \mathrm{E}-15$ & $2.50 \mathrm{E}-08$ & $2.19 \mathrm{E}+02$ \\
\hline $\mathrm{Au}-201$ & $7.31 \mathrm{E}-17$ & $9.74 \mathrm{E}-10$ & $8.53 \mathrm{E}+00$ \\
\hline $\mathrm{Au}-202$ & $2.81 \mathrm{E}-16$ & $3.74 \mathrm{E}-09$ & $3.28 \mathrm{E}+01$ \\
\hline $\mathrm{Hg}-190$ & $1.77 \mathrm{E}-16$ & $2.36 \mathrm{E}-09$ & $2.07 \mathrm{E}+01$ \\
\hline $\mathrm{Hg}-191 \mathrm{~m}$ & $1.38 \mathrm{E}-15$ & $1.84 \mathrm{E}-08$ & $1.61 \mathrm{E}+02$ \\
\hline $\mathrm{Hg}-192$ & $2.47 \mathrm{E}-16$ & $3.29 \mathrm{E}-09$ & $2.88 \mathrm{E}+01$ \\
\hline $\mathrm{Hg}-193$ & $7.65 \mathrm{E}-16$ & $1.02 \mathrm{E}-08$ & $8.93 \mathrm{E}+01$ \\
\hline $\mathrm{Hg}-193 \mathrm{~m}$ & $9.48 \mathrm{E}-16$ & $1.26 \mathrm{E}-08$ & $1.11 \mathrm{E}+02$ \\
\hline $\mathrm{Hg}-194$ & $1.62 \mathrm{E}-19$ & $2.16 \mathrm{E}-12$ & $1.89 \mathrm{E}-02$ \\
\hline $\mathrm{Hg}-195$ & $1.81 \mathrm{E}-16$ & $2.41 \mathrm{E}-09$ & $2.11 \mathrm{E}+01$ \\
\hline $\mathrm{Hg}-195 \mathrm{~m}$ & $1.83 \mathrm{E}-16$ & $2.44 \mathrm{E}-09$ & $2.14 \mathrm{E}+01$ \\
\hline $\mathrm{Hg}-197$ & $6.09 \mathrm{E}-17$ & $8.11 \mathrm{E}-10$ & $7.11 \mathrm{E}+00$ \\
\hline $\mathrm{Hg}-197 \mathrm{~m}$ & $8.38 \mathrm{E}-17$ & $1.12 \mathrm{E}-09$ & $9.78 \mathrm{E}+00$ \\
\hline $\mathrm{Hg}-199 \mathrm{~m}$ & $1.64 \mathrm{E}-16$ & $2.18 \mathrm{E}-09$ & $1.91 \mathrm{E}+01$ \\
\hline $\mathrm{Hg}-203$ & $2.22 \mathrm{E}-16$ & $2.96 \mathrm{E}-09$ & $2.59 \mathrm{E}+01$ \\
\hline $\mathrm{Hg}-205$ & $6.56 \mathrm{E}-17$ & $8.74 \mathrm{E}-10$ & $7.65 \mathrm{E}+00$ \\
\hline $\mathrm{Hg}-206$ & $1.49 \mathrm{E}-16$ & $1.98 \mathrm{E}-09$ & $1.74 \mathrm{E}+01$ \\
\hline $\mathrm{Hg}-207$ & $2.49 \mathrm{E}-15$ & $3.32 \mathrm{E}-08$ & $2.91 \mathrm{E}+02$ \\
\hline Tl-190 & $1.36 \mathrm{E}-15$ & $1.81 \mathrm{E}-08$ & $1.59 \mathrm{E}+02$ \\
\hline Tl-190m & $2.40 \mathrm{E}-15$ & $3.20 \mathrm{E}-08$ & $2.80 \mathrm{E}+02$ \\
\hline Tl-194 & $9.25 \mathrm{E}-16$ & $1.23 \mathrm{E}-08$ & $1.08 \mathrm{E}+02$ \\
\hline Tl-194m & $2.41 \mathrm{E}-15$ & $3.21 \mathrm{E}-08$ & $2.81 \mathrm{E}+02$ \\
\hline Tl-195 & $1.11 \mathrm{E}-15$ & $1.48 \mathrm{E}-08$ & $1.30 \mathrm{E}+02$ \\
\hline Tl-196 & $1.72 \mathrm{E}-15$ & $2.29 \mathrm{E}-08$ & $2.01 \mathrm{E}+02$ \\
\hline Tl-197 & $4.22 \mathrm{E}-16$ & $5.62 \mathrm{E}-09$ & $4.92 \mathrm{E}+01$ \\
\hline Tl-198 & $1.82 \mathrm{E}-15$ & $2.42 \mathrm{E}-08$ & $2.12 \mathrm{E}+02$ \\
\hline Tl-198m & $1.15 \mathrm{E}-15$ & $1.53 \mathrm{E}-08$ & $1.34 \mathrm{E}+02$ \\
\hline Tl-199 & $2.29 \mathrm{E}-16$ & $3.05 \mathrm{E}-09$ & $2.67 \mathrm{E}+01$ \\
\hline Tl-200 & $1.22 \mathrm{E}-15$ & $1.63 \mathrm{E}-08$ & $1.42 \mathrm{E}+02$ \\
\hline Tl-201 & 7.93E-17 & $1.06 \mathrm{E}-09$ & $9.25 \mathrm{E}+00$ \\
\hline Tl-202 & $4.37 \mathrm{E}-16$ & $5.82 \mathrm{E}-09$ & $5.10 \mathrm{E}+01$ \\
\hline T1-204 & $1.09 \mathrm{E}-17$ & $1.45 \mathrm{E}-10$ & $1.27 \mathrm{E}+00$ \\
\hline T1-206 & $6.12 \mathrm{E}-17$ & $8.15 \mathrm{E}-10$ & $7.14 \mathrm{E}+00$ \\
\hline Tl-206m & $2.29 \mathrm{E}-15$ & $3.05 \mathrm{E}-08$ & $2.67 \mathrm{E}+02$ \\
\hline Tl-207 & $5.61 \mathrm{E}-17$ & $7.47 \mathrm{E}-10$ & $6.55 \mathrm{E}+00$ \\
\hline Tl-208 & $2.96 \mathrm{E}-15$ & $3.94 \mathrm{E}-08$ & $3.45 \mathrm{E}+02$ \\
\hline Tl-209 & $2.02 \mathrm{E}-15$ & $2.69 \mathrm{E}-08$ & $2.36 \mathrm{E}+02$ \\
\hline Tl-210 & $2.64 \mathrm{E}-15$ & $3.52 \mathrm{E}-08$ & $3.08 \mathrm{E}+02$ \\
\hline
\end{tabular}


SRNL-STI-2013-00115

\begin{tabular}{|c|c|c|c|}
\hline Nuclide & $\begin{array}{c}\text { Ground } \\
\text { Shine Dose } \\
\text { Rate } \\
\text { Coefficient } \\
\left(\mathrm{Sv}-\mathrm{m}^{2} / \mathbf{B q}-\mathbf{s}\right)\end{array}$ & $\begin{array}{l}\text { Ground Shine } \\
\text { Dose Rate } \\
\text { Coefficient } \\
\text { mrem-m }{ }^{2} / \mathbf{p C i}-\mathrm{h}\end{array}$ & $\begin{array}{l}\text { Ground Shine } \\
\text { Dose Rate } \\
\text { Coefficient } \\
\text { mrem-m } / \mu \mathrm{Ci}-\mathrm{y}\end{array}$ \\
\hline $\mathrm{Pb}-194$ & $9.95 \mathrm{E}-16$ & $1.33 \mathrm{E}-08$ & $1.16 \mathrm{E}+02$ \\
\hline $\mathrm{Pb}-195 \mathrm{~m}$ & $1.57 \mathrm{E}-15$ & $2.09 \mathrm{E}-08$ & $1.83 \mathrm{E}+02$ \\
\hline $\mathrm{Pb}-196$ & $4.58 \mathrm{E}-16$ & $6.10 \mathrm{E}-09$ & $5.34 \mathrm{E}+01$ \\
\hline $\mathrm{Pb}-197$ & $1.40 \mathrm{E}-15$ & $1.86 \mathrm{E}-08$ & $1.63 \mathrm{E}+02$ \\
\hline $\mathrm{Pb}-197 \mathrm{~m}$ & $1.10 \mathrm{E}-15$ & $1.47 \mathrm{E}-08$ & $1.28 \mathrm{E}+02$ \\
\hline $\mathrm{Pb}-198$ & $4.05 \mathrm{E}-16$ & 5.39E-09 & $4.73 \mathrm{E}+01$ \\
\hline $\mathrm{Pb}-199$ & $9.54 \mathrm{E}-16$ & $1.27 \mathrm{E}-08$ & $1.11 \mathrm{E}+02$ \\
\hline $\mathrm{Pb}-200$ & $1.85 \mathrm{E}-16$ & $2.46 \mathrm{E}-09$ & $2.16 \mathrm{E}+01$ \\
\hline $\mathrm{Pb}-201$ & $7.05 \mathrm{E}-16$ & 9.39E-09 & $8.23 \mathrm{E}+01$ \\
\hline $\mathrm{Pb}-201 \mathrm{~m}$ & $3.79 \mathrm{E}-16$ & $5.05 \mathrm{E}-09$ & $4.42 \mathrm{E}+01$ \\
\hline $\mathrm{Pb}-202$ & $1.88 \mathrm{E}-19$ & $2.50 \mathrm{E}-12$ & $2.19 \mathrm{E}-02$ \\
\hline $\mathrm{Pb}-202 \mathrm{~m}$ & $1.89 \mathrm{E}-15$ & $2.52 \mathrm{E}-08$ & $2.21 \mathrm{E}+02$ \\
\hline $\mathrm{Pb}-203$ & $2.87 \mathrm{E}-16$ & $3.82 \mathrm{E}-09$ & $3.35 \mathrm{E}+01$ \\
\hline $\mathrm{Pb}-204 \mathrm{~m}$ & $1.96 \mathrm{E}-15$ & $2.61 \mathrm{E}-08$ & $2.29 \mathrm{E}+02$ \\
\hline $\mathrm{Pb}-205$ & $1.90 \mathrm{E}-19$ & $2.53 \mathrm{E}-12$ & $2.22 \mathrm{E}-02$ \\
\hline $\mathrm{Pb}-209$ & $3.19 \mathrm{E}-18$ & $4.25 \mathrm{E}-11$ & $3.72 \mathrm{E}-01$ \\
\hline $\mathrm{Pb}-210$ & $2.17 \mathrm{E}-18$ & $2.89 \mathrm{E}-11$ & $2.53 \mathrm{E}-01$ \\
\hline $\mathrm{Pb}-211$ & $1.08 \mathrm{E}-16$ & $1.44 \mathrm{E}-09$ & $1.26 \mathrm{E}+01$ \\
\hline $\mathrm{Pb}-212$ & $1.32 \mathrm{E}-16$ & $1.76 \mathrm{E}-09$ & $1.54 \mathrm{E}+01$ \\
\hline $\mathrm{Pb}-214$ & $2.43 \mathrm{E}-16$ & $3.24 \mathrm{E}-09$ & $2.84 \mathrm{E}+01$ \\
\hline Bi-197 & $1.60 \mathrm{E}-15$ & $2.13 \mathrm{E}-08$ & $1.87 \mathrm{E}+02$ \\
\hline Bi-200 & $2.31 \mathrm{E}-15$ & $3.08 \mathrm{E}-08$ & $2.70 \mathrm{E}+02$ \\
\hline Bi-201 & $1.58 \mathrm{E}-15$ & $2.10 \mathrm{E}-08$ & $1.84 \mathrm{E}+02$ \\
\hline Bi-202 & $2.58 \mathrm{E}-15$ & $3.44 \mathrm{E}-08$ & $3.01 \mathrm{E}+02$ \\
\hline Bi-203 & $2.17 \mathrm{E}-15$ & $2.89 \mathrm{E}-08$ & $2.53 \mathrm{E}+02$ \\
\hline $\mathrm{Bi}-204$ & $2.71 \mathrm{E}-15$ & $3.61 \mathrm{E}-08$ & $3.16 \mathrm{E}+02$ \\
\hline $\mathrm{Bi}-205$ & $1.53 \mathrm{E}-15$ & $2.04 \mathrm{E}-08$ & $1.79 \mathrm{E}+02$ \\
\hline Bi-206 & $3.05 \mathrm{E}-15$ & $4.06 \mathrm{E}-08$ & $3.56 \mathrm{E}+02$ \\
\hline Bi-207 & $1.45 \mathrm{E}-15$ & $1.93 \mathrm{E}-08$ & $1.69 \mathrm{E}+02$ \\
\hline Bi-208 & $2.21 \mathrm{E}-15$ & $2.94 \mathrm{E}-08$ & $2.58 \mathrm{E}+02$ \\
\hline $\mathrm{Bi}-210$ & $3.51 \mathrm{E}-17$ & $4.68 \mathrm{E}-10$ & $4.10 \mathrm{E}+00$ \\
\hline Bi-210m & $2.44 \mathrm{E}-16$ & $3.25 \mathrm{E}-09$ & $2.85 \mathrm{E}+01$ \\
\hline Bi-211 & $4.45 \mathrm{E}-17$ & 5.93E-10 & $5.19 \mathrm{E}+00$ \\
\hline Bi-212 & $1.54 \mathrm{E}-16$ & $2.05 \mathrm{E}-09$ & $1.80 \mathrm{E}+01$ \\
\hline Bi-212n & $6.03 \mathrm{E}-17$ & $8.03 \mathrm{E}-10$ & $7.04 \mathrm{E}+00$ \\
\hline Bi-213 & $1.64 \mathrm{E}-16$ & $2.18 \mathrm{E}-09$ & $1.91 \mathrm{E}+01$ \\
\hline Bi-214 & $1.42 \mathrm{E}-15$ & $1.89 \mathrm{E}-08$ & $1.66 \mathrm{E}+02$ \\
\hline $\mathrm{Bi}-215$ & $3.07 \mathrm{E}-16$ & 4.09E-09 & $3.58 \mathrm{E}+01$ \\
\hline Bi-216 & $8.46 \mathrm{E}-16$ & $1.13 \mathrm{E}-08$ & $9.87 \mathrm{E}+01$ \\
\hline
\end{tabular}


SRNL-STI-2013-00115

\begin{tabular}{|c|c|c|c|}
\hline Nuclide & $\begin{array}{c}\text { Ground } \\
\text { Shine Dose } \\
\text { Rate } \\
\text { Coefficient } \\
\left(\mathrm{Sv}-\mathrm{m}^{2} / \mathrm{Bq}-\mathrm{s}\right)\end{array}$ & $\begin{array}{l}\text { Ground Shine } \\
\text { Dose Rate } \\
\text { Coefficient } \\
\text { mrem-m²/pCi-h }\end{array}$ & $\begin{array}{c}\text { Ground Shine } \\
\text { Dose Rate } \\
\text { Coefficient } \\
\text { mrem-m }{ }^{2} / \mu \mathrm{Ci}-\mathrm{y}\end{array}$ \\
\hline Po-203 & $1.52 \mathrm{E}-15$ & $2.02 \mathrm{E}-08$ & $1.77 \mathrm{E}+02$ \\
\hline Po-204 & $1.08 \mathrm{E}-15$ & $1.44 \mathrm{E}-08$ & $1.26 \mathrm{E}+02$ \\
\hline Po-205 & $1.47 \mathrm{E}-15$ & $1.96 \mathrm{E}-08$ & $1.72 \mathrm{E}+02$ \\
\hline Po-206 & $1.11 \mathrm{E}-15$ & $1.48 \mathrm{E}-08$ & $1.30 \mathrm{E}+02$ \\
\hline Po-207 & $1.20 \mathrm{E}-15$ & $1.60 \mathrm{E}-08$ & $1.40 \mathrm{E}+02$ \\
\hline Po-208 & $2.00 \mathrm{E}-20$ & $2.66 \mathrm{E}-13$ & $2.33 \mathrm{E}-03$ \\
\hline Po-209 & $5.77 \mathrm{E}-18$ & 7.69E-11 & $6.73 \mathrm{E}-01$ \\
\hline Po-210 & $9.22 \mathrm{E}-21$ & $1.23 \mathrm{E}-13$ & $1.08 \mathrm{E}-03$ \\
\hline Po-211 & $7.76 \mathrm{E}-18$ & $1.03 \mathrm{E}-10$ & $9.05 \mathrm{E}-01$ \\
\hline Рo-212 & $0.00 \mathrm{E}+00$ & $0.00 \mathrm{E}+00$ & $0.00 \mathrm{E}+00$ \\
\hline Po-212m & $6.76 \mathrm{E}-17$ & $9.00 \mathrm{E}-10$ & $7.89 \mathrm{E}+00$ \\
\hline Po-213 & $3.57 \mathrm{E}-20$ & $4.76 \mathrm{E}-13$ & $4.17 \mathrm{E}-03$ \\
\hline Po-214 & $7.87 \mathrm{E}-20$ & $1.05 \mathrm{E}-12$ & $9.18 \mathrm{E}-03$ \\
\hline Po-215 & $1.68 \mathrm{E}-19$ & $2.24 \mathrm{E}-12$ & $1.96 \mathrm{E}-02$ \\
\hline Po-216 & $1.45 \mathrm{E}-20$ & $1.93 \mathrm{E}-13$ & $1.69 \mathrm{E}-03$ \\
\hline Po-218 & $6.65 \mathrm{E}-24$ & $8.86 \mathrm{E}-17$ & $7.76 \mathrm{E}-07$ \\
\hline At-204 & $2.25 \mathrm{E}-15$ & $3.00 \mathrm{E}-08$ & $2.63 \mathrm{E}+02$ \\
\hline At-205 & $1.09 \mathrm{E}-15$ & $1.45 \mathrm{E}-08$ & $1.27 \mathrm{E}+02$ \\
\hline At-206 & $2.36 \mathrm{E}-15$ & $3.14 \mathrm{E}-08$ & $2.75 \mathrm{E}+02$ \\
\hline At-207 & $1.85 \mathrm{E}-15$ & $2.46 \mathrm{E}-08$ & $2.16 \mathrm{E}+02$ \\
\hline At-208 & $2.83 \mathrm{E}-15$ & $3.77 \mathrm{E}-08$ & $3.30 \mathrm{E}+02$ \\
\hline At-209 & $2.15 \mathrm{E}-15$ & $2.86 \mathrm{E}-08$ & $2.51 \mathrm{E}+02$ \\
\hline At-210 & $2.70 \mathrm{E}-15$ & $3.60 \mathrm{E}-08$ & $3.15 \mathrm{E}+02$ \\
\hline At-211 & $3.06 \mathrm{E}-17$ & $4.08 \mathrm{E}-10$ & $3.57 \mathrm{E}+00$ \\
\hline At-215 & $1.63 \mathrm{E}-19$ & $2.17 \mathrm{E}-12$ & $1.90 \mathrm{E}-02$ \\
\hline At-216 & $2.19 \mathrm{E}-18$ & $2.92 \mathrm{E}-11$ & $2.56 \mathrm{E}-01$ \\
\hline At-217 & $2.27 \mathrm{E}-19$ & $3.02 \mathrm{E}-12$ & $2.65 \mathrm{E}-02$ \\
\hline At-218 & $1.25 \mathrm{E}-19$ & $1.67 \mathrm{E}-12$ & $1.46 \mathrm{E}-02$ \\
\hline At-219 & $0.00 \mathrm{E}+00$ & $0.00 \mathrm{E}+00$ & $0.00 \mathrm{E}+00$ \\
\hline At-220 & $5.48 \mathrm{E}-16$ & 7.30E-09 & $6.39 \mathrm{E}+01$ \\
\hline Rn-207 & $9.47 \mathrm{E}-16$ & $1.26 \mathrm{E}-08$ & $1.10 \mathrm{E}+02$ \\
\hline Rn-209 & $1.11 \mathrm{E}-15$ & $1.48 \mathrm{E}-08$ & $1.30 \mathrm{E}+02$ \\
\hline $\mathrm{Rn}-210$ & 5.71E-17 & $7.61 \mathrm{E}-10$ & $6.66 \mathrm{E}+00$ \\
\hline $\mathrm{Rn}-211$ & $1.74 \mathrm{E}-15$ & 2.32E-08 & $2.03 \mathrm{E}+02$ \\
\hline $\mathrm{Rn}-212$ & $3.23 \mathrm{E}-19$ & $4.30 \mathrm{E}-12$ & $3.77 \mathrm{E}-02$ \\
\hline $\mathrm{Rn}-215$ & $0.00 \mathrm{E}+00$ & $0.00 \mathrm{E}+00$ & $0.00 \mathrm{E}+00$ \\
\hline $\mathrm{Rn}-216$ & $0.00 \mathrm{E}+00$ & $0.00 \mathrm{E}+00$ & $0.00 \mathrm{E}+00$ \\
\hline Rn-217 & $0.00 \mathrm{E}+00$ & $0.00 \mathrm{E}+00$ & $0.00 \mathrm{E}+00$ \\
\hline $\mathrm{Rn}-218$ & 7.24E-19 & $9.64 \mathrm{E}-12$ & $8.45 \mathrm{E}-02$ \\
\hline
\end{tabular}


SRNL-STI-2013-00115

\begin{tabular}{|c|c|c|c|}
\hline Nuclide & $\begin{array}{c}\text { Ground } \\
\text { Shine Dose } \\
\text { Rate } \\
\text { Coefficient } \\
\text { (Sv-m } / \mathbf{B q}-\mathbf{s})\end{array}$ & $\begin{array}{l}\text { Ground Shine } \\
\text { Dose Rate } \\
\text { Coefficient } \\
\text { mrem-m }{ }^{2} / p C i-h\end{array}$ & $\begin{array}{l}\text { Ground Shine } \\
\text { Dose Rate } \\
\text { Coefficient } \\
\text { mrem-m }{ }^{2} / \mu \mathrm{Ci}-\mathrm{y}\end{array}$ \\
\hline Rn-219 & $5.50 \mathrm{E}-17$ & $7.33 \mathrm{E}-10$ & $6.42 \mathrm{E}+00$ \\
\hline $\mathrm{Rn}-220$ & $6.01 \mathrm{E}-19$ & $8.01 \mathrm{E}-12$ & $7.01 \mathrm{E}-02$ \\
\hline $\mathrm{Rn}-222$ & $3.72 \mathrm{E}-19$ & $4.96 \mathrm{E}-12$ & $4.34 \mathrm{E}-02$ \\
\hline $\mathrm{Rn}-223$ & $3.80 \mathrm{E}-16$ & $5.06 \mathrm{E}-09$ & $4.43 \mathrm{E}+01$ \\
\hline Fr-212 & $1.05 \mathrm{E}-15$ & $1.40 \mathrm{E}-08$ & $1.23 \mathrm{E}+02$ \\
\hline Fr-219 & $3.37 \mathrm{E}-18$ & $4.49 \mathrm{E}-11$ & $3.93 \mathrm{E}-01$ \\
\hline Fr-220 & $8.45 \mathrm{E}-18$ & $1.13 \mathrm{E}-10$ & $9.86 \mathrm{E}-01$ \\
\hline Fr-221 & $2.69 \mathrm{E}-17$ & $3.58 \mathrm{E}-10$ & $3.14 \mathrm{E}+00$ \\
\hline Fr-222 & $2.36 \mathrm{E}-16$ & $3.14 \mathrm{E}-09$ & $2.75 \mathrm{E}+01$ \\
\hline Fr-223 & $7.73 \mathrm{E}-17$ & $1.03 \mathrm{E}-09$ & $9.02 \mathrm{E}+00$ \\
\hline Fr-224 & $6.00 \mathrm{E}-16$ & 7.99E-09 & $7.00 \mathrm{E}+01$ \\
\hline Fr-227 & $5.03 \mathrm{E}-16$ & $6.70 \mathrm{E}-09$ & $5.87 \mathrm{E}+01$ \\
\hline Ra-219 & $1.59 \mathrm{E}-16$ & 2.12E-09 & $1.86 \mathrm{E}+01$ \\
\hline Ra-220 & $4.46 \mathrm{E}-18$ & 5.94E-11 & $5.20 \mathrm{E}-01$ \\
\hline Ra-221 & $3.27 \mathrm{E}-17$ & $4.36 \mathrm{E}-10$ & $3.82 \mathrm{E}+00$ \\
\hline Ra-222 & $8.65 \mathrm{E}-18$ & $1.15 \mathrm{E}-10$ & $1.01 \mathrm{E}+00$ \\
\hline Ra-223 & $1.27 \mathrm{E}-16$ & $1.69 \mathrm{E}-09$ & $1.48 \mathrm{E}+01$ \\
\hline Ra-224 & $9.60 \mathrm{E}-18$ & $1.28 \mathrm{E}-10$ & $1.12 \mathrm{E}+00$ \\
\hline Ra-225 & $1.10 \mathrm{E}-17$ & $1.47 \mathrm{E}-10$ & $1.28 \mathrm{E}+00$ \\
\hline Ra-226 & $6.68 \mathrm{E}-18$ & $8.90 \mathrm{E}-11$ & $7.79 \mathrm{E}-01$ \\
\hline Ra-227 & $1.69 \mathrm{E}-16$ & $2.25 \mathrm{E}-09$ & $1.97 \mathrm{E}+01$ \\
\hline Ra-228 & 7.34E-19 & $9.78 \mathrm{E}-12$ & $8.56 \mathrm{E}-02$ \\
\hline Ra-230 & $7.54 \mathrm{E}-17$ & $1.00 \mathrm{E}-09$ & $8.80 \mathrm{E}+00$ \\
\hline Ac-223 & $1.62 \mathrm{E}-17$ & $2.16 \mathrm{E}-10$ & $1.89 \mathrm{E}+00$ \\
\hline Ac-224 & $2.05 \mathrm{E}-16$ & $2.73 \mathrm{E}-09$ & $2.39 \mathrm{E}+01$ \\
\hline Ac- 225 & $1.32 \mathrm{E}-17$ & $1.76 \mathrm{E}-10$ & $1.54 \mathrm{E}+00$ \\
\hline Ac-226 & $1.37 \mathrm{E}-16$ & $1.82 \mathrm{E}-09$ & $1.60 \mathrm{E}+01$ \\
\hline Ac-227 & $2.37 \mathrm{E}-19$ & $3.16 \mathrm{E}-12$ & $2.77 \mathrm{E}-02$ \\
\hline Ac- 228 & $8.39 \mathrm{E}-16$ & $1.12 \mathrm{E}-08$ & $9.79 \mathrm{E}+01$ \\
\hline Ac- 230 & $5.91 \mathrm{E}-16$ & $7.87 \mathrm{E}-09$ & $6.90 \mathrm{E}+01$ \\
\hline Ac-231 & $4.48 \mathrm{E}-16$ & $5.97 \mathrm{E}-09$ & $5.23 \mathrm{E}+01$ \\
\hline Ac-232 & $1.14 \mathrm{E}-15$ & $1.52 \mathrm{E}-08$ & $1.33 \mathrm{E}+02$ \\
\hline Ac- 233 & $5.74 \mathrm{E}-16$ & $7.65 \mathrm{E}-09$ & $6.70 \mathrm{E}+01$ \\
\hline Th-223 & $6.36 \mathrm{E}-17$ & $8.47 \mathrm{E}-10$ & $7.42 \mathrm{E}+00$ \\
\hline Th-224 & $2.10 \mathrm{E}-17$ & $2.80 \mathrm{E}-10$ & $2.45 \mathrm{E}+00$ \\
\hline Th-226 & $7.21 \mathrm{E}-18$ & $9.60 \mathrm{E}-11$ & $8.41 \mathrm{E}-01$ \\
\hline Th-227 & $1.15 \mathrm{E}-16$ & $1.53 \mathrm{E}-09$ & $1.34 \mathrm{E}+01$ \\
\hline Th-228 & $2.16 \mathrm{E}-18$ & $2.88 \mathrm{E}-11$ & $2.52 \mathrm{E}-01$ \\
\hline Th-229 & $7.75 \mathrm{E}-17$ & $1.03 \mathrm{E}-09$ & $9.04 \mathrm{E}+00$ \\
\hline
\end{tabular}


SRNL-STI-2013-00115

\begin{tabular}{|c|c|c|c|}
\hline Nuclide & $\begin{array}{c}\text { Ground } \\
\text { Shine Dose } \\
\text { Rate } \\
\text { Coefficient } \\
\text { (Sv-m } / \mathbf{B q}-\mathbf{s})\end{array}$ & $\begin{array}{l}\text { Ground Shine } \\
\text { Dose Rate } \\
\text { Coefficient } \\
\text { mrem-m }{ }^{2} / p C i-h\end{array}$ & $\begin{array}{c}\text { Ground Shine } \\
\text { Dose Rate } \\
\text { Coefficient } \\
\text { mrem-m }{ }^{2} / \mu \mathrm{Ci}-\mathrm{y}\end{array}$ \\
\hline Th-230 & $6.41 \mathrm{E}-19$ & $8.54 \mathrm{E}-12$ & $7.48 \mathrm{E}-02$ \\
\hline Th-231 & $1.52 \mathrm{E}-17$ & $2.02 \mathrm{E}-10$ & $1.77 \mathrm{E}+00$ \\
\hline Th-232 & $4.53 \mathrm{E}-19$ & $6.03 \mathrm{E}-12$ & $5.29 \mathrm{E}-02$ \\
\hline Th-233 & $6.99 \mathrm{E}-17$ & $9.31 \mathrm{E}-10$ & $8.16 \mathrm{E}+00$ \\
\hline Th-234 & $8.19 \mathrm{E}-18$ & $1.09 \mathrm{E}-10$ & $9.56 \mathrm{E}-01$ \\
\hline Th-235 & $1.30 \mathrm{E}-16$ & 1.73E-09 & $1.52 \mathrm{E}+01$ \\
\hline Th-236 & $5.96 \mathrm{E}-17$ & 7.94E-10 & $6.95 \mathrm{E}+00$ \\
\hline $\mathrm{Pa}-227$ & $1.80 \mathrm{E}-17$ & $2.40 \mathrm{E}-10$ & $2.10 \mathrm{E}+00$ \\
\hline $\mathrm{Pa}-228$ & $1.26 \mathrm{E}-15$ & $1.68 \mathrm{E}-08$ & $1.47 \mathrm{E}+02$ \\
\hline $\mathrm{Pa}-229$ & $5.46 \mathrm{E}-17$ & $7.27 \mathrm{E}-10$ & $6.37 \mathrm{E}+00$ \\
\hline Pa-230 & $6.22 \mathrm{E}-16$ & $8.29 \mathrm{E}-09$ & $7.26 \mathrm{E}+01$ \\
\hline $\mathrm{Pa}-231$ & $3.47 \mathrm{E}-17$ & $4.62 \mathrm{E}-10$ & $4.05 \mathrm{E}+00$ \\
\hline $\mathrm{Pa}-232$ & $8.80 \mathrm{E}-16$ & $1.17 \mathrm{E}-08$ & $1.03 \mathrm{E}+02$ \\
\hline Pa-233 & $2.02 \mathrm{E}-16$ & 2.69E-09 & $2.36 \mathrm{E}+01$ \\
\hline $\mathrm{Pa}-234$ & $1.38 \mathrm{E}-15$ & $1.84 \mathrm{E}-08$ & $1.61 \mathrm{E}+02$ \\
\hline $\mathrm{Pa}-234 \mathrm{~m}$ & $1.12 \mathrm{E}-16$ & 1.49E-09 & $1.31 \mathrm{E}+01$ \\
\hline $\mathrm{Pa}-235$ & $5.05 \mathrm{E}-17$ & $6.73 \mathrm{E}-10$ & $5.89 \mathrm{E}+00$ \\
\hline Pa-236 & $9.20 \mathrm{E}-16$ & $1.23 \mathrm{E}-08$ & $1.07 \mathrm{E}+02$ \\
\hline Pa-237 & $6.40 \mathrm{E}-16$ & $8.52 \mathrm{E}-09$ & $7.47 \mathrm{E}+01$ \\
\hline $\mathrm{U}-227$ & $1.06 \mathrm{E}-16$ & $1.41 \mathrm{E}-09$ & $1.24 \mathrm{E}+01$ \\
\hline U-228 & $3.91 \mathrm{E}-18$ & $5.21 \mathrm{E}-11$ & $4.56 \mathrm{E}-01$ \\
\hline $\mathrm{U}-230$ & $1.52 \mathrm{E}-18$ & $2.02 \mathrm{E}-11$ & $1.77 \mathrm{E}-01$ \\
\hline U-231 & $6.67 \mathrm{E}-17$ & $8.88 \mathrm{E}-10$ & $7.78 \mathrm{E}+00$ \\
\hline $\mathrm{U}-232$ & $7.30 \mathrm{E}-19$ & $9.72 \mathrm{E}-12$ & $8.52 \mathrm{E}-02$ \\
\hline $\mathrm{U}-233$ & $4.76 \mathrm{E}-19$ & $6.34 \mathrm{E}-12$ & $5.55 \mathrm{E}-02$ \\
\hline U-234 & $5.80 \mathrm{E}-19$ & $7.73 \mathrm{E}-12$ & $6.77 \mathrm{E}-02$ \\
\hline U-235 & $1.49 \mathrm{E}-16$ & $1.98 \mathrm{E}-09$ & $1.74 \mathrm{E}+01$ \\
\hline U-235m & $0.00 \mathrm{E}+00$ & $0.00 \mathrm{E}+00$ & $0.00 \mathrm{E}+00$ \\
\hline U-236 & $4.82 \mathrm{E}-19$ & $6.42 \mathrm{E}-12$ & $5.62 \mathrm{E}-02$ \\
\hline $\mathrm{U}-237$ & $1.23 \mathrm{E}-16$ & $1.64 \mathrm{E}-09$ & $1.44 \mathrm{E}+01$ \\
\hline U-238 & $3.91 \mathrm{E}-19$ & $5.21 \mathrm{E}-12$ & $4.56 \mathrm{E}-02$ \\
\hline U-239 & $8.15 \mathrm{E}-17$ & $1.09 \mathrm{E}-09$ & $9.51 \mathrm{E}+00$ \\
\hline U-240 & $5.59 \mathrm{E}-18$ & $7.45 \mathrm{E}-11$ & $6.52 \mathrm{E}-01$ \\
\hline U-242 & $7.21 \mathrm{E}-17$ & $9.60 \mathrm{E}-10$ & $8.41 \mathrm{E}+00$ \\
\hline $\mathrm{Np}-232$ & $1.12 \mathrm{E}-15$ & $1.49 \mathrm{E}-08$ & $1.31 \mathrm{E}+02$ \\
\hline $\mathrm{Np}-233$ & $7.72 \mathrm{E}-17$ & $1.03 \mathrm{E}-09$ & $9.01 \mathrm{E}+00$ \\
\hline $\mathrm{Np}-234$ & $9.99 \mathrm{E}-16$ & $1.33 \mathrm{E}-08$ & $1.17 \mathrm{E}+02$ \\
\hline $\mathrm{Np}-235$ & $2.16 \mathrm{E}-18$ & $2.88 \mathrm{E}-11$ & $2.52 \mathrm{E}-01$ \\
\hline $\mathrm{Np}-236$ & $1.28 \mathrm{E}-16$ & $1.70 \mathrm{E}-09$ & $1.49 \mathrm{E}+01$ \\
\hline
\end{tabular}


SRNL-STI-2013-00115

\begin{tabular}{|c|c|c|c|}
\hline Nuclide & $\begin{array}{c}\text { Ground } \\
\text { Shine Dose } \\
\text { Rate } \\
\text { Coefficient } \\
\left(\mathrm{Sv}-\mathbf{m}^{2} / \mathbf{B q}-\mathbf{s}\right) \\
\end{array}$ & $\begin{array}{l}\text { Ground Shine } \\
\text { Dose Rate } \\
\text { Coefficient } \\
\text { mrem-m } / \mathbf{p C i} \text {-h }\end{array}$ & $\begin{array}{l}\text { Ground Shine } \\
\text { Dose Rate } \\
\text { Coefficient } \\
\text { mrem-m } / \mu \mathrm{Ci}-\mathrm{y}\end{array}$ \\
\hline Np-236m & 4.32E-17 & $5.75 \mathrm{E}-10$ & $5.04 \mathrm{E}+00$ \\
\hline $\mathrm{Np}-237$ & $2.44 \mathrm{E}-17$ & $3.25 \mathrm{E}-10$ & $2.85 \mathrm{E}+00$ \\
\hline $\mathrm{Np}-238$ & $5.63 \mathrm{E}-16$ & 7.50E-09 & $6.57 \mathrm{E}+01$ \\
\hline $\mathrm{Np}-239$ & $1.62 \mathrm{E}-16$ & $2.16 \mathrm{E}-09$ & $1.89 \mathrm{E}+01$ \\
\hline $\mathrm{Np}-240$ & $1.00 \mathrm{E}-15$ & $1.33 \mathrm{E}-08$ & $1.17 \mathrm{E}+02$ \\
\hline $\mathrm{Np}-240 \mathrm{~m}$ & $3.75 \mathrm{E}-16$ & $5.00 \mathrm{E}-09$ & $4.38 \mathrm{E}+01$ \\
\hline $\mathrm{Np}-241$ & $7.28 \mathrm{E}-17$ & $9.70 \mathrm{E}-10$ & $8.49 \mathrm{E}+00$ \\
\hline $\mathrm{Np}-242$ & $3.44 \mathrm{E}-16$ & $4.58 \mathrm{E}-09$ & $4.01 \mathrm{E}+01$ \\
\hline $\mathrm{Np}-242 \mathrm{~m}$ & $9.19 \mathrm{E}-16$ & $1.22 \mathrm{E}-08$ & $1.07 \mathrm{E}+02$ \\
\hline $\mathrm{Pu}-232$ & $5.31 \mathrm{E}-17$ & $7.07 \mathrm{E}-10$ & $6.20 \mathrm{E}+00$ \\
\hline $\mathrm{Pu}-234$ & $5.77 \mathrm{E}-17$ & $7.69 \mathrm{E}-10$ & $6.73 \mathrm{E}+00$ \\
\hline $\mathrm{Pu}-235$ & $7.98 \mathrm{E}-17$ & $1.06 \mathrm{E}-09$ & $9.31 \mathrm{E}+00$ \\
\hline $\mathrm{Pu}-236$ & $6.69 \mathrm{E}-19$ & $8.91 \mathrm{E}-12$ & 7.81E-02 \\
\hline $\mathrm{Pu}-237$ & $4.27 \mathrm{E}-17$ & $5.69 \mathrm{E}-10$ & $4.98 \mathrm{E}+00$ \\
\hline $\mathrm{Pu}-238$ & $5.99 \mathrm{E}-19$ & $7.98 \mathrm{E}-12$ & $6.99 \mathrm{E}-02$ \\
\hline $\mathrm{Pu}-239$ & $3.06 \mathrm{E}-19$ & $4.08 \mathrm{E}-12$ & $3.57 \mathrm{E}-02$ \\
\hline $\mathrm{Pu}-240$ & $5.68 \mathrm{E}-19$ & $7.57 \mathrm{E}-12$ & $6.63 \mathrm{E}-02$ \\
\hline $\mathrm{Pu}-241$ & $1.43 \mathrm{E}-21$ & $1.90 \mathrm{E}-14$ & $1.67 \mathrm{E}-04$ \\
\hline $\mathrm{Pu}-242$ & $5.56 \mathrm{E}-19$ & $7.41 \mathrm{E}-12$ & $6.49 \mathrm{E}-02$ \\
\hline $\mathrm{Pu}-243$ & $2.27 \mathrm{E}-17$ & $3.02 \mathrm{E}-10$ & $2.65 \mathrm{E}+00$ \\
\hline $\mathrm{Pu}-244$ & $1.92 \mathrm{E}-17$ & $2.56 \mathrm{E}-10$ & $2.24 \mathrm{E}+00$ \\
\hline $\mathrm{Pu}-245$ & $3.91 \mathrm{E}-16$ & $5.21 \mathrm{E}-09$ & $4.56 \mathrm{E}+01$ \\
\hline $\mathrm{Pu}-246$ & $1.25 \mathrm{E}-16$ & $1.67 \mathrm{E}-09$ & $1.46 \mathrm{E}+01$ \\
\hline Am-237 & $3.38 \mathrm{E}-16$ & $4.50 \mathrm{E}-09$ & $3.94 \mathrm{E}+01$ \\
\hline Am-238 & $8.34 \mathrm{E}-16$ & $1.11 \mathrm{E}-08$ & $9.73 \mathrm{E}+01$ \\
\hline Am-239 & $2.09 \mathrm{E}-16$ & $2.78 \mathrm{E}-09$ & $2.44 \mathrm{E}+01$ \\
\hline Am-240 & $9.60 \mathrm{E}-16$ & $1.28 \mathrm{E}-08$ & $1.12 \mathrm{E}+02$ \\
\hline Am-241 & $2.18 \mathrm{E}-17$ & $2.90 \mathrm{E}-10$ & $2.54 \mathrm{E}+00$ \\
\hline Am-242 & $1.61 \mathrm{E}-17$ & $2.14 \mathrm{E}-10$ & $1.88 \mathrm{E}+00$ \\
\hline Am-242m & $2.07 \mathrm{E}-18$ & $2.76 \mathrm{E}-11$ & $2.42 \mathrm{E}-01$ \\
\hline Am-243 & $4.96 \mathrm{E}-17$ & $6.61 \mathrm{E}-10$ & $5.79 \mathrm{E}+00$ \\
\hline Am-244 & $7.55 \mathrm{E}-16$ & $1.01 \mathrm{E}-08$ & $8.81 \mathrm{E}+01$ \\
\hline Am-244m & $7.01 \mathrm{E}-17$ & $9.34 \mathrm{E}-10$ & $8.18 \mathrm{E}+00$ \\
\hline Am-245 & $4.10 \mathrm{E}-17$ & $5.46 \mathrm{E}-10$ & $4.78 \mathrm{E}+00$ \\
\hline Am-246 & $7.28 \mathrm{E}-16$ & $9.70 \mathrm{E}-09$ & $8.49 \mathrm{E}+01$ \\
\hline $\mathrm{Am}-246 \mathrm{~m}$ & $9.59 \mathrm{E}-16$ & $1.28 \mathrm{E}-08$ & $1.12 \mathrm{E}+02$ \\
\hline Am-247 & $1.69 \mathrm{E}-16$ & $2.25 \mathrm{E}-09$ & $1.97 \mathrm{E}+01$ \\
\hline Cm-238 & $6.98 \mathrm{E}-17$ & $9.30 \mathrm{E}-10$ & $8.14 \mathrm{E}+00$ \\
\hline Cm-239 & $2.30 \mathrm{E}-16$ & $3.06 \mathrm{E}-09$ & $2.68 \mathrm{E}+01$ \\
\hline
\end{tabular}


SRNL-STI-2013-00115

\begin{tabular}{|c|c|c|c|}
\hline Nuclide & $\begin{array}{c}\text { Ground } \\
\text { Shine Dose } \\
\text { Rate } \\
\text { Coefficient } \\
\left(\mathrm{Sv}-\mathbf{m}^{2} / \mathbf{B q}-\mathbf{s}\right)\end{array}$ & $\begin{array}{l}\text { Ground Shine } \\
\text { Dose Rate } \\
\text { Coefficient } \\
\text { mrem-m²/pCi-h }\end{array}$ & $\begin{array}{c}\text { Ground Shine } \\
\text { Dose Rate } \\
\text { Coefficient } \\
\text { mrem-m }{ }^{2} / \mu \mathrm{Ci}-\mathrm{y}\end{array}$ \\
\hline $\mathrm{Cm}-240$ & $7.49 \mathrm{E}-19$ & $9.98 \mathrm{E}-12$ & $8.74 \mathrm{E}-02$ \\
\hline $\mathrm{Cm}-241$ & $4.64 \mathrm{E}-16$ & $6.18 \mathrm{E}-09$ & $5.41 \mathrm{E}+01$ \\
\hline $\mathrm{Cm}-242$ & $6.68 \mathrm{E}-19$ & $8.90 \mathrm{E}-12$ & 7.79E-02 \\
\hline $\mathrm{Cm}-243$ & $1.18 \mathrm{E}-16$ & $1.57 \mathrm{E}-09$ & $1.38 \mathrm{E}+01$ \\
\hline $\mathrm{Cm}-244$ & $5.85 \mathrm{E}-19$ & $7.79 \mathrm{E}-12$ & $6.83 \mathrm{E}-02$ \\
\hline $\mathrm{Cm}-245$ & $9.11 \mathrm{E}-17$ & $1.21 \mathrm{E}-09$ & $1.06 \mathrm{E}+01$ \\
\hline $\mathrm{Cm}-246$ & $3.87 \mathrm{E}-18$ & $5.15 \mathrm{E}-11$ & $4.52 \mathrm{E}-01$ \\
\hline $\mathrm{Cm}-247$ & $2.98 \mathrm{E}-16$ & 3.97E-09 & $3.48 \mathrm{E}+01$ \\
\hline $\mathrm{Cm}-248$ & $1.25 \mathrm{E}-15$ & $1.67 \mathrm{E}-08$ & $1.46 \mathrm{E}+02$ \\
\hline $\mathrm{Cm}-249$ & $3.30 \mathrm{E}-17$ & $4.40 \mathrm{E}-10$ & $3.85 \mathrm{E}+00$ \\
\hline $\mathrm{Cm}-250$ & $1.27 \mathrm{E}-14$ & $1.69 \mathrm{E}-07$ & $1.48 \mathrm{E}+03$ \\
\hline $\mathrm{Cm}-251$ & $1.47 \mathrm{E}-16$ & $1.96 \mathrm{E}-09$ & $1.72 \mathrm{E}+01$ \\
\hline Bk-245 & $2.06 \mathrm{E}-16$ & $2.74 \mathrm{E}-09$ & $2.40 \mathrm{E}+01$ \\
\hline Bk-246 & $7.93 \mathrm{E}-16$ & $1.06 \mathrm{E}-08$ & $9.25 \mathrm{E}+01$ \\
\hline Bk-247 & $1.32 \mathrm{E}-16$ & $1.76 \mathrm{E}-09$ & $1.54 \mathrm{E}+01$ \\
\hline $\mathrm{Bk}-248 \mathrm{~m}$ & $5.75 \mathrm{E}-17$ & $7.66 \mathrm{E}-10$ & $6.71 \mathrm{E}+00$ \\
\hline Bk-249 & $5.67 \mathrm{E}-21$ & $7.55 \mathrm{E}-14$ & $6.62 \mathrm{E}-04$ \\
\hline Bk-250 & $8.52 \mathrm{E}-16$ & $1.13 \mathrm{E}-08$ & $9.94 \mathrm{E}+01$ \\
\hline Bk-251 & $9.39 \mathrm{E}-17$ & $1.25 \mathrm{E}-09$ & $1.10 \mathrm{E}+01$ \\
\hline Cf-244 & $7.62 \mathrm{E}-19$ & $1.01 \mathrm{E}-11$ & $8.89 \mathrm{E}-02$ \\
\hline Cf-246 & $5.65 \mathrm{E}-19$ & $7.53 \mathrm{E}-12$ & $6.59 \mathrm{E}-02$ \\
\hline Cf-247 & $8.45 \mathrm{E}-17$ & 1.13E-09 & $9.86 \mathrm{E}+00$ \\
\hline Cf-248 & $9.49 \mathrm{E}-19$ & $1.26 \mathrm{E}-11$ & $1.11 \mathrm{E}-01$ \\
\hline Cf-249 & $3.08 \mathrm{E}-16$ & $4.10 \mathrm{E}-09$ & $3.59 \mathrm{E}+01$ \\
\hline Cf-250 & $9.75 \mathrm{E}-18$ & $1.30 \mathrm{E}-10$ & $1.14 \mathrm{E}+00$ \\
\hline Cf-251 & $1.07 \mathrm{E}-16$ & $1.43 \mathrm{E}-09$ & $1.25 \mathrm{E}+01$ \\
\hline Cf-252 & $4.32 \mathrm{E}-16$ & $5.75 \mathrm{E}-09$ & $5.04 \mathrm{E}+01$ \\
\hline Cf-253 & $2.12 \mathrm{E}-18$ & $2.82 \mathrm{E}-11$ & $2.47 \mathrm{E}-01$ \\
\hline Cf-254 & $1.60 \mathrm{E}-14$ & $2.13 \mathrm{E}-07$ & $1.87 \mathrm{E}+03$ \\
\hline Cf-255 & $5.78 \mathrm{E}-18$ & $7.70 \mathrm{E}-11$ & $6.74 \mathrm{E}-01$ \\
\hline Es-249 & $3.80 \mathrm{E}-16$ & $5.06 \mathrm{E}-09$ & $4.43 \mathrm{E}+01$ \\
\hline Es-250 & $1.12 \mathrm{E}-15$ & $1.49 \mathrm{E}-08$ & $1.31 \mathrm{E}+02$ \\
\hline Es-250m & $5.08 \mathrm{E}-16$ & $6.77 \mathrm{E}-09$ & $5.93 \mathrm{E}+01$ \\
\hline Es-251 & $8.31 \mathrm{E}-17$ & $1.11 \mathrm{E}-09$ & $9.70 \mathrm{E}+00$ \\
\hline Es-253 & $5.00 \mathrm{E}-19$ & $6.66 \mathrm{E}-12$ & $5.83 \mathrm{E}-02$ \\
\hline Es-254 & $8.85 \mathrm{E}-18$ & $1.18 \mathrm{E}-10$ & $1.03 \mathrm{E}+00$ \\
\hline Es-254m & $4.59 \mathrm{E}-16$ & $6.11 \mathrm{E}-09$ & $5.36 \mathrm{E}+01$ \\
\hline Es-255 & $6.83 \mathrm{E}-19$ & $9.10 \mathrm{E}-12$ & $7.97 \mathrm{E}-02$ \\
\hline Es-256 & $6.66 \mathrm{E}-17$ & $8.87 \mathrm{E}-10$ & $7.77 \mathrm{E}+00$ \\
\hline
\end{tabular}


SRNL-STI-2013-00115

\begin{tabular}{|c|c|c|c|}
\hline Nuclide & $\begin{array}{c}\text { Ground } \\
\text { Shine Dose } \\
\text { Rate } \\
\text { Coefficient } \\
\left(\mathbf{S v}-\mathbf{m}^{2} / \mathbf{B q}-\mathbf{s}\right)\end{array}$ & $\begin{array}{c}\text { Ground Shine } \\
\text { Dose Rate } \\
\text { Coefficient } \\
\mathbf{m r e m}^{2} / \mathbf{p C i}-\mathbf{h}\end{array}$ & $\begin{array}{c}\text { Ground Shine } \\
\text { Dose Rate } \\
\text { Coefficient } \\
\text { mrem-m } \mathbf{2} / \boldsymbol{\mu C i}-\mathbf{y}\end{array}$ \\
\hline Fm-251 & $1.40 \mathrm{E}-16$ & $1.86 \mathrm{E}-09$ & $1.63 \mathrm{E}+01$ \\
\hline Fm-252 & $8.98 \mathrm{E}-19$ & $1.20 \mathrm{E}-11$ & $1.05 \mathrm{E}-01$ \\
\hline Fm-253 & $5.61 \mathrm{E}-17$ & $7.47 \mathrm{E}-10$ & $6.55 \mathrm{E}+00$ \\
\hline Fm-254 & $7.25 \mathrm{E}-18$ & $9.66 \mathrm{E}-11$ & $8.46 \mathrm{E}-01$ \\
\hline Fm-255 & $7.16 \mathrm{E}-18$ & $9.54 \mathrm{E}-11$ & $8.35 \mathrm{E}-01$ \\
\hline Fm-256 & $1.17 \mathrm{E}-14$ & $1.56 \mathrm{E}-07$ & $1.37 \mathrm{E}+03$ \\
\hline Fm-257 & $1.32 \mathrm{E}-16$ & $1.76 \mathrm{E}-09$ & $1.54 \mathrm{E}+01$ \\
\hline
\end{tabular}




\section{APPENDIX D}

\section{DERIVED CONCENTRATION STANDARDS}

The data in Appendix D are the $95^{\text {th }}$ percentile and $50^{\text {th }}$ percentile DCS for ingestion, inhalation, submersion in air and water, and ground shine for the reference person. These DCS were calculated from the dose coefficients from Derived Concentration Standard report and in the DC_PACK3 toolbox, and the reference person intake values. 
Table D-1. 95th Percentile Ingestion DCS

\begin{tabular}{|c|c|c|c|c|c|c|c|c|c|c|c|c|}
\hline \multicolumn{13}{|c|}{$95 \%$} \\
\hline \multirow[t]{2}{*}{ Nuclide } & \multicolumn{2}{|c|}{ Water Ingestion DCS } & \multicolumn{2}{|c|}{ Meat Ingestion DCS } & \multicolumn{2}{|c|}{ Dairy Ingestion DCS } & \multicolumn{2}{|c|}{$\begin{array}{l}\text { Freshwater Fish } \\
\text { Ingestion DCS }\end{array}$} & \multicolumn{2}{|c|}{$\begin{array}{l}\text { Saltwater Shellfish } \\
\text { Ingestion DCS }\end{array}$} & \multicolumn{2}{|c|}{ Produce Ingestion DCS } \\
\hline & $(\mathrm{Bq} / \mathrm{L})$ & $(\mathrm{pCi} / \mathbf{L})$ & $(\mathrm{Bq} / \mathbf{k g})$ & $(\mathrm{pCi} / \mathbf{k g})$ & $(\mathbf{B q} / \mathbf{L})$ & $(\mathrm{pCi} / \mathrm{L})$ & $(\mathrm{Bq} / \mathrm{kg})$ & $(\mathrm{pCi} / \mathbf{k g})$ & $(\mathrm{Bq} / \mathbf{k g})$ & $(\mathrm{pCi} / \mathbf{k g})$ & $(\mathrm{Bq} / \mathrm{kg})$ & $(\mathrm{pCi} / \mathbf{k g})$ \\
\hline $\mathrm{H}-3$ & $5.92 \mathrm{E}+04$ & $1.60 \mathrm{E}+06$ & $5.88 \mathrm{E}+05$ & $1.59 \mathrm{E}+07$ & $1.82 \mathrm{E}+05$ & $4.93 \mathrm{E}+06$ & $2.16 \mathrm{E}+06$ & $5.85 \mathrm{E}+07$ & $5.95 \mathrm{E}+06$ & $1.61 \mathrm{E}+08$ & $1.47 \mathrm{E}+05$ & $1.61 \mathrm{E}+08$ \\
\hline $\mathrm{Be}-7$ & $3.57 \mathrm{E}+04$ & $9.66 \mathrm{E}+05$ & $3.55 \mathrm{E}+05$ & $9.59 \mathrm{E}+06$ & $1.10 \mathrm{E}+05$ & $2.98 \mathrm{E}+06$ & $1.31 \mathrm{E}+06$ & $3.53 \mathrm{E}+07$ & $3.59 \mathrm{E}+06$ & $9.71 \mathrm{E}+07$ & $8.87 \mathrm{E}+04$ & $9.71 \mathrm{E}+07$ \\
\hline Be-10 & $7.97 \mathrm{E}+02$ & $2.15 \mathrm{E}+04$ & $7.91 \mathrm{E}+03$ & $2.14 \mathrm{E}+05$ & $2.46 \mathrm{E}+03$ & $6.64 \mathrm{E}+04$ & $2.91 \mathrm{E}+04$ & $7.88 \mathrm{E}+05$ & $8.01 \mathrm{E}+04$ & $2.17 \mathrm{E}+06$ & $1.98 \mathrm{E}+03$ & $2.17 \mathrm{E}+06$ \\
\hline $\mathrm{C}-11$ & $4.01 \mathrm{E}+04$ & $1.08 \mathrm{E}+06$ & $3.98 \mathrm{E}+05$ & $1.08 \mathrm{E}+07$ & $1.24 \mathrm{E}+05$ & $3.34 \mathrm{E}+06$ & $1.47 \mathrm{E}+06$ & $3.96 \mathrm{E}+07$ & $4.03 \mathrm{E}+06$ & $1.09 \mathrm{E}+08$ & $9.96 \mathrm{E}+04$ & $1.09 \mathrm{E}+08$ \\
\hline C-14 & $1.96 \mathrm{E}+03$ & $5.31 \mathrm{E}+04$ & $1.95 \mathrm{E}+04$ & $5.27 \mathrm{E}+05$ & $6.05 \mathrm{E}+03$ & $1.64 \mathrm{E}+05$ & $7.18 \mathrm{E}+04$ & $1.94 \mathrm{E}+06$ & $1.97 \mathrm{E}+05$ & $5.34 \mathrm{E}+06$ & $4.88 \mathrm{E}+03$ & $5.34 \mathrm{E}+06$ \\
\hline $\mathrm{F}-18$ & $1.99 \mathrm{E}+04$ & $5.39 \mathrm{E}+05$ & $1.98 \mathrm{E}+05$ & $5.35 \mathrm{E}+06$ & $6.14 \mathrm{E}+04$ & $1.66 \mathrm{E}+06$ & $7.28 \mathrm{E}+05$ & $1.97 \mathrm{E}+07$ & $2.00 \mathrm{E}+06$ & $5.41 \mathrm{E}+07$ & $4.95 \mathrm{E}+04$ & $5.41 \mathrm{E}+07$ \\
\hline $\mathrm{Na}-22$ & $3.21 \mathrm{E}+02$ & $8.66 \mathrm{E}+03$ & $3.18 \mathrm{E}+03$ & $8.60 \mathrm{E}+04$ & $9.87 \mathrm{E}+02$ & $2.67 \mathrm{E}+04$ & $1.17 \mathrm{E}+04$ & $3.17 \mathrm{E}+05$ & $3.22 \mathrm{E}+04$ & $8.71 \mathrm{E}+05$ & $7.95 \mathrm{E}+02$ & $8.71 \mathrm{E}+05$ \\
\hline $\mathrm{Na}-24$ & $2.28 \mathrm{E}+03$ & $6.16 \mathrm{E}+04$ & $2.26 \mathrm{E}+04$ & $6.11 \mathrm{E}+05$ & $7.02 \mathrm{E}+03$ & $1.90 \mathrm{E}+05$ & $8.33 \mathrm{E}+04$ & $2.25 \mathrm{E}+06$ & $2.29 \mathrm{E}+05$ & $6.19 \mathrm{E}+06$ & $5.65 \mathrm{E}+03$ & $6.19 \mathrm{E}+06$ \\
\hline $\mathrm{Mg}-28$ & $4.41 \mathrm{E}+02$ & $1.19 \mathrm{E}+04$ & $4.38 \mathrm{E}+03$ & $1.18 \mathrm{E}+05$ & $1.36 \mathrm{E}+03$ & $3.67 \mathrm{E}+04$ & $1.61 \mathrm{E}+04$ & $4.36 \mathrm{E}+05$ & $4.43 \mathrm{E}+04$ & $1.20 \mathrm{E}+06$ & $1.09 \mathrm{E}+03$ & $1.20 \mathrm{E}+06$ \\
\hline Al-26 & $2.71 \mathrm{E}+02$ & $7.32 \mathrm{E}+03$ & $2.69 \mathrm{E}+03$ & $7.27 \mathrm{E}+04$ & $8.35 \mathrm{E}+02$ & $2.26 \mathrm{E}+04$ & $9.90 \mathrm{E}+03$ & $2.68 \mathrm{E}+05$ & $2.72 \mathrm{E}+04$ & $7.36 \mathrm{E}+05$ & $6.72 \mathrm{E}+02$ & $7.36 \mathrm{E}+05$ \\
\hline $\mathrm{Si}-31$ & $5.84 \mathrm{E}+03$ & $1.58 \mathrm{E}+05$ & $5.80 \mathrm{E}+04$ & $1.57 \mathrm{E}+06$ & $1.80 \mathrm{E}+04$ & $4.86 \mathrm{E}+05$ & $2.13 \mathrm{E}+05$ & $5.77 \mathrm{E}+06$ & $5.87 \mathrm{E}+05$ & $1.59 \mathrm{E}+07$ & $1.45 \mathrm{E}+04$ & $1.59 \mathrm{E}+07$ \\
\hline $\mathrm{Si}-32$ & $1.56 \mathrm{E}+03$ & $4.21 \mathrm{E}+04$ & $1.55 \mathrm{E}+04$ & $4.18 \mathrm{E}+05$ & $4.80 \mathrm{E}+03$ & $1.30 \mathrm{E}+05$ & $5.69 \mathrm{E}+04$ & $1.54 \mathrm{E}+06$ & $1.56 \mathrm{E}+05$ & $4.23 \mathrm{E}+06$ & $3.86 \mathrm{E}+03$ & $4.23 \mathrm{E}+06$ \\
\hline $\mathrm{P}-32$ & $3.67 \mathrm{E}+02$ & $9.92 \mathrm{E}+03$ & $3.64 \mathrm{E}+03$ & $9.84 \mathrm{E}+04$ & $1.13 \mathrm{E}+03$ & $3.05 \mathrm{E}+04$ & $1.34 \mathrm{E}+04$ & $3.62 \mathrm{E}+05$ & $3.69 \mathrm{E}+04$ & $9.97 \mathrm{E}+05$ & $9.10 \mathrm{E}+02$ & $9.97 \mathrm{E}+05$ \\
\hline P-33 & $3.69 \mathrm{E}+03$ & $9.97 \mathrm{E}+04$ & $3.66 \mathrm{E}+04$ & $9.90 \mathrm{E}+05$ & $1.14 \mathrm{E}+04$ & $3.07 \mathrm{E}+05$ & $1.35 \mathrm{E}+05$ & $3.65 \mathrm{E}+06$ & $3.71 \mathrm{E}+05$ & $1.00 \mathrm{E}+07$ & $9.16 \mathrm{E}+03$ & $1.00 \mathrm{E}+07$ \\
\hline S-35 & $7.15 \mathrm{E}+03$ & $1.93 \mathrm{E}+05$ & $7.10 \mathrm{E}+04$ & $1.92 \mathrm{E}+06$ & $2.20 \mathrm{E}+04$ & $5.95 \mathrm{E}+05$ & $2.61 \mathrm{E}+05$ & $7.06 \mathrm{E}+06$ & $7.18 \mathrm{E}+05$ & $1.94 \mathrm{E}+07$ & $1.77 \mathrm{E}+04$ & $1.94 \mathrm{E}+07$ \\
\hline S-38 & $2.87 \mathrm{E}+03$ & $7.75 \mathrm{E}+04$ & $2.84 \mathrm{E}+04$ & $7.69 \mathrm{E}+05$ & $8.83 \mathrm{E}+03$ & $2.39 \mathrm{E}+05$ & $1.05 \mathrm{E}+05$ & $2.83 \mathrm{E}+06$ & $2.88 \mathrm{E}+05$ & $7.78 \mathrm{E}+06$ & $7.11 \mathrm{E}+03$ & $7.78 \mathrm{E}+06$ \\
\hline
\end{tabular}


SRNL-STI-2013-00115

\begin{tabular}{|c|c|c|c|c|c|c|c|c|c|c|c|c|}
\hline \multirow[t]{2}{*}{ Nuclide } & \multicolumn{2}{|c|}{ Water Ingestion DCS } & \multicolumn{2}{|c|}{ Meat Ingestion DCS } & \multicolumn{2}{|c|}{ Dairy Ingestion DCS } & \multicolumn{2}{|c|}{$\begin{array}{c}\text { Freshwater Fish } \\
\text { Ingestion DCS }\end{array}$} & \multicolumn{2}{|c|}{$\begin{array}{l}\text { Saltwater Shellfish } \\
\text { Ingestion DCS }\end{array}$} & \multicolumn{2}{|c|}{ Produce Ingestion DCS } \\
\hline & $(\mathrm{Bq} / \mathrm{L})$ & $(\mathrm{pCi} / \mathrm{L})$ & $(\mathrm{Bq} / \mathrm{kg})$ & $(\mathrm{pCi} / \mathrm{kg})$ & $(\mathrm{Bq} / \mathrm{L})$ & $(\mathrm{pCi} / \mathrm{L})$ & $(\mathrm{Bq} / \mathbf{k g})$ & $(\mathrm{pCi} / \mathbf{k g})$ & $(\mathrm{Bq} / \mathbf{k g})$ & $(\mathrm{pCi} / \mathbf{k g})$ & $(\mathrm{Bq} / \mathbf{k g})$ & $(\mathrm{pCi} / \mathbf{k g})$ \\
\hline $\mathrm{Cl}-34 \mathrm{~m}$ & $9.08 \mathrm{E}+03$ & $2.45 \mathrm{E}+05$ & $9.01 \mathrm{E}+04$ & $2.44 \mathrm{E}+06$ & $2.80 \mathrm{E}+04$ & $7.56 \mathrm{E}+05$ & $3.32 \mathrm{E}+05$ & $8.97 \mathrm{E}+06$ & $9.12 \mathrm{E}+05$ & $2.47 \mathrm{E}+07$ & $2.25 \mathrm{E}+04$ & $2.47 \mathrm{E}+07$ \\
\hline $\mathrm{Cl}-36$ & $1.00 \mathrm{E}+03$ & $2.71 \mathrm{E}+04$ & $9.96 \mathrm{E}+03$ & $2.69 \mathrm{E}+05$ & $3.09 \mathrm{E}+03$ & $8.35 \mathrm{E}+04$ & $3.67 \mathrm{E}+04$ & $9.91 \mathrm{E}+05$ & $1.01 \mathrm{E}+05$ & $2.72 \mathrm{E}+06$ & $2.49 \mathrm{E}+03$ & $2.72 \mathrm{E}+06$ \\
\hline $\mathrm{Cl}-38$ & $7.97 \mathrm{E}+03$ & $2.15 \mathrm{E}+05$ & $7.91 \mathrm{E}+04$ & $2.14 \mathrm{E}+06$ & $2.46 \mathrm{E}+04$ & $6.64 \mathrm{E}+05$ & $2.91 \mathrm{E}+05$ & $7.88 \mathrm{E}+06$ & $8.01 \mathrm{E}+05$ & $2.17 \mathrm{E}+07$ & $1.98 \mathrm{E}+04$ & $2.17 \mathrm{E}+07$ \\
\hline $\mathrm{Cl}-39$ & $1.10 \mathrm{E}+04$ & $2.97 \mathrm{E}+05$ & $1.09 \mathrm{E}+05$ & $2.95 \mathrm{E}+06$ & $3.39 \mathrm{E}+04$ & $9.16 \mathrm{E}+05$ & $4.02 \mathrm{E}+05$ & $1.09 \mathrm{E}+07$ & $1.11 \mathrm{E}+06$ & $2.99 \mathrm{E}+07$ & $2.73 \mathrm{E}+04$ & $2.99 \mathrm{E}+07$ \\
\hline $\mathrm{K}-40$ & $1.51 \mathrm{E}+02$ & $4.09 \mathrm{E}+03$ & $1.50 \mathrm{E}+03$ & $4.06 \mathrm{E}+04$ & $4.66 \mathrm{E}+02$ & $1.26 \mathrm{E}+04$ & $5.53 \mathrm{E}+03$ & $1.49 \mathrm{E}+05$ & $1.52 \mathrm{E}+04$ & $4.11 \mathrm{E}+05$ & $3.75 \mathrm{E}+02$ & $4.11 \mathrm{E}+05$ \\
\hline $\mathrm{K}-42$ & $2.11 \mathrm{E}+03$ & $5.71 \mathrm{E}+04$ & $2.10 \mathrm{E}+04$ & $5.66 \mathrm{E}+05$ & $6.50 \mathrm{E}+03$ & $1.76 \mathrm{E}+05$ & $7.72 \mathrm{E}+04$ & $2.09 \mathrm{E}+06$ & $2.12 \mathrm{E}+05$ & $5.74 \mathrm{E}+06$ & $5.24 \mathrm{E}+03$ & $5.74 \mathrm{E}+06$ \\
\hline $\mathrm{K}-43$ & $3.89 \mathrm{E}+03$ & $1.05 \mathrm{E}+05$ & $3.86 \mathrm{E}+04$ & $1.04 \mathrm{E}+06$ & $1.20 \mathrm{E}+04$ & $3.24 \mathrm{E}+05$ & $1.42 \mathrm{E}+05$ & $3.84 \mathrm{E}+06$ & $3.91 \mathrm{E}+05$ & $1.06 \mathrm{E}+07$ & $9.65 \mathrm{E}+03$ & $1.06 \mathrm{E}+07$ \\
\hline $\mathrm{K}-44$ & $1.12 \mathrm{E}+04$ & $3.03 \mathrm{E}+05$ & $1.11 \mathrm{E}+05$ & $3.01 \mathrm{E}+06$ & $3.45 \mathrm{E}+04$ & $9.33 \mathrm{E}+05$ & $4.10 \mathrm{E}+05$ & $1.11 \mathrm{E}+07$ & $1.13 \mathrm{E}+06$ & $3.04 \mathrm{E}+07$ & $2.78 \mathrm{E}+04$ & $3.04 \mathrm{E}+07$ \\
\hline $\mathrm{K}-45$ & $1.92 \mathrm{E}+04$ & $5.19 \mathrm{E}+05$ & $1.91 \mathrm{E}+05$ & $5.15 \mathrm{E}+06$ & $5.91 \mathrm{E}+04$ & $1.60 \mathrm{E}+06$ & $7.01 \mathrm{E}+05$ & $1.90 \mathrm{E}+07$ & $1.93 \mathrm{E}+06$ & $5.21 \mathrm{E}+07$ & $4.76 \mathrm{E}+04$ & $5.21 \mathrm{E}+07$ \\
\hline $\mathrm{Ca}-41$ & $4.20 \mathrm{E}+03$ & $1.14 \mathrm{E}+05$ & $4.17 \mathrm{E}+04$ & $1.13 \mathrm{E}+06$ & $1.29 \mathrm{E}+04$ & $3.50 \mathrm{E}+05$ & $1.54 \mathrm{E}+05$ & $4.15 \mathrm{E}+06$ & $4.22 \mathrm{E}+05$ & $1.14 \mathrm{E}+07$ & $1.04 \mathrm{E}+04$ & $1.14 \mathrm{E}+07$ \\
\hline $\mathrm{Ca}-45$ & $1.20 \mathrm{E}+03$ & $3.23 \mathrm{E}+04$ & $1.19 \mathrm{E}+04$ & $3.21 \mathrm{E}+05$ & $3.68 \mathrm{E}+03$ & $9.96 \mathrm{E}+04$ & $4.37 \mathrm{E}+04$ & $1.18 \mathrm{E}+06$ & $1.20 \mathrm{E}+05$ & $3.25 \mathrm{E}+06$ & $2.97 \mathrm{E}+03$ & $3.25 \mathrm{E}+06$ \\
\hline $\mathrm{Ca}-47$ & $6.07 \mathrm{E}+02$ & $1.64 \mathrm{E}+04$ & $6.02 \mathrm{E}+03$ & $1.63 \mathrm{E}+05$ & $1.87 \mathrm{E}+03$ & $5.05 \mathrm{E}+04$ & $2.22 \mathrm{E}+04$ & $5.99 \mathrm{E}+05$ & $6.10 \mathrm{E}+04$ & $1.65 \mathrm{E}+06$ & $1.51 \mathrm{E}+03$ & $1.65 \mathrm{E}+06$ \\
\hline Sc-43 & $4.27 \mathrm{E}+03$ & $1.16 \mathrm{E}+05$ & $4.24 \mathrm{E}+04$ & $1.15 \mathrm{E}+06$ & $1.32 \mathrm{E}+04$ & $3.56 \mathrm{E}+05$ & $1.56 \mathrm{E}+05$ & $4.22 \mathrm{E}+06$ & $4.30 \mathrm{E}+05$ & $1.16 \mathrm{E}+07$ & $1.06 \mathrm{E}+04$ & $1.16 \mathrm{E}+07$ \\
\hline Sc-44 & $2.64 \mathrm{E}+03$ & $7.12 \mathrm{E}+04$ & $2.62 \mathrm{E}+04$ & $7.07 \mathrm{E}+05$ & $8.12 \mathrm{E}+03$ & $2.19 \mathrm{E}+05$ & $9.63 \mathrm{E}+04$ & $2.60 \mathrm{E}+06$ & $2.65 \mathrm{E}+05$ & $7.16 \mathrm{E}+06$ & $6.54 \mathrm{E}+03$ & $7.16 \mathrm{E}+06$ \\
\hline Sc- $44 \mathrm{~m}$ & $3.82 \mathrm{E}+02$ & $1.03 \mathrm{E}+04$ & $3.79 \mathrm{E}+03$ & $1.02 \mathrm{E}+05$ & $1.18 \mathrm{E}+03$ & $3.18 \mathrm{E}+04$ & $1.39 \mathrm{E}+04$ & $3.77 \mathrm{E}+05$ & $3.83 \mathrm{E}+04$ & $1.04 \mathrm{E}+06$ & $9.47 \mathrm{E}+02$ & $1.04 \mathrm{E}+06$ \\
\hline Sc-46 & $6.62 \mathrm{E}+02$ & $1.79 \mathrm{E}+04$ & $6.57 \mathrm{E}+03$ & $1.77 \mathrm{E}+05$ & $2.04 \mathrm{E}+03$ & $5.51 \mathrm{E}+04$ & $2.42 \mathrm{E}+04$ & $6.53 \mathrm{E}+05$ & $6.65 \mathrm{E}+04$ & $1.80 \mathrm{E}+06$ & $1.64 \mathrm{E}+03$ & $1.80 \mathrm{E}+06$ \\
\hline Sc-47 & $1.67 \mathrm{E}+03$ & $4.51 \mathrm{E}+04$ & $1.65 \mathrm{E}+04$ & $4.47 \mathrm{E}+05$ & $5.14 \mathrm{E}+03$ & $1.39 \mathrm{E}+05$ & $6.09 \mathrm{E}+04$ & $1.65 \mathrm{E}+06$ & $1.68 \mathrm{E}+05$ & $4.53 \mathrm{E}+06$ & $4.14 \mathrm{E}+03$ & $4.53 \mathrm{E}+06$ \\
\hline Sc-48 & $5.81 \mathrm{E}+02$ & $1.57 \mathrm{E}+04$ & $5.77 \mathrm{E}+03$ & $1.56 \mathrm{E}+05$ & $1.79 \mathrm{E}+03$ & $4.84 \mathrm{E}+04$ & $2.12 \mathrm{E}+04$ & $5.74 \mathrm{E}+05$ & $5.84 \mathrm{E}+04$ & $1.58 \mathrm{E}+06$ & $1.44 \mathrm{E}+03$ & $1.58 \mathrm{E}+06$ \\
\hline Sc-49 & $1.13 \mathrm{E}+04$ & $3.06 \mathrm{E}+05$ & $1.12 \mathrm{E}+05$ & $3.03 \mathrm{E}+06$ & $3.48 \mathrm{E}+04$ & $9.41 \mathrm{E}+05$ & $4.13 \mathrm{E}+05$ & $1.12 \mathrm{E}+07$ & $1.14 \mathrm{E}+06$ & $3.07 \mathrm{E}+07$ & $2.81 \mathrm{E}+04$ & $3.07 \mathrm{E}+07$ \\
\hline $\mathrm{Ti}-44$ & $1.68 \mathrm{E}+02$ & $4.54 \mathrm{E}+03$ & $1.67 \mathrm{E}+03$ & $4.50 \mathrm{E}+04$ & $5.17 \mathrm{E}+02$ & $1.40 \mathrm{E}+04$ & $6.13 \mathrm{E}+03$ & $1.66 \mathrm{E}+05$ & $1.69 \mathrm{E}+04$ & $4.56 \mathrm{E}+05$ & $4.17 \mathrm{E}+02$ & $4.56 \mathrm{E}+05$ \\
\hline Ti-45 & $6.19 \mathrm{E}+03$ & $1.67 \mathrm{E}+05$ & $6.14 \mathrm{E}+04$ & $1.66 \mathrm{E}+06$ & $1.91 \mathrm{E}+04$ & $5.15 \mathrm{E}+05$ & $2.26 \mathrm{E}+05$ & $6.11 \mathrm{E}+06$ & $6.22 \mathrm{E}+05$ & $1.68 \mathrm{E}+07$ & $1.54 \mathrm{E}+04$ & $1.68 \mathrm{E}+07$ \\
\hline V-47 & $1.49 \mathrm{E}+04$ & $4.02 \mathrm{E}+05$ & $1.47 \mathrm{E}+05$ & $3.99 \mathrm{E}+06$ & $4.58 \mathrm{E}+04$ & $1.24 \mathrm{E}+06$ & $5.43 \mathrm{E}+05$ & $1.47 \mathrm{E}+07$ & $1.49 \mathrm{E}+06$ & $4.04 \mathrm{E}+07$ & $3.69 \mathrm{E}+04$ & $4.04 \mathrm{E}+07$ \\
\hline V-48 & $4.90 \mathrm{E}+02$ & $1.32 \mathrm{E}+04$ & $4.86 \mathrm{E}+03$ & $1.31 \mathrm{E}+05$ & $1.51 \mathrm{E}+03$ & $4.08 \mathrm{E}+04$ & $1.79 \mathrm{E}+04$ & $4.84 \mathrm{E}+05$ & $4.92 \mathrm{E}+04$ & $1.33 \mathrm{E}+06$ & $1.22 \mathrm{E}+03$ & $1.33 \mathrm{E}+06$ \\
\hline
\end{tabular}


SRNL-STI-2013-00115

\begin{tabular}{|c|c|c|c|c|c|c|c|c|c|c|c|c|}
\hline \multirow[t]{2}{*}{ Nuclide } & \multicolumn{2}{|c|}{ Water Ingestion DCS } & \multicolumn{2}{|c|}{ Meat Ingestion DCS } & \multicolumn{2}{|c|}{ Dairy Ingestion DCS } & \multicolumn{2}{|c|}{$\begin{array}{c}\text { Freshwater Fish } \\
\text { Ingestion DCS }\end{array}$} & \multicolumn{2}{|c|}{$\begin{array}{l}\text { Saltwater Shellfish } \\
\text { Ingestion DCS }\end{array}$} & \multicolumn{2}{|c|}{ Produce Ingestion DCS } \\
\hline & $(\mathrm{Bq} / \mathrm{L})$ & $(\mathrm{pCi} / \mathrm{L})$ & $(\mathrm{Bq} / \mathrm{kg})$ & $(\mathrm{pCi} / \mathrm{kg})$ & $(\mathrm{Bq} / \mathrm{L})$ & $(\mathrm{pCi} / \mathrm{L})$ & $(\mathrm{Bq} / \mathbf{k g})$ & $(\mathrm{pCi} / \mathbf{k g})$ & $(\mathrm{Bq} / \mathbf{k g})$ & $(\mathrm{pCi} / \mathbf{k g})$ & $(\mathrm{Bq} / \mathbf{k g})$ & $(\mathrm{pCi} / \mathbf{k g})$ \\
\hline V-49 & $4.92 \mathrm{E}+04$ & $1.33 \mathrm{E}+06$ & $4.88 \mathrm{E}+05$ & $1.32 \mathrm{E}+07$ & $1.51 \mathrm{E}+05$ & $4.09 \mathrm{E}+06$ & $1.80 \mathrm{E}+06$ & $4.86 \mathrm{E}+07$ & $4.94 \mathrm{E}+06$ & $1.34 \mathrm{E}+08$ & $1.22 \mathrm{E}+05$ & $1.34 \mathrm{E}+08$ \\
\hline $\mathrm{V}-50$ & $3.32 \mathrm{E}+02$ & $8.96 \mathrm{E}+03$ & $3.29 \mathrm{E}+03$ & $8.90 \mathrm{E}+04$ & $1.02 \mathrm{E}+03$ & $2.76 \mathrm{E}+04$ & $1.21 \mathrm{E}+04$ & $3.28 \mathrm{E}+05$ & $3.33 \mathrm{E}+04$ & $9.01 \mathrm{E}+05$ & $8.23 \mathrm{E}+02$ & $9.01 \mathrm{E}+05$ \\
\hline $\mathrm{Cr}-48$ & $5.04 \mathrm{E}+03$ & $1.36 \mathrm{E}+05$ & $5.00 \mathrm{E}+04$ & $1.35 \mathrm{E}+06$ & $1.55 \mathrm{E}+04$ & $4.19 \mathrm{E}+05$ & $1.84 \mathrm{E}+05$ & $4.97 \mathrm{E}+06$ & $5.06 \mathrm{E}+05$ & $1.37 \mathrm{E}+07$ & $1.25 \mathrm{E}+04$ & $1.37 \mathrm{E}+07$ \\
\hline $\mathrm{Cr}-49$ & $1.53 \mathrm{E}+04$ & $4.13 \mathrm{E}+05$ & $1.52 \mathrm{E}+05$ & $4.10 \mathrm{E}+06$ & $4.71 \mathrm{E}+04$ & $1.27 \mathrm{E}+06$ & $5.59 \mathrm{E}+05$ & $1.51 \mathrm{E}+07$ & $1.54 \mathrm{E}+06$ & $4.16 \mathrm{E}+07$ & $3.80 \mathrm{E}+04$ & $4.16 \mathrm{E}+07$ \\
\hline $\mathrm{Cr}-51$ & $2.47 \mathrm{E}+04$ & $6.68 \mathrm{E}+05$ & $2.45 \mathrm{E}+05$ & $6.63 \mathrm{E}+06$ & $7.62 \mathrm{E}+04$ & $2.06 \mathrm{E}+06$ & $9.04 \mathrm{E}+05$ & $2.44 \mathrm{E}+07$ & $2.49 \mathrm{E}+06$ & $6.72 \mathrm{E}+07$ & $6.14 \mathrm{E}+04$ & $6.72 \mathrm{E}+07$ \\
\hline Mn-51 & $1.01 \mathrm{E}+04$ & $2.73 \mathrm{E}+05$ & $1.00 \mathrm{E}+05$ & $2.71 \mathrm{E}+06$ & $3.11 \mathrm{E}+04$ & $8.42 \mathrm{E}+05$ & $3.70 \mathrm{E}+05$ & $9.99 \mathrm{E}+06$ & $1.02 \mathrm{E}+06$ & $2.75 \mathrm{E}+07$ & $2.51 \mathrm{E}+04$ & $2.75 \mathrm{E}+07$ \\
\hline $\mathrm{Mn}-52$ & $5.50 \mathrm{E}+02$ & $1.49 \mathrm{E}+04$ & $5.46 \mathrm{E}+03$ & $1.48 \mathrm{E}+05$ & $1.70 \mathrm{E}+03$ & $4.58 \mathrm{E}+04$ & $2.01 \mathrm{E}+04$ & $5.44 \mathrm{E}+05$ & $5.53 \mathrm{E}+04$ & $1.49 \mathrm{E}+06$ & $1.37 \mathrm{E}+03$ & $1.49 \mathrm{E}+06$ \\
\hline $\mathrm{Mn}-52 \mathrm{~m}$ & $1.36 \mathrm{E}+04$ & $3.67 \mathrm{E}+05$ & $1.35 \mathrm{E}+05$ & $3.64 \mathrm{E}+06$ & $4.18 \mathrm{E}+04$ & $1.13 \mathrm{E}+06$ & $4.96 \mathrm{E}+05$ & $1.34 \mathrm{E}+07$ & $1.36 \mathrm{E}+06$ & $3.68 \mathrm{E}+07$ & $3.37 \mathrm{E}+04$ & $3.68 \mathrm{E}+07$ \\
\hline $\mathrm{Mn}-53$ & $3.00 \mathrm{E}+04$ & $8.12 \mathrm{E}+05$ & $2.98 \mathrm{E}+05$ & $8.06 \mathrm{E}+06$ & $9.25 \mathrm{E}+04$ & $2.50 \mathrm{E}+06$ & $1.10 \mathrm{E}+06$ & $2.97 \mathrm{E}+07$ & $3.02 \mathrm{E}+06$ & $8.16 \mathrm{E}+07$ & $7.46 \mathrm{E}+04$ & $8.16 \mathrm{E}+07$ \\
\hline Mn-54 & $1.40 \mathrm{E}+03$ & $3.78 \mathrm{E}+04$ & $1.39 \mathrm{E}+04$ & $3.75 \mathrm{E}+05$ & $4.30 \mathrm{E}+03$ & $1.16 \mathrm{E}+05$ & $5.11 \mathrm{E}+04$ & $1.38 \mathrm{E}+06$ & $1.40 \mathrm{E}+05$ & $3.80 \mathrm{E}+06$ & $3.47 \mathrm{E}+03$ & $3.80 \mathrm{E}+06$ \\
\hline Mn-56 & $3.65 \mathrm{E}+03$ & $9.86 \mathrm{E}+04$ & $3.62 \mathrm{E}+04$ & $9.78 \mathrm{E}+05$ & $1.12 \mathrm{E}+04$ & $3.04 \mathrm{E}+05$ & $1.33 \mathrm{E}+05$ & $3.60 \mathrm{E}+06$ & $3.67 \mathrm{E}+05$ & $9.91 \mathrm{E}+06$ & $9.05 \mathrm{E}+03$ & $9.91 \mathrm{E}+06$ \\
\hline $\mathrm{Fe}-52$ & $6.80 \mathrm{E}+02$ & $1.84 \mathrm{E}+04$ & $6.75 \mathrm{E}+03$ & $1.82 \mathrm{E}+05$ & $2.09 \mathrm{E}+03$ & $5.66 \mathrm{E}+04$ & $2.48 \mathrm{E}+04$ & $6.71 \mathrm{E}+05$ & $6.83 \mathrm{E}+04$ & $1.85 \mathrm{E}+06$ & $1.69 \mathrm{E}+03$ & $1.85 \mathrm{E}+06$ \\
\hline $\mathrm{Fe}-55$ & $2.26 \mathrm{E}+03$ & $6.10 \mathrm{E}+04$ & $2.24 \mathrm{E}+04$ & $6.06 \mathrm{E}+05$ & $6.95 \mathrm{E}+03$ & $1.88 \mathrm{E}+05$ & $8.25 \mathrm{E}+04$ & $2.23 \mathrm{E}+06$ & $2.27 \mathrm{E}+05$ & $6.13 \mathrm{E}+06$ & $5.60 \mathrm{E}+03$ & $6.13 \mathrm{E}+06$ \\
\hline $\mathrm{Fe}-59$ & $4.54 \mathrm{E}+02$ & $1.23 \mathrm{E}+04$ & $4.51 \mathrm{E}+03$ & $1.22 \mathrm{E}+05$ & $1.40 \mathrm{E}+03$ & $3.78 \mathrm{E}+04$ & $1.66 \mathrm{E}+04$ & $4.48 \mathrm{E}+05$ & $4.56 \mathrm{E}+04$ & $1.23 \mathrm{E}+06$ & $1.13 \mathrm{E}+03$ & $1.23 \mathrm{E}+06$ \\
\hline $\mathrm{Fe}-60$ & $8.40 \mathrm{E}+00$ & $2.27 \mathrm{E}+02$ & $8.34 \mathrm{E}+01$ & $2.25 \mathrm{E}+03$ & $2.59 \mathrm{E}+01$ & $7.00 \mathrm{E}+02$ & $3.07 \mathrm{E}+02$ & $8.30 \mathrm{E}+03$ & $8.45 \mathrm{E}+02$ & $2.28 \mathrm{E}+04$ & $2.09 \mathrm{E}+01$ & $2.28 \mathrm{E}+04$ \\
\hline $\mathrm{Co}-55$ & $9.79 \mathrm{E}+02$ & $2.65 \mathrm{E}+04$ & $9.72 \mathrm{E}+03$ & $2.63 \mathrm{E}+05$ & $3.02 \mathrm{E}+03$ & $8.15 \mathrm{E}+04$ & $3.58 \mathrm{E}+04$ & $9.67 \mathrm{E}+05$ & $9.84 \mathrm{E}+04$ & $2.66 \mathrm{E}+06$ & $2.43 \mathrm{E}+03$ & $2.66 \mathrm{E}+06$ \\
\hline Co-56 & $3.62 \mathrm{E}+02$ & $9.77 \mathrm{E}+03$ & $3.59 \mathrm{E}+03$ & $9.70 \mathrm{E}+04$ & $1.11 \mathrm{E}+03$ & $3.01 \mathrm{E}+04$ & $1.32 \mathrm{E}+04$ & $3.57 \mathrm{E}+05$ & $3.63 \mathrm{E}+04$ & $9.82 \mathrm{E}+05$ & $8.97 \mathrm{E}+02$ & $9.82 \mathrm{E}+05$ \\
\hline Co-57 & $3.97 \mathrm{E}+03$ & $1.07 \mathrm{E}+05$ & $3.94 \mathrm{E}+04$ & $1.07 \mathrm{E}+06$ & $1.22 \mathrm{E}+04$ & $3.31 \mathrm{E}+05$ & $1.45 \mathrm{E}+05$ & $3.92 \mathrm{E}+06$ & $3.99 \mathrm{E}+05$ & $1.08 \mathrm{E}+07$ & $9.86 \mathrm{E}+03$ & $1.08 \mathrm{E}+07$ \\
\hline Co-58 & $1.23 \mathrm{E}+03$ & $3.33 \mathrm{E}+04$ & $1.22 \mathrm{E}+04$ & $3.30 \mathrm{E}+05$ & $3.79 \mathrm{E}+03$ & $1.03 \mathrm{E}+05$ & $4.50 \mathrm{E}+04$ & $1.22 \mathrm{E}+06$ & $1.24 \mathrm{E}+05$ & $3.34 \mathrm{E}+06$ & $3.06 \mathrm{E}+03$ & $3.34 \mathrm{E}+06$ \\
\hline Co-58m & $4.01 \mathrm{E}+04$ & $1.08 \mathrm{E}+06$ & $3.98 \mathrm{E}+05$ & $1.08 \mathrm{E}+07$ & $1.24 \mathrm{E}+05$ & $3.34 \mathrm{E}+06$ & $1.47 \mathrm{E}+06$ & $3.96 \mathrm{E}+07$ & $4.03 \mathrm{E}+06$ & $1.09 \mathrm{E}+08$ & $9.96 \mathrm{E}+04$ & $1.09 \mathrm{E}+08$ \\
\hline Co-60 & $2.27 \mathrm{E}+02$ & $6.12 \mathrm{E}+03$ & $2.25 \mathrm{E}+03$ & $6.08 \mathrm{E}+04$ & $6.98 \mathrm{E}+02$ & $1.89 \mathrm{E}+04$ & $8.28 \mathrm{E}+03$ & $2.24 \mathrm{E}+05$ & $2.28 \mathrm{E}+04$ & $6.15 \mathrm{E}+05$ & $5.62 \mathrm{E}+02$ & $6.15 \mathrm{E}+05$ \\
\hline Co-60m & $5.48 \mathrm{E}+05$ & $1.48 \mathrm{E}+07$ & $5.44 \mathrm{E}+06$ & $1.47 \mathrm{E}+08$ & $1.69 \mathrm{E}+06$ & $4.56 \mathrm{E}+07$ & $2.00 \mathrm{E}+07$ & $5.41 \mathrm{E}+08$ & $5.51 \mathrm{E}+07$ & $1.49 \mathrm{E}+09$ & $1.36 \mathrm{E}+06$ & $1.49 \mathrm{E}+09$ \\
\hline $\mathrm{Co}-61$ & $1.24 \mathrm{E}+04$ & $3.36 \mathrm{E}+05$ & $1.23 \mathrm{E}+05$ & $3.34 \mathrm{E}+06$ & $3.83 \mathrm{E}+04$ & $1.04 \mathrm{E}+06$ & $4.55 \mathrm{E}+05$ & $1.23 \mathrm{E}+07$ & $1.25 \mathrm{E}+06$ & $3.38 \mathrm{E}+07$ & $3.09 \mathrm{E}+04$ & $3.38 \mathrm{E}+07$ \\
\hline
\end{tabular}

Page 170 of 298 
SRNL-STI-2013-00115

\begin{tabular}{|c|c|c|c|c|c|c|c|c|c|c|c|c|}
\hline \multirow[t]{2}{*}{ Nuclide } & \multicolumn{2}{|c|}{ Water Ingestion DCS } & \multicolumn{2}{|c|}{ Meat Ingestion DCS } & \multicolumn{2}{|c|}{ Dairy Ingestion DCS } & \multicolumn{2}{|c|}{$\begin{array}{c}\text { Freshwater Fish } \\
\text { Ingestion DCS }\end{array}$} & \multicolumn{2}{|c|}{$\begin{array}{l}\text { Saltwater Shellfish } \\
\text { Ingestion DCS }\end{array}$} & \multicolumn{2}{|c|}{ Produce Ingestion DCS } \\
\hline & $(\mathrm{Bq} / \mathrm{L})$ & $(\mathrm{pCi} / \mathrm{L})$ & $(\mathrm{Bq} / \mathrm{kg})$ & $(\mathrm{pCi} / \mathrm{kg})$ & $(\mathrm{Bq} / \mathrm{L})$ & $(\mathrm{pCi} / \mathrm{L})$ & $(\mathrm{Bq} / \mathbf{k g})$ & $(\mathrm{pCi} / \mathbf{k g})$ & $(\mathrm{Bq} / \mathbf{k g})$ & $(\mathrm{pCi} / \mathbf{k g})$ & $(\mathrm{Bq} / \mathbf{k g})$ & $(\mathrm{pCi} / \mathbf{k g})$ \\
\hline Co-62m & $1.92 \mathrm{E}+04$ & $5.19 \mathrm{E}+05$ & $1.91 \mathrm{E}+05$ & $5.15 \mathrm{E}+06$ & $5.91 \mathrm{E}+04$ & $1.60 \mathrm{E}+06$ & $7.01 \mathrm{E}+05$ & $1.90 \mathrm{E}+07$ & $1.93 \mathrm{E}+06$ & $5.21 \mathrm{E}+07$ & $4.76 \mathrm{E}+04$ & $5.21 \mathrm{E}+07$ \\
\hline $\mathrm{Ni}-56$ & $1.15 \mathrm{E}+03$ & $3.11 \mathrm{E}+04$ & $1.14 \mathrm{E}+04$ & $3.09 \mathrm{E}+05$ & $3.55 \mathrm{E}+03$ & $9.59 \mathrm{E}+04$ & $4.21 \mathrm{E}+04$ & $1.14 \mathrm{E}+06$ & $1.16 \mathrm{E}+05$ & $3.13 \mathrm{E}+06$ & $2.86 \mathrm{E}+03$ & $3.13 \mathrm{E}+06$ \\
\hline $\mathrm{Ni}-57$ & $1.06 \mathrm{E}+03$ & $2.87 \mathrm{E}+04$ & $1.06 \mathrm{E}+04$ & $2.85 \mathrm{E}+05$ & $3.27 \mathrm{E}+03$ & $8.85 \mathrm{E}+04$ & $3.89 \mathrm{E}+04$ & $1.05 \mathrm{E}+06$ & $1.07 \mathrm{E}+05$ & $2.89 \mathrm{E}+06$ & $2.64 \mathrm{E}+03$ & $2.89 \mathrm{E}+06$ \\
\hline $\mathrm{Ni}-59$ & $1.56 \mathrm{E}+04$ & $4.22 \mathrm{E}+05$ & $1.55 \mathrm{E}+05$ & $4.19 \mathrm{E}+06$ & $4.81 \mathrm{E}+04$ & $1.30 \mathrm{E}+06$ & $5.71 \mathrm{E}+05$ & $1.54 \mathrm{E}+07$ & $1.57 \mathrm{E}+06$ & $4.24 \mathrm{E}+07$ & $3.88 \mathrm{E}+04$ & $4.24 \mathrm{E}+07$ \\
\hline $\mathrm{Ni}-63$ & $6.28 \mathrm{E}+03$ & $1.70 \mathrm{E}+05$ & $6.24 \mathrm{E}+04$ & $1.69 \mathrm{E}+06$ & $1.94 \mathrm{E}+04$ & $5.23 \mathrm{E}+05$ & $2.30 \mathrm{E}+05$ & $6.20 \mathrm{E}+06$ & $6.31 \mathrm{E}+05$ & $1.71 \mathrm{E}+07$ & $1.56 \mathrm{E}+04$ & $1.71 \mathrm{E}+07$ \\
\hline $\mathrm{Ni}-65$ & $5.06 \mathrm{E}+03$ & $1.37 \mathrm{E}+05$ & $5.02 \mathrm{E}+04$ & $1.36 \mathrm{E}+06$ & $1.56 \mathrm{E}+04$ & $4.21 \mathrm{E}+05$ & $1.85 \mathrm{E}+05$ & $4.99 \mathrm{E}+06$ & $5.08 \mathrm{E}+05$ & $1.37 \mathrm{E}+07$ & $1.25 \mathrm{E}+04$ & $1.37 \mathrm{E}+07$ \\
\hline $\mathrm{Ni}-66$ & $3.00 \mathrm{E}+02$ & $8.10 \mathrm{E}+03$ & $2.97 \mathrm{E}+03$ & $8.04 \mathrm{E}+04$ & $9.23 \mathrm{E}+02$ & $2.50 \mathrm{E}+04$ & $1.10 \mathrm{E}+04$ & $2.96 \mathrm{E}+05$ & $3.01 \mathrm{E}+04$ & $8.14 \mathrm{E}+05$ & $7.44 \mathrm{E}+02$ & $8.14 \mathrm{E}+05$ \\
\hline $\mathrm{Cu}-60$ & $1.33 \mathrm{E}+04$ & $3.60 \mathrm{E}+05$ & $1.32 \mathrm{E}+05$ & $3.58 \mathrm{E}+06$ & $4.11 \mathrm{E}+04$ & $1.11 \mathrm{E}+06$ & $4.87 \mathrm{E}+05$ & $1.32 \mathrm{E}+07$ & $1.34 \mathrm{E}+06$ & $3.62 \mathrm{E}+07$ & $3.31 \mathrm{E}+04$ & $3.62 \mathrm{E}+07$ \\
\hline $\mathrm{Cu}-61$ & $8.35 \mathrm{E}+03$ & $2.26 \mathrm{E}+05$ & $8.29 \mathrm{E}+04$ & $2.24 \mathrm{E}+06$ & $2.57 \mathrm{E}+04$ & $6.95 \mathrm{E}+05$ & $3.05 \mathrm{E}+05$ & $8.24 \mathrm{E}+06$ & $8.39 \mathrm{E}+05$ & $2.27 \mathrm{E}+07$ & $2.07 \mathrm{E}+04$ & $2.27 \mathrm{E}+07$ \\
\hline $\mathrm{Cu}-64$ & $7.82 \mathrm{E}+03$ & $2.11 \mathrm{E}+05$ & $7.76 \mathrm{E}+04$ & $2.10 \mathrm{E}+06$ & $2.41 \mathrm{E}+04$ & $6.51 \mathrm{E}+05$ & $2.86 \mathrm{E}+05$ & $7.73 \mathrm{E}+06$ & $7.86 \mathrm{E}+05$ & $2.12 \mathrm{E}+07$ & $1.94 \mathrm{E}+04$ & $2.12 \mathrm{E}+07$ \\
\hline $\mathrm{Cu}-67$ & $2.86 \mathrm{E}+03$ & $7.73 \mathrm{E}+04$ & $2.84 \mathrm{E}+04$ & $7.67 \mathrm{E}+05$ & $8.81 \mathrm{E}+03$ & $2.38 \mathrm{E}+05$ & $1.04 \mathrm{E}+05$ & $2.82 \mathrm{E}+06$ & $2.87 \mathrm{E}+05$ & $7.77 \mathrm{E}+06$ & $7.10 \mathrm{E}+03$ & $7.77 \mathrm{E}+06$ \\
\hline $\mathrm{Zn}-62$ & $1.02 \mathrm{E}+03$ & $2.76 \mathrm{E}+04$ & $1.01 \mathrm{E}+04$ & $2.73 \mathrm{E}+05$ & $3.14 \mathrm{E}+03$ & $8.49 \mathrm{E}+04$ & $3.73 \mathrm{E}+04$ & $1.01 \mathrm{E}+06$ & $1.02 \mathrm{E}+05$ & $2.77 \mathrm{E}+06$ & $2.53 \mathrm{E}+03$ & $2.77 \mathrm{E}+06$ \\
\hline Zn-63 & $1.17 \mathrm{E}+04$ & $3.17 \mathrm{E}+05$ & $1.16 \mathrm{E}+05$ & $3.15 \mathrm{E}+06$ & $3.61 \mathrm{E}+04$ & $9.77 \mathrm{E}+05$ & $4.29 \mathrm{E}+05$ & $1.16 \mathrm{E}+07$ & $1.18 \mathrm{E}+06$ & $3.19 \mathrm{E}+07$ & $2.91 \mathrm{E}+04$ & $3.19 \mathrm{E}+07$ \\
\hline $\mathrm{Zn}-65$ & $2.61 \mathrm{E}+02$ & $7.05 \mathrm{E}+03$ & $2.59 \mathrm{E}+03$ & $7.00 \mathrm{E}+04$ & $8.03 \mathrm{E}+02$ & $2.17 \mathrm{E}+04$ & $9.53 \mathrm{E}+03$ & $2.58 \mathrm{E}+05$ & $2.62 \mathrm{E}+04$ & $7.08 \mathrm{E}+05$ & $6.47 \mathrm{E}+02$ & $7.08 \mathrm{E}+05$ \\
\hline Zn-69 & $3.00 \mathrm{E}+04$ & $8.12 \mathrm{E}+05$ & $2.98 \mathrm{E}+05$ & $8.06 \mathrm{E}+06$ & $9.25 \mathrm{E}+04$ & $2.50 \mathrm{E}+06$ & $1.10 \mathrm{E}+06$ & $2.97 \mathrm{E}+07$ & $3.02 \mathrm{E}+06$ & $8.16 \mathrm{E}+07$ & $7.46 \mathrm{E}+04$ & $8.16 \mathrm{E}+07$ \\
\hline Zn-69m & $2.87 \mathrm{E}+03$ & $7.76 \mathrm{E}+04$ & $2.85 \mathrm{E}+04$ & $7.71 \mathrm{E}+05$ & $8.85 \mathrm{E}+03$ & $2.39 \mathrm{E}+05$ & $1.05 \mathrm{E}+05$ & $2.84 \mathrm{E}+06$ & $2.89 \mathrm{E}+05$ & $7.80 \mathrm{E}+06$ & $7.13 \mathrm{E}+03$ & $7.80 \mathrm{E}+06$ \\
\hline $\mathrm{Zn}-71 \mathrm{~m}$ & $4.01 \mathrm{E}+03$ & $1.08 \mathrm{E}+05$ & $3.98 \mathrm{E}+04$ & $1.08 \mathrm{E}+06$ & $1.24 \mathrm{E}+04$ & $3.34 \mathrm{E}+05$ & $1.47 \mathrm{E}+05$ & $3.96 \mathrm{E}+06$ & $4.03 \mathrm{E}+05$ & $1.09 \mathrm{E}+07$ & $9.96 \mathrm{E}+03$ & $1.09 \mathrm{E}+07$ \\
\hline $\mathrm{Zn}-72$ & $6.83 \mathrm{E}+02$ & $1.85 \mathrm{E}+04$ & $6.78 \mathrm{E}+03$ & $1.83 \mathrm{E}+05$ & $2.11 \mathrm{E}+03$ & $5.69 \mathrm{E}+04$ & $2.50 \mathrm{E}+04$ & $6.75 \mathrm{E}+05$ & $6.87 \mathrm{E}+04$ & $1.86 \mathrm{E}+06$ & $1.70 \mathrm{E}+03$ & $1.86 \mathrm{E}+06$ \\
\hline Ga-65 & $2.56 \mathrm{E}+04$ & $6.92 \mathrm{E}+05$ & $2.54 \mathrm{E}+05$ & $6.87 \mathrm{E}+06$ & $7.88 \mathrm{E}+04$ & $2.13 \mathrm{E}+06$ & $9.35 \mathrm{E}+05$ & $2.53 \mathrm{E}+07$ & $2.57 \mathrm{E}+06$ & $6.95 \mathrm{E}+07$ & $6.35 \mathrm{E}+04$ & $6.95 \mathrm{E}+07$ \\
\hline Ga-66 & $7.82 \mathrm{E}+02$ & $2.11 \mathrm{E}+04$ & $7.76 \mathrm{E}+03$ & $2.10 \mathrm{E}+05$ & $2.41 \mathrm{E}+03$ & $6.51 \mathrm{E}+04$ & $2.86 \mathrm{E}+04$ & $7.73 \mathrm{E}+05$ & $7.86 \mathrm{E}+04$ & $2.12 \mathrm{E}+06$ & $1.94 \mathrm{E}+03$ & $2.12 \mathrm{E}+06$ \\
\hline Ga-67 & $4.78 \mathrm{E}+03$ & $1.29 \mathrm{E}+05$ & $4.75 \mathrm{E}+04$ & $1.28 \mathrm{E}+06$ & $1.47 \mathrm{E}+04$ & $3.98 \mathrm{E}+05$ & $1.75 \mathrm{E}+05$ & $4.73 \mathrm{E}+06$ & $4.81 \mathrm{E}+05$ & $1.30 \mathrm{E}+07$ & $1.19 \mathrm{E}+04$ & $1.30 \mathrm{E}+07$ \\
\hline Ga-68 & $9.15 \mathrm{E}+03$ & $2.47 \mathrm{E}+05$ & $9.08 \mathrm{E}+04$ & $2.45 \mathrm{E}+06$ & $2.82 \mathrm{E}+04$ & $7.61 \mathrm{E}+05$ & $3.34 \mathrm{E}+05$ & $9.03 \mathrm{E}+06$ & $9.19 \mathrm{E}+05$ & $2.48 \mathrm{E}+07$ & $2.27 \mathrm{E}+04$ & $2.48 \mathrm{E}+07$ \\
\hline Ga-70 & $2.95 \mathrm{E}+04$ & $7.97 \mathrm{E}+05$ & $2.93 \mathrm{E}+05$ & $7.91 \mathrm{E}+06$ & $9.08 \mathrm{E}+04$ & $2.45 \mathrm{E}+06$ & $1.08 \mathrm{E}+06$ & $2.91 \mathrm{E}+07$ & $2.96 \mathrm{E}+06$ & $8.01 \mathrm{E}+07$ & $7.31 \mathrm{E}+04$ & $8.01 \mathrm{E}+07$ \\
\hline
\end{tabular}


SRNL-STI-2013-00115

\begin{tabular}{|c|c|c|c|c|c|c|c|c|c|c|c|c|}
\hline \multirow[t]{2}{*}{ Nuclide } & \multicolumn{2}{|c|}{ Water Ingestion DCS } & \multicolumn{2}{|c|}{ Meat Ingestion DCS } & \multicolumn{2}{|c|}{ Dairy Ingestion DCS } & \multicolumn{2}{|c|}{$\begin{array}{c}\text { Freshwater Fish } \\
\text { Ingestion DCS }\end{array}$} & \multicolumn{2}{|c|}{$\begin{array}{l}\text { Saltwater Shellfish } \\
\text { Ingestion DCS }\end{array}$} & \multicolumn{2}{|c|}{ Produce Ingestion DCS } \\
\hline & $(\mathrm{Bq} / \mathrm{L})$ & $(\mathrm{pCi} / \mathrm{L})$ & $(\mathrm{Bq} / \mathrm{kg})$ & $(\mathrm{pCi} / \mathrm{kg})$ & $(\mathrm{Bq} / \mathrm{L})$ & $(\mathrm{pCi} / \mathrm{L})$ & $(\mathrm{Bq} / \mathbf{k g})$ & $(\mathrm{pCi} / \mathbf{k g})$ & $(\mathrm{Bq} / \mathbf{k g})$ & $(\mathrm{pCi} / \mathbf{k g})$ & $(\mathrm{Bq} / \mathbf{k g})$ & $(\mathrm{pCi} / \mathbf{k g})$ \\
\hline Ga-72 & $8.46 \mathrm{E}+02$ & $2.29 \mathrm{E}+04$ & $8.40 \mathrm{E}+03$ & $2.27 \mathrm{E}+05$ & $2.61 \mathrm{E}+03$ & $7.04 \mathrm{E}+04$ & $3.09 \mathrm{E}+04$ & $8.36 \mathrm{E}+05$ & $8.50 \mathrm{E}+04$ & $2.30 \mathrm{E}+06$ & $2.10 \mathrm{E}+03$ & $2.30 \mathrm{E}+06$ \\
\hline Ga-73 & $3.46 \mathrm{E}+03$ & $9.36 \mathrm{E}+04$ & $3.44 \mathrm{E}+04$ & $9.29 \mathrm{E}+05$ & $1.07 \mathrm{E}+04$ & $2.88 \mathrm{E}+05$ & $1.27 \mathrm{E}+05$ & $3.42 \mathrm{E}+06$ & $3.48 \mathrm{E}+05$ & $9.41 \mathrm{E}+06$ & $8.60 \mathrm{E}+03$ & $9.41 \mathrm{E}+06$ \\
\hline Ge-66 & $9.72 \mathrm{E}+03$ & $2.63 \mathrm{E}+05$ & $9.65 \mathrm{E}+04$ & $2.61 \mathrm{E}+06$ & $2.99 \mathrm{E}+04$ & $8.09 \mathrm{E}+05$ & $3.55 \mathrm{E}+05$ & $9.60 \mathrm{E}+06$ & $9.77 \mathrm{E}+05$ & $2.64 \mathrm{E}+07$ & $2.41 \mathrm{E}+04$ & $2.64 \mathrm{E}+07$ \\
\hline Ge-67 & $1.57 \mathrm{E}+04$ & $4.25 \mathrm{E}+05$ & $1.56 \mathrm{E}+05$ & $4.22 \mathrm{E}+06$ & $4.84 \mathrm{E}+04$ & $1.31 \mathrm{E}+06$ & $5.75 \mathrm{E}+05$ & $1.55 \mathrm{E}+07$ & $1.58 \mathrm{E}+06$ & $4.27 \mathrm{E}+07$ & $3.90 \mathrm{E}+04$ & $4.27 \mathrm{E}+07$ \\
\hline $\mathrm{Ge}-68$ & $7.36 \mathrm{E}+02$ & $1.99 \mathrm{E}+04$ & $7.31 \mathrm{E}+03$ & $1.97 \mathrm{E}+05$ & $2.27 \mathrm{E}+03$ & $6.13 \mathrm{E}+04$ & $2.69 \mathrm{E}+04$ & $7.27 \mathrm{E}+05$ & $7.40 \mathrm{E}+04$ & $2.00 \mathrm{E}+06$ & $1.83 \mathrm{E}+03$ & $2.00 \mathrm{E}+06$ \\
\hline Ge-69 & $4.77 \mathrm{E}+03$ & $1.29 \mathrm{E}+05$ & $4.73 \mathrm{E}+04$ & $1.28 \mathrm{E}+06$ & $1.47 \mathrm{E}+04$ & $3.97 \mathrm{E}+05$ & $1.74 \mathrm{E}+05$ & $4.71 \mathrm{E}+06$ & $4.79 \mathrm{E}+05$ & $1.29 \mathrm{E}+07$ & $1.18 \mathrm{E}+04$ & $1.29 \mathrm{E}+07$ \\
\hline Ge-71 & $7.87 \mathrm{E}+04$ & $2.13 \mathrm{E}+06$ & $7.81 \mathrm{E}+05$ & $2.11 \mathrm{E}+07$ & $2.42 \mathrm{E}+05$ & $6.55 \mathrm{E}+06$ & $2.88 \mathrm{E}+06$ & $7.78 \mathrm{E}+07$ & $7.91 \mathrm{E}+06$ & $2.14 \mathrm{E}+08$ & $1.95 \mathrm{E}+05$ & $2.14 \mathrm{E}+08$ \\
\hline Ge-75 & $2.01 \mathrm{E}+04$ & $5.43 \mathrm{E}+05$ & $1.99 \mathrm{E}+05$ & $5.39 \mathrm{E}+06$ & $6.19 \mathrm{E}+04$ & $1.67 \mathrm{E}+06$ & $7.34 \mathrm{E}+05$ & $1.98 \mathrm{E}+07$ & $2.02 \mathrm{E}+06$ & $5.46 \mathrm{E}+07$ & $4.99 \mathrm{E}+04$ & $5.46 \mathrm{E}+07$ \\
\hline Ge-77 & $2.95 \mathrm{E}+03$ & $7.98 \mathrm{E}+04$ & $2.93 \mathrm{E}+04$ & $7.93 \mathrm{E}+05$ & $9.10 \mathrm{E}+03$ & $2.46 \mathrm{E}+05$ & $1.08 \mathrm{E}+05$ & $2.92 \mathrm{E}+06$ & $2.97 \mathrm{E}+05$ & $8.02 \mathrm{E}+06$ & $7.33 \mathrm{E}+03$ & $8.02 \mathrm{E}+06$ \\
\hline $\mathrm{Ge}-78$ & $8.46 \mathrm{E}+03$ & $2.29 \mathrm{E}+05$ & $8.40 \mathrm{E}+04$ & $2.27 \mathrm{E}+06$ & $2.61 \mathrm{E}+04$ & $7.04 \mathrm{E}+05$ & $3.09 \mathrm{E}+05$ & $8.36 \mathrm{E}+06$ & $8.50 \mathrm{E}+05$ & $2.30 \mathrm{E}+07$ & $2.10 \mathrm{E}+04$ & $2.30 \mathrm{E}+07$ \\
\hline As-69 & $1.71 \mathrm{E}+04$ & $4.63 \mathrm{E}+05$ & $1.70 \mathrm{E}+05$ & $4.60 \mathrm{E}+06$ & $5.28 \mathrm{E}+04$ & $1.43 \mathrm{E}+06$ & $6.26 \mathrm{E}+05$ & $1.69 \mathrm{E}+07$ & $1.72 \mathrm{E}+06$ & $4.65 \mathrm{E}+07$ & $4.25 \mathrm{E}+04$ & $4.65 \mathrm{E}+07$ \\
\hline As-70 & $7.07 \mathrm{E}+03$ & $1.91 \mathrm{E}+05$ & $7.01 \mathrm{E}+04$ & $1.90 \mathrm{E}+06$ & $2.18 \mathrm{E}+04$ & $5.88 \mathrm{E}+05$ & $2.58 \mathrm{E}+05$ & $6.98 \mathrm{E}+06$ & $7.10 \mathrm{E}+05$ & $1.92 \mathrm{E}+07$ & $1.75 \mathrm{E}+04$ & $1.92 \mathrm{E}+07$ \\
\hline As-71 & $2.12 \mathrm{E}+03$ & $5.74 \mathrm{E}+04$ & $2.11 \mathrm{E}+04$ & $5.69 \mathrm{E}+05$ & $6.54 \mathrm{E}+03$ & $1.77 \mathrm{E}+05$ & $7.76 \mathrm{E}+04$ & $2.10 \mathrm{E}+06$ & $2.13 \mathrm{E}+05$ & $5.77 \mathrm{E}+06$ & $5.27 \mathrm{E}+03$ & $5.77 \mathrm{E}+06$ \\
\hline As-72 & $5.14 \mathrm{E}+02$ & $1.39 \mathrm{E}+04$ & $5.10 \mathrm{E}+03$ & $1.38 \mathrm{E}+05$ & $1.58 \mathrm{E}+03$ & $4.28 \mathrm{E}+04$ & $1.88 \mathrm{E}+04$ & $5.08 \mathrm{E}+05$ & $5.17 \mathrm{E}+04$ & $1.40 \mathrm{E}+06$ & $1.28 \mathrm{E}+03$ & $1.40 \mathrm{E}+06$ \\
\hline As-73 & $3.54 \mathrm{E}+03$ & $9.58 \mathrm{E}+04$ & $3.52 \mathrm{E}+04$ & $9.51 \mathrm{E}+05$ & $1.09 \mathrm{E}+04$ & $2.95 \mathrm{E}+05$ & $1.30 \mathrm{E}+05$ & $3.50 \mathrm{E}+06$ & $3.56 \mathrm{E}+05$ & $9.63 \mathrm{E}+06$ & $8.79 \mathrm{E}+03$ & $9.63 \mathrm{E}+06$ \\
\hline As-74 & $7.40 \mathrm{E}+02$ & $2.00 \mathrm{E}+04$ & $7.35 \mathrm{E}+03$ & $1.99 \mathrm{E}+05$ & $2.28 \mathrm{E}+03$ & $6.16 \mathrm{E}+04$ & $2.71 \mathrm{E}+04$ & $7.31 \mathrm{E}+05$ & $7.44 \mathrm{E}+04$ & $2.01 \mathrm{E}+06$ & $1.84 \mathrm{E}+03$ & $2.01 \mathrm{E}+06$ \\
\hline As-76 & $5.89 \mathrm{E}+02$ & $1.59 \mathrm{E}+04$ & $5.85 \mathrm{E}+03$ & $1.58 \mathrm{E}+05$ & $1.82 \mathrm{E}+03$ & $4.91 \mathrm{E}+04$ & $2.15 \mathrm{E}+04$ & $5.82 \mathrm{E}+05$ & $5.92 \mathrm{E}+04$ & $1.60 \mathrm{E}+06$ & $1.46 \mathrm{E}+03$ & $1.60 \mathrm{E}+06$ \\
\hline As-77 & $2.36 \mathrm{E}+03$ & $6.38 \mathrm{E}+04$ & $2.34 \mathrm{E}+04$ & $6.33 \mathrm{E}+05$ & $7.27 \mathrm{E}+03$ & $1.96 \mathrm{E}+05$ & $8.63 \mathrm{E}+04$ & $2.33 \mathrm{E}+06$ & $2.37 \mathrm{E}+05$ & $6.41 \mathrm{E}+06$ & $5.86 \mathrm{E}+03$ & $6.41 \mathrm{E}+06$ \\
\hline As-78 & $4.77 \mathrm{E}+03$ & $1.29 \mathrm{E}+05$ & $4.73 \mathrm{E}+04$ & $1.28 \mathrm{E}+06$ & $1.47 \mathrm{E}+04$ & $3.97 \mathrm{E}+05$ & $1.74 \mathrm{E}+05$ & $4.71 \mathrm{E}+06$ & $4.79 \mathrm{E}+05$ & $1.29 \mathrm{E}+07$ & $1.18 \mathrm{E}+04$ & $1.29 \mathrm{E}+07$ \\
\hline Se-70 & $9.95 \mathrm{E}+03$ & $2.69 \mathrm{E}+05$ & $9.88 \mathrm{E}+04$ & $2.67 \mathrm{E}+06$ & $3.07 \mathrm{E}+04$ & $8.28 \mathrm{E}+05$ & $3.64 \mathrm{E}+05$ & $9.83 \mathrm{E}+06$ & $1.00 \mathrm{E}+06$ & $2.70 \mathrm{E}+07$ & $2.47 \mathrm{E}+04$ & $2.70 \mathrm{E}+07$ \\
\hline Se-72 & $1.51 \mathrm{E}+02$ & $4.08 \mathrm{E}+03$ & $1.50 \mathrm{E}+03$ & $4.05 \mathrm{E}+04$ & $4.66 \mathrm{E}+02$ & $1.26 \mathrm{E}+04$ & $5.52 \mathrm{E}+03$ & $1.49 \mathrm{E}+05$ & $1.52 \mathrm{E}+04$ & $4.10 \mathrm{E}+05$ & $3.75 \mathrm{E}+02$ & $4.10 \mathrm{E}+05$ \\
\hline Se-73 & $4.47 \mathrm{E}+03$ & $1.21 \mathrm{E}+05$ & $4.44 \mathrm{E}+04$ & $1.20 \mathrm{E}+06$ & $1.38 \mathrm{E}+04$ & $3.72 \mathrm{E}+05$ & $1.64 \mathrm{E}+05$ & $4.42 \mathrm{E}+06$ & $4.50 \mathrm{E}+05$ & $1.22 \mathrm{E}+07$ & $1.11 \mathrm{E}+04$ & $1.22 \mathrm{E}+07$ \\
\hline Se-73m & $3.39 \mathrm{E}+04$ & $9.16 \mathrm{E}+05$ & $3.36 \mathrm{E}+05$ & $9.09 \mathrm{E}+06$ & $1.04 \mathrm{E}+05$ & $2.82 \mathrm{E}+06$ & $1.24 \mathrm{E}+06$ & $3.35 \mathrm{E}+07$ & $3.41 \mathrm{E}+06$ & $9.21 \mathrm{E}+07$ & $8.41 \mathrm{E}+04$ & $9.21 \mathrm{E}+07$ \\
\hline
\end{tabular}

Page 172 of 298 
SRNL-STI-2013-00115

\begin{tabular}{|c|c|c|c|c|c|c|c|c|c|c|c|c|}
\hline \multirow[t]{2}{*}{ Nuclide } & \multicolumn{2}{|c|}{ Water Ingestion DCS } & \multicolumn{2}{|c|}{ Meat Ingestion DCS } & \multicolumn{2}{|c|}{ Dairy Ingestion DCS } & \multicolumn{2}{|c|}{$\begin{array}{c}\text { Freshwater Fish } \\
\text { Ingestion DCS }\end{array}$} & \multicolumn{2}{|c|}{$\begin{array}{l}\text { Saltwater Shellfish } \\
\text { Ingestion DCS }\end{array}$} & \multicolumn{2}{|c|}{ Produce Ingestion DCS } \\
\hline & $(\mathrm{Bq} / \mathrm{L})$ & $(\mathrm{pCi} / \mathrm{L})$ & $(\mathrm{Bq} / \mathrm{kg})$ & $(\mathrm{pCi} / \mathrm{kg})$ & $(\mathrm{Bq} / \mathrm{L})$ & $(\mathrm{pCi} / \mathrm{L})$ & $(\mathrm{Bq} / \mathbf{k g})$ & $(\mathrm{pCi} / \mathbf{k g})$ & $(\mathrm{Bq} / \mathbf{k g})$ & $(\mathrm{pCi} / \mathbf{k g})$ & $(\mathrm{Bq} / \mathbf{k g})$ & $(\mathrm{pCi} / \mathbf{k g})$ \\
\hline $\mathrm{Se}-75$ & $3.71 \mathrm{E}+02$ & $1.00 \mathrm{E}+04$ & $3.69 \mathrm{E}+03$ & $9.96 \mathrm{E}+04$ & $1.14 \mathrm{E}+03$ & $3.09 \mathrm{E}+04$ & $1.36 \mathrm{E}+04$ & $3.67 \mathrm{E}+05$ & $3.73 \mathrm{E}+04$ & $1.01 \mathrm{E}+06$ & $9.21 \mathrm{E}+02$ & $1.01 \mathrm{E}+06$ \\
\hline Se-79 & $2.66 \mathrm{E}+02$ & $7.18 \mathrm{E}+03$ & $2.64 \mathrm{E}+03$ & $7.13 \mathrm{E}+04$ & $8.19 \mathrm{E}+02$ & $2.21 \mathrm{E}+04$ & $9.71 \mathrm{E}+03$ & $2.63 \mathrm{E}+05$ & $2.67 \mathrm{E}+04$ & $7.22 \mathrm{E}+05$ & $6.59 \mathrm{E}+02$ & $7.22 \mathrm{E}+05$ \\
\hline Se-81 & $3.45 \mathrm{E}+04$ & $9.31 \mathrm{E}+05$ & $3.42 \mathrm{E}+05$ & $9.24 \mathrm{E}+06$ & $1.06 \mathrm{E}+05$ & $2.87 \mathrm{E}+06$ & $1.26 \mathrm{E}+06$ & $3.40 \mathrm{E}+07$ & $3.46 \mathrm{E}+06$ & $9.36 \mathrm{E}+07$ & $8.55 \mathrm{E}+04$ & $9.36 \mathrm{E}+07$ \\
\hline Se- $81 \mathrm{~m}$ & $1.74 \mathrm{E}+04$ & $4.69 \mathrm{E}+05$ & $1.72 \mathrm{E}+05$ & $4.66 \mathrm{E}+06$ & $5.35 \mathrm{E}+04$ & $1.45 \mathrm{E}+06$ & $6.35 \mathrm{E}+05$ & $1.72 \mathrm{E}+07$ & $1.75 \mathrm{E}+06$ & $4.72 \mathrm{E}+07$ & $4.31 \mathrm{E}+04$ & $4.72 \mathrm{E}+07$ \\
\hline Se-83 & $2.12 \mathrm{E}+04$ & $5.73 \mathrm{E}+05$ & $2.10 \mathrm{E}+05$ & $5.68 \mathrm{E}+06$ & $6.53 \mathrm{E}+04$ & $1.76 \mathrm{E}+06$ & $7.74 \mathrm{E}+05$ & $2.09 \mathrm{E}+07$ & $2.13 \mathrm{E}+06$ & $5.76 \mathrm{E}+07$ & $5.26 \mathrm{E}+04$ & $5.76 \mathrm{E}+07$ \\
\hline $\mathrm{Br}-74$ & $1.16 \mathrm{E}+04$ & $3.14 \mathrm{E}+05$ & $1.15 \mathrm{E}+05$ & $3.12 \mathrm{E}+06$ & $3.58 \mathrm{E}+04$ & $9.68 \mathrm{E}+05$ & $4.25 \mathrm{E}+05$ & $1.15 \mathrm{E}+07$ & $1.17 \mathrm{E}+06$ & $3.16 \mathrm{E}+07$ & $2.88 \mathrm{E}+04$ & $3.16 \mathrm{E}+07$ \\
\hline $\mathrm{Br}-74 \mathrm{~m}$ & $7.07 \mathrm{E}+03$ & $1.91 \mathrm{E}+05$ & $7.01 \mathrm{E}+04$ & $1.90 \mathrm{E}+06$ & $2.18 \mathrm{E}+04$ & $5.88 \mathrm{E}+05$ & $2.58 \mathrm{E}+05$ & $6.98 \mathrm{E}+06$ & $7.10 \mathrm{E}+05$ & $1.92 \mathrm{E}+07$ & $1.75 \mathrm{E}+04$ & $1.92 \mathrm{E}+07$ \\
\hline $\mathrm{Br}-75$ & $1.21 \mathrm{E}+04$ & $3.26 \mathrm{E}+05$ & $1.20 \mathrm{E}+05$ & $3.24 \mathrm{E}+06$ & $3.72 \mathrm{E}+04$ & $1.01 \mathrm{E}+06$ & $4.41 \mathrm{E}+05$ & $1.19 \mathrm{E}+07$ & $1.21 \mathrm{E}+06$ & $3.28 \mathrm{E}+07$ & $3.00 \mathrm{E}+04$ & $3.28 \mathrm{E}+07$ \\
\hline $\mathrm{Br}-76$ & $2.10 \mathrm{E}+03$ & $5.68 \mathrm{E}+04$ & $2.09 \mathrm{E}+04$ & $5.64 \mathrm{E}+05$ & $6.47 \mathrm{E}+03$ & $1.75 \mathrm{E}+05$ & $7.68 \mathrm{E}+04$ & $2.08 \mathrm{E}+06$ & $2.11 \mathrm{E}+05$ & $5.71 \mathrm{E}+06$ & $5.21 \mathrm{E}+03$ & $5.71 \mathrm{E}+06$ \\
\hline $\mathrm{Br}-77$ & $1.04 \mathrm{E}+04$ & $2.80 \mathrm{E}+05$ & $1.03 \mathrm{E}+05$ & $2.78 \mathrm{E}+06$ & $3.19 \mathrm{E}+04$ & $8.63 \mathrm{E}+05$ & $3.79 \mathrm{E}+05$ & $1.02 \mathrm{E}+07$ & $1.04 \mathrm{E}+06$ & $2.82 \mathrm{E}+07$ & $2.57 \mathrm{E}+04$ & $2.82 \mathrm{E}+07$ \\
\hline $\mathrm{Br}-80$ & $2.98 \mathrm{E}+04$ & $8.04 \mathrm{E}+05$ & $2.95 \mathrm{E}+05$ & $7.98 \mathrm{E}+06$ & $9.17 \mathrm{E}+04$ & $2.48 \mathrm{E}+06$ & $1.09 \mathrm{E}+06$ & $2.94 \mathrm{E}+07$ & $2.99 \mathrm{E}+06$ & $8.08 \mathrm{E}+07$ & $7.38 \mathrm{E}+04$ & $8.08 \mathrm{E}+07$ \\
\hline $\mathrm{Br}-80 \mathrm{~m}$ & $8.02 \mathrm{E}+03$ & $2.17 \mathrm{E}+05$ & $7.96 \mathrm{E}+04$ & $2.15 \mathrm{E}+06$ & $2.47 \mathrm{E}+04$ & $6.68 \mathrm{E}+05$ & $2.93 \mathrm{E}+05$ & $7.93 \mathrm{E}+06$ & $8.06 \mathrm{E}+05$ & $2.18 \mathrm{E}+07$ & $1.99 \mathrm{E}+04$ & $2.18 \mathrm{E}+07$ \\
\hline $\mathrm{Br}-82$ & $1.84 \mathrm{E}+03$ & $4.98 \mathrm{E}+04$ & $1.83 \mathrm{E}+04$ & $4.94 \mathrm{E}+05$ & $5.68 \mathrm{E}+03$ & $1.53 \mathrm{E}+05$ & $6.73 \mathrm{E}+04$ & $1.82 \mathrm{E}+06$ & $1.85 \mathrm{E}+05$ & $5.01 \mathrm{E}+06$ & $4.57 \mathrm{E}+03$ & $5.01 \mathrm{E}+06$ \\
\hline $\mathrm{Br}-83$ & $2.08 \mathrm{E}+04$ & $5.62 \mathrm{E}+05$ & $2.06 \mathrm{E}+05$ & $5.58 \mathrm{E}+06$ & $6.41 \mathrm{E}+04$ & $1.73 \mathrm{E}+06$ & $7.60 \mathrm{E}+05$ & $2.05 \mathrm{E}+07$ & $2.09 \mathrm{E}+06$ & $5.65 \mathrm{E}+07$ & $5.16 \mathrm{E}+04$ & $5.65 \mathrm{E}+07$ \\
\hline $\mathrm{Br}-84$ & $1.05 \mathrm{E}+04$ & $2.85 \mathrm{E}+05$ & $1.05 \mathrm{E}+05$ & $2.83 \mathrm{E}+06$ & $3.25 \mathrm{E}+04$ & $8.78 \mathrm{E}+05$ & $3.85 \mathrm{E}+05$ & $1.04 \mathrm{E}+07$ & $1.06 \mathrm{E}+06$ & $2.86 \mathrm{E}+07$ & $2.62 \mathrm{E}+04$ & $2.86 \mathrm{E}+07$ \\
\hline $\mathrm{Rb}-78$ & $1.34 \mathrm{E}+04$ & $3.62 \mathrm{E}+05$ & $1.33 \mathrm{E}+05$ & $3.59 \mathrm{E}+06$ & $4.12 \mathrm{E}+04$ & $1.11 \mathrm{E}+06$ & $4.89 \mathrm{E}+05$ & $1.32 \mathrm{E}+07$ & $1.35 \mathrm{E}+06$ & $3.64 \mathrm{E}+07$ & $3.32 \mathrm{E}+04$ & $3.64 \mathrm{E}+07$ \\
\hline $\mathrm{Rb}-79$ & $1.89 \mathrm{E}+04$ & $5.11 \mathrm{E}+05$ & $1.88 \mathrm{E}+05$ & $5.07 \mathrm{E}+06$ & $5.82 \mathrm{E}+04$ & $1.57 \mathrm{E}+06$ & $6.91 \mathrm{E}+05$ & $1.87 \mathrm{E}+07$ & $1.90 \mathrm{E}+06$ & $5.13 \mathrm{E}+07$ & $4.69 \mathrm{E}+04$ & $5.13 \mathrm{E}+07$ \\
\hline $\mathrm{Rb}-81$ & $1.97 \mathrm{E}+04$ & $5.33 \mathrm{E}+05$ & $1.96 \mathrm{E}+05$ & $5.29 \mathrm{E}+06$ & $6.07 \mathrm{E}+04$ & $1.64 \mathrm{E}+06$ & $7.20 \mathrm{E}+05$ & $1.95 \mathrm{E}+07$ & $1.98 \mathrm{E}+06$ & $5.35 \mathrm{E}+07$ & $4.89 \mathrm{E}+04$ & $5.35 \mathrm{E}+07$ \\
\hline $\mathrm{Rb}-81 \mathrm{~m}$ & $9.95 \mathrm{E}+04$ & $2.69 \mathrm{E}+06$ & $9.88 \mathrm{E}+05$ & $2.67 \mathrm{E}+07$ & $3.07 \mathrm{E}+05$ & $8.28 \mathrm{E}+06$ & $3.64 \mathrm{E}+06$ & $9.83 \mathrm{E}+07$ & $1.00 \mathrm{E}+07$ & $2.70 \mathrm{E}+08$ & $2.47 \mathrm{E}+05$ & $2.70 \mathrm{E}+08$ \\
\hline $\mathrm{Rb}-82 \mathrm{~m}$ & $7.63 \mathrm{E}+03$ & $2.06 \mathrm{E}+05$ & $7.57 \mathrm{E}+04$ & $2.05 \mathrm{E}+06$ & $2.35 \mathrm{E}+04$ & $6.35 \mathrm{E}+05$ & $2.79 \mathrm{E}+05$ & $7.54 \mathrm{E}+06$ & $7.67 \mathrm{E}+05$ & $2.07 \mathrm{E}+07$ & $1.89 \mathrm{E}+04$ & $2.07 \mathrm{E}+07$ \\
\hline $\mathrm{Rb}-83$ & $5.81 \mathrm{E}+02$ & $1.57 \mathrm{E}+04$ & $5.77 \mathrm{E}+03$ & $1.56 \mathrm{E}+05$ & $1.79 \mathrm{E}+03$ & $4.84 \mathrm{E}+04$ & $2.12 \mathrm{E}+04$ & $5.74 \mathrm{E}+05$ & $5.84 \mathrm{E}+04$ & $1.58 \mathrm{E}+06$ & $1.44 \mathrm{E}+03$ & $1.58 \mathrm{E}+06$ \\
\hline $\mathrm{Rb}-84$ & $3.53 \mathrm{E}+02$ & $9.55 \mathrm{E}+03$ & $3.51 \mathrm{E}+03$ & $9.48 \mathrm{E}+04$ & $1.09 \mathrm{E}+03$ & $2.94 \mathrm{E}+04$ & $1.29 \mathrm{E}+04$ & $3.49 \mathrm{E}+05$ & $3.55 \mathrm{E}+04$ & $9.60 \mathrm{E}+05$ & $8.77 \mathrm{E}+02$ & $9.60 \mathrm{E}+05$ \\
\hline $\mathrm{Rb}-84 \mathrm{~m}$ & $1.36 \mathrm{E}+05$ & $3.67 \mathrm{E}+06$ & $1.35 \mathrm{E}+06$ & $3.64 \mathrm{E}+07$ & $4.18 \mathrm{E}+05$ & $1.13 \mathrm{E}+07$ & $4.96 \mathrm{E}+06$ & $1.34 \mathrm{E}+08$ & $1.36 \mathrm{E}+07$ & $3.69 \mathrm{E}+08$ & $3.37 \mathrm{E}+05$ & $3.69 \mathrm{E}+08$ \\
\hline
\end{tabular}

Page 173 of 298 
SRNL-STI-2013-00115

\begin{tabular}{|c|c|c|c|c|c|c|c|c|c|c|c|c|}
\hline \multirow[t]{2}{*}{ Nuclide } & \multicolumn{2}{|c|}{ Water Ingestion DCS } & \multicolumn{2}{|c|}{ Meat Ingestion DCS } & \multicolumn{2}{|c|}{ Dairy Ingestion DCS } & \multicolumn{2}{|c|}{$\begin{array}{c}\text { Freshwater Fish } \\
\text { Ingestion DCS }\end{array}$} & \multicolumn{2}{|c|}{$\begin{array}{l}\text { Saltwater Shellfish } \\
\text { Ingestion DCS }\end{array}$} & \multicolumn{2}{|c|}{ Produce Ingestion DCS } \\
\hline & $(\mathrm{Bq} / \mathrm{L})$ & $(\mathrm{pCi} / \mathrm{L})$ & $(\mathrm{Bq} / \mathbf{k g})$ & $(\mathrm{pCi} / \mathbf{k g})$ & $(\mathrm{Bq} / \mathrm{L})$ & $(\mathrm{pCi} / \mathrm{L})$ & $(\mathrm{Bq} / \mathrm{kg})$ & $(\mathrm{pCi} / \mathbf{k g})$ & $(\mathrm{Bq} / \mathbf{k g})$ & $(\mathrm{pCi} / \mathbf{k g})$ & $(\mathrm{Bq} / \mathrm{kg})$ & (pCi//kg) \\
\hline $\mathrm{Rb}-86$ & $3.26 \mathrm{E}+02$ & $8.82 \mathrm{E}+03$ & $3.24 \mathrm{E}+03$ & $8.76 \mathrm{E}+04$ & $1.01 \mathrm{E}+03$ & $2.72 \mathrm{E}+04$ & $1.19 \mathrm{E}+04$ & $3.22 \mathrm{E}+05$ & $3.28 \mathrm{E}+04$ & $8.87 \mathrm{E}+05$ & $8.10 \mathrm{E}+02$ & $8.87 \mathrm{E}+05$ \\
\hline $\mathrm{Rb}-87$ & $6.07 \mathrm{E}+02$ & $1.64 \mathrm{E}+04$ & $6.02 \mathrm{E}+03$ & $1.63 \mathrm{E}+05$ & $1.87 \mathrm{E}+03$ & $5.05 \mathrm{E}+04$ & $2.22 \mathrm{E}+04$ & $5.99 \mathrm{E}+05$ & $6.10 \mathrm{E}+04$ & $1.65 \mathrm{E}+06$ & $1.51 \mathrm{E}+03$ & $1.65 \mathrm{E}+06$ \\
\hline $\mathrm{Rb}-88$ & $1.02 \mathrm{E}+04$ & $2.76 \mathrm{E}+05$ & $1.01 \mathrm{E}+05$ & $2.73 \mathrm{E}+06$ & $3.14 \mathrm{E}+04$ & $8.49 \mathrm{E}+05$ & $3.73 \mathrm{E}+05$ & $1.01 \mathrm{E}+07$ & $1.02 \mathrm{E}+06$ & $2.77 \mathrm{E}+07$ & $2.53 \mathrm{E}+04$ & $2.77 \mathrm{E}+07$ \\
\hline $\mathrm{Rb}-89$ & $2.09 \mathrm{E}+04$ & $5.65 \mathrm{E}+05$ & $2.07 \mathrm{E}+05$ & $5.61 \mathrm{E}+06$ & $6.44 \mathrm{E}+04$ & $1.74 \mathrm{E}+06$ & $7.64 \mathrm{E}+05$ & $2.06 \mathrm{E}+07$ & $2.10 \mathrm{E}+06$ & $5.68 \mathrm{E}+07$ & $5.19 \mathrm{E}+04$ & $5.68 \mathrm{E}+07$ \\
\hline $\mathrm{Sr}-80$ & $2.48 \mathrm{E}+03$ & $6.71 \mathrm{E}+04$ & $2.46 \mathrm{E}+04$ & $6.66 \mathrm{E}+05$ & $7.65 \mathrm{E}+03$ & $2.07 \mathrm{E}+05$ & $9.07 \mathrm{E}+04$ & $2.45 \mathrm{E}+06$ & $2.50 \mathrm{E}+05$ & $6.74 \mathrm{E}+06$ & $6.16 \mathrm{E}+03$ & $6.74 \mathrm{E}+06$ \\
\hline Sr-81 & $1.41 \mathrm{E}+04$ & $3.80 \mathrm{E}+05$ & $1.40 \mathrm{E}+05$ & $3.77 \mathrm{E}+06$ & $4.33 \mathrm{E}+04$ & $1.17 \mathrm{E}+06$ & $5.14 \mathrm{E}+05$ & $1.39 \mathrm{E}+07$ & $1.41 \mathrm{E}+06$ & $3.82 \mathrm{E}+07$ & $3.49 \mathrm{E}+04$ & $3.82 \mathrm{E}+07$ \\
\hline $\mathrm{Sr}-82$ & $1.48 \mathrm{E}+02$ & $3.99 \mathrm{E}+03$ & $1.46 \mathrm{E}+03$ & $3.96 \mathrm{E}+04$ & $4.54 \mathrm{E}+02$ & $1.23 \mathrm{E}+04$ & $5.39 \mathrm{E}+03$ & $1.46 \mathrm{E}+05$ & $1.48 \mathrm{E}+04$ & $4.01 \mathrm{E}+05$ & $3.66 \mathrm{E}+02$ & $4.01 \mathrm{E}+05$ \\
\hline Sr-83 & $1.93 \mathrm{E}+03$ & $5.20 \mathrm{E}+04$ & $1.91 \mathrm{E}+04$ & $5.17 \mathrm{E}+05$ & $5.93 \mathrm{E}+03$ & $1.60 \mathrm{E}+05$ & $7.04 \mathrm{E}+04$ & $1.90 \mathrm{E}+06$ & $1.93 \mathrm{E}+05$ & $5.23 \mathrm{E}+06$ & $4.78 \mathrm{E}+03$ & $5.23 \mathrm{E}+06$ \\
\hline $\mathrm{Sr}-85$ & $1.57 \mathrm{E}+03$ & $4.23 \mathrm{E}+04$ & $1.55 \mathrm{E}+04$ & $4.20 \mathrm{E}+05$ & $4.83 \mathrm{E}+03$ & $1.30 \mathrm{E}+05$ & $5.72 \mathrm{E}+04$ & $1.55 \mathrm{E}+06$ & $1.57 \mathrm{E}+05$ & $4.25 \mathrm{E}+06$ & $3.89 \mathrm{E}+03$ & $4.25 \mathrm{E}+06$ \\
\hline $\mathrm{Sr}-85 \mathrm{~m}$ & $1.65 \mathrm{E}+05$ & $4.46 \mathrm{E}+06$ & $1.64 \mathrm{E}+06$ & $4.43 \mathrm{E}+07$ & $5.09 \mathrm{E}+05$ & $1.38 \mathrm{E}+07$ & $6.04 \mathrm{E}+06$ & $1.63 \mathrm{E}+08$ & $1.66 \mathrm{E}+07$ & $4.49 \mathrm{E}+08$ & $4.10 \mathrm{E}+05$ & $4.49 \mathrm{E}+08$ \\
\hline $\mathrm{Sr}-87 \mathrm{~m}$ & $3.22 \mathrm{E}+04$ & $8.71 \mathrm{E}+05$ & $3.20 \mathrm{E}+05$ & $8.64 \mathrm{E}+06$ & $9.93 \mathrm{E}+04$ & $2.68 \mathrm{E}+06$ & $1.18 \mathrm{E}+06$ & $3.18 \mathrm{E}+07$ & $3.24 \mathrm{E}+06$ & $8.75 \mathrm{E}+07$ & $8.00 \mathrm{E}+04$ & $8.75 \mathrm{E}+07$ \\
\hline Sr-89 & $3.45 \mathrm{E}+02$ & $9.31 \mathrm{E}+03$ & $3.42 \mathrm{E}+03$ & $9.24 \mathrm{E}+04$ & $1.06 \mathrm{E}+03$ & $2.87 \mathrm{E}+04$ & $1.26 \mathrm{E}+04$ & $3.40 \mathrm{E}+05$ & $3.46 \mathrm{E}+04$ & $9.36 \mathrm{E}+05$ & $8.55 \mathrm{E}+02$ & $9.36 \mathrm{E}+05$ \\
\hline Sr-90 & $3.45 \mathrm{E}+01$ & $9.34 \mathrm{E}+02$ & $3.43 \mathrm{E}+02$ & $9.27 \mathrm{E}+03$ & $1.06 \mathrm{E}+02$ & $2.88 \mathrm{E}+03$ & $1.26 \mathrm{E}+03$ & $3.41 \mathrm{E}+04$ & $3.47 \mathrm{E}+03$ & $9.38 \mathrm{E}+04$ & $8.57 \mathrm{E}+01$ & $9.38 \mathrm{E}+04$ \\
\hline Sr-91 & $1.47 \mathrm{E}+03$ & $3.97 \mathrm{E}+04$ & $1.46 \mathrm{E}+04$ & $3.94 \mathrm{E}+05$ & $4.52 \mathrm{E}+03$ & $1.22 \mathrm{E}+05$ & $5.37 \mathrm{E}+04$ & $1.45 \mathrm{E}+06$ & $1.48 \mathrm{E}+05$ & $3.99 \mathrm{E}+06$ & $3.64 \mathrm{E}+03$ & $3.99 \mathrm{E}+06$ \\
\hline Sr-92 & $2.29 \mathrm{E}+03$ & $6.18 \mathrm{E}+04$ & $2.27 \mathrm{E}+04$ & $6.13 \mathrm{E}+05$ & $7.04 \mathrm{E}+03$ & $1.90 \mathrm{E}+05$ & $8.36 \mathrm{E}+04$ & $2.26 \mathrm{E}+06$ & $2.30 \mathrm{E}+05$ & $6.21 \mathrm{E}+06$ & $5.67 \mathrm{E}+03$ & $6.21 \mathrm{E}+06$ \\
\hline $\mathrm{Y}-84 \mathrm{~m}$ & $6.95 \mathrm{E}+03$ & $1.88 \mathrm{E}+05$ & $6.90 \mathrm{E}+04$ & $1.86 \mathrm{E}+06$ & $2.14 \mathrm{E}+04$ & $5.79 \mathrm{E}+05$ & $2.54 \mathrm{E}+05$ & $6.86 \mathrm{E}+06$ & $6.98 \mathrm{E}+05$ & $1.89 \mathrm{E}+07$ & $1.72 \mathrm{E}+04$ & $1.89 \mathrm{E}+07$ \\
\hline Y-85 & $5.12 \mathrm{E}+03$ & $1.38 \mathrm{E}+05$ & $5.08 \mathrm{E}+04$ & $1.37 \mathrm{E}+06$ & $1.58 \mathrm{E}+04$ & $4.26 \mathrm{E}+05$ & $1.87 \mathrm{E}+05$ & $5.06 \mathrm{E}+06$ & $5.14 \mathrm{E}+05$ & $1.39 \mathrm{E}+07$ & $1.27 \mathrm{E}+04$ & $1.39 \mathrm{E}+07$ \\
\hline $\mathrm{Y}-85 \mathrm{~m}$ & $2.52 \mathrm{E}+03$ & $6.80 \mathrm{E}+04$ & $2.50 \mathrm{E}+04$ & $6.75 \mathrm{E}+05$ & $7.76 \mathrm{E}+03$ & $2.10 \mathrm{E}+05$ & $9.20 \mathrm{E}+04$ & $2.49 \mathrm{E}+06$ & $2.53 \mathrm{E}+05$ & $6.84 \mathrm{E}+06$ & $6.25 \mathrm{E}+03$ & $6.84 \mathrm{E}+06$ \\
\hline Y-86 & $1.02 \mathrm{E}+03$ & $2.76 \mathrm{E}+04$ & $1.01 \mathrm{E}+04$ & $2.73 \mathrm{E}+05$ & $3.14 \mathrm{E}+03$ & $8.49 \mathrm{E}+04$ & $3.73 \mathrm{E}+04$ & $1.01 \mathrm{E}+06$ & $1.02 \mathrm{E}+05$ & $2.77 \mathrm{E}+06$ & $2.53 \mathrm{E}+03$ & $2.77 \mathrm{E}+06$ \\
\hline $\mathrm{Y}-86 \mathrm{~m}$ & $1.73 \mathrm{E}+04$ & $4.69 \mathrm{E}+05$ & $1.72 \mathrm{E}+05$ & $4.65 \mathrm{E}+06$ & $5.34 \mathrm{E}+04$ & $1.44 \mathrm{E}+06$ & $6.34 \mathrm{E}+05$ & $1.71 \mathrm{E}+07$ & $1.74 \mathrm{E}+06$ & $4.71 \mathrm{E}+07$ & $4.30 \mathrm{E}+04$ & $4.71 \mathrm{E}+07$ \\
\hline Y-87 & $1.75 \mathrm{E}+03$ & $4.72 \mathrm{E}+04$ & $1.73 \mathrm{E}+04$ & $4.69 \mathrm{E}+05$ & $5.38 \mathrm{E}+03$ & $1.45 \mathrm{E}+05$ & $6.38 \mathrm{E}+04$ & $1.73 \mathrm{E}+06$ & $1.76 \mathrm{E}+05$ & $4.74 \mathrm{E}+06$ & $4.33 \mathrm{E}+03$ & $4.74 \mathrm{E}+06$ \\
\hline $\mathrm{Y}-87 \mathrm{~m}$ & $4.30 \mathrm{E}+03$ & $1.16 \mathrm{E}+05$ & $4.27 \mathrm{E}+04$ & $1.15 \mathrm{E}+06$ & $1.33 \mathrm{E}+04$ & $3.58 \mathrm{E}+05$ & $1.57 \mathrm{E}+05$ & $4.25 \mathrm{E}+06$ & $4.33 \mathrm{E}+05$ & $1.17 \mathrm{E}+07$ & $1.07 \mathrm{E}+04$ & $1.17 \mathrm{E}+07$ \\
\hline Y-88 & $7.73 \mathrm{E}+02$ & $2.09 \mathrm{E}+04$ & $7.67 \mathrm{E}+03$ & $2.07 \mathrm{E}+05$ & $2.38 \mathrm{E}+03$ & $6.43 \mathrm{E}+04$ & $2.82 \mathrm{E}+04$ & $7.63 \mathrm{E}+05$ & $7.76 \mathrm{E}+04$ & $2.10 \mathrm{E}+06$ & $1.92 \mathrm{E}+03$ & $2.10 \mathrm{E}+06$ \\
\hline
\end{tabular}

Page 174 of 298 
SRNL-STI-2013-00115

\begin{tabular}{|c|c|c|c|c|c|c|c|c|c|c|c|c|}
\hline \multirow[t]{2}{*}{ Nuclide } & \multicolumn{2}{|c|}{ Water Ingestion DCS } & \multicolumn{2}{|c|}{ Meat Ingestion DCS } & \multicolumn{2}{|c|}{ Dairy Ingestion DCS } & \multicolumn{2}{|c|}{$\begin{array}{l}\text { Freshwater Fish } \\
\text { Ingestion DCS }\end{array}$} & \multicolumn{2}{|c|}{$\begin{array}{l}\text { Saltwater Shellfish } \\
\text { Ingestion DCS }\end{array}$} & \multicolumn{2}{|c|}{ Produce Ingestion DCS } \\
\hline & $(\mathrm{Bq} / \mathrm{L})$ & $(\mathrm{pCi} / \mathrm{L})$ & $(\mathrm{Bq} / \mathrm{kg})$ & $(\mathrm{pCi} / \mathrm{kg})$ & $(\mathrm{Bq} / \mathrm{L})$ & $(\mathrm{pCi} / \mathrm{L})$ & $(\mathrm{Bq} / \mathbf{k g})$ & $(\mathrm{pCi} / \mathbf{k g})$ & $(\mathrm{Bq} / \mathbf{k g})$ & $(\mathrm{pCi} / \mathbf{k g})$ & $(\mathrm{Bq} / \mathbf{k g})$ & $(\mathrm{pCi} / \mathbf{k g})$ \\
\hline Y-90 & $3.36 \mathrm{E}+02$ & $9.09 \mathrm{E}+03$ & $3.34 \mathrm{E}+03$ & $9.02 \mathrm{E}+04$ & $1.04 \mathrm{E}+03$ & $2.80 \mathrm{E}+04$ & $1.23 \mathrm{E}+04$ & $3.32 \mathrm{E}+05$ & $3.38 \mathrm{E}+04$ & $9.13 \mathrm{E}+05$ & $8.34 \mathrm{E}+02$ & $9.13 \mathrm{E}+05$ \\
\hline $\mathrm{Y}-90 \mathrm{~m}$ & $5.29 \mathrm{E}+03$ & $1.43 \mathrm{E}+05$ & $5.25 \mathrm{E}+04$ & $1.42 \mathrm{E}+06$ & $1.63 \mathrm{E}+04$ & $4.41 \mathrm{E}+05$ & $1.93 \mathrm{E}+05$ & $5.23 \mathrm{E}+06$ & $5.32 \mathrm{E}+05$ & $1.44 \mathrm{E}+07$ & $1.31 \mathrm{E}+04$ & $1.44 \mathrm{E}+07$ \\
\hline Y-91 & $3.80 \mathrm{E}+02$ & $1.03 \mathrm{E}+04$ & $3.78 \mathrm{E}+03$ & $1.02 \mathrm{E}+05$ & $1.17 \mathrm{E}+03$ & $3.17 \mathrm{E}+04$ & $1.39 \mathrm{E}+04$ & $3.76 \mathrm{E}+05$ & $3.82 \mathrm{E}+04$ & $1.03 \mathrm{E}+06$ & $9.44 \mathrm{E}+02$ & $1.03 \mathrm{E}+06$ \\
\hline $\mathrm{Y}-91 \mathrm{~m}$ & $8.52 \mathrm{E}+04$ & $2.30 \mathrm{E}+06$ & $8.46 \mathrm{E}+05$ & $2.29 \mathrm{E}+07$ & $2.62 \mathrm{E}+05$ & $7.09 \mathrm{E}+06$ & $3.11 \mathrm{E}+06$ & $8.41 \mathrm{E}+07$ & $8.56 \mathrm{E}+06$ & $2.31 \mathrm{E}+08$ & $2.11 \mathrm{E}+05$ & $2.31 \mathrm{E}+08$ \\
\hline $\mathrm{Y}-92$ & $1.83 \mathrm{E}+03$ & $4.95 \mathrm{E}+04$ & $1.82 \mathrm{E}+04$ & $4.91 \mathrm{E}+05$ & $5.64 \mathrm{E}+03$ & $1.53 \mathrm{E}+05$ & $6.69 \mathrm{E}+04$ & $1.81 \mathrm{E}+06$ & $1.84 \mathrm{E}+05$ & $4.98 \mathrm{E}+06$ & $4.55 \mathrm{E}+03$ & $4.98 \mathrm{E}+06$ \\
\hline Y-93 & $7.77 \mathrm{E}+02$ & $2.10 \mathrm{E}+04$ & $7.72 \mathrm{E}+03$ & $2.09 \mathrm{E}+05$ & $2.39 \mathrm{E}+03$ & $6.47 \mathrm{E}+04$ & $2.84 \mathrm{E}+04$ & $7.68 \mathrm{E}+05$ & $7.81 \mathrm{E}+04$ & $2.11 \mathrm{E}+06$ & $1.93 \mathrm{E}+03$ & $2.11 \mathrm{E}+06$ \\
\hline Y-94 & $1.10 \mathrm{E}+04$ & $2.97 \mathrm{E}+05$ & $1.09 \mathrm{E}+05$ & $2.95 \mathrm{E}+06$ & $3.39 \mathrm{E}+04$ & $9.16 \mathrm{E}+05$ & $4.02 \mathrm{E}+05$ & $1.09 \mathrm{E}+07$ & $1.11 \mathrm{E}+06$ & $2.99 \mathrm{E}+07$ & $2.73 \mathrm{E}+04$ & $2.99 \mathrm{E}+07$ \\
\hline Y-95 & $2.18 \mathrm{E}+04$ & $5.89 \mathrm{E}+05$ & $2.16 \mathrm{E}+05$ & $5.84 \mathrm{E}+06$ & $6.71 \mathrm{E}+04$ & $1.81 \mathrm{E}+06$ & $7.96 \mathrm{E}+05$ & $2.15 \mathrm{E}+07$ & $2.19 \mathrm{E}+06$ & $5.92 \mathrm{E}+07$ & $5.41 \mathrm{E}+04$ & $5.92 \mathrm{E}+07$ \\
\hline $\mathrm{Zr}-86$ & $1.13 \mathrm{E}+03$ & $3.06 \mathrm{E}+04$ & $1.12 \mathrm{E}+04$ & $3.03 \mathrm{E}+05$ & $3.48 \mathrm{E}+03$ & $9.41 \mathrm{E}+04$ & $4.13 \mathrm{E}+04$ & $1.12 \mathrm{E}+06$ & $1.14 \mathrm{E}+05$ & $3.07 \mathrm{E}+06$ & $2.81 \mathrm{E}+03$ & $3.07 \mathrm{E}+06$ \\
\hline $\mathrm{Zr}-87$ & $4.96 \mathrm{E}+03$ & $1.34 \mathrm{E}+05$ & $4.92 \mathrm{E}+04$ & $1.33 \mathrm{E}+06$ & $1.53 \mathrm{E}+04$ & $4.13 \mathrm{E}+05$ & $1.81 \mathrm{E}+05$ & $4.89 \mathrm{E}+06$ & $4.98 \mathrm{E}+05$ & $1.35 \mathrm{E}+07$ & $1.23 \mathrm{E}+04$ & $1.35 \mathrm{E}+07$ \\
\hline $\mathrm{Zr}-88$ & $2.32 \mathrm{E}+03$ & $6.26 \mathrm{E}+04$ & $2.30 \mathrm{E}+04$ & $6.21 \mathrm{E}+05$ & $7.13 \mathrm{E}+03$ & $1.93 \mathrm{E}+05$ & $8.46 \mathrm{E}+04$ & $2.29 \mathrm{E}+06$ & $2.33 \mathrm{E}+05$ & $6.29 \mathrm{E}+06$ & $5.75 \mathrm{E}+03$ & $6.29 \mathrm{E}+06$ \\
\hline $\mathrm{Zr}-89$ & $1.22 \mathrm{E}+03$ & $3.30 \mathrm{E}+04$ & $1.21 \mathrm{E}+04$ & $3.27 \mathrm{E}+05$ & $3.76 \mathrm{E}+03$ & $1.02 \mathrm{E}+05$ & $4.46 \mathrm{E}+04$ & $1.20 \mathrm{E}+06$ & $1.23 \mathrm{E}+05$ & $3.31 \mathrm{E}+06$ & $3.03 \mathrm{E}+03$ & $3.31 \mathrm{E}+06$ \\
\hline $\mathrm{Zr}-93$ & $1.24 \mathrm{E}+03$ & $3.36 \mathrm{E}+04$ & $1.23 \mathrm{E}+04$ & $3.34 \mathrm{E}+05$ & $3.83 \mathrm{E}+03$ & $1.04 \mathrm{E}+05$ & $4.55 \mathrm{E}+04$ & $1.23 \mathrm{E}+06$ & $1.25 \mathrm{E}+05$ & $3.38 \mathrm{E}+06$ & $3.09 \mathrm{E}+03$ & $3.38 \mathrm{E}+06$ \\
\hline Zr-95 & $9.87 \mathrm{E}+02$ & $2.67 \mathrm{E}+04$ & $9.80 \mathrm{E}+03$ & $2.65 \mathrm{E}+05$ & $3.04 \mathrm{E}+03$ & $8.22 \mathrm{E}+04$ & $3.61 \mathrm{E}+04$ & $9.75 \mathrm{E}+05$ & $9.92 \mathrm{E}+04$ & $2.68 \mathrm{E}+06$ & $2.45 \mathrm{E}+03$ & $2.68 \mathrm{E}+06$ \\
\hline Zr-97 & $4.44 \mathrm{E}+02$ & $1.20 \mathrm{E}+04$ & $4.41 \mathrm{E}+03$ & $1.19 \mathrm{E}+05$ & $1.37 \mathrm{E}+03$ & $3.70 \mathrm{E}+04$ & $1.62 \mathrm{E}+04$ & $4.39 \mathrm{E}+05$ & $4.46 \mathrm{E}+04$ & $1.21 \mathrm{E}+06$ & $1.10 \mathrm{E}+03$ & $1.21 \mathrm{E}+06$ \\
\hline $\mathrm{Nb}-88$ & $1.32 \mathrm{E}+04$ & $3.56 \mathrm{E}+05$ & $1.31 \mathrm{E}+05$ & $3.54 \mathrm{E}+06$ & $4.06 \mathrm{E}+04$ & $1.10 \mathrm{E}+06$ & $4.82 \mathrm{E}+05$ & $1.30 \mathrm{E}+07$ & $1.33 \mathrm{E}+06$ & $3.58 \mathrm{E}+07$ & $3.27 \mathrm{E}+04$ & $3.58 \mathrm{E}+07$ \\
\hline $\mathrm{Nb}-89$ & $3.37 \mathrm{E}+03$ & $9.11 \mathrm{E}+04$ & $3.35 \mathrm{E}+04$ & $9.04 \mathrm{E}+05$ & $1.04 \mathrm{E}+04$ & $2.81 \mathrm{E}+05$ & $1.23 \mathrm{E}+05$ & $3.33 \mathrm{E}+06$ & $3.39 \mathrm{E}+05$ & $9.16 \mathrm{E}+06$ & $8.36 \mathrm{E}+03$ & $9.16 \mathrm{E}+06$ \\
\hline $\mathrm{Nb}-89 \mathrm{~m}$ & $6.91 \mathrm{E}+03$ & $1.87 \mathrm{E}+05$ & $6.86 \mathrm{E}+04$ & $1.85 \mathrm{E}+06$ & $2.13 \mathrm{E}+04$ & $5.75 \mathrm{E}+05$ & $2.53 \mathrm{E}+05$ & $6.83 \mathrm{E}+06$ & $6.94 \mathrm{E}+05$ & $1.88 \mathrm{E}+07$ & $1.71 \mathrm{E}+04$ & $1.88 \mathrm{E}+07$ \\
\hline $\mathrm{Nb}-90$ & $7.68 \mathrm{E}+02$ & $2.08 \mathrm{E}+04$ & $7.62 \mathrm{E}+03$ & $2.06 \mathrm{E}+05$ & $2.37 \mathrm{E}+03$ & $6.39 \mathrm{E}+04$ & $2.81 \mathrm{E}+04$ & $7.58 \mathrm{E}+05$ & $7.72 \mathrm{E}+04$ & $2.09 \mathrm{E}+06$ & $1.91 \mathrm{E}+03$ & $2.09 \mathrm{E}+06$ \\
\hline Nb-91 & $2.00 \mathrm{E}+04$ & $5.40 \mathrm{E}+05$ & $1.98 \mathrm{E}+05$ & $5.36 \mathrm{E}+06$ & $6.16 \mathrm{E}+04$ & $1.66 \mathrm{E}+06$ & $7.31 \mathrm{E}+05$ & $1.98 \mathrm{E}+07$ & $2.01 \mathrm{E}+06$ & $5.43 \mathrm{E}+07$ & $4.96 \mathrm{E}+04$ & $5.43 \mathrm{E}+07$ \\
\hline $\mathrm{Nb}-91 \mathrm{~m}$ & $2.20 \mathrm{E}+03$ & $5.95 \mathrm{E}+04$ & $2.19 \mathrm{E}+04$ & $5.91 \mathrm{E}+05$ & $6.78 \mathrm{E}+03$ & $1.83 \mathrm{E}+05$ & $8.05 \mathrm{E}+04$ & $2.17 \mathrm{E}+06$ & $2.21 \mathrm{E}+05$ & $5.98 \mathrm{E}+06$ & $5.46 \mathrm{E}+03$ & $5.98 \mathrm{E}+06$ \\
\hline $\mathrm{Nb}-92$ & $9.79 \mathrm{E}+02$ & $2.65 \mathrm{E}+04$ & $9.72 \mathrm{E}+03$ & $2.63 \mathrm{E}+05$ & $3.02 \mathrm{E}+03$ & $8.15 \mathrm{E}+04$ & $3.58 \mathrm{E}+04$ & $9.67 \mathrm{E}+05$ & $9.84 \mathrm{E}+04$ & $2.66 \mathrm{E}+06$ & $2.43 \mathrm{E}+03$ & $2.66 \mathrm{E}+06$ \\
\hline $\mathrm{Nb}-92 \mathrm{~m}$ & $1.99 \mathrm{E}+03$ & $5.37 \mathrm{E}+04$ & $1.97 \mathrm{E}+04$ & $5.33 \mathrm{E}+05$ & $6.12 \mathrm{E}+03$ & $1.65 \mathrm{E}+05$ & $7.26 \mathrm{E}+04$ & $1.96 \mathrm{E}+06$ & $2.00 \mathrm{E}+05$ & $5.40 \mathrm{E}+06$ & $4.93 \mathrm{E}+03$ & $5.40 \mathrm{E}+06$ \\
\hline
\end{tabular}


SRNL-STI-2013-00115

\begin{tabular}{|c|c|c|c|c|c|c|c|c|c|c|c|c|}
\hline \multirow[t]{2}{*}{ Nuclide } & \multicolumn{2}{|c|}{ Water Ingestion DCS } & \multicolumn{2}{|c|}{ Meat Ingestion DCS } & \multicolumn{2}{|c|}{ Dairy Ingestion DCS } & \multicolumn{2}{|c|}{$\begin{array}{c}\text { Freshwater Fish } \\
\text { Ingestion DCS }\end{array}$} & \multicolumn{2}{|c|}{$\begin{array}{l}\text { Saltwater Shellfish } \\
\text { Ingestion DCS }\end{array}$} & \multicolumn{2}{|c|}{ Produce Ingestion DCS } \\
\hline & $(\mathrm{Bq} / \mathrm{L})$ & $(\mathrm{pCi} / \mathrm{L})$ & $(\mathrm{Bq} / \mathbf{k g})$ & $(\mathrm{pCi} / \mathrm{kg})$ & $(\mathrm{Bq} / \mathrm{L})$ & $(\mathrm{pCi} / \mathrm{L})$ & $(\mathrm{Bq} / \mathbf{k g})$ & $(\mathrm{pCi} / \mathbf{k g})$ & $(\mathrm{Bq} / \mathbf{k g})$ & $(\mathrm{pCi} / \mathbf{k g})$ & $(\mathrm{Bq} / \mathbf{k g})$ & $(\mathrm{pCi} / \mathbf{k g})$ \\
\hline $\mathrm{Nb}-93 \mathrm{~m}$ & $6.99 \mathrm{E}+03$ & $1.89 \mathrm{E}+05$ & $6.94 \mathrm{E}+04$ & $1.87 \mathrm{E}+06$ & $2.15 \mathrm{E}+04$ & $5.82 \mathrm{E}+05$ & $2.55 \mathrm{E}+05$ & $6.90 \mathrm{E}+06$ & $7.02 \mathrm{E}+05$ & $1.90 \mathrm{E}+07$ & $1.73 \mathrm{E}+04$ & $1.90 \mathrm{E}+07$ \\
\hline $\mathrm{Nb}-94$ & $5.58 \mathrm{E}+02$ & $1.51 \mathrm{E}+04$ & $5.54 \mathrm{E}+03$ & $1.50 \mathrm{E}+05$ & $1.72 \mathrm{E}+03$ & $4.64 \mathrm{E}+04$ & $2.04 \mathrm{E}+04$ & $5.51 \mathrm{E}+05$ & $5.61 \mathrm{E}+04$ & $1.51 \mathrm{E}+06$ & $1.38 \mathrm{E}+03$ & $1.51 \mathrm{E}+06$ \\
\hline $\mathrm{Nb}-95$ & $1.66 \mathrm{E}+03$ & $4.48 \mathrm{E}+04$ & $1.65 \mathrm{E}+04$ & $4.45 \mathrm{E}+05$ & $5.11 \mathrm{E}+03$ & $1.38 \mathrm{E}+05$ & $6.06 \mathrm{E}+04$ & $1.64 \mathrm{E}+06$ & $1.67 \mathrm{E}+05$ & $4.50 \mathrm{E}+06$ & $4.12 \mathrm{E}+03$ & $4.50 \mathrm{E}+06$ \\
\hline $\mathrm{Nb}-95 \mathrm{~m}$ & $1.49 \mathrm{E}+03$ & $4.03 \mathrm{E}+04$ & $1.48 \mathrm{E}+04$ & $4.00 \mathrm{E}+05$ & $4.59 \mathrm{E}+03$ & $1.24 \mathrm{E}+05$ & $5.45 \mathrm{E}+04$ & $1.47 \mathrm{E}+06$ & $1.50 \mathrm{E}+05$ & $4.05 \mathrm{E}+06$ & $3.70 \mathrm{E}+03$ & $4.05 \mathrm{E}+06$ \\
\hline $\mathrm{Nb}-96$ & $8.76 \mathrm{E}+02$ & $2.37 \mathrm{E}+04$ & $8.69 \mathrm{E}+03$ & $2.35 \mathrm{E}+05$ & $2.70 \mathrm{E}+03$ & $7.29 \mathrm{E}+04$ & $3.20 \mathrm{E}+04$ & $8.65 \mathrm{E}+05$ & $8.80 \mathrm{E}+04$ & $2.38 \mathrm{E}+06$ & $2.17 \mathrm{E}+03$ & $2.38 \mathrm{E}+06$ \\
\hline $\mathrm{Nb}-97$ & $1.35 \mathrm{E}+04$ & $3.66 \mathrm{E}+05$ & $1.34 \mathrm{E}+05$ & $3.63 \mathrm{E}+06$ & $4.17 \mathrm{E}+04$ & $1.13 \mathrm{E}+06$ & $4.95 \mathrm{E}+05$ & $1.34 \mathrm{E}+07$ & $1.36 \mathrm{E}+06$ & $3.68 \mathrm{E}+07$ & $3.36 \mathrm{E}+04$ & $3.68 \mathrm{E}+07$ \\
\hline $\mathrm{Nb}-98 \mathrm{~m}$ & $8.76 \mathrm{E}+03$ & $2.37 \mathrm{E}+05$ & $8.69 \mathrm{E}+04$ & $2.35 \mathrm{E}+06$ & $2.70 \mathrm{E}+04$ & $7.29 \mathrm{E}+05$ & $3.20 \mathrm{E}+05$ & $8.65 \mathrm{E}+06$ & $8.80 \mathrm{E}+05$ & $2.38 \mathrm{E}+07$ & $2.17 \mathrm{E}+04$ & $2.38 \mathrm{E}+07$ \\
\hline Mo-90 & $4.49 \mathrm{E}+03$ & $1.21 \mathrm{E}+05$ & $4.46 \mathrm{E}+04$ & $1.20 \mathrm{E}+06$ & $1.38 \mathrm{E}+04$ & $3.74 \mathrm{E}+05$ & $1.64 \mathrm{E}+05$ & $4.44 \mathrm{E}+06$ & $4.51 \mathrm{E}+05$ & $1.22 \mathrm{E}+07$ & $1.11 \mathrm{E}+04$ & $1.22 \mathrm{E}+07$ \\
\hline Mo-91 & $1.55 \mathrm{E}+04$ & $4.19 \mathrm{E}+05$ & $1.54 \mathrm{E}+05$ & $4.16 \mathrm{E}+06$ & $4.77 \mathrm{E}+04$ & $1.29 \mathrm{E}+06$ & $5.66 \mathrm{E}+05$ & $1.53 \mathrm{E}+07$ & $1.56 \mathrm{E}+06$ & $4.21 \mathrm{E}+07$ & $3.84 \mathrm{E}+04$ & $4.21 \mathrm{E}+07$ \\
\hline Mo-93 & $3.99 \mathrm{E}+02$ & $1.08 \mathrm{E}+04$ & $3.96 \mathrm{E}+03$ & $1.07 \mathrm{E}+05$ & $1.23 \mathrm{E}+03$ & $3.32 \mathrm{E}+04$ & $1.46 \mathrm{E}+04$ & $3.94 \mathrm{E}+05$ & $4.01 \mathrm{E}+04$ & $1.08 \mathrm{E}+06$ & $9.89 \mathrm{E}+02$ & $1.08 \mathrm{E}+06$ \\
\hline Mo-93m & $8.46 \mathrm{E}+03$ & $2.29 \mathrm{E}+05$ & $8.40 \mathrm{E}+04$ & $2.27 \mathrm{E}+06$ & $2.61 \mathrm{E}+04$ & $7.04 \mathrm{E}+05$ & $3.09 \mathrm{E}+05$ & $8.36 \mathrm{E}+06$ & $8.50 \mathrm{E}+05$ & $2.30 \mathrm{E}+07$ & $2.10 \mathrm{E}+04$ & $2.30 \mathrm{E}+07$ \\
\hline Mo-99 & $1.61 \mathrm{E}+03$ & $4.35 \mathrm{E}+04$ & $1.60 \mathrm{E}+04$ & $4.32 \mathrm{E}+05$ & $4.96 \mathrm{E}+03$ & $1.34 \mathrm{E}+05$ & $5.88 \mathrm{E}+04$ & $1.59 \mathrm{E}+06$ & $1.62 \mathrm{E}+05$ & $4.37 \mathrm{E}+06$ & $3.99 \mathrm{E}+03$ & $4.37 \mathrm{E}+06$ \\
\hline Mo-101 & $2.33 \mathrm{E}+04$ & $6.31 \mathrm{E}+05$ & $2.32 \mathrm{E}+05$ & $6.26 \mathrm{E}+06$ & $7.19 \mathrm{E}+04$ & $1.94 \mathrm{E}+06$ & $8.53 \mathrm{E}+05$ & $2.30 \mathrm{E}+07$ & $2.35 \mathrm{E}+06$ & $6.34 \mathrm{E}+07$ & $5.79 \mathrm{E}+04$ & $6.34 \mathrm{E}+07$ \\
\hline Mo-102 & $1.33 \mathrm{E}+04$ & $3.61 \mathrm{E}+05$ & $1.32 \mathrm{E}+05$ & $3.58 \mathrm{E}+06$ & $4.11 \mathrm{E}+04$ & $1.11 \mathrm{E}+06$ & $4.88 \mathrm{E}+05$ & $1.32 \mathrm{E}+07$ & $1.34 \mathrm{E}+06$ & $3.62 \mathrm{E}+07$ & $3.31 \mathrm{E}+04$ & $3.62 \mathrm{E}+07$ \\
\hline Tc-93 & $1.41 \mathrm{E}+04$ & $3.80 \mathrm{E}+05$ & $1.40 \mathrm{E}+05$ & $3.77 \mathrm{E}+06$ & $4.33 \mathrm{E}+04$ & $1.17 \mathrm{E}+06$ & $5.14 \mathrm{E}+05$ & $1.39 \mathrm{E}+07$ & $1.41 \mathrm{E}+06$ & $3.82 \mathrm{E}+07$ & $3.49 \mathrm{E}+04$ & $3.82 \mathrm{E}+07$ \\
\hline Tc-93m & $3.03 \mathrm{E}+04$ & $8.18 \mathrm{E}+05$ & $3.00 \mathrm{E}+05$ & $8.12 \mathrm{E}+06$ & $9.32 \mathrm{E}+04$ & $2.52 \mathrm{E}+06$ & $1.11 \mathrm{E}+06$ & $2.99 \mathrm{E}+07$ & $3.04 \mathrm{E}+06$ & $8.22 \mathrm{E}+07$ & $7.51 \mathrm{E}+04$ & $8.22 \mathrm{E}+07$ \\
\hline Tc-94 & $4.92 \mathrm{E}+03$ & $1.33 \mathrm{E}+05$ & $4.88 \mathrm{E}+04$ & $1.32 \mathrm{E}+06$ & $1.51 \mathrm{E}+04$ & $4.09 \mathrm{E}+05$ & $1.80 \mathrm{E}+05$ & $4.86 \mathrm{E}+06$ & $4.94 \mathrm{E}+05$ & $1.34 \mathrm{E}+07$ & $1.22 \mathrm{E}+04$ & $1.34 \mathrm{E}+07$ \\
\hline Tc-94m & $9.15 \mathrm{E}+03$ & $2.47 \mathrm{E}+05$ & $9.08 \mathrm{E}+04$ & $2.45 \mathrm{E}+06$ & $2.82 \mathrm{E}+04$ & $7.61 \mathrm{E}+05$ & $3.34 \mathrm{E}+05$ & $9.03 \mathrm{E}+06$ & $9.19 \mathrm{E}+05$ & $2.48 \mathrm{E}+07$ & $2.27 \mathrm{E}+04$ & $2.48 \mathrm{E}+07$ \\
\hline Tc-95 & $5.50 \mathrm{E}+03$ & $1.49 \mathrm{E}+05$ & $5.46 \mathrm{E}+04$ & $1.48 \mathrm{E}+06$ & $1.70 \mathrm{E}+04$ & $4.58 \mathrm{E}+05$ & $2.01 \mathrm{E}+05$ & $5.44 \mathrm{E}+06$ & $5.53 \mathrm{E}+05$ & $1.49 \mathrm{E}+07$ & $1.37 \mathrm{E}+04$ & $1.49 \mathrm{E}+07$ \\
\hline Tc-95m & $1.73 \mathrm{E}+03$ & $4.68 \mathrm{E}+04$ & $1.72 \mathrm{E}+04$ & $4.64 \mathrm{E}+05$ & $5.33 \mathrm{E}+03$ & $1.44 \mathrm{E}+05$ & $6.32 \mathrm{E}+04$ & $1.71 \mathrm{E}+06$ & $1.74 \mathrm{E}+05$ & $4.70 \mathrm{E}+06$ & $4.29 \mathrm{E}+03$ & $4.70 \mathrm{E}+06$ \\
\hline Tc-96 & $9.01 \mathrm{E}+02$ & $2.44 \mathrm{E}+04$ & $8.95 \mathrm{E}+03$ & $2.42 \mathrm{E}+05$ & $2.78 \mathrm{E}+03$ & $7.50 \mathrm{E}+04$ & $3.29 \mathrm{E}+04$ & $8.90 \mathrm{E}+05$ & $9.06 \mathrm{E}+04$ & $2.45 \mathrm{E}+06$ & $2.24 \mathrm{E}+03$ & $2.45 \mathrm{E}+06$ \\
\hline Tc-96m & $7.87 \mathrm{E}+04$ & $2.13 \mathrm{E}+06$ & $7.81 \mathrm{E}+05$ & $2.11 \mathrm{E}+07$ & $2.42 \mathrm{E}+05$ & $6.55 \mathrm{E}+06$ & $2.88 \mathrm{E}+06$ & $7.78 \mathrm{E}+07$ & $7.91 \mathrm{E}+06$ & $2.14 \mathrm{E}+08$ & $1.95 \mathrm{E}+05$ & $2.14 \mathrm{E}+08$ \\
\hline Tc-97 & $1.31 \mathrm{E}+04$ & $3.55 \mathrm{E}+05$ & $1.30 \mathrm{E}+05$ & $3.52 \mathrm{E}+06$ & $4.05 \mathrm{E}+04$ & $1.09 \mathrm{E}+06$ & $4.80 \mathrm{E}+05$ & $1.30 \mathrm{E}+07$ & $1.32 \mathrm{E}+06$ & $3.57 \mathrm{E}+07$ & $3.26 \mathrm{E}+04$ & $3.57 \mathrm{E}+07$ \\
\hline
\end{tabular}

Page 176 of 298 
SRNL-STI-2013-00115

\begin{tabular}{|c|c|c|c|c|c|c|c|c|c|c|c|c|}
\hline \multirow[t]{2}{*}{ Nuclide } & \multicolumn{2}{|c|}{ Water Ingestion DCS } & \multicolumn{2}{|c|}{ Meat Ingestion DCS } & \multicolumn{2}{|c|}{ Dairy Ingestion DCS } & \multicolumn{2}{|c|}{$\begin{array}{c}\text { Freshwater Fish } \\
\text { Ingestion DCS }\end{array}$} & \multicolumn{2}{|c|}{$\begin{array}{l}\text { Saltwater Shellfish } \\
\text { Ingestion DCS }\end{array}$} & \multicolumn{2}{|c|}{ Produce Ingestion DCS } \\
\hline & $(\mathrm{Bq} / \mathrm{L})$ & $(\mathrm{pCi} / \mathrm{L})$ & $(\mathrm{Bq} / \mathrm{kg})$ & $(\mathrm{pCi} / \mathrm{kg})$ & $(\mathrm{Bq} / \mathrm{L})$ & $(\mathrm{pCi} / \mathrm{L})$ & $(\mathrm{Bq} / \mathbf{k g})$ & $(\mathrm{pCi} / \mathbf{k g})$ & $(\mathrm{Bq} / \mathbf{k g})$ & $(\mathrm{pCi} / \mathbf{k g})$ & $(\mathrm{Bq} / \mathbf{k g})$ & $(\mathrm{pCi} / \mathbf{k g})$ \\
\hline Tc-97m & $1.62 \mathrm{E}+03$ & $4.37 \mathrm{E}+04$ & $1.60 \mathrm{E}+04$ & $4.33 \mathrm{E}+05$ & $4.98 \mathrm{E}+03$ & $1.34 \mathrm{E}+05$ & $5.90 \mathrm{E}+04$ & $1.60 \mathrm{E}+06$ & $1.62 \mathrm{E}+05$ & $4.39 \mathrm{E}+06$ & $4.01 \mathrm{E}+03$ & $4.39 \mathrm{E}+06$ \\
\hline Tc-98 & $5.12 \mathrm{E}+02$ & $1.38 \mathrm{E}+04$ & $5.08 \mathrm{E}+03$ & $1.37 \mathrm{E}+05$ & $1.58 \mathrm{E}+03$ & $4.26 \mathrm{E}+04$ & $1.87 \mathrm{E}+04$ & $5.06 \mathrm{E}+05$ & $5.14 \mathrm{E}+04$ & $1.39 \mathrm{E}+06$ & $1.27 \mathrm{E}+03$ & $1.39 \mathrm{E}+06$ \\
\hline Tc-99 & $1.38 \mathrm{E}+03$ & $3.74 \mathrm{E}+04$ & $1.37 \mathrm{E}+04$ & $3.71 \mathrm{E}+05$ & $4.26 \mathrm{E}+03$ & $1.15 \mathrm{E}+05$ & $5.05 \mathrm{E}+04$ & $1.37 \mathrm{E}+06$ & $1.39 \mathrm{E}+05$ & $3.75 \mathrm{E}+06$ & $3.43 \mathrm{E}+03$ & $3.75 \mathrm{E}+06$ \\
\hline Tc-99m & $4.27 \mathrm{E}+04$ & $1.16 \mathrm{E}+06$ & $4.24 \mathrm{E}+05$ & $1.15 \mathrm{E}+07$ & $1.32 \mathrm{E}+05$ & $3.56 \mathrm{E}+06$ & $1.56 \mathrm{E}+06$ & $4.22 \mathrm{E}+07$ & $4.30 \mathrm{E}+06$ & $1.16 \mathrm{E}+08$ & $1.06 \mathrm{E}+05$ & $1.16 \mathrm{E}+08$ \\
\hline Tc-101 & $4.98 \mathrm{E}+04$ & $1.34 \mathrm{E}+06$ & $4.94 \mathrm{E}+05$ & $1.33 \mathrm{E}+07$ & $1.53 \mathrm{E}+05$ & $4.14 \mathrm{E}+06$ & $1.82 \mathrm{E}+06$ & $4.91 \mathrm{E}+07$ & $5.00 \mathrm{E}+06$ & $1.35 \mathrm{E}+08$ & $1.23 \mathrm{E}+05$ & $1.35 \mathrm{E}+08$ \\
\hline Tc-104 & $1.14 \mathrm{E}+04$ & $3.08 \mathrm{E}+05$ & $1.13 \mathrm{E}+05$ & $3.06 \mathrm{E}+06$ & $3.52 \mathrm{E}+04$ & $9.50 \mathrm{E}+05$ & $4.17 \mathrm{E}+05$ & $1.13 \mathrm{E}+07$ & $1.15 \mathrm{E}+06$ & $3.10 \mathrm{E}+07$ & $2.83 \mathrm{E}+04$ & $3.10 \mathrm{E}+07$ \\
\hline Ru-94 & $9.95 \mathrm{E}+03$ & $2.69 \mathrm{E}+05$ & $9.88 \mathrm{E}+04$ & $2.67 \mathrm{E}+06$ & $3.07 \mathrm{E}+04$ & $8.28 \mathrm{E}+05$ & $3.64 \mathrm{E}+05$ & $9.83 \mathrm{E}+06$ & $1.00 \mathrm{E}+06$ & $2.70 \mathrm{E}+07$ & $2.47 \mathrm{E}+04$ & $2.70 \mathrm{E}+07$ \\
\hline Ru-95 & $1.60 \mathrm{E}+04$ & $4.34 \mathrm{E}+05$ & $1.59 \mathrm{E}+05$ & $4.31 \mathrm{E}+06$ & $4.94 \mathrm{E}+04$ & $1.34 \mathrm{E}+06$ & $5.87 \mathrm{E}+05$ & $1.59 \mathrm{E}+07$ & $1.61 \mathrm{E}+06$ & $4.36 \mathrm{E}+07$ & $3.98 \mathrm{E}+04$ & $4.36 \mathrm{E}+07$ \\
\hline $\mathrm{Ru}-97$ & $6.41 \mathrm{E}+03$ & $1.73 \mathrm{E}+05$ & $6.36 \mathrm{E}+04$ & $1.72 \mathrm{E}+06$ & $1.97 \mathrm{E}+04$ & $5.34 \mathrm{E}+05$ & $2.34 \mathrm{E}+05$ & $6.33 \mathrm{E}+06$ & $6.44 \mathrm{E}+05$ & $1.74 \mathrm{E}+07$ & $1.59 \mathrm{E}+04$ & $1.74 \mathrm{E}+07$ \\
\hline Ru-103 & $1.32 \mathrm{E}+03$ & $3.57 \mathrm{E}+04$ & $1.31 \mathrm{E}+04$ & $3.55 \mathrm{E}+05$ & $4.07 \mathrm{E}+03$ & $1.10 \mathrm{E}+05$ & $4.83 \mathrm{E}+04$ & $1.31 \mathrm{E}+06$ & $1.33 \mathrm{E}+05$ & $3.59 \mathrm{E}+06$ & $3.28 \mathrm{E}+03$ & $3.59 \mathrm{E}+06$ \\
\hline Ru-105 & $3.31 \mathrm{E}+03$ & $8.94 \mathrm{E}+04$ & $3.28 \mathrm{E}+04$ & $8.87 \mathrm{E}+05$ & $1.02 \mathrm{E}+04$ & $2.75 \mathrm{E}+05$ & $1.21 \mathrm{E}+05$ & $3.27 \mathrm{E}+06$ & $3.32 \mathrm{E}+05$ & $8.99 \mathrm{E}+06$ & $8.21 \mathrm{E}+03$ & $8.99 \mathrm{E}+06$ \\
\hline Ru-106 & $1.30 \mathrm{E}+02$ & $3.51 \mathrm{E}+03$ & $1.29 \mathrm{E}+03$ & $3.48 \mathrm{E}+04$ & $4.00 \mathrm{E}+02$ & $1.08 \mathrm{E}+04$ & $4.74 \mathrm{E}+03$ & $1.28 \mathrm{E}+05$ & $1.30 \mathrm{E}+04$ & $3.52 \mathrm{E}+05$ & $3.22 \mathrm{E}+02$ & $3.52 \mathrm{E}+05$ \\
\hline Rh-97 & $2.01 \mathrm{E}+04$ & $5.43 \mathrm{E}+05$ & $1.99 \mathrm{E}+05$ & $5.39 \mathrm{E}+06$ & $6.19 \mathrm{E}+04$ & $1.67 \mathrm{E}+06$ & $7.34 \mathrm{E}+05$ & $1.98 \mathrm{E}+07$ & $2.02 \mathrm{E}+06$ & $5.46 \mathrm{E}+07$ & $4.99 \mathrm{E}+04$ & $5.46 \mathrm{E}+07$ \\
\hline $\mathrm{Rh}-97 \mathrm{~m}$ & $2.08 \mathrm{E}+04$ & $5.61 \mathrm{E}+05$ & $2.06 \mathrm{E}+05$ & $5.57 \mathrm{E}+06$ & $6.40 \mathrm{E}+04$ & $1.73 \mathrm{E}+06$ & $7.59 \mathrm{E}+05$ & $2.05 \mathrm{E}+07$ & $2.09 \mathrm{E}+06$ & $5.64 \mathrm{E}+07$ & $5.15 \mathrm{E}+04$ & $5.64 \mathrm{E}+07$ \\
\hline Rh-99 & $1.68 \mathrm{E}+03$ & $4.55 \mathrm{E}+04$ & $1.67 \mathrm{E}+04$ & $4.52 \mathrm{E}+05$ & $5.18 \mathrm{E}+03$ & $1.40 \mathrm{E}+05$ & $6.15 \mathrm{E}+04$ & $1.66 \mathrm{E}+06$ & $1.69 \mathrm{E}+05$ & $4.57 \mathrm{E}+06$ & $4.18 \mathrm{E}+03$ & $4.57 \mathrm{E}+06$ \\
\hline $\mathrm{Rh}-99 \mathrm{~m}$ & $1.47 \mathrm{E}+04$ & $3.97 \mathrm{E}+05$ & $1.46 \mathrm{E}+05$ & $3.94 \mathrm{E}+06$ & $4.53 \mathrm{E}+04$ & $1.22 \mathrm{E}+06$ & $5.37 \mathrm{E}+05$ & $1.45 \mathrm{E}+07$ & $1.48 \mathrm{E}+06$ & $3.99 \mathrm{E}+07$ & $3.65 \mathrm{E}+04$ & $3.99 \mathrm{E}+07$ \\
\hline Rh-100 & $1.47 \mathrm{E}+03$ & $3.96 \mathrm{E}+04$ & $1.46 \mathrm{E}+04$ & $3.93 \mathrm{E}+05$ & $4.52 \mathrm{E}+03$ & $1.22 \mathrm{E}+05$ & $5.36 \mathrm{E}+04$ & $1.45 \mathrm{E}+06$ & $1.47 \mathrm{E}+05$ & $3.98 \mathrm{E}+06$ & $3.64 \mathrm{E}+03$ & $3.98 \mathrm{E}+06$ \\
\hline Rh-101 & $1.80 \mathrm{E}+03$ & $4.87 \mathrm{E}+04$ & $1.79 \mathrm{E}+04$ & $4.84 \mathrm{E}+05$ & $5.55 \mathrm{E}+03$ & $1.50 \mathrm{E}+05$ & $6.59 \mathrm{E}+04$ & $1.78 \mathrm{E}+06$ & $1.81 \mathrm{E}+05$ & $4.90 \mathrm{E}+06$ & $4.47 \mathrm{E}+03$ & $4.90 \mathrm{E}+06$ \\
\hline $\mathrm{Rh}-101 \mathrm{~m}$ & $4.57 \mathrm{E}+03$ & $1.24 \mathrm{E}+05$ & $4.54 \mathrm{E}+04$ & $1.23 \mathrm{E}+06$ & $1.41 \mathrm{E}+04$ & $3.81 \mathrm{E}+05$ & $1.67 \mathrm{E}+05$ & $4.52 \mathrm{E}+06$ & $4.60 \mathrm{E}+05$ & $1.24 \mathrm{E}+07$ & $1.13 \mathrm{E}+04$ & $1.24 \mathrm{E}+07$ \\
\hline $\mathrm{Rh}-102$ & $7.82 \mathrm{E}+02$ & $2.11 \mathrm{E}+04$ & $7.76 \mathrm{E}+03$ & $2.10 \mathrm{E}+05$ & $2.41 \mathrm{E}+03$ & $6.51 \mathrm{E}+04$ & $2.86 \mathrm{E}+04$ & $7.73 \mathrm{E}+05$ & $7.86 \mathrm{E}+04$ & $2.12 \mathrm{E}+06$ & $1.94 \mathrm{E}+03$ & $2.12 \mathrm{E}+06$ \\
\hline $\mathrm{Rh}-102 \mathrm{~m}$ & $3.75 \mathrm{E}+02$ & $1.01 \mathrm{E}+04$ & $3.72 \mathrm{E}+03$ & $1.01 \mathrm{E}+05$ & $1.15 \mathrm{E}+03$ & $3.12 \mathrm{E}+04$ & $1.37 \mathrm{E}+04$ & $3.70 \mathrm{E}+05$ & $3.77 \mathrm{E}+04$ & $1.02 \mathrm{E}+06$ & $9.30 \mathrm{E}+02$ & $1.02 \mathrm{E}+06$ \\
\hline $\mathrm{Rh}-103 \mathrm{~m}$ & $2.43 \mathrm{E}+05$ & $6.58 \mathrm{E}+06$ & $2.42 \mathrm{E}+06$ & $6.53 \mathrm{E}+07$ & $7.50 \mathrm{E}+05$ & $2.03 \mathrm{E}+07$ & $8.90 \mathrm{E}+06$ & $2.40 \mathrm{E}+08$ & $2.45 \mathrm{E}+07$ & $6.61 \mathrm{E}+08$ & $6.04 \mathrm{E}+05$ & $6.61 \mathrm{E}+08$ \\
\hline Rh-105 & $2.48 \mathrm{E}+03$ & $6.70 \mathrm{E}+04$ & $2.46 \mathrm{E}+04$ & $6.65 \mathrm{E}+05$ & $7.63 \mathrm{E}+03$ & $2.06 \mathrm{E}+05$ & $9.05 \mathrm{E}+04$ & $2.45 \mathrm{E}+06$ & $2.49 \mathrm{E}+05$ & $6.73 \mathrm{E}+06$ & $6.15 \mathrm{E}+03$ & $6.73 \mathrm{E}+06$ \\
\hline
\end{tabular}

Page 177 of 298 
SRNL-STI-2013-00115

\begin{tabular}{|c|c|c|c|c|c|c|c|c|c|c|c|c|}
\hline \multirow[t]{2}{*}{ Nuclide } & \multicolumn{2}{|c|}{ Water Ingestion DCS } & \multicolumn{2}{|c|}{ Meat Ingestion DCS } & \multicolumn{2}{|c|}{ Dairy Ingestion DCS } & \multicolumn{2}{|c|}{$\begin{array}{l}\text { Freshwater Fish } \\
\text { Ingestion DCS }\end{array}$} & \multicolumn{2}{|c|}{$\begin{array}{l}\text { Saltwater Shellfish } \\
\text { Ingestion DCS }\end{array}$} & \multicolumn{2}{|c|}{ Produce Ingestion DCS } \\
\hline & $(\mathrm{Bq} / \mathrm{L})$ & $(\mathrm{pCi} / \mathrm{L})$ & $(\mathrm{Bq} / \mathrm{kg})$ & $(\mathrm{pCi} / \mathrm{kg})$ & $(\mathrm{Bq} / \mathrm{L})$ & $(\mathrm{pCi} / \mathrm{L})$ & $(\mathrm{Bq} / \mathbf{k g})$ & $(\mathrm{pCi} / \mathbf{k g})$ & $(\mathrm{Bq} / \mathbf{k g})$ & $(\mathrm{pCi} / \mathbf{k g})$ & $(\mathrm{Bq} / \mathbf{k g})$ & $(\mathrm{pCi} / \mathbf{k g})$ \\
\hline $\mathrm{Rh}-106 \mathrm{~m}$ & $5.71 \mathrm{E}+03$ & $1.54 \mathrm{E}+05$ & $5.66 \mathrm{E}+04$ & $1.53 \mathrm{E}+06$ & $1.76 \mathrm{E}+04$ & $4.75 \mathrm{E}+05$ & $2.09 \mathrm{E}+05$ & $5.64 \mathrm{E}+06$ & $5.73 \mathrm{E}+05$ & $1.55 \mathrm{E}+07$ & $1.42 \mathrm{E}+04$ & $1.55 \mathrm{E}+07$ \\
\hline $\mathrm{Rh}-107$ & $3.89 \mathrm{E}+04$ & $1.05 \mathrm{E}+06$ & $3.86 \mathrm{E}+05$ & $1.04 \mathrm{E}+07$ & $1.20 \mathrm{E}+05$ & $3.24 \mathrm{E}+06$ & $1.42 \mathrm{E}+06$ & $3.84 \mathrm{E}+07$ & $3.91 \mathrm{E}+06$ & $1.06 \mathrm{E}+08$ & $9.65 \mathrm{E}+04$ & $1.06 \mathrm{E}+08$ \\
\hline Pd-98 & $1.51 \mathrm{E}+04$ & $4.07 \mathrm{E}+05$ & $1.50 \mathrm{E}+05$ & $4.04 \mathrm{E}+06$ & $4.64 \mathrm{E}+04$ & $1.26 \mathrm{E}+06$ & $5.51 \mathrm{E}+05$ & $1.49 \mathrm{E}+07$ & $1.52 \mathrm{E}+06$ & $4.10 \mathrm{E}+07$ & $3.74 \mathrm{E}+04$ & $4.10 \mathrm{E}+07$ \\
\hline Pd-99 & $2.71 \mathrm{E}+04$ & $7.32 \mathrm{E}+05$ & $2.69 \mathrm{E}+05$ & $7.27 \mathrm{E}+06$ & $8.35 \mathrm{E}+04$ & $2.26 \mathrm{E}+06$ & $9.90 \mathrm{E}+05$ & $2.68 \mathrm{E}+07$ & $2.72 \mathrm{E}+06$ & $7.36 \mathrm{E}+07$ & $6.72 \mathrm{E}+04$ & $7.36 \mathrm{E}+07$ \\
\hline $\mathrm{Pd}-100$ & $1.05 \mathrm{E}+03$ & $2.85 \mathrm{E}+04$ & $1.05 \mathrm{E}+04$ & $2.83 \mathrm{E}+05$ & $3.25 \mathrm{E}+03$ & $8.78 \mathrm{E}+04$ & $3.85 \mathrm{E}+04$ & $1.04 \mathrm{E}+06$ & $1.06 \mathrm{E}+05$ & $2.86 \mathrm{E}+06$ & $2.62 \mathrm{E}+03$ & $2.86 \mathrm{E}+06$ \\
\hline Pd-101 & $1.02 \mathrm{E}+04$ & $2.76 \mathrm{E}+05$ & $1.01 \mathrm{E}+05$ & $2.73 \mathrm{E}+06$ & $3.14 \mathrm{E}+04$ & $8.49 \mathrm{E}+05$ & $3.73 \mathrm{E}+05$ & $1.01 \mathrm{E}+07$ & $1.02 \mathrm{E}+06$ & $2.77 \mathrm{E}+07$ & $2.53 \mathrm{E}+04$ & $2.77 \mathrm{E}+07$ \\
\hline $\mathrm{Pd}-103$ & $4.73 \mathrm{E}+03$ & $1.28 \mathrm{E}+05$ & $4.69 \mathrm{E}+04$ & $1.27 \mathrm{E}+06$ & $1.46 \mathrm{E}+04$ & $3.94 \mathrm{E}+05$ & $1.73 \mathrm{E}+05$ & $4.67 \mathrm{E}+06$ & $4.75 \mathrm{E}+05$ & $1.28 \mathrm{E}+07$ & $1.17 \mathrm{E}+04$ & $1.28 \mathrm{E}+07$ \\
\hline Pd-107 & $2.35 \mathrm{E}+04$ & $6.35 \mathrm{E}+05$ & $2.33 \mathrm{E}+05$ & $6.31 \mathrm{E}+06$ & $7.24 \mathrm{E}+04$ & $1.96 \mathrm{E}+06$ & $8.59 \mathrm{E}+05$ & $2.32 \mathrm{E}+07$ & $2.36 \mathrm{E}+06$ & $6.39 \mathrm{E}+07$ & $5.83 \mathrm{E}+04$ & $6.39 \mathrm{E}+07$ \\
\hline Pd-109 & $1.62 \mathrm{E}+03$ & $4.37 \mathrm{E}+04$ & $1.61 \mathrm{E}+04$ & $4.34 \mathrm{E}+05$ & $4.98 \mathrm{E}+03$ & $1.35 \mathrm{E}+05$ & $5.91 \mathrm{E}+04$ & $1.60 \mathrm{E}+06$ & $1.63 \mathrm{E}+05$ & $4.39 \mathrm{E}+06$ & $4.01 \mathrm{E}+03$ & $4.39 \mathrm{E}+06$ \\
\hline Pd-111 & $1.84 \mathrm{E}+04$ & $4.98 \mathrm{E}+05$ & $1.83 \mathrm{E}+05$ & $4.94 \mathrm{E}+06$ & $5.68 \mathrm{E}+04$ & $1.53 \mathrm{E}+06$ & $6.73 \mathrm{E}+05$ & $1.82 \mathrm{E}+07$ & $1.85 \mathrm{E}+06$ & $5.01 \mathrm{E}+07$ & $4.57 \mathrm{E}+04$ & $5.01 \mathrm{E}+07$ \\
\hline Pd-112 & $3.51 \mathrm{E}+02$ & $9.50 \mathrm{E}+03$ & $3.49 \mathrm{E}+03$ & $9.43 \mathrm{E}+04$ & $1.08 \mathrm{E}+03$ & $2.93 \mathrm{E}+04$ & $1.28 \mathrm{E}+04$ & $3.47 \mathrm{E}+05$ & $3.53 \mathrm{E}+04$ & $9.54 \mathrm{E}+05$ & $8.72 \mathrm{E}+02$ & $9.54 \mathrm{E}+05$ \\
\hline Ag-101 & $2.91 \mathrm{E}+04$ & $7.87 \mathrm{E}+05$ & $2.89 \mathrm{E}+05$ & $7.81 \mathrm{E}+06$ & $8.97 \mathrm{E}+04$ & $2.43 \mathrm{E}+06$ & $1.06 \mathrm{E}+06$ & $2.88 \mathrm{E}+07$ & $2.93 \mathrm{E}+06$ & $7.91 \mathrm{E}+07$ & $7.23 \mathrm{E}+04$ & $7.91 \mathrm{E}+07$ \\
\hline Ag-102 & $2.32 \mathrm{E}+04$ & $6.27 \mathrm{E}+05$ & $2.30 \mathrm{E}+05$ & $6.23 \mathrm{E}+06$ & $7.15 \mathrm{E}+04$ & $1.93 \mathrm{E}+06$ & $8.48 \mathrm{E}+05$ & $2.29 \mathrm{E}+07$ & $2.33 \mathrm{E}+06$ & $6.30 \mathrm{E}+07$ & $5.76 \mathrm{E}+04$ & $6.30 \mathrm{E}+07$ \\
\hline Ag-103 & $2.51 \mathrm{E}+04$ & $6.78 \mathrm{E}+05$ & $2.49 \mathrm{E}+05$ & $6.73 \mathrm{E}+06$ & $7.72 \mathrm{E}+04$ & $2.09 \mathrm{E}+06$ & $9.16 \mathrm{E}+05$ & $2.48 \mathrm{E}+07$ & $2.52 \mathrm{E}+06$ & $6.81 \mathrm{E}+07$ & $6.22 \mathrm{E}+04$ & $6.81 \mathrm{E}+07$ \\
\hline Ag-104 & $1.63 \mathrm{E}+04$ & $4.41 \mathrm{E}+05$ & $1.62 \mathrm{E}+05$ & $4.37 \mathrm{E}+06$ & $5.02 \mathrm{E}+04$ & $1.36 \mathrm{E}+06$ & $5.96 \mathrm{E}+05$ & $1.61 \mathrm{E}+07$ & $1.64 \mathrm{E}+06$ & $4.43 \mathrm{E}+07$ & $4.05 \mathrm{E}+04$ & $4.43 \mathrm{E}+07$ \\
\hline $\mathrm{Ag}-104 \mathrm{~m}$ & $1.42 \mathrm{E}+04$ & $3.85 \mathrm{E}+05$ & $1.41 \mathrm{E}+05$ & $3.82 \mathrm{E}+06$ & $4.38 \mathrm{E}+04$ & $1.18 \mathrm{E}+06$ & $5.20 \mathrm{E}+05$ & $1.41 \mathrm{E}+07$ & $1.43 \mathrm{E}+06$ & $3.87 \mathrm{E}+07$ & $3.53 \mathrm{E}+04$ & $3.87 \mathrm{E}+07$ \\
\hline Ag-105 & $2.10 \mathrm{E}+03$ & $5.69 \mathrm{E}+04$ & $2.09 \mathrm{E}+04$ & $5.65 \mathrm{E}+05$ & $6.48 \mathrm{E}+03$ & $1.75 \mathrm{E}+05$ & $7.69 \mathrm{E}+04$ & $2.08 \mathrm{E}+06$ & $2.12 \mathrm{E}+05$ & $5.72 \mathrm{E}+06$ & $5.22 \mathrm{E}+03$ & $5.72 \mathrm{E}+06$ \\
\hline Ag-106 & $2.95 \mathrm{E}+04$ & $7.98 \mathrm{E}+05$ & $2.93 \mathrm{E}+05$ & $7.93 \mathrm{E}+06$ & $9.10 \mathrm{E}+04$ & $2.46 \mathrm{E}+06$ & $1.08 \mathrm{E}+06$ & $2.92 \mathrm{E}+07$ & $2.97 \mathrm{E}+06$ & $8.02 \mathrm{E}+07$ & $7.33 \mathrm{E}+04$ & $8.02 \mathrm{E}+07$ \\
\hline Ag-106m & $6.80 \mathrm{E}+02$ & $1.84 \mathrm{E}+04$ & $6.75 \mathrm{E}+03$ & $1.82 \mathrm{E}+05$ & $2.09 \mathrm{E}+03$ & $5.66 \mathrm{E}+04$ & $2.48 \mathrm{E}+04$ & $6.71 \mathrm{E}+05$ & $6.83 \mathrm{E}+04$ & $1.85 \mathrm{E}+06$ & $1.69 \mathrm{E}+03$ & $1.85 \mathrm{E}+06$ \\
\hline $\mathrm{Ag}-108 \mathrm{~m}$ & $4.22 \mathrm{E}+02$ & $1.14 \mathrm{E}+04$ & $4.18 \mathrm{E}+03$ & $1.13 \mathrm{E}+05$ & $1.30 \mathrm{E}+03$ & $3.51 \mathrm{E}+04$ & $1.54 \mathrm{E}+04$ & $4.16 \mathrm{E}+05$ & $4.24 \mathrm{E}+04$ & $1.15 \mathrm{E}+06$ & $1.05 \mathrm{E}+03$ & $1.15 \mathrm{E}+06$ \\
\hline $\mathrm{Ag}-110 \mathrm{~m}$ & $3.50 \mathrm{E}+02$ & $9.47 \mathrm{E}+03$ & $3.48 \mathrm{E}+03$ & $9.40 \mathrm{E}+04$ & $1.08 \mathrm{E}+03$ & $2.92 \mathrm{E}+04$ & $1.28 \mathrm{E}+04$ & $3.46 \mathrm{E}+05$ & $3.52 \mathrm{E}+04$ & $9.52 \mathrm{E}+05$ & $8.69 \mathrm{E}+02$ & $9.52 \mathrm{E}+05$ \\
\hline $\mathrm{Ag}-111$ & $7.19 \mathrm{E}+02$ & $1.94 \mathrm{E}+04$ & $7.14 \mathrm{E}+03$ & $1.93 \mathrm{E}+05$ & $2.21 \mathrm{E}+03$ & $5.99 \mathrm{E}+04$ & $2.63 \mathrm{E}+04$ & $7.10 \mathrm{E}+05$ & $7.23 \mathrm{E}+04$ & $1.95 \mathrm{E}+06$ & $1.78 \mathrm{E}+03$ & $1.95 \mathrm{E}+06$ \\
\hline $\mathrm{Ag}-112$ & $2.17 \mathrm{E}+03$ & $5.87 \mathrm{E}+04$ & $2.15 \mathrm{E}+04$ & $5.82 \mathrm{E}+05$ & $6.69 \mathrm{E}+03$ & $1.81 \mathrm{E}+05$ & $7.93 \mathrm{E}+04$ & $2.14 \mathrm{E}+06$ & $2.18 \mathrm{E}+05$ & $5.90 \mathrm{E}+06$ & $5.39 \mathrm{E}+03$ & $5.90 \mathrm{E}+06$ \\
\hline
\end{tabular}

Page 178 of 298 
SRNL-STI-2013-00115

\begin{tabular}{|c|c|c|c|c|c|c|c|c|c|c|c|c|}
\hline \multirow[t]{2}{*}{ Nuclide } & \multicolumn{2}{|c|}{ Water Ingestion DCS } & \multicolumn{2}{|c|}{ Meat Ingestion DCS } & \multicolumn{2}{|c|}{ Dairy Ingestion DCS } & \multicolumn{2}{|c|}{$\begin{array}{c}\text { Freshwater Fish } \\
\text { Ingestion DCS }\end{array}$} & \multicolumn{2}{|c|}{$\begin{array}{l}\text { Saltwater Shellfish } \\
\text { Ingestion DCS }\end{array}$} & \multicolumn{2}{|c|}{ Produce Ingestion DCS } \\
\hline & $(\mathrm{Bq} / \mathrm{L})$ & $(\mathrm{pCi} / \mathrm{L})$ & $(\mathrm{Bq} / \mathrm{kg})$ & $(\mathrm{pCi} / \mathrm{kg})$ & $(\mathrm{Bq} / \mathrm{L})$ & $(\mathrm{pCi} / \mathrm{L})$ & $(\mathrm{Bq} / \mathbf{k g})$ & $(\mathrm{pCi} / \mathbf{k g})$ & $(\mathrm{Bq} / \mathbf{k g})$ & $(\mathrm{pCi} / \mathbf{k g})$ & $(\mathrm{Bq} / \mathbf{k g})$ & $(\mathrm{pCi} / \mathbf{k g})$ \\
\hline Ag-113 & $2.30 \mathrm{E}+03$ & $6.23 \mathrm{E}+04$ & $2.29 \mathrm{E}+04$ & $6.18 \mathrm{E}+05$ & $7.10 \mathrm{E}+03$ & $1.92 \mathrm{E}+05$ & $8.42 \mathrm{E}+04$ & $2.28 \mathrm{E}+06$ & $2.31 \mathrm{E}+05$ & $6.26 \mathrm{E}+06$ & $5.72 \mathrm{E}+03$ & $6.26 \mathrm{E}+06$ \\
\hline Ag-115 & $1.50 \mathrm{E}+04$ & $4.05 \mathrm{E}+05$ & $1.49 \mathrm{E}+05$ & $4.02 \mathrm{E}+06$ & $4.62 \mathrm{E}+04$ & $1.25 \mathrm{E}+06$ & $5.48 \mathrm{E}+05$ & $1.48 \mathrm{E}+07$ & $1.51 \mathrm{E}+06$ & $4.07 \mathrm{E}+07$ & $3.72 \mathrm{E}+04$ & $4.07 \mathrm{E}+07$ \\
\hline $\mathrm{Cd}-104$ & $9.28 \mathrm{E}+03$ & $2.51 \mathrm{E}+05$ & $9.21 \mathrm{E}+04$ & $2.49 \mathrm{E}+06$ & $2.86 \mathrm{E}+04$ & $7.73 \mathrm{E}+05$ & $3.39 \mathrm{E}+05$ & $9.17 \mathrm{E}+06$ & $9.33 \mathrm{E}+05$ & $2.52 \mathrm{E}+07$ & $2.30 \mathrm{E}+04$ & $2.52 \mathrm{E}+07$ \\
\hline Cd-105 & $2.27 \mathrm{E}+04$ & $6.15 \mathrm{E}+05$ & $2.26 \mathrm{E}+05$ & $6.10 \mathrm{E}+06$ & $7.00 \mathrm{E}+04$ & $1.89 \mathrm{E}+06$ & $8.31 \mathrm{E}+05$ & $2.25 \mathrm{E}+07$ & $2.29 \mathrm{E}+06$ & $6.18 \mathrm{E}+07$ & $5.64 \mathrm{E}+04$ & $6.18 \mathrm{E}+07$ \\
\hline $\mathrm{Cd}-107$ & $1.45 \mathrm{E}+04$ & $3.91 \mathrm{E}+05$ & $1.44 \mathrm{E}+05$ & $3.88 \mathrm{E}+06$ & $4.46 \mathrm{E}+04$ & $1.21 \mathrm{E}+06$ & $5.29 \mathrm{E}+05$ & $1.43 \mathrm{E}+07$ & $1.46 \mathrm{E}+06$ & $3.93 \mathrm{E}+07$ & $3.59 \mathrm{E}+04$ & $3.93 \mathrm{E}+07$ \\
\hline Cd-109 & $4.94 \mathrm{E}+02$ & $1.33 \mathrm{E}+04$ & $4.90 \mathrm{E}+03$ & $1.32 \mathrm{E}+05$ & $1.52 \mathrm{E}+03$ & $4.11 \mathrm{E}+04$ & $1.80 \mathrm{E}+04$ & $4.88 \mathrm{E}+05$ & $4.96 \mathrm{E}+04$ & $1.34 \mathrm{E}+06$ & $1.22 \mathrm{E}+03$ & $1.34 \mathrm{E}+06$ \\
\hline $\mathrm{Cd}-111 \mathrm{~m}$ & $6.87 \mathrm{E}+04$ & $1.86 \mathrm{E}+06$ & $6.82 \mathrm{E}+05$ & $1.84 \mathrm{E}+07$ & $2.12 \mathrm{E}+05$ & $5.72 \mathrm{E}+06$ & $2.51 \mathrm{E}+06$ & $6.79 \mathrm{E}+07$ & $6.91 \mathrm{E}+06$ & $1.87 \mathrm{E}+08$ & $1.71 \mathrm{E}+05$ & $1.87 \mathrm{E}+08$ \\
\hline Cd-113 & $4.73 \mathrm{E}+01$ & $1.28 \mathrm{E}+03$ & $4.69 \mathrm{E}+02$ & $1.27 \mathrm{E}+04$ & $1.46 \mathrm{E}+02$ & $3.94 \mathrm{E}+03$ & $1.73 \mathrm{E}+03$ & $4.67 \mathrm{E}+04$ & $4.75 \mathrm{E}+03$ & $1.28 \mathrm{E}+05$ & $1.17 \mathrm{E}+02$ & $1.28 \mathrm{E}+05$ \\
\hline $\mathrm{Cd}-113 \mathrm{~m}$ & $4.84 \mathrm{E}+01$ & $1.31 \mathrm{E}+03$ & $4.80 \mathrm{E}+02$ & $1.30 \mathrm{E}+04$ & $1.49 \mathrm{E}+02$ & $4.03 \mathrm{E}+03$ & $1.77 \mathrm{E}+03$ & $4.78 \mathrm{E}+04$ & $4.86 \mathrm{E}+03$ & $1.31 \mathrm{E}+05$ & $1.20 \mathrm{E}+02$ & $1.31 \mathrm{E}+05$ \\
\hline $\mathrm{Cd}-115$ & $6.55 \mathrm{E}+02$ & $1.77 \mathrm{E}+04$ & $6.50 \mathrm{E}+03$ & $1.76 \mathrm{E}+05$ & $2.02 \mathrm{E}+03$ & $5.45 \mathrm{E}+04$ & $2.39 \mathrm{E}+04$ & $6.47 \mathrm{E}+05$ & $6.58 \mathrm{E}+04$ & $1.78 \mathrm{E}+06$ & $1.62 \mathrm{E}+03$ & $1.78 \mathrm{E}+06$ \\
\hline $\mathrm{Cd}-115 \mathrm{~m}$ & $2.86 \mathrm{E}+02$ & $7.73 \mathrm{E}+03$ & $2.84 \mathrm{E}+03$ & $7.67 \mathrm{E}+04$ & $8.81 \mathrm{E}+02$ & $2.38 \mathrm{E}+04$ & $1.04 \mathrm{E}+04$ & $2.82 \mathrm{E}+05$ & $2.87 \mathrm{E}+04$ & $7.77 \mathrm{E}+05$ & $7.10 \mathrm{E}+02$ & $7.77 \mathrm{E}+05$ \\
\hline Cd-117 & $3.33 \mathrm{E}+03$ & $9.01 \mathrm{E}+04$ & $3.31 \mathrm{E}+04$ & $8.95 \mathrm{E}+05$ & $1.03 \mathrm{E}+04$ & $2.78 \mathrm{E}+05$ & $1.22 \mathrm{E}+05$ & $3.29 \mathrm{E}+06$ & $3.35 \mathrm{E}+05$ & $9.06 \mathrm{E}+06$ & $8.27 \mathrm{E}+03$ & $9.06 \mathrm{E}+06$ \\
\hline $\mathrm{Cd}-117 \mathrm{~m}$ & $3.35 \mathrm{E}+03$ & $9.06 \mathrm{E}+04$ & $3.33 \mathrm{E}+04$ & $8.99 \mathrm{E}+05$ & $1.03 \mathrm{E}+04$ & $2.79 \mathrm{E}+05$ & $1.23 \mathrm{E}+05$ & $3.31 \mathrm{E}+06$ & $3.37 \mathrm{E}+05$ & $9.11 \mathrm{E}+06$ & $8.32 \mathrm{E}+03$ & $9.11 \mathrm{E}+06$ \\
\hline Cd-118 & $4.98 \mathrm{E}+03$ & $1.34 \mathrm{E}+05$ & $4.94 \mathrm{E}+04$ & $1.33 \mathrm{E}+06$ & $1.53 \mathrm{E}+04$ & $4.14 \mathrm{E}+05$ & $1.82 \mathrm{E}+05$ & $4.91 \mathrm{E}+06$ & $5.00 \mathrm{E}+05$ & $1.35 \mathrm{E}+07$ & $1.23 \mathrm{E}+04$ & $1.35 \mathrm{E}+07$ \\
\hline In-107 & $2.35 \mathrm{E}+04$ & $6.35 \mathrm{E}+05$ & $2.33 \mathrm{E}+05$ & $6.31 \mathrm{E}+06$ & $7.24 \mathrm{E}+04$ & $1.96 \mathrm{E}+06$ & $8.59 \mathrm{E}+05$ & $2.32 \mathrm{E}+07$ & $2.36 \mathrm{E}+06$ & $6.39 \mathrm{E}+07$ & $5.83 \mathrm{E}+04$ & $6.39 \mathrm{E}+07$ \\
\hline In-108 & $1.28 \mathrm{E}+04$ & $3.46 \mathrm{E}+05$ & $1.27 \mathrm{E}+05$ & $3.43 \mathrm{E}+06$ & $3.94 \mathrm{E}+04$ & $1.07 \mathrm{E}+06$ & $4.68 \mathrm{E}+05$ & $1.26 \mathrm{E}+07$ & $1.29 \mathrm{E}+06$ & $3.48 \mathrm{E}+07$ & $3.18 \mathrm{E}+04$ & $3.48 \mathrm{E}+07$ \\
\hline In- $108 \mathrm{~m}$ & $1.17 \mathrm{E}+04$ & $3.17 \mathrm{E}+05$ & $1.16 \mathrm{E}+05$ & $3.15 \mathrm{E}+06$ & $3.61 \mathrm{E}+04$ & $9.77 \mathrm{E}+05$ & $4.29 \mathrm{E}+05$ & $1.16 \mathrm{E}+07$ & $1.18 \mathrm{E}+06$ & $3.19 \mathrm{E}+07$ & $2.91 \mathrm{E}+04$ & $3.19 \mathrm{E}+07$ \\
\hline In-109 & $1.67 \mathrm{E}+04$ & $4.51 \mathrm{E}+05$ & $1.65 \mathrm{E}+05$ & $4.47 \mathrm{E}+06$ & $5.14 \mathrm{E}+04$ & $1.39 \mathrm{E}+06$ & $6.09 \mathrm{E}+05$ & $1.65 \mathrm{E}+07$ & $1.68 \mathrm{E}+06$ & $4.53 \mathrm{E}+07$ & $4.14 \mathrm{E}+04$ & $4.53 \mathrm{E}+07$ \\
\hline In-110 & $4.10 \mathrm{E}+03$ & $1.11 \mathrm{E}+05$ & $4.07 \mathrm{E}+04$ & $1.10 \mathrm{E}+06$ & $1.26 \mathrm{E}+04$ & $3.42 \mathrm{E}+05$ & $1.50 \mathrm{E}+05$ & $4.05 \mathrm{E}+06$ & $4.13 \mathrm{E}+05$ & $1.11 \mathrm{E}+07$ & $1.02 \mathrm{E}+04$ & $1.11 \mathrm{E}+07$ \\
\hline In- $110 \mathrm{~m}$ & $9.28 \mathrm{E}+03$ & $2.51 \mathrm{E}+05$ & $9.21 \mathrm{E}+04$ & $2.49 \mathrm{E}+06$ & $2.86 \mathrm{E}+04$ & $7.73 \mathrm{E}+05$ & $3.39 \mathrm{E}+05$ & $9.17 \mathrm{E}+06$ & $9.33 \mathrm{E}+05$ & $2.52 \mathrm{E}+07$ & $2.30 \mathrm{E}+04$ & $2.52 \mathrm{E}+07$ \\
\hline In-111 & $3.28 \mathrm{E}+03$ & $8.87 \mathrm{E}+04$ & $3.26 \mathrm{E}+04$ & $8.80 \mathrm{E}+05$ & $1.01 \mathrm{E}+04$ & $2.73 \mathrm{E}+05$ & $1.20 \mathrm{E}+05$ & $3.24 \mathrm{E}+06$ & $3.30 \mathrm{E}+05$ & $8.91 \mathrm{E}+06$ & $8.14 \mathrm{E}+03$ & $8.91 \mathrm{E}+06$ \\
\hline In-112 & $8.88 \mathrm{E}+04$ & $2.40 \mathrm{E}+06$ & $8.82 \mathrm{E}+05$ & $2.38 \mathrm{E}+07$ & $2.74 \mathrm{E}+05$ & $7.40 \mathrm{E}+06$ & $3.25 \mathrm{E}+06$ & $8.78 \mathrm{E}+07$ & $8.93 \mathrm{E}+06$ & $2.41 \mathrm{E}+08$ & $2.20 \mathrm{E}+05$ & $2.41 \mathrm{E}+08$ \\
\hline In-112m & $5.46 \mathrm{E}+04$ & $1.47 \mathrm{E}+06$ & $5.41 \mathrm{E}+05$ & $1.46 \mathrm{E}+07$ & $1.68 \mathrm{E}+05$ & $4.54 \mathrm{E}+06$ & $1.99 \mathrm{E}+06$ & $5.39 \mathrm{E}+07$ & $5.48 \mathrm{E}+06$ & $1.48 \mathrm{E}+08$ & $1.35 \mathrm{E}+05$ & $1.48 \mathrm{E}+08$ \\
\hline
\end{tabular}


SRNL-STI-2013-00115

\begin{tabular}{|c|c|c|c|c|c|c|c|c|c|c|c|c|}
\hline \multirow[t]{2}{*}{ Nuclide } & \multicolumn{2}{|c|}{ Water Ingestion DCS } & \multicolumn{2}{|c|}{ Meat Ingestion DCS } & \multicolumn{2}{|c|}{ Dairy Ingestion DCS } & \multicolumn{2}{|c|}{$\begin{array}{c}\text { Freshwater Fish } \\
\text { Ingestion DCS }\end{array}$} & \multicolumn{2}{|c|}{$\begin{array}{l}\text { Saltwater Shellfish } \\
\text { Ingestion DCS }\end{array}$} & \multicolumn{2}{|c|}{ Produce Ingestion DCS } \\
\hline & $(\mathrm{Bq} / \mathrm{L})$ & $(\mathrm{pCi} / \mathrm{L})$ & $(\mathrm{Bq} / \mathbf{k g})$ & $(\mathrm{pCi} / \mathrm{kg})$ & $(\mathrm{Bq} / \mathrm{L})$ & $(\mathrm{pCi} / \mathrm{L})$ & $(\mathrm{Bq} / \mathbf{k g})$ & $(\mathrm{pCi} / \mathbf{k g})$ & $(\mathrm{Bq} / \mathbf{k g})$ & $(\mathrm{pCi} / \mathbf{k g})$ & $(\mathrm{Bq} / \mathbf{k g})$ & $(\mathrm{pCi} / \mathbf{k g})$ \\
\hline In-113m & $3.17 \mathrm{E}+04$ & $8.58 \mathrm{E}+05$ & $3.15 \mathrm{E}+05$ & $8.51 \mathrm{E}+06$ & $9.77 \mathrm{E}+04$ & $2.64 \mathrm{E}+06$ & $1.16 \mathrm{E}+06$ & $3.13 \mathrm{E}+07$ & $3.19 \mathrm{E}+06$ & $8.62 \mathrm{E}+07$ & $7.87 \mathrm{E}+04$ & $8.62 \mathrm{E}+07$ \\
\hline In- $114 \mathrm{~m}$ & $2.14 \mathrm{E}+02$ & $5.79 \mathrm{E}+03$ & $2.12 \mathrm{E}+03$ & $5.74 \mathrm{E}+04$ & $6.59 \mathrm{E}+02$ & $1.78 \mathrm{E}+04$ & $7.82 \mathrm{E}+03$ & $2.11 \mathrm{E}+05$ & $2.15 \mathrm{E}+04$ & $5.81 \mathrm{E}+05$ & $5.31 \mathrm{E}+02$ & $5.81 \mathrm{E}+05$ \\
\hline In-115 & $3.50 \mathrm{E}+01$ & $9.47 \mathrm{E}+02$ & $3.48 \mathrm{E}+02$ & $9.40 \mathrm{E}+03$ & $1.08 \mathrm{E}+02$ & $2.92 \mathrm{E}+03$ & $1.28 \mathrm{E}+03$ & $3.46 \mathrm{E}+04$ & $3.52 \mathrm{E}+03$ & $9.52 \mathrm{E}+04$ & $8.69 \mathrm{E}+01$ & $9.52 \mathrm{E}+04$ \\
\hline In- $115 \mathrm{~m}$ & $1.05 \mathrm{E}+04$ & $2.82 \mathrm{E}+05$ & $1.04 \mathrm{E}+05$ & $2.80 \mathrm{E}+06$ & $3.22 \mathrm{E}+04$ & $8.70 \mathrm{E}+05$ & $3.82 \mathrm{E}+05$ & $1.03 \mathrm{E}+07$ & $1.05 \mathrm{E}+06$ & $2.84 \mathrm{E}+07$ & $2.59 \mathrm{E}+04$ & $2.84 \mathrm{E}+07$ \\
\hline In-116m & $1.51 \mathrm{E}+04$ & $4.08 \mathrm{E}+05$ & $1.50 \mathrm{E}+05$ & $4.05 \mathrm{E}+06$ & $4.65 \mathrm{E}+04$ & $1.26 \mathrm{E}+06$ & $5.52 \mathrm{E}+05$ & $1.49 \mathrm{E}+07$ & $1.52 \mathrm{E}+06$ & $4.10 \mathrm{E}+07$ & $3.75 \mathrm{E}+04$ & $4.10 \mathrm{E}+07$ \\
\hline In-117 & $3.10 \mathrm{E}+04$ & $8.38 \mathrm{E}+05$ & $3.08 \mathrm{E}+05$ & $8.32 \mathrm{E}+06$ & $9.55 \mathrm{E}+04$ & $2.58 \mathrm{E}+06$ & $1.13 \mathrm{E}+06$ & $3.06 \mathrm{E}+07$ & $3.12 \mathrm{E}+06$ & $8.42 \mathrm{E}+07$ & $7.70 \mathrm{E}+04$ & $8.42 \mathrm{E}+07$ \\
\hline In- $117 \mathrm{~m}$ & $7.40 \mathrm{E}+03$ & $2.00 \mathrm{E}+05$ & $7.35 \mathrm{E}+04$ & $1.99 \mathrm{E}+06$ & $2.28 \mathrm{E}+04$ & $6.16 \mathrm{E}+05$ & $2.71 \mathrm{E}+05$ & $7.31 \mathrm{E}+06$ & $7.44 \mathrm{E}+05$ & $2.01 \mathrm{E}+07$ & $1.84 \mathrm{E}+04$ & $2.01 \mathrm{E}+07$ \\
\hline In-119m & $2.01 \mathrm{E}+04$ & $5.42 \mathrm{E}+05$ & $1.99 \mathrm{E}+05$ & $5.38 \mathrm{E}+06$ & $6.18 \mathrm{E}+04$ & $1.67 \mathrm{E}+06$ & $7.33 \mathrm{E}+05$ & $1.98 \mathrm{E}+07$ & $2.02 \mathrm{E}+06$ & $5.45 \mathrm{E}+07$ & $4.98 \mathrm{E}+04$ & $5.45 \mathrm{E}+07$ \\
\hline Sn-108 & $4.36 \mathrm{E}+04$ & $1.18 \mathrm{E}+06$ & $4.33 \mathrm{E}+05$ & $1.17 \mathrm{E}+07$ & $1.34 \mathrm{E}+05$ & $3.63 \mathrm{E}+06$ & $1.59 \mathrm{E}+06$ & $4.31 \mathrm{E}+07$ & $4.39 \mathrm{E}+06$ & $1.19 \mathrm{E}+08$ & $1.08 \mathrm{E}+05$ & $1.19 \mathrm{E}+08$ \\
\hline Sn-109 & $5.23 \mathrm{E}+04$ & $1.41 \mathrm{E}+06$ & $5.19 \mathrm{E}+05$ & $1.40 \mathrm{E}+07$ & $1.61 \mathrm{E}+05$ & $4.35 \mathrm{E}+06$ & $1.91 \mathrm{E}+06$ & $5.16 \mathrm{E}+07$ & $5.25 \mathrm{E}+06$ & $1.42 \mathrm{E}+08$ & $1.30 \mathrm{E}+05$ & $1.42 \mathrm{E}+08$ \\
\hline Sn-110 & $2.53 \mathrm{E}+03$ & $6.85 \mathrm{E}+04$ & $2.51 \mathrm{E}+04$ & $6.80 \mathrm{E}+05$ & $7.80 \mathrm{E}+03$ & $2.11 \mathrm{E}+05$ & $9.26 \mathrm{E}+04$ & $2.50 \mathrm{E}+06$ & $2.55 \mathrm{E}+05$ & $6.88 \mathrm{E}+06$ & $6.29 \mathrm{E}+03$ & $6.88 \mathrm{E}+06$ \\
\hline Sn-111 & $4.47 \mathrm{E}+04$ & $1.21 \mathrm{E}+06$ & $4.44 \mathrm{E}+05$ & $1.20 \mathrm{E}+07$ & $1.38 \mathrm{E}+05$ & $3.72 \mathrm{E}+06$ & $1.64 \mathrm{E}+06$ & $4.42 \mathrm{E}+07$ & $4.50 \mathrm{E}+06$ & $1.22 \mathrm{E}+08$ & $1.11 \mathrm{E}+05$ & $1.22 \mathrm{E}+08$ \\
\hline Sn-113 & $1.23 \mathrm{E}+03$ & $3.33 \mathrm{E}+04$ & $1.22 \mathrm{E}+04$ & $3.30 \mathrm{E}+05$ & $3.79 \mathrm{E}+03$ & $1.03 \mathrm{E}+05$ & $4.50 \mathrm{E}+04$ & $1.22 \mathrm{E}+06$ & $1.24 \mathrm{E}+05$ & $3.34 \mathrm{E}+06$ & $3.06 \mathrm{E}+03$ & $3.34 \mathrm{E}+06$ \\
\hline Sn-113m & $2.83 \mathrm{E}+05$ & $7.66 \mathrm{E}+06$ & $2.81 \mathrm{E}+06$ & $7.60 \mathrm{E}+07$ & $8.73 \mathrm{E}+05$ & $2.36 \mathrm{E}+07$ & $1.04 \mathrm{E}+07$ & $2.80 \mathrm{E}+08$ & $2.85 \mathrm{E}+07$ & $7.70 \mathrm{E}+08$ & $7.03 \mathrm{E}+05$ & $7.70 \mathrm{E}+08$ \\
\hline Sn-117m & $1.28 \mathrm{E}+03$ & $3.47 \mathrm{E}+04$ & $1.27 \mathrm{E}+04$ & $3.44 \mathrm{E}+05$ & $3.95 \mathrm{E}+03$ & $1.07 \mathrm{E}+05$ & $4.69 \mathrm{E}+04$ & $1.27 \mathrm{E}+06$ & $1.29 \mathrm{E}+05$ & $3.48 \mathrm{E}+06$ & $3.18 \mathrm{E}+03$ & $3.48 \mathrm{E}+06$ \\
\hline Sn-119m & $2.55 \mathrm{E}+03$ & $6.90 \mathrm{E}+04$ & $2.54 \mathrm{E}+04$ & $6.85 \mathrm{E}+05$ & $7.87 \mathrm{E}+03$ & $2.13 \mathrm{E}+05$ & $9.33 \mathrm{E}+04$ & $2.52 \mathrm{E}+06$ & $2.57 \mathrm{E}+05$ & $6.94 \mathrm{E}+06$ & $6.34 \mathrm{E}+03$ & $6.94 \mathrm{E}+06$ \\
\hline Sn-121 & $3.92 \mathrm{E}+03$ & $1.06 \mathrm{E}+05$ & $3.89 \mathrm{E}+04$ & $1.05 \mathrm{E}+06$ & $1.21 \mathrm{E}+04$ & $3.27 \mathrm{E}+05$ & $1.43 \mathrm{E}+05$ & $3.88 \mathrm{E}+06$ & $3.94 \mathrm{E}+05$ & $1.07 \mathrm{E}+07$ & $9.74 \mathrm{E}+03$ & $1.07 \mathrm{E}+07$ \\
\hline Sn-121m & $2.35 \mathrm{E}+03$ & $6.34 \mathrm{E}+04$ & $2.33 \mathrm{E}+04$ & $6.30 \mathrm{E}+05$ & $7.23 \mathrm{E}+03$ & $1.95 \mathrm{E}+05$ & $8.58 \mathrm{E}+04$ & $2.32 \mathrm{E}+06$ & $2.36 \mathrm{E}+05$ & $6.37 \mathrm{E}+06$ & $5.82 \mathrm{E}+03$ & $6.37 \mathrm{E}+06$ \\
\hline Sn-123 & $4.26 \mathrm{E}+02$ & $1.15 \mathrm{E}+04$ & $4.23 \mathrm{E}+03$ & $1.14 \mathrm{E}+05$ & $1.31 \mathrm{E}+03$ & $3.55 \mathrm{E}+04$ & $1.56 \mathrm{E}+04$ & $4.21 \mathrm{E}+05$ & $4.28 \mathrm{E}+04$ & $1.16 \mathrm{E}+06$ & $1.06 \mathrm{E}+03$ & $1.16 \mathrm{E}+06$ \\
\hline Sn-123m & $2.40 \mathrm{E}+04$ & $6.49 \mathrm{E}+05$ & $2.38 \mathrm{E}+05$ & $6.44 \mathrm{E}+06$ & $7.40 \mathrm{E}+04$ & $2.00 \mathrm{E}+06$ & $8.78 \mathrm{E}+05$ & $2.37 \mathrm{E}+07$ & $2.41 \mathrm{E}+06$ & $6.52 \mathrm{E}+07$ & $5.96 \mathrm{E}+04$ & $6.52 \mathrm{E}+07$ \\
\hline Sn-125 & $2.97 \mathrm{E}+02$ & $8.02 \mathrm{E}+03$ & $2.95 \mathrm{E}+03$ & $7.96 \mathrm{E}+04$ & $9.14 \mathrm{E}+02$ & $2.47 \mathrm{E}+04$ & $1.08 \mathrm{E}+04$ & $2.93 \mathrm{E}+05$ & $2.98 \mathrm{E}+04$ & $8.06 \mathrm{E}+05$ & $7.37 \mathrm{E}+02$ & $8.06 \mathrm{E}+05$ \\
\hline Sn-126 & $1.95 \mathrm{E}+02$ & $5.26 \mathrm{E}+03$ & $1.93 \mathrm{E}+03$ & $5.22 \mathrm{E}+04$ & $6.00 \mathrm{E}+02$ & $1.62 \mathrm{E}+04$ & $7.11 \mathrm{E}+03$ & $1.92 \mathrm{E}+05$ & $1.96 \mathrm{E}+04$ & $5.29 \mathrm{E}+05$ & $4.83 \mathrm{E}+02$ & $5.29 \mathrm{E}+05$ \\
\hline Sn-127 & $4.71 \mathrm{E}+03$ & $1.27 \mathrm{E}+05$ & $4.68 \mathrm{E}+04$ & $1.26 \mathrm{E}+06$ & $1.45 \mathrm{E}+04$ & $3.92 \mathrm{E}+05$ & $1.72 \mathrm{E}+05$ & $4.65 \mathrm{E}+06$ & $4.73 \mathrm{E}+05$ & $1.28 \mathrm{E}+07$ & $1.17 \mathrm{E}+04$ & $1.28 \mathrm{E}+07$ \\
\hline
\end{tabular}

Page 180 of 298 
SRNL-STI-2013-00115

\begin{tabular}{|c|c|c|c|c|c|c|c|c|c|c|c|c|}
\hline \multirow[t]{2}{*}{ Nuclide } & \multicolumn{2}{|c|}{ Water Ingestion DCS } & \multicolumn{2}{|c|}{ Meat Ingestion DCS } & \multicolumn{2}{|c|}{ Dairy Ingestion DCS } & \multicolumn{2}{|c|}{$\begin{array}{c}\text { Freshwater Fish } \\
\text { Ingestion DCS }\end{array}$} & \multicolumn{2}{|c|}{$\begin{array}{l}\text { Saltwater Shellfish } \\
\text { Ingestion DCS }\end{array}$} & \multicolumn{2}{|c|}{ Produce Ingestion DCS } \\
\hline & $(\mathrm{Bq} / \mathrm{L})$ & $(\mathrm{pCi} / \mathrm{L})$ & $(\mathrm{Bq} / \mathrm{kg})$ & $(\mathrm{pCi} / \mathrm{kg})$ & $(\mathrm{Bq} / \mathrm{L})$ & $(\mathrm{pCi} / \mathrm{L})$ & $(\mathrm{Bq} / \mathbf{k g})$ & $(\mathrm{pCi} / \mathbf{k g})$ & $(\mathrm{Bq} / \mathbf{k g})$ & $(\mathrm{pCi} / \mathbf{k g})$ & $(\mathrm{Bq} / \mathbf{k g})$ & $(\mathrm{pCi} / \mathbf{k g})$ \\
\hline Sn-128 & $6.10 \mathrm{E}+03$ & $1.65 \mathrm{E}+05$ & $6.05 \mathrm{E}+04$ & $1.64 \mathrm{E}+06$ & $1.88 \mathrm{E}+04$ & $5.08 \mathrm{E}+05$ & $2.23 \mathrm{E}+05$ & $6.02 \mathrm{E}+06$ & $6.13 \mathrm{E}+05$ & $1.66 \mathrm{E}+07$ & $1.51 \mathrm{E}+04$ & $1.66 \mathrm{E}+07$ \\
\hline $\mathrm{Sb}-115$ & $3.95 \mathrm{E}+04$ & $1.07 \mathrm{E}+06$ & $3.92 \mathrm{E}+05$ & $1.06 \mathrm{E}+07$ & $1.22 \mathrm{E}+05$ & $3.29 \mathrm{E}+06$ & $1.44 \mathrm{E}+06$ & $3.90 \mathrm{E}+07$ & $3.97 \mathrm{E}+06$ & $1.07 \mathrm{E}+08$ & $9.80 \mathrm{E}+04$ & $1.07 \mathrm{E}+08$ \\
\hline $\mathrm{Sb}-116$ & $3.14 \mathrm{E}+04$ & $8.49 \mathrm{E}+05$ & $3.12 \mathrm{E}+05$ & $8.43 \mathrm{E}+06$ & $9.68 \mathrm{E}+04$ & $2.61 \mathrm{E}+06$ & $1.15 \mathrm{E}+06$ & $3.10 \mathrm{E}+07$ & $3.16 \mathrm{E}+06$ & $8.53 \mathrm{E}+07$ & $7.79 \mathrm{E}+04$ & $8.53 \mathrm{E}+07$ \\
\hline $\mathrm{Sb}-116 \mathrm{~m}$ & $1.53 \mathrm{E}+04$ & $4.13 \mathrm{E}+05$ & $1.52 \mathrm{E}+05$ & $4.10 \mathrm{E}+06$ & $4.71 \mathrm{E}+04$ & $1.27 \mathrm{E}+06$ & $5.59 \mathrm{E}+05$ & $1.51 \mathrm{E}+07$ & $1.54 \mathrm{E}+06$ & $4.16 \mathrm{E}+07$ & $3.80 \mathrm{E}+04$ & $4.16 \mathrm{E}+07$ \\
\hline Sb-117 & $5.25 \mathrm{E}+04$ & $1.42 \mathrm{E}+06$ & $5.21 \mathrm{E}+05$ & $1.41 \mathrm{E}+07$ & $1.62 \mathrm{E}+05$ & $4.37 \mathrm{E}+06$ & $1.92 \mathrm{E}+06$ & $5.18 \mathrm{E}+07$ & $5.27 \mathrm{E}+06$ & $1.43 \mathrm{E}+08$ & $1.30 \mathrm{E}+05$ & $1.43 \mathrm{E}+08$ \\
\hline Sb-118m & $4.69 \mathrm{E}+03$ & $1.27 \mathrm{E}+05$ & $4.66 \mathrm{E}+04$ & $1.26 \mathrm{E}+06$ & $1.45 \mathrm{E}+04$ & $3.91 \mathrm{E}+05$ & $1.72 \mathrm{E}+05$ & $4.64 \mathrm{E}+06$ & $4.72 \mathrm{E}+05$ & $1.27 \mathrm{E}+07$ & $1.16 \mathrm{E}+04$ & $1.27 \mathrm{E}+07$ \\
\hline $\mathrm{Sb}-119$ & $1.11 \mathrm{E}+04$ & $3.00 \mathrm{E}+05$ & $1.10 \mathrm{E}+05$ & $2.98 \mathrm{E}+06$ & $3.42 \mathrm{E}+04$ & $9.25 \mathrm{E}+05$ & $4.06 \mathrm{E}+05$ & $1.10 \mathrm{E}+07$ & $1.12 \mathrm{E}+06$ & $3.02 \mathrm{E}+07$ & $2.76 \mathrm{E}+04$ & $3.02 \mathrm{E}+07$ \\
\hline $\mathrm{Sb}-120$ & $6.48 \mathrm{E}+04$ & $1.75 \mathrm{E}+06$ & $6.43 \mathrm{E}+05$ & $1.74 \mathrm{E}+07$ & $2.00 \mathrm{E}+05$ & $5.39 \mathrm{E}+06$ & $2.37 \mathrm{E}+06$ & $6.40 \mathrm{E}+07$ & $6.51 \mathrm{E}+06$ & $1.76 \mathrm{E}+08$ & $1.61 \mathrm{E}+05$ & $1.76 \mathrm{E}+08$ \\
\hline $\mathrm{Sb}-120 \mathrm{~m}$ & $7.97 \mathrm{E}+02$ & $2.15 \mathrm{E}+04$ & $7.91 \mathrm{E}+03$ & $2.14 \mathrm{E}+05$ & $2.46 \mathrm{E}+03$ & $6.64 \mathrm{E}+04$ & $2.91 \mathrm{E}+04$ & $7.88 \mathrm{E}+05$ & $8.01 \mathrm{E}+04$ & $2.17 \mathrm{E}+06$ & $1.98 \mathrm{E}+03$ & $2.17 \mathrm{E}+06$ \\
\hline $\mathrm{Sb}-122$ & $5.38 \mathrm{E}+02$ & $1.46 \mathrm{E}+04$ & $5.34 \mathrm{E}+03$ & $1.44 \mathrm{E}+05$ & $1.66 \mathrm{E}+03$ & $4.48 \mathrm{E}+04$ & $1.97 \mathrm{E}+04$ & $5.32 \mathrm{E}+05$ & $5.41 \mathrm{E}+04$ & $1.46 \mathrm{E}+06$ & $1.34 \mathrm{E}+03$ & $1.46 \mathrm{E}+06$ \\
\hline $\mathrm{Sb}-124$ & $3.69 \mathrm{E}+02$ & $9.97 \mathrm{E}+03$ & $3.66 \mathrm{E}+03$ & $9.90 \mathrm{E}+04$ & $1.14 \mathrm{E}+03$ & $3.07 \mathrm{E}+04$ & $1.35 \mathrm{E}+04$ & $3.65 \mathrm{E}+05$ & $3.71 \mathrm{E}+04$ & $1.00 \mathrm{E}+06$ & $9.16 \mathrm{E}+02$ & $1.00 \mathrm{E}+06$ \\
\hline $\mathrm{Sb}-124 \mathrm{n}$ & $9.79 \mathrm{E}+04$ & $2.65 \mathrm{E}+06$ & $9.72 \mathrm{E}+05$ & $2.63 \mathrm{E}+07$ & $3.02 \mathrm{E}+05$ & $8.15 \mathrm{E}+06$ & $3.58 \mathrm{E}+06$ & $9.67 \mathrm{E}+07$ & $9.84 \mathrm{E}+06$ & $2.66 \mathrm{E}+08$ & $2.43 \mathrm{E}+05$ & $2.66 \mathrm{E}+08$ \\
\hline $\mathrm{Sb}-125$ & $8.46 \mathrm{E}+02$ & $2.29 \mathrm{E}+04$ & $8.40 \mathrm{E}+03$ & $2.27 \mathrm{E}+05$ & $2.61 \mathrm{E}+03$ & $7.04 \mathrm{E}+04$ & $3.09 \mathrm{E}+04$ & $8.36 \mathrm{E}+05$ & $8.50 \mathrm{E}+04$ & $2.30 \mathrm{E}+06$ & $2.10 \mathrm{E}+03$ & $2.30 \mathrm{E}+06$ \\
\hline Sb-126 & $3.57 \mathrm{E}+02$ & $9.66 \mathrm{E}+03$ & $3.55 \mathrm{E}+03$ & $9.59 \mathrm{E}+04$ & $1.10 \mathrm{E}+03$ & $2.98 \mathrm{E}+04$ & $1.31 \mathrm{E}+04$ & $3.53 \mathrm{E}+05$ & $3.59 \mathrm{E}+04$ & $9.71 \mathrm{E}+05$ & $8.87 \mathrm{E}+02$ & $9.71 \mathrm{E}+05$ \\
\hline $\mathrm{Sb}-126 \mathrm{~m}$ & $2.49 \mathrm{E}+04$ & $6.72 \mathrm{E}+05$ & $2.47 \mathrm{E}+05$ & $6.67 \mathrm{E}+06$ & $7.66 \mathrm{E}+04$ & $2.07 \mathrm{E}+06$ & $9.09 \mathrm{E}+05$ & $2.46 \mathrm{E}+07$ & $2.50 \mathrm{E}+06$ & $6.76 \mathrm{E}+07$ & $6.17 \mathrm{E}+04$ & $6.76 \mathrm{E}+07$ \\
\hline $\mathrm{Sb}-127$ & $5.50 \mathrm{E}+02$ & $1.49 \mathrm{E}+04$ & $5.46 \mathrm{E}+03$ & $1.48 \mathrm{E}+05$ & $1.70 \mathrm{E}+03$ & $4.58 \mathrm{E}+04$ & $2.01 \mathrm{E}+04$ & $5.44 \mathrm{E}+05$ & $5.53 \mathrm{E}+04$ & $1.49 \mathrm{E}+06$ & $1.37 \mathrm{E}+03$ & $1.49 \mathrm{E}+06$ \\
\hline $\mathrm{Sb}-128$ & $1.17 \mathrm{E}+03$ & $3.17 \mathrm{E}+04$ & $1.16 \mathrm{E}+04$ & $3.15 \mathrm{E}+05$ & $3.61 \mathrm{E}+03$ & $9.77 \mathrm{E}+04$ & $4.29 \mathrm{E}+04$ & $1.16 \mathrm{E}+06$ & $1.18 \mathrm{E}+05$ & $3.19 \mathrm{E}+06$ & $2.91 \mathrm{E}+03$ & $3.19 \mathrm{E}+06$ \\
\hline $\mathrm{Sb}-128 \mathrm{~m}$ & $2.80 \mathrm{E}+04$ & $7.57 \mathrm{E}+05$ & $2.78 \mathrm{E}+05$ & $7.52 \mathrm{E}+06$ & $8.63 \mathrm{E}+04$ & $2.33 \mathrm{E}+06$ & $1.02 \mathrm{E}+06$ & $2.77 \mathrm{E}+07$ & $2.82 \mathrm{E}+06$ & $7.61 \mathrm{E}+07$ & $6.95 \mathrm{E}+04$ & $7.61 \mathrm{E}+07$ \\
\hline Sb-129 & $2.17 \mathrm{E}+03$ & $5.88 \mathrm{E}+04$ & $2.16 \mathrm{E}+04$ & $5.83 \mathrm{E}+05$ & $6.70 \mathrm{E}+03$ & $1.81 \mathrm{E}+05$ & $7.95 \mathrm{E}+04$ & $2.15 \mathrm{E}+06$ & $2.19 \mathrm{E}+05$ & $5.91 \mathrm{E}+06$ & $5.40 \mathrm{E}+03$ & $5.91 \mathrm{E}+06$ \\
\hline $\mathrm{Sb}-130$ & $1.02 \mathrm{E}+04$ & $2.76 \mathrm{E}+05$ & $1.01 \mathrm{E}+05$ & $2.73 \mathrm{E}+06$ & $3.14 \mathrm{E}+04$ & $8.49 \mathrm{E}+05$ & $3.73 \mathrm{E}+05$ & $1.01 \mathrm{E}+07$ & $1.02 \mathrm{E}+06$ & $2.77 \mathrm{E}+07$ & $2.53 \mathrm{E}+04$ & $2.77 \mathrm{E}+07$ \\
\hline $\mathrm{Sb}-131$ & $8.46 \mathrm{E}+03$ & $2.29 \mathrm{E}+05$ & $8.40 \mathrm{E}+04$ & $2.27 \mathrm{E}+06$ & $2.61 \mathrm{E}+04$ & $7.04 \mathrm{E}+05$ & $3.09 \mathrm{E}+05$ & $8.36 \mathrm{E}+06$ & $8.50 \mathrm{E}+05$ & $2.30 \mathrm{E}+07$ & $2.10 \mathrm{E}+04$ & $2.30 \mathrm{E}+07$ \\
\hline Te-114 & $1.43 \mathrm{E}+04$ & $3.86 \mathrm{E}+05$ & $1.42 \mathrm{E}+05$ & $3.83 \mathrm{E}+06$ & $4.40 \mathrm{E}+04$ & $1.19 \mathrm{E}+06$ & $5.22 \mathrm{E}+05$ & $1.41 \mathrm{E}+07$ & $1.44 \mathrm{E}+06$ & $3.88 \mathrm{E}+07$ & $3.54 \mathrm{E}+04$ & $3.88 \mathrm{E}+07$ \\
\hline Te-116 & $4.82 \mathrm{E}+03$ & $1.30 \mathrm{E}+05$ & $4.79 \mathrm{E}+04$ & $1.29 \mathrm{E}+06$ & $1.49 \mathrm{E}+04$ & $4.01 \mathrm{E}+05$ & $1.76 \mathrm{E}+05$ & $4.76 \mathrm{E}+06$ & $4.84 \mathrm{E}+05$ & $1.31 \mathrm{E}+07$ & $1.20 \mathrm{E}+04$ & $1.31 \mathrm{E}+07$ \\
\hline
\end{tabular}

Page 181 of 298 
SRNL-STI-2013-00115

\begin{tabular}{|c|c|c|c|c|c|c|c|c|c|c|c|c|}
\hline \multirow[t]{2}{*}{ Nuclide } & \multicolumn{2}{|c|}{ Water Ingestion DCS } & \multicolumn{2}{|c|}{ Meat Ingestion DCS } & \multicolumn{2}{|c|}{ Dairy Ingestion DCS } & \multicolumn{2}{|c|}{$\begin{array}{c}\text { Freshwater Fish } \\
\text { Ingestion DCS }\end{array}$} & \multicolumn{2}{|c|}{$\begin{array}{l}\text { Saltwater Shellfish } \\
\text { Ingestion DCS }\end{array}$} & \multicolumn{2}{|c|}{ Produce Ingestion DCS } \\
\hline & $(\mathrm{Bq} / \mathrm{L})$ & $(\mathrm{pCi} / \mathrm{L})$ & $(\mathrm{Bq} / \mathbf{k g})$ & $(\mathrm{pCi} / \mathrm{kg})$ & $(\mathrm{Bq} / \mathrm{L})$ & $(\mathrm{pCi} / \mathrm{L})$ & $(\mathrm{Bq} / \mathbf{k g})$ & $(\mathrm{pCi} / \mathbf{k g})$ & $(\mathrm{Bq} / \mathbf{k g})$ & $(\mathrm{pCi} / \mathbf{k g})$ & $(\mathrm{Bq} / \mathbf{k g})$ & $(\mathrm{pCi} / \mathbf{k g})$ \\
\hline Te-117 & $1.84 \mathrm{E}+04$ & $4.97 \mathrm{E}+05$ & $1.82 \mathrm{E}+05$ & $4.93 \mathrm{E}+06$ & $5.66 \mathrm{E}+04$ & $1.53 \mathrm{E}+06$ & $6.71 \mathrm{E}+05$ & $1.81 \mathrm{E}+07$ & $1.85 \mathrm{E}+06$ & $4.99 \mathrm{E}+07$ & $4.56 \mathrm{E}+04$ & $4.99 \mathrm{E}+07$ \\
\hline Te-118 & $3.01 \mathrm{E}+02$ & $8.14 \mathrm{E}+03$ & $2.99 \mathrm{E}+03$ & $8.08 \mathrm{E}+04$ & $9.28 \mathrm{E}+02$ & $2.51 \mathrm{E}+04$ & $1.10 \mathrm{E}+04$ & $2.97 \mathrm{E}+05$ & $3.03 \mathrm{E}+04$ & $8.18 \mathrm{E}+05$ & $7.47 \mathrm{E}+02$ & $8.18 \mathrm{E}+05$ \\
\hline Te-119 & $5.55 \mathrm{E}+03$ & $1.50 \mathrm{E}+05$ & $5.51 \mathrm{E}+04$ & $1.49 \mathrm{E}+06$ & $1.71 \mathrm{E}+04$ & $4.62 \mathrm{E}+05$ & $2.03 \mathrm{E}+05$ & $5.48 \mathrm{E}+06$ & $5.58 \mathrm{E}+05$ & $1.51 \mathrm{E}+07$ & $1.38 \mathrm{E}+04$ & $1.51 \mathrm{E}+07$ \\
\hline Te-119m & $1.39 \mathrm{E}+03$ & $3.75 \mathrm{E}+04$ & $1.38 \mathrm{E}+04$ & $3.72 \mathrm{E}+05$ & $4.28 \mathrm{E}+03$ & $1.16 \mathrm{E}+05$ & $5.07 \mathrm{E}+04$ & $1.37 \mathrm{E}+06$ & $1.40 \mathrm{E}+05$ & $3.77 \mathrm{E}+06$ & $3.44 \mathrm{E}+03$ & $3.77 \mathrm{E}+06$ \\
\hline Te-121 & $2.20 \mathrm{E}+03$ & $5.95 \mathrm{E}+04$ & $2.19 \mathrm{E}+04$ & $5.91 \mathrm{E}+05$ & $6.78 \mathrm{E}+03$ & $1.83 \mathrm{E}+05$ & $8.05 \mathrm{E}+04$ & $2.17 \mathrm{E}+06$ & $2.21 \mathrm{E}+05$ & $5.98 \mathrm{E}+06$ & $5.46 \mathrm{E}+03$ & $5.98 \mathrm{E}+06$ \\
\hline $\mathrm{Te}-121 \mathrm{~m}$ & $4.10 \mathrm{E}+02$ & $1.11 \mathrm{E}+04$ & $4.07 \mathrm{E}+03$ & $1.10 \mathrm{E}+05$ & $1.26 \mathrm{E}+03$ & $3.42 \mathrm{E}+04$ & $1.50 \mathrm{E}+04$ & $4.05 \mathrm{E}+05$ & $4.13 \mathrm{E}+04$ & $1.11 \mathrm{E}+06$ & $1.02 \mathrm{E}+03$ & $1.11 \mathrm{E}+06$ \\
\hline Te-123 & $8.35 \mathrm{E}+02$ & $2.26 \mathrm{E}+04$ & $8.29 \mathrm{E}+03$ & $2.24 \mathrm{E}+05$ & $2.57 \mathrm{E}+03$ & $6.95 \mathrm{E}+04$ & $3.05 \mathrm{E}+04$ & $8.24 \mathrm{E}+05$ & $8.39 \mathrm{E}+04$ & $2.27 \mathrm{E}+06$ & $2.07 \mathrm{E}+03$ & $2.27 \mathrm{E}+06$ \\
\hline Te-123m & $6.65 \mathrm{E}+02$ & $1.80 \mathrm{E}+04$ & $6.60 \mathrm{E}+03$ & $1.78 \mathrm{E}+05$ & $2.05 \mathrm{E}+03$ & $5.54 \mathrm{E}+04$ & $2.43 \mathrm{E}+04$ & $6.57 \mathrm{E}+05$ & $6.68 \mathrm{E}+04$ & $1.81 \mathrm{E}+06$ & $1.65 \mathrm{E}+03$ & $1.81 \mathrm{E}+06$ \\
\hline Te- $125 \mathrm{~m}$ & $1.02 \mathrm{E}+03$ & $2.76 \mathrm{E}+04$ & $1.01 \mathrm{E}+04$ & $2.73 \mathrm{E}+05$ & $3.14 \mathrm{E}+03$ & $8.49 \mathrm{E}+04$ & $3.73 \mathrm{E}+04$ & $1.01 \mathrm{E}+06$ & $1.02 \mathrm{E}+05$ & $2.77 \mathrm{E}+06$ & $2.53 \mathrm{E}+03$ & $2.77 \mathrm{E}+06$ \\
\hline Te-127 & $5.41 \mathrm{E}+03$ & $1.46 \mathrm{E}+05$ & $5.37 \mathrm{E}+04$ & $1.45 \mathrm{E}+06$ & $1.67 \mathrm{E}+04$ & $4.50 \mathrm{E}+05$ & $1.98 \mathrm{E}+05$ & $5.34 \mathrm{E}+06$ & $5.43 \mathrm{E}+05$ & $1.47 \mathrm{E}+07$ & $1.34 \mathrm{E}+04$ & $1.47 \mathrm{E}+07$ \\
\hline Te- $127 \mathrm{~m}$ & $3.66 \mathrm{E}+02$ & $9.89 \mathrm{E}+03$ & $3.63 \mathrm{E}+03$ & $9.81 \mathrm{E}+04$ & $1.13 \mathrm{E}+03$ & $3.05 \mathrm{E}+04$ & $1.34 \mathrm{E}+04$ & $3.61 \mathrm{E}+05$ & $3.68 \mathrm{E}+04$ & $9.94 \mathrm{E}+05$ & $9.08 \mathrm{E}+02$ & $9.94 \mathrm{E}+05$ \\
\hline Te-129 & $1.46 \mathrm{E}+04$ & $3.95 \mathrm{E}+05$ & $1.45 \mathrm{E}+05$ & $3.92 \mathrm{E}+06$ & $4.50 \mathrm{E}+04$ & $1.22 \mathrm{E}+06$ & $5.34 \mathrm{E}+05$ & $1.44 \mathrm{E}+07$ & $1.47 \mathrm{E}+06$ & $3.97 \mathrm{E}+07$ & $3.63 \mathrm{E}+04$ & $3.97 \mathrm{E}+07$ \\
\hline Te-129m & $2.93 \mathrm{E}+02$ & $7.91 \mathrm{E}+03$ & $2.90 \mathrm{E}+03$ & $7.85 \mathrm{E}+04$ & $9.02 \mathrm{E}+02$ & $2.44 \mathrm{E}+04$ & $1.07 \mathrm{E}+04$ & $2.89 \mathrm{E}+05$ & $2.94 \mathrm{E}+04$ & $7.95 \mathrm{E}+05$ & $7.26 \mathrm{E}+02$ & $7.95 \mathrm{E}+05$ \\
\hline Te-131 & $1.03 \mathrm{E}+04$ & $2.78 \mathrm{E}+05$ & $1.02 \mathrm{E}+05$ & $2.76 \mathrm{E}+06$ & $3.17 \mathrm{E}+04$ & $8.56 \mathrm{E}+05$ & $3.76 \mathrm{E}+05$ & $1.02 \mathrm{E}+07$ & $1.03 \mathrm{E}+06$ & $2.79 \mathrm{E}+07$ & $2.55 \mathrm{E}+04$ & $2.79 \mathrm{E}+07$ \\
\hline Te-131m & $4.62 \mathrm{E}+02$ & $1.25 \mathrm{E}+04$ & $4.59 \mathrm{E}+03$ & $1.24 \mathrm{E}+05$ & $1.42 \mathrm{E}+03$ & $3.85 \mathrm{E}+04$ & $1.69 \mathrm{E}+04$ & $4.57 \mathrm{E}+05$ & $4.65 \mathrm{E}+04$ & $1.26 \mathrm{E}+06$ & $1.15 \mathrm{E}+03$ & $1.26 \mathrm{E}+06$ \\
\hline Te-132 & $2.32 \mathrm{E}+02$ & $6.27 \mathrm{E}+03$ & $2.30 \mathrm{E}+03$ & $6.23 \mathrm{E}+04$ & $7.15 \mathrm{E}+02$ & $1.93 \mathrm{E}+04$ & $8.48 \mathrm{E}+03$ & $2.29 \mathrm{E}+05$ & $2.33 \mathrm{E}+04$ & $6.30 \mathrm{E}+05$ & $5.76 \mathrm{E}+02$ & $6.30 \mathrm{E}+05$ \\
\hline Te-133 & $1.23 \mathrm{E}+04$ & $3.33 \mathrm{E}+05$ & $1.22 \mathrm{E}+05$ & $3.30 \mathrm{E}+06$ & $3.79 \mathrm{E}+04$ & $1.03 \mathrm{E}+06$ & $4.50 \mathrm{E}+05$ & $1.22 \mathrm{E}+07$ & $1.24 \mathrm{E}+06$ & $3.34 \mathrm{E}+07$ & $3.06 \mathrm{E}+04$ & $3.34 \mathrm{E}+07$ \\
\hline Te-133m & $3.42 \mathrm{E}+03$ & $9.24 \mathrm{E}+04$ & $3.39 \mathrm{E}+04$ & $9.17 \mathrm{E}+05$ & $1.05 \mathrm{E}+04$ & $2.84 \mathrm{E}+05$ & $1.25 \mathrm{E}+05$ & $3.38 \mathrm{E}+06$ & $3.43 \mathrm{E}+05$ & $9.28 \mathrm{E}+06$ & $8.48 \mathrm{E}+03$ & $9.28 \mathrm{E}+06$ \\
\hline Te-134 & $9.35 \mathrm{E}+03$ & $2.53 \mathrm{E}+05$ & $9.28 \mathrm{E}+04$ & $2.51 \mathrm{E}+06$ & $2.88 \mathrm{E}+04$ & $7.79 \mathrm{E}+05$ & $3.42 \mathrm{E}+05$ & $9.24 \mathrm{E}+06$ & $9.40 \mathrm{E}+05$ & $2.54 \mathrm{E}+07$ & $2.32 \mathrm{E}+04$ & $2.54 \mathrm{E}+07$ \\
\hline $\mathrm{I}-118$ & $4.51 \mathrm{E}+03$ & $1.22 \mathrm{E}+05$ & $4.47 \mathrm{E}+04$ & $1.21 \mathrm{E}+06$ & $1.39 \mathrm{E}+04$ & $3.75 \mathrm{E}+05$ & $1.65 \mathrm{E}+05$ & $4.45 \mathrm{E}+06$ & $4.53 \mathrm{E}+05$ & $1.22 \mathrm{E}+07$ & $1.12 \mathrm{E}+04$ & $1.22 \mathrm{E}+07$ \\
\hline I-119 & $2.16 \mathrm{E}+04$ & $5.85 \mathrm{E}+05$ & $2.15 \mathrm{E}+05$ & $5.80 \mathrm{E}+06$ & $6.66 \mathrm{E}+04$ & $1.80 \mathrm{E}+06$ & $7.91 \mathrm{E}+05$ & $2.14 \mathrm{E}+07$ & $2.17 \mathrm{E}+06$ & $5.88 \mathrm{E}+07$ & $5.37 \mathrm{E}+04$ & $5.88 \mathrm{E}+07$ \\
\hline $\mathrm{I}-120$ & $3.03 \mathrm{E}+03$ & $8.20 \mathrm{E}+04$ & $3.01 \mathrm{E}+04$ & $8.14 \mathrm{E}+05$ & $9.34 \mathrm{E}+03$ & $2.53 \mathrm{E}+05$ & $1.11 \mathrm{E}+05$ & $3.00 \mathrm{E}+06$ & $3.05 \mathrm{E}+05$ & $8.24 \mathrm{E}+06$ & $7.53 \mathrm{E}+03$ & $8.24 \mathrm{E}+06$ \\
\hline $\mathrm{I}-120 \mathrm{~m}$ & $5.92 \mathrm{E}+03$ & $1.60 \mathrm{E}+05$ & $5.88 \mathrm{E}+04$ & $1.59 \mathrm{E}+06$ & $1.82 \mathrm{E}+04$ & $4.93 \mathrm{E}+05$ & $2.16 \mathrm{E}+05$ & $5.85 \mathrm{E}+06$ & $5.95 \mathrm{E}+05$ & $1.61 \mathrm{E}+07$ & $1.47 \mathrm{E}+04$ & $1.61 \mathrm{E}+07$ \\
\hline
\end{tabular}

Page 182 of 298 
SRNL-STI-2013-00115

\begin{tabular}{|c|c|c|c|c|c|c|c|c|c|c|c|c|}
\hline \multirow[t]{2}{*}{ Nuclide } & \multicolumn{2}{|c|}{ Water Ingestion DCS } & \multicolumn{2}{|c|}{ Meat Ingestion DCS } & \multicolumn{2}{|c|}{ Dairy Ingestion DCS } & \multicolumn{2}{|c|}{$\begin{array}{c}\text { Freshwater Fish } \\
\text { Ingestion DCS }\end{array}$} & \multicolumn{2}{|c|}{$\begin{array}{l}\text { Saltwater Shellfish } \\
\text { Ingestion DCS }\end{array}$} & \multicolumn{2}{|c|}{ Produce Ingestion DCS } \\
\hline & $(\mathrm{Bq} / \mathrm{L})$ & $(\mathrm{pCi} / \mathrm{L})$ & $(\mathrm{Bq} / \mathrm{kg})$ & $(\mathrm{pCi} / \mathrm{kg})$ & $(\mathrm{Bq} / \mathrm{L})$ & $(\mathrm{pCi} / \mathrm{L})$ & $(\mathrm{Bq} / \mathbf{k g})$ & $(\mathrm{pCi} / \mathbf{k g})$ & $(\mathrm{Bq} / \mathbf{k g})$ & $(\mathrm{pCi} / \mathbf{k g})$ & $(\mathrm{Bq} / \mathbf{k g})$ & $(\mathrm{pCi} / \mathbf{k g})$ \\
\hline $\mathrm{I}-121$ & $1.24 \mathrm{E}+04$ & $3.36 \mathrm{E}+05$ & $1.23 \mathrm{E}+05$ & $3.34 \mathrm{E}+06$ & $3.83 \mathrm{E}+04$ & $1.04 \mathrm{E}+06$ & $4.55 \mathrm{E}+05$ & $1.23 \mathrm{E}+07$ & $1.25 \mathrm{E}+06$ & $3.38 \mathrm{E}+07$ & $3.09 \mathrm{E}+04$ & $3.38 \mathrm{E}+07$ \\
\hline $\mathrm{I}-123$ & $3.84 \mathrm{E}+03$ & $1.04 \mathrm{E}+05$ & $3.81 \mathrm{E}+04$ & $1.03 \mathrm{E}+06$ & $1.18 \mathrm{E}+04$ & $3.20 \mathrm{E}+05$ & $1.40 \mathrm{E}+05$ & $3.79 \mathrm{E}+06$ & $3.86 \mathrm{E}+05$ & $1.04 \mathrm{E}+07$ & $9.53 \mathrm{E}+03$ & $1.04 \mathrm{E}+07$ \\
\hline $\mathrm{I}-124$ & $6.55 \mathrm{E}+01$ & $1.77 \mathrm{E}+03$ & $6.50 \mathrm{E}+02$ & $1.76 \mathrm{E}+04$ & $2.02 \mathrm{E}+02$ & $5.45 \mathrm{E}+03$ & $2.39 \mathrm{E}+03$ & $6.47 \mathrm{E}+04$ & $6.58 \mathrm{E}+03$ & $1.78 \mathrm{E}+05$ & $1.62 \mathrm{E}+02$ & $1.78 \mathrm{E}+05$ \\
\hline $\mathrm{I}-125$ & $6.58 \mathrm{E}+01$ & $1.78 \mathrm{E}+03$ & $6.53 \mathrm{E}+02$ & $1.77 \mathrm{E}+04$ & $2.03 \mathrm{E}+02$ & $5.48 \mathrm{E}+03$ & $2.41 \mathrm{E}+03$ & $6.50 \mathrm{E}+04$ & $6.61 \mathrm{E}+03$ & $1.79 \mathrm{E}+05$ & $1.63 \mathrm{E}+02$ & $1.79 \mathrm{E}+05$ \\
\hline $\mathrm{I}-126$ & $3.04 \mathrm{E}+01$ & $8.22 \mathrm{E}+02$ & $3.02 \mathrm{E}+02$ & $8.16 \mathrm{E}+03$ & $9.37 \mathrm{E}+01$ & $2.53 \mathrm{E}+03$ & $1.11 \mathrm{E}+03$ & $3.00 \mathrm{E}+04$ & $3.06 \mathrm{E}+03$ & $8.26 \mathrm{E}+04$ & $7.55 \mathrm{E}+01$ & $8.26 \mathrm{E}+04$ \\
\hline $\mathrm{I}-128$ & $2.00 \mathrm{E}+04$ & $5.40 \mathrm{E}+05$ & $1.98 \mathrm{E}+05$ & $5.36 \mathrm{E}+06$ & $6.15 \mathrm{E}+04$ & $1.66 \mathrm{E}+06$ & $7.30 \mathrm{E}+05$ & $1.97 \mathrm{E}+07$ & $2.01 \mathrm{E}+06$ & $5.42 \mathrm{E}+07$ & $4.95 \mathrm{E}+04$ & $5.42 \mathrm{E}+07$ \\
\hline $\mathrm{I}-129$ & $1.03 \mathrm{E}+01$ & $2.78 \mathrm{E}+02$ & $1.02 \mathrm{E}+02$ & $2.76 \mathrm{E}+03$ & $3.17 \mathrm{E}+01$ & $8.56 \mathrm{E}+02$ & $3.76 \mathrm{E}+02$ & $1.02 \mathrm{E}+04$ & $1.03 \mathrm{E}+03$ & $2.79 \mathrm{E}+04$ & $2.55 \mathrm{E}+01$ & $2.79 \mathrm{E}+04$ \\
\hline $\mathrm{I}-130$ & $4.51 \mathrm{E}+02$ & $1.22 \mathrm{E}+04$ & $4.47 \mathrm{E}+03$ & $1.21 \mathrm{E}+05$ & $1.39 \mathrm{E}+03$ & $3.75 \mathrm{E}+04$ & $1.65 \mathrm{E}+04$ & $4.45 \mathrm{E}+05$ & $4.53 \mathrm{E}+04$ & $1.22 \mathrm{E}+06$ & $1.12 \mathrm{E}+03$ & $1.22 \mathrm{E}+06$ \\
\hline $\mathrm{I}-131$ & $3.97 \mathrm{E}+01$ & $1.07 \mathrm{E}+03$ & $3.94 \mathrm{E}+02$ & $1.07 \mathrm{E}+04$ & $1.22 \mathrm{E}+02$ & $3.31 \mathrm{E}+03$ & $1.45 \mathrm{E}+03$ & $3.92 \mathrm{E}+04$ & $3.99 \mathrm{E}+03$ & $1.08 \mathrm{E}+05$ & $9.86 \mathrm{E}+01$ & $1.08 \mathrm{E}+05$ \\
\hline $\mathrm{I}-132$ & $3.07 \mathrm{E}+03$ & $8.30 \mathrm{E}+04$ & $3.05 \mathrm{E}+04$ & $8.24 \mathrm{E}+05$ & $9.46 \mathrm{E}+03$ & $2.56 \mathrm{E}+05$ & $1.12 \mathrm{E}+05$ & $3.03 \mathrm{E}+06$ & $3.09 \mathrm{E}+05$ & $8.34 \mathrm{E}+06$ & $7.62 \mathrm{E}+03$ & $8.34 \mathrm{E}+06$ \\
\hline $\mathrm{I}-132 \mathrm{~m}$ & $4.30 \mathrm{E}+03$ & $1.16 \mathrm{E}+05$ & $4.27 \mathrm{E}+04$ & $1.15 \mathrm{E}+06$ & $1.33 \mathrm{E}+04$ & $3.58 \mathrm{E}+05$ & $1.57 \mathrm{E}+05$ & $4.25 \mathrm{E}+06$ & $4.33 \mathrm{E}+05$ & $1.17 \mathrm{E}+07$ & $1.07 \mathrm{E}+04$ & $1.17 \mathrm{E}+07$ \\
\hline $\mathrm{I}-133$ & $1.88 \mathrm{E}+02$ & $5.09 \mathrm{E}+03$ & $1.87 \mathrm{E}+03$ & $5.06 \mathrm{E}+04$ & $5.81 \mathrm{E}+02$ & $1.57 \mathrm{E}+04$ & $6.89 \mathrm{E}+03$ & $1.86 \mathrm{E}+05$ & $1.89 \mathrm{E}+04$ & $5.12 \mathrm{E}+05$ & $4.68 \mathrm{E}+02$ & $5.12 \mathrm{E}+05$ \\
\hline $\mathrm{I}-134$ & $9.08 \mathrm{E}+03$ & $2.45 \mathrm{E}+05$ & $9.01 \mathrm{E}+04$ & $2.44 \mathrm{E}+06$ & $2.80 \mathrm{E}+04$ & $7.56 \mathrm{E}+05$ & $3.32 \mathrm{E}+05$ & $8.97 \mathrm{E}+06$ & $9.12 \mathrm{E}+05$ & $2.47 \mathrm{E}+07$ & $2.25 \mathrm{E}+04$ & $2.47 \mathrm{E}+07$ \\
\hline $\mathrm{I}-135$ & $9.49 \mathrm{E}+02$ & $2.57 \mathrm{E}+04$ & $9.42 \mathrm{E}+03$ & $2.55 \mathrm{E}+05$ & $2.92 \mathrm{E}+03$ & $7.90 \mathrm{E}+04$ & $3.47 \mathrm{E}+04$ & $9.38 \mathrm{E}+05$ & $9.54 \mathrm{E}+04$ & $2.58 \mathrm{E}+06$ & $2.36 \mathrm{E}+03$ & $2.58 \mathrm{E}+06$ \\
\hline Cs- 125 & $2.73 \mathrm{E}+04$ & $7.37 \mathrm{E}+05$ & $2.71 \mathrm{E}+05$ & $7.32 \mathrm{E}+06$ & $8.40 \mathrm{E}+04$ & $2.27 \mathrm{E}+06$ & $9.97 \mathrm{E}+05$ & $2.69 \mathrm{E}+07$ & $2.74 \mathrm{E}+06$ & $7.41 \mathrm{E}+07$ & $6.77 \mathrm{E}+04$ & $7.41 \mathrm{E}+07$ \\
\hline Cs-127 & $4.03 \mathrm{E}+04$ & $1.09 \mathrm{E}+06$ & $4.00 \mathrm{E}+05$ & $1.08 \mathrm{E}+07$ & $1.24 \mathrm{E}+05$ & $3.35 \mathrm{E}+06$ & $1.47 \mathrm{E}+06$ & $3.98 \mathrm{E}+07$ & $4.05 \mathrm{E}+06$ & $1.09 \mathrm{E}+08$ & $9.99 \mathrm{E}+04$ & $1.09 \mathrm{E}+08$ \\
\hline Cs-129 & $1.66 \mathrm{E}+04$ & $4.48 \mathrm{E}+05$ & $1.65 \mathrm{E}+05$ & $4.45 \mathrm{E}+06$ & $5.11 \mathrm{E}+04$ & $1.38 \mathrm{E}+06$ & $6.06 \mathrm{E}+05$ & $1.64 \mathrm{E}+07$ & $1.67 \mathrm{E}+06$ & $4.50 \mathrm{E}+07$ & $4.12 \mathrm{E}+04$ & $4.50 \mathrm{E}+07$ \\
\hline Cs-130 & $3.49 \mathrm{E}+04$ & $9.44 \mathrm{E}+05$ & $3.47 \mathrm{E}+05$ & $9.37 \mathrm{E}+06$ & $1.08 \mathrm{E}+05$ & $2.91 \mathrm{E}+06$ & $1.28 \mathrm{E}+06$ & $3.45 \mathrm{E}+07$ & $3.51 \mathrm{E}+06$ & $9.49 \mathrm{E}+07$ & $8.67 \mathrm{E}+04$ & $9.49 \mathrm{E}+07$ \\
\hline Cs-131 & $1.72 \mathrm{E}+04$ & $4.66 \mathrm{E}+05$ & $1.71 \mathrm{E}+05$ & $4.62 \mathrm{E}+06$ & $5.31 \mathrm{E}+04$ & $1.43 \mathrm{E}+06$ & $6.30 \mathrm{E}+05$ & $1.70 \mathrm{E}+07$ & $1.73 \mathrm{E}+06$ & $4.68 \mathrm{E}+07$ & $4.27 \mathrm{E}+04$ & $4.68 \mathrm{E}+07$ \\
\hline Cs-132 & $2.07 \mathrm{E}+03$ & $5.60 \mathrm{E}+04$ & $2.06 \mathrm{E}+04$ & $5.56 \mathrm{E}+05$ & $6.39 \mathrm{E}+03$ & $1.73 \mathrm{E}+05$ & $7.58 \mathrm{E}+04$ & $2.05 \mathrm{E}+06$ & $2.08 \mathrm{E}+05$ & $5.63 \mathrm{E}+06$ & $5.14 \mathrm{E}+03$ & $5.63 \mathrm{E}+06$ \\
\hline Cs-134 & $6.65 \mathrm{E}+01$ & $1.80 \mathrm{E}+03$ & $6.60 \mathrm{E}+02$ & $1.78 \mathrm{E}+04$ & $2.05 \mathrm{E}+02$ & $5.54 \mathrm{E}+03$ & $2.43 \mathrm{E}+03$ & $6.57 \mathrm{E}+04$ & $6.68 \mathrm{E}+03$ & $1.81 \mathrm{E}+05$ & $1.65 \mathrm{E}+02$ & $1.81 \mathrm{E}+05$ \\
\hline Cs- $134 \mathrm{~m}$ & $4.77 \mathrm{E}+04$ & $1.29 \mathrm{E}+06$ & $4.73 \mathrm{E}+05$ & $1.28 \mathrm{E}+07$ & $1.47 \mathrm{E}+05$ & $3.97 \mathrm{E}+06$ & $1.74 \mathrm{E}+06$ & $4.71 \mathrm{E}+07$ & $4.79 \mathrm{E}+06$ & $1.29 \mathrm{E}+08$ & $1.18 \mathrm{E}+05$ & $1.29 \mathrm{E}+08$ \\
\hline Cs-135 & $4.71 \mathrm{E}+02$ & $1.27 \mathrm{E}+04$ & $4.68 \mathrm{E}+03$ & $1.26 \mathrm{E}+05$ & $1.45 \mathrm{E}+03$ & $3.92 \mathrm{E}+04$ & $1.72 \mathrm{E}+04$ & $4.65 \mathrm{E}+05$ & $4.73 \mathrm{E}+04$ & $1.28 \mathrm{E}+06$ & $1.17 \mathrm{E}+03$ & $1.28 \mathrm{E}+06$ \\
\hline
\end{tabular}

Page 183 of 298 
SRNL-STI-2013-00115

\begin{tabular}{|c|c|c|c|c|c|c|c|c|c|c|c|c|}
\hline \multirow[t]{2}{*}{ Nuclide } & \multicolumn{2}{|c|}{ Water Ingestion DCS } & \multicolumn{2}{|c|}{ Meat Ingestion DCS } & \multicolumn{2}{|c|}{ Dairy Ingestion DCS } & \multicolumn{2}{|c|}{$\begin{array}{l}\text { Freshwater Fish } \\
\text { Ingestion DCS }\end{array}$} & \multicolumn{2}{|c|}{$\begin{array}{l}\text { Saltwater Shellfish } \\
\text { Ingestion DCS }\end{array}$} & \multicolumn{2}{|c|}{ Produce Ingestion DCS } \\
\hline & $(\mathrm{Bq} / \mathrm{L})$ & $(\mathrm{pCi} / \mathrm{L})$ & $(\mathrm{Bq} / \mathrm{kg})$ & $(\mathrm{pCi} / \mathrm{kg})$ & $(\mathrm{Bq} / \mathrm{L})$ & $(\mathrm{pCi} / \mathrm{L})$ & $(\mathrm{Bq} / \mathbf{k g})$ & $(\mathrm{pCi} / \mathbf{k g})$ & $(\mathrm{Bq} / \mathbf{k g})$ & $(\mathrm{pCi} / \mathbf{k g})$ & $(\mathrm{Bq} / \mathbf{k g})$ & $(\mathrm{pCi} / \mathbf{k g})$ \\
\hline Cs- $135 \mathrm{~m}$ & $5.25 \mathrm{E}+04$ & $1.42 \mathrm{E}+06$ & $5.21 \mathrm{E}+05$ & $1.41 \mathrm{E}+07$ & $1.62 \mathrm{E}+05$ & $4.37 \mathrm{E}+06$ & $1.92 \mathrm{E}+06$ & $5.18 \mathrm{E}+07$ & $5.27 \mathrm{E}+06$ & $1.43 \mathrm{E}+08$ & $1.30 \mathrm{E}+05$ & $1.43 \mathrm{E}+08$ \\
\hline Cs-136 & $3.61 \mathrm{E}+02$ & $9.74 \mathrm{E}+03$ & $3.58 \mathrm{E}+03$ & $9.67 \mathrm{E}+04$ & $1.11 \mathrm{E}+03$ & $3.00 \mathrm{E}+04$ & $1.32 \mathrm{E}+04$ & $3.56 \mathrm{E}+05$ & $3.62 \mathrm{E}+04$ & $9.79 \mathrm{E}+05$ & $8.95 \mathrm{E}+02$ & $9.79 \mathrm{E}+05$ \\
\hline Cs-137 & $9.35 \mathrm{E}+01$ & $2.53 \mathrm{E}+03$ & $9.28 \mathrm{E}+02$ & $2.51 \mathrm{E}+04$ & $2.88 \mathrm{E}+02$ & $7.79 \mathrm{E}+03$ & $3.42 \mathrm{E}+03$ & $9.24 \mathrm{E}+04$ & $9.40 \mathrm{E}+03$ & $2.54 \mathrm{E}+05$ & $2.32 \mathrm{E}+02$ & $2.54 \mathrm{E}+05$ \\
\hline Cs- 138 & $9.72 \mathrm{E}+03$ & $2.63 \mathrm{E}+05$ & $9.65 \mathrm{E}+04$ & $2.61 \mathrm{E}+06$ & $2.99 \mathrm{E}+04$ & $8.09 \mathrm{E}+05$ & $3.55 \mathrm{E}+05$ & $9.60 \mathrm{E}+06$ & $9.77 \mathrm{E}+05$ & $2.64 \mathrm{E}+07$ & $2.41 \mathrm{E}+04$ & $2.64 \mathrm{E}+07$ \\
\hline $\mathrm{Ba}-124$ & $1.35 \mathrm{E}+04$ & $3.64 \mathrm{E}+05$ & $1.34 \mathrm{E}+05$ & $3.62 \mathrm{E}+06$ & $4.15 \mathrm{E}+04$ & $1.12 \mathrm{E}+06$ & $4.92 \mathrm{E}+05$ & $1.33 \mathrm{E}+07$ & $1.35 \mathrm{E}+06$ & $3.66 \mathrm{E}+07$ & $3.34 \mathrm{E}+04$ & $3.66 \mathrm{E}+07$ \\
\hline Ba-126 & $3.70 \mathrm{E}+03$ & $1.00 \mathrm{E}+05$ & $3.67 \mathrm{E}+04$ & $9.93 \mathrm{E}+05$ & $1.14 \mathrm{E}+04$ & $3.08 \mathrm{E}+05$ & $1.35 \mathrm{E}+05$ & $3.66 \mathrm{E}+06$ & $3.72 \mathrm{E}+05$ & $1.01 \mathrm{E}+07$ & $9.19 \mathrm{E}+03$ & $1.01 \mathrm{E}+07$ \\
\hline Ba-127 & $3.79 \mathrm{E}+04$ & $1.02 \mathrm{E}+06$ & $3.76 \mathrm{E}+05$ & $1.02 \mathrm{E}+07$ & $1.17 \mathrm{E}+05$ & $3.16 \mathrm{E}+06$ & $1.39 \mathrm{E}+06$ & $3.75 \mathrm{E}+07$ & $3.81 \mathrm{E}+06$ & $1.03 \mathrm{E}+08$ & $9.41 \mathrm{E}+04$ & $1.03 \mathrm{E}+08$ \\
\hline $\mathrm{Ba}-128$ & $3.49 \mathrm{E}+02$ & $9.44 \mathrm{E}+03$ & $3.47 \mathrm{E}+03$ & $9.37 \mathrm{E}+04$ & $1.08 \mathrm{E}+03$ & $2.91 \mathrm{E}+04$ & $1.28 \mathrm{E}+04$ & $3.45 \mathrm{E}+05$ & $3.51 \mathrm{E}+04$ & $9.49 \mathrm{E}+05$ & $8.67 \mathrm{E}+02$ & $9.49 \mathrm{E}+05$ \\
\hline Ba-129 & $1.99 \mathrm{E}+04$ & $5.37 \mathrm{E}+05$ & $1.97 \mathrm{E}+05$ & $5.33 \mathrm{E}+06$ & $6.12 \mathrm{E}+04$ & $1.65 \mathrm{E}+06$ & $7.26 \mathrm{E}+05$ & $1.96 \mathrm{E}+07$ & $2.00 \mathrm{E}+06$ & $5.40 \mathrm{E}+07$ & $4.93 \mathrm{E}+04$ & $5.40 \mathrm{E}+07$ \\
\hline Ba-129m & $1.41 \mathrm{E}+04$ & $3.82 \mathrm{E}+05$ & $1.40 \mathrm{E}+05$ & $3.79 \mathrm{E}+06$ & $4.35 \mathrm{E}+04$ & $1.18 \mathrm{E}+06$ & $5.16 \mathrm{E}+05$ & $1.39 \mathrm{E}+07$ & $1.42 \mathrm{E}+06$ & $3.83 \mathrm{E}+07$ & $3.50 \mathrm{E}+04$ & $3.83 \mathrm{E}+07$ \\
\hline Ba-131 & $2.05 \mathrm{E}+03$ & $5.53 \mathrm{E}+04$ & $2.03 \mathrm{E}+04$ & $5.49 \mathrm{E}+05$ & $6.30 \mathrm{E}+03$ & $1.70 \mathrm{E}+05$ & $7.48 \mathrm{E}+04$ & $2.02 \mathrm{E}+06$ & $2.06 \mathrm{E}+05$ & $5.56 \mathrm{E}+06$ & $5.08 \mathrm{E}+03$ & $5.56 \mathrm{E}+06$ \\
\hline Ba-131m & $1.87 \mathrm{E}+05$ & $5.05 \mathrm{E}+06$ & $1.86 \mathrm{E}+06$ & $5.02 \mathrm{E}+07$ & $5.76 \mathrm{E}+05$ & $1.56 \mathrm{E}+07$ & $6.84 \mathrm{E}+06$ & $1.85 \mathrm{E}+08$ & $1.88 \mathrm{E}+07$ & $5.08 \mathrm{E}+08$ & $4.64 \mathrm{E}+05$ & $5.08 \mathrm{E}+08$ \\
\hline Ba-133 & $5.10 \mathrm{E}+02$ & $1.38 \mathrm{E}+04$ & $5.06 \mathrm{E}+03$ & $1.37 \mathrm{E}+05$ & $1.57 \mathrm{E}+03$ & $4.24 \mathrm{E}+04$ & $1.86 \mathrm{E}+04$ & $5.03 \mathrm{E}+05$ & $5.12 \mathrm{E}+04$ & $1.38 \mathrm{E}+06$ & $1.26 \mathrm{E}+03$ & $1.38 \mathrm{E}+06$ \\
\hline Ba-133m & $1.73 \mathrm{E}+03$ & $4.68 \mathrm{E}+04$ & $1.72 \mathrm{E}+04$ & $4.64 \mathrm{E}+05$ & $5.33 \mathrm{E}+03$ & $1.44 \mathrm{E}+05$ & $6.32 \mathrm{E}+04$ & $1.71 \mathrm{E}+06$ & $1.74 \mathrm{E}+05$ & $4.70 \mathrm{E}+06$ & $4.29 \mathrm{E}+03$ & $4.70 \mathrm{E}+06$ \\
\hline Ba- $135 \mathrm{~m}$ & $2.20 \mathrm{E}+03$ & $5.94 \mathrm{E}+04$ & $2.18 \mathrm{E}+04$ & $5.90 \mathrm{E}+05$ & $6.77 \mathrm{E}+03$ & $1.83 \mathrm{E}+05$ & $8.03 \mathrm{E}+04$ & $2.17 \mathrm{E}+06$ & $2.21 \mathrm{E}+05$ & $5.97 \mathrm{E}+06$ & $5.45 \mathrm{E}+03$ & $5.97 \mathrm{E}+06$ \\
\hline Ba-139 & $7.58 \mathrm{E}+03$ & $2.05 \mathrm{E}+05$ & $7.53 \mathrm{E}+04$ & $2.03 \mathrm{E}+06$ & $2.34 \mathrm{E}+04$ & $6.31 \mathrm{E}+05$ & $2.77 \mathrm{E}+05$ & $7.49 \mathrm{E}+06$ & $7.62 \mathrm{E}+05$ & $2.06 \mathrm{E}+07$ & $1.88 \mathrm{E}+04$ & $2.06 \mathrm{E}+07$ \\
\hline Ba-140 & $3.43 \mathrm{E}+02$ & $9.26 \mathrm{E}+03$ & $3.40 \mathrm{E}+03$ & $9.19 \mathrm{E}+04$ & $1.06 \mathrm{E}+03$ & $2.85 \mathrm{E}+04$ & $1.25 \mathrm{E}+04$ & $3.38 \mathrm{E}+05$ & $3.44 \mathrm{E}+04$ & $9.31 \mathrm{E}+05$ & $8.50 \mathrm{E}+02$ & $9.31 \mathrm{E}+05$ \\
\hline Ba-141 & $1.26 \mathrm{E}+04$ & $3.41 \mathrm{E}+05$ & $1.25 \mathrm{E}+05$ & $3.39 \mathrm{E}+06$ & $3.89 \mathrm{E}+04$ & $1.05 \mathrm{E}+06$ & $4.61 \mathrm{E}+05$ & $1.25 \mathrm{E}+07$ & $1.27 \mathrm{E}+06$ & $3.43 \mathrm{E}+07$ & $3.13 \mathrm{E}+04$ & $3.43 \mathrm{E}+07$ \\
\hline Ba-142 & $2.79 \mathrm{E}+04$ & $7.54 \mathrm{E}+05$ & $2.77 \mathrm{E}+05$ & $7.48 \mathrm{E}+06$ & $8.59 \mathrm{E}+04$ & $2.32 \mathrm{E}+06$ & $1.02 \mathrm{E}+06$ & $2.75 \mathrm{E}+07$ & $2.80 \mathrm{E}+06$ & $7.57 \mathrm{E}+07$ & $6.92 \mathrm{E}+04$ & $7.57 \mathrm{E}+07$ \\
\hline La-129 & $3.52 \mathrm{E}+04$ & $9.52 \mathrm{E}+05$ & $3.50 \mathrm{E}+05$ & $9.45 \mathrm{E}+06$ & $1.09 \mathrm{E}+05$ & $2.93 \mathrm{E}+06$ & $1.29 \mathrm{E}+06$ & $3.48 \mathrm{E}+07$ & $3.54 \mathrm{E}+06$ & $9.57 \mathrm{E}+07$ & $8.74 \mathrm{E}+04$ & $9.57 \mathrm{E}+07$ \\
\hline La-131 & $2.83 \mathrm{E}+04$ & $7.66 \mathrm{E}+05$ & $2.81 \mathrm{E}+05$ & $7.60 \mathrm{E}+06$ & $8.73 \mathrm{E}+04$ & $2.36 \mathrm{E}+06$ & $1.04 \mathrm{E}+06$ & $2.80 \mathrm{E}+07$ & $2.85 \mathrm{E}+06$ & $7.70 \mathrm{E}+07$ & $7.03 \mathrm{E}+04$ & $7.70 \mathrm{E}+07$ \\
\hline La-132 & $2.30 \mathrm{E}+03$ & $6.21 \mathrm{E}+04$ & $2.28 \mathrm{E}+04$ & $6.17 \mathrm{E}+05$ & $7.08 \mathrm{E}+03$ & $1.91 \mathrm{E}+05$ & $8.40 \mathrm{E}+04$ & $2.27 \mathrm{E}+06$ & $2.31 \mathrm{E}+05$ & $6.24 \mathrm{E}+06$ & $5.71 \mathrm{E}+03$ & $6.24 \mathrm{E}+06$ \\
\hline La-132m & $2.58 \mathrm{E}+04$ & $6.96 \mathrm{E}+05$ & $2.56 \mathrm{E}+05$ & $6.91 \mathrm{E}+06$ & $7.93 \mathrm{E}+04$ & $2.14 \mathrm{E}+06$ & $9.41 \mathrm{E}+05$ & $2.54 \mathrm{E}+07$ & $2.59 \mathrm{E}+06$ & $6.99 \mathrm{E}+07$ & $6.39 \mathrm{E}+04$ & $6.99 \mathrm{E}+07$ \\
\hline
\end{tabular}

Page 184 of 298 
SRNL-STI-2013-00115

\begin{tabular}{|c|c|c|c|c|c|c|c|c|c|c|c|c|}
\hline \multirow{2}{*}{ Nuclide } & \multicolumn{2}{|c|}{ Water Ingestion DCS } & \multicolumn{2}{|c|}{ Meat Ingestion DCS } & \multicolumn{2}{|c|}{ Dairy Ingestion DCS } & \multicolumn{2}{|c|}{$\begin{array}{c}\text { Freshwater Fish } \\
\text { Ingestion DCS }\end{array}$} & \multicolumn{2}{|c|}{$\begin{array}{l}\text { Saltwater Shellfish } \\
\text { Ingestion DCS }\end{array}$} & \multicolumn{2}{|c|}{ Produce Ingestion DCS } \\
\hline & $(\mathrm{Bq} / \mathrm{L})$ & $(\mathrm{pCi} / \mathrm{L})$ & $(\mathrm{Bq} / \mathrm{kg})$ & $(\mathrm{pCi} / \mathrm{kg})$ & $(\mathrm{Bq} / \mathrm{L})$ & $(\mathrm{pCi} / \mathrm{L})$ & $(\mathrm{Bq} / \mathbf{k g})$ & $(\mathrm{pCi} / \mathbf{k g})$ & $(\mathrm{Bq} / \mathbf{k g})$ & $(\mathrm{pCi} / \mathbf{k g})$ & $(\mathrm{Bq} / \mathbf{k g})$ & $(\mathrm{pCi} / \mathbf{k g})$ \\
\hline La-133 & $2.95 \mathrm{E}+04$ & $7.98 \mathrm{E}+05$ & $2.93 \mathrm{E}+05$ & $7.93 \mathrm{E}+06$ & $9.10 \mathrm{E}+04$ & $2.46 \mathrm{E}+06$ & $1.08 \mathrm{E}+06$ & $2.92 \mathrm{E}+07$ & $2.97 \mathrm{E}+06$ & $8.02 \mathrm{E}+07$ & $7.33 \mathrm{E}+04$ & $8.02 \mathrm{E}+07$ \\
\hline La-135 & $3.02 \mathrm{E}+04$ & $8.16 \mathrm{E}+05$ & $3.00 \mathrm{E}+05$ & $8.10 \mathrm{E}+06$ & $9.30 \mathrm{E}+04$ & $2.51 \mathrm{E}+06$ & $1.10 \mathrm{E}+06$ & $2.98 \mathrm{E}+07$ & $3.03 \mathrm{E}+06$ & $8.20 \mathrm{E}+07$ & $7.49 \mathrm{E}+04$ & $8.20 \mathrm{E}+07$ \\
\hline La-137 & $1.12 \mathrm{E}+04$ & $3.03 \mathrm{E}+05$ & $1.11 \mathrm{E}+05$ & $3.01 \mathrm{E}+06$ & $3.45 \mathrm{E}+04$ & $9.33 \mathrm{E}+05$ & $4.10 \mathrm{E}+05$ & $1.11 \mathrm{E}+07$ & $1.13 \mathrm{E}+06$ & $3.04 \mathrm{E}+07$ & $2.78 \mathrm{E}+04$ & $3.04 \mathrm{E}+07$ \\
\hline La-138 & $9.08 \mathrm{E}+02$ & $2.45 \mathrm{E}+04$ & $9.01 \mathrm{E}+03$ & $2.44 \mathrm{E}+05$ & $2.80 \mathrm{E}+03$ & $7.56 \mathrm{E}+04$ & $3.32 \mathrm{E}+04$ & $8.97 \mathrm{E}+05$ & $9.12 \mathrm{E}+04$ & $2.47 \mathrm{E}+06$ & $2.25 \mathrm{E}+03$ & $2.47 \mathrm{E}+06$ \\
\hline La-140 & $4.66 \mathrm{E}+02$ & $1.26 \mathrm{E}+04$ & $4.62 \mathrm{E}+03$ & $1.25 \mathrm{E}+05$ & $1.43 \mathrm{E}+03$ & $3.88 \mathrm{E}+04$ & $1.70 \mathrm{E}+04$ & $4.60 \mathrm{E}+05$ & $4.68 \mathrm{E}+04$ & $1.27 \mathrm{E}+06$ & $1.16 \mathrm{E}+03$ & $1.27 \mathrm{E}+06$ \\
\hline La-141 & $2.44 \mathrm{E}+03$ & $6.59 \mathrm{E}+04$ & $2.42 \mathrm{E}+04$ & $6.54 \mathrm{E}+05$ & $7.51 \mathrm{E}+03$ & $2.03 \mathrm{E}+05$ & $8.91 \mathrm{E}+04$ & $2.41 \mathrm{E}+06$ & $2.45 \mathrm{E}+05$ & $6.62 \mathrm{E}+06$ & $6.05 \mathrm{E}+03$ & $6.62 \mathrm{E}+06$ \\
\hline La-142 & $5.38 \mathrm{E}+03$ & $1.46 \mathrm{E}+05$ & $5.34 \mathrm{E}+04$ & $1.44 \mathrm{E}+06$ & $1.66 \mathrm{E}+04$ & $4.48 \mathrm{E}+05$ & $1.97 \mathrm{E}+05$ & $5.32 \mathrm{E}+06$ & $5.41 \mathrm{E}+05$ & $1.46 \mathrm{E}+07$ & $1.34 \mathrm{E}+04$ & $1.46 \mathrm{E}+07$ \\
\hline La-143 & $1.66 \mathrm{E}+04$ & $4.48 \mathrm{E}+05$ & $1.64 \mathrm{E}+05$ & $4.44 \mathrm{E}+06$ & $5.10 \mathrm{E}+04$ & $1.38 \mathrm{E}+06$ & $6.05 \mathrm{E}+05$ & $1.64 \mathrm{E}+07$ & $1.66 \mathrm{E}+06$ & $4.50 \mathrm{E}+07$ & $4.11 \mathrm{E}+04$ & $4.50 \mathrm{E}+07$ \\
\hline Ce-130 & $1.33 \mathrm{E}+04$ & $3.59 \mathrm{E}+05$ & $1.32 \mathrm{E}+05$ & $3.56 \mathrm{E}+06$ & $4.09 \mathrm{E}+04$ & $1.11 \mathrm{E}+06$ & $4.85 \mathrm{E}+05$ & $1.31 \mathrm{E}+07$ & $1.33 \mathrm{E}+06$ & $3.61 \mathrm{E}+07$ & $3.29 \mathrm{E}+04$ & $3.61 \mathrm{E}+07$ \\
\hline Ce-131 & $3.44 \mathrm{E}+04$ & $9.29 \mathrm{E}+05$ & $3.41 \mathrm{E}+05$ & $9.22 \mathrm{E}+06$ & $1.06 \mathrm{E}+05$ & $2.86 \mathrm{E}+06$ & $1.26 \mathrm{E}+06$ & $3.39 \mathrm{E}+07$ & $3.45 \mathrm{E}+06$ & $9.33 \mathrm{E}+07$ & $8.53 \mathrm{E}+04$ & $9.33 \mathrm{E}+07$ \\
\hline Ce-132 & $2.81 \mathrm{E}+03$ & $7.61 \mathrm{E}+04$ & $2.79 \mathrm{E}+04$ & $7.55 \mathrm{E}+05$ & $8.67 \mathrm{E}+03$ & $2.34 \mathrm{E}+05$ & $1.03 \mathrm{E}+05$ & $2.78 \mathrm{E}+06$ & $2.83 \mathrm{E}+05$ & $7.64 \mathrm{E}+06$ & $6.98 \mathrm{E}+03$ & $7.64 \mathrm{E}+06$ \\
\hline Ce-133 & $1.03 \mathrm{E}+04$ & $2.78 \mathrm{E}+05$ & $1.02 \mathrm{E}+05$ & $2.76 \mathrm{E}+06$ & $3.17 \mathrm{E}+04$ & $8.56 \mathrm{E}+05$ & $3.76 \mathrm{E}+05$ & $1.02 \mathrm{E}+07$ & $1.03 \mathrm{E}+06$ & $2.79 \mathrm{E}+07$ & $2.55 \mathrm{E}+04$ & $2.79 \mathrm{E}+07$ \\
\hline $\mathrm{Ce}-133 \mathrm{~m}$ & $4.62 \mathrm{E}+03$ & $1.25 \mathrm{E}+05$ & $4.59 \mathrm{E}+04$ & $1.24 \mathrm{E}+06$ & $1.42 \mathrm{E}+04$ & $3.85 \mathrm{E}+05$ & $1.69 \mathrm{E}+05$ & $4.57 \mathrm{E}+06$ & $4.65 \mathrm{E}+05$ & $1.26 \mathrm{E}+07$ & $1.15 \mathrm{E}+04$ & $1.26 \mathrm{E}+07$ \\
\hline $\mathrm{Ce}-134$ & $3.43 \mathrm{E}+02$ & $9.26 \mathrm{E}+03$ & $3.40 \mathrm{E}+03$ & $9.19 \mathrm{E}+04$ & $1.06 \mathrm{E}+03$ & $2.85 \mathrm{E}+04$ & $1.25 \mathrm{E}+04$ & $3.38 \mathrm{E}+05$ & $3.44 \mathrm{E}+04$ & $9.31 \mathrm{E}+05$ & $8.50 \mathrm{E}+02$ & $9.31 \mathrm{E}+05$ \\
\hline Ce-135 & $3.71 \mathrm{E}+03$ & $1.00 \mathrm{E}+05$ & $3.69 \mathrm{E}+04$ & $9.96 \mathrm{E}+05$ & $1.14 \mathrm{E}+04$ & $3.09 \mathrm{E}+05$ & $1.36 \mathrm{E}+05$ & $3.67 \mathrm{E}+06$ & $3.73 \mathrm{E}+05$ & $1.01 \mathrm{E}+07$ & $9.21 \mathrm{E}+03$ & $1.01 \mathrm{E}+07$ \\
\hline $\mathrm{Ce}-137$ & $3.48 \mathrm{E}+04$ & $9.42 \mathrm{E}+05$ & $3.46 \mathrm{E}+05$ & $9.35 \mathrm{E}+06$ & $1.07 \mathrm{E}+05$ & $2.90 \mathrm{E}+06$ & $1.27 \mathrm{E}+06$ & $3.44 \mathrm{E}+07$ & $3.50 \mathrm{E}+06$ & $9.46 \mathrm{E}+07$ & $8.65 \mathrm{E}+04$ & $9.46 \mathrm{E}+07$ \\
\hline $\mathrm{Ce}-137 \mathrm{~m}$ & $1.64 \mathrm{E}+03$ & $4.43 \mathrm{E}+04$ & $1.63 \mathrm{E}+04$ & $4.40 \mathrm{E}+05$ & $5.05 \mathrm{E}+03$ & $1.36 \mathrm{E}+05$ & $5.99 \mathrm{E}+04$ & $1.62 \mathrm{E}+06$ & $1.65 \mathrm{E}+05$ & $4.45 \mathrm{E}+06$ & $4.07 \mathrm{E}+03$ & $4.45 \mathrm{E}+06$ \\
\hline Ce-139 & $3.55 \mathrm{E}+03$ & $9.60 \mathrm{E}+04$ & $3.53 \mathrm{E}+04$ & $9.53 \mathrm{E}+05$ & $1.09 \mathrm{E}+04$ & $2.96 \mathrm{E}+05$ & $1.30 \mathrm{E}+05$ & $3.51 \mathrm{E}+06$ & $3.57 \mathrm{E}+05$ & $9.65 \mathrm{E}+06$ & $8.82 \mathrm{E}+03$ & $9.65 \mathrm{E}+06$ \\
\hline Ce-141 & $1.27 \mathrm{E}+03$ & $3.44 \mathrm{E}+04$ & $1.26 \mathrm{E}+04$ & $3.41 \mathrm{E}+05$ & $3.92 \mathrm{E}+03$ & $1.06 \mathrm{E}+05$ & $4.65 \mathrm{E}+04$ & $1.26 \mathrm{E}+06$ & $1.28 \mathrm{E}+05$ & $3.45 \mathrm{E}+06$ & $3.16 \mathrm{E}+03$ & $3.45 \mathrm{E}+06$ \\
\hline Ce-143 & $8.08 \mathrm{E}+02$ & $2.18 \mathrm{E}+04$ & $8.02 \mathrm{E}+03$ & $2.17 \mathrm{E}+05$ & $2.49 \mathrm{E}+03$ & $6.72 \mathrm{E}+04$ & $2.95 \mathrm{E}+04$ & $7.98 \mathrm{E}+05$ & $8.12 \mathrm{E}+04$ & $2.19 \mathrm{E}+06$ & $2.00 \mathrm{E}+03$ & $2.19 \mathrm{E}+06$ \\
\hline Ce-144 & $1.72 \mathrm{E}+02$ & $4.64 \mathrm{E}+03$ & $1.70 \mathrm{E}+03$ & $4.60 \mathrm{E}+04$ & $5.28 \mathrm{E}+02$ & $1.43 \mathrm{E}+04$ & $6.27 \mathrm{E}+03$ & $1.69 \mathrm{E}+05$ & $1.72 \mathrm{E}+04$ & $4.66 \mathrm{E}+05$ & $4.26 \mathrm{E}+02$ & $4.66 \mathrm{E}+05$ \\
\hline Pr-134 & $1.93 \mathrm{E}+04$ & $5.20 \mathrm{E}+05$ & $1.91 \mathrm{E}+05$ & $5.17 \mathrm{E}+06$ & $5.93 \mathrm{E}+04$ & $1.60 \mathrm{E}+06$ & $7.04 \mathrm{E}+05$ & $1.90 \mathrm{E}+07$ & $1.93 \mathrm{E}+06$ & $5.23 \mathrm{E}+07$ & $4.78 \mathrm{E}+04$ & $5.23 \mathrm{E}+07$ \\
\hline Pr- $134 \mathrm{~m}$ & $1.11 \mathrm{E}+04$ & $3.00 \mathrm{E}+05$ & $1.10 \mathrm{E}+05$ & $2.98 \mathrm{E}+06$ & $3.42 \mathrm{E}+04$ & $9.25 \mathrm{E}+05$ & $4.06 \mathrm{E}+05$ & $1.10 \mathrm{E}+07$ & $1.12 \mathrm{E}+06$ & $3.02 \mathrm{E}+07$ & $2.76 \mathrm{E}+04$ & $3.02 \mathrm{E}+07$ \\
\hline
\end{tabular}

Page 185 of 298 
SRNL-STI-2013-00115

\begin{tabular}{|c|c|c|c|c|c|c|c|c|c|c|c|c|}
\hline \multirow[t]{2}{*}{ Nuclide } & \multicolumn{2}{|c|}{ Water Ingestion DCS } & \multicolumn{2}{|c|}{ Meat Ingestion DCS } & \multicolumn{2}{|c|}{ Dairy Ingestion DCS } & \multicolumn{2}{|c|}{$\begin{array}{c}\text { Freshwater Fish } \\
\text { Ingestion DCS }\end{array}$} & \multicolumn{2}{|c|}{$\begin{array}{l}\text { Saltwater Shellfish } \\
\text { Ingestion DCS }\end{array}$} & \multicolumn{2}{|c|}{ Produce Ingestion DCS } \\
\hline & $(\mathrm{Bq} / \mathrm{L})$ & $(\mathrm{pCi} / \mathrm{L})$ & $(\mathrm{Bq} / \mathrm{kg})$ & $(\mathrm{pCi} / \mathrm{kg})$ & $(\mathrm{Bq} / \mathrm{L})$ & $(\mathrm{pCi} / \mathrm{L})$ & $(\mathrm{Bq} / \mathbf{k g})$ & $(\mathrm{pCi} / \mathbf{k g})$ & $(\mathrm{Bq} / \mathbf{k g})$ & $(\mathrm{pCi} / \mathbf{k g})$ & $(\mathrm{Bq} / \mathbf{k g})$ & $(\mathrm{pCi} / \mathbf{k g})$ \\
\hline Pr-135 & $2.17 \mathrm{E}+04$ & $5.87 \mathrm{E}+05$ & $2.15 \mathrm{E}+05$ & $5.82 \mathrm{E}+06$ & $6.69 \mathrm{E}+04$ & $1.81 \mathrm{E}+06$ & $7.93 \mathrm{E}+05$ & $2.14 \mathrm{E}+07$ & $2.18 \mathrm{E}+06$ & $5.90 \mathrm{E}+07$ & $5.39 \mathrm{E}+04$ & $5.90 \mathrm{E}+07$ \\
\hline Pr-136 & $2.78 \mathrm{E}+04$ & $7.52 \mathrm{E}+05$ & $2.76 \mathrm{E}+05$ & $7.46 \mathrm{E}+06$ & $8.57 \mathrm{E}+04$ & $2.32 \mathrm{E}+06$ & $1.02 \mathrm{E}+06$ & $2.75 \mathrm{E}+07$ & $2.80 \mathrm{E}+06$ & $7.56 \mathrm{E}+07$ & $6.90 \mathrm{E}+04$ & $7.56 \mathrm{E}+07$ \\
\hline Pr-137 & $2.55 \mathrm{E}+04$ & $6.90 \mathrm{E}+05$ & $2.54 \mathrm{E}+05$ & $6.85 \mathrm{E}+06$ & $7.87 \mathrm{E}+04$ & $2.13 \mathrm{E}+06$ & $9.33 \mathrm{E}+05$ & $2.52 \mathrm{E}+07$ & $2.57 \mathrm{E}+06$ & $6.94 \mathrm{E}+07$ & $6.34 \mathrm{E}+04$ & $6.94 \mathrm{E}+07$ \\
\hline Pr- $138 \mathrm{~m}$ & $7.45 \mathrm{E}+03$ & $2.01 \mathrm{E}+05$ & $7.39 \mathrm{E}+04$ & $2.00 \mathrm{E}+06$ & $2.29 \mathrm{E}+04$ & $6.20 \mathrm{E}+05$ & $2.72 \mathrm{E}+05$ & $7.36 \mathrm{E}+06$ & $7.49 \mathrm{E}+05$ & $2.02 \mathrm{E}+07$ & $1.85 \mathrm{E}+04$ & $2.02 \mathrm{E}+07$ \\
\hline Pr-139 & $2.87 \mathrm{E}+04$ & $7.75 \mathrm{E}+05$ & $2.84 \mathrm{E}+05$ & $7.69 \mathrm{E}+06$ & $8.83 \mathrm{E}+04$ & $2.39 \mathrm{E}+06$ & $1.05 \mathrm{E}+06$ & $2.83 \mathrm{E}+07$ & $2.88 \mathrm{E}+06$ & $7.78 \mathrm{E}+07$ & $7.11 \mathrm{E}+04$ & $7.78 \mathrm{E}+07$ \\
\hline Pr-142 & $6.83 \mathrm{E}+02$ & $1.85 \mathrm{E}+04$ & $6.78 \mathrm{E}+03$ & $1.83 \mathrm{E}+05$ & $2.11 \mathrm{E}+03$ & $5.69 \mathrm{E}+04$ & $2.50 \mathrm{E}+04$ & $6.75 \mathrm{E}+05$ & $6.87 \mathrm{E}+04$ & $1.86 \mathrm{E}+06$ & $1.70 \mathrm{E}+03$ & $1.86 \mathrm{E}+06$ \\
\hline Pr- $142 \mathrm{~m}$ & $5.36 \mathrm{E}+04$ & $1.45 \mathrm{E}+06$ & $5.32 \mathrm{E}+05$ & $1.44 \mathrm{E}+07$ & $1.65 \mathrm{E}+05$ & $4.46 \mathrm{E}+06$ & $1.96 \mathrm{E}+06$ & $5.30 \mathrm{E}+07$ & $5.39 \mathrm{E}+06$ & $1.46 \mathrm{E}+08$ & $1.33 \mathrm{E}+05$ & $1.46 \mathrm{E}+08$ \\
\hline Pr-143 & $7.68 \mathrm{E}+02$ & $2.08 \mathrm{E}+04$ & $7.62 \mathrm{E}+03$ & $2.06 \mathrm{E}+05$ & $2.37 \mathrm{E}+03$ & $6.39 \mathrm{E}+04$ & $2.81 \mathrm{E}+04$ & $7.58 \mathrm{E}+05$ & $7.72 \mathrm{E}+04$ & $2.09 \mathrm{E}+06$ & $1.91 \mathrm{E}+03$ & $2.09 \mathrm{E}+06$ \\
\hline Pr-144 & $1.82 \mathrm{E}+04$ & $4.93 \mathrm{E}+05$ & $1.81 \mathrm{E}+05$ & $4.89 \mathrm{E}+06$ & $5.62 \mathrm{E}+04$ & $1.52 \mathrm{E}+06$ & $6.66 \mathrm{E}+05$ & $1.80 \mathrm{E}+07$ & $1.83 \mathrm{E}+06$ & $4.95 \mathrm{E}+07$ & $4.53 \mathrm{E}+04$ & $4.95 \mathrm{E}+07$ \\
\hline Pr-145 & $2.29 \mathrm{E}+03$ & $6.18 \mathrm{E}+04$ & $2.27 \mathrm{E}+04$ & $6.13 \mathrm{E}+05$ & $7.04 \mathrm{E}+03$ & $1.90 \mathrm{E}+05$ & $8.36 \mathrm{E}+04$ & $2.26 \mathrm{E}+06$ & $2.30 \mathrm{E}+05$ & $6.21 \mathrm{E}+06$ & $5.67 \mathrm{E}+03$ & $6.21 \mathrm{E}+06$ \\
\hline Pr-146 & $1.20 \mathrm{E}+04$ & $3.23 \mathrm{E}+05$ & $1.19 \mathrm{E}+05$ & $3.21 \mathrm{E}+06$ & $3.68 \mathrm{E}+04$ & $9.96 \mathrm{E}+05$ & $4.37 \mathrm{E}+05$ & $1.18 \mathrm{E}+07$ & $1.20 \mathrm{E}+06$ & $3.25 \mathrm{E}+07$ & $2.97 \mathrm{E}+04$ & $3.25 \mathrm{E}+07$ \\
\hline Pr-147 & $2.75 \mathrm{E}+04$ & $7.44 \mathrm{E}+05$ & $2.73 \mathrm{E}+05$ & $7.38 \mathrm{E}+06$ & $8.48 \mathrm{E}+04$ & $2.29 \mathrm{E}+06$ & $1.01 \mathrm{E}+06$ & $2.72 \mathrm{E}+07$ & $2.77 \mathrm{E}+06$ & $7.47 \mathrm{E}+07$ & $6.83 \mathrm{E}+04$ & $7.47 \mathrm{E}+07$ \\
\hline Nd-135 & $1.60 \mathrm{E}+04$ & $4.33 \mathrm{E}+05$ & $1.59 \mathrm{E}+05$ & $4.29 \mathrm{E}+06$ & $4.93 \mathrm{E}+04$ & $1.33 \mathrm{E}+06$ & $5.85 \mathrm{E}+05$ & $1.58 \mathrm{E}+07$ & $1.61 \mathrm{E}+06$ & $4.35 \mathrm{E}+07$ & $3.97 \mathrm{E}+04$ & $4.35 \mathrm{E}+07$ \\
\hline Nd-136 & $9.57 \mathrm{E}+03$ & $2.59 \mathrm{E}+05$ & $9.50 \mathrm{E}+04$ & $2.57 \mathrm{E}+06$ & $2.95 \mathrm{E}+04$ & $7.97 \mathrm{E}+05$ & $3.50 \mathrm{E}+05$ & $9.45 \mathrm{E}+06$ & $9.62 \mathrm{E}+05$ & $2.60 \mathrm{E}+07$ & $2.37 \mathrm{E}+04$ & $2.60 \mathrm{E}+07$ \\
\hline Nd-137 & $1.77 \mathrm{E}+04$ & $4.78 \mathrm{E}+05$ & $1.76 \mathrm{E}+05$ & $4.75 \mathrm{E}+06$ & $5.45 \mathrm{E}+04$ & $1.47 \mathrm{E}+06$ & $6.47 \mathrm{E}+05$ & $1.75 \mathrm{E}+07$ & $1.78 \mathrm{E}+06$ & $4.81 \mathrm{E}+07$ & $4.39 \mathrm{E}+04$ & $4.81 \mathrm{E}+07$ \\
\hline Nd-138 & $1.43 \mathrm{E}+03$ & $3.86 \mathrm{E}+04$ & $1.42 \mathrm{E}+04$ & $3.83 \mathrm{E}+05$ & $4.40 \mathrm{E}+03$ & $1.19 \mathrm{E}+05$ & $5.22 \mathrm{E}+04$ & $1.41 \mathrm{E}+06$ & $1.44 \mathrm{E}+05$ & $3.88 \mathrm{E}+06$ & $3.54 \mathrm{E}+03$ & $3.88 \mathrm{E}+06$ \\
\hline Nd-139 & $4.56 \mathrm{E}+04$ & $1.23 \mathrm{E}+06$ & $4.52 \mathrm{E}+05$ & $1.22 \mathrm{E}+07$ & $1.40 \mathrm{E}+05$ & $3.79 \mathrm{E}+06$ & $1.67 \mathrm{E}+06$ & $4.50 \mathrm{E}+07$ & $4.58 \mathrm{E}+06$ & $1.24 \mathrm{E}+08$ & $1.13 \mathrm{E}+05$ & $1.24 \mathrm{E}+08$ \\
\hline Nd-139m & $4.01 \mathrm{E}+03$ & $1.08 \mathrm{E}+05$ & $3.98 \mathrm{E}+04$ & $1.08 \mathrm{E}+06$ & $1.24 \mathrm{E}+04$ & $3.34 \mathrm{E}+05$ & $1.47 \mathrm{E}+05$ & $3.96 \mathrm{E}+06$ & $4.03 \mathrm{E}+05$ & $1.09 \mathrm{E}+07$ & $9.96 \mathrm{E}+03$ & $1.09 \mathrm{E}+07$ \\
\hline Nd-140 & $4.59 \mathrm{E}+02$ & $1.24 \mathrm{E}+04$ & $4.56 \mathrm{E}+03$ & $1.23 \mathrm{E}+05$ & $1.41 \mathrm{E}+03$ & $3.82 \mathrm{E}+04$ & $1.68 \mathrm{E}+04$ & $4.53 \mathrm{E}+05$ & $4.61 \mathrm{E}+04$ & $1.25 \mathrm{E}+06$ & $1.14 \mathrm{E}+03$ & $1.25 \mathrm{E}+06$ \\
\hline Nd-141 & $1.10 \mathrm{E}+05$ & $2.97 \mathrm{E}+06$ & $1.09 \mathrm{E}+06$ & $2.95 \mathrm{E}+07$ & $3.39 \mathrm{E}+05$ & $9.16 \mathrm{E}+06$ & $4.02 \mathrm{E}+06$ & $1.09 \mathrm{E}+08$ & $1.11 \mathrm{E}+07$ & $2.99 \mathrm{E}+08$ & $2.73 \mathrm{E}+05$ & $2.99 \mathrm{E}+08$ \\
\hline Nd-144 & $2.36 \mathrm{E}+01$ & $6.37 \mathrm{E}+02$ & $2.34 \mathrm{E}+02$ & $6.32 \mathrm{E}+03$ & $7.26 \mathrm{E}+01$ & $1.96 \mathrm{E}+03$ & $8.61 \mathrm{E}+02$ & $2.33 \mathrm{E}+04$ & $2.37 \mathrm{E}+03$ & $6.40 \mathrm{E}+04$ & $5.85 \mathrm{E}+01$ & $6.40 \mathrm{E}+04$ \\
\hline Nd-147 & $8.40 \mathrm{E}+02$ & $2.27 \mathrm{E}+04$ & $8.34 \mathrm{E}+03$ & $2.25 \mathrm{E}+05$ & $2.59 \mathrm{E}+03$ & $7.00 \mathrm{E}+04$ & $3.07 \mathrm{E}+04$ & $8.30 \mathrm{E}+05$ & $8.45 \mathrm{E}+04$ & $2.28 \mathrm{E}+06$ & $2.09 \mathrm{E}+03$ & $2.28 \mathrm{E}+06$ \\
\hline Nd-149 & $7.36 \mathrm{E}+03$ & $1.99 \mathrm{E}+05$ & $7.31 \mathrm{E}+04$ & $1.97 \mathrm{E}+06$ & $2.27 \mathrm{E}+04$ & $6.13 E+05$ & $2.69 \mathrm{E}+05$ & $7.27 \mathrm{E}+06$ & $7.40 \mathrm{E}+05$ & $2.00 \mathrm{E}+07$ & $1.83 \mathrm{E}+04$ & $2.00 \mathrm{E}+07$ \\
\hline
\end{tabular}

Page 186 of 298 
SRNL-STI-2013-00115

\begin{tabular}{|c|c|c|c|c|c|c|c|c|c|c|c|c|}
\hline \multirow[t]{2}{*}{ Nuclide } & \multicolumn{2}{|c|}{ Water Ingestion DCS } & \multicolumn{2}{|c|}{ Meat Ingestion DCS } & \multicolumn{2}{|c|}{ Dairy Ingestion DCS } & \multicolumn{2}{|c|}{$\begin{array}{l}\text { Freshwater Fish } \\
\text { Ingestion DCS }\end{array}$} & \multicolumn{2}{|c|}{$\begin{array}{l}\text { Saltwater Shellfish } \\
\text { Ingestion DCS }\end{array}$} & \multicolumn{2}{|c|}{ Produce Ingestion DCS } \\
\hline & $(\mathrm{Bq} / \mathrm{L})$ & $(\mathrm{pCi} / \mathrm{L})$ & $(\mathrm{Bq} / \mathrm{kg})$ & $(\mathrm{pCi} / \mathrm{kg})$ & $(\mathrm{Bq} / \mathrm{L})$ & $(\mathrm{pCi} / \mathrm{L})$ & $(\mathrm{Bq} / \mathbf{k g})$ & $(\mathrm{pCi} / \mathbf{k g})$ & $(\mathrm{Bq} / \mathbf{k g})$ & $(\mathrm{pCi} / \mathbf{k g})$ & $(\mathrm{Bq} / \mathbf{k g})$ & $(\mathrm{pCi} / \mathbf{k g})$ \\
\hline $\mathrm{Nd}-151$ & $3.26 \mathrm{E}+04$ & $8.80 \mathrm{E}+05$ & $3.23 \mathrm{E}+05$ & $8.73 \mathrm{E}+06$ & $1.00 \mathrm{E}+05$ & $2.71 \mathrm{E}+06$ & $1.19 \mathrm{E}+06$ & $3.22 \mathrm{E}+07$ & $3.27 \mathrm{E}+06$ & $8.84 \mathrm{E}+07$ & $8.08 \mathrm{E}+04$ & $8.84 \mathrm{E}+07$ \\
\hline Nd-152 & $1.91 \mathrm{E}+04$ & $5.17 \mathrm{E}+05$ & $1.90 \mathrm{E}+05$ & $5.13 \mathrm{E}+06$ & $5.89 \mathrm{E}+04$ & $1.59 \mathrm{E}+06$ & $6.99 \mathrm{E}+05$ & $1.89 \mathrm{E}+07$ & $1.92 \mathrm{E}+06$ & $5.20 \mathrm{E}+07$ & $4.75 \mathrm{E}+04$ & $5.20 \mathrm{E}+07$ \\
\hline Pm-141 & $2.67 \mathrm{E}+04$ & $7.21 \mathrm{E}+05$ & $2.65 \mathrm{E}+05$ & $7.16 \mathrm{E}+06$ & $8.22 \mathrm{E}+04$ & $2.22 \mathrm{E}+06$ & $9.75 \mathrm{E}+05$ & $2.64 \mathrm{E}+07$ & $2.68 \mathrm{E}+06$ & $7.25 \mathrm{E}+07$ & $6.62 \mathrm{E}+04$ & $7.25 \mathrm{E}+07$ \\
\hline Pm-143 & $4.15 \mathrm{E}+03$ & $1.12 \mathrm{E}+05$ & $4.12 \mathrm{E}+04$ & $1.11 \mathrm{E}+06$ & $1.28 \mathrm{E}+04$ & $3.45 \mathrm{E}+05$ & $1.52 \mathrm{E}+05$ & $4.10 \mathrm{E}+06$ & $4.17 \mathrm{E}+05$ & $1.13 \mathrm{E}+07$ & $1.03 \mathrm{E}+04$ & $1.13 \mathrm{E}+07$ \\
\hline $\mathrm{Pm}-144$ & $1.00 \mathrm{E}+03$ & $2.71 \mathrm{E}+04$ & $9.96 \mathrm{E}+03$ & $2.69 \mathrm{E}+05$ & $3.09 \mathrm{E}+03$ & $8.35 \mathrm{E}+04$ & $3.67 \mathrm{E}+04$ & $9.91 \mathrm{E}+05$ & $1.01 \mathrm{E}+05$ & $2.72 \mathrm{E}+06$ & $2.49 \mathrm{E}+03$ & $2.72 \mathrm{E}+06$ \\
\hline Pm-145 & $8.40 \mathrm{E}+03$ & $2.27 \mathrm{E}+05$ & $8.34 \mathrm{E}+04$ & $2.25 \mathrm{E}+06$ & $2.59 \mathrm{E}+04$ & $7.00 \mathrm{E}+05$ & $3.07 \mathrm{E}+05$ & $8.30 \mathrm{E}+06$ & $8.45 \mathrm{E}+05$ & $2.28 \mathrm{E}+07$ & $2.09 \mathrm{E}+04$ & $2.28 \mathrm{E}+07$ \\
\hline Pm-146 & $1.05 \mathrm{E}+03$ & $2.85 \mathrm{E}+04$ & $1.05 \mathrm{E}+04$ & $2.83 \mathrm{E}+05$ & $3.25 \mathrm{E}+03$ & $8.78 \mathrm{E}+04$ & $3.85 \mathrm{E}+04$ & $1.04 \mathrm{E}+06$ & $1.06 \mathrm{E}+05$ & $2.86 \mathrm{E}+06$ & $2.62 \mathrm{E}+03$ & $2.86 \mathrm{E}+06$ \\
\hline Pm-147 & $3.43 \mathrm{E}+03$ & $9.26 \mathrm{E}+04$ & $3.40 \mathrm{E}+04$ & $9.19 \mathrm{E}+05$ & $1.06 \mathrm{E}+04$ & $2.85 \mathrm{E}+05$ & $1.25 \mathrm{E}+05$ & $3.38 \mathrm{E}+06$ & $3.44 \mathrm{E}+05$ & $9.31 \mathrm{E}+06$ & $8.50 \mathrm{E}+03$ & $9.31 \mathrm{E}+06$ \\
\hline Pm-148 & $3.38 \mathrm{E}+02$ & $9.13 \mathrm{E}+03$ & $3.35 \mathrm{E}+03$ & $9.07 \mathrm{E}+04$ & $1.04 \mathrm{E}+03$ & $2.81 \mathrm{E}+04$ & $1.24 \mathrm{E}+04$ & $3.34 \mathrm{E}+05$ & $3.40 \mathrm{E}+04$ & $9.18 \mathrm{E}+05$ & $8.39 \mathrm{E}+02$ & $9.18 \mathrm{E}+05$ \\
\hline Pm-148m & $5.46 \mathrm{E}+02$ & $1.47 \mathrm{E}+04$ & $5.41 \mathrm{E}+03$ & $1.46 \mathrm{E}+05$ & $1.68 \mathrm{E}+03$ & $4.54 \mathrm{E}+04$ & $1.99 \mathrm{E}+04$ & $5.39 \mathrm{E}+05$ & $5.48 \mathrm{E}+04$ & $1.48 \mathrm{E}+06$ & $1.35 \mathrm{E}+03$ & $1.48 \mathrm{E}+06$ \\
\hline Pm-149 & $9.08 \mathrm{E}+02$ & $2.45 \mathrm{E}+04$ & $9.01 \mathrm{E}+03$ & $2.44 \mathrm{E}+05$ & $2.80 \mathrm{E}+03$ & $7.56 \mathrm{E}+04$ & $3.32 \mathrm{E}+04$ & $8.97 \mathrm{E}+05$ & $9.12 \mathrm{E}+04$ & $2.47 \mathrm{E}+06$ & $2.25 \mathrm{E}+03$ & $2.47 \mathrm{E}+06$ \\
\hline Pm-150 & $3.56 \mathrm{E}+03$ & $9.63 \mathrm{E}+04$ & $3.54 \mathrm{E}+04$ & $9.56 \mathrm{E}+05$ & $1.10 \mathrm{E}+04$ & $2.97 \mathrm{E}+05$ & $1.30 \mathrm{E}+05$ & $3.52 \mathrm{E}+06$ & $3.58 \mathrm{E}+05$ & $9.68 \mathrm{E}+06$ & $8.84 \mathrm{E}+03$ & $9.68 \mathrm{E}+06$ \\
\hline Pm-151 & $1.25 \mathrm{E}+03$ & $3.37 \mathrm{E}+04$ & $1.24 \mathrm{E}+04$ & $3.35 \mathrm{E}+05$ & $3.84 \mathrm{E}+03$ & $1.04 \mathrm{E}+05$ & $4.56 \mathrm{E}+04$ & $1.23 \mathrm{E}+06$ & $1.25 \mathrm{E}+05$ & $3.39 \mathrm{E}+06$ & $3.10 \mathrm{E}+03$ & $3.39 \mathrm{E}+06$ \\
\hline Sm-140 & $9.64 \mathrm{E}+03$ & $2.61 \mathrm{E}+05$ & $9.57 \mathrm{E}+04$ & $2.59 \mathrm{E}+06$ & $2.97 \mathrm{E}+04$ & $8.03 \mathrm{E}+05$ & $3.52 \mathrm{E}+05$ & $9.52 \mathrm{E}+06$ & $9.69 \mathrm{E}+05$ & $2.62 \mathrm{E}+07$ & $2.39 \mathrm{E}+04$ & $2.62 \mathrm{E}+07$ \\
\hline Sm-141 & $2.42 \mathrm{E}+04$ & $6.54 \mathrm{E}+05$ & $2.40 \mathrm{E}+05$ & $6.49 \mathrm{E}+06$ & $7.45 \mathrm{E}+04$ & $2.01 \mathrm{E}+06$ & $8.84 \mathrm{E}+05$ & $2.39 \mathrm{E}+07$ & $2.43 \mathrm{E}+06$ & $6.57 \mathrm{E}+07$ & $6.00 \mathrm{E}+04$ & $6.57 \mathrm{E}+07$ \\
\hline Sm-141m & $1.52 \mathrm{E}+04$ & $4.11 \mathrm{E}+05$ & $1.51 \mathrm{E}+05$ & $4.08 \mathrm{E}+06$ & $4.68 \mathrm{E}+04$ & $1.27 \mathrm{E}+06$ & $5.56 \mathrm{E}+05$ & $1.50 \mathrm{E}+07$ & $1.53 \mathrm{E}+06$ & $4.13 \mathrm{E}+07$ & $3.77 \mathrm{E}+04$ & $4.13 \mathrm{E}+07$ \\
\hline Sm-142 & $5.12 \mathrm{E}+03$ & $1.38 \mathrm{E}+05$ & $5.08 \mathrm{E}+04$ & $1.37 \mathrm{E}+06$ & $1.58 \mathrm{E}+04$ & $4.26 \mathrm{E}+05$ & $1.87 \mathrm{E}+05$ & $5.06 \mathrm{E}+06$ & $5.14 \mathrm{E}+05$ & $1.39 \mathrm{E}+07$ & $1.27 \mathrm{E}+04$ & $1.39 \mathrm{E}+07$ \\
\hline Sm-145 & $4.35 \mathrm{E}+03$ & $1.18 \mathrm{E}+05$ & $4.32 \mathrm{E}+04$ & $1.17 \mathrm{E}+06$ & $1.34 \mathrm{E}+04$ & $3.62 \mathrm{E}+05$ & $1.59 \mathrm{E}+05$ & $4.30 \mathrm{E}+06$ & $4.37 \mathrm{E}+05$ & $1.18 \mathrm{E}+07$ & $1.08 \mathrm{E}+04$ & $1.18 \mathrm{E}+07$ \\
\hline Sm-146 & $1.77 \mathrm{E}+01$ & $4.80 \mathrm{E}+02$ & $1.76 \mathrm{E}+02$ & $4.76 \mathrm{E}+03$ & $5.47 \mathrm{E}+01$ & $1.48 \mathrm{E}+03$ & $6.48 \mathrm{E}+02$ & $1.75 \mathrm{E}+04$ & $1.78 \mathrm{E}+03$ & $4.82 \mathrm{E}+04$ & $4.40 \mathrm{E}+01$ & $4.82 \mathrm{E}+04$ \\
\hline Sm-147 & $1.94 \mathrm{E}+01$ & $5.25 \mathrm{E}+02$ & $1.93 \mathrm{E}+02$ & $5.21 \mathrm{E}+03$ & $5.99 \mathrm{E}+01$ & $1.62 \mathrm{E}+03$ & $7.10 \mathrm{E}+02$ & $1.92 \mathrm{E}+04$ & $1.95 \mathrm{E}+03$ & $5.28 \mathrm{E}+04$ & $4.82 \mathrm{E}+01$ & $5.28 \mathrm{E}+04$ \\
\hline Sm-148 & $2.26 \mathrm{E}+01$ & $6.11 \mathrm{E}+02$ & $2.24 \mathrm{E}+02$ & $6.07 \mathrm{E}+03$ & $6.97 \mathrm{E}+01$ & $1.88 \mathrm{E}+03$ & $8.26 \mathrm{E}+02$ & $2.23 \mathrm{E}+04$ & $2.27 \mathrm{E}+03$ & $6.14 \mathrm{E}+04$ & $5.61 \mathrm{E}+01$ & $6.14 \mathrm{E}+04$ \\
\hline Sm-151 & $9.21 \mathrm{E}+03$ & $2.49 \mathrm{E}+05$ & $9.14 \mathrm{E}+04$ & $2.47 \mathrm{E}+06$ & $2.84 \mathrm{E}+04$ & $7.67 \mathrm{E}+05$ & $3.37 \mathrm{E}+05$ & $9.10 \mathrm{E}+06$ & $9.26 \mathrm{E}+05$ & $2.50 \mathrm{E}+07$ & $2.29 \mathrm{E}+04$ & $2.50 \mathrm{E}+07$ \\
\hline Sm-153 & $1.23 \mathrm{E}+03$ & $3.33 \mathrm{E}+04$ & $1.22 \mathrm{E}+04$ & $3.30 \mathrm{E}+05$ & $3.79 \mathrm{E}+03$ & $1.03 \mathrm{E}+05$ & $4.50 \mathrm{E}+04$ & $1.22 \mathrm{E}+06$ & $1.24 \mathrm{E}+05$ & $3.34 \mathrm{E}+06$ & $3.06 \mathrm{E}+03$ & $3.34 \mathrm{E}+06$ \\
\hline
\end{tabular}

Page 187 of 298 
SRNL-STI-2013-00115

\begin{tabular}{|c|c|c|c|c|c|c|c|c|c|c|c|c|}
\hline \multirow[t]{2}{*}{ Nuclide } & \multicolumn{2}{|c|}{ Water Ingestion DCS } & \multicolumn{2}{|c|}{ Meat Ingestion DCS } & \multicolumn{2}{|c|}{ Dairy Ingestion DCS } & \multicolumn{2}{|c|}{$\begin{array}{l}\text { Freshwater Fish } \\
\text { Ingestion DCS }\end{array}$} & \multicolumn{2}{|c|}{$\begin{array}{l}\text { Saltwater Shellfish } \\
\text { Ingestion DCS }\end{array}$} & \multicolumn{2}{|c|}{ Produce Ingestion DCS } \\
\hline & $(\mathrm{Bq} / \mathbf{L})$ & $(\mathrm{pCi} / \mathrm{L})$ & $(\mathrm{Bq} / \mathrm{kg})$ & $(\mathrm{pCi} / \mathrm{kg})$ & $(\mathrm{Bq} / \mathrm{L})$ & $(\mathrm{pCi} / \mathrm{L})$ & $(\mathrm{Bq} / \mathrm{kg})$ & $(\mathrm{pCi} / \mathbf{k g})$ & $(\mathrm{Bq} / \mathbf{k g})$ & $(\mathrm{pCi} / \mathrm{kg})$ & $(\mathrm{Bq} / \mathrm{kg})$ & $(\mathrm{pCi} / \mathrm{kg})$ \\
\hline Sm-155 & $3.14 \mathrm{E}+04$ & $8.49 \mathrm{E}+05$ & $3.12 \mathrm{E}+05$ & $8.43 \mathrm{E}+06$ & $9.68 \mathrm{E}+04$ & $2.61 \mathrm{E}+06$ & $1.15 \mathrm{E}+06$ & $3.10 \mathrm{E}+07$ & $3.16 \mathrm{E}+06$ & $8.53 \mathrm{E}+07$ & $7.79 \mathrm{E}+04$ & $8.53 \mathrm{E}+07$ \\
\hline Sm-156 & $3.57 \mathrm{E}+03$ & $9.66 \mathrm{E}+04$ & $3.55 \mathrm{E}+04$ & $9.59 \mathrm{E}+05$ & $1.10 \mathrm{E}+04$ & $2.98 \mathrm{E}+05$ & $1.31 \mathrm{E}+05$ & $3.53 \mathrm{E}+06$ & $3.59 \mathrm{E}+05$ & $9.71 \mathrm{E}+06$ & $8.87 \mathrm{E}+03$ & $9.71 \mathrm{E}+06$ \\
\hline Eu-145 & $1.49 \mathrm{E}+03$ & $4.02 \mathrm{E}+04$ & $1.48 \mathrm{E}+04$ & $3.99 \mathrm{E}+05$ & $4.58 \mathrm{E}+03$ & $1.24 \mathrm{E}+05$ & $5.44 \mathrm{E}+04$ & $1.47 \mathrm{E}+06$ & $1.50 \mathrm{E}+05$ & $4.04 \mathrm{E}+06$ & $3.69 \mathrm{E}+03$ & $4.04 \mathrm{E}+06$ \\
\hline Eu-146 & $8.24 \mathrm{E}+02$ & $2.23 \mathrm{E}+04$ & $8.18 \mathrm{E}+03$ & $2.21 \mathrm{E}+05$ & $2.54 \mathrm{E}+03$ & $6.86 \mathrm{E}+04$ & $3.01 \mathrm{E}+04$ & $8.14 \mathrm{E}+05$ & $8.28 \mathrm{E}+04$ & $2.24 \mathrm{E}+06$ & $2.04 \mathrm{E}+03$ & $2.24 \mathrm{E}+06$ \\
\hline Eu-147 & $2.17 \mathrm{E}+03$ & $5.86 \mathrm{E}+04$ & $2.15 \mathrm{E}+04$ & $5.81 \mathrm{E}+05$ & $6.67 \mathrm{E}+03$ & $1.80 \mathrm{E}+05$ & $7.92 \mathrm{E}+04$ & $2.14 \mathrm{E}+06$ & $2.18 \mathrm{E}+05$ & $5.89 \mathrm{E}+06$ & $5.38 \mathrm{E}+03$ & $5.89 \mathrm{E}+06$ \\
\hline Eu-148 & $7.68 \mathrm{E}+02$ & $2.08 \mathrm{E}+04$ & $7.62 \mathrm{E}+03$ & $2.06 \mathrm{E}+05$ & $2.37 \mathrm{E}+03$ & $6.39 \mathrm{E}+04$ & $2.81 \mathrm{E}+04$ & $7.58 \mathrm{E}+05$ & $7.72 \mathrm{E}+04$ & $2.09 \mathrm{E}+06$ & $1.91 \mathrm{E}+03$ & $2.09 \mathrm{E}+06$ \\
\hline Eu-149 & $5.79 \mathrm{E}+03$ & $1.56 \mathrm{E}+05$ & $5.74 \mathrm{E}+04$ & $1.55 \mathrm{E}+06$ & $1.78 \mathrm{E}+04$ & $4.82 \mathrm{E}+05$ & $2.11 \mathrm{E}+05$ & $5.71 \mathrm{E}+06$ & $5.81 \mathrm{E}+05$ & $1.57 \mathrm{E}+07$ & $1.44 \mathrm{E}+04$ & $1.57 \mathrm{E}+07$ \\
\hline Eu-150 & $7.92 \mathrm{E}+02$ & $2.14 \mathrm{E}+04$ & $7.86 \mathrm{E}+03$ & $2.13 \mathrm{E}+05$ & $2.44 \mathrm{E}+03$ & $6.60 \mathrm{E}+04$ & $2.90 \mathrm{E}+04$ & $7.82 \mathrm{E}+05$ & $7.96 \mathrm{E}+04$ & $2.15 \mathrm{E}+06$ & $1.97 \mathrm{E}+03$ & $2.15 \mathrm{E}+06$ \\
\hline $\mathrm{Eu}-150 \mathrm{~m}$ & $2.36 \mathrm{E}+03$ & $6.38 \mathrm{E}+04$ & $2.34 \mathrm{E}+04$ & $6.33 \mathrm{E}+05$ & $7.27 \mathrm{E}+03$ & $1.96 \mathrm{E}+05$ & $8.63 \mathrm{E}+04$ & $2.33 \mathrm{E}+06$ & $2.37 \mathrm{E}+05$ & $6.41 \mathrm{E}+06$ & $5.86 \mathrm{E}+03$ & $6.41 \mathrm{E}+06$ \\
\hline Eu-152 & $7.15 \mathrm{E}+02$ & $1.93 \mathrm{E}+04$ & $7.10 \mathrm{E}+03$ & $1.92 \mathrm{E}+05$ & $2.20 \mathrm{E}+03$ & $5.95 \mathrm{E}+04$ & $2.61 \mathrm{E}+04$ & $7.06 \mathrm{E}+05$ & $7.18 \mathrm{E}+04$ & $1.94 \mathrm{E}+06$ & $1.77 \mathrm{E}+03$ & $1.94 \mathrm{E}+06$ \\
\hline $\mathrm{Eu}-152 \mathrm{~m}$ & $1.82 \mathrm{E}+03$ & $4.93 \mathrm{E}+04$ & $1.81 \mathrm{E}+04$ & $4.89 \mathrm{E}+05$ & $5.62 \mathrm{E}+03$ & $1.52 \mathrm{E}+05$ & $6.66 \mathrm{E}+04$ & $1.80 \mathrm{E}+06$ & $1.83 \mathrm{E}+05$ & $4.95 \mathrm{E}+06$ & $4.53 \mathrm{E}+03$ & $4.95 \mathrm{E}+06$ \\
\hline Eu-152n & $7.07 \mathrm{E}+04$ & $1.91 \mathrm{E}+06$ & $7.01 \mathrm{E}+05$ & $1.90 \mathrm{E}+07$ & $2.18 \mathrm{E}+05$ & $5.88 \mathrm{E}+06$ & $2.58 \mathrm{E}+06$ & $6.98 \mathrm{E}+07$ & $7.10 \mathrm{E}+06$ & $1.92 \mathrm{E}+08$ & $1.75 \mathrm{E}+05$ & $1.92 \mathrm{E}+08$ \\
\hline Eu-154 & $4.77 \mathrm{E}+02$ & $1.29 \mathrm{E}+04$ & $4.73 \mathrm{E}+03$ & $1.28 \mathrm{E}+05$ & $1.47 \mathrm{E}+03$ & $3.97 \mathrm{E}+04$ & $1.74 \mathrm{E}+04$ & $4.71 \mathrm{E}+05$ & $4.79 \mathrm{E}+04$ & $1.29 \mathrm{E}+06$ & $1.18 \mathrm{E}+03$ & $1.29 \mathrm{E}+06$ \\
\hline Eu-154m & $1.17 \mathrm{E}+05$ & $3.17 \mathrm{E}+06$ & $1.16 \mathrm{E}+06$ & $3.15 \mathrm{E}+07$ & $3.61 \mathrm{E}+05$ & $9.77 \mathrm{E}+06$ & $4.29 \mathrm{E}+06$ & $1.16 \mathrm{E}+08$ & $1.18 \mathrm{E}+07$ & $3.19 \mathrm{E}+08$ & $2.91 \mathrm{E}+05$ & $3.19 \mathrm{E}+08$ \\
\hline $\mathrm{Eu}-155$ & $2.75 \mathrm{E}+03$ & $7.44 \mathrm{E}+04$ & $2.73 \mathrm{E}+04$ & $7.38 \mathrm{E}+05$ & $8.48 \mathrm{E}+03$ & $2.29 \mathrm{E}+05$ & $1.01 \mathrm{E}+05$ & $2.72 \mathrm{E}+06$ & $2.77 \mathrm{E}+05$ & $7.47 \mathrm{E}+06$ & $6.83 \mathrm{E}+03$ & $7.47 \mathrm{E}+06$ \\
\hline Eu-156 & $4.05 \mathrm{E}+02$ & $1.09 \mathrm{E}+04$ & $4.02 \mathrm{E}+03$ & $1.09 \mathrm{E}+05$ & $1.25 \mathrm{E}+03$ & $3.37 \mathrm{E}+04$ & $1.48 \mathrm{E}+04$ & $4.00 \mathrm{E}+05$ & $4.07 \mathrm{E}+04$ & $1.10 \mathrm{E}+06$ & $1.01 \mathrm{E}+03$ & $1.10 \mathrm{E}+06$ \\
\hline Eu-157 & $1.49 \mathrm{E}+03$ & $4.02 \mathrm{E}+04$ & $1.47 \mathrm{E}+04$ & $3.99 \mathrm{E}+05$ & $4.58 \mathrm{E}+03$ & $1.24 \mathrm{E}+05$ & $5.43 \mathrm{E}+04$ & $1.47 \mathrm{E}+06$ & $1.49 \mathrm{E}+05$ & $4.04 \mathrm{E}+06$ & $3.69 \mathrm{E}+03$ & $4.04 \mathrm{E}+06$ \\
\hline Eu-158 & $1.03 \mathrm{E}+04$ & $2.78 \mathrm{E}+05$ & $1.02 \mathrm{E}+05$ & $2.76 \mathrm{E}+06$ & $3.17 \mathrm{E}+04$ & $8.56 \mathrm{E}+05$ & $3.76 \mathrm{E}+05$ & $1.02 \mathrm{E}+07$ & $1.03 \mathrm{E}+06$ & $2.79 \mathrm{E}+07$ & $2.55 \mathrm{E}+04$ & $2.79 \mathrm{E}+07$ \\
\hline Eu-159 & $1.89 \mathrm{E}+04$ & $5.12 \mathrm{E}+05$ & $1.88 \mathrm{E}+05$ & $5.08 \mathrm{E}+06$ & $5.83 \mathrm{E}+04$ & $1.58 \mathrm{E}+06$ & $6.92 \mathrm{E}+05$ & $1.87 \mathrm{E}+07$ & $1.90 \mathrm{E}+06$ & $5.14 \mathrm{E}+07$ & $4.70 \mathrm{E}+04$ & $5.14 \mathrm{E}+07$ \\
\hline Gd-145 & $2.83 \mathrm{E}+04$ & $7.64 \mathrm{E}+05$ & $2.81 \mathrm{E}+05$ & $7.58 \mathrm{E}+06$ & $8.71 \mathrm{E}+04$ & $2.35 \mathrm{E}+06$ & $1.03 \mathrm{E}+06$ & $2.79 \mathrm{E}+07$ & $2.84 \mathrm{E}+06$ & $7.68 \mathrm{E}+07$ & $7.01 \mathrm{E}+04$ & $7.68 \mathrm{E}+07$ \\
\hline Gd-146 & $9.87 \mathrm{E}+02$ & $2.67 \mathrm{E}+04$ & $9.80 \mathrm{E}+03$ & $2.65 \mathrm{E}+05$ & $3.04 \mathrm{E}+03$ & $8.22 \mathrm{E}+04$ & $3.61 \mathrm{E}+04$ & $9.75 \mathrm{E}+05$ & $9.92 \mathrm{E}+04$ & $2.68 \mathrm{E}+06$ & $2.45 \mathrm{E}+03$ & $2.68 \mathrm{E}+06$ \\
\hline Gd-147 & $1.53 \mathrm{E}+03$ & $4.12 \mathrm{E}+04$ & $1.51 \mathrm{E}+04$ & $4.09 \mathrm{E}+05$ & $4.70 \mathrm{E}+03$ & $1.27 \mathrm{E}+05$ & $5.58 \mathrm{E}+04$ & $1.51 \mathrm{E}+06$ & $1.53 \mathrm{E}+05$ & $4.15 \mathrm{E}+06$ & $3.79 \mathrm{E}+03$ & $4.15 \mathrm{E}+06$ \\
\hline Gd-148 & $1.73 \mathrm{E}+01$ & $4.68 \mathrm{E}+02$ & $1.72 \mathrm{E}+02$ & $4.64 \mathrm{E}+03$ & $5.33 \mathrm{E}+01$ & $1.44 \mathrm{E}+03$ & $6.32 \mathrm{E}+02$ & $1.71 \mathrm{E}+04$ & $1.74 \mathrm{E}+03$ & $4.70 \mathrm{E}+04$ & $4.29 \mathrm{E}+01$ & $4.70 \mathrm{E}+04$ \\
\hline
\end{tabular}


SRNL-STI-2013-00115

\begin{tabular}{|c|c|c|c|c|c|c|c|c|c|c|c|c|}
\hline \multirow[t]{2}{*}{ Nuclide } & \multicolumn{2}{|c|}{ Water Ingestion DCS } & \multicolumn{2}{|c|}{ Meat Ingestion DCS } & \multicolumn{2}{|c|}{ Dairy Ingestion DCS } & \multicolumn{2}{|c|}{$\begin{array}{c}\text { Freshwater Fish } \\
\text { Ingestion DCS }\end{array}$} & \multicolumn{2}{|c|}{$\begin{array}{l}\text { Saltwater Shellfish } \\
\text { Ingestion DCS }\end{array}$} & \multicolumn{2}{|c|}{ Produce Ingestion DCS } \\
\hline & $(\mathrm{Bq} / \mathrm{L})$ & $(\mathrm{pCi} / \mathrm{L})$ & $(\mathrm{Bq} / \mathrm{kg})$ & $(\mathrm{pCi} / \mathbf{k g})$ & $(\mathrm{Bq} / \mathrm{L})$ & $(\mathrm{pCi} / \mathrm{L})$ & $(\mathrm{Bq} / \mathrm{kg})$ & $(\mathrm{pCi} / \mathbf{k g})$ & $(\mathrm{Bq} / \mathbf{k g})$ & $(\mathrm{pCi} / \mathbf{k g})$ & $(\mathrm{Bq} / \mathrm{kg})$ & (pCi//kg) \\
\hline Gd-149 & $1.74 \mathrm{E}+03$ & $4.70 \mathrm{E}+04$ & $1.73 \mathrm{E}+04$ & $4.67 \mathrm{E}+05$ & $5.36 \mathrm{E}+03$ & $1.45 \mathrm{E}+05$ & $6.36 \mathrm{E}+04$ & $1.72 \mathrm{E}+06$ & $1.75 \mathrm{E}+05$ & $4.73 \mathrm{E}+06$ & $4.32 \mathrm{E}+03$ & $4.73 \mathrm{E}+06$ \\
\hline Gd-150 & $1.83 \mathrm{E}+01$ & $4.96 \mathrm{E}+02$ & $1.82 \mathrm{E}+02$ & $4.92 \mathrm{E}+03$ & $5.65 \mathrm{E}+01$ & $1.53 \mathrm{E}+03$ & $6.70 \mathrm{E}+02$ & $1.81 \mathrm{E}+04$ & $1.84 \mathrm{E}+03$ & $4.98 \mathrm{E}+04$ & $4.55 \mathrm{E}+01$ & $4.98 \mathrm{E}+04$ \\
\hline Gd-151 & $4.05 \mathrm{E}+03$ & $1.09 \mathrm{E}+05$ & $4.02 \mathrm{E}+04$ & $1.09 \mathrm{E}+06$ & $1.25 \mathrm{E}+04$ & $3.37 \mathrm{E}+05$ & $1.48 \mathrm{E}+05$ & $4.00 \mathrm{E}+06$ & $4.07 \mathrm{E}+05$ & $1.10 \mathrm{E}+07$ & $1.01 \mathrm{E}+04$ & $1.10 \mathrm{E}+07$ \\
\hline Gd-152 & $2.34 \mathrm{E}+01$ & $6.32 \mathrm{E}+02$ & $2.32 \mathrm{E}+02$ & $6.27 \mathrm{E}+03$ & $7.20 \mathrm{E}+01$ & $1.95 \mathrm{E}+03$ & $8.54 \mathrm{E}+02$ & $2.31 \mathrm{E}+04$ & $2.35 \mathrm{E}+03$ & $6.35 \mathrm{E}+04$ & $5.80 \mathrm{E}+01$ & $6.35 \mathrm{E}+04$ \\
\hline Gd-153 & $3.33 \mathrm{E}+03$ & $8.99 \mathrm{E}+04$ & $3.30 \mathrm{E}+04$ & $8.92 \mathrm{E}+05$ & $1.02 \mathrm{E}+04$ & $2.77 \mathrm{E}+05$ & $1.22 \mathrm{E}+05$ & $3.28 \mathrm{E}+06$ & $3.34 \mathrm{E}+05$ & $9.03 \mathrm{E}+06$ & $8.25 \mathrm{E}+03$ & $9.03 \mathrm{E}+06$ \\
\hline Gd-159 & $1.79 \mathrm{E}+03$ & $4.84 \mathrm{E}+04$ & $1.78 \mathrm{E}+04$ & $4.80 \mathrm{E}+05$ & $5.51 \mathrm{E}+03$ & $1.49 \mathrm{E}+05$ & $6.54 \mathrm{E}+04$ & $1.77 \mathrm{E}+06$ & $1.80 \mathrm{E}+05$ & $4.86 \mathrm{E}+06$ & $4.44 \mathrm{E}+03$ & $4.86 \mathrm{E}+06$ \\
\hline $\mathrm{Tb}-147$ & $7.68 \mathrm{E}+03$ & $2.08 \mathrm{E}+05$ & $7.62 \mathrm{E}+04$ & $2.06 \mathrm{E}+06$ & $2.37 \mathrm{E}+04$ & $6.39 \mathrm{E}+05$ & $2.81 \mathrm{E}+05$ & $7.58 \mathrm{E}+06$ & $7.72 \mathrm{E}+05$ & $2.09 \mathrm{E}+07$ & $1.91 \mathrm{E}+04$ & $2.09 \mathrm{E}+07$ \\
\hline Tb-148 & $7.63 \mathrm{E}+03$ & $2.06 \mathrm{E}+05$ & $7.57 \mathrm{E}+04$ & $2.05 \mathrm{E}+06$ & $2.35 \mathrm{E}+04$ & $6.35 \mathrm{E}+05$ & $2.79 \mathrm{E}+05$ & $7.54 \mathrm{E}+06$ & $7.67 \mathrm{E}+05$ & $2.07 \mathrm{E}+07$ & $1.89 \mathrm{E}+04$ & $2.07 \mathrm{E}+07$ \\
\hline Tb-149 & $5.18 \mathrm{E}+03$ & $1.40 \mathrm{E}+05$ & $5.14 \mathrm{E}+04$ & $1.39 \mathrm{E}+06$ & $1.60 \mathrm{E}+04$ & $4.31 \mathrm{E}+05$ & $1.89 \mathrm{E}+05$ & $5.12 \mathrm{E}+06$ & $5.21 \mathrm{E}+05$ & $1.41 \mathrm{E}+07$ & $1.29 \mathrm{E}+04$ & $1.41 \mathrm{E}+07$ \\
\hline Tb-150 & $4.43 \mathrm{E}+03$ & $1.20 \mathrm{E}+05$ & $4.39 \mathrm{E}+04$ & $1.19 \mathrm{E}+06$ & $1.36 \mathrm{E}+04$ & $3.69 \mathrm{E}+05$ & $1.62 \mathrm{E}+05$ & $4.37 \mathrm{E}+06$ & $4.45 \mathrm{E}+05$ & $1.20 \mathrm{E}+07$ & $1.10 \mathrm{E}+04$ & $1.20 \mathrm{E}+07$ \\
\hline Tb-151 & $2.67 \mathrm{E}+03$ & $7.21 \mathrm{E}+04$ & $2.65 \mathrm{E}+04$ & $7.16 \mathrm{E}+05$ & $8.22 \mathrm{E}+03$ & $2.22 \mathrm{E}+05$ & $9.75 \mathrm{E}+04$ & $2.64 \mathrm{E}+06$ & $2.68 \mathrm{E}+05$ & $7.25 \mathrm{E}+06$ & $6.62 \mathrm{E}+03$ & $7.25 \mathrm{E}+06$ \\
\hline Tb-152 & $1.35 \mathrm{E}+03$ & $3.66 \mathrm{E}+04$ & $1.34 \mathrm{E}+04$ & $3.63 \mathrm{E}+05$ & $4.17 \mathrm{E}+03$ & $1.13 \mathrm{E}+05$ & $4.95 \mathrm{E}+04$ & $1.34 \mathrm{E}+06$ & $1.36 \mathrm{E}+05$ & $3.68 \mathrm{E}+06$ & $3.36 \mathrm{E}+03$ & $3.68 \mathrm{E}+06$ \\
\hline Tb-153 & $3.29 \mathrm{E}+03$ & $8.89 \mathrm{E}+04$ & $3.27 \mathrm{E}+04$ & $8.83 \mathrm{E}+05$ & $1.01 \mathrm{E}+04$ & $2.74 \mathrm{E}+05$ & $1.20 \mathrm{E}+05$ & $3.25 \mathrm{E}+06$ & $3.31 \mathrm{E}+05$ & $8.94 \mathrm{E}+06$ & $8.17 \mathrm{E}+03$ & $8.94 \mathrm{E}+06$ \\
\hline Tb-154 & $1.57 \mathrm{E}+03$ & $4.24 \mathrm{E}+04$ & $1.56 \mathrm{E}+04$ & $4.21 \mathrm{E}+05$ & $4.84 \mathrm{E}+03$ & $1.31 \mathrm{E}+05$ & $5.74 \mathrm{E}+04$ & $1.55 \mathrm{E}+06$ & $1.58 \mathrm{E}+05$ & $4.27 \mathrm{E}+06$ & $3.90 \mathrm{E}+03$ & $4.27 \mathrm{E}+06$ \\
\hline Tb-155 & $3.54 \mathrm{E}+03$ & $9.58 \mathrm{E}+04$ & $3.52 \mathrm{E}+04$ & $9.51 \mathrm{E}+05$ & $1.09 \mathrm{E}+04$ & $2.95 \mathrm{E}+05$ & $1.30 \mathrm{E}+05$ & $3.50 \mathrm{E}+06$ & $3.56 \mathrm{E}+05$ & $9.63 \mathrm{E}+06$ & $8.79 \mathrm{E}+03$ & $9.63 \mathrm{E}+06$ \\
\hline Tb-156 & $8.40 \mathrm{E}+02$ & $2.27 \mathrm{E}+04$ & $8.34 \mathrm{E}+03$ & $2.25 \mathrm{E}+05$ & $2.59 \mathrm{E}+03$ & $7.00 \mathrm{E}+04$ & $3.07 \mathrm{E}+04$ & $8.30 \mathrm{E}+05$ & $8.45 \mathrm{E}+04$ & $2.28 \mathrm{E}+06$ & $2.09 \mathrm{E}+03$ & $2.28 \mathrm{E}+06$ \\
\hline Tb-156m & $5.84 \mathrm{E}+03$ & $1.58 \mathrm{E}+05$ & $5.80 \mathrm{E}+04$ & $1.57 \mathrm{E}+06$ & $1.80 \mathrm{E}+04$ & $4.86 \mathrm{E}+05$ & $2.13 \mathrm{E}+05$ & $5.77 \mathrm{E}+06$ & $5.87 \mathrm{E}+05$ & $1.59 \mathrm{E}+07$ & $1.45 \mathrm{E}+04$ & $1.59 \mathrm{E}+07$ \\
\hline Tb-156n & $1.07 \mathrm{E}+04$ & $2.90 \mathrm{E}+05$ & $1.06 \mathrm{E}+05$ & $2.88 \mathrm{E}+06$ & $3.30 \mathrm{E}+04$ & $8.93 \mathrm{E}+05$ & $3.92 \mathrm{E}+05$ & $1.06 \mathrm{E}+07$ & $1.08 \mathrm{E}+06$ & $2.91 \mathrm{E}+07$ & $2.66 \mathrm{E}+04$ & $2.91 \mathrm{E}+07$ \\
\hline Tb-157 & $2.36 \mathrm{E}+04$ & $6.38 \mathrm{E}+05$ & $2.34 \mathrm{E}+05$ & $6.33 \mathrm{E}+06$ & $7.27 \mathrm{E}+04$ & $1.96 \mathrm{E}+06$ & $8.63 \mathrm{E}+05$ & $2.33 \mathrm{E}+07$ & $2.37 \mathrm{E}+06$ & $6.41 \mathrm{E}+07$ & $5.86 \mathrm{E}+04$ & $6.41 \mathrm{E}+07$ \\
\hline Tb-158 & $8.58 \mathrm{E}+02$ & $2.32 \mathrm{E}+04$ & $8.51 \mathrm{E}+03$ & $2.30 \mathrm{E}+05$ & $2.64 \mathrm{E}+03$ & $7.14 \mathrm{E}+04$ & $3.13 \mathrm{E}+04$ & $8.47 \mathrm{E}+05$ & $8.62 \mathrm{E}+04$ & $2.33 \mathrm{E}+06$ & $2.13 \mathrm{E}+03$ & $2.33 \mathrm{E}+06$ \\
\hline Tb-160 & $5.79 \mathrm{E}+02$ & $1.56 \mathrm{E}+04$ & $5.74 \mathrm{E}+03$ & $1.55 \mathrm{E}+05$ & $1.78 \mathrm{E}+03$ & $4.82 \mathrm{E}+04$ & $2.11 \mathrm{E}+04$ & $5.71 \mathrm{E}+05$ & $5.81 \mathrm{E}+04$ & $1.57 \mathrm{E}+06$ & $1.44 \mathrm{E}+03$ & $1.57 \mathrm{E}+06$ \\
\hline Tb-161 & $1.22 \mathrm{E}+03$ & $3.30 \mathrm{E}+04$ & $1.21 \mathrm{E}+04$ & $3.27 \mathrm{E}+05$ & $3.76 \mathrm{E}+03$ & $1.02 \mathrm{E}+05$ & $4.46 \mathrm{E}+04$ & $1.20 \mathrm{E}+06$ & $1.23 \mathrm{E}+05$ & $3.31 \mathrm{E}+06$ & $3.03 \mathrm{E}+03$ & $3.31 \mathrm{E}+06$ \\
\hline Tb-163 & $4.43 \mathrm{E}+04$ & $1.20 \mathrm{E}+06$ & $4.39 \mathrm{E}+05$ & $1.19 \mathrm{E}+07$ & $1.36 \mathrm{E}+05$ & $3.69 \mathrm{E}+06$ & $1.62 \mathrm{E}+06$ & $4.37 \mathrm{E}+07$ & $4.45 \mathrm{E}+06$ & $1.20 \mathrm{E}+08$ & $1.10 \mathrm{E}+05$ & $1.20 \mathrm{E}+08$ \\
\hline
\end{tabular}


SRNL-STI-2013-00115

\begin{tabular}{|c|c|c|c|c|c|c|c|c|c|c|c|c|}
\hline \multirow[t]{2}{*}{ Nuclide } & \multicolumn{2}{|c|}{ Water Ingestion DCS } & \multicolumn{2}{|c|}{ Meat Ingestion DCS } & \multicolumn{2}{|c|}{ Dairy Ingestion DCS } & \multicolumn{2}{|c|}{$\begin{array}{c}\text { Freshwater Fish } \\
\text { Ingestion DCS }\end{array}$} & \multicolumn{2}{|c|}{$\begin{array}{l}\text { Saltwater Shellfish } \\
\text { Ingestion DCS }\end{array}$} & \multicolumn{2}{|c|}{ Produce Ingestion DCS } \\
\hline & $(\mathrm{Bq} / \mathrm{L})$ & $(\mathrm{pCi} / \mathrm{L})$ & $(\mathrm{Bq} / \mathrm{kg})$ & $(\mathrm{pCi} / \mathbf{k g})$ & $(\mathrm{Bq} / \mathrm{L})$ & $(\mathrm{pCi} / \mathrm{L})$ & $(\mathrm{Bq} / \mathrm{kg})$ & $(\mathrm{pCi} / \mathbf{k g})$ & $(\mathrm{Bq} / \mathbf{k g})$ & $(\mathrm{pCi} / \mathbf{k g})$ & $(\mathrm{Bq} / \mathrm{kg})$ & (pCi//kg) \\
\hline Dy-151 & $4.94 \mathrm{E}+04$ & $1.33 \mathrm{E}+06$ & $4.90 \mathrm{E}+05$ & $1.32 \mathrm{E}+07$ & $1.52 \mathrm{E}+05$ & $4.11 \mathrm{E}+06$ & $1.80 \mathrm{E}+06$ & $4.88 \mathrm{E}+07$ & $4.96 \mathrm{E}+06$ & $1.34 \mathrm{E}+08$ & $1.22 \mathrm{E}+05$ & $1.34 \mathrm{E}+08$ \\
\hline Dy-152 & $8.76 \mathrm{E}+03$ & $2.37 \mathrm{E}+05$ & $8.69 \mathrm{E}+04$ & $2.35 \mathrm{E}+06$ & $2.70 \mathrm{E}+04$ & $7.29 \mathrm{E}+05$ & $3.20 \mathrm{E}+05$ & $8.65 \mathrm{E}+06$ & $8.80 \mathrm{E}+05$ & $2.38 \mathrm{E}+07$ & $2.17 \mathrm{E}+04$ & $2.38 \mathrm{E}+07$ \\
\hline Dy-153 & $5.36 \mathrm{E}+03$ & $1.45 \mathrm{E}+05$ & $5.32 \mathrm{E}+04$ & $1.44 \mathrm{E}+06$ & $1.65 \mathrm{E}+04$ & $4.46 \mathrm{E}+05$ & $1.96 \mathrm{E}+05$ & $5.30 \mathrm{E}+06$ & $5.39 \mathrm{E}+05$ & $1.46 \mathrm{E}+07$ & $1.33 \mathrm{E}+04$ & $1.46 \mathrm{E}+07$ \\
\hline Dy-154 & $1.71 \mathrm{E}+01$ & $4.62 \mathrm{E}+02$ & $1.70 \mathrm{E}+02$ & $4.59 \mathrm{E}+03$ & $5.27 \mathrm{E}+01$ & $1.42 \mathrm{E}+03$ & $6.25 \mathrm{E}+02$ & $1.69 \mathrm{E}+04$ & $1.72 \mathrm{E}+03$ & $4.65 \mathrm{E}+04$ & $4.25 \mathrm{E}+01$ & $4.65 \mathrm{E}+04$ \\
\hline Dy-155 & $6.76 \mathrm{E}+03$ & $1.83 \mathrm{E}+05$ & $6.71 \mathrm{E}+04$ & $1.81 \mathrm{E}+06$ & $2.08 \mathrm{E}+04$ & $5.63 \mathrm{E}+05$ & $2.47 \mathrm{E}+05$ & $6.68 \mathrm{E}+06$ & $6.79 \mathrm{E}+05$ & $1.84 \mathrm{E}+07$ & $1.68 \mathrm{E}+04$ & $1.84 \mathrm{E}+07$ \\
\hline Dy-157 & $1.60 \mathrm{E}+04$ & $4.33 \mathrm{E}+05$ & $1.59 \mathrm{E}+05$ & $4.30 \mathrm{E}+06$ & $4.94 \mathrm{E}+04$ & $1.33 \mathrm{E}+06$ & $5.86 \mathrm{E}+05$ & $1.58 \mathrm{E}+07$ & $1.61 \mathrm{E}+06$ & $4.35 \mathrm{E}+07$ & $3.98 \mathrm{E}+04$ & $4.35 \mathrm{E}+07$ \\
\hline Dy-159 & $8.82 \mathrm{E}+03$ & $2.38 \mathrm{E}+05$ & $8.76 \mathrm{E}+04$ & $2.37 \mathrm{E}+06$ & $2.72 \mathrm{E}+04$ & $7.34 \mathrm{E}+05$ & $3.22 \mathrm{E}+05$ & $8.71 \mathrm{E}+06$ & $8.87 \mathrm{E}+05$ & $2.40 \mathrm{E}+07$ & $2.19 \mathrm{E}+04$ & $2.40 \mathrm{E}+07$ \\
\hline Dy-165 & $8.35 \mathrm{E}+03$ & $2.26 \mathrm{E}+05$ & $8.29 \mathrm{E}+04$ & $2.24 \mathrm{E}+06$ & $2.57 \mathrm{E}+04$ & $6.95 \mathrm{E}+05$ & $3.05 \mathrm{E}+05$ & $8.24 \mathrm{E}+06$ & $8.39 \mathrm{E}+05$ & $2.27 \mathrm{E}+07$ & $2.07 \mathrm{E}+04$ & $2.27 \mathrm{E}+07$ \\
\hline Dy-166 & $5.43 \mathrm{E}+02$ & $1.47 \mathrm{E}+04$ & $5.39 \mathrm{E}+03$ & $1.46 \mathrm{E}+05$ & $1.67 \mathrm{E}+03$ & $4.52 \mathrm{E}+04$ & $1.98 \mathrm{E}+04$ & $5.36 \mathrm{E}+05$ & $5.46 \mathrm{E}+04$ & $1.48 \mathrm{E}+06$ & $1.35 \mathrm{E}+03$ & $1.48 \mathrm{E}+06$ \\
\hline Ho-154 & $2.30 \mathrm{E}+04$ & $6.23 \mathrm{E}+05$ & $2.29 \mathrm{E}+05$ & $6.18 \mathrm{E}+06$ & $7.10 \mathrm{E}+04$ & $1.92 \mathrm{E}+06$ & $8.42 \mathrm{E}+05$ & $2.28 \mathrm{E}+07$ & $2.31 \mathrm{E}+06$ & $6.26 \mathrm{E}+07$ & $5.72 \mathrm{E}+04$ & $6.26 \mathrm{E}+07$ \\
\hline Ho-155 & $2.42 \mathrm{E}+04$ & $6.55 \mathrm{E}+05$ & $2.41 \mathrm{E}+05$ & $6.50 \mathrm{E}+06$ & $7.47 \mathrm{E}+04$ & $2.02 \mathrm{E}+06$ & $8.86 \mathrm{E}+05$ & $2.39 \mathrm{E}+07$ & $2.44 \mathrm{E}+06$ & $6.59 \mathrm{E}+07$ & $6.02 \mathrm{E}+04$ & $6.59 \mathrm{E}+07$ \\
\hline Ho-156 & $9.79 \mathrm{E}+03$ & $2.65 \mathrm{E}+05$ & $9.72 \mathrm{E}+04$ & $2.63 \mathrm{E}+06$ & $3.02 \mathrm{E}+04$ & $8.15 \mathrm{E}+05$ & $3.58 \mathrm{E}+05$ & $9.67 \mathrm{E}+06$ & $9.84 \mathrm{E}+05$ & $2.66 \mathrm{E}+07$ & $2.43 \mathrm{E}+04$ & $2.66 \mathrm{E}+07$ \\
\hline Ho-157 & $1.33 \mathrm{E}+05$ & $3.61 \mathrm{E}+06$ & $1.32 \mathrm{E}+06$ & $3.58 \mathrm{E}+07$ & $4.11 \mathrm{E}+05$ & $1.11 \mathrm{E}+07$ & $4.88 \mathrm{E}+06$ & $1.32 \mathrm{E}+08$ & $1.34 \mathrm{E}+07$ & $3.62 \mathrm{E}+08$ & $3.31 \mathrm{E}+05$ & $3.62 \mathrm{E}+08$ \\
\hline Ho-159 & $1.14 \mathrm{E}+05$ & $3.08 \mathrm{E}+06$ & $1.13 \mathrm{E}+06$ & $3.06 \mathrm{E}+07$ & $3.52 \mathrm{E}+05$ & $9.50 \mathrm{E}+06$ & $4.17 \mathrm{E}+06$ & $1.13 \mathrm{E}+08$ & $1.15 \mathrm{E}+07$ & $3.10 \mathrm{E}+08$ & $2.83 \mathrm{E}+05$ & $3.10 \mathrm{E}+08$ \\
\hline Ho-160 & $5.84 \mathrm{E}+04$ & $1.58 \mathrm{E}+06$ & $5.80 \mathrm{E}+05$ & $1.57 \mathrm{E}+07$ & $1.80 \mathrm{E}+05$ & $4.86 \mathrm{E}+06$ & $2.13 \mathrm{E}+06$ & $5.77 \mathrm{E}+07$ & $5.87 \mathrm{E}+06$ & $1.59 \mathrm{E}+08$ & $1.45 \mathrm{E}+05$ & $1.59 \mathrm{E}+08$ \\
\hline Ho-161 & $7.27 \mathrm{E}+04$ & $1.97 \mathrm{E}+06$ & $7.22 \mathrm{E}+05$ & $1.95 \mathrm{E}+07$ & $2.24 \mathrm{E}+05$ & $6.06 \mathrm{E}+06$ & $2.66 \mathrm{E}+06$ & $7.18 \mathrm{E}+07$ & $7.31 \mathrm{E}+06$ & $1.98 \mathrm{E}+08$ & $1.80 \mathrm{E}+05$ & $1.98 \mathrm{E}+08$ \\
\hline Ho-162 & $2.93 \mathrm{E}+05$ & $7.93 \mathrm{E}+06$ & $2.91 \mathrm{E}+06$ & $7.87 \mathrm{E}+07$ & $9.04 \mathrm{E}+05$ & $2.44 \mathrm{E}+07$ & $1.07 \mathrm{E}+07$ & $2.90 \mathrm{E}+08$ & $2.95 \mathrm{E}+07$ & $7.97 \mathrm{E}+08$ & $7.28 \mathrm{E}+05$ & $7.97 \mathrm{E}+08$ \\
\hline Ho- $162 \mathrm{~m}$ & $3.80 \mathrm{E}+04$ & $1.03 \mathrm{E}+06$ & $3.78 \mathrm{E}+05$ & $1.02 \mathrm{E}+07$ & $1.17 \mathrm{E}+05$ & $3.17 \mathrm{E}+06$ & $1.39 \mathrm{E}+06$ & $3.76 \mathrm{E}+07$ & $3.82 \mathrm{E}+06$ & $1.03 \mathrm{E}+08$ & $9.44 \mathrm{E}+04$ & $1.03 \mathrm{E}+08$ \\
\hline Ho-163 & $3.11 \mathrm{E}+05$ & $8.40 \mathrm{E}+06$ & $3.09 \mathrm{E}+06$ & $8.34 \mathrm{E}+07$ & $9.58 \mathrm{E}+05$ & $2.59 \mathrm{E}+07$ & $1.14 \mathrm{E}+07$ & $3.07 \mathrm{E}+08$ & $3.13 \mathrm{E}+07$ & $8.45 \mathrm{E}+08$ & $7.72 \mathrm{E}+05$ & $8.45 \mathrm{E}+08$ \\
\hline Ho-164 & $9.79 \mathrm{E}+04$ & $2.65 \mathrm{E}+06$ & $9.72 \mathrm{E}+05$ & $2.63 \mathrm{E}+07$ & $3.02 \mathrm{E}+05$ & $8.15 \mathrm{E}+06$ & $3.58 \mathrm{E}+06$ & $9.67 \mathrm{E}+07$ & $9.84 \mathrm{E}+06$ & $2.66 \mathrm{E}+08$ & $2.43 \mathrm{E}+05$ & $2.66 \mathrm{E}+08$ \\
\hline Ho-164m & $5.58 \mathrm{E}+04$ & $1.51 \mathrm{E}+06$ & $5.54 \mathrm{E}+05$ & $1.50 \mathrm{E}+07$ & $1.72 \mathrm{E}+05$ & $4.64 \mathrm{E}+06$ & $2.04 \mathrm{E}+06$ & $5.51 \mathrm{E}+07$ & $5.61 \mathrm{E}+06$ & $1.51 \mathrm{E}+08$ & $1.38 \mathrm{E}+05$ & $1.51 \mathrm{E}+08$ \\
\hline Ho-166 & $6.44 \mathrm{E}+02$ & $1.74 \mathrm{E}+04$ & $6.40 \mathrm{E}+03$ & $1.73 \mathrm{E}+05$ & $1.99 \mathrm{E}+03$ & $5.37 \mathrm{E}+04$ & $2.36 \mathrm{E}+04$ & $6.37 \mathrm{E}+05$ & $6.48 \mathrm{E}+04$ & $1.75 \mathrm{E}+06$ & $1.60 \mathrm{E}+03$ & $1.75 \mathrm{E}+06$ \\
\hline Ho-166m & $4.88 \mathrm{E}+02$ & $1.32 \mathrm{E}+04$ & $4.84 \mathrm{E}+03$ & $1.31 \mathrm{E}+05$ & $1.50 \mathrm{E}+03$ & $4.06 \mathrm{E}+04$ & $1.78 \mathrm{E}+04$ & $4.82 \mathrm{E}+05$ & $4.90 \mathrm{E}+04$ & $1.32 \mathrm{E}+06$ & $1.21 \mathrm{E}+03$ & $1.32 \mathrm{E}+06$ \\
\hline
\end{tabular}

Page 190 of 298 
SRNL-STI-2013-00115

\begin{tabular}{|c|c|c|c|c|c|c|c|c|c|c|c|c|}
\hline \multirow[t]{2}{*}{ Nuclide } & \multicolumn{2}{|c|}{ Water Ingestion DCS } & \multicolumn{2}{|c|}{ Meat Ingestion DCS } & \multicolumn{2}{|c|}{ Dairy Ingestion DCS } & \multicolumn{2}{|c|}{$\begin{array}{c}\text { Freshwater Fish } \\
\text { Ingestion DCS }\end{array}$} & \multicolumn{2}{|c|}{$\begin{array}{l}\text { Saltwater Shellfish } \\
\text { Ingestion DCS }\end{array}$} & \multicolumn{2}{|c|}{ Produce Ingestion DCS } \\
\hline & $(\mathrm{Bq} / \mathrm{L})$ & $(\mathrm{pCi} / \mathrm{L})$ & $(\mathrm{Bq} / \mathrm{kg})$ & $(\mathrm{pCi} / \mathrm{kg})$ & $(\mathrm{Bq} / \mathrm{L})$ & $(\mathrm{pCi} / \mathrm{L})$ & $(\mathrm{Bq} / \mathbf{k g})$ & $(\mathrm{pCi} / \mathbf{k g})$ & $(\mathrm{Bq} / \mathbf{k g})$ & $(\mathrm{pCi} / \mathbf{k g})$ & $(\mathrm{Bq} / \mathbf{k g})$ & $(\mathrm{pCi} / \mathbf{k g})$ \\
\hline Ho-167 & $1.06 \mathrm{E}+04$ & $2.87 \mathrm{E}+05$ & $1.06 \mathrm{E}+05$ & $2.85 \mathrm{E}+06$ & $3.27 \mathrm{E}+04$ & $8.85 \mathrm{E}+05$ & $3.89 \mathrm{E}+05$ & $1.05 \mathrm{E}+07$ & $1.07 \mathrm{E}+06$ & $2.89 \mathrm{E}+07$ & $2.64 \mathrm{E}+04$ & $2.89 \mathrm{E}+07$ \\
\hline Er-156 & $2.75 \mathrm{E}+04$ & $7.44 \mathrm{E}+05$ & $2.73 \mathrm{E}+05$ & $7.38 \mathrm{E}+06$ & $8.48 \mathrm{E}+04$ & $2.29 \mathrm{E}+06$ & $1.01 \mathrm{E}+06$ & $2.72 \mathrm{E}+07$ & $2.77 \mathrm{E}+06$ & $7.47 \mathrm{E}+07$ & $6.83 \mathrm{E}+04$ & $7.47 \mathrm{E}+07$ \\
\hline Er-159 & $4.26 \mathrm{E}+04$ & $1.15 \mathrm{E}+06$ & $4.23 \mathrm{E}+05$ & $1.14 \mathrm{E}+07$ & $1.31 \mathrm{E}+05$ & $3.55 \mathrm{E}+06$ & $1.56 \mathrm{E}+06$ & $4.21 \mathrm{E}+07$ & $4.28 \mathrm{E}+06$ & $1.16 \mathrm{E}+08$ & $1.06 \mathrm{E}+05$ & $1.16 \mathrm{E}+08$ \\
\hline Er-161 & $1.15 \mathrm{E}+04$ & $3.11 \mathrm{E}+05$ & $1.14 \mathrm{E}+05$ & $3.09 \mathrm{E}+06$ & $3.55 \mathrm{E}+04$ & $9.59 \mathrm{E}+05$ & $4.21 \mathrm{E}+05$ & $1.14 \mathrm{E}+07$ & $1.16 \mathrm{E}+06$ & $3.13 \mathrm{E}+07$ & $2.86 \mathrm{E}+04$ & $3.13 \mathrm{E}+07$ \\
\hline Er-163 & $3.67 \mathrm{E}+05$ & $9.92 \mathrm{E}+06$ & $3.64 \mathrm{E}+06$ & $9.84 \mathrm{E}+07$ & $1.13 \mathrm{E}+06$ & $3.05 \mathrm{E}+07$ & $1.34 \mathrm{E}+07$ & $3.62 \mathrm{E}+08$ & $3.69 \mathrm{E}+07$ & $9.97 \mathrm{E}+08$ & $9.10 \mathrm{E}+05$ & $9.97 \mathrm{E}+08$ \\
\hline Er-165 & $4.84 \mathrm{E}+04$ & $1.31 \mathrm{E}+06$ & $4.80 \mathrm{E}+05$ & $1.30 \mathrm{E}+07$ & $1.49 \mathrm{E}+05$ & $4.03 \mathrm{E}+06$ & $1.77 \mathrm{E}+06$ & $4.78 \mathrm{E}+07$ & $4.86 \mathrm{E}+06$ & $1.31 \mathrm{E}+08$ & $1.20 \mathrm{E}+05$ & $1.31 \mathrm{E}+08$ \\
\hline Er-169 & $2.41 \mathrm{E}+03$ & $6.51 \mathrm{E}+04$ & $2.39 \mathrm{E}+04$ & $6.47 \mathrm{E}+05$ & $7.43 \mathrm{E}+03$ & $2.01 \mathrm{E}+05$ & $8.81 \mathrm{E}+04$ & $2.38 \mathrm{E}+06$ & $2.42 \mathrm{E}+05$ & $6.55 \mathrm{E}+06$ & $5.98 \mathrm{E}+03$ & $6.55 \mathrm{E}+06$ \\
\hline Er-171 & $2.56 \mathrm{E}+03$ & $6.93 \mathrm{E}+04$ & $2.55 \mathrm{E}+04$ & $6.88 \mathrm{E}+05$ & $7.90 \mathrm{E}+03$ & $2.14 \mathrm{E}+05$ & $9.37 \mathrm{E}+04$ & $2.53 \mathrm{E}+06$ & $2.58 \mathrm{E}+05$ & $6.97 \mathrm{E}+06$ & $6.36 \mathrm{E}+03$ & $6.97 \mathrm{E}+06$ \\
\hline Er-172 & $8.88 \mathrm{E}+02$ & $2.40 \mathrm{E}+04$ & $8.82 \mathrm{E}+03$ & $2.38 \mathrm{E}+05$ & $2.74 \mathrm{E}+03$ & $7.40 \mathrm{E}+04$ & $3.25 \mathrm{E}+04$ & $8.78 \mathrm{E}+05$ & $8.93 \mathrm{E}+04$ & $2.41 \mathrm{E}+06$ & $2.20 \mathrm{E}+03$ & $2.41 \mathrm{E}+06$ \\
\hline Tm-161 & $2.46 \mathrm{E}+04$ & $6.66 \mathrm{E}+05$ & $2.44 \mathrm{E}+05$ & $6.61 \mathrm{E}+06$ & $7.59 \mathrm{E}+04$ & $2.05 \mathrm{E}+06$ & $9.00 \mathrm{E}+05$ & $2.43 \mathrm{E}+07$ & $2.48 \mathrm{E}+06$ & $6.69 \mathrm{E}+07$ & $6.11 \mathrm{E}+04$ & $6.69 \mathrm{E}+07$ \\
\hline Tm-162 & $2.41 \mathrm{E}+04$ & $6.50 \mathrm{E}+05$ & $2.39 \mathrm{E}+05$ & $6.45 \mathrm{E}+06$ & $7.41 \mathrm{E}+04$ & $2.00 \mathrm{E}+06$ & $8.79 \mathrm{E}+05$ & $2.38 \mathrm{E}+07$ & $2.42 \mathrm{E}+06$ & $6.53 \mathrm{E}+07$ & $5.97 \mathrm{E}+04$ & $6.53 \mathrm{E}+07$ \\
\hline Tm-163 & $1.78 \mathrm{E}+04$ & $4.82 \mathrm{E}+05$ & $1.77 \mathrm{E}+05$ & $4.79 \mathrm{E}+06$ & $5.50 \mathrm{E}+04$ & $1.49 \mathrm{E}+06$ & $6.52 \mathrm{E}+05$ & $1.76 \mathrm{E}+07$ & $1.79 \mathrm{E}+06$ & $4.85 \mathrm{E}+07$ & $4.43 \mathrm{E}+04$ & $4.85 \mathrm{E}+07$ \\
\hline Tm-165 & $2.67 \mathrm{E}+03$ & $7.23 \mathrm{E}+04$ & $2.65 \mathrm{E}+04$ & $7.18 \mathrm{E}+05$ & $8.24 \mathrm{E}+03$ & $2.23 \mathrm{E}+05$ & $9.78 \mathrm{E}+04$ & $2.64 \mathrm{E}+06$ & $2.69 \mathrm{E}+05$ & $7.27 \mathrm{E}+06$ & $6.64 \mathrm{E}+03$ & $7.27 \mathrm{E}+06$ \\
\hline Tm-166 & $3.44 \mathrm{E}+03$ & $9.29 \mathrm{E}+04$ & $3.41 \mathrm{E}+04$ & $9.22 \mathrm{E}+05$ & $1.06 \mathrm{E}+04$ & $2.86 \mathrm{E}+05$ & $1.26 \mathrm{E}+05$ & $3.39 \mathrm{E}+06$ & $3.45 \mathrm{E}+05$ & $9.33 \mathrm{E}+06$ & $8.53 \mathrm{E}+03$ & $9.33 \mathrm{E}+06$ \\
\hline Tm-167 & $1.57 \mathrm{E}+03$ & $4.25 \mathrm{E}+04$ & $1.56 \mathrm{E}+04$ & $4.22 \mathrm{E}+05$ & $4.84 \mathrm{E}+03$ & $1.31 \mathrm{E}+05$ & $5.75 \mathrm{E}+04$ & $1.55 \mathrm{E}+06$ & $1.58 \mathrm{E}+05$ & $4.27 \mathrm{E}+06$ & $3.90 \mathrm{E}+03$ & $4.27 \mathrm{E}+06$ \\
\hline Tm-168 & $9.28 \mathrm{E}+02$ & $2.51 \mathrm{E}+04$ & $9.21 \mathrm{E}+03$ & $2.49 \mathrm{E}+05$ & $2.86 \mathrm{E}+03$ & $7.73 \mathrm{E}+04$ & $3.39 \mathrm{E}+04$ & $9.17 \mathrm{E}+05$ & $9.33 \mathrm{E}+04$ & $2.52 \mathrm{E}+06$ & $2.30 \mathrm{E}+03$ & $2.52 \mathrm{E}+06$ \\
\hline Tm-170 & $6.87 \mathrm{E}+02$ & $1.86 \mathrm{E}+04$ & $6.82 \mathrm{E}+03$ & $1.84 \mathrm{E}+05$ & $2.12 \mathrm{E}+03$ & $5.72 \mathrm{E}+04$ & $2.51 \mathrm{E}+04$ & $6.79 \mathrm{E}+05$ & $6.91 \mathrm{E}+04$ & $1.87 \mathrm{E}+06$ & $1.71 \mathrm{E}+03$ & $1.87 \mathrm{E}+06$ \\
\hline Tm-171 & $8.40 \mathrm{E}+03$ & $2.27 \mathrm{E}+05$ & $8.34 \mathrm{E}+04$ & $2.25 \mathrm{E}+06$ & $2.59 \mathrm{E}+04$ & $7.00 \mathrm{E}+05$ & $3.07 \mathrm{E}+05$ & $8.30 \mathrm{E}+06$ & $8.45 \mathrm{E}+05$ & $2.28 \mathrm{E}+07$ & $2.09 \mathrm{E}+04$ & $2.28 \mathrm{E}+07$ \\
\hline Tm-172 & $5.36 \mathrm{E}+02$ & $1.45 \mathrm{E}+04$ & $5.32 \mathrm{E}+03$ & $1.44 \mathrm{E}+05$ & $1.65 \mathrm{E}+03$ & $4.46 \mathrm{E}+04$ & $1.96 \mathrm{E}+04$ & $5.30 \mathrm{E}+05$ & $5.39 \mathrm{E}+04$ & $1.46 \mathrm{E}+06$ & $1.33 \mathrm{E}+03$ & $1.46 \mathrm{E}+06$ \\
\hline Tm-173 & $3.06 \mathrm{E}+03$ & $8.26 \mathrm{E}+04$ & $3.03 \mathrm{E}+04$ & $8.20 \mathrm{E}+05$ & $9.41 \mathrm{E}+03$ & $2.54 \mathrm{E}+05$ & $1.12 \mathrm{E}+05$ & $3.02 \mathrm{E}+06$ & $3.07 \mathrm{E}+05$ & $8.30 \mathrm{E}+06$ & $7.58 \mathrm{E}+03$ & $8.30 \mathrm{E}+06$ \\
\hline Tm-175 & $3.64 \mathrm{E}+04$ & $9.83 \mathrm{E}+05$ & $3.61 \mathrm{E}+05$ & $9.76 \mathrm{E}+06$ & $1.12 \mathrm{E}+05$ & $3.03 \mathrm{E}+06$ & $1.33 \mathrm{E}+06$ & $3.59 \mathrm{E}+07$ & $3.65 \mathrm{E}+06$ & $9.88 \mathrm{E}+07$ & $9.02 \mathrm{E}+04$ & $9.88 \mathrm{E}+07$ \\
\hline Yb-162 & $3.07 \mathrm{E}+04$ & $8.30 \mathrm{E}+05$ & $3.05 \mathrm{E}+05$ & $8.24 \mathrm{E}+06$ & $9.46 \mathrm{E}+04$ & $2.56 \mathrm{E}+06$ & $1.12 \mathrm{E}+06$ & $3.03 \mathrm{E}+07$ & $3.09 \mathrm{E}+06$ & $8.34 \mathrm{E}+07$ & $7.62 \mathrm{E}+04$ & $8.34 \mathrm{E}+07$ \\
\hline Yb-163 & $6.01 \mathrm{E}+04$ & $1.62 \mathrm{E}+06$ & $5.96 \mathrm{E}+05$ & $1.61 \mathrm{E}+07$ & $1.85 \mathrm{E}+05$ & $5.00 \mathrm{E}+06$ & $2.20 \mathrm{E}+06$ & $5.93 \mathrm{E}+07$ & $6.04 \mathrm{E}+06$ & $1.63 \mathrm{E}+08$ & $1.49 \mathrm{E}+05$ & $1.63 \mathrm{E}+08$ \\
\hline
\end{tabular}

Page 191 of 298 
SRNL-STI-2013-00115

\begin{tabular}{|c|c|c|c|c|c|c|c|c|c|c|c|c|}
\hline \multirow[t]{2}{*}{ Nuclide } & \multicolumn{2}{|c|}{ Water Ingestion DCS } & \multicolumn{2}{|c|}{ Meat Ingestion DCS } & \multicolumn{2}{|c|}{ Dairy Ingestion DCS } & \multicolumn{2}{|c|}{$\begin{array}{c}\text { Freshwater Fish } \\
\text { Ingestion DCS }\end{array}$} & \multicolumn{2}{|c|}{$\begin{array}{l}\text { Saltwater Shellfish } \\
\text { Ingestion DCS }\end{array}$} & \multicolumn{2}{|c|}{ Produce Ingestion DCS } \\
\hline & $(\mathrm{Bq} / \mathrm{L})$ & $(\mathrm{pCi} / \mathrm{L})$ & $(\mathrm{Bq} / \mathrm{kg})$ & $(\mathrm{pCi} / \mathrm{kg})$ & $(\mathrm{Bq} / \mathrm{L})$ & $(\mathrm{pCi} / \mathrm{L})$ & $(\mathrm{Bq} / \mathbf{k g})$ & $(\mathrm{pCi} / \mathbf{k g})$ & $(\mathrm{Bq} / \mathbf{k g})$ & $(\mathrm{pCi} / \mathbf{k g})$ & $(\mathrm{Bq} / \mathbf{k g})$ & $(\mathrm{pCi} / \mathbf{k g})$ \\
\hline $\mathrm{Yb}-164$ & $1.02 \mathrm{E}+04$ & $2.76 \mathrm{E}+05$ & $1.01 \mathrm{E}+05$ & $2.73 \mathrm{E}+06$ & $3.14 \mathrm{E}+04$ & $8.49 \mathrm{E}+05$ & $3.73 \mathrm{E}+05$ & $1.01 \mathrm{E}+07$ & $1.02 \mathrm{E}+06$ & $2.77 \mathrm{E}+07$ & $2.53 \mathrm{E}+04$ & $2.77 \mathrm{E}+07$ \\
\hline $\mathrm{Yb}-166$ & $1.01 \mathrm{E}+03$ & $2.73 \mathrm{E}+04$ & $1.00 \mathrm{E}+04$ & $2.71 \mathrm{E}+05$ & $3.11 \mathrm{E}+03$ & $8.42 \mathrm{E}+04$ & $3.70 \mathrm{E}+04$ & $9.99 \mathrm{E}+05$ & $1.02 \mathrm{E}+05$ & $2.75 \mathrm{E}+06$ & $2.51 \mathrm{E}+03$ & $2.75 \mathrm{E}+06$ \\
\hline $\mathrm{Yb}-167$ & $1.38 \mathrm{E}+05$ & $3.73 \mathrm{E}+06$ & $1.37 \mathrm{E}+06$ & $3.70 \mathrm{E}+07$ & $4.25 \mathrm{E}+05$ & $1.15 \mathrm{E}+07$ & $5.04 \mathrm{E}+06$ & $1.36 \mathrm{E}+08$ & $1.39 \mathrm{E}+07$ & $3.75 \mathrm{E}+08$ & $3.43 \mathrm{E}+05$ & $3.75 \mathrm{E}+08$ \\
\hline Yb-169 & $1.13 \mathrm{E}+03$ & $3.06 \mathrm{E}+04$ & $1.12 \mathrm{E}+04$ & $3.03 \mathrm{E}+05$ & $3.48 \mathrm{E}+03$ & $9.41 \mathrm{E}+04$ & $4.13 \mathrm{E}+04$ & $1.12 \mathrm{E}+06$ & $1.14 \mathrm{E}+05$ & $3.07 \mathrm{E}+06$ & $2.81 \mathrm{E}+03$ & $3.07 \mathrm{E}+06$ \\
\hline $\mathrm{Yb}-175$ & $2.06 \mathrm{E}+03$ & $5.57 \mathrm{E}+04$ & $2.05 \mathrm{E}+04$ & $5.53 \mathrm{E}+05$ & $6.35 \mathrm{E}+03$ & $1.72 \mathrm{E}+05$ & $7.54 \mathrm{E}+04$ & $2.04 \mathrm{E}+06$ & $2.07 \mathrm{E}+05$ & $5.60 \mathrm{E}+06$ & $5.12 \mathrm{E}+03$ & $5.60 \mathrm{E}+06$ \\
\hline $\mathrm{Yb}-177$ & $9.95 \mathrm{E}+03$ & $2.69 \mathrm{E}+05$ & $9.88 \mathrm{E}+04$ & $2.67 \mathrm{E}+06$ & $3.07 \mathrm{E}+04$ & $8.28 \mathrm{E}+05$ & $3.64 \mathrm{E}+05$ & $9.83 \mathrm{E}+06$ & $1.00 \mathrm{E}+06$ & $2.70 \mathrm{E}+07$ & $2.47 \mathrm{E}+04$ & $2.70 \mathrm{E}+07$ \\
\hline $\mathrm{Yb}-178$ & $7.87 \mathrm{E}+03$ & $2.13 \mathrm{E}+05$ & $7.81 \mathrm{E}+04$ & $2.11 \mathrm{E}+06$ & $2.42 \mathrm{E}+04$ & $6.55 \mathrm{E}+05$ & $2.88 \mathrm{E}+05$ & $7.78 \mathrm{E}+06$ & $7.91 \mathrm{E}+05$ & $2.14 \mathrm{E}+07$ & $1.95 \mathrm{E}+04$ & $2.14 \mathrm{E}+07$ \\
\hline Lu-165 & $4.36 \mathrm{E}+04$ & $1.18 \mathrm{E}+06$ & $4.33 \mathrm{E}+05$ & $1.17 \mathrm{E}+07$ & $1.34 \mathrm{E}+05$ & $3.63 \mathrm{E}+06$ & $1.59 \mathrm{E}+06$ & $4.31 \mathrm{E}+07$ & $4.39 \mathrm{E}+06$ & $1.19 \mathrm{E}+08$ & $1.08 \mathrm{E}+05$ & $1.19 \mathrm{E}+08$ \\
\hline Lu-167 & $2.03 \mathrm{E}+04$ & $5.47 \mathrm{E}+05$ & $2.01 \mathrm{E}+05$ & $5.43 \mathrm{E}+06$ & $6.24 \mathrm{E}+04$ & $1.69 \mathrm{E}+06$ & $7.40 \mathrm{E}+05$ & $2.00 \mathrm{E}+07$ & $2.04 \mathrm{E}+06$ & $5.50 \mathrm{E}+07$ & $5.03 \mathrm{E}+04$ & $5.50 \mathrm{E}+07$ \\
\hline Lu-169 & $1.84 \mathrm{E}+03$ & $4.97 \mathrm{E}+04$ & $1.82 \mathrm{E}+04$ & $4.93 \mathrm{E}+05$ & $5.66 \mathrm{E}+03$ & $1.53 \mathrm{E}+05$ & $6.71 \mathrm{E}+04$ & $1.81 \mathrm{E}+06$ & $1.85 \mathrm{E}+05$ & $4.99 \mathrm{E}+06$ & $4.56 \mathrm{E}+03$ & $4.99 \mathrm{E}+06$ \\
\hline Lu-170 & $1.02 \mathrm{E}+03$ & $2.76 \mathrm{E}+04$ & $1.01 \mathrm{E}+04$ & $2.73 \mathrm{E}+05$ & $3.14 \mathrm{E}+03$ & $8.49 \mathrm{E}+04$ & $3.73 \mathrm{E}+04$ & $1.01 \mathrm{E}+06$ & $1.02 \mathrm{E}+05$ & $2.77 \mathrm{E}+06$ & $2.53 \mathrm{E}+03$ & $2.77 \mathrm{E}+06$ \\
\hline Lu-171 & $1.37 \mathrm{E}+03$ & $3.71 \mathrm{E}+04$ & $1.36 \mathrm{E}+04$ & $3.69 \mathrm{E}+05$ & $4.23 \mathrm{E}+03$ & $1.14 \mathrm{E}+05$ & $5.02 \mathrm{E}+04$ & $1.36 \mathrm{E}+06$ & $1.38 \mathrm{E}+05$ & $3.73 \mathrm{E}+06$ & $3.41 \mathrm{E}+03$ & $3.73 \mathrm{E}+06$ \\
\hline Lu-172 & $7.36 \mathrm{E}+02$ & $1.99 \mathrm{E}+04$ & $7.31 \mathrm{E}+03$ & $1.97 \mathrm{E}+05$ & $2.27 \mathrm{E}+03$ & $6.13 \mathrm{E}+04$ & $2.69 \mathrm{E}+04$ & $7.27 \mathrm{E}+05$ & $7.40 \mathrm{E}+04$ & $2.00 \mathrm{E}+06$ & $1.83 \mathrm{E}+03$ & $2.00 \mathrm{E}+06$ \\
\hline Lu-173 & $2.55 \mathrm{E}+03$ & $6.90 \mathrm{E}+04$ & $2.54 \mathrm{E}+04$ & $6.85 \mathrm{E}+05$ & $7.87 \mathrm{E}+03$ & $2.13 \mathrm{E}+05$ & $9.33 \mathrm{E}+04$ & $2.52 \mathrm{E}+06$ & $2.57 \mathrm{E}+05$ & $6.94 \mathrm{E}+06$ & $6.34 \mathrm{E}+03$ & $6.94 \mathrm{E}+06$ \\
\hline Lu-174 & $3.25 \mathrm{E}+03$ & $8.78 \mathrm{E}+04$ & $3.22 \mathrm{E}+04$ & $8.71 \mathrm{E}+05$ & $1.00 \mathrm{E}+04$ & $2.70 \mathrm{E}+05$ & $1.19 \mathrm{E}+05$ & $3.21 \mathrm{E}+06$ & $3.26 \mathrm{E}+05$ & $8.82 \mathrm{E}+06$ & $8.06 \mathrm{E}+03$ & $8.82 \mathrm{E}+06$ \\
\hline Lu-174m & $1.67 \mathrm{E}+03$ & $4.50 \mathrm{E}+04$ & $1.65 \mathrm{E}+04$ & $4.47 \mathrm{E}+05$ & $5.13 \mathrm{E}+03$ & $1.39 \mathrm{E}+05$ & $6.08 \mathrm{E}+04$ & $1.64 \mathrm{E}+06$ & $1.67 \mathrm{E}+05$ & $4.52 \mathrm{E}+06$ & $4.13 \mathrm{E}+03$ & $4.52 \mathrm{E}+06$ \\
\hline Lu-176 & $5.14 \mathrm{E}+02$ & $1.39 \mathrm{E}+04$ & $5.10 \mathrm{E}+03$ & $1.38 \mathrm{E}+05$ & $1.58 \mathrm{E}+03$ & $4.28 \mathrm{E}+04$ & $1.88 \mathrm{E}+04$ & $5.08 \mathrm{E}+05$ & $5.17 \mathrm{E}+04$ & $1.40 \mathrm{E}+06$ & $1.28 \mathrm{E}+03$ & $1.40 \mathrm{E}+06$ \\
\hline $\mathrm{Lu}-176 \mathrm{~m}$ & $5.48 \mathrm{E}+03$ & $1.48 \mathrm{E}+05$ & $5.44 \mathrm{E}+04$ & $1.47 \mathrm{E}+06$ & $1.69 \mathrm{E}+04$ & $4.56 \mathrm{E}+05$ & $2.00 \mathrm{E}+05$ & $5.41 \mathrm{E}+06$ & $5.51 \mathrm{E}+05$ & $1.49 \mathrm{E}+07$ & $1.36 \mathrm{E}+04$ & $1.49 \mathrm{E}+07$ \\
\hline Lu-177 & $1.70 \mathrm{E}+03$ & $4.59 \mathrm{E}+04$ & $1.69 \mathrm{E}+04$ & $4.56 \mathrm{E}+05$ & $5.23 \mathrm{E}+03$ & $1.41 \mathrm{E}+05$ & $6.21 \mathrm{E}+04$ & $1.68 \mathrm{E}+06$ & $1.71 \mathrm{E}+05$ & $4.62 \mathrm{E}+06$ & $4.22 \mathrm{E}+03$ & $4.62 \mathrm{E}+06$ \\
\hline Lu-177m & $5.48 \mathrm{E}+02$ & $1.48 \mathrm{E}+04$ & $5.44 \mathrm{E}+03$ & $1.47 \mathrm{E}+05$ & $1.69 \mathrm{E}+03$ & $4.56 \mathrm{E}+04$ & $2.00 \mathrm{E}+04$ & $5.41 \mathrm{E}+05$ & $5.51 \mathrm{E}+04$ & $1.49 \mathrm{E}+06$ & $1.36 \mathrm{E}+03$ & $1.49 \mathrm{E}+06$ \\
\hline Lu-178 & $1.99 \mathrm{E}+04$ & $5.38 \mathrm{E}+05$ & $1.98 \mathrm{E}+05$ & $5.34 \mathrm{E}+06$ & $6.13 \mathrm{E}+04$ & $1.66 \mathrm{E}+06$ & $7.27 \mathrm{E}+05$ & $1.97 \mathrm{E}+07$ & $2.00 \mathrm{E}+06$ & $5.41 \mathrm{E}+07$ & $4.94 \mathrm{E}+04$ & $5.41 \mathrm{E}+07$ \\
\hline $\mathrm{Lu}-178 \mathrm{~m}$ & $2.83 \mathrm{E}+04$ & $7.66 \mathrm{E}+05$ & $2.81 \mathrm{E}+05$ & $7.60 \mathrm{E}+06$ & $8.73 \mathrm{E}+04$ & $2.36 \mathrm{E}+06$ & $1.04 \mathrm{E}+06$ & $2.80 \mathrm{E}+07$ & $2.85 \mathrm{E}+06$ & $7.70 \mathrm{E}+07$ & $7.03 \mathrm{E}+04$ & $7.70 \mathrm{E}+07$ \\
\hline Lu-179 & $4.19 \mathrm{E}+03$ & $1.13 \mathrm{E}+05$ & $4.16 \mathrm{E}+04$ & $1.12 \mathrm{E}+06$ & $1.29 \mathrm{E}+04$ & $3.49 \mathrm{E}+05$ & $1.53 \mathrm{E}+05$ & $4.14 \mathrm{E}+06$ & $4.21 \mathrm{E}+05$ & $1.14 \mathrm{E}+07$ & $1.04 \mathrm{E}+04$ & $1.14 \mathrm{E}+07$ \\
\hline
\end{tabular}

Page 192 of 298 
SRNL-STI-2013-00115

\begin{tabular}{|c|c|c|c|c|c|c|c|c|c|c|c|c|}
\hline \multirow[t]{2}{*}{ Nuclide } & \multicolumn{2}{|c|}{ Water Ingestion DCS } & \multicolumn{2}{|c|}{ Meat Ingestion DCS } & \multicolumn{2}{|c|}{ Dairy Ingestion DCS } & \multicolumn{2}{|c|}{$\begin{array}{c}\text { Freshwater Fish } \\
\text { Ingestion DCS }\end{array}$} & \multicolumn{2}{|c|}{$\begin{array}{l}\text { Saltwater Shellfish } \\
\text { Ingestion DCS }\end{array}$} & \multicolumn{2}{|c|}{ Produce Ingestion DCS } \\
\hline & $(\mathrm{Bq} / \mathrm{L})$ & $(\mathrm{pCi} / \mathrm{L})$ & $(\mathrm{Bq} / \mathrm{kg})$ & $(\mathrm{pCi} / \mathrm{kg})$ & $(\mathrm{Bq} / \mathrm{L})$ & $(\mathrm{pCi} / \mathrm{L})$ & $(\mathrm{Bq} / \mathbf{k g})$ & $(\mathrm{pCi} / \mathbf{k g})$ & $(\mathrm{Bq} / \mathbf{k g})$ & $(\mathrm{pCi} / \mathbf{k g})$ & $(\mathrm{Bq} / \mathbf{k g})$ & $(\mathrm{pCi} / \mathbf{k g})$ \\
\hline Hf-170 & $2.35 \mathrm{E}+03$ & $6.34 \mathrm{E}+04$ & $2.33 \mathrm{E}+04$ & $6.30 \mathrm{E}+05$ & $7.23 \mathrm{E}+03$ & $1.95 \mathrm{E}+05$ & $8.58 \mathrm{E}+04$ & $2.32 \mathrm{E}+06$ & $2.36 \mathrm{E}+05$ & $6.37 \mathrm{E}+06$ & $5.82 \mathrm{E}+03$ & $6.37 \mathrm{E}+06$ \\
\hline Hf-172 & $8.52 \mathrm{E}+02$ & $2.30 \mathrm{E}+04$ & $8.46 \mathrm{E}+03$ & $2.29 \mathrm{E}+05$ & $2.62 \mathrm{E}+03$ & $7.09 \mathrm{E}+04$ & $3.11 \mathrm{E}+04$ & $8.41 \mathrm{E}+05$ & $8.56 \mathrm{E}+04$ & $2.31 \mathrm{E}+06$ & $2.11 \mathrm{E}+03$ & $2.31 \mathrm{E}+06$ \\
\hline Hf-173 & $4.29 \mathrm{E}+03$ & $1.16 \mathrm{E}+05$ & $4.26 \mathrm{E}+04$ & $1.15 \mathrm{E}+06$ & $1.32 \mathrm{E}+04$ & $3.57 \mathrm{E}+05$ & $1.57 \mathrm{E}+05$ & $4.24 \mathrm{E}+06$ & $4.31 \mathrm{E}+05$ & $1.16 \mathrm{E}+07$ & $1.06 \mathrm{E}+04$ & $1.16 \mathrm{E}+07$ \\
\hline Hf-174 & $3.95 \mathrm{E}+00$ & $1.07 \mathrm{E}+02$ & $3.92 \mathrm{E}+01$ & $1.06 \mathrm{E}+03$ & $1.22 \mathrm{E}+01$ & $3.29 \mathrm{E}+02$ & $1.44 \mathrm{E}+02$ & $3.90 \mathrm{E}+03$ & $3.97 \mathrm{E}+02$ & $1.07 \mathrm{E}+04$ & $9.80 \mathrm{E}+00$ & $1.07 \mathrm{E}+04$ \\
\hline Hf-175 & $2.36 \mathrm{E}+03$ & $6.37 \mathrm{E}+04$ & $2.34 \mathrm{E}+04$ & $6.32 \mathrm{E}+05$ & $7.26 \mathrm{E}+03$ & $1.96 \mathrm{E}+05$ & $8.61 \mathrm{E}+04$ & $2.33 \mathrm{E}+06$ & $2.37 \mathrm{E}+05$ & $6.40 \mathrm{E}+06$ & $5.85 \mathrm{E}+03$ & $6.40 \mathrm{E}+06$ \\
\hline Hf- $177 \mathrm{~m}$ & $1.15 \mathrm{E}+04$ & $3.11 \mathrm{E}+05$ & $1.14 \mathrm{E}+05$ & $3.09 \mathrm{E}+06$ & $3.55 \mathrm{E}+04$ & $9.59 \mathrm{E}+05$ & $4.21 \mathrm{E}+05$ & $1.14 \mathrm{E}+07$ & $1.16 \mathrm{E}+06$ & $3.13 \mathrm{E}+07$ & $2.86 \mathrm{E}+04$ & $3.13 \mathrm{E}+07$ \\
\hline Hf- $178 \mathrm{~m}$ & $2.50 \mathrm{E}+02$ & $6.76 \mathrm{E}+03$ & $2.48 \mathrm{E}+03$ & $6.71 \mathrm{E}+04$ & $7.71 \mathrm{E}+02$ & $2.08 \mathrm{E}+04$ & $9.15 \mathrm{E}+03$ & $2.47 \mathrm{E}+05$ & $2.52 \mathrm{E}+04$ & $6.80 \mathrm{E}+05$ & $6.21 \mathrm{E}+02$ & $6.80 \mathrm{E}+05$ \\
\hline Hf-179m & $7.40 \mathrm{E}+02$ & $2.00 \mathrm{E}+04$ & $7.35 \mathrm{E}+03$ & $1.99 \mathrm{E}+05$ & $2.28 \mathrm{E}+03$ & $6.16 \mathrm{E}+04$ & $2.71 \mathrm{E}+04$ & $7.31 \mathrm{E}+05$ & $7.44 \mathrm{E}+04$ & $2.01 \mathrm{E}+06$ & $1.84 \mathrm{E}+03$ & $2.01 \mathrm{E}+06$ \\
\hline Hf- $180 \mathrm{~m}$ & $5.63 \mathrm{E}+03$ & $1.52 \mathrm{E}+05$ & $5.59 \mathrm{E}+04$ & $1.51 \mathrm{E}+06$ & $1.73 \mathrm{E}+04$ & $4.69 \mathrm{E}+05$ & $2.06 \mathrm{E}+05$ & $5.56 \mathrm{E}+06$ & $5.66 \mathrm{E}+05$ & $1.53 \mathrm{E}+07$ & $1.40 \mathrm{E}+04$ & $1.53 \mathrm{E}+07$ \\
\hline Hf-181 & $8.29 \mathrm{E}+02$ & $2.24 \mathrm{E}+04$ & $8.23 \mathrm{E}+03$ & $2.22 \mathrm{E}+05$ & $2.55 \mathrm{E}+03$ & $6.90 \mathrm{E}+04$ & $3.03 \mathrm{E}+04$ & $8.19 \mathrm{E}+05$ & $8.33 \mathrm{E}+04$ & $2.25 \mathrm{E}+06$ & $2.06 \mathrm{E}+03$ & $2.25 \mathrm{E}+06$ \\
\hline Hf-182 & $3.63 \mathrm{E}+02$ & $9.80 \mathrm{E}+03$ & $3.60 \mathrm{E}+03$ & $9.73 \mathrm{E}+04$ & $1.12 \mathrm{E}+03$ & $3.02 \mathrm{E}+04$ & $1.33 \mathrm{E}+04$ & $3.58 \mathrm{E}+05$ & $3.64 \mathrm{E}+04$ & $9.85 \mathrm{E}+05$ & $9.00 \mathrm{E}+02$ & $9.85 \mathrm{E}+05$ \\
\hline Hf- $182 \mathrm{~m}$ & $2.09 \mathrm{E}+04$ & $5.66 \mathrm{E}+05$ & $2.08 \mathrm{E}+05$ & $5.62 \mathrm{E}+06$ & $6.45 \mathrm{E}+04$ & $1.74 \mathrm{E}+06$ & $7.65 \mathrm{E}+05$ & $2.07 \mathrm{E}+07$ & $2.10 \mathrm{E}+06$ & $5.69 \mathrm{E}+07$ & $5.20 \mathrm{E}+04$ & $5.69 \mathrm{E}+07$ \\
\hline Hf-183 & $1.26 \mathrm{E}+04$ & $3.41 \mathrm{E}+05$ & $1.25 \mathrm{E}+05$ & $3.38 \mathrm{E}+06$ & $3.88 \mathrm{E}+04$ & $1.05 \mathrm{E}+06$ & $4.61 \mathrm{E}+05$ & $1.24 \mathrm{E}+07$ & $1.27 \mathrm{E}+06$ & $3.42 \mathrm{E}+07$ & $3.13 \mathrm{E}+04$ & $3.42 \mathrm{E}+07$ \\
\hline Hf- 184 & $1.76 \mathrm{E}+03$ & $4.76 \mathrm{E}+04$ & $1.75 \mathrm{E}+04$ & $4.73 \mathrm{E}+05$ & $5.43 \mathrm{E}+03$ & $1.47 \mathrm{E}+05$ & $6.44 \mathrm{E}+04$ & $1.74 \mathrm{E}+06$ & $1.77 \mathrm{E}+05$ & $4.79 \mathrm{E}+06$ & $4.37 \mathrm{E}+03$ & $4.79 \mathrm{E}+06$ \\
\hline Тa-172 & $1.65 \mathrm{E}+04$ & $4.45 \mathrm{E}+05$ & $1.64 \mathrm{E}+05$ & $4.42 \mathrm{E}+06$ & $5.07 \mathrm{E}+04$ & $1.37 \mathrm{E}+06$ & $6.02 \mathrm{E}+05$ & $1.63 \mathrm{E}+07$ & $1.66 \mathrm{E}+06$ & $4.47 \mathrm{E}+07$ & $4.09 \mathrm{E}+04$ & $4.47 \mathrm{E}+07$ \\
\hline Ta-173 & $8.64 \mathrm{E}+03$ & $2.33 \mathrm{E}+05$ & $8.57 \mathrm{E}+04$ & $2.32 \mathrm{E}+06$ & $2.66 \mathrm{E}+04$ & $7.19 \mathrm{E}+05$ & $3.16 \mathrm{E}+05$ & $8.53 \mathrm{E}+06$ & $8.68 \mathrm{E}+05$ & $2.35 \mathrm{E}+07$ & $2.14 \mathrm{E}+04$ & $2.35 \mathrm{E}+07$ \\
\hline Ta-174 & $1.31 \mathrm{E}+04$ & $3.53 \mathrm{E}+05$ & $1.30 \mathrm{E}+05$ & $3.51 \mathrm{E}+06$ & $4.03 \mathrm{E}+04$ & $1.09 \mathrm{E}+06$ & $4.78 \mathrm{E}+05$ & $1.29 \mathrm{E}+07$ & $1.31 \mathrm{E}+06$ & $3.55 \mathrm{E}+07$ & $3.25 \mathrm{E}+04$ & $3.55 \mathrm{E}+07$ \\
\hline Ta-175 & $4.09 \mathrm{E}+03$ & $1.11 \mathrm{E}+05$ & $4.06 \mathrm{E}+04$ & $1.10 \mathrm{E}+06$ & $1.26 \mathrm{E}+04$ & $3.41 \mathrm{E}+05$ & $1.50 \mathrm{E}+05$ & $4.04 \mathrm{E}+06$ & $4.11 \mathrm{E}+05$ & $1.11 \mathrm{E}+07$ & $1.02 \mathrm{E}+04$ & $1.11 \mathrm{E}+07$ \\
\hline Ta-176 & $3.11 \mathrm{E}+03$ & $8.40 \mathrm{E}+04$ & $3.09 \mathrm{E}+04$ & $8.34 \mathrm{E}+05$ & $9.58 \mathrm{E}+03$ & $2.59 \mathrm{E}+05$ & $1.14 \mathrm{E}+05$ & $3.07 \mathrm{E}+06$ & $3.13 \mathrm{E}+05$ & $8.45 \mathrm{E}+06$ & $7.72 \mathrm{E}+03$ & $8.45 \mathrm{E}+06$ \\
\hline Ta-177 & $8.52 \mathrm{E}+03$ & $2.30 \mathrm{E}+05$ & $8.46 \mathrm{E}+04$ & $2.29 \mathrm{E}+06$ & $2.62 \mathrm{E}+04$ & $7.09 \mathrm{E}+05$ & $3.11 \mathrm{E}+05$ & $8.41 \mathrm{E}+06$ & $8.56 \mathrm{E}+05$ & $2.31 \mathrm{E}+07$ & $2.11 \mathrm{E}+04$ & $2.31 \mathrm{E}+07$ \\
\hline Ta-178m & $1.12 \mathrm{E}+04$ & $3.03 \mathrm{E}+05$ & $1.11 \mathrm{E}+05$ & $3.01 \mathrm{E}+06$ & $3.45 \mathrm{E}+04$ & $9.33 \mathrm{E}+05$ & $4.10 \mathrm{E}+05$ & $1.11 \mathrm{E}+07$ & $1.13 \mathrm{E}+06$ & $3.04 \mathrm{E}+07$ & $2.78 \mathrm{E}+04$ & $3.04 \mathrm{E}+07$ \\
\hline Тa-179 & $1.56 \mathrm{E}+04$ & $4.22 \mathrm{E}+05$ & $1.55 \mathrm{E}+05$ & $4.19 \mathrm{E}+06$ & $4.81 \mathrm{E}+04$ & $1.30 \mathrm{E}+06$ & $5.70 \mathrm{E}+05$ & $1.54 \mathrm{E}+07$ & $1.57 \mathrm{E}+06$ & $4.24 \mathrm{E}+07$ & $3.87 \mathrm{E}+04$ & $4.24 \mathrm{E}+07$ \\
\hline Ta-180 & $1.65 \mathrm{E}+04$ & $4.45 \mathrm{E}+05$ & $1.63 \mathrm{E}+05$ & $4.41 \mathrm{E}+06$ & $5.07 \mathrm{E}+04$ & $1.37 \mathrm{E}+06$ & $6.01 \mathrm{E}+05$ & $1.63 \mathrm{E}+07$ & $1.65 \mathrm{E}+06$ & $4.47 \mathrm{E}+07$ & $4.08 \mathrm{E}+04$ & $4.47 \mathrm{E}+07$ \\
\hline
\end{tabular}

Page 193 of 298 
SRNL-STI-2013-00115

\begin{tabular}{|c|c|c|c|c|c|c|c|c|c|c|c|c|}
\hline \multirow[t]{2}{*}{ Nuclide } & \multicolumn{2}{|c|}{ Water Ingestion DCS } & \multicolumn{2}{|c|}{ Meat Ingestion DCS } & \multicolumn{2}{|c|}{ Dairy Ingestion DCS } & \multicolumn{2}{|c|}{$\begin{array}{l}\text { Freshwater Fish } \\
\text { Ingestion DCS }\end{array}$} & \multicolumn{2}{|c|}{$\begin{array}{l}\text { Saltwater Shellfish } \\
\text { Ingestion DCS }\end{array}$} & \multicolumn{2}{|c|}{ Produce Ingestion DCS } \\
\hline & $(\mathrm{Bq} / \mathrm{L})$ & $(\mathrm{pCi} / \mathrm{L})$ & $(\mathrm{Bq} / \mathrm{kg})$ & $(\mathrm{pCi} / \mathrm{kg})$ & $(\mathrm{Bq} / \mathrm{L})$ & $(\mathrm{pCi} / \mathrm{L})$ & $(\mathrm{Bq} / \mathbf{k g})$ & $(\mathrm{pCi} / \mathbf{k g})$ & $(\mathrm{Bq} / \mathbf{k g})$ & $(\mathrm{pCi} / \mathbf{k g})$ & $(\mathrm{Bq} / \mathbf{k g})$ & $(\mathrm{pCi} / \mathbf{k g})$ \\
\hline Ta-182 & $6.25 \mathrm{E}+02$ & $1.69 \mathrm{E}+04$ & $6.20 \mathrm{E}+03$ & $1.68 \mathrm{E}+05$ & $1.93 \mathrm{E}+03$ & $5.20 \mathrm{E}+04$ & $2.28 \mathrm{E}+04$ & $6.17 \mathrm{E}+05$ & $6.28 \mathrm{E}+04$ & $1.70 \mathrm{E}+06$ & $1.55 \mathrm{E}+03$ & $1.70 \mathrm{E}+06$ \\
\hline Ta- $182 \mathrm{~m}$ & $7.63 \mathrm{E}+04$ & $2.06 \mathrm{E}+06$ & $7.57 \mathrm{E}+05$ & $2.05 \mathrm{E}+07$ & $2.35 \mathrm{E}+05$ & $6.35 \mathrm{E}+06$ & $2.79 \mathrm{E}+06$ & $7.54 \mathrm{E}+07$ & $7.67 \mathrm{E}+06$ & $2.07 \mathrm{E}+08$ & $1.89 \mathrm{E}+05$ & $2.07 \mathrm{E}+08$ \\
\hline Ta-183 & $6.76 \mathrm{E}+02$ & $1.83 \mathrm{E}+04$ & $6.71 \mathrm{E}+03$ & $1.81 \mathrm{E}+05$ & $2.08 \mathrm{E}+03$ & $5.63 \mathrm{E}+04$ & $2.47 \mathrm{E}+04$ & $6.68 \mathrm{E}+05$ & $6.79 \mathrm{E}+04$ & $1.84 \mathrm{E}+06$ & $1.68 \mathrm{E}+03$ & $1.84 \mathrm{E}+06$ \\
\hline Ta-184 & $1.39 \mathrm{E}+03$ & $3.76 \mathrm{E}+04$ & $1.38 \mathrm{E}+04$ & $3.73 \mathrm{E}+05$ & $4.28 \mathrm{E}+03$ & $1.16 \mathrm{E}+05$ & $5.08 \mathrm{E}+04$ & $1.37 \mathrm{E}+06$ & $1.40 \mathrm{E}+05$ & $3.77 \mathrm{E}+06$ & $3.45 \mathrm{E}+03$ & $3.77 \mathrm{E}+06$ \\
\hline Ta-185 & $1.34 \mathrm{E}+04$ & $3.61 \mathrm{E}+05$ & $1.33 \mathrm{E}+05$ & $3.59 \mathrm{E}+06$ & 4.12E+04 & $1.11 \mathrm{E}+06$ & $4.89 \mathrm{E}+05$ & $1.32 \mathrm{E}+07$ & $1.34 \mathrm{E}+06$ & $3.63 \mathrm{E}+07$ & $3.32 \mathrm{E}+04$ & $3.63 \mathrm{E}+07$ \\
\hline Ta-186 & $2.67 \mathrm{E}+04$ & $7.21 \mathrm{E}+05$ & $2.65 \mathrm{E}+05$ & $7.16 \mathrm{E}+06$ & $8.22 \mathrm{E}+04$ & $2.22 \mathrm{E}+06$ & $9.75 \mathrm{E}+05$ & $2.64 \mathrm{E}+07$ & $2.68 \mathrm{E}+06$ & $7.25 \mathrm{E}+07$ & $6.62 \mathrm{E}+04$ & $7.25 \mathrm{E}+07$ \\
\hline $\mathrm{W}-177$ & $1.73 \mathrm{E}+04$ & $4.68 \mathrm{E}+05$ & $1.72 \mathrm{E}+05$ & $4.64 \mathrm{E}+06$ & $5.33 \mathrm{E}+04$ & $1.44 \mathrm{E}+06$ & $6.32 \mathrm{E}+05$ & $1.71 \mathrm{E}+07$ & $1.74 \mathrm{E}+06$ & $4.70 \mathrm{E}+07$ & $4.29 \mathrm{E}+04$ & $4.70 \mathrm{E}+07$ \\
\hline W-178 & $3.79 \mathrm{E}+03$ & $1.02 \mathrm{E}+05$ & $3.76 \mathrm{E}+04$ & $1.02 \mathrm{E}+06$ & $1.17 \mathrm{E}+04$ & $3.16 \mathrm{E}+05$ & $1.39 \mathrm{E}+05$ & $3.75 \mathrm{E}+06$ & $3.81 \mathrm{E}+05$ & $1.03 \mathrm{E}+07$ & $9.41 \mathrm{E}+03$ & $1.03 \mathrm{E}+07$ \\
\hline W-179 & $2.64 \mathrm{E}+05$ & $7.12 \mathrm{E}+06$ & $2.62 \mathrm{E}+06$ & $7.07 \mathrm{E}+07$ & $8.12 \mathrm{E}+05$ & $2.19 \mathrm{E}+07$ & $9.63 \mathrm{E}+06$ & $2.60 \mathrm{E}+08$ & $2.65 \mathrm{E}+07$ & $7.16 \mathrm{E}+08$ & $6.54 \mathrm{E}+05$ & $7.16 \mathrm{E}+08$ \\
\hline W-181 & $1.10 \mathrm{E}+04$ & $2.97 \mathrm{E}+05$ & $1.09 \mathrm{E}+05$ & $2.95 \mathrm{E}+06$ & $3.39 \mathrm{E}+04$ & $9.16 \mathrm{E}+05$ & $4.02 \mathrm{E}+05$ & $1.09 \mathrm{E}+07$ & $1.11 \mathrm{E}+06$ & $2.99 \mathrm{E}+07$ & $2.73 \mathrm{E}+04$ & $2.99 \mathrm{E}+07$ \\
\hline W-185 & $2.05 \mathrm{E}+03$ & $5.55 \mathrm{E}+04$ & $2.04 \mathrm{E}+04$ & $5.51 \mathrm{E}+05$ & $6.32 \mathrm{E}+03$ & $1.71 \mathrm{E}+05$ & $7.50 \mathrm{E}+04$ & $2.03 \mathrm{E}+06$ & $2.06 \mathrm{E}+05$ & $5.57 \mathrm{E}+06$ & $5.09 \mathrm{E}+03$ & $5.57 \mathrm{E}+06$ \\
\hline W-187 & $1.56 \mathrm{E}+03$ & $4.21 \mathrm{E}+04$ & $1.55 \mathrm{E}+04$ & $4.18 \mathrm{E}+05$ & $4.80 \mathrm{E}+03$ & $1.30 \mathrm{E}+05$ & $5.70 \mathrm{E}+04$ & $1.54 \mathrm{E}+06$ & $1.57 \mathrm{E}+05$ & $4.23 \mathrm{E}+06$ & $3.87 \mathrm{E}+03$ & $4.23 \mathrm{E}+06$ \\
\hline W-188 & $4.36 \mathrm{E}+02$ & $1.18 \mathrm{E}+04$ & $4.33 \mathrm{E}+03$ & $1.17 \mathrm{E}+05$ & $1.34 \mathrm{E}+03$ & $3.63 \mathrm{E}+04$ & $1.59 \mathrm{E}+04$ & $4.31 \mathrm{E}+05$ & $4.39 \mathrm{E}+04$ & $1.19 \mathrm{E}+06$ & $1.08 \mathrm{E}+03$ & $1.19 \mathrm{E}+06$ \\
\hline W-190 & $1.12 \mathrm{E}+04$ & $3.03 \mathrm{E}+05$ & $1.11 \mathrm{E}+05$ & $3.01 \mathrm{E}+06$ & $3.45 \mathrm{E}+04$ & $9.33 \mathrm{E}+05$ & $4.10 \mathrm{E}+05$ & $1.11 \mathrm{E}+07$ & $1.13 \mathrm{E}+06$ & $3.04 \mathrm{E}+07$ & $2.78 \mathrm{E}+04$ & $3.04 \mathrm{E}+07$ \\
\hline Re-178 & $3.38 \mathrm{E}+04$ & $9.13 \mathrm{E}+05$ & $3.35 \mathrm{E}+05$ & $9.07 \mathrm{E}+06$ & $1.04 \mathrm{E}+05$ & $2.81 \mathrm{E}+06$ & $1.24 \mathrm{E}+06$ & $3.34 \mathrm{E}+07$ & $3.40 \mathrm{E}+06$ & $9.18 \mathrm{E}+07$ & $8.39 \mathrm{E}+04$ & $9.18 \mathrm{E}+07$ \\
\hline Re-179 & $7.40 \mathrm{E}+04$ & $2.00 \mathrm{E}+06$ & $7.35 \mathrm{E}+05$ & $1.99 \mathrm{E}+07$ & $2.28 \mathrm{E}+05$ & $6.16 \mathrm{E}+06$ & $2.71 \mathrm{E}+06$ & $7.31 \mathrm{E}+07$ & $7.44 \mathrm{E}+06$ & $2.01 \mathrm{E}+08$ & $1.84 \mathrm{E}+05$ & $2.01 \mathrm{E}+08$ \\
\hline Re-181 & $2.19 \mathrm{E}+03$ & $5.92 \mathrm{E}+04$ & $2.17 \mathrm{E}+04$ & $5.87 \mathrm{E}+05$ & $6.75 \mathrm{E}+03$ & $1.82 \mathrm{E}+05$ & $8.00 \mathrm{E}+04$ & $2.16 \mathrm{E}+06$ & $2.20 \mathrm{E}+05$ & $5.95 \mathrm{E}+06$ & $5.43 \mathrm{E}+03$ & $5.95 \mathrm{E}+06$ \\
\hline Re-182 & $6.65 \mathrm{E}+02$ & $1.80 \mathrm{E}+04$ & $6.60 \mathrm{E}+03$ & $1.78 \mathrm{E}+05$ & $2.05 \mathrm{E}+03$ & $5.54 \mathrm{E}+04$ & $2.43 \mathrm{E}+04$ & $6.57 \mathrm{E}+05$ & $6.68 \mathrm{E}+04$ & $1.81 \mathrm{E}+06$ & $1.65 \mathrm{E}+03$ & $1.81 \mathrm{E}+06$ \\
\hline Re- $182 \mathrm{~m}$ & $3.31 \mathrm{E}+03$ & $8.94 \mathrm{E}+04$ & $3.28 \mathrm{E}+04$ & $8.87 \mathrm{E}+05$ & $1.02 \mathrm{E}+04$ & $2.75 \mathrm{E}+05$ & $1.21 \mathrm{E}+05$ & $3.27 \mathrm{E}+06$ & $3.32 \mathrm{E}+05$ & $8.99 \mathrm{E}+06$ & $8.21 \mathrm{E}+03$ & $8.99 \mathrm{E}+06$ \\
\hline Re-183 & $9.49 \mathrm{E}+02$ & $2.57 \mathrm{E}+04$ & $9.42 \mathrm{E}+03$ & $2.55 \mathrm{E}+05$ & $2.92 \mathrm{E}+03$ & $7.90 \mathrm{E}+04$ & $3.47 \mathrm{E}+04$ & $9.38 \mathrm{E}+05$ & $9.54 \mathrm{E}+04$ & $2.58 \mathrm{E}+06$ & $2.36 \mathrm{E}+03$ & $2.58 \mathrm{E}+06$ \\
\hline Re-184 & $9.64 \mathrm{E}+02$ & $2.61 \mathrm{E}+04$ & $9.57 \mathrm{E}+03$ & $2.59 \mathrm{E}+05$ & $2.97 \mathrm{E}+03$ & $8.03 \mathrm{E}+04$ & $3.52 \mathrm{E}+04$ & $9.52 \mathrm{E}+05$ & $9.69 \mathrm{E}+04$ & $2.62 \mathrm{E}+06$ & $2.39 \mathrm{E}+03$ & $2.62 \mathrm{E}+06$ \\
\hline $\mathrm{Re}-184 \mathrm{~m}$ & $6.28 \mathrm{E}+02$ & $1.70 \mathrm{E}+04$ & $6.24 \mathrm{E}+03$ & $1.69 \mathrm{E}+05$ & $1.94 \mathrm{E}+03$ & $5.23 \mathrm{E}+04$ & $2.30 \mathrm{E}+04$ & $6.20 \mathrm{E}+05$ & $6.31 \mathrm{E}+04$ & $1.71 \mathrm{E}+06$ & $1.56 \mathrm{E}+03$ & $1.71 \mathrm{E}+06$ \\
\hline Re-186 & $6.25 \mathrm{E}+02$ & $1.69 \mathrm{E}+04$ & $6.20 \mathrm{E}+03$ & $1.68 \mathrm{E}+05$ & $1.93 \mathrm{E}+03$ & $5.20 \mathrm{E}+04$ & $2.28 \mathrm{E}+04$ & $6.17 \mathrm{E}+05$ & $6.28 \mathrm{E}+04$ & $1.70 \mathrm{E}+06$ & $1.55 \mathrm{E}+03$ & $1.70 \mathrm{E}+06$ \\
\hline
\end{tabular}

Page 194 of 298 
SRNL-STI-2013-00115

\begin{tabular}{|c|c|c|c|c|c|c|c|c|c|c|c|c|}
\hline \multirow[t]{2}{*}{ Nuclide } & \multicolumn{2}{|c|}{ Water Ingestion DCS } & \multicolumn{2}{|c|}{ Meat Ingestion DCS } & \multicolumn{2}{|c|}{ Dairy Ingestion DCS } & \multicolumn{2}{|c|}{$\begin{array}{c}\text { Freshwater Fish } \\
\text { Ingestion DCS }\end{array}$} & \multicolumn{2}{|c|}{$\begin{array}{l}\text { Saltwater Shellfish } \\
\text { Ingestion DCS }\end{array}$} & \multicolumn{2}{|c|}{ Produce Ingestion DCS } \\
\hline & $(\mathrm{Bq} / \mathrm{L})$ & $(\mathrm{pCi} / \mathrm{L})$ & $(\mathrm{Bq} / \mathrm{kg})$ & $(\mathrm{pCi} / \mathbf{k g})$ & $(\mathrm{Bq} / \mathrm{L})$ & $(\mathrm{pCi} / \mathrm{L})$ & $(\mathrm{Bq} / \mathrm{kg})$ & $(\mathrm{pCi} / \mathbf{k g})$ & $(\mathrm{Bq} / \mathrm{kg})$ & $(\mathrm{pCi} / \mathbf{k g})$ & $(\mathrm{Bq} / \mathrm{kg})$ & (pCi//kg) \\
\hline Re-186m & $4.09 \mathrm{E}+02$ & $1.11 \mathrm{E}+04$ & $4.06 \mathrm{E}+03$ & $1.10 \mathrm{E}+05$ & $1.26 \mathrm{E}+03$ & $3.41 \mathrm{E}+04$ & $1.50 \mathrm{E}+04$ & $4.04 \mathrm{E}+05$ & $4.11 \mathrm{E}+04$ & $1.11 \mathrm{E}+06$ & $1.02 \mathrm{E}+03$ & $1.11 \mathrm{E}+06$ \\
\hline Re-187 & $1.89 \mathrm{E}+05$ & $5.10 \mathrm{E}+06$ & $1.87 \mathrm{E}+06$ & $5.06 \mathrm{E}+07$ & $5.81 \mathrm{E}+05$ & $1.57 \mathrm{E}+07$ & $6.90 \mathrm{E}+06$ & $1.86 \mathrm{E}+08$ & $1.90 \mathrm{E}+07$ & $5.13 \mathrm{E}+08$ & $4.68 \mathrm{E}+05$ & $5.13 \mathrm{E}+08$ \\
\hline Re-188 & $6.48 \mathrm{E}+02$ & $1.75 \mathrm{E}+04$ & $6.43 \mathrm{E}+03$ & $1.74 \mathrm{E}+05$ & $2.00 \mathrm{E}+03$ & $5.39 \mathrm{E}+04$ & $2.37 \mathrm{E}+04$ & $6.40 \mathrm{E}+05$ & $6.51 \mathrm{E}+04$ & $1.76 \mathrm{E}+06$ & $1.61 \mathrm{E}+03$ & $1.76 \mathrm{E}+06$ \\
\hline Re- $188 \mathrm{~m}$ & $2.99 \mathrm{E}+04$ & $8.08 \mathrm{E}+05$ & $2.97 \mathrm{E}+05$ & $8.02 \mathrm{E}+06$ & $9.21 \mathrm{E}+04$ & $2.49 \mathrm{E}+06$ & $1.09 \mathrm{E}+06$ & $2.95 \mathrm{E}+07$ & $3.00 \mathrm{E}+06$ & $8.12 \mathrm{E}+07$ & $7.42 \mathrm{E}+04$ & $8.12 \mathrm{E}+07$ \\
\hline Re-189 & $1.20 \mathrm{E}+03$ & $3.23 \mathrm{E}+04$ & $1.19 \mathrm{E}+04$ & $3.21 \mathrm{E}+05$ & $3.68 \mathrm{E}+03$ & $9.96 \mathrm{E}+04$ & $4.37 \mathrm{E}+04$ & $1.18 \mathrm{E}+06$ & $1.20 \mathrm{E}+05$ & $3.25 \mathrm{E}+06$ & $2.97 \mathrm{E}+03$ & $3.25 \mathrm{E}+06$ \\
\hline Re-190m & $2.47 \mathrm{E}+03$ & $6.67 \mathrm{E}+04$ & $2.45 \mathrm{E}+04$ & $6.62 \mathrm{E}+05$ & $7.60 \mathrm{E}+03$ & $2.05 \mathrm{E}+05$ & $9.02 \mathrm{E}+04$ & $2.44 \mathrm{E}+06$ & $2.48 \mathrm{E}+05$ & $6.70 \mathrm{E}+06$ & $6.12 \mathrm{E}+03$ & $6.70 \mathrm{E}+06$ \\
\hline Os-180 & $5.20 \mathrm{E}+04$ & $1.41 \mathrm{E}+06$ & $5.17 \mathrm{E}+05$ & $1.40 \mathrm{E}+07$ & $1.60 \mathrm{E}+05$ & $4.33 \mathrm{E}+06$ & $1.90 \mathrm{E}+06$ & $5.14 \mathrm{E}+07$ & $5.23 \mathrm{E}+06$ & $1.41 \mathrm{E}+08$ & $1.29 \mathrm{E}+05$ & $1.41 \mathrm{E}+08$ \\
\hline Os-181 & $1.05 \mathrm{E}+04$ & $2.85 \mathrm{E}+05$ & $1.05 \mathrm{E}+05$ & $2.83 \mathrm{E}+06$ & $3.25 \mathrm{E}+04$ & $8.78 \mathrm{E}+05$ & $3.85 \mathrm{E}+05$ & $1.04 \mathrm{E}+07$ & $1.06 \mathrm{E}+06$ & $2.86 \mathrm{E}+07$ & $2.62 \mathrm{E}+04$ & $2.86 \mathrm{E}+07$ \\
\hline Os-182 & $1.66 \mathrm{E}+03$ & $4.48 \mathrm{E}+04$ & $1.65 \mathrm{E}+04$ & $4.45 \mathrm{E}+05$ & $5.11 \mathrm{E}+03$ & $1.38 \mathrm{E}+05$ & $6.06 \mathrm{E}+04$ & $1.64 \mathrm{E}+06$ & $1.67 \mathrm{E}+05$ & $4.50 \mathrm{E}+06$ & $4.12 \mathrm{E}+03$ & $4.50 \mathrm{E}+06$ \\
\hline Os-183 & $4.22 \mathrm{E}+03$ & $1.14 \mathrm{E}+05$ & $4.18 \mathrm{E}+04$ & $1.13 \mathrm{E}+06$ & $1.30 \mathrm{E}+04$ & $3.51 \mathrm{E}+05$ & $1.54 \mathrm{E}+05$ & $4.16 \mathrm{E}+06$ & $4.24 \mathrm{E}+05$ & $1.15 \mathrm{E}+07$ & $1.05 \mathrm{E}+04$ & $1.15 \mathrm{E}+07$ \\
\hline Os- $183 \mathrm{~m}$ & $4.71 \mathrm{E}+03$ & $1.27 \mathrm{E}+05$ & $4.68 \mathrm{E}+04$ & $1.26 \mathrm{E}+06$ & $1.45 \mathrm{E}+04$ & $3.92 \mathrm{E}+05$ & $1.72 \mathrm{E}+05$ & $4.65 \mathrm{E}+06$ & $4.73 \mathrm{E}+05$ & $1.28 \mathrm{E}+07$ & $1.17 \mathrm{E}+04$ & $1.28 \mathrm{E}+07$ \\
\hline Os-185 & $1.96 \mathrm{E}+03$ & $5.29 \mathrm{E}+04$ & $1.94 \mathrm{E}+04$ & $5.25 \mathrm{E}+05$ & $6.03 \mathrm{E}+03$ & $1.63 \mathrm{E}+05$ & $7.16 \mathrm{E}+04$ & $1.93 \mathrm{E}+06$ & $1.97 \mathrm{E}+05$ & $5.32 \mathrm{E}+06$ & $4.86 \mathrm{E}+03$ & $5.32 \mathrm{E}+06$ \\
\hline Os-186 & $2.90 \mathrm{E}+01$ & $7.84 \mathrm{E}+02$ & $2.88 \mathrm{E}+02$ & $7.78 \mathrm{E}+03$ & $8.93 \mathrm{E}+01$ & $2.41 \mathrm{E}+03$ & $1.06 \mathrm{E}+03$ & $2.86 \mathrm{E}+04$ & $2.91 \mathrm{E}+03$ & $7.88 \mathrm{E}+04$ & $7.19 \mathrm{E}+01$ & $7.88 \mathrm{E}+04$ \\
\hline Os-189m & $5.27 \mathrm{E}+04$ & $1.42 \mathrm{E}+06$ & $5.23 \mathrm{E}+05$ & $1.41 \mathrm{E}+07$ & $1.62 \mathrm{E}+05$ & $4.39 \mathrm{E}+06$ & $1.93 \mathrm{E}+06$ & $5.21 \mathrm{E}+07$ & $5.30 \mathrm{E}+06$ & $1.43 \mathrm{E}+08$ & $1.31 \mathrm{E}+05$ & $1.43 \mathrm{E}+08$ \\
\hline Os-191 & $1.57 \mathrm{E}+03$ & $4.23 \mathrm{E}+04$ & $1.55 \mathrm{E}+04$ & $4.20 \mathrm{E}+05$ & $4.83 \mathrm{E}+03$ & $1.30 \mathrm{E}+05$ & $5.72 \mathrm{E}+04$ & $1.55 \mathrm{E}+06$ & $1.57 \mathrm{E}+05$ & $4.25 \mathrm{E}+06$ & $3.89 \mathrm{E}+03$ & $4.25 \mathrm{E}+06$ \\
\hline Os-191m & $9.15 \mathrm{E}+03$ & $2.47 \mathrm{E}+05$ & $9.08 \mathrm{E}+04$ & $2.45 \mathrm{E}+06$ & $2.82 \mathrm{E}+04$ & $7.61 \mathrm{E}+05$ & $3.34 \mathrm{E}+05$ & $9.03 \mathrm{E}+06$ & $9.19 \mathrm{E}+05$ & $2.48 \mathrm{E}+07$ & $2.27 \mathrm{E}+04$ & $2.48 \mathrm{E}+07$ \\
\hline Os-193 & $1.09 \mathrm{E}+03$ & $2.95 \mathrm{E}+04$ & $1.08 \mathrm{E}+04$ & $2.93 \mathrm{E}+05$ & $3.36 \mathrm{E}+03$ & $9.08 \mathrm{E}+04$ & $3.99 \mathrm{E}+04$ & $1.08 \mathrm{E}+06$ & $1.10 \mathrm{E}+05$ & $2.96 \mathrm{E}+06$ & $2.71 \mathrm{E}+03$ & $2.96 \mathrm{E}+06$ \\
\hline Os-194 & $3.66 \mathrm{E}+02$ & $9.89 \mathrm{E}+03$ & $3.63 \mathrm{E}+03$ & $9.81 \mathrm{E}+04$ & $1.13 \mathrm{E}+03$ & $3.05 \mathrm{E}+04$ & $1.34 \mathrm{E}+04$ & $3.61 \mathrm{E}+05$ & $3.68 \mathrm{E}+04$ & $9.94 \mathrm{E}+05$ & $9.08 \mathrm{E}+02$ & $9.94 \mathrm{E}+05$ \\
\hline Os-196 & $8.40 \mathrm{E}+03$ & $2.27 \mathrm{E}+05$ & $8.34 \mathrm{E}+04$ & $2.25 \mathrm{E}+06$ & $2.59 \mathrm{E}+04$ & $7.00 \mathrm{E}+05$ & $3.07 \mathrm{E}+05$ & $8.30 \mathrm{E}+06$ & $8.45 \mathrm{E}+05$ & $2.28 \mathrm{E}+07$ & $2.09 \mathrm{E}+04$ & $2.28 \mathrm{E}+07$ \\
\hline Ir-182 & $1.79 \mathrm{E}+04$ & $4.84 \mathrm{E}+05$ & $1.78 \mathrm{E}+05$ & $4.80 \mathrm{E}+06$ & $5.51 \mathrm{E}+04$ & $1.49 \mathrm{E}+06$ & $6.54 \mathrm{E}+05$ & $1.77 \mathrm{E}+07$ & $1.80 \mathrm{E}+06$ & $4.86 \mathrm{E}+07$ & $4.44 \mathrm{E}+04$ & $4.86 \mathrm{E}+07$ \\
\hline Ir-183 & $1.83 \mathrm{E}+04$ & $4.94 \mathrm{E}+05$ & $1.81 \mathrm{E}+05$ & $4.90 \mathrm{E}+06$ & $5.63 \mathrm{E}+04$ & $1.52 \mathrm{E}+06$ & $6.67 \mathrm{E}+05$ & $1.80 \mathrm{E}+07$ & $1.84 \mathrm{E}+06$ & $4.96 \mathrm{E}+07$ & $4.53 \mathrm{E}+04$ & $4.96 \mathrm{E}+07$ \\
\hline Ir-184 & $5.10 \mathrm{E}+03$ & $1.38 \mathrm{E}+05$ & $5.06 \mathrm{E}+04$ & $1.37 \mathrm{E}+06$ & $1.57 \mathrm{E}+04$ & $4.24 \mathrm{E}+05$ & $1.86 \mathrm{E}+05$ & $5.03 \mathrm{E}+06$ & $5.12 \mathrm{E}+05$ & $1.38 \mathrm{E}+07$ & $1.26 \mathrm{E}+04$ & $1.38 \mathrm{E}+07$ \\
\hline Ir-185 & $2.91 \mathrm{E}+03$ & $7.85 \mathrm{E}+04$ & $2.88 \mathrm{E}+04$ & $7.80 \mathrm{E}+05$ & $8.95 \mathrm{E}+03$ & $2.42 \mathrm{E}+05$ & $1.06 \mathrm{E}+05$ & $2.87 \mathrm{E}+06$ & $2.92 \mathrm{E}+05$ & $7.89 \mathrm{E}+06$ & $7.21 \mathrm{E}+03$ & $7.89 \mathrm{E}+06$ \\
\hline
\end{tabular}

Page 195 of 298 
SRNL-STI-2013-00115

\begin{tabular}{|c|c|c|c|c|c|c|c|c|c|c|c|c|}
\hline \multirow[t]{2}{*}{ Nuclide } & \multicolumn{2}{|c|}{ Water Ingestion DCS } & \multicolumn{2}{|c|}{ Meat Ingestion DCS } & \multicolumn{2}{|c|}{ Dairy Ingestion DCS } & \multicolumn{2}{|c|}{$\begin{array}{c}\text { Freshwater Fish } \\
\text { Ingestion DCS }\end{array}$} & \multicolumn{2}{|c|}{$\begin{array}{l}\text { Saltwater Shellfish } \\
\text { Ingestion DCS }\end{array}$} & \multicolumn{2}{|c|}{ Produce Ingestion DCS } \\
\hline & $(\mathrm{Bq} / \mathrm{L})$ & $(\mathrm{pCi} / \mathrm{L})$ & $(\mathrm{Bq} / \mathrm{kg})$ & $(\mathrm{pCi} / \mathbf{k g})$ & $(\mathrm{Bq} / \mathrm{L})$ & $(\mathrm{pCi} / \mathrm{L})$ & $(\mathrm{Bq} / \mathrm{kg})$ & $(\mathrm{pCi} / \mathbf{k g})$ & $(\mathrm{Bq} / \mathbf{k g})$ & $(\mathrm{pCi} / \mathbf{k g})$ & $(\mathrm{Bq} / \mathrm{kg})$ & (pCi//kg) \\
\hline Ir-186 & $1.71 \mathrm{E}+03$ & $4.62 \mathrm{E}+04$ & $1.70 \mathrm{E}+04$ & $4.59 \mathrm{E}+05$ & $5.27 \mathrm{E}+03$ & $1.42 \mathrm{E}+05$ & $6.25 \mathrm{E}+04$ & $1.69 \mathrm{E}+06$ & $1.72 \mathrm{E}+05$ & $4.65 \mathrm{E}+06$ & $4.25 \mathrm{E}+03$ & $4.65 \mathrm{E}+06$ \\
\hline Ir-186m & $1.33 \mathrm{E}+04$ & $3.58 \mathrm{E}+05$ & $1.32 \mathrm{E}+05$ & $3.56 \mathrm{E}+06$ & $4.08 \mathrm{E}+04$ & $1.10 \mathrm{E}+06$ & $4.85 \mathrm{E}+05$ & $1.31 \mathrm{E}+07$ & $1.33 \mathrm{E}+06$ & $3.60 \mathrm{E}+07$ & $3.29 \mathrm{E}+04$ & $3.60 \mathrm{E}+07$ \\
\hline Ir-187 & $8.18 \mathrm{E}+03$ & $2.21 \mathrm{E}+05$ & $8.12 \mathrm{E}+04$ & $2.20 \mathrm{E}+06$ & $2.52 \mathrm{E}+04$ & $6.81 \mathrm{E}+05$ & $2.99 \mathrm{E}+05$ & $8.08 \mathrm{E}+06$ & $8.22 \mathrm{E}+05$ & $2.22 \mathrm{E}+07$ & $2.03 \mathrm{E}+04$ & $2.22 \mathrm{E}+07$ \\
\hline Ir-188 & $1.29 \mathrm{E}+03$ & $3.49 \mathrm{E}+04$ & $1.28 \mathrm{E}+04$ & $3.47 \mathrm{E}+05$ & $3.98 \mathrm{E}+03$ & $1.08 \mathrm{E}+05$ & $4.73 \mathrm{E}+04$ & $1.28 \mathrm{E}+06$ & $1.30 \mathrm{E}+05$ & $3.51 \mathrm{E}+06$ & $3.21 \mathrm{E}+03$ & $3.51 \mathrm{E}+06$ \\
\hline Ir-189 & $3.80 \mathrm{E}+03$ & $1.03 \mathrm{E}+05$ & $3.78 \mathrm{E}+04$ & $1.02 \mathrm{E}+06$ & $1.17 \mathrm{E}+04$ & $3.17 \mathrm{E}+05$ & $1.39 \mathrm{E}+05$ & $3.76 \mathrm{E}+06$ & $3.82 \mathrm{E}+05$ & $1.03 \mathrm{E}+07$ & $9.44 \mathrm{E}+03$ & $1.03 \mathrm{E}+07$ \\
\hline Ir-190 & $9.15 \mathrm{E}+02$ & $2.47 \mathrm{E}+04$ & $9.08 \mathrm{E}+03$ & $2.45 \mathrm{E}+05$ & $2.82 \mathrm{E}+03$ & $7.61 \mathrm{E}+04$ & $3.34 \mathrm{E}+04$ & $9.03 \mathrm{E}+05$ & $9.19 \mathrm{E}+04$ & $2.48 \mathrm{E}+06$ & $2.27 \mathrm{E}+03$ & $2.48 \mathrm{E}+06$ \\
\hline Ir-190m & $1.35 \mathrm{E}+05$ & $3.66 \mathrm{E}+06$ & $1.34 \mathrm{E}+06$ & $3.63 \mathrm{E}+07$ & $4.17 \mathrm{E}+05$ & $1.13 \mathrm{E}+07$ & $4.95 \mathrm{E}+06$ & $1.34 \mathrm{E}+08$ & $1.36 \mathrm{E}+07$ & $3.68 \mathrm{E}+08$ & $3.36 \mathrm{E}+05$ & $3.68 \mathrm{E}+08$ \\
\hline Ir-190n & $8.24 \mathrm{E}+03$ & $2.23 \mathrm{E}+05$ & $8.18 \mathrm{E}+04$ & $2.21 \mathrm{E}+06$ & $2.54 \mathrm{E}+04$ & $6.86 \mathrm{E}+05$ & $3.01 \mathrm{E}+05$ & $8.14 \mathrm{E}+06$ & $8.28 \mathrm{E}+05$ & $2.24 \mathrm{E}+07$ & $2.04 \mathrm{E}+04$ & $2.24 \mathrm{E}+07$ \\
\hline Ir-192 & $6.83 \mathrm{E}+02$ & $1.85 \mathrm{E}+04$ & $6.78 \mathrm{E}+03$ & $1.83 \mathrm{E}+05$ & $2.11 \mathrm{E}+03$ & $5.69 \mathrm{E}+04$ & $2.50 \mathrm{E}+04$ & $6.75 \mathrm{E}+05$ & $6.87 \mathrm{E}+04$ & $1.86 \mathrm{E}+06$ & $1.70 \mathrm{E}+03$ & $1.86 \mathrm{E}+06$ \\
\hline Ir-192n & $1.00 \mathrm{E}+03$ & $2.71 \mathrm{E}+04$ & $9.96 \mathrm{E}+03$ & $2.69 \mathrm{E}+05$ & $3.09 \mathrm{E}+03$ & $8.35 \mathrm{E}+04$ & $3.67 \mathrm{E}+04$ & $9.91 \mathrm{E}+05$ & $1.01 \mathrm{E}+05$ & $2.72 \mathrm{E}+06$ & $2.49 \mathrm{E}+03$ & $2.72 \mathrm{E}+06$ \\
\hline Ir-193m & $3.11 \mathrm{E}+03$ & $8.40 \mathrm{E}+04$ & $3.09 \mathrm{E}+04$ & $8.34 \mathrm{E}+05$ & $9.58 \mathrm{E}+03$ & $2.59 \mathrm{E}+05$ & $1.14 \mathrm{E}+05$ & $3.07 \mathrm{E}+06$ & $3.13 \mathrm{E}+05$ & $8.45 \mathrm{E}+06$ & $7.72 \mathrm{E}+03$ & $8.45 \mathrm{E}+06$ \\
\hline Ir-194 & $6.76 \mathrm{E}+02$ & $1.83 \mathrm{E}+04$ & $6.71 \mathrm{E}+03$ & $1.81 \mathrm{E}+05$ & $2.08 \mathrm{E}+03$ & $5.63 \mathrm{E}+04$ & $2.47 \mathrm{E}+04$ & $6.68 \mathrm{E}+05$ & $6.79 \mathrm{E}+04$ & $1.84 \mathrm{E}+06$ & $1.68 \mathrm{E}+03$ & $1.84 \mathrm{E}+06$ \\
\hline Ir-194m & $4.73 \mathrm{E}+02$ & $1.28 \mathrm{E}+04$ & $4.69 \mathrm{E}+03$ & $1.27 \mathrm{E}+05$ & $1.46 \mathrm{E}+03$ & $3.94 \mathrm{E}+04$ & $1.73 \mathrm{E}+04$ & $4.67 \mathrm{E}+05$ & $4.75 \mathrm{E}+04$ & $1.28 \mathrm{E}+06$ & $1.17 \mathrm{E}+03$ & $1.28 \mathrm{E}+06$ \\
\hline Ir-195 & $9.01 \mathrm{E}+03$ & $2.44 \mathrm{E}+05$ & $8.95 \mathrm{E}+04$ & $2.42 \mathrm{E}+06$ & $2.78 \mathrm{E}+04$ & $7.50 \mathrm{E}+05$ & $3.29 \mathrm{E}+05$ & $8.90 \mathrm{E}+06$ & $9.06 \mathrm{E}+05$ & $2.45 \mathrm{E}+07$ & $2.24 \mathrm{E}+04$ & $2.45 \mathrm{E}+07$ \\
\hline Ir-195m & $6.80 \mathrm{E}+03$ & $1.84 \mathrm{E}+05$ & $6.75 \mathrm{E}+04$ & $1.82 \mathrm{E}+06$ & $2.09 \mathrm{E}+04$ & $5.66 \mathrm{E}+05$ & $2.48 \mathrm{E}+05$ & $6.71 \mathrm{E}+06$ & $6.83 \mathrm{E}+05$ & $1.85 \mathrm{E}+07$ & $1.69 \mathrm{E}+04$ & $1.85 \mathrm{E}+07$ \\
\hline Ir-196m & $8.70 \mathrm{E}+03$ & $2.35 \mathrm{E}+05$ & $8.63 \mathrm{E}+04$ & $2.33 \mathrm{E}+06$ & $2.68 \mathrm{E}+04$ & $7.24 \mathrm{E}+05$ & $3.18 \mathrm{E}+05$ & $8.59 \mathrm{E}+06$ & $8.74 \mathrm{E}+05$ & $2.36 \mathrm{E}+07$ & $2.16 \mathrm{E}+04$ & $2.36 \mathrm{E}+07$ \\
\hline Pt-184 & $3.20 \mathrm{E}+04$ & $8.64 \mathrm{E}+05$ & $3.17 \mathrm{E}+05$ & $8.58 \mathrm{E}+06$ & $9.85 \mathrm{E}+04$ & $2.66 \mathrm{E}+06$ & $1.17 \mathrm{E}+06$ & $3.16 \mathrm{E}+07$ & $3.21 \mathrm{E}+06$ & $8.68 \mathrm{E}+07$ & $7.93 \mathrm{E}+04$ & $8.68 \mathrm{E}+07$ \\
\hline Pt-186 & $8.88 \mathrm{E}+03$ & $2.40 \mathrm{E}+05$ & $8.82 \mathrm{E}+04$ & $2.38 \mathrm{E}+06$ & $2.74 \mathrm{E}+04$ & $7.40 \mathrm{E}+05$ & $3.25 \mathrm{E}+05$ & $8.78 \mathrm{E}+06$ & $8.93 \mathrm{E}+05$ & $2.41 \mathrm{E}+07$ & $2.20 \mathrm{E}+04$ & $2.41 \mathrm{E}+07$ \\
\hline Pt-187 & $1.11 \mathrm{E}+04$ & $3.00 \mathrm{E}+05$ & $1.10 \mathrm{E}+05$ & $2.98 \mathrm{E}+06$ & $3.42 \mathrm{E}+04$ & $9.25 \mathrm{E}+05$ & $4.06 \mathrm{E}+05$ & $1.10 \mathrm{E}+07$ & $1.12 \mathrm{E}+06$ & $3.02 \mathrm{E}+07$ & $2.76 \mathrm{E}+04$ & $3.02 \mathrm{E}+07$ \\
\hline Pt-188 & $1.13 \mathrm{E}+03$ & $3.06 \mathrm{E}+04$ & $1.12 \mathrm{E}+04$ & $3.03 \mathrm{E}+05$ & $3.48 \mathrm{E}+03$ & $9.41 \mathrm{E}+04$ & $4.13 \mathrm{E}+04$ & $1.12 \mathrm{E}+06$ & $1.14 \mathrm{E}+05$ & $3.07 \mathrm{E}+06$ & $2.81 \mathrm{E}+03$ & $3.07 \mathrm{E}+06$ \\
\hline Pt-189 & $4.80 \mathrm{E}+03$ & $1.30 \mathrm{E}+05$ & $4.77 \mathrm{E}+04$ & $1.29 \mathrm{E}+06$ & $1.48 \mathrm{E}+04$ & $4.00 \mathrm{E}+05$ & $1.76 \mathrm{E}+05$ & $4.74 \mathrm{E}+06$ & $4.83 \mathrm{E}+05$ & $1.30 \mathrm{E}+07$ & $1.19 \mathrm{E}+04$ & $1.30 \mathrm{E}+07$ \\
\hline Pt-190 & $1.33 \mathrm{E}+02$ & $3.58 \mathrm{E}+03$ & $1.32 \mathrm{E}+03$ & $3.56 \mathrm{E}+04$ & $4.08 \mathrm{E}+02$ & $1.10 \mathrm{E}+04$ & $4.85 \mathrm{E}+03$ & $1.31 \mathrm{E}+05$ & $1.33 \mathrm{E}+04$ & $3.60 \mathrm{E}+05$ & $3.29 \mathrm{E}+02$ & $3.60 \mathrm{E}+05$ \\
\hline Pt-191 & $2.55 \mathrm{E}+03$ & $6.89 \mathrm{E}+04$ & $2.53 \mathrm{E}+04$ & $6.84 \mathrm{E}+05$ & $7.85 \mathrm{E}+03$ & $2.12 \mathrm{E}+05$ & $9.31 \mathrm{E}+04$ & $2.52 \mathrm{E}+06$ & $2.56 \mathrm{E}+05$ & $6.92 \mathrm{E}+06$ & $6.32 \mathrm{E}+03$ & $6.92 \mathrm{E}+06$ \\
\hline
\end{tabular}

Page 196 of 298 
SRNL-STI-2013-00115

\begin{tabular}{|c|c|c|c|c|c|c|c|c|c|c|c|c|}
\hline \multirow[t]{2}{*}{ Nuclide } & \multicolumn{2}{|c|}{ Water Ingestion DCS } & \multicolumn{2}{|c|}{ Meat Ingestion DCS } & \multicolumn{2}{|c|}{ Dairy Ingestion DCS } & \multicolumn{2}{|c|}{$\begin{array}{c}\text { Freshwater Fish } \\
\text { Ingestion DCS }\end{array}$} & \multicolumn{2}{|c|}{$\begin{array}{l}\text { Saltwater Shellfish } \\
\text { Ingestion DCS }\end{array}$} & \multicolumn{2}{|c|}{ Produce Ingestion DCS } \\
\hline & $(\mathrm{Bq} / \mathrm{L})$ & $(\mathrm{pCi} / \mathrm{L})$ & $(\mathrm{Bq} / \mathrm{kg})$ & $(\mathrm{pCi} / \mathrm{kg})$ & $(\mathrm{Bq} / \mathrm{L})$ & $(\mathrm{pCi} / \mathrm{L})$ & $(\mathrm{Bq} / \mathbf{k g})$ & $(\mathrm{pCi} / \mathbf{k g})$ & $(\mathrm{Bq} / \mathbf{k g})$ & $(\mathrm{pCi} / \mathbf{k g})$ & $(\mathrm{Bq} / \mathbf{k g})$ & $(\mathrm{pCi} / \mathbf{k g})$ \\
\hline Pt-193 & $2.52 \mathrm{E}+04$ & $6.82 \mathrm{E}+05$ & $2.50 \mathrm{E}+05$ & $6.77 \mathrm{E}+06$ & $7.77 \mathrm{E}+04$ & $2.10 \mathrm{E}+06$ & $9.22 \mathrm{E}+05$ & $2.49 \mathrm{E}+07$ & $2.54 \mathrm{E}+06$ & $6.85 \mathrm{E}+07$ & $6.26 \mathrm{E}+04$ & $6.85 \mathrm{E}+07$ \\
\hline Pt-193m & $1.97 \mathrm{E}+03$ & $5.34 \mathrm{E}+04$ & $1.96 \mathrm{E}+04$ & $5.30 \mathrm{E}+05$ & $6.08 \mathrm{E}+03$ & $1.64 \mathrm{E}+05$ & $7.22 \mathrm{E}+04$ & $1.95 \mathrm{E}+06$ & $1.98 \mathrm{E}+05$ & $5.36 \mathrm{E}+06$ & $4.90 \mathrm{E}+03$ & $5.36 \mathrm{E}+06$ \\
\hline Pt- $195 \mathrm{~m}$ & $1.41 \mathrm{E}+03$ & $3.81 \mathrm{E}+04$ & $1.40 \mathrm{E}+04$ & $3.78 \mathrm{E}+05$ & $4.34 \mathrm{E}+03$ & $1.17 \mathrm{E}+05$ & $5.15 \mathrm{E}+04$ & $1.39 \mathrm{E}+06$ & $1.42 \mathrm{E}+05$ & $3.83 \mathrm{E}+06$ & $3.50 \mathrm{E}+03$ & $3.83 \mathrm{E}+06$ \\
\hline Pt-197 & $2.09 \mathrm{E}+03$ & $5.65 \mathrm{E}+04$ & $2.07 \mathrm{E}+04$ & $5.61 \mathrm{E}+05$ & $6.44 \mathrm{E}+03$ & $1.74 \mathrm{E}+05$ & $7.64 \mathrm{E}+04$ & $2.06 \mathrm{E}+06$ & $2.10 \mathrm{E}+05$ & $5.68 \mathrm{E}+06$ & $5.19 \mathrm{E}+03$ & $5.68 \mathrm{E}+06$ \\
\hline Pt-197m & $1.07 \mathrm{E}+04$ & $2.90 \mathrm{E}+05$ & $1.06 \mathrm{E}+05$ & $2.88 \mathrm{E}+06$ & $3.30 \mathrm{E}+04$ & $8.93 \mathrm{E}+05$ & $3.92 \mathrm{E}+05$ & $1.06 \mathrm{E}+07$ & $1.08 \mathrm{E}+06$ & $2.91 \mathrm{E}+07$ & $2.66 \mathrm{E}+04$ & $2.91 \mathrm{E}+07$ \\
\hline Pt-199 & $2.32 \mathrm{E}+04$ & $6.28 \mathrm{E}+05$ & $2.31 \mathrm{E}+05$ & $6.24 \mathrm{E}+06$ & $7.16 \mathrm{E}+04$ & $1.94 \mathrm{E}+06$ & $8.50 \mathrm{E}+05$ & $2.30 \mathrm{E}+07$ & $2.34 \mathrm{E}+06$ & $6.31 \mathrm{E}+07$ & $5.77 \mathrm{E}+04$ & $6.31 \mathrm{E}+07$ \\
\hline Pt-200 & $7.73 \mathrm{E}+02$ & $2.09 \mathrm{E}+04$ & $7.67 \mathrm{E}+03$ & $2.07 \mathrm{E}+05$ & $2.38 \mathrm{E}+03$ & $6.43 \mathrm{E}+04$ & $2.82 \mathrm{E}+04$ & $7.63 \mathrm{E}+05$ & $7.76 \mathrm{E}+04$ & $2.10 \mathrm{E}+06$ & $1.92 \mathrm{E}+03$ & $2.10 \mathrm{E}+06$ \\
\hline Pt-202 & $2.05 \mathrm{E}+02$ & $5.54 \mathrm{E}+03$ & $2.03 \mathrm{E}+03$ & $5.50 \mathrm{E}+04$ & $6.31 \mathrm{E}+02$ & $1.71 \mathrm{E}+04$ & $7.49 \mathrm{E}+03$ & $2.02 \mathrm{E}+05$ & $2.06 \mathrm{E}+04$ & $5.57 \mathrm{E}+05$ & $5.08 \mathrm{E}+02$ & $5.57 \mathrm{E}+05$ \\
\hline $\mathrm{Au}-186$ & $2.10 \mathrm{E}+04$ & $5.69 \mathrm{E}+05$ & $2.09 \mathrm{E}+05$ & $5.65 \mathrm{E}+06$ & $6.48 \mathrm{E}+04$ & $1.75 \mathrm{E}+06$ & $7.69 \mathrm{E}+05$ & $2.08 \mathrm{E}+07$ & $2.12 \mathrm{E}+06$ & $5.72 \mathrm{E}+07$ & $5.22 \mathrm{E}+04$ & $5.72 \mathrm{E}+07$ \\
\hline $\mathrm{Au}-190$ & $2.30 \mathrm{E}+04$ & $6.21 \mathrm{E}+05$ & $2.28 \mathrm{E}+05$ & $6.17 \mathrm{E}+06$ & $7.08 \mathrm{E}+04$ & $1.91 \mathrm{E}+06$ & $8.40 \mathrm{E}+05$ & $2.27 \mathrm{E}+07$ & $2.31 \mathrm{E}+06$ & $6.24 \mathrm{E}+07$ & $5.71 \mathrm{E}+04$ & $6.24 \mathrm{E}+07$ \\
\hline $\mathrm{Au}-191$ & $1.31 \mathrm{E}+04$ & $3.55 \mathrm{E}+05$ & $1.30 \mathrm{E}+05$ & $3.52 \mathrm{E}+06$ & $4.05 \mathrm{E}+04$ & $1.09 \mathrm{E}+06$ & $4.80 \mathrm{E}+05$ & $1.30 \mathrm{E}+07$ & $1.32 \mathrm{E}+06$ & $3.57 \mathrm{E}+07$ & $3.26 \mathrm{E}+04$ & $3.57 \mathrm{E}+07$ \\
\hline $\mathrm{Au}-192$ & $5.68 \mathrm{E}+03$ & $1.53 \mathrm{E}+05$ & $5.64 \mathrm{E}+04$ & $1.52 \mathrm{E}+06$ & $1.75 \mathrm{E}+04$ & $4.73 \mathrm{E}+05$ & $2.08 \mathrm{E}+05$ & $5.61 \mathrm{E}+06$ & $5.71 \mathrm{E}+05$ & $1.54 \mathrm{E}+07$ & $1.41 \mathrm{E}+04$ & $1.54 \mathrm{E}+07$ \\
\hline $\mathrm{Au}-193$ & $7.27 \mathrm{E}+03$ & $1.97 \mathrm{E}+05$ & $7.22 \mathrm{E}+04$ & $1.95 \mathrm{E}+06$ & $2.24 \mathrm{E}+04$ & $6.06 \mathrm{E}+05$ & $2.66 \mathrm{E}+05$ & $7.18 \mathrm{E}+06$ & $7.31 \mathrm{E}+05$ & $1.98 \mathrm{E}+07$ & $1.80 \mathrm{E}+04$ & $1.98 \mathrm{E}+07$ \\
\hline Au-194 & $2.41 \mathrm{E}+03$ & $6.51 \mathrm{E}+04$ & $2.39 \mathrm{E}+04$ & $6.47 \mathrm{E}+05$ & $7.43 \mathrm{E}+03$ & $2.01 \mathrm{E}+05$ & $8.81 \mathrm{E}+04$ & $2.38 \mathrm{E}+06$ & $2.42 \mathrm{E}+05$ & $6.55 \mathrm{E}+06$ & $5.98 \mathrm{E}+03$ & $6.55 \mathrm{E}+06$ \\
\hline $\mathrm{Au}-195$ & $3.50 \mathrm{E}+03$ & $9.47 \mathrm{E}+04$ & $3.48 \mathrm{E}+04$ & $9.40 \mathrm{E}+05$ & $1.08 \mathrm{E}+04$ & $2.92 \mathrm{E}+05$ & $1.28 \mathrm{E}+05$ & $3.46 \mathrm{E}+06$ & $3.52 \mathrm{E}+05$ & $9.52 \mathrm{E}+06$ & $8.69 \mathrm{E}+03$ & $9.52 \mathrm{E}+06$ \\
\hline $\mathrm{Au}-196$ & $2.74 \mathrm{E}+03$ & $7.40 \mathrm{E}+04$ & $2.72 \mathrm{E}+04$ & $7.35 \mathrm{E}+05$ & $8.44 \mathrm{E}+03$ & $2.28 \mathrm{E}+05$ & $1.00 \mathrm{E}+05$ & $2.71 \mathrm{E}+06$ & $2.75 \mathrm{E}+05$ & $7.44 \mathrm{E}+06$ & $6.80 \mathrm{E}+03$ & $7.44 \mathrm{E}+06$ \\
\hline $\mathrm{Au}-196 \mathrm{~m}$ & $2.36 \mathrm{E}+03$ & $6.37 \mathrm{E}+04$ & $2.34 \mathrm{E}+04$ & $6.32 \mathrm{E}+05$ & $7.26 \mathrm{E}+03$ & $1.96 \mathrm{E}+05$ & $8.61 \mathrm{E}+04$ & $2.33 \mathrm{E}+06$ & $2.37 \mathrm{E}+05$ & $6.40 \mathrm{E}+06$ & $5.85 \mathrm{E}+03$ & $6.40 \mathrm{E}+06$ \\
\hline $\mathrm{Au}-198$ & $8.95 \mathrm{E}+02$ & $2.42 \mathrm{E}+04$ & $8.88 \mathrm{E}+03$ & $2.40 \mathrm{E}+05$ & $2.76 \mathrm{E}+03$ & $7.45 \mathrm{E}+04$ & $3.27 \mathrm{E}+04$ & $8.84 \mathrm{E}+05$ & $8.99 \mathrm{E}+04$ & $2.43 \mathrm{E}+06$ & $2.22 \mathrm{E}+03$ & $2.43 \mathrm{E}+06$ \\
\hline $\mathrm{Au}-198 \mathrm{~m}$ & $7.73 \mathrm{E}+02$ & $2.09 \mathrm{E}+04$ & $7.67 \mathrm{E}+03$ & $2.07 \mathrm{E}+05$ & $2.38 \mathrm{E}+03$ & $6.43 \mathrm{E}+04$ & $2.82 \mathrm{E}+04$ & $7.63 \mathrm{E}+05$ & $7.76 \mathrm{E}+04$ & $2.10 \mathrm{E}+06$ & $1.92 \mathrm{E}+03$ & $2.10 \mathrm{E}+06$ \\
\hline $\mathrm{Au}-199$ & $2.05 \mathrm{E}+03$ & $5.53 \mathrm{E}+04$ & $2.03 \mathrm{E}+04$ & $5.49 \mathrm{E}+05$ & $6.30 \mathrm{E}+03$ & $1.70 \mathrm{E}+05$ & $7.48 \mathrm{E}+04$ & $2.02 \mathrm{E}+06$ & $2.06 \mathrm{E}+05$ & $5.56 \mathrm{E}+06$ & $5.08 \mathrm{E}+03$ & $5.56 \mathrm{E}+06$ \\
\hline $\mathrm{Au}-200$ & $1.37 \mathrm{E}+04$ & $3.70 \mathrm{E}+05$ & $1.36 \mathrm{E}+05$ & $3.67 \mathrm{E}+06$ & $4.22 \mathrm{E}+04$ & $1.14 \mathrm{E}+06$ & $5.01 \mathrm{E}+05$ & $1.35 \mathrm{E}+07$ & $1.38 \mathrm{E}+06$ & $3.72 \mathrm{E}+07$ & $3.40 \mathrm{E}+04$ & $3.72 \mathrm{E}+07$ \\
\hline $\mathrm{Au}-200 \mathrm{~m}$ & $9.42 \mathrm{E}+02$ & $2.55 \mathrm{E}+04$ & $9.35 \mathrm{E}+03$ & $2.53 \mathrm{E}+05$ & $2.90 \mathrm{E}+03$ & $7.84 \mathrm{E}+04$ & $3.44 \mathrm{E}+04$ & $9.31 \mathrm{E}+05$ & $9.47 \mathrm{E}+04$ & $2.56 \mathrm{E}+06$ & $2.34 \mathrm{E}+03$ & $2.56 \mathrm{E}+06$ \\
\hline $\mathrm{Au}-201$ & $3.80 \mathrm{E}+04$ & $1.03 \mathrm{E}+06$ & $3.78 \mathrm{E}+05$ & $1.02 \mathrm{E}+07$ & $1.17 \mathrm{E}+05$ & $3.17 \mathrm{E}+06$ & $1.39 \mathrm{E}+06$ & $3.76 \mathrm{E}+07$ & $3.82 \mathrm{E}+06$ & $1.03 \mathrm{E}+08$ & $9.44 \mathrm{E}+04$ & $1.03 \mathrm{E}+08$ \\
\hline
\end{tabular}

Page 197 of 298 
SRNL-STI-2013-00115

\begin{tabular}{|c|c|c|c|c|c|c|c|c|c|c|c|c|}
\hline \multirow[t]{2}{*}{ Nuclide } & \multicolumn{2}{|c|}{ Water Ingestion DCS } & \multicolumn{2}{|c|}{ Meat Ingestion DCS } & \multicolumn{2}{|c|}{ Dairy Ingestion DCS } & \multicolumn{2}{|c|}{$\begin{array}{l}\text { Freshwater Fish } \\
\text { Ingestion DCS }\end{array}$} & \multicolumn{2}{|c|}{$\begin{array}{l}\text { Saltwater Shellfish } \\
\text { Ingestion DCS }\end{array}$} & \multicolumn{2}{|c|}{ Produce Ingestion DCS } \\
\hline & $(\mathbf{B q} / \mathbf{L})$ & $(\mathrm{pCi} / \mathrm{L})$ & $(\mathrm{Bq} / \mathrm{kg})$ & $(\mathrm{pCi} / \mathrm{kg})$ & $(\mathrm{Bq} / \mathrm{L})$ & $(\mathrm{pCi} / \mathrm{L})$ & $(\mathrm{Bq} / \mathrm{kg})$ & $(\mathrm{pCi} / \mathbf{k g})$ & $(\mathrm{Bq} / \mathbf{k g})$ & $(\mathrm{pCi} / \mathrm{kg})$ & $(\mathrm{Bq} / \mathrm{kg})$ & $(\mathrm{pCi} / \mathrm{kg})$ \\
\hline Hg-190 & $4.47 \mathrm{E}+04$ & $1.21 \mathrm{E}+06$ & $4.44 \mathrm{E}+05$ & $1.20 \mathrm{E}+07$ & $1.38 \mathrm{E}+05$ & $3.72 \mathrm{E}+06$ & $1.64 \mathrm{E}+06$ & $4.42 \mathrm{E}+07$ & $4.50 \mathrm{E}+06$ & $1.22 \mathrm{E}+08$ & $1.11 \mathrm{E}+05$ & $1.22 \mathrm{E}+08$ \\
\hline $\mathrm{Hg}-191 \mathrm{~m}$ & $1.81 \mathrm{E}+04$ & $4.89 \mathrm{E}+05$ & $1.79 \mathrm{E}+05$ & $4.85 \mathrm{E}+06$ & $5.57 \mathrm{E}+04$ & $1.51 \mathrm{E}+06$ & $6.61 \mathrm{E}+05$ & $1.79 \mathrm{E}+07$ & $1.82 \mathrm{E}+06$ & $4.91 \mathrm{E}+07$ & $4.49 \mathrm{E}+04$ & $4.91 \mathrm{E}+07$ \\
\hline Hg-192 & $4.27 \mathrm{E}+03$ & $1.16 \mathrm{E}+05$ & $4.24 \mathrm{E}+04$ & $1.15 \mathrm{E}+06$ & $1.32 \mathrm{E}+04$ & $3.56 \mathrm{E}+05$ & $1.56 \mathrm{E}+05$ & $4.22 \mathrm{E}+06$ & $4.30 \mathrm{E}+05$ & $1.16 \mathrm{E}+07$ & $1.06 \mathrm{E}+04$ & $1.16 \mathrm{E}+07$ \\
\hline Hg-193 & $8.95 \mathrm{E}+03$ & $2.42 \mathrm{E}+05$ & $8.88 \mathrm{E}+04$ & $2.40 \mathrm{E}+06$ & $2.76 \mathrm{E}+04$ & $7.45 \mathrm{E}+05$ & $3.27 \mathrm{E}+05$ & $8.84 \mathrm{E}+06$ & $8.99 \mathrm{E}+05$ & $2.43 \mathrm{E}+07$ & $2.22 \mathrm{E}+04$ & $2.43 \mathrm{E}+07$ \\
\hline $\mathrm{Hg}-193 \mathrm{~m}$ & $2.21 \mathrm{E}+03$ & $5.98 \mathrm{E}+04$ & $2.20 \mathrm{E}+04$ & $5.94 \mathrm{E}+05$ & $6.82 \mathrm{E}+03$ & $1.84 \mathrm{E}+05$ & $8.09 \mathrm{E}+04$ & $2.19 \mathrm{E}+06$ & $2.22 \mathrm{E}+05$ & $6.01 \mathrm{E}+06$ & $5.49 \mathrm{E}+03$ & $6.01 \mathrm{E}+06$ \\
\hline Hg-194 & $7.97 \mathrm{E}+02$ & $2.15 \mathrm{E}+04$ & $7.91 \mathrm{E}+03$ & $2.14 \mathrm{E}+05$ & $2.46 \mathrm{E}+03$ & $6.64 \mathrm{E}+04$ & $2.91 \mathrm{E}+04$ & $7.88 \mathrm{E}+05$ & $8.01 \mathrm{E}+04$ & $2.17 \mathrm{E}+06$ & $1.98 \mathrm{E}+03$ & $2.17 \mathrm{E}+06$ \\
\hline Hg-195 & $9.08 \mathrm{E}+03$ & $2.45 \mathrm{E}+05$ & $9.01 \mathrm{E}+04$ & $2.44 \mathrm{E}+06$ & $2.80 \mathrm{E}+04$ & $7.56 \mathrm{E}+05$ & $3.32 \mathrm{E}+05$ & $8.97 \mathrm{E}+06$ & $9.12 \mathrm{E}+05$ & $2.47 \mathrm{E}+07$ & $2.25 \mathrm{E}+04$ & $2.47 \mathrm{E}+07$ \\
\hline $\mathrm{Hg}-195 \mathrm{~m}$ & $1.67 \mathrm{E}+03$ & $4.50 \mathrm{E}+04$ & $1.65 \mathrm{E}+04$ & $4.47 \mathrm{E}+05$ & $5.13 \mathrm{E}+03$ & $1.39 \mathrm{E}+05$ & $6.08 \mathrm{E}+04$ & $1.64 \mathrm{E}+06$ & $1.67 \mathrm{E}+05$ & $4.52 \mathrm{E}+06$ & $4.13 \mathrm{E}+03$ & $4.52 \mathrm{E}+06$ \\
\hline $\mathrm{Hg}-197$ & $3.67 \mathrm{E}+03$ & $9.92 \mathrm{E}+04$ & $3.64 \mathrm{E}+04$ & $9.84 \mathrm{E}+05$ & $1.13 \mathrm{E}+04$ & $3.05 \mathrm{E}+05$ & $1.34 \mathrm{E}+05$ & $3.62 \mathrm{E}+06$ & $3.69 \mathrm{E}+05$ & $9.97 \mathrm{E}+06$ & $9.10 \mathrm{E}+03$ & $9.97 \mathrm{E}+06$ \\
\hline $\mathrm{Hg}-197 \mathrm{~m}$ & $1.89 \mathrm{E}+03$ & $5.11 \mathrm{E}+04$ & $1.88 \mathrm{E}+04$ & $5.07 \mathrm{E}+05$ & $5.82 \mathrm{E}+03$ & $1.57 \mathrm{E}+05$ & $6.91 \mathrm{E}+04$ & $1.87 \mathrm{E}+06$ & $1.90 \mathrm{E}+05$ & $5.13 \mathrm{E}+06$ & $4.69 \mathrm{E}+03$ & $5.13 \mathrm{E}+06$ \\
\hline $\mathrm{Hg}-199 \mathrm{~m}$ & $3.03 \mathrm{E}+04$ & $8.18 \mathrm{E}+05$ & $3.00 \mathrm{E}+05$ & $8.12 \mathrm{E}+06$ & $9.32 \mathrm{E}+04$ & $2.52 \mathrm{E}+06$ & $1.11 \mathrm{E}+06$ & $2.99 \mathrm{E}+07$ & $3.04 \mathrm{E}+06$ & $8.22 \mathrm{E}+07$ & $7.51 \mathrm{E}+04$ & $8.22 \mathrm{E}+07$ \\
\hline $\mathrm{Hg}-203$ & $1.72 \mathrm{E}+03$ & $4.65 \mathrm{E}+04$ & $1.71 \mathrm{E}+04$ & $4.62 \mathrm{E}+05$ & $5.30 \mathrm{E}+03$ & $1.43 \mathrm{E}+05$ & $6.29 \mathrm{E}+04$ & $1.70 \mathrm{E}+06$ & $1.73 \mathrm{E}+05$ & $4.67 \mathrm{E}+06$ & $4.27 \mathrm{E}+03$ & $4.67 \mathrm{E}+06$ \\
\hline $\mathrm{Tl}-194$ & $2.06 \mathrm{E}+04$ & $5.56 \mathrm{E}+05$ & $2.04 \mathrm{E}+05$ & $5.52 \mathrm{E}+06$ & $6.33 \mathrm{E}+04$ & $1.71 \mathrm{E}+06$ & $7.51 \mathrm{E}+05$ & $2.03 \mathrm{E}+07$ & $2.07 \mathrm{E}+06$ & $5.58 \mathrm{E}+07$ & $5.10 \mathrm{E}+04$ & $5.58 \mathrm{E}+07$ \\
\hline $\mathrm{Tl}-194 \mathrm{~m}$ & $2.51 \mathrm{E}+04$ & $6.79 \mathrm{E}+05$ & $2.49 \mathrm{E}+05$ & $6.74 \mathrm{E}+06$ & $7.74 \mathrm{E}+04$ & $2.09 \mathrm{E}+06$ & $9.18 \mathrm{E}+05$ & $2.48 \mathrm{E}+07$ & $2.53 \mathrm{E}+06$ & $6.83 \mathrm{E}+07$ & $6.24 \mathrm{E}+04$ & $6.83 \mathrm{E}+07$ \\
\hline Tl-195 & $4.01 \mathrm{E}+04$ & $1.08 \mathrm{E}+06$ & $3.98 \mathrm{E}+05$ & $1.08 \mathrm{E}+07$ & $1.24 \mathrm{E}+05$ & $3.34 \mathrm{E}+06$ & $1.47 \mathrm{E}+06$ & $3.96 \mathrm{E}+07$ & $4.03 \mathrm{E}+06$ & $1.09 \mathrm{E}+08$ & $9.96 \mathrm{E}+04$ & $1.09 \mathrm{E}+08$ \\
\hline Tl-196 & $1.94 \mathrm{E}+04$ & $5.24 \mathrm{E}+05$ & $1.92 \mathrm{E}+05$ & $5.20 \mathrm{E}+06$ & $5.97 \mathrm{E}+04$ & $1.61 \mathrm{E}+06$ & $7.08 \mathrm{E}+05$ & $1.91 \mathrm{E}+07$ & $1.95 \mathrm{E}+06$ & $5.26 \mathrm{E}+07$ & $4.81 \mathrm{E}+04$ & $5.26 \mathrm{E}+07$ \\
\hline Tl-197 & $4.20 \mathrm{E}+04$ & $1.14 \mathrm{E}+06$ & $4.17 \mathrm{E}+05$ & $1.13 \mathrm{E}+07$ & $1.29 \mathrm{E}+05$ & $3.50 \mathrm{E}+06$ & $1.54 \mathrm{E}+06$ & $4.15 \mathrm{E}+07$ & $4.22 \mathrm{E}+06$ & $1.14 \mathrm{E}+08$ & $1.04 \mathrm{E}+05$ & $1.14 \mathrm{E}+08$ \\
\hline Tl-198 & $1.38 \mathrm{E}+04$ & $3.72 \mathrm{E}+05$ & $1.37 \mathrm{E}+05$ & $3.70 \mathrm{E}+06$ & $4.24 \mathrm{E}+04$ & $1.15 \mathrm{E}+06$ & $5.03 \mathrm{E}+05$ & $1.36 \mathrm{E}+07$ & $1.38 \mathrm{E}+06$ & $3.74 \mathrm{E}+07$ & $3.42 \mathrm{E}+04$ & $3.74 \mathrm{E}+07$ \\
\hline $\mathrm{Tl}-198 \mathrm{~m}$ & $1.78 \mathrm{E}+04$ & $4.80 \mathrm{E}+05$ & $1.76 \mathrm{E}+05$ & $4.77 \mathrm{E}+06$ & $5.47 \mathrm{E}+04$ & $1.48 \mathrm{E}+06$ & $6.49 \mathrm{E}+05$ & $1.76 \mathrm{E}+07$ & $1.79 \mathrm{E}+06$ & $4.83 \mathrm{E}+07$ & $4.41 \mathrm{E}+04$ & $4.83 \mathrm{E}+07$ \\
\hline Tl-199 & $3.57 \mathrm{E}+04$ & $9.66 \mathrm{E}+05$ & $3.55 \mathrm{E}+05$ & $9.59 \mathrm{E}+06$ & $1.10 \mathrm{E}+05$ & $2.98 \mathrm{E}+06$ & $1.31 \mathrm{E}+06$ & $3.53 \mathrm{E}+07$ & $3.59 \mathrm{E}+06$ & $9.71 \mathrm{E}+07$ & $8.87 \mathrm{E}+04$ & $9.71 \mathrm{E}+07$ \\
\hline Tl-200 & $5.04 \mathrm{E}+03$ & $1.36 \mathrm{E}+05$ & $5.00 \mathrm{E}+04$ & $1.35 \mathrm{E}+06$ & $1.55 \mathrm{E}+04$ & $4.19 \mathrm{E}+05$ & $1.84 \mathrm{E}+05$ & $4.97 \mathrm{E}+06$ & $5.06 \mathrm{E}+05$ & $1.37 \mathrm{E}+07$ & $1.25 \mathrm{E}+04$ & $1.37 \mathrm{E}+07$ \\
\hline Tl-201 & $9.79 \mathrm{E}+03$ & $2.65 \mathrm{E}+05$ & $9.72 \mathrm{E}+04$ & $2.63 \mathrm{E}+06$ & $3.02 \mathrm{E}+04$ & $8.15 \mathrm{E}+05$ & $3.58 \mathrm{E}+05$ & $9.67 \mathrm{E}+06$ & $9.84 \mathrm{E}+05$ & $2.66 \mathrm{E}+07$ & $2.43 \mathrm{E}+04$ & $2.66 \mathrm{E}+07$ \\
\hline $\mathrm{Tl}-202$ & $2.22 \mathrm{E}+03$ & $6.00 \mathrm{E}+04$ & $2.20 \mathrm{E}+04$ & $5.96 \mathrm{E}+05$ & $6.84 \mathrm{E}+03$ & $1.85 \mathrm{E}+05$ & $8.12 \mathrm{E}+04$ & $2.19 \mathrm{E}+06$ & $2.23 \mathrm{E}+05$ & $6.03 \mathrm{E}+06$ & $5.51 \mathrm{E}+03$ & $6.03 \mathrm{E}+06$ \\
\hline
\end{tabular}

Page 198 of 298 
SRNL-STI-2013-00115

\begin{tabular}{|c|c|c|c|c|c|c|c|c|c|c|c|c|}
\hline \multirow[t]{2}{*}{ Nuclide } & \multicolumn{2}{|c|}{ Water Ingestion DCS } & \multicolumn{2}{|c|}{ Meat Ingestion DCS } & \multicolumn{2}{|c|}{ Dairy Ingestion DCS } & \multicolumn{2}{|c|}{$\begin{array}{c}\text { Freshwater Fish } \\
\text { Ingestion DCS }\end{array}$} & \multicolumn{2}{|c|}{$\begin{array}{l}\text { Saltwater Shellfish } \\
\text { Ingestion DCS }\end{array}$} & \multicolumn{2}{|c|}{ Produce Ingestion DCS } \\
\hline & $(\mathrm{Bq} / \mathrm{L})$ & $(\mathrm{pCi} / \mathrm{L})$ & $(\mathrm{Bq} / \mathrm{kg})$ & $(\mathrm{pCi} / \mathrm{kg})$ & $(\mathrm{Bq} / \mathrm{L})$ & $(\mathrm{pCi} / \mathrm{L})$ & $(\mathrm{Bq} / \mathbf{k g})$ & $(\mathrm{pCi} / \mathbf{k g})$ & $(\mathrm{Bq} / \mathbf{k g})$ & $(\mathrm{pCi} / \mathbf{k g})$ & $(\mathrm{Bq} / \mathbf{k g})$ & $(\mathrm{pCi} / \mathbf{k g})$ \\
\hline Tl-204 & $7.73 \mathrm{E}+02$ & $2.09 \mathrm{E}+04$ & $7.67 \mathrm{E}+03$ & $2.07 \mathrm{E}+05$ & $2.38 \mathrm{E}+03$ & $6.43 \mathrm{E}+04$ & $2.82 \mathrm{E}+04$ & $7.63 \mathrm{E}+05$ & $7.76 \mathrm{E}+04$ & $2.10 \mathrm{E}+06$ & $1.92 \mathrm{E}+03$ & $2.10 \mathrm{E}+06$ \\
\hline $\mathrm{Pb}-194$ & $4.68 \mathrm{E}+04$ & $1.26 \mathrm{E}+06$ & $4.64 \mathrm{E}+05$ & $1.25 \mathrm{E}+07$ & $1.44 \mathrm{E}+05$ & $3.89 \mathrm{E}+06$ & $1.71 \mathrm{E}+06$ & $4.62 \mathrm{E}+07$ & $4.70 \mathrm{E}+06$ & $1.27 \mathrm{E}+08$ & $1.16 \mathrm{E}+05$ & $1.27 \mathrm{E}+08$ \\
\hline $\mathrm{Pb}-195 \mathrm{~m}$ & $3.89 \mathrm{E}+04$ & $1.05 \mathrm{E}+06$ & $3.86 \mathrm{E}+05$ & $1.04 \mathrm{E}+07$ & $1.20 \mathrm{E}+05$ & $3.24 \mathrm{E}+06$ & $1.42 \mathrm{E}+06$ & $3.84 \mathrm{E}+07$ & $3.91 \mathrm{E}+06$ & $1.06 \mathrm{E}+08$ & $9.65 \mathrm{E}+04$ & $1.06 \mathrm{E}+08$ \\
\hline $\mathrm{Pb}-196$ & $3.61 \mathrm{E}+04$ & $9.74 \mathrm{E}+05$ & $3.58 \mathrm{E}+05$ & $9.67 \mathrm{E}+06$ & $1.11 \mathrm{E}+05$ & $3.00 \mathrm{E}+06$ & $1.32 \mathrm{E}+06$ & $3.56 \mathrm{E}+07$ & $3.62 \mathrm{E}+06$ & $9.79 \mathrm{E}+07$ & $8.95 \mathrm{E}+04$ & $9.79 \mathrm{E}+07$ \\
\hline $\mathrm{Pb}-197 \mathrm{~m}$ & $2.17 \mathrm{E}+04$ & $5.87 \mathrm{E}+05$ & $2.15 \mathrm{E}+05$ & $5.82 \mathrm{E}+06$ & $6.69 \mathrm{E}+04$ & $1.81 \mathrm{E}+06$ & $7.93 \mathrm{E}+05$ & $2.14 \mathrm{E}+07$ & $2.18 \mathrm{E}+06$ & $5.90 \mathrm{E}+07$ & $5.39 \mathrm{E}+04$ & $5.90 \mathrm{E}+07$ \\
\hline $\mathrm{Pb}-198$ & $1.28 \mathrm{E}+04$ & $3.46 \mathrm{E}+05$ & $1.27 \mathrm{E}+05$ & $3.44 \mathrm{E}+06$ & $3.95 \mathrm{E}+04$ & $1.07 \mathrm{E}+06$ & $4.68 \mathrm{E}+05$ & $1.27 \mathrm{E}+07$ & $1.29 \mathrm{E}+06$ & $3.48 \mathrm{E}+07$ & $3.18 \mathrm{E}+04$ & $3.48 \mathrm{E}+07$ \\
\hline $\mathrm{Pb}-199$ & $2.54 \mathrm{E}+04$ & $6.87 \mathrm{E}+05$ & $2.52 \mathrm{E}+05$ & $6.82 \mathrm{E}+06$ & $7.84 \mathrm{E}+04$ & $2.12 \mathrm{E}+06$ & $9.30 \mathrm{E}+05$ & $2.51 \mathrm{E}+07$ & $2.56 \mathrm{E}+06$ & $6.91 \mathrm{E}+07$ & $6.31 \mathrm{E}+04$ & $6.91 \mathrm{E}+07$ \\
\hline $\mathrm{Pb}-200$ & $2.61 \mathrm{E}+03$ & $7.06 \mathrm{E}+04$ & $2.59 \mathrm{E}+04$ & $7.01 \mathrm{E}+05$ & $8.05 \mathrm{E}+03$ & $2.18 \mathrm{E}+05$ & $9.55 \mathrm{E}+04$ & $2.58 \mathrm{E}+06$ & $2.63 \mathrm{E}+05$ & $7.10 \mathrm{E}+06$ & $6.48 \mathrm{E}+03$ & $7.10 \mathrm{E}+06$ \\
\hline $\mathrm{Pb}-201$ & $6.31 \mathrm{E}+03$ & $1.71 \mathrm{E}+05$ & $6.27 \mathrm{E}+04$ & $1.69 \mathrm{E}+06$ & $1.94 \mathrm{E}+04$ & $5.26 \mathrm{E}+05$ & $2.31 \mathrm{E}+05$ & $6.24 \mathrm{E}+06$ & $6.35 \mathrm{E}+05$ & $1.71 \mathrm{E}+07$ & $1.57 \mathrm{E}+04$ & $1.71 \mathrm{E}+07$ \\
\hline $\mathrm{Pb}-202$ & $5.95 \mathrm{E}+01$ & $1.61 \mathrm{E}+03$ & $5.91 \mathrm{E}+02$ & $1.60 \mathrm{E}+04$ & $1.83 \mathrm{E}+02$ & $4.95 \mathrm{E}+03$ & $2.17 \mathrm{E}+03$ & $5.88 \mathrm{E}+04$ & $5.98 \mathrm{E}+03$ & $1.62 \mathrm{E}+05$ & $1.48 \mathrm{E}+02$ & $1.62 \mathrm{E}+05$ \\
\hline $\mathrm{Pb}-202 \mathrm{~m}$ & $6.91 \mathrm{E}+03$ & $1.87 \mathrm{E}+05$ & $6.86 \mathrm{E}+04$ & $1.85 \mathrm{E}+06$ & $2.13 \mathrm{E}+04$ & $5.75 \mathrm{E}+05$ & $2.53 \mathrm{E}+05$ & $6.83 \mathrm{E}+06$ & $6.94 \mathrm{E}+05$ & $1.88 \mathrm{E}+07$ & $1.71 \mathrm{E}+04$ & $1.88 \mathrm{E}+07$ \\
\hline $\mathrm{Pb}-203$ & $4.05 \mathrm{E}+03$ & $1.09 \mathrm{E}+05$ & $4.02 \mathrm{E}+04$ & $1.09 \mathrm{E}+06$ & $1.25 \mathrm{E}+04$ & $3.37 \mathrm{E}+05$ & $1.48 \mathrm{E}+05$ & $4.00 \mathrm{E}+06$ & $4.07 \mathrm{E}+05$ & $1.10 \mathrm{E}+07$ & $1.01 \mathrm{E}+04$ & $1.10 \mathrm{E}+07$ \\
\hline $\mathrm{Pb}-204 \mathrm{~m}$ & $2.07 \mathrm{E}+04$ & $5.59 \mathrm{E}+05$ & $2.05 \mathrm{E}+05$ & $5.55 \mathrm{E}+06$ & $6.38 \mathrm{E}+04$ & $1.72 \mathrm{E}+06$ & $7.56 \mathrm{E}+05$ & $2.04 \mathrm{E}+07$ & $2.08 \mathrm{E}+06$ & $5.62 \mathrm{E}+07$ & $5.14 \mathrm{E}+04$ & $5.62 \mathrm{E}+07$ \\
\hline $\mathrm{Pb}-205$ & $3.53 \mathrm{E}+03$ & $9.55 \mathrm{E}+04$ & $3.51 \mathrm{E}+04$ & $9.48 \mathrm{E}+05$ & $1.09 \mathrm{E}+04$ & $2.94 \mathrm{E}+05$ & $1.29 \mathrm{E}+05$ & $3.49 \mathrm{E}+06$ & $3.55 \mathrm{E}+05$ & $9.60 \mathrm{E}+06$ & $8.77 \mathrm{E}+03$ & $9.60 \mathrm{E}+06$ \\
\hline $\mathrm{Pb}-209$ & $1.67 \mathrm{E}+04$ & $4.50 \mathrm{E}+05$ & $1.65 \mathrm{E}+05$ & $4.47 \mathrm{E}+06$ & $5.13 \mathrm{E}+04$ & $1.39 \mathrm{E}+06$ & $6.08 \mathrm{E}+05$ & $1.64 \mathrm{E}+07$ & $1.67 \mathrm{E}+06$ & $4.52 \mathrm{E}+07$ & $4.13 \mathrm{E}+04$ & $4.52 \mathrm{E}+07$ \\
\hline $\mathrm{Pb}-210$ & $1.22 \mathrm{E}+00$ & $3.30 \mathrm{E}+01$ & $1.21 \mathrm{E}+01$ & $3.27 \mathrm{E}+02$ & $3.76 \mathrm{E}+00$ & $1.02 \mathrm{E}+02$ & $4.46 \mathrm{E}+01$ & $1.20 \mathrm{E}+03$ & $1.23 \mathrm{E}+02$ & $3.31 \mathrm{E}+03$ & $3.03 \mathrm{E}+00$ & $3.31 \mathrm{E}+03$ \\
\hline $\mathrm{Pb}-211$ & $4.75 \mathrm{E}+03$ & $1.28 \mathrm{E}+05$ & $4.71 \mathrm{E}+04$ & $1.27 \mathrm{E}+06$ & $1.46 \mathrm{E}+04$ & $3.95 \mathrm{E}+05$ & $1.73 \mathrm{E}+05$ & $4.69 \mathrm{E}+06$ & $4.77 \mathrm{E}+05$ & $1.29 \mathrm{E}+07$ & $1.18 \mathrm{E}+04$ & $1.29 \mathrm{E}+07$ \\
\hline $\mathrm{Pb}-212$ & $1.21 \mathrm{E}+02$ & $3.26 \mathrm{E}+03$ & $1.20 \mathrm{E}+03$ & $3.24 \mathrm{E}+04$ & $3.72 \mathrm{E}+02$ & $1.01 \mathrm{E}+04$ & $4.41 \mathrm{E}+03$ & $1.19 \mathrm{E}+05$ & $1.21 \mathrm{E}+04$ & $3.28 \mathrm{E}+05$ & $3.00 \mathrm{E}+02$ & $3.28 \mathrm{E}+05$ \\
\hline $\mathrm{Pb}-214$ & $6.25 \mathrm{E}+03$ & $1.69 \mathrm{E}+05$ & $6.20 \mathrm{E}+04$ & $1.68 \mathrm{E}+06$ & $1.93 \mathrm{E}+04$ & $5.20 \mathrm{E}+05$ & $2.28 \mathrm{E}+05$ & $6.17 \mathrm{E}+06$ & $6.28 \mathrm{E}+05$ & $1.70 \mathrm{E}+07$ & $1.55 \mathrm{E}+04$ & $1.70 \mathrm{E}+07$ \\
\hline Bi-200 & $1.74 \mathrm{E}+04$ & $4.69 \mathrm{E}+05$ & $1.72 \mathrm{E}+05$ & $4.66 \mathrm{E}+06$ & $5.35 \mathrm{E}+04$ & $1.45 \mathrm{E}+06$ & $6.35 \mathrm{E}+05$ & $1.72 \mathrm{E}+07$ & $1.75 \mathrm{E}+06$ & $4.72 \mathrm{E}+07$ & $4.31 \mathrm{E}+04$ & $4.72 \mathrm{E}+07$ \\
\hline Bi-201 & $8.35 \mathrm{E}+03$ & $2.26 \mathrm{E}+05$ & $8.29 \mathrm{E}+04$ & $2.24 \mathrm{E}+06$ & $2.57 \mathrm{E}+04$ & $6.95 \mathrm{E}+05$ & $3.05 \mathrm{E}+05$ & $8.24 \mathrm{E}+06$ & $8.39 \mathrm{E}+05$ & $2.27 \mathrm{E}+07$ & $2.07 \mathrm{E}+04$ & $2.27 \mathrm{E}+07$ \\
\hline Bi-202 & $9.87 \mathrm{E}+03$ & $2.67 \mathrm{E}+05$ & $9.80 \mathrm{E}+04$ & $2.65 \mathrm{E}+06$ & $3.04 \mathrm{E}+04$ & $8.22 \mathrm{E}+05$ & $3.61 \mathrm{E}+05$ & $9.75 \mathrm{E}+06$ & $9.92 \mathrm{E}+05$ & $2.68 \mathrm{E}+07$ & $2.45 \mathrm{E}+04$ & $2.68 \mathrm{E}+07$ \\
\hline Bi-203 & $1.99 \mathrm{E}+03$ & $5.37 \mathrm{E}+04$ & $1.97 \mathrm{E}+04$ & $5.33 \mathrm{E}+05$ & $6.12 \mathrm{E}+03$ & $1.65 \mathrm{E}+05$ & $7.26 \mathrm{E}+04$ & $1.96 \mathrm{E}+06$ & $2.00 \mathrm{E}+05$ & $5.40 \mathrm{E}+06$ & $4.93 \mathrm{E}+03$ & $5.40 \mathrm{E}+06$ \\
\hline
\end{tabular}

Page 199 of 298 
SRNL-STI-2013-00115

\begin{tabular}{|c|c|c|c|c|c|c|c|c|c|c|c|c|}
\hline \multirow[t]{2}{*}{ Nuclide } & \multicolumn{2}{|c|}{ Water Ingestion DCS } & \multicolumn{2}{|c|}{ Meat Ingestion DCS } & \multicolumn{2}{|c|}{ Dairy Ingestion DCS } & \multicolumn{2}{|c|}{$\begin{array}{c}\text { Freshwater Fish } \\
\text { Ingestion DCS }\end{array}$} & \multicolumn{2}{|c|}{$\begin{array}{l}\text { Saltwater Shellfish } \\
\text { Ingestion DCS }\end{array}$} & \multicolumn{2}{|c|}{ Produce Ingestion DCS } \\
\hline & $(\mathrm{Bq} / \mathrm{L})$ & $(\mathrm{pCi} / \mathrm{L})$ & $(\mathrm{Bq} / \mathrm{kg})$ & $(\mathrm{pCi} / \mathrm{kg})$ & $(\mathrm{Bq} / \mathrm{L})$ & $(\mathrm{pCi} / \mathrm{L})$ & $(\mathrm{Bq} / \mathbf{k g})$ & $(\mathrm{pCi} / \mathbf{k g})$ & $(\mathrm{Bq} / \mathbf{k g})$ & $(\mathrm{pCi} / \mathbf{k g})$ & $(\mathrm{Bq} / \mathbf{k g})$ & $(\mathrm{pCi} / \mathbf{k g})$ \\
\hline Bi-204 & $1.72 \mathrm{E}+03$ & $4.66 \mathrm{E}+04$ & $1.71 \mathrm{E}+04$ & $4.62 \mathrm{E}+05$ & $5.31 \mathrm{E}+03$ & $1.43 \mathrm{E}+05$ & $6.30 \mathrm{E}+04$ & $1.70 \mathrm{E}+06$ & $1.73 \mathrm{E}+05$ & $4.68 \mathrm{E}+06$ & $4.27 \mathrm{E}+03$ & $4.68 \mathrm{E}+06$ \\
\hline $\mathrm{Bi}-205$ & $1.08 \mathrm{E}+03$ & $2.92 \mathrm{E}+04$ & $1.07 \mathrm{E}+04$ & $2.90 \mathrm{E}+05$ & $3.33 \mathrm{E}+03$ & $9.00 \mathrm{E}+04$ & $3.95 \mathrm{E}+04$ & $1.07 \mathrm{E}+06$ & $1.09 \mathrm{E}+05$ & $2.94 \mathrm{E}+06$ & $2.68 \mathrm{E}+03$ & $2.94 \mathrm{E}+06$ \\
\hline $\mathrm{Bi}-206$ & $5.04 \mathrm{E}+02$ & $1.36 \mathrm{E}+04$ & $5.00 \mathrm{E}+03$ & $1.35 \mathrm{E}+05$ & $1.55 \mathrm{E}+03$ & $4.19 \mathrm{E}+04$ & $1.84 \mathrm{E}+04$ & $4.97 \mathrm{E}+05$ & $5.06 \mathrm{E}+04$ & $1.37 \mathrm{E}+06$ & $1.25 \mathrm{E}+03$ & $1.37 \mathrm{E}+06$ \\
\hline Bi-207 & $7.54 \mathrm{E}+02$ & $2.04 \mathrm{E}+04$ & $7.48 \mathrm{E}+03$ & $2.02 \mathrm{E}+05$ & $2.32 \mathrm{E}+03$ & $6.28 \mathrm{E}+04$ & $2.75 \mathrm{E}+04$ & $7.45 \mathrm{E}+05$ & $7.58 \mathrm{E}+04$ & $2.05 \mathrm{E}+06$ & $1.87 \mathrm{E}+03$ & $2.05 \mathrm{E}+06$ \\
\hline Bi-208 & $8.64 \mathrm{E}+02$ & $2.33 \mathrm{E}+04$ & $8.57 \mathrm{E}+03$ & $2.32 \mathrm{E}+05$ & $2.66 \mathrm{E}+03$ & $7.19 \mathrm{E}+04$ & $3.16 \mathrm{E}+04$ & $8.53 \mathrm{E}+05$ & $8.68 \mathrm{E}+04$ & $2.35 \mathrm{E}+06$ & $2.14 \mathrm{E}+03$ & $2.35 \mathrm{E}+06$ \\
\hline $\mathrm{Bi}-210$ & $6.91 \mathrm{E}+02$ & $1.87 \mathrm{E}+04$ & $6.86 \mathrm{E}+03$ & $1.85 \mathrm{E}+05$ & $2.13 \mathrm{E}+03$ & $5.75 \mathrm{E}+04$ & $2.53 \mathrm{E}+04$ & $6.83 \mathrm{E}+05$ & $6.94 \mathrm{E}+04$ & $1.88 \mathrm{E}+06$ & $1.71 \mathrm{E}+03$ & $1.88 \mathrm{E}+06$ \\
\hline $\mathrm{Bi}-210 \mathrm{~m}$ & $6.19 \mathrm{E}+01$ & $1.67 \mathrm{E}+03$ & $6.14 \mathrm{E}+02$ & $1.66 \mathrm{E}+04$ & $1.91 \mathrm{E}+02$ & $5.15 \mathrm{E}+03$ & $2.26 \mathrm{E}+03$ & $6.11 \mathrm{E}+04$ & $6.22 \mathrm{E}+03$ & $1.68 \mathrm{E}+05$ & $1.54 \mathrm{E}+02$ & $1.68 \mathrm{E}+05$ \\
\hline Bi-212 & $3.53 \mathrm{E}+03$ & $9.55 \mathrm{E}+04$ & $3.51 \mathrm{E}+04$ & $9.48 \mathrm{E}+05$ & $1.09 \mathrm{E}+04$ & $2.94 \mathrm{E}+05$ & $1.29 \mathrm{E}+05$ & $3.49 \mathrm{E}+06$ & $3.55 \mathrm{E}+05$ & $9.60 \mathrm{E}+06$ & $8.77 \mathrm{E}+03$ & $9.60 \mathrm{E}+06$ \\
\hline Bi-213 & $4.64 \mathrm{E}+03$ & $1.25 \mathrm{E}+05$ & $4.61 \mathrm{E}+04$ & $1.25 \mathrm{E}+06$ & $1.43 \mathrm{E}+04$ & $3.86 \mathrm{E}+05$ & $1.70 \mathrm{E}+05$ & $4.58 \mathrm{E}+06$ & $4.66 \mathrm{E}+05$ & $1.26 \mathrm{E}+07$ & $1.15 \mathrm{E}+04$ & $1.26 \mathrm{E}+07$ \\
\hline Bi-214 & $8.35 \mathrm{E}+03$ & $2.26 \mathrm{E}+05$ & $8.29 \mathrm{E}+04$ & $2.24 \mathrm{E}+06$ & $2.57 \mathrm{E}+04$ & $6.95 \mathrm{E}+05$ & $3.05 \mathrm{E}+05$ & $8.24 \mathrm{E}+06$ & $8.39 \mathrm{E}+05$ & $2.27 \mathrm{E}+07$ & $2.07 \mathrm{E}+04$ & $2.27 \mathrm{E}+07$ \\
\hline Po-203 & $1.83 \mathrm{E}+04$ & $4.95 \mathrm{E}+05$ & $1.82 \mathrm{E}+05$ & $4.91 \mathrm{E}+06$ & $5.64 \mathrm{E}+04$ & $1.53 \mathrm{E}+06$ & $6.69 \mathrm{E}+05$ & $1.81 \mathrm{E}+07$ & $1.84 \mathrm{E}+06$ & $4.98 \mathrm{E}+07$ & $4.55 \mathrm{E}+04$ & $4.98 \mathrm{E}+07$ \\
\hline Po-204 & $3.30 \mathrm{E}+03$ & $8.92 \mathrm{E}+04$ & $3.27 \mathrm{E}+04$ & $8.85 \mathrm{E}+05$ & $1.02 \mathrm{E}+04$ & $2.75 \mathrm{E}+05$ & $1.21 \mathrm{E}+05$ & $3.26 \mathrm{E}+06$ & $3.32 \mathrm{E}+05$ & $8.96 \mathrm{E}+06$ & $8.19 \mathrm{E}+03$ & $8.96 \mathrm{E}+06$ \\
\hline Po-205 & $1.76 \mathrm{E}+04$ & $4.76 \mathrm{E}+05$ & $1.75 \mathrm{E}+05$ & $4.73 \mathrm{E}+06$ & $5.43 \mathrm{E}+04$ & $1.47 \mathrm{E}+06$ & $6.44 \mathrm{E}+05$ & $1.74 \mathrm{E}+07$ & $1.77 \mathrm{E}+06$ & $4.79 \mathrm{E}+07$ & $4.37 \mathrm{E}+04$ & $4.79 \mathrm{E}+07$ \\
\hline Po-206 & $1.91 \mathrm{E}+02$ & $5.17 \mathrm{E}+03$ & $1.90 \mathrm{E}+03$ & $5.13 \mathrm{E}+04$ & $5.89 \mathrm{E}+02$ & $1.59 \mathrm{E}+04$ & $6.99 \mathrm{E}+03$ & $1.89 \mathrm{E}+05$ & $1.92 \mathrm{E}+04$ & $5.20 \mathrm{E}+05$ & $4.75 \mathrm{E}+02$ & $5.20 \mathrm{E}+05$ \\
\hline Po-207 & $7.15 \mathrm{E}+03$ & $1.93 \mathrm{E}+05$ & $7.10 \mathrm{E}+04$ & $1.92 \mathrm{E}+06$ & $2.20 \mathrm{E}+04$ & $5.95 \mathrm{E}+05$ & $2.61 \mathrm{E}+05$ & $7.06 \mathrm{E}+06$ & $7.18 \mathrm{E}+05$ & $1.94 \mathrm{E}+07$ & $1.77 \mathrm{E}+04$ & $1.94 \mathrm{E}+07$ \\
\hline Po-208 & $2.81 \mathrm{E}+00$ & $7.59 \mathrm{E}+01$ & $2.79 \mathrm{E}+01$ & $7.53 \mathrm{E}+02$ & $8.65 \mathrm{E}+00$ & $2.34 \mathrm{E}+02$ & $1.03 \mathrm{E}+02$ & $2.77 \mathrm{E}+03$ & $2.82 \mathrm{E}+02$ & $7.63 \mathrm{E}+03$ & $6.97 \mathrm{E}+00$ & $7.63 \mathrm{E}+03$ \\
\hline Po-209 & $2.83 \mathrm{E}+00$ & $7.64 \mathrm{E}+01$ & $2.81 \mathrm{E}+01$ & $7.58 \mathrm{E}+02$ & $8.71 \mathrm{E}+00$ & $2.35 \mathrm{E}+02$ & $1.03 \mathrm{E}+02$ & $2.79 \mathrm{E}+03$ & $2.84 \mathrm{E}+02$ & $7.68 \mathrm{E}+03$ & $7.01 \mathrm{E}+00$ & $7.68 \mathrm{E}+03$ \\
\hline Po-210 & $3.49 \mathrm{E}+00$ & $9.44 \mathrm{E}+01$ & $3.47 \mathrm{E}+01$ & $9.37 \mathrm{E}+02$ & $1.08 \mathrm{E}+01$ & $2.91 \mathrm{E}+02$ & $1.28 \mathrm{E}+02$ & $3.45 \mathrm{E}+03$ & $3.51 \mathrm{E}+02$ & $9.49 \mathrm{E}+03$ & $8.67 \mathrm{E}+00$ & $9.49 \mathrm{E}+03$ \\
\hline At-205 & $1.58 \mathrm{E}+04$ & $4.28 \mathrm{E}+05$ & $1.57 \mathrm{E}+05$ & $4.25 \mathrm{E}+06$ & $4.88 \mathrm{E}+04$ & $1.32 \mathrm{E}+06$ & $5.79 \mathrm{E}+05$ & $1.56 \mathrm{E}+07$ & $1.59 \mathrm{E}+06$ & $4.30 \mathrm{E}+07$ & $3.93 \mathrm{E}+04$ & $4.30 \mathrm{E}+07$ \\
\hline At-206 & $1.43 \mathrm{E}+04$ & $3.86 \mathrm{E}+05$ & $1.42 \mathrm{E}+05$ & $3.84 \mathrm{E}+06$ & $4.40 \mathrm{E}+04$ & $1.19 \mathrm{E}+06$ & $5.22 \mathrm{E}+05$ & $1.41 \mathrm{E}+07$ & $1.44 \mathrm{E}+06$ & $3.88 \mathrm{E}+07$ & $3.55 \mathrm{E}+04$ & $3.88 \mathrm{E}+07$ \\
\hline At-207 & $4.12 \mathrm{E}+03$ & $1.11 \mathrm{E}+05$ & $4.09 \mathrm{E}+04$ & $1.10 \mathrm{E}+06$ & $1.27 \mathrm{E}+04$ & $3.43 \mathrm{E}+05$ & $1.51 \mathrm{E}+05$ & $4.07 \mathrm{E}+06$ & $4.14 \mathrm{E}+05$ & $1.12 \mathrm{E}+07$ & $1.02 \mathrm{E}+04$ & $1.12 \mathrm{E}+07$ \\
\hline At-208 & $1.05 \mathrm{E}+04$ & $2.85 \mathrm{E}+05$ & $1.05 \mathrm{E}+05$ & $2.83 \mathrm{E}+06$ & $3.25 \mathrm{E}+04$ & $8.78 \mathrm{E}+05$ & $3.85 \mathrm{E}+05$ & $1.04 \mathrm{E}+07$ & $1.06 \mathrm{E}+06$ & $2.86 \mathrm{E}+07$ & $2.62 \mathrm{E}+04$ & $2.86 \mathrm{E}+07$ \\
\hline At-209 & $2.47 \mathrm{E}+03$ & $6.67 \mathrm{E}+04$ & $2.45 \mathrm{E}+04$ & $6.62 \mathrm{E}+05$ & $7.60 \mathrm{E}+03$ & $2.05 \mathrm{E}+05$ & $9.02 \mathrm{E}+04$ & $2.44 \mathrm{E}+06$ & $2.48 \mathrm{E}+05$ & $6.70 \mathrm{E}+06$ & $6.12 \mathrm{E}+03$ & $6.70 \mathrm{E}+06$ \\
\hline
\end{tabular}

Page 200 of 298 
SRNL-STI-2013-00115

\begin{tabular}{|c|c|c|c|c|c|c|c|c|c|c|c|c|}
\hline \multirow[t]{2}{*}{ Nuclide } & \multicolumn{2}{|c|}{ Water Ingestion DCS } & \multicolumn{2}{|c|}{ Meat Ingestion DCS } & \multicolumn{2}{|c|}{ Dairy Ingestion DCS } & \multicolumn{2}{|c|}{$\begin{array}{c}\text { Freshwater Fish } \\
\text { Ingestion DCS }\end{array}$} & \multicolumn{2}{|c|}{$\begin{array}{l}\text { Saltwater Shellfish } \\
\text { Ingestion DCS }\end{array}$} & \multicolumn{2}{|c|}{ Produce Ingestion DCS } \\
\hline & $(\mathrm{Bq} / \mathrm{L})$ & $(\mathrm{pCi} / \mathrm{L})$ & $(\mathrm{Bq} / \mathrm{kg})$ & $(\mathrm{pCi} / \mathrm{kg})$ & $(\mathrm{Bq} / \mathrm{L})$ & $(\mathrm{pCi} / \mathrm{L})$ & $(\mathrm{Bq} / \mathbf{k g})$ & $(\mathrm{pCi} / \mathbf{k g})$ & $(\mathrm{Bq} / \mathbf{k g})$ & $(\mathrm{pCi} / \mathbf{k g})$ & $(\mathrm{Bq} / \mathbf{k g})$ & $(\mathrm{pCi} / \mathbf{k g})$ \\
\hline At-210 & $1.06 \mathrm{E}+03$ & $2.87 \mathrm{E}+04$ & $1.06 \mathrm{E}+04$ & $2.85 \mathrm{E}+05$ & $3.27 \mathrm{E}+03$ & $8.85 \mathrm{E}+04$ & $3.89 \mathrm{E}+04$ & $1.05 \mathrm{E}+06$ & $1.07 \mathrm{E}+05$ & $2.89 \mathrm{E}+06$ & $2.64 \mathrm{E}+03$ & $2.89 \mathrm{E}+06$ \\
\hline At-211 & $8.40 \mathrm{E}+01$ & $2.27 \mathrm{E}+03$ & $8.34 \mathrm{E}+02$ & $2.25 \mathrm{E}+04$ & $2.59 \mathrm{E}+02$ & $7.00 \mathrm{E}+03$ & $3.07 \mathrm{E}+03$ & $8.30 \mathrm{E}+04$ & $8.45 \mathrm{E}+03$ & $2.28 \mathrm{E}+05$ & $2.09 \mathrm{E}+02$ & $2.28 \mathrm{E}+05$ \\
\hline Fr-212 & $1.41 \mathrm{E}+03$ & $3.81 \mathrm{E}+04$ & $1.40 \mathrm{E}+04$ & $3.78 \mathrm{E}+05$ & $4.34 \mathrm{E}+03$ & $1.17 \mathrm{E}+05$ & $5.15 \mathrm{E}+04$ & $1.39 \mathrm{E}+06$ & $1.42 \mathrm{E}+05$ & $3.83 \mathrm{E}+06$ & $3.50 \mathrm{E}+03$ & $3.83 \mathrm{E}+06$ \\
\hline Fr-222 & $1.39 \mathrm{E}+03$ & $3.76 \mathrm{E}+04$ & $1.38 \mathrm{E}+04$ & $3.73 \mathrm{E}+05$ & $4.29 \mathrm{E}+03$ & $1.16 \mathrm{E}+05$ & $5.08 \mathrm{E}+04$ & $1.37 \mathrm{E}+06$ & $1.40 \mathrm{E}+05$ & $3.78 \mathrm{E}+06$ & $3.45 \mathrm{E}+03$ & $3.78 \mathrm{E}+06$ \\
\hline Fr-223 & $3.85 \mathrm{E}+02$ & $1.04 \mathrm{E}+04$ & $3.82 \mathrm{E}+03$ & $1.03 \mathrm{E}+05$ & $1.19 \mathrm{E}+03$ & $3.21 \mathrm{E}+04$ & $1.41 \mathrm{E}+04$ & $3.80 \mathrm{E}+05$ & $3.87 \mathrm{E}+04$ & $1.05 \mathrm{E}+06$ & $9.56 \mathrm{E}+02$ & $1.05 \mathrm{E}+06$ \\
\hline $\mathrm{Ra}-223$ & $5.73 \mathrm{E}+00$ & $1.55 \mathrm{E}+02$ & $5.69 \mathrm{E}+01$ & $1.54 \mathrm{E}+03$ & $1.77 \mathrm{E}+01$ & $4.77 \mathrm{E}+02$ & $2.09 \mathrm{E}+02$ & $5.66 \mathrm{E}+03$ & $5.76 \mathrm{E}+02$ & $1.56 \mathrm{E}+04$ & $1.42 \mathrm{E}+01$ & $1.56 \mathrm{E}+04$ \\
\hline Ra-224 & $9.87 \mathrm{E}+00$ & $2.67 \mathrm{E}+02$ & $9.80 \mathrm{E}+01$ & $2.65 \mathrm{E}+03$ & $3.04 \mathrm{E}+01$ & $8.22 \mathrm{E}+02$ & $3.61 \mathrm{E}+02$ & $9.75 \mathrm{E}+03$ & $9.92 \mathrm{E}+02$ & $2.68 \mathrm{E}+04$ & $2.45 \mathrm{E}+01$ & $2.68 \mathrm{E}+04$ \\
\hline Ra-225 & $5.23 \mathrm{E}+00$ & $1.41 \mathrm{E}+02$ & $5.19 \mathrm{E}+01$ & $1.40 \mathrm{E}+03$ & $1.61 \mathrm{E}+01$ & $4.35 \mathrm{E}+02$ & $1.91 \mathrm{E}+02$ & $5.16 \mathrm{E}+03$ & $5.25 \mathrm{E}+02$ & $1.42 \mathrm{E}+04$ & $1.30 \mathrm{E}+01$ & $1.42 \mathrm{E}+04$ \\
\hline Ra-226 & $2.75 \mathrm{E}+00$ & $7.42 \mathrm{E}+01$ & $2.73 \mathrm{E}+01$ & $7.37 \mathrm{E}+02$ & $8.46 \mathrm{E}+00$ & $2.29 \mathrm{E}+02$ & $1.00 \mathrm{E}+02$ & $2.71 \mathrm{E}+03$ & $2.76 \mathrm{E}+02$ & $7.46 \mathrm{E}+03$ & $6.81 \mathrm{E}+00$ & $7.46 \mathrm{E}+03$ \\
\hline Ra-227 & $1.12 \mathrm{E}+04$ & $3.03 \mathrm{E}+05$ & $1.11 \mathrm{E}+05$ & $3.01 \mathrm{E}+06$ & $3.45 \mathrm{E}+04$ & $9.33 \mathrm{E}+05$ & $4.10 \mathrm{E}+05$ & $1.11 \mathrm{E}+07$ & $1.13 \mathrm{E}+06$ & $3.04 \mathrm{E}+07$ & $2.78 \mathrm{E}+04$ & $3.04 \mathrm{E}+07$ \\
\hline Ra-228 & 7.77E-01 & $2.10 \mathrm{E}+01$ & $7.72 \mathrm{E}+00$ & $2.09 \mathrm{E}+02$ & $2.39 \mathrm{E}+00$ & $6.47 \mathrm{E}+01$ & $2.84 \mathrm{E}+01$ & $7.68 \mathrm{E}+02$ & $7.81 \mathrm{E}+01$ & $2.11 \mathrm{E}+03$ & $1.93 \mathrm{E}+00$ & $2.11 \mathrm{E}+03$ \\
\hline Ra-230 & $5.16 \mathrm{E}+03$ & $1.39 \mathrm{E}+05$ & $5.12 \mathrm{E}+04$ & $1.38 \mathrm{E}+06$ & $1.59 \mathrm{E}+04$ & $4.30 \mathrm{E}+05$ & $1.89 \mathrm{E}+05$ & $5.10 \mathrm{E}+06$ & $5.19 \mathrm{E}+05$ & $1.40 \mathrm{E}+07$ & $1.28 \mathrm{E}+04$ & $1.40 \mathrm{E}+07$ \\
\hline Ac-224 & $4.47 \mathrm{E}+02$ & $1.21 \mathrm{E}+04$ & $4.44 \mathrm{E}+03$ & $1.20 \mathrm{E}+05$ & $1.38 \mathrm{E}+03$ & $3.72 \mathrm{E}+04$ & $1.64 \mathrm{E}+04$ & $4.42 \mathrm{E}+05$ & $4.50 \mathrm{E}+04$ & $1.22 \mathrm{E}+06$ & $1.11 \mathrm{E}+03$ & $1.22 \mathrm{E}+06$ \\
\hline Ac- 225 & $2.38 \mathrm{E}+01$ & $6.43 \mathrm{E}+02$ & $2.36 \mathrm{E}+02$ & $6.38 \mathrm{E}+03$ & $7.33 \mathrm{E}+01$ & $1.98 \mathrm{E}+03$ & $8.69 \mathrm{E}+02$ & $2.35 \mathrm{E}+04$ & $2.39 \mathrm{E}+03$ & $6.46 \mathrm{E}+04$ & $5.90 \mathrm{E}+01$ & $6.46 \mathrm{E}+04$ \\
\hline Ac-226 & $8.58 \mathrm{E}+01$ & $2.32 \mathrm{E}+03$ & $8.51 \mathrm{E}+02$ & $2.30 \mathrm{E}+04$ & $2.64 \mathrm{E}+02$ & $7.14 \mathrm{E}+03$ & $3.13 \mathrm{E}+03$ & $8.47 \mathrm{E}+04$ & $8.62 \mathrm{E}+03$ & $2.33 \mathrm{E}+05$ & $2.13 \mathrm{E}+02$ & $2.33 \mathrm{E}+05$ \\
\hline Ac- 227 & $3.17 \mathrm{E}+00$ & $8.58 \mathrm{E}+01$ & $3.15 \mathrm{E}+01$ & $8.51 \mathrm{E}+02$ & $9.77 \mathrm{E}+00$ & $2.64 \mathrm{E}+02$ & $1.16 \mathrm{E}+02$ & $3.13 \mathrm{E}+03$ & $3.19 \mathrm{E}+02$ & $8.62 \mathrm{E}+03$ & $7.87 \mathrm{E}+00$ & $8.62 \mathrm{E}+03$ \\
\hline Ac-228 & $2.42 \mathrm{E}+03$ & $6.54 \mathrm{E}+04$ & $2.40 \mathrm{E}+04$ & $6.49 \mathrm{E}+05$ & $7.45 \mathrm{E}+03$ & $2.01 \mathrm{E}+05$ & $8.84 \mathrm{E}+04$ & $2.39 \mathrm{E}+06$ & $2.43 \mathrm{E}+05$ & $6.57 \mathrm{E}+06$ & $6.00 \mathrm{E}+03$ & $6.57 \mathrm{E}+06$ \\
\hline Th-226 & $2.61 \mathrm{E}+03$ & $7.06 \mathrm{E}+04$ & $2.59 \mathrm{E}+04$ & $7.01 \mathrm{E}+05$ & $8.05 \mathrm{E}+03$ & $2.18 \mathrm{E}+05$ & $9.55 \mathrm{E}+04$ & $2.58 \mathrm{E}+06$ & $2.63 \mathrm{E}+05$ & $7.10 \mathrm{E}+06$ & $6.48 \mathrm{E}+03$ & $7.10 \mathrm{E}+06$ \\
\hline Th-227 & $8.46 \mathrm{E}+01$ & $2.29 \mathrm{E}+03$ & $8.40 \mathrm{E}+02$ & $2.27 \mathrm{E}+04$ & $2.61 \mathrm{E}+02$ & $7.04 \mathrm{E}+03$ & $3.09 \mathrm{E}+03$ & $8.36 \mathrm{E}+04$ & $8.50 \mathrm{E}+03$ & $2.30 \mathrm{E}+05$ & $2.10 \mathrm{E}+02$ & $2.30 \mathrm{E}+05$ \\
\hline Th-228 & $1.07 \mathrm{E}+01$ & $2.90 \mathrm{E}+02$ & $1.06 \mathrm{E}+02$ & $2.88 \mathrm{E}+03$ & $3.30 \mathrm{E}+01$ & $8.93 \mathrm{E}+02$ & $3.92 \mathrm{E}+02$ & $1.06 \mathrm{E}+04$ & $1.08 \mathrm{E}+03$ & $2.91 \mathrm{E}+04$ & $2.66 \mathrm{E}+01$ & $2.91 \mathrm{E}+04$ \\
\hline Th-229 & $2.04 \mathrm{E}+00$ & $5.52 \mathrm{E}+01$ & $2.03 \mathrm{E}+01$ & $5.48 \mathrm{E}+02$ & $6.29 \mathrm{E}+00$ & $1.70 \mathrm{E}+02$ & $7.46 \mathrm{E}+01$ & $2.02 \mathrm{E}+03$ & $2.05 \mathrm{E}+02$ & $5.55 \mathrm{E}+03$ & $5.07 \mathrm{E}+00$ & $5.55 \mathrm{E}+03$ \\
\hline Th-230 & $4.92 \mathrm{E}+00$ & $1.33 \mathrm{E}+02$ & $4.88 \mathrm{E}+01$ & $1.32 \mathrm{E}+03$ & $1.51 \mathrm{E}+01$ & $4.09 \mathrm{E}+02$ & $1.80 \mathrm{E}+02$ & $4.86 \mathrm{E}+03$ & $4.94 \mathrm{E}+02$ & $1.34 \mathrm{E}+04$ & $1.22 \mathrm{E}+01$ & $1.34 \mathrm{E}+04$ \\
\hline Th-231 & $2.70 \mathrm{E}+03$ & $7.29 \mathrm{E}+04$ & $2.68 \mathrm{E}+04$ & $7.24 \mathrm{E}+05$ & $8.31 \mathrm{E}+03$ & $2.25 \mathrm{E}+05$ & $9.86 \mathrm{E}+04$ & $2.66 \mathrm{E}+06$ & $2.71 \mathrm{E}+05$ & $7.33 \mathrm{E}+06$ & $6.70 \mathrm{E}+03$ & $7.33 \mathrm{E}+06$ \\
\hline
\end{tabular}

Page 201 of 298 
SRNL-STI-2013-00115

\begin{tabular}{|c|c|c|c|c|c|c|c|c|c|c|c|c|}
\hline \multirow[t]{2}{*}{ Nuclide } & \multicolumn{2}{|c|}{ Water Ingestion DCS } & \multicolumn{2}{|c|}{ Meat Ingestion DCS } & \multicolumn{2}{|c|}{ Dairy Ingestion DCS } & \multicolumn{2}{|c|}{$\begin{array}{c}\text { Freshwater Fish } \\
\text { Ingestion DCS }\end{array}$} & \multicolumn{2}{|c|}{$\begin{array}{l}\text { Saltwater Shellfish } \\
\text { Ingestion DCS }\end{array}$} & \multicolumn{2}{|c|}{ Produce Ingestion DCS } \\
\hline & $(\mathrm{Bq} / \mathrm{L})$ & $(\mathrm{pCi} / \mathrm{L})$ & $(\mathrm{Bq} / \mathbf{k g})$ & $(\mathrm{pCi} / \mathrm{kg})$ & $(\mathrm{Bq} / \mathrm{L})$ & $(\mathrm{pCi} / \mathrm{L})$ & $(\mathrm{Bq} / \mathbf{k g})$ & $(\mathrm{pCi} / \mathbf{k g})$ & $(\mathrm{Bq} / \mathbf{k g})$ & $(\mathrm{pCi} / \mathbf{k g})$ & $(\mathrm{Bq} / \mathbf{k g})$ & $(\mathrm{pCi} / \mathbf{k g})$ \\
\hline Th-232 & $4.47 \mathrm{E}+00$ & $1.21 \mathrm{E}+02$ & $4.44 \mathrm{E}+01$ & $1.20 \mathrm{E}+03$ & $1.38 \mathrm{E}+01$ & $3.72 \mathrm{E}+02$ & $1.64 \mathrm{E}+02$ & $4.42 \mathrm{E}+03$ & $4.50 \mathrm{E}+02$ & $1.22 \mathrm{E}+04$ & $1.11 \mathrm{E}+01$ & $1.22 \mathrm{E}+04$ \\
\hline Th-233 & $4.24 \mathrm{E}+04$ & $1.15 \mathrm{E}+06$ & $4.21 \mathrm{E}+05$ & $1.14 \mathrm{E}+07$ & $1.31 \mathrm{E}+05$ & $3.53 \mathrm{E}+06$ & $1.55 \mathrm{E}+06$ & $4.19 \mathrm{E}+07$ & $4.27 \mathrm{E}+06$ & $1.15 \mathrm{E}+08$ & $1.05 \mathrm{E}+05$ & $1.15 \mathrm{E}+08$ \\
\hline Th-234 & $2.66 \mathrm{E}+02$ & $7.18 \mathrm{E}+03$ & $2.64 \mathrm{E}+03$ & $7.13 \mathrm{E}+04$ & $8.19 \mathrm{E}+02$ & $2.21 \mathrm{E}+04$ & $9.71 \mathrm{E}+03$ & $2.63 \mathrm{E}+05$ & $2.67 \mathrm{E}+04$ & $7.22 \mathrm{E}+05$ & $6.59 \mathrm{E}+02$ & $7.22 \mathrm{E}+05$ \\
\hline Th-236 & $1.06 \mathrm{E}+04$ & $2.87 \mathrm{E}+05$ & $1.06 \mathrm{E}+05$ & $2.85 \mathrm{E}+06$ & $3.27 \mathrm{E}+04$ & $8.85 \mathrm{E}+05$ & $3.89 \mathrm{E}+05$ & $1.05 \mathrm{E}+07$ & $1.07 \mathrm{E}+06$ & $2.89 \mathrm{E}+07$ & $2.64 \mathrm{E}+04$ & $2.89 \mathrm{E}+07$ \\
\hline $\mathrm{Pa}-227$ & $2.02 \mathrm{E}+03$ & $5.45 \mathrm{E}+04$ & $2.00 \mathrm{E}+04$ & $5.41 \mathrm{E}+05$ & $6.21 \mathrm{E}+03$ & $1.68 \mathrm{E}+05$ & $7.37 \mathrm{E}+04$ & $1.99 \mathrm{E}+06$ & $2.03 \mathrm{E}+05$ & $5.48 \mathrm{E}+06$ & $5.00 \mathrm{E}+03$ & $5.48 \mathrm{E}+06$ \\
\hline $\mathrm{Pa}-228$ & $1.15 \mathrm{E}+03$ & $3.11 \mathrm{E}+04$ & $1.14 \mathrm{E}+04$ & $3.09 \mathrm{E}+05$ & $3.55 \mathrm{E}+03$ & $9.59 \mathrm{E}+04$ & $4.21 \mathrm{E}+04$ & $1.14 \mathrm{E}+06$ & $1.16 \mathrm{E}+05$ & $3.13 \mathrm{E}+06$ & $2.86 \mathrm{E}+03$ & $3.13 \mathrm{E}+06$ \\
\hline Pa-229 & $1.10 \mathrm{E}+04$ & $2.97 \mathrm{E}+05$ & $1.09 \mathrm{E}+05$ & $2.95 \mathrm{E}+06$ & $3.39 \mathrm{E}+04$ & $9.16 \mathrm{E}+05$ & $4.02 \mathrm{E}+05$ & $1.09 \mathrm{E}+07$ & $1.11 \mathrm{E}+06$ & $2.99 \mathrm{E}+07$ & $2.73 \mathrm{E}+04$ & $2.99 \mathrm{E}+07$ \\
\hline Pa-230 & $1.03 \mathrm{E}+03$ & $2.78 \mathrm{E}+04$ & $1.02 \mathrm{E}+04$ & $2.76 \mathrm{E}+05$ & $3.17 \mathrm{E}+03$ & $8.56 \mathrm{E}+04$ & $3.76 \mathrm{E}+04$ & $1.02 \mathrm{E}+06$ & $1.03 \mathrm{E}+05$ & $2.79 \mathrm{E}+06$ & $2.55 \mathrm{E}+03$ & $2.79 \mathrm{E}+06$ \\
\hline $\mathrm{Pa}-231$ & $2.23 \mathrm{E}+00$ & $6.01 \mathrm{E}+01$ & $2.21 \mathrm{E}+01$ & $5.97 \mathrm{E}+02$ & $6.85 \mathrm{E}+00$ & $1.85 \mathrm{E}+02$ & $8.13 \mathrm{E}+01$ & $2.20 \mathrm{E}+03$ & $2.24 \mathrm{E}+02$ & $6.04 \mathrm{E}+03$ & $5.52 \mathrm{E}+00$ & $6.04 \mathrm{E}+03$ \\
\hline $\mathrm{Pa}-232$ & $1.40 \mathrm{E}+03$ & $3.79 \mathrm{E}+04$ & $1.39 \mathrm{E}+04$ & $3.76 \mathrm{E}+05$ & $4.32 \mathrm{E}+03$ & $1.17 \mathrm{E}+05$ & $5.12 \mathrm{E}+04$ & $1.39 \mathrm{E}+06$ & $1.41 \mathrm{E}+05$ & $3.81 \mathrm{E}+06$ & $3.48 \mathrm{E}+03$ & $3.81 \mathrm{E}+06$ \\
\hline $\mathrm{Pa}-233$ & $9.42 \mathrm{E}+02$ & $2.55 \mathrm{E}+04$ & $9.35 \mathrm{E}+03$ & $2.53 \mathrm{E}+05$ & $2.90 \mathrm{E}+03$ & $7.84 \mathrm{E}+04$ & $3.44 \mathrm{E}+04$ & $9.31 \mathrm{E}+05$ & $9.47 \mathrm{E}+04$ & $2.56 \mathrm{E}+06$ & $2.34 \mathrm{E}+03$ & $2.56 \mathrm{E}+06$ \\
\hline $\mathrm{Pa}-234$ & $2.24 \mathrm{E}+03$ & $6.05 \mathrm{E}+04$ & $2.22 \mathrm{E}+04$ & $6.00 \mathrm{E}+05$ & $6.89 \mathrm{E}+03$ & $1.86 \mathrm{E}+05$ & $8.18 \mathrm{E}+04$ & $2.21 \mathrm{E}+06$ & $2.25 \mathrm{E}+05$ & $6.08 \mathrm{E}+06$ & $5.55 \mathrm{E}+03$ & $6.08 \mathrm{E}+06$ \\
\hline $\mathrm{Pa}-235$ & $3.48 \mathrm{E}+04$ & $9.42 \mathrm{E}+05$ & $3.46 \mathrm{E}+05$ & $9.35 \mathrm{E}+06$ & $1.07 \mathrm{E}+05$ & $2.90 \mathrm{E}+06$ & $1.27 \mathrm{E}+06$ & $3.44 \mathrm{E}+07$ & $3.50 \mathrm{E}+06$ & $9.46 \mathrm{E}+07$ & $8.65 \mathrm{E}+04$ & $9.46 \mathrm{E}+07$ \\
\hline $\mathrm{U}-230$ & $1.73 \mathrm{E}+01$ & $4.66 \mathrm{E}+02$ & $1.71 \mathrm{E}+02$ & $4.63 \mathrm{E}+03$ & $5.31 \mathrm{E}+01$ & $1.44 \mathrm{E}+03$ & $6.30 \mathrm{E}+02$ & $1.70 \mathrm{E}+04$ & $1.73 \mathrm{E}+03$ & $4.69 \mathrm{E}+04$ & $4.28 \mathrm{E}+01$ & $4.69 \mathrm{E}+04$ \\
\hline U-231 & $2.69 \mathrm{E}+03$ & $7.26 \mathrm{E}+04$ & $2.67 \mathrm{E}+04$ & $7.21 \mathrm{E}+05$ & $8.28 \mathrm{E}+03$ & $2.24 \mathrm{E}+05$ & $9.82 \mathrm{E}+04$ & $2.65 \mathrm{E}+06$ & $2.70 \mathrm{E}+05$ & $7.30 \mathrm{E}+06$ & $6.67 \mathrm{E}+03$ & $7.30 \mathrm{E}+06$ \\
\hline U-232 & $3.08 \mathrm{E}+00$ & $8.32 \mathrm{E}+01$ & $3.06 \mathrm{E}+01$ & $8.26 \mathrm{E}+02$ & $9.48 \mathrm{E}+00$ & $2.56 \mathrm{E}+02$ & $1.13 \mathrm{E}+02$ & $3.04 \mathrm{E}+03$ & $3.09 \mathrm{E}+02$ & $8.36 \mathrm{E}+03$ & $7.64 \mathrm{E}+00$ & $8.36 \mathrm{E}+03$ \\
\hline U-233 & $2.07 \mathrm{E}+01$ & $5.58 \mathrm{E}+02$ & $2.05 \mathrm{E}+02$ & $5.54 \mathrm{E}+03$ & $6.36 \mathrm{E}+01$ & $1.72 \mathrm{E}+03$ & $7.55 \mathrm{E}+02$ & $2.04 \mathrm{E}+04$ & $2.08 \mathrm{E}+03$ & $5.61 \mathrm{E}+04$ & $5.13 \mathrm{E}+01$ & $5.61 \mathrm{E}+04$ \\
\hline U-234 & $2.14 \mathrm{E}+01$ & $5.79 \mathrm{E}+02$ & $2.12 \mathrm{E}+02$ & $5.74 \mathrm{E}+03$ & $6.59 \mathrm{E}+01$ & $1.78 \mathrm{E}+03$ & $7.82 \mathrm{E}+02$ & $2.11 \mathrm{E}+04$ & $2.15 \mathrm{E}+03$ & $5.81 \mathrm{E}+04$ & $5.31 \mathrm{E}+01$ & $5.81 \mathrm{E}+04$ \\
\hline U-235 & $2.27 \mathrm{E}+01$ & $6.12 \mathrm{E}+02$ & $2.25 \mathrm{E}+02$ & $6.08 \mathrm{E}+03$ & $6.98 \mathrm{E}+01$ & $1.89 \mathrm{E}+03$ & $8.28 \mathrm{E}+02$ & $2.24 \mathrm{E}+04$ & $2.28 \mathrm{E}+03$ & $6.15 \mathrm{E}+04$ & $5.62 \mathrm{E}+01$ & $6.15 \mathrm{E}+04$ \\
\hline $\mathrm{U}-235 \mathrm{~m}$ & $2.14 \mathrm{E}+08$ & $5.78 \mathrm{E}+09$ & $2.12 \mathrm{E}+09$ & $5.73 \mathrm{E}+10$ & $6.58 \mathrm{E}+08$ & $1.78 \mathrm{E}+10$ & $7.81 \mathrm{E}+09$ & $2.11 \mathrm{E}+11$ & $2.15 \mathrm{E}+10$ & $5.80 \mathrm{E}+11$ & $5.30 \mathrm{E}+08$ & $5.80 \mathrm{E}+11$ \\
\hline U-236 & $2.27 \mathrm{E}+01$ & $6.15 \mathrm{E}+02$ & $2.26 \mathrm{E}+02$ & $6.10 \mathrm{E}+03$ & $7.00 \mathrm{E}+01$ & $1.89 \mathrm{E}+03$ & $8.31 \mathrm{E}+02$ & $2.25 \mathrm{E}+04$ & $2.29 \mathrm{E}+03$ & $6.18 \mathrm{E}+04$ & $5.64 \mathrm{E}+01$ & $6.18 \mathrm{E}+04$ \\
\hline U-237 & $1.17 \mathrm{E}+03$ & $3.17 \mathrm{E}+04$ & $1.16 \mathrm{E}+04$ & $3.15 \mathrm{E}+05$ & $3.61 \mathrm{E}+03$ & $9.77 \mathrm{E}+04$ & $4.29 \mathrm{E}+04$ & $1.16 \mathrm{E}+06$ & $1.18 \mathrm{E}+05$ & $3.19 \mathrm{E}+06$ & $2.91 \mathrm{E}+03$ & $3.19 \mathrm{E}+06$ \\
\hline U-238 & $2.37 \mathrm{E}+01$ & $6.42 \mathrm{E}+02$ & $2.36 \mathrm{E}+02$ & $6.37 \mathrm{E}+03$ & $7.31 \mathrm{E}+01$ & $1.98 \mathrm{E}+03$ & $8.67 \mathrm{E}+02$ & $2.34 \mathrm{E}+04$ & $2.39 \mathrm{E}+03$ & $6.45 \mathrm{E}+04$ & $5.89 \mathrm{E}+01$ & $6.45 \mathrm{E}+04$ \\
\hline
\end{tabular}

Page 202 of 298 
SRNL-STI-2013-00115

\begin{tabular}{|c|c|c|c|c|c|c|c|c|c|c|c|c|}
\hline \multirow{2}{*}{ Nuclide } & \multicolumn{2}{|c|}{ Water Ingestion DCS } & \multicolumn{2}{|c|}{ Meat Ingestion DCS } & \multicolumn{2}{|c|}{ Dairy Ingestion DCS } & \multicolumn{2}{|c|}{$\begin{array}{c}\text { Freshwater Fish } \\
\text { Ingestion DCS }\end{array}$} & \multicolumn{2}{|c|}{$\begin{array}{l}\text { Saltwater Shellfish } \\
\text { Ingestion DCS }\end{array}$} & \multicolumn{2}{|c|}{ Produce Ingestion DCS } \\
\hline & $(\mathrm{Bq} / \mathrm{L})$ & $(\mathrm{pCi} / \mathrm{L})$ & $(\mathrm{Bq} / \mathrm{kg})$ & $(\mathrm{pCi} / \mathrm{kg})$ & $(\mathrm{Bq} / \mathrm{L})$ & $(\mathrm{pCi} / \mathrm{L})$ & $(\mathrm{Bq} / \mathbf{k g})$ & $(\mathrm{pCi} / \mathrm{kg})$ & $(\mathrm{Bq} / \mathbf{k g})$ & $(\mathrm{pCi} / \mathbf{k g})$ & $(\mathrm{Bq} / \mathbf{k g})$ & $(\mathrm{pCi} / \mathbf{k g})$ \\
\hline U-239 & $3.33 \mathrm{E}+04$ & $9.01 \mathrm{E}+05$ & $3.31 \mathrm{E}+05$ & $8.95 \mathrm{E}+06$ & $1.03 \mathrm{E}+05$ & $2.78 \mathrm{E}+06$ & $1.22 \mathrm{E}+06$ & $3.29 \mathrm{E}+07$ & $3.35 \mathrm{E}+06$ & $9.06 \mathrm{E}+07$ & $8.27 \mathrm{E}+04$ & $9.06 \mathrm{E}+07$ \\
\hline $\mathrm{U}-240$ & $8.29 \mathrm{E}+02$ & $2.24 \mathrm{E}+04$ & $8.23 \mathrm{E}+03$ & $2.22 \mathrm{E}+05$ & $2.55 \mathrm{E}+03$ & $6.90 \mathrm{E}+04$ & $3.03 \mathrm{E}+04$ & $8.19 \mathrm{E}+05$ & $8.33 \mathrm{E}+04$ & $2.25 \mathrm{E}+06$ & $2.06 \mathrm{E}+03$ & $2.25 \mathrm{E}+06$ \\
\hline $\mathrm{U}-242$ & $1.76 \mathrm{E}+04$ & $4.75 \mathrm{E}+05$ & $1.75 \mathrm{E}+05$ & $4.72 \mathrm{E}+06$ & $5.42 \mathrm{E}+04$ & $1.46 \mathrm{E}+06$ & $6.43 \mathrm{E}+05$ & $1.74 \mathrm{E}+07$ & $1.77 \mathrm{E}+06$ & $4.78 \mathrm{E}+07$ & $4.37 \mathrm{E}+04$ & $4.78 \mathrm{E}+07$ \\
\hline $\mathrm{Np}-232$ & $1.00 \mathrm{E}+05$ & $2.71 \mathrm{E}+06$ & $9.96 \mathrm{E}+05$ & $2.69 \mathrm{E}+07$ & $3.09 \mathrm{E}+05$ & $8.35 \mathrm{E}+06$ & $3.67 \mathrm{E}+06$ & $9.91 \mathrm{E}+07$ & $1.01 \mathrm{E}+07$ & $2.72 \mathrm{E}+08$ & $2.49 \mathrm{E}+05$ & $2.72 \mathrm{E}+08$ \\
\hline $\mathrm{Np}-233$ & $4.23 \mathrm{E}+05$ & $1.14 \mathrm{E}+07$ & $4.20 \mathrm{E}+06$ & $1.13 \mathrm{E}+08$ & $1.30 \mathrm{E}+06$ & $3.52 \mathrm{E}+07$ & $1.55 \mathrm{E}+07$ & $4.18 \mathrm{E}+08$ & $4.25 \mathrm{E}+07$ & $1.15 \mathrm{E}+09$ & $1.05 \mathrm{E}+06$ & $1.15 \mathrm{E}+09$ \\
\hline $\mathrm{Np}-234$ & $1.51 \mathrm{E}+03$ & $4.07 \mathrm{E}+04$ & $1.49 \mathrm{E}+04$ & $4.04 \mathrm{E}+05$ & $4.64 \mathrm{E}+03$ & $1.25 \mathrm{E}+05$ & $5.50 \mathrm{E}+04$ & $1.49 \mathrm{E}+06$ & $1.51 \mathrm{E}+05$ & $4.09 \mathrm{E}+06$ & $3.74 \mathrm{E}+03$ & $4.09 \mathrm{E}+06$ \\
\hline $\mathrm{Np}-235$ & $1.56 \mathrm{E}+04$ & $4.22 \mathrm{E}+05$ & $1.55 \mathrm{E}+05$ & $4.19 \mathrm{E}+06$ & $4.81 \mathrm{E}+04$ & $1.30 \mathrm{E}+06$ & $5.71 \mathrm{E}+05$ & $1.54 \mathrm{E}+07$ & $1.57 \mathrm{E}+06$ & $4.24 \mathrm{E}+07$ & $3.88 \mathrm{E}+04$ & $4.24 \mathrm{E}+07$ \\
\hline Np-236 & $4.61 \mathrm{E}+01$ & $1.25 \mathrm{E}+03$ & $4.57 \mathrm{E}+02$ & $1.24 \mathrm{E}+04$ & $1.42 \mathrm{E}+02$ & $3.84 \mathrm{E}+03$ & $1.68 \mathrm{E}+03$ & $4.55 \mathrm{E}+04$ & $4.63 \mathrm{E}+03$ & $1.25 \mathrm{E}+05$ & $1.14 \mathrm{E}+02$ & $1.25 \mathrm{E}+05$ \\
\hline $\mathrm{Np}-236 \mathrm{~m}$ & $4.66 \mathrm{E}+03$ & $1.26 \mathrm{E}+05$ & $4.62 \mathrm{E}+04$ & $1.25 \mathrm{E}+06$ & $1.43 \mathrm{E}+04$ & $3.88 \mathrm{E}+05$ & $1.70 \mathrm{E}+05$ & $4.60 \mathrm{E}+06$ & $4.68 \mathrm{E}+05$ & $1.27 \mathrm{E}+07$ & $1.16 \mathrm{E}+04$ & $1.27 \mathrm{E}+07$ \\
\hline $\mathrm{Np}-237$ & $9.95 \mathrm{E}+00$ & $2.69 \mathrm{E}+02$ & $9.88 \mathrm{E}+01$ & $2.67 \mathrm{E}+03$ & $3.07 \mathrm{E}+01$ & $8.28 \mathrm{E}+02$ & $3.64 \mathrm{E}+02$ & $9.83 \mathrm{E}+03$ & $1.00 \mathrm{E}+03$ & $2.70 \mathrm{E}+04$ & $2.47 \mathrm{E}+01$ & $2.70 \mathrm{E}+04$ \\
\hline $\mathrm{Np}-238$ & $1.04 \mathrm{E}+03$ & $2.80 \mathrm{E}+04$ & $1.03 \mathrm{E}+04$ & $2.78 \mathrm{E}+05$ & $3.19 \mathrm{E}+03$ & $8.63 \mathrm{E}+04$ & $3.79 \mathrm{E}+04$ & $1.02 \mathrm{E}+06$ & $1.04 \mathrm{E}+05$ & $2.82 \mathrm{E}+06$ & $2.57 \mathrm{E}+03$ & $2.82 \mathrm{E}+06$ \\
\hline Np-239 & $1.12 \mathrm{E}+03$ & $3.03 \mathrm{E}+04$ & $1.11 \mathrm{E}+04$ & $3.01 \mathrm{E}+05$ & $3.45 \mathrm{E}+03$ & $9.33 \mathrm{E}+04$ & $4.10 \mathrm{E}+04$ & $1.11 \mathrm{E}+06$ & $1.13 \mathrm{E}+05$ & $3.04 \mathrm{E}+06$ & $2.78 \mathrm{E}+03$ & $3.04 \mathrm{E}+06$ \\
\hline $\mathrm{Np}-240$ & $1.30 \mathrm{E}+04$ & $3.51 \mathrm{E}+05$ & $1.29 \mathrm{E}+05$ & $3.48 \mathrm{E}+06$ & $4.00 \mathrm{E}+04$ & $1.08 \mathrm{E}+06$ & $4.74 \mathrm{E}+05$ & $1.28 \mathrm{E}+07$ & $1.30 \mathrm{E}+06$ & $3.52 \mathrm{E}+07$ & $3.22 \mathrm{E}+04$ & $3.52 \mathrm{E}+07$ \\
\hline Np-241 & $5.95 \mathrm{E}+04$ & $1.61 \mathrm{E}+06$ & $5.91 \mathrm{E}+05$ & $1.60 \mathrm{E}+07$ & $1.83 \mathrm{E}+05$ & $4.95 \mathrm{E}+06$ & $2.17 \mathrm{E}+06$ & $5.88 \mathrm{E}+07$ & $5.98 \mathrm{E}+06$ & $1.62 \mathrm{E}+08$ & $1.48 \mathrm{E}+05$ & $1.62 \mathrm{E}+08$ \\
\hline $\mathrm{Pu}-232$ & $6.76 \mathrm{E}+03$ & $1.83 \mathrm{E}+05$ & $6.71 \mathrm{E}+04$ & $1.81 \mathrm{E}+06$ & $2.08 \mathrm{E}+04$ & $5.63 \mathrm{E}+05$ & $2.47 \mathrm{E}+05$ & $6.68 \mathrm{E}+06$ & $6.79 \mathrm{E}+05$ & $1.84 \mathrm{E}+07$ & $1.68 \mathrm{E}+04$ & $1.84 \mathrm{E}+07$ \\
\hline $\mathrm{Pu}-234$ & $5.98 \mathrm{E}+03$ & $1.62 \mathrm{E}+05$ & $5.94 \mathrm{E}+04$ & $1.60 \mathrm{E}+06$ & $1.84 \mathrm{E}+04$ & $4.98 \mathrm{E}+05$ & $2.19 \mathrm{E}+05$ & $5.91 \mathrm{E}+06$ & $6.01 \mathrm{E}+05$ & $1.62 \mathrm{E}+07$ & $1.48 \mathrm{E}+04$ & $1.62 \mathrm{E}+07$ \\
\hline $\mathrm{Pu}-235$ & $4.22 \mathrm{E}+05$ & $1.14 \mathrm{E}+07$ & $4.18 \mathrm{E}+06$ & $1.13 \mathrm{E}+08$ & $1.30 \mathrm{E}+06$ & $3.51 \mathrm{E}+07$ & $1.54 \mathrm{E}+07$ & $4.16 \mathrm{E}+08$ & $4.24 \mathrm{E}+07$ & $1.15 \mathrm{E}+09$ & $1.05 \mathrm{E}+06$ & $1.15 \mathrm{E}+09$ \\
\hline $\mathrm{Pu}-236$ & $1.13 \mathrm{E}+01$ & $3.06 \mathrm{E}+02$ & $1.12 \mathrm{E}+02$ & $3.03 \mathrm{E}+03$ & $3.48 \mathrm{E}+01$ & $9.41 \mathrm{E}+02$ & $4.13 \mathrm{E}+02$ & $1.12 \mathrm{E}+04$ & $1.14 \mathrm{E}+03$ & $3.07 \mathrm{E}+04$ & $2.81 \mathrm{E}+01$ & $3.07 \mathrm{E}+04$ \\
\hline $\mathrm{Pu}-237$ & $8.24 \mathrm{E}+03$ & $2.23 \mathrm{E}+05$ & $8.18 \mathrm{E}+04$ & $2.21 \mathrm{E}+06$ & $2.54 \mathrm{E}+04$ & $6.86 \mathrm{E}+05$ & $3.01 \mathrm{E}+05$ & $8.14 \mathrm{E}+06$ & $8.28 \mathrm{E}+05$ & $2.24 \mathrm{E}+07$ & $2.04 \mathrm{E}+04$ & $2.24 \mathrm{E}+07$ \\
\hline $\mathrm{Pu}-238$ & $4.73 \mathrm{E}+00$ & $1.28 \mathrm{E}+02$ & $4.69 \mathrm{E}+01$ & $1.27 \mathrm{E}+03$ & $1.46 \mathrm{E}+01$ & $3.94 \mathrm{E}+02$ & $1.73 \mathrm{E}+02$ & $4.67 \mathrm{E}+03$ & $4.75 \mathrm{E}+02$ & $1.28 \mathrm{E}+04$ & $1.17 \mathrm{E}+01$ & $1.28 \mathrm{E}+04$ \\
\hline $\mathrm{Pu}-239$ & $4.32 \mathrm{E}+00$ & $1.17 \mathrm{E}+02$ & $4.29 \mathrm{E}+01$ & $1.16 \mathrm{E}+03$ & $1.33 \mathrm{E}+01$ & $3.60 \mathrm{E}+02$ & $1.58 \mathrm{E}+02$ & $4.27 \mathrm{E}+03$ & $4.34 \mathrm{E}+02$ & $1.17 \mathrm{E}+04$ & $1.07 \mathrm{E}+01$ & $1.17 \mathrm{E}+04$ \\
\hline $\mathrm{Pu}-240$ & $4.32 \mathrm{E}+00$ & $1.17 \mathrm{E}+02$ & $4.29 \mathrm{E}+01$ & $1.16 \mathrm{E}+03$ & $1.33 \mathrm{E}+01$ & $3.60 \mathrm{E}+02$ & $1.58 \mathrm{E}+02$ & $4.27 \mathrm{E}+03$ & $4.34 \mathrm{E}+02$ & $1.17 \mathrm{E}+04$ & $1.07 \mathrm{E}+01$ & $1.17 \mathrm{E}+04$ \\
\hline $\mathrm{Pu}-241$ & $2.39 \mathrm{E}+02$ & $6.45 \mathrm{E}+03$ & $2.37 \mathrm{E}+03$ & $6.40 \mathrm{E}+04$ & $7.35 \mathrm{E}+02$ & $1.99 \mathrm{E}+04$ & $8.72 \mathrm{E}+03$ & $2.36 \mathrm{E}+05$ & $2.40 \mathrm{E}+04$ & $6.48 \mathrm{E}+05$ & $5.92 \mathrm{E}+02$ & $6.48 \mathrm{E}+05$ \\
\hline
\end{tabular}


SRNL-STI-2013-00115

\begin{tabular}{|c|c|c|c|c|c|c|c|c|c|c|c|c|}
\hline \multirow{2}{*}{ Nuclide } & \multicolumn{2}{|c|}{ Water Ingestion DCS } & \multicolumn{2}{|c|}{ Meat Ingestion DCS } & \multicolumn{2}{|c|}{ Dairy Ingestion DCS } & \multicolumn{2}{|c|}{$\begin{array}{c}\text { Freshwater Fish } \\
\text { Ingestion DCS }\end{array}$} & \multicolumn{2}{|c|}{$\begin{array}{l}\text { Saltwater Shellfish } \\
\text { Ingestion DCS }\end{array}$} & \multicolumn{2}{|c|}{ Produce Ingestion DCS } \\
\hline & $(\mathrm{Bq} / \mathrm{L})$ & $(\mathrm{pCi} / \mathrm{L})$ & $(\mathrm{Bq} / \mathrm{kg})$ & $(\mathrm{pCi} / \mathrm{kg})$ & $(\mathrm{Bq} / \mathrm{L})$ & $(\mathrm{pCi} / \mathrm{L})$ & $(\mathrm{Bq} / \mathbf{k g})$ & $(\mathrm{pCi} / \mathbf{k g})$ & $(\mathrm{Bq} / \mathbf{k g})$ & $(\mathrm{pCi} / \mathbf{k g})$ & $(\mathrm{Bq} / \mathbf{k g})$ & $(\mathrm{pCi} / \mathbf{k g})$ \\
\hline $\mathrm{Pu}-242$ & $4.54 \mathrm{E}+00$ & $1.23 \mathrm{E}+02$ & $4.51 \mathrm{E}+01$ & $1.22 \mathrm{E}+03$ & $1.40 \mathrm{E}+01$ & $3.78 \mathrm{E}+02$ & $1.66 \mathrm{E}+02$ & $4.48 \mathrm{E}+03$ & $4.56 \mathrm{E}+02$ & $1.23 \mathrm{E}+04$ & $1.13 \mathrm{E}+01$ & $1.23 \mathrm{E}+04$ \\
\hline $\mathrm{Pu}-243$ & $1.06 \mathrm{E}+04$ & $2.87 \mathrm{E}+05$ & $1.06 \mathrm{E}+05$ & $2.85 \mathrm{E}+06$ & $3.27 \mathrm{E}+04$ & $8.85 \mathrm{E}+05$ & $3.89 \mathrm{E}+05$ & $1.05 \mathrm{E}+07$ & $1.07 \mathrm{E}+06$ & $2.89 \mathrm{E}+07$ & $2.64 \mathrm{E}+04$ & $2.89 \mathrm{E}+07$ \\
\hline $\mathrm{Pu}-244$ & $4.56 \mathrm{E}+00$ & $1.23 \mathrm{E}+02$ & $4.52 \mathrm{E}+01$ & $1.22 \mathrm{E}+03$ & $1.40 \mathrm{E}+01$ & $3.79 \mathrm{E}+02$ & $1.67 \mathrm{E}+02$ & $4.50 \mathrm{E}+03$ & $4.58 \mathrm{E}+02$ & $1.24 \mathrm{E}+04$ & $1.13 \mathrm{E}+01$ & $1.24 \mathrm{E}+04$ \\
\hline $\mathrm{Pu}-245$ & $1.33 \mathrm{E}+03$ & $3.60 \mathrm{E}+04$ & $1.32 \mathrm{E}+04$ & $3.57 \mathrm{E}+05$ & $4.10 \mathrm{E}+03$ & $1.11 \mathrm{E}+05$ & $4.87 \mathrm{E}+04$ & $1.32 \mathrm{E}+06$ & $1.34 \mathrm{E}+05$ & $3.62 \mathrm{E}+06$ & $3.30 \mathrm{E}+03$ & $3.62 \mathrm{E}+06$ \\
\hline $\mathrm{Pu}-246$ & $3.27 \mathrm{E}+02$ & $8.85 \mathrm{E}+03$ & $3.25 \mathrm{E}+03$ & $8.78 \mathrm{E}+04$ & $1.01 \mathrm{E}+03$ & $2.73 \mathrm{E}+04$ & $1.20 \mathrm{E}+04$ & $3.23 \mathrm{E}+05$ & $3.29 \mathrm{E}+04$ & $8.89 \mathrm{E}+05$ & $8.12 \mathrm{E}+02$ & $8.89 \mathrm{E}+05$ \\
\hline Am-237 & $5.23 \mathrm{E}+04$ & $1.41 \mathrm{E}+06$ & $5.19 \mathrm{E}+05$ & $1.40 \mathrm{E}+07$ & $1.61 \mathrm{E}+05$ & $4.35 \mathrm{E}+06$ & $1.91 \mathrm{E}+06$ & $5.16 \mathrm{E}+07$ & $5.25 \mathrm{E}+06$ & $1.42 \mathrm{E}+08$ & $1.30 \mathrm{E}+05$ & $1.42 \mathrm{E}+08$ \\
\hline Am-238 & $3.07 \mathrm{E}+04$ & $8.30 \mathrm{E}+05$ & $3.05 \mathrm{E}+05$ & $8.24 \mathrm{E}+06$ & $9.46 \mathrm{E}+04$ & $2.56 \mathrm{E}+06$ & $1.12 \mathrm{E}+06$ & $3.03 \mathrm{E}+07$ & $3.09 \mathrm{E}+06$ & $8.34 \mathrm{E}+07$ & $7.62 \mathrm{E}+04$ & $8.34 \mathrm{E}+07$ \\
\hline Am-239 & $3.75 \mathrm{E}+03$ & $1.01 \mathrm{E}+05$ & $3.72 \mathrm{E}+04$ & $1.01 \mathrm{E}+06$ & $1.15 \mathrm{E}+04$ & $3.12 \mathrm{E}+05$ & $1.37 \mathrm{E}+05$ & $3.70 \mathrm{E}+06$ & $3.77 \mathrm{E}+05$ & $1.02 \mathrm{E}+07$ & $9.30 \mathrm{E}+03$ & $1.02 \mathrm{E}+07$ \\
\hline Am-240 & $1.63 \mathrm{E}+03$ & $4.41 \mathrm{E}+04$ & $1.62 \mathrm{E}+04$ & $4.37 \mathrm{E}+05$ & $5.02 \mathrm{E}+03$ & $1.36 \mathrm{E}+05$ & $5.96 \mathrm{E}+04$ & $1.61 \mathrm{E}+06$ & $1.64 \mathrm{E}+05$ & $4.43 \mathrm{E}+06$ & $4.05 \mathrm{E}+03$ & $4.43 \mathrm{E}+06$ \\
\hline Am-241 & $5.23 \mathrm{E}+00$ & $1.41 \mathrm{E}+02$ & $5.19 \mathrm{E}+01$ & $1.40 \mathrm{E}+03$ & $1.61 \mathrm{E}+01$ & $4.35 \mathrm{E}+02$ & $1.91 \mathrm{E}+02$ & $5.16 \mathrm{E}+03$ & $5.25 \mathrm{E}+02$ & $1.42 \mathrm{E}+04$ & $1.30 \mathrm{E}+01$ & $1.42 \mathrm{E}+04$ \\
\hline Am-242 & $2.95 \mathrm{E}+03$ & $7.97 \mathrm{E}+04$ & $2.93 \mathrm{E}+04$ & $7.91 \mathrm{E}+05$ & $9.08 \mathrm{E}+03$ & $2.45 \mathrm{E}+05$ & $1.08 \mathrm{E}+05$ & $2.91 \mathrm{E}+06$ & $2.96 \mathrm{E}+05$ & $8.01 \mathrm{E}+06$ & $7.31 \mathrm{E}+03$ & $8.01 \mathrm{E}+06$ \\
\hline Am-242m & $5.76 \mathrm{E}+00$ & $1.56 \mathrm{E}+02$ & $5.72 \mathrm{E}+01$ & $1.54 \mathrm{E}+03$ & $1.77 \mathrm{E}+01$ & $4.79 \mathrm{E}+02$ & $2.10 \mathrm{E}+02$ & $5.69 \mathrm{E}+03$ & $5.79 \mathrm{E}+02$ & $1.56 \mathrm{E}+04$ & $1.43 \mathrm{E}+01$ & $1.56 \mathrm{E}+04$ \\
\hline Am-243 & $5.27 \mathrm{E}+00$ & $1.42 \mathrm{E}+02$ & $5.23 \mathrm{E}+01$ & $1.41 \mathrm{E}+03$ & $1.62 \mathrm{E}+01$ & $4.39 \mathrm{E}+02$ & $1.93 \mathrm{E}+02$ & $5.21 \mathrm{E}+03$ & $5.30 \mathrm{E}+02$ & $1.43 \mathrm{E}+04$ & $1.31 \mathrm{E}+01$ & $1.43 \mathrm{E}+04$ \\
\hline Am-244 & $2.03 \mathrm{E}+03$ & $5.47 \mathrm{E}+04$ & $2.01 \mathrm{E}+04$ & $5.43 \mathrm{E}+05$ & $6.24 \mathrm{E}+03$ & $1.69 \mathrm{E}+05$ & $7.40 \mathrm{E}+04$ & $2.00 \mathrm{E}+06$ & $2.04 \mathrm{E}+05$ & $5.50 \mathrm{E}+06$ & $5.03 \mathrm{E}+03$ & $5.50 \mathrm{E}+06$ \\
\hline Am-244m & $3.10 \mathrm{E}+04$ & $8.38 \mathrm{E}+05$ & $3.08 \mathrm{E}+05$ & $8.32 \mathrm{E}+06$ & $9.55 \mathrm{E}+04$ & $2.58 \mathrm{E}+06$ & $1.13 \mathrm{E}+06$ & $3.06 \mathrm{E}+07$ & $3.12 \mathrm{E}+06$ & $8.42 \mathrm{E}+07$ & $7.70 \mathrm{E}+04$ & $8.42 \mathrm{E}+07$ \\
\hline Am-245 & $1.47 \mathrm{E}+04$ & $3.98 \mathrm{E}+05$ & $1.46 \mathrm{E}+05$ & $3.95 \mathrm{E}+06$ & $4.53 \mathrm{E}+04$ & $1.23 \mathrm{E}+06$ & $5.38 \mathrm{E}+05$ & $1.45 \mathrm{E}+07$ & $1.48 \mathrm{E}+06$ & $4.00 \mathrm{E}+07$ & $3.65 \mathrm{E}+04$ & $4.00 \mathrm{E}+07$ \\
\hline Am-246 & $1.46 \mathrm{E}+04$ & $3.95 \mathrm{E}+05$ & $1.45 \mathrm{E}+05$ & $3.92 \mathrm{E}+06$ & $4.50 \mathrm{E}+04$ & $1.22 \mathrm{E}+06$ & $5.34 \mathrm{E}+05$ & $1.44 \mathrm{E}+07$ & $1.47 \mathrm{E}+06$ & $3.97 \mathrm{E}+07$ & $3.63 \mathrm{E}+04$ & $3.97 \mathrm{E}+07$ \\
\hline Am-246m & $2.72 \mathrm{E}+04$ & $7.34 \mathrm{E}+05$ & $2.70 \mathrm{E}+05$ & $7.29 \mathrm{E}+06$ & $8.37 \mathrm{E}+04$ & $2.26 \mathrm{E}+06$ & $9.92 \mathrm{E}+05$ & $2.68 \mathrm{E}+07$ & $2.73 \mathrm{E}+06$ & $7.38 \mathrm{E}+07$ & $6.74 \mathrm{E}+04$ & $7.38 \mathrm{E}+07$ \\
\hline Am-247 & $3.04 \mathrm{E}+04$ & $8.22 \mathrm{E}+05$ & $3.02 \mathrm{E}+05$ & $8.16 \mathrm{E}+06$ & $9.37 \mathrm{E}+04$ & $2.53 \mathrm{E}+06$ & $1.11 \mathrm{E}+06$ & $3.00 \mathrm{E}+07$ & $3.06 \mathrm{E}+06$ & $8.26 \mathrm{E}+07$ & $7.55 \mathrm{E}+04$ & $8.26 \mathrm{E}+07$ \\
\hline $\mathrm{Cm}-238$ & $1.52 \mathrm{E}+04$ & $4.11 \mathrm{E}+05$ & $1.51 \mathrm{E}+05$ & $4.08 \mathrm{E}+06$ & $4.69 \mathrm{E}+04$ & $1.27 \mathrm{E}+06$ & $5.56 \mathrm{E}+05$ & $1.50 \mathrm{E}+07$ & $1.53 \mathrm{E}+06$ & $4.14 \mathrm{E}+07$ & $3.78 \mathrm{E}+04$ & $4.14 \mathrm{E}+07$ \\
\hline Cm-239 & $1.14 \mathrm{E}+04$ & $3.08 \mathrm{E}+05$ & $1.13 \mathrm{E}+05$ & $3.06 \mathrm{E}+06$ & $3.52 \mathrm{E}+04$ & $9.50 \mathrm{E}+05$ & $4.17 \mathrm{E}+05$ & $1.13 \mathrm{E}+07$ & $1.15 \mathrm{E}+06$ & $3.10 \mathrm{E}+07$ & $2.83 \mathrm{E}+04$ & $3.10 \mathrm{E}+07$ \\
\hline $\mathrm{Cm}-240$ & $1.11 \mathrm{E}+02$ & $3.00 \mathrm{E}+03$ & $1.10 \mathrm{E}+03$ & $2.98 \mathrm{E}+04$ & $3.42 \mathrm{E}+02$ & $9.25 \mathrm{E}+03$ & $4.06 \mathrm{E}+03$ & $1.10 \mathrm{E}+05$ & $1.12 \mathrm{E}+04$ & $3.02 \mathrm{E}+05$ & $2.76 \mathrm{E}+02$ & $3.02 \mathrm{E}+05$ \\
\hline $\mathrm{Cm}-241$ & $1.00 \mathrm{E}+03$ & $2.71 \mathrm{E}+04$ & $9.96 \mathrm{E}+03$ & $2.69 \mathrm{E}+05$ & $3.09 \mathrm{E}+03$ & $8.35 \mathrm{E}+04$ & $3.67 \mathrm{E}+04$ & $9.91 \mathrm{E}+05$ & $1.01 \mathrm{E}+05$ & $2.72 \mathrm{E}+06$ & $2.49 \mathrm{E}+03$ & $2.72 \mathrm{E}+06$ \\
\hline
\end{tabular}

Page 204 of 298 
SRNL-STI-2013-00115

\begin{tabular}{|c|c|c|c|c|c|c|c|c|c|c|c|c|}
\hline \multirow[t]{2}{*}{ Nuclide } & \multicolumn{2}{|c|}{ Water Ingestion DCS } & \multicolumn{2}{|c|}{ Meat Ingestion DCS } & \multicolumn{2}{|c|}{ Dairy Ingestion DCS } & \multicolumn{2}{|c|}{$\begin{array}{c}\text { Freshwater Fish } \\
\text { Ingestion DCS }\end{array}$} & \multicolumn{2}{|c|}{$\begin{array}{l}\text { Saltwater Shellfish } \\
\text { Ingestion DCS }\end{array}$} & \multicolumn{2}{|c|}{ Produce Ingestion DCS } \\
\hline & $(\mathrm{Bq} / \mathrm{L})$ & $(\mathrm{pCi} / \mathrm{L})$ & $(\mathrm{Bq} / \mathbf{k g})$ & $(\mathrm{pCi} / \mathrm{kg})$ & $(\mathrm{Bq} / \mathrm{L})$ & $(\mathrm{pCi} / \mathrm{L})$ & $(\mathrm{Bq} / \mathbf{k g})$ & $(\mathrm{pCi} / \mathbf{k g})$ & $(\mathrm{Bq} / \mathbf{k g})$ & $(\mathrm{pCi} / \mathbf{k g})$ & $(\mathrm{Bq} / \mathbf{k g})$ & $(\mathrm{pCi} / \mathbf{k g})$ \\
\hline $\mathrm{Cm}-242$ & $6.48 \mathrm{E}+01$ & $1.75 \mathrm{E}+03$ & $6.43 \mathrm{E}+02$ & $1.74 \mathrm{E}+04$ & $2.00 \mathrm{E}+02$ & $5.39 \mathrm{E}+03$ & $2.37 \mathrm{E}+03$ & $6.40 \mathrm{E}+04$ & $6.51 \mathrm{E}+03$ & $1.76 \mathrm{E}+05$ & $1.61 \mathrm{E}+02$ & $1.76 \mathrm{E}+05$ \\
\hline $\mathrm{Cm}-243$ & $6.91 \mathrm{E}+00$ & $1.87 \mathrm{E}+02$ & $6.86 \mathrm{E}+01$ & $1.85 \mathrm{E}+03$ & $2.13 \mathrm{E}+01$ & $5.75 \mathrm{E}+02$ & $2.53 \mathrm{E}+02$ & $6.83 \mathrm{E}+03$ & $6.94 \mathrm{E}+02$ & $1.88 \mathrm{E}+04$ & $1.71 \mathrm{E}+01$ & $1.88 \mathrm{E}+04$ \\
\hline $\mathrm{Cm}-244$ & $8.24 \mathrm{E}+00$ & $2.23 \mathrm{E}+02$ & $8.18 \mathrm{E}+01$ & $2.21 \mathrm{E}+03$ & $2.54 \mathrm{E}+01$ & $6.86 \mathrm{E}+02$ & $3.01 \mathrm{E}+02$ & $8.14 \mathrm{E}+03$ & $8.28 \mathrm{E}+02$ & $2.24 \mathrm{E}+04$ & $2.04 \mathrm{E}+01$ & $2.24 \mathrm{E}+04$ \\
\hline $\mathrm{Cm}-245$ & $5.14 \mathrm{E}+00$ & $1.39 \mathrm{E}+02$ & $5.10 \mathrm{E}+01$ & $1.38 \mathrm{E}+03$ & $1.58 \mathrm{E}+01$ & $4.28 \mathrm{E}+02$ & $1.88 \mathrm{E}+02$ & $5.08 \mathrm{E}+03$ & $5.17 \mathrm{E}+02$ & $1.40 \mathrm{E}+04$ & $1.28 \mathrm{E}+01$ & $1.40 \mathrm{E}+04$ \\
\hline $\mathrm{Cm}-246$ & $5.16 \mathrm{E}+00$ & $1.39 \mathrm{E}+02$ & $5.12 \mathrm{E}+01$ & $1.38 \mathrm{E}+03$ & $1.59 \mathrm{E}+01$ & $4.30 \mathrm{E}+02$ & $1.89 \mathrm{E}+02$ & $5.10 \mathrm{E}+03$ & $5.19 \mathrm{E}+02$ & $1.40 \mathrm{E}+04$ & $1.28 \mathrm{E}+01$ & $1.40 \mathrm{E}+04$ \\
\hline $\mathrm{Cm}-247$ & $5.60 \mathrm{E}+00$ & $1.51 \mathrm{E}+02$ & $5.56 \mathrm{E}+01$ & $1.50 \mathrm{E}+03$ & $1.73 \mathrm{E}+01$ & $4.66 \mathrm{E}+02$ & $2.05 \mathrm{E}+02$ & $5.53 \mathrm{E}+03$ & $5.63 \mathrm{E}+02$ & $1.52 \mathrm{E}+04$ & $1.39 \mathrm{E}+01$ & $1.52 \mathrm{E}+04$ \\
\hline $\mathrm{Cm}-248$ & $1.38 \mathrm{E}+00$ & $3.72 \mathrm{E}+01$ & $1.37 \mathrm{E}+01$ & $3.70 \mathrm{E}+02$ & $4.24 \mathrm{E}+00$ & $1.15 \mathrm{E}+02$ & $5.03 \mathrm{E}+01$ & $1.36 \mathrm{E}+03$ & $1.38 \mathrm{E}+02$ & $3.74 \mathrm{E}+03$ & $3.42 \mathrm{E}+00$ & $3.74 \mathrm{E}+03$ \\
\hline $\mathrm{Cm}-249$ & $2.94 \mathrm{E}+04$ & $7.95 \mathrm{E}+05$ & $2.92 \mathrm{E}+05$ & $7.89 \mathrm{E}+06$ & $9.06 \mathrm{E}+04$ & $2.45 \mathrm{E}+06$ & $1.07 \mathrm{E}+06$ & $2.90 \mathrm{E}+07$ & $2.96 \mathrm{E}+06$ & $7.99 \mathrm{E}+07$ & $7.30 \mathrm{E}+04$ & $7.99 \mathrm{E}+07$ \\
\hline $\mathrm{Cm}-250$ & $2.01 \mathrm{E}-01$ & $5.44 \mathrm{E}+00$ & $2.00 \mathrm{E}+00$ & $5.40 \mathrm{E}+01$ & $6.20 \mathrm{E}-01$ & $1.68 \mathrm{E}+01$ & $7.36 \mathrm{E}+00$ & $1.99 \mathrm{E}+02$ & $2.02 \mathrm{E}+01$ & $5.47 \mathrm{E}+02$ & $4.99 \mathrm{E}-01$ & $5.47 \mathrm{E}+02$ \\
\hline $\mathrm{Cm}-251$ & $3.21 \mathrm{E}+04$ & $8.69 \mathrm{E}+05$ & $3.19 \mathrm{E}+05$ & $8.62 \mathrm{E}+06$ & $9.90 \mathrm{E}+04$ & $2.68 \mathrm{E}+06$ & $1.17 \mathrm{E}+06$ & $3.17 \mathrm{E}+07$ & $3.23 \mathrm{E}+06$ & $8.73 \mathrm{E}+07$ & $7.98 \mathrm{E}+04$ & $8.73 \mathrm{E}+07$ \\
\hline Bk-245 & $1.59 \mathrm{E}+03$ & $4.29 \mathrm{E}+04$ & $1.58 \mathrm{E}+04$ & $4.26 \mathrm{E}+05$ & $4.89 \mathrm{E}+03$ & $1.32 \mathrm{E}+05$ & $5.81 \mathrm{E}+04$ & $1.57 \mathrm{E}+06$ & $1.60 \mathrm{E}+05$ & $4.31 \mathrm{E}+06$ & $3.94 \mathrm{E}+03$ & $4.31 \mathrm{E}+06$ \\
\hline Bk-246 & $2.14 \mathrm{E}+03$ & $5.78 \mathrm{E}+04$ & $2.12 \mathrm{E}+04$ & $5.73 \mathrm{E}+05$ & $6.58 \mathrm{E}+03$ & $1.78 \mathrm{E}+05$ & $7.81 \mathrm{E}+04$ & $2.11 \mathrm{E}+06$ & $2.15 \mathrm{E}+05$ & $5.80 \mathrm{E}+06$ & $5.30 \mathrm{E}+03$ & $5.80 \mathrm{E}+06$ \\
\hline Bk-247 & $2.81 \mathrm{E}+00$ & $7.59 \mathrm{E}+01$ & $2.79 \mathrm{E}+01$ & $7.53 \mathrm{E}+02$ & $8.65 \mathrm{E}+00$ & $2.34 \mathrm{E}+02$ & $1.03 \mathrm{E}+02$ & $2.77 \mathrm{E}+03$ & $2.82 \mathrm{E}+02$ & $7.63 \mathrm{E}+03$ & $6.97 \mathrm{E}+00$ & $7.63 \mathrm{E}+03$ \\
\hline Bk-248m & $2.06 \mathrm{E}+03$ & $5.56 \mathrm{E}+04$ & $2.04 \mathrm{E}+04$ & $5.52 \mathrm{E}+05$ & $6.33 \mathrm{E}+03$ & $1.71 \mathrm{E}+05$ & $7.51 \mathrm{E}+04$ & $2.03 \mathrm{E}+06$ & $2.07 \mathrm{E}+05$ & $5.58 \mathrm{E}+06$ & $5.10 \mathrm{E}+03$ & $5.58 \mathrm{E}+06$ \\
\hline Bk-249 & $9.95 \mathrm{E}+02$ & $2.69 \mathrm{E}+04$ & $9.88 \mathrm{E}+03$ & $2.67 \mathrm{E}+05$ & $3.07 \mathrm{E}+03$ & $8.28 \mathrm{E}+04$ & $3.64 \mathrm{E}+04$ & $9.83 \mathrm{E}+05$ & $1.00 \mathrm{E}+05$ & $2.70 \mathrm{E}+06$ & $2.47 \mathrm{E}+03$ & $2.70 \mathrm{E}+06$ \\
\hline Bk-250 & $6.80 \mathrm{E}+03$ & $1.84 \mathrm{E}+05$ & $6.75 \mathrm{E}+04$ & $1.82 \mathrm{E}+06$ & $2.09 \mathrm{E}+04$ & $5.66 \mathrm{E}+05$ & $2.48 \mathrm{E}+05$ & $6.71 \mathrm{E}+06$ & $6.83 \mathrm{E}+05$ & $1.85 \mathrm{E}+07$ & $1.69 \mathrm{E}+04$ & $1.85 \mathrm{E}+07$ \\
\hline Bk-251 & $2.43 \mathrm{E}+04$ & $6.58 \mathrm{E}+05$ & $2.42 \mathrm{E}+05$ & $6.53 \mathrm{E}+06$ & $7.50 \mathrm{E}+04$ & $2.03 \mathrm{E}+06$ & $8.90 \mathrm{E}+05$ & $2.40 \mathrm{E}+07$ & $2.45 \mathrm{E}+06$ & $6.61 \mathrm{E}+07$ & $6.04 \mathrm{E}+04$ & $6.61 \mathrm{E}+07$ \\
\hline Cf-244 & $1.30 \mathrm{E}+04$ & $3.52 \mathrm{E}+05$ & $1.29 \mathrm{E}+05$ & $3.49 \mathrm{E}+06$ & $4.01 \mathrm{E}+04$ & $1.08 \mathrm{E}+06$ & $4.76 \mathrm{E}+05$ & $1.29 \mathrm{E}+07$ & $1.31 \mathrm{E}+06$ & $3.54 \mathrm{E}+07$ & $3.23 \mathrm{E}+04$ & $3.54 \mathrm{E}+07$ \\
\hline Cf-246 & $2.69 \mathrm{E}+02$ & $7.26 \mathrm{E}+03$ & $2.67 \mathrm{E}+03$ & $7.21 \mathrm{E}+04$ & $8.28 \mathrm{E}+02$ & $2.24 \mathrm{E}+04$ & $9.82 \mathrm{E}+03$ & $2.65 \mathrm{E}+05$ & $2.70 \mathrm{E}+04$ & $7.30 \mathrm{E}+05$ & $6.67 \mathrm{E}+02$ & $7.30 \mathrm{E}+05$ \\
\hline Cf-247 & $4.29 \mathrm{E}+04$ & $1.16 \mathrm{E}+06$ & $4.26 \mathrm{E}+05$ & $1.15 \mathrm{E}+07$ & $1.32 \mathrm{E}+05$ & $3.57 \mathrm{E}+06$ & $1.57 \mathrm{E}+06$ & $4.24 \mathrm{E}+07$ & $4.31 \mathrm{E}+06$ & $1.16 \mathrm{E}+08$ & $1.06 \mathrm{E}+05$ & $1.16 \mathrm{E}+08$ \\
\hline Cf-248 & $2.66 \mathrm{E}+01$ & $7.18 \mathrm{E}+02$ & $2.64 \mathrm{E}+02$ & $7.13 \mathrm{E}+03$ & $8.19 \mathrm{E}+01$ & $2.21 \mathrm{E}+03$ & $9.71 \mathrm{E}+02$ & $2.63 \mathrm{E}+04$ & $2.67 \mathrm{E}+03$ & $7.22 \mathrm{E}+04$ & $6.59 \mathrm{E}+01$ & $7.22 \mathrm{E}+04$ \\
\hline Cf-249 & $2.79 \mathrm{E}+00$ & $7.54 \mathrm{E}+01$ & $2.77 \mathrm{E}+01$ & $7.48 \mathrm{E}+02$ & $8.59 \mathrm{E}+00$ & $2.32 \mathrm{E}+02$ & $1.02 \mathrm{E}+02$ & $2.75 \mathrm{E}+03$ & $2.80 \mathrm{E}+02$ & $7.57 \mathrm{E}+03$ & $6.92 \mathrm{E}+00$ & $7.57 \mathrm{E}+03$ \\
\hline Cf- 250 & $5.60 \mathrm{E}+00$ & $1.51 \mathrm{E}+02$ & $5.56 \mathrm{E}+01$ & $1.50 \mathrm{E}+03$ & $1.73 \mathrm{E}+01$ & $4.66 \mathrm{E}+02$ & $2.05 \mathrm{E}+02$ & $5.53 \mathrm{E}+03$ & $5.63 \mathrm{E}+02$ & $1.52 \mathrm{E}+04$ & $1.39 \mathrm{E}+01$ & $1.52 \mathrm{E}+04$ \\
\hline
\end{tabular}

Page 205 of 298 
SRNL-STI-2013-00115

\begin{tabular}{|c|c|c|c|c|c|c|c|c|c|c|c|c|}
\hline \multirow[t]{2}{*}{ Nuclide } & \multicolumn{2}{|c|}{ Water Ingestion DCS } & \multicolumn{2}{|c|}{ Meat Ingestion DCS } & \multicolumn{2}{|c|}{ Dairy Ingestion DCS } & \multicolumn{2}{|c|}{$\begin{array}{c}\text { Freshwater Fish } \\
\text { Ingestion DCS }\end{array}$} & \multicolumn{2}{|c|}{$\begin{array}{l}\text { Saltwater Shellfish } \\
\text { Ingestion DCS }\end{array}$} & \multicolumn{2}{|c|}{ Produce Ingestion DCS } \\
\hline & $(\mathrm{Bq} / \mathrm{L})$ & $(\mathrm{pCi} / \mathrm{L})$ & $(\mathrm{Bq} / \mathrm{kg})$ & $(\mathrm{pCi} / \mathbf{k g})$ & $(\mathrm{Bq} / \mathrm{L})$ & $(\mathrm{pCi} / \mathrm{L})$ & $(\mathrm{Bq} / \mathrm{kg})$ & $(\mathrm{pCi} / \mathbf{k g})$ & $(\mathrm{Bq} / \mathrm{kg})$ & $(\mathrm{pCi} / \mathbf{k g})$ & $(\mathrm{Bq} / \mathrm{kg})$ & $(\mathrm{pCi} / \mathbf{k g})$ \\
\hline Cf-251 & $2.73 \mathrm{E}+00$ & $7.39 \mathrm{E}+01$ & $2.71 \mathrm{E}+01$ & $7.33 \mathrm{E}+02$ & $8.42 \mathrm{E}+00$ & $2.28 \mathrm{E}+02$ & $9.99 \mathrm{E}+01$ & $2.70 \mathrm{E}+03$ & $2.75 \mathrm{E}+02$ & $7.43 \mathrm{E}+03$ & $6.78 \mathrm{E}+00$ & $7.43 \mathrm{E}+03$ \\
\hline Cf-252 & $8.24 \mathrm{E}+00$ & $2.23 \mathrm{E}+02$ & $8.18 \mathrm{E}+01$ & $2.21 \mathrm{E}+03$ & $2.54 \mathrm{E}+01$ & $6.86 \mathrm{E}+02$ & $3.01 \mathrm{E}+02$ & $8.14 \mathrm{E}+03$ & $8.28 \mathrm{E}+02$ & $2.24 \mathrm{E}+04$ & $2.04 \mathrm{E}+01$ & $2.24 \mathrm{E}+04$ \\
\hline Cf-253 & $4.51 \mathrm{E}+02$ & $1.22 \mathrm{E}+04$ & $4.47 \mathrm{E}+03$ & $1.21 \mathrm{E}+05$ & $1.39 \mathrm{E}+03$ & $3.75 \mathrm{E}+04$ & $1.65 \mathrm{E}+04$ & $4.45 \mathrm{E}+05$ & $4.53 \mathrm{E}+04$ & $1.22 \mathrm{E}+06$ & $1.12 \mathrm{E}+03$ & $1.22 \mathrm{E}+06$ \\
\hline Cf- 254 & $2.07 \mathrm{E}+00$ & $5.58 \mathrm{E}+01$ & $2.05 \mathrm{E}+01$ & $5.54 \mathrm{E}+02$ & $6.36 \mathrm{E}+00$ & $1.72 \mathrm{E}+02$ & $7.55 \mathrm{E}+01$ & $2.04 \mathrm{E}+03$ & $2.08 \mathrm{E}+02$ & $5.61 \mathrm{E}+03$ & $5.13 \mathrm{E}+00$ & $5.61 \mathrm{E}+03$ \\
\hline Cf-255 & $2.28 \mathrm{E}+04$ & $6.16 \mathrm{E}+05$ & $2.26 \mathrm{E}+05$ & $6.11 \mathrm{E}+06$ & $7.02 \mathrm{E}+04$ & $1.90 \mathrm{E}+06$ & $8.33 \mathrm{E}+05$ & $2.25 \mathrm{E}+07$ & $2.29 \mathrm{E}+06$ & $6.19 \mathrm{E}+07$ & $5.65 \mathrm{E}+04$ & $6.19 \mathrm{E}+07$ \\
\hline Es-249 & $4.56 \mathrm{E}+04$ & $1.23 \mathrm{E}+06$ & $4.52 \mathrm{E}+05$ & $1.22 \mathrm{E}+07$ & $1.40 \mathrm{E}+05$ & $3.79 \mathrm{E}+06$ & $1.67 \mathrm{E}+06$ & $4.50 \mathrm{E}+07$ & $4.58 \mathrm{E}+06$ & $1.24 \mathrm{E}+08$ & $1.13 \mathrm{E}+05$ & $1.24 \mathrm{E}+08$ \\
\hline Es-250 & $2.01 \mathrm{E}+03$ & $5.42 \mathrm{E}+04$ & $1.99 \mathrm{E}+04$ & $5.38 \mathrm{E}+05$ & $6.18 \mathrm{E}+03$ & $1.67 \mathrm{E}+05$ & $7.33 \mathrm{E}+04$ & $1.98 \mathrm{E}+06$ & $2.02 \mathrm{E}+05$ & $5.45 \mathrm{E}+06$ & $4.98 \mathrm{E}+03$ & $5.45 \mathrm{E}+06$ \\
\hline Es- $250 \mathrm{~m}$ & $3.16 \mathrm{E}+04$ & $8.55 \mathrm{E}+05$ & $3.14 \mathrm{E}+05$ & $8.49 \mathrm{E}+06$ & $9.75 \mathrm{E}+04$ & $2.63 \mathrm{E}+06$ & $1.16 \mathrm{E}+06$ & $3.13 \mathrm{E}+07$ & $3.18 \mathrm{E}+06$ & $8.60 \mathrm{E}+07$ & $7.85 \mathrm{E}+04$ & $8.60 \mathrm{E}+07$ \\
\hline Es-251 & $5.08 \mathrm{E}+03$ & $1.37 \mathrm{E}+05$ & $5.04 \mathrm{E}+04$ & $1.36 \mathrm{E}+06$ & $1.56 \mathrm{E}+04$ & $4.23 \mathrm{E}+05$ & $1.86 \mathrm{E}+05$ & $5.01 \mathrm{E}+06$ & $5.10 \mathrm{E}+05$ & $1.38 \mathrm{E}+07$ & $1.26 \mathrm{E}+04$ & $1.38 \mathrm{E}+07$ \\
\hline Es-253 & $1.35 \mathrm{E}+02$ & $3.65 \mathrm{E}+03$ & $1.34 \mathrm{E}+03$ & $3.62 \mathrm{E}+04$ & $4.16 \mathrm{E}+02$ & $1.12 \mathrm{E}+04$ & $4.94 \mathrm{E}+03$ & $1.33 \mathrm{E}+05$ & $1.36 \mathrm{E}+04$ & $3.67 \mathrm{E}+05$ & $3.35 \mathrm{E}+02$ & $3.67 \mathrm{E}+05$ \\
\hline Es-254 & $2.71 \mathrm{E}+01$ & $7.32 \mathrm{E}+02$ & $2.69 \mathrm{E}+02$ & $7.27 \mathrm{E}+03$ & $8.35 \mathrm{E}+01$ & $2.26 \mathrm{E}+03$ & $9.90 \mathrm{E}+02$ & $2.68 \mathrm{E}+04$ & $2.72 \mathrm{E}+03$ & $7.36 \mathrm{E}+04$ & $6.72 \mathrm{E}+01$ & $7.36 \mathrm{E}+04$ \\
\hline Es- $254 \mathrm{~m}$ & $2.08 \mathrm{E}+02$ & $5.61 \mathrm{E}+03$ & $2.06 \mathrm{E}+03$ & $5.57 \mathrm{E}+04$ & $6.40 \mathrm{E}+02$ & $1.73 \mathrm{E}+04$ & $7.59 \mathrm{E}+03$ & $2.05 \mathrm{E}+05$ & $2.09 \mathrm{E}+04$ & $5.64 \mathrm{E}+05$ & $5.15 \mathrm{E}+02$ & $5.64 \mathrm{E}+05$ \\
\hline Es-255 & $1.26 \mathrm{E}+02$ & $3.39 \mathrm{E}+03$ & $1.25 \mathrm{E}+03$ & $3.37 \mathrm{E}+04$ & $3.87 \mathrm{E}+02$ & $1.04 \mathrm{E}+04$ & $4.59 \mathrm{E}+03$ & $1.24 \mathrm{E}+05$ & $1.26 \mathrm{E}+04$ & $3.41 \mathrm{E}+05$ & $3.11 \mathrm{E}+02$ & $3.41 \mathrm{E}+05$ \\
\hline Es-256 & $3.21 \mathrm{E}+02$ & $8.69 \mathrm{E}+03$ & $3.19 \mathrm{E}+03$ & $8.62 \mathrm{E}+04$ & $9.90 \mathrm{E}+02$ & $2.68 \mathrm{E}+04$ & $1.17 \mathrm{E}+04$ & $3.17 \mathrm{E}+05$ & $3.23 \mathrm{E}+04$ & $8.73 \mathrm{E}+05$ & $7.98 \mathrm{E}+02$ & $8.73 \mathrm{E}+05$ \\
\hline Fm-251 & $1.27 \mathrm{E}+04$ & $3.42 \mathrm{E}+05$ & $1.26 \mathrm{E}+05$ & $3.39 \mathrm{E}+06$ & $3.90 \mathrm{E}+04$ & $1.05 \mathrm{E}+06$ & $4.62 \mathrm{E}+05$ & $1.25 \mathrm{E}+07$ & $1.27 \mathrm{E}+06$ & $3.44 \mathrm{E}+07$ & $3.14 \mathrm{E}+04$ & $3.44 \mathrm{E}+07$ \\
\hline Fm-252 & $3.10 \mathrm{E}+02$ & $8.38 \mathrm{E}+03$ & $3.08 \mathrm{E}+03$ & $8.32 \mathrm{E}+04$ & $9.55 \mathrm{E}+02$ & $2.58 \mathrm{E}+04$ & $1.13 \mathrm{E}+04$ & $3.06 \mathrm{E}+05$ & $3.12 \mathrm{E}+04$ & $8.42 \mathrm{E}+05$ & $7.70 \mathrm{E}+02$ & $8.42 \mathrm{E}+05$ \\
\hline Fm-253 & $7.15 \mathrm{E}+02$ & $1.93 \mathrm{E}+04$ & $7.10 \mathrm{E}+03$ & $1.92 \mathrm{E}+05$ & $2.20 \mathrm{E}+03$ & $5.95 \mathrm{E}+04$ & $2.61 \mathrm{E}+04$ & $7.06 \mathrm{E}+05$ & $7.18 \mathrm{E}+04$ & $1.94 \mathrm{E}+06$ & $1.77 \mathrm{E}+03$ & $1.94 \mathrm{E}+06$ \\
\hline $\mathrm{Fm}-254$ & $1.98 \mathrm{E}+03$ & $5.34 \mathrm{E}+04$ & $1.96 \mathrm{E}+04$ & $5.30 \mathrm{E}+05$ & $6.09 \mathrm{E}+03$ & $1.65 \mathrm{E}+05$ & $7.23 \mathrm{E}+04$ & $1.95 \mathrm{E}+06$ & $1.99 \mathrm{E}+05$ & $5.37 \mathrm{E}+06$ & $4.91 \mathrm{E}+03$ & $5.37 \mathrm{E}+06$ \\
\hline Fm-255 & $3.50 \mathrm{E}+02$ & $9.47 \mathrm{E}+03$ & $3.48 \mathrm{E}+03$ & $9.40 \mathrm{E}+04$ & $1.08 \mathrm{E}+03$ & $2.92 \mathrm{E}+04$ & $1.28 \mathrm{E}+04$ & $3.46 \mathrm{E}+05$ & $3.52 \mathrm{E}+04$ & $9.52 \mathrm{E}+05$ & $8.69 \mathrm{E}+02$ & $9.52 \mathrm{E}+05$ \\
\hline Fm-256 & $4.98 \mathrm{E}+01$ & $1.34 \mathrm{E}+03$ & $4.94 \mathrm{E}+02$ & $1.33 \mathrm{E}+04$ & $1.53 \mathrm{E}+02$ & $4.14 \mathrm{E}+03$ & $1.82 \mathrm{E}+03$ & $4.91 \mathrm{E}+04$ & $5.00 \mathrm{E}+03$ & $1.35 \mathrm{E}+05$ & $1.23 \mathrm{E}+02$ & $1.35 \mathrm{E}+05$ \\
\hline Fm-257 & $4.23 \mathrm{E}+01$ & $1.14 \mathrm{E}+03$ & $4.20 \mathrm{E}+02$ & $1.13 \mathrm{E}+04$ & $1.30 \mathrm{E}+02$ & $3.52 \mathrm{E}+03$ & $1.55 \mathrm{E}+03$ & $4.18 \mathrm{E}+04$ & $4.25 \mathrm{E}+03$ & $1.15 \mathrm{E}+05$ & $1.05 \mathrm{E}+02$ & $1.15 \mathrm{E}+05$ \\
\hline
\end{tabular}

Page 206 of 298 
Table D-2. Inhalation DCS

\begin{tabular}{|c|c|c|}
\hline Nuclide & $\begin{array}{c}\text { Inhalation } \\
\text { DCS } \\
\left(\mathbf{B q} / \mathbf{m}^{3}\right) \\
\end{array}$ & $\begin{array}{c}\text { Inhalation } \\
\text { DCS } \\
\left(\mathbf{p C i} / \mathbf{m}^{3}\right) \\
\end{array}$ \\
\hline $\mathrm{H}-3$ & $8.10 \mathrm{E}+03$ & $2.19 \mathrm{E}+05$ \\
\hline $\mathrm{Be}-7$ & $2.44 \mathrm{E}+03$ & $6.60 \mathrm{E}+04$ \\
\hline Be-10 & $4.27 \mathrm{E}+00$ & $1.15 \mathrm{E}+02$ \\
\hline $\mathrm{C}-11$ & $6.10 \mathrm{E}+04$ & $1.65 \mathrm{E}+06$ \\
\hline C-14 & $2.33 \mathrm{E}+04$ & $6.30 \mathrm{E}+05$ \\
\hline F-18 & $2.35 \mathrm{E}+03$ & $6.36 \mathrm{E}+04$ \\
\hline $\mathrm{Na}-22$ & $4.96 \mathrm{E}+00$ & $1.34 \mathrm{E}+02$ \\
\hline $\mathrm{Na}-24$ & $2.68 \mathrm{E}+02$ & $7.24 \mathrm{E}+03$ \\
\hline $\mathrm{Mg}-28$ & $1.04 \mathrm{E}+02$ & $2.82 \mathrm{E}+03$ \\
\hline $\mathrm{Al}-26$ & $1.37 \mathrm{E}+00$ & $3.70 \mathrm{E}+01$ \\
\hline Si-31 & $1.68 \mathrm{E}+03$ & $4.54 \mathrm{E}+04$ \\
\hline Si-32 & $1.35 \mathrm{E}+00$ & $3.64 \mathrm{E}+01$ \\
\hline $\mathrm{P}-32$ & $3.57 \mathrm{E}+01$ & $9.64 \mathrm{E}+02$ \\
\hline P-33 & $7.97 \mathrm{E}+01$ & $2.15 \mathrm{E}+03$ \\
\hline S-35 & $1.00 \mathrm{E}+02$ & $2.71 \mathrm{E}+03$ \\
\hline S-38 & $4.69 \mathrm{E}+02$ & $1.27 \mathrm{E}+04$ \\
\hline $\mathrm{Cl}-34 \mathrm{~m}$ & $2.84 \mathrm{E}+03$ & $7.68 \mathrm{E}+04$ \\
\hline Cl-36 & $3.92 \mathrm{E}+00$ & $1.06 \mathrm{E}+02$ \\
\hline Cl-38 & $2.74 \mathrm{E}+03$ & $7.41 \mathrm{E}+04$ \\
\hline Cl-39 & $2.70 \mathrm{E}+03$ & $7.29 \mathrm{E}+04$ \\
\hline K-40 & $1.76 \mathrm{E}+00$ & $4.76 \mathrm{E}+01$ \\
\hline K-42 & $3.83 \mathrm{E}+02$ & $1.04 \mathrm{E}+04$ \\
\hline K-43 & $3.62 \mathrm{E}+02$ & $9.78 \mathrm{E}+03$ \\
\hline K-44 & $3.92 \mathrm{E}+03$ & $1.06 \mathrm{E}+05$ \\
\hline K-45 & $5.94 \mathrm{E}+03$ & $1.61 \mathrm{E}+05$ \\
\hline $\mathrm{Ca}-41$ & $1.23 \mathrm{E}+03$ & $3.33 \mathrm{E}+04$ \\
\hline $\mathrm{Ca}-45$ & $5.23 \mathrm{E}+01$ & $1.41 \mathrm{E}+03$ \\
\hline $\mathrm{Ca}-47$ & $7.30 \mathrm{E}+01$ & $1.97 \mathrm{E}+03$ \\
\hline Sc-43 & $1.05 \mathrm{E}+03$ & $2.83 \mathrm{E}+04$ \\
\hline Sc-44 & $7.01 \mathrm{E}+02$ & $1.89 \mathrm{E}+04$ \\
\hline Sc-44m & $9.25 \mathrm{E}+01$ & $2.50 \mathrm{E}+03$ \\
\hline Sc-46 & $2.06 \mathrm{E}+01$ & $5.56 \mathrm{E}+02$ \\
\hline Sc-47 & $1.92 \mathrm{E}+02$ & $5.20 \mathrm{E}+03$ \\
\hline Sc-48 & $1.20 \mathrm{E}+02$ & $3.25 \mathrm{E}+03$ \\
\hline
\end{tabular}




\begin{tabular}{|c|c|c|}
\hline Nuclide & $\begin{array}{c}\text { Inhalation } \\
\text { DCS } \\
\left(\mathbf{B q} / \mathbf{m}^{3}\right)\end{array}$ & $\begin{array}{c}\text { Inhalation } \\
\text { DCS } \\
\left(\mathbf{p C i} / \mathbf{m}^{3}\right)\end{array}$ \\
\hline Sc-49 & $3.35 \mathrm{E}+03$ & $9.04 \mathrm{E}+04$ \\
\hline Ti-44 & $1.17 \mathrm{E}+00$ & $3.18 \mathrm{E}+01$ \\
\hline $\mathrm{Ti}-45$ & $1.41 \mathrm{E}+03$ & $3.80 \mathrm{E}+04$ \\
\hline $\mathrm{V}-47$ & $4.27 \mathrm{E}+03$ & $1.15 \mathrm{E}+05$ \\
\hline $\mathrm{V}-48$ & $5.06 \mathrm{E}+01$ & $1.37 \mathrm{E}+03$ \\
\hline$V-49$ & $1.96 \mathrm{E}+03$ & $5.30 \mathrm{E}+04$ \\
\hline $\mathrm{V}-50$ & $2.34 \mathrm{E}+00$ & $6.31 \mathrm{E}+01$ \\
\hline $\mathrm{Cr}-48$ & $6.43 \mathrm{E}+02$ & $1.74 \mathrm{E}+04$ \\
\hline $\mathrm{Cr}-49$ & $3.74 \mathrm{E}+03$ & $1.01 \mathrm{E}+05$ \\
\hline $\mathrm{Cr}-51$ & $3.60 \mathrm{E}+03$ & $9.73 E+04$ \\
\hline Mn-51 & $3.02 \mathrm{E}+03$ & $8.17 \mathrm{E}+04$ \\
\hline Mn-52 & $9.41 \mathrm{E}+01$ & $2.54 \mathrm{E}+03$ \\
\hline $\mathrm{Mn}-52 \mathrm{~m}$ & $4.40 \mathrm{E}+03$ & $1.19 \mathrm{E}+05$ \\
\hline $\mathrm{Mn}-53$ & $4.28 \mathrm{E}+02$ & $1.16 \mathrm{E}+04$ \\
\hline $\mathrm{Mn}-54$ & $4.34 \mathrm{E}+01$ & $1.17 \mathrm{E}+03$ \\
\hline Mn-56 & $1.01 \mathrm{E}+03$ & $2.74 \mathrm{E}+04$ \\
\hline $\mathrm{Fe}-52$ & $2.13 \mathrm{E}+02$ & $5.76 \mathrm{E}+03$ \\
\hline $\mathrm{Fe}-55$ & $3.58 \mathrm{E}+02$ & $9.66 \mathrm{E}+03$ \\
\hline $\mathrm{Fe}-59$ & $3.79 \mathrm{E}+01$ & $1.02 \mathrm{E}+03$ \\
\hline Fe-60 & $1.08 \mathrm{E}+00$ & $2.91 \mathrm{E}+01$ \\
\hline $\mathrm{Co}-55$ & $2.61 \mathrm{E}+02$ & $7.05 \mathrm{E}+03$ \\
\hline Co-56 & $2.89 \mathrm{E}+01$ & $7.82 \mathrm{E}+02$ \\
\hline Co-57 & $2.53 \mathrm{E}+02$ & $6.83 E+03$ \\
\hline Co-58 & $8.78 \mathrm{E}+01$ & $2.37 \mathrm{E}+03$ \\
\hline $\mathrm{Co}-58 \mathrm{~m}$ & $1.00 \mathrm{E}+04$ & $2.71 \mathrm{E}+05$ \\
\hline Co-60 & $1.40 \mathrm{E}+01$ & $3.77 \mathrm{E}+02$ \\
\hline Co-60m & $1.15 \mathrm{E}+05$ & $3.11 \mathrm{E}+06$ \\
\hline Co-61 & $2.80 \mathrm{E}+03$ & $7.57 \mathrm{E}+04$ \\
\hline $\mathrm{Co}-62 \mathrm{~m}$ & $6.40 \mathrm{E}+03$ & $1.73 \mathrm{E}+05$ \\
\hline $\mathrm{Ni}-56$ & $1.55 \mathrm{E}+02$ & $4.18 \mathrm{E}+03$ \\
\hline $\mathrm{Ni}-57$ & $2.55 \mathrm{E}+02$ & $6.89 \mathrm{E}+03$ \\
\hline Ni-59 & $1.06 \mathrm{E}+03$ & $2.85 \mathrm{E}+04$ \\
\hline $\mathrm{Ni}-63$ & $2.88 \mathrm{E}+02$ & $7.78 \mathrm{E}+03$ \\
\hline $\mathrm{Ni}-65$ & $1.55 \mathrm{E}+03$ & $4.18 \mathrm{E}+04$ \\
\hline $\mathrm{Ni}-66$ & $8.05 \mathrm{E}+01$ & $2.18 \mathrm{E}+03$ \\
\hline $\mathrm{Cu}-60$ & $3.70 \mathrm{E}+03$ & $1.00 \mathrm{E}+05$ \\
\hline $\mathrm{Cu}-61$ & $1.73 \mathrm{E}+03$ & $4.68 \mathrm{E}+04$ \\
\hline $\mathrm{Cu}-64$ & $1.15 \mathrm{E}+03$ & $3.11 \mathrm{E}+04$ \\
\hline
\end{tabular}




\begin{tabular}{|c|c|c|}
\hline Nuclide & $\begin{array}{c}\text { Inhalation } \\
\text { DCS } \\
\left(\mathbf{B q} / \mathbf{m}^{3}\right)\end{array}$ & $\begin{array}{c}\text { Inhalation } \\
\text { DCS } \\
\left(\mathbf{p C i} / \mathbf{m}^{3}\right)\end{array}$ \\
\hline $\mathrm{Cu}-67$ & $2.36 \mathrm{E}+02$ & $6.39 \mathrm{E}+03$ \\
\hline $\mathrm{Zn}-62$ & $2.57 \mathrm{E}+02$ & $6.95 \mathrm{E}+03$ \\
\hline $\mathrm{Zn}-63$ & $3.69 \mathrm{E}+03$ & $9.98 \mathrm{E}+04$ \\
\hline $\mathrm{Zn}-65$ & $8.68 \mathrm{E}+01$ & $2.35 \mathrm{E}+03$ \\
\hline Zn-69 & $5.16 \mathrm{E}+03$ & $1.39 \mathrm{E}+05$ \\
\hline $\mathrm{Zn}-69 \mathrm{~m}$ & $5.33 \mathrm{E}+02$ & $1.44 \mathrm{E}+04$ \\
\hline $\mathrm{Zn}-71 \mathrm{~m}$ & $8.49 \mathrm{E}+02$ & $2.30 \mathrm{E}+04$ \\
\hline $\mathrm{Zn}-72$ & $1.10 \mathrm{E}+02$ & $2.97 \mathrm{E}+03$ \\
\hline Ga-65 & $7.58 \mathrm{E}+03$ & $2.05 \mathrm{E}+05$ \\
\hline Ga-66 & $2.74 \mathrm{E}+02$ & $7.41 \mathrm{E}+03$ \\
\hline Ga-67 & $5.19 \mathrm{E}+02$ & $1.40 \mathrm{E}+04$ \\
\hline Ga-68 & $2.54 \mathrm{E}+03$ & $6.88 \mathrm{E}+04$ \\
\hline Ga-70 & $8.01 \mathrm{E}+03$ & $2.17 \mathrm{E}+05$ \\
\hline Ga-72 & $2.33 \mathrm{E}+02$ & $6.28 \mathrm{E}+03$ \\
\hline Ga-73 & $8.54 \mathrm{E}+02$ & $2.31 \mathrm{E}+04$ \\
\hline Ge-66 & $1.42 \mathrm{E}+03$ & $3.84 \mathrm{E}+04$ \\
\hline Ge-67 & $5.26 \mathrm{E}+03$ & $1.42 \mathrm{E}+05$ \\
\hline Ge-68 & $4.66 \mathrm{E}+00$ & $1.26 \mathrm{E}+02$ \\
\hline Ge-69 & $5.33 \mathrm{E}+02$ & $1.44 \mathrm{E}+04$ \\
\hline Ge-71 & $1.09 \mathrm{E}+04$ & $2.93 \mathrm{E}+05$ \\
\hline Ge-75 & $3.49 \mathrm{E}+03$ & $9.43 \mathrm{E}+04$ \\
\hline Ge-77 & $3.47 \mathrm{E}+02$ & $9.38 \mathrm{E}+03$ \\
\hline Ge-78 & $1.36 \mathrm{E}+03$ & $3.67 \mathrm{E}+04$ \\
\hline As-69 & $6.08 \mathrm{E}+03$ & $1.64 \mathrm{E}+05$ \\
\hline As-70 & $1.81 \mathrm{E}+03$ & $4.89 E+04$ \\
\hline As-71 & $3.44 \mathrm{E}+02$ & $9.30 \mathrm{E}+03$ \\
\hline As-72 & $1.37 \mathrm{E}+02$ & $3.70 \mathrm{E}+03$ \\
\hline As-73 & $1.03 \mathrm{E}+02$ & $2.78 \mathrm{E}+03$ \\
\hline As-74 & $5.62 \mathrm{E}+01$ & $1.52 \mathrm{E}+03$ \\
\hline As-76 & $1.71 \mathrm{E}+02$ & $4.63 \mathrm{E}+03$ \\
\hline As-77 & $3.23 \mathrm{E}+02$ & $8.74 \mathrm{E}+03$ \\
\hline As-78 & $1.45 \mathrm{E}+03$ & $3.91 \mathrm{E}+04$ \\
\hline Se-70 & $3.36 \mathrm{E}+03$ & $9.08 \mathrm{E}+04$ \\
\hline Se-72 & $5.50 \mathrm{E}+01$ & $1.49 \mathrm{E}+03$ \\
\hline Se-73 & $1.47 \mathrm{E}+03$ & $3.98 \mathrm{E}+04$ \\
\hline $\mathrm{Se}-73 \mathrm{~m}$ & $1.30 \mathrm{E}+04$ & $3.52 \mathrm{E}+05$ \\
\hline $\mathrm{Se}-75$ & $1.26 \mathrm{E}+02$ & $3.41 \mathrm{E}+03$ \\
\hline Se-79 & $9.30 \mathrm{E}+01$ & $2.51 \mathrm{E}+03$ \\
\hline
\end{tabular}




\begin{tabular}{|c|c|c|}
\hline Nuclide & $\begin{array}{c}\text { Inhalation } \\
\text { DCS } \\
\left(\mathbf{B q} / \mathbf{m}^{3}\right)\end{array}$ & $\begin{array}{c}\text { Inhalation } \\
\text { DCS } \\
\left(\mathbf{p C i} / \mathbf{m}^{3}\right)\end{array}$ \\
\hline Se- 81 & $1.63 \mathrm{E}+04$ & $4.39 \mathrm{E}+05$ \\
\hline $\mathrm{Se}-81 \mathrm{~m}$ & $7.70 \mathrm{E}+03$ & $2.08 \mathrm{E}+05$ \\
\hline $\mathrm{Se}-83$ & $7.07 \mathrm{E}+03$ & $1.91 \mathrm{E}+05$ \\
\hline $\mathrm{Br}-74$ & $3.29 \mathrm{E}+03$ & $8.89 \mathrm{E}+04$ \\
\hline $\mathrm{Br}-74 \mathrm{~m}$ & $1.94 \mathrm{E}+03$ & $5.24 \mathrm{E}+04$ \\
\hline $\mathrm{Br}-75$ & $2.36 \mathrm{E}+03$ & $6.37 \mathrm{E}+04$ \\
\hline $\mathrm{Br}-76$ & $3.07 \mathrm{E}+02$ & $8.30 \mathrm{E}+03$ \\
\hline $\mathrm{Br}-77$ & $1.46 \mathrm{E}+03$ & $3.95 \mathrm{E}+04$ \\
\hline $\mathrm{Br}-80$ & $9.03 \mathrm{E}+03$ & $2.44 \mathrm{E}+05$ \\
\hline $\mathrm{Br}-80 \mathrm{~m}$ & $1.25 \mathrm{E}+03$ & $3.38 \mathrm{E}+04$ \\
\hline Br-82 & $2.02 \mathrm{E}+02$ & $5.47 \mathrm{E}+03$ \\
\hline $\mathrm{Br}-83$ & $2.59 \mathrm{E}+03$ & $6.99 \mathrm{E}+04$ \\
\hline $\mathrm{Br}-84$ & $3.38 \mathrm{E}+03$ & $9.14 \mathrm{E}+04$ \\
\hline $\mathrm{Rb}-78$ & $4.40 \mathrm{E}+03$ & $1.19 \mathrm{E}+05$ \\
\hline $\mathrm{Rb}-79$ & $4.57 \mathrm{E}+03$ & $1.23 \mathrm{E}+05$ \\
\hline $\mathrm{Rb}-81$ & $1.87 \mathrm{E}+03$ & $5.06 \mathrm{E}+04$ \\
\hline $\mathrm{Rb}-81 \mathrm{~m}$ & $8.14 \mathrm{E}+03$ & $2.20 \mathrm{E}+05$ \\
\hline $\mathrm{Rb}-82 \mathrm{~m}$ & $8.78 \mathrm{E}+02$ & $2.37 \mathrm{E}+04$ \\
\hline $\mathrm{Rb}-83$ & $1.00 \mathrm{E}+02$ & $2.71 \mathrm{E}+03$ \\
\hline $\mathrm{Rb}-84$ & $4.82 \mathrm{E}+01$ & $1.30 \mathrm{E}+03$ \\
\hline $\mathrm{Rb}-84 \mathrm{~m}$ & $1.55 \mathrm{E}+04$ & $4.18 \mathrm{E}+05$ \\
\hline $\mathrm{Rb}-86$ & $2.96 \mathrm{E}+01$ & $8.01 \mathrm{E}+02$ \\
\hline $\mathrm{Rb}-87$ & $9.30 \mathrm{E}+00$ & $2.51 \mathrm{E}+02$ \\
\hline $\mathrm{Rb}-88$ & $4.69 \mathrm{E}+03$ & $1.27 \mathrm{E}+05$ \\
\hline $\mathrm{Rb}-89$ & $5.92 \mathrm{E}+03$ & $1.60 \mathrm{E}+05$ \\
\hline Sr-80 & $8.63 \mathrm{E}+02$ & $2.33 \mathrm{E}+04$ \\
\hline Sr-81 & $4.20 \mathrm{E}+03$ & $1.14 \mathrm{E}+05$ \\
\hline Sr-82 & $1.53 \mathrm{E}+01$ & $4.14 \mathrm{E}+02$ \\
\hline Sr-83 & $3.98 \mathrm{E}+02$ & $1.07 \mathrm{E}+04$ \\
\hline Sr- 85 & $2.10 \mathrm{E}+02$ & $5.67 \mathrm{E}+03$ \\
\hline Sr- $85 \mathrm{~m}$ & $3.15 \mathrm{E}+04$ & $8.51 \mathrm{E}+05$ \\
\hline Sr- $87 \mathrm{~m}$ & $6.51 \mathrm{E}+03$ & $1.76 \mathrm{E}+05$ \\
\hline Sr-89 & $2.29 \mathrm{E}+01$ & $6.18 \mathrm{E}+02$ \\
\hline Sr-90 & $3.99 \mathrm{E}+00$ & $1.08 \mathrm{E}+02$ \\
\hline Sr-91 & $3.52 \mathrm{E}+02$ & $9.51 \mathrm{E}+03$ \\
\hline Sr-92 & $6.25 \mathrm{E}+02$ & $1.69 E+04$ \\
\hline $\mathrm{Y}-84 \mathrm{~m}$ & $2.08 \mathrm{E}+03$ & $5.63 \mathrm{E}+04$ \\
\hline $\mathrm{Y}-85$ & $1.35 \mathrm{E}+03$ & $3.64 \mathrm{E}+04$ \\
\hline
\end{tabular}

Page 210 of 298 


\begin{tabular}{|c|c|c|}
\hline Nuclide & $\begin{array}{c}\text { Inhalation } \\
\text { DCS } \\
\left(\mathbf{B q} / \mathbf{m}^{3}\right)\end{array}$ & $\begin{array}{c}\text { Inhalation } \\
\text { DCS } \\
\left(\mathbf{p C i} / \mathbf{m}^{3}\right)\end{array}$ \\
\hline$Y-85 \mathrm{~m}$ & $7.66 \mathrm{E}+02$ & $2.07 \mathrm{E}+04$ \\
\hline $\mathrm{Y}-86$ & $2.75 \mathrm{E}+02$ & $7.42 \mathrm{E}+03$ \\
\hline $\mathrm{Y}-86 \mathrm{~m}$ & $4.57 \mathrm{E}+03$ & $1.23 \mathrm{E}+05$ \\
\hline $\mathrm{Y}-87$ & $3.37 \mathrm{E}+02$ & $9.10 \mathrm{E}+03$ \\
\hline Y-87m & $9.41 \mathrm{E}+02$ & $2.54 \mathrm{E}+04$ \\
\hline $\mathrm{Y}-88$ & $2.27 \mathrm{E}+01$ & $6.13 E+02$ \\
\hline$Y-90$ & $8.83 \mathrm{E}+01$ & $2.39 \mathrm{E}+03$ \\
\hline Y-90m & $1.30 \mathrm{E}+03$ & $3.52 \mathrm{E}+04$ \\
\hline $\mathrm{Y}-91$ & $1.58 \mathrm{E}+01$ & $4.26 \mathrm{E}+02$ \\
\hline $\mathrm{Y}-91 \mathrm{~m}$ & $1.17 \mathrm{E}+04$ & $3.18 \mathrm{E}+05$ \\
\hline Y-92 & $7.27 \mathrm{E}+02$ & $1.96 \mathrm{E}+04$ \\
\hline Y-93 & $3.00 \mathrm{E}+02$ & $8.12 \mathrm{E}+03$ \\
\hline Y-94 & $4.75 \mathrm{E}+03$ & $1.28 \mathrm{E}+05$ \\
\hline$Y-95$ & $8.63 \mathrm{E}+03$ & $2.33 \mathrm{E}+05$ \\
\hline $\mathrm{Zr}-86$ & $3.08 \mathrm{E}+02$ & $8.33 \mathrm{E}+03$ \\
\hline $\mathrm{Zr}-87$ & $1.53 \mathrm{E}+03$ & $4.14 \mathrm{E}+04$ \\
\hline $\mathrm{Zr}-88$ & $5.46 \mathrm{E}+01$ & $1.48 \mathrm{E}+03$ \\
\hline $\mathrm{Zr}-89$ & $2.54 \mathrm{E}+02$ & $6.87 \mathrm{E}+03$ \\
\hline Zr-93 & $1.73 \mathrm{E}+01$ & $4.68 \mathrm{E}+02$ \\
\hline Zr-95 & $2.95 \mathrm{E}+01$ & $7.98 \mathrm{E}+02$ \\
\hline Zr-97 & $1.42 \mathrm{E}+02$ & $3.84 \mathrm{E}+03$ \\
\hline $\mathrm{Nb}-88$ & $4.41 \mathrm{E}+03$ & $1.19 \mathrm{E}+05$ \\
\hline $\mathrm{Nb}-89$ & $1.14 \mathrm{E}+03$ & $3.08 \mathrm{E}+04$ \\
\hline $\mathrm{Nb}-89 \mathrm{~m}$ & $1.95 \mathrm{E}+03$ & $5.26 \mathrm{E}+04$ \\
\hline $\mathrm{Nb}-90$ & $2.05 \mathrm{E}+02$ & $5.53 \mathrm{E}+03$ \\
\hline $\mathrm{Nb}-91$ & $5.16 \mathrm{E}+02$ & $1.39 \mathrm{E}+04$ \\
\hline $\mathrm{Nb}-91 \mathrm{~m}$ & $4.28 \mathrm{E}+01$ & $1.16 \mathrm{E}+03$ \\
\hline $\mathrm{Nb}-92$ & $2.86 \mathrm{E}+01$ & $7.73 \mathrm{E}+02$ \\
\hline $\mathrm{Nb}-92 \mathrm{~m}$ & $3.13 \mathrm{E}+02$ & $8.45 \mathrm{E}+03$ \\
\hline $\mathrm{Nb}-93 \mathrm{~m}$ & $2.56 \mathrm{E}+02$ & $6.91 \mathrm{E}+03$ \\
\hline $\mathrm{Nb}-94$ & $1.32 \mathrm{E}+01$ & $3.58 \mathrm{E}+02$ \\
\hline $\mathrm{Nb}-95$ & $9.53 \mathrm{E}+01$ & $2.57 \mathrm{E}+03$ \\
\hline $\mathrm{Nb}-95 \mathrm{~m}$ & $1.74 \mathrm{E}+02$ & $4.71 \mathrm{E}+03$ \\
\hline $\mathrm{Nb}-96$ & $2.09 \mathrm{E}+02$ & $5.65 \mathrm{E}+03$ \\
\hline $\mathrm{Nb}-97$ & $3.09 \mathrm{E}+03$ & $8.35 \mathrm{E}+04$ \\
\hline $\mathrm{Nb}-98 \mathrm{~m}$ & $2.30 \mathrm{E}+03$ & $6.23 E+04$ \\
\hline Mo-90 & $3.91 \mathrm{E}+02$ & $1.06 \mathrm{E}+04$ \\
\hline Мo-91 & $6.54 \mathrm{E}+03$ & $1.77 \mathrm{E}+05$ \\
\hline
\end{tabular}




\begin{tabular}{|c|c|c|}
\hline Nuclide & $\begin{array}{c}\text { Inhalation } \\
\text { DCS } \\
\left(\mathbf{B q} / \mathbf{m}^{3}\right)\end{array}$ & $\begin{array}{c}\text { Inhalation } \\
\text { DCS } \\
\left(\mathbf{p C i} / \mathbf{m}^{3}\right)\end{array}$ \\
\hline Мo-93 & $2.58 \mathrm{E}+02$ & $6.97 \mathrm{E}+03$ \\
\hline Mo-93m & $7.62 \mathrm{E}+02$ & $2.06 \mathrm{E}+04$ \\
\hline Мо-99 & $1.52 \mathrm{E}+02$ & $4.10 \mathrm{E}+03$ \\
\hline Mo-101 & $5.28 \mathrm{E}+03$ & $1.43 \mathrm{E}+05$ \\
\hline Mo-102 & $5.41 \mathrm{E}+03$ & $1.46 \mathrm{E}+05$ \\
\hline Tc-93 & $2.83 \mathrm{E}+03$ & $7.64 \mathrm{E}+04$ \\
\hline Tc-93m & $6.30 \mathrm{E}+03$ & $1.70 \mathrm{E}+05$ \\
\hline Tc-94 & $1.02 \mathrm{E}+03$ & $2.76 \mathrm{E}+04$ \\
\hline Tc-94m & $2.80 \mathrm{E}+03$ & $7.57 \mathrm{E}+04$ \\
\hline Tc-95 & $1.19 \mathrm{E}+03$ & $3.22 \mathrm{E}+04$ \\
\hline Tc-95m & $1.50 \mathrm{E}+02$ & $4.06 \mathrm{E}+03$ \\
\hline Tc-96 & $1.88 \mathrm{E}+02$ & $5.09 \mathrm{E}+03$ \\
\hline Tc-96m & $1.76 \mathrm{E}+04$ & $4.75 \mathrm{E}+05$ \\
\hline Tc-97 & $6.20 \mathrm{E}+02$ & $1.68 \mathrm{E}+04$ \\
\hline Tc-97m & $4.40 \mathrm{E}+01$ & $1.19 \mathrm{E}+03$ \\
\hline Tc-98 & $1.80 \mathrm{E}+01$ & $4.85 \mathrm{E}+02$ \\
\hline Tc-99 & $3.54 \mathrm{E}+01$ & $9.55 \mathrm{E}+02$ \\
\hline Tc-99m & $6.98 \mathrm{E}+03$ & $1.89 E+05$ \\
\hline Tc-101 & $1.12 \mathrm{E}+04$ & $3.02 \mathrm{E}+05$ \\
\hline Tc-104 & $4.48 \mathrm{E}+03$ & $1.21 \mathrm{E}+05$ \\
\hline Ru-94 & $3.00 \mathrm{E}+03$ & $8.11 \mathrm{E}+04$ \\
\hline $\mathrm{Ru}-95$ & $3.64 \mathrm{E}+03$ & $9.84 \mathrm{E}+04$ \\
\hline Ru-97 & $1.26 \mathrm{E}+03$ & $3.41 \mathrm{E}+04$ \\
\hline Ru-103 & $5.99 \mathrm{E}+01$ & $1.62 \mathrm{E}+03$ \\
\hline Ru-105 & $7.17 \mathrm{E}+02$ & $1.94 \mathrm{E}+04$ \\
\hline Ru-106 & $5.04 \mathrm{E}+00$ & $1.36 \mathrm{E}+02$ \\
\hline Rh-97 & $5.50 \mathrm{E}+03$ & $1.49 \mathrm{E}+05$ \\
\hline $\mathrm{Rh}-97 \mathrm{~m}$ & $4.73 \mathrm{E}+03$ & $1.28 \mathrm{E}+05$ \\
\hline Rh-99 & $1.11 \mathrm{E}+02$ & $3.00 \mathrm{E}+03$ \\
\hline Rh-99m & $3.18 \mathrm{E}+03$ & $8.60 \mathrm{E}+04$ \\
\hline Rh-100 & $3.77 \mathrm{E}+02$ & $1.02 \mathrm{E}+04$ \\
\hline Rh-101 & $2.81 \mathrm{E}+01$ & $7.60 \mathrm{E}+02$ \\
\hline $\mathrm{Rh}-101 \mathrm{~m}$ & $6.57 \mathrm{E}+02$ & $1.77 \mathrm{E}+04$ \\
\hline Rh-102 & $1.95 \mathrm{E}+01$ & $5.27 \mathrm{E}+02$ \\
\hline Rh-102m & $7.34 \mathrm{E}+00$ & $1.98 \mathrm{E}+02$ \\
\hline Rh-103m & $5.04 \mathrm{E}+04$ & $1.36 \mathrm{E}+06$ \\
\hline Rh-105 & $3.89 \mathrm{E}+02$ & $1.05 \mathrm{E}+04$ \\
\hline Rh-106m & $1.18 \mathrm{E}+03$ & $3.20 \mathrm{E}+04$ \\
\hline
\end{tabular}




\begin{tabular}{|c|c|c|}
\hline Nuclide & $\begin{array}{c}\text { Inhalation } \\
\text { DCS } \\
\left(\mathbf{B q} / \mathbf{m}^{3}\right)\end{array}$ & $\begin{array}{c}\text { Inhalation } \\
\text { DCS } \\
\left(\mathbf{p C i} / \mathbf{m}^{3}\right)\end{array}$ \\
\hline Rh-107 & $8.01 \mathrm{E}+03$ & $2.17 \mathrm{E}+05$ \\
\hline Pd-98 & $4.66 \mathrm{E}+03$ & $1.26 \mathrm{E}+05$ \\
\hline Pd-99 & $6.85 \mathrm{E}+03$ & $1.85 \mathrm{E}+05$ \\
\hline Pd-100 & $1.67 \mathrm{E}+02$ & $4.50 \mathrm{E}+03$ \\
\hline Pd-101 & $2.12 \mathrm{E}+03$ & $5.73 \mathrm{E}+04$ \\
\hline Pd-103 & $3.13 \mathrm{E}+02$ & $8.45 \mathrm{E}+03$ \\
\hline Pd-107 & $2.37 \mathrm{E}+02$ & $6.41 \mathrm{E}+03$ \\
\hline Pd-109 & $3.55 \mathrm{E}+02$ & $9.60 \mathrm{E}+03$ \\
\hline Pd-111 & $4.98 \mathrm{E}+03$ & $1.34 \mathrm{E}+05$ \\
\hline Pd-112 & $1.18 \mathrm{E}+02$ & $3.20 \mathrm{E}+03$ \\
\hline Ag-101 & $9.77 \mathrm{E}+03$ & $2.64 \mathrm{E}+05$ \\
\hline Ag-102 & $7.27 \mathrm{E}+03$ & $1.96 \mathrm{E}+05$ \\
\hline Ag-103 & $5.33 \mathrm{E}+03$ & $1.44 \mathrm{E}+05$ \\
\hline Ag-104 & $3.51 \mathrm{E}+03$ & $9.49 \mathrm{E}+04$ \\
\hline Ag-104m & $4.52 \mathrm{E}+03$ & $1.22 \mathrm{E}+05$ \\
\hline Ag-105 & $1.84 \mathrm{E}+02$ & $4.97 \mathrm{E}+03$ \\
\hline Ag-106 & $8.93 \mathrm{E}+03$ & $2.41 \mathrm{E}+05$ \\
\hline Ag-108m & $1.88 \mathrm{E}+01$ & $5.09 \mathrm{E}+02$ \\
\hline $\mathrm{Ag}-110 \mathrm{~m}$ & $1.80 \mathrm{E}+01$ & $4.87 \mathrm{E}+02$ \\
\hline Ag-111 & $8.93 \mathrm{E}+01$ & $2.41 \mathrm{E}+03$ \\
\hline Ag-112 & $7.93 \mathrm{E}+02$ & $2.14 \mathrm{E}+04$ \\
\hline Ag-113 & $8.54 \mathrm{E}+02$ & $2.31 \mathrm{E}+04$ \\
\hline Ag-115 & $4.88 \mathrm{E}+03$ & $1.32 \mathrm{E}+05$ \\
\hline Cd-104 & $2.67 \mathrm{E}+03$ & $7.21 \mathrm{E}+04$ \\
\hline Cd-105 & $5.46 \mathrm{E}+03$ & $1.48 \mathrm{E}+05$ \\
\hline Cd-107 & $1.65 \mathrm{E}+03$ & $4.45 \mathrm{E}+04$ \\
\hline Cd-109 & $1.69 \mathrm{E}+01$ & $4.57 \mathrm{E}+02$ \\
\hline $\mathrm{Cd}-111 \mathrm{~m}$ & $5.87 \mathrm{E}+03$ & $1.59 \mathrm{E}+05$ \\
\hline $\mathrm{Cd}-113$ & $1.26 \mathrm{E}+00$ & $3.41 \mathrm{E}+01$ \\
\hline Cd-113m & $1.34 \mathrm{E}+00$ & $3.61 \mathrm{E}+01$ \\
\hline $\mathrm{Cd}-115$ & $1.25 \mathrm{E}+02$ & $3.38 \mathrm{E}+03$ \\
\hline Cd-115m & $1.84 \mathrm{E}+01$ & $4.97 \mathrm{E}+02$ \\
\hline $\mathrm{Cd}-117$ & $7.74 \mathrm{E}+02$ & $2.09 \mathrm{E}+04$ \\
\hline $\mathrm{Cd}-117 \mathrm{~m}$ & $6.40 \mathrm{E}+02$ & $1.73 \mathrm{E}+04$ \\
\hline Cd-118 & $1.63 \mathrm{E}+03$ & $4.40 \mathrm{E}+04$ \\
\hline In-107 & $5.11 \mathrm{E}+03$ & $1.38 \mathrm{E}+05$ \\
\hline In-108 & $2.74 \mathrm{E}+03$ & $7.40 \mathrm{E}+04$ \\
\hline In-108m & $3.45 \mathrm{E}+03$ & $9.32 \mathrm{E}+04$ \\
\hline
\end{tabular}




\begin{tabular}{|c|c|c|}
\hline Nuclide & $\begin{array}{c}\text { Inhalation } \\
\text { DCS } \\
\left(\mathbf{B q} / \mathbf{m}^{\mathbf{3}}\right)\end{array}$ & $\begin{array}{c}\text { Inhalation } \\
\text { DCS } \\
\left(\mathbf{p C i} / \mathbf{m}^{3}\right)\end{array}$ \\
\hline In-109 & $3.33 \mathrm{E}+03$ & $9.00 \mathrm{E}+04$ \\
\hline In-110 & $9.53 \mathrm{E}+02$ & $2.57 \mathrm{E}+04$ \\
\hline In-110m & $2.60 \mathrm{E}+03$ & $7.03 \mathrm{E}+04$ \\
\hline In-111 & $5.44 \mathrm{E}+02$ & $1.47 \mathrm{E}+04$ \\
\hline In-112 & $1.66 \mathrm{E}+04$ & $4.48 \mathrm{E}+05$ \\
\hline In-112m & $6.01 \mathrm{E}+03$ & $1.62 \mathrm{E}+05$ \\
\hline In-113m & $6.25 \mathrm{E}+03$ & $1.69 \mathrm{E}+05$ \\
\hline In-114m & $1.04 \mathrm{E}+01$ & $2.82 \mathrm{E}+02$ \\
\hline In-115 & $3.82 \mathrm{E}-01$ & $1.03 \mathrm{E}+01$ \\
\hline In-115m & $2.11 \mathrm{E}+03$ & $5.70 \mathrm{E}+04$ \\
\hline In-116m & $2.77 \mathrm{E}+03$ & $7.47 \mathrm{E}+04$ \\
\hline In-117 & $4.43 \mathrm{E}+03$ & $1.20 \mathrm{E}+05$ \\
\hline In-117m & $1.75 \mathrm{E}+03$ & $4.72 \mathrm{E}+04$ \\
\hline In-119m & $7.13 \mathrm{E}+03$ & $1.93 \mathrm{E}+05$ \\
\hline Sn-108 & $1.12 \mathrm{E}+04$ & $3.02 \mathrm{E}+05$ \\
\hline Sn-109 & $1.09 \mathrm{E}+04$ & $2.93 \mathrm{E}+05$ \\
\hline Sn-110 & $7.66 \mathrm{E}+02$ & $2.07 \mathrm{E}+04$ \\
\hline Sn-111 & $1.02 \mathrm{E}+04$ & $2.76 \mathrm{E}+05$ \\
\hline Sn-113 & $3.52 \mathrm{E}+01$ & $9.51 \mathrm{E}+02$ \\
\hline Sn-113m & $3.01 \mathrm{E}+04$ & $8.14 \mathrm{E}+05$ \\
\hline Sn-117m & $5.04 \mathrm{E}+01$ & $1.36 \mathrm{E}+03$ \\
\hline Sn-119m & $4.12 \mathrm{E}+01$ & $1.11 \mathrm{E}+03$ \\
\hline Sn-121 & $5.50 \mathrm{E}+02$ & $1.49 \mathrm{E}+04$ \\
\hline $\mathrm{Sn}-121 \mathrm{~m}$ & $9.83 \mathrm{E}+00$ & $2.66 \mathrm{E}+02$ \\
\hline Sn-123 & $1.10 \mathrm{E}+01$ & $2.97 \mathrm{E}+02$ \\
\hline Sn-123m & $4.69 \mathrm{E}+03$ & $1.27 \mathrm{E}+05$ \\
\hline Sn-125 & $3.94 \mathrm{E}+01$ & $1.06 \mathrm{E}+03$ \\
\hline Sn-126 & $9.41 \mathrm{E}-01$ & $2.54 \mathrm{E}+01$ \\
\hline Sn-127 & $9.41 \mathrm{E}+02$ & $2.54 \mathrm{E}+04$ \\
\hline Sn-128 & $1.37 \mathrm{E}+03$ & $3.70 \mathrm{E}+04$ \\
\hline $\mathrm{Sb}-115$ & $9.95 \mathrm{E}+03$ & $2.69 \mathrm{E}+05$ \\
\hline $\mathrm{Sb}-116$ & $9.19 \mathrm{E}+03$ & $2.48 \mathrm{E}+05$ \\
\hline $\mathrm{Sb}-116 \mathrm{~m}$ & $2.86 \mathrm{E}+03$ & $7.72 \mathrm{E}+04$ \\
\hline Sb-117 & $7.85 \mathrm{E}+03$ & $2.12 \mathrm{E}+05$ \\
\hline $\mathrm{Sb}-118 \mathrm{~m}$ & $1.06 \mathrm{E}+03$ & $2.87 \mathrm{E}+04$ \\
\hline Sb-119 & $3.39 \mathrm{E}+03$ & $9.16 \mathrm{E}+04$ \\
\hline Sb-120 & $1.86 \mathrm{E}+04$ & $5.03 \mathrm{E}+05$ \\
\hline $\mathrm{Sb}-120 \mathrm{~m}$ & $1.30 \mathrm{E}+02$ & $3.52 \mathrm{E}+03$ \\
\hline
\end{tabular}

Page 214 of 298 


\begin{tabular}{|c|c|c|}
\hline Nuclide & $\begin{array}{c}\text { Inhalation } \\
\text { DCS } \\
\left(\mathbf{B q} / \mathbf{m}^{3}\right)\end{array}$ & $\begin{array}{c}\text { Inhalation } \\
\text { DCS } \\
\left(\mathbf{p C i} / \mathbf{m}^{3}\right)\end{array}$ \\
\hline $\mathrm{Sb}-122$ & $1.28 \mathrm{E}+02$ & $3.46 \mathrm{E}+03$ \\
\hline $\mathrm{Sb}-124$ & $2.19 \mathrm{E}+01$ & $5.91 \mathrm{E}+02$ \\
\hline $\mathrm{Sb}-124 n$ & $2.09 \mathrm{E}+04$ & $5.65 \mathrm{E}+05$ \\
\hline $\mathrm{Sb}-125$ & $2.93 \mathrm{E}+01$ & $7.92 \mathrm{E}+02$ \\
\hline $\mathrm{Sb}-126$ & $4.41 \mathrm{E}+01$ & $1.19 \mathrm{E}+03$ \\
\hline $\mathrm{Sb}-126 \mathrm{~m}$ & $6.79 \mathrm{E}+03$ & $1.84 \mathrm{E}+05$ \\
\hline $\mathrm{Sb}-127$ & $8.14 \mathrm{E}+01$ & $2.20 \mathrm{E}+03$ \\
\hline Sb-128 & $3.10 \mathrm{E}+02$ & $8.38 \mathrm{E}+03$ \\
\hline Sb-128m & $9.08 \mathrm{E}+03$ & $2.46 \mathrm{E}+05$ \\
\hline Sb-129 & $5.52 \mathrm{E}+02$ & $1.49 \mathrm{E}+04$ \\
\hline $\mathrm{Sb}-130$ & $2.52 \mathrm{E}+03$ & $6.81 \mathrm{E}+04$ \\
\hline Te-114 & $4.68 \mathrm{E}+03$ & $1.26 \mathrm{E}+05$ \\
\hline Te-116 & $1.09 \mathrm{E}+03$ & $2.95 \mathrm{E}+04$ \\
\hline Te-117 & $4.16 \mathrm{E}+03$ & $1.12 \mathrm{E}+05$ \\
\hline Te-118 & $5.83 \mathrm{E}+01$ & $1.58 \mathrm{E}+03$ \\
\hline Te-119 & $1.24 \mathrm{E}+03$ & $3.35 \mathrm{E}+04$ \\
\hline Te-119m & $2.55 \mathrm{E}+02$ & $6.90 \mathrm{E}+03$ \\
\hline Te-121 & $3.27 \mathrm{E}+02$ & $8.83 E+03$ \\
\hline $\mathrm{Te}-121 \mathrm{~m}$ & $3.30 \mathrm{E}+01$ & $8.93 E+02$ \\
\hline Te-123 & $2.68 \mathrm{E}+02$ & $7.24 \mathrm{E}+03$ \\
\hline Te-123m & $3.56 \mathrm{E}+01$ & $9.62 \mathrm{E}+02$ \\
\hline Te-125m & $4.22 \mathrm{E}+01$ & $1.14 \mathrm{E}+03$ \\
\hline Te-127 & $1.03 \mathrm{E}+03$ & $2.80 \mathrm{E}+04$ \\
\hline $\mathrm{Te}-127 \mathrm{~m}$ & $1.90 \mathrm{E}+01$ & $5.13 \mathrm{E}+02$ \\
\hline Te-129 & $3.59 \mathrm{E}+03$ & $9.71 E+04$ \\
\hline Te-129m & $2.15 \mathrm{E}+01$ & $5.82 \mathrm{E}+02$ \\
\hline Te-131 & $4.62 \mathrm{E}+03$ & $1.25 \mathrm{E}+05$ \\
\hline Te-131m & $1.25 \mathrm{E}+02$ & $3.38 \mathrm{E}+03$ \\
\hline Te-132 & $6.30 \mathrm{E}+01$ & $1.70 \mathrm{E}+03$ \\
\hline Te-133 & $6.65 \mathrm{E}+03$ & $1.80 \mathrm{E}+05$ \\
\hline Te-133m & $1.67 \mathrm{E}+03$ & $4.51 \mathrm{E}+04$ \\
\hline $\mathrm{Te}-134$ & $2.07 \mathrm{E}+03$ & $5.59 \mathrm{E}+04$ \\
\hline I-118 & $7.70 \mathrm{E}+02$ & $2.08 \mathrm{E}+04$ \\
\hline $\mathrm{I}-119$ & $2.76 \mathrm{E}+03$ & $7.46 \mathrm{E}+04$ \\
\hline $\mathrm{I}-120$ & $4.82 \mathrm{E}+02$ & $1.30 \mathrm{E}+04$ \\
\hline $\mathrm{I}-120 \mathrm{~m}$ & $9.65 \mathrm{E}+02$ & $2.61 \mathrm{E}+04$ \\
\hline I-121 & $1.63 \mathrm{E}+03$ & $4.40 \mathrm{E}+04$ \\
\hline I-123 & $5.48 \mathrm{E}+02$ & $1.48 \mathrm{E}+04$ \\
\hline
\end{tabular}




\begin{tabular}{|c|c|c|}
\hline Nuclide & $\begin{array}{c}\text { Inhalation } \\
\text { DCS } \\
\left(\mathbf{B q} / \mathbf{m}^{3}\right)\end{array}$ & $\begin{array}{c}\text { Inhalation } \\
\text { DCS } \\
\left(\mathbf{p C i} / \mathbf{m}^{3}\right)\end{array}$ \\
\hline $\mathrm{I}-124$ & $9.95 \mathrm{E}+00$ & $2.69 \mathrm{E}+02$ \\
\hline I- 125 & $9.47 \mathrm{E}+00$ & $2.56 \mathrm{E}+02$ \\
\hline I-126 & $4.54 \mathrm{E}+00$ & $1.23 \mathrm{E}+02$ \\
\hline I-129 & $1.45 \mathrm{E}+00$ & $3.91 \mathrm{E}+01$ \\
\hline $\mathrm{I}-130$ & $6.62 \mathrm{E}+01$ & $1.79 E+03$ \\
\hline $\mathrm{I}-131$ & $5.99 \mathrm{E}+00$ & $1.62 \mathrm{E}+02$ \\
\hline $\mathrm{I}-132$ & $4.04 \mathrm{E}+02$ & $1.09 \mathrm{E}+04$ \\
\hline $\mathrm{I}-132 \mathrm{~m}$ & $5.28 \mathrm{E}+02$ & $1.43 \mathrm{E}+04$ \\
\hline I-133 & $2.85 \mathrm{E}+01$ & $7.71 \mathrm{E}+02$ \\
\hline I-134 & $9.65 \mathrm{E}+02$ & $2.61 \mathrm{E}+04$ \\
\hline $\mathrm{I}-135$ & $1.35 \mathrm{E}+02$ & $3.64 \mathrm{E}+03$ \\
\hline Cs-125 & $1.16 \mathrm{E}+04$ & $3.13 \mathrm{E}+05$ \\
\hline Cs-127 & $6.28 \mathrm{E}+03$ & $1.70 \mathrm{E}+05$ \\
\hline Cs-129 & $3.03 \mathrm{E}+03$ & $8.18 \mathrm{E}+04$ \\
\hline Cs-130 & $1.70 \mathrm{E}+04$ & $4.59 \mathrm{E}+05$ \\
\hline Cs-131 & $4.73 \mathrm{E}+03$ & $1.28 \mathrm{E}+05$ \\
\hline Cs-132 & $5.70 \mathrm{E}+02$ & $1.54 \mathrm{E}+04$ \\
\hline Cs-134 & $2.38 \mathrm{E}+01$ & $6.44 \mathrm{E}+02$ \\
\hline Cs-134m & $9.25 \mathrm{E}+03$ & $2.50 \mathrm{E}+05$ \\
\hline Cs-135 & $1.71 \mathrm{E}+02$ & $4.63 \mathrm{E}+03$ \\
\hline Cs-135m & $1.08 \mathrm{E}+04$ & $2.91 \mathrm{E}+05$ \\
\hline Cs-136 & $1.13 \mathrm{E}+02$ & $3.06 \mathrm{E}+03$ \\
\hline Cs-137 & $3.40 \mathrm{E}+01$ & $9.18 \mathrm{E}+02$ \\
\hline Cs-138 & $4.99 \mathrm{E}+03$ & $1.35 \mathrm{E}+05$ \\
\hline $\mathrm{Ba}-124$ & $5.94 \mathrm{E}+03$ & $1.61 \mathrm{E}+05$ \\
\hline $\mathrm{Ba}-126$ & $1.21 \mathrm{E}+03$ & $3.27 \mathrm{E}+04$ \\
\hline Ba-127 & $1.20 \mathrm{E}+04$ & $3.25 \mathrm{E}+05$ \\
\hline Ba-128 & $1.00 \mathrm{E}+02$ & $2.71 \mathrm{E}+03$ \\
\hline $\mathrm{Ba}-129$ & $4.72 \mathrm{E}+03$ & $1.28 \mathrm{E}+05$ \\
\hline $\mathrm{Ba}-129 \mathrm{~m}$ & $2.87 \mathrm{E}+03$ & $7.76 \mathrm{E}+04$ \\
\hline Ba-131 & $1.85 \mathrm{E}+02$ & $4.99 \mathrm{E}+03$ \\
\hline $\mathrm{Ba}-131 \mathrm{~m}$ & $1.84 \mathrm{E}+04$ & $4.98 \mathrm{E}+05$ \\
\hline $\mathrm{Ba}-133$ & $4.25 \mathrm{E}+01$ & $1.15 \mathrm{E}+03$ \\
\hline Ba-133m & $3.14 \mathrm{E}+02$ & $8.48 \mathrm{E}+03$ \\
\hline Ba-135m & $4.05 \mathrm{E}+02$ & $1.09 \mathrm{E}+04$ \\
\hline Ba-139 & $2.33 \mathrm{E}+03$ & $6.30 \mathrm{E}+04$ \\
\hline Ba-140 & $2.74 \mathrm{E}+01$ & $7.41 \mathrm{E}+02$ \\
\hline Ba-141 & $3.93 \mathrm{E}+03$ & $1.06 \mathrm{E}+05$ \\
\hline
\end{tabular}

Page 216 of 298 


\begin{tabular}{|c|c|c|}
\hline Nuclide & $\begin{array}{c}\text { Inhalation } \\
\text { DCS } \\
\left(\mathbf{B q} / \mathbf{m}^{\mathbf{3}}\right)\end{array}$ & $\begin{array}{c}\text { Inhalation } \\
\text { DCS } \\
\left(\mathbf{p C i} / \mathbf{m}^{3}\right)\end{array}$ \\
\hline Ba-142 & $6.38 \mathrm{E}+03$ & $1.72 \mathrm{E}+05$ \\
\hline La-129 & $1.06 \mathrm{E}+04$ & $2.85 \mathrm{E}+05$ \\
\hline La-131 & $5.58 \mathrm{E}+03$ & $1.51 \mathrm{E}+05$ \\
\hline La-132 & $7.13 \mathrm{E}+02$ & $1.93 \mathrm{E}+04$ \\
\hline La-132m & $6.23 \mathrm{E}+03$ & $1.68 \mathrm{E}+05$ \\
\hline La-133 & $7.51 \mathrm{E}+03$ & $2.03 \mathrm{E}+05$ \\
\hline La-135 & $8.49 \mathrm{E}+03$ & $2.30 \mathrm{E}+05$ \\
\hline La-137 & $1.66 \mathrm{E}+01$ & $4.48 \mathrm{E}+02$ \\
\hline La-138 & $9.65 \mathrm{E}-01$ & $2.61 \mathrm{E}+01$ \\
\hline La-140 & $1.16 \mathrm{E}+02$ & $3.13 \mathrm{E}+03$ \\
\hline La-141 & $8.10 \mathrm{E}+02$ & $2.19 \mathrm{E}+04$ \\
\hline La-142 & $1.46 \mathrm{E}+03$ & $3.95 \mathrm{E}+04$ \\
\hline La-143 & $5.77 \mathrm{E}+03$ & $1.56 \mathrm{E}+05$ \\
\hline Ce-130 & $3.82 \mathrm{E}+03$ & $1.03 \mathrm{E}+05$ \\
\hline Ce-131 & $9.65 \mathrm{E}+03$ & $2.61 \mathrm{E}+05$ \\
\hline Ce-132 & $9.47 \mathrm{E}+02$ & $2.56 \mathrm{E}+04$ \\
\hline Ce-133 & $2.65 \mathrm{E}+03$ & $7.16 \mathrm{E}+04$ \\
\hline $\mathrm{Ce}-133 \mathrm{~m}$ & $1.13 \mathrm{E}+03$ & $3.06 \mathrm{E}+04$ \\
\hline $\mathrm{Ce}-134$ & $9.77 \mathrm{E}+01$ & $2.64 \mathrm{E}+03$ \\
\hline Ce-135 & $9.59 \mathrm{E}+02$ & $2.59 \mathrm{E}+04$ \\
\hline $\mathrm{Ce}-137$ & $1.19 \mathrm{E}+04$ & $3.22 \mathrm{E}+05$ \\
\hline $\mathrm{Ce}-137 \mathrm{~m}$ & $3.24 \mathrm{E}+02$ & $8.76 \mathrm{E}+03$ \\
\hline Ce-139 & $8.27 \mathrm{E}+01$ & $2.23 \mathrm{E}+03$ \\
\hline Ce-141 & $4.41 \mathrm{E}+01$ & $1.19 \mathrm{E}+03$ \\
\hline Ce-143 & $1.77 \mathrm{E}+02$ & $4.78 \mathrm{E}+03$ \\
\hline Ce-144 & $3.86 \mathrm{E}+00$ & $1.04 \mathrm{E}+02$ \\
\hline Pr-134 & $6.20 \mathrm{E}+03$ & $1.68 \mathrm{E}+05$ \\
\hline $\operatorname{Pr}-134 \mathrm{~m}$ & $4.08 \mathrm{E}+03$ & $1.10 \mathrm{E}+05$ \\
\hline Pr-135 & $6.38 \mathrm{E}+03$ & $1.72 \mathrm{E}+05$ \\
\hline Pr-136 & $9.30 \mathrm{E}+03$ & $2.51 \mathrm{E}+05$ \\
\hline Pr-137 & $6.79 \mathrm{E}+03$ & $1.84 \mathrm{E}+05$ \\
\hline $\operatorname{Pr}-138 \mathrm{~m}$ & $1.72 \mathrm{E}+03$ & $4.65 E+04$ \\
\hline Pr-139 & $6.48 \mathrm{E}+03$ & $1.75 \mathrm{E}+05$ \\
\hline Pr-142 & $2.34 \mathrm{E}+02$ & $6.33 \mathrm{E}+03$ \\
\hline Pr-142m & $1.84 \mathrm{E}+04$ & $4.97 \mathrm{E}+05$ \\
\hline Pr-143 & $5.74 \mathrm{E}+01$ & $1.55 \mathrm{E}+03$ \\
\hline Pr-144 & $7.17 \mathrm{E}+03$ & $1.94 \mathrm{E}+05$ \\
\hline Pr-145 & $7.77 \mathrm{E}+02$ & $2.10 \mathrm{E}+04$ \\
\hline
\end{tabular}

Page 217 of 298 


\begin{tabular}{|c|c|c|}
\hline Nuclide & $\begin{array}{c}\text { Inhalation } \\
\text { DCS } \\
\left(\mathbf{B q} / \mathbf{m}^{\mathbf{3}}\right)\end{array}$ & $\begin{array}{c}\text { Inhalation } \\
\text { DCS } \\
\left(\mathbf{p C i} / \mathbf{m}^{3}\right)\end{array}$ \\
\hline Pr-146 & $4.36 \mathrm{E}+03$ & $1.18 \mathrm{E}+05$ \\
\hline Pr-147 & $7.37 \mathrm{E}+03$ & $1.99 \mathrm{E}+05$ \\
\hline Nd-135 & $4.91 \mathrm{E}+03$ & $1.33 \mathrm{E}+05$ \\
\hline Nd-136 & $2.56 \mathrm{E}+03$ & $6.91 \mathrm{E}+04$ \\
\hline $\mathrm{Nd}-136$ & $2.45 \mathrm{E}+03$ & $6.62 \mathrm{E}+04$ \\
\hline $\mathrm{Nd}-137$ & $7.74 \mathrm{E}+03$ & $2.09 \mathrm{E}+05$ \\
\hline Nd-137 & $4.61 \mathrm{E}+03$ & $1.25 \mathrm{E}+05$ \\
\hline Nd-138 & $5.16 \mathrm{E}+02$ & $1.39 \mathrm{E}+04$ \\
\hline Nd-139 & $1.25 \mathrm{E}+04$ & $3.38 \mathrm{E}+05$ \\
\hline Nd-139 & $1.19 \mathrm{E}+04$ & $3.22 \mathrm{E}+05$ \\
\hline $\mathrm{Nd}-139 \mathrm{~m}$ & $1.41 \mathrm{E}+03$ & $3.80 \mathrm{E}+04$ \\
\hline $\mathrm{Nd}-139 \mathrm{~m}$ & $8.63 \mathrm{E}+02$ & $2.33 \mathrm{E}+04$ \\
\hline $\mathrm{Nd}-140$ & $1.17 \mathrm{E}+02$ & $3.18 \mathrm{E}+03$ \\
\hline Nd-141 & $2.54 \mathrm{E}+04$ & $6.87 \mathrm{E}+05$ \\
\hline Nd-141 & $2.45 \mathrm{E}+04$ & $6.62 \mathrm{E}+05$ \\
\hline Nd-144 & 7.77E-03 & $2.10 \mathrm{E}-01$ \\
\hline Nd-144 & $1.92 \mathrm{E}-02$ & $5.20 \mathrm{E}-01$ \\
\hline $\mathrm{Nd}-147$ & $5.85 \mathrm{E}+01$ & $1.58 \mathrm{E}+03$ \\
\hline Nd-149 & $1.47 \mathrm{E}+03$ & $3.98 \mathrm{E}+04$ \\
\hline Nd-151 & $7.62 \mathrm{E}+03$ & $2.06 \mathrm{E}+05$ \\
\hline $\mathrm{Nd}-152$ & $5.58 \mathrm{E}+03$ & $1.51 \mathrm{E}+05$ \\
\hline Pm-141 & $9.03 \mathrm{E}+03$ & $2.44 \mathrm{E}+05$ \\
\hline Pm-143 & $4.78 \mathrm{E}+01$ & $1.29 \mathrm{E}+03$ \\
\hline Pm-144 & $8.18 \mathrm{E}+00$ & $2.21 \mathrm{E}+02$ \\
\hline Pm-145 & $1.81 \mathrm{E}+01$ & $4.90 \mathrm{E}+02$ \\
\hline Pm-146 & $3.27 \mathrm{E}+00$ & $8.83 \mathrm{E}+01$ \\
\hline Pm-147 & $1.96 \mathrm{E}+01$ & $5.30 \mathrm{E}+02$ \\
\hline Pm-148 & $6.23 \mathrm{E}+01$ & $1.68 \mathrm{E}+03$ \\
\hline Pm-148m & $2.48 \mathrm{E}+01$ & $6.71 \mathrm{E}+02$ \\
\hline Pm-149 & $1.86 \mathrm{E}+02$ & $5.04 \mathrm{E}+03$ \\
\hline Pm-150 & $1.00 \mathrm{E}+03$ & $2.71 \mathrm{E}+04$ \\
\hline Pm-151 & $2.82 \mathrm{E}+02$ & $7.61 \mathrm{E}+03$ \\
\hline Sm-140 & $3.99 \mathrm{E}+03$ & $1.08 \mathrm{E}+05$ \\
\hline Sm-141 & $8.22 \mathrm{E}+03$ & $2.22 \mathrm{E}+05$ \\
\hline Sm-141m & $4.02 \mathrm{E}+03$ & $1.09 \mathrm{E}+05$ \\
\hline Sm-142 & $1.76 \mathrm{E}+03$ & $4.76 \mathrm{E}+04$ \\
\hline Sm-145 & $4.73 \mathrm{E}+01$ & $1.28 \mathrm{E}+03$ \\
\hline Sm-146 & $5.85 \mathrm{E}-03$ & $1.58 \mathrm{E}-01$ \\
\hline
\end{tabular}




\begin{tabular}{|c|c|c|}
\hline Nuclide & $\begin{array}{c}\text { Inhalation } \\
\text { DCS } \\
\left(\mathbf{B q} / \mathbf{m}^{3}\right)\end{array}$ & $\begin{array}{c}\text { Inhalation } \\
\text { DCS } \\
\left(\mathbf{p C i} / \mathbf{m}^{3}\right)\end{array}$ \\
\hline Sm-147 & $6.40 \mathrm{E}-03$ & $1.73 \mathrm{E}-01$ \\
\hline Sm-148 & $7.44 \mathrm{E}-03$ & $2.01 \mathrm{E}-01$ \\
\hline $\mathrm{Sm}-151$ & $1.59 \mathrm{E}+01$ & $4.29 \mathrm{E}+02$ \\
\hline $\mathrm{Sm}-153$ & $1.98 \mathrm{E}+02$ & $5.35 \mathrm{E}+03$ \\
\hline Sm-155 & $7.44 \mathrm{E}+03$ & $2.01 \mathrm{E}+05$ \\
\hline Sm-156 & $5.62 \mathrm{E}+02$ & $1.52 \mathrm{E}+04$ \\
\hline Eu-145 & $2.57 \mathrm{E}+02$ & $6.93 \mathrm{E}+03$ \\
\hline Eu-146 & $1.68 \mathrm{E}+02$ & $4.54 \mathrm{E}+03$ \\
\hline Eu-147 & $1.19 \mathrm{E}+02$ & $3.22 \mathrm{E}+03$ \\
\hline Eu-148 & $3.77 \mathrm{E}+01$ & $1.02 \mathrm{E}+03$ \\
\hline Eu-149 & $2.44 \mathrm{E}+02$ & $6.60 \mathrm{E}+03$ \\
\hline Eu-150 & $1.17 \mathrm{E}+00$ & $3.15 \mathrm{E}+01$ \\
\hline Eu-150m & $6.40 \mathrm{E}+02$ & $1.73 \mathrm{E}+04$ \\
\hline Eu-152 & $1.57 \mathrm{E}+00$ & $4.25 \mathrm{E}+01$ \\
\hline Eu-152m & $6.06 \mathrm{E}+02$ & $1.64 \mathrm{E}+04$ \\
\hline Eu-152n & $1.55 \mathrm{E}+04$ & $4.18 \mathrm{E}+05$ \\
\hline Eu-154 & $1.36 \mathrm{E}+00$ & $3.67 \mathrm{E}+01$ \\
\hline Eu-154m & $3.28 \mathrm{E}+04$ & $8.87 \mathrm{E}+05$ \\
\hline Eu-155 & $1.13 \mathrm{E}+01$ & $3.06 \mathrm{E}+02$ \\
\hline Eu-156 & $3.54 \mathrm{E}+01$ & $9.58 \mathrm{E}+02$ \\
\hline Eu-157 & $4.11 \mathrm{E}+02$ & $1.11 \mathrm{E}+04$ \\
\hline Eu-158 & $2.68 \mathrm{E}+03$ & $7.23 \mathrm{E}+04$ \\
\hline Eu-159 & $5.48 \mathrm{E}+03$ & $1.48 \mathrm{E}+05$ \\
\hline Gd-145 & $7.41 \mathrm{E}+03$ & $2.00 \mathrm{E}+05$ \\
\hline Gd-146 & $2.01 \mathrm{E}+01$ & $5.42 \mathrm{E}+02$ \\
\hline Gd-147 & $3.00 \mathrm{E}+02$ & $8.12 \mathrm{E}+03$ \\
\hline Gd-148 & $5.83 \mathrm{E}-03$ & $1.58 \mathrm{E}-01$ \\
\hline Gd-149 & $1.49 \mathrm{E}+02$ & $4.02 \mathrm{E}+03$ \\
\hline Gd-150 & $6.10 \mathrm{E}-03$ & $1.65 \mathrm{E}-01$ \\
\hline Gd-151 & $1.19 \mathrm{E}+02$ & $3.22 \mathrm{E}+03$ \\
\hline Gd-152 & $7.77 \mathrm{E}-03$ & $2.10 \mathrm{E}-01$ \\
\hline Gd-153 & $5.90 \mathrm{E}+01$ & $1.59 \mathrm{E}+03$ \\
\hline Gd-159 & $4.45 \mathrm{E}+02$ & $1.20 \mathrm{E}+04$ \\
\hline Tb-147 & $1.77 \mathrm{E}+03$ & $4.79 \mathrm{E}+04$ \\
\hline Tb-148 & $1.89 \mathrm{E}+03$ & $5.10 \mathrm{E}+04$ \\
\hline Tb-149 & $3.21 \mathrm{E}+01$ & $8.67 \mathrm{E}+02$ \\
\hline Tb-150 & $1.21 \mathrm{E}+03$ & $3.27 \mathrm{E}+04$ \\
\hline Tb-151 & $5.28 \mathrm{E}+02$ & $1.43 \mathrm{E}+04$ \\
\hline
\end{tabular}




\begin{tabular}{|c|c|c|}
\hline Nuclide & $\begin{array}{c}\text { Inhalation } \\
\text { DCS } \\
\left(\mathbf{B q} / \mathbf{m}^{3}\right)\end{array}$ & $\begin{array}{c}\text { Inhalation } \\
\text { DCS } \\
\left(\mathbf{p C i} / \mathbf{m}^{3}\right)\end{array}$ \\
\hline Tb-152 & $4.09 \mathrm{E}+02$ & $1.11 \mathrm{E}+04$ \\
\hline Tb-153 & $5.74 \mathrm{E}+02$ & $1.55 \mathrm{E}+04$ \\
\hline Tb-154 & $3.63 \mathrm{E}+02$ & $9.82 \mathrm{E}+03$ \\
\hline Tb-155 & $4.46 \mathrm{E}+02$ & $1.21 \mathrm{E}+04$ \\
\hline Tb-156 & $1.09 \mathrm{E}+02$ & $2.93 \mathrm{E}+03$ \\
\hline $\mathrm{Tb}-156 \mathrm{~m}$ & $6.71 \mathrm{E}+02$ & $1.81 \mathrm{E}+04$ \\
\hline $\mathrm{Tb}-156 \mathrm{n}$ & $1.22 \mathrm{E}+03$ & $3.30 \mathrm{E}+04$ \\
\hline Tb-157 & $4.57 \mathrm{E}+01$ & $1.23 \mathrm{E}+03$ \\
\hline Tb-158 & $1.42 \mathrm{E}+00$ & $3.84 \mathrm{E}+01$ \\
\hline Tb-160 & $1.70 \mathrm{E}+01$ & $4.61 \mathrm{E}+02$ \\
\hline Tb-161 & $9.83 \mathrm{E}+01$ & $2.66 \mathrm{E}+03$ \\
\hline Tb-163 & $7.70 \mathrm{E}+03$ & $2.08 \mathrm{E}+05$ \\
\hline Dy-151 & $1.03 \mathrm{E}+03$ & $2.80 \mathrm{E}+04$ \\
\hline Dy-152 & $2.09 \mathrm{E}+03$ & $5.66 \mathrm{E}+04$ \\
\hline Dy-153 & $1.03 \mathrm{E}+03$ & $2.80 \mathrm{E}+04$ \\
\hline Dy-154 & $5.70 \mathrm{E}-03$ & $1.54 \mathrm{E}-01$ \\
\hline Dy-155 & $1.43 \mathrm{E}+03$ & $3.87 \mathrm{E}+04$ \\
\hline Dy-157 & $4.14 \mathrm{E}+03$ & $1.12 \mathrm{E}+05$ \\
\hline Dy-159 & $2.90 \mathrm{E}+02$ & $7.85 \mathrm{E}+03$ \\
\hline Dy-165 & $2.08 \mathrm{E}+03$ & $5.62 \mathrm{E}+04$ \\
\hline Dy-166 & $6.51 \mathrm{E}+01$ & $1.76 \mathrm{E}+03$ \\
\hline Нo-154 & $8.59 \mathrm{E}+03$ & $2.32 \mathrm{E}+05$ \\
\hline Нo- 155 & $5.44 \mathrm{E}+03$ & $1.47 \mathrm{E}+05$ \\
\hline Но-156 & $2.38 \mathrm{E}+03$ & $6.44 \mathrm{E}+04$ \\
\hline Но-157 & $2.66 \mathrm{E}+04$ & $7.19 \mathrm{E}+05$ \\
\hline Но-159 & $1.97 \mathrm{E}+04$ & $5.31 \mathrm{E}+05$ \\
\hline Но- 160 & $9.59 \mathrm{E}+03$ & $2.59 \mathrm{E}+05$ \\
\hline Но-161 & $1.74 \mathrm{E}+04$ & $4.70 \mathrm{E}+05$ \\
\hline Ho-162 & $4.56 \mathrm{E}+04$ & $1.23 \mathrm{E}+06$ \\
\hline Ho-162m & $6.23 \mathrm{E}+03$ & $1.68 \mathrm{E}+05$ \\
\hline Ho-163 & $5.54 \mathrm{E}+02$ & $1.50 \mathrm{E}+04$ \\
\hline Но-164 & $1.53 \mathrm{E}+04$ & $4.14 \mathrm{E}+05$ \\
\hline Ho- $164 \mathrm{~m}$ & $1.12 \mathrm{E}+04$ & $3.04 \mathrm{E}+05$ \\
\hline Ho-166 & $1.86 \mathrm{E}+02$ & $5.02 \mathrm{E}+03$ \\
\hline Но-166m & $5.28 \mathrm{E}-01$ & $1.43 \mathrm{E}+01$ \\
\hline Ho-167 & $1.71 \mathrm{E}+03$ & $4.63 E+04$ \\
\hline Er-156 & $6.57 \mathrm{E}+03$ & $1.77 \mathrm{E}+05$ \\
\hline Er-159 & $7.55 \mathrm{E}+03$ & $2.04 \mathrm{E}+05$ \\
\hline
\end{tabular}




\begin{tabular}{|c|c|c|}
\hline Nuclide & $\begin{array}{c}\text { Inhalation } \\
\text { DCS } \\
\left(\mathbf{B q} / \mathbf{m}^{3}\right)\end{array}$ & $\begin{array}{c}\text { Inhalation } \\
\text { DCS } \\
\left(\mathbf{p C i} / \mathbf{m}^{3}\right)\end{array}$ \\
\hline Er-161 & $2.43 \mathrm{E}+03$ & $6.57 \mathrm{E}+04$ \\
\hline Er-163 & $1.00 \mathrm{E}+05$ & $2.71 \mathrm{E}+06$ \\
\hline Er-165 & $1.50 \mathrm{E}+04$ & $4.06 \mathrm{E}+05$ \\
\hline Er-169 & $1.22 \mathrm{E}+02$ & $3.30 \mathrm{E}+03$ \\
\hline Er-171 & $5.79 E+02$ & $1.56 \mathrm{E}+04$ \\
\hline Er-172 & $1.12 \mathrm{E}+02$ & $3.04 \mathrm{E}+03$ \\
\hline Tm-161 & $5.11 \mathrm{E}+03$ & $1.38 \mathrm{E}+05$ \\
\hline Tm-162 & $6.51 \mathrm{E}+03$ & $1.76 \mathrm{E}+05$ \\
\hline Tm-163 & $3.45 \mathrm{E}+03$ & $9.32 \mathrm{E}+04$ \\
\hline Tm-165 & $5.70 \mathrm{E}+02$ & $1.54 \mathrm{E}+04$ \\
\hline Tm-166 & $7.17 \mathrm{E}+02$ & $1.94 \mathrm{E}+04$ \\
\hline Tm-167 & $1.06 \mathrm{E}+02$ & $2.87 \mathrm{E}+03$ \\
\hline Tm-168 & $2.79 \mathrm{E}+01$ & $7.54 \mathrm{E}+02$ \\
\hline Tm-170 & $1.55 \mathrm{E}+01$ & $4.18 \mathrm{E}+02$ \\
\hline Tm-171 & $6.30 \mathrm{E}+01$ & $1.70 \mathrm{E}+03$ \\
\hline Tm-172 & $1.10 \mathrm{E}+02$ & $2.97 \mathrm{E}+03$ \\
\hline Tm-173 & $6.94 \mathrm{E}+02$ & $1.88 \mathrm{E}+04$ \\
\hline Tm-175 & $6.91 \mathrm{E}+03$ & $1.87 \mathrm{E}+05$ \\
\hline Yb-162 & $7.48 \mathrm{E}+03$ & $2.02 \mathrm{E}+05$ \\
\hline Yb-163 & $1.53 \mathrm{E}+04$ & $4.14 \mathrm{E}+05$ \\
\hline Yb-164 & $2.94 \mathrm{E}+03$ & $7.95 \mathrm{E}+04$ \\
\hline Yb-166 & $1.76 \mathrm{E}+02$ & $4.74 \mathrm{E}+03$ \\
\hline Yb-167 & $1.93 \mathrm{E}+04$ & $5.22 \mathrm{E}+05$ \\
\hline Yb-169 & $4.16 \mathrm{E}+01$ & $1.12 \mathrm{E}+03$ \\
\hline Yb-175 & $1.93 \mathrm{E}+02$ & $5.21 \mathrm{E}+03$ \\
\hline Yb-177 & $1.85 \mathrm{E}+03$ & $5.00 \mathrm{E}+04$ \\
\hline Yb-178 & $1.81 \mathrm{E}+03$ & $4.89 \mathrm{E}+04$ \\
\hline Lu-165 & $9.59 \mathrm{E}+03$ & $2.59 \mathrm{E}+05$ \\
\hline Lu-167 & $3.43 \mathrm{E}+03$ & $9.26 \mathrm{E}+04$ \\
\hline Lu-169 & $3.03 \mathrm{E}+02$ & $8.18 \mathrm{E}+03$ \\
\hline Lu-170 & $2.06 \mathrm{E}+02$ & $5.58 \mathrm{E}+03$ \\
\hline Lu-171 & $1.41 \mathrm{E}+02$ & $3.80 \mathrm{E}+03$ \\
\hline Lu-172 & $8.68 \mathrm{E}+01$ & $2.35 \mathrm{E}+03$ \\
\hline Lu-173 & $2.96 \mathrm{E}+01$ & $8.01 \mathrm{E}+02$ \\
\hline Lu-174 & $1.93 \mathrm{E}+01$ & $5.21 \mathrm{E}+02$ \\
\hline Lu-174m & $3.22 \mathrm{E}+01$ & $8.69 \mathrm{E}+02$ \\
\hline Lu-176 & $9.70 \mathrm{E}-01$ & $2.62 \mathrm{E}+01$ \\
\hline Lu-176m & $1.18 \mathrm{E}+03$ & $3.20 \mathrm{E}+04$ \\
\hline
\end{tabular}




\begin{tabular}{|c|c|c|}
\hline Nuclide & $\begin{array}{c}\text { Inhalation } \\
\text { DCS } \\
\text { (Bq/m }\end{array}$ & $\begin{array}{c}\text { Inhalation } \\
\text { DCS } \\
\text { (pCi/m }^{3} \text { ) }\end{array}$ \\
\hline Lu-177 & $1.18 \mathrm{E}+02$ & $3.20 \mathrm{E}+03$ \\
\hline Lu-177m & $8.88 \mathrm{E}+00$ & $2.40 \mathrm{E}+02$ \\
\hline Lu-178 & $5.30 \mathrm{E}+03$ & $1.43 \mathrm{E}+05$ \\
\hline Lu-178m & $4.21 \mathrm{E}+03$ & $1.14 \mathrm{E}+05$ \\
\hline Lu-179 & $1.18 \mathrm{E}+03$ & $3.20 \mathrm{E}+04$ \\
\hline Hf-170 & $4.58 \mathrm{E}+02$ & $1.24 \mathrm{E}+04$ \\
\hline Hf-172 & $4.22 \mathrm{E}+00$ & $1.14 \mathrm{E}+02$ \\
\hline Hf-173 & $7.70 \mathrm{E}+02$ & $2.08 \mathrm{E}+04$ \\
\hline Hf-174 & $4.88 \mathrm{E}-03$ & $1.32 \mathrm{E}-01$ \\
\hline Hf-175 & $1.01 \mathrm{E}+02$ & $2.72 \mathrm{E}+03$ \\
\hline Hf-177m & $1.41 \mathrm{E}+03$ & $3.80 \mathrm{E}+04$ \\
\hline Hf-178m & $6.79 \mathrm{E}-01$ & $1.84 \mathrm{E}+01$ \\
\hline Hf-179m & $3.19 \mathrm{E}+01$ & $8.62 \mathrm{E}+02$ \\
\hline Hf-180m & $9.36 \mathrm{E}+02$ & $2.53 \mathrm{E}+04$ \\
\hline Hf-181 & $2.40 \mathrm{E}+01$ & $6.48 \mathrm{E}+02$ \\
\hline Hf-182 & $5.14 \mathrm{E}-01$ & $1.39 \mathrm{E}+01$ \\
\hline Hf-182m & $2.56 \mathrm{E}+03$ & $6.91 \mathrm{E}+04$ \\
\hline Hf-183 & $2.14 \mathrm{E}+03$ & $5.78 \mathrm{E}+04$ \\
\hline Hf-184 & $3.83 \mathrm{E}+02$ & $1.04 \mathrm{E}+04$ \\
\hline Ta-172 & $3.63 \mathrm{E}+03$ & $9.82 \mathrm{E}+04$ \\
\hline Ta-173 & $1.67 \mathrm{E}+03$ & $4.51 \mathrm{E}+04$ \\
\hline Ta-174 & $2.74 \mathrm{E}+03$ & $7.40 \mathrm{E}+04$ \\
\hline Ta-175 & $8.73 \mathrm{E}+02$ & $2.36 \mathrm{E}+04$ \\
\hline Ta-176 & $6.48 \mathrm{E}+02$ & $1.75 \mathrm{E}+04$ \\
\hline Ta-177 & $1.28 \mathrm{E}+03$ & $3.46 \mathrm{E}+04$ \\
\hline Ta-178m & $1.74 \mathrm{E}+03$ & $4.71 \mathrm{E}+04$ \\
\hline Ta-179 & $2.86 \mathrm{E}+02$ & $7.73 \mathrm{E}+03$ \\
\hline Ta-180 & $3.02 \mathrm{E}+03$ & $8.15 \mathrm{E}+04$ \\
\hline Ta-182 & $1.37 \mathrm{E}+01$ & $3.70 \mathrm{E}+02$ \\
\hline Ta-182m & $6.01 \mathrm{E}+03$ & $1.62 \mathrm{E}+05$ \\
\hline Ta-183 & $6.25 \mathrm{E}+01$ & $1.69 \mathrm{E}+03$ \\
\hline Ta-184 & $3.06 \mathrm{E}+02$ & $8.28 \mathrm{E}+03$ \\
\hline Ta-185 & $2.69 \mathrm{E}+03$ & $7.28 \mathrm{E}+04$ \\
\hline Ta-186 & $7.30 \mathrm{E}+03$ & $1.97 \mathrm{E}+05$ \\
\hline W-177 & $2.99 \mathrm{E}+03$ & $8.09 \mathrm{E}+04$ \\
\hline W-178 & $1.68 \mathrm{E}+02$ & $4.55 \mathrm{E}+03$ \\
\hline W-179 & $9.65 \mathrm{E}+04$ & $2.61 \mathrm{E}+06$ \\
\hline W-181 & $4.81 \mathrm{E}+02$ & $1.30 \mathrm{E}+04$ \\
\hline & & \\
\hline
\end{tabular}




\begin{tabular}{|c|c|c|}
\hline Nuclide & $\begin{array}{c}\text { Inhalation } \\
\text { DCS } \\
\left(\mathbf{B q} / \mathbf{m}^{\mathbf{3}}\right)\end{array}$ & $\begin{array}{c}\text { Inhalation } \\
\text { DCS } \\
\left(\mathbf{p C i} / \mathbf{m}^{3}\right)\end{array}$ \\
\hline W-185 & $3.69 \mathrm{E}+01$ & $9.96 \mathrm{E}+02$ \\
\hline W-187 & $3.23 \mathrm{E}+02$ & $8.74 \mathrm{E}+03$ \\
\hline W-188 & $9.08 \mathrm{E}+00$ & $2.46 \mathrm{E}+02$ \\
\hline W-190 & $1.83 \mathrm{E}+03$ & $4.94 \mathrm{E}+04$ \\
\hline Re-178 & $8.22 \mathrm{E}+03$ & $2.22 \mathrm{E}+05$ \\
\hline Re-179 & $1.18 \mathrm{E}+04$ & $3.20 \mathrm{E}+05$ \\
\hline Re-181 & $4.98 \mathrm{E}+02$ & $1.34 \mathrm{E}+04$ \\
\hline Re-182 & $1.06 \mathrm{E}+02$ & $2.85 \mathrm{E}+03$ \\
\hline Re-182m & $6.10 \mathrm{E}+02$ & $1.65 \mathrm{E}+04$ \\
\hline Re-183 & $3.97 \mathrm{E}+01$ & $1.07 \mathrm{E}+03$ \\
\hline Re-184 & $6.08 \mathrm{E}+01$ & $1.64 \mathrm{E}+03$ \\
\hline Re-184m & $1.40 \mathrm{E}+01$ & $3.77 \mathrm{E}+02$ \\
\hline Re-186 & $1.19 \mathrm{E}+02$ & $3.22 \mathrm{E}+03$ \\
\hline Re-186m & $2.43 \mathrm{E}+00$ & $6.57 \mathrm{E}+01$ \\
\hline Re-187 & $3.67 \mathrm{E}+03$ & $9.91 \mathrm{E}+04$ \\
\hline Re-188 & $2.34 \mathrm{E}+02$ & $6.32 \mathrm{E}+03$ \\
\hline Re-188m & $8.83 \mathrm{E}+03$ & $2.39 \mathrm{E}+05$ \\
\hline Re-189 & $3.09 \mathrm{E}+02$ & $8.35 \mathrm{E}+03$ \\
\hline Re-190m & $6.40 \mathrm{E}+02$ & $1.73 E+04$ \\
\hline Os-180 & $8.83 \mathrm{E}+03$ & $2.39 \mathrm{E}+05$ \\
\hline Os-181 & $2.07 \mathrm{E}+03$ & $5.60 \mathrm{E}+04$ \\
\hline Os-182 & $3.33 \mathrm{E}+02$ & $9.00 \mathrm{E}+03$ \\
\hline Os-183 & $7.10 \mathrm{E}+02$ & $1.92 \mathrm{E}+04$ \\
\hline Os-183m & $8.98 \mathrm{E}+02$ & $2.43 \mathrm{E}+04$ \\
\hline Os-185 & $9.03 \mathrm{E}+01$ & $2.44 \mathrm{E}+03$ \\
\hline Os-186 & $3.46 \mathrm{E}-02$ & $9.36 \mathrm{E}-01$ \\
\hline Os-189m & $2.43 \mathrm{E}+04$ & $6.58 \mathrm{E}+05$ \\
\hline Os-191 & $7.07 \mathrm{E}+01$ & $1.91 \mathrm{E}+03$ \\
\hline Os-191m & $8.49 \mathrm{E}+02$ & $2.30 \mathrm{E}+04$ \\
\hline Os-193 & $2.47 \mathrm{E}+02$ & $6.68 \mathrm{E}+03$ \\
\hline Os-194 & $1.72 \mathrm{E}+00$ & $4.65 \mathrm{E}+01$ \\
\hline Os-196 & $2.35 \mathrm{E}+03$ & $6.36 \mathrm{E}+04$ \\
\hline Ir-182 & $5.09 \mathrm{E}+03$ & $1.38 \mathrm{E}+05$ \\
\hline Ir-183 & $3.32 \mathrm{E}+03$ & $8.97 \mathrm{E}+04$ \\
\hline Ir-184 & $1.05 \mathrm{E}+03$ & $2.83 \mathrm{E}+04$ \\
\hline Ir-185 & $5.66 \mathrm{E}+02$ & $1.53 \mathrm{E}+04$ \\
\hline Ir-186 & $3.71 \mathrm{E}+02$ & $1.00 \mathrm{E}+04$ \\
\hline Ir-186m & $2.62 \mathrm{E}+03$ & $7.07 \mathrm{E}+04$ \\
\hline
\end{tabular}




\begin{tabular}{|c|c|c|}
\hline Nuclide & $\begin{array}{c}\text { Inhalation } \\
\text { DCS } \\
\left(\mathbf{B q} / \mathbf{m}^{\mathbf{3}}\right)\end{array}$ & $\begin{array}{c}\text { Inhalation } \\
\text { DCS } \\
\left(\mathbf{p C i} / \mathbf{m}^{3}\right)\end{array}$ \\
\hline Ir-187 & $1.72 \mathrm{E}+03$ & $4.64 \mathrm{E}+04$ \\
\hline Ir-188 & $2.74 \mathrm{E}+02$ & $7.40 \mathrm{E}+03$ \\
\hline Ir-189 & $2.50 \mathrm{E}+02$ & $6.75 \mathrm{E}+03$ \\
\hline Ir-190 & $1.00 \mathrm{E}+02$ & $2.71 \mathrm{E}+03$ \\
\hline $\mathrm{Ir}-190 \mathrm{~m}$ & $2.24 \mathrm{E}+04$ & $6.05 \mathrm{E}+05$ \\
\hline Ir-190n & $1.57 \mathrm{E}+03$ & $4.25 \mathrm{E}+04$ \\
\hline Ir-192 & $2.14 \mathrm{E}+01$ & $5.78 \mathrm{E}+02$ \\
\hline Ir-192n & $2.55 \mathrm{E}+00$ & $6.90 \mathrm{E}+01$ \\
\hline Ir-193m & $1.16 \mathrm{E}+02$ & $3.13 \mathrm{E}+03$ \\
\hline Ir-194 & $2.31 \mathrm{E}+02$ & $6.26 \mathrm{E}+03$ \\
\hline Ir-194m & $1.17 \mathrm{E}+01$ & $3.18 \mathrm{E}+02$ \\
\hline Ir-195 & $1.88 \mathrm{E}+03$ & $5.08 \mathrm{E}+04$ \\
\hline Ir-195m & $1.06 \mathrm{E}+03$ & $2.87 \mathrm{E}+04$ \\
\hline Pt-184 & $5.11 \mathrm{E}+03$ & $1.38 \mathrm{E}+05$ \\
\hline Pt-186 & $1.85 \mathrm{E}+03$ & $4.99 \mathrm{E}+04$ \\
\hline Pt-187 & $1.88 \mathrm{E}+03$ & $5.09 \mathrm{E}+04$ \\
\hline Pt-188 & $6.85 \mathrm{E}+01$ & $1.85 \mathrm{E}+03$ \\
\hline Pt-189 & $7.58 \mathrm{E}+02$ & $2.05 \mathrm{E}+04$ \\
\hline Pt-190 & $2.80 \mathrm{E}-02$ & 7.57E-01 \\
\hline Pt-191 & $3.71 \mathrm{E}+02$ & $1.00 \mathrm{E}+04$ \\
\hline Pt-193 & $2.15 \mathrm{E}+02$ & $5.81 \mathrm{E}+03$ \\
\hline Pt-193m & $1.45 \mathrm{E}+02$ & $3.91 \mathrm{E}+03$ \\
\hline Pt-195m & $1.21 \mathrm{E}+02$ & $3.27 \mathrm{E}+03$ \\
\hline Pt-197 & $3.63 \mathrm{E}+02$ & $9.80 \mathrm{E}+03$ \\
\hline Pt-197m & $1.70 \mathrm{E}+03$ & $4.59 E+04$ \\
\hline Pt-199 & $4.62 \mathrm{E}+03$ & $1.25 \mathrm{E}+05$ \\
\hline Pt-202 & $5.99 \mathrm{E}+01$ & $1.62 \mathrm{E}+03$ \\
\hline $\mathrm{Au}-186$ & $6.43 \mathrm{E}+03$ & $1.74 \mathrm{E}+05$ \\
\hline $\mathrm{Au}-190$ & $5.39 \mathrm{E}+03$ & $1.46 \mathrm{E}+05$ \\
\hline $\mathrm{Au}-191$ & $2.07 \mathrm{E}+03$ & $5.59 \mathrm{E}+04$ \\
\hline $\mathrm{Au}-192$ & $1.36 \mathrm{E}+03$ & $3.67 \mathrm{E}+04$ \\
\hline $\mathrm{Au}-193$ & $1.27 \mathrm{E}+03$ & $3.43 \mathrm{E}+04$ \\
\hline Au-194 & $5.60 \mathrm{E}+02$ & $1.51 \mathrm{E}+04$ \\
\hline $\mathrm{Au}-195$ & $7.81 \mathrm{E}+01$ & $2.11 \mathrm{E}+03$ \\
\hline Au-196 & $4.13 \mathrm{E}+02$ & $1.12 \mathrm{E}+04$ \\
\hline $\mathrm{Au}-196 \mathrm{~m}$ & $3.13 \mathrm{E}+02$ & $8.45 \mathrm{E}+03$ \\
\hline Au-198 & $1.59 \mathrm{E}+02$ & $4.29 \mathrm{E}+03$ \\
\hline $\mathrm{Au}-198 \mathrm{~m}$ & $7.62 \mathrm{E}+01$ & $2.06 \mathrm{E}+03$ \\
\hline
\end{tabular}




\begin{tabular}{|c|c|c|}
\hline Nuclide & $\begin{array}{c}\text { Inhalation } \\
\text { DCS } \\
\left(\mathbf{B q} / \mathbf{m}^{3}\right)\end{array}$ & $\begin{array}{c}\text { Inhalation } \\
\text { DCS } \\
\left(\mathbf{p C i} / \mathbf{m}^{3}\right)\end{array}$ \\
\hline Au-199 & $1.74 \mathrm{E}+02$ & $4.70 \mathrm{E}+03$ \\
\hline $\mathrm{Au}-200$ & $3.77 \mathrm{E}+03$ & $1.02 \mathrm{E}+05$ \\
\hline $\mathrm{Au}-200 \mathrm{~m}$ & $1.97 \mathrm{E}+02$ & $5.31 \mathrm{E}+03$ \\
\hline $\mathrm{Au}-201$ & $7.70 \mathrm{E}+03$ & $2.08 \mathrm{E}+05$ \\
\hline Hg-190 & $1.88 \mathrm{E}+03$ & $5.07 \mathrm{E}+04$ \\
\hline $\mathrm{Hg}-191 \mathrm{~m}$ & $4.50 \mathrm{E}+02$ & $1.22 \mathrm{E}+04$ \\
\hline Hg-192 & $1.45 \mathrm{E}+02$ & $3.91 \mathrm{E}+03$ \\
\hline $\mathrm{Hg}-193$ & $1.66 \mathrm{E}+02$ & $4.47 \mathrm{E}+03$ \\
\hline Hg-193m & $4.81 \mathrm{E}+01$ & $1.30 \mathrm{E}+03$ \\
\hline Hg-194 & $3.68 \mathrm{E}+00$ & $9.94 \mathrm{E}+01$ \\
\hline Hg-195 & $9.59 \mathrm{E}+01$ & $2.59 \mathrm{E}+03$ \\
\hline $\mathrm{Hg}-195 \mathrm{~m}$ & $1.78 \mathrm{E}+01$ & $4.80 \mathrm{E}+02$ \\
\hline $\mathrm{Hg}-197$ & $3.09 \mathrm{E}+01$ & $8.35 \mathrm{E}+02$ \\
\hline $\mathrm{Hg}-197 \mathrm{~m}$ & $2.45 \mathrm{E}+01$ & $6.62 \mathrm{E}+02$ \\
\hline Hg-199m & $8.18 \mathrm{E}+02$ & $2.21 \mathrm{E}+04$ \\
\hline $\mathrm{Hg}-203$ & $2.03 \mathrm{E}+01$ & $5.48 \mathrm{E}+02$ \\
\hline T1-194 & $6.79 \mathrm{E}+03$ & $1.84 \mathrm{E}+05$ \\
\hline Tl-194m & $4.41 \mathrm{E}+03$ & $1.19 E+05$ \\
\hline T1-195 & $5.24 \mathrm{E}+03$ & $1.42 \mathrm{E}+05$ \\
\hline T1-196 & $3.32 \mathrm{E}+03$ & $8.99 \mathrm{E}+04$ \\
\hline T1-197 & $3.47 \mathrm{E}+03$ & $9.38 \mathrm{E}+04$ \\
\hline T1-198 & $1.71 \mathrm{E}+03$ & $4.63 E+04$ \\
\hline Tl-198m & $1.93 \mathrm{E}+03$ & $5.22 \mathrm{E}+04$ \\
\hline T1-199 & $2.69 \mathrm{E}+03$ & $7.27 \mathrm{E}+04$ \\
\hline T1-200 & $7.30 \mathrm{E}+02$ & $1.97 \mathrm{E}+04$ \\
\hline T1-201 & $7.10 \mathrm{E}+02$ & $1.92 \mathrm{E}+04$ \\
\hline T1-202 & $3.40 \mathrm{E}+02$ & $9.20 \mathrm{E}+03$ \\
\hline T1-204 & $7.62 \mathrm{E}+00$ & $2.06 \mathrm{E}+02$ \\
\hline $\mathrm{Pb}-194$ & $1.03 \mathrm{E}+04$ & $2.80 \mathrm{E}+05$ \\
\hline $\mathrm{Pb}-195 \mathrm{~m}$ & $5.96 \mathrm{E}+03$ & $1.61 \mathrm{E}+05$ \\
\hline $\mathrm{Pb}-196$ & $5.43 \mathrm{E}+03$ & $1.47 \mathrm{E}+05$ \\
\hline $\mathrm{Pb}-197 \mathrm{~m}$ & $2.96 \mathrm{E}+03$ & $8.01 \mathrm{E}+04$ \\
\hline $\mathrm{Pb}-198$ & $2.12 \mathrm{E}+03$ & $5.73 \mathrm{E}+04$ \\
\hline $\mathrm{Pb}-199$ & $4.50 \mathrm{E}+03$ & $1.22 \mathrm{E}+05$ \\
\hline $\mathrm{Pb}-200$ & $4.14 \mathrm{E}+02$ & $1.12 \mathrm{E}+04$ \\
\hline $\mathrm{Pb}-201$ & $1.17 \mathrm{E}+03$ & $3.18 \mathrm{E}+04$ \\
\hline $\mathrm{Pb}-202$ & $7.93 \mathrm{E}+00$ & $2.14 \mathrm{E}+02$ \\
\hline $\mathrm{Pb}-202 \mathrm{~m}$ & $1.32 \mathrm{E}+03$ & $3.58 \mathrm{E}+04$ \\
\hline
\end{tabular}




\begin{tabular}{|c|c|c|}
\hline Nuclide & $\begin{array}{c}\text { Inhalation } \\
\text { DCS } \\
\left(\mathbf{B q} / \mathbf{m}^{3}\right)\end{array}$ & $\begin{array}{c}\text { Inhalation } \\
\text { DCS } \\
\left(\mathbf{p C i} / \mathbf{m}^{3}\right)\end{array}$ \\
\hline $\mathrm{Pb}-203$ & $6.54 \mathrm{E}+02$ & $1.77 \mathrm{E}+04$ \\
\hline $\mathrm{Pb}-204 \mathrm{~m}$ & $4.54 \mathrm{E}+03$ & $1.23 \mathrm{E}+05$ \\
\hline $\mathrm{Pb}-205$ & $6.06 \mathrm{E}+02$ & $1.64 \mathrm{E}+04$ \\
\hline $\mathrm{Pb}-209$ & $2.42 \mathrm{E}+03$ & $6.54 \mathrm{E}+04$ \\
\hline $\mathrm{Pb}-210$ & $1.29 \mathrm{E}-01$ & $3.49 \mathrm{E}+00$ \\
\hline $\mathrm{Pb}-211$ & $1.24 \mathrm{E}+01$ & $3.35 \mathrm{E}+02$ \\
\hline $\mathrm{Pb}-212$ & $8.40 \mathrm{E}-01$ & $2.27 \mathrm{E}+01$ \\
\hline $\mathrm{Pb}-214$ & $1.06 \mathrm{E}+01$ & $2.87 \mathrm{E}+02$ \\
\hline $\mathrm{Bi}-200$ & $3.56 \mathrm{E}+03$ & $9.62 \mathrm{E}+04$ \\
\hline $\mathrm{Bi}-201$ & $1.74 \mathrm{E}+03$ & $4.70 \mathrm{E}+04$ \\
\hline Bi-202 & $2.08 \mathrm{E}+03$ & $5.61 \mathrm{E}+04$ \\
\hline Bi-203 & $4.57 \mathrm{E}+02$ & $1.23 \mathrm{E}+04$ \\
\hline Bi-204 & $4.10 \mathrm{E}+02$ & $1.11 \mathrm{E}+04$ \\
\hline Bi-205 & $1.27 \mathrm{E}+02$ & $3.43 \mathrm{E}+03$ \\
\hline Bi-206 & $7.27 \mathrm{E}+01$ & $1.96 \mathrm{E}+03$ \\
\hline Bi-207 & $3.83 \mathrm{E}+00$ & $1.04 \mathrm{E}+02$ \\
\hline Bi-208 & $4.08 \mathrm{E}+00$ & $1.10 \mathrm{E}+02$ \\
\hline $\mathrm{Bi}-210$ & $1.07 \mathrm{E}+00$ & $2.89 \mathrm{E}+01$ \\
\hline Bi-210m & $1.46 \mathrm{E}-02$ & $3.95 \mathrm{E}-01$ \\
\hline Bi-212 & $4.26 \mathrm{E}+00$ & $1.15 \mathrm{E}+02$ \\
\hline $\mathrm{Bi}-213$ & $4.40 \mathrm{E}+00$ & $1.19 \mathrm{E}+02$ \\
\hline $\mathrm{Bi}-214$ & $9.08 \mathrm{E}+00$ & $2.46 \mathrm{E}+02$ \\
\hline Po-203 & $3.03 \mathrm{E}+03$ & $8.18 \mathrm{E}+04$ \\
\hline Po-204 & $3.69 \mathrm{E}+02$ & $9.96 \mathrm{E}+03$ \\
\hline Po-205 & $3.00 \mathrm{E}+03$ & $8.11 \mathrm{E}+04$ \\
\hline Po-206 & $2.49 \mathrm{E}+00$ & $6.74 \mathrm{E}+01$ \\
\hline Po-207 & $1.49 \mathrm{E}+03$ & $4.02 \mathrm{E}+04$ \\
\hline Рo-208 & $3.76 \mathrm{E}-02$ & $1.02 \mathrm{E}+00$ \\
\hline Po-209 & $3.89 \mathrm{E}-02$ & $1.05 \mathrm{E}+00$ \\
\hline Рo-210 & 4.34E-02 & $1.17 \mathrm{E}+00$ \\
\hline At-205 & $2.00 \mathrm{E}+02$ & $5.39 \mathrm{E}+03$ \\
\hline At-206 & $5.96 \mathrm{E}+02$ & $1.61 \mathrm{E}+04$ \\
\hline At-207 & $6.48 \mathrm{E}+01$ & $1.75 \mathrm{E}+03$ \\
\hline At-208 & $2.38 \mathrm{E}+02$ & $6.44 \mathrm{E}+03$ \\
\hline At-209 & $5.14 \mathrm{E}+01$ & $1.39 E+03$ \\
\hline At-210 & $1.34 \mathrm{E}+01$ & $3.61 \mathrm{E}+02$ \\
\hline At-211 & $1.20 \mathrm{E}+00$ & $3.25 \mathrm{E}+01$ \\
\hline Fr-212 & $2.24 \mathrm{E}+01$ & $6.05 \mathrm{E}+02$ \\
\hline
\end{tabular}

Page 226 of 298 


\begin{tabular}{|c|c|c|}
\hline Nuclide & $\begin{array}{c}\text { Inhalation } \\
\text { DCS } \\
\left(\mathbf{B q} / \mathbf{m}^{3}\right)\end{array}$ & $\begin{array}{c}\text { Inhalation } \\
\text { DCS } \\
\left(\mathbf{p C i} / \mathbf{m}^{3}\right)\end{array}$ \\
\hline Fr-222 & $5.07 \mathrm{E}+00$ & $1.37 \mathrm{E}+02$ \\
\hline Fr-223 & $1.17 \mathrm{E}+01$ & $3.18 \mathrm{E}+02$ \\
\hline Ra-223 & $1.94 \mathrm{E}-02$ & $5.25 \mathrm{E}-01$ \\
\hline $\mathrm{Ra}-224$ & $4.85 \mathrm{E}-02$ & $1.31 \mathrm{E}+00$ \\
\hline Ra-225 & 2.29E-02 & $6.18 \mathrm{E}-01$ \\
\hline Ra-226 & 4.09E-02 & $1.11 \mathrm{E}+00$ \\
\hline Ra-227 & $5.44 \mathrm{E}+02$ & $1.47 \mathrm{E}+04$ \\
\hline $\mathrm{Ra}-228$ & $5.07 \mathrm{E}-02$ & $1.37 \mathrm{E}+00$ \\
\hline Ra-230 & $1.21 \mathrm{E}+03$ & $3.27 \mathrm{E}+04$ \\
\hline Ac-224 & $1.26 \mathrm{E}+00$ & $3.41 \mathrm{E}+01$ \\
\hline Ac-225 & $1.70 \mathrm{E}-02$ & $4.60 \mathrm{E}-01$ \\
\hline Ac- 226 & $1.11 \mathrm{E}-01$ & $3.00 \mathrm{E}+00$ \\
\hline Ac- 227 & $9.70 \mathrm{E}-04$ & $2.62 \mathrm{E}-02$ \\
\hline Ac-228 & $9.70 \mathrm{E}+00$ & $2.62 \mathrm{E}+02$ \\
\hline Th-228 & $3.59 \mathrm{E}-03$ & $9.71 \mathrm{E}-02$ \\
\hline Th-229 & $2.07 \mathrm{E}-03$ & $5.59 \mathrm{E}-02$ \\
\hline Th-230 & $1.06 \mathrm{E}-02$ & $2.87 \mathrm{E}-01$ \\
\hline Th-232 & $6.10 \mathrm{E}-03$ & $1.65 \mathrm{E}-01$ \\
\hline Th-234 & $1.82 \mathrm{E}+01$ & $4.91 \mathrm{E}+02$ \\
\hline $\mathrm{Pa}-227$ & $1.82 \mathrm{E}+00$ & $4.93 \mathrm{E}+01$ \\
\hline $\mathrm{Pa}-228$ & $2.01 \mathrm{E}+00$ & $5.43 \mathrm{E}+01$ \\
\hline $\mathrm{Pa}-229$ & $2.01 \mathrm{E}+01$ & $5.43 \mathrm{E}+02$ \\
\hline $\mathrm{Pa}-230$ & $2.13 \mathrm{E}-01$ & $5.75 \mathrm{E}+00$ \\
\hline $\mathrm{Pa}-231$ & $6.59 \mathrm{E}-04$ & $1.78 \mathrm{E}-02$ \\
\hline $\mathrm{Pa}-232$ & $6.01 \mathrm{E}+01$ & $1.62 \mathrm{E}+03$ \\
\hline $\mathrm{Pa}-233$ & $3.43 \mathrm{E}+01$ & $9.26 \mathrm{E}+02$ \\
\hline $\mathrm{Pa}-234$ & $3.93 \mathrm{E}+02$ & $1.06 \mathrm{E}+04$ \\
\hline $\mathrm{Pa}-235$ & $7.89 \mathrm{E}+03$ & $2.13 \mathrm{E}+05$ \\
\hline U-230 & $1.06 \mathrm{E}-02$ & $2.87 \mathrm{E}-01$ \\
\hline U-231 & $2.95 \mathrm{E}+02$ & $7.97 \mathrm{E}+03$ \\
\hline U-232 & $1.81 \mathrm{E}-02$ & $4.90 \mathrm{E}-01$ \\
\hline U-233 & $4.02 \mathrm{E}-02$ & $1.09 \mathrm{E}+00$ \\
\hline U-234 & $4.10 \mathrm{E}-02$ & $1.11 \mathrm{E}+00$ \\
\hline U-235 & $4.62 \mathrm{E}-02$ & $1.25 \mathrm{E}+00$ \\
\hline $\mathrm{U}-235 \mathrm{~m}$ & $1.73 \mathrm{E}+08$ & $4.69 E+09$ \\
\hline U-236 & 4.48E-02 & $1.21 \mathrm{E}+00$ \\
\hline U-237 & $8.49 \mathrm{E}+01$ & $2.30 \mathrm{E}+03$ \\
\hline U-238 & $4.98 \mathrm{E}-02$ & $1.34 \mathrm{E}+00$ \\
\hline
\end{tabular}




\begin{tabular}{|c|c|c|}
\hline Nuclide & $\begin{array}{c}\text { Inhalation } \\
\text { DCS } \\
\text { (Bq/m }\end{array}$ & $\begin{array}{c}\text { Inhalation } \\
\text { DCS } \\
\text { (pCi/m }\end{array}$ \\
\hline $\mathrm{U}-239$ & $5.96 \mathrm{E}+03$ & $1.61 \mathrm{E}+05$ \\
\hline $\mathrm{U}-240$ & $2.53 \mathrm{E}+02$ & $6.84 \mathrm{E}+03$ \\
\hline $\mathrm{U}-242$ & $4.87 \mathrm{E}+03$ & $1.32 \mathrm{E}+05$ \\
\hline $\mathrm{Np}-232$ & $3.00 \mathrm{E}+03$ & $8.12 \mathrm{E}+04$ \\
\hline $\mathrm{Np}-233$ & $7.10 \mathrm{E}+04$ & $1.92 \mathrm{E}+06$ \\
\hline $\mathrm{Np}-234$ & $3.07 \mathrm{E}+02$ & $8.30 \mathrm{E}+03$ \\
\hline $\mathrm{Np}-235$ & $2.89 \mathrm{E}+02$ & $7.81 \mathrm{E}+03$ \\
\hline $\mathrm{Np}-236$ & $3.46 \mathrm{E}-02$ & $9.36 \mathrm{E}-01$ \\
\hline $\mathrm{Np}-236 \mathrm{~m}$ & $2.75 \mathrm{E}+01$ & $7.43 \mathrm{E}+02$ \\
\hline $\mathrm{Np}-237$ & $6.79 \mathrm{E}-03$ & $1.84 \mathrm{E}-01$ \\
\hline $\mathrm{Np}-238$ & $6.98 \mathrm{E}+01$ & $1.89 \mathrm{E}+03$ \\
\hline $\mathrm{Np}-239$ & $1.45 \mathrm{E}+02$ & $3.91 \mathrm{E}+03$ \\
\hline $\mathrm{Np}-240$ & $1.95 \mathrm{E}+03$ & $5.26 \mathrm{E}+04$ \\
\hline $\mathrm{Np}-241$ & $1.12 \mathrm{E}+04$ & $3.02 \mathrm{E}+05$ \\
\hline $\mathrm{Pu}-232$ & $6.40 \mathrm{E}+00$ & $1.73 \mathrm{E}+02$ \\
\hline $\mathrm{Pu}-234$ & $6.98 \mathrm{E}+00$ & $1.89 \mathrm{E}+02$ \\
\hline $\mathrm{Pu}-235$ & $8.31 \mathrm{E}+04$ & $2.25 \mathrm{E}+06$ \\
\hline $\mathrm{Pu}-236$ & $7.62 \mathrm{E}-03$ & $2.06 \mathrm{E}-01$ \\
\hline $\mathrm{Pu}-237$ & $3.92 \mathrm{E}+02$ & $1.06 \mathrm{E}+04$ \\
\hline $\mathrm{Pu}-238$ & $3.36 \mathrm{E}-03$ & $9.08 \mathrm{E}-02$ \\
\hline $\mathrm{Pu}-239$ & $3.10 \mathrm{E}-03$ & $8.38 \mathrm{E}-02$ \\
\hline $\mathrm{Pu}-240$ & $3.10 \mathrm{E}-03$ & $8.38 \mathrm{E}-02$ \\
\hline $\mathrm{Pu}-241$ & $1.75 \mathrm{E}-01$ & $4.72 \mathrm{E}+00$ \\
\hline $\mathrm{Pu}-242$ & $3.26 \mathrm{E}-03$ & $8.82 \mathrm{E}-02$ \\
\hline $\mathrm{Pu}-243$ & $1.67 \mathrm{E}+03$ & $4.50 \mathrm{E}+04$ \\
\hline $\mathrm{Pu}-244$ & $3.32 \mathrm{E}-03$ & $8.99 \mathrm{E}-02$ \\
\hline $\mathrm{Pu}-245$ & $3.30 \mathrm{E}+02$ & $8.91 \mathrm{E}+03$ \\
\hline $\mathrm{Pu}-246$ & $3.18 \mathrm{E}+01$ & $8.60 \mathrm{E}+02$ \\
\hline $\mathrm{Am}-237$ & $5.43 \mathrm{E}+03$ & $1.47 \mathrm{E}+05$ \\
\hline $\mathrm{Am}-238$ & $1.67 \mathrm{E}+03$ & $4.53 \mathrm{E}+04$ \\
\hline $\mathrm{Am}-239$ & $5.96 \mathrm{E}+02$ & $1.61 \mathrm{E}+04$ \\
\hline $\mathrm{Am}-240$ & $3.06 \mathrm{E}+02$ & $8.28 \mathrm{E}+03$ \\
\hline $\mathrm{Am}-241$ & $3.71 \mathrm{E}-03$ & $1.00 \mathrm{E}-01$ \\
\hline $\mathrm{Am}-242$ & $8.18 \mathrm{E}+00$ & $2.21 \mathrm{E}+02$ \\
\hline $\mathrm{Am}-242 \mathrm{~m}$ & $4.26 \mathrm{E}-03$ & $1.15 \mathrm{E}-01$ \\
\hline $\mathrm{Am}-244 \mathrm{~m}$ & $1.79 \mathrm{E}+03$ & $4.83 \mathrm{E}+04$ \\
\hline & & \\
\hline
\end{tabular}




\begin{tabular}{|c|c|c|}
\hline Nuclide & $\begin{array}{c}\text { Inhalation } \\
\text { DCS } \\
\left(\mathbf{B q} / \mathbf{m}^{3}\right)\end{array}$ & $\begin{array}{c}\text { Inhalation } \\
\text { DCS } \\
\left(\mathbf{p C i} / \mathbf{m}^{3}\right)\end{array}$ \\
\hline Am-245 & $2.55 \mathrm{E}+03$ & $6.90 \mathrm{E}+04$ \\
\hline Am-246 & $1.85 \mathrm{E}+03$ & $5.01 \mathrm{E}+04$ \\
\hline Am-246m & $5.85 \mathrm{E}+03$ & $1.58 \mathrm{E}+05$ \\
\hline Am-247 & $5.17 \mathrm{E}+03$ & $1.40 \mathrm{E}+05$ \\
\hline $\mathrm{Cm}-238$ & $8.01 \mathrm{E}+01$ & $2.17 \mathrm{E}+03$ \\
\hline Cm-239 & $2.06 \mathrm{E}+03$ & $5.56 \mathrm{E}+04$ \\
\hline $\mathrm{Cm}-240$ & $4.56 \mathrm{E}-02$ & $1.23 \mathrm{E}+00$ \\
\hline $\mathrm{Cm}-241$ & $3.91 \mathrm{E}+00$ & $1.06 \mathrm{E}+02$ \\
\hline $\mathrm{Cm}-242$ & $2.73 \mathrm{E}-02$ & $7.38 \mathrm{E}-01$ \\
\hline $\mathrm{Cm}-243$ & $4.84 \mathrm{E}-03$ & $1.31 \mathrm{E}-01$ \\
\hline $\mathrm{Cm}-244$ & $5.72 \mathrm{E}-03$ & $1.55 \mathrm{E}-01$ \\
\hline $\mathrm{Cm}-246$ & $3.66 \mathrm{E}-03$ & $9.89 \mathrm{E}-02$ \\
\hline CM-248 & $1.04 \mathrm{E}-03$ & $2.82 \mathrm{E}-02$ \\
\hline Bk-245 & $6.30 \mathrm{E}+01$ & $1.70 \mathrm{E}+03$ \\
\hline Bk-246 & $4.68 \mathrm{E}+02$ & $1.26 \mathrm{E}+04$ \\
\hline Bk-247 & $8.83 \mathrm{E}-04$ & $2.39 \mathrm{E}-02$ \\
\hline Bk-248m & $5.56 \mathrm{E}+00$ & $1.50 \mathrm{E}+02$ \\
\hline Bk-249 & $3.55 \mathrm{E}-01$ & $9.60 \mathrm{E}+00$ \\
\hline $\mathrm{Bk}-250$ & $6.71 \mathrm{E}+01$ & $1.81 \mathrm{E}+03$ \\
\hline Bk-251 & $3.07 \mathrm{E}+03$ & $8.30 \mathrm{E}+04$ \\
\hline Cf-244 & $1.04 \mathrm{E}+01$ & $2.82 \mathrm{E}+02$ \\
\hline Cf-246 & $3.18 \mathrm{E}-01$ & $8.58 \mathrm{E}+00$ \\
\hline Cf-247 & $3.38 \mathrm{E}+03$ & $9.14 \mathrm{E}+04$ \\
\hline Cf-248 & $1.60 \mathrm{E}-02$ & $4.31 \mathrm{E}-01$ \\
\hline Cf-249 & $2.14 \mathrm{E}-03$ & 5.79E-02 \\
\hline Cf-250 & $4.33 \mathrm{E}-03$ & $1.17 \mathrm{E}-01$ \\
\hline Cf-251 & $2.11 \mathrm{E}-03$ & $5.70 \mathrm{E}-02$ \\
\hline Cf-252 & $6.94 \mathrm{E}-03$ & $1.88 \mathrm{E}-01$ \\
\hline Cf-253 & $1.07 \mathrm{E}-01$ & $2.89 \mathrm{E}+00$ \\
\hline Cf-254 & $3.34 \mathrm{E}-03$ & $9.02 \mathrm{E}-02$ \\
\hline Cf-255 & $2.50 \mathrm{E}+01$ & $6.77 \mathrm{E}+02$ \\
\hline Es-249 & $6.28 \mathrm{E}+02$ & $1.70 \mathrm{E}+04$ \\
\hline Es-250 & $2.54 \mathrm{E}+01$ & $6.86 \mathrm{E}+02$ \\
\hline Es-250m & $9.83 \mathrm{E}+01$ & $2.66 \mathrm{E}+03$ \\
\hline Es-251 & $6.73 \mathrm{E}+01$ & $1.82 \mathrm{E}+03$ \\
\hline Es-253 & $4.56 \mathrm{E}-02$ & $1.23 \mathrm{E}+00$ \\
\hline Es-254 & $1.27 \mathrm{E}-02$ & $3.43 \mathrm{E}-01$ \\
\hline Es-254m & $2.77 \mathrm{E}-01$ & $7.49 \mathrm{E}+00$ \\
\hline
\end{tabular}


SRNL-STI-2013-00115

\begin{tabular}{|c|c|c|}
\hline Nuclide & $\begin{array}{c}\text { Inhalation } \\
\text { DCS } \\
\left(\mathbf{B q} / \mathbf{m}^{3}\right)\end{array}$ & $\begin{array}{c}\text { Inhalation } \\
\text { DCS } \\
\left(\mathbf{p C i} / \mathbf{m}^{3}\right)\end{array}$ \\
\hline Es-255 & $3.16 \mathrm{E}-02$ & 8.53E-01 \\
\hline Es-256 & $3.43 \mathrm{E}+00$ & $9.28 \mathrm{E}+01$ \\
\hline Fm-251 & $7.23 \mathrm{E}+01$ & $1.96 \mathrm{E}+03$ \\
\hline Fm-252 & $3.75 \mathrm{E}-01$ & $1.01 \mathrm{E}+01$ \\
\hline Fm-253 & $3.14 \mathrm{E}-01$ & $8.48 \mathrm{E}+00$ \\
\hline Fm-254 & $2.10 \mathrm{E}+00$ & $5.68 \mathrm{E}+01$ \\
\hline Fm-255 & $4.90 \mathrm{E}-01$ & $1.32 \mathrm{E}+01$ \\
\hline Fm-256 & $5.48 \mathrm{E}-01$ & $1.48 \mathrm{E}+01$ \\
\hline Fm-257 & $1.71 \mathrm{E}-02$ & 4.62E-01 \\
\hline
\end{tabular}


Table D-3 Air Immersion and Water Submersion DCS

\begin{tabular}{|c|c|c|c|c|}
\hline Nuclide & $\begin{array}{c}\text { Air } \\
\text { Immersion } \\
\text { DCS } \\
\left(\mathbf{B q} / \mathbf{m}^{3}\right)\end{array}$ & $\begin{array}{c}\text { Air } \\
\text { Immersion } \\
\text { DCS } \\
\left(\mu \mathrm{Ci} / \mathbf{c m}^{3}\right)\end{array}$ & $\begin{array}{c}\text { Water } \\
\text { Submersion } \\
\text { DCS } \\
\left(\mathbf{B q} / \mathbf{m}^{\mathbf{3}}\right)\end{array}$ & $\begin{array}{c}\text { Water } \\
\text { Submersion } \\
\text { DCS } \\
\left(\mu \mathrm{Ci} / \mathbf{m}^{3}\right)\end{array}$ \\
\hline $\mathrm{Be}-7$ & $1.43 \mathrm{E}+04$ & $3.88 \mathrm{E}-07$ & $6.59 \mathrm{E}+06$ & $5.65 \mathrm{E}+01$ \\
\hline $\mathrm{Be}-10$ & $2.28 \mathrm{E}+05$ & $6.17 \mathrm{E}-06$ & $2.05 E+08$ & $1.75 E+03$ \\
\hline $\mathrm{C}-10$ & $4.01 \mathrm{E}+02$ & $1.08 \mathrm{E}-08$ & $1.85 \mathrm{E}+05$ & $1.59 \mathrm{E}+00$ \\
\hline $\mathrm{C}-11$ & $6.95 \mathrm{E}+02$ & $1.88 \mathrm{E}-08$ & $3.20 \mathrm{E}+05$ & $2.75 \mathrm{E}+00$ \\
\hline C-14 & $1.22 \mathrm{E}+07$ & $3.30 \mathrm{E}-04$ & $1.10 \mathrm{E}+10$ & $9.40 \mathrm{E}+04$ \\
\hline $\mathrm{N}-13$ & $6.94 \mathrm{E}+02$ & $1.88 \mathrm{E}-08$ & $3.20 \mathrm{E}+05$ & $2.74 \mathrm{E}+00$ \\
\hline $\mathrm{N}-16$ & $1.22 \mathrm{E}+02$ & $3.31 \mathrm{E}-09$ & $5.63 \mathrm{E}+04$ & $4.83 \mathrm{E}-01$ \\
\hline O-14 & $1.95 \mathrm{E}+02$ & $5.26 \mathrm{E}-09$ & $9.01 \mathrm{E}+04$ & $7.72 \mathrm{E}-01$ \\
\hline O-15 & $6.89 \mathrm{E}+02$ & $1.86 \mathrm{E}-08$ & $3.19 \mathrm{E}+05$ & $2.73 \mathrm{E}+00$ \\
\hline O-19 & $6.89 \mathrm{E}+02$ & $1.86 \mathrm{E}-08$ & $3.23 \mathrm{E}+05$ & $2.76 \mathrm{E}+00$ \\
\hline F-17 & $6.89 \mathrm{E}+02$ & $1.86 \mathrm{E}-08$ & $3.19 \mathrm{E}+05$ & $2.73 \mathrm{E}+00$ \\
\hline F-18 & $7.19 \mathrm{E}+02$ & $1.94 \mathrm{E}-08$ & $3.31 \mathrm{E}+05$ & $2.84 \mathrm{E}+00$ \\
\hline $\mathrm{Ne}-19$ & $6.85 \mathrm{E}+02$ & $1.85 \mathrm{E}-08$ & $3.18 \mathrm{E}+05$ & $2.72 \mathrm{E}+00$ \\
\hline $\mathrm{Ne}-24$ & $1.28 \mathrm{E}+03$ & $3.46 \mathrm{E}-08$ & $5.95 \mathrm{E}+05$ & $5.10 \mathrm{E}+00$ \\
\hline $\mathrm{Na}-22$ & $3.11 \mathrm{E}+02$ & $8.40 \mathrm{E}-09$ & $1.44 \mathrm{E}+05$ & $1.24 \mathrm{E}+00$ \\
\hline $\mathrm{Na}-24$ & $1.52 \mathrm{E}+02$ & $4.12 \mathrm{E}-09$ & $7.03 E+04$ & $6.03 \mathrm{E}-01$ \\
\hline Mg-27 & $7.62 \mathrm{E}+02$ & $2.06 \mathrm{E}-08$ & $3.53 \mathrm{E}+05$ & $3.03 \mathrm{E}+00$ \\
\hline Mg-28 & $4.97 \mathrm{E}+02$ & $1.34 \mathrm{E}-08$ & $2.30 \mathrm{E}+05$ & $1.97 \mathrm{E}+00$ \\
\hline $\mathrm{Al}-26$ & $2.48 \mathrm{E}+02$ & $6.70 \mathrm{E}-09$ & $1.14 \mathrm{E}+05$ & $9.78 \mathrm{E}-01$ \\
\hline $\mathrm{Al}-28$ & $3.57 \mathrm{E}+02$ & $9.65 \mathrm{E}-09$ & $1.66 \mathrm{E}+05$ & $1.42 \mathrm{E}+00$ \\
\hline $\mathrm{Al}-29$ & $4.73 \mathrm{E}+02$ & $1.28 \mathrm{E}-08$ & $2.20 \mathrm{E}+05$ & $1.89 \mathrm{E}+00$ \\
\hline Si-31 & $6.57 \mathrm{E}+04$ & $1.77 \mathrm{E}-06$ & $5.16 \mathrm{E}+07$ & $4.43 \mathrm{E}+02$ \\
\hline $\mathrm{Si}-32$ & $3.02 \mathrm{E}+06$ & $8.16 \mathrm{E}-05$ & $2.78 \mathrm{E}+09$ & $2.38 \mathrm{E}+04$ \\
\hline P-30 & $6.76 \mathrm{E}+02$ & $1.83 \mathrm{E}-08$ & $3.14 \mathrm{E}+05$ & $2.69 \mathrm{E}+00$ \\
\hline P-32 & $5.92 \mathrm{E}+04$ & $1.60 \mathrm{E}-06$ & $4.92 \mathrm{E}+07$ & $4.21 \mathrm{E}+02$ \\
\hline P-33 & $2.20 \mathrm{E}+06$ & $5.95 \mathrm{E}-05$ & $2.02 \mathrm{E}+09$ & $1.73 \mathrm{E}+04$ \\
\hline S-35 & $1.03 \mathrm{E}+07$ & $2.79 \mathrm{E}-04$ & $9.33 \mathrm{E}+09$ & $7.99 \mathrm{E}+04$ \\
\hline S-37 & $2.06 \mathrm{E}+02$ & $5.57 \mathrm{E}-09$ & $9.49 \mathrm{E}+04$ & $8.14 \mathrm{E}-01$ \\
\hline S-38 & $3.74 \mathrm{E}+02$ & $1.01 \mathrm{E}-08$ & $1.73 \mathrm{E}+05$ & $1.49 \mathrm{E}+00$ \\
\hline Cl-34 & $6.65 \mathrm{E}+02$ & $1.80 \mathrm{E}-08$ & $3.11 \mathrm{E}+05$ & $2.66 \mathrm{E}+00$ \\
\hline $\mathrm{Cl}-34 \mathrm{~m}$ & $3.08 \mathrm{E}+02$ & $8.32 \mathrm{E}-09$ & $1.42 \mathrm{E}+05$ & $1.21 \mathrm{E}+00$ \\
\hline $\mathrm{Cl}-36$ & $1.91 \mathrm{E}+05$ & $5.16 \mathrm{E}-06$ & $1.63 \mathrm{E}+08$ & $1.40 \mathrm{E}+03$ \\
\hline Cl-38 & $4.31 \mathrm{E}+02$ & $1.16 \mathrm{E}-08$ & $2.01 \mathrm{E}+05$ & $1.72 \mathrm{E}+00$ \\
\hline $\mathrm{Cl}-39$ & $4.55 \mathrm{E}+02$ & $1.23 \mathrm{E}-08$ & $2.11 \mathrm{E}+05$ & $1.81 \mathrm{E}+00$ \\
\hline $\mathrm{Cl}-40$ & $1.52 \mathrm{E}+02$ & 4.10E-09 & $7.00 \mathrm{E}+04$ & $6.00 \mathrm{E}-01$ \\
\hline
\end{tabular}


SRNL-STI-2013-00115

\begin{tabular}{|c|c|c|c|c|}
\hline Nuclide & $\begin{array}{c}\text { Air } \\
\text { Immersion } \\
\text { DCS } \\
\left(\mathbf{B q} / \mathbf{m}^{3}\right)\end{array}$ & $\begin{array}{c}\text { Air } \\
\text { Immersion } \\
\text { DCS } \\
\left(\mu \mathrm{Ci} / \mathbf{c m}^{3}\right)\end{array}$ & $\begin{array}{c}\text { Water } \\
\text { Submersion } \\
\text { DCS } \\
\left(\mathbf{B q} / \mathbf{m}^{3}\right)\end{array}$ & $\begin{array}{c}\text { Water } \\
\text { Submersion } \\
\text { DCS } \\
\left(\mu \mathrm{Ci} / \mathbf{m}^{3}\right)\end{array}$ \\
\hline Ar-37 & $5.18 \mathrm{E}+07$ & $1.40 \mathrm{E}-03$ & N/A & $\mathrm{N} / \mathrm{A}$ \\
\hline Ar-39 & $2.76 \mathrm{E}+05$ & $7.45 \mathrm{E}-06$ & $2.48 \mathrm{E}+08$ & $2.12 \mathrm{E}+03$ \\
\hline Ar-41 & $5.16 \mathrm{E}+02$ & $1.39 \mathrm{E}-08$ & $2.38 \mathrm{E}+05$ & $2.04 \mathrm{E}+00$ \\
\hline Ar-42 & $2.52 \mathrm{E}+05$ & $6.80 \mathrm{E}-06$ & $2.25 \mathrm{E}+08$ & $1.93 \mathrm{E}+03$ \\
\hline Ar-43 & $4.20 \mathrm{E}+02$ & $1.14 \mathrm{E}-08$ & $1.96 \mathrm{E}+05$ & $1.68 \mathrm{E}+00$ \\
\hline Ar-44 & $3.35 \mathrm{E}+02$ & $9.06 \mathrm{E}-09$ & $1.55 \mathrm{E}+05$ & $1.33 \mathrm{E}+00$ \\
\hline K-38 & $2.03 \mathrm{E}+02$ & 5.49E-09 & $9.44 \mathrm{E}+04$ & $8.09 \mathrm{E}-01$ \\
\hline $\mathrm{K}-40$ & $3.99 \mathrm{E}+03$ & $1.08 \mathrm{E}-07$ & $1.89 \mathrm{E}+06$ & $1.62 \mathrm{E}+01$ \\
\hline K-42 & $2.13 \mathrm{E}+03$ & $5.75 \mathrm{E}-08$ & $1.02 \mathrm{E}+06$ & $8.74 \mathrm{E}+00$ \\
\hline K-43 & $7.32 \mathrm{E}+02$ & $1.98 \mathrm{E}-08$ & $3.38 \mathrm{E}+05$ & $2.89 \mathrm{E}+00$ \\
\hline K-44 & $2.66 \mathrm{E}+02$ & $7.20 \mathrm{E}-09$ & $1.23 \mathrm{E}+05$ & $1.06 \mathrm{E}+00$ \\
\hline K-45 & $3.50 \mathrm{E}+02$ & $9.47 \mathrm{E}-09$ & $1.63 \mathrm{E}+05$ & $1.39 \mathrm{E}+00$ \\
\hline K-46 & $2.16 \mathrm{E}+02$ & $5.83 \mathrm{E}-09$ & $1.00 \mathrm{E}+05$ & $8.57 \mathrm{E}-01$ \\
\hline $\mathrm{Ca}-45$ & $2.09 \mathrm{E}+06$ & $5.64 \mathrm{E}-05$ & $1.91 \mathrm{E}+09$ & $1.64 \mathrm{E}+04$ \\
\hline $\mathrm{Ca}-47$ & $6.32 \mathrm{E}+02$ & $1.71 \mathrm{E}-08$ & $2.94 \mathrm{E}+05$ & $2.52 \mathrm{E}+00$ \\
\hline Ca-49 & $1.90 \mathrm{E}+02$ & 5.13E-09 & $8.78 \mathrm{E}+04$ & $7.53 \mathrm{E}-01$ \\
\hline Sc-42m & $1.60 \mathrm{E}+02$ & $4.33 \mathrm{E}-09$ & $7.41 \mathrm{E}+04$ & $6.35 \mathrm{E}-01$ \\
\hline Sc-43 & $7.19 \mathrm{E}+02$ & $1.94 \mathrm{E}-08$ & $3.32 \mathrm{E}+05$ & $2.85 \mathrm{E}+00$ \\
\hline Sc-44 & $3.21 \mathrm{E}+02$ & $8.68 \mathrm{E}-09$ & $1.48 \mathrm{E}+05$ & $1.27 \mathrm{E}+00$ \\
\hline Sc-44m & $2.60 \mathrm{E}+03$ & 7.02E-08 & $1.19 \mathrm{E}+06$ & $1.02 \mathrm{E}+01$ \\
\hline Sc-46 & $3.38 \mathrm{E}+02$ & $9.15 \mathrm{E}-09$ & $1.56 \mathrm{E}+05$ & $1.34 \mathrm{E}+00$ \\
\hline Sc-47 & $6.75 \mathrm{E}+03$ & $1.82 \mathrm{E}-07$ & $3.08 \mathrm{E}+06$ & $2.64 \mathrm{E}+01$ \\
\hline Sc-48 & $2.01 \mathrm{E}+02$ & $5.42 \mathrm{E}-09$ & $9.27 \mathrm{E}+04$ & $7.95 \mathrm{E}-01$ \\
\hline Sc-49 & $4.45 \mathrm{E}+04$ & $1.20 \mathrm{E}-06$ & $3.45 \mathrm{E}+07$ & $2.95 \mathrm{E}+02$ \\
\hline Sc-50 & $2.06 \mathrm{E}+02$ & $5.57 \mathrm{E}-09$ & $9.55 \mathrm{E}+04$ & $8.19 \mathrm{E}-01$ \\
\hline Ti-44 & $6.50 \mathrm{E}+03$ & $1.76 \mathrm{E}-07$ & $2.88 \mathrm{E}+06$ & $2.47 \mathrm{E}+01$ \\
\hline Ti-45 & $8.13 \mathrm{E}+02$ & $2.20 \mathrm{E}-08$ & $3.75 \mathrm{E}+05$ & $3.22 \mathrm{E}+00$ \\
\hline Ti-51 & $1.85 \mathrm{E}+03$ & $5.01 \mathrm{E}-08$ & $8.66 \mathrm{E}+05$ & $7.43 \mathrm{E}+00$ \\
\hline Ti-52 & $5.51 \mathrm{E}+03$ & $1.49 \mathrm{E}-07$ & $2.60 \mathrm{E}+06$ & $2.23 \mathrm{E}+01$ \\
\hline V-47 & $7.05 \mathrm{E}+02$ & $1.90 \mathrm{E}-08$ & $3.26 \mathrm{E}+05$ & $2.80 \mathrm{E}+00$ \\
\hline V-48 & $2.33 \mathrm{E}+02$ & $6.30 \mathrm{E}-09$ & $1.07 \mathrm{E}+05$ & $9.21 \mathrm{E}-01$ \\
\hline V-50 & $4.62 \mathrm{E}+02$ & $1.25 \mathrm{E}-08$ & $2.13 \mathrm{E}+05$ & $1.82 \mathrm{E}+00$ \\
\hline $\mathrm{V}-52$ & $4.50 \mathrm{E}+02$ & $1.22 \mathrm{E}-08$ & $2.09 \mathrm{E}+05$ & $1.79 \mathrm{E}+00$ \\
\hline V-53 & $6.43 \mathrm{E}+02$ & $1.74 \mathrm{E}-08$ & $2.99 \mathrm{E}+05$ & $2.56 \mathrm{E}+00$ \\
\hline $\mathrm{Cr}-48$ & $1.69 \mathrm{E}+03$ & $4.56 \mathrm{E}-08$ & $7.70 \mathrm{E}+05$ & $6.60 \mathrm{E}+00$ \\
\hline $\mathrm{Cr}-49$ & $6.78 \mathrm{E}+02$ & $1.83 \mathrm{E}-08$ & $3.11 \mathrm{E}+05$ & $2.66 \mathrm{E}+00$ \\
\hline $\mathrm{Cr}-51$ & $2.26 \mathrm{E}+04$ & $6.12 \mathrm{E}-07$ & $1.04 \mathrm{E}+07$ & $8.91 \mathrm{E}+01$ \\
\hline
\end{tabular}




\begin{tabular}{|c|c|c|c|c|}
\hline Nuclide & $\begin{array}{c}\text { Air } \\
\text { Immersion } \\
\text { DCS } \\
\left(\mathbf{B q} / \mathbf{m}^{3}\right)\end{array}$ & $\begin{array}{c}\text { Air } \\
\text { Immersion } \\
\text { DCS } \\
\left(\mu \mathrm{Ci} / \mathbf{c m}^{3}\right)\end{array}$ & $\begin{array}{c}\text { Water } \\
\text { Submersion } \\
\text { DCS } \\
\left(\mathbf{B q} / \mathbf{m}^{3}\right)\end{array}$ & $\begin{array}{c}\text { Water } \\
\text { Submersion } \\
\text { DCS } \\
\left(\mu \mathrm{Ci} / \mathbf{m}^{3}\right)\end{array}$ \\
\hline Cr-55 & $3.17 \mathrm{E}+04$ & $8.57 \mathrm{E}-07$ & $2.44 \mathrm{E}+07$ & $2.09 \mathrm{E}+02$ \\
\hline Cr-56 & $9.14 \mathrm{E}+03$ & $2.47 \mathrm{E}-07$ & $4.31 \mathrm{E}+06$ & $3.69 \mathrm{E}+01$ \\
\hline Mn-50m & $1.45 \mathrm{E}+02$ & $3.93 \mathrm{E}-09$ & $6.73 \mathrm{E}+04$ & $5.77 \mathrm{E}-01$ \\
\hline Mn-51 & $7.02 \mathrm{E}+02$ & $1.90 \mathrm{E}-08$ & $3.25 \mathrm{E}+05$ & $2.78 \mathrm{E}+00$ \\
\hline Mn-52m & $2.81 \mathrm{E}+02$ & $7.58 \mathrm{E}-09$ & $1.29 \mathrm{E}+05$ & $1.11 \mathrm{E}+00$ \\
\hline Mn-52 & $1.96 \mathrm{E}+02$ & $5.29 \mathrm{E}-09$ & $9.03 \mathrm{E}+04$ & $7.74 \mathrm{E}-01$ \\
\hline Mn-54 & $8.28 \mathrm{E}+02$ & $2.24 \mathrm{E}-08$ & $3.82 \mathrm{E}+05$ & $3.27 \mathrm{E}+00$ \\
\hline $\mathrm{Mn}-56$ & $3.88 \mathrm{E}+02$ & $1.05 \mathrm{E}-08$ & $1.80 \mathrm{E}+05$ & $1.54 \mathrm{E}+00$ \\
\hline Mn-57 & $5.98 \mathrm{E}+03$ & $1.62 \mathrm{E}-07$ & $2.96 \mathrm{E}+06$ & $2.54 \mathrm{E}+01$ \\
\hline Mn-58m & $2.76 \mathrm{E}+02$ & $7.45 \mathrm{E}-09$ & $1.28 \mathrm{E}+05$ & $1.10 \mathrm{E}+00$ \\
\hline $\mathrm{Fe}-52$ & $9.67 \mathrm{E}+02$ & $2.61 \mathrm{E}-08$ & $4.45 \mathrm{E}+05$ & $3.81 \mathrm{E}+00$ \\
\hline $\mathrm{Fe}-53$ & $5.93 \mathrm{E}+02$ & $1.60 \mathrm{E}-08$ & $2.76 \mathrm{E}+05$ & $2.36 \mathrm{E}+00$ \\
\hline Fe-53m & $2.20 \mathrm{E}+02$ & $5.95 \mathrm{E}-09$ & $1.02 \mathrm{E}+05$ & $8.71 \mathrm{E}-01$ \\
\hline Fe-55 & $4.74 \mathrm{E}+12$ & $1.28 \mathrm{E}+02$ & $2.13 \mathrm{E}+15$ & $1.82 \mathrm{E}+10$ \\
\hline Fe-59 & $5.64 \mathrm{E}+02$ & $1.52 \mathrm{E}-08$ & $2.60 \mathrm{E}+05$ & $2.23 \mathrm{E}+00$ \\
\hline Fe-60 & $4.58 \mathrm{E}+06$ & $1.24 \mathrm{E}-04$ & $4.18 \mathrm{E}+09$ & $3.58 \mathrm{E}+04$ \\
\hline $\mathrm{Fe}-61$ & $4.75 \mathrm{E}+02$ & $1.28 \mathrm{E}-08$ & $2.20 \mathrm{E}+05$ & $1.89 \mathrm{E}+00$ \\
\hline $\mathrm{Fe}-62$ & $1.37 \mathrm{E}+03$ & $3.69 \mathrm{E}-08$ & $6.37 \mathrm{E}+05$ & $5.46 \mathrm{E}+00$ \\
\hline Co-54m & $1.71 \mathrm{E}+02$ & $4.63 \mathrm{E}-09$ & $7.95 \mathrm{E}+04$ & $6.81 \mathrm{E}-01$ \\
\hline Co-55 & $3.45 \mathrm{E}+02$ & $9.34 \mathrm{E}-09$ & $1.59 \mathrm{E}+05$ & $7 \mathrm{E}+00$ \\
\hline Co-56 & & & $8.30 \mathrm{E}+04$ & \\
\hline Co-57 & $6.37 \mathrm{E}+03$ & $1.72 \mathrm{E}-07$ & $2.86 \mathrm{E}+06$ & $2.45 \mathrm{E}+01$ \\
\hline Co-58 & $7.14 \mathrm{E}+02$ & $1.93 \mathrm{E}-08$ & $3.29 \mathrm{E}+05$ & $2.82 \mathrm{E}+00$ \\
\hline Co-58m & $5.21 \mathrm{E}+08$ & $1.41 \mathrm{E}-02$ & $2.22 \mathrm{E}+11$ & $1.90 \mathrm{E}+06$ \\
\hline Co-60 & $2.66 \mathrm{E}+02$ & $7.20 \mathrm{E}-09$ & $1.23 \mathrm{E}+05$ & $1.05 \mathrm{E}+00$ \\
\hline Co-60m & $1.63 \mathrm{E}+05$ & $4.42 \mathrm{E}-06$ & $7.50 \mathrm{E}+07$ & $6.42 \mathrm{E}+02$ \\
\hline Co-61 & $7.81 \mathrm{E}+03$ & $2.11 \mathrm{E}-07$ & $3.66 \mathrm{E}+06$ & $3.13 \mathrm{E}+01$ \\
\hline Co-62 & $4.00 \mathrm{E}+02$ & $1.08 \mathrm{E}-08$ & $1.87 \mathrm{E}+05$ & $1.60 \mathrm{E}+00$ \\
\hline Co-62m & $2.44 \mathrm{E}+02$ & $6.59 \mathrm{E}-09$ & $1.13 \mathrm{E}+05$ & $9.67 \mathrm{E}-01$ \\
\hline $\mathrm{Ni}-56$ & $4.05 \mathrm{E}+02$ & $1.10 \mathrm{E}-08$ & $1.87 \mathrm{E}+05$ & $1.60 \mathrm{E}+00$ \\
\hline $\mathrm{Ni}-57$ & $3.45 \mathrm{E}+02$ & $9.33 \mathrm{E}-09$ & $1.59 \mathrm{E}+05$ & $1.37 \mathrm{E}+00$ \\
\hline $\mathrm{Ni}-59$ & & & $2.11 \mathrm{E}+10$ & $1.81 \mathrm{E}+05$ \\
\hline $\mathrm{Ni}-65$ & $1.17 \mathrm{E}+03$ & $3.15 \mathrm{E}-08$ & $5.44 \mathrm{E}+05$ & $4.66 \mathrm{E}+00$ \\
\hline Ni-66 & $2.33 \mathrm{E}+06$ & $6.30 \mathrm{E}-05$ & $2.14 \mathrm{E}+09$ & $1.84 \mathrm{E}+04$ \\
\hline $\mathrm{Cu}-57$ & $5.61 \mathrm{E}+02$ & $1.52 \mathrm{E}-08$ & $2.66 \mathrm{E}+05$ & $2.28 \mathrm{E}+00$ \\
\hline $\mathrm{Cu}-59$ & $4.75 \mathrm{E}+02$ & $1.28 \mathrm{E}-08$ & $2.20 \mathrm{E}+05$ & $1.89 \mathrm{E}+00$ \\
\hline $\mathrm{Cu}-60$ & $1.69 \mathrm{E}+02$ & $4.56 \mathrm{E}-09$ & $7.81 \mathrm{E}+04$ & $6.69 \mathrm{E}-01$ \\
\hline
\end{tabular}




\begin{tabular}{|c|c|c|c|c|}
\hline Nuclide & $\begin{array}{c}\text { Air } \\
\text { Immersion } \\
\text { DCS } \\
\left(\mathbf{B q} / \mathbf{m}^{3}\right)\end{array}$ & $\begin{array}{c}\text { Air } \\
\text { Immersion } \\
\text { DCS } \\
\left(\mu \mathrm{Ci} / \mathbf{c m}^{3}\right)\end{array}$ & $\begin{array}{c}\text { Water } \\
\text { Submersion } \\
\text { DCS } \\
\left(\mathbf{B q} / \mathbf{m}^{3}\right)\end{array}$ & $\begin{array}{c}\text { Water } \\
\text { Submersion } \\
\text { DCS } \\
\left(\mu \mathrm{Ci} / \mathbf{m}^{3}\right)\end{array}$ \\
\hline $\mathrm{Cu}-61$ & $8.57 \mathrm{E}+02$ & $2.32 \mathrm{E}-08$ & $3.95 \mathrm{E}+05$ & $3.39 \mathrm{E}+00$ \\
\hline $\mathrm{Cu}-62$ & $6.89 \mathrm{E}+02$ & $1.86 \mathrm{E}-08$ & $3.20 \mathrm{E}+05$ & $2.75 \mathrm{E}+00$ \\
\hline $\mathrm{Cu}-64$ & $3.83 \mathrm{E}+03$ & $1.03 \mathrm{E}-07$ & $1.76 \mathrm{E}+06$ & $1.51 \mathrm{E}+01$ \\
\hline $\mathrm{Cu}-66$ & $5.77 \mathrm{E}+03$ & $1.56 \mathrm{E}-07$ & $2.86 \mathrm{E}+06$ & $2.45 \mathrm{E}+01$ \\
\hline $\mathrm{Cu}-67$ & $6.46 \mathrm{E}+03$ & $1.75 \mathrm{E}-07$ & $2.94 \mathrm{E}+06$ & $2.52 \mathrm{E}+01$ \\
\hline Cu-69 & $1.25 \mathrm{E}+03$ & $3.39 \mathrm{E}-08$ & $5.86 \mathrm{E}+05$ & $5.02 \mathrm{E}+00$ \\
\hline $\mathrm{Zn}-60$ & $4.59 \mathrm{E}+02$ & $1.24 \mathrm{E}-08$ & $2.13 \mathrm{E}+05$ & $1.82 \mathrm{E}+00$ \\
\hline Zn-61 & $4.39 \mathrm{E}+02$ & $1.19 \mathrm{E}-08$ & $2.05 \mathrm{E}+05$ & $1.75 \mathrm{E}+00$ \\
\hline Zn-62 & $1.63 \mathrm{E}+03$ & 4.42E-08 & $7.53 \mathrm{E}+05$ & $6.46 \mathrm{E}+00$ \\
\hline Zn-63 & $6.35 \mathrm{E}+02$ & $1.72 \mathrm{E}-08$ & $2.94 \mathrm{E}+05$ & $2.52 \mathrm{E}+00$ \\
\hline Zn-65 & $1.17 \mathrm{E}+03$ & $3.15 \mathrm{E}-08$ & $5.39 \mathrm{E}+05$ & $4.62 \mathrm{E}+00$ \\
\hline $\mathrm{Zn}-69 \mathrm{~m}$ & $1.72 \mathrm{E}+03$ & $4.66 \mathrm{E}-08$ & $7.91 \mathrm{E}+05$ & $6.78 \mathrm{E}+00$ \\
\hline Zn-69 & $1.59 \mathrm{E}+05$ & $4.29 \mathrm{E}-06$ & $1.39 \mathrm{E}+08$ & $1.19 \mathrm{E}+03$ \\
\hline $\mathrm{Zn}-71 \mathrm{~m}$ & $4.50 \mathrm{E}+02$ & $1.22 \mathrm{E}-08$ & $2.07 \mathrm{E}+05$ & $1.78 \mathrm{E}+00$ \\
\hline $\mathrm{Zn}-71$ & $2.09 \mathrm{E}+03$ & 5.64E-08 & $9.85 \mathrm{E}+05$ & $8.44 \mathrm{E}+00$ \\
\hline $\mathrm{Zn}-72$ & $5.13 \mathrm{E}+03$ & $1.39 \mathrm{E}-07$ & $2.31 \mathrm{E}+06$ & $1.98 \mathrm{E}+01$ \\
\hline Ga-64 & $1.93 \mathrm{E}+02$ & $5.23 \mathrm{E}-09$ & $8.93 \mathrm{E}+04$ & $7.66 \mathrm{E}-01$ \\
\hline Ga-65 & $6.06 \mathrm{E}+02$ & $1.64 \mathrm{E}-08$ & $2.81 \mathrm{E}+05$ & $2.40 \mathrm{E}+00$ \\
\hline Ga-66 & $2.56 \mathrm{E}+02$ & $6.91 \mathrm{E}-09$ & $1.18 \mathrm{E}+05$ & $1.01 \mathrm{E}+00$ \\
\hline Ga-67 & $4.83 \mathrm{E}+03$ & $1.30 \mathrm{E}-07$ & $2.19 \mathrm{E}+06$ & $1.87 \mathrm{E}+01$ \\
\hline Ga-68 & $7.39 \mathrm{E}+02$ & $2.00 \mathrm{E}-08$ & $3.42 \mathrm{E}+05$ & $2.93 \mathrm{E}+00$ \\
\hline Ga-70 & $3.83 \mathrm{E}+04$ & $1.04 \mathrm{E}-06$ & $2.40 \mathrm{E}+07$ & $2.06 \mathrm{E}+02$ \\
\hline Ga-72 & $2.42 \mathrm{E}+02$ & $6.54 \mathrm{E}-09$ & $1.12 \mathrm{E}+05$ & $9.60 \mathrm{E}-01$ \\
\hline Ga-73 & $2.03 \mathrm{E}+03$ & 5.49E-08 & $9.41 \mathrm{E}+05$ & $8.06 \mathrm{E}+00$ \\
\hline Ga-74 & $2.05 \mathrm{E}+02$ & $5.53 \mathrm{E}-09$ & $9.47 \mathrm{E}+04$ & $8.11 \mathrm{E}-01$ \\
\hline Ge-66 & $1.07 \mathrm{E}+03$ & $2.89 \mathrm{E}-08$ & $4.91 \mathrm{E}+05$ & $4.21 \mathrm{E}+00$ \\
\hline Ge-67 & $4.85 \mathrm{E}+02$ & $1.31 \mathrm{E}-08$ & $2.25 \mathrm{E}+05$ & $1.93 \mathrm{E}+00$ \\
\hline Ge-68 & $3.57 \mathrm{E}+08$ & $9.66 \mathrm{E}-03$ & $1.55 \mathrm{E}+11$ & $1.33 \mathrm{E}+06$ \\
\hline Ge-69 & $7.24 \mathrm{E}+02$ & $1.96 \mathrm{E}-08$ & $3.34 \mathrm{E}+05$ & $2.86 \mathrm{E}+00$ \\
\hline Ge-71 & $3.52 \mathrm{E}+08$ & $9.52 \mathrm{E}-03$ & $1.52 \mathrm{E}+11$ & $1.31 \mathrm{E}+06$ \\
\hline Ge-75 & $1.73 \mathrm{E}+04$ & $4.68 \mathrm{E}-07$ & $8.55 \mathrm{E}+06$ & $7.33 \mathrm{E}+01$ \\
\hline Ge-77 & $6.41 \mathrm{E}+02$ & $1.73 \mathrm{E}-08$ & $2.96 \mathrm{E}+05$ & $2.54 \mathrm{E}+00$ \\
\hline Ge-78 & $2.58 \mathrm{E}+03$ & $6.97 \mathrm{E}-08$ & $1.18 \mathrm{E}+06$ & $1.01 \mathrm{E}+01$ \\
\hline As-68 & $1.80 \mathrm{E}+02$ & $4.87 \mathrm{E}-09$ & $8.34 \mathrm{E}+04$ & $7.15 \mathrm{E}-01$ \\
\hline As-69 & $6.04 \mathrm{E}+02$ & $1.63 \mathrm{E}-08$ & $2.81 \mathrm{E}+05$ & $2.40 \mathrm{E}+00$ \\
\hline As-70 & $1.59 \mathrm{E}+02$ & 4.29E-09 & $7.34 \mathrm{E}+04$ & $6.29 \mathrm{E}-01$ \\
\hline As-71 & $1.24 \mathrm{E}+03$ & $3.36 \mathrm{E}-08$ & $5.70 \mathrm{E}+05$ & $4.89 \mathrm{E}+00$ \\
\hline
\end{tabular}




\begin{tabular}{|c|c|c|c|c|}
\hline Nuclide & $\begin{array}{c}\text { Air } \\
\text { Immersion } \\
\text { DCS } \\
\left(\mathbf{B q} / \mathbf{m}^{3}\right)\end{array}$ & $\begin{array}{c}\text { Air } \\
\text { Immersion } \\
\text { DCS } \\
\left(\mu \mathrm{Ci} / \mathbf{c m}^{3}\right)\end{array}$ & $\begin{array}{c}\text { Water } \\
\text { Submersion } \\
\text { DCS } \\
\left(\mathbf{B q} / \mathbf{m}^{3}\right)\end{array}$ & $\begin{array}{c}\text { Water } \\
\text { Submersion } \\
\text { DCS } \\
\left(\mu \mathrm{Ci} / \mathbf{m}^{3}\right)\end{array}$ \\
\hline As-72 & $3.86 \mathrm{E}+02$ & $1.04 \mathrm{E}-08$ & $1.79 \mathrm{E}+05$ & $1.54 \mathrm{E}+00$ \\
\hline As-73 & $2.06 \mathrm{E}+05$ & $5.57 \mathrm{E}-06$ & $8.93 \mathrm{E}+07$ & $7.66 \mathrm{E}+02$ \\
\hline As-74 & $9.33 \mathrm{E}+02$ & $2.52 \mathrm{E}-08$ & $4.30 \mathrm{E}+05$ & $3.68 \mathrm{E}+00$ \\
\hline As-76 & $1.59 \mathrm{E}+03$ & $4.29 \mathrm{E}-08$ & $7.46 \mathrm{E}+05$ & $6.39 \mathrm{E}+00$ \\
\hline As-77 & $6.52 \mathrm{E}+04$ & $1.76 \mathrm{E}-06$ & $3.40 \mathrm{E}+07$ & $2.91 \mathrm{E}+02$ \\
\hline As-78 & $5.06 \mathrm{E}+02$ & $1.37 \mathrm{E}-08$ & $2.35 \mathrm{E}+05$ & $2.01 \mathrm{E}+00$ \\
\hline As-79 & $1.41 \mathrm{E}+04$ & $3.81 \mathrm{E}-07$ & $7.55 \mathrm{E}+06$ & $6.47 \mathrm{E}+01$ \\
\hline Se-70 & $1.01 \mathrm{E}+03$ & $2.73 \mathrm{E}-08$ & $4.64 \mathrm{E}+05$ & $3.98 \mathrm{E}+00$ \\
\hline Se-71 & $4.29 \mathrm{E}+02$ & $1.16 \mathrm{E}-08$ & $1.98 \mathrm{E}+05$ & $1.70 \mathrm{E}+00$ \\
\hline Se-72 & $5.20 \mathrm{E}+04$ & $1.40 \mathrm{E}-06$ & $2.25 \mathrm{E}+07$ & $1.93 \mathrm{E}+02$ \\
\hline Se-73 & $6.59 \mathrm{E}+02$ & $1.78 \mathrm{E}-08$ & $3.02 \mathrm{E}+05$ & $2.59 \mathrm{E}+00$ \\
\hline Se-73m & $2.69 \mathrm{E}+03$ & $7.26 \mathrm{E}-08$ & $1.24 \mathrm{E}+06$ & $1.07 \mathrm{E}+01$ \\
\hline Se-75 & $1.91 \mathrm{E}+03$ & $5.16 \mathrm{E}-08$ & $8.71 \mathrm{E}+05$ & $7.47 \mathrm{E}+00$ \\
\hline Se-77m & $8.57 \mathrm{E}+03$ & $2.32 \mathrm{E}-07$ & $3.90 \mathrm{E}+06$ & $3.34 \mathrm{E}+01$ \\
\hline Se-79 & $1.04 \mathrm{E}+07$ & $2.81 \mathrm{E}-04$ & $9.38 \mathrm{E}+09$ & $8.04 \mathrm{E}+04$ \\
\hline Se-79m & $8.81 \mathrm{E}+04$ & $2.38 \mathrm{E}-06$ & $3.96 \mathrm{E}+07$ & $3.39 \mathrm{E}+02$ \\
\hline Se-81 & $3.89 \mathrm{E}+04$ & $1.05 \mathrm{E}-06$ & $2.38 \mathrm{E}+07$ & $2.04 \mathrm{E}+02$ \\
\hline Se-81m & $5.72 \mathrm{E}+04$ & $1.55 \mathrm{E}-06$ & $2.58 \mathrm{E}+07$ & $2.21 \mathrm{E}+02$ \\
\hline Se-83 & $2.56 \mathrm{E}+02$ & $6.91 \mathrm{E}-09$ & $1.18 \mathrm{E}+05$ & $1.01 \mathrm{E}+00$ \\
\hline Se- $83 m$ & $6.63 \mathrm{E}+02$ & $1.79 \mathrm{E}-08$ & $3.11 \mathrm{E}+05$ & $2.66 \mathrm{E}+00$ \\
\hline Se-84 & $1.67 \mathrm{E}+03$ & $4.51 \mathrm{E}-08$ & $7.75 \mathrm{E}+05$ & $6.64 \mathrm{E}+00$ \\
\hline $\mathrm{Br}-72$ & $2.25 \mathrm{E}+02$ & $6.08 \mathrm{E}-09$ & $1.05 \mathrm{E}+05$ & $8.97 \mathrm{E}-01$ \\
\hline $\mathrm{Br}-73$ & $4.86 \mathrm{E}+02$ & $1.31 \mathrm{E}-08$ & $2.25 \mathrm{E}+05$ & $1.93 \mathrm{E}+00$ \\
\hline $\mathrm{Br}-74$ & $1.38 \mathrm{E}+02$ & $3.73 \mathrm{E}-09$ & $6.37 \mathrm{E}+04$ & $5.46 \mathrm{E}-01$ \\
\hline Br-74m & $1.59 \mathrm{E}+02$ & 4.29E-09 & $7.32 \mathrm{E}+04$ & $6.28 \mathrm{E}-01$ \\
\hline $\mathrm{Br}-75$ & $5.92 \mathrm{E}+02$ & $1.60 \mathrm{E}-08$ & $2.73 \mathrm{E}+05$ & $2.34 \mathrm{E}+00$ \\
\hline $\mathrm{Br}-76$ & $2.37 \mathrm{E}+02$ & $6.40 \mathrm{E}-09$ & $1.09 \mathrm{E}+05$ & $9.34 \mathrm{E}-01$ \\
\hline $\mathrm{Br}-76 \mathrm{~m}$ & $3.28 \mathrm{E}+04$ & $8.87 \mathrm{E}-07$ & $1.45 \mathrm{E}+07$ & $1.24 \mathrm{E}+02$ \\
\hline $\mathrm{Br}-77$ & $2.26 \mathrm{E}+03$ & $6.12 \mathrm{E}-08$ & $1.04 \mathrm{E}+06$ & $8.91 \mathrm{E}+00$ \\
\hline $\mathrm{Br}-77 \mathrm{~m}$ & $5.32 \mathrm{E}+04$ & $1.44 \mathrm{E}-06$ & $2.40 \mathrm{E}+07$ & $2.06 \mathrm{E}+02$ \\
\hline $\mathrm{Br}-78$ & $6.76 \mathrm{E}+02$ & $1.83 \mathrm{E}-08$ & $3.14 \mathrm{E}+05$ & $2.69 \mathrm{E}+00$ \\
\hline $\mathrm{Br}-80$ & $7.97 \mathrm{E}+03$ & & $3.92 \mathrm{E}+06$ & $3.36 \mathrm{E}+01$ \\
\hline $\mathrm{Br}-80 \mathrm{~m}$ & $1.33 \mathrm{E}+05$ & $3.60 \mathrm{E}-06$ & $5.70 \mathrm{E}+07$ & $4.89 \mathrm{E}+02$ \\
\hline $\mathrm{Br}-82$ & $2.60 \mathrm{E}+02$ & 7.02E-09 & $1.20 \mathrm{E}+05$ & $1.03 \mathrm{E}+00$ \\
\hline Br- $82 m$ & $1.99 \mathrm{E}+05$ & $5.39 \mathrm{E}-06$ & $9.94 \mathrm{E}+07$ & $8.52 \mathrm{E}+02$ \\
\hline $\mathrm{Br}-83$ & $6.22 \mathrm{E}+04$ & $1.68 \mathrm{E}-06$ & $3.53 \mathrm{E}+07$ & $3.03 \mathrm{E}+02$ \\
\hline $\mathrm{Br}-84$ & $3.57 \mathrm{E}+02$ & $9.65 \mathrm{E}-09$ & $1.65 \mathrm{E}+05$ & $1.42 \mathrm{E}+00$ \\
\hline
\end{tabular}




\begin{tabular}{|c|c|c|c|c|}
\hline Nuclide & $\begin{array}{c}\text { Air } \\
\text { Immersion } \\
\text { DCS } \\
\left(\mathbf{B q} / \mathbf{m}^{3}\right)\end{array}$ & $\begin{array}{c}\text { Air } \\
\text { Immersion } \\
\text { DCS } \\
\left(\mu \mathrm{Ci} / \mathbf{c m}^{3}\right)\end{array}$ & $\begin{array}{c}\text { Water } \\
\text { Submersion } \\
\text { DCS } \\
\left(\mathbf{B q} / \mathbf{m}^{3}\right)\end{array}$ & $\begin{array}{c}\text { Water } \\
\text { Submersion } \\
\text { DCS } \\
\left(\mu \mathrm{Ci} / \mathbf{m}^{3}\right)\end{array}$ \\
\hline $\mathrm{Br}-84 \mathrm{~m}$ & $2.42 \mathrm{E}+02$ & $6.54 \mathrm{E}-09$ & $1.12 \mathrm{E}+05$ & $9.60 \mathrm{E}-01$ \\
\hline $\mathrm{Br}-85$ & $7.97 \mathrm{E}+03$ & $2.15 \mathrm{E}-07$ & $4.06 \mathrm{E}+06$ & $3.48 \mathrm{E}+01$ \\
\hline Kr-74 & $6.75 \mathrm{E}+02$ & $1.82 \mathrm{E}-08$ & $3.11 \mathrm{E}+05$ & $2.66 \mathrm{E}+00$ \\
\hline $\mathrm{Kr}-75$ & $5.42 \mathrm{E}+02$ & $1.46 \mathrm{E}-08$ & $2.52 \mathrm{E}+05$ & $2.16 \mathrm{E}+00$ \\
\hline $\mathrm{Kr}-76$ & $1.73 \mathrm{E}+03$ & $4.68 \mathrm{E}-08$ & $7.93 \mathrm{E}+05$ & $6.79 \mathrm{E}+00$ \\
\hline $\mathrm{Kr}-77$ & $6.85 \mathrm{E}+02$ & $1.85 \mathrm{E}-08$ & $3.17 \mathrm{E}+05$ & $2.72 \mathrm{E}+00$ \\
\hline $\mathrm{Kr}-79$ & $2.86 \mathrm{E}+03$ & $7.72 \mathrm{E}-08$ & $1.31 \mathrm{E}+06$ & $1.12 \mathrm{E}+01$ \\
\hline $\mathrm{Kr}-81$ & $8.30 \mathrm{E}+05$ & $2.24 \mathrm{E}-05$ & $3.78 \mathrm{E}+08$ & $3.24 \mathrm{E}+03$ \\
\hline Kr-81m & $5.69 \mathrm{E}+03$ & $1.54 \mathrm{E}-07$ & $2.60 \mathrm{E}+06$ & $2.23 \mathrm{E}+01$ \\
\hline $\mathrm{Kr}-83 \mathrm{~m}$ & $2.88 \mathrm{E}+07$ & 7.79E-04 & $1.23 \mathrm{E}+10$ & $1.06 \mathrm{E}+05$ \\
\hline $\mathrm{Kr}-85$ & $1.32 \mathrm{E}+05$ & $3.56 \mathrm{E}-06$ & $8.46 \mathrm{E}+07$ & $7.25 \mathrm{E}+02$ \\
\hline $\mathrm{Kr}-85 \mathrm{~m}$ & $4.63 \mathrm{E}+03$ & $1.25 \mathrm{E}-07$ & $2.13 \mathrm{E}+06$ & $1.82 \mathrm{E}+01$ \\
\hline $\mathrm{Kr}-87$ & $7.99 \mathrm{E}+02$ & $2.16 \mathrm{E}-08$ & $3.73 \mathrm{E}+05$ & $3.19 \mathrm{E}+00$ \\
\hline $\mathrm{Kr}-88$ & $3.27 \mathrm{E}+02$ & $8.83 \mathrm{E}-09$ & $1.51 \mathrm{E}+05$ & $1.29 \mathrm{E}+00$ \\
\hline Kr-89 & $3.32 \mathrm{E}+02$ & 8.96E-09 & $1.54 \mathrm{E}+05$ & $1.32 \mathrm{E}+00$ \\
\hline Rb-77 & $4.47 \mathrm{E}+02$ & $1.21 \mathrm{E}-08$ & $2.07 \mathrm{E}+05$ & $1.78 \mathrm{E}+00$ \\
\hline $\mathrm{Rb}-78$ & $1.55 \mathrm{E}+02$ & $4.20 \mathrm{E}-09$ & $7.19 \mathrm{E}+04$ & $6.16 \mathrm{E}-01$ \\
\hline $\mathrm{Rb}-78 \mathrm{~m}$ & $2.09 \mathrm{E}+02$ & 5.64E-09 & $9.67 \mathrm{E}+04$ & $8.29 \mathrm{E}-01$ \\
\hline Rb-79 & $4.86 \mathrm{E}+02$ & $1.31 \mathrm{E}-08$ & $2.25 \mathrm{E}+05$ & $1.93 \mathrm{E}+00$ \\
\hline $\mathrm{Rb}-80$ & $5.72 \mathrm{E}+02$ & $1.55 \mathrm{E}-08$ & $2.66 \mathrm{E}+05$ & $2.28 \mathrm{E}+00$ \\
\hline Rb-81 & $1.41 \mathrm{E}+03$ & & $6.48 \mathrm{E}+05$ & $5.56 \mathrm{E}+00$ \\
\hline $\mathrm{Rb}-81 \mathrm{~m}$ & $2.96 \mathrm{E}+04$ & $8.01 \mathrm{E}-07$ & $1.36 \mathrm{E}+07$ & $1.17 \mathrm{E}+02$ \\
\hline Rb-82 & $6.23 \mathrm{E}+02$ & $1.68 \mathrm{E}-08$ & $2.91 \mathrm{E}+05$ & $2.49 \mathrm{E}+00$ \\
\hline $\mathrm{Rb}-82 \mathrm{~m}$ & $2.37 \mathrm{E}+02$ & $6.40 \mathrm{E}-09$ & $1.09 \mathrm{E}+05$ & $9.34 \mathrm{E}-01$ \\
\hline Rb-83 & $1.47 \mathrm{E}+03$ & $3.97 \mathrm{E}-08$ & $6.76 \mathrm{E}+05$ & $5.79 \mathrm{E}+00$ \\
\hline $\mathrm{Rb}-84$ & $7.68 \mathrm{E}+02$ & $2.08 \mathrm{E}-08$ & $3.54 \mathrm{E}+05$ & $3.04 \mathrm{E}+00$ \\
\hline $\mathrm{Rb}-84 \mathrm{~m}$ & $1.89 \mathrm{E}+03$ & $5.10 \mathrm{E}-08$ & $8.66 \mathrm{E}+05$ & $7.43 \mathrm{E}+00$ \\
\hline Rb-86 & $6.50 \mathrm{E}+03$ & $1.76 \mathrm{E}-07$ & $3.14 \mathrm{E}+06$ & $2.69 \mathrm{E}+01$ \\
\hline $\mathrm{Rb}-86 \mathrm{~m}$ & $1.30 \mathrm{E}+03$ & $3.51 \mathrm{E}-08$ & $5.98 \mathrm{E}+05$ & $5.13 \mathrm{E}+00$ \\
\hline $\mathrm{Rb}-87$ & $8.88 \mathrm{E}+05$ & 2.40E-05 & $8.13 \mathrm{E}+08$ & $6.97 \mathrm{E}+03$ \\
\hline $\mathrm{Rb}-88$ & $9.41 \mathrm{E}+02$ & $2.54 \mathrm{E}-08$ & $4.45 \mathrm{E}+05$ & $3.82 \mathrm{E}+00$ \\
\hline $\mathrm{Rb}-89$ & $2.91 \mathrm{E}+02$ & 7.86E-09 & $1.34 \mathrm{E}+05$ & $1.15 \mathrm{E}+00$ \\
\hline $\mathrm{Rb}-90$ & $2.94 \mathrm{E}+02$ & 7.94E-09 & $1.36 \mathrm{E}+05$ & $1.17 \mathrm{E}+00$ \\
\hline Rb-90m & $1.95 \mathrm{E}+02$ & $5.26 \mathrm{E}-09$ & $9.03 \mathrm{E}+04$ & 7.74E-01 \\
\hline Sr-79 & $5.88 \mathrm{E}+02$ & $1.59 \mathrm{E}-08$ & $2.73 \mathrm{E}+05$ & $2.34 \mathrm{E}+00$ \\
\hline $\mathrm{Sr}-80$ & $1.65 \mathrm{E}+03$ & $4.46 \mathrm{E}-08$ & $7.60 \mathrm{E}+05$ & $6.52 \mathrm{E}+00$ \\
\hline $\mathrm{Sr}-81$ & $5.07 \mathrm{E}+02$ & $1.37 \mathrm{E}-08$ & $2.35 \mathrm{E}+05$ & $2.01 \mathrm{E}+00$ \\
\hline
\end{tabular}




\begin{tabular}{|c|c|c|c|c|}
\hline Nuclide & $\begin{array}{c}\text { Air } \\
\text { Immersion } \\
\text { DCS } \\
\left(\mathbf{B q} / \mathbf{m}^{3}\right)\end{array}$ & $\begin{array}{c}\text { Air } \\
\text { Immersion } \\
\text { DCS } \\
\left(\mu \mathrm{Ci} / \mathbf{c m}^{3}\right)\end{array}$ & $\begin{array}{c}\text { Water } \\
\text { Submersion } \\
\text { DCS } \\
\left(\mathbf{B q} / \mathbf{m}^{3}\right)\end{array}$ & $\begin{array}{c}\text { Water } \\
\text { Submersion } \\
\text { DCS } \\
\left(\mu \mathrm{Ci} / \mathbf{m}^{3}\right)\end{array}$ \\
\hline Sr-82 & $6.70 \mathrm{E}+06$ & $1.81 \mathrm{E}-04$ & $2.88 \mathrm{E}+09$ & $2.47 \mathrm{E}+04$ \\
\hline Sr-83 & $8.57 \mathrm{E}+02$ & $2.32 \mathrm{E}-08$ & $3.95 \mathrm{E}+05$ & $3.39 \mathrm{E}+00$ \\
\hline Sr-85 & $1.45 \mathrm{E}+03$ & $3.91 \mathrm{E}-08$ & $6.65 \mathrm{E}+05$ & $5.70 \mathrm{E}+00$ \\
\hline Sr- $85 \mathrm{~m}$ & $3.37 \mathrm{E}+03$ & $9.10 \mathrm{E}-08$ & $1.53 \mathrm{E}+06$ & $1.31 \mathrm{E}+01$ \\
\hline Sr- $87 \mathrm{~m}$ & $2.25 \mathrm{E}+03$ & $6.08 \mathrm{E}-08$ & $1.03 \mathrm{E}+06$ & $8.85 \mathrm{E}+00$ \\
\hline Sr-89 & $7.22 \mathrm{E}+04$ & $1.95 \mathrm{E}-06$ & $6.03 \mathrm{E}+07$ & $5.17 \mathrm{E}+02$ \\
\hline Sr-90 & $3.23 \mathrm{E}+05$ & $8.72 \mathrm{E}-06$ & $2.91 \mathrm{E}+08$ & $2.49 \mathrm{E}+03$ \\
\hline Sr-91 & $9.55 \mathrm{E}+02$ & $2.58 \mathrm{E}-08$ & $4.44 \mathrm{E}+05$ & $3.81 \mathrm{E}+00$ \\
\hline Sr-92 & $4.95 \mathrm{E}+02$ & $1.34 \mathrm{E}-08$ & $2.28 \mathrm{E}+05$ & $1.96 \mathrm{E}+00$ \\
\hline Sr-93 & $2.96 \mathrm{E}+02$ & $8.01 \mathrm{E}-09$ & $1.37 \mathrm{E}+05$ & $1.18 \mathrm{E}+00$ \\
\hline Sr-94 & $4.58 \mathrm{E}+02$ & $1.24 \mathrm{E}-08$ & $2.13 \mathrm{E}+05$ & $1.82 \mathrm{E}+00$ \\
\hline Y-81 & $5.93 \mathrm{E}+02$ & $1.60 \mathrm{E}-08$ & $2.76 \mathrm{E}+05$ & $2.36 \mathrm{E}+00$ \\
\hline Y-83 & $5.15 \mathrm{E}+02$ & $1.39 \mathrm{E}-08$ & $2.38 \mathrm{E}+05$ & $2.04 \mathrm{E}+00$ \\
\hline Y-83m & $8.41 \mathrm{E}+02$ & $2.27 \mathrm{E}-08$ & $3.89 \mathrm{E}+05$ & $3.33 \mathrm{E}+00$ \\
\hline $\mathrm{Y}-84 \mathrm{~m}$ & $1.72 \mathrm{E}+02$ & $4.66 \mathrm{E}-09$ & $7.97 \mathrm{E}+04$ & $6.83 \mathrm{E}-01$ \\
\hline Y-85 & $6.54 \mathrm{E}+02$ & $1.77 \mathrm{E}-08$ & $3.02 \mathrm{E}+05$ & $2.59 \mathrm{E}+00$ \\
\hline Y-85m & $5.13 \mathrm{E}+02$ & $1.39 \mathrm{E}-08$ & $2.37 \mathrm{E}+05$ & $2.03 \mathrm{E}+00$ \\
\hline Y-86 & $1.89 \mathrm{E}+02$ & $5.10 \mathrm{E}-09$ & $8.71 \mathrm{E}+04$ & $7.47 \mathrm{E}-01$ \\
\hline Y-86m & $3.30 \mathrm{E}+03$ & $8.93 \mathrm{E}-08$ & $1.51 \mathrm{E}+06$ & $1.29 \mathrm{E}+01$ \\
\hline Y-87 & $1.63 \mathrm{E}+03$ & $4.42 \mathrm{E}-08$ & $7.50 \mathrm{E}+05$ & $6.42 \mathrm{E}+00$ \\
\hline $\mathrm{Y}-87 \mathrm{~m}$ & $2.35 \mathrm{E}+03$ & $6.35 \mathrm{E}-08$ & $1.08 \mathrm{E}+06$ & $9.24 \mathrm{E}+00$ \\
\hline $\mathrm{Y}-88$ & $2.44 \mathrm{E}+02$ & $6.59 \mathrm{E}-09$ & $1.12 \mathrm{E}+05$ & $9.64 \mathrm{E}-01$ \\
\hline Y-89m & $7.62 \mathrm{E}+02$ & $2.06 \mathrm{E}-08$ & $3.52 \mathrm{E}+05$ & $3.01 \mathrm{E}+00$ \\
\hline Y-90 & $4.01 \mathrm{E}+04$ & $1.08 \mathrm{E}-06$ & $3.22 \mathrm{E}+07$ & $2.76 \mathrm{E}+02$ \\
\hline Y-90m & $1.13 \mathrm{E}+03$ & $3.06 \mathrm{E}-08$ & $5.20 \mathrm{E}+05$ & $4.46 \mathrm{E}+00$ \\
\hline Y-91 & $5.28 \mathrm{E}+04$ & $1.43 \mathrm{E}-06$ & $3.68 \mathrm{E}+07$ & $3.16 \mathrm{E}+02$ \\
\hline Y-91m & $1.34 \mathrm{E}+03$ & $3.63 \mathrm{E}-08$ & $6.18 \mathrm{E}+05$ & $5.30 \mathrm{E}+00$ \\
\hline Y-92 & $2.40 \mathrm{E}+03$ & $6.49 \mathrm{E}-08$ & $1.16 \mathrm{E}+06$ & $9.92 \mathrm{E}+00$ \\
\hline Y-93 & $5.63 \mathrm{E}+03$ & $1.52 \mathrm{E}-07$ & $2.81 \mathrm{E}+06$ & $2.40 \mathrm{E}+01$ \\
\hline Y-94 & $8.30 \mathrm{E}+02$ & 2.24E-08 & $3.91 \mathrm{E}+05$ & $3.35 \mathrm{E}+00$ \\
\hline Y-95 & $5.55 \mathrm{E}+02$ & $1.50 \mathrm{E}-08$ & $2.58 \mathrm{E}+05$ & $2.21 \mathrm{E}+00$ \\
\hline $\mathrm{Zr}-85$ & $4.70 \mathrm{E}+02$ & $1.27 \mathrm{E}-08$ & $2.19 \mathrm{E}+05$ & $1.87 \mathrm{E}+00$ \\
\hline $\mathrm{Zr}-86$ & $2.64 \mathrm{E}+03$ & $7.14 \mathrm{E}-08$ & $1.21 \mathrm{E}+06$ & $1.03 \mathrm{E}+01$ \\
\hline $\begin{array}{l}\mathrm{Zr}-87 \\
\end{array}$ & $7.51 \mathrm{E}+02$ & $2.03 \mathrm{E}-08$ & $3.48 \mathrm{E}+05$ & $2.99 \mathrm{E}+00$ \\
\hline Zr- 88 & $1.88 \mathrm{E}+03$ & $5.07 \mathrm{E}-08$ & $8.62 \mathrm{E}+05$ & $7.38 \mathrm{E}+00$ \\
\hline $\mathrm{Zr}-89$ & $5.99 \mathrm{E}+02$ & $1.62 \mathrm{E}-08$ & $2.76 \mathrm{E}+05$ & $2.36 \mathrm{E}+00$ \\
\hline $\mathrm{Zr}-89 \mathrm{~m}$ & $1.10 \mathrm{E}+03$ & $2.98 \mathrm{E}-08$ & $5.08 \mathrm{E}+05$ & $4.36 \mathrm{E}+00$ \\
\hline
\end{tabular}




\begin{tabular}{|c|c|c|c|c|}
\hline Nuclide & $\begin{array}{c}\text { Air } \\
\text { Immersion } \\
\text { DCS } \\
\left(\mathbf{B q} / \mathbf{m}^{3}\right)\end{array}$ & $\begin{array}{c}\text { Air } \\
\text { Immersion } \\
\text { DCS } \\
\left(\mu \mathrm{Ci} / \mathbf{c m}^{3}\right)\end{array}$ & $\begin{array}{c}\text { Water } \\
\text { Submersion } \\
\text { DCS } \\
\left(\mathbf{B q} / \mathbf{m}^{3}\right)\end{array}$ & $\begin{array}{c}\text { Water } \\
\text { Submersion } \\
\text { DCS } \\
\left(\mu \mathrm{Ci} / \mathbf{m}^{3}\right)\end{array}$ \\
\hline $\mathrm{Zr}-93$ & $4.92 \mathrm{E}+10$ & $1.33 \mathrm{E}+00$ & $4.70 \mathrm{E}+13$ & $4.03 \mathrm{E}+08$ \\
\hline Zr-95 & $9.52 \mathrm{E}+02$ & $2.57 \mathrm{E}-08$ & $4.39 \mathrm{E}+05$ & $3.76 \mathrm{E}+00$ \\
\hline Zr-97 & $7.79 \mathrm{E}+02$ & $2.11 \mathrm{E}-08$ & $3.61 \mathrm{E}+05$ & $3.09 \mathrm{E}+00$ \\
\hline $\mathrm{Nb}-87$ & $5.72 \mathrm{E}+02$ & $1.55 \mathrm{E}-08$ & $2.66 \mathrm{E}+05$ & $2.28 \mathrm{E}+00$ \\
\hline $\mathrm{Nb}-88$ & $1.63 \mathrm{E}+02$ & $4.42 \mathrm{E}-09$ & $7.55 \mathrm{E}+04$ & $6.47 \mathrm{E}-01$ \\
\hline $\mathrm{Nb}-88 \mathrm{~m}$ & $1.66 \mathrm{E}+02$ & 4.49E-09 & $7.68 \mathrm{E}+04$ & $6.58 \mathrm{E}-01$ \\
\hline $\mathrm{Nb}-89$ & $4.87 \mathrm{E}+02$ & $1.32 \mathrm{E}-08$ & $2.26 \mathrm{E}+05$ & $1.94 \mathrm{E}+00$ \\
\hline $\mathrm{Nb}-89 \mathrm{~m}$ & $5.39 \mathrm{E}+02$ & $1.46 \mathrm{E}-08$ & $2.50 \mathrm{E}+05$ & $2.14 \mathrm{E}+00$ \\
\hline $\mathrm{Nb}-90$ & $1.55 \mathrm{E}+02$ & $4.18 \mathrm{E}-09$ & $7.14 \mathrm{E}+04$ & $6.12 \mathrm{E}-01$ \\
\hline $\mathrm{Nb}-91$ & $3.75 \mathrm{E}+05$ & $1.01 \mathrm{E}-05$ & $1.70 \mathrm{E}+08$ & $1.46 \mathrm{E}+03$ \\
\hline $\mathrm{Nb}-91 \mathrm{~m}$ & $2.64 \mathrm{E}+04$ & $7.14 \mathrm{E}-07$ & $1.22 \mathrm{E}+07$ & $1.05 \mathrm{E}+02$ \\
\hline $\mathrm{Nb}-92$ & $4.64 \mathrm{E}+02$ & $1.25 \mathrm{E}-08$ & $2.14 \mathrm{E}+05$ & $1.84 \mathrm{E}+00$ \\
\hline Nb-92m & $7.14 \mathrm{E}+02$ & $1.93 \mathrm{E}-08$ & $3.29 \mathrm{E}+05$ & $2.82 \mathrm{E}+00$ \\
\hline $\mathrm{Nb}-93 \mathrm{~m}$ & $1.04 \mathrm{E}+07$ & $2.82 \mathrm{E}-04$ & $4.45 \mathrm{E}+09$ & $3.81 \mathrm{E}+04$ \\
\hline Nb-94 & $4.45 \mathrm{E}+02$ & $1.20 \mathrm{E}-08$ & $2.05 \mathrm{E}+05$ & $1.75 \mathrm{E}+00$ \\
\hline $\mathrm{Nb}-94 \mathrm{~m}$ & $1.47 \mathrm{E}+05$ & $3.99 \mathrm{E}-06$ & $6.79 \mathrm{E}+07$ & $5.82 \mathrm{E}+02$ \\
\hline $\mathrm{Nb}-95$ & $9.09 \mathrm{E}+02$ & $2.46 \mathrm{E}-08$ & $4.19 \mathrm{E}+05$ & $3.59 \mathrm{E}+00$ \\
\hline Nb-95m & $1.12 \mathrm{E}+04$ & $3.03 \mathrm{E}-07$ & $5.20 \mathrm{E}+06$ & $4.46 \mathrm{E}+01$ \\
\hline Nb-96 & $2.81 \mathrm{E}+02$ & $7.58 \mathrm{E}-09$ & $1.29 \mathrm{E}+05$ & $1.11 \mathrm{E}+00$ \\
\hline $\mathrm{Nb}-97$ & $1.04 \mathrm{E}+03$ & $2.82 \mathrm{E}-08$ & $4.83 \mathrm{E}+05$ & $4.14 \mathrm{E}+00$ \\
\hline $\mathrm{Nb}-98 \mathrm{~m}$ & $2.40 \mathrm{E}+02$ & 6.49E-09 & $1.11 \mathrm{E}+05$ & $9.50 \mathrm{E}-01$ \\
\hline $\mathrm{Nb}-99$ & $3.81 \mathrm{E}+03$ & $1.03 \mathrm{E}-07$ & $1.84 \mathrm{E}+06$ & $1.58 \mathrm{E}+01$ \\
\hline $\mathrm{Nb}-99 \mathrm{~m}$ & $8.21 \mathrm{E}+02$ & $2.22 \mathrm{E}-08$ & $3.84 \mathrm{E}+05$ & $3.29 \mathrm{E}+00$ \\
\hline Mo-89 & $5.56 \mathrm{E}+02$ & $1.50 \mathrm{E}-08$ & $2.60 \mathrm{E}+05$ & $2.23 \mathrm{E}+00$ \\
\hline Mo-90 & $8.66 \mathrm{E}+02$ & $2.34 \mathrm{E}-08$ & $3.97 \mathrm{E}+05$ & $3.41 \mathrm{E}+00$ \\
\hline Mo-91 & $7.05 \mathrm{E}+02$ & $1.90 \mathrm{E}-08$ & $3.28 \mathrm{E}+05$ & \\
\hline Mo-91m & $4.92 \mathrm{E}+02$ & $1.33 \mathrm{E}-08$ & $2.26 \mathrm{E}+05$ & $1.94 \mathrm{E}+00$ \\
\hline Mo-93 & $1.87 \mathrm{E}+06$ & $5.04 \mathrm{E}-05$ & $7.95 \mathrm{E}+08$ & $6.81 \mathrm{E}+03$ \\
\hline Mo-93m & $2.91 \mathrm{E}+02$ & $7.86 \mathrm{E}-09$ & $1.34 \mathrm{E}+05$ & $1.15 \mathrm{E}+00$ \\
\hline Mo-99 & $4.58 \mathrm{E}+03$ & $1.24 \mathrm{E}-07$ & $2.14 \mathrm{E}+06$ & $1.84 \mathrm{E}+01$ \\
\hline Mo-101 & $4.55 \mathrm{E}+02$ & $1.23 \mathrm{E}-08$ & $2.10 \mathrm{E}+05$ & $1.80 \mathrm{E}+00$ \\
\hline Mo-102 & $3.11 \mathrm{E}+04$ & & $1.58 \mathrm{E}+07$ & $1.35 \mathrm{E}+02$ \\
\hline Tc-91 & $2.64 \mathrm{E}+02$ & $7.14 \mathrm{E}-09$ & $1.22 \mathrm{E}+05$ & $1.05 \mathrm{E}+00$ \\
\hline Tc-91m & $4.80 \mathrm{E}+02$ & $1.30 \mathrm{E}-08$ & $2.23 \mathrm{E}+05$ & $1.91 \mathrm{E}+00$ \\
\hline Tc-92 & $1.77 \mathrm{E}+02$ & $4.79 \mathrm{E}-09$ & $8.17 \mathrm{E}+04$ & $7.00 \mathrm{E}-01$ \\
\hline Tc-93 & $4.25 \mathrm{E}+02$ & $1.15 \mathrm{E}-08$ & $1.96 \mathrm{E}+05$ & $1.68 \mathrm{E}+00$ \\
\hline Tc-93m & $6.80 \mathrm{E}+02$ & $1.84 \mathrm{E}-08$ & $3.14 \mathrm{E}+05$ & $2.69 \mathrm{E}+00$ \\
\hline
\end{tabular}




\begin{tabular}{|c|c|c|c|c|}
\hline Nuclide & $\begin{array}{c}\text { Air } \\
\text { Immersion } \\
\text { DCS } \\
\left(\mathbf{B q} / \mathbf{m}^{3}\right)\end{array}$ & $\begin{array}{c}\text { Air } \\
\text { Immersion } \\
\text { DCS } \\
\left(\mu \mathrm{Ci} / \mathbf{c m}^{3}\right)\end{array}$ & $\begin{array}{c}\text { Water } \\
\text { Submersion } \\
\text { DCS } \\
\left(\mathbf{B q} / \mathbf{m}^{3}\right)\end{array}$ & $\begin{array}{c}\text { Water } \\
\text { Submersion } \\
\text { DCS } \\
\left(\mu \mathrm{Ci} / \mathbf{m}^{3}\right)\end{array}$ \\
\hline Tc-94 & $2.62 \mathrm{E}+02$ & $7.08 \mathrm{E}-09$ & $1.21 \mathrm{E}+05$ & $1.03 \mathrm{E}+00$ \\
\hline Tc-94m & $3.48 \mathrm{E}+02$ & $9.41 \mathrm{E}-09$ & $1.61 \mathrm{E}+05$ & $1.38 \mathrm{E}+00$ \\
\hline Tc-95 & $8.83 \mathrm{E}+02$ & $2.39 \mathrm{E}-08$ & $4.08 \mathrm{E}+05$ & $3.49 \mathrm{E}+00$ \\
\hline Tc-95m & $1.04 \mathrm{E}+03$ & $2.80 \mathrm{E}-08$ & $4.78 \mathrm{E}+05$ & $4.09 \mathrm{E}+00$ \\
\hline Tc-96 & $2.78 \mathrm{E}+02$ & 7.52E-09 & $1.28 \mathrm{E}+05$ & $1.10 \mathrm{E}+00$ \\
\hline Tc-96m & $1.63 \mathrm{E}+04$ & $4.42 \mathrm{E}-07$ & $7.53 \mathrm{E}+06$ & $6.46 \mathrm{E}+01$ \\
\hline Tc-97 & $1.43 \mathrm{E}+06$ & $3.88 \mathrm{E}-05$ & $6.10 \mathrm{E}+08$ & $5.23 \mathrm{E}+03$ \\
\hline Tc- $97 \mathrm{~m}$ & $8.62 \mathrm{E}+05$ & $2.33 \mathrm{E}-05$ & $3.91 \mathrm{E}+08$ & $3.36 \mathrm{E}+03$ \\
\hline Tc-98 & $4.95 \mathrm{E}+02$ & $1.34 \mathrm{E}-08$ & $2.28 \mathrm{E}+05$ & $1.96 \mathrm{E}+00$ \\
\hline Tc-99 & $1.10 \mathrm{E}+06$ & $2.98 \mathrm{E}-05$ & $1.01 \mathrm{E}+09$ & $8.65 \mathrm{E}+03$ \\
\hline Tc-99m & $6.02 \mathrm{E}+03$ & $1.63 \mathrm{E}-07$ & $2.71 \mathrm{E}+06$ & $2.32 \mathrm{E}+01$ \\
\hline Tc-101 & $2.09 \mathrm{E}+03$ & $5.64 \mathrm{E}-08$ & $9.67 \mathrm{E}+05$ & $8.29 \mathrm{E}+00$ \\
\hline Tc-102 & $5.42 \mathrm{E}+03$ & $1.46 \mathrm{E}-07$ & $2.88 \mathrm{E}+06$ & $2.47 \mathrm{E}+01$ \\
\hline $\mathrm{Tc}-102 \mathrm{~m}$ & $2.69 \mathrm{E}+02$ & $7.26 \mathrm{E}-09$ & $1.24 \mathrm{E}+05$ & $1.06 \mathrm{E}+00$ \\
\hline Tc-104 & $2.88 \mathrm{E}+02$ & 7.79E-09 & $1.34 \mathrm{E}+05$ & $1.15 \mathrm{E}+00$ \\
\hline Tc-105 & $8.41 \mathrm{E}+02$ & $2.27 \mathrm{E}-08$ & $3.91 \mathrm{E}+05$ & $3.36 \mathrm{E}+00$ \\
\hline Ru-92 & $3.36 \mathrm{E}+02$ & $9.08 \mathrm{E}-09$ & $1.55 \mathrm{E}+05$ & $1.33 \mathrm{E}+00$ \\
\hline Ru-94 & $1.39 \mathrm{E}+03$ & $3.76 \mathrm{E}-08$ & $6.39 \mathrm{E}+05$ & $5.48 \mathrm{E}+00$ \\
\hline Ru-95 & $5.58 \mathrm{E}+02$ & $1.51 \mathrm{E}-08$ & $2.58 \mathrm{E}+05$ & $2.21 \mathrm{E}+00$ \\
\hline Ru-97 & $3.18 \mathrm{E}+03$ & $8.60 \mathrm{E}-08$ & $1.45 \mathrm{E}+06$ & $1.25 \mathrm{E}+01$ \\
\hline Ru-103 & $1.43 \mathrm{E}+03$ & $3.88 \mathrm{E}-08$ & $6.61 \mathrm{E}+05$ & $5.66 \mathrm{E}+00$ \\
\hline Ru-105 & $9.35 \mathrm{E}+02$ & $2.53 \mathrm{E}-08$ & $4.32 \mathrm{E}+05$ & $3.70 \mathrm{E}+00$ \\
\hline Ru-107 & $1.88 \mathrm{E}+03$ & $5.07 \mathrm{E}-08$ & $8.88 \mathrm{E}+05$ & $7.61 \mathrm{E}+00$ \\
\hline Ru-108 & $1.09 \mathrm{E}+04$ & $2.94 \mathrm{E}-07$ & $5.20 \mathrm{E}+06$ & $4.46 \mathrm{E}+01$ \\
\hline Rh-94 & $1.75 \mathrm{E}+02$ & 4.73E-09 & $8.15 \mathrm{E}+04$ & $6.99 \mathrm{E}-01$ \\
\hline Rh-95 & $2.62 \mathrm{E}+02$ & $7.08 \mathrm{E}-09$ & $1.21 \mathrm{E}+05$ & $1.04 \mathrm{E}+00$ \\
\hline Rh-95m & $7.39 \mathrm{E}+02$ & $2.00 \mathrm{E}-08$ & $3.41 \mathrm{E}+05$ & $2.92 \mathrm{E}+00$ \\
\hline Rh-96 & $1.75 \mathrm{E}+02$ & $4.73 \mathrm{E}-09$ & $8.07 \mathrm{E}+04$ & $6.92 \mathrm{E}-01$ \\
\hline Rh-96m & $5.26 \mathrm{E}+02$ & $1.42 \mathrm{E}-08$ & $2.44 \mathrm{E}+05$ & $2.09 \mathrm{E}+00$ \\
\hline Rh-97 & $4.80 \mathrm{E}+02$ & $1.30 \mathrm{E}-08$ & $2.22 \mathrm{E}+05$ & $1.90 \mathrm{E}+00$ \\
\hline Rh-97m & $2.99 \mathrm{E}+02$ & 8.09E-09 & $1.37 \mathrm{E}+05$ & $1.18 \mathrm{E}+00$ \\
\hline Rh-98 & $3.80 \mathrm{E}+02$ & $1.03 \mathrm{E}-08$ & $1.76 \mathrm{E}+05$ & $1.51 \mathrm{E}+00$ \\
\hline Rh-99 & $1.31 \mathrm{E}+03$ & $3.54 \mathrm{E}-08$ & $6.01 \mathrm{E}+05$ & $5.15 \mathrm{E}+00$ \\
\hline Rh-99m & $1.10 \mathrm{E}+03$ & $2.97 \mathrm{E}-08$ & $5.05 \mathrm{E}+05$ & $4.33 \mathrm{E}+00$ \\
\hline Rh-100 & $2.40 \mathrm{E}+02$ & $6.49 \mathrm{E}-09$ & $1.11 \mathrm{E}+05$ & 9.54E-01 \\
\hline Rh-100m & $1.56 \mathrm{E}+04$ & $4.22 \mathrm{E}-07$ & $7.16 \mathrm{E}+06$ & $6.13 \mathrm{E}+01$ \\
\hline Rh-101 & $2.71 \mathrm{E}+03$ & $7.32 \mathrm{E}-08$ & $1.23 \mathrm{E}+06$ & $1.05 \mathrm{E}+01$ \\
\hline
\end{tabular}




\begin{tabular}{|c|c|c|c|c|}
\hline Nuclide & $\begin{array}{c}\text { Air } \\
\text { Immersion } \\
\text { DCS } \\
\left(\mathbf{B q} / \mathbf{m}^{3}\right)\end{array}$ & $\begin{array}{c}\text { Air } \\
\text { Immersion } \\
\text { DCS } \\
\left(\mu \mathrm{Ci} / \mathbf{c m}^{3}\right)\end{array}$ & $\begin{array}{c}\text { Water } \\
\text { Submersion } \\
\text { DCS } \\
\left(\mathbf{B q} / \mathbf{m}^{3}\right)\end{array}$ & $\begin{array}{c}\text { Water } \\
\text { Submersion } \\
\text { DCS } \\
\left(\mu \mathrm{Ci} / \mathbf{m}^{3}\right)\end{array}$ \\
\hline Rh-101m & $2.62 \mathrm{E}+03$ & $7.08 \mathrm{E}-08$ & $1.20 \mathrm{E}+06$ & $1.03 \mathrm{E}+01$ \\
\hline Rh-102 & $1.42 \mathrm{E}+03$ & $3.83 \mathrm{E}-08$ & $6.52 \mathrm{E}+05$ & $5.59 \mathrm{E}+00$ \\
\hline Rh-102m & $3.26 \mathrm{E}+02$ & $8.80 \mathrm{E}-09$ & $1.50 \mathrm{E}+05$ & $1.29 \mathrm{E}+00$ \\
\hline Rh-103m & $5.65 \mathrm{E}+06$ & $1.53 \mathrm{E}-04$ & $2.40 \mathrm{E}+09$ & $2.06 \mathrm{E}+04$ \\
\hline Rh-104 & $2.26 \mathrm{E}+04$ & $6.12 \mathrm{E}-07$ & $1.40 \mathrm{E}+07$ & $1.20 \mathrm{E}+02$ \\
\hline Rh-104m & $3.47 \mathrm{E}+04$ & $9.38 \mathrm{E}-07$ & $1.52 \mathrm{E}+07$ & $1.30 \mathrm{E}+02$ \\
\hline Rh-105 & $9.14 \mathrm{E}+03$ & $2.47 \mathrm{E}-07$ & $4.23 \mathrm{E}+06$ & $3.63 \mathrm{E}+01$ \\
\hline Rh-106 & $2.96 \mathrm{E}+03$ & $8.01 \mathrm{E}-08$ & $1.44 \mathrm{E}+06$ & $1.24 \mathrm{E}+01$ \\
\hline Rh-106m & $2.40 \mathrm{E}+02$ & 6.49E-09 & $1.11 \mathrm{E}+05$ & $9.50 \mathrm{E}-01$ \\
\hline Rh-107 & $2.25 \mathrm{E}+03$ & $6.08 \mathrm{E}-08$ & $1.04 \mathrm{E}+06$ & $8.91 \mathrm{E}+00$ \\
\hline Rh-108 & $1.97 \mathrm{E}+03$ & $5.32 \mathrm{E}-08$ & $9.49 \mathrm{E}+05$ & $8.14 \mathrm{E}+00$ \\
\hline Rh-109 & $2.30 \mathrm{E}+03$ & $6.21 \mathrm{E}-08$ & $1.07 \mathrm{E}+06$ & $9.21 \mathrm{E}+00$ \\
\hline Pd-96 & $4.87 \mathrm{E}+02$ & $1.32 \mathrm{E}-08$ & $2.25 \mathrm{E}+05$ & $1.93 \mathrm{E}+00$ \\
\hline Pd-97 & $2.83 \mathrm{E}+02$ & $7.65 \mathrm{E}-09$ & $1.30 \mathrm{E}+05$ & $1.12 \mathrm{E}+00$ \\
\hline Pd-98 & $1.80 \mathrm{E}+03$ & $4.87 \mathrm{E}-08$ & $8.24 \mathrm{E}+05$ & $7.06 \mathrm{E}+00$ \\
\hline Pd-99 & $5.39 \mathrm{E}+02$ & $1.46 \mathrm{E}-08$ & $2.48 \mathrm{E}+05$ & $2.12 \mathrm{E}+00$ \\
\hline Pd-100 & $8.71 \mathrm{E}+03$ & $2.35 \mathrm{E}-07$ & $3.87 \mathrm{E}+06$ & $3.32 \mathrm{E}+01$ \\
\hline Pd-101 & $2.14 \mathrm{E}+03$ & 5.79E-08 & $9.85 \mathrm{E}+05$ & $8.44 \mathrm{E}+00$ \\
\hline Pd-103 & $5.99 \mathrm{E}+05$ & $1.62 \mathrm{E}-05$ & $2.56 \mathrm{E}+08$ & $2.19 \mathrm{E}+03$ \\
\hline Pd-109 & $7.55 \mathrm{E}+04$ & $2.04 \mathrm{E}-06$ & & \\
\hline Pd-109m & $6.91 \mathrm{E}+03$ & $1.87 \mathrm{E}-07$ & & $2.69 \mathrm{E}+01$ \\
\hline Pd-111 & $1.10 \mathrm{E}+04$ & $2.97 \mathrm{E}-07$ & $5.64 \mathrm{E}+06$ & $4.84 \mathrm{E}+01$ \\
\hline Pd-112 & $1.18 \mathrm{E}+06$ & $3.20 \mathrm{E}-05$ & $7.32 \mathrm{E}+08$ & $6.28 \mathrm{E}+03$ \\
\hline Pd-114 & $2.13 \mathrm{E}+04$ & $5.75 \mathrm{E}-07$ & $1.10 \mathrm{E}+07$ & $9.40 \mathrm{E}+01$ \\
\hline Ag-99 & $2.94 \mathrm{E}+02$ & 7.94E-09 & $1.36 \mathrm{E}+05$ & $1.17 \mathrm{E}+00$ \\
\hline $\mathrm{Ag}-100 \mathrm{~m}$ & $2.38 \mathrm{E}+02$ & $6.44 \mathrm{E}-09$ & $1.10 \mathrm{E}+05$ & $9.47 \mathrm{E}-01$ \\
\hline Ag-101 & $4.41 \mathrm{E}+02$ & $1.19 \mathrm{E}-08$ & $2.03 \mathrm{E}+05$ & $1.74 \mathrm{E}+00$ \\
\hline $\mathrm{Ag}-102$ & $1.98 \mathrm{E}+02$ & 5.36E-09 & $9.14 \mathrm{E}+04$ & $\begin{array}{l}7.83 \mathrm{E}-01 \\
\end{array}$ \\
\hline $\mathrm{Ag}-102 \mathrm{~m}$ & $3.26 \mathrm{E}+02$ & $8.82 \mathrm{E}-09$ & $1.51 \mathrm{E}+05$ & $1.29 \mathrm{E}+00$ \\
\hline Ag-103 & $8.37 \mathrm{E}+02$ & $2.26 \mathrm{E}-08$ & $3.85 \mathrm{E}+05$ & $3.30 \mathrm{E}+00$ \\
\hline Ag-104 & $2.56 \mathrm{E}+02$ & $6.91 \mathrm{E}-09$ & $1.17 \mathrm{E}+05$ & $1.01 \mathrm{E}+00$ \\
\hline Ag-104m & $3.75 \mathrm{E}+02$ & $1.01 \mathrm{E}-08$ & & $1.49 \mathrm{E}+00$ \\
\hline Ag-105 & $1.43 \mathrm{E}+03$ & $3.88 \mathrm{E}-08$ & $6.59 \mathrm{E}+05$ & $5.65 \mathrm{E}+00$ \\
\hline $\mathrm{Ag}-105 \mathrm{~m}$ & $7.17 \mathrm{E}+05$ & $1.94 \mathrm{E}-05$ & $3.29 \mathrm{E}+08$ & $2.82 \mathrm{E}+03$ \\
\hline Ag-106 & $1.01 \mathrm{E}+03$ & $2.74 \mathrm{E}-08$ & $4.68 \mathrm{E}+05$ & $4.01 \mathrm{E}+00$ \\
\hline Ag-106m & $2.46 \mathrm{E}+02$ & $6.64 \mathrm{E}-09$ & $1.14 \mathrm{E}+05$ & $9.74 \mathrm{E}-01$ \\
\hline Ag-108 & $2.50 \mathrm{E}+04$ & $6.75 \mathrm{E}-07$ & $1.37 \mathrm{E}+07$ & $1.17 \mathrm{E}+02$ \\
\hline
\end{tabular}




\begin{tabular}{|c|c|c|c|c|}
\hline Nuclide & $\begin{array}{c}\text { Air } \\
\text { Immersion } \\
\text { DCS } \\
\left(\mathbf{B q} / \mathbf{m}^{3}\right)\end{array}$ & $\begin{array}{c}\text { Air } \\
\text { Immersion } \\
\text { DCS } \\
\left(\mu \mathrm{Ci} / \mathbf{c m}^{3}\right)\end{array}$ & $\begin{array}{c}\text { Water } \\
\text { Submersion } \\
\text { DCS } \\
\left(\mathbf{B q} / \mathbf{m}^{3}\right)\end{array}$ & $\begin{array}{c}\text { Water } \\
\text { Submersion } \\
\text { DCS } \\
\left(\mu \mathrm{Ci} / \mathbf{m}^{3}\right)\end{array}$ \\
\hline Ag-108m & $4.39 \mathrm{E}+02$ & $1.19 \mathrm{E}-08$ & $2.02 \mathrm{E}+05$ & $1.73 \mathrm{E}+00$ \\
\hline Ag-109m & $2.01 \mathrm{E}+05$ & $5.42 \mathrm{E}-06$ & $8.91 \mathrm{E}+07$ & $7.63 \mathrm{E}+02$ \\
\hline Ag-110 & $1.29 \mathrm{E}+04$ & $3.48 \mathrm{E}-07$ & $7.24 \mathrm{E}+06$ & $6.20 \mathrm{E}+01$ \\
\hline $\mathrm{Ag}-110 \mathrm{~m}$ & $2.48 \mathrm{E}+02$ & $6.70 \mathrm{E}-09$ & $1.14 \mathrm{E}+05$ & $9.81 \mathrm{E}-01$ \\
\hline Ag-111 & $2.28 \mathrm{E}+04$ & $6.17 \mathrm{E}-07$ & $1.13 \mathrm{E}+07$ & $9.71 \mathrm{E}+01$ \\
\hline $\mathrm{Ag}-111 \mathrm{~m}$ & $1.89 \mathrm{E}+05$ & $5.10 \mathrm{E}-06$ & $8.55 \mathrm{E}+07$ & $7.33 \mathrm{E}+02$ \\
\hline Ag-112 & $9.35 \mathrm{E}+02$ & $2.53 \mathrm{E}-08$ & $4.38 \mathrm{E}+05$ & $3.75 \mathrm{E}+00$ \\
\hline $\mathrm{Ag}-113$ & $8.28 \mathrm{E}+03$ & $2.24 \mathrm{E}-07$ & $4.09 \mathrm{E}+06$ & $3.51 \mathrm{E}+01$ \\
\hline $\mathrm{Ag}-113 \mathrm{~m}$ & $3.32 \mathrm{E}+03$ & $8.97 \mathrm{E}-08$ & $1.53 \mathrm{E}+06$ & $1.31 \mathrm{E}+01$ \\
\hline Ag-114 & $2.17 \mathrm{E}+03$ & $5.87 \mathrm{E}-08$ & $1.07 \mathrm{E}+06$ & $9.15 \mathrm{E}+00$ \\
\hline Ag-115 & $1.32 \mathrm{E}+03$ & $3.57 \mathrm{E}-08$ & $6.19 \mathrm{E}+05$ & $5.31 \mathrm{E}+00$ \\
\hline Ag-116 & $2.96 \mathrm{E}+02$ & $8.01 \mathrm{E}-09$ & $1.38 \mathrm{E}+05$ & $1.19 \mathrm{E}+00$ \\
\hline Ag-117 & $4.88 \mathrm{E}+02$ & $1.32 \mathrm{E}-08$ & $2.26 \mathrm{E}+05$ & $1.94 \mathrm{E}+00$ \\
\hline Cd-101 & $2.71 \mathrm{E}+02$ & 7.32E-09 & $1.25 \mathrm{E}+05$ & $1.07 \mathrm{E}+00$ \\
\hline Cd-102 & $8.50 \mathrm{E}+02$ & $2.30 \mathrm{E}-08$ & $3.91 \mathrm{E}+05$ & $3.36 \mathrm{E}+00$ \\
\hline Cd-103 & $3.17 \mathrm{E}+02$ & $8.57 \mathrm{E}-09$ & $1.46 \mathrm{E}+05$ & $1.25 \mathrm{E}+00$ \\
\hline Cd-104 & $3.14 \mathrm{E}+03$ & $8.49 \mathrm{E}-08$ & $1.43 \mathrm{E}+06$ & $1.23 \mathrm{E}+01$ \\
\hline Cd-105 & $5.17 \mathrm{E}+02$ & $1.40 \mathrm{E}-08$ & $2.38 \mathrm{E}+05$ & $2.04 \mathrm{E}+00$ \\
\hline Cd-107 & $6.47 \mathrm{E}+04$ & $1.75 \mathrm{E}-06$ & $2.91 \mathrm{E}+07$ & $2.49 \mathrm{E}+02$ \\
\hline Cd-109 & $1.40 \mathrm{E}+05$ & $3.78 \mathrm{E}-06$ & $6.10 \mathrm{E}+07$ & $5.23 \mathrm{E}+02$ \\
\hline Cd-111m & $2.64 \mathrm{E}+03$ & $7.14 \mathrm{E}-08$ & $1.20 \mathrm{E}+06$ & $1.03 \mathrm{E}+01$ \\
\hline Cd-113 & $1.27 \mathrm{E}+06$ & $3.44 \mathrm{E}-05$ & $1.17 \mathrm{E}+09$ & $9.99 \mathrm{E}+03$ \\
\hline $\mathrm{Cd}-113 \mathrm{~m}$ & $3.42 \mathrm{E}+05$ & $9.24 \mathrm{E}-06$ & $2.99 \mathrm{E}+08$ & $2.56 \mathrm{E}+03$ \\
\hline $\mathrm{Cd}-115$ & $3.63 \mathrm{E}+03$ & $9.82 \mathrm{E}-08$ & $1.69 \mathrm{E}+06$ & $1.45 \mathrm{E}+01$ \\
\hline $\mathrm{Cd}-115 \mathrm{~m}$ & $1.59 \mathrm{E}+04$ & $4.31 \mathrm{E}-07$ & $8.19 \mathrm{E}+06$ & $7.02 \mathrm{E}+01$ \\
\hline Cd-117 & $6.21 \mathrm{E}+02$ & $1.68 \mathrm{E}-08$ & $2.88 \mathrm{E}+05$ & $2.47 \mathrm{E}+00$ \\
\hline $\mathrm{Cd}-117 \mathrm{~m}$ & $3.21 \mathrm{E}+02$ & $8.67 \mathrm{E}-09$ & $1.48 \mathrm{E}+05$ & $1.27 \mathrm{E}+00$ \\
\hline $\mathrm{Cd}-118$ & $4.37 \mathrm{E}+05$ & $1.18 \mathrm{E}-05$ & $3.95 \mathrm{E}+08$ & $3.39 \mathrm{E}+03$ \\
\hline Cd-119 & $3.98 \mathrm{E}+02$ & $1.08 \mathrm{E}-08$ & $1.84 \mathrm{E}+05$ & $1.58 \mathrm{E}+00$ \\
\hline Cd-119m & $2.86 \mathrm{E}+02$ & $7.72 \mathrm{E}-09$ & $1.32 \mathrm{E}+05$ & $1.13 \mathrm{E}+00$ \\
\hline In-103 & $2.44 \mathrm{E}+02$ & $6.59 \mathrm{E}-09$ & $1.12 \mathrm{E}+05$ & $9.64 \mathrm{E}-01$ \\
\hline In-105 & $3.54 \mathrm{E}+02$ & $9.55 \mathrm{E}-09$ & $1.63 \mathrm{E}+05$ & $1.40 \mathrm{E}+00$ \\
\hline In-106m & $2.35 \mathrm{E}+02$ & $6.35 \mathrm{E}-09$ & $1.09 \mathrm{E}+05$ & $9.31 \mathrm{E}-01$ \\
\hline In-106 & $1.95 \mathrm{E}+02$ & $5.26 \mathrm{E}-09$ & $8.98 \mathrm{E}+04$ & $7.70 \mathrm{E}-01$ \\
\hline In-107 & $4.42 \mathrm{E}+02$ & $1.19 \mathrm{E}-08$ & $2.03 \mathrm{E}+05$ & $1.74 \mathrm{E}+00$ \\
\hline In-108m & $2.35 \mathrm{E}+02$ & $6.35 \mathrm{E}-09$ & $1.09 \mathrm{E}+05$ & $9.31 \mathrm{E}-01$ \\
\hline In-108 & $1.75 \mathrm{E}+02$ & $4.73 \mathrm{E}-09$ & $8.07 \mathrm{E}+04$ & $6.92 \mathrm{E}-01$ \\
\hline
\end{tabular}




\begin{tabular}{|c|c|c|c|c|}
\hline Nuclide & $\begin{array}{c}\text { Air } \\
\text { Immersion } \\
\text { DCS } \\
\left(\mathbf{B q} / \mathbf{m}^{3}\right)\end{array}$ & $\begin{array}{c}\text { Air } \\
\text { Immersion } \\
\text { DCS } \\
\left(\mu \mathrm{Ci} / \mathbf{c m}^{3}\right)\end{array}$ & $\begin{array}{c}\text { Water } \\
\text { Submersion } \\
\text { DCS } \\
\left(\mathbf{B q} / \mathbf{m}^{3}\right)\end{array}$ & $\begin{array}{c}\text { Water } \\
\text { Submersion } \\
\text { DCS } \\
\left(\mu \mathrm{Ci} / \mathbf{m}^{3}\right)\end{array}$ \\
\hline In-109m & $1.16 \mathrm{E}+03$ & $3.13 \mathrm{E}-08$ & $5.33 \mathrm{E}+05$ & $4.57 \mathrm{E}+00$ \\
\hline In-109 & $1.11 \mathrm{E}+03$ & $3.00 \mathrm{E}-08$ & $5.09 \mathrm{E}+05$ & $4.36 \mathrm{E}+00$ \\
\hline In-110m & $4.37 \mathrm{E}+02$ & $1.18 \mathrm{E}-08$ & $2.02 \mathrm{E}+05$ & $1.73 \mathrm{E}+00$ \\
\hline In-110 & $2.25 \mathrm{E}+02$ & $6.08 \mathrm{E}-09$ & $1.04 \mathrm{E}+05$ & $8.88 \mathrm{E}-01$ \\
\hline In-111m & $1.51 \mathrm{E}+03$ & $4.08 \mathrm{E}-08$ & $6.97 \mathrm{E}+05$ & $5.97 \mathrm{E}+00$ \\
\hline In-111 & $1.88 \mathrm{E}+03$ & $5.07 \mathrm{E}-08$ & $8.57 \mathrm{E}+05$ & $7.34 \mathrm{E}+00$ \\
\hline In-112m & $3.22 \mathrm{E}+04$ & $8.69 \mathrm{E}-07$ & $1.47 \mathrm{E}+07$ & $1.26 \mathrm{E}+02$ \\
\hline In-112 & $2.66 \mathrm{E}+03$ & $7.20 \mathrm{E}-08$ & $1.24 \mathrm{E}+06$ & $1.06 \mathrm{E}+01$ \\
\hline In-113m & $2.81 \mathrm{E}+03$ & $7.58 \mathrm{E}-08$ & $1.29 \mathrm{E}+06$ & $1.10 \mathrm{E}+01$ \\
\hline In-114m & $9.73 \mathrm{E}+03$ & $2.63 \mathrm{E}-07$ & $4.49 \mathrm{E}+06$ & $3.84 \mathrm{E}+01$ \\
\hline In-114 & $4.36 \mathrm{E}+04$ & $1.18 \mathrm{E}-06$ & $3.21 \mathrm{E}+07$ & $2.75 \mathrm{E}+02$ \\
\hline In-115m & $4.57 \mathrm{E}+03$ & $1.23 \mathrm{E}-07$ & $2.11 \mathrm{E}+06$ & $1.81 \mathrm{E}+01$ \\
\hline In-115 & $4.80 \mathrm{E}+05$ & $1.30 \mathrm{E}-05$ & $4.35 \mathrm{E}+08$ & $3.73 \mathrm{E}+03$ \\
\hline In-116m & $2.69 \mathrm{E}+02$ & $7.26 \mathrm{E}-09$ & $1.24 \mathrm{E}+05$ & $1.07 \mathrm{E}+00$ \\
\hline In-117m & $7.77 \mathrm{E}+03$ & $2.10 \mathrm{E}-07$ & $3.67 \mathrm{E}+06$ & $3.15 \mathrm{E}+01$ \\
\hline In-117 & $1.03 \mathrm{E}+03$ & $2.78 \mathrm{E}-08$ & $4.74 \mathrm{E}+05$ & $4.06 \mathrm{E}+00$ \\
\hline In-118m & $2.42 \mathrm{E}+02$ & $6.54 \mathrm{E}-09$ & $1.12 \mathrm{E}+05$ & $9.60 \mathrm{E}-01$ \\
\hline In-118 & $5.54 \mathrm{E}+03$ & $1.50 \mathrm{E}-07$ & $2.94 \mathrm{E}+06$ & $2.52 \mathrm{E}+01$ \\
\hline In-119m & $8.15 \mathrm{E}+03$ & $2.20 \mathrm{E}-07$ & $4.16 \mathrm{E}+06$ & $3.56 \mathrm{E}+01$ \\
\hline In-119 & $8.96 \mathrm{E}+02$ & $2.42 \mathrm{E}-08$ & $4.16 \mathrm{E}+05$ & $3.56 \mathrm{E}+00$ \\
\hline In-121m & $8.09 \mathrm{E}+03$ & $2.19 \mathrm{E}-07$ & $4.35 \mathrm{E}+06$ & $3.73 \mathrm{E}+01$ \\
\hline In-121 & $7.27 \mathrm{E}+02$ & $1.97 \mathrm{E}-08$ & $3.38 \mathrm{E}+05$ & $2.90 \mathrm{E}+00$ \\
\hline Sn-106 & $5.87 \mathrm{E}+02$ & $1.59 \mathrm{E}-08$ & $2.71 \mathrm{E}+05$ & $2.32 \mathrm{E}+00$ \\
\hline Sn-108 & $1.07 \mathrm{E}+03$ & $2.90 \mathrm{E}-08$ & $4.92 \mathrm{E}+05$ & $4.21 \mathrm{E}+00$ \\
\hline Sn-109 & $3.02 \mathrm{E}+02$ & $8.16 \mathrm{E}-09$ & $1.39 \mathrm{E}+05$ & $1.19 \mathrm{E}+00$ \\
\hline Sn-110 & $2.62 \mathrm{E}+03$ & $7.08 \mathrm{E}-08$ & $1.20 \mathrm{E}+06$ & $1.03 \mathrm{E}+01$ \\
\hline Sn-111 & $1.43 \mathrm{E}+03$ & $3.88 \mathrm{E}-08$ & $6.62 \mathrm{E}+05$ & $5.67 \mathrm{E}+00$ \\
\hline Sn-113m & $3.11 \mathrm{E}+05$ & $8.40 \mathrm{E}-06$ & $1.33 \mathrm{E}+08$ & $1.14 \mathrm{E}+03$ \\
\hline Sn-113 & $9.19 \mathrm{E}+04$ & $2.48 \mathrm{E}-06$ & $4.10 \mathrm{E}+07$ & $3.52 \mathrm{E}+02$ \\
\hline Sn-117m & $5.17 \mathrm{E}+03$ & $1.40 \mathrm{E}-07$ & $2.35 \mathrm{E}+06$ & $2.01 \mathrm{E}+01$ \\
\hline Sn-119m & $3.44 \mathrm{E}+05$ & $9.29 \mathrm{E}-06$ & $1.46 \mathrm{E}+08$ & $1.25 \mathrm{E}+03$ \\
\hline Sn-121m & $5.97 \mathrm{E}+05$ & $1.61 \mathrm{E}-05$ & & $2.43 \mathrm{E}+03$ \\
\hline Sn-121 & $7.97 \mathrm{E}+05$ & $2.15 \mathrm{E}-05$ & $7.27 \mathrm{E}+08$ & $6.23 \mathrm{E}+03$ \\
\hline Sn-123m & $5.11 \mathrm{E}+03$ & $1.38 \mathrm{E}-07$ & $2.37 \mathrm{E}+06$ & $2.03 \mathrm{E}+01$ \\
\hline Sn-123 & $4.52 \mathrm{E}+04$ & $1.22 \mathrm{E}-06$ & $2.76 \mathrm{E}+07$ & $2.36 \mathrm{E}+02$ \\
\hline Sn-125m & $1.98 \mathrm{E}+03$ & $5.36 \mathrm{E}-08$ & $9.27 \mathrm{E}+05$ & $7.95 \mathrm{E}+00$ \\
\hline Sn-125 & $1.93 \mathrm{E}+03$ & $5.23 \mathrm{E}-08$ & $9.09 \mathrm{E}+05$ & $7.79 \mathrm{E}+00$ \\
\hline
\end{tabular}




\begin{tabular}{|c|c|c|c|c|}
\hline Nuclide & $\begin{array}{c}\text { Air } \\
\text { Immersion } \\
\text { DCS } \\
\left(\mathbf{B q} / \mathbf{m}^{3}\right)\end{array}$ & $\begin{array}{c}\text { Air } \\
\text { Immersion } \\
\text { DCS } \\
\left(\mu \mathrm{Ci} / \mathbf{c m}^{3}\right)\end{array}$ & $\begin{array}{c}\text { Water } \\
\text { Submersion } \\
\text { DCS } \\
\left(\mathbf{B q} / \mathbf{m}^{3}\right)\end{array}$ & $\begin{array}{c}\text { Water } \\
\text { Submersion } \\
\text { DCS } \\
\left(\mu \mathrm{Ci} / \mathbf{m}^{3}\right)\end{array}$ \\
\hline Sn-126 & $1.74 \mathrm{E}+04$ & $4.71 \mathrm{E}-07$ & $7.75 \mathrm{E}+06$ & $6.64 \mathrm{E}+01$ \\
\hline $\mathrm{Sn}-127 \mathrm{~m}$ & $1.19 \mathrm{E}+03$ & $3.21 \mathrm{E}-08$ & $5.54 \mathrm{E}+05$ & $4.75 \mathrm{E}+00$ \\
\hline Sn-127 & $3.52 \mathrm{E}+02$ & $9.50 \mathrm{E}-09$ & $1.63 \mathrm{E}+05$ & $1.39 \mathrm{E}+00$ \\
\hline Sn-128 & $1.26 \mathrm{E}+03$ & $3.40 \mathrm{E}-08$ & $5.78 \mathrm{E}+05$ & $4.95 \mathrm{E}+00$ \\
\hline Sn-129 & $6.66 \mathrm{E}+02$ & $1.80 \mathrm{E}-08$ & $3.11 \mathrm{E}+05$ & $2.66 \mathrm{E}+00$ \\
\hline $\mathrm{Sn}-130 \mathrm{~m}$ & $7.55 \mathrm{E}+02$ & $2.04 \mathrm{E}-08$ & $3.53 \mathrm{E}+05$ & $3.02 \mathrm{E}+00$ \\
\hline Sn-130 & $7.62 \mathrm{E}+02$ & $2.06 \mathrm{E}-08$ & $3.51 \mathrm{E}+05$ & $3.01 \mathrm{E}+00$ \\
\hline Sb-111 & $4.69 \mathrm{E}+02$ & $1.27 \mathrm{E}-08$ & $2.17 \mathrm{E}+05$ & $1.86 \mathrm{E}+00$ \\
\hline Sb-113 & $5.55 \mathrm{E}+02$ & $1.50 \mathrm{E}-08$ & $2.56 \mathrm{E}+05$ & $2.19 \mathrm{E}+00$ \\
\hline Sb-114 & $2.50 \mathrm{E}+02$ & $6.75 \mathrm{E}-09$ & $1.16 \mathrm{E}+05$ & $9.92 \mathrm{E}-01$ \\
\hline Sb-115 & $8.05 \mathrm{E}+02$ & $2.18 \mathrm{E}-08$ & $3.71 \mathrm{E}+05$ & $3.18 \mathrm{E}+00$ \\
\hline Sb-116 & $2.94 \mathrm{E}+02$ & 7.94E-09 & $1.36 \mathrm{E}+05$ & $1.17 \mathrm{E}+00$ \\
\hline Sb-116m & $2.22 \mathrm{E}+02$ & $5.99 \mathrm{E}-09$ & $1.02 \mathrm{E}+05$ & $8.74 \mathrm{E}-01$ \\
\hline Sb-117 & $4.39 \mathrm{E}+03$ & $1.19 \mathrm{E}-07$ & $1.99 \mathrm{E}+06$ & $1.71 \mathrm{E}+01$ \\
\hline Sb-118 & $8.69 \mathrm{E}+02$ & $2.35 \mathrm{E}-08$ & $4.03 \mathrm{E}+05$ & $3.46 \mathrm{E}+00$ \\
\hline Sb-118m & $2.62 \mathrm{E}+02$ & $7.08 \mathrm{E}-09$ & $1.21 \mathrm{E}+05$ & $1.04 \mathrm{E}+00$ \\
\hline Sb-119 & $2.10 \mathrm{E}+05$ & $5.68 \mathrm{E}-06$ & $8.93 \mathrm{E}+07$ & $7.66 \mathrm{E}+02$ \\
\hline Sb-120 & $1.59 \mathrm{E}+03$ & $4.29 \mathrm{E}-08$ & $7.34 \mathrm{E}+05$ & $6.29 \mathrm{E}+00$ \\
\hline $\mathrm{Sb}-120 \mathrm{~m}$ & $2.81 \mathrm{E}+02$ & $7.58 \mathrm{E}-09$ & $1.29 \mathrm{E}+05$ & $1.10 \mathrm{E}+00$ \\
\hline Sb-122 & $1.55 \mathrm{E}+03$ & $4.20 \mathrm{E}-08$ & $7.22 \mathrm{E}+05$ & $6.19 \mathrm{E}+00$ \\
\hline Sb-122m & $1.79 \mathrm{E}+04$ & 4.84E-07 & $7.85 \mathrm{E}+06$ & $6.73 \mathrm{E}+01$ \\
\hline Sb-124 & $3.60 \mathrm{E}+02$ & $9.74 \mathrm{E}-09$ & $1.67 \mathrm{E}+05$ & $1.43 \mathrm{E}+00$ \\
\hline $\mathrm{Sb}-124 \mathrm{~m}$ & $1.60 \mathrm{E}+03$ & $4.33 \mathrm{E}-08$ & $7.37 \mathrm{E}+05$ & $6.32 \mathrm{E}+00$ \\
\hline $\mathrm{Sb}-124 \mathrm{n}$ & $5.63 \mathrm{E}+09$ & $1.52 \mathrm{E}-01$ & $2.40 \mathrm{E}+12$ & $2.06 \mathrm{E}+07$ \\
\hline Sb-125 & $1.67 \mathrm{E}+03$ & $4.51 \mathrm{E}-08$ & $7.70 \mathrm{E}+05$ & $6.60 \mathrm{E}+00$ \\
\hline Sb-126 & $2.54 \mathrm{E}+02$ & $6.86 \mathrm{E}-09$ & $1.17 \mathrm{E}+05$ & $1.00 \mathrm{E}+00$ \\
\hline $\mathrm{Sb}-126 \mathrm{~m}$ & $4.52 \mathrm{E}+02$ & $1.22 \mathrm{E}-08$ & $2.09 \mathrm{E}+05$ & $1.79 \mathrm{E}+00$ \\
\hline Sb-127 & $1.01 \mathrm{E}+03$ & $2.73 \mathrm{E}-08$ & $4.66 \mathrm{E}+05$ & $4.00 \mathrm{E}+00$ \\
\hline Sb-128 & $2.25 \mathrm{E}+02$ & $6.08 \mathrm{E}-09$ & $1.04 \mathrm{E}+05$ & $8.91 \mathrm{E}-01$ \\
\hline $\mathrm{Sb}-128 \mathrm{~m}$ & $3.63 \mathrm{E}+02$ & $9.82 \mathrm{E}-09$ & $1.68 \mathrm{E}+05$ & $1.44 \mathrm{E}+00$ \\
\hline Sb-129 & $4.64 \mathrm{E}+02$ & $1.25 \mathrm{E}-08$ & $2.14 \mathrm{E}+05$ & $1.84 \mathrm{E}+00$ \\
\hline Sb-130 & $2.10 \mathrm{E}+02$ & & & \\
\hline $\mathrm{Sb}-130 \mathrm{~m}$ & $2.52 \mathrm{E}+02$ & $6.80 \mathrm{E}-09$ & $1.17 \mathrm{E}+05$ & $9.99 \mathrm{E}-01$ \\
\hline Sb-131 & $3.22 \mathrm{E}+02$ & $8.70 \mathrm{E}-09$ & $1.49 \mathrm{E}+05$ & $1.28 \mathrm{E}+00$ \\
\hline Sb-133 & $2.38 \mathrm{E}+02$ & $6.44 \mathrm{E}-09$ & $1.10 \mathrm{E}+05$ & $9.47 \mathrm{E}-01$ \\
\hline Te-113 & $3.02 \mathrm{E}+02$ & $8.16 \mathrm{E}-09$ & $1.40 \mathrm{E}+05$ & $1.20 \mathrm{E}+00$ \\
\hline Te-114 & $5.36 \mathrm{E}+02$ & $1.45 \mathrm{E}-08$ & $2.48 \mathrm{E}+05$ & $2.12 \mathrm{E}+00$ \\
\hline
\end{tabular}




\begin{tabular}{|c|c|c|c|c|}
\hline Nuclide & $\begin{array}{c}\text { Air } \\
\text { Immersion } \\
\text { DCS } \\
\left(\mathbf{B q} / \mathbf{m}^{3}\right)\end{array}$ & $\begin{array}{c}\text { Air } \\
\text { Immersion } \\
\text { DCS } \\
\left(\mu \mathrm{Ci} / \mathbf{c m}^{3}\right)\end{array}$ & $\begin{array}{c}\text { Water } \\
\text { Submersion } \\
\text { DCS } \\
\left(\mathbf{B q} / \mathbf{m}^{3}\right)\end{array}$ & $\begin{array}{c}\text { Water } \\
\text { Submersion } \\
\text { DCS } \\
\left(\mu \mathrm{Ci} / \mathbf{m}^{3}\right)\end{array}$ \\
\hline Te-115 & $3.02 \mathrm{E}+02$ & $8.16 \mathrm{E}-09$ & $1.40 \mathrm{E}+05$ & $1.20 \mathrm{E}+00$ \\
\hline Te-115m & $2.60 \mathrm{E}+02$ & 7.02E-09 & $1.20 \mathrm{E}+05$ & $1.03 \mathrm{E}+00$ \\
\hline Te-116 & $8.66 \mathrm{E}+03$ & $2.34 \mathrm{E}-07$ & $3.92 \mathrm{E}+06$ & $3.36 \mathrm{E}+01$ \\
\hline Te-117 & $4.37 \mathrm{E}+02$ & $1.18 \mathrm{E}-08$ & $2.02 \mathrm{E}+05$ & $1.73 \mathrm{E}+00$ \\
\hline Te-118 & $2.10 \mathrm{E}+05$ & $5.68 \mathrm{E}-06$ & $8.91 \mathrm{E}+07$ & $7.63 \mathrm{E}+02$ \\
\hline Te-119 & $9.24 \mathrm{E}+02$ & $2.50 \mathrm{E}-08$ & $4.26 \mathrm{E}+05$ & $3.65 \mathrm{E}+00$ \\
\hline Te-119m & $4.55 \mathrm{E}+02$ & $1.23 \mathrm{E}-08$ & $2.10 \mathrm{E}+05$ & $1.80 \mathrm{E}+00$ \\
\hline Te-121 & $1.26 \mathrm{E}+03$ & $3.41 \mathrm{E}-08$ & $5.82 \mathrm{E}+05$ & $4.99 \mathrm{E}+00$ \\
\hline Te-121m & $3.52 \mathrm{E}+03$ & $9.50 \mathrm{E}-08$ & $1.60 \mathrm{E}+06$ & $1.37 \mathrm{E}+01$ \\
\hline Te-123 & $1.21 \mathrm{E}+08$ & $3.26 \mathrm{E}-03$ & $5.12 \mathrm{E}+10$ & $4.39 \mathrm{E}+05$ \\
\hline Te-123m & $5.46 \mathrm{E}+03$ & $1.48 \mathrm{E}-07$ & $2.48 \mathrm{E}+06$ & $2.12 \mathrm{E}+01$ \\
\hline Te-125m & $9.44 \mathrm{E}+04$ & $2.55 \mathrm{E}-06$ & $4.08 \mathrm{E}+07$ & $3.49 \mathrm{E}+02$ \\
\hline Te-127 & $9.47 \mathrm{E}+04$ & $2.56 \mathrm{E}-06$ & $5.27 \mathrm{E}+07$ & $4.51 \mathrm{E}+02$ \\
\hline Te-127m & $2.83 \mathrm{E}+05$ & $7.65 \mathrm{E}-06$ & $1.24 \mathrm{E}+08$ & $1.07 \mathrm{E}+03$ \\
\hline Te-129 & $1.06 \mathrm{E}+04$ & $2.87 \mathrm{E}-07$ & $5.17 \mathrm{E}+06$ & $4.43 \mathrm{E}+01$ \\
\hline Te-129m & $2.02 \mathrm{E}+04$ & $5.46 \mathrm{E}-07$ & $9.73 \mathrm{E}+06$ & $8.34 \mathrm{E}+01$ \\
\hline Te-131 & $1.65 \mathrm{E}+03$ & $4.46 \mathrm{E}-08$ & $7.66 \mathrm{E}+05$ & $6.56 \mathrm{E}+00$ \\
\hline Te-131m & $4.74 \mathrm{E}+02$ & $1.28 \mathrm{E}-08$ & $2.19 \mathrm{E}+05$ & $1.87 \mathrm{E}+00$ \\
\hline Te-132 & $3.39 \mathrm{E}+03$ & $9.17 \mathrm{E}-08$ & $1.55 \mathrm{E}+06$ & $1.33 \mathrm{E}+01$ \\
\hline Te-133 & $5.59 \mathrm{E}+02$ & & & $1 \mathrm{E}+00$ \\
\hline Te-133m & $3.67 \mathrm{E}+02$ & & $1.70 \mathrm{E}+05$ & $1.45 \mathrm{E}+00$ \\
\hline Te-134 & $8.19 \mathrm{E}+02$ & $2.21 \mathrm{E}-08$ & $3.77 \mathrm{E}+05$ & $3.23 \mathrm{E}+00$ \\
\hline I-118 & $3.38 \mathrm{E}+02$ & 9.14E-09 & $1.57 \mathrm{E}+05$ & $1.35 \mathrm{E}+00$ \\
\hline $\mathrm{I}-118 \mathrm{~m}$ & $1.85 \mathrm{E}+02$ & $5.01 \mathrm{E}-09$ & $8.55 \mathrm{E}+04$ & 7.33E-01 \\
\hline I-119 & $7.81 \mathrm{E}+02$ & $2.11 \mathrm{E}-08$ & $3.61 \mathrm{E}+05$ & $3.09 \mathrm{E}+00$ \\
\hline $\mathrm{I}-120$ & $2.48 \mathrm{E}+02$ & $6.70 \mathrm{E}-09$ & $1.15 \mathrm{E}+05$ & $9.85 \mathrm{E}-01$ \\
\hline $\mathrm{I}-120 \mathrm{~m}$ & $1.95 \mathrm{E}+02$ & $5.26 \mathrm{E}-09$ & $8.98 \mathrm{E}+04$ & $7.70 \mathrm{E}-01$ \\
\hline $\mathrm{I}-121$ & $1.88 \mathrm{E}+03$ & $5.07 \mathrm{E}-08$ & $8.57 \mathrm{E}+05$ & $7.34 \mathrm{E}+00$ \\
\hline $\mathrm{I}-122$ & $7.22 \mathrm{E}+02$ & $1.95 \mathrm{E}-08$ & $3.36 \mathrm{E}+05$ & $2.88 \mathrm{E}+00$ \\
\hline I-123 & $4.84 \mathrm{E}+03$ & $1.31 \mathrm{E}-07$ & $2.19 \mathrm{E}+06$ & $1.87 \mathrm{E}+01$ \\
\hline I-124 & $6.21 \mathrm{E}+02$ & $1.68 \mathrm{E}-08$ & $2.86 \mathrm{E}+05$ & $2.45 \mathrm{E}+00$ \\
\hline I-125 & & $2.27 \mathrm{E}-06$ & $3.57 \mathrm{E}+07$ & $3.06 \mathrm{E}+02$ \\
\hline $\mathrm{I}-126$ & $1.65 \mathrm{E}+03$ & $4.46 \mathrm{E}-08$ & $7.60 \mathrm{E}+05$ & $6.52 \mathrm{E}+00$ \\
\hline $\mathrm{I}-128$ & $8.93 \mathrm{E}+03$ & $2.41 \mathrm{E}-07$ & $4.43 \mathrm{E}+06$ & $3.80 \mathrm{E}+01$ \\
\hline I-129 & $1.11 \mathrm{E}+05$ & $3.00 \mathrm{E}-06$ & $4.75 \mathrm{E}+07$ & $4.07 \mathrm{E}+02$ \\
\hline $\mathrm{I}-130$ & $3.28 \mathrm{E}+02$ & $8.85 \mathrm{E}-09$ & $1.51 \mathrm{E}+05$ & $1.29 \mathrm{E}+00$ \\
\hline $\mathrm{I}-130 \mathrm{~m}$ & $6.50 \mathrm{E}+03$ & $1.76 \mathrm{E}-07$ & $3.02 \mathrm{E}+06$ & $2.59 \mathrm{E}+01$ \\
\hline
\end{tabular}




\begin{tabular}{|c|c|c|c|c|}
\hline Nuclide & $\begin{array}{c}\text { Air } \\
\text { Immersion } \\
\text { DCS } \\
\left(\mathbf{B q} / \mathbf{m}^{3}\right)\end{array}$ & $\begin{array}{c}\text { Air } \\
\text { Immersion } \\
\text { DCS } \\
\left(\mu \mathrm{Ci} / \mathbf{c m}^{3}\right)\end{array}$ & $\begin{array}{c}\text { Water } \\
\text { Submersion } \\
\text { DCS } \\
\left(\mathbf{B q} / \mathbf{m}^{3}\right)\end{array}$ & $\begin{array}{c}\text { Water } \\
\text { Submersion } \\
\text { DCS } \\
\left(\mu \mathrm{Ci} / \mathbf{m}^{3}\right)\end{array}$ \\
\hline I-131 & $1.87 \mathrm{E}+03$ & $5.04 \mathrm{E}-08$ & $8.59 \mathrm{E}+05$ & $7.36 \mathrm{E}+00$ \\
\hline $\mathrm{I}-132$ & $3.05 \mathrm{E}+02$ & $8.24 \mathrm{E}-09$ & $1.40 \mathrm{E}+05$ & $1.20 \mathrm{E}+00$ \\
\hline $\mathrm{I}-132 \mathrm{~m}$ & $2.11 \mathrm{E}+03$ & 5.71E-08 & $9.73 \mathrm{E}+05$ & $8.34 \mathrm{E}+00$ \\
\hline $\mathrm{I}-133$ & $1.14 \mathrm{E}+03$ & $3.08 \mathrm{E}-08$ & $5.27 \mathrm{E}+05$ & $4.51 \mathrm{E}+00$ \\
\hline $\mathrm{I}-134$ & $2.62 \mathrm{E}+02$ & 7.08E-09 & $1.21 \mathrm{E}+05$ & $1.04 \mathrm{E}+00$ \\
\hline $\mathrm{I}-134 \mathrm{~m}$ & $2.66 \mathrm{E}+03$ & $7.20 \mathrm{E}-08$ & $1.22 \mathrm{E}+06$ & $1.05 \mathrm{E}+01$ \\
\hline $\mathrm{I}-135$ & $4.19 \mathrm{E}+02$ & $1.13 \mathrm{E}-08$ & $1.93 \mathrm{E}+05$ & $1.66 \mathrm{E}+00$ \\
\hline $\mathrm{Xe}-120$ & $1.92 \mathrm{E}+03$ & $5.19 \mathrm{E}-08$ & $8.81 \mathrm{E}+05$ & $7.55 \mathrm{E}+00$ \\
\hline Xe-121 & $4.56 \mathrm{E}+02$ & $1.23 \mathrm{E}-08$ & $2.11 \mathrm{E}+05$ & $1.81 \mathrm{E}+00$ \\
\hline $\mathrm{Xe}-122$ & $1.45 \mathrm{E}+04$ & $3.91 \mathrm{E}-07$ & $6.59 \mathrm{E}+06$ & $5.65 \mathrm{E}+01$ \\
\hline Xe-123 & $1.11 \mathrm{E}+03$ & $3.01 \mathrm{E}-08$ & $5.11 \mathrm{E}+05$ & $4.38 \mathrm{E}+00$ \\
\hline Xe-125 & $2.94 \mathrm{E}+03$ & $7.94 \mathrm{E}-08$ & $1.34 \mathrm{E}+06$ & $1.15 \mathrm{E}+01$ \\
\hline Xe-127 & $2.81 \mathrm{E}+03$ & $7.58 \mathrm{E}-08$ & $1.28 \mathrm{E}+06$ & $1.10 \mathrm{E}+01$ \\
\hline $\mathrm{Xe}-127 \mathrm{~m}$ & $4.83 \mathrm{E}+03$ & $1.30 \mathrm{E}-07$ & $2.19 \mathrm{E}+06$ & $1.87 \mathrm{E}+01$ \\
\hline Xe-129m & $3.45 \mathrm{E}+04$ & 9.34E-07 & $1.58 \mathrm{E}+07$ & $1.35 \mathrm{E}+02$ \\
\hline $\mathrm{Xe}-131 \mathrm{~m}$ & $8.88 \mathrm{E}+04$ & $2.40 \mathrm{E}-06$ & $4.15 \mathrm{E}+07$ & $3.56 \mathrm{E}+02$ \\
\hline Xe-133 & $2.31 \mathrm{E}+04$ & $6.26 \mathrm{E}-07$ & $1.04 \mathrm{E}+07$ & $8.88 \mathrm{E}+01$ \\
\hline Xe-133m & $2.46 \mathrm{E}+04$ & $6.64 \mathrm{E}-07$ & $1.15 \mathrm{E}+07$ & $9.88 \mathrm{E}+01$ \\
\hline Xe-135 & $2.88 \mathrm{E}+03$ & $7.79 \mathrm{E}-08$ & $1.33 \mathrm{E}+06$ & $1.14 \mathrm{E}+01$ \\
\hline $\mathrm{Xe}-135 \mathrm{~m}$ & $1.68 \mathrm{E}+03$ & $4.53 \mathrm{E}-08$ & $7.75 \mathrm{E}+05$ & $6.64 \mathrm{E}+00$ \\
\hline Xe-137 & $3.05 \mathrm{E}+03$ & $8.24 \mathrm{E}-08$ & $1.50 \mathrm{E}+06$ & $1.28 \mathrm{E}+01$ \\
\hline Xe-138 & $5.80 \mathrm{E}+02$ & $1.57 \mathrm{E}-08$ & $2.69 \mathrm{E}+05$ & $2.30 \mathrm{E}+00$ \\
\hline Cs-121 & $5.86 \mathrm{E}+02$ & $1.58 \mathrm{E}-08$ & $2.73 \mathrm{E}+05$ & $2.34 \mathrm{E}+00$ \\
\hline Cs-121m & $5.89 \mathrm{E}+02$ & $1.59 \mathrm{E}-08$ & $2.73 \mathrm{E}+05$ & $2.34 \mathrm{E}+00$ \\
\hline Cs-123 & $6.48 \mathrm{E}+02$ & $1.75 \mathrm{E}-08$ & $2.99 \mathrm{E}+05$ & $2.56 \mathrm{E}+00$ \\
\hline Cs-124 & $5.84 \mathrm{E}+02$ & $1.58 \mathrm{E}-08$ & $2.73 \mathrm{E}+05$ & $2.34 \mathrm{E}+00$ \\
\hline Cs-125 & $9.41 \mathrm{E}+02$ & $2.54 \mathrm{E}-08$ & $4.34 \mathrm{E}+05$ & $3.72 \mathrm{E}+00$ \\
\hline Cs-126 & $5.99 \mathrm{E}+02$ & $1.62 \mathrm{E}-08$ & $2.78 \mathrm{E}+05$ & $2.38 \mathrm{E}+00$ \\
\hline Cs-127 & $1.72 \mathrm{E}+03$ & $4.66 \mathrm{E}-08$ & $7.91 \mathrm{E}+05$ & $6.78 \mathrm{E}+00$ \\
\hline Cs-128 & $7.85 \mathrm{E}+02$ & $2.12 \mathrm{E}-08$ & $3.64 \mathrm{E}+05$ & $3.12 \mathrm{E}+00$ \\
\hline Cs-129 & $2.83 \mathrm{E}+03$ & $7.65 \mathrm{E}-08$ & $1.29 \mathrm{E}+06$ & $1.10 \mathrm{E}+01$ \\
\hline Cs-130 & $1.42 \mathrm{E}+03$ & $3.84 \mathrm{E}-08$ & $6.57 \mathrm{E}+05$ & $5.63 \mathrm{E}+00$ \\
\hline Cs-130m & $1.58 \mathrm{E}+04$ & $4.26 \mathrm{E}-07$ & $7.00 \mathrm{E}+06$ & $6.00 \mathrm{E}+01$ \\
\hline Cs-131 & $1.32 \mathrm{E}+05$ & $3.57 \mathrm{E}-06$ & $5.63 \mathrm{E}+07$ & $4.83 \mathrm{E}+02$ \\
\hline Cs-132 & $1.01 \mathrm{E}+03$ & $2.72 \mathrm{E}-08$ & $4.63 \mathrm{E}+05$ & $3.97 \mathrm{E}+00$ \\
\hline Cs-134 & $4.49 \mathrm{E}+02$ & $1.21 \mathrm{E}-08$ & $2.07 \mathrm{E}+05$ & $1.78 \mathrm{E}+00$ \\
\hline Cs- $134 \mathrm{~m}$ & $3.96 \mathrm{E}+04$ & $1.07 \mathrm{E}-06$ & $1.79 \mathrm{E}+07$ & $1.54 \mathrm{E}+02$ \\
\hline
\end{tabular}




\begin{tabular}{|c|c|c|c|c|}
\hline Nuclide & $\begin{array}{c}\text { Air } \\
\text { Immersion } \\
\text { DCS } \\
\left(\mathbf{B q} / \mathbf{m}^{3}\right)\end{array}$ & $\begin{array}{c}\text { Air } \\
\text { Immersion } \\
\text { DCS } \\
\left(\mu \mathrm{Ci} / \mathbf{c m}^{3}\right)\end{array}$ & $\begin{array}{c}\text { Water } \\
\text { Submersion } \\
\text { DCS } \\
\left(\mathbf{B q} / \mathbf{m}^{3}\right)\end{array}$ & $\begin{array}{c}\text { Water } \\
\text { Submersion } \\
\text { DCS } \\
\left(\mu \mathrm{Ci} / \mathbf{m}^{3}\right)\end{array}$ \\
\hline Cs-135 & $1.46 \mathrm{E}+06$ & $3.95 \mathrm{E}-05$ & $1.34 \mathrm{E}+09$ & $1.15 \mathrm{E}+04$ \\
\hline Cs-135m & $4.34 \mathrm{E}+02$ & $1.17 \mathrm{E}-08$ & $1.99 \mathrm{E}+05$ & $1.71 \mathrm{E}+00$ \\
\hline Cs-136 & $3.23 \mathrm{E}+02$ & $8.74 \mathrm{E}-09$ & $1.49 \mathrm{E}+05$ & $1.28 \mathrm{E}+00$ \\
\hline Cs-137 & $3.37 \mathrm{E}+05$ & $9.12 \mathrm{E}-06$ & $3.02 \mathrm{E}+08$ & $2.59 \mathrm{E}+03$ \\
\hline Cs-138 & $2.76 \mathrm{E}+02$ & $7.45 \mathrm{E}-09$ & $1.28 \mathrm{E}+05$ & $1.10 \mathrm{E}+00$ \\
\hline Cs-138m & $1.65 \mathrm{E}+03$ & $4.46 \mathrm{E}-08$ & $7.66 \mathrm{E}+05$ & $6.56 \mathrm{E}+00$ \\
\hline Cs-139 & $1.91 \mathrm{E}+03$ & $5.16 \mathrm{E}-08$ & $9.19 \mathrm{E}+05$ & $7.88 \mathrm{E}+00$ \\
\hline Cs-140 & $3.57 \mathrm{E}+02$ & 9.64E-09 & $1.66 \mathrm{E}+05$ & $1.42 \mathrm{E}+00$ \\
\hline Ba-124 & $1.26 \mathrm{E}+03$ & $3.41 \mathrm{E}-08$ & $5.81 \mathrm{E}+05$ & $4.98 \mathrm{E}+00$ \\
\hline Ba-126 & $1.24 \mathrm{E}+03$ & $3.35 \mathrm{E}-08$ & $5.69 \mathrm{E}+05$ & $4.88 \mathrm{E}+00$ \\
\hline Ba-127 & $9.67 \mathrm{E}+02$ & $2.61 \mathrm{E}-08$ & $4.48 \mathrm{E}+05$ & $3.84 \mathrm{E}+00$ \\
\hline Ba-128 & $1.50 \mathrm{E}+04$ & 4.04E-07 & $6.79 \mathrm{E}+06$ & $5.82 \mathrm{E}+01$ \\
\hline Ba-129 & $2.20 \mathrm{E}+03$ & $5.95 \mathrm{E}-08$ & $1.01 \mathrm{E}+06$ & $8.68 \mathrm{E}+00$ \\
\hline Ba-129m & $4.40 \mathrm{E}+02$ & 1.19E-08 & $2.03 \mathrm{E}+05$ & $1.74 \mathrm{E}+00$ \\
\hline Ba-131 & $1.59 \mathrm{E}+03$ & 4.29E-08 & $7.27 \mathrm{E}+05$ & $6.23 \mathrm{E}+00$ \\
\hline Ba-131m & $1.20 \mathrm{E}+04$ & $3.23 \mathrm{E}-07$ & $5.35 \mathrm{E}+06$ & $4.58 \mathrm{E}+01$ \\
\hline Ba-133 & $1.96 \mathrm{E}+03$ & $5.29 \mathrm{E}-08$ & $8.91 \mathrm{E}+05$ & $7.63 \mathrm{E}+00$ \\
\hline Ba-133m & $1.27 \mathrm{E}+04$ & $3.44 \mathrm{E}-07$ & $5.92 \mathrm{E}+06$ & $5.07 \mathrm{E}+01$ \\
\hline Ba-135m & $1.47 \mathrm{E}+04$ & $3.97 \mathrm{E}-07$ & $6.82 \mathrm{E}+06$ & $5.84 \mathrm{E}+01$ \\
\hline Ba-137m & $1.18 \mathrm{E}+03$ & 3.19E-08 & $44 \mathrm{E}+05$ & $4.66 \mathrm{E}+00$ \\
\hline Ba-139 & $1.19 \mathrm{E}+04$ & $3.21 \mathrm{E}-07$ & $6.13 \mathrm{E}+06$ & $5.26 \mathrm{E}+01$ \\
\hline Ba-140 & $3.93 \mathrm{E}+03$ & $1.06 \mathrm{E}-07$ & $1.82 \mathrm{E}+06$ & $1.56 \mathrm{E}+01$ \\
\hline Ba-141 & $7.34 \mathrm{E}+02$ & $1.98 \mathrm{E}-08$ & $3.40 \mathrm{E}+05$ & $2.92 \mathrm{E}+00$ \\
\hline Ba-142 & $6.55 \mathrm{E}+02$ & $1.77 \mathrm{E}-08$ & $3.02 \mathrm{E}+05$ & $2.59 \mathrm{E}+00$ \\
\hline La-128 & $2.44 \mathrm{E}+02$ & $6.59 \mathrm{E}-09$ & $1.12 \mathrm{E}+05$ & 9.64E-01 \\
\hline La-129 & $7.72 \mathrm{E}+02$ & $2.09 \mathrm{E}-08$ & $3.56 \mathrm{E}+05$ & $3.05 \mathrm{E}+00$ \\
\hline La-130 & $3.08 \mathrm{E}+02$ & $8.32 \mathrm{E}-09$ & $1.42 \mathrm{E}+05$ & $1.22 \mathrm{E}+00$ \\
\hline La-131 & $1.10 \mathrm{E}+03$ & $2.98 \mathrm{E}-08$ & $5.07 \mathrm{E}+05$ & $4.34 \mathrm{E}+00$ \\
\hline La-132 & $3.40 \mathrm{E}+02$ & $9.18 \mathrm{E}-09$ & $1.57 \mathrm{E}+05$ & $1.35 \mathrm{E}+00$ \\
\hline La-132m & $1.07 \mathrm{E}+03$ & $2.91 \mathrm{E}-08$ & $4.94 \mathrm{E}+05$ & $4.23 \mathrm{E}+00$ \\
\hline La-133 & $4.96 \mathrm{E}+03$ & $1.34 \mathrm{E}-07$ & $2.28 \mathrm{E}+06$ & $1.96 \mathrm{E}+01$ \\
\hline La-134 & $9.73 \mathrm{E}+02$ & & & $3.87 \mathrm{E}+00$ \\
\hline La-135 & $4.10 \mathrm{E}+04$ & 1.11E-06 & $1.83 \mathrm{E}+07$ & $1.57 \mathrm{E}+02$ \\
\hline La-136 & $1.77 \mathrm{E}+03$ & 4.79E-08 & $8.21 \mathrm{E}+05$ & $7.04 \mathrm{E}+00$ \\
\hline La-137 & $1.03 \mathrm{E}+05$ & $2.79 \mathrm{E}-06$ & $4.40 \mathrm{E}+07$ & $3.77 \mathrm{E}+02$ \\
\hline La-138 & $5.45 \mathrm{E}+02$ & $1.47 \mathrm{E}-08$ & $2.52 \mathrm{E}+05$ & $2.16 \mathrm{E}+00$ \\
\hline La-140 & $2.86 \mathrm{E}+02$ & $7.72 \mathrm{E}-09$ & $1.33 \mathrm{E}+05$ & $1.14 \mathrm{E}+00$ \\
\hline
\end{tabular}




\begin{tabular}{|c|c|c|c|c|}
\hline Nuclide & $\begin{array}{c}\text { Air } \\
\text { Immersion } \\
\text { DCS } \\
\left(\mathbf{B q} / \mathbf{m}^{3}\right)\end{array}$ & $\begin{array}{c}\text { Air } \\
\text { Immersion } \\
\text { DCS } \\
\left(\mu \mathrm{Ci} / \mathbf{c m}^{3}\right)\end{array}$ & $\begin{array}{c}\text { Water } \\
\text { Submersion } \\
\text { DCS } \\
\left(\mathbf{B q} / \mathbf{m}^{3}\right)\end{array}$ & $\begin{array}{c}\text { Water } \\
\text { Submersion } \\
\text { DCS } \\
\left(\mu \mathrm{Ci} / \mathbf{m}^{3}\right)\end{array}$ \\
\hline La-141 & $1.48 \mathrm{E}+04$ & $4.00 \mathrm{E}-07$ & $8.21 \mathrm{E}+06$ & $7.04 \mathrm{E}+01$ \\
\hline La-142 & $2.66 \mathrm{E}+02$ & $7.20 \mathrm{E}-09$ & $1.24 \mathrm{E}+05$ & $1.06 \mathrm{E}+00$ \\
\hline La-143 & $2.28 \mathrm{E}+03$ & $6.17 \mathrm{E}-08$ & $1.09 \mathrm{E}+06$ & $9.34 \mathrm{E}+00$ \\
\hline Ce-130 & $1.48 \mathrm{E}+03$ & $4.00 \mathrm{E}-08$ & $6.80 \mathrm{E}+05$ & $5.83 \mathrm{E}+00$ \\
\hline Ce-131 & $4.27 \mathrm{E}+02$ & $1.15 \mathrm{E}-08$ & $1.97 \mathrm{E}+05$ & $1.69 \mathrm{E}+00$ \\
\hline Ce-132 & $2.91 \mathrm{E}+03$ & $7.86 \mathrm{E}-08$ & $1.32 \mathrm{E}+06$ & $1.13 \mathrm{E}+01$ \\
\hline Ce-133 & $1.39 \mathrm{E}+03$ & $3.76 \mathrm{E}-08$ & $6.39 \mathrm{E}+05$ & $5.48 \mathrm{E}+00$ \\
\hline $\mathrm{Ce}-133 \mathrm{~m}$ & $3.99 \mathrm{E}+02$ & $1.08 \mathrm{E}-08$ & $1.83 \mathrm{E}+05$ & $1.57 \mathrm{E}+00$ \\
\hline Ce-134 & $7.55 \mathrm{E}+04$ & $2.04 \mathrm{E}-06$ & $3.26 \mathrm{E}+07$ & $2.80 \mathrm{E}+02$ \\
\hline Ce-135 & $8.78 \mathrm{E}+02$ & $2.37 \mathrm{E}-08$ & $4.04 \mathrm{E}+05$ & $3.46 \mathrm{E}+00$ \\
\hline Ce-137 & $3.74 \mathrm{E}+04$ & $1.01 \mathrm{E}-06$ & $1.67 \mathrm{E}+07$ & $1.43 \mathrm{E}+02$ \\
\hline Ce-137m & $1.63 \mathrm{E}+04$ & $4.42 \mathrm{E}-07$ & $7.59 \mathrm{E}+06$ & $6.50 \mathrm{E}+01$ \\
\hline Ce-139 & $5.30 \mathrm{E}+03$ & $1.43 \mathrm{E}-07$ & $2.40 \mathrm{E}+06$ & $2.06 \mathrm{E}+01$ \\
\hline Ce-141 & $1.01 \mathrm{E}+04$ & $2.74 \mathrm{E}-07$ & $4.62 \mathrm{E}+06$ & $3.96 \mathrm{E}+01$ \\
\hline Ce-143 & $2.66 \mathrm{E}+03$ & $7.20 \mathrm{E}-08$ & $1.23 \mathrm{E}+06$ & $1.05 \mathrm{E}+01$ \\
\hline Ce-144 & $4.31 \mathrm{E}+04$ & $1.17 \mathrm{E}-06$ & $1.97 \mathrm{E}+07$ & $1.69 \mathrm{E}+02$ \\
\hline $\mathrm{Ce}-145$ & $8.71 \mathrm{E}+02$ & $2.35 \mathrm{E}-08$ & $4.03 \mathrm{E}+05$ & $3.45 \mathrm{E}+00$ \\
\hline Pr-134 & $2.20 \mathrm{E}+02$ & 5.95E-09 & $1.02 \mathrm{E}+05$ & $8.71 \mathrm{E}-01$ \\
\hline Pr-134m & $2.94 \mathrm{E}+02$ & $7.94 \mathrm{E}-09$ & $1.36 \mathrm{E}+05$ & $1.16 \mathrm{E}+00$ \\
\hline Pr-135 & $8.15 \mathrm{E}+02$ & $2.20 \mathrm{E}-08$ & $77 \mathrm{E}+05$ & $3.23 \mathrm{E}+00$ \\
\hline Pr-136 & & & $1.47 \mathrm{E}+05$ & $1.26 \mathrm{E}+00$ \\
\hline Pr-137 & $1.96 \mathrm{E}+03$ & 5.29E-08 & $9.06 \mathrm{E}+05$ & $7.76 \mathrm{E}+00$ \\
\hline Pr-138 & $8.50 \mathrm{E}+02$ & $2.30 \mathrm{E}-08$ & $3.95 \mathrm{E}+05$ & $3.39 \mathrm{E}+00$ \\
\hline Pr-138m & $2.81 \mathrm{E}+02$ & $7.58 \mathrm{E}-09$ & $1.29 \mathrm{E}+05$ & $1.11 \mathrm{E}+00$ \\
\hline Pr-139 & $6.22 \mathrm{E}+03$ & $1.68 \mathrm{E}-07$ & $2.86 \mathrm{E}+06$ & $2.45 \mathrm{E}+01$ \\
\hline Pr-140 & $1.30 \mathrm{E}+03$ & $3.51 \mathrm{E}-08$ & $6.02 \mathrm{E}+05$ & $5.16 \mathrm{E}+00$ \\
\hline Pr-142 & $9.09 \mathrm{E}+03$ & $2.46 \mathrm{E}-07$ & $4.57 \mathrm{E}+06$ & $3.92 \mathrm{E}+01$ \\
\hline Pr-143 & $1.63 \mathrm{E}+05$ & $4.39 \mathrm{E}-06$ & $1.43 \mathrm{E}+08$ & $1.22 \mathrm{E}+03$ \\
\hline Pr-144 & $1.26 \mathrm{E}+04$ & $3.41 \mathrm{E}-07$ & $7.13 \mathrm{E}+06$ & $6.11 \mathrm{E}+01$ \\
\hline Pr-144m & $1.26 \mathrm{E}+05$ & $3.40 \mathrm{E}-06$ & $5.51 \mathrm{E}+07$ & $4.72 \mathrm{E}+02$ \\
\hline Pr-145 & $2.31 \mathrm{E}+04$ & $6.26 \mathrm{E}-07$ & $1.29 \mathrm{E}+07$ & $1.10 \mathrm{E}+02$ \\
\hline Pr-146 & & & & $2.56 \mathrm{E}+00$ \\
\hline Pr-147 & $1.45 \mathrm{E}+03$ & $3.93 \mathrm{E}-08$ & $6.76 \mathrm{E}+05$ & $5.79 \mathrm{E}+00$ \\
\hline Pr-148 & $6.57 \mathrm{E}+02$ & $1.77 \mathrm{E}-08$ & $3.08 \mathrm{E}+05$ & $2.64 \mathrm{E}+00$ \\
\hline Pr-148m & $7.26 \mathrm{E}+02$ & $1.96 \mathrm{E}-08$ & $3.39 \mathrm{E}+05$ & $2.90 \mathrm{E}+00$ \\
\hline Nd-134 & $1.37 \mathrm{E}+03$ & $3.69 \mathrm{E}-08$ & $6.27 \mathrm{E}+05$ & $5.37 \mathrm{E}+00$ \\
\hline Nd-135 & $5.63 \mathrm{E}+02$ & $1.52 \mathrm{E}-08$ & $2.60 \mathrm{E}+05$ & $2.23 \mathrm{E}+00$ \\
\hline
\end{tabular}




\begin{tabular}{|c|c|c|c|c|}
\hline Nuclide & $\begin{array}{c}\text { Air } \\
\text { Immersion } \\
\text { DCS } \\
\left(\mathbf{B q} / \mathbf{m}^{3}\right)\end{array}$ & $\begin{array}{c}\text { Air } \\
\text { Immersion } \\
\text { DCS } \\
\left(\mu \mathrm{Ci} / \mathbf{c m}^{3}\right)\end{array}$ & $\begin{array}{c}\text { Water } \\
\text { Submersion } \\
\text { DCS } \\
\left(\mathbf{B q} / \mathbf{m}^{3}\right)\end{array}$ & $\begin{array}{c}\text { Water } \\
\text { Submersion } \\
\text { DCS } \\
\left(\mu \mathrm{Ci} / \mathbf{m}^{3}\right)\end{array}$ \\
\hline Nd-136 & $2.88 \mathrm{E}+03$ & $7.79 \mathrm{E}-08$ & $1.32 \mathrm{E}+06$ & $1.13 \mathrm{E}+01$ \\
\hline Nd-137 & $5.95 \mathrm{E}+02$ & $1.61 \mathrm{E}-08$ & $2.73 \mathrm{E}+05$ & $2.34 \mathrm{E}+00$ \\
\hline Nd-138 & $2.94 \mathrm{E}+04$ & 7.94E-07 & $1.30 \mathrm{E}+07$ & $1.11 \mathrm{E}+02$ \\
\hline Nd-139 & $1.63 \mathrm{E}+03$ & 4.39E-08 & $7.51 \mathrm{E}+05$ & $6.44 \mathrm{E}+00$ \\
\hline $\mathrm{Nd}-139 \mathrm{~m}$ & $4.41 \mathrm{E}+02$ & 1.19E-08 & $2.03 \mathrm{E}+05$ & $1.74 \mathrm{E}+00$ \\
\hline Nd-140 & $7.14 \mathrm{E}+04$ & $1.93 \mathrm{E}-06$ & $3.05 \mathrm{E}+07$ & $2.61 \mathrm{E}+02$ \\
\hline Nd-141 & $1.20 \mathrm{E}+04$ & $3.25 \mathrm{E}-07$ & $5.48 \mathrm{E}+06$ & $4.69 \mathrm{E}+01$ \\
\hline $\mathrm{Nd}-141 \mathrm{~m}$ & $1.00 \mathrm{E}+03$ & $2.71 \mathrm{E}-08$ & $4.63 \mathrm{E}+05$ & $3.97 \mathrm{E}+00$ \\
\hline Nd-147 & $5.52 \mathrm{E}+03$ & $1.49 \mathrm{E}-07$ & $2.54 \mathrm{E}+06$ & $2.17 \mathrm{E}+01$ \\
\hline Nd-149 & $1.95 \mathrm{E}+03$ & $5.26 \mathrm{E}-08$ & $8.98 \mathrm{E}+05$ & $7.70 \mathrm{E}+00$ \\
\hline Nd-151 & $8.07 \mathrm{E}+02$ & $2.18 \mathrm{E}-08$ & $3.73 \mathrm{E}+05$ & $3.20 \mathrm{E}+00$ \\
\hline $\mathrm{Nd}-152$ & $4.35 \mathrm{E}+03$ & $1.18 \mathrm{E}-07$ & $2.02 \mathrm{E}+06$ & $1.73 \mathrm{E}+01$ \\
\hline $\mathrm{Pm}-136$ & $2.54 \mathrm{E}+02$ & $6.86 \mathrm{E}-09$ & $1.18 \mathrm{E}+05$ & $1.01 \mathrm{E}+00$ \\
\hline Pm-137m & $3.96 \mathrm{E}+02$ & $1.07 \mathrm{E}-08$ & $1.82 \mathrm{E}+05$ & $1.56 \mathrm{E}+00$ \\
\hline Pm-139 & $7.39 \mathrm{E}+02$ & $2.00 \mathrm{E}-08$ & $3.43 \mathrm{E}+05$ & $2.94 \mathrm{E}+00$ \\
\hline $\mathrm{Pm}-140$ & $6.43 \mathrm{E}+02$ & $1.74 \mathrm{E}-08$ & $3.02 \mathrm{E}+05$ & $2.59 \mathrm{E}+00$ \\
\hline Pm-140m & $2.28 \mathrm{E}+02$ & $6.17 \mathrm{E}-09$ & $1.05 \mathrm{E}+05$ & $9.03 \mathrm{E}-01$ \\
\hline Pm-141 & $9.47 \mathrm{E}+02$ & $2.56 \mathrm{E}-08$ & $4.38 \mathrm{E}+05$ & $3.75 \mathrm{E}+00$ \\
\hline Pm-142 & $8.03 \mathrm{E}+02$ & $2.17 \mathrm{E}-08$ & $3.74 \mathrm{E}+05$ & $3.20 \mathrm{E}+00$ \\
\hline Pm-143 & $2.35 \mathrm{E}+03$ & & & $9.28 \mathrm{E}+00$ \\
\hline Pm-144 & $4.56 \mathrm{E}+02$ & & & $1.80 \mathrm{E}+00$ \\
\hline Pm-145 & $5.77 \mathrm{E}+04$ & $1.56 \mathrm{E}-06$ & $2.48 \mathrm{E}+07$ & $2.12 \mathrm{E}+02$ \\
\hline Pm-146 & $9.52 \mathrm{E}+02$ & $2.57 \mathrm{E}-08$ & $4.39 \mathrm{E}+05$ & $3.76 \mathrm{E}+00$ \\
\hline Pm-147 & $3.66 \mathrm{E}+06$ & $9.90 \mathrm{E}-05$ & $3.29 \mathrm{E}+09$ & $2.82 \mathrm{E}+04$ \\
\hline Pm-148 & $1.15 \mathrm{E}+03$ & $3.11 \mathrm{E}-08$ & $5.35 \mathrm{E}+05$ & $4.58 \mathrm{E}+00$ \\
\hline Pm-148m & $3.53 \mathrm{E}+02$ & $9.53 \mathrm{E}-09$ & $1.63 \mathrm{E}+05$ & $1.39 \mathrm{E}+00$ \\
\hline Pm-149 & $4.17 \mathrm{E}+04$ & $1.13 \mathrm{E}-06$ & $2.25 \mathrm{E}+07$ & $1.93 \mathrm{E}+02$ \\
\hline $\mathrm{Pm}-150$ & $4.55 \mathrm{E}+02$ & $1.23 \mathrm{E}-08$ & $2.11 \mathrm{E}+05$ & $1.81 \mathrm{E}+00$ \\
\hline Pm-151 & $2.20 \mathrm{E}+03$ & $5.95 \mathrm{E}-08$ & $1.02 \mathrm{E}+06$ & $8.71 \mathrm{E}+00$ \\
\hline Pm-152 & $2.19 \mathrm{E}+03$ & $5.91 \mathrm{E}-08$ & $1.04 \mathrm{E}+06$ & $8.94 \mathrm{E}+00$ \\
\hline Pm-152m & $4.49 \mathrm{E}+02$ & $1.21 \mathrm{E}-08$ & $2.07 \mathrm{E}+05$ & $1.78 \mathrm{E}+00$ \\
\hline Pm-153 & $9.33 \mathrm{E}+03$ & & & $3.87 \mathrm{E}+01$ \\
\hline $\mathrm{Pm}-154$ & $3.63 \mathrm{E}+02$ & $9.82 \mathrm{E}-09$ & $1.69 \mathrm{E}+05$ & $1.45 \mathrm{E}+00$ \\
\hline $\mathrm{Pm}-154 \mathrm{~m}$ & $3.73 \mathrm{E}+02$ & $1.01 \mathrm{E}-08$ & $1.72 \mathrm{E}+05$ & $1.48 \mathrm{E}+00$ \\
\hline Sm-139 & $4.78 \mathrm{E}+02$ & $1.29 \mathrm{E}-08$ & $2.20 \mathrm{E}+05$ & $1.89 \mathrm{E}+00$ \\
\hline Sm-140 & $1.25 \mathrm{E}+03$ & $3.39 \mathrm{E}-08$ & $5.78 \mathrm{E}+05$ & $4.95 \mathrm{E}+00$ \\
\hline Sm-141 & $4.90 \mathrm{E}+02$ & $1.32 \mathrm{E}-08$ & $2.26 \mathrm{E}+05$ & $1.94 \mathrm{E}+00$ \\
\hline
\end{tabular}




\begin{tabular}{|c|c|c|c|c|}
\hline Nuclide & $\begin{array}{c}\text { Air } \\
\text { Immersion } \\
\text { DCS } \\
\left(\mathbf{B q} / \mathbf{m}^{3}\right)\end{array}$ & $\begin{array}{c}\text { Air } \\
\text { Immersion } \\
\text { DCS } \\
\left(\mu \mathrm{Ci} / \mathbf{c m}^{3}\right)\end{array}$ & $\begin{array}{c}\text { Water } \\
\text { Submersion } \\
\text { DCS } \\
\left(\mathbf{B q} / \mathbf{m}^{3}\right)\end{array}$ & $\begin{array}{c}\text { Water } \\
\text { Submersion } \\
\text { DCS } \\
\left(\mu \mathrm{Ci} / \mathbf{m}^{3}\right)\end{array}$ \\
\hline Sm-141m & $3.55 \mathrm{E}+02$ & 9.61E-09 & $1.64 \mathrm{E}+05$ & $1.41 \mathrm{E}+00$ \\
\hline Sm-142 & $7.60 \mathrm{E}+03$ & $2.06 \mathrm{E}-07$ & $3.48 \mathrm{E}+06$ & $2.98 \mathrm{E}+01$ \\
\hline Sm-143 & $1.34 \mathrm{E}+03$ & $3.63 \mathrm{E}-08$ & $6.21 \mathrm{E}+05$ & $5.32 \mathrm{E}+00$ \\
\hline Sm-143m & $1.02 \mathrm{E}+03$ & $2.76 \mathrm{E}-08$ & $4.70 \mathrm{E}+05$ & $4.03 \mathrm{E}+00$ \\
\hline Sm-145 & $2.58 \mathrm{E}+04$ & $6.97 \mathrm{E}-07$ & $1.11 \mathrm{E}+07$ & $9.50 \mathrm{E}+01$ \\
\hline Sm-151 & $1.20 \mathrm{E}+09$ & $3.23 \mathrm{E}-02$ & $5.11 \mathrm{E}+11$ & $4.38 \mathrm{E}+06$ \\
\hline Sm-153 & $1.50 \mathrm{E}+04$ & 4.04E-07 & $6.82 \mathrm{E}+06$ & $5.84 \mathrm{E}+01$ \\
\hline Sm-155 & $7.16 \mathrm{E}+03$ & $1.93 \mathrm{E}-07$ & $3.36 \mathrm{E}+06$ & $2.88 \mathrm{E}+01$ \\
\hline Sm-156 & $6.70 \mathrm{E}+03$ & $1.81 \mathrm{E}-07$ & $3.05 \mathrm{E}+06$ & $2.61 \mathrm{E}+01$ \\
\hline Sm-157 & $1.66 \mathrm{E}+03$ & 4.49E-08 & $7.75 \mathrm{E}+05$ & $6.64 \mathrm{E}+00$ \\
\hline Eu-142 & $5.49 \mathrm{E}+02$ & $1.48 \mathrm{E}-08$ & $2.58 \mathrm{E}+05$ & $2.21 \mathrm{E}+00$ \\
\hline Eu-142m & $2.01 \mathrm{E}+02$ & $5.42 \mathrm{E}-09$ & $9.27 \mathrm{E}+04$ & $7.95 \mathrm{E}-01$ \\
\hline Eu-143 & $6.03 \mathrm{E}+02$ & $1.63 \mathrm{E}-08$ & $2.81 \mathrm{E}+05$ & $2.40 \mathrm{E}+00$ \\
\hline Eu-144 & $6.12 \mathrm{E}+02$ & $1.65 \mathrm{E}-08$ & $2.86 \mathrm{E}+05$ & $2.45 \mathrm{E}+00$ \\
\hline Eu-145 & $5.33 \mathrm{E}+02$ & $1.44 \mathrm{E}-08$ & $2.46 \mathrm{E}+05$ & $2.11 \mathrm{E}+00$ \\
\hline Eu-146 & $2.86 \mathrm{E}+02$ & $7.72 \mathrm{E}-09$ & $1.32 \mathrm{E}+05$ & $1.13 \mathrm{E}+00$ \\
\hline Eu-147 & $1.56 \mathrm{E}+03$ & $4.22 \mathrm{E}-08$ & $7.17 \mathrm{E}+05$ & $6.15 \mathrm{E}+00$ \\
\hline Eu-148 & $3.14 \mathrm{E}+02$ & $8.49 \mathrm{E}-09$ & $1.45 \mathrm{E}+05$ & $1.24 \mathrm{E}+00$ \\
\hline Eu-149 & $1.56 \mathrm{E}+04$ & $4.22 \mathrm{E}-07$ & $7.00 \mathrm{E}+06$ & $6.00 \mathrm{E}+01$ \\
\hline Eu-150 & $4.58 \mathrm{E}+02$ & $1.24 \mathrm{E}-08$ & $2.10 \mathrm{E}+05$ & $1.80 \mathrm{E}+00$ \\
\hline Eu-150m & $1.36 \mathrm{E}+04$ & $3.68 \mathrm{E}-07$ & $6.50 \mathrm{E}+06$ & $5.57 \mathrm{E}+01$ \\
\hline Eu-152 & $5.89 \mathrm{E}+02$ & $1.59 \mathrm{E}-08$ & $2.71 \mathrm{E}+05$ & $2.32 \mathrm{E}+00$ \\
\hline Eu-152m & $2.31 \mathrm{E}+03$ & $6.26 \mathrm{E}-08$ & $1.08 \mathrm{E}+06$ & $9.24 \mathrm{E}+00$ \\
\hline Eu-152n & $1.21 \mathrm{E}+04$ & $3.26 \mathrm{E}-07$ & $5.36 \mathrm{E}+06$ & $4.59 \mathrm{E}+01$ \\
\hline Eu-154 & $5.49 \mathrm{E}+02$ & $1.48 \mathrm{E}-08$ & $2.54 \mathrm{E}+05$ & $2.17 \mathrm{E}+00$ \\
\hline Eu-154m & $1.47 \mathrm{E}+04$ & $3.99 \mathrm{E}-07$ & $6.51 \mathrm{E}+06$ & $5.58 \mathrm{E}+01$ \\
\hline $\mathrm{Eu}-155$ & $1.46 \mathrm{E}+04$ & $3.95 \mathrm{E}-07$ & $6.51 \mathrm{E}+06$ & $5.58 \mathrm{E}+01$ \\
\hline Eu-156 & $5.34 \mathrm{E}+02$ & $1.44 \mathrm{E}-08$ & $2.48 \mathrm{E}+05$ & $2.12 \mathrm{E}+00$ \\
\hline Eu-157 & $2.58 \mathrm{E}+03$ & $6.97 \mathrm{E}-08$ & $1.19 \mathrm{E}+06$ & $1.02 \mathrm{E}+01$ \\
\hline Eu-158 & $5.16 \mathrm{E}+02$ & $1.40 \mathrm{E}-08$ & $2.40 \mathrm{E}+05$ & $2.06 \mathrm{E}+00$ \\
\hline Eu-159 & $2.40 \mathrm{E}+03$ & $6.49 \mathrm{E}-08$ & $1.12 \mathrm{E}+06$ & $9.60 \mathrm{E}+00$ \\
\hline Gd-142 & $6.63 \mathrm{E}+02$ & $1.79 \mathrm{E}-08$ & & $2.64 \mathrm{E}+00$ \\
\hline Gd-143m & $3.25 \mathrm{E}+02$ & $8.77 \mathrm{E}-09$ & $1.50 \mathrm{E}+05$ & $1.29 \mathrm{E}+00$ \\
\hline Gd-144 & $7.46 \mathrm{E}+02$ & $2.02 \mathrm{E}-08$ & $3.45 \mathrm{E}+05$ & $2.96 \mathrm{E}+00$ \\
\hline Gd-145 & $2.69 \mathrm{E}+02$ & $7.26 \mathrm{E}-09$ & $1.24 \mathrm{E}+05$ & $1.07 \mathrm{E}+00$ \\
\hline Gd-145m & $1.03 \mathrm{E}+03$ & $2.78 \mathrm{E}-08$ & $4.75 E+05$ & $4.07 \mathrm{E}+00$ \\
\hline Gd-146 & $3.63 \mathrm{E}+03$ & $9.81 \mathrm{E}-08$ & $1.63 \mathrm{E}+06$ & $1.39 \mathrm{E}+01$ \\
\hline
\end{tabular}




\begin{tabular}{|c|c|c|c|c|}
\hline Nuclide & $\begin{array}{c}\text { Air } \\
\text { Immersion } \\
\text { DCS } \\
\left(\mathbf{B q} / \mathbf{m}^{3}\right)\end{array}$ & $\begin{array}{c}\text { Air } \\
\text { Immersion } \\
\text { DCS } \\
\left(\mu \mathrm{Ci} / \mathbf{c m}^{3}\right)\end{array}$ & $\begin{array}{c}\text { Water } \\
\text { Submersion } \\
\text { DCS } \\
\left(\mathbf{B q} / \mathbf{m}^{\mathbf{3}}\right)\end{array}$ & $\begin{array}{c}\text { Water } \\
\text { Submersion } \\
\text { DCS } \\
\left(\mu \mathrm{Ci} / \mathbf{m}^{3}\right)\end{array}$ \\
\hline Gd-147 & $5.04 \mathrm{E}+02$ & $1.36 \mathrm{E}-08$ & $2.31 \mathrm{E}+05$ & $1.98 \mathrm{E}+00$ \\
\hline Gd-149 & $1.42 \mathrm{E}+03$ & $3.83 \mathrm{E}-08$ & $6.48 \mathrm{E}+05$ & $5.56 \mathrm{E}+00$ \\
\hline Gd-151 & $1.47 \mathrm{E}+04$ & 3.99E-07 & $6.59 \mathrm{E}+06$ & $5.65 \mathrm{E}+01$ \\
\hline Gd-153 & $1.02 \mathrm{E}+04$ & $2.76 \mathrm{E}-07$ & $4.50 \mathrm{E}+06$ & $3.85 \mathrm{E}+01$ \\
\hline Gd-159 & $1.35 \mathrm{E}+04$ & $3.65 \mathrm{E}-07$ & $6.41 \mathrm{E}+06$ & $5.49 \mathrm{E}+01$ \\
\hline Gd-162 & $1.70 \mathrm{E}+03$ & 4.61E-08 & $7.89 \mathrm{E}+05$ & $6.76 \mathrm{E}+00$ \\
\hline Tb-146 & $1.82 \mathrm{E}+02$ & $4.93 \mathrm{E}-09$ & $8.41 \mathrm{E}+04$ & $7.21 \mathrm{E}-01$ \\
\hline Tb-147 & $3.11 \mathrm{E}+02$ & $8.40 \mathrm{E}-09$ & $1.44 \mathrm{E}+05$ & $1.24 \mathrm{E}+00$ \\
\hline Tb-147m & $3.48 \mathrm{E}+02$ & $9.41 \mathrm{E}-09$ & $1.61 \mathrm{E}+05$ & $1.38 \mathrm{E}+00$ \\
\hline Tb-148 & $2.86 \mathrm{E}+02$ & 7.72E-09 & $1.32 \mathrm{E}+05$ & $1.13 \mathrm{E}+00$ \\
\hline Tb-148m & $2.23 \mathrm{E}+02$ & $6.04 \mathrm{E}-09$ & $1.03 \mathrm{E}+05$ & $8.79 \mathrm{E}-01$ \\
\hline Tb-149 & $5.05 \mathrm{E}+02$ & $1.36 \mathrm{E}-08$ & $2.33 \mathrm{E}+05$ & $2.00 \mathrm{E}+00$ \\
\hline Tb-149m & $5.11 \mathrm{E}+02$ & $1.38 \mathrm{E}-08$ & $2.37 \mathrm{E}+05$ & $2.03 \mathrm{E}+00$ \\
\hline $\mathrm{Tb}-150$ & $2.71 \mathrm{E}+02$ & 7.32E-09 & $25 \mathrm{E}+05$ & $1.07 \mathrm{E}+00$ \\
\hline $\mathrm{Tb}-150 \mathrm{~m}$ & $2.83 \mathrm{E}+02$ & 7.65E-09 & $1.30 \mathrm{E}+05$ & $1.11 \mathrm{E}+00$ \\
\hline Tb-151 & $7.31 \mathrm{E}+02$ & $1.97 \mathrm{E}-08$ & $3.35 \mathrm{E}+05$ & $2.87 \mathrm{E}+00$ \\
\hline Tb-151m & $9.91 \mathrm{E}+03$ & $2.68 \mathrm{E}-07$ & $4.54 \mathrm{E}+06$ & $3.89 \mathrm{E}+01$ \\
\hline Tb-152 & $4.54 \mathrm{E}+02$ & $1.23 \mathrm{E}-08$ & $2.10 \mathrm{E}+05$ & $1.80 \mathrm{E}+00$ \\
\hline $\mathrm{Tb}-152 \mathrm{~m}$ & $9.67 \mathrm{E}+02$ & $2.61 \mathrm{E}-08$ & $4.43 \mathrm{E}+05$ & $3.80 \mathrm{E}+00$ \\
\hline Tb-153 & & $6.35 \mathrm{E}-08$ & & $5 \mathrm{E}+00$ \\
\hline Tb-154 & $2.86 \mathrm{E}+02$ & 7.72E-09 & $1.32 \mathrm{E}+05$ & $1.13 \mathrm{E}+00$ \\
\hline Tb-155 & $5.03 \mathrm{E}+03$ & $1.36 \mathrm{E}-07$ & $2.26 \mathrm{E}+06$ & $1.94 \mathrm{E}+01$ \\
\hline Tb-156 & $3.57 \mathrm{E}+02$ & $9.66 \mathrm{E}-09$ & $1.64 \mathrm{E}+05$ & $1.41 \mathrm{E}+00$ \\
\hline Tb-156m & $3.43 \mathrm{E}+04$ & $9.28 \mathrm{E}-07$ & $1.49 \mathrm{E}+07$ & $1.28 \mathrm{E}+02$ \\
\hline Tb-156n & $3.18 \mathrm{E}+05$ & $8.60 \mathrm{E}-06$ & $1.41 \mathrm{E}+08$ & $1.21 \mathrm{E}+03$ \\
\hline Tb-157 & $3.23 \mathrm{E}+05$ & $8.72 \mathrm{E}-06$ & $1.39 \mathrm{E}+08$ & $1.19 \mathrm{E}+03$ \\
\hline Tb-158 & $8.78 \mathrm{E}+02$ & $2.37 \mathrm{E}-08$ & $4.04 \mathrm{E}+05$ & $3.47 \mathrm{E}+00$ \\
\hline Tb-160 & $6.10 \mathrm{E}+02$ & $1.65 \mathrm{E}-08$ & $2.81 \mathrm{E}+05$ & $2.40 \mathrm{E}+00$ \\
\hline Tb-161 & $3.47 \mathrm{E}+04$ & $9.38 \mathrm{E}-07$ & $1.58 \mathrm{E}+07$ & $1.35 \mathrm{E}+02$ \\
\hline Tb-162 & $6.29 \mathrm{E}+02$ & $1.70 \mathrm{E}-08$ & $2.91 \mathrm{E}+05$ & $2.49 \mathrm{E}+00$ \\
\hline Tb-163 & $9.03 \mathrm{E}+02$ & $2.44 \mathrm{E}-08$ & $4.16 \mathrm{E}+05$ & $3.57 \mathrm{E}+00$ \\
\hline Tb-164 & & & & $1.10 \mathrm{E}+00$ \\
\hline Tb-165 & $7.85 \mathrm{E}+02$ & $2.12 \mathrm{E}-08$ & $3.66 \mathrm{E}+05$ & $3.13 \mathrm{E}+00$ \\
\hline Dy-148 & $1.01 \mathrm{E}+03$ & $2.72 \mathrm{E}-08$ & $4.63 \mathrm{E}+05$ & $3.97 \mathrm{E}+00$ \\
\hline Dy-149 & $4.22 \mathrm{E}+02$ & $1.14 \mathrm{E}-08$ & $1.95 \mathrm{E}+05$ & $1.67 \mathrm{E}+00$ \\
\hline Dy-150 & $2.69 \mathrm{E}+03$ & $7.26 \mathrm{E}-08$ & $1.23 \mathrm{E}+06$ & $1.06 \mathrm{E}+01$ \\
\hline Dy-151 & $5.05 \mathrm{E}+02$ & $1.36 \mathrm{E}-08$ & $2.33 \mathrm{E}+05$ & $2.00 \mathrm{E}+00$ \\
\hline
\end{tabular}




\begin{tabular}{|c|c|c|c|c|}
\hline Nuclide & $\begin{array}{c}\text { Air } \\
\text { Immersion } \\
\text { DCS } \\
\left(\mathbf{B q} / \mathbf{m}^{3}\right)\end{array}$ & $\begin{array}{c}\text { Air } \\
\text { Immersion } \\
\text { DCS } \\
\left(\mu \mathrm{Ci} / \mathbf{c m}^{3}\right)\end{array}$ & $\begin{array}{c}\text { Water } \\
\text { Submersion } \\
\text { DCS } \\
\left(\mathbf{B q} / \mathbf{m}^{\mathbf{3}}\right)\end{array}$ & $\begin{array}{c}\text { Water } \\
\text { Submersion } \\
\text { DCS } \\
\left(\mu \mathrm{Ci} / \mathbf{m}^{3}\right)\end{array}$ \\
\hline Dy-152 & $2.69 \mathrm{E}+03$ & $7.26 \mathrm{E}-08$ & $1.22 \mathrm{E}+06$ & $1.05 \mathrm{E}+01$ \\
\hline Dy-153 & $8.30 \mathrm{E}+02$ & $2.24 \mathrm{E}-08$ & $3.80 \mathrm{E}+05$ & $3.26 \mathrm{E}+00$ \\
\hline Dy-155 & $1.07 \mathrm{E}+03$ & $2.90 \mathrm{E}-08$ & $4.92 \mathrm{E}+05$ & $4.21 \mathrm{E}+00$ \\
\hline Dy-157 & $2.20 \mathrm{E}+03$ & $5.95 \mathrm{E}-08$ & $1.01 \mathrm{E}+06$ & $8.63 \mathrm{E}+00$ \\
\hline Dy-159 & $3.17 \mathrm{E}+04$ & $8.57 \mathrm{E}-07$ & $1.37 \mathrm{E}+07$ & $1.17 \mathrm{E}+02$ \\
\hline Dy-165 & $2.28 \mathrm{E}+04$ & $6.17 \mathrm{E}-07$ & $1.16 \mathrm{E}+07$ & $9.95 \mathrm{E}+01$ \\
\hline Dy-165m & $4.48 \mathrm{E}+04$ & $1.21 \mathrm{E}-06$ & $2.05 \mathrm{E}+07$ & $1.75 \mathrm{E}+02$ \\
\hline Dy-166 & $2.42 \mathrm{E}+04$ & $6.54 \mathrm{E}-07$ & $1.09 \mathrm{E}+07$ & $9.34 \mathrm{E}+01$ \\
\hline Dy-167 & $1.32 \mathrm{E}+03$ & $3.57 \mathrm{E}-08$ & $6.13 \mathrm{E}+05$ & $5.26 \mathrm{E}+00$ \\
\hline Dy-168 & $1.83 \mathrm{E}+03$ & $4.95 \mathrm{E}-08$ & $8.48 \mathrm{E}+05$ & $7.27 \mathrm{E}+00$ \\
\hline Ho- 150 & $3.63 \mathrm{E}+02$ & $9.82 \mathrm{E}-09$ & $1.69 \mathrm{E}+05$ & $1.45 \mathrm{E}+00$ \\
\hline Ho-153 & $6.88 \mathrm{E}+02$ & $1.86 \mathrm{E}-08$ & $3.17 \mathrm{E}+05$ & $2.72 \mathrm{E}+00$ \\
\hline Ho-153m & $6.72 \mathrm{E}+02$ & $1.82 \mathrm{E}-08$ & $3.11 \mathrm{E}+05$ & $2.66 \mathrm{E}+00$ \\
\hline Ho-154 & $3.67 \mathrm{E}+02$ & $9.93 \mathrm{E}-09$ & $1.70 \mathrm{E}+05$ & $1.45 \mathrm{E}+00$ \\
\hline Ho- $154 \mathrm{~m}$ & $2.91 \mathrm{E}+02$ & $7.86 \mathrm{E}-09$ & $1.34 \mathrm{E}+05$ & $1.15 \mathrm{E}+00$ \\
\hline Ho-155 & $1.17 \mathrm{E}+03$ & $3.16 \mathrm{E}-08$ & $5.38 \mathrm{E}+05$ & $4.61 \mathrm{E}+00$ \\
\hline Ho-156 & $3.24 \mathrm{E}+02$ & $8.76 \mathrm{E}-09$ & $1.50 \mathrm{E}+05$ & $1.28 \mathrm{E}+00$ \\
\hline Ho-157 & $1.29 \mathrm{E}+03$ & $3.48 \mathrm{E}-08$ & $5.89 \mathrm{E}+05$ & $5.05 \mathrm{E}+00$ \\
\hline Ho-159 & $2.09 \mathrm{E}+03$ & 5.64E-08 & $9.44 \mathrm{E}+05$ & $8.09 \mathrm{E}+00$ \\
\hline Ho-160 & $4.15 \mathrm{E}+02$ & $1.12 \mathrm{E}-08$ & $91 \mathrm{E}+05$ & $4 \mathrm{E}+00$ \\
\hline Ho-161 & $2.38 \mathrm{E}+04$ & $6.44 \mathrm{E}-07$ & $1.04 \mathrm{E}+07$ & $8.88 \mathrm{E}+01$ \\
\hline Ho-162 & $4.85 \mathrm{E}+03$ & $1.31 \mathrm{E}-07$ & $2.22 \mathrm{E}+06$ & $1.90 \mathrm{E}+01$ \\
\hline Ho- $162 \mathrm{~m}$ & $1.28 \mathrm{E}+03$ & $3.46 \mathrm{E}-08$ & $5.87 \mathrm{E}+05$ & $5.03 \mathrm{E}+00$ \\
\hline Ho-164 & $3.94 \mathrm{E}+04$ & $1.07 \mathrm{E}-06$ & $1.80 \mathrm{E}+07$ & $1.54 \mathrm{E}+02$ \\
\hline Ho- $164 \mathrm{~m}$ & $2.99 \mathrm{E}+04$ & 8.09E-07 & $1.30 \mathrm{E}+07$ & $1.11 \mathrm{E}+02$ \\
\hline Ho-166 & $1.81 \mathrm{E}+04$ & $4.90 \mathrm{E}-07$ & $9.49 \mathrm{E}+06$ & $8.14 \mathrm{E}+01$ \\
\hline Ho- $166 \mathrm{~m}$ & $4.35 \mathrm{E}+02$ & $1.18 \mathrm{E}-08$ & $1.99 \mathrm{E}+05$ & $1.71 \mathrm{E}+00$ \\
\hline Ho-167 & $1.98 \mathrm{E}+03$ & $5.36 \mathrm{E}-08$ & $9.11 \mathrm{E}+05$ & $7.81 \mathrm{E}+00$ \\
\hline Ho-168 & $7.81 \mathrm{E}+02$ & $2.11 \mathrm{E}-08$ & $3.63 \mathrm{E}+05$ & $3.11 \mathrm{E}+00$ \\
\hline Нo- $168 \mathrm{~m}$ & $2.11 \mathrm{E}+05$ & 5.71E-06 & $9.16 \mathrm{E}+07$ & $7.85 \mathrm{E}+02$ \\
\hline Ho-170 & $4.05 \mathrm{E}+02$ & $1.09 \mathrm{E}-08$ & $1.88 \mathrm{E}+05$ & $1.61 \mathrm{E}+00$ \\
\hline Er-154 & $1.42 \mathrm{E}+04$ & & & \\
\hline Er-156 & $1.84 \mathrm{E}+04$ & $4.98 \mathrm{E}-07$ & $8.15 \mathrm{E}+06$ & $6.99 \mathrm{E}+01$ \\
\hline Er-159 & $7.27 \mathrm{E}+02$ & $1.97 \mathrm{E}-08$ & $3.35 \mathrm{E}+05$ & $2.87 \mathrm{E}+00$ \\
\hline Er-161 & $7.11 \mathrm{E}+02$ & $1.92 \mathrm{E}-08$ & $3.27 \mathrm{E}+05$ & $2.80 \mathrm{E}+00$ \\
\hline Er-163 & $3.23 \mathrm{E}+04$ & $8.73 \mathrm{E}-07$ & $1.40 \mathrm{E}+07$ & $1.20 \mathrm{E}+02$ \\
\hline Er-165 & $3.52 \mathrm{E}+04$ & $9.51 \mathrm{E}-07$ & $1.52 \mathrm{E}+07$ & $1.31 \mathrm{E}+02$ \\
\hline
\end{tabular}




\begin{tabular}{|c|c|c|c|c|}
\hline Nuclide & $\begin{array}{c}\text { Air } \\
\text { Immersion } \\
\text { DCS } \\
\left(\mathbf{B q} / \mathbf{m}^{3}\right)\end{array}$ & $\begin{array}{c}\text { Air } \\
\text { Immersion } \\
\text { DCS } \\
\left(\mu \mathrm{Ci} / \mathbf{c m}^{3}\right)\end{array}$ & $\begin{array}{c}\text { Water } \\
\text { Submersion } \\
\text { DCS } \\
\left(\mathbf{B q} / \mathbf{m}^{3}\right)\end{array}$ & $\begin{array}{c}\text { Water } \\
\text { Submersion } \\
\text { DCS } \\
\left(\mu \mathrm{Ci} / \mathbf{m}^{3}\right)\end{array}$ \\
\hline Er-167m & $7.87 \mathrm{E}+03$ & $2.13 \mathrm{E}-07$ & $3.59 \mathrm{E}+06$ & $3.08 \mathrm{E}+01$ \\
\hline Er-169 & $1.07 \mathrm{E}+06$ & $2.91 \mathrm{E}-05$ & $9.79 \mathrm{E}+08$ & $8.39 \mathrm{E}+03$ \\
\hline Er-171 & $1.97 \mathrm{E}+03$ & 5.32E-08 & $9.06 \mathrm{E}+05$ & $7.76 \mathrm{E}+00$ \\
\hline Er-172 & $1.42 \mathrm{E}+03$ & $3.83 \mathrm{E}-08$ & $6.50 \mathrm{E}+05$ & $5.57 \mathrm{E}+00$ \\
\hline Er-173 & $8.50 \mathrm{E}+02$ & $2.30 \mathrm{E}-08$ & $3.92 \mathrm{E}+05$ & $3.36 \mathrm{E}+00$ \\
\hline Tm-161 & $5.38 \mathrm{E}+02$ & $1.46 \mathrm{E}-08$ & $2.48 \mathrm{E}+05$ & $2.12 \mathrm{E}+00$ \\
\hline Tm-162 & $3.47 \mathrm{E}+02$ & $9.38 \mathrm{E}-09$ & $1.60 \mathrm{E}+05$ & $1.37 \mathrm{E}+00$ \\
\hline Tm-163 & $5.26 \mathrm{E}+02$ & $1.42 \mathrm{E}-08$ & $2.42 \mathrm{E}+05$ & $2.07 \mathrm{E}+00$ \\
\hline Tm-164 & $8.88 \mathrm{E}+02$ & $2.40 \mathrm{E}-08$ & $4.12 \mathrm{E}+05$ & $3.53 \mathrm{E}+00$ \\
\hline Tm-165 & $1.32 \mathrm{E}+03$ & $3.57 \mathrm{E}-08$ & $6.06 \mathrm{E}+05$ & $5.20 \mathrm{E}+00$ \\
\hline Tm-166 & $3.41 \mathrm{E}+02$ & $9.22 \mathrm{E}-09$ & $1.57 \mathrm{E}+05$ & $1.35 \mathrm{E}+00$ \\
\hline Tm-167 & $5.78 \mathrm{E}+03$ & $1.56 \mathrm{E}-07$ & $2.62 \mathrm{E}+06$ & $2.25 \mathrm{E}+01$ \\
\hline Tm-168 & $5.75 \mathrm{E}+02$ & $1.56 \mathrm{E}-08$ & $2.64 \mathrm{E}+05$ & $2.26 \mathrm{E}+00$ \\
\hline Tm-170 & $9.76 \mathrm{E}+04$ & $2.64 \mathrm{E}-06$ & $6.17 \mathrm{E}+07$ & $5.29 \mathrm{E}+02$ \\
\hline Tm-171 & $1.87 \mathrm{E}+06$ & 5.04E-05 & $8.15 \mathrm{E}+08$ & $6.99 \mathrm{E}+03$ \\
\hline Tm-172 & $1.38 \mathrm{E}+03$ & $3.74 \mathrm{E}-08$ & $6.45 \mathrm{E}+05$ & $5.52 \mathrm{E}+00$ \\
\hline Tm-173 & $1.84 \mathrm{E}+03$ & $4.98 \mathrm{E}-08$ & $8.48 \mathrm{E}+05$ & $7.27 \mathrm{E}+00$ \\
\hline Tm-174 & $3.94 \mathrm{E}+02$ & $1.06 \mathrm{E}-08$ & $1.81 \mathrm{E}+05$ & $1.55 \mathrm{E}+00$ \\
\hline Tm-175 & $6.39 \mathrm{E}+02$ & $1.73 \mathrm{E}-08$ & $2.96 \mathrm{E}+05$ & $2.54 \mathrm{E}+00$ \\
\hline Tm-176 & $3.38 \mathrm{E}+02$ & $9.13 \mathrm{E}-09$ & $1.56 \mathrm{E}+05$ & \\
\hline Yb-162 & $3.17 \mathrm{E}+03$ & $8.57 \mathrm{E}-08$ & $1.43 \mathrm{E}+06$ & \\
\hline Yb-163 & $9.64 \mathrm{E}+02$ & $2.60 \mathrm{E}-08$ & $4.45 \mathrm{E}+05$ & $3.81 \mathrm{E}+00$ \\
\hline Yb-164 & $1.97 \mathrm{E}+04$ & $5.32 \mathrm{E}-07$ & $8.71 \mathrm{E}+06$ & $7.47 \mathrm{E}+01$ \\
\hline $\mathrm{Yb}-165$ & $2.31 \mathrm{E}+03$ & $6.26 \mathrm{E}-08$ & $1.05 \mathrm{E}+06$ & $9.03 \mathrm{E}+00$ \\
\hline Yb-166 & $1.34 \mathrm{E}+04$ & $3.62 \mathrm{E}-07$ & $5.85 \mathrm{E}+06$ & $5.01 \mathrm{E}+01$ \\
\hline Yb-167 & $3.32 \mathrm{E}+03$ & $8.97 \mathrm{E}-08$ & $1.48 \mathrm{E}+06$ & $1.27 \mathrm{E}+01$ \\
\hline Yb-169 & $2.66 \mathrm{E}+03$ & $7.20 \mathrm{E}-08$ & $1.20 \mathrm{E}+06$ & $1.03 \mathrm{E}+01$ \\
\hline Yb-175 & $1.83 \mathrm{E}+04$ & $4.95 \mathrm{E}-07$ & $8.50 \mathrm{E}+06$ & $7.29 \mathrm{E}+01$ \\
\hline Yb-177 & $3.48 \mathrm{E}+03$ & $9.41 \mathrm{E}-08$ & $1.63 \mathrm{E}+06$ & $1.39 \mathrm{E}+01$ \\
\hline Yb-178 & $1.80 \mathrm{E}+04$ & $4.87 \mathrm{E}-07$ & $8.48 \mathrm{E}+06$ & $7.27 \mathrm{E}+01$ \\
\hline Yb-179 & $7.21 \mathrm{E}+02$ & $1.95 \mathrm{E}-08$ & $3.33 \mathrm{E}+05$ & $2.85 \mathrm{E}+00$ \\
\hline Lu-165 & $6.32 \mathrm{E}+02$ & & & $2.49 \mathrm{E}+00$ \\
\hline Lu-167 & $3.99 \mathrm{E}+02$ & $1.08 \mathrm{E}-08$ & $1.84 \mathrm{E}+05$ & $1.58 \mathrm{E}+00$ \\
\hline Lu-169 & $5.22 \mathrm{E}+02$ & $1.41 \mathrm{E}-08$ & $2.40 \mathrm{E}+05$ & $2.06 \mathrm{E}+00$ \\
\hline Lu-169m & $1.26 \mathrm{E}+09$ & $3.40 \mathrm{E}-02$ & $5.44 \mathrm{E}+11$ & $4.66 \mathrm{E}+06$ \\
\hline Lu-170 & $2.54 \mathrm{E}+02$ & $6.86 \mathrm{E}-09$ & $1.17 \mathrm{E}+05$ & $1.00 \mathrm{E}+00$ \\
\hline Lu-171 & $1.14 \mathrm{E}+03$ & $3.07 \mathrm{E}-08$ & $5.21 \mathrm{E}+05$ & $4.46 \mathrm{E}+00$ \\
\hline
\end{tabular}




\begin{tabular}{|c|c|c|c|c|}
\hline Nuclide & $\begin{array}{c}\text { Air } \\
\text { Immersion } \\
\text { DCS } \\
\left(\mathbf{B q} / \mathbf{m}^{3}\right)\end{array}$ & $\begin{array}{c}\text { Air } \\
\text { Immersion } \\
\text { DCS } \\
\left(\mu \mathrm{Ci} / \mathbf{c m}^{3}\right)\end{array}$ & $\begin{array}{c}\text { Water } \\
\text { Submersion } \\
\text { DCS } \\
\left(\mathbf{B q} / \mathbf{m}^{3}\right)\end{array}$ & $\begin{array}{c}\text { Water } \\
\text { Submersion } \\
\text { DCS } \\
\left(\mu \mathrm{Ci} / \mathbf{m}^{3}\right)\end{array}$ \\
\hline Lu-171m & $3.22 \mathrm{E}+06$ & $8.69 \mathrm{E}-05$ & $1.42 \mathrm{E}+09$ & $1.21 \mathrm{E}+04$ \\
\hline Lu-172 & $3.54 \mathrm{E}+02$ & $9.56 \mathrm{E}-09$ & $1.63 \mathrm{E}+05$ & $1.40 \mathrm{E}+00$ \\
\hline Lu-172m & $8.13 \mathrm{E}+08$ & $2.20 \mathrm{E}-02$ & $3.51 \mathrm{E}+11$ & $3.01 \mathrm{E}+06$ \\
\hline Lu-173 & $4.90 \mathrm{E}+03$ & $1.32 \mathrm{E}-07$ & $2.20 \mathrm{E}+06$ & $1.89 \mathrm{E}+01$ \\
\hline Lu-174 & $7.11 \mathrm{E}+03$ & $1.92 \mathrm{E}-07$ & $3.22 \mathrm{E}+06$ & $2.76 \mathrm{E}+01$ \\
\hline Lu-174m & $1.78 \mathrm{E}+04$ & $4.81 \mathrm{E}-07$ & $7.85 \mathrm{E}+06$ & $6.73 \mathrm{E}+01$ \\
\hline Lu-176 & $1.53 \mathrm{E}+03$ & $4.14 \mathrm{E}-08$ & $7.02 \mathrm{E}+05$ & $6.01 \mathrm{E}+00$ \\
\hline Lu-176m & $4.11 \mathrm{E}+04$ & $1.11 \mathrm{E}-06$ & $2.25 \mathrm{E}+07$ & $1.93 \mathrm{E}+02$ \\
\hline Lu-177 & $2.11 \mathrm{E}+04$ & $5.71 \mathrm{E}-07$ & $9.76 \mathrm{E}+06$ & $8.36 \mathrm{E}+01$ \\
\hline Lu-177m & $7.50 \mathrm{E}+02$ & $2.03 \mathrm{E}-08$ & $3.41 \mathrm{E}+05$ & $2.93 \mathrm{E}+00$ \\
\hline Lu-178 & $4.92 \mathrm{E}+03$ & $1.33 \mathrm{E}-07$ & $2.37 \mathrm{E}+06$ & $2.03 \mathrm{E}+01$ \\
\hline Lu-178m & $7.00 \mathrm{E}+02$ & $1.89 \mathrm{E}-08$ & $3.21 \mathrm{E}+05$ & $2.75 \mathrm{E}+00$ \\
\hline Lu-179 & $1.95 \mathrm{E}+04$ & $5.26 \mathrm{E}-07$ & $9.82 \mathrm{E}+06$ & $8.41 \mathrm{E}+01$ \\
\hline Lu-180 & $4.47 \mathrm{E}+02$ & $1.21 \mathrm{E}-08$ & $2.07 \mathrm{E}+05$ & $1.78 \mathrm{E}+00$ \\
\hline Lu-181 & $1.23 \mathrm{E}+03$ & $3.32 \mathrm{E}-08$ & $5.69 \mathrm{E}+05$ & $4.88 \mathrm{E}+00$ \\
\hline Hf-167 & $1.16 \mathrm{E}+03$ & $3.14 \mathrm{E}-08$ & $5.37 \mathrm{E}+05$ & $4.60 \mathrm{E}+00$ \\
\hline Hf-169 & $1.14 \mathrm{E}+03$ & $3.08 \mathrm{E}-08$ & $5.24 \mathrm{E}+05$ & $4.49 \mathrm{E}+00$ \\
\hline Hf-170 & $1.73 \mathrm{E}+03$ & $4.68 \mathrm{E}-08$ & $7.91 \mathrm{E}+05$ & $6.78 \mathrm{E}+00$ \\
\hline Hf-172 & $1.03 \mathrm{E}+04$ & $2.77 \mathrm{E}-07$ & $4.52 \mathrm{E}+06$ & $3.87 \mathrm{E}+01$ \\
\hline Hf-173 & $1.96 \mathrm{E}+03$ & $5.29 \mathrm{E}-08$ & & $7.61 \mathrm{E}+00$ \\
\hline Hf-175 & & & & $8.44 \mathrm{E}+00$ \\
\hline Hf-177m & $3.22 \mathrm{E}+02$ & $8.71 \mathrm{E}-09$ & $1.47 \mathrm{E}+05$ & $1.26 \mathrm{E}+00$ \\
\hline Hf- $178 \mathrm{~m}$ & $3.24 \mathrm{E}+02$ & $8.76 \mathrm{E}-09$ & $1.49 \mathrm{E}+05$ & $1.28 \mathrm{E}+00$ \\
\hline Hf-179m & $8.09 \mathrm{E}+02$ & $2.19 \mathrm{E}-08$ & $3.69 \mathrm{E}+05$ & $3.16 \mathrm{E}+00$ \\
\hline Hf-180m & $7.43 \mathrm{E}+02$ & $2.01 \mathrm{E}-08$ & $3.40 \mathrm{E}+05$ & $2.91 \mathrm{E}+00$ \\
\hline Hf-181 & $1.37 \mathrm{E}+03$ & $3.69 \mathrm{E}-08$ & $6.28 \mathrm{E}+05$ & $5.38 \mathrm{E}+00$ \\
\hline Hf-182 & $3.05 \mathrm{E}+03$ & $8.24 \mathrm{E}-08$ & $1.40 \mathrm{E}+06$ & $1.20 \mathrm{E}+01$ \\
\hline Hf-182m & $7.95 \mathrm{E}+02$ & $2.15 \mathrm{E}-08$ & $3.65 \mathrm{E}+05$ & $3.13 \mathrm{E}+00$ \\
\hline Hf-183 & $9.06 \mathrm{E}+02$ & $2.45 \mathrm{E}-08$ & $4.18 \mathrm{E}+05$ & $3.59 \mathrm{E}+00$ \\
\hline Hf-184 & $3.21 \mathrm{E}+03$ & $8.67 \mathrm{E}-08$ & $1.47 \mathrm{E}+06$ & $1.26 \mathrm{E}+01$ \\
\hline Тa-170 & $6.50 \mathrm{E}+02$ & $1.76 \mathrm{E}-08$ & $3.02 \mathrm{E}+05$ & $2.59 \mathrm{E}+00$ \\
\hline Ta-172 & $4.05 \mathrm{E}+02$ & $1.10 \mathrm{E}-08$ & & $1.61 \mathrm{E}+00$ \\
\hline Ta-173 & $1.24 \mathrm{E}+03$ & $3.35 \mathrm{E}-08$ & $5.68 \mathrm{E}+05$ & $4.87 \mathrm{E}+00$ \\
\hline Ta-174 & $7.11 \mathrm{E}+02$ & $1.92 \mathrm{E}-08$ & $3.28 \mathrm{E}+05$ & $2.81 \mathrm{E}+00$ \\
\hline Ta-175 & $6.23 \mathrm{E}+02$ & $1.68 \mathrm{E}-08$ & $2.86 \mathrm{E}+05$ & $2.45 \mathrm{E}+00$ \\
\hline Тa-176 & $2.96 \mathrm{E}+02$ & $8.01 \mathrm{E}-09$ & $1.37 \mathrm{E}+05$ & $1.17 \mathrm{E}+00$ \\
\hline Тa-177 & $1.46 \mathrm{E}+04$ & $3.95 \mathrm{E}-07$ & $6.47 \mathrm{E}+06$ & $5.55 \mathrm{E}+01$ \\
\hline
\end{tabular}




\begin{tabular}{|c|c|c|c|c|}
\hline Nuclide & $\begin{array}{c}\text { Air } \\
\text { Immersion } \\
\text { DCS } \\
\left(\mathbf{B q} / \mathbf{m}^{3}\right)\end{array}$ & $\begin{array}{c}\text { Air } \\
\text { Immersion } \\
\text { DCS } \\
\left(\mu \mathrm{Ci} / \mathbf{c m}^{3}\right)\end{array}$ & $\begin{array}{c}\text { Water } \\
\text { Submersion } \\
\text { DCS } \\
\left(\mathbf{B q} / \mathbf{m}^{3}\right)\end{array}$ & $\begin{array}{c}\text { Water } \\
\text { Submersion } \\
\text { DCS } \\
\left(\mu \mathrm{Ci} / \mathbf{m}^{3}\right)\end{array}$ \\
\hline Тa-178 & $6.72 \mathrm{E}+03$ & $1.82 \mathrm{E}-07$ & $3.05 \mathrm{E}+06$ & $2.61 \mathrm{E}+01$ \\
\hline Ta-178m & $6.46 \mathrm{E}+02$ & $1.75 \mathrm{E}-08$ & $2.94 \mathrm{E}+05$ & $2.52 \mathrm{E}+00$ \\
\hline Ta-179 & $4.56 \mathrm{E}+04$ & $1.23 \mathrm{E}-06$ & $1.99 \mathrm{E}+07$ & $1.71 \mathrm{E}+02$ \\
\hline Ta-180 & $2.23 \mathrm{E}+04$ & $6.04 \mathrm{E}-07$ & $9.85 \mathrm{E}+06$ & $8.44 \mathrm{E}+01$ \\
\hline Ta-182 & $5.30 \mathrm{E}+02$ & $1.43 \mathrm{E}-08$ & $2.44 \mathrm{E}+05$ & $2.09 \mathrm{E}+00$ \\
\hline Ta- $182 \mathrm{~m}$ & $3.02 \mathrm{E}+03$ & $8.16 \mathrm{E}-08$ & $1.37 \mathrm{E}+06$ & $1.18 \mathrm{E}+01$ \\
\hline Ta-183 & $2.64 \mathrm{E}+03$ & $7.14 \mathrm{E}-08$ & $1.21 \mathrm{E}+06$ & $1.03 \mathrm{E}+01$ \\
\hline Ta-184 & $4.49 \mathrm{E}+02$ & $1.21 \mathrm{E}-08$ & $2.06 \mathrm{E}+05$ & $1.76 \mathrm{E}+00$ \\
\hline Ta-185 & $4.73 \mathrm{E}+03$ & $1.28 \mathrm{E}-07$ & $2.22 \mathrm{E}+06$ & $1.90 \mathrm{E}+01$ \\
\hline Ta-186 & $4.93 \mathrm{E}+02$ & $1.33 \mathrm{E}-08$ & $2.28 \mathrm{E}+05$ & $1.96 \mathrm{E}+00$ \\
\hline W-177 & $7.95 \mathrm{E}+02$ & $2.15 \mathrm{E}-08$ & $3.64 \mathrm{E}+05$ & $3.12 \mathrm{E}+00$ \\
\hline W-178 & $7.32 \mathrm{E}+04$ & $1.98 \mathrm{E}-06$ & $3.21 \mathrm{E}+07$ & $2.75 \mathrm{E}+02$ \\
\hline W-179 & $2.19 \mathrm{E}+04$ & $5.91 \mathrm{E}-07$ & $9.55 \mathrm{E}+06$ & $8.19 \mathrm{E}+01$ \\
\hline $\mathrm{W}-179 \mathrm{~m}$ & $1.59 \mathrm{E}+04$ & $4.31 \mathrm{E}-07$ & $7.22 \mathrm{E}+06$ & $6.19 \mathrm{E}+01$ \\
\hline W-181 & $2.76 \mathrm{E}+04$ & $7.45 \mathrm{E}-07$ & $21 \mathrm{E}+07$ & $1.03 \mathrm{E}+02$ \\
\hline W-185 & $6.39 \mathrm{E}+05$ & $1.73 \mathrm{E}-05$ & $5.59 \mathrm{E}+08$ & $4.79 \mathrm{E}+03$ \\
\hline W-185m & $3.40 \mathrm{E}+04$ & $9.19 \mathrm{E}-07$ & $1.55 \mathrm{E}+07$ & $1.33 \mathrm{E}+02$ \\
\hline W-187 & $1.59 \mathrm{E}+03$ & $4.29 \mathrm{E}-08$ & $7.32 \mathrm{E}+05$ & $6.28 \mathrm{E}+00$ \\
\hline W-188 & $2.88 \mathrm{E}+05$ & 7.79E-06 & $1.52 \mathrm{E}+08$ & $1.31 \mathrm{E}+03$ \\
\hline W-190 & $5.51 \mathrm{E}+03$ & $1.49 \mathrm{E}-07$ & $2.52 \mathrm{E}+06$ & $2.16 \mathrm{E}+01$ \\
\hline Re-178 & $3.88 \mathrm{E}+02$ & $1.05 \mathrm{E}-08$ & $1.79 \mathrm{E}+05$ & $1.54 \mathrm{E}+00$ \\
\hline Re-179 & $6.47 \mathrm{E}+02$ & $1.75 \mathrm{E}-08$ & $2.96 \mathrm{E}+05$ & $2.54 \mathrm{E}+00$ \\
\hline Re-180 & $5.83 \mathrm{E}+02$ & $1.58 \mathrm{E}-08$ & $2.69 \mathrm{E}+05$ & $2.30 \mathrm{E}+00$ \\
\hline Re-181 & $9.01 \mathrm{E}+02$ & $2.43 \mathrm{E}-08$ & $4.14 \mathrm{E}+05$ & $3.55 \mathrm{E}+00$ \\
\hline Re-182 & $3.91 \mathrm{E}+02$ & $1.06 \mathrm{E}-08$ & $1.80 \mathrm{E}+05$ & $1.54 \mathrm{E}+00$ \\
\hline Re-182m & $5.67 \mathrm{E}+02$ & $1.53 \mathrm{E}-08$ & $2.60 \mathrm{E}+05$ & $2.23 \mathrm{E}+00$ \\
\hline Re-183 & $5.73 \mathrm{E}+03$ & $1.55 \mathrm{E}-07$ & $2.56 \mathrm{E}+06$ & $2.19 \mathrm{E}+01$ \\
\hline Re-184 & $7.95 \mathrm{E}+02$ & $2.15 \mathrm{E}-08$ & $3.65 \mathrm{E}+05$ & $3.13 \mathrm{E}+00$ \\
\hline Re- $184 m$ & $1.93 \mathrm{E}+03$ & $5.23 \mathrm{E}-08$ & $8.86 \mathrm{E}+05$ & $7.59 \mathrm{E}+00$ \\
\hline Re-186 & $3.19 \mathrm{E}+04$ & $8.61 \mathrm{E}-07$ & $1.59 \mathrm{E}+07$ & $1.37 \mathrm{E}+02$ \\
\hline Re-186m & $7.44 \mathrm{E}+04$ & $2.01 \mathrm{E}-06$ & $3.26 \mathrm{E}+07$ & $2.80 \mathrm{E}+02$ \\
\hline Re-188 & $9.64 \mathrm{E}+03$ & & & \\
\hline Re-188m & $1.40 \mathrm{E}+04$ & 3.79E-07 & $6.19 \mathrm{E}+06$ & $5.31 \mathrm{E}+01$ \\
\hline Re-189 & $1.24 \mathrm{E}+04$ & $3.36 \mathrm{E}-07$ & $5.87 \mathrm{E}+06$ & $5.03 \mathrm{E}+01$ \\
\hline Re-190 & $5.25 \mathrm{E}+02$ & $1.42 \mathrm{E}-08$ & $2.42 \mathrm{E}+05$ & $2.07 \mathrm{E}+00$ \\
\hline Re-190m & $7.68 \mathrm{E}+02$ & $2.08 \mathrm{E}-08$ & $3.54 \mathrm{E}+05$ & $3.03 \mathrm{E}+00$ \\
\hline Os-180 & $6.79 \mathrm{E}+03$ & $1.84 \mathrm{E}-07$ & $3.08 \mathrm{E}+06$ & $2.64 \mathrm{E}+01$ \\
\hline
\end{tabular}




\begin{tabular}{|c|c|c|c|c|}
\hline Nuclide & $\begin{array}{c}\text { Air } \\
\text { Immersion } \\
\text { DCS } \\
\left(\mathbf{B q} / \mathbf{m}^{3}\right)\end{array}$ & $\begin{array}{c}\text { Air } \\
\text { Immersion } \\
\text { DCS } \\
\left(\mu \mathrm{Ci} / \mathbf{c m}^{3}\right)\end{array}$ & $\begin{array}{c}\text { Water } \\
\text { Submersion } \\
\text { DCS } \\
\left(\mathbf{B q} / \mathbf{m}^{3}\right)\end{array}$ & $\begin{array}{c}\text { Water } \\
\text { Submersion } \\
\text { DCS } \\
\left(\mu \mathrm{Ci} / \mathbf{m}^{3}\right)\end{array}$ \\
\hline Os-181 & $5.03 \mathrm{E}+02$ & $1.36 \mathrm{E}-08$ & $2.31 \mathrm{E}+05$ & $1.98 \mathrm{E}+00$ \\
\hline Os-182 & $1.74 \mathrm{E}+03$ & 4.71E-08 & $7.97 \mathrm{E}+05$ & $6.83 \mathrm{E}+00$ \\
\hline Os-183 & $1.19 \mathrm{E}+03$ & $3.22 \mathrm{E}-08$ & $5.44 \mathrm{E}+05$ & $4.66 \mathrm{E}+00$ \\
\hline Os-183m & $6.85 \mathrm{E}+02$ & $1.85 \mathrm{E}-08$ & $3.17 \mathrm{E}+05$ & $2.72 \mathrm{E}+00$ \\
\hline Os-185 & $1.04 \mathrm{E}+03$ & $2.80 \mathrm{E}-08$ & $4.77 \mathrm{E}+05$ & $4.09 \mathrm{E}+00$ \\
\hline Os-189m & $3.08 \mathrm{E}+08$ & $8.32 \mathrm{E}-03$ & $1.33 \mathrm{E}+11$ & $1.14 \mathrm{E}+06$ \\
\hline Os-190m & $4.50 \mathrm{E}+02$ & $1.22 \mathrm{E}-08$ & $2.07 \mathrm{E}+05$ & $1.78 \mathrm{E}+00$ \\
\hline Os-191 & $1.07 \mathrm{E}+04$ & $2.91 \mathrm{E}-07$ & $4.79 \mathrm{E}+06$ & $4.11 \mathrm{E}+01$ \\
\hline Os-191m & $1.74 \mathrm{E}+05$ & $4.71 \mathrm{E}-06$ & $7.64 \mathrm{E}+07$ & $6.55 \mathrm{E}+02$ \\
\hline Os-193 & $1.05 \mathrm{E}+04$ & $2.83 \mathrm{E}-07$ & $4.95 \mathrm{E}+06$ & $4.24 \mathrm{E}+01$ \\
\hline Os-194 & $6.23 \mathrm{E}+05$ & $1.68 \mathrm{E}-05$ & $2.69 \mathrm{E}+08$ & $2.30 \mathrm{E}+03$ \\
\hline Os-196 & $8.71 \mathrm{E}+03$ & $2.35 \mathrm{E}-07$ & $4.10 \mathrm{E}+06$ & $3.52 \mathrm{E}+01$ \\
\hline Ir-180 & $4.38 \mathrm{E}+02$ & $1.18 \mathrm{E}-08$ & $2.03 \mathrm{E}+05$ & $1.74 \mathrm{E}+00$ \\
\hline Ir-182 & $4.94 \mathrm{E}+02$ & $1.33 \mathrm{E}-08$ & $2.28 \mathrm{E}+05$ & $1.96 \mathrm{E}+00$ \\
\hline Ir-183 & $5.79 \mathrm{E}+02$ & $1.56 \mathrm{E}-08$ & $2.66 \mathrm{E}+05$ & $2.28 \mathrm{E}+00$ \\
\hline Ir-184 & $3.52 \mathrm{E}+02$ & $9.51 \mathrm{E}-09$ & $1.62 \mathrm{E}+05$ & $1.39 \mathrm{E}+00$ \\
\hline Ir-185 & $8.03 \mathrm{E}+02$ & $2.17 \mathrm{E}-08$ & $3.69 \mathrm{E}+05$ & $3.16 \mathrm{E}+00$ \\
\hline Ir-186 & $4.16 \mathrm{E}+02$ & 1.12E-08 & $1.91 \mathrm{E}+05$ & $1.64 \mathrm{E}+00$ \\
\hline Ir-186m & $5.46 \mathrm{E}+02$ & $1.48 \mathrm{E}-08$ & $2.52 \mathrm{E}+05$ & $2.16 \mathrm{E}+00$ \\
\hline Ir-187 & $2.26 \mathrm{E}+03$ & $6.12 \mathrm{E}-08$ & $03 \mathrm{E}+06$ & $8.85 \mathrm{E}+00$ \\
\hline Ir-188 & $3.14 \mathrm{E}+02$ & 8.49E-09 & $1.45 \mathrm{E}+05$ & $1.24 \mathrm{E}+00$ \\
\hline Ir-189 & $1.19 \mathrm{E}+04$ & $3.21 \mathrm{E}-07$ & $5.29 \mathrm{E}+06$ & $4.54 \mathrm{E}+01$ \\
\hline Ir-190 & $4.87 \mathrm{E}+02$ & $1.32 \mathrm{E}-08$ & $2.23 \mathrm{E}+05$ & $1.91 \mathrm{E}+00$ \\
\hline Ir-190m & $2.78 \mathrm{E}+08$ & $7.52 \mathrm{E}-03$ & $1.20 \mathrm{E}+11$ & $1.03 \mathrm{E}+06$ \\
\hline Ir-190n & $1.74 \mathrm{E}+04$ & $4.71 \mathrm{E}-07$ & $7.70 \mathrm{E}+06$ & $6.60 \mathrm{E}+01$ \\
\hline Ir-191m & $1.19 \mathrm{E}+04$ & $3.22 \mathrm{E}-07$ & $5.32 \mathrm{E}+06$ & $4.56 \mathrm{E}+01$ \\
\hline Ir-192 & $8.78 \mathrm{E}+02$ & $2.37 \mathrm{E}-08$ & $4.03 \mathrm{E}+05$ & $3.46 \mathrm{E}+00$ \\
\hline Ir-192m & $1.18 \mathrm{E}+07$ & $3.20 \mathrm{E}-04$ & $5.36 \mathrm{E}+09$ & $4.59 \mathrm{E}+04$ \\
\hline Ir-192n & $4.77 \mathrm{E}+05$ & $1.29 \mathrm{E}-05$ & $3.30 \mathrm{E}+08$ & $2.83 \mathrm{E}+03$ \\
\hline Ir-193m & $3.05 \mathrm{E}+06$ & $8.24 \mathrm{E}-05$ & $1.37 \mathrm{E}+09$ & $1.17 \mathrm{E}+04$ \\
\hline Ir-194 & $6.65 \mathrm{E}+03$ & $1.80 \mathrm{E}-07$ & $3.26 \mathrm{E}+06$ & $2.79 \mathrm{E}+01$ \\
\hline Ir-194m & $3.05 \mathrm{E}+02$ & $8.24 \mathrm{E}-09$ & $1.40 \mathrm{E}+05$ & $1.20 \mathrm{E}+00$ \\
\hline Ir-195 & $1.44 \mathrm{E}+04$ & $3.90 \mathrm{E}-07$ & $6.72 \mathrm{E}+06$ & $5.76 \mathrm{E}+01$ \\
\hline Ir-195m & $1.95 \mathrm{E}+03$ & $5.26 \mathrm{E}-08$ & $8.91 \mathrm{E}+05$ & $7.63 \mathrm{E}+00$ \\
\hline Ir-196 & $2.73 \mathrm{E}+03$ & 7.39E-08 & $1.31 \mathrm{E}+06$ & $1.12 \mathrm{E}+01$ \\
\hline Ir-196m & $2.88 \mathrm{E}+02$ & $7.79 \mathrm{E}-09$ & $1.33 \mathrm{E}+05$ & $1.14 \mathrm{E}+00$ \\
\hline Pt-184 & $1.05 \mathrm{E}+03$ & $2.84 \mathrm{E}-08$ & $4.78 \mathrm{E}+05$ & $4.10 \mathrm{E}+00$ \\
\hline
\end{tabular}




\begin{tabular}{|c|c|c|c|c|}
\hline Nuclide & $\begin{array}{c}\text { Air } \\
\text { Immersion } \\
\text { DCS } \\
\left(\mathbf{B q} / \mathbf{m}^{3}\right)\end{array}$ & $\begin{array}{c}\text { Air } \\
\text { Immersion } \\
\text { DCS } \\
\left(\mu \mathrm{Ci} / \mathbf{c m}^{3}\right)\end{array}$ & $\begin{array}{c}\text { Water } \\
\text { Submersion } \\
\text { DCS } \\
\left(\mathbf{B q} / \mathbf{m}^{3}\right)\end{array}$ & $\begin{array}{c}\text { Water } \\
\text { Submersion } \\
\text { DCS } \\
\left(\mu \mathrm{Ci} / \mathbf{m}^{3}\right)\end{array}$ \\
\hline Pt-186 & $1.06 \mathrm{E}+03$ & $2.87 \mathrm{E}-08$ & $4.87 \mathrm{E}+05$ & $4.17 \mathrm{E}+00$ \\
\hline Pt-187 & $1.19 \mathrm{E}+03$ & $3.22 \mathrm{E}-08$ & $5.46 \mathrm{E}+05$ & $4.68 \mathrm{E}+00$ \\
\hline Pt-188 & $3.94 \mathrm{E}+03$ & $1.07 \mathrm{E}-07$ & $1.78 \mathrm{E}+06$ & $1.53 \mathrm{E}+01$ \\
\hline Pt-189 & $1.54 \mathrm{E}+03$ & $4.16 \mathrm{E}-08$ & $7.03 \mathrm{E}+05$ & $6.03 \mathrm{E}+00$ \\
\hline Pt-191 & $2.69 \mathrm{E}+03$ & $7.26 \mathrm{E}-08$ & $1.22 \mathrm{E}+06$ & $1.05 \mathrm{E}+01$ \\
\hline Pt-193 & $1.12 \mathrm{E}+08$ & $3.03 \mathrm{E}-03$ & $4.83 \mathrm{E}+10$ & $4.14 \mathrm{E}+05$ \\
\hline Pt-193m & $8.62 \mathrm{E}+04$ & $2.33 \mathrm{E}-06$ & $3.94 \mathrm{E}+07$ & $3.38 \mathrm{E}+02$ \\
\hline Pt-195m & $1.29 \mathrm{E}+04$ & $3.50 \mathrm{E}-07$ & $5.74 \mathrm{E}+06$ & $4.92 \mathrm{E}+01$ \\
\hline Pt-197 & $3.20 \mathrm{E}+04$ & $8.65 \mathrm{E}-07$ & $1.51 \mathrm{E}+07$ & $1.29 \mathrm{E}+02$ \\
\hline Pt-197m & $9.73 \mathrm{E}+03$ & $2.63 \mathrm{E}-07$ & $4.50 \mathrm{E}+06$ & $3.85 \mathrm{E}+01$ \\
\hline Pt-199 & $3.44 \mathrm{E}+03$ & $9.29 \mathrm{E}-08$ & $1.61 \mathrm{E}+06$ & $1.38 \mathrm{E}+01$ \\
\hline Pt-200 & $1.36 \mathrm{E}+04$ & $3.68 \mathrm{E}-07$ & $6.23 \mathrm{E}+06$ & $5.34 \mathrm{E}+01$ \\
\hline Pt-202 & $6.30 \mathrm{E}+04$ & $1.70 \mathrm{E}-06$ & $5.24 \mathrm{E}+07$ & $4.49 \mathrm{E}+02$ \\
\hline Au-186 & $4.62 \mathrm{E}+02$ & $1.25 \mathrm{E}-08$ & $2.13 \mathrm{E}+05$ & $1.82 \mathrm{E}+00$ \\
\hline Au-187 & $6.39 \mathrm{E}+02$ & $1.73 \mathrm{E}-08$ & $2.94 \mathrm{E}+05$ & $2.52 \mathrm{E}+00$ \\
\hline $\mathrm{Au}-190$ & $2.73 \mathrm{E}+02$ & 7.39E-09 & $1.26 \mathrm{E}+05$ & $1.08 \mathrm{E}+00$ \\
\hline $\mathrm{Au}-191$ & $1.24 \mathrm{E}+03$ & $3.36 \mathrm{E}-08$ & $5.68 \mathrm{E}+05$ & $4.87 \mathrm{E}+00$ \\
\hline Au-192 & $3.41 \mathrm{E}+02$ & $9.23 \mathrm{E}-09$ & $1.57 \mathrm{E}+05$ & $1.35 \mathrm{E}+00$ \\
\hline $\mathrm{Au}-193$ & $4.95 \mathrm{E}+03$ & $1.34 \mathrm{E}-07$ & $2.23 \mathrm{E}+06$ & $1.91 \mathrm{E}+01$ \\
\hline $\mathrm{Au}-193 \mathrm{~m}$ & $3.79 \mathrm{E}+03$ & $1.02 \mathrm{E}-07$ & $1.73 \mathrm{E}+06$ & $1.49 \mathrm{E}+01$ \\
\hline Au-194 & $6.59 \mathrm{E}+02$ & $1.78 \mathrm{E}-08$ & $3.02 \mathrm{E}+05$ & $2.59 \mathrm{E}+00$ \\
\hline Au-195 & $1.17 \mathrm{E}+04$ & $3.17 \mathrm{E}-07$ & $5.20 \mathrm{E}+06$ & $4.46 \mathrm{E}+01$ \\
\hline $\mathrm{Au}-195 \mathrm{~m}$ & $3.72 \mathrm{E}+03$ & $1.01 \mathrm{E}-07$ & $1.70 \mathrm{E}+06$ & $1.45 \mathrm{E}+01$ \\
\hline $\mathrm{Au}-196$ & $1.57 \mathrm{E}+03$ & 4.24E-08 & $7.17 \mathrm{E}+05$ & $6.15 \mathrm{E}+00$ \\
\hline $\mathrm{Au}-196 \mathrm{~m}$ & $3.27 \mathrm{E}+03$ & $8.84 \mathrm{E}-08$ & $1.48 \mathrm{E}+06$ & $1.27 \mathrm{E}+01$ \\
\hline $\mathrm{Au}-198$ & $1.76 \mathrm{E}+03$ & $4.76 \mathrm{E}-08$ & $8.13 \mathrm{E}+05$ & $6.97 \mathrm{E}+00$ \\
\hline $\mathrm{Au}-198 \mathrm{~m}$ & $1.43 \mathrm{E}+03$ & $3.88 \mathrm{E}-08$ & $6.51 \mathrm{E}+05$ & $5.58 \mathrm{E}+00$ \\
\hline Au-199 & $7.99 \mathrm{E}+03$ & $2.16 \mathrm{E}-07$ & $3.63 \mathrm{E}+06$ & $3.11 \mathrm{E}+01$ \\
\hline Au-200 & $2.38 \mathrm{E}+03$ & $6.44 \mathrm{E}-08$ & $1.12 \mathrm{E}+06$ & $9.57 \mathrm{E}+00$ \\
\hline $\mathrm{Au}-200 \mathrm{~m}$ & $3.59 \mathrm{E}+02$ & $9.69 \mathrm{E}-09$ & $1.65 \mathrm{E}+05$ & $1.42 \mathrm{E}+00$ \\
\hline $\mathrm{Au}-201$ & $1.77 \mathrm{E}+04$ & $4.79 \mathrm{E}-07$ & $8.76 \mathrm{E}+06$ & $7.51 \mathrm{E}+01$ \\
\hline $\mathrm{Au}-202$ & $3.56 \mathrm{E}+03$ & & & $1.47 \mathrm{E}+01$ \\
\hline Hg-190 & $4.10 \mathrm{E}+03$ & $1.11 \mathrm{E}-07$ & $1.84 \mathrm{E}+06$ & $1.58 \mathrm{E}+01$ \\
\hline $\mathrm{Hg}-191 \mathrm{~m}$ & $4.71 \mathrm{E}+02$ & $1.27 \mathrm{E}-08$ & $2.17 \mathrm{E}+05$ & $1.86 \mathrm{E}+00$ \\
\hline Hg-192 & $2.88 \mathrm{E}+03$ & 7.79E-08 & $1.30 \mathrm{E}+06$ & $1.11 \mathrm{E}+01$ \\
\hline Hg-193 & $8.32 \mathrm{E}+02$ & $2.25 \mathrm{E}-08$ & $3.83 \mathrm{E}+05$ & $3.28 \mathrm{E}+00$ \\
\hline Hg-193m & $6.79 \mathrm{E}+02$ & $1.84 \mathrm{E}-08$ & $3.14 \mathrm{E}+05$ & $2.69 \mathrm{E}+00$ \\
\hline
\end{tabular}




\begin{tabular}{|c|c|c|c|c|}
\hline Nuclide & $\begin{array}{c}\text { Air } \\
\text { Immersion } \\
\text { DCS } \\
\left(\mathbf{B q} / \mathbf{m}^{3}\right)\end{array}$ & $\begin{array}{c}\text { Air } \\
\text { Immersion } \\
\text { DCS } \\
\left(\mu \mathrm{Ci} / \mathbf{c m}^{3}\right)\end{array}$ & $\begin{array}{c}\text { Water } \\
\text { Submersion } \\
\text { DCS } \\
\left(\mathbf{B q} / \mathbf{m}^{3}\right)\end{array}$ & $\begin{array}{c}\text { Water } \\
\text { Submersion } \\
\text { DCS } \\
\left(\mu \mathrm{Ci} / \mathbf{m}^{3}\right)\end{array}$ \\
\hline Hg-194 & $7.06 \mathrm{E}+07$ & $1.91 \mathrm{E}-03$ & $3.05 \mathrm{E}+10$ & $2.61 \mathrm{E}+05$ \\
\hline Hg-195 & $3.85 \mathrm{E}+03$ & $1.04 \mathrm{E}-07$ & $1.75 \mathrm{E}+06$ & $1.50 \mathrm{E}+01$ \\
\hline $\mathrm{Hg}-195 \mathrm{~m}$ & $3.79 \mathrm{E}+03$ & $1.02 \mathrm{E}-07$ & $1.73 \mathrm{E}+06$ & $1.49 \mathrm{E}+01$ \\
\hline $\mathrm{Hg}-197$ & $1.33 \mathrm{E}+04$ & $3.59 \mathrm{E}-07$ & $5.86 \mathrm{E}+06$ & $5.02 \mathrm{E}+01$ \\
\hline $\mathrm{Hg}-197 \mathrm{~m}$ & $8.46 \mathrm{E}+03$ & 2.29E-07 & $3.83 \mathrm{E}+06$ & $3.28 \mathrm{E}+01$ \\
\hline $\mathrm{Hg}-199 \mathrm{~m}$ & $4.21 \mathrm{E}+03$ & $1.14 \mathrm{E}-07$ & $1.92 \mathrm{E}+06$ & $1.65 \mathrm{E}+01$ \\
\hline $\mathrm{Hg}-203$ & $3.05 \mathrm{E}+03$ & $8.24 \mathrm{E}-08$ & $1.40 \mathrm{E}+06$ & $1.20 \mathrm{E}+01$ \\
\hline $\mathrm{Hg}-205$ & $5.12 \mathrm{E}+04$ & $1.38 \mathrm{E}-06$ & $3.29 \mathrm{E}+07$ & $2.82 \mathrm{E}+02$ \\
\hline Hg-206 & $5.70 \mathrm{E}+03$ & $1.54 \mathrm{E}-07$ & $2.66 \mathrm{E}+06$ & $2.28 \mathrm{E}+01$ \\
\hline Hg-207 & $2.48 \mathrm{E}+02$ & $6.70 \mathrm{E}-09$ & $1.14 \mathrm{E}+05$ & $9.81 \mathrm{E}-01$ \\
\hline Tl-190 & $5.34 \mathrm{E}+02$ & $1.44 \mathrm{E}-08$ & $2.48 \mathrm{E}+05$ & $2.12 \mathrm{E}+00$ \\
\hline Tl-190m & $2.86 \mathrm{E}+02$ & $7.72 \mathrm{E}-09$ & $1.32 \mathrm{E}+05$ & $1.13 \mathrm{E}+00$ \\
\hline Tl-194 & $7.73 \mathrm{E}+02$ & $2.09 \mathrm{E}-08$ & $3.57 \mathrm{E}+05$ & $3.06 \mathrm{E}+00$ \\
\hline Tl-194m & $2.81 \mathrm{E}+02$ & $7.58 \mathrm{E}-09$ & $1.29 \mathrm{E}+05$ & $1.10 \mathrm{E}+00$ \\
\hline Tl-195 & $5.52 \mathrm{E}+02$ & $1.49 \mathrm{E}-08$ & $2.54 \mathrm{E}+05$ & $2.17 \mathrm{E}+00$ \\
\hline Tl-196 & $3.60 \mathrm{E}+02$ & $9.74 \mathrm{E}-09$ & $1.66 \mathrm{E}+05$ & $1.42 \mathrm{E}+00$ \\
\hline T1-197 & $1.57 \mathrm{E}+03$ & 4.24E-08 & $7.21 \mathrm{E}+05$ & $6.18 \mathrm{E}+00$ \\
\hline Tl-198 & $3.34 \mathrm{E}+02$ & $9.02 \mathrm{E}-09$ & $1.54 \mathrm{E}+05$ & $1.32 \mathrm{E}+00$ \\
\hline Tl-198m & $5.92 \mathrm{E}+02$ & $1.60 \mathrm{E}-08$ & $2.71 \mathrm{E}+05$ & $2.32 \mathrm{E}+00$ \\
\hline Tl-199 & $3.08 \mathrm{E}+03$ & $8.32 \mathrm{E}-08$ & & $1.20 \mathrm{E}+01$ \\
\hline Tl-200 & $5.30 \mathrm{E}+02$ & & $2.44 \mathrm{E}+05$ & $2.09 \mathrm{E}+00$ \\
\hline T1-201 & $9.73 \mathrm{E}+03$ & $2.63 \mathrm{E}-07$ & $4.33 \mathrm{E}+06$ & $3.71 \mathrm{E}+01$ \\
\hline T1-202 & $1.59 \mathrm{E}+03$ & $4.31 \mathrm{E}-08$ & $7.27 \mathrm{E}+05$ & $6.23 \mathrm{E}+00$ \\
\hline T1-204 & $1.81 \mathrm{E}+05$ & $4.90 \mathrm{E}-06$ & $1.30 \mathrm{E}+08$ & $1.11 \mathrm{E}+03$ \\
\hline Tl-206 & $7.99 \mathrm{E}+04$ & $2.16 \mathrm{E}-06$ & $6.68 \mathrm{E}+07$ & $5.72 \mathrm{E}+02$ \\
\hline Tl-206m & $2.91 \mathrm{E}+02$ & $7.86 \mathrm{E}-09$ & $1.34 \mathrm{E}+05$ & $1.15 \mathrm{E}+00$ \\
\hline Tl-207 & $6.88 \mathrm{E}+04$ & $1.86 \mathrm{E}-06$ & $4.88 \mathrm{E}+07$ & $4.18 \mathrm{E}+02$ \\
\hline Tl-208 & $1.89 \mathrm{E}+02$ & $5.10 \mathrm{E}-09$ & $8.71 \mathrm{E}+04$ & $7.47 \mathrm{E}-01$ \\
\hline T1-209 & $3.11 \mathrm{E}+02$ & $8.40 \mathrm{E}-09$ & $1.44 \mathrm{E}+05$ & $1.24 \mathrm{E}+00$ \\
\hline Tl-210 & $2.40 \mathrm{E}+02$ & $6.49 \mathrm{E}-09$ & $1.11 \mathrm{E}+05$ & 9.54E-01 \\
\hline $\mathrm{Pb}-194$ & $6.42 \mathrm{E}+02$ & $1.73 \mathrm{E}-08$ & $2.96 \mathrm{E}+05$ & $2.54 \mathrm{E}+00$ \\
\hline $\mathrm{Pb}-195 \mathrm{~m}$ & $4.29 \mathrm{E}+02$ & $1.16 \mathrm{E}-08$ & & $1.69 \mathrm{E}+00$ \\
\hline $\mathrm{Pb}-196$ & $1.51 \mathrm{E}+03$ & $4.08 \mathrm{E}-08$ & $6.89 \mathrm{E}+05$ & $5.91 \mathrm{E}+00$ \\
\hline $\mathrm{Pb}-197$ & $4.44 \mathrm{E}+02$ & $1.20 \mathrm{E}-08$ & $2.05 \mathrm{E}+05$ & $1.75 \mathrm{E}+00$ \\
\hline $\mathrm{Pb}-197 \mathrm{~m}$ & $6.07 \mathrm{E}+02$ & $1.64 \mathrm{E}-08$ & $2.78 \mathrm{E}+05$ & $2.38 \mathrm{E}+00$ \\
\hline Pb-198 & $1.70 \mathrm{E}+03$ & $4.61 \mathrm{E}-08$ & $7.79 \mathrm{E}+05$ & $6.68 \mathrm{E}+00$ \\
\hline $\mathrm{Pb}-199$ & $6.62 \mathrm{E}+02$ & $1.79 \mathrm{E}-08$ & $3.05 \mathrm{E}+05$ & $2.61 \mathrm{E}+00$ \\
\hline
\end{tabular}




\begin{tabular}{|c|c|c|c|c|}
\hline Nuclide & $\begin{array}{c}\text { Air } \\
\text { Immersion } \\
\text { DCS } \\
\left(\mathbf{B q} / \mathbf{m}^{3}\right)\end{array}$ & $\begin{array}{c}\text { Air } \\
\text { Immersion } \\
\text { DCS } \\
\left(\mu \mathrm{Ci} / \mathbf{c m}^{3}\right)\end{array}$ & $\begin{array}{c}\text { Water } \\
\text { Submersion } \\
\text { DCS } \\
\left(\mathbf{B q} / \mathbf{m}^{3}\right)\end{array}$ & $\begin{array}{c}\text { Water } \\
\text { Submersion } \\
\text { DCS } \\
\left(\mu \mathrm{Ci} / \mathbf{m}^{3}\right)\end{array}$ \\
\hline $\mathrm{Pb}-200$ & $3.89 \mathrm{E}+03$ & $1.05 \mathrm{E}-07$ & $1.75 \mathrm{E}+06$ & $1.50 \mathrm{E}+01$ \\
\hline $\mathrm{Pb}-201$ & $9.49 \mathrm{E}+02$ & $2.57 \mathrm{E}-08$ & $4.36 \mathrm{E}+05$ & $3.73 \mathrm{E}+00$ \\
\hline $\mathrm{Pb}-201 \mathrm{~m}$ & $1.95 \mathrm{E}+03$ & $5.26 \mathrm{E}-08$ & $8.96 \mathrm{E}+05$ & $7.68 \mathrm{E}+00$ \\
\hline $\mathrm{Pb}-202$ & $6.54 \mathrm{E}+07$ & $1.77 \mathrm{E}-03$ & $2.83 \mathrm{E}+10$ & $2.43 \mathrm{E}+05$ \\
\hline $\mathrm{Pb}-202 \mathrm{~m}$ & $3.50 \mathrm{E}+02$ & $9.46 \mathrm{E}-09$ & $1.61 \mathrm{E}+05$ & $1.38 \mathrm{E}+00$ \\
\hline $\mathrm{Pb}-203$ & $2.42 \mathrm{E}+03$ & $6.54 \mathrm{E}-08$ & $1.10 \mathrm{E}+06$ & $9.44 \mathrm{E}+00$ \\
\hline $\mathrm{Pb}-204 \mathrm{~m}$ & $3.36 \mathrm{E}+02$ & $9.07 \mathrm{E}-09$ & $1.55 \mathrm{E}+05$ & $1.33 \mathrm{E}+00$ \\
\hline $\mathrm{Pb}-205$ & $6.45 \mathrm{E}+07$ & $1.74 \mathrm{E}-03$ & $2.78 \mathrm{E}+10$ & $2.38 \mathrm{E}+05$ \\
\hline $\mathrm{Pb}-209$ & $3.17 \mathrm{E}+05$ & $8.57 \mathrm{E}-06$ & $2.83 \mathrm{E}+08$ & $2.43 \mathrm{E}+03$ \\
\hline $\mathrm{Pb}-210$ & $6.73 \mathrm{E}+05$ & $1.82 \mathrm{E}-05$ & $2.91 \mathrm{E}+08$ & $2.49 \mathrm{E}+03$ \\
\hline $\mathrm{Pb}-211$ & $9.85 \mathrm{E}+03$ & $2.66 \mathrm{E}-07$ & $4.75 \mathrm{E}+06$ & $4.07 \mathrm{E}+01$ \\
\hline $\mathrm{Pb}-212$ & $5.19 \mathrm{E}+03$ & $1.40 \mathrm{E}-07$ & $2.37 \mathrm{E}+06$ & $2.03 \mathrm{E}+01$ \\
\hline $\mathrm{Pb}-214$ & $2.86 \mathrm{E}+03$ & $7.72 \mathrm{E}-08$ & $1.32 \mathrm{E}+06$ & $1.13 \mathrm{E}+01$ \\
\hline Bi-197 & $4.04 \mathrm{E}+02$ & $1.09 \mathrm{E}-08$ & $1.87 \mathrm{E}+05$ & $1.60 \mathrm{E}+00$ \\
\hline $\mathrm{Bi}-200$ & $2.88 \mathrm{E}+02$ & 7.79E-09 & $1.33 \mathrm{E}+05$ & $1.14 \mathrm{E}+00$ \\
\hline Bi-201 & $3.91 \mathrm{E}+02$ & $1.06 \mathrm{E}-08$ & $1.80 \mathrm{E}+05$ & $1.54 \mathrm{E}+00$ \\
\hline Bi-202 & $2.52 \mathrm{E}+02$ & $6.80 \mathrm{E}-09$ & $1.16 \mathrm{E}+05$ & $9.92 \mathrm{E}-01$ \\
\hline Bi-203 & $2.81 \mathrm{E}+02$ & $7.58 \mathrm{E}-09$ & $1.30 \mathrm{E}+05$ & $1.11 \mathrm{E}+00$ \\
\hline Bi-204 & $2.35 \mathrm{E}+02$ & $6.35 \mathrm{E}-09$ & $1.09 \mathrm{E}+05$ & $9.31 \mathrm{E}-01$ \\
\hline $\mathrm{Bi}-205$ & $3.97 \mathrm{E}+02$ & $1.07 \mathrm{E}-08$ & $1.83 \mathrm{E}+05$ & $1.57 \mathrm{E}+00$ \\
\hline Bi-206 & $2.10 \mathrm{E}+02$ & $5.68 \mathrm{E}-09$ & $9.67 \mathrm{E}+04$ & $8.29 \mathrm{E}-01$ \\
\hline Bi-207 & $4.51 \mathrm{E}+02$ & $1.22 \mathrm{E}-08$ & $2.07 \mathrm{E}+05$ & $1.78 \mathrm{E}+00$ \\
\hline Bi-208 & $2.35 \mathrm{E}+02$ & $6.35 \mathrm{E}-09$ & $1.08 \mathrm{E}+05$ & $9.28 \mathrm{E}-01$ \\
\hline Bi-210 & $1.23 \mathrm{E}+05$ & $3.32 \mathrm{E}-06$ & $1.06 \mathrm{E}+08$ & $9.12 \mathrm{E}+02$ \\
\hline $\mathrm{Bi}-210 \mathrm{~m}$ & $2.78 \mathrm{E}+03$ & $7.52 \mathrm{E}-08$ & $1.27 \mathrm{E}+06$ & $1.09 \mathrm{E}+01$ \\
\hline $\mathrm{Bi}-211$ & $1.53 \mathrm{E}+04$ & $4.14 \mathrm{E}-07$ & $7.03 \mathrm{E}+06$ & $6.03 \mathrm{E}+01$ \\
\hline Bi-212 & $6.13 \mathrm{E}+03$ & $1.66 \mathrm{E}-07$ & $2.94 \mathrm{E}+06$ & $2.52 \mathrm{E}+01$ \\
\hline Bi-212n & $8.15 \mathrm{E}+04$ & $2.20 \mathrm{E}-06$ & $6.88 \mathrm{E}+07$ & $5.90 \mathrm{E}+02$ \\
\hline Bi-213 & $5.34 \mathrm{E}+03$ & $1.44 \mathrm{E}-07$ & $2.52 \mathrm{E}+06$ & $2.16 \mathrm{E}+01$ \\
\hline Bi-214 & $4.46 \mathrm{E}+02$ & $1.21 \mathrm{E}-08$ & $2.06 \mathrm{E}+05$ & $1.76 \mathrm{E}+00$ \\
\hline Bi-215 & $2.69 \mathrm{E}+03$ & $7.26 \mathrm{E}-08$ & $1.26 \mathrm{E}+06$ & $1.08 \mathrm{E}+01$ \\
\hline $\mathrm{Bi}-216$ & $9.30 \mathrm{E}+02$ & & & $3.72 \mathrm{E}+00$ \\
\hline Po-203 & $4.20 \mathrm{E}+02$ & $1.14 \mathrm{E}-08$ & $1.93 \mathrm{E}+05$ & $1.66 \mathrm{E}+00$ \\
\hline Po-204 & $6.15 \mathrm{E}+02$ & $1.66 \mathrm{E}-08$ & $2.83 \mathrm{E}+05$ & $2.43 \mathrm{E}+00$ \\
\hline Po-205 & $4.34 \mathrm{E}+02$ & $1.17 \mathrm{E}-08$ & $1.99 \mathrm{E}+05$ & $1.71 \mathrm{E}+00$ \\
\hline Po-206 & $5.95 \mathrm{E}+02$ & $1.61 \mathrm{E}-08$ & $2.73 E+05$ & $2.34 \mathrm{E}+00$ \\
\hline Po-207 & $5.41 \mathrm{E}+02$ & $1.46 \mathrm{E}-08$ & $2.50 \mathrm{E}+05$ & $2.14 \mathrm{E}+00$ \\
\hline
\end{tabular}




\begin{tabular}{|c|c|c|c|c|}
\hline Nuclide & $\begin{array}{c}\text { Air } \\
\text { Immersion } \\
\text { DCS } \\
\left(\mathbf{B q} / \mathbf{m}^{3}\right)\end{array}$ & $\begin{array}{c}\text { Air } \\
\text { Immersion } \\
\text { DCS } \\
\left(\mu \mathrm{Ci} / \mathbf{c m}^{3}\right)\end{array}$ & $\begin{array}{c}\text { Water } \\
\text { Submersion } \\
\text { DCS } \\
\left(\mathbf{B q} / \mathbf{m}^{3}\right)\end{array}$ & $\begin{array}{c}\text { Water } \\
\text { Submersion } \\
\text { DCS } \\
\left(\mu \mathrm{Ci} / \mathbf{m}^{3}\right)\end{array}$ \\
\hline Po-208 & $3.39 \mathrm{E}+07$ & $9.16 \mathrm{E}-04$ & $1.55 \mathrm{E}+10$ & $1.33 \mathrm{E}+05$ \\
\hline Po-209 & $1.15 \mathrm{E}+05$ & $3.11 \mathrm{E}-06$ & $5.28 \mathrm{E}+07$ & $4.52 \mathrm{E}+02$ \\
\hline Po-210 & $7.13 \mathrm{E}+07$ & $1.93 \mathrm{E}-03$ & $3.29 \mathrm{E}+10$ & $2.82 \mathrm{E}+05$ \\
\hline Po-211 & $8.50 \mathrm{E}+04$ & $2.30 \mathrm{E}-06$ & $3.92 \mathrm{E}+07$ & $3.36 \mathrm{E}+02$ \\
\hline Po-212m & $7.95 \mathrm{E}+03$ & $2.15 \mathrm{E}-07$ & $3.67 \mathrm{E}+06$ & $3.14 \mathrm{E}+01$ \\
\hline Po-213 & $1.85 \mathrm{E}+07$ & $5.01 \mathrm{E}-04$ & $8.55 \mathrm{E}+09$ & $7.33 \mathrm{E}+04$ \\
\hline Po-214 & $8.34 \mathrm{E}+06$ & $2.26 \mathrm{E}-04$ & $3.85 \mathrm{E}+09$ & $3.30 \mathrm{E}+04$ \\
\hline Po-215 & $4.07 \mathrm{E}+06$ & $1.10 \mathrm{E}-04$ & $1.87 \mathrm{E}+09$ & $1.60 \mathrm{E}+04$ \\
\hline Po-216 & $4.53 \mathrm{E}+07$ & $1.22 \mathrm{E}-03$ & $2.09 \mathrm{E}+10$ & $1.79 \mathrm{E}+05$ \\
\hline Po-218 & $1.21 \mathrm{E}+10$ & $3.27 \mathrm{E}-01$ & $1.11 \mathrm{E}+13$ & $9.50 \mathrm{E}+07$ \\
\hline At-204 & $3.05 \mathrm{E}+02$ & $8.24 \mathrm{E}-09$ & $1.40 \mathrm{E}+05$ & $1.20 \mathrm{E}+00$ \\
\hline At-205 & $6.07 \mathrm{E}+02$ & $1.64 \mathrm{E}-08$ & $2.81 \mathrm{E}+05$ & $2.40 \mathrm{E}+00$ \\
\hline At-206 & $2.83 \mathrm{E}+02$ & $7.65 \mathrm{E}-09$ & $1.30 \mathrm{E}+05$ & $1.11 \mathrm{E}+00$ \\
\hline At-207 & $3.40 \mathrm{E}+02$ & $9.18 \mathrm{E}-09$ & $1.56 \mathrm{E}+05$ & $1.34 \mathrm{E}+00$ \\
\hline At-208 & $2.26 \mathrm{E}+02$ & $6.12 \mathrm{E}-09$ & $1.05 \mathrm{E}+05$ & $8.97 \mathrm{E}-01$ \\
\hline At-209 & $3.08 \mathrm{E}+02$ & $8.32 \mathrm{E}-09$ & $1.42 \mathrm{E}+05$ & $1.21 \mathrm{E}+00$ \\
\hline At-210 & $2.26 \mathrm{E}+02$ & $6.12 \mathrm{E}-09$ & $1.05 \mathrm{E}+05$ & $8.97 \mathrm{E}-01$ \\
\hline At-211 & $2.50 \mathrm{E}+04$ & $6.75 \mathrm{E}-07$ & $1.11 \mathrm{E}+07$ & $9.50 \mathrm{E}+01$ \\
\hline At-215 & $4.21 \mathrm{E}+06$ & $1.14 \mathrm{E}-04$ & $1.93 \mathrm{E}+09$ & $1.66 \mathrm{E}+04$ \\
\hline At-216 & $3.33 \mathrm{E}+05$ & $9.00 \mathrm{E}-06$ & $1.50 \mathrm{E}+08$ & $1.28 \mathrm{E}+03$ \\
\hline At-217 & $2.99 \mathrm{E}+06$ & $8.09 \mathrm{E}-05$ & $1.37 \mathrm{E}+09$ & $1.18 \mathrm{E}+04$ \\
\hline At-218 & $3.24 \mathrm{E}+07$ & $8.75 \mathrm{E}-04$ & $2.54 \mathrm{E}+10$ & $2.17 \mathrm{E}+05$ \\
\hline At-220 & $1.52 \mathrm{E}+03$ & 4.10E-08 & $7.11 \mathrm{E}+05$ & $6.09 \mathrm{E}+00$ \\
\hline Rn-207 & $7.19 \mathrm{E}+02$ & $1.94 \mathrm{E}-08$ & $3.31 \mathrm{E}+05$ & $2.83 \mathrm{E}+00$ \\
\hline Rn-209 & $5.80 \mathrm{E}+02$ & $1.57 \mathrm{E}-08$ & $2.66 \mathrm{E}+05$ & $2.28 \mathrm{E}+00$ \\
\hline Rn-210 & $2.50 \mathrm{E}+03$ & $6.75 \mathrm{E}-08$ & $5.38 \mathrm{E}+06$ & $4.61 \mathrm{E}+01$ \\
\hline Rn-211 & $3.68 \mathrm{E}+02$ & 9.94E-09 & $1.70 \mathrm{E}+05$ & $1.45 \mathrm{E}+00$ \\
\hline Rn-212 & $2.30 \mathrm{E}+03$ & $6.21 \mathrm{E}-08$ & $9.55 \mathrm{E}+08$ & $8.19 \mathrm{E}+03$ \\
\hline Rn-215 & $1.67 \mathrm{E}+03$ & $4.51 \mathrm{E}-08$ & $\mathrm{~N} / \mathrm{A}$ & N/A \\
\hline Rn-216 & $1.79 \mathrm{E}+03$ & 4.84E-08 & N/A & N/A \\
\hline Rn-217 & $1.87 \mathrm{E}+03$ & $5.04 \mathrm{E}-08$ & $\mathrm{~N} / \mathrm{A}$ & $\mathrm{N} / \mathrm{A}$ \\
\hline Rn-218 & $2.01 \mathrm{E}+03$ & & $4.29 \mathrm{E}+08$ & $3.68 \mathrm{E}+03$ \\
\hline Rn-219 & $2.14 \mathrm{E}+03$ & 5.79E-08 & $5.66 \mathrm{E}+06$ & $4.85 \mathrm{E}+01$ \\
\hline Rn-220 & $2.30 \mathrm{E}+03$ & $6.21 \mathrm{E}-08$ & $5.20 \mathrm{E}+08$ & $4.46 \mathrm{E}+03$ \\
\hline Rn-222 & $2.64 \mathrm{E}+03$ & 7.14E-08 & $8.43 \mathrm{E}+08$ & $7.23 \mathrm{E}+03$ \\
\hline Rn-223 & $2.03 \mathrm{E}+03$ & $5.49 \mathrm{E}-08$ & $9.47 \mathrm{E}+05$ & $8.11 \mathrm{E}+00$ \\
\hline Fr-212 & $6.03 \mathrm{E}+02$ & $1.63 \mathrm{E}-08$ & $2.78 \mathrm{E}+05$ & $2.38 \mathrm{E}+00$ \\
\hline
\end{tabular}




\begin{tabular}{|c|c|c|c|c|}
\hline Nuclide & $\begin{array}{c}\text { Air } \\
\text { Immersion } \\
\text { DCS } \\
\left(\mathbf{B q} / \mathbf{m}^{3}\right)\end{array}$ & $\begin{array}{c}\text { Air } \\
\text { Immersion } \\
\text { DCS } \\
\left(\mu \mathrm{Ci} / \mathbf{c m}^{3}\right)\end{array}$ & $\begin{array}{c}\text { Water } \\
\text { Submersion } \\
\text { DCS } \\
\left(\mathbf{B q} / \mathbf{m}^{3}\right)\end{array}$ & $\begin{array}{c}\text { Water } \\
\text { Submersion } \\
\text { DCS } \\
\left(\mu \mathrm{Ci} / \mathbf{m}^{3}\right)\end{array}$ \\
\hline Fr-219 & $2.02 \mathrm{E}+05$ & $5.46 \mathrm{E}-06$ & $9.30 \mathrm{E}+07$ & $7.97 \mathrm{E}+02$ \\
\hline Fr-220 & $9.01 \mathrm{E}+04$ & $2.43 \mathrm{E}-06$ & $4.04 \mathrm{E}+07$ & $3.46 \mathrm{E}+02$ \\
\hline Fr-221 & $2.54 \mathrm{E}+04$ & $6.86 \mathrm{E}-07$ & $1.15 \mathrm{E}+07$ & $9.88 \mathrm{E}+01$ \\
\hline Fr-222 & $3.88 \mathrm{E}+03$ & $1.05 \mathrm{E}-07$ & $1.82 \mathrm{E}+06$ & $1.56 \mathrm{E}+01$ \\
\hline Fr-223 & $1.47 \mathrm{E}+04$ & 3.99E-07 & $6.97 \mathrm{E}+06$ & $5.97 \mathrm{E}+01$ \\
\hline Fr-224 & $1.21 \mathrm{E}+03$ & $3.27 \mathrm{E}-08$ & $5.63 \mathrm{E}+05$ & $4.83 \mathrm{E}+00$ \\
\hline Fr-227 & $1.59 \mathrm{E}+03$ & $4.29 \mathrm{E}-08$ & $7.34 \mathrm{E}+05$ & $6.29 \mathrm{E}+00$ \\
\hline $\mathrm{Ra}-219$ & $4.30 \mathrm{E}+03$ & $1.16 \mathrm{E}-07$ & $1.97 \mathrm{E}+06$ & $1.69 \mathrm{E}+01$ \\
\hline Ra-220 & $1.53 \mathrm{E}+05$ & $4.14 \mathrm{E}-06$ & $7.03 \mathrm{E}+07$ & $6.03 \mathrm{E}+02$ \\
\hline $\mathrm{Ra}-221$ & $2.16 \mathrm{E}+04$ & $5.83 \mathrm{E}-07$ & $9.79 \mathrm{E}+06$ & $8.39 \mathrm{E}+01$ \\
\hline Ra-222 & $7.85 \mathrm{E}+04$ & $2.12 \mathrm{E}-06$ & $3.60 \mathrm{E}+07$ & $3.08 \mathrm{E}+02$ \\
\hline Ra-223 & $5.52 \mathrm{E}+03$ & $1.49 \mathrm{E}-07$ & $2.50 \mathrm{E}+06$ & $2.14 \mathrm{E}+01$ \\
\hline Ra-224 & $7.03 \mathrm{E}+04$ & $1.90 \mathrm{E}-06$ & $3.21 \mathrm{E}+07$ & $2.75 \mathrm{E}+02$ \\
\hline $\mathrm{Ra}-225$ & $1.28 \mathrm{E}+05$ & $3.47 \mathrm{E}-06$ & $5.84 \mathrm{E}+07$ & $5.00 \mathrm{E}+02$ \\
\hline Ra-226 & $1.02 \mathrm{E}+05$ & $2.76 \mathrm{E}-06$ & $4.64 \mathrm{E}+07$ & $3.97 \mathrm{E}+02$ \\
\hline Ra-227 & $4.98 \mathrm{E}+03$ & $1.35 \mathrm{E}-07$ & $2.31 \mathrm{E}+06$ & $1.98 \mathrm{E}+01$ \\
\hline Ra-228 & $1.10 \mathrm{E}+07$ & $2.97 \mathrm{E}-04$ & $4.68 \mathrm{E}+09$ & $4.01 \mathrm{E}+04$ \\
\hline Ra-230 & $9.52 \mathrm{E}+03$ & $2.57 \mathrm{E}-07$ & $4.39 \mathrm{E}+06$ & $3.76 \mathrm{E}+01$ \\
\hline Ac-223 & $4.36 \mathrm{E}+04$ & $1.18 \mathrm{E}-06$ & $1.98 \mathrm{E}+07$ & $1.70 \mathrm{E}+02$ \\
\hline Ac- 224 & $3.40 \mathrm{E}+03$ & $9.19 \mathrm{E}-08$ & & $1.32 \mathrm{E}+01$ \\
\hline Ac-225 & $5.60 \mathrm{E}+04$ & $1.51 \mathrm{E}-06$ & $2.52 \mathrm{E}+07$ & $2.16 \mathrm{E}+02$ \\
\hline Ac-226 & $5.58 \mathrm{E}+03$ & $1.51 \mathrm{E}-07$ & $2.58 \mathrm{E}+06$ & $2.21 \mathrm{E}+01$ \\
\hline Ac-227 & $8.69 \mathrm{E}+06$ & $2.35 \mathrm{E}-04$ & $3.84 \mathrm{E}+09$ & $3.29 \mathrm{E}+04$ \\
\hline Ac-228 & $7.91 \mathrm{E}+02$ & $2.14 \mathrm{E}-08$ & $3.66 \mathrm{E}+05$ & $3.13 \mathrm{E}+00$ \\
\hline Ac-230 & $1.19 \mathrm{E}+03$ & $3.22 \mathrm{E}-08$ & $5.56 \mathrm{E}+05$ & $4.77 \mathrm{E}+00$ \\
\hline Ac-231 & $1.72 \mathrm{E}+03$ & $4.66 \mathrm{E}-08$ & $7.97 \mathrm{E}+05$ & $6.83 \mathrm{E}+00$ \\
\hline Ac-232 & $5.61 \mathrm{E}+02$ & $1.52 \mathrm{E}-08$ & $2.60 \mathrm{E}+05$ & $2.23 \mathrm{E}+00$ \\
\hline Ac-233 & $1.38 \mathrm{E}+03$ & $3.74 \mathrm{E}-08$ & $6.45 \mathrm{E}+05$ & $5.52 \mathrm{E}+00$ \\
\hline Th-223 & $1.14 \mathrm{E}+04$ & $3.08 \mathrm{E}-07$ & $5.13 \mathrm{E}+06$ & $4.40 \mathrm{E}+01$ \\
\hline Th-224 & $3.25 \mathrm{E}+04$ & 8.79E-07 & $1.48 \mathrm{E}+07$ & $1.27 \mathrm{E}+02$ \\
\hline Th-226 & $9.88 \mathrm{E}+04$ & $2.67 \mathrm{E}-06$ & $4.48 \mathrm{E}+07$ & $3.84 \mathrm{E}+02$ \\
\hline Th-227 & $6.07 \mathrm{E}+03$ & $1.64 \mathrm{E}-07$ & $2.76 \mathrm{E}+06$ & $2.36 \mathrm{E}+01$ \\
\hline Th-228 & $3.84 \mathrm{E}+05$ & $1.04 \mathrm{E}-05$ & $1.72 \mathrm{E}+08$ & $1.48 \mathrm{E}+03$ \\
\hline Th-229 & $9.55 \mathrm{E}+03$ & $2.58 \mathrm{E}-07$ & $4.29 \mathrm{E}+06$ & $3.67 \mathrm{E}+01$ \\
\hline Th-230 & $2.09 \mathrm{E}+06$ & $5.64 \mathrm{E}-05$ & $9.27 \mathrm{E}+08$ & $7.95 \mathrm{E}+03$ \\
\hline Th-231 & $6.85 \mathrm{E}+04$ & $1.85 \mathrm{E}-06$ & $3.11 \mathrm{E}+07$ & $2.66 \mathrm{E}+02$ \\
\hline Th-232 & $4.01 \mathrm{E}+06$ & $1.08 \mathrm{E}-04$ & $1.76 \mathrm{E}+09$ & $1.51 \mathrm{E}+04$ \\
\hline
\end{tabular}




\begin{tabular}{|c|c|c|c|c|}
\hline Nuclide & $\begin{array}{c}\text { Air } \\
\text { Immersion } \\
\text { DCS } \\
\left(\mathbf{B q} / \mathbf{m}^{3}\right)\end{array}$ & $\begin{array}{c}\text { Air } \\
\text { Immersion } \\
\text { DCS } \\
\left(\mu \mathrm{Ci} / \mathbf{c m}^{3}\right)\end{array}$ & $\begin{array}{c}\text { Water } \\
\text { Submersion } \\
\text { DCS } \\
\left(\mathbf{B q} / \mathbf{m}^{3}\right)\end{array}$ & $\begin{array}{c}\text { Water } \\
\text { Submersion } \\
\text { DCS } \\
\left(\mu \mathrm{Ci} / \mathbf{m}^{3}\right)\end{array}$ \\
\hline Th-233 & $1.77 \mathrm{E}+04$ & $4.79 \mathrm{E}-07$ & $8.74 \mathrm{E}+06$ & $7.49 \mathrm{E}+01$ \\
\hline Th-234 & $9.85 \mathrm{E}+04$ & $2.66 \mathrm{E}-06$ & $4.39 \mathrm{E}+07$ & $3.76 \mathrm{E}+02$ \\
\hline Th-235 & $1.08 \mathrm{E}+04$ & $2.92 \mathrm{E}-07$ & $5.41 \mathrm{E}+06$ & $4.64 \mathrm{E}+01$ \\
\hline Th-236 & $1.89 \mathrm{E}+04$ & $5.10 \mathrm{E}-07$ & $9.22 \mathrm{E}+06$ & $7.90 \mathrm{E}+01$ \\
\hline $\mathrm{Pa}-227$ & $4.42 \mathrm{E}+04$ & 1.19E-06 & $1.97 \mathrm{E}+07$ & $1.69 \mathrm{E}+02$ \\
\hline $\mathrm{Pa}-228$ & $5.11 \mathrm{E}+02$ & $1.38 \mathrm{E}-08$ & $2.35 \mathrm{E}+05$ & $2.01 \mathrm{E}+00$ \\
\hline $\mathrm{Pa}-229$ & $1.36 \mathrm{E}+04$ & $3.68 \mathrm{E}-07$ & $6.07 \mathrm{E}+06$ & $5.21 \mathrm{E}+01$ \\
\hline $\mathrm{Pa}-230$ & $1.06 \mathrm{E}+03$ & $2.87 \mathrm{E}-08$ & $4.88 \mathrm{E}+05$ & $4.18 \mathrm{E}+00$ \\
\hline $\mathrm{Pa}-231$ & $2.19 \mathrm{E}+04$ & $5.91 \mathrm{E}-07$ & $9.97 \mathrm{E}+06$ & $8.55 \mathrm{E}+01$ \\
\hline $\mathrm{Pa}-232$ & $7.44 \mathrm{E}+02$ & $2.01 \mathrm{E}-08$ & $3.43 \mathrm{E}+05$ & $2.94 \mathrm{E}+00$ \\
\hline $\mathrm{Pa}-233$ & $3.42 \mathrm{E}+03$ & $9.25 \mathrm{E}-08$ & $1.56 \mathrm{E}+06$ & $1.34 \mathrm{E}+01$ \\
\hline $\mathrm{Pa}-234$ & $4.75 \mathrm{E}+02$ & $1.28 \mathrm{E}-08$ & $2.19 \mathrm{E}+05$ & $1.87 \mathrm{E}+00$ \\
\hline $\mathrm{Pa}-234 \mathrm{~m}$ & $2.23 \mathrm{E}+04$ & $6.04 \mathrm{E}-07$ & $1.29 \mathrm{E}+07$ & $1.11 \mathrm{E}+02$ \\
\hline $\mathrm{Pa}-235$ & $9.47 \mathrm{E}+04$ & $2.56 \mathrm{E}-06$ & $8.07 \mathrm{E}+07$ & $6.92 \mathrm{E}+02$ \\
\hline $\mathrm{Pa}-236$ & $7.27 \mathrm{E}+02$ & $1.97 \mathrm{E}-08$ & $3.38 \mathrm{E}+05$ & $2.90 \mathrm{E}+00$ \\
\hline $\mathrm{Pa}-237$ & $1.12 \mathrm{E}+03$ & $3.04 \mathrm{E}-08$ & $5.22 \mathrm{E}+05$ & $4.48 \mathrm{E}+00$ \\
\hline U-227 & $6.54 \mathrm{E}+03$ & $1.77 \mathrm{E}-07$ & $2.96 \mathrm{E}+06$ & $2.54 \mathrm{E}+01$ \\
\hline $\mathrm{U}-228$ & $1.99 \mathrm{E}+05$ & 5.39E-06 & $8.98 \mathrm{E}+07$ & $7.70 \mathrm{E}+02$ \\
\hline U-230 & $6.95 \mathrm{E}+05$ & $1.88 \mathrm{E}-05$ & $3.11 \mathrm{E}+08$ & $2.66 \mathrm{E}+03$ \\
\hline U-231 & $1.19 \mathrm{E}+04$ & $3.22 \mathrm{E}-07$ & & $4.56 \mathrm{E}+01$ \\
\hline U-232 & $2.94 \mathrm{E}+06$ & & $1.30 \mathrm{E}+09$ & $1.12 \mathrm{E}+04$ \\
\hline U-233 & $2.99 \mathrm{E}+06$ & $8.09 \mathrm{E}-05$ & $1.35 \mathrm{E}+09$ & $1.16 \mathrm{E}+04$ \\
\hline U-234 & $5.16 \mathrm{E}+06$ & $1.40 \mathrm{E}-04$ & $2.26 \mathrm{E}+09$ & $1.94 \mathrm{E}+04$ \\
\hline U-235 & $4.62 \mathrm{E}+03$ & $1.25 \mathrm{E}-07$ & $2.10 \mathrm{E}+06$ & $1.80 \mathrm{E}+01$ \\
\hline U-236 & $8.39 \mathrm{E}+06$ & $2.27 \mathrm{E}-04$ & $3.65 \mathrm{E}+09$ & $3.13 \mathrm{E}+04$ \\
\hline U-237 & $6.01 \mathrm{E}+03$ & $1.62 \mathrm{E}-07$ & $2.71 \mathrm{E}+06$ & $2.32 \mathrm{E}+01$ \\
\hline U-238 & $9.91 \mathrm{E}+06$ & $2.68 \mathrm{E}-04$ & $4.33 \mathrm{E}+09$ & $3.71 \mathrm{E}+04$ \\
\hline U-239 & $1.51 \mathrm{E}+04$ & $4.08 \mathrm{E}-07$ & $7.19 \mathrm{E}+06$ & $6.16 \mathrm{E}+01$ \\
\hline U-240 & $1.53 \mathrm{E}+05$ & $4.14 \mathrm{E}-06$ & $7.37 \mathrm{E}+07$ & $6.32 \mathrm{E}+02$ \\
\hline U-242 & $1.62 \mathrm{E}+04$ & $4.37 \mathrm{E}-07$ & $7.87 \mathrm{E}+06$ & $6.74 \mathrm{E}+01$ \\
\hline $\mathrm{Np}-232$ & $5.93 \mathrm{E}+02$ & $1.60 \mathrm{E}-08$ & $2.73 \mathrm{E}+05$ & $2.34 \mathrm{E}+00$ \\
\hline $\mathrm{Np}-233$ & $9.38 \mathrm{E}+03$ & & & $3.61 \mathrm{E}+01$ \\
\hline $\mathrm{Np}-234$ & $6.06 \mathrm{E}+02$ & $1.64 \mathrm{E}-08$ & $2.81 \mathrm{E}+05$ & $2.40 \mathrm{E}+00$ \\
\hline Np-235 & $1.12 \mathrm{E}+06$ & $3.02 \mathrm{E}-05$ & $4.93 \mathrm{E}+08$ & $4.23 \mathrm{E}+03$ \\
\hline $\mathrm{Np}-236$ & $5.69 \mathrm{E}+03$ & $1.54 \mathrm{E}-07$ & $2.58 \mathrm{E}+06$ & $2.21 \mathrm{E}+01$ \\
\hline $\mathrm{Np}-236 \mathrm{~m}$ & $1.67 \mathrm{E}+04$ & $4.51 \mathrm{E}-07$ & $7.59 \mathrm{E}+06$ & $6.50 \mathrm{E}+01$ \\
\hline Np-237 & $3.69 \mathrm{E}+04$ & $9.97 \mathrm{E}-07$ & $1.64 \mathrm{E}+07$ & $1.41 \mathrm{E}+02$ \\
\hline
\end{tabular}




\begin{tabular}{|c|c|c|c|c|}
\hline Nuclide & $\begin{array}{c}\text { Air } \\
\text { Immersion } \\
\text { DCS } \\
\left(\mathbf{B q} / \mathbf{m}^{3}\right)\end{array}$ & $\begin{array}{c}\text { Air } \\
\text { Immersion } \\
\text { DCS } \\
\left(\mu \mathrm{Ci} / \mathbf{c m}^{3}\right)\end{array}$ & $\begin{array}{c}\text { Water } \\
\text { Submersion } \\
\text { DCS } \\
\left(\mathbf{B q} / \mathbf{m}^{3}\right)\end{array}$ & $\begin{array}{c}\text { Water } \\
\text { Submersion } \\
\text { DCS } \\
\left(\mu \mathrm{Ci} / \mathbf{m}^{3}\right)\end{array}$ \\
\hline Np-238 & $1.17 \mathrm{E}+03$ & $3.15 \mathrm{E}-08$ & $5.39 \mathrm{E}+05$ & $4.62 \mathrm{E}+00$ \\
\hline Np-239 & $4.31 \mathrm{E}+03$ & $1.17 \mathrm{E}-07$ & $1.96 \mathrm{E}+06$ & $1.68 \mathrm{E}+01$ \\
\hline $\mathrm{Np}-240$ & $6.70 \mathrm{E}+02$ & $1.81 \mathrm{E}-08$ & $3.08 \mathrm{E}+05$ & $2.64 \mathrm{E}+00$ \\
\hline $\mathrm{Np}-240 \mathrm{~m}$ & $2.13 \mathrm{E}+03$ & $5.75 \mathrm{E}-08$ & $9.91 \mathrm{E}+05$ & $8.49 \mathrm{E}+00$ \\
\hline $\mathrm{Np}-241$ & $1.77 \mathrm{E}+04$ & 4.79E-07 & $8.64 \mathrm{E}+06$ & $7.40 \mathrm{E}+01$ \\
\hline Np-242 & $2.38 \mathrm{E}+03$ & $6.44 \mathrm{E}-08$ & $1.12 \mathrm{E}+06$ & $9.64 \mathrm{E}+00$ \\
\hline $\mathrm{Np}-242 \mathrm{~m}$ & $7.62 \mathrm{E}+02$ & $2.06 \mathrm{E}-08$ & $3.53 \mathrm{E}+05$ & $3.02 \mathrm{E}+00$ \\
\hline $\mathrm{Pu}-232$ & $1.37 \mathrm{E}+04$ & $3.71 \mathrm{E}-07$ & $6.15 \mathrm{E}+06$ & $5.27 \mathrm{E}+01$ \\
\hline $\mathrm{Pu}-234$ & $1.27 \mathrm{E}+04$ & $3.43 \mathrm{E}-07$ & $5.68 \mathrm{E}+06$ & $4.87 \mathrm{E}+01$ \\
\hline $\mathrm{Pu}-235$ & $9.16 \mathrm{E}+03$ & $2.48 \mathrm{E}-07$ & $4.12 \mathrm{E}+06$ & $3.53 \mathrm{E}+01$ \\
\hline $\mathrm{Pu}-236$ & $7.32 \mathrm{E}+06$ & $1.98 \mathrm{E}-04$ & $3.17 \mathrm{E}+09$ & $2.72 \mathrm{E}+04$ \\
\hline Pu-237 & $1.77 \mathrm{E}+04$ & $4.79 \mathrm{E}-07$ & $7.93 \mathrm{E}+06$ & $6.79 \mathrm{E}+01$ \\
\hline $\mathrm{Pu}-238$ & $9.44 \mathrm{E}+06$ & $2.55 \mathrm{E}-04$ & $4.06 \mathrm{E}+09$ & $3.48 \mathrm{E}+04$ \\
\hline $\mathrm{Pu}-239$ & $8.41 \mathrm{E}+06$ & $27 \mathrm{E}-04$ & $3.73 \mathrm{E}+09$ & $3.19 \mathrm{E}+04$ \\
\hline $\mathrm{Pu}-240$ & $9.64 \mathrm{E}+06$ & $2.60 \mathrm{E}-04$ & $4.14 \mathrm{E}+09$ & $3.55 \mathrm{E}+04$ \\
\hline Pu-241 & $5.16 \mathrm{E}+08$ & $1.39 \mathrm{E}-02$ & $2.31 \mathrm{E}+11$ & $1.98 \mathrm{E}+06$ \\
\hline $\mathrm{Pu}-242$ & $4.93 \mathrm{E}+06$ & $1.33 \mathrm{E}-04$ & $2.22 \mathrm{E}+09$ & $1.90 \mathrm{E}+04$ \\
\hline $\mathrm{Pu}-243$ & $3.29 \mathrm{E}+04$ & $8.88 \mathrm{E}-07$ & $1.52 \mathrm{E}+07$ & $1.31 \mathrm{E}+02$ \\
\hline $\mathrm{Pu}-244$ & $3.27 \mathrm{E}+04$ & $8.84 \mathrm{E}-07$ & $1.52 \mathrm{E}+07$ & $1.30 \mathrm{E}+02$ \\
\hline $\mathrm{Pu}-245$ & $1.75 \mathrm{E}+03$ & $73 \mathrm{E}-08$ & $8.09 \mathrm{E}+05$ & $6.93 \mathrm{E}+00$ \\
\hline $\mathrm{Pu}-246$ & & $1.58 \mathrm{E}-07$ & $2.64 \mathrm{E}+06$ & $2.26 \mathrm{E}+01$ \\
\hline Am-237 & $2.05 \mathrm{E}+03$ & $5.53 \mathrm{E}-08$ & $9.30 \mathrm{E}+05$ & $7.97 \mathrm{E}+00$ \\
\hline Am-238 & $7.75 \mathrm{E}+02$ & $2.10 \mathrm{E}-08$ & $3.57 \mathrm{E}+05$ & $3.06 \mathrm{E}+00$ \\
\hline Am-239 & $3.38 \mathrm{E}+03$ & $9.15 \mathrm{E}-08$ & $1.53 \mathrm{E}+06$ & $1.31 \mathrm{E}+01$ \\
\hline Am-240 & $6.76 \mathrm{E}+02$ & $1.83 \mathrm{E}-08$ & $3.11 \mathrm{E}+05$ & $2.66 \mathrm{E}+00$ \\
\hline Am-241 & $4.72 \mathrm{E}+04$ & $1.28 \mathrm{E}-06$ & $2.06 \mathrm{E}+07$ & $1.76 \mathrm{E}+02$ \\
\hline Am-242 & $5.19 \mathrm{E}+04$ & $1.40 \mathrm{E}-06$ & $2.50 \mathrm{E}+07$ & $2.14 \mathrm{E}+02$ \\
\hline Am-242m & $1.60 \mathrm{E}+06$ & $4.33 \mathrm{E}-05$ & $7.00 \mathrm{E}+08$ & $6.00 \mathrm{E}+03$ \\
\hline Am-243 & $1.65 \mathrm{E}+04$ & $4.46 \mathrm{E}-07$ & $7.31 \mathrm{E}+06$ & $6.26 \mathrm{E}+01$ \\
\hline Am-244 & $8.86 \mathrm{E}+02$ & $2.39 \mathrm{E}-08$ & $4.08 \mathrm{E}+05$ & $3.50 \mathrm{E}+00$ \\
\hline $\mathrm{Am}-244 \mathrm{~m}$ & $3.05 \mathrm{E}+04$ & $8.24 \mathrm{E}-07$ & $1.68 \mathrm{E}+07$ & $1.44 \mathrm{E}+02$ \\
\hline Am-245 & $2.19 \mathrm{E}+04$ & & & $8.97 \mathrm{E}+01$ \\
\hline Am-246 & $9.70 \mathrm{E}+02$ & $2.62 \mathrm{E}-08$ & $4.47 \mathrm{E}+05$ & $3.83 \mathrm{E}+00$ \\
\hline Am-246m & $6.94 \mathrm{E}+02$ & $1.88 \mathrm{E}-08$ & $3.22 \mathrm{E}+05$ & $2.76 \mathrm{E}+00$ \\
\hline Am-247 & $5.42 \mathrm{E}+03$ & $1.46 \mathrm{E}-07$ & $2.54 \mathrm{E}+06$ & $2.17 \mathrm{E}+01$ \\
\hline $\mathrm{Cm}-238$ & $1.04 \mathrm{E}+04$ & $2.81 \mathrm{E}-07$ & $4.66 \mathrm{E}+06$ & $4.00 \mathrm{E}+01$ \\
\hline $\mathrm{Cm}-239$ & $3.02 \mathrm{E}+03$ & $8.16 \mathrm{E}-08$ & $1.37 \mathrm{E}+06$ & $1.17 \mathrm{E}+01$ \\
\hline
\end{tabular}




\begin{tabular}{|c|c|c|c|c|}
\hline Nuclide & $\begin{array}{c}\text { Air } \\
\text { Immersion } \\
\text { DCS } \\
\left(\mathbf{B q} / \mathbf{m}^{3}\right)\end{array}$ & $\begin{array}{c}\text { Air } \\
\text { Immersion } \\
\text { DCS } \\
\left(\mu \mathrm{Ci} / \mathbf{c m}^{3}\right)\end{array}$ & $\begin{array}{c}\text { Water } \\
\text { Submersion } \\
\text { DCS } \\
\left(\mathbf{B q} / \mathbf{m}^{3}\right)\end{array}$ & $\begin{array}{c}\text { Water } \\
\text { Submersion } \\
\text { DCS } \\
\left(\mu \mathrm{Ci} / \mathbf{m}^{3}\right)\end{array}$ \\
\hline $\mathrm{Cm}-240$ & $6.98 \mathrm{E}+06$ & $1.89 \mathrm{E}-04$ & $2.99 \mathrm{E}+09$ & $2.56 \mathrm{E}+04$ \\
\hline $\mathrm{Cm}-241$ & $1.50 \mathrm{E}+03$ & 4.04E-08 & $6.85 \mathrm{E}+05$ & $5.87 \mathrm{E}+00$ \\
\hline $\mathrm{Cm}-242$ & $8.13 \mathrm{E}+06$ & $2.20 \mathrm{E}-04$ & $3.49 \mathrm{E}+09$ & $2.99 \mathrm{E}+04$ \\
\hline $\mathrm{Cm}-243$ & $5.95 \mathrm{E}+03$ & $1.61 \mathrm{E}-07$ & $2.71 \mathrm{E}+06$ & $2.32 \mathrm{E}+01$ \\
\hline $\mathrm{Cm}-244$ & $7.93 \mathrm{E}+06$ & $2.14 \mathrm{E}-04$ & $3.44 \mathrm{E}+09$ & $2.95 \mathrm{E}+04$ \\
\hline $\mathrm{Cm}-245$ & $7.93 \mathrm{E}+03$ & $2.14 \mathrm{E}-07$ & $3.56 \mathrm{E}+06$ & $3.05 \mathrm{E}+01$ \\
\hline $\mathrm{Cm}-246$ & $1.77 \mathrm{E}+05$ & $4.79 \mathrm{E}-06$ & $8.19 \mathrm{E}+07$ & $7.02 \mathrm{E}+02$ \\
\hline $\mathrm{Cm}-247$ & $2.30 \mathrm{E}+03$ & $6.21 \mathrm{E}-08$ & $1.05 \mathrm{E}+06$ & $9.03 \mathrm{E}+00$ \\
\hline $\mathrm{Cm}-248$ & $4.95 \mathrm{E}+02$ & $1.34 \mathrm{E}-08$ & $2.28 \mathrm{E}+05$ & $1.96 \mathrm{E}+00$ \\
\hline $\mathrm{Cm}-249$ & $3.11 \mathrm{E}+04$ & $8.40 \mathrm{E}-07$ & $1.55 \mathrm{E}+07$ & $1.33 \mathrm{E}+02$ \\
\hline $\mathrm{Cm}-250$ & $4.86 \mathrm{E}+01$ & $1.31 \mathrm{E}-09$ & $2.25 \mathrm{E}+04$ & $1.93 \mathrm{E}-01$ \\
\hline $\mathrm{Cm}-251$ & $6.10 \mathrm{E}+03$ & $1.65 \mathrm{E}-07$ & $2.88 \mathrm{E}+06$ & $2.47 \mathrm{E}+01$ \\
\hline Bk-245 & $3.41 \mathrm{E}+03$ & $9.23 \mathrm{E}-08$ & $1.55 \mathrm{E}+06$ & $1.33 \mathrm{E}+01$ \\
\hline Bk-246 & $8.30 \mathrm{E}+02$ & $2.24 \mathrm{E}-08$ & $3.82 \mathrm{E}+05$ & $3.27 \mathrm{E}+00$ \\
\hline Bk-247 & $5.29 \mathrm{E}+03$ & $1.43 \mathrm{E}-07$ & $2.40 \mathrm{E}+06$ & $2.06 \mathrm{E}+01$ \\
\hline Bk-248m & $1.39 \mathrm{E}+04$ & $3.76 \mathrm{E}-07$ & $6.46 \mathrm{E}+06$ & $5.53 \mathrm{E}+01$ \\
\hline Bk-249 & $7.26 \mathrm{E}+07$ & $1.96 \mathrm{E}-03$ & $5.85 \mathrm{E}+10$ & $5.01 \mathrm{E}+05$ \\
\hline Bk-250 & $7.59 \mathrm{E}+02$ & $2.05 \mathrm{E}-08$ & $3.51 \mathrm{E}+05$ & $3.01 \mathrm{E}+00$ \\
\hline Bk-251 & $8.91 \mathrm{E}+03$ & $2.41 \mathrm{E}-07$ & $4.12 \mathrm{E}+06$ & $3.53 \mathrm{E}+01$ \\
\hline Cf-244 & $7.34 \mathrm{E}+06$ & $1.98 \mathrm{E}-04$ & $3.14 \mathrm{E}+09$ & $2.69 \mathrm{E}+04$ \\
\hline Cf-246 & $6.47 \mathrm{E}+06$ & $1.75 \mathrm{E}-04$ & $2.83 \mathrm{E}+09$ & $2.43 \mathrm{E}+04$ \\
\hline Cf-247 & $8.86 \mathrm{E}+03$ & $2.39 \mathrm{E}-07$ & $3.99 \mathrm{E}+06$ & $3.42 \mathrm{E}+01$ \\
\hline Cf-248 & $1.59 \mathrm{E}+06$ & $4.29 \mathrm{E}-05$ & $7.21 \mathrm{E}+08$ & $6.18 \mathrm{E}+03$ \\
\hline Cf-249 & $2.22 \mathrm{E}+03$ & 5.99E-08 & $1.02 \mathrm{E}+06$ & $8.74 \mathrm{E}+00$ \\
\hline Cf-250 & $6.58 \mathrm{E}+04$ & $1.78 \mathrm{E}-06$ & $3.05 \mathrm{E}+07$ & $2.61 \mathrm{E}+02$ \\
\hline Cf-251 & $6.55 \mathrm{E}+03$ & $1.77 \mathrm{E}-07$ & $2.96 \mathrm{E}+06$ & $2.54 \mathrm{E}+01$ \\
\hline Cf-252 & $1.42 \mathrm{E}+03$ & $3.84 \mathrm{E}-08$ & $6.59 \mathrm{E}+05$ & $5.65 \mathrm{E}+00$ \\
\hline Cf- 253 & $1.01 \mathrm{E}+06$ & $2.72 \mathrm{E}-05$ & $5.62 \mathrm{E}+08$ & $4.82 \mathrm{E}+03$ \\
\hline Cf-254 & $3.85 \mathrm{E}+01$ & $1.04 \mathrm{E}-09$ & $1.78 \mathrm{E}+04$ & $1.53 \mathrm{E}-01$ \\
\hline Cf- 255 & $2.73 \mathrm{E}+05$ & $7.39 \mathrm{E}-06$ & $2.44 \mathrm{E}+08$ & $2.09 \mathrm{E}+03$ \\
\hline Es-249 & $1.79 \mathrm{E}+03$ & $4.84 \mathrm{E}-08$ & $8.21 \mathrm{E}+05$ & $7.04 \mathrm{E}+00$ \\
\hline Es-250 & $6.04 \mathrm{E}+02$ & & $2.78 \mathrm{E}+05$ & $2.38 \mathrm{E}+00$ \\
\hline Es-250m & $1.27 \mathrm{E}+03$ & $3.44 \mathrm{E}-08$ & $5.85 \mathrm{E}+05$ & $5.01 \mathrm{E}+00$ \\
\hline Es-251 & $8.86 \mathrm{E}+03$ & $2.39 \mathrm{E}-07$ & $3.98 \mathrm{E}+06$ & $3.41 \mathrm{E}+01$ \\
\hline Es-253 & $2.11 \mathrm{E}+06$ & $5.71 \mathrm{E}-05$ & $9.61 \mathrm{E}+08$ & $8.24 \mathrm{E}+03$ \\
\hline Es-254 & $2.16 \mathrm{E}+05$ & $5.83 \mathrm{E}-06$ & $9.52 \mathrm{E}+07$ & $8.16 \mathrm{E}+02$ \\
\hline Es- $254 \mathrm{~m}$ & $1.49 \mathrm{E}+03$ & $4.02 \mathrm{E}-08$ & $6.88 \mathrm{E}+05$ & $5.90 \mathrm{E}+00$ \\
\hline
\end{tabular}


SRNL-STI-2013-00115

\begin{tabular}{|c|c|c|c|c|}
\hline Nuclide & $\begin{array}{c}\text { Air } \\
\text { Immersion } \\
\mathbf{D C S} \\
\left(\mathbf{B q} / \mathbf{m}^{\mathbf{3}}\right)\end{array}$ & $\begin{array}{c}\text { Air } \\
\text { Immersion } \\
\mathbf{D C S} \\
\left(\boldsymbol{\mu C i} / \mathbf{c m}^{\mathbf{3}}\right)\end{array}$ & $\begin{array}{c}\text { Water } \\
\text { Submersion } \\
\mathbf{D C S} \\
\mathbf{( B q / \mathbf { m } ^ { \mathbf { 3 } } )}\end{array}$ & $\begin{array}{c}\text { Water } \\
\text { Submersion } \\
\mathbf{D C S} \\
\left(\boldsymbol{\mu C i} / \mathbf{m}^{\mathbf{3}}\right)\end{array}$ \\
\hline Es-255 & $6.41 \mathrm{E}+05$ & $1.73 \mathrm{E}-05$ & $3.54 \mathrm{E}+08$ & $3.03 \mathrm{E}+03$ \\
\hline Es-256 & $7.32 \mathrm{E}+04$ & $1.98 \mathrm{E}-06$ & $5.99 \mathrm{E}+07$ & $5.14 \mathrm{E}+02$ \\
\hline Fm-251 & $4.99 \mathrm{E}+03$ & $1.35 \mathrm{E}-07$ & $2.26 \mathrm{E}+06$ & $1.94 \mathrm{E}+01$ \\
\hline Fm-252 & $1.85 \mathrm{E}+06$ & $5.01 \mathrm{E}-05$ & $8.39 \mathrm{E}+08$ & $7.19 \mathrm{E}+03$ \\
\hline Fm-253 & $1.37 \mathrm{E}+04$ & $3.69 \mathrm{E}-07$ & $6.16 \mathrm{E}+06$ & $5.28 \mathrm{E}+01$ \\
\hline Fm-254 & $9.14 \mathrm{E}+04$ & $2.47 \mathrm{E}-06$ & $4.23 \mathrm{E}+07$ & $3.62 \mathrm{E}+02$ \\
\hline Fm-255 & $3.35 \mathrm{E}+05$ & $9.05 \mathrm{E}-06$ & $1.47 \mathrm{E}+08$ & $1.26 \mathrm{E}+03$ \\
\hline Fm-256 & $5.22 \mathrm{E}+01$ & $1.41 \mathrm{E}-09$ & $2.42 \mathrm{E}+04$ & $2.07 \mathrm{E}-01$ \\
\hline Fm-257 & $5.27 \mathrm{E}+03$ & $1.42 \mathrm{E}-07$ & $2.40 \mathrm{E}+06$ & $2.06 \mathrm{E}+01$ \\
\hline
\end{tabular}


Table D-4 Ground Shine DCS

\begin{tabular}{|c|c|c|}
\hline Nuclide & $\begin{array}{c}\text { Ground } \\
\text { Shine DCS } \\
\left(\mathbf{B q} / \mathbf{m}^{2}\right)\end{array}$ & $\begin{array}{c}\text { Ground } \\
\text { Shine DCS } \\
\left(\mu \mathrm{Ci} / \mathbf{m}^{2}\right)\end{array}$ \\
\hline Be-7 & $6.66 \mathrm{E}+05$ & $5.71 \mathrm{E}-12$ \\
\hline Be-10 & $9.22 \mathrm{E}+06$ & $7.90 \mathrm{E}-11$ \\
\hline $\mathrm{C}-10$ & $1.80 \mathrm{E}+04$ & $1.54 \mathrm{E}-13$ \\
\hline C-11 & $3.17 \mathrm{E}+04$ & $2.72 \mathrm{E}-13$ \\
\hline C-14 & $2.48 \mathrm{E}+09$ & $2.12 \mathrm{E}-08$ \\
\hline $\mathrm{N}-13$ & $3.08 \mathrm{E}+04$ & $2.64 \mathrm{E}-13$ \\
\hline $\mathrm{N}-16$ & $9.22 \mathrm{E}+03$ & $7.90 \mathrm{E}-14$ \\
\hline O-14 & $1.05 \mathrm{E}+04$ & $8.97 \mathrm{E}-14$ \\
\hline O-15 & $2.96 \mathrm{E}+04$ & $2.54 \mathrm{E}-13$ \\
\hline O-19 & $3.08 \mathrm{E}+04$ & $2.64 \mathrm{E}-13$ \\
\hline F-17 & $2.96 \mathrm{E}+04$ & $2.54 \mathrm{E}-13$ \\
\hline F-18 & $3.34 \mathrm{E}+04$ & $2.86 \mathrm{E}-13$ \\
\hline $\mathrm{Ne}-19$ & $2.91 \mathrm{E}+04$ & $2.49 \mathrm{E}-13$ \\
\hline $\mathrm{Ne}-24$ & $5.16 \mathrm{E}+04$ & $4.42 \mathrm{E}-13$ \\
\hline $\mathrm{Na}-22$ & $1.55 \mathrm{E}+04$ & $1.33 \mathrm{E}-13$ \\
\hline $\mathrm{Na}-24$ & $8.83 \mathrm{E}+03$ & $7.57 \mathrm{E}-14$ \\
\hline $\mathrm{Mg}-27$ & $3.43 \mathrm{E}+04$ & $2.94 \mathrm{E}-13$ \\
\hline $\mathrm{Mg}-28$ & $2.52 \mathrm{E}+04$ & $2.16 \mathrm{E}-13$ \\
\hline Al-26 & $1.28 \mathrm{E}+04$ & $1.10 \mathrm{E}-13$ \\
\hline Al-28 & $1.85 \mathrm{E}+04$ & $1.59 \mathrm{E}-13$ \\
\hline Al-29 & $2.31 \mathrm{E}+04$ & $1.98 \mathrm{E}-13$ \\
\hline $\mathrm{Si}-31$ & $4.45 \mathrm{E}+05$ & $3.81 \mathrm{E}-12$ \\
\hline Si-32 & $1.10 \mathrm{E}+09$ & $9.47 \mathrm{E}-09$ \\
\hline $\mathrm{P}-30$ & $2.81 \mathrm{E}+04$ & $2.40 \mathrm{E}-13$ \\
\hline P-32 & $3.72 \mathrm{E}+05$ & $3.19 \mathrm{E}-12$ \\
\hline $\mathrm{P}-33$ & $8.71 \mathrm{E}+08$ & $7.47 \mathrm{E}-09$ \\
\hline S-35 & $2.38 \mathrm{E}+09$ & $2.04 \mathrm{E}-08$ \\
\hline S-37 & $1.28 \mathrm{E}+04$ & $1.10 \mathrm{E}-13$ \\
\hline S-38 & $2.09 \mathrm{E}+04$ & $1.79 \mathrm{E}-13$ \\
\hline $\mathrm{Cl}-34$ & $2.71 \mathrm{E}+04$ & $2.32 \mathrm{E}-13$ \\
\hline $\mathrm{Cl}-34 \mathrm{~m}$ & $1.66 \mathrm{E}+04$ & $1.42 \mathrm{E}-13$ \\
\hline $\mathrm{Cl}-36$ & $2.86 \mathrm{E}+06$ & $2.45 \mathrm{E}-11$ \\
\hline $\mathrm{Cl}-38$ & $2.26 \mathrm{E}+04$ & $1.94 \mathrm{E}-13$ \\
\hline Cl-39 & $2.23 \mathrm{E}+04$ & $1.91 \mathrm{E}-13$ \\
\hline $\mathrm{Cl}-40$ & $8.81 \mathrm{E}+03$ & $7.55 \mathrm{E}-14$ \\
\hline Ar-39 & $1.26 \mathrm{E}+07$ & $1.08 \mathrm{E}-10$ \\
\hline Ar-41 & $2.60 \mathrm{E}+04$ & $2.23 \mathrm{E}-13$ \\
\hline
\end{tabular}




\begin{tabular}{|c|c|c|}
\hline Nuclide & $\begin{array}{c}\text { Ground } \\
\text { Shine DCS } \\
\left(\mathbf{B q} / \mathbf{m}^{2}\right)\end{array}$ & $\begin{array}{c}\text { Ground } \\
\text { Shine DCS } \\
\left(\mu \mathrm{Ci} / \mathbf{m}^{2}\right)\end{array}$ \\
\hline Ar-42 & $7.77 \mathrm{E}+06$ & $6.66 \mathrm{E}-11$ \\
\hline Ar-43 & $2.09 \mathrm{E}+04$ & $1.79 \mathrm{E}-13$ \\
\hline Ar-44 & $1.78 \mathrm{E}+04$ & $1.53 \mathrm{E}-13$ \\
\hline K-38 & $1.07 \mathrm{E}+04$ & $9.15 \mathrm{E}-14$ \\
\hline $\mathrm{K}-40$ & $1.55 \mathrm{E}+05$ & $1.33 \mathrm{E}-12$ \\
\hline $\mathrm{K}-42$ & $7.93 \mathrm{E}+04$ & $6.79 \mathrm{E}-13$ \\
\hline $\mathrm{K}-43$ & $3.39 \mathrm{E}+04$ & $2.91 \mathrm{E}-13$ \\
\hline K-44 & $1.42 \mathrm{E}+04$ & $1.22 \mathrm{E}-13$ \\
\hline $\mathrm{K}-45$ & $1.82 \mathrm{E}+04$ & $1.56 \mathrm{E}-13$ \\
\hline $\mathrm{K}-46$ & $1.19 \mathrm{E}+04$ & $1.02 \mathrm{E}-13$ \\
\hline $\mathrm{Ca}-45$ & $8.39 \mathrm{E}+08$ & 7.19E-09 \\
\hline $\mathrm{Ca}-47$ & $3.20 \mathrm{E}+04$ & $2.75 \mathrm{E}-13$ \\
\hline Ca-49 & $1.19 \mathrm{E}+04$ & $1.02 \mathrm{E}-13$ \\
\hline $\mathrm{Sc}-42 \mathrm{~m}$ & $7.87 \mathrm{E}+03$ & $6.74 \mathrm{E}-14$ \\
\hline Sc-43 & $3.23 \mathrm{E}+04$ & $2.77 \mathrm{E}-13$ \\
\hline Sc-44 & $1.52 \mathrm{E}+04$ & $1.31 \mathrm{E}-13$ \\
\hline Sc- $44 m$ & $1.24 \mathrm{E}+05$ & $1.06 \mathrm{E}-12$ \\
\hline $\mathrm{Sc}-46$ & $1.69 \mathrm{E}+04$ & $1.45 \mathrm{E}-13$ \\
\hline Sc-47 & $3.17 \mathrm{E}+05$ & $2.72 \mathrm{E}-12$ \\
\hline Sc-48 & $1.02 \mathrm{E}+04$ & $8.74 \mathrm{E}-14$ \\
\hline Sc-49 & $3.14 \mathrm{E}+05$ & $2.69 \mathrm{E}-12$ \\
\hline Sc-50 & $1.02 \mathrm{E}+04$ & $8.77 \mathrm{E}-14$ \\
\hline Ti-44 & $2.58 \mathrm{E}+05$ & $2.21 \mathrm{E}-12$ \\
\hline Ti-45 & $3.66 \mathrm{E}+04$ & $3.14 \mathrm{E}-13$ \\
\hline Ti-51 & $6.98 \mathrm{E}+04$ & $5.99 \mathrm{E}-13$ \\
\hline Ti-52 & $1.55 \mathrm{E}+05$ & $1.33 \mathrm{E}-12$ \\
\hline $\mathrm{V}-47$ & $3.02 \mathrm{E}+04$ & $2.59 \mathrm{E}-13$ \\
\hline $\mathrm{V}-48$ & $1.17 \mathrm{E}+04$ & $1.00 \mathrm{E}-13$ \\
\hline $\mathrm{V}-50$ & $2.48 \mathrm{E}+04$ & $2.12 \mathrm{E}-13$ \\
\hline $\mathrm{V}-52$ & $2.22 \mathrm{E}+04$ & $1.90 \mathrm{E}-13$ \\
\hline $\mathrm{V}-53$ & $2.91 \mathrm{E}+04$ & $2.49 \mathrm{E}-13$ \\
\hline Cr-48 & $7.83 \mathrm{E}+04$ & $6.71 \mathrm{E}-13$ \\
\hline Cr-49 & $2.96 \mathrm{E}+04$ & $2.54 \mathrm{E}-13$ \\
\hline $\mathrm{Cr}-51$ & $1.06 \mathrm{E}+06$ & $9.09 \mathrm{E}-12$ \\
\hline Cr-55 & $2.46 \mathrm{E}+05$ & $2.11 \mathrm{E}-12$ \\
\hline Cr-56 & $2.14 \mathrm{E}+05$ & $1.84 \mathrm{E}-12$ \\
\hline $\mathrm{Mn}-50 \mathrm{~m}$ & $7.09 \mathrm{E}+03$ & $6.08 \mathrm{E}-14$ \\
\hline Mn-51 & $2.96 \mathrm{E}+04$ & $2.54 \mathrm{E}-13$ \\
\hline
\end{tabular}




\begin{tabular}{|c|c|c|}
\hline Nuclide & $\begin{array}{c}\text { Ground } \\
\text { Shine DCS } \\
\left(\mathrm{Bq} / \mathbf{m}^{2}\right)\end{array}$ & $\begin{array}{c}\text { Ground } \\
\text { Shine DCS } \\
\left(\mu \mathrm{Ci} / \mathbf{m}^{2}\right)\end{array}$ \\
\hline $\mathrm{Mn}-52 \mathrm{~m}$ & $1.34 \mathrm{E}+04$ & $1.15 \mathrm{E}-13$ \\
\hline $\mathrm{Mn}-52$ & $9.88 \mathrm{E}+03$ & $8.47 \mathrm{E}-14$ \\
\hline Mn-54 & $4.02 \mathrm{E}+04$ & $3.44 \mathrm{E}-13$ \\
\hline Mn-56 & $1.96 \mathrm{E}+04$ & $1.68 \mathrm{E}-13$ \\
\hline Mn-57 & $1.46 \mathrm{E}+05$ & $1.25 \mathrm{E}-12$ \\
\hline $\mathrm{Mn}-58 \mathrm{~m}$ & $1.34 \mathrm{E}+04$ & $1.15 \mathrm{E}-13$ \\
\hline $\mathrm{Fe}-52$ & $4.47 \mathrm{E}+04$ & $3.83 \mathrm{E}-13$ \\
\hline Fe-53 & $2.54 \mathrm{E}+04$ & $2.17 \mathrm{E}-13$ \\
\hline Fe-53m & $1.13 \mathrm{E}+04$ & $9.67 \mathrm{E}-14$ \\
\hline $\mathrm{Fe}-55$ & $2.19 \mathrm{E}+14$ & $1.87 \mathrm{E}-03$ \\
\hline Fe-59 & $2.88 \mathrm{E}+04$ & $2.47 \mathrm{E}-13$ \\
\hline Fe-60 & $1.38 \mathrm{E}+09$ & $1.19 \mathrm{E}-08$ \\
\hline Fe-61 & $2.26 \mathrm{E}+04$ & $1.94 \mathrm{E}-13$ \\
\hline Fe-62 & $5.42 \mathrm{E}+04$ & $4.65 \mathrm{E}-13$ \\
\hline Co-54m & $8.24 \mathrm{E}+03$ & $7.06 \mathrm{E}-14$ \\
\hline Co-55 & $1.65 \mathrm{E}+04$ & $1.42 \mathrm{E}-13$ \\
\hline Co-56 & $9.70 \mathrm{E}+03$ & $8.31 \mathrm{E}-14$ \\
\hline Co-57 & $2.91 \mathrm{E}+05$ & $2.49 \mathrm{E}-12$ \\
\hline Co-58 & $3.44 \mathrm{E}+04$ & $2.95 \mathrm{E}-13$ \\
\hline Co-58m & $4.75 \mathrm{E}+09$ & 4.07E-08 \\
\hline Co-60 & $1.38 \mathrm{E}+04$ & $1.18 \mathrm{E}-13$ \\
\hline Co-60m & $7.48 \mathrm{E}+06$ & $6.41 \mathrm{E}-11$ \\
\hline Co-61 & $2.33 \mathrm{E}+05$ & $2.00 \mathrm{E}-12$ \\
\hline Co-62 & $1.97 \mathrm{E}+04$ & $1.69 \mathrm{E}-13$ \\
\hline Co-62m & $1.23 \mathrm{E}+04$ & $1.06 \mathrm{E}-13$ \\
\hline $\mathrm{Ni}-56$ & $1.97 \mathrm{E}+04$ & $1.69 \mathrm{E}-13$ \\
\hline $\mathrm{Ni}-57$ & $1.78 \mathrm{E}+04$ & $1.53 \mathrm{E}-13$ \\
\hline $\mathrm{Ni}-59$ & $2.14 \mathrm{E}+09$ & $1.84 \mathrm{E}-08$ \\
\hline $\mathrm{Ni}-65$ & $5.50 \mathrm{E}+04$ & $4.71 \mathrm{E}-13$ \\
\hline Ni-66 & $9.19 \mathrm{E}+08$ & 7.88E-09 \\
\hline $\mathrm{Cu}-57$ & $2.33 \mathrm{E}+04$ & $2.00 \mathrm{E}-13$ \\
\hline $\mathrm{Cu}-59$ & $2.09 \mathrm{E}+04$ & $1.79 \mathrm{E}-13$ \\
\hline $\mathrm{Cu}-60$ & $8.69 \mathrm{E}+03$ & $7.45 \mathrm{E}-14$ \\
\hline $\mathrm{Cu}-61$ & $3.89 \mathrm{E}+04$ & $3.33 \mathrm{E}-13$ \\
\hline $\mathrm{Cu}-62$ & $2.86 \mathrm{E}+04$ & $2.45 \mathrm{E}-13$ \\
\hline $\mathrm{Cu}-64$ & $1.78 \mathrm{E}+05$ & $1.53 \mathrm{E}-12$ \\
\hline $\mathrm{Cu}-66$ & $1.47 \mathrm{E}+05$ & $1.26 \mathrm{E}-12$ \\
\hline $\mathrm{Cu}-67$ & $3.02 \mathrm{E}+05$ & $2.59 \mathrm{E}-12$ \\
\hline
\end{tabular}




\begin{tabular}{|c|c|c|}
\hline Nuclide & $\begin{array}{c}\text { Ground } \\
\text { Shine DCS } \\
\left(\mathbf{B q} / \mathbf{m}^{2}\right)\end{array}$ & $\begin{array}{c}\text { Ground } \\
\text { Shine DCS } \\
\left(\mu \mathrm{Ci} / \mathbf{m}^{2}\right)\end{array}$ \\
\hline $\mathrm{Cu}-69$ & $5.31 \mathrm{E}+04$ & $4.55 \mathrm{E}-13$ \\
\hline $\mathrm{Zn}-60$ & $1.99 \mathrm{E}+04$ & $1.71 \mathrm{E}-13$ \\
\hline $\mathrm{Zn}-61$ & $1.97 \mathrm{E}+04$ & $1.69 \mathrm{E}-13$ \\
\hline $\mathrm{Zn}-62$ & $7.57 \mathrm{E}+04$ & $6.49 \mathrm{E}-13$ \\
\hline $\mathrm{Zn}-63$ & $2.73 \mathrm{E}+04$ & $2.34 \mathrm{E}-13$ \\
\hline $\mathrm{Zn}-65$ & $5.90 \mathrm{E}+04$ & $5.06 \mathrm{E}-13$ \\
\hline $\mathrm{Zn}-69 \mathrm{~m}$ & $7.97 \mathrm{E}+04$ & $6.83 \mathrm{E}-13$ \\
\hline $\mathrm{Zn}-69$ & $1.52 \mathrm{E}+06$ & $1.30 \mathrm{E}-11$ \\
\hline $\mathrm{Zn}-71 \mathrm{~m}$ & $2.06 \mathrm{E}+04$ & $1.76 \mathrm{E}-13$ \\
\hline $\mathrm{Zn}-71$ & $7.57 \mathrm{E}+04$ & $6.49 \mathrm{E}-13$ \\
\hline $\mathrm{Zn}-72$ & $2.38 \mathrm{E}+05$ & $2.04 \mathrm{E}-12$ \\
\hline Ga-64 & $9.97 \mathrm{E}+03$ & $8.55 \mathrm{E}-14$ \\
\hline Ga-65 & $2.64 \mathrm{E}+04$ & $2.26 \mathrm{E}-13$ \\
\hline Ga-66 & $1.40 \mathrm{E}+04$ & $1.20 \mathrm{E}-13$ \\
\hline Ga-67 & $2.22 \mathrm{E}+05$ & $1.90 \mathrm{E}-12$ \\
\hline Ga-68 & $3.18 \mathrm{E}+04$ & $2.73 \mathrm{E}-13$ \\
\hline Ga-70 & $3.75 \mathrm{E}+05$ & $3.22 \mathrm{E}-12$ \\
\hline Ga-72 & $1.28 \mathrm{E}+04$ & $1.10 \mathrm{E}-13$ \\
\hline Ga-73 & $8.55 \mathrm{E}+04$ & 7.33E-13 \\
\hline Ga-74 & $1.09 \mathrm{E}+04$ & $9.37 \mathrm{E}-14$ \\
\hline Ge-66 & $4.95 \mathrm{E}+04$ & $4.24 \mathrm{E}-13$ \\
\hline Ge-67 & $2.16 \mathrm{E}+04$ & $1.85 \mathrm{E}-13$ \\
\hline Ge-68 & $8.78 \mathrm{E}+08$ & 7.53E-09 \\
\hline Ge-69 & $3.53 \mathrm{E}+04$ & $3.02 \mathrm{E}-13$ \\
\hline Ge-71 & $8.66 \mathrm{E}+08$ & 7.43E-09 \\
\hline Ge-75 & $4.34 \mathrm{E}+05$ & $3.72 \mathrm{E}-12$ \\
\hline Ge-77 & $2.94 \mathrm{E}+04$ & $2.52 \mathrm{E}-13$ \\
\hline Ge-78 & $1.19 \mathrm{E}+05$ & $1.02 \mathrm{E}-12$ \\
\hline As-68 & $8.78 \mathrm{E}+03$ & $7.53 \mathrm{E}-14$ \\
\hline As-69 & $2.60 \mathrm{E}+04$ & $2.23 \mathrm{E}-13$ \\
\hline As-70 & $7.85 \mathrm{E}+03$ & $6.73 \mathrm{E}-14$ \\
\hline As-71 & $5.83 \mathrm{E}+04$ & $5.00 \mathrm{E}-13$ \\
\hline As-72 & $1.76 \mathrm{E}+04$ & $1.51 \mathrm{E}-13$ \\
\hline As-73 & $6.17 \mathrm{E}+06$ & $5.29 \mathrm{E}-11$ \\
\hline As-74 & $4.25 \mathrm{E}+04$ & $3.64 \mathrm{E}-13$ \\
\hline As-76 & $6.18 \mathrm{E}+04$ & $5.30 \mathrm{E}-13$ \\
\hline As-77 & $2.38 \mathrm{E}+06$ & $2.04 \mathrm{E}-11$ \\
\hline As-78 & $2.38 \mathrm{E}+04$ & $2.04 \mathrm{E}-13$ \\
\hline
\end{tabular}




\begin{tabular}{|c|c|c|}
\hline Nuclide & $\begin{array}{c}\text { Ground } \\
\text { Shine DCS } \\
\left(\mathbf{B q} / \mathbf{m}^{2}\right)\end{array}$ & $\begin{array}{c}\text { Ground } \\
\text { Shine DCS } \\
\left(\mu \mathrm{Ci} / \mathbf{m}^{2}\right)\end{array}$ \\
\hline As-79 & $2.30 \mathrm{E}+05$ & $1.97 \mathrm{E}-12$ \\
\hline $\mathrm{Se}-70$ & $4.56 \mathrm{E}+04$ & $3.90 \mathrm{E}-13$ \\
\hline Se-71 & $1.91 \mathrm{E}+04$ & $1.64 \mathrm{E}-13$ \\
\hline Se-72 & $1.30 \mathrm{E}+06$ & $1.12 \mathrm{E}-11$ \\
\hline $\mathrm{Se}-73$ & $2.94 \mathrm{E}+04$ & $2.52 \mathrm{E}-13$ \\
\hline $\mathrm{Se}-73 \mathrm{~m}$ & $1.19 \mathrm{E}+05$ & $1.02 \mathrm{E}-12$ \\
\hline $\mathrm{Se}-75$ & $8.91 \mathrm{E}+04$ & $7.63 \mathrm{E}-13$ \\
\hline $\mathrm{Se}-77 \mathrm{~m}$ & $4.02 \mathrm{E}+05$ & $3.45 \mathrm{E}-12$ \\
\hline $\mathrm{Se}-79$ & $2.19 \mathrm{E}+09$ & $1.87 \mathrm{E}-08$ \\
\hline $\mathrm{Se}-79 \mathrm{~m}$ & $3.70 \mathrm{E}+06$ & $3.17 \mathrm{E}-11$ \\
\hline Se-81 & $3.95 \mathrm{E}+05$ & $3.39 \mathrm{E}-12$ \\
\hline $\mathrm{Se}-81 \mathrm{~m}$ & $2.50 \mathrm{E}+06$ & $2.14 \mathrm{E}-11$ \\
\hline $\mathrm{Se}-83$ & $1.29 \mathrm{E}+04$ & $1.11 \mathrm{E}-13$ \\
\hline $\mathrm{Se}-83 \mathrm{~m}$ & $3.05 \mathrm{E}+04$ & $2.61 \mathrm{E}-13$ \\
\hline $\mathrm{Se}-84$ & $6.85 \mathrm{E}+04$ & $5.87 \mathrm{E}-13$ \\
\hline $\mathrm{Br}-72$ & $1.06 \mathrm{E}+04$ & $9.09 \mathrm{E}-14$ \\
\hline Br-73 & $2.10 \mathrm{E}+04$ & $1.80 \mathrm{E}-13$ \\
\hline Br-74 & $7.73 \mathrm{E}+03$ & $6.63 \mathrm{E}-14$ \\
\hline $\mathrm{Br}-74 \mathrm{~m}$ & $8.24 \mathrm{E}+03$ & $7.06 \mathrm{E}-14$ \\
\hline $\mathrm{Br}-75$ & $2.64 \mathrm{E}+04$ & $2.26 \mathrm{E}-13$ \\
\hline $\mathrm{Br}-76$ & $1.23 \mathrm{E}+04$ & $1.05 \mathrm{E}-13$ \\
\hline $\mathrm{Br}-76 \mathrm{~m}$ & $9.82 \mathrm{E}+05$ & $8.41 \mathrm{E}-12$ \\
\hline Br-77 & $1.06 \mathrm{E}+05$ & $9.09 \mathrm{E}-13$ \\
\hline $\mathrm{Br}-77 \mathrm{~m}$ & $2.31 \mathrm{E}+06$ & $1.98 \mathrm{E}-11$ \\
\hline Br-78 & $2.86 \mathrm{E}+04$ & $2.45 \mathrm{E}-13$ \\
\hline $\mathrm{Br}-80$ & $1.98 \mathrm{E}+05$ & $1.70 \mathrm{E}-12$ \\
\hline $\mathrm{Br}-80 \mathrm{~m}$ & $2.30 \mathrm{E}+06$ & $1.97 \mathrm{E}-11$ \\
\hline Br-82 & $1.28 \mathrm{E}+04$ & $1.10 \mathrm{E}-13$ \\
\hline $\mathrm{Br}-82 \mathrm{~m}$ & $4.75 \mathrm{E}+06$ & $4.07 \mathrm{E}-11$ \\
\hline $\mathrm{Br}-83$ & $1.10 \mathrm{E}+06$ & $9.44 \mathrm{E}-12$ \\
\hline $\mathrm{Br}-84$ & $1.92 \mathrm{E}+04$ & $1.65 \mathrm{E}-13$ \\
\hline Br-84m & $1.19 \mathrm{E}+04$ & $1.02 \mathrm{E}-13$ \\
\hline Br-85 & $1.73 \mathrm{E}+05$ & $1.49 \mathrm{E}-12$ \\
\hline $\mathrm{Kr}-74$ & $2.96 \mathrm{E}+04$ & $2.54 \mathrm{E}-13$ \\
\hline $\mathrm{Kr}-75$ & $2.31 \mathrm{E}+04$ & $1.98 \mathrm{E}-13$ \\
\hline $\mathrm{Kr}-76$ & $8.01 \mathrm{E}+04$ & $6.86 \mathrm{E}-13$ \\
\hline $\mathrm{Kr}-77$ & $2.99 \mathrm{E}+04$ & $2.56 \mathrm{E}-13$ \\
\hline $\mathrm{Kr}-79$ & $1.34 \mathrm{E}+05$ & $1.15 \mathrm{E}-12$ \\
\hline
\end{tabular}




\begin{tabular}{|c|c|c|}
\hline Nuclide & $\begin{array}{c}\text { Ground } \\
\text { Shine DCS } \\
\left(\mathbf{B q} / \mathbf{m}^{2}\right)\end{array}$ & $\begin{array}{c}\text { Ground } \\
\text { Shine DCS } \\
\left(\mu \mathrm{Ci} / \mathbf{m}^{2}\right)\end{array}$ \\
\hline $\mathrm{Kr}-81$ & $2.02 \mathrm{E}+07$ & $1.73 \mathrm{E}-10$ \\
\hline $\mathrm{Kr}-81 \mathrm{~m}$ & $2.69 \mathrm{E}+05$ & $2.30 \mathrm{E}-12$ \\
\hline $\mathrm{Kr}-83 \mathrm{~m}$ & $9.73 \mathrm{E}+07$ & $8.34 \mathrm{E}-10$ \\
\hline $\mathrm{Kr}-85$ & $3.02 \mathrm{E}+06$ & $2.59 \mathrm{E}-11$ \\
\hline $\mathrm{Kr}-85 \mathrm{~m}$ & $2.03 \mathrm{E}+05$ & $1.74 \mathrm{E}-12$ \\
\hline $\mathrm{Kr}-87$ & $3.78 \mathrm{E}+04$ & $3.24 \mathrm{E}-13$ \\
\hline $\mathrm{Kr}-88$ & $1.84 \mathrm{E}+04$ & $1.58 \mathrm{E}-13$ \\
\hline $\mathrm{Kr}-89$ & $1.72 \mathrm{E}+04$ & $1.48 \mathrm{E}-13$ \\
\hline $\mathrm{Rb}-77$ & $1.96 \mathrm{E}+04$ & $1.68 \mathrm{E}-13$ \\
\hline $\mathrm{Rb}-78$ & $8.64 \mathrm{E}+03$ & $7.40 \mathrm{E}-14$ \\
\hline $\mathrm{Rb}-78 \mathrm{~m}$ & $1.02 \mathrm{E}+04$ & $8.74 \mathrm{E}-14$ \\
\hline $\mathrm{Rb}-79$ & $2.17 \mathrm{E}+04$ & $1.86 \mathrm{E}-13$ \\
\hline $\mathrm{Rb}-80$ & $2.38 \mathrm{E}+04$ & $2.04 \mathrm{E}-13$ \\
\hline $\mathrm{Rb}-81$ & $6.47 \mathrm{E}+04$ & $5.55 \mathrm{E}-13$ \\
\hline $\mathrm{Rb}-81 \mathrm{~m}$ & $1.30 \mathrm{E}+06$ & $1.12 \mathrm{E}-11$ \\
\hline $\mathrm{Rb}-82$ & $2.62 \mathrm{E}+04$ & $2.25 \mathrm{E}-13$ \\
\hline $\mathrm{Rb}-82 \mathrm{~m}$ & $1.16 \mathrm{E}+04$ & $9.92 \mathrm{E}-14$ \\
\hline $\mathrm{Rb}-83$ & $6.83 \mathrm{E}+04$ & $5.86 \mathrm{E}-13$ \\
\hline $\mathrm{Rb}-84$ & $3.64 \mathrm{E}+04$ & $3.12 \mathrm{E}-13$ \\
\hline $\mathrm{Rb}-84 \mathrm{~m}$ & $8.86 \mathrm{E}+04$ & $7.59 \mathrm{E}-13$ \\
\hline $\mathrm{Rb}-86$ & $1.92 \mathrm{E}+05$ & $1.65 \mathrm{E}-12$ \\
\hline $\mathrm{Rb}-86 \mathrm{~m}$ & $6.06 \mathrm{E}+04$ & $5.20 \mathrm{E}-13$ \\
\hline $\mathrm{Rb}-87$ & $4.02 \mathrm{E}+08$ & 3.44E-09 \\
\hline $\mathrm{Rb}-88$ & $4.24 \mathrm{E}+04$ & $3.63 \mathrm{E}-13$ \\
\hline $\mathrm{Rb}-89$ & $1.50 \mathrm{E}+04$ & $1.28 \mathrm{E}-13$ \\
\hline $\mathrm{Rb}-90$ & $1.72 \mathrm{E}+04$ & $1.48 \mathrm{E}-13$ \\
\hline $\mathrm{Rb}-90 \mathrm{~m}$ & $1.07 \mathrm{E}+04$ & $9.21 \mathrm{E}-14$ \\
\hline Sr-79 & $2.44 \mathrm{E}+04$ & $2.09 \mathrm{E}-13$ \\
\hline Sr-80 & $7.68 \mathrm{E}+04$ & $6.58 \mathrm{E}-13$ \\
\hline Sr-81 & $2.22 \mathrm{E}+04$ & $1.90 \mathrm{E}-13$ \\
\hline Sr-82 & $2.09 \mathrm{E}+07$ & $1.79 \mathrm{E}-10$ \\
\hline Sr-83 & $4.07 \mathrm{E}+04$ & $3.48 \mathrm{E}-13$ \\
\hline Sr-85 & $6.72 \mathrm{E}+04$ & $5.76 \mathrm{E}-13$ \\
\hline Sr- $85 \mathrm{~m}$ & $1.59 \mathrm{E}+05$ & $1.36 \mathrm{E}-12$ \\
\hline Sr- $87 \mathrm{~m}$ & $1.05 \mathrm{E}+05$ & 8.97E-13 \\
\hline Sr-89 & $4.61 \mathrm{E}+05$ & $3.95 \mathrm{E}-12$ \\
\hline Sr-90 & $1.93 \mathrm{E}+07$ & $1.66 \mathrm{E}-10$ \\
\hline Sr-91 & $4.31 \mathrm{E}+04$ & $3.70 \mathrm{E}-13$ \\
\hline
\end{tabular}




\begin{tabular}{|c|c|c|}
\hline Nuclide & $\begin{array}{c}\text { Ground } \\
\text { Shine DCS } \\
\left(\mathbf{B q} / \mathbf{m}^{2}\right)\end{array}$ & $\begin{array}{c}\text { Ground } \\
\text { Shine DCS } \\
\left(\mu \mathrm{Ci} / \mathbf{m}^{2}\right)\end{array}$ \\
\hline Sr-92 & $2.60 \mathrm{E}+04$ & $2.23 \mathrm{E}-13$ \\
\hline Sr-93 & $1.46 \mathrm{E}+04$ & $1.25 \mathrm{E}-13$ \\
\hline Sr-94 & $2.26 \mathrm{E}+04$ & $1.94 \mathrm{E}-13$ \\
\hline$Y-81$ & $2.46 \mathrm{E}+04$ & $2.11 \mathrm{E}-13$ \\
\hline$Y-83$ & $2.25 \mathrm{E}+04$ & $1.93 \mathrm{E}-13$ \\
\hline Y-83m & $3.60 \mathrm{E}+04$ & $3.09 \mathrm{E}-13$ \\
\hline$Y-84 m$ & $8.21 \mathrm{E}+03$ & $7.04 \mathrm{E}-14$ \\
\hline $\mathrm{Y}-85$ & $2.91 \mathrm{E}+04$ & $2.49 \mathrm{E}-13$ \\
\hline $\mathrm{Y}-85 \mathrm{~m}$ & $2.46 \mathrm{E}+04$ & $2.11 \mathrm{E}-13$ \\
\hline $\mathrm{Y}-86$ & $9.58 \mathrm{E}+03$ & $8.21 \mathrm{E}-14$ \\
\hline $\mathrm{Y}-86 \mathrm{~m}$ & $1.56 \mathrm{E}+05$ & $1.34 \mathrm{E}-12$ \\
\hline $\mathrm{Y}-87$ & $7.55 \mathrm{E}+04$ & $6.47 \mathrm{E}-13$ \\
\hline $\mathrm{Y}-87 \mathrm{~m}$ & $1.09 \mathrm{E}+05$ & $9.34 \mathrm{E}-13$ \\
\hline Y-88 & $1.32 \mathrm{E}+04$ & $1.13 \mathrm{E}-13$ \\
\hline$Y-89 m$ & $3.73 \mathrm{E}+04$ & $3.20 \mathrm{E}-13$ \\
\hline Y-90 & $2.88 \mathrm{E}+05$ & $2.47 \mathrm{E}-12$ \\
\hline $\mathrm{Y}-90 \mathrm{~m}$ & $5.27 \mathrm{E}+04$ & $4.51 \mathrm{E}-13$ \\
\hline Y-91 & $4.27 \mathrm{E}+05$ & $3.66 \mathrm{E}-12$ \\
\hline Y-91m & $6.24 \mathrm{E}+04$ & $5.35 \mathrm{E}-13$ \\
\hline $\mathrm{Y}-92$ & $8.28 \mathrm{E}+04$ & $7.10 \mathrm{E}-13$ \\
\hline Y-93 & $1.46 \mathrm{E}+05$ & $1.25 \mathrm{E}-12$ \\
\hline Y-94 & $3.56 \mathrm{E}+04$ & $3.05 \mathrm{E}-13$ \\
\hline Y-95 & $2.91 \mathrm{E}+04$ & $2.49 \mathrm{E}-13$ \\
\hline Zr-85 & $2.06 \mathrm{E}+04$ & $1.76 \mathrm{E}-13$ \\
\hline Zr-86 & $1.21 \mathrm{E}+05$ & $1.03 \mathrm{E}-12$ \\
\hline Zr-87 & $3.23 \mathrm{E}+04$ & $2.77 \mathrm{E}-13$ \\
\hline $\mathrm{Zr}-88$ & $8.66 \mathrm{E}+04$ & $7.43 \mathrm{E}-13$ \\
\hline Zr-89 & $2.88 \mathrm{E}+04$ & $2.47 \mathrm{E}-13$ \\
\hline $\mathrm{Zr}-89 \mathrm{~m}$ & $5.25 \mathrm{E}+04$ & $4.50 \mathrm{E}-13$ \\
\hline Zr-95 & $4.56 \mathrm{E}+04$ & $3.90 \mathrm{E}-13$ \\
\hline Zr-97 & $3.45 \mathrm{E}+04$ & $2.96 \mathrm{E}-13$ \\
\hline $\mathrm{Nb}-87$ & $2.40 \mathrm{E}+04$ & $2.06 \mathrm{E}-13$ \\
\hline $\mathrm{Nb}-88$ & $7.72 \mathrm{E}+03$ & $6.61 \mathrm{E}-14$ \\
\hline $\mathrm{Nb}-88 \mathrm{~m}$ & $7.95 \mathrm{E}+03$ & $6.81 \mathrm{E}-14$ \\
\hline $\mathrm{Nb}-89$ & $2.31 \mathrm{E}+04$ & $1.98 \mathrm{E}-13$ \\
\hline $\mathrm{Nb}-89 \mathrm{~m}$ & $2.37 \mathrm{E}+04$ & $2.03 \mathrm{E}-13$ \\
\hline Nb-90 & $8.39 \mathrm{E}+03$ & 7.19E-14 \\
\hline $\mathrm{Nb}-91$ & $6.69 \mathrm{E}+06$ & $5.73 \mathrm{E}-11$ \\
\hline
\end{tabular}




\begin{tabular}{|c|c|c|}
\hline Nuclide & $\begin{array}{c}\text { Ground } \\
\text { Shine DCS } \\
\left(\mathbf{B q} / \mathbf{m}^{2}\right)\end{array}$ & $\begin{array}{c}\text { Ground } \\
\text { Shine DCS } \\
\left(\mu \mathrm{Ci} / \mathbf{m}^{2}\right)\end{array}$ \\
\hline $\mathrm{Nb}-91 \mathrm{~m}$ & $1.21 \mathrm{E}+06$ & $1.04 \mathrm{E}-11$ \\
\hline $\mathrm{Nb}-92$ & $2.23 \mathrm{E}+04$ & $1.91 \mathrm{E}-13$ \\
\hline $\mathrm{Nb}-92 \mathrm{~m}$ & $3.51 \mathrm{E}+04$ & $3.01 \mathrm{E}-13$ \\
\hline $\mathrm{Nb}-93 \mathrm{~m}$ & $4.64 \mathrm{E}+07$ & $3.98 \mathrm{E}-10$ \\
\hline $\mathrm{Nb}-94$ & $2.14 \mathrm{E}+04$ & $1.84 \mathrm{E}-13$ \\
\hline $\mathrm{Nb}-94 \mathrm{~m}$ & $4.55 \mathrm{E}+06$ & $3.90 \mathrm{E}-11$ \\
\hline $\mathrm{Nb}-95$ & $4.37 \mathrm{E}+04$ & $3.74 \mathrm{E}-13$ \\
\hline $\mathrm{Nb}-95 \mathrm{~m}$ & $5.07 \mathrm{E}+05$ & $4.35 \mathrm{E}-12$ \\
\hline $\mathrm{Nb}-96$ & $1.36 \mathrm{E}+04$ & $1.17 \mathrm{E}-13$ \\
\hline $\mathrm{Nb}-97$ & $4.65 \mathrm{E}+04$ & $3.98 \mathrm{E}-13$ \\
\hline $\mathrm{Nb}-98 \mathrm{~m}$ & $1.17 \mathrm{E}+04$ & $1.00 \mathrm{E}-13$ \\
\hline $\mathrm{Nb}-99$ & $1.03 \mathrm{E}+05$ & $8.82 \mathrm{E}-13$ \\
\hline $\mathrm{Nb}-99 \mathrm{~m}$ & $3.95 \mathrm{E}+04$ & $3.38 \mathrm{E}-13$ \\
\hline Мо-89 & $2.37 \mathrm{E}+04$ & $2.03 \mathrm{E}-13$ \\
\hline Мo-90 & $4.05 \mathrm{E}+04$ & $3.47 \mathrm{E}-13$ \\
\hline Мo-91 & $2.94 \mathrm{E}+04$ & $2.52 \mathrm{E}-13$ \\
\hline Мo-91m & $2.33 \mathrm{E}+04$ & $2.00 \mathrm{E}-13$ \\
\hline Mo-93 & $8.28 \mathrm{E}+06$ & $7.10 \mathrm{E}-11$ \\
\hline Mo-93m & $1.49 \mathrm{E}+04$ & $1.28 \mathrm{E}-13$ \\
\hline Мo-99 & $1.79 \mathrm{E}+05$ & $1.54 \mathrm{E}-12$ \\
\hline Mo-101 & $2.26 \mathrm{E}+04$ & $1.94 \mathrm{E}-13$ \\
\hline Mo-102 & $7.19 \mathrm{E}+05$ & $6.16 \mathrm{E}-12$ \\
\hline Tc-91 & $1.32 \mathrm{E}+04$ & $1.13 \mathrm{E}-13$ \\
\hline Tc-91m & $2.07 \mathrm{E}+04$ & $1.78 \mathrm{E}-13$ \\
\hline Tc-92 & $8.55 \mathrm{E}+03$ & 7.33E-14 \\
\hline Tc-93 & $2.23 \mathrm{E}+04$ & $1.91 \mathrm{E}-13$ \\
\hline Tc-93m & $3.82 \mathrm{E}+04$ & $3.27 \mathrm{E}-13$ \\
\hline Tc-94 & $1.26 \mathrm{E}+04$ & $1.08 \mathrm{E}-13$ \\
\hline Tc-94m & $1.66 \mathrm{E}+04$ & $1.42 \mathrm{E}-13$ \\
\hline Tc-95 & $4.24 \mathrm{E}+04$ & $3.63 \mathrm{E}-13$ \\
\hline Tc-95m & $4.93 \mathrm{E}+04$ & $4.23 \mathrm{E}-13$ \\
\hline Tc-96 & $1.34 \mathrm{E}+04$ & $1.15 \mathrm{E}-13$ \\
\hline Tc-96m & $7.62 \mathrm{E}+05$ & $6.53 \mathrm{E}-12$ \\
\hline Tc-97 & $6.95 \mathrm{E}+06$ & $5.96 \mathrm{E}-11$ \\
\hline Tc-97m & $7.26 \mathrm{E}+06$ & $6.22 \mathrm{E}-11$ \\
\hline Tc-98 & $2.37 \mathrm{E}+04$ & $2.03 \mathrm{E}-13$ \\
\hline Tc-99 & $4.84 \mathrm{E}+08$ & $4.15 \mathrm{E}-09$ \\
\hline Tc-99m & $2.78 \mathrm{E}+05$ & $2.38 \mathrm{E}-12$ \\
\hline
\end{tabular}




\begin{tabular}{|c|c|c|}
\hline Nuclide & $\begin{array}{c}\text { Ground } \\
\text { Shine DCS } \\
\left(\mathbf{B q} / \mathbf{m}^{2}\right)\end{array}$ & $\begin{array}{c}\text { Ground } \\
\text { Shine DCS } \\
\left(\mu \mathrm{Ci} / \mathbf{m}^{2}\right)\end{array}$ \\
\hline Tc-101 & $8.66 \mathrm{E}+04$ & $7.43 \mathrm{E}-13$ \\
\hline Tc-102 & $1.22 \mathrm{E}+05$ & $1.05 \mathrm{E}-12$ \\
\hline Tc- $102 \mathrm{~m}$ & $1.36 \mathrm{E}+04$ & $1.16 \mathrm{E}-13$ \\
\hline Tc-104 & $1.46 \mathrm{E}+04$ & $1.25 \mathrm{E}-13$ \\
\hline Tc-105 & $3.65 \mathrm{E}+04$ & $3.13 \mathrm{E}-13$ \\
\hline Ru-92 & $1.59 \mathrm{E}+04$ & $1.36 \mathrm{E}-13$ \\
\hline Ru-94 & $6.54 \mathrm{E}+04$ & $5.60 \mathrm{E}-13$ \\
\hline Ru-95 & $2.73 \mathrm{E}+04$ & $2.34 \mathrm{E}-13$ \\
\hline Ru-97 & $1.46 \mathrm{E}+05$ & $1.25 \mathrm{E}-12$ \\
\hline Ru-103 & $6.69 \mathrm{E}+04$ & $5.73 \mathrm{E}-13$ \\
\hline Ru-105 & $4.24 \mathrm{E}+04$ & $3.63 \mathrm{E}-13$ \\
\hline Ru-107 & $7.17 \mathrm{E}+04$ & $6.15 \mathrm{E}-13$ \\
\hline Ru-108 & $3.02 \mathrm{E}+05$ & $2.59 \mathrm{E}-12$ \\
\hline Rh-94 & $8.57 \mathrm{E}+03$ & $7.34 \mathrm{E}-14$ \\
\hline Rh-95 & $1.29 \mathrm{E}+04$ & $1.11 \mathrm{E}-13$ \\
\hline Rh-95m & $3.85 \mathrm{E}+04$ & $3.30 \mathrm{E}-13$ \\
\hline Rh-96 & $8.41 \mathrm{E}+03$ & $7.21 \mathrm{E}-14$ \\
\hline Rh-96m & $2.56 \mathrm{E}+04$ & $2.19 \mathrm{E}-13$ \\
\hline Rh-97 & $2.23 \mathrm{E}+04$ & $1.91 \mathrm{E}-13$ \\
\hline Rh-97m & $1.61 \mathrm{E}+04$ & $1.38 \mathrm{E}-13$ \\
\hline Rh-98 & $1.71 \mathrm{E}+04$ & $1.47 \mathrm{E}-13$ \\
\hline Rh-99 & $6.06 \mathrm{E}+04$ & $5.20 \mathrm{E}-13$ \\
\hline Rh-99m & $5.22 \mathrm{E}+04$ & $4.47 \mathrm{E}-13$ \\
\hline Rh-100 & $1.29 \mathrm{E}+04$ & $1.10 \mathrm{E}-13$ \\
\hline $\mathrm{Rh}-100 \mathrm{~m}$ & $6.16 \mathrm{E}+05$ & $5.28 \mathrm{E}-12$ \\
\hline Rh-101 & $1.23 \mathrm{E}+05$ & $1.06 \mathrm{E}-12$ \\
\hline Rh-101m & $1.20 \mathrm{E}+05$ & $1.03 \mathrm{E}-12$ \\
\hline Rh-102 & $6.42 \mathrm{E}+04$ & $5.50 \mathrm{E}-13$ \\
\hline Rh-102m & $1.56 \mathrm{E}+04$ & $1.34 \mathrm{E}-13$ \\
\hline Rh-103m & $3.84 \mathrm{E}+07$ & $3.29 \mathrm{E}-10$ \\
\hline Rh-104 & $2.48 \mathrm{E}+05$ & $2.12 \mathrm{E}-12$ \\
\hline Rh-104m & $9.24 \mathrm{E}+05$ & $7.92 \mathrm{E}-12$ \\
\hline Rh-105 & $4.30 \mathrm{E}+05$ & $3.68 \mathrm{E}-12$ \\
\hline Rh-106 & $9.16 \mathrm{E}+04$ & $7.85 \mathrm{E}-13$ \\
\hline Rh-106m & $1.18 \mathrm{E}+04$ & $1.01 \mathrm{E}-13$ \\
\hline Rh-107 & $9.38 \mathrm{E}+04$ & $8.04 \mathrm{E}-13$ \\
\hline Rh-108 & $6.62 \mathrm{E}+04$ & $5.67 \mathrm{E}-13$ \\
\hline Rh-109 & $8.11 \mathrm{E}+04$ & $6.95 \mathrm{E}-13$ \\
\hline
\end{tabular}




\begin{tabular}{|c|c|c|}
\hline Nuclide & $\begin{array}{c}\text { Ground } \\
\text { Shine DCS } \\
\left(\mathbf{B q} / \mathbf{m}^{2}\right)\end{array}$ & $\begin{array}{c}\text { Ground } \\
\text { Shine DCS } \\
\left(\mu \mathrm{Ci} / \mathbf{m}^{2}\right)\end{array}$ \\
\hline $\mathrm{Pd}-96$ & $2.30 \mathrm{E}+04$ & $1.97 \mathrm{E}-13$ \\
\hline $\mathrm{Pd}-97$ & $1.40 \mathrm{E}+04$ & $1.20 \mathrm{E}-13$ \\
\hline Pd-98 & $8.30 \mathrm{E}+04$ & $7.11 \mathrm{E}-13$ \\
\hline Pd-99 & $2.56 \mathrm{E}+04$ & $2.19 \mathrm{E}-13$ \\
\hline Pd-100 & $3.18 \mathrm{E}+05$ & $2.72 \mathrm{E}-12$ \\
\hline Pd-101 & $9.88 \mathrm{E}+04$ & $8.47 \mathrm{E}-13$ \\
\hline Pd-103 & $4.14 \mathrm{E}+06$ & $3.55 \mathrm{E}-11$ \\
\hline Pd-109 & $8.52 \mathrm{E}+05$ & $7.31 \mathrm{E}-12$ \\
\hline $\mathrm{Pd}-109 \mathrm{~m}$ & $3.17 \mathrm{E}+05$ & $2.72 \mathrm{E}-12$ \\
\hline Pd-111 & $2.17 \mathrm{E}+05$ & $1.86 \mathrm{E}-12$ \\
\hline Pd-112 & $1.43 \mathrm{E}+07$ & $1.22 \mathrm{E}-10$ \\
\hline Pd-114 & $3.79 \mathrm{E}+05$ & $3.25 \mathrm{E}-12$ \\
\hline Ag-99 & $1.39 \mathrm{E}+04$ & $1.19 \mathrm{E}-13$ \\
\hline Ag-100m & $1.13 \mathrm{E}+04$ & $9.67 \mathrm{E}-14$ \\
\hline Ag-101 & $2.02 \mathrm{E}+04$ & $1.73 \mathrm{E}-13$ \\
\hline Ag-102 & $9.79 \mathrm{E}+03$ & $8.39 \mathrm{E}-14$ \\
\hline $\mathrm{Ag}-102 \mathrm{~m}$ & $1.75 \mathrm{E}+04$ & $1.50 \mathrm{E}-13$ \\
\hline Ag-103 & $3.94 \mathrm{E}+04$ & $3.38 \mathrm{E}-13$ \\
\hline Ag-104 & $1.25 \mathrm{E}+04$ & $1.07 \mathrm{E}-13$ \\
\hline Ag-104m & $1.82 \mathrm{E}+04$ & $1.56 \mathrm{E}-13$ \\
\hline Ag-105 & $6.63 \mathrm{E}+04$ & $5.69 \mathrm{E}-13$ \\
\hline Ag-105m & $3.30 \mathrm{E}+07$ & $2.83 \mathrm{E}-10$ \\
\hline Ag-106 & $4.36 \mathrm{E}+04$ & $3.73 \mathrm{E}-13$ \\
\hline Ag-106m & $1.21 \mathrm{E}+04$ & $1.03 \mathrm{E}-13$ \\
\hline Ag-108 & $3.53 \mathrm{E}+05$ & $3.03 \mathrm{E}-12$ \\
\hline Ag-108m & $2.06 \mathrm{E}+04$ & $1.76 \mathrm{E}-13$ \\
\hline Ag-109m & $4.28 \mathrm{E}+06$ & $3.67 \mathrm{E}-11$ \\
\hline Ag-110 & $1.95 \mathrm{E}+05$ & $1.67 \mathrm{E}-12$ \\
\hline $\mathrm{Ag}-110 \mathrm{~m}$ & $1.22 \mathrm{E}+04$ & $1.05 \mathrm{E}-13$ \\
\hline Ag-111 & $5.99 \mathrm{E}+05$ & $5.14 \mathrm{E}-12$ \\
\hline Ag-111m & $5.48 \mathrm{E}+06$ & $4.69 \mathrm{E}-11$ \\
\hline Ag-112 & $4.11 \mathrm{E}+04$ & $3.52 \mathrm{E}-13$ \\
\hline $\mathrm{Ag}-113$ & $1.99 \mathrm{E}+05$ & $1.71 \mathrm{E}-12$ \\
\hline $\mathrm{Ag}-113 \mathrm{~m}$ & $1.42 \mathrm{E}+05$ & $1.22 \mathrm{E}-12$ \\
\hline Ag-114 & $7.36 \mathrm{E}+04$ & $6.31 \mathrm{E}-13$ \\
\hline Ag-115 & $5.72 \mathrm{E}+04$ & $4.91 \mathrm{E}-13$ \\
\hline Ag-116 & $1.53 \mathrm{E}+04$ & $1.31 \mathrm{E}-13$ \\
\hline Ag-117 & $2.48 \mathrm{E}+04$ & $2.12 \mathrm{E}-13$ \\
\hline
\end{tabular}




\begin{tabular}{|c|c|c|}
\hline Nuclide & $\begin{array}{c}\text { Ground } \\
\text { Shine DCS } \\
\left(\mathrm{Bq} / \mathbf{m}^{2}\right)\end{array}$ & $\begin{array}{c}\text { Ground } \\
\text { Shine DCS } \\
\left(\mu \mathrm{Ci} / \mathbf{m}^{2}\right)\end{array}$ \\
\hline Cd-101 & $1.33 \mathrm{E}+04$ & $1.14 \mathrm{E}-13$ \\
\hline Cd-102 & $4.01 \mathrm{E}+04$ & $3.44 \mathrm{E}-13$ \\
\hline $\mathrm{Cd}-103$ & $1.66 \mathrm{E}+04$ & $1.42 \mathrm{E}-13$ \\
\hline Cd-104 & $1.38 \mathrm{E}+05$ & $1.19 \mathrm{E}-12$ \\
\hline Cd-105 & $2.64 \mathrm{E}+04$ & $2.26 \mathrm{E}-13$ \\
\hline Cd-107 & $1.39 \mathrm{E}+06$ & $1.19 \mathrm{E}-11$ \\
\hline Cd-109 & $1.92 \mathrm{E}+06$ & $1.65 \mathrm{E}-11$ \\
\hline $\mathrm{Cd}-111 \mathrm{~m}$ & $1.22 \mathrm{E}+05$ & $1.05 \mathrm{E}-12$ \\
\hline $\mathrm{Cd}-113$ & $5.52 \mathrm{E}+08$ & 4.73E-09 \\
\hline Cd-113m & $1.78 \mathrm{E}+07$ & $1.53 \mathrm{E}-10$ \\
\hline $\mathrm{Cd}-115$ & $1.53 \mathrm{E}+05$ & $1.31 \mathrm{E}-12$ \\
\hline $\mathrm{Cd}-115 \mathrm{~m}$ & $3.11 \mathrm{E}+05$ & $2.66 \mathrm{E}-12$ \\
\hline Cd-117 & $3.08 \mathrm{E}+04$ & $2.64 \mathrm{E}-13$ \\
\hline $\mathrm{Cd}-117 \mathrm{~m}$ & $1.72 \mathrm{E}+04$ & $1.48 \mathrm{E}-13$ \\
\hline Cd-118 & $5.47 \mathrm{E}+07$ & $4.69 \mathrm{E}-10$ \\
\hline $\mathrm{Cd}-119$ & $2.03 \mathrm{E}+04$ & $1.74 \mathrm{E}-13$ \\
\hline $\mathrm{Cd}-119 \mathrm{~m}$ & $1.47 \mathrm{E}+04$ & $1.26 \mathrm{E}-13$ \\
\hline In-103 & $1.19 \mathrm{E}+04$ & $1.02 \mathrm{E}-13$ \\
\hline In-105 & $1.67 \mathrm{E}+04$ & $1.43 \mathrm{E}-13$ \\
\hline In-106m & $1.16 \mathrm{E}+04$ & $9.95 \mathrm{E}-14$ \\
\hline In-106 & $9.14 \mathrm{E}+03$ & $7.83 \mathrm{E}-14$ \\
\hline In-107 & $2.22 \mathrm{E}+04$ & $1.90 \mathrm{E}-13$ \\
\hline In-108m & $1.26 \mathrm{E}+04$ & $1.08 \mathrm{E}-13$ \\
\hline In-108 & $8.66 \mathrm{E}+03$ & 7.43E-14 \\
\hline In-109m & $5.41 \mathrm{E}+04$ & $4.64 \mathrm{E}-13$ \\
\hline In-109 & $5.34 \mathrm{E}+04$ & $4.58 \mathrm{E}-13$ \\
\hline $\mathrm{In}-110 \mathrm{~m}$ & $2.05 \mathrm{E}+04$ & $1.75 \mathrm{E}-13$ \\
\hline In-110 & $1.09 \mathrm{E}+04$ & $9.31 \mathrm{E}-14$ \\
\hline In-111m & $6.95 \mathrm{E}+04$ & $5.96 \mathrm{E}-13$ \\
\hline In-111 & $8.59 \mathrm{E}+04$ & 7.36E-13 \\
\hline In-112m & $1.13 \mathrm{E}+06$ & $9.71 \mathrm{E}-12$ \\
\hline In-112 & $1.16 \mathrm{E}+05$ & $9.92 \mathrm{E}-13$ \\
\hline In-113m & $1.29 \mathrm{E}+05$ & $1.10 \mathrm{E}-12$ \\
\hline In-114m & $4.36 \mathrm{E}+05$ & $3.73 \mathrm{E}-12$ \\
\hline In-114 & $3.29 \mathrm{E}+05$ & $2.82 \mathrm{E}-12$ \\
\hline In-115m & $2.09 \mathrm{E}+05$ & $1.79 \mathrm{E}-12$ \\
\hline In-115 & $8.48 \mathrm{E}+07$ & $7.27 \mathrm{E}-10$ \\
\hline In-116m & $1.40 \mathrm{E}+04$ & $1.20 \mathrm{E}-13$ \\
\hline
\end{tabular}




\begin{tabular}{|c|c|c|}
\hline Nuclide & $\begin{array}{c}\text { Ground } \\
\text { Shine DCS } \\
\left(\mathrm{Bq} / \mathbf{m}^{2}\right)\end{array}$ & $\begin{array}{c}\text { Ground } \\
\text { Shine DCS } \\
\left(\mu \mathrm{Ci} / \mathbf{m}^{2}\right)\end{array}$ \\
\hline In-117m & $2.54 \mathrm{E}+05$ & $2.17 \mathrm{E}-12$ \\
\hline In-117 & $4.77 \mathrm{E}+04$ & $4.09 \mathrm{E}-13$ \\
\hline In-118m & $1.20 \mathrm{E}+04$ & $1.03 \mathrm{E}-13$ \\
\hline In-118 & $1.26 \mathrm{E}+05$ & $1.08 \mathrm{E}-12$ \\
\hline In-119m & $1.79 \mathrm{E}+05$ & $1.54 \mathrm{E}-12$ \\
\hline In-119 & $3.97 \mathrm{E}+04$ & $3.40 \mathrm{E}-13$ \\
\hline In- $121 \mathrm{~m}$ & $1.50 \mathrm{E}+05$ & $1.28 \mathrm{E}-12$ \\
\hline In-121 & $3.21 \mathrm{E}+04$ & $2.75 \mathrm{E}-13$ \\
\hline Sn-106 & $2.78 \mathrm{E}+04$ & $2.38 \mathrm{E}-13$ \\
\hline Sn-108 & $4.95 \mathrm{E}+04$ & $4.25 \mathrm{E}-13$ \\
\hline Sn-109 & $1.59 \mathrm{E}+04$ & $1.37 \mathrm{E}-13$ \\
\hline Sn-110 & $1.18 \mathrm{E}+05$ & $1.01 \mathrm{E}-12$ \\
\hline Sn-111 & $6.63 \mathrm{E}+04$ & $5.69 \mathrm{E}-13$ \\
\hline Sn-113m & $3.40 \mathrm{E}+06$ & $2.92 \mathrm{E}-11$ \\
\hline Sn-113 & $1.87 \mathrm{E}+06$ & $1.60 \mathrm{E}-11$ \\
\hline $\mathrm{Sn}-117 \mathrm{~m}$ & $2.26 \mathrm{E}+05$ & $1.94 \mathrm{E}-12$ \\
\hline Sn-119m & $3.23 \mathrm{E}+06$ & $2.77 \mathrm{E}-11$ \\
\hline Sn-121m & $8.69 \mathrm{E}+06$ & $7.45 \mathrm{E}-11$ \\
\hline $\mathrm{Sn}-121$ & $3.50 \mathrm{E}+08$ & 3.00E-09 \\
\hline Sn- $123 \mathrm{~m}$ & $1.81 \mathrm{E}+05$ & $1.55 \mathrm{E}-12$ \\
\hline Sn-123 & $4.84 \mathrm{E}+05$ & $4.15 \mathrm{E}-12$ \\
\hline $\mathrm{Sn}-125 \mathrm{~m}$ & $7.50 \mathrm{E}+04$ & $6.42 \mathrm{E}-13$ \\
\hline Sn-125 & $7.91 \mathrm{E}+04$ & $6.78 \mathrm{E}-13$ \\
\hline Sn-126 & $6.58 \mathrm{E}+05$ & $5.64 \mathrm{E}-12$ \\
\hline Sn- $127 m$ & $4.77 \mathrm{E}+04$ & $4.09 \mathrm{E}-13$ \\
\hline Sn-127 & $1.76 \mathrm{E}+04$ & $1.51 \mathrm{E}-13$ \\
\hline Sn-128 & $5.59 \mathrm{E}+04$ & $4.79 \mathrm{E}-13$ \\
\hline Sn-129 & $2.94 \mathrm{E}+04$ & $2.52 \mathrm{E}-13$ \\
\hline Sn-130m & $3.29 \mathrm{E}+04$ & $2.82 \mathrm{E}-13$ \\
\hline Sn-130 & $3.45 \mathrm{E}+04$ & $2.96 \mathrm{E}-13$ \\
\hline Sb-111 & $2.05 \mathrm{E}+04$ & $1.75 \mathrm{E}-13$ \\
\hline Sb-113 & $2.46 \mathrm{E}+04$ & $2.11 \mathrm{E}-13$ \\
\hline Sb-114 & $1.21 \mathrm{E}+04$ & $1.04 \mathrm{E}-13$ \\
\hline Sb-115 & $3.64 \mathrm{E}+04$ & $3.12 \mathrm{E}-13$ \\
\hline $\mathrm{Sb}-116$ & $1.47 \mathrm{E}+04$ & $1.26 \mathrm{E}-13$ \\
\hline $\mathrm{Sb}-116 \mathrm{~m}$ & $1.10 \mathrm{E}+04$ & $9.40 \mathrm{E}-14$ \\
\hline Sb-117 & $1.91 \mathrm{E}+05$ & $1.64 \mathrm{E}-12$ \\
\hline Sb-118 & $3.66 \mathrm{E}+04$ & $3.13 \mathrm{E}-13$ \\
\hline
\end{tabular}




\begin{tabular}{|c|c|c|}
\hline Nuclide & $\begin{array}{c}\text { Ground } \\
\text { Shine DCS } \\
\left(\mathbf{B q} / \mathbf{m}^{2}\right)\end{array}$ & $\begin{array}{c}\text { Ground } \\
\text { Shine DCS } \\
\left(\mu \mathrm{Ci} / \mathbf{m}^{2}\right)\end{array}$ \\
\hline $\mathrm{Sb}-118 \mathrm{~m}$ & $1.32 \mathrm{E}+04$ & $1.13 \mathrm{E}-13$ \\
\hline $\mathrm{Sb}-119$ & $2.03 \mathrm{E}+06$ & $1.74 \mathrm{E}-11$ \\
\hline Sb-120 & $6.79 \mathrm{E}+04$ & $5.82 \mathrm{E}-13$ \\
\hline $\mathrm{Sb}-120 \mathrm{~m}$ & $1.39 \mathrm{E}+04$ & $1.19 \mathrm{E}-13$ \\
\hline $\mathrm{Sb}-122$ & $6.50 \mathrm{E}+04$ & $5.57 \mathrm{E}-13$ \\
\hline $\mathrm{Sb}-122 \mathrm{~m}$ & $5.39 \mathrm{E}+05$ & $4.62 \mathrm{E}-12$ \\
\hline $\mathrm{Sb}-124$ & $1.83 \mathrm{E}+04$ & $1.57 \mathrm{E}-13$ \\
\hline $\mathrm{Sb}-124 \mathrm{~m}$ & $7.37 \mathrm{E}+04$ & $6.32 \mathrm{E}-13$ \\
\hline $\mathrm{Sb}-124 n$ & $5.57 \mathrm{E}+10$ & $4.78 \mathrm{E}-07$ \\
\hline $\mathrm{Sb}-125$ & $7.66 \mathrm{E}+04$ & $6.56 \mathrm{E}-13$ \\
\hline $\mathrm{Sb}-126$ & $1.20 \mathrm{E}+04$ & $1.03 \mathrm{E}-13$ \\
\hline $\mathrm{Sb}-126 \mathrm{~m}$ & $2.05 \mathrm{E}+04$ & $1.75 \mathrm{E}-13$ \\
\hline Sb-127 & $4.66 \mathrm{E}+04$ & $4.00 \mathrm{E}-13$ \\
\hline $\mathrm{Sb}-128$ & $1.06 \mathrm{E}+04$ & $9.12 \mathrm{E}-14$ \\
\hline Sb-128m & $1.65 \mathrm{E}+04$ & $1.42 \mathrm{E}-13$ \\
\hline Sb-129 & $2.28 \mathrm{E}+04$ & $1.96 \mathrm{E}-13$ \\
\hline Sb-130 & $1.01 \mathrm{E}+04$ & $8.63 \mathrm{E}-14$ \\
\hline Sb-130m & $1.19 \mathrm{E}+04$ & $1.02 \mathrm{E}-13$ \\
\hline $\mathrm{Sb}-131$ & $1.62 \mathrm{E}+04$ & $1.39 \mathrm{E}-13$ \\
\hline $\mathrm{Sb}-133$ & $1.25 \mathrm{E}+04$ & $1.07 \mathrm{E}-13$ \\
\hline Te-113 & $1.43 \mathrm{E}+04$ & $1.22 \mathrm{E}-13$ \\
\hline $\mathrm{Te}-114$ & $2.69 \mathrm{E}+04$ & $2.30 \mathrm{E}-13$ \\
\hline Te-115 & $1.45 \mathrm{E}+04$ & $1.25 \mathrm{E}-13$ \\
\hline Te-115m & $1.28 \mathrm{E}+04$ & $1.10 \mathrm{E}-13$ \\
\hline Te-116 & $3.27 \mathrm{E}+05$ & $2.80 \mathrm{E}-12$ \\
\hline Te-117 & $2.19 \mathrm{E}+04$ & $1.87 \mathrm{E}-13$ \\
\hline Te-118 & $2.23 \mathrm{E}+06$ & $1.91 \mathrm{E}-11$ \\
\hline Te-119 & $4.40 \mathrm{E}+04$ & $3.77 \mathrm{E}-13$ \\
\hline Te-119m & $2.30 \mathrm{E}+04$ & $1.97 \mathrm{E}-13$ \\
\hline Te-121 & $5.80 \mathrm{E}+04$ & $4.97 \mathrm{E}-13$ \\
\hline Te- $121 \mathrm{~m}$ & $1.60 \mathrm{E}+05$ & $1.37 \mathrm{E}-12$ \\
\hline Te-123 & $1.28 \mathrm{E}+09$ & $1.10 \mathrm{E}-08$ \\
\hline Te-123m & $2.40 \mathrm{E}+05$ & $2.06 \mathrm{E}-12$ \\
\hline Te-125m & $1.18 \mathrm{E}+06$ & $1.01 \mathrm{E}-11$ \\
\hline Te-127 & $3.02 \mathrm{E}+06$ & $2.59 \mathrm{E}-11$ \\
\hline $\mathrm{Te}-127 \mathrm{~m}$ & $3.71 \mathrm{E}+06$ & $3.18 \mathrm{E}-11$ \\
\hline Te-129 & $2.73 \mathrm{E}+05$ & $2.34 \mathrm{E}-12$ \\
\hline Te-129m & $5.44 \mathrm{E}+05$ & $4.66 \mathrm{E}-12$ \\
\hline
\end{tabular}




\begin{tabular}{|c|c|c|}
\hline Nuclide & $\begin{array}{c}\text { Ground } \\
\text { Shine DCS } \\
\left(\mathbf{B q} / \mathbf{m}^{2}\right)\end{array}$ & $\begin{array}{c}\text { Ground } \\
\text { Shine DCS } \\
\left(\mu \mathrm{Ci} / \mathbf{m}^{2}\right)\end{array}$ \\
\hline Te-131 & $6.72 \mathrm{E}+04$ & $5.76 \mathrm{E}-13$ \\
\hline Te-131m & $2.33 \mathrm{E}+04$ & $2.00 \mathrm{E}-13$ \\
\hline Te-132 & $1.49 \mathrm{E}+05$ & $1.28 \mathrm{E}-12$ \\
\hline Te-133 & $2.69 \mathrm{E}+04$ & $2.30 \mathrm{E}-13$ \\
\hline Te-133m & $1.80 \mathrm{E}+04$ & $1.54 \mathrm{E}-13$ \\
\hline Te-134 & $3.84 \mathrm{E}+04$ & $3.29 \mathrm{E}-13$ \\
\hline $\mathrm{I}-118$ & $1.52 \mathrm{E}+04$ & $1.31 \mathrm{E}-13$ \\
\hline $\mathrm{I}-118 \mathrm{~m}$ & $8.64 \mathrm{E}+03$ & $7.40 \mathrm{E}-14$ \\
\hline I-119 & $3.44 \mathrm{E}+04$ & $2.95 \mathrm{E}-13$ \\
\hline $\mathrm{I}-120$ & $1.26 \mathrm{E}+04$ & $1.08 \mathrm{E}-13$ \\
\hline $\mathrm{I}-120 \mathrm{~m}$ & $9.35 \mathrm{E}+03$ & $8.02 \mathrm{E}-14$ \\
\hline I-121 & $8.46 \mathrm{E}+04$ & $7.25 \mathrm{E}-13$ \\
\hline $\mathrm{I}-122$ & $3.05 \mathrm{E}+04$ & $2.61 \mathrm{E}-13$ \\
\hline I-123 & $2.06 \mathrm{E}+05$ & $1.76 \mathrm{E}-12$ \\
\hline $\mathrm{I}-124$ & $3.02 \mathrm{E}+04$ & $2.59 \mathrm{E}-13$ \\
\hline $\mathrm{I}-125$ & $9.94 \mathrm{E}+05$ & $8.52 \mathrm{E}-12$ \\
\hline $\mathrm{I}-126$ & $7.50 \mathrm{E}+04$ & $6.42 \mathrm{E}-13$ \\
\hline I-128 & $2.06 \mathrm{E}+05$ & $1.76 \mathrm{E}-12$ \\
\hline I-129 & $1.59 \mathrm{E}+06$ & $1.37 \mathrm{E}-11$ \\
\hline $\mathrm{I}-130$ & $1.55 \mathrm{E}+04$ & $1.33 \mathrm{E}-13$ \\
\hline $\mathrm{I}-130 \mathrm{~m}$ & $2.64 \mathrm{E}+05$ & $2.26 \mathrm{E}-12$ \\
\hline I-131 & $8.69 \mathrm{E}+04$ & $7.45 \mathrm{E}-13$ \\
\hline $\mathrm{I}-132$ & $1.45 \mathrm{E}+04$ & $1.25 \mathrm{E}-13$ \\
\hline $\mathrm{I}-132 \mathrm{~m}$ & $9.70 \mathrm{E}+04$ & $8.31 \mathrm{E}-13$ \\
\hline I-133 & $5.10 \mathrm{E}+04$ & 4.37E-13 \\
\hline I-134 & $1.27 \mathrm{E}+04$ & $1.09 \mathrm{E}-13$ \\
\hline $\mathrm{I}-134 \mathrm{~m}$ & $1.18 \mathrm{E}+05$ & $1.01 \mathrm{E}-12$ \\
\hline I-135 & $2.16 \mathrm{E}+04$ & $1.85 \mathrm{E}-13$ \\
\hline Xe-120 & $8.55 \mathrm{E}+04$ & 7.33E-13 \\
\hline Xe-121 & $2.26 \mathrm{E}+04$ & $1.94 \mathrm{E}-13$ \\
\hline $\mathrm{Xe}-122$ & $5.28 \mathrm{E}+05$ & $4.52 \mathrm{E}-12$ \\
\hline $\mathrm{Xe}-123$ & $5.21 \mathrm{E}+04$ & $4.46 \mathrm{E}-13$ \\
\hline $\mathrm{Xe}-125$ & $1.28 \mathrm{E}+05$ & $1.10 \mathrm{E}-12$ \\
\hline $\mathrm{Xe}-127$ & $1.24 \mathrm{E}+05$ & $1.06 \mathrm{E}-12$ \\
\hline $\mathrm{Xe}-127 \mathrm{~m}$ & $2.10 \mathrm{E}+05$ & $1.80 \mathrm{E}-12$ \\
\hline Xe-129m & $7.60 \mathrm{E}+05$ & $6.52 \mathrm{E}-12$ \\
\hline $\mathrm{Xe}-131 \mathrm{~m}$ & $1.95 \mathrm{E}+06$ & $1.67 \mathrm{E}-11$ \\
\hline $\mathrm{Xe}-133$ & $7.81 \mathrm{E}+05$ & $6.69 \mathrm{E}-12$ \\
\hline
\end{tabular}




\begin{tabular}{|c|c|c|}
\hline Nuclide & $\begin{array}{c}\text { Ground } \\
\text { Shine DCS } \\
\left(\mathrm{Bq} / \mathbf{m}^{2}\right)\end{array}$ & $\begin{array}{c}\text { Ground } \\
\text { Shine DCS } \\
\left(\mu \mathrm{Ci} / \mathbf{m}^{2}\right)\end{array}$ \\
\hline $\mathrm{Xe}-133 \mathrm{~m}$ & $8.98 \mathrm{E}+05$ & $7.70 \mathrm{E}-12$ \\
\hline $\mathrm{Xe}-135$ & $1.27 \mathrm{E}+05$ & $1.09 \mathrm{E}-12$ \\
\hline $\mathrm{Xe}-135 \mathrm{~m}$ & $7.64 \mathrm{E}+04$ & $6.55 \mathrm{E}-13$ \\
\hline $\mathrm{Xe}-137$ & $9.11 \mathrm{E}+04$ & $7.81 \mathrm{E}-13$ \\
\hline $\mathrm{Xe}-138$ & $2.96 \mathrm{E}+04$ & $2.54 \mathrm{E}-13$ \\
\hline Cs-121 & $2.48 \mathrm{E}+04$ & $2.12 \mathrm{E}-13$ \\
\hline Cs-121m & $2.54 \mathrm{E}+04$ & $2.17 \mathrm{E}-13$ \\
\hline Cs-123 & $2.81 \mathrm{E}+04$ & $2.40 \mathrm{E}-13$ \\
\hline Cs-124 & $2.48 \mathrm{E}+04$ & $2.12 \mathrm{E}-13$ \\
\hline Cs-125 & $4.21 \mathrm{E}+04$ & $3.60 \mathrm{E}-13$ \\
\hline Cs-126 & $2.58 \mathrm{E}+04$ & $2.21 \mathrm{E}-13$ \\
\hline Cs-127 & $7.77 \mathrm{E}+04$ & $6.66 \mathrm{E}-13$ \\
\hline Cs-128 & $3.35 \mathrm{E}+04$ & $2.87 \mathrm{E}-13$ \\
\hline Cs-129 & $1.22 \mathrm{E}+05$ & $1.05 \mathrm{E}-12$ \\
\hline Cs-130 & $6.04 \mathrm{E}+04$ & $5.18 \mathrm{E}-13$ \\
\hline Cs-130m & $5.07 \mathrm{E}+05$ & $4.34 \mathrm{E}-12$ \\
\hline Cs-131 & $1.76 \mathrm{E}+06$ & $1.51 \mathrm{E}-11$ \\
\hline Cs-132 & $4.68 \mathrm{E}+04$ & $4.01 \mathrm{E}-13$ \\
\hline Cs-134 & $2.14 \mathrm{E}+04$ & $1.84 \mathrm{E}-13$ \\
\hline $\mathrm{Cs}-134 \mathrm{~m}$ & $1.41 \mathrm{E}+06$ & $1.21 \mathrm{E}-11$ \\
\hline Cs-135 & $6.27 \mathrm{E}+08$ & $5.37 \mathrm{E}-09$ \\
\hline Cs-135m & $2.09 \mathrm{E}+04$ & $1.79 \mathrm{E}-13$ \\
\hline Cs-136 & $1.59 \mathrm{E}+04$ & $1.37 \mathrm{E}-13$ \\
\hline Cs-137 & $1.01 \mathrm{E}+07$ & $8.68 \mathrm{E}-11$ \\
\hline Cs-138 & $1.40 \mathrm{E}+04$ & $1.20 \mathrm{E}-13$ \\
\hline Cs-138m & $7.79 \mathrm{E}+04$ & $6.68 \mathrm{E}-13$ \\
\hline Cs-139 & $7.32 \mathrm{E}+04$ & $6.28 \mathrm{E}-13$ \\
\hline Cs-140 & $1.82 \mathrm{E}+04$ & $1.56 \mathrm{E}-13$ \\
\hline Ba-124 & $5.73 \mathrm{E}+04$ & $4.91 \mathrm{E}-13$ \\
\hline $\mathrm{Ba}-126$ & $5.87 \mathrm{E}+04$ & $5.03 \mathrm{E}-13$ \\
\hline Ba-127 & $4.21 \mathrm{E}+04$ & $3.60 \mathrm{E}-13$ \\
\hline Ba-128 & $5.41 \mathrm{E}+05$ & $4.64 \mathrm{E}-12$ \\
\hline Ba-129 & $9.82 \mathrm{E}+04$ & $8.41 \mathrm{E}-13$ \\
\hline $\mathrm{Ba}-129 \mathrm{~m}$ & $2.16 \mathrm{E}+04$ & $1.85 \mathrm{E}-13$ \\
\hline Ba-131 & $7.13 \mathrm{E}+04$ & $6.11 \mathrm{E}-13$ \\
\hline $\mathrm{Ba}-131 \mathrm{~m}$ & $4.71 \mathrm{E}+05$ & $4.04 \mathrm{E}-12$ \\
\hline Ba-133 & $8.50 \mathrm{E}+04$ & 7.29E-13 \\
\hline Ba-133m & $5.22 \mathrm{E}+05$ & $4.47 \mathrm{E}-12$ \\
\hline
\end{tabular}




\begin{tabular}{|c|c|c|}
\hline Nuclide & $\begin{array}{c}\text { Ground } \\
\text { Shine DCS } \\
\left(\mathbf{B q} / \mathbf{m}^{2}\right)\end{array}$ & $\begin{array}{c}\text { Ground } \\
\text { Shine DCS } \\
\left(\mu \mathrm{Ci} / \mathbf{m}^{2}\right)\end{array}$ \\
\hline Ba-135m & $5.89 \mathrm{E}+05$ & $5.05 \mathrm{E}-12$ \\
\hline Ba-137m & $5.50 \mathrm{E}+04$ & $4.71 \mathrm{E}-13$ \\
\hline Ba-139 & $2.13 \mathrm{E}+05$ & $1.82 \mathrm{E}-12$ \\
\hline Ba-140 & $1.66 \mathrm{E}+05$ & $1.42 \mathrm{E}-12$ \\
\hline Ba-141 & $3.28 \mathrm{E}+04$ & $2.81 \mathrm{E}-13$ \\
\hline Ba-142 & $3.14 \mathrm{E}+04$ & $2.69 \mathrm{E}-13$ \\
\hline La-128 & $1.14 \mathrm{E}+04$ & $9.74 \mathrm{E}-14$ \\
\hline La-129 & $3.37 \mathrm{E}+04$ & $2.89 \mathrm{E}-13$ \\
\hline La-130 & $1.44 \mathrm{E}+04$ & $1.24 \mathrm{E}-13$ \\
\hline La-131 & $4.92 \mathrm{E}+04$ & $4.22 \mathrm{E}-13$ \\
\hline La-132 & $1.68 \mathrm{E}+04$ & $1.44 \mathrm{E}-13$ \\
\hline La-132m & $4.99 \mathrm{E}+04$ & $4.27 \mathrm{E}-13$ \\
\hline La-133 & $2.10 \mathrm{E}+05$ & $1.80 \mathrm{E}-12$ \\
\hline La-134 & $4.10 \mathrm{E}+04$ & $3.52 \mathrm{E}-13$ \\
\hline La-135 & $1.04 \mathrm{E}+06$ & $8.91 \mathrm{E}-12$ \\
\hline La-136 & $7.51 \mathrm{E}+04$ & $6.44 \mathrm{E}-13$ \\
\hline La-137 & $1.58 \mathrm{E}+06$ & $1.35 \mathrm{E}-11$ \\
\hline La-138 & $2.83 \mathrm{E}+04$ & $2.43 \mathrm{E}-13$ \\
\hline La-140 & $1.47 \mathrm{E}+04$ & $1.26 \mathrm{E}-13$ \\
\hline La-141 & $2.26 \mathrm{E}+05$ & $1.94 \mathrm{E}-12$ \\
\hline La-142 & $1.47 \mathrm{E}+04$ & $1.26 \mathrm{E}-13$ \\
\hline La-143 & $8.43 \mathrm{E}+04$ & $7.23 \mathrm{E}-13$ \\
\hline Ce-130 & $6.82 \mathrm{E}+04$ & $5.84 \mathrm{E}-13$ \\
\hline Ce-131 & $2.01 \mathrm{E}+04$ & $1.72 \mathrm{E}-13$ \\
\hline Ce-132 & $1.27 \mathrm{E}+05$ & $1.09 \mathrm{E}-12$ \\
\hline Ce-133 & $5.78 \mathrm{E}+04$ & $4.95 \mathrm{E}-13$ \\
\hline Ce-133m & $1.97 \mathrm{E}+04$ & $1.69 \mathrm{E}-13$ \\
\hline Ce-134 & $1.38 \mathrm{E}+06$ & $1.18 \mathrm{E}-11$ \\
\hline Ce-135 & $4.09 \mathrm{E}+04$ & $3.51 \mathrm{E}-13$ \\
\hline Ce-137 & $9.76 \mathrm{E}+05$ & $8.36 \mathrm{E}-12$ \\
\hline $\mathrm{Ce}-137 \mathrm{~m}$ & $6.38 \mathrm{E}+05$ & $5.47 \mathrm{E}-12$ \\
\hline Ce-139 & $2.22 \mathrm{E}+05$ & $1.90 \mathrm{E}-12$ \\
\hline Ce-141 & $4.55 \mathrm{E}+05$ & $3.90 \mathrm{E}-12$ \\
\hline Ce-143 & $1.06 \mathrm{E}+05$ & $9.09 \mathrm{E}-13$ \\
\hline Ce-144 & $1.83 \mathrm{E}+06$ & $1.57 \mathrm{E}-11$ \\
\hline Ce-145 & $3.77 \mathrm{E}+04$ & $3.24 \mathrm{E}-13$ \\
\hline Pr-134 & $1.03 \mathrm{E}+04$ & $8.82 \mathrm{E}-14$ \\
\hline Pr-134m & $1.38 \mathrm{E}+04$ & $1.19 \mathrm{E}-13$ \\
\hline
\end{tabular}




\begin{tabular}{|c|c|c|}
\hline Nuclide & $\begin{array}{c}\text { Ground } \\
\text { Shine DCS } \\
\left(\mathbf{B q} / \mathbf{m}^{2}\right)\end{array}$ & $\begin{array}{c}\text { Ground } \\
\text { Shine DCS } \\
\left(\mu \mathrm{Ci} / \mathbf{m}^{2}\right)\end{array}$ \\
\hline Pr-135 & $3.57 \mathrm{E}+04$ & $3.06 \mathrm{E}-13$ \\
\hline $\operatorname{Pr}-136$ & $1.52 \mathrm{E}+04$ & $1.31 \mathrm{E}-13$ \\
\hline Pr-137 & $8.55 \mathrm{E}+04$ & 7.33E-13 \\
\hline $\operatorname{Pr}-138$ & $3.52 \mathrm{E}+04$ & $3.02 \mathrm{E}-13$ \\
\hline Pr-138m & $1.35 \mathrm{E}+04$ & $1.16 \mathrm{E}-13$ \\
\hline Pr-139 & $2.56 \mathrm{E}+05$ & $2.19 \mathrm{E}-12$ \\
\hline Pr-140 & $5.41 \mathrm{E}+04$ & $4.64 \mathrm{E}-13$ \\
\hline Pr-142 & $2.16 \mathrm{E}+05$ & $1.85 \mathrm{E}-12$ \\
\hline Pr-143 & $1.52 \mathrm{E}+06$ & $1.31 \mathrm{E}-11$ \\
\hline Pr-144 & $1.97 \mathrm{E}+05$ & $1.69 \mathrm{E}-12$ \\
\hline Pr-144m & $2.91 \mathrm{E}+06$ & $2.49 \mathrm{E}-11$ \\
\hline Pr-145 & $3.21 \mathrm{E}+05$ & $2.75 \mathrm{E}-12$ \\
\hline Pr-146 & $2.99 \mathrm{E}+04$ & $2.56 \mathrm{E}-13$ \\
\hline Pr-147 & $5.77 \mathrm{E}+04$ & $4.94 \mathrm{E}-13$ \\
\hline Pr-148 & $2.96 \mathrm{E}+04$ & $2.54 \mathrm{E}-13$ \\
\hline $\operatorname{Pr}-148 \mathrm{~m}$ & $3.02 \mathrm{E}+04$ & $2.59 \mathrm{E}-13$ \\
\hline $\mathrm{Nd}-134$ & $6.04 \mathrm{E}+04$ & $5.18 \mathrm{E}-13$ \\
\hline Nd-135 & $2.44 \mathrm{E}+04$ & $2.09 \mathrm{E}-13$ \\
\hline Nd-136 & $1.22 \mathrm{E}+05$ & $1.05 \mathrm{E}-12$ \\
\hline Nd-137 & $2.81 \mathrm{E}+04$ & $2.40 \mathrm{E}-13$ \\
\hline Nd-138 & $8.34 \mathrm{E}+05$ & $7.15 \mathrm{E}-12$ \\
\hline Nd-139 & $7.16 \mathrm{E}+04$ & $6.13 \mathrm{E}-13$ \\
\hline Nd-139m & $2.14 \mathrm{E}+04$ & $1.84 \mathrm{E}-13$ \\
\hline Nd-140 & $1.33 \mathrm{E}+06$ & $1.14 \mathrm{E}-11$ \\
\hline $\mathrm{Nd}-141$ & $4.57 \mathrm{E}+05$ & $3.92 \mathrm{E}-12$ \\
\hline Nd-141m & $4.75 \mathrm{E}+04$ & $4.07 \mathrm{E}-13$ \\
\hline Nd-147 & $2.26 \mathrm{E}+05$ & $1.94 \mathrm{E}-12$ \\
\hline $\mathrm{Nd}-149$ & $8.05 \mathrm{E}+04$ & $6.90 \mathrm{E}-13$ \\
\hline $\mathrm{Nd}-151$ & $3.70 \mathrm{E}+04$ & $3.17 \mathrm{E}-13$ \\
\hline Nd-152 & $1.81 \mathrm{E}+05$ & $1.55 \mathrm{E}-12$ \\
\hline Pm-136 & $1.14 \mathrm{E}+04$ & $9.81 \mathrm{E}-14$ \\
\hline $\mathrm{Pm}-137 \mathrm{~m}$ & $1.77 \mathrm{E}+04$ & $1.52 \mathrm{E}-13$ \\
\hline Pm-139 & $3.17 \mathrm{E}+04$ & $2.72 \mathrm{E}-13$ \\
\hline Pm-140 & $2.69 \mathrm{E}+04$ & $2.30 \mathrm{E}-13$ \\
\hline Pm-140m & $1.07 \mathrm{E}+04$ & $9.15 \mathrm{E}-14$ \\
\hline Pm-141 & $4.17 \mathrm{E}+04$ & $3.58 \mathrm{E}-13$ \\
\hline Pm-142 & $3.35 \mathrm{E}+04$ & $2.87 \mathrm{E}-13$ \\
\hline Pm-143 & $1.07 \mathrm{E}+05$ & $9.15 \mathrm{E}-13$ \\
\hline
\end{tabular}




\begin{tabular}{|c|c|c|}
\hline Nuclide & $\begin{array}{c}\text { Ground } \\
\text { Shine DCS } \\
\left(\mathbf{B q} / \mathbf{m}^{2}\right)\end{array}$ & $\begin{array}{c}\text { Ground } \\
\text { Shine DCS } \\
\left(\mu \mathrm{Ci} / \mathbf{m}^{2}\right)\end{array}$ \\
\hline Pm-144 & $2.13 \mathrm{E}+04$ & $1.82 \mathrm{E}-13$ \\
\hline Pm-145 & $1.20 \mathrm{E}+06$ & $1.03 \mathrm{E}-11$ \\
\hline Pm-146 & $4.42 \mathrm{E}+04$ & $3.79 \mathrm{E}-13$ \\
\hline Pm-147 & $1.13 \mathrm{E}+09$ & $9.67 \mathrm{E}-09$ \\
\hline $\mathrm{Pm}-148$ & $5.19 \mathrm{E}+04$ & $4.45 \mathrm{E}-13$ \\
\hline Pm-148m & $1.67 \mathrm{E}+04$ & $1.43 \mathrm{E}-13$ \\
\hline Pm-149 & $7.66 \mathrm{E}+05$ & $6.56 \mathrm{E}-12$ \\
\hline Pm-150 & $2.20 \mathrm{E}+04$ & $1.89 \mathrm{E}-13$ \\
\hline Pm-151 & $9.76 \mathrm{E}+04$ & $8.36 \mathrm{E}-13$ \\
\hline Pm-152 & $7.85 \mathrm{E}+04$ & $6.73 \mathrm{E}-13$ \\
\hline Pm-152m & $2.11 \mathrm{E}+04$ & $1.81 \mathrm{E}-13$ \\
\hline Pm-153 & $2.14 \mathrm{E}+05$ & $1.84 \mathrm{E}-12$ \\
\hline Pm-154 & $1.87 \mathrm{E}+04$ & $1.60 \mathrm{E}-13$ \\
\hline Pm-154m & $1.81 \mathrm{E}+04$ & $1.55 \mathrm{E}-13$ \\
\hline Sm-139 & $2.14 \mathrm{E}+04$ & $1.84 \mathrm{E}-13$ \\
\hline $\mathrm{Sm}-140$ & $5.80 \mathrm{E}+04$ & $4.97 \mathrm{E}-13$ \\
\hline Sm-141 & $2.26 \mathrm{E}+04$ & $1.94 \mathrm{E}-13$ \\
\hline Sm-141m & $1.70 \mathrm{E}+04$ & $1.46 \mathrm{E}-13$ \\
\hline Sm-142 & $2.99 \mathrm{E}+05$ & $2.56 \mathrm{E}-12$ \\
\hline Sm-143 & $5.65 \mathrm{E}+04$ & $4.84 \mathrm{E}-13$ \\
\hline Sm-143m & $4.80 \mathrm{E}+04$ & $4.12 \mathrm{E}-13$ \\
\hline Sm-145 & $5.81 \mathrm{E}+05$ & $4.98 \mathrm{E}-12$ \\
\hline Sm-151 & $8.32 \mathrm{E}+09$ & $7.13 \mathrm{E}-08$ \\
\hline Sm-153 & $5.07 \mathrm{E}+05$ & $4.35 \mathrm{E}-12$ \\
\hline Sm-155 & $2.05 \mathrm{E}+05$ & $1.75 \mathrm{E}-12$ \\
\hline Sm-156 & $2.96 \mathrm{E}+05$ & $2.54 \mathrm{E}-12$ \\
\hline Sm-157 & $6.57 \mathrm{E}+04$ & $5.63 \mathrm{E}-13$ \\
\hline Eu-142 & $2.33 \mathrm{E}+04$ & $2.00 \mathrm{E}-13$ \\
\hline Eu-142m & $9.33 \mathrm{E}+03$ & $7.99 \mathrm{E}-14$ \\
\hline Eu-143 & $2.69 \mathrm{E}+04$ & $2.30 \mathrm{E}-13$ \\
\hline Eu-144 & $2.62 \mathrm{E}+04$ & $2.25 \mathrm{E}-13$ \\
\hline Eu-145 & $2.69 \mathrm{E}+04$ & $2.30 \mathrm{E}-13$ \\
\hline Eu-146 & $1.42 \mathrm{E}+04$ & $1.21 \mathrm{E}-13$ \\
\hline Eu-147 & $7.24 \mathrm{E}+04$ & $6.20 \mathrm{E}-13$ \\
\hline Eu-148 & $1.51 \mathrm{E}+04$ & $1.29 \mathrm{E}-13$ \\
\hline Eu-149 & $5.42 \mathrm{E}+05$ & $4.65 \mathrm{E}-12$ \\
\hline Eu-150 & $2.16 \mathrm{E}+04$ & $1.85 \mathrm{E}-13$ \\
\hline Eu-150m & $4.52 \mathrm{E}+05$ & $3.88 \mathrm{E}-12$ \\
\hline
\end{tabular}




\begin{tabular}{|c|c|c|}
\hline Nuclide & $\begin{array}{c}\text { Ground } \\
\text { Shine DCS } \\
\left(\mathbf{B q} / \mathbf{m}^{2}\right)\end{array}$ & $\begin{array}{c}\text { Ground } \\
\text { Shine DCS } \\
\left(\mu \mathrm{Ci} / \mathbf{m}^{2}\right)\end{array}$ \\
\hline Eu-152 & $2.91 \mathrm{E}+04$ & $2.49 \mathrm{E}-13$ \\
\hline Eu-152m & $9.44 \mathrm{E}+04$ & $8.09 \mathrm{E}-13$ \\
\hline $\mathrm{Eu}-152 \mathrm{n}$ & $4.88 \mathrm{E}+05$ & $4.18 \mathrm{E}-12$ \\
\hline Eu-154 & $2.71 \mathrm{E}+04$ & $2.32 \mathrm{E}-13$ \\
\hline Eu-154m & $5.30 \mathrm{E}+05$ & $4.54 \mathrm{E}-12$ \\
\hline Eu-155 & $5.88 \mathrm{E}+05$ & $5.04 \mathrm{E}-12$ \\
\hline Eu-156 & $2.73 \mathrm{E}+04$ & $2.34 \mathrm{E}-13$ \\
\hline Eu-157 & $1.04 \mathrm{E}+05$ & $8.91 \mathrm{E}-13$ \\
\hline Eu-158 & $2.44 \mathrm{E}+04$ & $2.09 \mathrm{E}-13$ \\
\hline Eu-159 & $8.34 \mathrm{E}+04$ & $7.15 \mathrm{E}-13$ \\
\hline Gd-142 & $2.99 \mathrm{E}+04$ & $2.56 \mathrm{E}-13$ \\
\hline Gd-143m & $1.51 \mathrm{E}+04$ & $1.29 \mathrm{E}-13$ \\
\hline Gd-144 & $3.53 \mathrm{E}+04$ & $3.03 \mathrm{E}-13$ \\
\hline Gd-145 & $1.45 \mathrm{E}+04$ & $1.24 \mathrm{E}-13$ \\
\hline Gd-145m & $4.75 \mathrm{E}+04$ & $4.07 \mathrm{E}-13$ \\
\hline Gd-146 & $1.42 \mathrm{E}+05$ & $1.21 \mathrm{E}-12$ \\
\hline Gd-147 & $2.42 \mathrm{E}+04$ & $2.07 \mathrm{E}-13$ \\
\hline Gd-149 & $6.42 \mathrm{E}+04$ & $5.50 \mathrm{E}-13$ \\
\hline Gd-151 & $5.14 \mathrm{E}+05$ & $4.40 \mathrm{E}-12$ \\
\hline Gd-153 & $3.44 \mathrm{E}+05$ & $2.95 \mathrm{E}-12$ \\
\hline Gd-159 & $4.55 \mathrm{E}+05$ & $3.90 \mathrm{E}-12$ \\
\hline Gd-162 & $7.59 \mathrm{E}+04$ & $6.50 \mathrm{E}-13$ \\
\hline Tb-146 & $9.27 \mathrm{E}+03$ & $7.95 \mathrm{E}-14$ \\
\hline Tb-147 & $1.55 \mathrm{E}+04$ & $1.33 \mathrm{E}-13$ \\
\hline $\mathrm{Tb}-147 \mathrm{~m}$ & $1.79 \mathrm{E}+04$ & $1.54 \mathrm{E}-13$ \\
\hline Tb-148 & $1.40 \mathrm{E}+04$ & $1.20 \mathrm{E}-13$ \\
\hline $\mathrm{Tb}-148 \mathrm{~m}$ & $1.06 \mathrm{E}+04$ & $9.06 \mathrm{E}-14$ \\
\hline Tb-149 & $2.52 \mathrm{E}+04$ & $2.16 \mathrm{E}-13$ \\
\hline Tb-149m & $2.38 \mathrm{E}+04$ & $2.04 \mathrm{E}-13$ \\
\hline $\mathrm{Tb}-150$ & $1.43 \mathrm{E}+04$ & $1.23 \mathrm{E}-13$ \\
\hline $\mathrm{Tb}-150 \mathrm{~m}$ & $1.32 \mathrm{E}+04$ & $1.13 \mathrm{E}-13$ \\
\hline $\mathrm{Tb}-151$ & $3.41 \mathrm{E}+04$ & $2.92 \mathrm{E}-13$ \\
\hline $\mathrm{Tb}-151 \mathrm{~m}$ & $4.34 \mathrm{E}+05$ & $3.72 \mathrm{E}-12$ \\
\hline Tb-152 & $2.28 \mathrm{E}+04$ & $1.96 \mathrm{E}-13$ \\
\hline $\mathrm{Tb}-152 \mathrm{~m}$ & $4.44 \mathrm{E}+04$ & $3.81 \mathrm{E}-13$ \\
\hline $\mathrm{Tb}-153$ & $1.04 \mathrm{E}+05$ & $8.91 \mathrm{E}-13$ \\
\hline $\mathrm{Tb}-154$ & $1.57 \mathrm{E}+04$ & $1.35 \mathrm{E}-13$ \\
\hline $\mathrm{Tb}-155$ & $1.99 \mathrm{E}+05$ & $1.71 \mathrm{E}-12$ \\
\hline
\end{tabular}




\begin{tabular}{|c|c|c|}
\hline Nuclide & $\begin{array}{c}\text { Ground } \\
\text { Shine DCS } \\
\left(\mathbf{B q} / \mathbf{m}^{2} \mathbf{)}\right.\end{array}$ & $\begin{array}{c}\text { Ground } \\
\text { Shine DCS } \\
\left(\boldsymbol{\mu C} \mathbf{\text { i }} \mathbf{m}^{\mathbf{}} \mathbf{)}\right.\end{array}$ \\
\hline Tb-156 & $1.77 \mathrm{E}+04$ & $1.52 \mathrm{E}-13$ \\
\hline Tb-156m & $9.70 \mathrm{E}+05$ & $8.31 \mathrm{E}-12$ \\
\hline Tb-156n & $9.88 \mathrm{E}+06$ & $8.47 \mathrm{E}-11$ \\
\hline Tb-157 & $7.85 \mathrm{E}+06$ & $6.73 \mathrm{E}-11$ \\
\hline Tb-158 & $4.21 \mathrm{E}+04$ & $3.61 \mathrm{E}-13$ \\
\hline Tb-160 & $2.99 \mathrm{E}+04$ & $2.56 \mathrm{E}-13$ \\
\hline Tb-161 & $1.05 \mathrm{E}+06$ & $8.97 \mathrm{E}-12$ \\
\hline Tb-162 & $2.91 \mathrm{E}+04$ & $2.49 \mathrm{E}-13$ \\
\hline Tb-163 & $4.10 \mathrm{E}+04$ & $3.51 \mathrm{E}-13$ \\
\hline Tb-164 & $1.35 \mathrm{E}+04$ & $1.16 \mathrm{E}-13$ \\
\hline Tb-165 & $3.67 \mathrm{E}+04$ & $3.15 \mathrm{E}-13$ \\
\hline Dy-148 & $4.66 \mathrm{E}+04$ & $3.99 \mathrm{E}-13$ \\
\hline Dy-149 & $2.13 \mathrm{E}+04$ & $1.82 \mathrm{E}-13$ \\
\hline Dy-150 & $1.21 \mathrm{E}+05$ & $1.03 \mathrm{E}-12$ \\
\hline Dy-151 & $2.50 \mathrm{E}+04$ & $2.14 \mathrm{E}-13$ \\
\hline Dy-152 & $1.20 \mathrm{E}+05$ & $1.03 \mathrm{E}-12$ \\
\hline Dy-153 & $3.94 \mathrm{E}+04$ & $3.38 \mathrm{E}-13$ \\
\hline Dy-155 & $5.13 \mathrm{E}+04$ & $4.40 \mathrm{E}-13$ \\
\hline Dy-157 & $9.79 \mathrm{E}+04$ & $8.39 \mathrm{E}-13$ \\
\hline Dy-159 & $8.11 \mathrm{E}+05$ & $6.95 \mathrm{E}-12$ \\
\hline Dy-165 & $4.57 \mathrm{E}+05$ & $3.92 \mathrm{E}-12$ \\
\hline Dy-165m & $1.85 \mathrm{E}+06$ & $1.59 \mathrm{E}-11$ \\
\hline Dy-166 & $8.43 \mathrm{E}+05$ & $7.23 \mathrm{E}-12$ \\
\hline Dy-167 & $5.46 \mathrm{E}+04$ & $4.68 \mathrm{E}-13$ \\
\hline Dy-168 & $7.91 \mathrm{E}+04$ & $6.78 \mathrm{E}-13$ \\
\hline Ho-150 & $1.61 \mathrm{E}+04$ & $1.38 \mathrm{E}-13$ \\
\hline Ho-153 & $3.08 \mathrm{E}+04$ & $2.64 \mathrm{E}-13$ \\
\hline Ho-153m & $2.96 \mathrm{E}+04$ & $2.54 \mathrm{E}-13$ \\
\hline Ho-154 & $1.69 \mathrm{E}+04$ & $1.45 \mathrm{E}-13$ \\
\hline Ho-154m & $1.34 \mathrm{E}+04$ & $1.15 \mathrm{E}-13$ \\
\hline Ho-155 & $5.38 \mathrm{E}+04$ & $4.61 \mathrm{E}-13$ \\
\hline Ho-156 & $1.59 \mathrm{E}+04$ & $1.36 \mathrm{E}-13$ \\
\hline Ho-157 & $5.82 \mathrm{E}+04$ & $4.99 \mathrm{E}-13$ \\
\hline Ho-159 & $9.01 \mathrm{E}+04$ & $7.72 \mathrm{E}-13$ \\
\hline Ho-162m & $6.16 \mathrm{E}+04$ & $5.28 \mathrm{E}-13$ \\
\hline
\end{tabular}




\begin{tabular}{|c|c|c|}
\hline Nuclide & $\begin{array}{c}\text { Ground } \\
\text { Shine DCS } \\
\mathbf{( B q}^{\mathbf{2}} \mathbf{\text { ) }}\end{array}$ & $\begin{array}{c}\text { Ground } \\
\text { Shine DCS } \\
\mathbf{( \mu C i}^{\mathbf{2}} \mathbf{)}\end{array}$ \\
\hline Ho-164 & $9.44 \mathrm{E}+05$ & $8.09 \mathrm{E}-12$ \\
\hline Ho-164m & $8.21 \mathrm{E}+05$ & $7.04 \mathrm{E}-12$ \\
\hline Ho-166 & $2.99 \mathrm{E}+05$ & $2.56 \mathrm{E}-12$ \\
\hline Ho-166m & $2.07 \mathrm{E}+04$ & $1.78 \mathrm{E}-13$ \\
\hline Ho-167 & $9.01 \mathrm{E}+04$ & $7.72 \mathrm{E}-13$ \\
\hline Ho-168 & $3.46 \mathrm{E}+04$ & $2.96 \mathrm{E}-13$ \\
\hline Ho-168m & $5.85 \mathrm{E}+06$ & $5.01 \mathrm{E}-11$ \\
\hline Ho-170 & $1.89 \mathrm{E}+04$ & $1.62 \mathrm{E}-13$ \\
\hline Er-154 & $4.72 \mathrm{E}+05$ & $4.04 \mathrm{E}-12$ \\
\hline Er-156 & $5.59 \mathrm{E}+05$ & $4.79 \mathrm{E}-12$ \\
\hline Er-159 & $3.53 \mathrm{E}+04$ & $3.03 \mathrm{E}-13$ \\
\hline Er-161 & $3.43 \mathrm{E}+04$ & $2.94 \mathrm{E}-13$ \\
\hline Er-163 & $9.14 \mathrm{E}+05$ & $7.83 \mathrm{E}-12$ \\
\hline Er-165 & $9.76 \mathrm{E}+05$ & $8.36 \mathrm{E}-12$ \\
\hline Er-167m & $3.60 \mathrm{E}+05$ & $3.08 \mathrm{E}-12$ \\
\hline Er-169 & $4.54 \mathrm{E}+08$ & $3.89 \mathrm{E}-09$ \\
\hline Er-171 & $8.41 \mathrm{E}+04$ & $7.21 \mathrm{E}-13$ \\
\hline Er-172 & $6.47 \mathrm{E}+04$ & $5.55 \mathrm{E}-13$ \\
\hline Er-173 & $3.74 \mathrm{E}+04$ & $3.21 \mathrm{E}-13$ \\
\hline Tm-161 & $2.66 \mathrm{E}+04$ & $2.28 \mathrm{E}-13$ \\
\hline Tm-162 & $1.78 \mathrm{E}+04$ & $1.53 \mathrm{E}-13$ \\
\hline Tm-163 & $2.64 \mathrm{E}+04$ & $2.26 \mathrm{E}-13$ \\
\hline Tm-164 & $4.04 \mathrm{E}+04$ & $3.47 \mathrm{E}-13$ \\
\hline Tm-165 & $6.06 \mathrm{E}+04$ & $5.20 \mathrm{E}-13$ \\
\hline Tm-166 & $1.77 \mathrm{E}+04$ & $1.52 \mathrm{E}-13$ \\
\hline Tm-167 & $2.38 \mathrm{E}+05$ & $2.04 \mathrm{E}-12$ \\
\hline Tm-168 & $2.71 \mathrm{E}+04$ & $2.32 \mathrm{E}-13$ \\
\hline Tm-170 & $1.27 \mathrm{E}+06$ & $1.09 \mathrm{E}-11$ \\
\hline Tm-171 & $5.97 \mathrm{E}+07$ & $5.12 \mathrm{E}-10$ \\
\hline Tm-172 & $6.55 \mathrm{E}+04$ & $5.61 \mathrm{E}-13$ \\
\hline Tm-173 & $8.21 \mathrm{E}+04$ & $7.04 \mathrm{E}-13$ \\
\hline Tm-174 & $1.87 \mathrm{E}+04$ & $1.60 \mathrm{E}-13$ \\
\hline Tm-175 & $2.96 \mathrm{E}+04$ & $2.54 \mathrm{E}-13$ \\
\hline Tm-176 & $1.70 \mathrm{E}+04$ & $1.45 \mathrm{E}-13$ \\
\hline Yb-162 & $1.38 \mathrm{E}+05$ & $1.18 \mathrm{E}-12$ \\
\hline Yb-164 & $4.50 \mathrm{E}+04$ & $3.86 \mathrm{E}-13$ \\
\hline Yb-165 & $1.01 \mathrm{E}+05$ & $8.68 \mathrm{E}-13$ \\
\hline
\end{tabular}




\begin{tabular}{|c|c|c|}
\hline Nuclide & $\begin{array}{c}\text { Ground } \\
\text { Shine DCS } \\
\left(\mathbf{B q} / \mathbf{m}^{2}\right)\end{array}$ & $\begin{array}{c}\text { Ground } \\
\text { Shine DCS } \\
\left(\mu \mathrm{Ci} / \mathbf{m}^{2}\right)\end{array}$ \\
\hline Yb-166 & $4.23 \mathrm{E}+05$ & $3.63 \mathrm{E}-12$ \\
\hline Yb-167 & $1.33 \mathrm{E}+05$ & $1.14 \mathrm{E}-12$ \\
\hline Yb-169 & $1.07 \mathrm{E}+05$ & $9.21 \mathrm{E}-13$ \\
\hline Yb-175 & $8.59 \mathrm{E}+05$ & $7.36 \mathrm{E}-12$ \\
\hline Yb-177 & $1.42 \mathrm{E}+05$ & $1.22 \mathrm{E}-12$ \\
\hline Yb-178 & $8.11 \mathrm{E}+05$ & $6.95 \mathrm{E}-12$ \\
\hline Yb-179 & $3.14 \mathrm{E}+04$ & $2.69 \mathrm{E}-13$ \\
\hline Lu-165 & $2.99 \mathrm{E}+04$ & $2.56 \mathrm{E}-13$ \\
\hline Lu-167 & $2.07 \mathrm{E}+04$ & $1.78 \mathrm{E}-13$ \\
\hline Lu-169 & $2.64 \mathrm{E}+04$ & $2.26 \mathrm{E}-13$ \\
\hline Lu-169m & $3.38 \mathrm{E}+09$ & $2.90 \mathrm{E}-08$ \\
\hline Lu-170 & $1.40 \mathrm{E}+04$ & $1.20 \mathrm{E}-13$ \\
\hline Lu-171 & $5.22 \mathrm{E}+04$ & $4.48 \mathrm{E}-13$ \\
\hline $\mathrm{Lu}-171 \mathrm{~m}$ & $1.10 \mathrm{E}+08$ & $9.40 \mathrm{E}-10$ \\
\hline Lu-172 & $1.74 \mathrm{E}+04$ & $1.49 \mathrm{E}-13$ \\
\hline Lu-172m & $6.97 \mathrm{E}+09$ & 5.97E-08 \\
\hline Lu-173 & $1.93 \mathrm{E}+05$ & $1.66 \mathrm{E}-12$ \\
\hline Lu-174 & $3.08 \mathrm{E}+05$ & $2.64 \mathrm{E}-12$ \\
\hline $\mathrm{Lu}-174 \mathrm{~m}$ & $6.10 \mathrm{E}+05$ & $5.23 \mathrm{E}-12$ \\
\hline Lu-176 & $7.09 \mathrm{E}+04$ & $6.08 \mathrm{E}-13$ \\
\hline $\mathrm{Lu}-176 \mathrm{~m}$ & $5.64 \mathrm{E}+05$ & $4.84 \mathrm{E}-12$ \\
\hline Lu-177 & $9.88 \mathrm{E}+05$ & $8.47 \mathrm{E}-12$ \\
\hline $\mathrm{Lu}-177 \mathrm{~m}$ & $3.42 \mathrm{E}+04$ & $2.93 \mathrm{E}-13$ \\
\hline Lu-178 & $1.59 \mathrm{E}+05$ & $1.36 \mathrm{E}-12$ \\
\hline Lu-178m & $3.14 \mathrm{E}+04$ & $2.69 \mathrm{E}-13$ \\
\hline Lu-179 & $3.98 \mathrm{E}+05$ & $3.41 \mathrm{E}-12$ \\
\hline Lu-180 & $2.17 \mathrm{E}+04$ & $1.86 \mathrm{E}-13$ \\
\hline Lu-181 & $5.04 \mathrm{E}+04$ & $4.32 \mathrm{E}-13$ \\
\hline Hf-167 & $4.96 \mathrm{E}+04$ & $4.25 \mathrm{E}-13$ \\
\hline Hf-169 & $5.10 \mathrm{E}+04$ & $4.37 \mathrm{E}-13$ \\
\hline Hf-170 & $7.75 \mathrm{E}+04$ & $6.64 \mathrm{E}-13$ \\
\hline Hf-172 & $3.56 \mathrm{E}+05$ & $3.05 \mathrm{E}-12$ \\
\hline Hf-173 & $8.74 \mathrm{E}+04$ & $7.49 \mathrm{E}-13$ \\
\hline Hf-175 & $9.64 \mathrm{E}+04$ & $8.26 \mathrm{E}-13$ \\
\hline Hf-177m & $1.49 \mathrm{E}+04$ & $1.28 \mathrm{E}-13$ \\
\hline Hf-178m & $1.50 \mathrm{E}+04$ & $1.29 \mathrm{E}-13$ \\
\hline Hf-179m & $3.69 \mathrm{E}+04$ & $3.16 \mathrm{E}-13$ \\
\hline Hf-180m & $3.41 \mathrm{E}+04$ & $2.93 \mathrm{E}-13$ \\
\hline
\end{tabular}




\begin{tabular}{|c|c|c|}
\hline Nuclide & $\begin{array}{c}\text { Ground } \\
\text { Shine DCS } \\
\left(\mathbf{B q} / \mathbf{m}^{2}\right)\end{array}$ & $\begin{array}{c}\text { Ground } \\
\text { Shine DCS } \\
\left(\mu \mathrm{Ci} / \mathbf{m}^{2}\right)\end{array}$ \\
\hline Hf-181 & $6.32 \mathrm{E}+04$ & $5.41 \mathrm{E}-13$ \\
\hline Hf-182 & $1.42 \mathrm{E}+05$ & $1.22 \mathrm{E}-12$ \\
\hline Hf-182m & $3.69 \mathrm{E}+04$ & $3.16 \mathrm{E}-13$ \\
\hline Hf-183 & $4.10 \mathrm{E}+04$ & $3.52 \mathrm{E}-13$ \\
\hline Hf-184 & $1.34 \mathrm{E}+05$ & $1.15 \mathrm{E}-12$ \\
\hline Ta-170 & $2.78 \mathrm{E}+04$ & $2.38 \mathrm{E}-13$ \\
\hline Ta-172 & $1.96 \mathrm{E}+04$ & $1.68 \mathrm{E}-13$ \\
\hline Ta-173 & $5.85 \mathrm{E}+04$ & $5.01 \mathrm{E}-13$ \\
\hline Ta-174 & $3.38 \mathrm{E}+04$ & $2.90 \mathrm{E}-13$ \\
\hline Ta-175 & $3.14 \mathrm{E}+04$ & $2.69 \mathrm{E}-13$ \\
\hline Тa-176 & $1.59 \mathrm{E}+04$ & $1.36 \mathrm{E}-13$ \\
\hline Ta-177 & $5.38 \mathrm{E}+05$ & $4.61 \mathrm{E}-12$ \\
\hline Ta-178 & $2.91 \mathrm{E}+05$ & $2.49 \mathrm{E}-12$ \\
\hline Ta-178m & $2.94 \mathrm{E}+04$ & $2.52 \mathrm{E}-13$ \\
\hline Ta-179 & $1.50 \mathrm{E}+06$ & $1.28 \mathrm{E}-11$ \\
\hline Ta-180 & $7.59 \mathrm{E}+05$ & $6.50 \mathrm{E}-12$ \\
\hline Ta-182 & $2.66 \mathrm{E}+04$ & $2.28 \mathrm{E}-13$ \\
\hline Ta-182m & $1.34 \mathrm{E}+05$ & $1.15 \mathrm{E}-12$ \\
\hline Ta-183 & $1.17 \mathrm{E}+05$ & $1.01 \mathrm{E}-12$ \\
\hline Ta-184 & $2.10 \mathrm{E}+04$ & $1.80 \mathrm{E}-13$ \\
\hline Ta-185 & $1.47 \mathrm{E}+05$ & $1.26 \mathrm{E}-12$ \\
\hline Ta-186 & $2.19 \mathrm{E}+04$ & $1.87 \mathrm{E}-13$ \\
\hline W-177 & $3.73 \mathrm{E}+04$ & $3.20 \mathrm{E}-13$ \\
\hline $\mathrm{W}-178$ & $2.48 \mathrm{E}+06$ & $2.12 \mathrm{E}-11$ \\
\hline W-179 & $6.98 \mathrm{E}+05$ & $5.99 \mathrm{E}-12$ \\
\hline $\mathrm{W}-179 \mathrm{~m}$ & $6.50 \mathrm{E}+05$ & $5.57 \mathrm{E}-12$ \\
\hline W-181 & $9.33 \mathrm{E}+05$ & $7.99 \mathrm{E}-12$ \\
\hline W-185 & $1.90 \mathrm{E}+08$ & $1.63 \mathrm{E}-09$ \\
\hline $\mathrm{W}-185 \mathrm{~m}$ & $1.47 \mathrm{E}+06$ & $1.26 \mathrm{E}-11$ \\
\hline W-187 & $7.19 \mathrm{E}+04$ & $6.16 \mathrm{E}-13$ \\
\hline W-188 & $1.75 \mathrm{E}+07$ & $1.50 \mathrm{E}-10$ \\
\hline W-190 & $2.06 \mathrm{E}+05$ & $1.76 \mathrm{E}-12$ \\
\hline Re-178 & $2.01 \mathrm{E}+04$ & $1.72 \mathrm{E}-13$ \\
\hline Re-179 & $3.17 \mathrm{E}+04$ & $2.72 \mathrm{E}-13$ \\
\hline Re-180 & $2.81 \mathrm{E}+04$ & $2.40 \mathrm{E}-13$ \\
\hline Re-181 & $4.23 \mathrm{E}+04$ & $3.62 \mathrm{E}-13$ \\
\hline Re-182 & $1.92 \mathrm{E}+04$ & $1.65 \mathrm{E}-13$ \\
\hline $\mathrm{Re}-182 \mathrm{~m}$ & $2.83 \mathrm{E}+04$ & $2.43 \mathrm{E}-13$ \\
\hline
\end{tabular}




\begin{tabular}{|c|c|c|}
\hline Nuclide & $\begin{array}{c}\text { Ground } \\
\text { Shine DCS } \\
\mathbf{( B q}^{\mathbf{2}} \mathbf{\text { ) }}\end{array}$ & $\begin{array}{c}\text { Ground } \\
\text { Shine DCS } \\
\mathbf{( \mu C i}^{\mathbf{2}} \mathbf{)}\end{array}$ \\
\hline Re-183 & $2.31 \mathrm{E}+05$ & $1.98 \mathrm{E}-12$ \\
\hline Re-184 & $3.80 \mathrm{E}+04$ & $3.25 \mathrm{E}-13$ \\
\hline Re-184m & $9.01 \mathrm{E}+04$ & $7.72 \mathrm{E}-13$ \\
\hline Re-186 & $7.31 \mathrm{E}+05$ & $6.26 \mathrm{E}-12$ \\
\hline Re-186m & $2.42 \mathrm{E}+06$ & $2.07 \mathrm{E}-11$ \\
\hline Re-188 & $2.14 \mathrm{E}+05$ & $1.84 \mathrm{E}-12$ \\
\hline Re-188m & $5.34 \mathrm{E}+05$ & $4.58 \mathrm{E}-12$ \\
\hline Re-189 & $4.44 \mathrm{E}+05$ & $3.81 \mathrm{E}-12$ \\
\hline Re-190 & $2.37 \mathrm{E}+04$ & $2.03 \mathrm{E}-13$ \\
\hline Re-190m & $3.47 \mathrm{E}+04$ & $2.98 \mathrm{E}-13$ \\
\hline Os-180 & $2.83 \mathrm{E}+05$ & $2.43 \mathrm{E}-12$ \\
\hline Os-181 & $2.50 \mathrm{E}+04$ & $2.14 \mathrm{E}-13$ \\
\hline Os-182 & $7.89 \mathrm{E}+04$ & $6.76 \mathrm{E}-13$ \\
\hline Os-183 & $5.44 \mathrm{E}+04$ & $4.66 \mathrm{E}-13$ \\
\hline Os-183m & $3.41 \mathrm{E}+04$ & $2.92 \mathrm{E}-13$ \\
\hline Os-185 & $4.85 \mathrm{E}+04$ & $4.16 \mathrm{E}-13$ \\
\hline Os-189m & $7.87 \mathrm{E}+08$ & $6.74 \mathrm{E}-09$ \\
\hline Os-190m & $2.10 \mathrm{E}+04$ & $1.80 \mathrm{E}-13$ \\
\hline Os-191 & $4.46 \mathrm{E}+05$ & $3.82 \mathrm{E}-12$ \\
\hline Os-191m & $6.29 \mathrm{E}+06$ & $5.39 \mathrm{E}-11$ \\
\hline Os-193 & $3.52 \mathrm{E}+05$ & $3.01 \mathrm{E}-12$ \\
\hline Os-194 & $1.43 \mathrm{E}+07$ & $1.23 \mathrm{E}-10$ \\
\hline Os-196 & $3.02 \mathrm{E}+05$ & $2.59 \mathrm{E}-12$ \\
\hline Ir-180 & $1.96 \mathrm{E}+04$ & $1.68 \mathrm{E}-13$ \\
\hline Ir-182 & $2.23 \mathrm{E}+04$ & $1.91 \mathrm{E}-13$ \\
\hline Ir-183 & $2.91 \mathrm{E}+04$ & $2.49 \mathrm{E}-13$ \\
\hline Ir-184 & $1.72 \mathrm{E}+04$ & $1.48 \mathrm{E}-13$ \\
\hline Ir-185 & $4.13 \mathrm{E}+04$ & $3.54 \mathrm{E}-13$ \\
\hline Ir-186 & $2.07 \mathrm{E}+04$ & $1.78 \mathrm{E}-13$ \\
\hline Ir-186m & $2.76 \mathrm{E}+04$ & $2.36 \mathrm{E}-13$ \\
\hline Ir-187 & $1.04 \mathrm{E}+05$ & $8.88 \mathrm{E}-13$ \\
\hline Ir-188 & $1.70 \mathrm{E}+04$ & $1.46 \mathrm{E}-13$ \\
\hline Ir-189 & $4.70 \mathrm{E}+05$ & $4.03 \mathrm{E}-12$ \\
\hline Ir-190 & $2.26 \mathrm{E}+04$ & $1.94 \mathrm{E}-13$ \\
\hline Ir-190m & $7.16 \mathrm{E}+08$ & $6.13 \mathrm{E}-09$ \\
\hline Ir-192 & $6.51 \mathrm{E}+05$ & $5.58 \mathrm{E}-12$ \\
\hline
\end{tabular}




\begin{tabular}{|c|c|c|}
\hline Nuclide & $\begin{array}{c}\text { Ground } \\
\text { Shine DCS } \\
\left(\mathbf{B q} / \mathbf{m}^{2}\right)\end{array}$ & $\begin{array}{c}\text { Ground } \\
\text { Shine DCS } \\
\left(\mu \mathrm{Ci} / \mathbf{m}^{2}\right)\end{array}$ \\
\hline Ir-192m & $1.49 \mathrm{E}+08$ & $1.28 \mathrm{E}-09$ \\
\hline Ir-192n & $3.73 \mathrm{E}+07$ & $3.20 \mathrm{E}-10$ \\
\hline Ir-193m & $9.97 \mathrm{E}+07$ & $8.55 \mathrm{E}-10$ \\
\hline Ir-194 & $1.75 \mathrm{E}+05$ & $1.50 \mathrm{E}-12$ \\
\hline Ir-194m & $1.43 \mathrm{E}+04$ & $1.22 \mathrm{E}-13$ \\
\hline Ir-195 & $4.30 \mathrm{E}+05$ & $3.68 \mathrm{E}-12$ \\
\hline Ir-195m & $8.81 \mathrm{E}+04$ & $7.55 \mathrm{E}-13$ \\
\hline Ir-196 & $9.11 \mathrm{E}+04$ & $7.81 \mathrm{E}-13$ \\
\hline Ir-196m & $1.34 \mathrm{E}+04$ & $1.15 \mathrm{E}-13$ \\
\hline Pt-184 & $4.75 \mathrm{E}+04$ & $4.07 \mathrm{E}-13$ \\
\hline Pt-186 & $4.93 \mathrm{E}+04$ & $4.23 \mathrm{E}-13$ \\
\hline Pt-187 & $5.50 \mathrm{E}+04$ & $4.71 \mathrm{E}-13$ \\
\hline Pt-188 & $1.72 \mathrm{E}+05$ & $1.48 \mathrm{E}-12$ \\
\hline Pt-189 & $7.06 \mathrm{E}+04$ & $6.05 \mathrm{E}-13$ \\
\hline Pt-191 & $1.17 \mathrm{E}+05$ & $1.01 \mathrm{E}-12$ \\
\hline Pt-193 & $2.96 \mathrm{E}+08$ & $2.54 \mathrm{E}-09$ \\
\hline Pt-193m & $3.50 \mathrm{E}+06$ & $3.00 \mathrm{E}-11$ \\
\hline Pt-195m & $5.11 \mathrm{E}+05$ & $4.38 \mathrm{E}-12$ \\
\hline Pt-197 & $1.31 \mathrm{E}+06$ & $1.12 \mathrm{E}-11$ \\
\hline Pt-197m & $4.36 \mathrm{E}+05$ & $3.74 \mathrm{E}-12$ \\
\hline Pt-199 & $1.29 \mathrm{E}+05$ & $1.10 \mathrm{E}-12$ \\
\hline $\mathrm{Pt}-200$ & $5.87 \mathrm{E}+05$ & $5.03 \mathrm{E}-12$ \\
\hline Pt-202 & $4.07 \mathrm{E}+05$ & $3.49 \mathrm{E}-12$ \\
\hline $\mathrm{Au}-186$ & $2.11 \mathrm{E}+04$ & $1.81 \mathrm{E}-13$ \\
\hline $\mathrm{Au}-187$ & $3.25 \mathrm{E}+04$ & $2.79 \mathrm{E}-13$ \\
\hline $\mathrm{Au}-190$ & $1.50 \mathrm{E}+04$ & $1.29 \mathrm{E}-13$ \\
\hline $\mathrm{Au}-191$ & $5.72 \mathrm{E}+04$ & $4.91 \mathrm{E}-13$ \\
\hline $\mathrm{Au}-192$ & $1.83 \mathrm{E}+04$ & $1.57 \mathrm{E}-13$ \\
\hline $\mathrm{Au}-193$ & $2.13 \mathrm{E}+05$ & $1.82 \mathrm{E}-12$ \\
\hline $\mathrm{Au}-193 \mathrm{~m}$ & $1.76 \mathrm{E}+05$ & $1.51 \mathrm{E}-12$ \\
\hline Au-194 & $3.36 \mathrm{E}+04$ & $2.88 \mathrm{E}-13$ \\
\hline $\mathrm{Au}-195$ & $4.58 \mathrm{E}+05$ & $3.92 \mathrm{E}-12$ \\
\hline $\mathrm{Au}-195 \mathrm{~m}$ & $1.72 \mathrm{E}+05$ & $1.48 \mathrm{E}-12$ \\
\hline $\mathrm{Au}-196$ & $7.16 \mathrm{E}+04$ & $6.13 \mathrm{E}-13$ \\
\hline Au-196m & $1.47 \mathrm{E}+05$ & $1.26 \mathrm{E}-12$ \\
\hline $\mathrm{Au}-198$ & $7.83 \mathrm{E}+04$ & $6.71 \mathrm{E}-13$ \\
\hline $\mathrm{Au}-198 \mathrm{~m}$ & $6.58 \mathrm{E}+04$ & $5.64 \mathrm{E}-13$ \\
\hline Au-199 & $3.68 \mathrm{E}+05$ & $3.16 \mathrm{E}-12$ \\
\hline
\end{tabular}




\begin{tabular}{|c|c|c|}
\hline Nuclide & $\begin{array}{c}\text { Ground } \\
\text { Shine DCS } \\
\left(\mathrm{Bq} / \mathbf{m}^{2}\right)\end{array}$ & $\begin{array}{c}\text { Ground } \\
\text { Shine DCS } \\
\left(\mu \mathrm{Ci} / \mathbf{m}^{2}\right)\end{array}$ \\
\hline $\mathrm{Au}-200$ & $9.41 \mathrm{E}+04$ & $8.06 \mathrm{E}-13$ \\
\hline $\mathrm{Au}-200 \mathrm{~m}$ & $1.69 \mathrm{E}+04$ & $1.45 \mathrm{E}-13$ \\
\hline $\mathrm{Au}-201$ & $4.34 \mathrm{E}+05$ & $3.72 \mathrm{E}-12$ \\
\hline $\mathrm{Au}-202$ & $1.13 \mathrm{E}+05$ & $9.67 \mathrm{E}-13$ \\
\hline Hg-190 & $1.79 \mathrm{E}+05$ & $1.54 \mathrm{E}-12$ \\
\hline $\mathrm{Hg}-191 \mathrm{~m}$ & $2.30 \mathrm{E}+04$ & $1.97 \mathrm{E}-13$ \\
\hline $\mathrm{Hg}-192$ & $1.28 \mathrm{E}+05$ & $1.10 \mathrm{E}-12$ \\
\hline $\mathrm{Hg}-193$ & $4.15 \mathrm{E}+04$ & $3.55 \mathrm{E}-13$ \\
\hline $\mathrm{Hg}-193 \mathrm{~m}$ & $3.34 \mathrm{E}+04$ & $2.87 \mathrm{E}-13$ \\
\hline $\mathrm{Hg}-194$ & $1.96 \mathrm{E}+08$ & $1.68 \mathrm{E}-09$ \\
\hline $\mathrm{Hg}-195$ & $1.75 \mathrm{E}+05$ & $1.50 \mathrm{E}-12$ \\
\hline $\mathrm{Hg}-195 \mathrm{~m}$ & $1.73 \mathrm{E}+05$ & $1.49 \mathrm{E}-12$ \\
\hline Hg-197 & $5.21 \mathrm{E}+05$ & $4.46 \mathrm{E}-12$ \\
\hline $\mathrm{Hg}-197 \mathrm{~m}$ & $3.78 \mathrm{E}+05$ & $3.24 \mathrm{E}-12$ \\
\hline $\mathrm{Hg}-199 \mathrm{~m}$ & $1.93 \mathrm{E}+05$ & $1.66 \mathrm{E}-12$ \\
\hline $\mathrm{Hg}-203$ & $1.43 \mathrm{E}+05$ & $1.22 \mathrm{E}-12$ \\
\hline $\mathrm{Hg}-205$ & $4.83 \mathrm{E}+05$ & 4.14E-12 \\
\hline $\mathrm{Hg}-206$ & $2.13 \mathrm{E}+05$ & $1.82 \mathrm{E}-12$ \\
\hline Hg-207 & $1.27 \mathrm{E}+04$ & $1.09 \mathrm{E}-13$ \\
\hline T1-190 & $2.33 \mathrm{E}+04$ & $2.00 \mathrm{E}-13$ \\
\hline $\mathrm{Tl}-190 \mathrm{~m}$ & $1.32 \mathrm{E}+04$ & $1.13 \mathrm{E}-13$ \\
\hline Tl-194 & $3.43 \mathrm{E}+04$ & $2.94 \mathrm{E}-13$ \\
\hline $\mathrm{Tl}-194 \mathrm{~m}$ & $1.32 \mathrm{E}+04$ & $1.13 \mathrm{E}-13$ \\
\hline T1-195 & $2.86 \mathrm{E}+04$ & $2.45 \mathrm{E}-13$ \\
\hline T1-196 & $1.84 \mathrm{E}+04$ & $1.58 \mathrm{E}-13$ \\
\hline T1-197 & $7.51 \mathrm{E}+04$ & $6.44 \mathrm{E}-13$ \\
\hline T1-198 & $1.74 \mathrm{E}+04$ & $1.49 \mathrm{E}-13$ \\
\hline Tl-198m & $2.76 \mathrm{E}+04$ & $2.36 \mathrm{E}-13$ \\
\hline Tl-199 & $1.38 \mathrm{E}+05$ & $1.19 \mathrm{E}-12$ \\
\hline Tl-200 & $2.60 \mathrm{E}+04$ & $2.23 \mathrm{E}-13$ \\
\hline T1-201 & $4.00 \mathrm{E}+05$ & $3.43 \mathrm{E}-12$ \\
\hline T1-202 & $7.26 \mathrm{E}+04$ & $6.22 \mathrm{E}-13$ \\
\hline T1-204 & $2.91 \mathrm{E}+06$ & $2.49 \mathrm{E}-11$ \\
\hline T1-206 & $5.18 \mathrm{E}+05$ & $4.44 \mathrm{E}-12$ \\
\hline T1-206m & $1.38 \mathrm{E}+04$ & $1.19 \mathrm{E}-13$ \\
\hline T1-207 & $5.65 \mathrm{E}+05$ & $4.84 \mathrm{E}-12$ \\
\hline T1-208 & $1.07 \mathrm{E}+04$ & $9.18 \mathrm{E}-14$ \\
\hline T1-209 & $1.57 \mathrm{E}+04$ & $1.35 \mathrm{E}-13$ \\
\hline
\end{tabular}




\begin{tabular}{|c|c|c|}
\hline Nuclide & $\begin{array}{c}\text { Ground } \\
\text { Shine DCS } \\
\mathbf{( B q}^{\mathbf{2}} \mathbf{\text { ) }}\end{array}$ & $\begin{array}{c}\text { Ground } \\
\text { Shine DCS } \\
\mathbf{( \mu C i}^{\mathbf{2}} \mathbf{)}\end{array}$ \\
\hline $\mathrm{Tl}-210$ & $1.20 \mathrm{E}+04$ & $1.03 \mathrm{E}-13$ \\
\hline $\mathrm{Pb}-194$ & $3.19 \mathrm{E}+04$ & $2.73 \mathrm{E}-13$ \\
\hline $\mathrm{Pb}-195 \mathrm{~m}$ & $2.02 \mathrm{E}+04$ & $1.73 \mathrm{E}-13$ \\
\hline $\mathrm{Pb}-196$ & $6.92 \mathrm{E}+04$ & $5.93 \mathrm{E}-13$ \\
\hline $\mathrm{Pb}-197$ & $2.26 \mathrm{E}+04$ & $1.94 \mathrm{E}-13$ \\
\hline $\mathrm{Pb}-197 \mathrm{~m}$ & $2.88 \mathrm{E}+04$ & $2.47 \mathrm{E}-13$ \\
\hline $\mathrm{Pb}-198$ & $7.83 \mathrm{E}+04$ & $6.71 \mathrm{E}-13$ \\
\hline $\mathrm{Pb}-199$ & $3.32 \mathrm{E}+04$ & $2.85 \mathrm{E}-13$ \\
\hline $\mathrm{Pb}-200$ & $1.71 \mathrm{E}+05$ & $1.47 \mathrm{E}-12$ \\
\hline $\mathrm{Pb}-201$ & $4.50 \mathrm{E}+04$ & $3.85 \mathrm{E}-13$ \\
\hline $\mathrm{Pb}-201 \mathrm{~m}$ & $8.37 \mathrm{E}+04$ & $7.17 \mathrm{E}-13$ \\
\hline $\mathrm{Pb}-202$ & $1.69 \mathrm{E}+08$ & $1.45 \mathrm{E}-09$ \\
\hline $\mathrm{Pb}-202 \mathrm{~m}$ & $1.68 \mathrm{E}+04$ & $1.44 \mathrm{E}-13$ \\
\hline $\mathrm{Pb}-203$ & $1.10 \mathrm{E}+05$ & $9.47 \mathrm{E}-13$ \\
\hline $\mathrm{Pb}-204 \mathrm{~m}$ & $1.62 \mathrm{E}+04$ & $1.39 \mathrm{E}-13$ \\
\hline $\mathrm{Pb}-205$ & $1.67 \mathrm{E}+08$ & $1.43 \mathrm{E}-09$ \\
\hline $\mathrm{Pb}-209$ & $9.94 \mathrm{E}+06$ & $8.52 \mathrm{E}-11$ \\
\hline $\mathrm{Pb}-210$ & $1.46 \mathrm{E}+07$ & $1.25 \mathrm{E}-10$ \\
\hline $\mathrm{Pb}-211$ & $2.94 \mathrm{E}+05$ & $2.52 \mathrm{E}-12$ \\
\hline $\mathrm{Pb}-212$ & $2.40 \mathrm{E}+05$ & $2.06 \mathrm{E}-12$ \\
\hline $\mathrm{Pb}-214$ & $1.30 \mathrm{E}+05$ & $1.12 \mathrm{E}-12$ \\
\hline $\mathrm{Bi}-197$ & $1.98 \mathrm{E}+04$ & $1.70 \mathrm{E}-13$ \\
\hline $\mathrm{Bi}-200$ & $1.37 \mathrm{E}+04$ & $1.18 \mathrm{E}-13$ \\
\hline $\mathrm{Bi}-201$ & $2.01 \mathrm{E}+04$ & $1.72 \mathrm{E}-13$ \\
\hline $\mathrm{Bi}-202$ & $1.23 \mathrm{E}+04$ & $1.05 \mathrm{E}-13$ \\
\hline $\mathrm{Bi}-203$ & $1.46 \mathrm{E}+04$ & $1.25 \mathrm{E}-13$ \\
\hline $\mathrm{Bi}-204$ & $1.17 \mathrm{E}+04$ & $1.00 \mathrm{E}-13$ \\
\hline $\mathrm{Bi}-205$ & $2.07 \mathrm{E}+04$ & $1.78 \mathrm{E}-13$ \\
\hline $\mathrm{Bi}-206$ & $1.04 \mathrm{E}+04$ & $8.91 \mathrm{E}-14$ \\
\hline $\mathrm{Bi}-207$ & $2.19 \mathrm{E}+04$ & $1.87 \mathrm{E}-13$ \\
\hline $\mathrm{Bi}-208$ & $1.43 \mathrm{E}+04$ & $1.23 \mathrm{E}-13$ \\
\hline $\mathrm{Bi}-210$ & $9.03 \mathrm{E}+05$ & $7.74 \mathrm{E}-12$ \\
\hline $\mathrm{Bi}-210 \mathrm{~m}$ & $1.30 \mathrm{E}+05$ & $1.11 \mathrm{E}-12$ \\
\hline $\mathrm{Bi}-211$ & $7.13 \mathrm{E}+05$ & $6.11 \mathrm{E}-12$ \\
\hline $\mathrm{Bi}-212$ & $2.06 \mathrm{E}+05$ & $1.76 \mathrm{E}-12$ \\
\hline $\mathrm{Bi}-213$ & $1.93 \mathrm{E}+05$ & $1.66 \mathrm{E}-12$ \\
\hline
\end{tabular}




\begin{tabular}{|c|c|c|}
\hline Nuclide & $\begin{array}{c}\text { Ground } \\
\text { Shine DCS } \\
\left(\mathbf{B q} / \mathbf{m}^{2}\right)\end{array}$ & $\begin{array}{c}\text { Ground } \\
\text { Shine DCS } \\
\left(\mu \mathrm{Ci} / \mathbf{m}^{2}\right)\end{array}$ \\
\hline Bi-215 & $1.03 \mathrm{E}+05$ & $8.85 \mathrm{E}-13$ \\
\hline Bi-216 & $3.75 \mathrm{E}+04$ & $3.21 \mathrm{E}-13$ \\
\hline Po-203 & $2.09 \mathrm{E}+04$ & $1.79 \mathrm{E}-13$ \\
\hline Po-204 & $2.94 \mathrm{E}+04$ & $2.52 \mathrm{E}-13$ \\
\hline Po-205 & $2.16 \mathrm{E}+04$ & $1.85 \mathrm{E}-13$ \\
\hline Po-206 & $2.86 \mathrm{E}+04$ & $2.45 \mathrm{E}-13$ \\
\hline Рo-207 & $2.64 \mathrm{E}+04$ & $2.26 \mathrm{E}-13$ \\
\hline Po-208 & $1.59 \mathrm{E}+09$ & $1.36 \mathrm{E}-08$ \\
\hline Po-209 & $5.50 \mathrm{E}+06$ & $4.71 \mathrm{E}-11$ \\
\hline Po-210 & $3.44 \mathrm{E}+09$ & $2.95 \mathrm{E}-08$ \\
\hline Po-211 & $4.09 \mathrm{E}+06$ & $3.50 \mathrm{E}-11$ \\
\hline Po-212m & $4.69 \mathrm{E}+05$ & $4.02 \mathrm{E}-12$ \\
\hline Рo-213 & $8.88 \mathrm{E}+08$ & 7.61E-09 \\
\hline Рo-214 & $4.03 \mathrm{E}+08$ & $3.45 \mathrm{E}-09$ \\
\hline Po-215 & $1.89 \mathrm{E}+08$ & $1.62 \mathrm{E}-09$ \\
\hline Po-216 & $2.19 \mathrm{E}+09$ & $1.87 \mathrm{E}-08$ \\
\hline Po-218 & $4.77 \mathrm{E}+12$ & 4.09E-05 \\
\hline At-204 & $1.41 \mathrm{E}+04$ & $1.21 \mathrm{E}-13$ \\
\hline At-205 & $2.91 \mathrm{E}+04$ & $2.49 \mathrm{E}-13$ \\
\hline At-206 & $1.34 \mathrm{E}+04$ & $1.15 \mathrm{E}-13$ \\
\hline At-207 & $1.71 \mathrm{E}+04$ & $1.47 \mathrm{E}-13$ \\
\hline At-208 & $1.12 \mathrm{E}+04$ & $9.60 \mathrm{E}-14$ \\
\hline At-209 & $1.47 \mathrm{E}+04$ & $1.26 \mathrm{E}-13$ \\
\hline At-210 & $1.17 \mathrm{E}+04$ & $1.01 \mathrm{E}-13$ \\
\hline At-211 & $1.04 \mathrm{E}+06$ & $8.88 \mathrm{E}-12$ \\
\hline At-215 & $1.95 \mathrm{E}+08$ & $1.67 \mathrm{E}-09$ \\
\hline At-216 & $1.45 \mathrm{E}+07$ & $1.24 \mathrm{E}-10$ \\
\hline At-217 & $1.40 \mathrm{E}+08$ & $1.20 \mathrm{E}-09$ \\
\hline At-218 & $2.54 \mathrm{E}+08$ & $2.17 \mathrm{E}-09$ \\
\hline At-220 & $5.79 \mathrm{E}+04$ & $4.96 \mathrm{E}-13$ \\
\hline Rn-207 & $3.35 \mathrm{E}+04$ & $2.87 \mathrm{E}-13$ \\
\hline Rn-209 & $2.86 \mathrm{E}+04$ & $2.45 \mathrm{E}-13$ \\
\hline Rn-210 & $5.55 \mathrm{E}+05$ & $4.76 \mathrm{E}-12$ \\
\hline Rn-211 & $1.82 \mathrm{E}+04$ & $1.56 \mathrm{E}-13$ \\
\hline Rn-212 & $9.82 \mathrm{E}+07$ & $8.41 \mathrm{E}-10$ \\
\hline Rn-218 & $4.38 \mathrm{E}+07$ & $3.75 \mathrm{E}-10$ \\
\hline Rn-219 & $5.77 \mathrm{E}+05$ & $4.94 \mathrm{E}-12$ \\
\hline $\mathrm{Rn}-220$ & $5.28 \mathrm{E}+07$ & $4.52 \mathrm{E}-10$ \\
\hline
\end{tabular}




\begin{tabular}{|c|c|c|}
\hline Nuclide & $\begin{array}{c}\text { Ground } \\
\text { Shine DCS } \\
\left(\mathbf{B q} / \mathbf{m}^{2}\right)\end{array}$ & $\begin{array}{c}\text { Ground } \\
\text { Shine DCS } \\
\left(\mu \mathrm{Ci} / \mathbf{m}^{2}\right)\end{array}$ \\
\hline Rn-222 & $8.52 \mathrm{E}+07$ & $7.31 \mathrm{E}-10$ \\
\hline $\mathrm{Rn}-223$ & $8.34 \mathrm{E}+04$ & $7.15 \mathrm{E}-13$ \\
\hline Fr-212 & $3.02 \mathrm{E}+04$ & $2.59 \mathrm{E}-13$ \\
\hline Fr-219 & $9.41 \mathrm{E}+06$ & $8.06 \mathrm{E}-11$ \\
\hline Fr-220 & $3.75 \mathrm{E}+06$ & $3.22 \mathrm{E}-11$ \\
\hline Fr-221 & $1.18 \mathrm{E}+06$ & $1.01 \mathrm{E}-11$ \\
\hline Fr-222 & $1.34 \mathrm{E}+05$ & $1.15 \mathrm{E}-12$ \\
\hline Fr-223 & $4.10 \mathrm{E}+05$ & $3.52 \mathrm{E}-12$ \\
\hline Fr-224 & $5.28 \mathrm{E}+04$ & $4.53 \mathrm{E}-13$ \\
\hline Fr-227 & $6.30 \mathrm{E}+04$ & $5.40 \mathrm{E}-13$ \\
\hline Ra-219 & $1.99 \mathrm{E}+05$ & $1.71 \mathrm{E}-12$ \\
\hline Ra-220 & $7.11 \mathrm{E}+06$ & $6.09 \mathrm{E}-11$ \\
\hline Ra-221 & $9.70 \mathrm{E}+05$ & $8.31 \mathrm{E}-12$ \\
\hline Ra-222 & $3.67 \mathrm{E}+06$ & $3.14 \mathrm{E}-11$ \\
\hline Ra-223 & $2.50 \mathrm{E}+05$ & $2.14 \mathrm{E}-12$ \\
\hline Ra-224 & $3.30 \mathrm{E}+06$ & $2.83 \mathrm{E}-11$ \\
\hline Ra-225 & $2.88 \mathrm{E}+06$ & $2.47 \mathrm{E}-11$ \\
\hline Ra-226 & $4.75 \mathrm{E}+06$ & $4.07 \mathrm{E}-11$ \\
\hline Ra-227 & $1.88 \mathrm{E}+05$ & $1.61 \mathrm{E}-12$ \\
\hline Ra-228 & $4.32 \mathrm{E}+07$ & $3.70 \mathrm{E}-10$ \\
\hline Ra-230 & $4.21 \mathrm{E}+05$ & $3.60 \mathrm{E}-12$ \\
\hline Ac-223 & $1.96 \mathrm{E}+06$ & $1.68 \mathrm{E}-11$ \\
\hline Ac-224 & $1.55 \mathrm{E}+05$ & $1.33 \mathrm{E}-12$ \\
\hline Ac-225 & $2.40 \mathrm{E}+06$ & $2.06 \mathrm{E}-11$ \\
\hline Ac- -226 & $2.31 \mathrm{E}+05$ & $1.98 \mathrm{E}-12$ \\
\hline Ac-227 & $1.34 \mathrm{E}+08$ & $1.15 \mathrm{E}-09$ \\
\hline Ac-228 & $3.78 \mathrm{E}+04$ & $3.24 \mathrm{E}-13$ \\
\hline Ac- 230 & $5.37 \mathrm{E}+04$ & $4.60 \mathrm{E}-13$ \\
\hline Ac-231 & $7.08 \mathrm{E}+04$ & $6.07 \mathrm{E}-13$ \\
\hline Ac-232 & $2.78 \mathrm{E}+04$ & $2.38 \mathrm{E}-13$ \\
\hline Ac- 233 & $5.52 \mathrm{E}+04$ & $4.73 \mathrm{E}-13$ \\
\hline Th-223 & $4.99 \mathrm{E}+05$ & $4.27 \mathrm{E}-12$ \\
\hline Th-224 & $1.51 \mathrm{E}+06$ & $1.29 \mathrm{E}-11$ \\
\hline Th-226 & $4.40 \mathrm{E}+06$ & $3.77 \mathrm{E}-11$ \\
\hline Th-227 & $2.76 \mathrm{E}+05$ & $2.36 \mathrm{E}-12$ \\
\hline Th-228 & $1.47 \mathrm{E}+07$ & $1.26 \mathrm{E}-10$ \\
\hline Th-229 & $4.09 \mathrm{E}+05$ & $3.51 \mathrm{E}-12$ \\
\hline Th-230 & $4.95 \mathrm{E}+07$ & $4.24 \mathrm{E}-10$ \\
\hline
\end{tabular}




\begin{tabular}{|c|c|c|}
\hline Nuclide & $\begin{array}{c}\text { Ground } \\
\text { Shine DCS } \\
\mathbf{( B q}^{\mathbf{2}} \mathbf{\text { ) }}\end{array}$ & $\begin{array}{c}\text { Ground } \\
\text { Shine DCS } \\
\left(\boldsymbol{\mu} \mathbf{C} / \mathbf{m}^{\mathbf{2}} \mathbf{)}\right.\end{array}$ \\
\hline Th-231 & $2.09 \mathrm{E}+06$ & $1.79 \mathrm{E}-11$ \\
\hline Th-232 & $7.00 \mathrm{E}+07$ & $6.00 \mathrm{E}-10$ \\
\hline Th-233 & $4.54 \mathrm{E}+05$ & $3.89 \mathrm{E}-12$ \\
\hline Th-234 & $3.87 \mathrm{E}+06$ & $3.32 \mathrm{E}-11$ \\
\hline Th-235 & $2.44 \mathrm{E}+05$ & $2.09 \mathrm{E}-12$ \\
\hline $\mathrm{Th}-236$ & $5.32 \mathrm{E}+05$ & $4.56 \mathrm{E}-12$ \\
\hline $\mathrm{Pa}-227$ & $1.76 \mathrm{E}+06$ & $1.51 \mathrm{E}-11$ \\
\hline $\mathrm{Pa}-228$ & $2.52 \mathrm{E}+04$ & $2.16 \mathrm{E}-13$ \\
\hline $\mathrm{Pa}-229$ & $5.81 \mathrm{E}+05$ & $4.98 \mathrm{E}-12$ \\
\hline $\mathrm{Pa}-230$ & $5.10 \mathrm{E}+04$ & $4.37 \mathrm{E}-13$ \\
\hline $\mathrm{Pa}-231$ & $9.14 \mathrm{E}+05$ & $7.83 \mathrm{E}-12$ \\
\hline $\mathrm{Pa}-232$ & $3.60 \mathrm{E}+04$ & $3.09 \mathrm{E}-13$ \\
\hline $\mathrm{Pa}-233$ & $1.57 \mathrm{E}+05$ & $1.35 \mathrm{E}-12$ \\
\hline $\mathrm{Pa}-234$ & $2.30 \mathrm{E}+04$ & $1.97 \mathrm{E}-13$ \\
\hline $\mathrm{Pa}-234 \mathrm{~m}$ & $2.83 \mathrm{E}+05$ & $2.43 \mathrm{E}-12$ \\
\hline $\mathrm{Pa}-235$ & $6.28 \mathrm{E}+05$ & $5.38 \mathrm{E}-12$ \\
\hline $\mathrm{Pa}-236$ & $3.45 \mathrm{E}+04$ & $2.95 \mathrm{E}-13$ \\
\hline $\mathrm{Pa}-237$ & $4.95 \mathrm{E}+04$ & $4.25 \mathrm{E}-13$ \\
\hline $\mathrm{U}-227$ & $2.99 \mathrm{E}+05$ & $2.56 \mathrm{E}-12$ \\
\hline $\mathrm{U}-228$ & $8.11 \mathrm{E}+06$ & $6.95 \mathrm{E}-11$ \\
\hline $\mathrm{U}-230$ & $2.09 \mathrm{E}+07$ & $1.79 \mathrm{E}-10$ \\
\hline $\mathrm{U}-231$ & $4.75 \mathrm{E}+05$ & $4.07 \mathrm{E}-12$ \\
\hline $\mathrm{U}-232$ & $4.34 \mathrm{E}+07$ & $3.72 \mathrm{E}-10$ \\
\hline $\mathrm{U}-233$ & $6.66 \mathrm{E}+07$ & $5.71 \mathrm{E}-10$ \\
\hline $\mathrm{U}-234$ & $5.47 \mathrm{E}+07$ & $4.69 \mathrm{E}-10$ \\
\hline $\mathrm{U}-235$ & $2.13 \mathrm{E}+05$ & $1.82 \mathrm{E}-12$ \\
\hline $\mathrm{U}-236$ & $6.58 \mathrm{E}+07$ & $5.64 \mathrm{E}-10$ \\
\hline $\mathrm{U}-237$ & $2.58 \mathrm{E}+05$ & $2.21 \mathrm{E}-12$ \\
\hline $\mathrm{U}-238$ & $8.11 \mathrm{E}+07$ & $6.95 \mathrm{E}-10$ \\
\hline $\mathrm{U}-239$ & $3.89 \mathrm{E}+05$ & $3.33 \mathrm{E}-12$ \\
\hline $\mathrm{U}-240$ & $5.67 \mathrm{E}+06$ & $4.86 \mathrm{E}-11$ \\
\hline $\mathrm{U}-242$ & $4.40 \mathrm{E}+05$ & $3.77 \mathrm{E}-12$ \\
\hline $\mathrm{Np}-232$ & $2.83 \mathrm{E}+04$ & $2.43 \mathrm{E}-13$ \\
\hline $\mathrm{Np}-233$ & $4.11 \mathrm{E}+05$ & $3.52 \mathrm{E}-12$ \\
\hline $\mathrm{Np}-234$ & $3.17 \mathrm{E}+04$ & $2.72 \mathrm{E}-13$ \\
\hline $\mathrm{Np}-235$ & $1.47 \mathrm{E}+07$ & $1.26 \mathrm{E}-10$ \\
\hline $\mathrm{Np}-236$ & $2.48 \mathrm{E}+05$ & $2.12 \mathrm{E}-12$ \\
\hline $\mathrm{Np}-236 \mathrm{~m}$ & $7.34 \mathrm{E}+05$ & $6.29 \mathrm{E}-12$ \\
\hline
\end{tabular}




\begin{tabular}{|c|c|c|}
\hline Nuclide & $\begin{array}{c}\text { Ground } \\
\text { Shine DCS } \\
\mathbf{( B q}^{\mathbf{2}} \mathbf{\text { ) }}\end{array}$ & $\begin{array}{c}\text { Ground } \\
\text { Shine DCS } \\
\mathbf{( \mu C i}^{\mathbf{2}} \mathbf{\text { ) }}\end{array}$ \\
\hline $\mathrm{Np}-237$ & $1.30 \mathrm{E}+06$ & $1.11 \mathrm{E}-11$ \\
\hline $\mathrm{Np}-238$ & $5.63 \mathrm{E}+04$ & $4.83 \mathrm{E}-13$ \\
\hline $\mathrm{Np}-239$ & $1.96 \mathrm{E}+05$ & $1.68 \mathrm{E}-12$ \\
\hline $\mathrm{Np}-240$ & $3.17 \mathrm{E}+04$ & $2.72 \mathrm{E}-13$ \\
\hline $\mathrm{Np}-240 \mathrm{~m}$ & $8.46 \mathrm{E}+04$ & $7.25 \mathrm{E}-13$ \\
\hline $\mathrm{Np}-241$ & $4.36 \mathrm{E}+05$ & $3.73 \mathrm{E}-12$ \\
\hline $\mathrm{Np}-242$ & $9.22 \mathrm{E}+04$ & $7.90 \mathrm{E}-13$ \\
\hline $\mathrm{Np}-242 \mathrm{~m}$ & $3.45 \mathrm{E}+04$ & $2.96 \mathrm{E}-13$ \\
\hline $\mathrm{Pu}-232$ & $5.97 \mathrm{E}+05$ & $5.12 \mathrm{E}-12$ \\
\hline $\mathrm{Pu}-234$ & $5.50 \mathrm{E}+05$ & $4.71 \mathrm{E}-12$ \\
\hline $\mathrm{Pu}-235$ & $3.97 \mathrm{E}+05$ & $3.41 \mathrm{E}-12$ \\
\hline $\mathrm{Pu}-236$ & $4.74 \mathrm{E}+07$ & $4.06 \mathrm{E}-10$ \\
\hline $\mathrm{Pu}-237$ & $7.43 \mathrm{E}+05$ & $6.36 \mathrm{E}-12$ \\
\hline $\mathrm{Pu}-238$ & $5.29 \mathrm{E}+07$ & $4.54 \mathrm{E}-10$ \\
\hline $\mathrm{Pu}-239$ & $1.04 \mathrm{E}+08$ & $8.88 \mathrm{E}-10$ \\
\hline $\mathrm{Pu}-240$ & $5.58 \mathrm{E}+07$ & $4.78 \mathrm{E}-10$ \\
\hline $\mathrm{Pu}-241$ & $2.22 \mathrm{E}+10$ & $1.90 \mathrm{E}-07$ \\
\hline $\mathrm{Pu}-242$ & $5.70 \mathrm{E}+07$ & $4.89 \mathrm{E}-10$ \\
\hline $\mathrm{Pu}-243$ & $1.40 \mathrm{E}+06$ & $1.20 \mathrm{E}-11$ \\
\hline $\mathrm{Pu}-244$ & $1.65 \mathrm{E}+06$ & $1.42 \mathrm{E}-11$ \\
\hline $\mathrm{Pu}-245$ & $8.11 \mathrm{E}+04$ & $6.95 \mathrm{E}-13$ \\
\hline $\mathrm{Pu}-246$ & $2.54 \mathrm{E}+05$ & $2.17 \mathrm{E}-12$ \\
\hline $\mathrm{Am}-237$ & $9.38 \mathrm{E}+04$ & $8.04 \mathrm{E}-13$ \\
\hline $\mathrm{Am}-238$ & $3.80 \mathrm{E}+04$ & $3.26 \mathrm{E}-13$ \\
\hline $\mathrm{Am}-239$ & $1.52 \mathrm{E}+05$ & $1.30 \mathrm{E}-12$ \\
\hline $\mathrm{Am}-240$ & $3.30 \mathrm{E}+04$ & $2.83 \mathrm{E}-13$ \\
\hline $\mathrm{Am}-241$ & $1.45 \mathrm{E}+06$ & $1.25 \mathrm{E}-11$ \\
\hline $\mathrm{Am}-242$ & $1.97 \mathrm{E}+06$ & $1.69 \mathrm{E}-11$ \\
\hline $\mathrm{Am}-242 \mathrm{~m}$ & $1.53 \mathrm{E}+07$ & $1.31 \mathrm{E}-10$ \\
\hline $\mathrm{Am}-243$ & $6.39 \mathrm{E}+05$ & $5.48 \mathrm{E}-12$ \\
\hline $\mathrm{Am}-244$ & $4.20 \mathrm{E}+04$ & $3.60 \mathrm{E}-13$ \\
\hline $\mathrm{Am}-244 \mathrm{~m}$ & $4.52 \mathrm{E}+05$ & $3.88 \mathrm{E}-12$ \\
\hline $\mathrm{Am}-245$ & $7.73 \mathrm{E}+05$ & $6.63 \mathrm{E}-12$ \\
\hline $\mathrm{Am}-246$ & $4.36 \mathrm{E}+04$ & $3.73 \mathrm{E}-13$ \\
\hline $\mathrm{Am}-246 \mathrm{~m}$ & $3.31 \mathrm{E}+04$ & $2.83 \mathrm{E}-13$ \\
\hline $\mathrm{Cm}-238$ & $4.54 \mathrm{E}+05$ & $3.89 \mathrm{E}-12$ \\
\hline
\end{tabular}




\begin{tabular}{|c|c|c|}
\hline Nuclide & $\begin{array}{c}\text { Ground } \\
\text { Shine DCS } \\
\left(\mathbf{B q} / \mathbf{m}^{2}\right)\end{array}$ & $\begin{array}{c}\text { Ground } \\
\text { Shine DCS } \\
\left(\mu \mathrm{Ci} / \mathbf{m}^{2}\right)\end{array}$ \\
\hline $\mathrm{Cm}-240$ & $4.23 \mathrm{E}+07$ & $3.63 \mathrm{E}-10$ \\
\hline $\mathrm{Cm}-241$ & $6.83 \mathrm{E}+04$ & $5.86 \mathrm{E}-13$ \\
\hline $\mathrm{Cm}-242$ & $4.75 \mathrm{E}+07$ & $4.07 \mathrm{E}-10$ \\
\hline $\mathrm{Cm}-243$ & $2.69 \mathrm{E}+05$ & $2.30 \mathrm{E}-12$ \\
\hline $\mathrm{Cm}-244$ & $5.42 \mathrm{E}+07$ & $4.65 \mathrm{E}-10$ \\
\hline $\mathrm{Cm}-245$ & $3.48 \mathrm{E}+05$ & $2.98 \mathrm{E}-12$ \\
\hline $\mathrm{Cm}-246$ & $8.19 \mathrm{E}+06$ & $7.02 \mathrm{E}-11$ \\
\hline $\mathrm{Cm}-247$ & $1.06 \mathrm{E}+05$ & $9.12 \mathrm{E}-13$ \\
\hline $\mathrm{Cm}-248$ & $2.54 \mathrm{E}+04$ & $2.17 \mathrm{E}-13$ \\
\hline Cm-249 & $9.61 \mathrm{E}+05$ & $8.24 \mathrm{E}-12$ \\
\hline $\mathrm{Cm}-250$ & $2.50 \mathrm{E}+03$ & $2.14 \mathrm{E}-14$ \\
\hline $\mathrm{Cm}-251$ & $2.16 \mathrm{E}+05$ & $1.85 \mathrm{E}-12$ \\
\hline $\mathrm{Bk}-245$ & $1.54 \mathrm{E}+05$ & $1.32 \mathrm{E}-12$ \\
\hline Bk-246 & $4.00 \mathrm{E}+04$ & $3.43 \mathrm{E}-13$ \\
\hline $\mathrm{Bk}-247$ & $2.40 \mathrm{E}+05$ & $2.06 \mathrm{E}-12$ \\
\hline $\mathrm{Bk}-248 \mathrm{~m}$ & $5.51 \mathrm{E}+05$ & $4.73 \mathrm{E}-12$ \\
\hline Bk-249 & $5.59 \mathrm{E}+09$ & 4.79E-08 \\
\hline Bk-250 & $3.72 \mathrm{E}+04$ & $3.19 \mathrm{E}-13$ \\
\hline Bk-251 & $3.38 \mathrm{E}+05$ & $2.89 \mathrm{E}-12$ \\
\hline Cf-244 & $4.16 \mathrm{E}+07$ & $3.57 \mathrm{E}-10$ \\
\hline Cf-246 & $5.61 \mathrm{E}+07$ & $4.81 \mathrm{E}-10$ \\
\hline Cf-247 & $3.75 \mathrm{E}+05$ & $3.22 \mathrm{E}-12$ \\
\hline Cf-248 & $3.34 \mathrm{E}+07$ & $2.86 \mathrm{E}-10$ \\
\hline Cf-249 & $1.03 \mathrm{E}+05$ & $8.82 \mathrm{E}-13$ \\
\hline Cf-250 & $3.25 \mathrm{E}+06$ & $2.79 \mathrm{E}-11$ \\
\hline Cf-251 & $2.96 \mathrm{E}+05$ & $2.54 \mathrm{E}-12$ \\
\hline Cf-252 & $7.34 \mathrm{E}+04$ & $6.29 \mathrm{E}-13$ \\
\hline Cf-253 & $1.50 \mathrm{E}+07$ & $1.28 \mathrm{E}-10$ \\
\hline Cf-254 & $1.98 \mathrm{E}+03$ & $1.70 \mathrm{E}-14$ \\
\hline Cf-255 & $5.49 \mathrm{E}+06$ & $4.70 \mathrm{E}-11$ \\
\hline Es-249 & $8.34 \mathrm{E}+04$ & $7.15 \mathrm{E}-13$ \\
\hline Es-250 & $2.83 \mathrm{E}+04$ & $2.43 \mathrm{E}-13$ \\
\hline Es-250m & $6.24 \mathrm{E}+04$ & $5.35 \mathrm{E}-13$ \\
\hline Es-251 & $3.82 \mathrm{E}+05$ & $3.27 \mathrm{E}-12$ \\
\hline Es-253 & $6.34 \mathrm{E}+07$ & $5.44 \mathrm{E}-10$ \\
\hline Es-254 & $3.58 \mathrm{E}+06$ & $3.07 \mathrm{E}-11$ \\
\hline Es-254m & $6.91 \mathrm{E}+04$ & $5.92 \mathrm{E}-13$ \\
\hline Es-255 & $4.64 \mathrm{E}+07$ & $3.98 \mathrm{E}-10$ \\
\hline
\end{tabular}


SRNL-STI-2013-00115

\begin{tabular}{|c|c|c|}
\hline Nuclide & $\begin{array}{c}\text { Ground } \\
\text { Shine DCS } \\
\left(\mathbf{B q} / \mathbf{m}^{2}\right)\end{array}$ & $\begin{array}{c}\text { Ground } \\
\text { Shine DCS } \\
\left(\boldsymbol{\mu} \mathbf{C i} / \mathbf{m}^{\mathbf{2}}\right)\end{array}$ \\
\hline Es-256 & $4.76 \mathrm{E}+05$ & $4.08 \mathrm{E}-12$ \\
\hline Fm-251 & $2.26 \mathrm{E}+05$ & $1.94 \mathrm{E}-12$ \\
\hline Fm-252 & $3.53 \mathrm{E}+07$ & $3.03 \mathrm{E}-10$ \\
\hline Fm-253 & $5.65 \mathrm{E}+05$ & $4.84 \mathrm{E}-12$ \\
\hline Fm-254 & $4.37 \mathrm{E}+06$ & $3.75 \mathrm{E}-11$ \\
\hline Fm-255 & $4.43 \mathrm{E}+06$ & $3.80 \mathrm{E}-11$ \\
\hline Fm-256 & $2.71 \mathrm{E}+03$ & $2.32 \mathrm{E}-14$ \\
\hline Fm-257 & $2.40 \mathrm{E}+05$ & $2.06 \mathrm{E}-12$ \\
\hline
\end{tabular}


cc: J.J. Mayer

A.M. Murray

E.B. Farfan

G.R. Whitney

B.T. Butcher

K.H. Rosenberger

A.J. Meyer

K.L. Dixon 